\title{
sustainability
}

Enhancement of

Public Real-Estate

Assets and

CulturalHeritage

Management Plans and Models,

Innovative Practices and Tools

in Supporting the Local

Sustainable Development

Edited by

Lucia Della Spina and Francesco Calabrò

Printed Edition of the Special Issue Published in Sustainability 


\section{Enhancement of Public Real-Estate Assets and Cultural Heritage}





\section{Enhancement of Public Real-Estate Assets and Cultural Heritage}

Management Plans and Models, Innovative Practices and Tools in Supporting the Local Sustainable Development

Special Issue Editors

Lucia Della Spina

Francesco Calabrò 
Lucia Della Spina

Mediterranea University of Reggio Calabria

Italy
Francesco Calabrò

Mediterranea University of Reggio Calabria

Italy

\section{Editorial Office}

MDPI

St. Alban-Anlage 66

4052 Basel, Switzerland

This is a reprint of articles from the Special Issue published online in the open access journal Sustainability (ISSN 2071-1050) (available at: https://www.mdpi.com/journal/sustainability/ special_issues/pra).

For citation purposes, cite each article independently as indicated on the article page online and as indicated below:

LastName, A.A.; LastName, B.B.; LastName, C.C. Article Title. Journal Name Year, Article Number, Page Range.

ISBN 978-3-03936-304-9 (Hbk)

ISBN 978-3-03936-305-6 (PDF)

(C) 2020 by the authors. Articles in this book are Open Access and distributed under the Creative Commons Attribution (CC BY) license, which allows users to download, copy and build upon published articles, as long as the author and publisher are properly credited, which ensures maximum dissemination and a wider impact of our publications.

The book as a whole is distributed by MDPI under the terms and conditions of the Creative Commons license CC BY-NC-ND. 


\section{Contents}

About the Special Issue Editors $\ldots \ldots \ldots \ldots \ldots \ldots \ldots \ldots \ldots \ldots$

Preface to "Enhancement of Public Real-estate Assets and Cultural Heritage" . . . . . . . . xi

Francesco Calabrò and Lucia Della Spina

The Public-Private Partnership for the Enhancement of Unused Public Buildings:

An Experimental Model of Economic Feasibility Project

Reprinted from: Sustainability 2019, 11, 5662, doi:10.3390/su11205662 . . . . . . . . . . . 1

\section{Lucia Della Spina}

Adaptive Sustainable Reuse for Cultural Heritage: A Multiple Criteria Decision Aiding Approach Supporting Urban Development Processes

Reprinted from: Sustainability 2020, 12, 1363, doi:10.3390/su12041363 . . . . . . . . . . . . . 27

Ma del Pilar Muñoz Dueñas, Antonio Vaamonde Liste and Maria do Rosário Cabrita

The Survival of Cultural Firms: A Study of Multiple Accounting Parameters in Spain

Reprinted from: Sustainability 2020, 12, 1159, doi:10.3390/su12031159 . . . . . . . . . . . . 47

Luisa Sturiale, Alessandro Scuderi, Giuseppe Timpanaro and Benedetto Matarazzo

Sustainable Use and Conservation of the Environmental Resources of the Etna Park (UNESCO

Heritage): Evaluation Model Supporting Sustainable Local Development Strategies

Reprinted from: Sustainability 2020, 12, 1453, doi:10.3390/su12041453 . . . . . . . . . . . . . 61

Antonio Nesticò and Piera Somma

Comparative Analysis of Multi-Criteria Methods for the Enhancement of Historical Buildings

Reprinted from: Sustainability 2019, 11, 4526, doi:10.3390/su11174526 . . . . . . . . . . . 77

Sebastiano Carbonara and Davide Stefano

An Operational Protocol for the Valorisation of Public Real Estate Assets in Italy

Reprinted from: Sustainability 2020, 12, 732, doi:10.3390/su12020732 . . . . . . . . . . . . . 97

Esad Muminović, Uroš Radosavljević and Džemila Beganović

Strategic Planning and Management Model for the Regeneration of Historic Urban Landscapes:

The Case of Historic Center of Novi Pazar in Serbia

Reprinted from: Sustainability 2020, 12, 1323, doi:10.3390/su12041323 . . . . . . . . . . . . . 113

Paola Pellegrini and Ezio Micelli

Paradoxes of the Italian Historic Centres between Underutilisation and Planning Policies for Sustainability

Reprinted from: Sustainability 2019, 11, 2614, doi:10.3390/su11092614 . . . . . . . . . . . . . 147

Fabiana Forte, Vincenzo Del Giudice, Pierfrancesco De Paola and Federica Troisi

Valuation of the Vocationality of Cultural Heritage: The Vesuvian Villas

Reprinted from: Sustainability 2020, 12, 943, doi:10.3390/su12030943 . . . . . . . . . . . . . . 163

Maria Rosaria Guarini, Pierluigi Morano and Francesco Sica

Historical School Buildings. A Multi-Criteria Approach for Urban Sustainable Projects

Reprinted from: Sustainability 2020, 12, 1076, doi:10.3390/su12031076 . . . . . . . . . . . . . 183 
María José Piñeira Mantiñán, Francisco R. Durán Villa and Ramón López Rodríguez

Citizen Action as a Driving Force of Change. The Meninas of Canido, Art in the Street as an Urban Dynamizer

Reprinted from: Sustainability 2020, 12,740, doi:10.3390/su12020740 . . . . . . . . . . . . . . 207

\section{Chiara D'Alpaos and Maria Rosa Valluzzi}

Protection of Cultural Heritage Buildings and Artistic Assets from Seismic Hazard:

A Hierarchical Approach

Reprinted from: Sustainability 2020, 12, 1608, doi:10.3390/su12041608 . . . . . . . . . . . . . . . . 229

Stefania De Medici, Pasquale De Toro and Francesca Nocca

Cultural Heritage and Sustainable Development: Impact Assessment of Two Adaptive Reuse

Projects in Siracusa, Sicily

Reprinted from: Sustainability 2020, 12,311, doi:10.3390/su12010311 . . . . . . . . . . . . . . . . . 243

Giuliano Marella and Valentina Antoniucci

Time Overrun in Public Works-Evidence from North-East Italy

Reprinted from: Sustainability 2019, 11, 7057, doi:10.3390/su11247057 . . . . . . . . . . . . . . . 267

Francesca Torrieri, Marina Fumo, Michele Sarnataro and Gigliola Ausiello

An Integrated Decision Support System for the Sustainable Reuse of the Former Monastery of

"Ritiro del Carmine" in Campania Region

Reprinted from: Sustainability 2019, 11,5244, doi:10.3390/su11195244 . . . . . . . . . . . . . . . . 285

\section{María Griñán Montealegre}

Solutions for the Sustainable Management of a Cultural Landscape in Danger:

Mar Menor, Spain

Reprinted from: Sustainability 2020, 12,335, doi:10.3390/su12010335 . . . . . . . . . . . . . . . . . 307

\section{Marta Bottero, Chiara D'Alpaos and Alessia Marello}

An Application of the A'WOT Analysis for the Management of Cultural Heritage Assets:

The Case of the Historical Farmhouses in the Aglié Castle (Turin)

Reprinted from: Sustainability 2020, 12, 1071, doi:10.3390/su12031071 . . . . . . . . . . . . . . . 323

Giovanna Acampa, Mariolina Grasso, Giorgia Marino and Claudia Mariaserena Parisi

Tourist Flow Management: Social Impact Evaluation through Social Network Analysis

Reprinted from: Sustainability 2020, 12,731, doi:10.3390/su12020731 . . . . . . . . . . . . . . . . . 341

\section{Maria Rosa Valluzzi, Sergio Calò and Gianluca Giacometti}

Correlation of Vulnerability and Damage between Artistic Assets and Structural Elements: The DataBAES Archive for the Conservation Planning of $\mathrm{CH}$ Masonry Buildings in Seismic Areas Reprinted from: Sustainability 2020, 12,653, doi:10.3390/su12020653 . . . . . . . . . . . . . . . . . 357

\section{Luc Beal, Hugues Séraphin, Giuseppe Modica, Manuela Pilato and Marco Platania}

Analysing the Mediating Effect of Heritage Between Locals and Visitors: An Exploratory Study Using Mission Patrimoine as a Case Study

Reprinted from: Sustainability 2019, 11,3015, doi:10.3390/su11113015 . . . . . . . . . . . . . . . . 377

Stefano Capolongo, Leopoldo Sdino, Marta Dell'Ovo, Rossella Moioli and

Stefano Della Torre

How to Assess Urban Regeneration Proposals by Considering Conflicting Values

Reprinted from: Sustainability 2019, 11,3877, doi:10.3390/su11143877 . . . . . . . . . . . . . . . . 393 
Luigi Petti, Claudia Trillo and Busisiwe Ncube Makore

Cultural Heritage and Sustainable Development Targets: A Possible Harmonisation? Insights from the European Perspective

Reprinted from: Sustainability 2020, 12, 926, doi:10.3390/su12030926 . . . . . . . . . . . . . . 409

Carmelina Bevilacqua, Yapeng Ou, Pasquale Pizzimenti and Guglielmo Minervino

New Public Institutional Forms and Social Innovation in Urban Governance: Insights from the

"Mayor's Office of New Urban Mechanics" (MONUM) in Boston

Reprinted from: Sustainability 2020, 12, 23, doi:10.3390/su12010023 . . . . . . . . . . . . . . 433

JoséÁlvarez-García, Claudia Patricia Maldonado-Erazo, María de la Cruz Del Río-Rama and Francisco Javier Castellano-Álvarez

Cultural Heritage and Tourism Basis for Regional Development: Mapping of

Scientific Coverage

Reprinted from: Sustainability 2019, 11, 6034, doi:10.3390/su11216034 . . . . . . . . . . . . . 457

Salvatore Giuffrida, Filippo Gagliano, Enrico Giannitrapani, Carmelo Marisca, Grazia Napoli and Maria Rosa Trovato

Promoting Research and Landscape Experience in the Management of the Archaeological

Networks. A Project-Valuation Experiment in Italy

Reprinted from: Sustainability 2020, 12, 4022, doi:10.3390/su12104022 . . . . . . . . . . . . . . 479

Fabiana Forte, Vincenzo Del Giudice, Pierfrancesco De Paola and Federica Troisi

Erratum: Forte, F., et al. Valuation of the Vocationality of Cultural Heritage: The Vesuvian Villas. Sustainability 2020, 12, 943

Reprinted from: Sustainability 2020, 12, 2069, doi:10.3390/su12052069 . . . . . . . . . . . . . . . 515 



\section{About the Special Issue Editors}

Lucia Della Spina, Ph.D., Aggregate Professor at the University of Reggio Calabria (Italy) since 2002 (SSD ICAR/22). She graduated in Architecture (University of Reggio Calabria, 1989) and obtained her Ph.D. in Assessment Methods in Urban Planning and Architectural Design (University of Reggio Calabria, 1994). In 1995, she received a scholarship from the Institute for Housing and Social Infrastructure (IRIS), Bari (Italy). From 1999 to 2003, she was a postdoctoral researcher with the research program on "Evaluation Processes in Complex Urban Programs" with the Department of Architecture of Mediterranea University of Reggio Calabria. She conducts management tasks for the University of Reggio Calabria (Member of the Board of Directors of the spin-off URBAN LAB S.r.l. as of 2020; delegate for the orientation activities as of 2019; member of the Administrative Board from 2016 to 2018). Since 2019, she has been a member of the committee of: the Ph.D. program in Architettura; Urban Regeneration and Economic Development from 2013 to 2018; and Conservation of Architectural and Environmental Heritage from 2008 to 2012. Since 2011, she has been scientific co-director of LaborEst-Economic Evaluations and Real Estate Appraisals Lab (http://www.laborest.unirc.it/) and co-Editor-in-Chief of LaborEst, an open access scientific journal by Mediterranea University of Reggio Calabria (Italy) http://pkp.unirc.it/ojs/index.php/LaborEst. She has been on the scientific organizing committee of the International Symposium "New Metropolitan Perspectives" since 2014, biennial symposium (4th edition) http:/ / www.nmp.unirc.it/. Since 2018, she has been a member of the Scientific Committee for the II level University Master in Strategic Assessment and Planning of the City and Territory at University of Reggio Calabria. In 2013, she was Visiting Professor at the School of Public Affairs San Diego University (U.S.); in 2016, at the Universidade Trás-os-Montes Alto Douro (UTAD) (Portugal); and in 2017 at the University of Salford, Manchester (U.K.). Since 2020, she has been an Editorial Scientific Committee Member for Valori e Valutazioni (Section Board for Cultural Heritage and Intangibles Valuation) a peer-reviewed and open access journal of the Italian Real Estate Appraisal and Investment Decision Society (SIEV). Since 2019, she has been an Editorial Board Member for Sustainability (Section Board for Economic, Business and Management Aspects of Sustainability) and Land, an open access journal by MDPI. Since 2007, she has been an ordinary member of the Italian Society of Valuation and Assessment (SIEV). Since 1990, she has conducted scientific research activities with the Architecture Department of the University of Reggio Calabria, mainly in the following areas: economic evaluation of the feasibility and sustainability of public and private programs and projects, economic evaluation of environmental and cultural heritage, complex decision-making processes and integrated evaluations, governance of local development processes, and plans and models for managing cultural and environmental assets. She is responsible for research projects for public and private bodies and is the author of numerous publications registered in the SCOPUS and WoS databases*.

*SCOPUS Author ID: 55988148100

*WOS ResearcherID: K-6682-2015

*ORCID: 0000-0001-8754-3523

*GOOGLE scholar: https://scholar.google.com/citations?

hl=it\&user=FxndRHUAAAAJ\&view_op=list_works\&sortby=pubdate 
Francesco Calabrò, Ph.D., Researcher and Aggregate Professor at the University of Reggio Calabria (Italy) since 2007 (SSD ICAR/22). He teaches Real Estate Appraisal and Economic Assessment of Plans and Projects (ICAR SSD 22) in the degree courses in Architecture and Engineering at the Mediterranea University of Reggio Calabria. He graduated in Architecture in 1995 and, in 2003, he was awarded a Ph.D. in Conservation of Architectural and Environmental Heritage (estimate curriculum) - XIII cycle. Since 1996, he has conducted scientific research as an Adjunct Professor and Research Fellow with the Department of Urban and Architectural Heritage through a structured process, but consistent with the contents of the specification ICAR/22. He is the head of 2nd level University Post Graduate Programme in Strategic Valuation and Planning of City and Territory. Since 2011, he has been a scientific co-director of LaborEst-Economic Evaluations and Real Estate Appraisals Lab (http://www.laborest.unirc.it/) and co-Editor-in-Chief of LaborEst, an open access scientific journal by Mediterranea University of Reggio Calabria (Italy) (http://pkp.unirc.it/ojs/index.php/LaborEst)/ He has been a Scientific Coordinator, along with other colleagues, of the International Symposium "New Metropolitan Perspectives" since 2014, biennial symposium (4 edition) http://www.nmp.unirc.it/. In 2013, he was a Visiting Scholar at the School of Public Affairs San Diego University (U.S.); in 2016, at Universidade Trás-os-Montes Alto Douro (UTAD) (Portugal); and in 2017, at the University of Salford, Manchester (U.K.). Since 2019, he has been a member of the committee of Ph.D. program in Architettura. His research activities of local and national interest, in partnership with both public and private entities, are mainly aimed at economic evaluation of the feasibility and sustainability of complex programs of urban transformation and for the enhancement of historic centers, public and private investments in real estate and infrastructure, and plans and models for managing cultural and environmental assets. This research also includes estimating the real estate market, building production, and the quality in the building process. He is an ordinary member of the SIEV-Italian Society of Valuation and Assessment, a Member of the Board of ICOMOS Italia, and a Member of ICOMOS Italia Scientific Committees on Conservation Economics and Intangible Heritage. He is the President of the Urban Lab university spin-off. He has joined national and international scientific conferences and has several publications registered in SCOPUS, WoS, and Google Scholar 


\section{Preface to "Enhancement of Public Real-estate Assets and Cultural Heritage"}

We live in an era in which the demand for public goods is increasing with progressively ongoing physical and functional deterioration of existing goods, whether they are of absolute intrinsic value or provide secure social service (mobility infrastructure, social, commercial, leisure and culture equipment, etc.). In this scenario, future territorial redevelopment projects can be based on an open system of social attractors, composed of appropriately selected and enhanced public utility assets to promote their knowledge and use. Research has received incentives and resources to advance these projects.

Identifying effective models for the recovery, financing, and shared management of heritage intended as a common good, which is often unused, is essential for transforming what is now recognized as a cost for society into productive investment, not only from economic but also social, cultural, and environmental points of view, from a circular perspective. We foresee cultural heritage as a potential element of a territorial system that continually redefines itself, and sustains and reproduces from within. The re-use of cultural heritage can be considered an essential contribution in the realization of a model of economy and city/circular territory, capable of preventing the waste of environmental (soils, materials, energy, etc.) and cultural resources (tangible and intangible heritage, knowledge, etc.), and capable of transforming waste into new economic, environmental, cultural, and social resources.

The nature of public goods considered by the enhancement projects and the strong articulation that characterizes the redevelopment projects of a territory requires complex approaches. For public goods, enhancement projects involve determining the profile of greater social preference with respect to alternative options, to balance heterogeneous and often antagonistic factors. For this purpose, choices derived from the analysis of trade-offs of the type equity/efficiency, conservation/transformation, environment/economy, use/protection, public administration/civil society, public sector/private sector, and individual interest/collective interest are decisive, as are the decisions arising from new problems such as the participation of the community in decision-making processes and the prefiguration of shared project scenarios; the definition of compatible horizons with respect to short-, medium-, and long-term choices; or the systemization of various public goods and the interrelation of this system with the needs and supply in its territory.

The complexity of the projects in the city, territory, or environment in question requires technical, scientific, and humanistic knowledge and skills that are capable of recomposing cognitive areas has progressively been separated and turned away by the hyperspecialism of recent years, producing often distorted and harmful effects.

Today, the different areas of planning are called upon to overcome their self-reference and to seek interrelationships with the many types of knowledge involved to achieve a more advanced cognitive balance that responds to the needs of the contemporary world. Full awareness of having to operate in a cognitive quadrant is required based on conditions of uncertainty, risk, indeterminacy, instability, discontinuity, and a multiplicity of knowledge.

Studies have shown that the scenario in which projects are implemented for the enhancement of public goods is problematic. The difficulties inherent in the prevision of the effects and impacts of the projects imply numerous conditions of risk and uncertainty.

In this context, assessment can be used to determine the resolutive value of the existing problems 
as its cognitive system can help build or select implementation strategies of greater efficiency, equity, securit,y and social preferability.

The concept of evaluation must be widely applied, including when and where to apply it to the areas to be investigated, the points of view to be considered, the type of judgment to be made, and the methods and references to be followed to express decisions and establish the extent of the phenomena considered.

The first consideration is that the project implementation strategy should preidenitfy the contribution of the evaluation, not as a set of separate, occasional, and inorganic moments, but as a structured, logical, and interactive process applied to all phases of the implementation process, to all decision-making nodes, and to all alternative hypotheses. All decisions made through the evaluation process must maintain consistency.

The second consideration is that the choices connected with outlining the feasible scenarios of complex projects, while continuing to use one-dimensional assessments-traditional and otherwise - should lead to a multidimensional assessment.

The third consideration is that the implementation strategy of the project should include evaluation approaches capable of addressing the problems of distributive justice.

The projects undertaken for the enhancement of public goods involve a wide range of interests, different and often conflicting, which must be considered if the basic objective is to achieve the most balanced design solution. Therefore, the configuration of the project scenarios should arise from collective visions and concerted actions, for which social participation and the active role of civil society are becoming increasingly decisive.

This situation urges the use of evaluations conducted with consideration of several points of view, expressed by the project stakeholders, including public, private, and civil society, all this with the aims of having knowledge of the effects and impacts produced by the project and of providing, if necessary, their redistribution with shared criteria.

Starting from these reflections, the contributions presented in this book outline rich and varied experiences and innovative tools on the enhancement of public and cultural real estate assets in support of a model of sustainable development and capable of promoting re-use of cultural heritage in European cities and cultural landscapes with circular economy logic as a model of sustainable development. From this point of view, cultural capital is the driver of the regeneration process on local, urban, or metropolitan scales, in which the transversal interconnections between the production cycles of the adaptive re-use of the available heritage, both in the adaptation and in the management phase, configure a circular process of multidimensional production of value.

Lucia Della Spina, Francesco Calabrò Special Issue Editors 




\title{
The Public-Private Partnership for the Enhancement of Unused Public Buildings: An Experimental Model of Economic Feasibility Project
}

\author{
Francesco Calabrò and Lucia Della Spina * \\ PAU Department, Mediterranea University of Reggio Calabria, 89124 Reggio Calabria, Italy; \\ francesco.calabro@unirc.it \\ * Correspondence: lucia.dellaspina@unirc.it
}

Received: 10 September 2019; Accepted: 7 October 2019; Published: 14 October 2019

\begin{abstract}
This article is part of the debate on the economic evaluation of urban regeneration projects to be implemented through partnership forms between public and private subjects. It illustrates the results of the research activity carried out by the authors, aimed at developing innovative tools to verify the economic feasibility and the sustainability of projects for the reuse of unused public buildings. Particularly, the study made it possible to develop an experimental model of economic feasibility project to be used in the. The model aims at verifying if the economic conditions are satisfied, and which ones, if any, are appealing for the private involvement within the realization and/or management of collective utility interventions. Significant points of the model are: (1) The inclusion of real estate re-use projects in the wider context of urban and territorial regeneration; (2) the adoption of criteria to assess costs and revenues remarkably eligible, in the authors' opinion, to understand the effective economic feasibility and/or sustainability of reuse projects, even under the framework of reliable techniques as the 'Cash Flow Analysis' and the 'Discounted Cash Flow Analysis'.
\end{abstract}

Keywords: economic feasibility; economic sustainability; project feasibility investment profitability; unused public buildings

\section{Introduction}

Nowadays, the inadequacy of public resources availability for the exploitation of unused public buildings, similarly to what happens to other sectors of the Public Administration, leads to seek for innovative solutions that allow to pursue the economic improvement of those buildings [1]: Following that purpose, some public-private partnerships might be considered useful relevant to those who work in the field of services of architecture and engineering.

Such partnerships, the need to guarantee the economic-financial balance in the processes of valorization of real estate becomes fundamental.

At the moment, the prevailing approach for such questions draws the methodological basis from disciplines dealing with the economic dynamics of companies. This approach allows us to evaluate the expected revenues properly, but it shows some weaknesses in terms of cost estimation, as it will be deepened ahead in the article.

In the last decades, the estimation culture in Italy has contributed significantly to develop methodologies and techniques economic evaluation of the projects, starting from its classical scientific base and enriching it with contributions coming from other related disciplines. It is to this relatively recent disciplinary evolution that this work intends to connect.

This contribution objective is to provide an easy-to-use tool in order to verify the feasibility and the economic sustainability of hypotheses of re-use of buildings unused public, in case it is intended to involve private subjects for the realization and/or management of the interventions. The model 
explained below can also be used in the case of important architectural heritage, but it is not intended for residential target or for regeneration initiatives entirely carried out by public bodies [2].

It is to be hoped that this approach can be of help to the decision-making process, from the start-up phase of the planning activity, matching with the technical and the economic feasibility project, under the Italian law.

Part First-Public-Private Partnerships for the Exploitation of Unused Public Buildings

\section{The Cooperation between Public and Private Subjects}

\subsection{The Technical and Economic Feasibility Project}

The technical and economic feasibility project as a level of planning was introduced by the Code of public contracts (otherwise known as "Procurement Code"), as regulated by the Legislative Decree 18 April 2016 n. 50 e s.m.i. [3]. It replaces two design levels previously provided for by the legislation, the feasibility study and the preliminary project. Similar tools for verifying the feasibility of projects are also present in the legislation of many OECD countries, such as Canada, Germany and France.

The Article 23-Levels of the project for the procurements-establishes that planning for public works is organized, according to three levels of subsequent technical in-depth analysis: the technical and economic feasibility of the project, the final project, and the executive project.

The technical and economic feasibility project, according to the provisions of paragraph 5 of the art. 23, identifies, among several solutions, the one that presents the best balance between costs and benefits for the community, in relation to the specific needs to be met and the services to be provided.

\subsection{The Public-Private Partnership}

The cooperation between public and private subjects to realize works and provide public utility services is an old matter. Even at the time of the ancient Roman Republic, it was a common practice to grant private citizens for the provision of public services and, often, for the achievement of works useful to the purpose of the allowance itself [4].

The current legislation on public works, in force as of March 2018, refers to Legislative Decree 18 April 2016 n. 50 "Codice dei contratti di appalto e di concessione delle amministrazioni aggiudicatrici e degli enti aggiudicatori aventi ad oggetto l'acquisizione di servizi, forniture, lavori e opere, nonchè i concorsi pubblici di progettazione", as amended by D. Lgs. 19 April 2017, n. 56.

Article n. 180 of the Code above mentioned regulates, the public-private partnership contract, as defined in art. n. 3: The agreement for pecuniary interest, stipulated in writing, with which one or more contracting stations, grant one or more economic operators, for a fixed period, depending on the duration of the amortization of the investment or the financing methods established, a complex of activities consisting in the realization, transformation, maintenance and operational management of a work, in exchange for its availability, or its economic exploitation, or the provision of a service that is connected to the use of the work itself, with risk-taking according to identified methods in the contract, by the operator.

According to the Department for Regional Affairs of the Presidency of the Council of Ministers, the enhancement of public real estate assets can represent an important opportunity to trigger urban regeneration processes and to promote local development: It must be approached as a logical consequence of the primary declination of an idea of city and territory, that must be well defined in the purposes of public decision makers. In this context, some arguments are hinged that describe administrative revolution, implemented in Italy with the entry into force of law no. 56 of 7 April 2014 (Delrio law) "Provisions on metropolitan cities, on Provinces, on unions and mergers of Municipalities". It means to consider city and territory, and the enhancement of public real estate assets as a conceptual "unicum". A coherent idea of the city will also be based on careful development of the public real estate and vice versa a good action of development is certainly part of a coherent idea of a city [5]. 


\subsection{A Classification of Subjects' Nature, Activities and Costs}

As previously highlighted, Public Administration searches forms of PPPs with increasing frequency, to satisfy the needs of the local communities, while respecting the growing budget constraints.

The most important distinction, when considering involving private entities into partnership agreements with the Public Administration, is among the characteristics of the expected activities, not among the subjects (Table 1): There are, in fact, numerous cases of the subject with not-for-profit nature, but that carry out economic activities. In such cases, the cost structure between a not-for-profit or profit subject does not change, but the tax regime to which they are subordinate; in addition, of course, the prohibition for not-for-profit organizations to distribute profits [6-8].

Table 1. Nature of entities and of activities.

\begin{tabular}{cc}
\hline Nature of Activities & Nature of Managing Subject \\
\hline Public services & Public \\
$\begin{array}{c}\text { Activities of public interest without economic relevance } \\
\text { Activities of public interest with economic revenues, such to } \\
\text { be considered economic activities }\end{array}$ & $\begin{array}{c}\text { Private not-for-profit } \\
\text { Private not-for-profit } \\
\text { Private for-profit }\end{array}$ \\
\hline
\end{tabular}

Each type of subject identified has different characteristics. In the case of public bodies, it is well known the firmness along with the management phase, due to procedural constraints imposed by the current legislation, which is often associated with the difficulties to acquire the necessary skills. The private for-profit entities normally are characterized by greater operational efficiency, which, however, must match an adequate return on investment, with the risk to overshadow the community's interests, which should be prominent in the case of public interest services management; furthermore, these subjects have a cost structure characterized by high fixed expenses for human resources. The not-for-profit organizations arise from determined values shared by the members: Usually, these subjects carry out volunteer activities without economic relevance, but they also have the opportunity to manage economic activities linked to their objectives. In organizational terms, they are characterized by an intermediate level of efficiency between public entities and private for-profit, due, on the one hand, by the absence of the procedural constraints typical of public bodies, on the other hand by the voluntary nature of members participation, when it comes without economic returns activities (Table 2) [9].

Table 2. Features of managing subjects.

\begin{tabular}{cll}
\hline Managing Subject & & \multicolumn{1}{c}{ Features } \\
\hline Public & - & Management rigidity \\
& - & Growing budget constraints \\
& - & Lack of adequate skills \\
& - & Managerial management logics \\
Private for-profit & - & Fair flexibility \\
& - & High fixed costs for human resources \\
& - & Poor attention to public interests \\
Private not-for-profit & - & Presh flexibility \\
& - & Moderate fixed costs of management \\
\hline
\end{tabular}

Private entities involvement, with the purpose of cultural heritage enhancement, contributes to focus the attention on the economic dimension of these processes, since it entails the need for such subjects to maintain the financial feasibility of businesses and, in the case of for-profit activities, to ensure adequate levels of profit. 
The purpose of this paper is precisely to verify the conditions that ensure the economic and financial balance in the enhancement processes in the public-private partnerships, in relation to managing entities with different nature, which are associated with different management models: For that aim, therefore, it is particularly important to understand how the cost structure changes if management models change too, while we can consider invariable the structure of revenues.

\subsection{Kinds of Entities and of Activities}

Considering different kinds of activities and managing entities, we can suppose the three following models (Table 3):

Table 3. Managing models by type of subject.

\begin{tabular}{cc}
\hline Managing Model & Kinds of Entity and Activities \\
\hline Model P-Profit & For-profit Entity, for-profit activity \\
Model NP-Not-for-profit & Not-for-profit Entity, not-for-profit activity \\
Model M-Mixed & Not-for-profit Entity, for-profit activity \\
\hline
\end{tabular}

The first model does not require an in-depth analysis: It responds to the classical model of business entities, for which the economic and financial balance occurs only if an adequate level of profit is ensured. Given the fixed costs for human resources that characterize it, this model is inapplicable where the business volume is not so significant as to generate substantial revenues.

In the not-for-profit model, the managing entity, carrying out not-for-profit activities, benefits from the volunteer commitment of the members and other parties at no charge except in certain cases, in addition to the reimbursement of any costs incurred. Another significant cost item for human resources, absent in this model, is the management, of the production aspects [10]. Thereby, the fixed cost of human resources is greatly reduced, sometimes transforming this type in variable cost (being occasional performances), significantly reducing the operating costs in the case of limited flows of users (Figure 1). It is a working model when a community considers a given asset particularly important, but its management does not generate sufficient revenues to cover the operating costs for the entity involved.

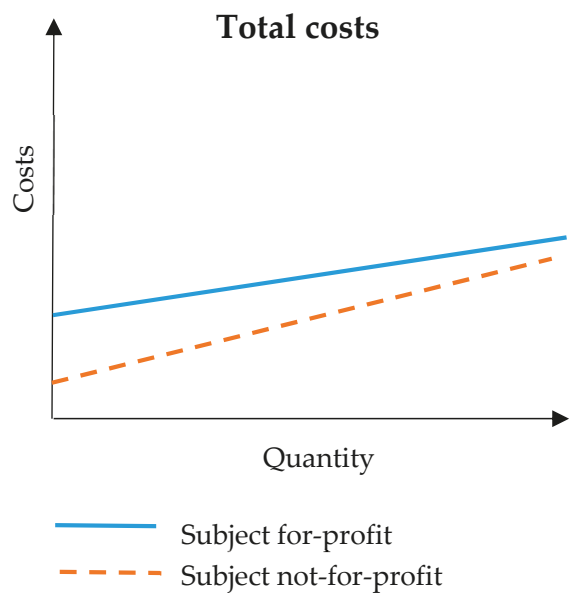

Figure 1. Trend of total costs by type of subject.

In the mixed model, however, the subject not-for-profit, carries on activities with significant revenues. In that case, it can be compared to a for-profit subject, in terms of cost structure, since the involved human resources have to be paid, as established by law, for those particular tasks. The main 
differences are: There is not profit, since any surplus is reinvested in the same activity and it cannot be distributed among members; the cost of the managerial duties can be considered, but is not a constant for the model.

It should be noted that the management model for the same asset can change over time. For example, in the start-up phase, a not-for-profit entity undertakes the management as a volunteer activity. Thereafter, if the demand increases adequately, it can turn the activity into business or acting as a start-up with entrepreneurial purposes.

In both cases, the management model choice does not depend exclusively on the economic and financial equilibrium: Even with adequate profits, it is essential to analyze the territory potential which can be positively exploited through the involved subjects, It may happen, for instance, the lack entrepreneurial stakeholders to manage a specific asset, or political interests may prevail, and that can act in favor of a particular model.

The economic and financial equilibrium assessment of physical asset management, however, allows the decision maker to make weighted choices, thereby reducing the possibility of failure, also bringing more clearness in the decision-making process.

\subsection{The Nature of Costs}

Firstly, we suggest a distinction among different types of cost that private entities can sustain, with respect to the development of architectural heritage, essentially referable to two macro-categories [11]: Investment costs and management costs.

In fact, the enhancement of real estate requires a starting investment to make the asset working, including the masonry work and the structures fitting with the safety standards, and the equipment and the facilities for its fruition (Figure 2). With respect, the intrinsic characteristics, the condition of preservation and its future use, such investment may be more unbalanced towards the building works or, conversely, toward equipment and furniture [12,13].

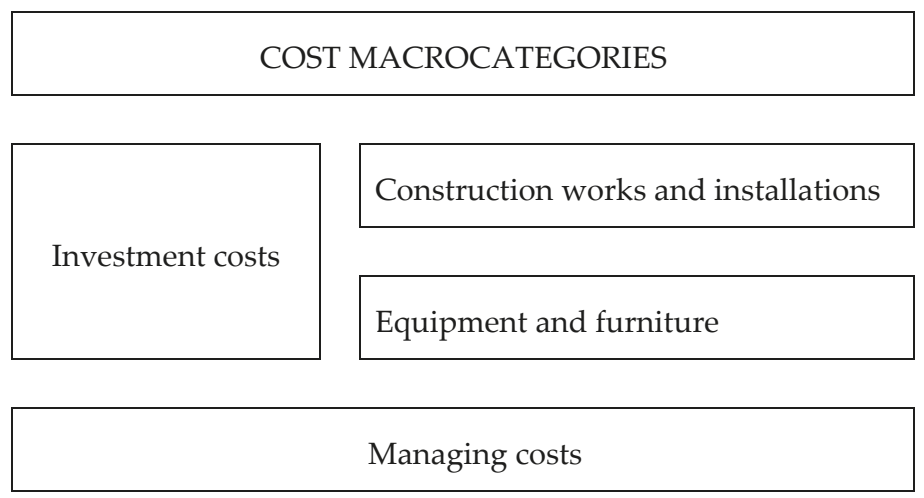

Figure 2. Cost categories for building enhancement.

Once the starting investment is completed, the management phase begins, involving costs, including human resources, utilities, maintenance, depreciation and amortization, and other goods and services [14]. It is to point out that, to assess the economic sustainability of the projects, among the management costs, the depreciation of equipment and furniture and the provisions for extraordinary maintenance, are particularly significant: Following this ratio, the replacement of equipment and furniture and the asset usability over time, are ensured, thus, allowing the sustainability of the initiative.

Operating costs, in turn, can be divided into two categories: Fixed costs and variable costs. Moreover, fixed costs are not related to production volumes, as in the case of the variable costs. Some of 
the items listed above are characterized by the presence of a fixed component and a variable, such as utilities or some kinds of human resources.

As seen above, the Human resources item, usually among those with higher incidence, changes significantly following with the different management models: In the case of for-profit activities, for instance, it represents one of the major fixed management costs.

In the case of for-profit subjects, the economic-financial balance occurred only with an adequate level of profit: For this reason, during financial sustainability tests, this item can also be treated as an operating cost and subtracted from revenues.

\section{Part Second-The Economic Evaluation of Feasibility Projects}

\section{Monetary Evaluations of Projects in the PPP}

\subsection{The Companies' Balance Sheet: Financial Statement and Cash Flows}

The accounting of companies serves to measure exchanges and the relationship with the external environment in monetary and economic terms: The balance sheet represents the summary document.

Information on the accounts has fiscal purposes, but it also serves to assess the company's "state of health" and its activities, reporting any unstable situations.

The Civil Code regulates the accounting report; in particular, the Art. 2423-Drafting the financial statements provides that the managers, annually, must prepare the financial statements, consisting of the balance sheet (art. 2424), the income statement (art. 2425), the cash flow statement and the explanatory note (art. 2427) [15]

The income and the expenses shown in the Cash Flow Statement represent the cash flows actually recorded in the year for the different categories of business of the company. For these reasons, the cash flow report is an analysis of cash flows [16].

We consider cash flows since the economic balance, given by revenues = costs, cannot be achieved daily: While some costs and revenues have a fairly uniform distribution over time, others occur at unpredictable intervals; indeed, some expenses. The flows can be:

- On entry, then we talk about cash inflow;

- Outgoing, then we talk about cash outflow.

- A company's cash flows can be traced back to three categories $[17,18]$ :

- Operating activity, which generally includes those operations connected with the purchase, the production, the distribution of goods and the provision of services, even if referable to ancillary operations, as well as other operations not included in the investment and financing activities.

- Investment activity, which includes the purchasing and sale transactions of tangible, intangible and financial assets and non-fixed financial assets.

- Financing activity, which includes the operations of obtaining and returning cash in the form of risk capital or debt capital.

\subsubsection{Operational Activity}

Cash flows from operating activities generally comprise the flows coming from the acquisition, the production and the distribution of goods and from the supply services, even if referable to ancillary operations, and the other flows not included in the investment and financing activities.

Some examples of cash flows generated or absorbed by operating activities are:

- Collections from the sale of products and from the provision of services;

- Collections from royalties, commissions, fees, insurance reimbursements and other revenues;

- Payments for the purchase of raw materials, semi-finished products, goods and other production factors;

- Payments for the acquisition of services; 
- Payments to, and on behalf of, employees;

- Payments and tax refunds;

- Receipts for financial income.

\subsubsection{Investment Activity}

The cash flow of the investment activity includes the flows that derive from the purchasing and sale of tangible, intangible and financial assets and not-immobilized financial assets.

By way of example, the financial flows generated or absorbed by the investment activity derive from:

- Purchasing or sale of buildings, plants, equipment or other tangible assets (including tangible assets of internal construction);

- Purchasing or sale of intangible assets, such as patents, trademarks, concessions; these payments also include those relating to capitalized multi-year charges;

- Acquisitions or sale of investments in subsidiaries and associated companies;

- Acquisitions or disposals of other investments;

- Acquisitions or sale of other securities, including government bonds and bonds;

- Disbursements of advances and loans made to third parties and proceeds from refunds.

\subsubsection{Financing Activities}

The financial flows of the financing activity include those flows deriving from obtaining or compensation of liquid assets in the form of risk capital or debt capital.

By way of example, the financial flows generated or absorbed by the financing activity are:

- Collections deriving from the issue of stocks or units belonging to the risk capital;

- Payment of dividends;

- Payments to refund the risk capital, including the form of the purchasing of treasury stocks;

- Collections or payments coming from the issue or redemption of bonds, fixed-income securities, the opening or restitution of mortgages and other short or long-term loans;

- Increasing or decreasing of debts including those ones in the short or medium term, of financial nature.

\subsection{Monetary Techniques for the Economic Evaluation of Projects}

A project is only a small part of any private subject activity, being profit or non-profit. The assessment of its sustainability or feasibility aims to verify:

- Its economic-financial balance over time (sustainability);

- Its ability to generate wealth for the person who realizes it (feasibility).

In the first case, it is necessary to identify the equilibrium conditions in the current year, in the second one the entire useful life of the project must be taken into consideration.

The techniques used in the two cases, therefore, differ exclusively for the time-based variable. Thus, for the sustainability assessment, the Cash Flow Analysis (CFA) will be used, for the feasibility forecast, instead, the Discounted Cash Flow Analysis (DCFA).

\subsubsection{Balance Evaluation of the Project Management Phase: Cash Flows Analysis}

In the case of projects for the enhancement of public buildings that provide, as a form of public-private partnership, only the assignment in management, without the need for investments by private parties, it is sufficient to resort to an examination of cash flows (Cash Flow Analysis-CFA) in the running year, using an analysis structure illustrated later, whose result must be equal to or greater than zero. 


\subsubsection{Evaluation of the Profitability Evaluation of the Project: Discounted Cash Flow Analysis}

Discounted cash flow or discounted cash flow is an evaluation method to assess the investment profitability, based on the current value of flows, according to a risk-adjusted rate, generated by the investment.

Usually, a project implementation does not represent an immediate operation:

- Costs and revenues associated with the transformation are articulated over time;

- Values occurring at different time ranges are not homogeneous, it is, therefore, not possible to make an immediate comparison of costs and revenues, both for a single project and among different projects.

It is, therefore, necessary to address two problems:

- Assessing costs and revenues for each year of the project;

- Making homogeneous balances between revenues and costs, under a shared time reference, reporting them up-to-date events.

The discounted cash flow analysis criterion is based on the economic principle of the anticipation. The main indicators to verify the profitability of investments are the Net Present Value (NPV) and the Internal Rate of Return (IRR).

The models that use discounted cash flow are widely consolidated, both in the international literature, relating to the valuation of real estate investments and in the manuals used for the professional practice, and in the Italian literature [19-22] to which we refer for further details.

The DCFA is considered the standard tool used in the valuation of real estate investments. The theory that supports it is shared within the community of scholars and professionals, and its results depend substantially on the quality of the inputs, which in general applies to any economic model.

However, it is characterized by some critical aspects, often underestimated; first of all, the uncertainty of the future scenario, from the decision-making point of view, despite the deterministic nature of the inputs can be corrected through sensitivity analysis and simulation models. In these analyzes, necessary decision-making flexibility is required with respect to the current economic scenarios, characterized by a high level of uncertainty relative to some of the variables of the system, endogenous, but above all exogenous.

\section{Evaluation Techniques and Project Profitability}

\subsection{Feasibility and Sustainability of Projects}

According to some authors [23], project feasibility depends on the conditions for effective cooperation between the actors called to implement it.

A project feasibility is ensured when all the subjects involved reach their goals. In particular:

- The public administration manages to regulate the transformation of the city effectively, and to satisfy the needs of citizens;

- The private profit partner gets a normal market profit from the project;

- $\quad$ The private non-profit partner carries out actions consistent with its own social goals.

With reference to Table 4, in the case of projects for the enhancement, in private-public partnership, of unused public buildings, it is possible to find substantially three cases:

- $\quad$ The private partner supports, entirely or partially the investment and project management costs (Band A,) in a context of profit activity;

- The private partner, profit or non-profit, exclusively assumes the management of a recovered and re-used property with public resources (Band B);

- $\quad$ The project is implemented and managed by public entities (Band C). 
In the first two cases the economic evaluation of the project aims at verifying:

- In the first case, profitability, that is the ability over time to adequately remunerate the capital invested;

- In the second case, the managerial balance, i.e. the ability to guarantee the mere sustainability over time of the functions envisaged.

To clarify, by now, the term "feasibility" will be used when the need arises to verify the profitability of an investment; instead, the term "sustainability" will be used (referring to its economic dimension), when it will be necessary to verify the balance during the project management phase.

Table 4. Criteria for selecting the type of managing subject.

\begin{tabular}{|c|c|c|}
\hline Type of Managing Subject & Sources of Financing & $\begin{array}{c}\text { Criteria for Economic Feasibility and/or } \\
\text { Sustainability Verification }\end{array}$ \\
\hline $\begin{array}{l}\text { Private for-profit } \\
\quad \text { (Band A.1) }\end{array}$ & $\begin{array}{l}\text { Investments: Private } \\
\text { Management: Private }\end{array}$ & $\begin{array}{l}\text { Revenues must cover management costs } \\
\text { and fully and adequately remunerate the } \\
\text { capital invested }\end{array}$ \\
\hline $\begin{array}{l}\text { Private for-profit } \\
\quad \text { (Band A.2) }\end{array}$ & $\begin{array}{c}\text { Investments: Private with public } \\
\text { co-financing } \\
\text { Management: Private }\end{array}$ & $\begin{array}{c}\text { Revenues must cover operating costs and } \\
\text { adequately remunerate the quote of } \\
\text { equity capital }\end{array}$ \\
\hline $\begin{array}{l}\text { Private for-profit } \\
\quad \text { (Band B.1) }\end{array}$ & $\begin{array}{l}\text { Investments: Public } \\
\text { Management: Private } \\
\text { for-profit }\end{array}$ & $\begin{array}{c}\text { Revenues must cover operating costs with } \\
\text { an adequate profit margin }\end{array}$ \\
\hline $\begin{array}{l}\text { Private not-for-profit } \\
\text { (Band B.2) }\end{array}$ & $\begin{array}{l}\text { Investments: Public } \\
\text { Management: Private } \\
\text { not-for-profit } \\
\text { Investments: Public }\end{array}$ & Revenues must only cover operating costs \\
\hline $\begin{array}{l}\text { Private not-for-profit } \\
\text { (Band B.3) }\end{array}$ & $\begin{array}{l}\text { Management: Private } \\
\text { not-for-profit with public } \\
\text { co-financing }\end{array}$ & $\begin{array}{l}\text { Revenues must cover a significant portion } \\
\text { of management costs }\end{array}$ \\
\hline $\begin{array}{l}\text { Public } \\
\text { (Band C) }\end{array}$ & $\begin{array}{l}\text { Investments: Public } \\
\text { Management: Public }\end{array}$ & $\begin{array}{l}\text { The usability of the asset must also be } \\
\text { guaranteed in case of limited or } \\
\text { zero revenues }\end{array}$ \\
\hline
\end{tabular}

\subsection{Profitability in the Hypotheses of Re-Use of Unused Public Buildings}

Considering the asset capacity to generate revenue, six different conditions of profitability can be assumed [24]:

Band A

Band A.1 High profitability

Band A.2 Medium to high profitability

\section{Band B}

Band B.1 Average profitability

Band B.2 Lower-middle profitability

Band B.3 Low profitability

\section{Band C}

Band C.1 Insufficient or nothing profitability

The case of insufficient or zero profitability, implies the absence of the minimum conditions for any form of public-private partnership and entrusts the public bodies exclusively, as responsible for making a specific case of asset available. This assumption, however, is quite difficult to be, due essentially to the public sector progressive decline in the delivery of resources $[25,26]$. 
Each of the first five of profitability conditions can be associated with five different public-private partnerships procedures, with the related management models (Table 5).

Table 5. Distribution of investment and managing costs between public and private entities.

\begin{tabular}{|c|c|c|c|c|}
\hline \multicolumn{3}{|c|}{ Investment Costs } & \multicolumn{2}{|c|}{ Managing Costs } \\
\hline \multicolumn{5}{|c|}{ Band $A$} \\
\hline \multicolumn{5}{|l|}{ Band A.1 High profitability } \\
\hline Band A.2 Medium to high profitability & $1-\mu$ & $\mu$ & & \\
\hline \multicolumn{5}{|c|}{ Band B } \\
\hline \multicolumn{5}{|l|}{ Band B.1 Average profitability } \\
\hline \multicolumn{5}{|l|}{ Band B.2 Lower-middle profitability } \\
\hline Band B.3 Low profitability & & & $1-\varepsilon$ & $\varepsilon$ \\
\hline \multicolumn{5}{|c|}{ Band C } \\
\hline Band C Insufficient or not profitability & & & & \\
\hline
\end{tabular}

Legenda: 므: Private for-profit; ㅁ: Private not-for-profit, activities not-for-profit; ㅍ: Public.

\subsection{The Choice of the Evaluation Technique}

For each of the profitability bands referred to in Table 5, different techniques for verifying the feasibility and economic sustainability of the projects respond to the different purposes of the assessment.

Band $A$ (high and medium high profitability): In this case, the assessment of the economic feasibility aims at verifying whether the incoming cash flows generated over time by the project, in addition to fully covering the operating costs, also manage to adequately remunerate the risk-sharing of the invested capital In this case, the financial analysis will be developed for a reasonable period of time, equal to the life cycle of the project; the technique to be used is the Discounted Cash Flow Analysis-DCFA or Discounted Cash Flow Analysis. If the project fails to generate such cash flows, remunerating the initial investment entirely, it is possible to reiterate the evaluation, hypothesizing alternative scenarios, characterized by different relationships between risk capital and public contribution, in order to identify the minimum threshold for the public contribution, for which the project is still economically feasible.

Band $B$ (average, medium-low and low profitability): In this case, the evaluation aims to verify the mere economic sustainability referred to a time period equal to the life cycle of the project. Therefore, the incoming cash flows generated annually by the project must cover the relative management costs [27]. The most suitable valuation technique, in this case, is the Cash Flow Analysis-CFA or Cash Flow Analysis: It differs from DCFA for the time horizon, which, in this case, is equal to 1 and refers to a full year of the project; further differences concern some of the items that are taken into consideration by the two techniques, as will be seen better below. The technique uses a particular form of an income statement, referring to the specific project, whose result must be equal to or greater than zero. It can also be applied repeatedly to alternative scenarios, characterized by subjects of different nature (profit, non-profit) according to the scheme illustrated below.

Band C (Insufficient or no profitability): The evaluation must provide the public decision maker with features to understand the social utility of the project. Frequently, the most used technique is the Cost Benefit Analysis-CBA or Cost Benefit Analysis [28]: The evaluation verifies if the direct and indirect benefits, internal and external, deriving from the project, are higher than the related costs, and therefore, the community benefits from its implementation. One of this technique limits, particularly relevant in this historical phase lacking of public resources, is that, even if the utility of a project is demonstrated, the uncertainty about its economic feasibility: A compared analysis between alternative projects, for instance, could help understanding which of them has the best relationship between the reachable benefits and the costs to be incurred (efficiency measure) or which project could maximizes the benefits (measure of effectiveness) [29]. This case can be considered beyond the public-private partnership ratio, and therefore, it will not be further investigated, referring to the copious scientific production on the subject for possible further investigations, including Florio et al., 2003; Pennisi and Scandizzo, 2003 [30,31]. 
Summary (Table 6):

- $\quad$ Range A (High and Medium High Profitability): Discounted Cash Flow Analysis-DCFA or Discounted Cash Flow Analysis; time horizon: Project life cycle;

- Range B (Average, medium-low and low profitability): Cash Flow Analysis-CFA or Cash Flow Analysis; time horizon: Full year of operation;

- Range C (Insufficient or no profitability): Cost Benefit Analysis-CBA or Cost Benefit Analysis; time horizon: Project life cycle.

Table 6. Aims and techniques of evaluation.

\begin{tabular}{cc}
\hline Aim & Technique \\
\hline $\begin{array}{c}\text { Verification of the profitability of an investment (feasibility) } \\
\text { Verification of the management balance of a project } \\
\text { (economic sustainability) }\end{array}$ & Discounted Cash Flow Analysis-DCFA \\
Verification of the public convenience of a project & Cash Flow Analysis-CFA \\
\hline
\end{tabular}

Whatever the question, the evaluation process is often repeated, in order to evaluate both alternative solutions and scenarios starting from a single solution. The technique to be used is chosen by the evaluator based on his experience, linked to the specific case.

\section{The Financial Economic Plan of a Project}

\subsection{The Financial Economic Plan Structure}

A fundamental tool to express a judgment of convenience regarding the feasibility/economic sustainability of a project to be carried out in public-private partnership is the economic financial plan (PEF), which includes the estimate of revenues, investments and management costs assumed by the private subject involved [32].

The PEF can be divided into 4 phases:

- Phase 1. Estimation of Investment Costs

- Phase 2. Estimation of Revenues

- Phase 3. Estimation of management costs

- Phase 4. Validation of economic feasibility and/or sustainability.

The last phase of the PEF, therefore, is the validation of the project feasibility and/or economic sustainability: This can be done by using one of the two kinds of the Cash Flow Analysis, for the full year if we refer to sustainability, or referring to the life cycle of the project to assess the economic feasibility.

Phase 1. Investment costs appraisal

The investment appraisal is divided into three parts, corresponding to different types of expenditure:

- $\quad$ Part I-recovery and re-functionalization of buildings investments;

- Part II—building practicability investments;

- Part III—communication and marketing investments.

The required investment to recover and possibly redefine the property can be estimated by drawing up an economic framework, in accordance with the provisions of art. 16 of the D.P.R. 5 October 2010, n. 207, Regulation for the execution and implementation of the Code of public contracts, and $€$ equivalent to the estimate of Production Cost [33,34].

As already mentioned above, economic and financial plan prevailing approach draws the methodological bases from the disciplines dealing with the companies' economic dynamics. 
However, if we take into consideration, for example, the authoritative guide published by the UVAL, number 30/2014 of its own series [Materials] [35], such approach leads to some uncertainty: It is enough to note, only to highlight an aspect between the many, how the costs for labor are treated in the estimate of the investment costs of table III.5, page 23 of the guide, shown below, in clear contrast to all the coded methods of calculating costs in the building process (Table 7).

Table 7. Investment costs according to UVAL.

\begin{tabular}{|c|c|c|c|}
\hline \multirow[t]{14}{*}{5} & & Investment Costs & Euro \\
\hline & + & Civil works & 6.000 .000 \\
\hline & + & Systems and equipment & 3.000 .000 \\
\hline & + & Expropriations ( $<20 \%$ of eligible expenses) & 1.000 .000 \\
\hline & + & Manpower & 500.000 \\
\hline & + & Design & 100.000 \\
\hline & + & Other (general expenses) & 500.000 \\
\hline & $=$ & Sub-total initial investment & 11.100 .000 \\
\hline & & Unforeseen events (percentage share $5-10 \%$ ) & $7 \%$ \\
\hline & + & Unforeseen events & 777.000 \\
\hline & + & Investment not eligible for public grant & 100.000 \\
\hline & + & Extraordinary maintenance during exercise & 200.000 \\
\hline & $=$ & Total investment cost & 12.177 .000 \\
\hline & & of which Eligible costs & 11.877 .000 \\
\hline
\end{tabular}

The cost of works evaluation, or construction cost, will take place through synthetic-comparative procedures, since it is developed within a preliminary phase of design choices definition, through the use of parametric, or mixed, appraisals for functional elements or significant samples.

It is also possible to identify the various items of the works, possibly subdivided by macro-category of works (consolidations, masonry works, installations, external arrangements, parking lots, etc.).

It is clear that, in this case, since these are public owned properties, the costs for their acquisition are zero. However, if the administration intends to use a property potentially important for the community, such as a building of historical value, but still privately owned, the acquisition-related costs should be assessed since the beginning, according to the provisions of the Consolidation Act on expropriations for public utility [36].

As regards the need to make the buildings usable, it will be required to estimate the furnishings and equipment, hardware and software costs, with the related value-added tax, if not recoverable by the investor. Similarly, depending on the function, the communication and marketing initial investments have to be estimated.

This phase ends with the formulation the investment capital composition, subdividing it into a share of the private investor's own capital, the share of debt capital and any share of public co-financing, in the form of capital grants.

Phase 2. Revenues appraisal

The revenue assessment phase is divided into sub-categories:

- Identification of goods to be produced or services to be provided;

- Estimation of their unit sales price;

- Identification of the target reference environment;

- Demand to be satisfied according to the referring target;

- Revenues appraisal.

Clearly, revenues generated by projects depends on the demand, which in turn depends on a series of factors [37], such as: 
- The potential user pool (an asset located in a big city with strong tourist attendance, for example, has a higher potential pool than one located in a small town with a low visitors attendance);

- The asset planned use and the related functions it brings with;

- The existence of similar assets and/or functions in its proximity;

- The intrinsic attractiveness of the considered asset;

- The communication and marketing strategies effectiveness and the availability of resources to be allocated for these activities.

Phase 3. management costs appraisal

The management cost appraisal phase implies the management model definition, and manager identification. This makes it possible to identify the human resources essential for the project management analytically, and to assess the annual costs related. In addition to workers costs. Then, other management costs occur in the fully operational year, such as consumables, services, etc.

Particularly interesting is the cost items evaluation, in order to figure out the analysis to be carried out subsequently, whether annual (CFA) or multi-year (DCFA): As already said before in the two cases, cost items are treated differently, such as provisions for extraordinary maintenance and financial charges.

Phase 4. feasibility and/or economic sustainability check

The PEF last section consists of the cash flows analysis; as seen, this activity can be aimed at verifying:

- The feasibility of the investment, through the evaluation of its profitability, through the use of the Discounted Cash Flow Analysis-DCFA or Discounted Cash Flow Analysis;

- The project management sustainability, through the check of the budget balance in the fully operational year, through the Cash Flow Analysis.

\subsection{The Cash Flow Analysis for the Projects Economic Evaluation}

To be able to proceed with the project feasibility and/or economic sustainability evaluation, it is necessary to build a cash flow analysis structure that is simultaneously consistent with the purpose of the assessment and with the indications of national and international accounting principles, regarding financial reporting previously mentioned (OIC 10 and IAS 7). Below are two diagrams, one for the Cash-Flow Analysis-CFA, the other for the Discounted Cash-Flow Analysis-DCFA meeting these requirements.

\subsubsection{Project Equilibrium Evaluation in the Management Phase: The Cash-Flow Analysis}

Considering a project for the public buildings improvement, through a public-private partnership that provide the private assignment of the management phase, without any investments from them: To evaluate the project equilibrium, the cash flows (Cash Flow Analysis) in the full year is sufficient, and the result must be equal to or greater than zero (Table 8).

The difference between income and revenues, in the case of for-profit activities, provides the result before taxes, while in the case of non-profit activities, it provides a management surplus, to be used in following years for purposes consistent with the management entity purposes. 
Table 8. Scheme of Cash Flow Analysis.

\begin{tabular}{l}
\hline A) Inflows: \\
\hline 1) revenue from sales and project performance \\
\hline $\begin{array}{l}\text { 2) other revenue and income (government grants for management, fundraising, membership fees, other } \\
\text { private contributions, etc.) }\end{array}$ \\
\hline B) Outflows: \\
\hline 3) for raw materials, ancillary materials, consumables and goods \\
\hline 4) for services (utilities; repairs; cleaning; other routine maintenance services) \\
\hline 5) for the use of third-party assets \\
\hline 6) for human resources: \\
\hline $\begin{array}{l}\text { (a) wages and salaries; (b) social security contributions; (c) severance pay; (d) retirement and similar } \\
\text { treatme€ (e) other costs; }\end{array}$ \\
\hline 7) for setting up a contingency fund \\
\hline 8) other management charges \\
\hline 9) Investments planned for replacement of tangible fixed assets (equipment and furnishings) \\
\hline 10) Investments planned for replacement or renewal of intangible assets \\
\hline 11) Establishment of a fund for extraordinary property maintenance \\
\hline
\end{tabular}

\subsubsection{Profitability Evaluation of a Project: The Discounted Cash-Flow Analysis-DCFA}

In the case of projects for the enhancement of public buildings that provide the private entities involvement, with money investment, the DCFA is used. To this purpose, it is possible to hypothesize the following scheme for the Analysis of Discounted Cash Flow (Table 9): 
Table 9. Scheme of Discounted Cash Flow Analysis.

\begin{tabular}{|c|c|c|c|c|c|c|}
\hline & \multirow[b]{2}{*}{0 (Investim) } & \multicolumn{5}{|c|}{ Years } \\
\hline & & 1 & 2 & 3 (Regime) & n & TOT \\
\hline \multicolumn{7}{|l|}{ A) Inflows: } \\
\hline \multicolumn{7}{|l|}{ 1) Revenues from sales } \\
\hline \multicolumn{7}{|l|}{$\begin{array}{l}\text { 2a) Other revenue and income: Investment: } \\
\text { Public co-financing quota-capital contribution }\end{array}$} \\
\hline \multicolumn{7}{|l|}{$\begin{array}{l}\text { 2b) Other revenue and income: The terminal } \\
\text { value of the building }\end{array}$} \\
\hline \multicolumn{7}{|l|}{ Total A-Inflows } \\
\hline \multicolumn{7}{|l|}{ B) Outflows: } \\
\hline \multicolumn{7}{|l|}{$\begin{array}{l}\text { 3) for raw materials, ancillary materials, } \\
\text { consumables and goods }\end{array}$} \\
\hline \multicolumn{7}{|l|}{$\begin{array}{l}\text { 4) for services (utilities; repairs; cleaning; other } \\
\text { routine maintenance services) }\end{array}$} \\
\hline \multicolumn{7}{|l|}{ 5) for the use of third-party assets } \\
\hline \multicolumn{7}{|l|}{ 6) for human resources: } \\
\hline \multicolumn{7}{|l|}{$\begin{array}{l}\text { (a) wages and salaries; (b) social security } \\
\text { contributions; (c) severance pay; (d) } \\
\text { retirement and similar trea€nt; (e) } \\
\text { other costs; }\end{array}$} \\
\hline \multicolumn{7}{|l|}{ 7) for setting up a contingency fund } \\
\hline \multicolumn{7}{|l|}{ 8) other management charges } \\
\hline \multicolumn{7}{|l|}{$\begin{array}{l}\text { 9) Investments planned for replacement of } \\
\text { tangible fixed assets (equipment and furnishings) }\end{array}$} \\
\hline $\begin{array}{l}\text { 10) Investments planned for replacement or } \\
\text { renewal of intangible assets }\end{array}$ & & & & & & \\
\hline
\end{tabular}

11) Investments planned for extraordinary property maintenance

12) Initial investments (equity ratio)

13) Financing activities: Mortgage interest and other financial charges

\section{Total B-Outflows}

\section{C) Difference between inflows and outflows-Result before taxes on the project's operating-ncome (A-B)}

14) taxes on gross profits

15) net profit (loss) of the project in the year

15b) actualized net profit (loss) of the project in the year (NPV)

\subsection{Cash Flows Detection Criteria}

In order to use the diagrams shown above, some in-depth details are needed: 


\section{2) Other revenues and income}

With respect revenues, the most important aspect concerns the investment residual value at the end of the project life cycle: This value must be considered in the case of application of the DCFA, by inserting it in the item (2) Other revenue and income, for the evaluation of the investment profitability and it might vary, in relation to the possible provision of extraordinary maintenance interventions, during the life cycle or not.

A further aspect to be considered for an investment profitability evaluation is the inclusion of other revenues and income, again in item (2), of any public co-financing quota: This item must be considered in those cases where revenues do not allow to fully recover the capital invested (Profitability class A.2).

The case of the project operational sustainability evaluation, under item (2): Other revenues and income can be added to any other public contributions for management reasons or from fundraising activities (membership fees, fundraising, other voluntary contributions private individuals, etc.).

4) Costs for services

Within this cost item, in particular, exits for utilities (electricity, gas, telephone, etc.) and exits for routine maintenance must be considered, starting first of all from cleaning costs.

There are different definitions of maintenance.

The definition of maintenance as it appears in the UNI EN 13306 [38] standard, for example, defines maintenance interventions as those characterized by the "combination of all technical, administrative and management actions, foreseen during the life cycle of an entity, intended to maintain it or bring it back to a state in which it can perform the required function".

The most relevant distinction, according to the authors, to be stressed, is that between ordinary and extraordinary maintenance.

The D.P.R. 380/2001 - Consolidated text for buildings [39], in art. 3, defines "ordinary maintenance interventions", the building interventions that concern the works of repair, renovation and replacement of the finishes of the buildings and those necessary to integrate or maintain the existing technological systems.

From an economic point of view, ordinary maintenance has a recurring nature (for example cleaning, painting, repair, replacement of parts damaged by use) and it is carried out to keep tangible assets in good working order through interventions that aim to guarantee their life expected profit, as well as the original capacity and productivity; it also includes repairs, and can also be attributed to fixed assets.

According to the national accounting principles issued by the Italian Accounting Body-OIC, in particular, the principle OIC 16 [40], ordinary maintenance costs are those incurred by the company to preserve the efficiency of the asset. The asset to be improved, therefore, has to keep its useful life and the original productive capacity unchanged. This category includes the costs incurred to repair faults, for the replacement of consumables, painting, cleaning, etc. The purchase of consumables, therefore, unlike the others, must be attributed to item 3).

\section{6) Workers costs}

As specified in the first part, the manager can also affect the costs of human resources.

In some cases, indeed, the activities planned do not produce sufficient income to cover all management costs, including those for human resources involved.

In such cases, a community, considering the value of those initiatives for the entire society involved, may decide to take charge of their performance anyway, through not-for-profit organizations, carrying out no-profit activities. Acting like this, there will be significant cost reductions, even if some member might still be paid, if the conditions are met. 
9), 10) Planned investments for the replacement of tangible and intangible fixed assets

A balance of economic activity is guaranteed over time if it generates incomes to restore the capital assets value over time, even for the purpose of replacing them [41].

It is, therefore, advisable to consider a periodic investment intended to replace the furnishings, equipment and intangible assets; together with the establishment of a fund for the extraordinary maintenance of the buildings, point 11), that becomes crucial to maintain the usability purpose of the building and its services over time.

11) Scheduled investments or establishment of a fund for the buildings extraordinary maintenance

In order to guarantee the sustainability of the activities over time, in the case of the management balance assessment through the cash flows analysis, considering the year of operation, a fund that allows periodic interventions of extraordinary maintenance must be established in advance $\mathrm{f}$.

On the contrary, in the case of an investment profitability evaluation through the DCFA application, the prevision of sums for extraordinary maintenance interventions is discretionary and depends on the time range taken into consideration: Considering a life cycle of 10 years, for example, such sums may not be envisaged; conversely, if they are considered, they will positively affect the residual asset value at the end of the measured life cycle.

From an economic point of view, extraordinary maintenance costs are expenditures for the growth, upgrading or improvement of the structural asset elements, translating into a significant and measurable increase:

1. Capacity;

2. Productivity;

3. Security;

4. Useful life;

5. Asset compliance with the expected purposes [42].

The above-mentioned art. Three of the Consolidated Law on Building, on the other hand, defines as "extraordinary maintenance interventions", the works and the modifications necessary to renew and replace even structural parts of buildings, as well as to create and integrate hygienic-sanitary and technological services, maintaining volumes and surfaces of the single real estate unit and not involving changes of use.

12) Initial investments (equity ratio)

For the investment profitability evaluation through the DCFA, it is necessary to add the share of private capital, belonging to the initial investment, among the outgoing cash flows, considering the time of implementation. This amount also includes any capital obtained from the private entity through the use of credit (mortgages etc.).

In this way, it will be possible to verify the actual trend of the cash flows and to estimate even more correctly any financial charges.

If the difference between all the discounted incoming and outgoing cash flows is positive or not, it will be possible to check if the revenues produced by the project will cover all the outflows and if they will allow the initially invested risk capital recovery, remunerating it adequately.

13) Interest and other financial charges;

This output item can be considered optional. Indeed, according to some authors, any financial charges fall within the subjective convenience judgment that the private entity expresses when decides to enter a partnership agreement with a public body.

This study, however, looks at the public decision-maker perspective, who must decide whether, and under what conditions, to entrust private subjects to build and manage ì a development project: Thus, the assessment has to consider any payable interest, which may be one of the variables within 
alternative forecast scenarios, aimed at verifying the conditions of convenience in the long run, considering different compositions of the capital investment.

Of course, interests on debt capital must be calculated to check the investment profitability. Nevertheless, this item is difficult to quantify, since it depends on variables external to the project (company financial capacity, cost of money, etc.); hence, an ordinary assessment has to be delivered, considering the specific space-time conditions in which the project will be carried out.

As a first step, the composition of the capital should be identified for initial investments, distinguishing it between the capital owned by investors and the debt capital, which will cause the interest expense charges.

On the other hand, no financial burdens must be borne by the private partner in the case of the mere management balance check.

14) Income taxes for the year;

In the case of profitability investment evaluation, the taxes on the gross profits generated by the project must also be considered; the net profits, once the taxes have been paid, represent the remuneration of the invested capital and the corresponding risks, i.e. the investment profitability.

Moreover, in this case, the appraisal of taxes implies significant margins of uncertainty, since the real amount paid depends on the other variables of the company's balance sheet, and as before, an ordinary assessment is mandatory considering all the other variable as nil, therefore, as if the company paid taxes exclusively for the gross profit deriving from the project.

Part Third-An Experimental Model of "Project of Economic Feasibility for the Enhancement of Unused Public Buildings in the Strategic Planning and the Integrated Projects"

\section{The SOSTEC Model}

The Laboratory LaborEst and the spin-off Urban Lab, both active at the Mediterranea University of Reggio Calabria, have developed an experimental model of "economic feasibility project for the development of unused public buildings" called SOSTEC; this model can be used when the public decision maker intends to verify whether the economic conditions exist for the use of private-public partnership agreements to implement and/or to manage a project.

The model is aimed at verifying the feasibility/economic sustainability of reuse hypotheses of unused public buildings, which is consistent with, and derived from, an overall idea of territorial development. The model, which can also be used in the case of buildings with a certain cultural value, is divided into three sections (Table 10):

- Section A-cognitive surveys

- Section B-reuse hypothesis

- Section C-financial economic plan.

The model structure allows to derive the reuse hypotheses from the knowledge of the territory dynamics and to verify the feasibility/sustainability of the formulated hypotheses.

Indeed, the model internalizes not only the usual socio-economic surveys (demographic trend, labor market, infrastructure and mobility system, cultural and environmental heritage, etc.), but also the stakeholders' point of view and this information, which derives from the programs in progress or already finished.

Particularly, as far as the programming is concerned, the references are assumed for the strategy and the objectives already identified by the local community, to develop coherent hypotheses, and the other programmed actions, with which eventually operate in synergy [43]. This cognitive framework should not be taken as a constraint, but as an element of awareness: The re-use of the specific building can also follow a different direction compared to the framework of the interventions already planned with other tools, but a similar choice should be motivated and conscious [44,45]. 
From the design point of view, (Section B-reuse hypothesis) choices to implement the model are synthetic: It is sufficient a functional program, equipped with the physical quantities of spaces intended for the different functions: These hypotheses allow to verify, at first glance, the coherence between the intrinsic characteristics of the building and the hypotheses of reuse formulated.

From the economic point of view (Section C-financial economic plan), the model provides for the preliminary evaluation of investment costs (Works for the recovery and re-functionalization of buildings; Furniture, hardware and software equipment for the usability of buildings; communication and marketing; etc.) followed by the economic dynamics analysis of the management phase. These dynamics are influenced, among others, by the type of manager entrusted to manage the asset, that can be a profit or not-for-profit subject: This hypothesis also determines economic implications, as explained later in the article.

The main purpose of the model, as mentioned, is the verification of the economic feasibility/sustainability of public-private partnership hypotheses: It serves, in other words, to verify the existence of sufficient conditions of convenience for private subjects, in the project realization and/or management in compliance with the expected public objectives, from which the work itself originates.

The term "feasibility", used in this article, applies to verify the profitability of an investment; instead, the term "sustainability" is used (making exclusive reference to its economic dimension), with the attempt to verify the balance during the management phase of a project.

The financial economic plan, therefore, will have the purpose of verifying the feasibility/sustainability of the reuse hypotheses and, consequently, identifying the economic conditions that can be placed at the basis of the partnership agreement.

Table 10. Structure of the SOSTEC Model.

\begin{tabular}{|c|c|c|c|}
\hline \multirow{10}{*}{ SECTION A } & \multirow{10}{*}{$\begin{array}{l}\text { COGNITIVE } \\
\text { SURVEYS }\end{array}$} & \multirow{10}{*}{$\Longrightarrow$} & Territorial framework \\
\hline & & & The territorial context \\
\hline & & & $\begin{array}{l}\text { Census of tangible and intangible } \\
\text { cultural heritage }\end{array}$ \\
\hline & & & The infrastructure and mobility system \\
\hline & & & Existing programming \\
\hline & & & Description of the asset to be enhanced \\
\hline & & & Recognition of already completed projects \\
\hline & & & Stakeholders' point of view \\
\hline & & & Best Practices Identification \\
\hline & & & Problems and vocations identification \\
\hline \multirow{4}{*}{ SECTION B } & \multirow{4}{*}{$\begin{array}{c}\text { REUSE } \\
\text { HYPOTHESIS }\end{array}$} & \multirow{4}{*}{$\Longrightarrow$} & Idea-force of the project \\
\hline & & & Objectives of the project \\
\hline & & & Intervention hypothesis \\
\hline & & & Functional schemes and substance \\
\hline \multirow{4}{*}{ SECTION C } & \multirow{4}{*}{$\begin{array}{l}\text { FINANCIAL } \\
\text { ECONOMIC } \\
\text { PLAN }\end{array}$} & \multirow{4}{*}{$\Longrightarrow$} & Estimation of investment costs \\
\hline & & & Estimation of revenues \\
\hline & & & Estimation of management costs \\
\hline & & & $\begin{array}{l}\text { Verification of economic feasibility and/or } \\
\text { sustainability of the project }\end{array}$ \\
\hline
\end{tabular}

\subsection{Section A-Cognitive Surveys}

The first section is divided into a series of sub-sections:

\section{A.1 Territorial framework}

The territory of the Municipality to which the cognitive investigation refers is described highlighting its position on a provincial and regional scale, associated with the description of graphic representations on appropriate cartography. 


\section{A.2 The territorial context}

Economic and social aspects, productive fabric, etc. of the territory considered are analyzed. Specifically, data relating to population, employment (main sectors), agriculture and local production, number of companies by category, accommodation services, places of culture, non-profit associations, tourist flows (attendances and arrivals) are reported.

\section{A.3 Census of tangible and intangible cultural heritage}

In this section, material and immaterial cultural heritage of the Municipality considered are listed and mapped. Specifically, the following are identified: Material heritage archaeological areas, defensive architecture, religious architecture, noble palaces, historical centers, rural architecture, national and regional parks, S.I.C. (Sites of Community Importance), Z.P.S. (Special Protection Areas), geo-sites. Intangible heritage: Oral traditions, particular languages, social and ritual practices, artisan skills and the spaces associated with them, peculiar productions (including food and wine) part of that specific cultural identity.

\section{A.4 The infrastructure and mobility system}

This sub-section is devoted to the infrastructural and mobility systems, identifying, also on maps, the main hubs: Airports; railroad; ports; network of roads; mobility by public transport.

\section{A.5 Existing programming}

Through this sub-section it is possible to carry out a survey of existing or recently concluded programs, to develop strategies already in the territory, the needs, the objectives and any solutions already identified previously.

In the following phases it will be possible to select project coherent with this framework, but also to decide to change the priorities, the needs, the objectives and the solutions, in consistency with the analysis conducted.

\section{A.6 Description of the asset to be enhanced}

At this point, the cognitive investigation focuses attention on the asset to be valued, starting from a brief description. Then, the main identification data are reported: Name; type of asset; year of construction; the presence of constraints; public or private property; location; photos; level of decay; usability; reachability by public transport; availability of information (in the case of cultural heritage).

\section{A.7 Recognition of already completed projects}

In this sub-section there is a recognition of the projects already carried out on the asset considered for the redevelopment initiative, but also the general program to which the enhancement project must be coherent and, hopefully, helpful to achieve its objectives.

\section{A.8 The stakeholders' point of view}

Every investment has an impact beyond the finalized goods or services produced. Therefore, stakeholders have a specific role, and also interests, in the project implementation, helping future scenarios. The model foresees interviews with institutional stakeholders, such as local administrators, but also entrepreneurs, professionals, municipal technicians, associations. Interviews are useful to analyze perceptions and expectations from all categories of stakeholders involved. Following some examples:

- What are the main problems of the territory?

- What are the territory strengths and resources? Which hypothesis of reuse of the building can be strategically functional to improve its value? Why? 
- What are the weak points, the critical points, the limits within the urban and rural territory that can hinder the development, and the hypothesis of re-use of the building? How can they be overcome?

- Can there be private profit or non-profit entities interested in building enhancement? If yes, which ones?

\section{A.9 Identification of Best Practices}

Once the cultural object of the simulation has been chosen, it can be useful to identify and describe a best practice, or an experience of success comparable to similar assets, in order to highlight those positive elements that allowed to obtain the best result, with respect to the contexts and the set objectives, taking into account previous cases of enhancement projects and projects to improve accessibility. It can also be useful to analyze management models and kinds of PPPs.

The best practices analysis not always provide the expected results: Solutions and models are often designed for completely different contexts and situations, not always useful for specific projects. In any case, the knowledge of how similar cases could provide some useful indications, as long as there is awareness of the limits of this methodology.

\section{A.10 Identification of problems and vocations}

The cognitive analysis conducted allows the identification of main issues, but also the territory vocation and the assets to be enhanced.

\subsection{Section B-Reuse Hypothesis}

\section{B.1 Project strong-point}

In this first sub-section the idea that the project intends to pursue according to the resources of the territory, the economic fabric, etc., must be briefly described.

\section{B.2 Project objectives}

In detail, a strong idea of the project should be explained, describing the specific objectives, specifying which needs the project intends to satisfy through the asset development project.

\section{B.3 Intervention hypothesis}

Considering the cognitive investigations carried out, and the outcomes analyzed the first hypothesis of intervention can be drawn, referring to those activities to be localized, and the subjects to be involved (profit and not-for-profit, private individuals, institutions, etc.).

\section{B.4 Functional schemes and consistency}

The meta-design idea drawn by the intervention hypothesis must be graphically depicted through plan diagrams, on adequate scales, describing the functions to be added, by attaching a legend to match the assigned surfaces with the given functions.

\subsection{Section C-Financial Economic Plan (FEP)}

As already explained above, the FEP is divided into 4 phases:

Phase C.1. Investment costs assessment (Works for the recovery and re-functionalization of buildings; Hardware and software furniture and equipment for the usability of buildings; communication and marketing; etc.).

Phase C.2. Revenues assessment (Identification of goods to be produced or services to be provided; estimate of their unitary sale price; identification of the target audience; demand assessment revenues assessment).

Phase C.3. management costs assessment (management model and manager description; human resources plan, management costs appraisal, such as consumables, services, workers, etc.). 
Phase C.4. Project economic feasibility and/or sustainability.

\subsubsection{Phase C.1. Investment Costs Assessment}

Preliminarily, it is necessary to identify all the investment items, possibly subdivided by macro-category of works (masonry, consolidation, parking, etc.) and by type (furnishings, equipment, software, technical expenses). The estimate will be made through the synthetic procedures for the estimation of the construction/production cost, as concern the building works, and the equipment and furnishings too, that will have to be carried out according to the building re-use hypothesis, with respect the different rooms it is composed by.

Then, the capital composition has to be analyzed, establish public and private investment shares; as concern the private share, it the equity the debt share has to be established, to calculate a possible annual loan installment, to be added to the Discounted cash flow analysis.

At the end of this phase, it is necessary to hypothesize the residual value of the building at the end of the life cycle, that will be added among the revenues, in the case of the profit management entity, jointly to the public investment shares.

\subsubsection{Phase C.2. Revenues Assessment}

After the investment costs assessment, the private subject has to be established, if profit or not-for-profit entity of management, to proceed with the subsequent $\mathrm{f}$ costs and revenues assessments.

The revenues appraisal requires, first of all, the identification of goods to be produced, or the services to be provided, and the estimate of their unity sale price.

Then, it is possible to figure out the demand, through the definition of the reference target of the proposed project.

Among the revenues, in the case of no-profit management entity, membership fees have to be considered; other private contributions and fundraising; any government grants for management.

\subsubsection{Phase C.3. Management Costs Assessment}

To assess management costs, the management model has to be established in advance. In order to identify the optimum management model for the proposed project, its sustainability has to be verified, both with a private for-profit entity and a not-for-profit one, also specifying the additional subjects eventually involved in the management phase. The management model is based on the use of an organization chart to list the activities, the foreseen functions and the role assigned to each human resource involved, specifying the taken legal form (consortium, partnership agreement, etc.)

Consistently with the management model, the different items of management costs (consumables, services, maintenance etc.) are evaluated, identified through synthetic procedures and surveys, clarifying the reference sources (national labor contracts, best practices, etc.).

\subsubsection{Phase C.4. Project Economic Feasibility and/or Sustainability}

Basing on the revenues and costs assessments, the economic sustainability of the intervention can be verified in the management phase (non-profit subject), or the investment feasibility in terms of profitability (profit subject) by alternatively drawing up one of the two economic accounts exposed before (Scheme of Cash Flow Analysis and Scheme of Discounted Cash Flow Analysis).

\section{Conclusions}

The SOSTEC model was conceived as a tool. Even though it was still experimental in character, it was intended for public administrations needs to re-develop unused real estate, not for residential use, with the involvement of private entities.

The model, can be helpful for existing buildings enhancement, also of historical and architectural value, and suitable for development projects of areas susceptible to transformation, and allows to: 
- Identify destinations of use that meet the needs of the territory, local development policies and the intrinsic characteristics of the asset to be enhanced;

- Identify the possible ways of private subjects involvement: If there are those real conditions, such as sufficient profitability, for their involvement in the investment or rather providing support for the management phase;

- Determine the economic conditions base of the partnership: Discerning the need for, public participation, by co-financing or by managing the investment or if, instead, the revenues generated by the project are sufficient to guarantee its feasibility, (with private investments), or its sustainability, (with private management).

The identification of the type of private entity involvement, through the SOSTEC model, takes place by reiterating the evaluation, in order to identify feasible and/or sustainable solutions and to exclude those that do not possess these requirements.

One of the SOSTEC model objectives is also the homogenization of those items included within the different cash flow analysis methods: In detail, the article aimed at highlighting the main differences introduced by the cash flow analysis scheme, updated or not, focused to assess the urban projects feasibility and sustainability, in compliance with the company accounting, such as the Balance Sheet.

Therefore, the model will be conveniently tested through the case studies application.

Moreover, by these tests, it is expected:

- $\quad$ To verify the model completeness as concern the fact-finding phase;

- To understand the different item effects on the final results, in input and output, introduced by the cash flow analysis, discounted and not discounted, usually different from those within the Balance Sheet.

The research activity will also continue toward the integration of monetary techniques, used in the model described above, with qualitative criteria that improve the capacity of the hypothesized solutions in order to respond to the citizens' real needs and the territories socio-economic dynamics.

Author Contributions: 1. Introduction, 3. Monetary evaluations of projects in the PPP, and 7. Conclusions, L.D.S.; 2. The cooperation between public and private subjects, 4 . Evaluation techniques and project profitability, and 5. The Financial Economic Plan of a project, F.C.; 6. The SOSTEC model, F.C. and L.D.S.

Funding: The present study has not received any type of financing.

Conflicts of Interest: The authors declare that they have no conflict of interest with regard to the present study.

\section{References}

1. Donolo, C. Dalle politiche pubbliche alle pratiche sociali nella produzione di beni pubblici? Osservazioni su una nuova generazione di policies. Stato Mercato 2005, 25, 33-66.

2. Calabrò, F.; Della Spina, L. II Partenariato Pubblico Privato per la valorizzazione degli immobili pubblici inutilizzati. LaborEst 2018, 16 IS, 3-4.

3. Decreto Legislativo 18 Aprile 2016, n. 50 e s.m.i., Codice dei Contratti di Appalto e di Concessione Delle Amministrazioni Aggiudicatrici e Degli enti Aggiudicatori Aventi ad Oggetto L'acquisizione di Servizi, Forniture, Lavori e Opere, Nonchè i Concorsi Pubblici di Progettazione. 2016.

4. Merola, G.D. Autonomia Locale e Governo Imperiale: Fiscalità e Amministrazione Nelle Province Asiane; Edipuglia: Bari, Italy, 2001.

5. Studio-Linee Guida "Strategie e strumenti per la valorizzazione del patrimonio immobiliare pubblico" a cura di "Studiare Sviluppo", società in house del Ministero dell'Economia e Finanze, per conto del Dipartimento per gli Affari regionali, le Autonome e lo Sport (DARAS) della Presidenza del Consiglio dei Ministri. 2016.

6. Bilancia, P. (Ed.) La Valorizzazione dei Beni Culturali. Modelli Giuridici di Gestione Integrata; FrancoAngeli: Milano, Italy, 2006; Volume 642.

7. Propersi, A. II Sistema di Rendicontazione Negli Enti non Profit. Dal Bilancio D'esercizio al Bilancio di Missione; Vita e Pensiero: Milano, Italy, 2004. 
8. Francesconi, A. Comunicare il Valore Dell'azienda non Profit; Wolters Kluwer: Milano, Italia, 2007.

9. Fiorentini, G.; Preite, D. Azienda Pubblica e Azienda non Profit: II Ruolo del Bilancio Sociale Nel Decentramento; Azienda pubblica: Parma, Italy, 2000.

10. Fazzi, L.; Giorgetti, G. II Bilancio Sociale Per le Organizzazioni non Profit: Teoria e Pratica; Guerini e Associati: Milano, Italy, 2005.

11. Pelfrey, S. Cost categories, behavior patterns, and break-even analysis. J. Nurs. Adm. 1990, 20, 10-14. [CrossRef] [PubMed]

12. Carbonara, S.; Cerasa, D.; Sclocco, T.; Spacone, E. A preliminary estimate of the rebuilding costs for the towns of the Abruzzo Region affected by the April 2009 earthquake: An alternate approach to current legislative procedures. In International Conference on Computational Science and Its Applications; Springer: Cham, Switzerland, 2015; pp. 269-283. [CrossRef]

13. Scrivo, R.; Rugolo, A. Sostenibilità e fattibilità nella programmazione delle opera pubbliche. Metodologie e strumenti per un centro regionale di controllo dei costi negli appalti pubblici. LaborEst 2016. [CrossRef]

14. Baraldi, S.; Cifalinò, A.; Sacco, P. I Sistemi di Programmazione e Controllo; Giappicheddi: Torino, Italy, 2011; pp. $15-29$.

15. Codice Civile italiano, Artt. 2423-2427.

16. Del Pozzo, A. Controllo Finanziario e Rischio di Default; FrancoAngeli: Milano, Italy, 2011.

17. Organismo Italiano di Contabilità-OIC. Principio Contabile OIC 10; OIC: Rome, Italy.

18. International Accounting Standards IAS 7.

19. Realfonzo, A. Teoria e Metodo Dell'estimo Urbano; Nuova Italia Scientifica: Rome, Italy, 1994.

20. Prizzon, F. Gli Investimenti Immobiliari; Celid: Torino, Italy, 1995.

21. Conferenza Delle Regioni e Delle Province Autonome, Linee Guida per la Redazione di Studi di Fattibilità; ITACA: Rome, Italy, 2013.

22. Morano, P.; Tajani, F. The Break-Even Analysis applied to urban renewal investments: A model to evaluate the share of social housing financially sustainable for private investors. Habitat Int. 2017, 59, 10-20. [CrossRef]

23. Roscelli, R. Manuale di Estimo; UTET Università: Novara, Italy, 2014.

24. Tramontana, C.; Calabrò, F.; Cassalia, G.; Rizzuto, M.C. Economic sustainability in the management of archaeological areas: The case of Bova Marina (Reggio Calabria, Italy). In New Metropolitan Perspectives; Calabrò, F., Della Spina, L., Bevilacqua, C., Eds.; Springer: Cham, Switzerland, 2019; Volume 101, pp. $288-297$. [CrossRef]

25. Aas, C.; Ladkin, A.; Fletcher, J. Stakeholder collaboration and heritage management. Ann. Tour. Res. 2005, 32, 28-48. [CrossRef]

26. Franch, M. Le frontiere manageriali per la valorizzazione della cultura e dell'arte. Sinergie Ital. J. Manag. 2010, 82, 95-107.

27. Kaplan, R.S.; Atkinson, A.A.; Morris, D.J. Advanced Management Accounting; Prentice Hall: Upper Saddle River, NJ, USA, 1998; Volume 3.

28. Mollica, E. Principi e Metodi di Valutazione Economica dei Progetti di Recupero; Rubbettino: Soveria Mannelli, Italy, 1995.

29. Nesticò, A.; Macchiaroli, M.; Pipolo, O. Costs and benefits in the recovery of historic buildings: The application of an economic model. Sustainability 2015, 7, 14661-14676. [CrossRef]

30. Florio, M.; Finzi, U.; Genco, M.; Levarlet, F.; Maffii, S.; Tracogna, A.; Vignetti, S. Guida All'analisi Costi-Benefici dei Progetti di Investimento; Fondi Strutturali, Fondo di Coesione e ISPA: Roma, Italy, 2003.

31. Pennisi, G.; Scandizzo, P. Valutare L'incertezza: L'analisi Costi-Benefici nel 21. Secolo; Giappichelli: Torino, Italy, 2003.

32. CIPE-Unità Tecnica Finanza di Progetto. La Valutazione Della Convenienza Economico-Finanziaria Nella Realizzazione e Gestione Degli Investimenti Pubblici con il Ricorso Alla Finanza Privata. 2002.

33. Decreto del Presidente Della Repubblica del 5 Ottobre 2010, n. 207, Regolamento di Esecuzione ed Attuazione del Decreto Legislativo 12 Aprile 2006, n. 163, Recante Codice dei Contratti Pubblici Relativi a Lavori, Servizi e Forniture in Attuazione Delle Direttive 2004/17/CE e 2004/18/CE. 2010.

34. Calabrò, F.; Della Spina, L. Processo edilizio e stima dei costi. LaborEst 2015. [CrossRef]

35. Gori, G.; Lattarulo, P.; Maiolo, S.; Petrina, F.; Rosignoli, S.; Rubino, P. Lo studio di fattibilità nei progetti locali realizzati in forma partenariale: Una guida e uno strumento. Mater. UVAL 2014, 30, 47-62.

36. Decreto del Presidente Della Repubblica dell's Giugno 2001, n. 327, Testo Unico Sulle Espropriazioni per Pubblica Utilità. 2001. 
37. Cochrane, J.; Tapper, R. Tourism's contribution to world heritage site management. In Managing World Heritage Sites; Routledge: Abingdon-on-Thames, UK, 2006; pp. 97-109.

38. Norma UNI EN 13306. Manutenzione-Terminologia di manutenzione.

39. Decreto del Presidente Della Repubblica del 6 Giugno 2001, n. 380-Testo Unico Edilizia, Art. 3. 2001.

40. Principio OIC 16 .

41. Ziani, L. Voce: "Ammortamento", Dizionario di Economia e Finanza; Treccani: Roma, Italy, 2012.

42. Cherubini, D. Le Spese di Manutenzione-Trattamento Civilistico e Fiscale. 2007. Available online: http://www.blustring.it.

43. Della Spina, L.; Calabrò, F. Decision Support Model for Conservation, Reuse and Valorization of the Historic Cultural Heritage. In Computational Science and Its Applications_ICCSA 2018; ICCSA 2018. Lecture Notes in Computer Science; Gervasi, O., Ed.; Springer: Cham, Switzerland, 2018; Volume 10962. [CrossRef]

44. Giuffrida, S.; Napoli, G.; Trovato, M.R. Industrial Areas and the City. Equalization and Compensation in a Value-Oriented Allocation Pattern. In International Conference on Computational Science and Its Applications; Springer International Publishing: Berlin, Germany, 2016; pp. 79-94.

45. Guarini, M.R. Self-renovation in Rome: Ex ante, in itinere and ex post evaluation. In Computational Science and Its Applications_-ICCSA 2016; ICCSA 2016. Lecture Notes in Computer Science; Gervasi, O., Ed.; Springer: Cham, Switzerland, 2016; Volume 9789, pp. 204-218. [CrossRef]

(C) 2019 by the authors. Licensee MDPI, Basel, Switzerland. This article is an open access article distributed under the terms and conditions of the Creative Commons Attribution (CC BY) license (http://creativecommons.org/licenses/by/4.0/). 

Article

\title{
Adaptive Sustainable Reuse for Cultural Heritage: A Multiple Criteria Decision Aiding Approach Supporting Urban Development Processes
}

\author{
Lucia Della Spina \\ Department of Heritage, Architecture and Urban Planning, Mediterranea University of Reggio Calabria, \\ 89125 Reggio Calabria (RC), Italy; lucia.dellaspina@unirc.it
}

Received: 20 December 2019; Accepted: 5 February 2020; Published: 13 February 2020

\begin{abstract}
The European Union identifies the cultural heritage of cities as the main driver of development strategies. From this perspective, adaptive reuse can play a decisive role not only in terms of increasing the life cycle of the heritage but also as an urban strategy capable of generating new economic, cultural, and social values, thus supporting innovative dynamics of local development. The aim is to propose an integrated evaluation model based on the combined use of multi-criteria techniques, which helps to classify adaptive reuse strategies of unused cultural heritage assets and supports decision-makers in the implementation of development strategies in vulnerable contexts. The case study focuses on the potential reuse of some historical fortifications located along the coasts of the Strait of Messina in Southern Italy. The results obtained show that the proposed model can be a useful decision support tool, in contexts characterized by high complexity, able to guarantee the transparency of the decision-making process, and in which it is necessary to highlight the elements that influence the dynamics of the choice for the construction of shared development strategies.
\end{abstract}

Keywords: cultural heritage conservation; adaptive reuse; multi-criteria decision aid; strategic assessment

\section{Introduction and Overview}

In the current scientific debate, cities represent the contexts in which resources, capital, skills, and talents are concentrated and, at the same time, places where many challenges are concentrated regarding environmental (pollution, waste, climate change), economic (unemployment, social exclusion, well-being), political (instability in governance processes, lack of strategic planning), and cultural (training, creativity and innovation) dimensions. The city, therefore, allows to experiment the paradigm shift from linear economy to circular economy, in which to promote synergetic, fair and inclusive processes capable of activating new forms of urban productivity and social and economic innovation.

In particular, the European Commission identifies the cultural heritage of cities as the main driver of development and supports strategies in which it is considered one of the fundamental elements of possible transformations, which can be activated through mixed top down/bottom up approaches in the short and long term. From this perspective, adaptive reuse can play a decisive role not only in terms of increasing the life cycle of the heritage but also as an urban strategy capable of generating new, even divergent values (economic, aesthetic, cultural, educational, political), supporting innovative dynamics of local development and which underline the importance of preserving cultural heritage and the instrumental values for its livelihood [1] in a "circular" perspective [2]. In fact, if you look at cultural heritage as a potential element of an "self-sustainability" territorial system [3], which continually redefines itself and sustains and reproduces itself from within, it is possible to identify the re-use of cultural heritage as an essential contribution in the realization of a model of economy and of "circular" cities and territories, capable of eliminating the waste of environmental resources (soils, materials, 
energy, etc.) and cultural resources (tangible and intangible heritage, knowledge, etc.), and capable of transforming "waste" into new economic, environmental, cultural, and social resources.

Cultural heritage, in fact, can be considered not only a legacy to be handed down to posterity but also a central resource for triggering processes of local and global development. At the moment, however, the conservation and enhancement of the heritage that has a cultural and landscape value represent a burden for the community. However, the inequality between the quantity of material and cultural heritage available and the increasingly limited availability of public resources for conservation have led to the total or partial abandonment of cultural heritage. Even where conservation measures are carried out, the potential impact of this public investment is countered by an absent or short-sighted management program.

Today, in the field of reusing historical heritage, scientific research has developed methods to assess the compatibility of new functions with the morphological and dimensional characteristics of existing buildings and with the objectives of urban development and revitalization [4-6]. In the planning and technical and economic feasibility study phase, the purpose of these methods is to determine the most compatible use. In the planning phase, the compatibility assessment allows decision-makers to identify new appropriate uses, compatible with the layout and the original meaning of buildings and historic places, a new use that will bring benefits such as the improvement of the building, market value, use, environmental, cultural and social value [5,7]. The desire to protect the identity of a historic building leads planners to estimate the quantity and amount of transformations required by each function in order to find the most compatible solution. However, the choice of the new function must also take into account the economic and social objectives and the effects of reuse on the urban environment.

The lack of public resources has, in fact, pushed many local administrations to favor new forms of enhancement of public buildings in able to promote the "adaptive reuse" of a historic building in order to preserve its social, historical, and cultural values but also to stimulate the experimentation of new circular businesses, financing and governance models in heritage conservation, creating synergies between multiple actors, and reducing the use of resources and regenerating values, capital, and knowledge

Decisions on the increasingly scarce allocation of public resources for the conservation or development of cultural heritage are based on a series of multiple and heterogeneous criteria, which are often in conflict, as well as on the preferences of various stakeholder. In this context, multi-criteria approaches provide an adequate theoretical and methodological framework to deal with the complexity that characterizes the adaptive reuse strategies of cultural heritage.

In the literature, many multi-criteria methods have been developed in recent decades [8] with applications to real cases (including examples of reuse) and in different sectors to support decision-making processes [9]. "Multi-criteria analyses" are very useful tools in the ex-ante evaluation phase, in which different options must be evaluated in order to determine and classify the alternatives. Therefore, some multi-criteria evaluation methods have been developed as "decision support systems": they do not automatically offer a choice, but rather provide help to decision-makers who face complex situations and problems characterized by a certain degree of uncertainty and information of different nature.

In order to evaluate the overall effectiveness of the alternative candidates and select the best option, the multi-criteria method requires four phases-(i) defining a set of attributes that most influence the choice of the alternative, (ii) a decision matrix, (iii) weighting of the criteria using an appropriate method, and (iv) evaluate alternatives by classifying them from best to worst.

It is possible to find several examples of the application of the Multi-Criteria Decision Aid (MCDA) methods [8] for the conservation and reuse of cultural heritage [10-15]. From an analysis of the main scientific literature with applications for MCDA in decision problems regarding cultural heritage, emerges the importance of the use of combined methodologies [16-21], which are particularly useful for dealing with complex situations [22-24], and are capable of structuring more inclusive decision-making processes aimed at a greater sharing of choices [25-27]. 
Many applications of MCDA are in the domain of cultural heritage. From the point of view of field application, the analysis shows that there are works in different research domains and that few works exist for the evaluation of cultural assets with reference to the strategies for their reuse [28]. As regards applications in the literature, multi-criteria analysis provides a theoretical and operational framework useful for supporting the design and implementation of public policy strategies in complex contexts with different values at stake and often conflicting [11-13]. The robustness of the multi-criteria methodologies is due to a series of main factors-heterogeneous criteria capable of managing both quantitative and qualitative information, multiple actors can be easily involved, the final aggregation of the different positions on adaptive reuse strategies [9-13], and the research for a solution of greater compromise that reflects the preferences of most points of view but also that of minority points [29].

This document addresses the issue of adaptive reuse of cultural heritage in a context of multi-criteria decision making and contributes to the cited literature. In detail, a decision aid system is developed, that identifies context-aware enhancement scenarios, oriented to the construction of new tangible and intangible values for the cultural heritage abandoned located in Southern Italy. What is proposed is an integrated evaluation model, based on the use of different multi-criteria methodologies, to define a classification between different scenarios and development strategies for unused historical heritage located in vulnerable contexts. The methodology is able to support the choice of an alternative reuse and to define a "shared strategy" based on a "bottom-up" approach.

The application to the case study concerns the potential reuse of six different historical fortifications (built at the end of the 18th century) located along the coasts of the Strait of Messina in Southern Italy, which are characterized by particular architectural and technical characteristics and by enormous spaces suitable for being redeveloped for new uses.

This requires the definition of the needs that must be met by the new functions assigned to the historical asset and the identification of the structural and cultural constraints to its transformation. From this perspective, a key problem is that of prior identification of the most suitable assessment methods for the decision problem.

The choice of methodology used here derives from the complementary relationship activated between the techniques of the Systems Thinking Approach [30-33] and the multi-criteria and multi-group evaluation methods, which allows us to outline a decision-making process that makes use of the specifics of each one in order to improve the knowledge of the context, to make explicit the preferences of the different stakeholders, to build shared visions of the future, and identify the preferable scenarios considering both the "qualitative" data and the "quantitative" data. The application of combined evaluation methodologies is justified by an increasingly widespread practice [30-37] aimed at overcoming the limits of each single method, to consider a multi-dimensional and plural perspective, to activate inclusive decision-making processes, and to improve the quality of the process oriented to the construction of shared choices.

The evaluation process implemented also integrates the contribution of different knowledge. It is based on the sharing of responsibilities between the different stakeholders and the concertation of choices through the complementarity between experiences and competences of different domains.

The main objective is to provide a decision support tool to design and implementation of adaptive reuse strategies of abandoned military heritage in a vulnerable context [37-39].

Furthermore, the combined use of multi-criteria techniques helps to classify unused adaptive cultural heritage reuse strategies and is able to support decision makers in implementing development strategies in vulnerable contexts. The application to the case study focuses on the potential reuse of some historical fortifications located along the coasts of the Strait of Messina. The results obtained show that the proposed model can be a useful decision support tool in contexts characterized by high complexity and that it is able to guarantee the transparency of the decision-making process for the construction of shared development strategies, in which it is necessary to highlight the elements that influence the dynamics of choice toward the best reuse project that reaches an optimal balance between conservation and usability [40-42]. 
In detail, the document is organized into five sections. Section 2 describes the methodological framework and the theoretical references of the techniques used. Section 3 presents the application to the case. Section 4 discusses the results obtained, while Section 5 traces the conclusions and identifies the research prospects.

\section{Materials and Methods}

\section{Methodological Background}

In the field of decision making, Multiple Criteria Decision Aid (MCDA) has become a widespread tool capable of dealing with problems involving more than one goal. A number of theorists $[43,44]$ and empirical studies [45-47] have shown that the MCDA can manage the complexity of the entire process and through a participatory process can help avoid conflicts and make transparent decisions more effective, involving different points of view in the initial phase of the project/problem [48].

These decision support methods, in addition to the possibility of considering data of different nature (quantitative and qualitative), offer the opportunity to assign different weights to the criteria identified, manage conflicts between objectives, and deduce priorities among alternative options.

Many Multi-Criteria Decision-Making (MCDM) methods and techniques have been proposed by researchers in the past decades. Some of the most important ones are Weighted Sum Model (WSM) [49], Weighted Product Model (WPM) [50], Weighted Aggregated Sum Product Assessment (WASPAS) [51], Analytical Hierarchy Process (AHP) [52], ELimination Et Choix TRaduisant la REalité (ELECTRE) [53], Technique for Order of Preference By Similarity to Ideal Solution (TOPSIS) [54], Preference Ranking Organization Method for Enrichment of Evaluations (PROMETHEE) [55], Complex Proportional Assessment (COPRAS) [56], Visekriterijumska Optimizacija i kompromisno Resenje (VIKOR) [57], Multiobjective Optimization by Ratio Analysis plus Full Multiplicative Form (MULTIMOORA) [58], Additive Ratio Assessment (ARAS) [59], Evaluation Based on Distance from Average Solution (EDAS) [60], and Combinative Distance-Based Assessment (CODAS).

The choice of a particular multi-criteria method depends on the specific problem and user needs. It can be pointed out that the number of existing methods is already large enough and is still increasing, and choosing the "right" method is not an easy task. To facilitate the selection of the appropriate method for decision making, a list of quality criteria has been developed [9] that can be used to reveal the strengths and weaknesses of MCDA methods compared to their application to specific problems. For the evaluation of the individual methods, quality criteria were used to highlight differences in the characteristics of the methods presented. The study also highlighted that selecting the best method is not possible. In managing complex situations, all methods show similar performances with respect to the aspects and scales that can be considered.

All the MCDM methods mentioned above have advantages and disadvantages - their evaluation is not the purpose of this document. In this document, a hybrid method to manage multi-criteria decision problems has been proposed. Furthermore, in multi-criteria analysis models, determining the weights of the criteria is another of the key problems of the decision-making process as the weights of the criteria can significantly influence the outcome of the decision-making process. It is therefore clear that particular attention must be paid to the factors of objectivity in attributing the weights to the criteria.

A study on the advantages and disadvantages of individual criteria weighting methods is provided by Roberts and Goodwin [61]; it is also recognized that the values of the weights of the criteria are significantly conditioned by the methods used for their determination [61-65].

In the literature, it is possible to find a classification [53] of the methods for determining the weights of the criteria, but there is no agreement on the best method for determining the weights of the criteria. In the literature, most authors suggest a classification of the models for determining the weights of the criteria distinguishing subjective and objective models. Subjective approaches mirror the decision maker's subjective opinion and intuition. With such an approach, the decision maker 
directly influences the outcome of the decision-making process, since the weights of the criteria are determined on the basis of information obtained from the decision maker or experts involved in the decision-making process. Objective approaches neglect the decision maker's opinion and are based on determining the weights of the criteria based on the information contained in a decision matrix that applies certain mathematical models.

The best-known subjective methods for weighting the values of the criteria are the Analytic Hierarchy Process (AHP) method, the Step-Wise Weight Assessment Ratio Analysis (SWARA), the Best Worst Method (BWM), and the Full Consistency Method (FUCOM). Each of these methods has a wide application in the various areas of science, technology, and in solving real life problems.

It can be pointed out that, despite being a relatively old method, the AHP method is still used in a large number of publications. This confirms the conclusions of Zavadskas et al. [66] that the AHP method is the most commonly used method for determining the weights of the criteria and/or classifying alternatives.

The Analytic Hierarchy Process (AHP) method [67] is certainly one of the most commonly applied methods based on pairwise comparisons to determine the weights of alternative criteria and/or rankings [67]. The validation of the results in the AHP model is based on the degree of consistency, the value of which is limited to 0.10 . In the AHP method, $n(n-1) / 2$ pairwise must be performed. It should also be added that a large number of comparisons complicate the application of the model, especially in cases of a greater number of criteria. According to some authors [68], it is almost impossible to perform fully coherent pairwise comparisons in the AHP method if more than nine criteria exist. This problem is usually overcome by dividing the criteria into sub-criteria, which further complicates the model.

Therefore, this document, in order to classify different reuse alternatives [69-71], combines different multi-criteria methods in the different stages of the decision-making process in order to develop a "quick" tool to improve the quality and reliability of the decision-making process itself, especially in the planning decision phase. The Figure 1 presents the methodological framework of the decision support process used in the work.

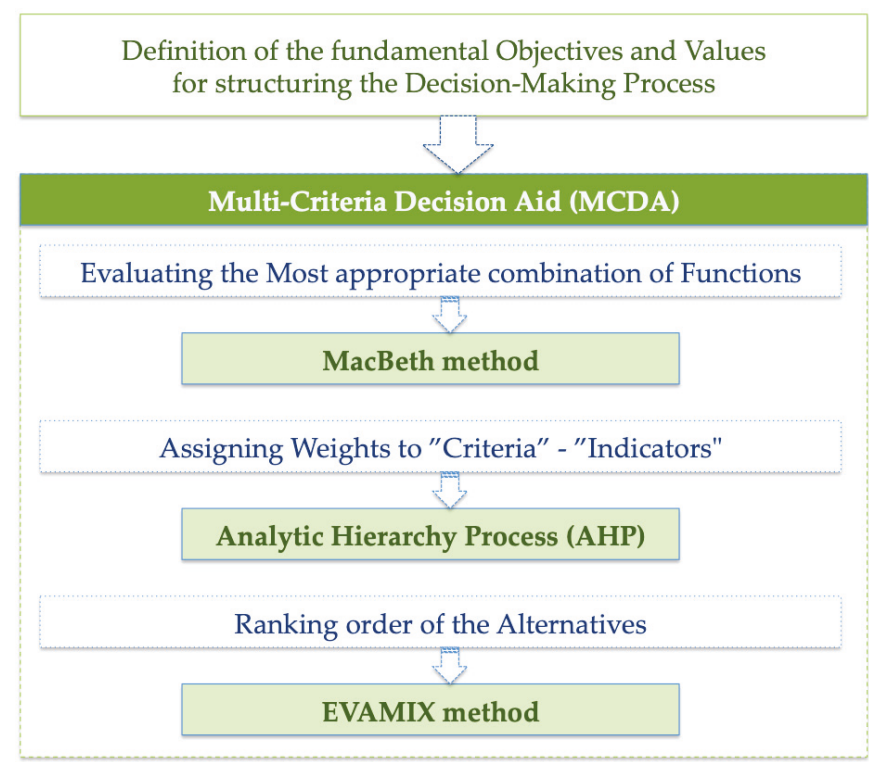

Figure 1. Methodological framework of the decision support process. 
In detail, to address the complexity of the decision problem under consideration and to support the choice about the highest and best adaptive reuse of defensive cultural heritage under study, an assessment has been implemented through the combination and integration of three multi-criteria methods: the MacBeth method [72-74], the Analytic Hierarchy Process (AHP) [68,75], and the Evaluation of Mixed data (EVAMIX) [75-79].

The combination and integration of multi-criteria methodologies [80-82] also allows the decision maker (especially in the planning phase) to classify the alternatives from the best to the worst, using a simple, effective and logical decision-making method.

In addition, the EVAMIX method was chosen as it is a fairly effective method compared to other approaches due to its simplicity of approach and the fact that it requires less time regarding the necessary mathematical calculations. To the knowledge of the authors of the article, this study represents the first experimentation relating to the combination of techniques of this type.

The application of the MacBeth evaluation method (Measuring Attractiveness by a Categorical Based Evaluation Technique) [72-74] was useful to evaluate the multidimensional impacts that the different functional choices (resulting from knowledge and participatory phases) can have on the general objective-increase the multidimensional productivity of the city. The elaboration of the data collected in the preliminary phase of knowledge and participation (focus groups and questionnaires) allowed to define the priorities regarding the functions to be included in the alternative scenarios. In the MacBeth method, the evaluation of priorities is based on pairwise comparisons and adopts an intervallic scale [8].

The Evaluation of Mixed data (EVAMIX) method [76-79], from a procedural point of view, allows the construction of an " $\mathrm{m} \times \mathrm{n}$ " evaluation matrix, characterized by " $\mathrm{m}$ " criteria and " $\mathrm{n}$ " alternative options, where the components are both qualitative rather than quantitative [83,84], whose lines express the performance of each alternative with respect to a specific criterion (see APPENDIX A Matrix of Evaluation). Definite 2.0 software was used to implement the EVAMIX method [85].

From a procedural point of view, the EVAMIX method consists of seven steps, for the analytical-mathematical procedure it's possible refer to the reference literature [65-68].

In practice, the EVAMIX method consists of the following passages [76-79]:

(1) distinction between ordinal and cardinal criteria;

(2) calculation of dominance scores for all ordinal and cardinal criteria;

(3) calculation of standardized dominance scores for all ordinal and cardinal criteria;

(4) calculation of overall dominance scores;

(5) calculation of evaluation scores.

The first step is the construction of an evaluation matrix E, which is a mxn matrix, characterized by $m$ evaluation criteria and $n$ alternative options. The qualitative or quantitative data, contained in the matrix, express the performance of each alternative with respect to a given criterion.

The set of criteria is divided into two subsets- the set $O$ is the set of ordinal criteria (qualitative) and the set $C$ is the set of cardinal criteria (quantitative). This leads to two distinct evaluation matrices-EO (ordinal criteria/alternative options) and CE (cardinal criteria/alternative options).

Therefore, the differences between the alternatives can be expressed through two measures of dominance, the first based on ordinal criteria, the second on cardinal criteria. In particular, in order to construct the cardinal score of dominance, the components of EC are standardized by a common unit; this allows all quantitative criteria to be expressed on a scale from 0 to 1 . The weights can be assigned to both criteria, both quantitative and qualitative.

In the second step, it is possible to calculate the "dominance scores" for all ordinal and cardinal criteria; these scores reflect the degree to which one option exceeds the other.

Subsequently (step 3), the dominance scores are standardized with the same unit of measurement to be comparable to each other, and two "standardized dominance measures" are deduced for all ordinal and cardinal criteria. 
Thus (step 4), a "measure of overall dominance" is calculated for each pair of alternatives, which translates the degree to which an option dominates over another option.

Finally (step 5), starting from the overall dominance measure, the "evaluation scores" are calculated for each alternative: the result is a complete classification of the alternatives, where the optional alternative is the one with the evaluation score higher.

The AHP Method, developed by Thomas Lorie Saaty [68,75], structures decision making in the form of hierarchy. From a procedural point of view, this method consists of the following three phases:

(1) construction of an adequate hierarchy;

(2) definition of priorities between elements of the hierarchy by comparisons in pairs;

(3) verification of the logical coherence of pairwise comparisons.

In the first step, the AHP method helps to break down complex systems into simple structures. This simplification is made possible by a logical process that aims at the construction of adequate hierarchies.

The simplest hierarchy model consists of three levels. The first level is that of the main objective (called "Objective") of the decision problem; the second and third levels include criteria and alternatives. However, it is possible to develop more complex (i.e., multi-level) hierarchies including a number of sub-criteria. This means that the factors that influence the decision are organized in gradual steps from the general, in the upper level of the hierarchy, to the particular, in the lower levels.

In the AHP method, pairwise comparisons (i.e., comparison of elements in pairs with respect to a given criterion) are used to establish priorities (or weights) between elements of the same hierarchical level (step 2). These elements are compared in pairs with respect to the elements corresponding to the next higher level. This comparison translates into a matrix of pairwise comparisons.

To represent the relative importance of one element with respect to another, a suitable evaluation scale called the "Saaty scale" is introduced. It defines and explains the values 1 to 9 assigned to the judgments to compare pairs of elements of each level with respect to a criterion in the next higher level.

Pairwise comparisons are organized in appropriate matrices, since each of them is related to "priority vectors" (expressed on a 0-1 scale) and, when aggregated, they provide a complete classification of alternatives.

For pairwise comparisons, both quantitative and qualitative data can be used. As for the third phase, it should be noted that when comparing the elements, a certain degree of inconsistency can arise: in the AHP approach, a "coherence ratio" is calculated for each matrix of pairwise comparisons to verify the degree of inconsistency. A consistency ratio of 0.10 or less is considered acceptable; if this ratio is greater than 0.10 , it is necessary to reformulate the judgments using new pairwise comparisons.

\section{Application}

\subsection{The Case Study and Presentation of the Alternatives}

This research relates to the reuse of defensive historical and cultural heritage located in Southern Italy. In particular, the study concerns the "Forti Umbertini" System located along the shores of the "Stretto di Messina" (Figure 2) of which it is possible to see some representative images in Figure 3. 


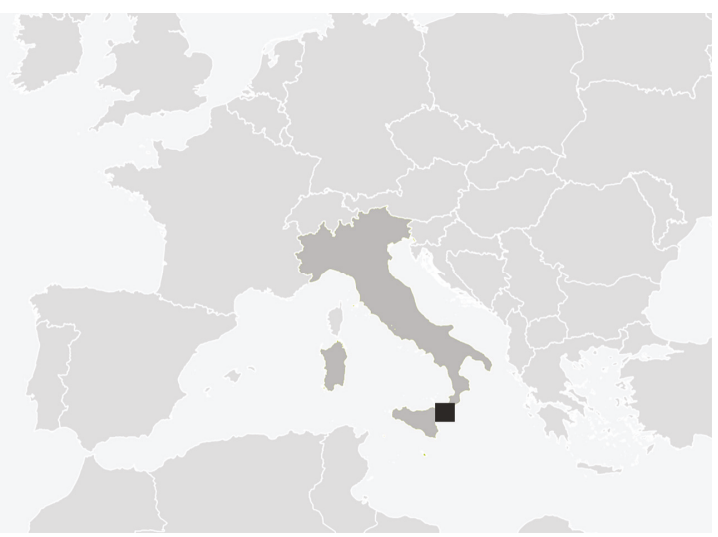

Figure 2. Location of the "Stretto di Messina" in South Italy.
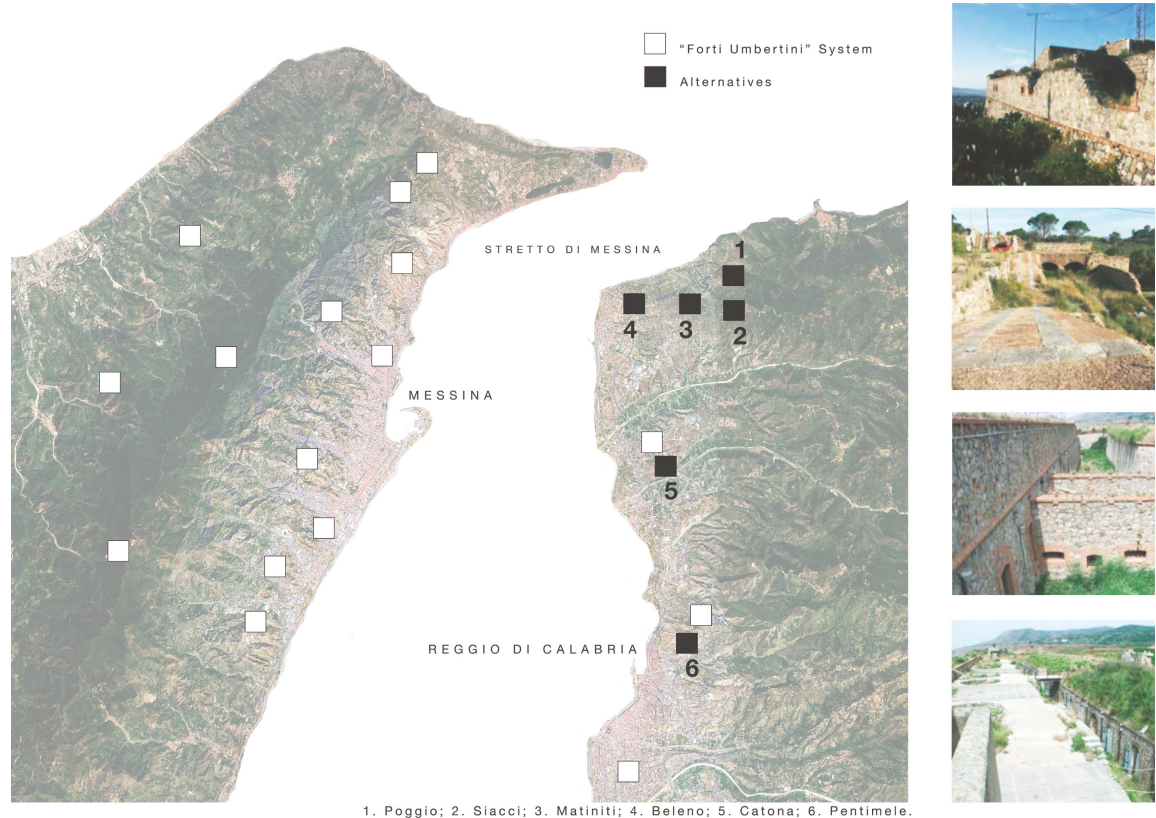

1. Poggio; 2. Siaceli, 3,-Matiniti, 4. Beleno; 5 . Catona; 6. Pentimele.
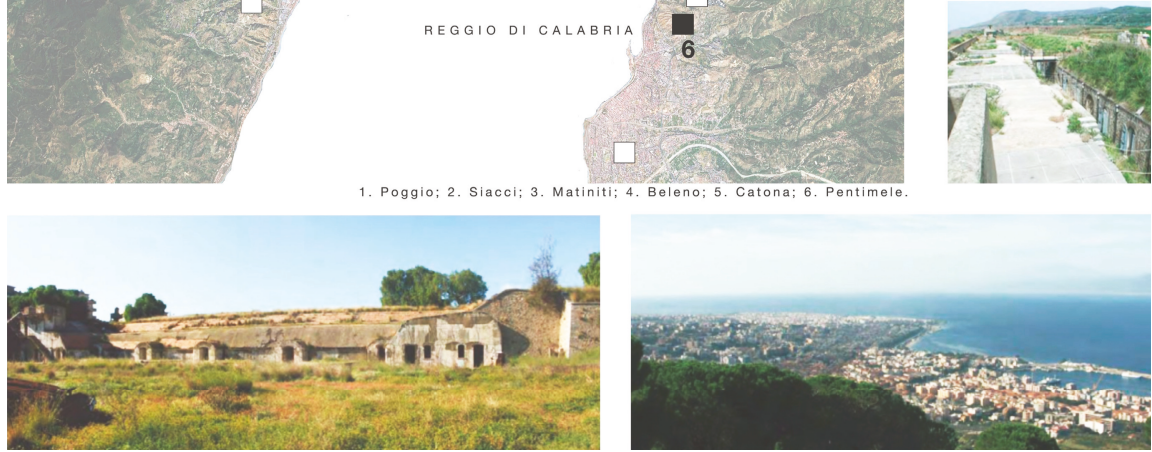

Figure 3. The "Forti Umbertini" System (source: authors' processing).

The system of the forts was built after the Italian Unification in 1860, as part of a system of military fortifications that controlled the entire eastern hill line of the Strait. It includes twenty-two fortified historic buildings, now entered into the common imagination with the term "Forti Umbertini." They were sentinels defending the waterway with the aim of not interrupting the continuity of defense 
of the new national territory. Built by the new unified Italian State in order to defend the Strait of Messina from external enemies. They form a unique system in the world consisting of twenty fortified positions placed on the two shores of Sicily and Calabria. Since the king of the time was Umberto I, they are also known as "Forti Umbertini." These camouflaged architectures ensured the defense of the territory until World War Two.

The fortified structures, which survived the 1908 earthquake and the two World Wars unscathed, since the military administration handed over deliveries to the State Property Agency, have been, for military servitude, kept away for many years from the attention of citizens and have suffered the scarce attention of local administrations that have never grasped their great tourist and economic potential.

Starting from the analysis of the network of Forts located in the area [86] we have identified six alternative buildings not yet used and enhanced, thus allowing the introduction of new functions and destinations. The Figure 3 shows the map with the alternatives considered in the evaluation process.

\subsection{Implementation of Multicriteria Methods to the Case Study}

The main objective of the research is to identify among the six forts what is most suitable for hosting new functions/uses.

In order to assess the suitability of the cultural heritage selected for adaptive reuse, through a participatory process four potential reuse scenarios have been identified, which represent four different alternative uses.

In the present study, the evaluation was conducted through the integration of three multi-criteria approaches-the MacBeth method, AHP, and EVAMIX [76-79]. The MacBeth method [60-62] was helpful when evaluating the most appropriate combination of functions to enhance the historical heritage and resources of the territory, the EVAMIX method [6] was used to deduce a classification among the alternatives, characterized by qualitative and quantitative information. The AHP method $[68,75]$ was used to assign weights to "criteria" and "indicators."

\subsubsection{MacBeth Implementation of the Method}

The preliminary assessment phase was useful for processing the data collected, through focus groups and questionnaires, and defining the alternatives/functions to be included in the cultural heritage under study.

The participatory process has allowed users to acquire local information, analyze possible conflicting behaviors, and produce more preferable and shared alternatives. In this phase, four groups of stakeholders have been identified:

- Institutions: Calabria Region, Metropolitan city, Municipality, Superintendence;

- Technical-professional organizations: Professional Association of Geologists, Professional Association of Architects, Professional Association of Engineers;

- Professionals in real estate and cultural heritage;

- Association of Builders Construction of Reggio Calabria (ACEN), Industrial Union, Trade Union;

- Tourism and Trading;

- Community: Residents, Representatives of associations.

The questionnaires were administered to the community and to the association's representatives; focus groups involved representatives of institutions and technical-professional organizations.

Below is the list of functions deduced from the participatory process.

1. Co-working spaces;

2. Spaces for higher education;

3. Multimedia library;

4. Spaces for theater activities;

5. Music school; 


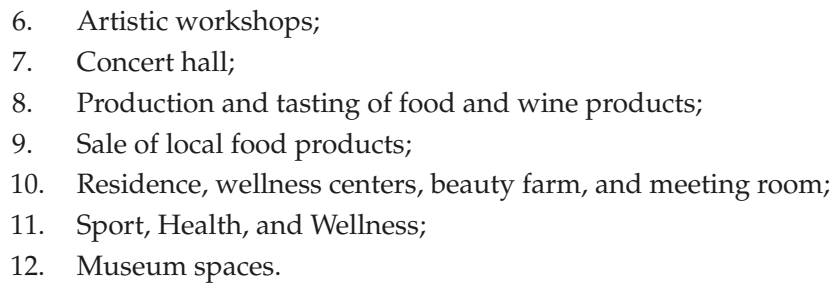

For the evaluation of the priorities of the alternative functions to be used for the implementation of the scenarios, the MacBeth evaluation method [72-74] wich was chosen for the possibility of processing qualitative judgments from various data sources (focus groups and questionnaires). This method uses a synthetic matrix, which links options (functions) to potential multi-dimensional impacts on defined impact categories/criteria.

The impact indicators have been extracted, through focus groups with expert actors, and take into account the multidimensional impacts (economic, social, environmental, and cultural) on the wider territorial area according to the systemic perspective of the landscape proposed in the UNESCO recommendation on the historical urban landscape $[87,88]$. The categories of impact/criteria are i. Tourism and recreation; ii. Creative, cultural and innovative activities; iii. Local typical productions; iv. Environment and natural capital; v. Social and community cohesion, vi. Real estate; vii. Financial performance; viii. Welfare; ix. Cultural value of the property/landscape, which have been identified in relation to the general objective aimed at increasing productivity in more dimensions of the territory interested [89].

A judgment matrix was then elaborated with pairwise comparisons between the different alternative functions with respect to each indicator. Impacts of each function on each category of indicators were determined starting from the qualitative judgments expressed using the seven semantic categories of MacBeth (no impact; very weak; weak; moderate; strong; very strong; extreme).

After the phase referring to each fundamental criterion and to the attribution of the weights, the final aggregation phase was elaborated and a final classification of preferences was elaborated in relation to the general objective. Below, the MacBeth scale represents the final classification of the impacts that each option/function has on the criteria.

11. Sport, Health and Wellness;

6. Artistic workshops;

5. Music school;

4. Spaces for the theater;

7. Concert hall;

10. Residence, wellness centers, beauty farm, and meeting room;

2. Spaces for higher education;

9. Sale of local food products;

8. Production and tasting of food and wine products;

1. Co-working spaces;

3. Multimedia library;

12. Museum spaces.

The group of experts (entrepreneurs, investors, and professionals in the real estate, tourism, and cultural heritage sectors) subsequently interacted and combined the various selected functions (among those that were compatible) and designed four adaptive reuse scenarios, representative of potential changes in current use on the economic, social, environmental and cultural plan. 


\subsubsection{EVAMIX Implementation of the Method}

In relation to the chosen functions and in order to assess the suitability for adaptive reuse of the six selected forts, four potential reuse scenarios have been implemented. These scenarios represent the potential uses that can be successfully hosted in the military fortifications in question. The scenarios are the result of the combination of the functions selected through the MacBeth Method [72,73,73].

In relation to each decision scenario, the six alternative buildings have been evaluated and classified from the most to the least suitable for use in relation to each scenario, based on the criteria chosen based on the characteristics of the alternatives to be evaluated.

To structure the EVAMIX decision process [76-79] we proceeded according to the following phases:

Step 1. Identification of the assessment scenarios. Four different scenarios have been designed by the group of experts, as a result from of the integration of multiple functions chosen among those classified and judged most suitable and representative of potential changes in term of economic, social, environmental and cultural terms development. The following scenarios represent real and potential uses that can be successfully hosted in abandoned fortifications:

- Artistic/Cultural Park: art gallery, studios/residences/artists' workshops (designers, architects, painters, sculptors, etc.).

- Design Center: co-working spaces, multi-functional equipped classrooms with spaces for conferences and university events and high education, museums, bars, and restaurants.

- Food Farm: theme park dedicated to agribusiness. Meeting areas for schools, universities and companies. The idea is to offer study courses on the production techniques of local products and wine and food itineraries of the Strait Region.

- Health and Wellness Center: heliotherapy, thalassotherapy, kiosks and bars, green public areas, recreational paths for childcare, urban agriculture

Step 2. Construction of the criteria tree. The set of criteria used in the evaluation process was formulated by a focus group of experts including entrepreneurs, investors and professionals in the real estate, tourism and cultural heritage sectors, in which the different points of view were discussed. Subsequently the research group has elaborated and translated the information in a limited number of criteria [68], clearly defined based on the characteristics of the alternatives to be evaluated, choosing above all the relevant ones (coherence with the objectives of the project) because a large number of indicators could make the evaluation process more complex and less effective.

The structure of the criteria considers the relevant aspects of the decision-making problem under consideration-environmental quality, economic aspects, context infrastructuring, and architectural features-in relation to the main strategic objective of the decision maker (DM) to create a new catalyst for the entire urban development process of the Strait area.

Step 3. Construction of the evaluation matrix. A $11 \times 6$ matrix characterized by mxn (eleven evaluation criteria $x$ six alternative options) whose components are cardinal (quantitative) and ordinal (qualitative) data, which express the impact/performance of each alternative with respect to a given criterion. The evaluation matrix is shown in Appendix A.

Step 4. Criteria weighting. To assess the importance of the criteria, we made some interviews with the group of experts to indicate the weights for each of the criteria. Then AHP method was therefore implemented of the comparison in pairs [68,75], through the use of the "Saaty scale," to establish priorities (weights) and compare pairs of elements of each level with respect to a criterion in the next higher level. During the brainstorming process, with the help of a moderator, the experts reached consensus on the final set of weights.

The overall set of weights of criteria and sub-criteria is displayed in Appendix A. Note that, in the evaluation matrix, the values enclosed in round brackets are weights assigned in the AHP assessment $[68,75]$.

Step 5. Classification of alternatives. In order to obtain the priority vectors and the final ranking, the EVAMIX method was implemented in the Definite 2.0 software [85]. 
In order to classify the adaptive reuse alternatives with respect to each scenario, it was simulated the investment decision of potential Decision Makers (DM) who expressed their expert judgment [90]. To this end, on the basis of their relevant experience and strong interest in the reuse of historical-cultural buildings, the group of experts was asked to identify which of the four adaptive reuse scenarios is the most suitable for the six alternatives of historic fortifications under study.

The results and the ranking order of alternatives are discussed in the following section.

\section{Results and Discussion}

Figures 4 and 5 show the overall values of the EVAMIX assessment and the ranking of alternatives for the different uses considered in the study. According to the results obtained, alternative 1. Poggio is the most suitable building to be reused for different purposes and scenarios considered: Design Center, Artistic/Cultural Park, Health and Wellness Center, and Food Farm.

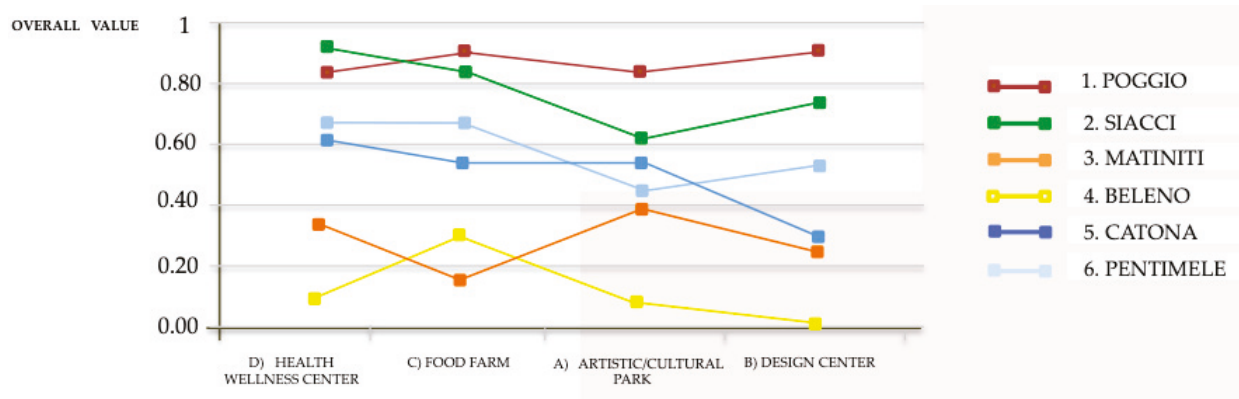

Figure 4. Evaluation of Mixed data (EVAMIX) method: overall value for the different scenarios.

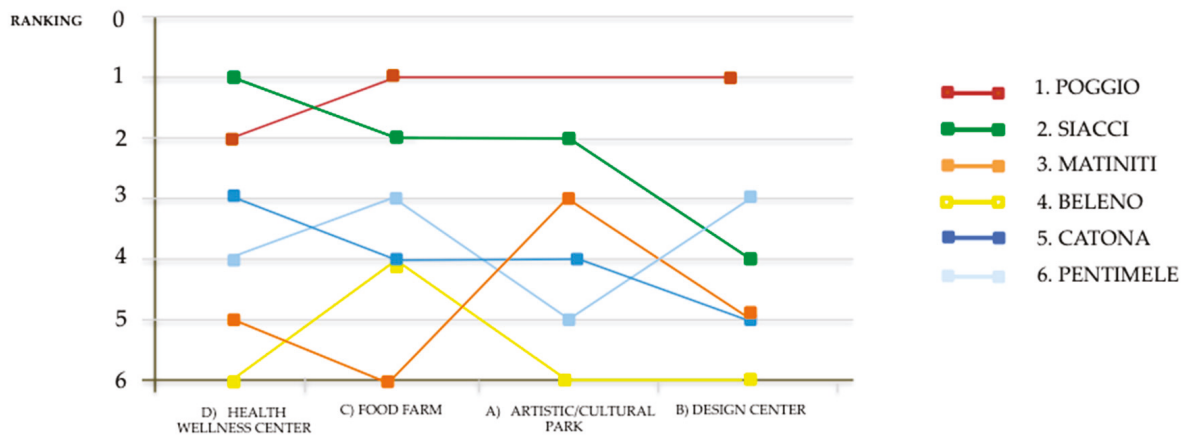

Figure 5. EVAMIX method: ranking of the alternatives for the different scenarios.

From the results obtained, it is possible to say that the "1. Poggio" alternative is the best alternative for three out of four scenarios. The alternative "2. Siacci" also has excellent performance, since it is classified as the best alternative for the scenario: Health and Wellness Center and as the second best alternative for the scenarios: Design Center, Artistic/Cultural Park, Agriturismo. Furthermore, it should be noted that the "Beleno" is the alternative classified as the worst alternative in three out of four scenarios.

This result is influenced by the greater weight (relative importance) attributed by the experts to the criterion "Project Sustainability" (relative to the indicators: Degradation level, architectural characteristics and Flexibility of spaces to new functions, Total property availability), which in relation to the potential for reuse are of great importance [91] (Appendix A). 
After obtaining a classification of alternatives and checking the consistency obtained in the results, it is useful to carry out a sensitivity analysis on the final outcome of the model. Sensitivity analysis concerns a type of "what if" question, which allows us to check if the final answer is stable when the model inputs are changed. It is of particular interest to see if these changes change the order of the alternatives.

In the present study, sensitivity analysis was performed regarding the weights of the criteria. In particular, the weight of one criterion at a time was increased up to $60 \%$ while the weights of the other criteria were kept equal to $20 \%$. The evaluation model was performed considering again the weights and the final priorities of the alternatives were recalculated.

The Figure 6 represents the results of the sensitivity analysis performed. As can see, three alternatives-Poggio, Siacci, and Pentimele-have the best performances among the scenarios considered, while the Beleno alternative is ranked last.

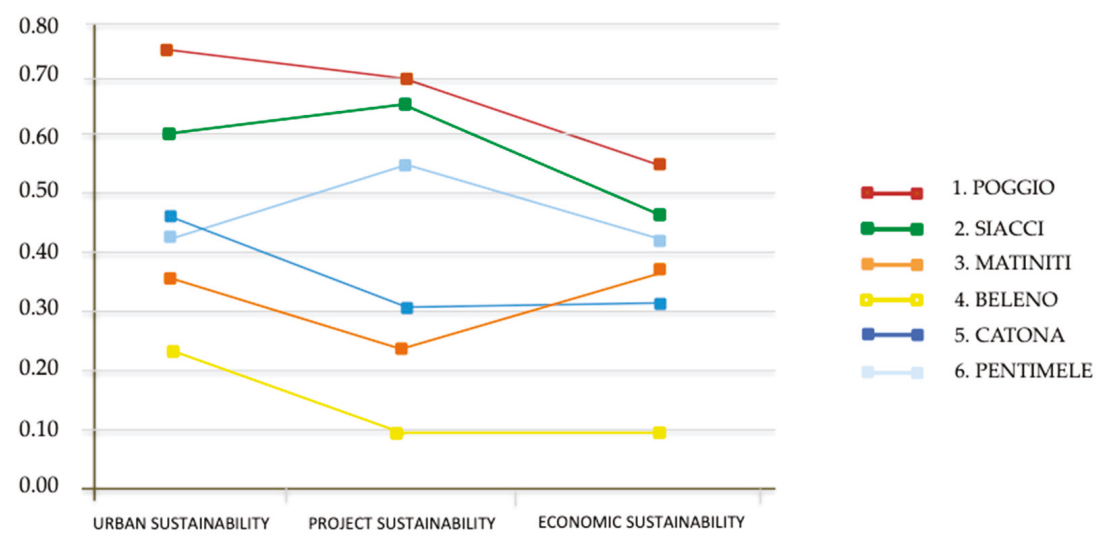

Figure 6. EVAMIX method: results of the sensitivity analysis.

\section{Conclusions}

This document proposes a methodology that integrates different multi-criteria evaluation methods to support the decision-making process regarding the choice of sustainable alternative functions, aimed at the adaptive reuse of the historical-cultural heritage [92]. This theme is of particular importance because the phenomenon of the reuse of unused or abandoned historical and cultural heritage is becoming increasingly important, especially in Italy, where there are thousands of architectural quality assets that constitute a real resource for the territory.

The multi-criteria decision support method uses qualitative and quantitative criteria to evaluate four different reuse scenarios for the redesign of the "Forti Umbertini" system located in Southern Italy. The different scenarios were designed by a group of experts who also assigned the weights to the different criteria involved in the evaluation model.

The nature of the public goods considered by the enhancement projects and the strong articulation that characterizes the territorial redevelopment projects require complex approaches.

The application developed in the present study proved to be very advantageous, especially when planning interventions, to support complex decisions, about alternative scenarios, in which it is necessary to make a choice regarding alternative scenarios that take into account different points of view and the involvement of experts in the evaluation process.

For this purpose, the evaluation process was structured in order to incorporate the participation of all stakeholders in defining a "shared solution" capable of meeting the needs of the local community, and the same time being sustainable over time from an economic point of view. 
The methodological approach based on the integration of participatory processes and the multi-criteria analyzes, in a proposed multidimensional perspective, allowed to include the opinions of the stakeholders in the decision-making process. In this study, the involvement of the local community is one of the key factors for the success of this type of process and represented a fundamental support for the decision-making process. The integration between the community and specialist knowledge guarantees a higher level of acceptability of the results, reaching greater consensus, outlining strategies that are as shared as possible. It helps guide strategic choices better. The involvement of all stakeholders and actors facilitated the acquisition of information and knowledge that supported the decision-making process; it also ensured credibility and transparency in the process. In addition, this integrated evaluation process, in addition to considering the point of view of the various stakeholders, has enabled the generation of alternatives (possible functions) to be considered. Through the participation of all interested parties, the implementation and integration of multi-criteria methodologies, it was possible to define a "shared solution" and obtain a ranking of the alternatives for each decision scenario.

According to the results obtained, the "Poggio" alternative is the most suitable building to be reused for different purposes. This is due to the fact that the building is in good condition and the space configuration is very flexible to accommodate new and differentiated functions. The "Siacci" alternative contains the best features to be reused as a Health and Wellness Center due to the possibility of a large area that can be used for complementary functions. On the contrary, the "Beleno" alternative is classified in the last position in all the decision scenarios considered.

The results obtained, validated by the sensitivity analysis, are consistent in defining the most suitable building for adaptive reuse with respect to specific uses.

This study could have interesting implications, as it could represent a useful tool for policy makers in the process of planning and designing a general plan aimed at re-launching and enhancing the immense heritage with a view to creating a circular economy and promoting the economic development of the territory under investigation [93].

Today, the new challenge for local authorities is to regenerate abandoned heritage buildings while involving various stakeholders and the local community in order to create new governance models that are able to guarantee economic sustainability and conservation of values of historical and cultural heritage [94-96], as in the case of the "Forti Umbertini" under study, which have always been a symbol for the Strait community.

According to this perspective, future research in this field can be oriented towards the search for new management models, based on the concept of "heritage as common goods" [97-99], based on agreements between public promoters and Profit or No-Profit associations for the management of common goods $[95,96]$.

Funding: This research received no external funding.

Conflicts of Interest: The author declare no conflict of interest. 


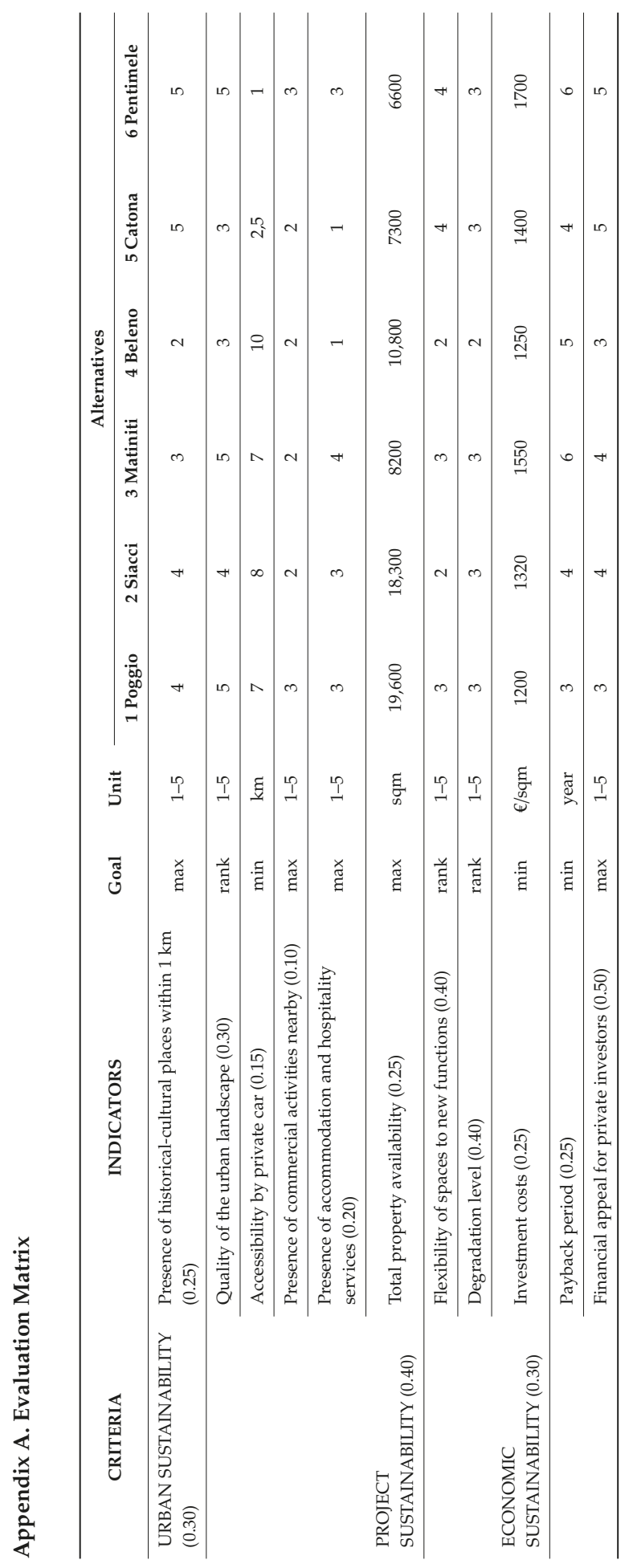




\section{References}

1. Klamer, A. The values of cultural heritage. In Handbook on the Economics of Cultural Heritage; Rizzo, I., Mignosa, A., Eds.; Edward Elgar: Cheltenham, UK, 2013.

2. Fusco Girard, L.; Gravagnuolo, A. Circular economy and cultural heritage/landscape regeneration. Circular business, financing and governance models for a competitive Europe. BDC 2017, 2017, 35-52.

3. Zeleny, M. Autopoiesis and self-sustainability in economic systems. Hum. Syst. Manag. 1997, 16, 251-262. [CrossRef]

4. Kincaid, D. Adapting Buildings for Changing Uses: Guidelines for Change of Use Refurbishment; Spon: New York, NY, USA, 2002.

5. Wang, H.; Zeng, Z. A multi-objective decision-making process for reuse selection of historic buildings. Expert Syst. Appl. 2010, 37, 1241-1249. [CrossRef]

6. Fuentes, J.M. Methodological bases for documenting and reusing vernacular farm architecture. J. Cult. Herit. 2010, 11, 119-129. [CrossRef]

7. Simons, R.A.; Choi, E. Adaptive Reuse of Religious Buildings and Schools in the US: Determinants of Project Outcomes. Int. Real Estate Rev. 2010, 13, 79-108.

8. Ishizaka, A.; Nemery, P. Multi-Criteria Decision Analysis: Methods and Software; Wiley: Chichester, UK, 2013.

9. De Montis, A.; De Toro, P.; Droste-Franke, B.; Omann, I.; Stagl, S. Assessing the quality of different MCDA methods. In Alternatives for Environmental Evaluation; Getzner, M., Spash, C.L., Stagl, S., Eds.; Routledge: Abingdon-on-Thames, UK, 2004; pp. 99-133.

10. Ferretti, V.; Bottero, M.; Mondini, G. Decision making and cultural heritage: An application of the Multi-Attribute Value Theory for the reuse of historical buildings. J. Cult. Herit. 2014, 15, 644-655. [CrossRef]

11. Plevoets, B.; Van Cleempoel, K. Adaptive reuse as a strategy towards conservation of cultural heritage: A literature review. WIT Trans. Built Environ. 2011, 118, 155-164.

12. Elsorady, D. The economic value of heritage properties in Alexandria, Egypt. J. Cult. Herit. 2014, 15, 511-521. [CrossRef]

13. Giove, S.; Rosato, P.; Breil, M. An application of Multicriteria Decision Making to built heritage. The redevelopment of Venice Arsenale. J. Multi. Crit. Decis. Anal. 2011, 17, 85-99. [CrossRef]

14. Misırlısoy, D.; Gunçe, K. Adaptive reuse strategies for heritage buildings: A holistic approach. Sustain. Cities Soc. 2016, 26, 91-98. [CrossRef]

15. Bryan, B.A.; Grandgirard, A.; Ward, J.R. Quantifying and exploring strategic regional priorities for managing natural capital and ecosystem services given multiple stakeholder perspectives. Ecosystems 2010, 13, 539-555. [CrossRef]

16. Thórhallsdóttir, T.E. Environment and energy in Iceland: A comparative analysis of values and impacts. Environ. Impact Asses 2007, 27, 522-544. [CrossRef]

17. Hamadouche, M.A.; Mederbal, K.; Kouri, L.; Regagba, Z.; Fekir, Y.; Anteur, D. GIS-based multicriteria analysis: An approach to select priority areas for preservation in the Ahaggar National Park, Algeria. Arab J. Geosci. 2014, 1-16. [CrossRef]

18. Palmas, C.; Abis, E.; von Haaren, C.; Lovett, A. Renewables in residential development: An integrated GIS-based multicriteria approach for decentralized micro-renewable energy production in new settlement development: A case study of the eastern metropolitan area of Cagliari, Sardinia, Italy. Energy Sustain. Soc. 2012, 2, 1-15. [CrossRef]

19. Di Bitonto, P.; Laterza, M.; Roselli, T.; Rossano, V. Multicriteria retrieval in cultural heritage recommendation systems. In KES 2010, Part II, LNAI 6277; Setchi, R., Setchi, R., Jordanov, I., Howlett, R.J., Jain, L.C., Eds.; Springer: Berlin, Germany, 2010; pp. 64-73.

20. Paolillo, P.L.; Benedetti, A.; Baresi, U.; Terlizzi, L.; Graj, G. An assessment-based process for modifying the built fabric of historic centres: The case of Como in Lombardy. In Computational Science and Its Applications - ICCSA 2011 (vol 6782); Murgante, B., Gervasi, O., Iglesias, A., Taniar, D., Apduhan, B.O., Eds.; Springer: Berlin, Germany, 2011; pp. 162-176.

21. Tarragüel, A.A.; Krol, B.; van Westen, C. Analysing the possible impact of landslides and avalanches on cultural heritage in Upper Svaneti, Georgia. J. Cult. Herit. 2012, 13, 453-461. [CrossRef] 
22. Cerreta, M.; Panaro, S.; Cannatella, D. Multidimensional spatial decision-making process: Local shared values in action. In ICCSA 2012, Part II, LNCS 7334; Murgante, B., Gervasi, O., Misra, S., Nedjah, N., Rocha, A.M.A.C., Taniar, D., Apduhan, B.O., Eds.; Springer: Berlin, Germany, 2012; pp. 54-70.

23. Girard, L.F.; De Toro, P. Integrated spatial assessment: A multicriteria approach to sustainable development of cultural and environmental heritage in San Marco dei Cavoti, Italy. CEJOR 2007, 15, 281-299. [CrossRef]

24. Fuentes, J.M. Basi metodologiche per documentare e riutilizzare l'architettura della fattoria vernacolare; Elsevier: Amsterdam, The Netherlands, 2010; pp. 119-129.

25. Dutta, M.; Husain, Z. An application of Multicriteria Decision Making to built heritage. The case of Calcutta. J. Cult. Herit. 2009, 10, 237-243. [CrossRef]

26. Geraedts, R.P.; van der Voordt, T.; Remøy, H. Conversion Potential Assessment Tools. In Building Resilience in Urban Settlements through Sustainable Change of Use; Remøy, H., Wilkinson, S.J., Eds.; Wiley-Blackwell: Hoboken, NJ, USA, 2018; pp. 121-151. ISBN 978-1-119-23142-4.

27. Celadyn, M. Interior Architectural Design for Adaptive Reuse in Application of Environmental Sustainability Principles. Sustainability 2019, 11, 3820. [CrossRef]

28. Pinto, M.R.; De Medici, S.; Senia, C.; Fabbricatti, K.; De Toro, P. Building reuse: Multi-criteria assessment for compatible design. Int. J. Des. Sci. Technol. IJDST 2017, 22, 165-193.

29. Roy, B. Multicriteria methodology for decision aiding; Springer Science \& Business Media: Berlin, Germany, 2013; Volume 12.

30. Bánáthy, B.H. Guided Evolution of Society: A Systems View (Contemporary Systems Thinking); Springer: Berlin, Germany, 2000.

31. Jackson, M. Systems Thinking: Creating Holisms for Managers; Wiley: Chichester, UK, 2003.

32. Checkland, P.; Poulter, J. Learning for Action; Wiley: Chichester, UK, 2006.

33. Ackoff, R.L. Systems Thinking for Curious Managers; Triarchy Press: Gillingham, UK, 2010.

34. Allmendinger, P.; Haughton, G. Soft spaces, fuzzy boundaries, and metagovernance: The new spatial $\mathrm{p}$ lanning in the Thames Gateway. Environ. Plan. A 2009, 41, 617-633. [CrossRef]

35. Carlsson-Kanyama, A.K.; Dreborg, H.; Moll, H.; Padovan, D. Participative backcasting: A tool for involving stakeholders in local sustainability planning. Futures 2007, 40, 34-46. [CrossRef]

36. Fusco Girard, L.; Cerreta, M.; De Toro, P. Integrated planning and integrated evaluation. Theoretical references and methodological approaches. In Beyond Benefit Cost Analysis. ACCOUNTING for Non-Market Values in Planning Evaluation; Miller, D., Patassini, D., Eds.; Ashgate: Aldershot, UK, 2005; pp. 175-205.

37. Pearce, D.W.; Turner, R.K. Economics of Natural Resources and the Environment; Johns Hopkins University Press: Baltimore, MD, USA, 1990.

38. European Commission. Towards an Integrated Approach to Cultural Heritage for Europe. 2014. Available online: http://ec.europa.eu/culture/library/publications/2014-heritage-communication_en.pdf (accessed on 22 July 2014).

39. Della Spina, L.; Giorno, C.; Galati Casmiro, R. Bottom-Up Processes for Culture-Led Urban Regeneration Scenarios. In Computational Science and Its Applications - ICCSA 2019, (Lecture Notes in Computer Science Book, Volume 11622); Misra, S., Gervasi, O., Murgante, B., Stankova, E., Korkhov, V., Torre, C., Rocha, A.M.A.C., Taniar, D., Apduhan, B.O., Tarantino, E., Eds.; Springer: Cham, Switzerland, 2019; pp. 93-107. [CrossRef]

40. Throsby, D. Investment in urban heritage conservation in developing countries: Concepts, methods and data. City Cult. Soc. 2016, 7, 81-86. [CrossRef]

41. Della Spina, L. Evaluation Decision Support Models: Highest and Best Use Choice. Procedia Soc. Behav. Sci. 2016, 223, 936-943. [CrossRef]

42. Della Spina, L.; Calabrò, F. Decision Support Model for Conservation, Reuse and Valorization of the Historic Cultural Heritage. In Computational Science and Its Applications - ICCSA 2018. Lecture Notes in Computer Science Book Series; Gervasi, O., Murgante, B., Misra, S., Stankova, E., Torre, C.M., Rocha, A.M.A.C., Taniar, D., Apduhan, B.O., Tarantino, E., Ryu, Y., Eds.; Springer: Cham, Switzerland, 2018; volume 10962, pp. 3-17. [CrossRef]

43. Munda, G. Multiple Criteria Evaluation in a Fuzzy Environment - Theory and Applications in Ecological Economics; Springer Physika Verlag: Heidelberg, Germany, 1995.

44. Castells, N.; Munda, G. International environmental issues: Towards a new integrated assessment approach. In Valuation and the Environment - Theory, Method and Practice; O'Connor, M., Spash, C., Eds.; Edward Elgar: Cheltenham, UK, 1999; pp. 309-327. 
45. Janssen, R. Multiobjective Decision Support for Environmental Management; Kluwer Academic: Dortrecht, The Netherlands, 1992.

46. Andreoli, M.; Tellarini, V. Farm sustainability evaluation: Methodology and practice. Agric. Ecosyst. Environ. 2000, 77, 43-52. [CrossRef]

47. Mendoza, G.A.; Prabhu, R. Multiple criteria decision making approaches to assessing forest sustainability using criteria and indicators: A case study. For. Ecol. Manag. 2000, 131, 107-126. [CrossRef]

48. Roy, B. Decision-aid and decision-making. Eur. J. Oper. Res. 1990, 45, 324-331. [CrossRef]

49. Fishburn, P.C. Additive Utilities with Incomplete Product Sets: Application to Priorities and Assignments. Oper. Res. 1967, 15, 537-542. [CrossRef]

50. Miller, D.W.; Starr, M.K. Executive Decisions and Operations Research; Prentice-Hall: Upper Saddle River, NJ, USA, 1969.

51. Zavadskas, E.K.; Turskis, Z.; Antucheviciene, J.; Zakarevicius, A. Optimization of Weighted Aggregated Sum Product Assessment. Elektron. Elektrotech. 2012, 122, 3-6. [CrossRef]

52. Saaty, T.L. How to Make a Decision: The Analytic Hierarchy Process. Eur. J. Oper. Res. 1981, 48, 9-26. [CrossRef]

53. Roy, B. Classement et choix en présence de points de vue multiples. RAIRO Oper. Res. Rech. Opér. 1968, 2, 57-75. [CrossRef]

54. Hwang, C.L.; Yoon, K. Multiple Attribute Decision Making: Methods and Applications: A State-of-the-Art Survey; Springer-Verlag: Dortrecht, The Netherlands, 1981.

55. Brans, J.P.; Vincke, P. Note-A Preference Ranking Organisation Method. Manag. Sci. 1985, 31, 647-656. [CrossRef]

56. Zavadskas, E.; Kaklauskas, A. Determination of an Efficient Contractor by Using the New Method of Multicriteria Assessment. International Symposium for "The Organization and Management of Construction"; Langford, D.A., Retik, A., Eds.; Shaping Theory and Practice: London, UK, 1996; pp. 94-104.

57. Opricovic, S. Multicriteria Optimization of Civil Engineering Systems; Faculty of Civil Engineering: Belgrade, Serbia, 1998.

58. Brauers, W.K.M.; Zavadskas, E.K. Project Management by Multimoora as an Instrument for Transition Economies. Technol. Econ. Dev. Econ. 2010, 16, 5-24. [CrossRef]

59. Zavadskas, E.K.; Turskis, Z. A New Additive Ratio Assessment (ARAS) Method in Multicriteria Decision-Making. Technol. Econ. Dev. Econ. 2010, 16, 159-172. [CrossRef]

60. Keshavarz Ghorabaee, M.; Zavadskas, E.K.; Olfat, L.; Turskis, Z. Multi-criteria Inventory Classification Using a New Method of Evaluation Based on Distance from Average Solution (EDAS). Informatica 2015, 26, 435-451. [CrossRef]

61. Roberts, R.; Goodwin, P. Weight approximations in multi-attribute decision models. J. Multicrit. Decis. Anal. 2002, 11, 291-303. [CrossRef]

62. Solymosi, T.; Dompi, J. Method for determining the weights of criteria: The centralized weights. Eur. J. Oper. Res. 1985, 26, 35-41. [CrossRef]

63. Cook, W.D. Distance-based and ad hoc consensus models in ordinal preference ranking. Eur. J. Oper. Res. 2006, 172, 369-385. [CrossRef]

64. Weber, M.; Borcherding, K. Behavioral influences on weight judgments in multiattribute decision making. Eur. J. Oper. Res. 1993, 67, 1-12. [CrossRef]

65. Tzeng, G.-H.; Chen, T.-Y.; Wang, J.C. A weight-assessing method with habitual domains. Eur. J. Oper. Res. 1998, 110, 342-367. [CrossRef]

66. Zavadskas, E.K.; Govindan, K.; Antucheviciene, J.; Turskis, Z. Hybrid multiple criteria decision-making methods: A review of applications for sustainability issues. Econ. Res. Ekon. Istraž. 2016, 29, 857-887. [CrossRef]

67. Saaty, T.L. The Analytic Hierarchy Process for Decision in a Complex World; RWS Publications: Pittsburgh, PA, USA, 1980.

68. Zhu, G.N.; Hu, J.; Qi, J.; Gu, C.C.; Peng, J.H. An integrated AHP and VIKOR for design concept evaluation based on rough number. Adv. Eng. Inform. 2015, 29, 408-418. [CrossRef]

69. Giacomini, C.; Longo, G.; Lunardi, A.; Padoano, E. AHP-Aided Evaluation of Logistic and Transport Solutions in a Seaport. In Applications and Theory of Analytic Hierarchy Process-Decision Making for Strategic Decisions; Tech: Bruchsal, Germany, 2016; pp. 115-141. 
70. Raymundo, H.; Reis, J.G.M. Passenger Transport Drawbacks: An Analysis of Its "Disutilities" Applying the AHP Approach in a Case Study in Tokyo, Japan. In Proceedings of the IFIP International Conference on Advances in Production Management Systems, Riga, Latvia, 17-20 October 2018; pp. 545-552.

71. Olivková, I. Methodology for Assessment of Electronic Payment Systems in Transport Using AHP Method. In Proceedings of the International Conference on Reliability and Statistics in Transportation and Communication, Riga, Latvia, 17-20 October 2018; pp. 290-299.

72. Bana, E.; Costa, C.A.; Vansnick, J.C. MACBETH-An interactive path towards the construction of cardinal value functions. Int. Trans. Oper. Res. 1994, 1, 489-500. [CrossRef]

73. Bana e Costa, C.A.; Ensslin, L.; Corrêa, E.C.; Vansnick, J.-C. Decision support systems in action: Integrated application in a multicriteria decision aid process. Eur. J. Oper. Res. 1999, 113, 315-335. [CrossRef]

74. Bana e Costa, C.A.; Corrêa, É.C.; De Corte, J.-M.; Vansnick, J.-C. Facilitating bid evaluation in public call for tenders: A socio-technical approach. Omega 2002, 30, 227-242. [CrossRef]

75. Saaty, T.L. Multicriteria Decision Making: The Analytic Hierarchy Process; RWS Publications: Pittsburgh, PA, USA, 1992.

76. Voogd, H. Multicriteria Evaluation with Mixed Qualitative and Quantitative Data. Environ. Plan. Bull. 1982, 9, 221-236. [CrossRef]

77. Voogd, H. Multicriteria Evaluation for Urban and Regional Planning; Pion: Paris, France, 1983.

78. Alinezhad, A.; Khalili, J. EVAMIX Method. In New Methods and Applications in Multiple Attribute Decision Making (MADM); International Series in Operations Research \& Management Science (Volume 277); Springer: Cham, Switzerland, 2019.

79. Martel, J.M.; Matarazzo, B. Other Outranking Approaches. In Multiple Criteria Decision Analysis: State of the Art Surveys; Salvatore, F.J., Ehrgott, G.M., Eds.; Springer: New York, NY, USA, 2005; pp. 197-262.

80. Chatterjee, P.; Stević, Ž. A two-phase fuzzy AHP-fuzzy TOPSIS model for supplier evaluation in manufacturing environment. Oper. Res. Eng. Sci. Theory Appl. 2019, 2, 72-90. [CrossRef]

81. Hassanpour, M.; Pamucar, D. Evaluation of Iranian household appliance industries using MCDM models. Oper. Res. Eng. Sci. Theory Appl. 2019, 2, 1-25. [CrossRef]

82. Popovic, M.; Kuzmanović, M.; Savić, G. A comparative empirical study of Analytic Hierarchy Process and Conjoint analysis: Literature review. Decis. Mak. Appl. Manag. Eng. 2018, 1, 153-163. [CrossRef]

83. Creswell, J.W. Research Design: Qualitative, Quantitative, and Mixed Methods Approaches, 2nd ed.; Sage: Thousand Oaks, CA, USA, 2003.

84. Tashakkori, A.; Teddlie, C. Mixed Methodology: Combining Qualitative and Quantitative Approaches; Sage: Thousand Oaks, CA, USA, 1998.

85. Herwijnen, M.V.; Janssen, R. DEFINITE, A support system for decisions on a finite set of alternatives; Springer: Dordrecht, The Netherlands, 1995.

86. Lo Curzio, M.; Caruso, V.; D’Angelo, M. La Fortificazione Permanente dello Stretto di Messina: Storia, Conservazione e Restauro di un Patrimonio Architettonico e Ambientale; EDAS: Leonia, NJ, USA, 2006.

87. United Nations Educational, Scientific and Cultural Organization. Recommendation on the Historic Urban Landscape, Including a Glossary of Definitions. Available online: https://www.portal.unesco.org (accessed on 10 November 2011).

88. Della Spina, L. Integrated Evaluation and Multi-methodological Approaches for the Enhancement of the Cultural Landscape. In Computational Science and Its Applications - ICCSA 2017; Springer: Cham, Switzerland, 2017; Lecture Notes in Computer Science; Volume 10404. [CrossRef]

89. Nocca, F. Il ruolo del patrimonio culturale nello sviluppo sostenibile: Indicatori multidimensionali come strumento decisionale. Sostenibilità 2017, 9, 1882.

90. Dias, L.C.; Antunes, C.H.; Dantas, G.; de Castro, N.; Zamboni, L. A multi-criteria approach to sort and rank policies based on Delphi qualitative assessments and ELECTRE TRI: The case of smart grids in Brazil. Omega 2018, 76, 100-111. [CrossRef]

91. Antoniucci, V.; Marella, G. The influence of building typology on the economic feasibility of urban developments. Int. J. Appl. Eng. Res. 2017, 12, 4946-4954.

92. Augusto, M.; Lisboa, J.; Yasin, M.; Figueira, J.R. Benchmarking in a multiple criteria performance context: An application and a conceptual framework. Eur. J. Oper. Res. 2008, 184, 244-254. [CrossRef] 
93. Della Spina, L. The Integrated Evaluation as a Driving Tool for Cultural-Heritage Enhancement Strategies. In Smart and Sustainable Planning for Cities and Regions. SSPCR 2017. Green Energy and Technology; Bisello, A., Vettorato, D., Laconte, P., Costa, S., Eds.; Springer: Berlin, Germany, 2018; pp. 1-11. [CrossRef]

94. Bottero, M.; D'Alpaos, C.; Oppio, A. Ranking of adaptive reuse strategies for abandoned industrial heritage in vulnerable contexts: A multiple criteria decision aiding approach. Sustainability 2019, 11, 785. [CrossRef]

95. Calabrò, F.; Della Spina, L. The Public-Private Partnership for the Enhancement of Unused Public Buildings: An Experimental Model of Economic Feasibility Project. Sustainability 2019, 11, 5662. [CrossRef]

96. Calabrò, F.; Della Spina, L. The Projects' Economic Feasibility within Strategic Planning, Integrated Planning, Cultural Planning and Management Plans. An Experimental Model for the Valorisation of Public Buildings in Public-Private Partnerships. LaborEst 2018, 16 IS, 1-40.

97. Carbonara, S. Il recupero dell'edilizia privata nell'Abruzzo post-sisma: Un'analisi delle procedure di stima. Territorio 2014. [CrossRef]

98. Carbonara, S. The effect of infrastructural works on urban property values: The asse attrezzato in pescara, italy. In Computational Science and Its Applications - ICCSA 2012; Springer: Berlin/Heidelberg, Germany, 2012; pp. $128-143$.

99. Carbonara, S.; Stefano, D. An Operational Protocol for the Valorisation of Public Real Estate Assets in Italy. Sustainability 2020, 12, 732. [CrossRef]

(C) 2020 by the author. Licensee MDPI, Basel, Switzerland. This article is an open access article distributed under the terms and conditions of the Creative Commons Attribution (CC BY) license (http://creativecommons.org/licenses/by/4.0/). 


\title{
The Survival of Cultural Firms: A Study of Multiple Accounting Parameters in Spain
}

\author{
Ma del Pilar Muñoz Dueñas ${ }^{1, *}$, Antonio Vaamonde Liste ${ }^{1}$ and Maria do Rosário Cabrita ${ }^{2}$ \\ 1 School of Business Studies, University of Vigo, 36208 Vigo, Spain; vaamonde@uvigo.es \\ 2 Unidade de Investigação e Desenvolvimento em Engenharia Mecânica e Industrial (UNIDEMI), \\ Department of Mechanical and Industrial Engineering, Faculty of Science and Technology (FCT), \\ NOVA School of Science and Technology, New University of Lisbon, 2829-516 Caparica, Portugal; \\ m.cabrita@fct.unl.pt \\ * Correspondence: pilar.munoz@uvigo.es
}

Received: 17 December 2019; Accepted: 3 February 2020; Published: 6 February 2020

\begin{abstract}
Cultural firms are an important development factor in economic and social terms. Their objectives are often aimed at maintaining and disseminating the traditions and values of societies. The prosperity of these firms in a nation ensures that its tangible and intangible cultural heritage is made known to other nations and generations. Despite their importance, little is known about their survival and the factors associated with it. This paper analyses data from 6951 Spanish firms, of which 2105 are cultural firms. We have studied the survival of non-cultural firms in comparison with cultural firms and also the impact that profitability, solvency and indebtedness may have on their survival. We have used the Kaplan-Meier method in order to assess their survival and the Harrington-Fleming test and the Cox regression model to check the statistical significance of variables. These variables are key factors influencing the survival of cultural enterprises. Particularly, low solvency in firms increases by twenty the risk of disappearance. This paper contributes to literature highlighting some of the key factors for the survival of cultural enterprises. It provides administrations with a roadmap in order to implement measures for the promotion of the cultural industry, favouring the process of enhancement of cultural heritage.
\end{abstract}

Keywords: cultural firms; accounting parameters; survival analysis; Kaplan-Meier curves

\section{Introduction}

The cultural and creative industry (henceforth cultural firms/enterprises) is a main actor in the labour market and a major contributor to regional and national gross domestic product (GDP). This industry is increasingly recognised around the world for its great growth potential [1-3] and its impact on territorial wealth [4-6]. Moreover, according to the European Commission, such firms become key drivers of creativity and economic and social innovation in other sectors [7]. For this reason, cultural firms constitute a strategic industry in Europe, where some of them are world leaders and competitive exporters in a wide range of fields. The EU trade balance for cultural goods grew from EUR 6.3 billion in 2012 to EUR 8.6 billion in 2017. Furthermore, in 2018, there were 8.7 million people in cultural employment across the EU-28 (3.8\% of all employment) [8].

This industry has a very particular characteristic associated with its business models that makes it more fragile than other industries. In the case of cultural firms, it is difficult to predict the value of use in advance as they are subject to high levels of novelty [9]. Save for a few exceptions, traditional methods make no allowance to determine a price for cultural products $[10,11]$. In many cases, the valuation of cultural products is determined by their uniqueness or prototype characteristics; if their originality is involved [12,13], the prototype of a cultural product itself becomes the end product, thereby raising the risk of failure of the company offering it [14]. 
Another particularity of cultural firms refers to their costs and sustainability. The existence of sunk costs (hours of rehearsal in performances or song recordings, for example) and the ongoing increasing costs of these industries, called "cost illness" [15], have traditionally led researchers to assume productivity was scarce $[15,16]$. Therefore, the sustainability of these firms is assumed to be mainly associated with contributions made by public or private patrons, and the parameters to design business policies may consider these particularities to minimize the weakness of cultural firms.

Despite the growing importance of cultural firms, little is known about their survival and the associated factors. Some studies have analysed separately the effect of specific factors in specific cultural sub-sectors, for example, the length of performance runs on stage, initial income from the box office, and nominations for prizes in firms that are active in music, theatre or cinema $[17,18]$. But there are few studies that have analysed all the firms in the cultural sector in a comprehensive manner.

Several studies related to the survival of manufacturing firms have found it is associated with size, innovation, profitability, solvency, indebtedness and subsidies, among others. For instance, Segarra and Castejón [19] point out that innovation and technological progress is significantly associated with the survival of Spanish manufacturing firms. Correa et al. [20] and Mateut et al. [21] focused on the impact of solvency in the Spanish case. Delmar et al. [22] found that the profitability of Swedish firms is the main factor associated with their survival and growth. Guimaraes [23] analyzed the effect of indebtedness in small enterprises in Brazil; Pellegrini and Muccigrosso [24] and Shin [25] analysed the effect of subsidies in the survival of Italian and Korean firms, respectively. Analyses of these or other factors related to the survival of cultural enterprises are scarce or practically non-existent.

Unquestionably, studies on the survival of firms are essential. They should study what factors are making these firms enter or exit the market and evaluate the impact of business dynamics on productivity growth. In fact, several studies related to the survival of firms have found it is associated with profitability, solvency and indebtedness, among others [23,26]. However, as the cultural industry was not previously analysed in this regard, this study aims to provide a comparative analysis between cultural and non-cultural firms, providing empirical evidence of the variables that determine the survival of cultural firms in Spain. Profitability, solvency and indebtedness are the variables analysed and are used to study how they influence survival.

This paper is organized as follows. Section 2 presents the research methodology. Results and analysis are presented in Section 3. Finally, Section 4 offers the main conclusions.

\section{Methodology}

In order to analyse what factors may affect the survival of cultural firms, the concept of the cultural firm is defined and the parameters that must be considered are described. We will describe the database used as well as the statistical methodology that allow us to carry out the analysis of the survival of cultural enterprises.

\subsection{Definition of Cultural Firm}

There is a wide variety of definitions for the cultural firm [27,28]. The European Commission [7] (p.6) defines them as those that produce and distribute goods or services that have an attribute, use or purpose that incorporates or transmits cultural expressions regardless of the value they can incorporate. The Department of Studies, Foresight and Statistics (DEPS in France) [29] (p. 7), dependent on the French Government, defined them as a set of economic activities that combine the functions of conception, creation and production with the industrial functions of large-scale manufacture and marketing, using physical or communication products. The Department for Culture, Media and Sport (DMCS) [30] (p. 5), in the United Kingdom, defines them as "those industries that have their origin in the individual creativity, skill and talent and that have a potential for wealth and employment creation through the generation and exploitation of intellectual property". They are also seen as an industry that originates from creative or cultural accumulation through the formation and application of intellectual property and has the potential to enhance our living environment [31]. These definitions 
may be valid when undertaking research of the cultural sector because they are generic and cover a broad interdisciplinary field of activities.

In Spain, the National Institute of Statistics (INE), following the European statistical nomenclature, classifies economic activities and groups them according to the so-called CNAE code (National Economic Activities Classification). However, there is no specific code that brings together the wide range of activities within the cultural sector. The set of cultural activities is divided into different groups established in the CNAE.

Thus, for this paper, we will consider that cultural enterprises are those focusing on cultural, artistic and/or both tangible and intangible heritage activities. This includes activities relating to publishing, libraries, archives, museums, film activities, video, radio and television, shows, recorded music and other manifestations of intangible cultural heritage.

\subsection{Parameters}

According to the literature on firm duration mentioned in the previous section, there are a number of determining factors in the survival of firms. For our study, the indicator for the survival of the firms is their age. Therefore, the variable of interest is the age of the firms, defined as the time that elapses from their creation until their disappearance or until the moment at which the study is conducted if they continue to remain active in the market. We selected as explanatory variables of their survival, their economic profit, their solvency and their indebtedness. We opted to use accounting information, one of the main approaches used by the literature on the failure of firms [32,33].

Profitability is associated with survival [26,34,35]. According to Pérez Goróstegui [36], economic profitability is the profitability generated by each one of the company assets and it is calculated as the ratio of its gross profit to its total assets.

Solvency also has an impact $[20,37,38]$. The most solvent firms have the lowest risk of disappearing. Solvency is defined as the ability of the company to respond to its long-term debts. Solvency indicators are very good for predicting business failure and they often bring the prognosis forward by two or three years before this situation happens [20].

Finally, we considered that the debts are the proportion of debt a company supports against its own resources. Authors such as Mata et al. and Guimaraes [23,38] have analysed indebtedness. They find that moderate indebtedness offers greater protection to creditors in the event of insolvency. If the company has little indebtedness, an increase in debt has a positive impact on the survival of the company. Therefore, if there is already a high level of indebtedness, an increase in debt increases the probability of failure of firms, either because the ability of the company to meet the payment obligations of the debt is decreased, or because a highly leveraged company may be subject to predation by rival firms whose leverage is low.

The analysed firms are categorized considering their values for the accounting parameters: positive and negative profitability, low and high solvency, and low and high indebtedness.

\subsection{Data Collection}

The data used in this paper is a sample from the ARDÁN database. ARDÁN is a business information service that creates databases of firms from all sectors in Spain with economic information from the annual accounts: balance sheet, profit and loss account, annual report, statement of changes in equity and management report, filed with the Companies Registry.

For our research, we selected firms randomly using an automated procedure based on the generation of random numbers. The resulting sample comprises of a total of 6951 Spanish enterprises, being 4846 non-cultural companies and 2105 cultural companies according to the definition provided in the previous section. However, some firms of the sample were not finally considered for the study because relevant data was not available: the company status (e.g., winding-up process, creditors contest), age or accounting variables (company's profitability, solvency and indebtedness). This uncompleted profile was verified for 1055 non-cultural firms and 699 cultural firms. Consequently, we analysed 
a total of 1406 cultural firms and 3791 non-cultural firms. Figure 1 shows the histogram of the age distribution of cultural firms. Most are about 20 years old, and few of them surpass 100 years old.

Accounting information on the 2016 Annual Accounts of the mentioned enterprises is included in the database. We analysed the factors affecting the survival of cultural enterprises in this selected year.

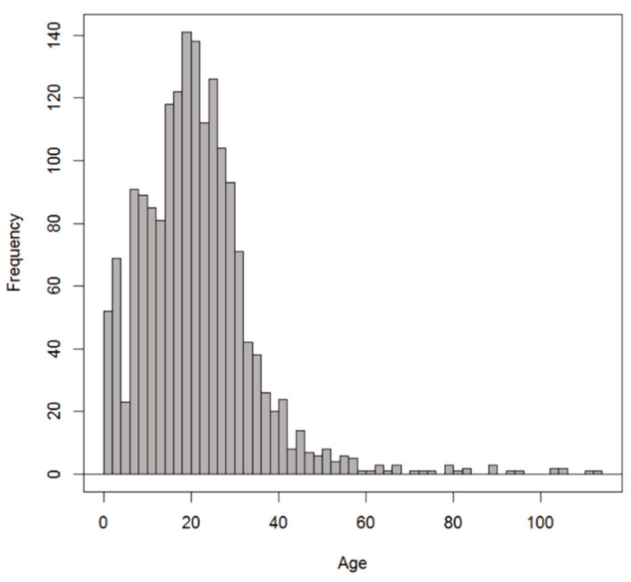

Figure 1. Age distribution of the cultural enterprises.

\subsection{Analysis}

The exploitation of data has been made using the statistical package R. A summary for each variable is shown through a box plot. We then analysed the survival of firms using the statistical Kaplan-Meier method [39]. This model constructs a survival table that indicates the estimated probability of surviving over a given period, conditioned by the current age. The same table shows the firms' age, the number of firms at risk, the number of firms that do not survive in that period, the probability of surviving in the given year, its standard error and confidence intervals at $95 \%$. The graphical representation of this curve, through a step function, is the Kaplan-Meier survival curve.

In order to analyse in detail the accounting parameters in non-cultural firms and the effect of the considered categories on their survival, the Harrington-Fleming test [40] is used. This test has been specifically designed to compare survival curves. It allows the assessment of the effect of the considered factors on survival. The null hypothesis to be compared is that there is no difference between the survival curves of the categories of enterprises. A $p$-value under 0.05 indicates that the effect is statistically significant.

The impact of the considered accounting parameters in the survival of cultural firms in Spain is revealed by the Cox regression model [41]. This model estimates the hazard ratio (HR), which determines whether every analysed parameter has an influence on the survival function. The Cox regression analyses the accounting parameters of cultural firms where the impact of the explanatory variables is proportional, i.e., that $\mathrm{HR}$ is constant for any age, a hypothesis that has been proven in the sample data. This test has been applied to non-cultural firms in order to evaluate differences and similarities between both types of firms.

\section{Results and Analysis}

Once the cultural enterprises were separated from the non-cultural ones, we analysed whether there are differences between the survival of both groups and, if so, what the factors are that influence survival. 


\subsection{Comparative Assessment of the Survival of Cultural Enterprises Against Non-Cultural Ones}

For 2016, the estimated survival of cultural enterprises by Kaplan-Meier is shown in Table 1 where the number of existing firms by age range can be seen together with the number of firms that disappear in the year under review, as well as an estimate of the probability of survival; for example, in 2016, there were 298 firms that were 30 years old or older; 7 disappeared throughout this year. The estimate of the probability of survival is $96.8 \%$. The probability of survival of a cultural enterprise over 50 years old is $92.6 \%$.

The same analysis was performed for non-cultural enterprises. The table of survival of these firms (Table 2) indicates that the probability of survival of a non-cultural company aged 30 years or over is $98.9 \%$ and the probability of survival of firms aged 50 years or older is $96.7 \%$.

Table 1. Life table of cultural enterprises.

\begin{tabular}{|c|c|c|c|c|c|c|}
\hline \multirow{2}{*}{$\begin{array}{l}\text { Age of the } \\
\text { Company } \\
\text { in Years }\end{array}$} & \multirow{2}{*}{$\begin{array}{l}\text { Existing } \\
\text { Firms }\end{array}$} & \multirow{2}{*}{$\begin{array}{l}\text { Firms that } \\
\text { Disappear }\end{array}$} & \multirow{2}{*}{$\begin{array}{l}\text { Probability } \\
\text { of Survival }\end{array}$} & \multirow{2}{*}{$\begin{array}{l}\text { Standard } \\
\text { Error }\end{array}$} & \multicolumn{2}{|c|}{ The Confidence Interval } \\
\hline & & & & & $\begin{array}{c}\text { Lower Limit } \\
95 \%\end{array}$ & $\begin{array}{c}\text { Upper Limit } \\
95 \%\end{array}$ \\
\hline$\geq 0$ & 1406 & 0 & 1.000 & 0.000000 & 1.000 & 1.000 \\
\hline$\geq 5$ & 1324 & 1 & 0.999 & 0.000726 & 0.998 & 1.000 \\
\hline$\geq 10$ & 1216 & 3 & 0.997 & 0.001544 & 0.994 & 1.000 \\
\hline$\geq 15$ & 1043 & 4 & 0.993 & 0.002383 & 0.989 & 0.998 \\
\hline$\geq 20$ & 807 & 3 & 0.990 & 0.003132 & 0.984 & 0.996 \\
\hline$\geq 25$ & 540 & 3 & 0.985 & 0.004051 & 0.977 & 0.993 \\
\hline$\geq 30$ & 298 & 7 & 0.968 & 0.007603 & 0.953 & 0.983 \\
\hline$\geq 35$ & 165 & 1 & 0.963 & 0.009358 & 0.944 & 0.981 \\
\hline$\geq 40$ & 101 & 1 & 0.954 & 0.012722 & 0.929 & 0.979 \\
\hline$\geq 45$ & 69 & 2 & 0.926 & 0.022884 & 0.882 & 0.972 \\
\hline$\geq 50$ & 48 & 0 & 0.926 & 0.022884 & 0.882 & 0.972 \\
\hline$\geq 65$ & 21 & 0 & 0.926 & 0.022884 & 0.882 & 0.972 \\
\hline$\geq 80$ & 14 & 1 & 0.880 & 0.050098 & 0.787 & 0.98 \\
\hline
\end{tabular}

Table 2. Life table of non-cultural enterprises.

\begin{tabular}{|c|c|c|c|c|c|c|}
\hline \multirow{2}{*}{$\begin{array}{l}\text { Age of the } \\
\text { Company } \\
\text { in Years }\end{array}$} & \multirow{2}{*}{$\begin{array}{l}\text { Existing } \\
\text { Firms }\end{array}$} & \multirow{2}{*}{$\begin{array}{l}\text { Firms that } \\
\text { Disappear }\end{array}$} & \multirow{2}{*}{$\begin{array}{l}\text { Probability } \\
\text { of Survival }\end{array}$} & \multirow{2}{*}{$\begin{array}{l}\text { Standard } \\
\text { Error }\end{array}$} & \multicolumn{2}{|c|}{ The Confidence Interval } \\
\hline & & & & & $\begin{array}{c}\text { Lower Limit } \\
95 \%\end{array}$ & $\begin{array}{c}\text { Upper Limit } \\
95 \%\end{array}$ \\
\hline$\geq 0$ & 3791 & 0 & 1.000 & 0.000000 & 1.000 & 1.000 \\
\hline$\geq 5$ & 3501 & 1 & 1.000 & 0.000265 & 0.999 & 1.000 \\
\hline$\geq 10$ & 3264 & 5 & 0.998 & 0.000714 & 0.997 & 1.000 \\
\hline$\geq 15$ & 2798 & 1 & 0.998 & 0.000792 & 0.996 & 0.999 \\
\hline$\geq 20$ & 2162 & 7 & 0.995 & 0.001325 & 0.993 & 0.998 \\
\hline$\geq 25$ & 1407 & 5 & 0.992 & 0.001977 & 0.988 & 0.996 \\
\hline$\geq 30$ & 805 & 3 & 0.989 & 0.002671 & 0.984 & 0.994 \\
\hline$\geq 35$ & 445 & 4 & 0.981 & 0.004910 & 0.971 & 0.990 \\
\hline$\geq 40$ & 254 & 1 & 0.977 & 0.005798 & 0.966 & 0.989 \\
\hline$\geq 45$ & 152 & 2 & 0.967 & 0.009254 & 0.949 & 0.986 \\
\hline$\geq 50$ & 97 & 0 & 0.967 & 0.009254 & 0.949 & 0.986 \\
\hline$\geq 65$ & 39 & 1 & 0.951 & 0.018868 & 0.914 & 0.988 \\
\hline$\geq 80$ & 20 & 1 & 0.915 & 0.039033 & 0.842 & 0.995 \\
\hline
\end{tabular}

The survival curves for both groups, cultural and non-cultural firms are shown in Figure 2. As can be seen, the curve for cultural enterprises is systematically lower; the older the firms, the greater the difference. This shows that cultural firms have lower survival rates. In order to verify that the survival difference between cultural and non-cultural enterprises is statistically significant, we applied the 
Harrington-Fleming test. As shown in Table 3, the $p$-value of the test is 0.003 , indicating that survival is significantly different between cultural and non-cultural enterprises.

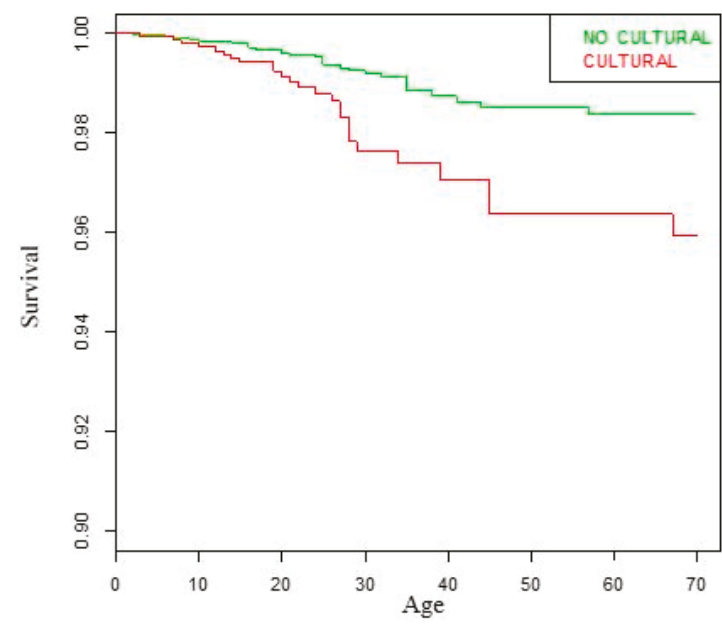

Figure 2. Survival curve for cultural/non-cultural enterprises.

Table 3. Harrington-Fleming Test.

\begin{tabular}{cc}
\hline Variable & $p^{*}$ \\
\hline Cultural & 0.003 \\
\hline \multicolumn{2}{c}{${ }^{*}=$ significant at $5 \%}$.
\end{tabular}

We checked to see if the characteristic of being a cultural enterprise is an influential factor in the survival of firms, applying the Cox regression model that allows us to detect the relationship between the risk of a certain event; in our case, the disappearance of the company and one or several variables, whether or not it is cultural.

The results of the model are shown in Table 4. As can be seen, the regression coefficient is positive (coefficient $=0.77$ ), indicating that the risk of disappearance increases in cultural enterprises compared with non-cultural ones where it is at the reference level. As for the $p$-value obtained ( $p=0.0038)$, its effect is statistically significant. The HR is greater than 1, indicating more risk of disappearance in cultural enterprises. In particular, the HR is 2.161 , which means that the risk of disappearance of a cultural company is 2.161 times that of a non-cultural one.

Table 4. Cox's model.

\begin{tabular}{cccc}
\hline Variable & Coefficient & HR & $p^{*}$ \\
\hline Cultural & 0.770 & 2.161 & 0.038 \\
\hline \multicolumn{4}{c}{${ }^{*}=$ significant at $5 \%}$.
\end{tabular}




\subsection{Analysis of Accounting Factors that Influence the Survival of Cultural Enterprises}

\subsubsection{Profitability}

We considered two levels of profitability in cultural enterprises: negative and positive profitability. First, we made a comparison between both groups of cultural enterprises through a box plot. Figure 3 shows the age of both groups of enterprises on the basis of their negative or positive profitability. The median of the group of firms with positive profitability is greater than that of the group of firms with negative profitability. This suggests that the cultural firms with positive profitability tend to be older.

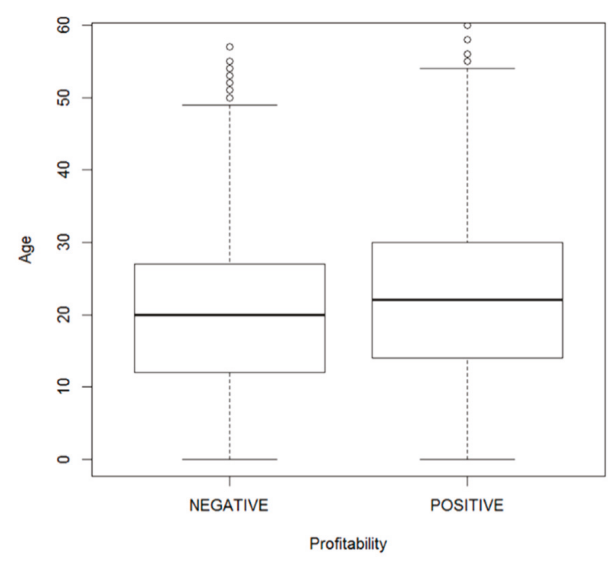

Figure 3. Box plot for profitability.

The Kaplan-Meier survival curve for both levels of profitability is shown in Figure 4. This figure shows that the survival rate of both groups of cultural enterprises is different. It is noted that cultural enterprises with lower profitability have lower survival rates. This is consistent with studies for firms in other industrial sectors.

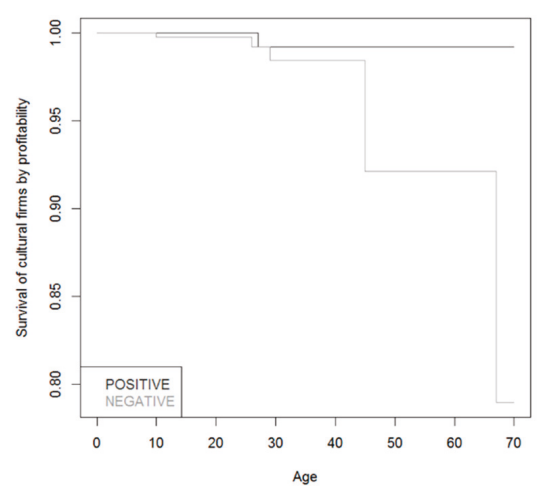

Figure 4. Survival curve for the cultural firms by profitability.

Table 5 shows the results of the Harrington-Fleming test. The obtained $p$-value $(p=0.0483)$ is lower than the significance level commonly used (0.05). This indicates that the effect of profitability on the survival of cultural enterprises is statistically significant. 
Table 5. Harrington-Fleming test.

\begin{tabular}{cc}
\hline Variable & $p^{*}$ \\
\hline Profitability (negative) & 0.0483 \\
\hline
\end{tabular}

We checked to see if the profitability factor is an influential factor in the survival of cultural enterprises by applying the Cox regression model. The results are shown in Table 6. The regression coefficient is positive (coefficient $=1.481$ ). This result indicates that the risk of disappearance of cultural enterprises that have negative economic profitability rates is higher compared to those with positive profitability rates, which is the reference level. The obtained $p$-value $(p=0.0487)$ shows the statistically significant effect. The HR is 4.39638 , indicating that the risk of disappearance of cultural enterprises with negative rates of economic profitability is 4.396 times higher than in those with positive rates of profitability.

Table 6. Cox's model.

\begin{tabular}{cccc}
\hline Variable & Coefficient & HR & $p^{*}$ \\
\hline Profitability (negative) & 1.481 & 4.39638 & 0.0487 \\
\hline \multicolumn{4}{c}{${ }^{*}=$ significant at $5 \%}$.
\end{tabular}

\subsubsection{Solvency}

We have compared two groups according to their solvency: low level, up to the first quartile, versus high level.

Figure 5 shows how the median age of cultural enterprises with high solvency is over 20 years, while in the case of firms with low solvency, the median age is around 15 years. This indicates that the firms with greater solvency tend to be older.

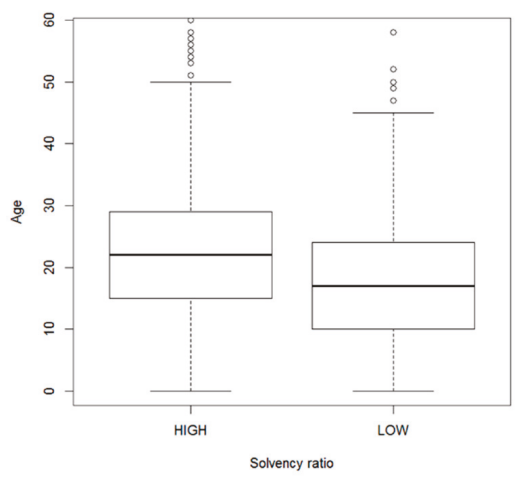

Figure 5. Box plot for solvency ratio.

Figure 6 represents the survival curve for the cultural firms according to the solvency levels mentioned above. Cultural enterprises with low solvency ratios have lower survival rates; this difference increases with age. Therefore, there is a greater risk of disappearance in those cultural enterprises with low solvency ratios than in those cultural enterprises with high solvency ratios. 


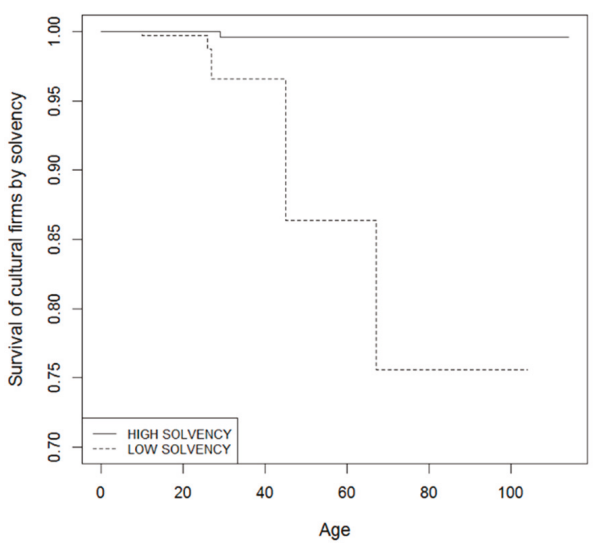

Figure 6. Survival curve for the cultural firms by solvency.

Table 7 shows the results of the Harrington-Fleming test. The obtained $p$-value $(p=0.000114)$ indicates that the difference between both groups of solvency levels is significant.

We checked to see if the low solvency level is an influential factor in the survival of cultural enterprises by applying the Cox regression model. The results of this model are shown in Table 8 . It shows that the obtained $p$-value for the test $(p=0.000301)$ is clearly lower than the usual level of significance. This indicates that the solvency factor is statistically significant. This confirms that solvency is an important factor in the survival of cultural enterprises. As the value of the HR collected in the table (19.58647) shows, the risk of disappearance in cultural enterprises with a low solvency ratio is multiplied by almost twenty.

Table 7. Harrington-Fleming test.

\begin{tabular}{cc}
\hline Variable & $p^{*}$ \\
\hline Solvency (low) & 0.000114 \\
\hline \multicolumn{2}{c}{${ }^{*}=$ significant at $5 \%}$.
\end{tabular}

Table 8. Cox's model.

\begin{tabular}{cccc}
\hline Variable & Coefficient & HR & $p^{*}$ \\
\hline Solvency (low) & 2.9748 & 19.58647 & 0.000301 \\
\hline \multicolumn{5}{c}{$*$ significant at $5 \%}$.
\end{tabular}

\subsubsection{Indebtedness}

Two groups of indebtedness are considered: low and high. There is a high level of indebtedness when its ratio exceeds the mean value; otherwise, we considered it low. Table 9 shows the results of the statistics that describe this ratio for the cultural firms analysed. From these data, we determined that cultural enterprises exceeding the value of 4.362 have a high debt ratio.

Table 9. Descriptive statistics of the debt ratio.

\begin{tabular}{cccccc}
\hline Min. & 1St Qu. & Median & Mean & 3rd Qu. & Max. \\
\hline 0.000 & 0.420 & 1.120 & 4.362 & 2.740 & 98.85 \\
\hline
\end{tabular}

Then we compared cultural enterprises according to their indebtedness levels. Figure 7 shows the box plot of high and low groups. These show how cultural enterprises with low debt ratios have a higher 
median age, around 25 years, compared with those whose debt ratios are high, for which the median age is just over 20 years. This seems to indicate that cultural enterprises with low debt ratios tend to be older.

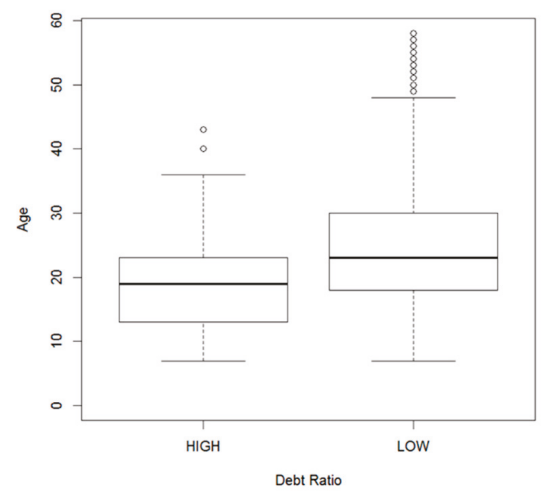

Figure 7. Box plot for debt ratio.

The survival curve for the cultural firms according to their level of indebtedness is represented in Figure 8. As can be seen, the survival curves for the cultural firms with high and low ratios of indebtedness are different. Cultural enterprises with higher debt ratios have survival rates lower than those whose ratio is low.

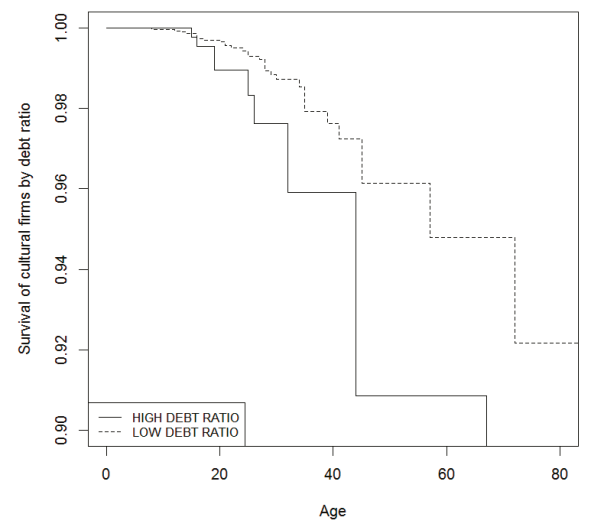

Figure 8. Survival curve for the cultural firms by indebtedness.

Table 10 presents the results of the Harrington-Fleming test. The $p$-value obtained $(p=0.0171)$ shows that this factor is significant because its significance level is lower than the level commonly used (0.05). This indicates that the effect of indebtedness on the survival of cultural enterprises is statistically significant.

Table 10. Harrington-Fleming test.

\begin{tabular}{cc}
\hline Variable & $p^{*}$ \\
\hline Indebtedness (High) & 0.0171 \\
\hline \multicolumn{2}{c}{${ }^{*}=$ significant at $5 \%}$.
\end{tabular}


We applied the Cox regression model to verify that this factor is influential in the survival of the firms analysed. Table 11 shows the results of this model and indicates that the regression coefficient is positive (coefficient $=1.1729$ ), which shows that the risk of disappearance of cultural enterprises with high debt ratios is greater compared to those with low debt rates, which is the reference level. The $p$-value (0.0308) indicates that its effect is statistically significant. In particular, as indicated by the HR, the more indebted firms have a risk of disappearance 3.2314 times greater than the less indebted ones.

Table 11. Cox's model.

\begin{tabular}{cccc}
\hline & Coefficient & HR & $p^{*}$ \\
\hline Indebtedness (high) & 1.1729 & 3.2314 & 0.0308 \\
\hline \multicolumn{4}{c}{$=$ significant at $5 \%}$.
\end{tabular}

\subsection{Analysis of Accounting Factors that Influence the Survival of Non-Cultural Enterprises}

In order to evaluate similarities and differences in accounting parameters between cultural and non-cultural firms, profitability, solvency and indebtedness have been obtained for the 3791 non-cultural firms. Applying the Cox regression model, we studied the relationship between these parameters and the firms' risk of disappearance.

The results from the model for profitability are shown in Table 12. It shows that the regression coefficient is positive (coefficient $=1.2224$ ), indicating that the risk of disappearance increases in non-cultural firms whose profitability is negative compared to those whose profitability is positive at the reference level. The $p$-value obtained $(p=0.000729)$ shows the effect is statistically significant. An HR greater than 1 indicates a greater risk of disappearance. In this case, HR has a value of 3.395, which indicates that the risk of disappearance of a non-cultural firm with negative profitability is 3.3952 times higher than that of a firm with positive profitability. The effect is not as significant as for cultural firms.

Table 12. Cox's model for non-cultural firms.

\begin{tabular}{cccc}
\hline Variable & Coefficient & HR & $p^{*}$ \\
\hline Profitability (negative) & 1.2224 & 3.3952 & 0.000729 \\
\hline Solvency (low) & 0.9773 & 2.6572 & 0.00722 \\
\hline & $*$ = significant at $5 \%$. &
\end{tabular}

Applying the same Cox regression model, we checked to see if the low solvency level is an influential factor in the survival of non-cultural enterprises. The results of this model are shown in Table 12. The resulting $p$-value $(p=0.00722)$ reveals that this parameter is statistically significant. It is confirmed that solvency affects the survival of non-cultural enterprises. Considering the HR (2.6572), the risk of disappearance in non-cultural enterprises with a low solvency ratio is doubled in relation to highly solvent companies.

It might be highlighted that solvency is a risk factor for the survival of firms, but especially for cultural firms: $\mathrm{HR}=19.586$ against $\mathrm{HR}=2.657$ in non-cultural firms.

Finally, for the third factor under consideration, indebtedness, the same model applied to non-cultural firms in Spain shows there is no statistical significance $(p=0.382)$ in the analysed period.

\section{Conclusions}

This article has examined the survival of cultural enterprises against non-cultural ones. In addition, the effect of different accounting factors on the survival of the firms has also been studied. 
The conducted study has shown, by using the survival curve of Kaplan and Meier, that the cultural orientation of the firms' business activity affects their survival. In effect, we have found that the fact of belonging to the group of cultural firms increases the risk of disappearance and that this risk is statistically significant.

We have also analysed the effect of different accounting variables on the survival of cultural enterprises. This study has shown that profitability, solvency and indebtedness are variables that have an influence.

The statistical significance of the above factors has been verified with the Harrington-Fleming test and the Cox regression model. The study shows statistically robust empirical evidence as regards the effect of the variables considered. Similarly, we have shown that profitability, solvency and indebtedness ratios have predictive capabilities to anticipate business failure. In particular, we have seen how the risk of disappearance of highly indebted cultural enterprises is three times that of those not indebted; that cultural enterprises with negative profitability have a risk of disappearance four times higher compared to those with positive profitability; and, finally, that firms with low solvency ratios have a risk of disappearance of almost twenty times higher than those whose solvency ratio is high.

Additionally, we have analyzed the behavior of these ratios in non-cultural firms to reveal differences in behaviour. The results show that the risk of disappearance of companies with low profitability is slightly higher in cultural firms than in non-cultural ones. The behaviour of cultural and non-cultural firms strongly differs in indebtedness: cultural firms with high and low indebtedness show a substantial difference in their risk of disappearance, while this parameter has no statistical significance in non-cultural companies. However, the behaviour of both types of companies especially differs in conditions of low solvency: the risk of disappearance in non-cultural companies with low solvency is 2.7 times higher than companies with high solvency. This index achieves 19.6 in cultural companies.

In view of the results, solvency is the ratio that reveals a risky situation for cultural enterprises with greater intensity, which seems to be consistent with the very nature of such enterprises and spreads the belief of their fragility. For this reason, the evaluation of the position and management of the solvency ratio in cultural enterprises is of utmost importance to the stakeholders in this sector.

This type of analysis is interesting from a triple perspective. First, these studies are relevant for cultural enterprises because they allow the design of business policies to minimise the weakness of cultural enterprises. Second, they are important for administrators because they allow a better understanding of the behaviour of cultural firms and the factors that influence their survival in order to develop effective policies to support a sustainable cultural and creative industry. Third, and finally, because the prosperity of these firms will result in the enhancement of cultural heritage. Due to the importance of this type of study, for future research, it would be interesting to undertake a longitudinal study to see these variables over a longer period.

Author Contributions: Conceptualization, M.d.P.M.D. and A.V.L.; formal analysis, M.d.P.M.D.; A.V.L. and M.d.R.C.; methodology, M.d.P.M.D.; A.V.L. and M.d.R.C.; project administration, M.d.P.M.D. and A.V.L. and M.d.R.C.; Writing-review \& editing, M.d.P.M.D., A.V.L. and M.d.R.C. All authors have read and agreed to the published version of the manuscript.

Funding: This Research was funded by European Union though Atlantic CultureScape, grant number eapa_744/2018. APC was funded by EU.

Acknowledgments: This research has been carried out in the framework of the Project AtlanticCultureScape eapa_744/2018; Start Date: 2019-04-12; End date: 2022-03-31.

Conflicts of Interest: The authors declare no conflict of interest.

\section{References}

1. Cooke, P.; De Propris, L. A policy agenda for EU smart growth: The role of creative and cultural industries. Policy Stud. 2011, 32, 365-375. [CrossRef]

2. O'Connor, J. Intermediaries and imaginaries in the cultural and creative industries. Reg. Stud. 2015, 49, 374-387. [CrossRef] 
3. Lu, W.M.; Kweh, Q.L.; He, D.S.; Shih, J.M. Performance analysis of the cultural and creative industry: A network-based approach. Nav. Res. Logist. (NRL) 2017, 64, 662-676. [CrossRef]

4. De-Miguel-Molina, B.; Hervas-Oliver, J.L.; Boix, R.; De-Miguel-Molina, M. The Importance of Creative Industry Agglomerations in Explaining the Wealth of European Regions. Eur. Plan. Stud. 2012, 20, 1263-1280. [CrossRef]

5. Power, D. Priority Sector Report: Creative and Cultural Industries; European Commission, Ed.; Publications Office of the European Union: Bruxeles, Belgium, 2011.

6. Fanea-Ivanovici, M. Culture as a Prerequisite for Sustainable Development. An Investigation into the Process of Cultural Content Digitisation in Romania. Sustainability 2018, 10, 1859. [CrossRef]

7. European Commission. Liberar el Potencial de las Industrias Culturales y Creativas; European Commission: Bruselas, Belgium, 2010.

8. Eurostat. Culture Statistic. Available online: https://ec.europa.eu/eurostat/documents/3217494/10177894/KS01-19-712-EN-N.pdf/915f828b-daae-1cca-ba54-a87e90d6b68b (accessed on 10 October 2019).

9. O'Connor, J. Las industrias creativas y culturales: Una historia crítica. Ekonomiaz 2011, 78, $24-47$.

10. Hirschman, E.C.; Holbrook, M.B. The experiential aspects of consumption: Consumer fantasies, feelings, and fun. J. Consum. Res. 1982, 9, 132-140.

11. Lampel, J.; Lant, T.; Shamsie, J. Balancing act: Learning from organizing practices in cultural industries. Organ. Sci. 2000, 11, 263-269. [CrossRef]

12. Becker, H.S. Arts and crafts. Am. J. Sociol. 1978, 83, 862-889. [CrossRef]

13. Hirschman, E.C. Aesthetics, ideologies and the limits of the marketing concept. J. Mark. 1983, 47, 45-55. [CrossRef]

14. Greffe, X.; Simonnet, V. Les entreprises culturelles sont-elles soutenables? Revue d'économie Politique 2010, 120, 57-86. [CrossRef]

15. Baumol, W.; Bowen, W. Performing Arts: The Economic Dilemma; Twentieth Century Fund: New York, NY, USA, 1966.

16. Zallo, R. La Economía de la Cultura (y la Comunicación) como objeto de estudio. Zer 2007, 22, $215-234$. Available online: https://www.ehu.eus/ojs/index.php/Zer/article/download/3682/3314 (accessed on 19 October 2019).

17. Giles, D.E. Survival of the hippest: Life at the top of the hot 100. Appl. Econ. 2007, 39, 1877-1887. [CrossRef]

18. Madison, D. Increasing returns to information and the survival of Broadway theatre productions. Appl. Econ. Lett. 2004, 11, 639-643. [CrossRef]

19. Segarra, A.; Callejón, M. New Firms' Survival and Market Turbulence: New Evidence from Spain. Rev. Ind. Organ. 2002, 20, 1-14. [CrossRef]

20. Correa, A.; Acosta, M.; Gónzalez, A.L. La insolvencia empresarial: Un análisis empírico para la pequeña y mediana empresa. Rev. Contab.-Span. Account. Rev. 2003, 12, 47-49.

21. Mateut, S.; Bougheas, S.; Mizen, P. Trade credit, bank lending and monetary policy transmission. Eur. Econ. Rev. 2006, 50, 603-629. [CrossRef]

22. Delmar, F.; McKelvie, A.; Wennberg, K. Untangling the relationships among growth, profitability and survival in new firms. Technovation 2013, 33, 276-291. [CrossRef]

23. Guimaraes Barbosa, E. Determinants of Small Business Survival: The Case of Very Small Enterprises of the Traditional Manufacturing Sectors in Brazil. 2016. Available online: https://ssrn.com/abstract=2802706 (accessed on 9 March 2019).

24. Pellegrini, G.; Muccigrosso, T. Do subsidized new firms survive longer?. Evidence from a counterfactual approach. Reg. Stud. 2017, 51, 1483-1493. [CrossRef]

25. Shin, K.; Park, G.; Choi, J.; Choy, M. Factors affecting the survival of SMEs: A study of biotechnology firms in South Korea. Sustainability 2017, 9, 108. [CrossRef]

26. Mata, J.; Portugal, P. The Survival of New Domestic and Foreign Owned Firms. Strateg. Manag. J. 2002, 23, 323-343. [CrossRef]

27. United Nations Conference on Trade and Development (UNCTAD). Informe sobre la Economía Creativa; UNCTAD: Geneve, Switzerland, 2010.

28. Peltoniemi, M. Cultural industries: Product-market characteristics, management challenges and industry dynamics. Int. J. Manag. Rev. 2014, 17, 41-68. [CrossRef] 
29. Département des études, de la prospective et des statistiques (DEPS). Aperçu Statistique des Industries Culturelles; Ministère de la Culture: Paris, Francia, 2006.

30. Department for Culture, Media and Sport (DMCS). Annual Report; HMSO: London, UK, 2001.

31. Shih, T.Y. Determinants of enterprises radical innovation and performance: Insights into strategic orientation of cultural and creative enterprises. Sustainability 2018, 10, 1871. [CrossRef]

32. Altman, E. Financial ratios, discriminant analysis and the prediction of corporate bankruptcy. J. Financ. 1968, 23, 589-609. [CrossRef]

33. Beccetti, L.; Sierra, J. Bankruptcy risk and productive efficiency in manufacturing firms. J. Bank. Financ. 2003, 27, 2099-2120. [CrossRef]

34. Lizárraga Dallo, F. Utilidad de la información contable en el proceso de fracaso: Análisis del sector industrial de la mediana empresa. REFC 1997, 93, 871-915.

35. Markman, G.D.; Gartner, W.B. The effects of Hyper Growth on Firm Profitability. J. Priv. Equity 2002, 5, 58-65. [CrossRef]

36. Pérez Gorostegui, E. Curso de Economía de la Empresa; Ramón Areces: Madrid, España, 2017.

37. Hale, G.; Santos, J. The decision to first enter the public bond market: The role of firm reputation, funding choices, and bank relationships. J. Bank. Financ. 2008, 32, 1928-1940. [CrossRef]

38. Mata, J.; Portugal, P.; Guimaraes, P. The survival of new plants: Entry conditions and post-entry evolution. Int. J. Ind. Organ. 1995, 13, 459-482. [CrossRef]

39. Kaplan, E.; Meier, P. Nonparametric estimation from incomplete observations. J. Am. Stat. Assoc. 1958, 53, 457-481. [CrossRef]

40. Harrington, D.; Fleming, T. A class of rank test procedure for censored survival data. Biometrika 1982, 60, 553-566. [CrossRef]

41. Cox, D.R. Regression models and life-tables. J. R. Stat. Soc. Ser. B. 1972, 34, 187-220. [CrossRef]

(C) 2020 by the authors. Licensee MDPI, Basel, Switzerland. This article is an open access article distributed under the terms and conditions of the Creative Commons Attribution (CC BY) license (http://creativecommons.org/licenses/by/4.0/). 
Article

\title{
Sustainable Use and Conservation of the Environmental Resources of the Etna Park (UNESCO Heritage): Evaluation Model Supporting Sustainable Local Development Strategies
}

\author{
Luisa Sturiale ${ }^{1, *}$, Alessandro Scuderi ${ }^{2}$, Giuseppe Timpanaro ${ }^{2}$ and Benedetto Matarazzo ${ }^{3}$ \\ 1 Department of Civil engineering and Architecture (DICAR), University of Catania, 95127 Catania, Italy \\ 2 Agriculture, Food and Environment Department (D3A), University of Catania, 95127 Catania, Italy; \\ scuderia@unict.it (A.S.); giuseppe.timpanaro@unict.it (G.T.) \\ 3 University of Catania, 95127 Catania, Italy; matarazzo@unict.it \\ * Correspondence: luisa.sturiale@unict.it; Tel.: +39-095-7382720
}

Received: 29 November 2019; Accepted: 10 February 2020; Published: 15 February 2020

\begin{abstract}
Rural areas are recognized as multifunctional spaces, where traditional agro-silvo-pastoral and other human activities (unrelated rural tourism, ecotourism, processing industries of agricultural and or extractive products, land maintenance, trade in local products, etc.) take place alongside each other. The integrated endogenous development model, established to mitigate the effects of human activity in protected areas, relies on the enhancement of specific resources of individual territories through the active participation of the community to promote local development. This model is intrinsically connected with the model of sustainable development, based on three cornerstones: environmental, social, and economic sustainability. The difficulty in achieving a reasonable balance among these values relates primarily to areas subject to protection (i.e., Parks and Natural Reserves). Ultimately, the environmental culture emphasizes the sustainability of natural resources, obviously in relation to these values and to the vulnerability of these areas. This paper outlines some relationships between environmental protection and the exercise of agricultural activities and other human activities in protected areas by using the theory of "rough sets". The study aims to show that in the complex context of Etna Park (recognized World Heritage of Humanity by UNESCO in 2013), the model developed by the "rough sets" could provide useful guidance to policy makers to formulate local development strategies according to a model of the sustainable management of protected areas.
\end{abstract}

Keywords: landscape management; agriculture; evaluation; multi criteria decision aide (MCDA); rough sets; multifunctionality

\section{Introduction}

In contemporary society, the need for human progress and for nature conservation are often perceived as conflicting values, which define obvious issues and contradictions in land management [1-3]. The process of industrialization, urbanization, and the associated socio-cultural changes that started in the nineteenth century has culminated in the bipolarization of the relationship between progress and nature [4]. On one hand, the urban-industrial condition, in which one lives and works, on the other, the natural condition in which one takes refuge [5]. The first concept of a protected area took shape according to the American model, dominant until the start of the 1980s and was defined as: "a different, exceptional place, made of uncontaminated nature, where the intervention of man is almost completely absent. The local community is completely excluded from the use of the territory, and seen as a threat to the preservation of natural ecosystems, while visitors are allowed access, only to benefit from them for tourism" [3]. This idea of protecting the natural environment is understood as a safeguard action 
through the imposition of specific legislative constraints provided for protected areas [6]. Over the years in Italy, the legislative orientation passed by a vision of protection to a vision of protection and sustainable use, with the aim of conserving the natural environment (as enshrined in National Law No. 394/1991).

There are 871 protected areas in Italy, covering approximately $32,000 \mathrm{~km}^{2}$. A reality of enormous value both from an environmental point of view and in terms of history, culture, and traditions [7]. Protected areas constitute a great laboratory for new innovative and eco-compatible practices, which, in recent years, have been at the center of an interesting socio-economic awakening. In terms of sustainability and the economy of local communities, this is the true essence of the Italian production model [8]. For this reason, attention to protected natural areas is in harmony with the theme of development and the revival of the economy [9]. A winning model is needed that can generate a transition for local economies toward sustainable growth [10-14].

The role of a multi-functional environment [15] in which traditional agriculture remains very important also relies on other activities that may or may not be related to those of agritourism, rural tourism, ecotourism, processing industries for agricultural products, handicrafts, land maintenance, trade in local products, and so forth [16-18].

At the same time, the integrated endogenous development model is making headway and affirming itself, which validates the use of specific resources such as individual territories through the mobilization of the local community. This endogenous development model proposes change based on innovation, but with the conservation of local social values and natural resources $[19,20]$.

This model is intrinsically connected with the theory of sustainable development, variously formulated, although the most widely known is the definition formulated in the famous Brundtland Report in 1987 (WCED) [21], which is based on three benchmarks [9,22]:

- $\quad$ environmental sustainability (sustainable use of natural resources: air, soil, water);

- $\quad$ social and cultural sustainability (health and hygiene; food standards, health and safety); and

- $\quad$ economic sustainability (income for economic operators).

These are conditions, all equally important, that can, however, also be in conflict for certain activities, especially based on organizational and operational methods and with reference to different territorial areas [23-25].

In the latter case, it is clear that the greatest difficulty in achieving a reasonable balance between these three conditions relates primarily to areas subject to protection (i.e., Parks and Natural Reserves), which above all emphasizes environmental culture and the sustainability of natural resources $[18,19,26]$.

The research outlines some relationships between environmental protection and the exercise of agricultural activities in the delicate environment of protected areas, and exposes some ideas on the theory of rough sets, which are believed to contribute to the resolution of the relationships that are sometimes rather complex in certain situations. The application of this decision model was carried out in a study of Etna Park, which surrounds the largest active volcano in Europe. The area was recognized as a UNESCO World Heritage Site in 2013 where the issues of progress and protection are at the center of debate amongst local operators. At times, there are tensions due to this dichotomy, with stakeholders declaring the need for evaluation methods and decision support, precisely because of the complexity of the activities in the area and because of the vulnerable natural and socio-cultural environment.

\section{Relationship between Protection and Use in Protected Areas in Italy}

The factors that have allowed an increasing number of protected areas in Italy are both political and institutional [27]. In the former case, it is important to underline the gradual change in the philosophy on which the establishment of a park is based, since we have moved from a restrictive and protectionist concept in managing protected areas, to a more flexible approach of sustainable and balanced management of natural resources, which is more compatible with economic growth in the area [28]. In Italy, there are a total of 871 protected areas of over three million hectares, which 
accounts for around $22 \%$ of the entire country. Based on the American classification of wildland economics and benefits, Italy's protected areas are rich in so-called non-market values (conservation of biodiversity, well-being, exchange of carbon dioxide in the forests, ability to regenerate the layers of pure water, mitigating the effects of climate change, landscape, etc.). This is not the case, however, as none of the Italian parks bear comparison with the gross domestic product (GDP) produced by parks in the European Union (EU). The Italian National Parks has pushed the growth of wealth in many areas in the north, while the south, they have still not managed to enhance them in economic terms. The Italian National Parks guarantee 3.2\% of the country's wealth. The natural heritage of the Italian National Parks covers a total area of 34 thousand square $\mathrm{km}$ within the territory of 527 different municipalities. Tourism, eco-tourism, the chain of eco-sustainability, and agriculture are the economic wealth. There is a "park effect", that is, a greater ability to create wealth and well-being by companies located in areas subject to environmental protection. It is a new development model based on the mix of economic growth, environmental sustainability, quality production, and respect for the knowledge and well-being of the territories.

In legal terms, there are several weak points, as there are few national parks that could beclassified as "active", in the sense that they operate at full capacity. According to Law 394/1991, parks must have a territorial plan and the regulation must be perfectly in force. Therefore, a push toward the strengthening of the role played by protected areas in the sustainable development of local communities (in terms of the management and use of natural landscapes and cultural resources as well as the positive effects in terms of employment and income this would have) could be derived from Decree 119 of 2017 (which reforms Law 394/91, and provides for the simplification of procedures) [6,15].

Turning to the relative consistency of all protected areas in Italy, the available documentation for 2018 reports that protected areas across national territories amounts to about $22 \%$, although in some regions this is higher (i.e., in Lombardy, Trentino Alto Adige, Abruzzo, Campania) [29].

Protected areas established by private or public initiatives, and then managed by environmental associations must be also be included in that figure. In particular: (a) protected areas managed by the WWF (World Wildlife Fund), which amount to 94 units (oases, areas, and shelters) covering an area of about 30,000 hectares; (b) those managed by the LIPU (Italian Bird Protection League), equal to 43 structures (36 oases and reserves, six recovery centers, and a museum); (c) those managed by the FAI (Italian Environmental Fund) that are engaged in the conservation of 12 areas of natural interest, covering 95 hectares; and (d) those managed by the "Italian Wilderness Association", who have proposed 15 areas of "wild space" that have been recognized.

As stated in Article 1 of Law 394/91 (currently in force), the main purpose of the legislation consists of maintaining the balance of the ecosystem both by preserving the set of biotic and abiotic elements in protected areas and by developing management methods to achieve harmonization between the natural environment and human progress. That is to say, a sort of integration between anthropic and natural elements to safeguard vulnerable architectural, archaeological, and landscapes as well as preserving traditional economic activities in particular, agricultural ones [18,30,31]. Article 1 also adds that eco-compatible production activities can be promoted in these areas. It is therefore clear that in terms of priority, the preservation of the balance of the ecosystem is in a pivotal position, and one with which economic activities must be compatible.

Agriculture and its associated activities play an eminent role in consolidating the combination of protection and enjoyment, which has relevance not only for protected areas, but for the entire rural area. Guidelines on the subject issued by the OECD (Organization for Economic Cooperation and Development) and the EU have their own application in relation to the degree of anthropization of the areas concerned and with specific reference to protected areas (integral reserves, general areas of protection, and areas of economic promotion).

On a theoretical level, this regulatory combination does not present difficulties to the extent that economic activities do not give rise to negative or positive externalities in the ecosystem. However, the 
same cannot be said in operational terms as the performance of economic activities can also impact the environment in ways that are not always considered neutral $[32,33]$.

With specific reference to protected areas, and bearing in mind the priority of protecting the ecosystem whilst also associating it with the use of agricultural areas, asynoptic framework (deliberately simplified, but undoubtedly emblematic) can be reasonably constructed $[3,15,18]$.

The primary purpose of protection consists of conserving the ecosystem balance, which is attributable to the macro-variables listed under (a)- $(\mathrm{g})$; (see Table 1 below), and that must not be impacted by negative externalities from economic activities in the area.

Table 1. Synoptic framework of some protection activity fruition reports.

\begin{tabular}{ll}
\hline Ecosystem Protection & Territorial Use \\
\hline (a) Physical, geological, geomorphological components & - Land transformations affecting component (a) \\
(b) Biodiversity & - Crop changes affecting component (b) (crop conversions) \\
(c) Traditional agroforestry landscape & - Modifications of the cultivation framework with effects on (c). \\
& - Production methods that cause changes in the relative \\
(d) Physical-chemical component of air, soil, water & composition of (d) (physical-chemical composition of air, soil \\
(e) Hydrogeological and hydraulic structure & and water) \\
(f) Rural and architectural building heritage & - Interventions in the area that affect on (e). \\
(g) Forest and forest mass & - Restructuring, modernization, building extensions that \\
\hline
\end{tabular}

Source: Our elaboration.

In the case of agro-silvo-pastoral human activity, this impacts the environment in one or more ways reported in the "fruition" part of the table. Therefore, this activity cannot always be included among those that give rise to positive externalities.

Indeed, such activity can affect biological diversity and traditional landscapes, affecting orography and hydrogeological structure, causing pollution of various kinds (physical, chemical, acoustic, etc.). It can also modify rural construction and architectural heritage, reducing the scale of forests, or the "lungs" of the environment, as they are known. Given that in protected areas the fundamental objective is to maintain these essential ecosystem macro-variables, it is essential to develop strategies for the use of these areas that allow for activities without compromising protection.

The existence of binomial protection/fruition appears completely evident, especially with reference to protected areas that are more or less strongly anthropized and that provide vital economic activities for local populations, already present before the establishment of these areas subject to constraints [6]. This is the case with the Sicilian Regional Parks (Etna, Madonie, and Monti Nebrodi) and more generally with most of the Italian protected areas.

It is precisely in these circumstances that the problems of sustainable development are exacerbated, when the emphasis is on the environmental benchmark, attributing a subordinate role to the other activities (especially economic activity).

This position can lead to conflicting situations if the territories have deep-rooted human settlements and are also sites with a multiplicity of economic activities, sources of income, and employment on which the local population is dependent for its survival [22].

A notable case is Etna Park, which 30 years after its establishment, is now adopting a Territorial Coordination Plan. This plan will see the complex merging of the local ecosystem and the economic activities traditionally practiced in the region. At present, the plan has been drawn up and is in the process of being adopted with a view to integrating the economic, political, and environmental problems that intertwine, generating a block of activities [34].

The general objectives of the plan and of the regulations are those aimed at forming a unitary instrument of the government of the park territory thatis flexible and capable of combining different development interests against the socio-economic and cultural background of the populations established in the park municipalities with the priority interests of naturalistic, environmental, landscape protection, and the anthropic cultural values that determined the institution of the park itself. 
From an environmental point of view, Etna Park, recognized as a UNESCO Heritage site in 2013, has the largest active volcano in Europe (Figure 1). The recurrent magmatic eruptions have shaped and reshaped the territory over the centuries, widening the sterile surfaces covered with lava and narrowing those usable for primary activities. The eruptions have recreated the landscape with the formation of volcanic cones (of great landscape value), but which are extremely vulnerable due to anthropic activity. Ranging from $550 \mathrm{~m}$ to over $3300 \mathrm{~m}$, the site is populated by a multitude of plant species (stratified by altimetric bands), together with variegated terrain (volcanic), which contributes to the formation of natural and agroforestry landscapes of great value that are characterized by panoramas of incomparable beauty. UNESCO has registered Etna Park on the World Heritage List as a "Natural Site", giving this brief description (Decision: 37 COM 8B.15): "Mount Etna World Heritage Site (19,237 ha) comprises the most strictly protected and scientifically important area of Mount Etna, and forms part of the Parco dell'Etna Regional Nature Park. Mount Etna is renowned for its exceptional level of volcanic activity, and the documentation of its activity over at least 2700 years. Its notoriety, scientific importance, and cultural and educational value are of global significance".

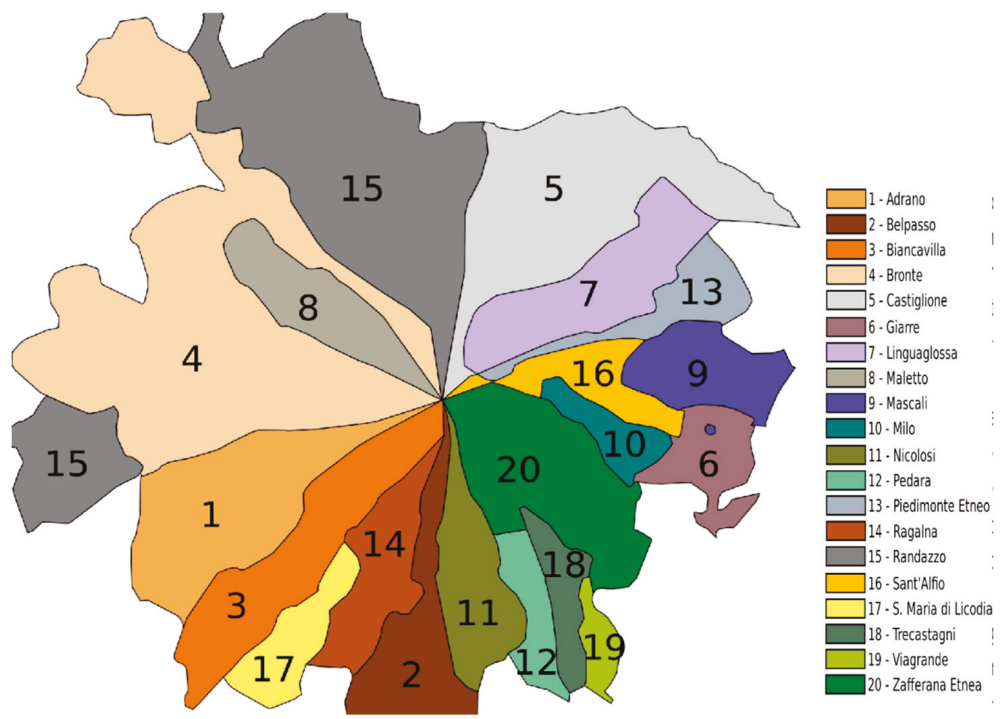

Figure 1. Communal territories that fall within Etna Park.

The Parco dell'Etna (Etna Park) was established as a Regional Nature Park by Decree of the President of the Sicilian Regional Authority in May 1987. Etna Park comprises a zone defined as an integral reserve, in addition to which, nine Natura 2000 sites overlap the area to various degrees, providing additional protection for $77 \%$ of the site under EU legislation. The territory of the district is characterized by excellent lands for agricultural production, thanks to the particular fertility of volcanic debris. The inhabited area reaches up to $900 \mathrm{~m}$, while the cultivated areas and woodlands reach over $1500 \mathrm{~m}$. The area is managed and coordinated by Ente Parco dell' Etna, established as the managing authority for Etna Park by Decree of the President of the Sicilian Regional Authority in May 1987, working in close cooperation with the Regional Authority of State Forests and the Regional Corps of Forest Rangers (Corpo Forestale). Management of the Park is guided by a long-term management plan and Triennial Intervention Programs.

All this gives rise to remarkable biological diversity, both in flora and fauna, a fundamental inheritance for society that the Park enriches or at least "conserves" as a precious germplasm for future generations [35]. 
The Park zoning shows that areas A (integral reserve) and B (natural reserve) constitute 77.5\% of the entire Park surface, while the remaining part is represented by zones $C$ and D (protection and control, respectively) (See Figure 2).

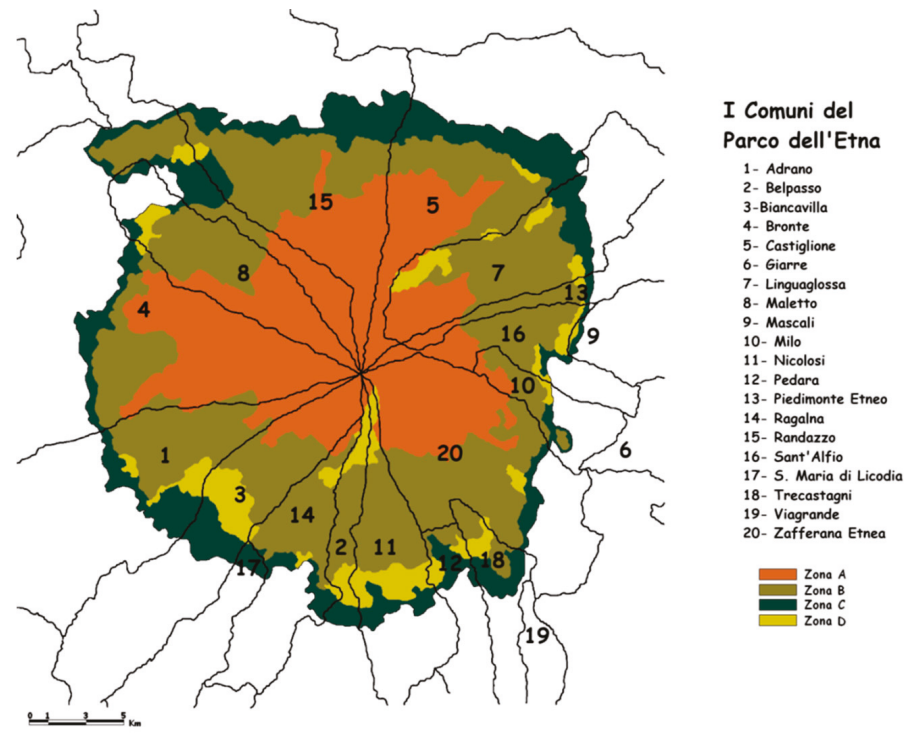

Figure 2. Delimitation of the areas A, B, C, D at Etna Park.

The "Mount Etna" site has a core zone coinciding with " $\mathrm{A}$ " of the Park and a buffer zone coinciding with " $\mathrm{B}$ " of the Park. The UNESCO site management plan coincides with the Natura 2000 site management plan where a project is underway to revise and update the UNESCO Site Management Plan.

With reference to agroforestry in Etna Park, this appears to be increasing from zone A to that of D (Figure 2), resulting in a total of 30,830 hectares, equal to $53.1 \%$ of the entire area of the Park. Obviously, the forest area is prevalent in zones A and B, while the agrarian areas are located in B, C, and D.

Centuries-old traditions of agricultural activities exist within the Park, albeit progressively reducing, but with the expansion of "abandoned" agricultural areas, pastoral forest and undergrowth have increased. However, Etna Park is also a privileged destination for mountain tourists throughout the year. In relation to the worldwide development of the Park, this represents an expansive trend because tourism is associated with traditional tourism (i.e., food, wine, and more generally rural tourism) [34].

Finally, the flourishing trade in local products should be added: agricultural, zootechnical, and artisanal items, based on direct (producer-consumer) interaction, and seen by the continuous growth of agricultural revenue with the intensification of the tourist wine trade.

As predicted in the study, there is a very diverse range of economic activity present in the Park (vital on a social level), and one that certainly does not favor the resolution of the relationship between protection and use. The same applies for the multiplicity of co-operative activities and the corresponding professionals involved regarding the complexities within the activity.

Analyzing agricultural activities in the Park, the research carried out in the field by $[26,35,36]$ highlighted the presence of numerous trees and herbaceous species. The information acquired allowed us to deduce that the effects of agricultural activity on the macro-elements of the ecosystem are not perfectly quantifiable, but to state that although the qualitative cause-effect relationships are known, inevitably, they are surrounded by a certain imprecision. 
This conclusion justifies the search for methods of analysis that compare protection parameters with those of fruition, and that can offer significant assessment for decision-makers, allowing them to choose options that safeguard protection but that do not stifle progress.

\section{Method-The Theory of Rough Sets}

There are several theories and mathematical techniques which, over time, have been developed to analyze problems characterized by uncertainty and in accuracy. Included in these theories is the theory of probability, the theory of evidence, discriminatory analysis, and the theory of fuzzy sets. The theory of rough sets can also be added to this group, as it has been proven to be a suitable tool to analyze data characterized by a certain vagueness and inaccuracy and to highlight the possible cause-effect relationships that exist between them. These relationships are not immediately evident, but can be useful in the context of choice. The theory of rough sets has different points of intersection with the theories above-mentioned and in some applications, it is complementary.

The theory of rough sets was introduced by Pawlak (1997) [37] and has been the subject of in-depth studies by various researchers and applied to different fields as well as to decision problems relating to cultural heritage. One particularly interesting aspect is the analysis of problems of multi-attribute classification and more recently, in the analysis of multi-criteria decision problems such as classification, choice, and sorting; making methodological changes to the original theory by [38-40].

This theory is based on the premise that information is associated with each unit of a given universe, which are expressed by employing determined attributes that describe the aforesaid units. For example, if the units represent the agricultural companies whose objective is to reduce negative externalities on the ecosystem, the information is represented by the activity location, the cultivation method adopted, etc. The units characterized by the same description are considered to be indiscernible (or similar), depending on the information available [41,42].

As demonstrated by several researchers [43-45], the relationship of indiscernibility generated constitutes the mathematical foundation for the theory of rough sets, the bricks (or granules) with which the knowledge reality is constructed.

Each set of indiscernible units is defined as an elementary set and represents an atom of knowledge of the universe. Any subset $Y$ of the universe can be defined in terms of granules, union of elementary sets, or an approximation. In the latter case, the subset can be characterized by two ordinary sets: lower approximation and upper approximation. The "rough set" is defined by the previously mentioned approximations, which coincide in the case of ordinary sets, and the difference between the lower and upper approximations represents the frontier [37,42] of the rough set.

The lesser approximation of $\mathrm{Y}$ is constituted by all the elementary sets included in $\mathrm{Y}$, whose elements surely belong to $Y$; the upper approximation is given by all the elementary sets that have a non-empty intersection with $Y$, whose elements can belong to $Y$. The elements that belong to the border of the rough sets cannot therefore be characterized with certainty as belonging to $\mathrm{Y}$ using the available information. According to the approach of rough sets I, therefore, a concept described in an imprecise way can be replaced by a pair of precise concepts, its inferior and superior approximations [40].

The peculiarity of rough sets is that it deals with an imprecise representation of reality due to the granularity of knowledge, a consequence of the indiscernibility between objects having the same description (granules) [41].

This methodology, because of its peculiar characteristics, is particularly interesting for application to different concrete problems. This approach allows the possible cause-effect relationships between the available data to be highlighted, underlining the importance and the strategic role of some information and the irrelevance of others [40].

Only qualitative data are required, and it is not necessary to perform a prior analysis on the consistency of the data, while quantitative data must be discretized. This method takes into account inconsistent information that is not automatically eliminated a priori, as it is possible to obtain elements from both positive and negative examples. It is also possible to gain a posteriori information on the 
importance that some attributes have in the analysis and the results are presented in the form of decisional rules of the type if, then..., [43]. The set of decision-making rules obtained constitutes the global model of the decision maker's preferences based on the set of examples provided and can be used as a support to the decision-making process [40].

In order to apply the theory of rough sets to choices relating to the use of natural resources in protected areas and, in particular, to the compatibility between agriculture and the protection of the ecosystem, the main concepts that characterize the issues will be illustrated.

The information in the units that make up the universe of the analysis are provided in the form of a table (defined as an information table). Each table row refers to a different unit and each column to the different attributes being examined; each cell, therefore, will indicate the evaluation, quantitative or qualitative, of the unit placed in the row through the attribute of the corresponding column. With reference to the quantitative evaluation of a given $q$ attribute, the relative domain is subdivided into coded sub-intervals [40].

The information table is:

"... the 4-upla $S=<\mathrm{V}, \mathrm{Q}, \mathrm{V}, \mathrm{f}\rangle$,

where $\mathrm{U}$ is the finite set of objects (universe), $\mathrm{Q}$ is a finite set of attributes, $\mathrm{V}=\mathrm{U} \mathrm{Vq}$ and $\mathrm{Vq}$ is the domain of the attribute.

$Q, f: U x Q \rightarrow V$ is a $q \in Q$ function that $(x, y) \in V q$ for each $q \in Q, x \in U$, defined as the function of information.

Each element $x$ of the universe $U$ will be described by a vector where each element represents the value that the relative attribute assumes with reference to $\mathrm{x}$. This vector is called the description of $\mathrm{x}$ in terms of the evaluations of the attributes of $Q$ and indicated DesQ (x) [40]. It is thus possible to obtain a description of $x \in U$ in terms of any non-empty subset $P \subseteq Q$.

Each subset (not empty) of $\mathrm{P}$ attributes is associated with an indiscernible relation on $\mathrm{U}$, indicated with Ip:

Ip $=\{(x, y) \in U \times U: f q(x)=f q(y), q \in P\}$.

Obviously, the binary relation of indiscernibility thus defined is a relation of equivalence (reflexive, symmetrical and transitive).

If $(x, y) \in I p$, it is stated that objects $x$ and $y$ are P-indiscernible. The equivalence classes of the Ip relation are defined as $\mathrm{P}$-elementary sets.

If $\mathrm{P}=\mathrm{Q}$, the Q-elementary sets are defined as "atoms".

It is necessary to develop two other key concepts when using rough sets to aid definition: lower approximation and upper approximation.

A subset $P$ of attributes $Q, P \subseteq Q$, and $X$ is defined as a non-empty subset of $U$. The lower $\mathrm{P}$-assessment of $\mathrm{X}(\mathrm{PLX})$ is defined as the subset of $\mathrm{U}$, having as its elements all the objects belonging to the P-elementary sets contained in the $X$ set, and only those.

The subset of $U$ is defined as the upper P-approximation of $X(P U X)$, made up of all the objects belonging to the P-elementary sets with at least one element in common with set $\mathrm{X}$, and only those [40].

The difference between these two sets is referred to as the X-frontier, indicated by BNp (X):

$\operatorname{BNp}(X)=$ PUX - PLX

If an object $x$ belongs to the lower $\mathrm{P}$-axis of $\mathrm{X}$, it is certainly an element of $\mathrm{X}$, while if $\mathrm{x}$ belongs to the upper P-axis, it can belong to set $\mathrm{X}$.

If the frontier of $X$ is empty, then set $X$ is an ordinary set, obtained by the union of a certain number of P-elementary sets. If the frontier of $\mathrm{X}$ is not empty, set $\mathrm{X}$ is an approximate set (rough) with respect to $\mathrm{P}$, definable through the lower and upper P-approximations.

This approach leads to an imprecise representation of reality due to the "granularity" of knowledge, which is in fact, the key concept of the theory of rough sets. Reality is represented by granules that correspond to the P-elementary sets; whose elements are P-indisputable because they have the same description in terms of the attributes of $\mathrm{P}$. The size of these granules depends, obviously, on the number of attributes used for the description of the objects in the original information and on the domain of 
each attribute. The representation of reality depends on the knowledge possessed with reference to it and on the ability to classify the information obtained.

Another particularly important aspect for operational applications is the possible presence of superfluous data in the information table, which can be eliminated without altering the content of the original table [42].

It is possible to determine a reduced, minimal subset of attributes that allow the same quality of approximations of the units of $U$ obtainable by employing the complete set of attributes of $P$. In a table of the information can exist attributes smaller than $\mathrm{P}$, and it is defined as the nucleus (core) of $\mathrm{P}$, the set containing all the indispensable attributes of $\mathrm{P}$. Being included in each reduced $\mathrm{P}$, the nucleus is configured as the most important subset of attributes of $Q$, for which none of its elements can be removed, otherwise the quality of the information would deteriorate.

$\mathrm{Q}$ is reduced in concrete applications because it is often difficult or expensive to acquire assessments of certain attributes. However, these assessments can be left out if they are superfluous, allowing significant savings of time and resources.

If the $Q$ attributes in the information table are divided into conditional attributes (set $C$ ) and decision-making attributes (set D), then it is defined as the decision table. It can be expressed as a set of decisional rules, as it tends to underline the functional dependence between conditional and decisional attributes. In operative applications, the tendency is to employ the least possible number of conditional attributes, without deteriorating the quality of the approximation of the classification induced by the decisional attributes, in such a way as to allow decisions to be made using less information. Very often the calculation of the decisional rules is complex, so that in applications with concrete cases, it is sufficient to know the minimal set of attributes. This method allows the same information to be used, resulting in more comprehensible results even if the sample is dimensionally reduced.

The decision rules are configured as logical propositions of the type if ... then, in which the antecedent is given by values assumed by one or more conditional attributes and the consequent is represented by the partitions generated by one or more decisional attributes. There are two types of decisional rules: exact (or certain), if the partitions generated by the decisional attributes contain those corresponding to the considered conditional attributes; and approximate (or uncertain) in the opposite case.

The expression used to define the decision-making rule not only has the advantage of being perfectly understandable to the decision-maker, but also has the advantage of being the simplest and most natural way to build a model of preferences in light of a sufficiently large sample of explanatory comparisons.

From the decisional point of view, the construction of the preference model does not require any parameters, but simply uses the examples provided by the decision-maker and the rules obtained can be adopted as support to the decision-making process. It is important to note that it is possible to verify the information contained in the original decision table and to implement it, acquiring further elements also in the form of decisional rules, following interviews or discussions with experts.

The concepts exposed constitute the key points of the original theory of rough sets, to which methodological adaptations have been made to apply it to the multicriteria problems of classification, choice, and sorting. The methods applied by previous researchers using this theory suggest considering the ordinary properties of the criteria and information on the decision-maker preferences and to proceed to the comparison by pairs of action.

\section{Results: The Rough Sets Approach in the "Etna Park" Study}

The theory of rough sets has been successfully applied to various real problems of classification in research fields. The most significant of these are medicine; engineering; credit; market research; financial analysis; and economics. This study proposes that the theory of rough sets can be applied to a very complex and difficult field to explore such as environmental economics. Focusing on the related environmental issues, and in particular, the use of agricultural land in protected areas, 
this methodology supports decision-making rules that can aid the definition of strategies for the management of agricultural activities that are compatible with both environmental protection and the maintenance of the ecosystem.

The subject area of this research was Etna Park, to which the theory of rough sets was applied, with a view to solving decisional problems related to the choice of crops to be adopted within the protected area (see Figure 3). These decisions need to be compatible with the maintenance of the ecosystem balance, which has been altered to an extent due to the increase in human activity.

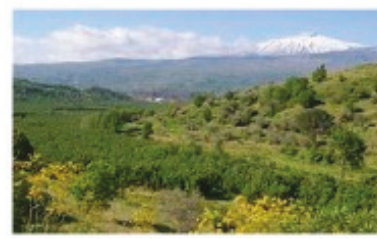

Meseinut archerch in the tina perk

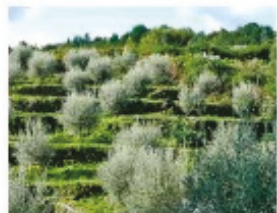

Gitive rase an Etina igoo Marte tina!

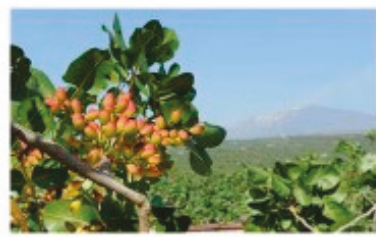

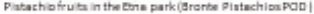

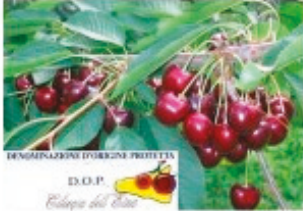

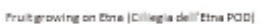

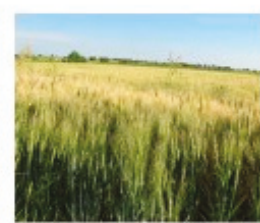

Cecesis in the Ems parkiounum wheat!

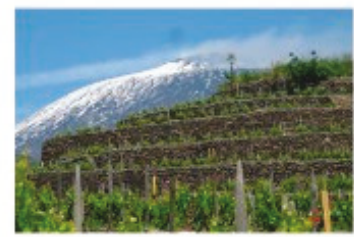

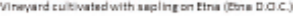

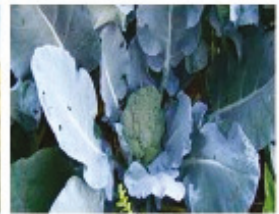

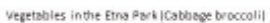

Figure 3. Landscapes and agricultural productions in Etna Park.

On the basis of information provided by experts and scholars, an information table has been drawn up with the $x$ units that make up universe $D$, in the individual lines. The sex units represent the main types of farms in the Park, (see Table 2 below), whose details are represented by the following:

Table 2. Average profitability expressed as EBITDA * referring the main Etna park crops.

\begin{tabular}{cc}
\hline Crops & EBITDA $\mathbf{~ / h a ~}$ \\
\hline x1. hazelnuts; & 650 \\
x2. pistachios; & 1.700 \\
x3. fruit (pear, apple, cherry, peach); & 2.700 \\
x4. wine grapes; & 3.000 \\
x5. olives; & 1.200 \\
x6. vegetables; & 4.500 \\
x7. cereals; & 180
\end{tabular}

Source: Our elaboration. * EBITDA (Earnings Before Interests Taxes Depreciation and Amortization).

The information table below was produced by inserting the attributes that mainly influence the equilibrium of the ecosystem as a result of agricultural activity. Only five conditional attributes were considered amongst the various possible ones, four of which were qualitative and one was quantitative (see Table 3). The column attributes are as follows:

c1. Ability to maintain biodiversity (quality attribute).

c2. Influence on the landscape (quality attribute).

c3. Environmental pollution due to conventional production methods (qualitative attribute).

c4. Alterations in the ecosystem equilibrium with the adoption of the organic production methods (qualitative attribute).

c5. Profitability expressed as EBITDA (Earnings Before Interests Taxes Depreciation and Amortization), in the agricultural year (2017/18) obtained without subsidies or income supplements 
provided for in community and national legislation for the various production sectors (quantitative allocation).

$\mathrm{U}=\{1,2,3,4,5,6,7\}, \mathrm{Q}=\{\mathrm{cl}, \mathrm{c} 2, \mathrm{c} 3, \mathrm{c} 4, \mathrm{c5}, \mathrm{d}\}, \mathrm{V} 1=\{$ weak, medium, high $\}, \mathrm{V} 2=\{$ weak, medium, strong $\}, \mathrm{V} 3=($ low, medium, high $\}, \mathrm{V} 4=\{$ low, medium, high $\}, \mathrm{V} 5=\{$ low, medium, high $\}, \mathrm{Vd}=\{\mathrm{A}, \mathrm{B}$, C, D\}.

Table 3. Information and evaluation table.

\begin{tabular}{ccccccc}
\hline U & c1 & c2 & c3 & c4 & c5 & d \\
\hline x1 & high & strong & medium & low & low & A \\
x2 & medium & strong & medium & low & low & B \\
x3 & weak & medium & high & medium & medium & D \\
x4 & weak & medium & medium & low & medium & C \\
x5 & weak & medium & medium & low & low & C \\
x6 & weak & weak & high & medium & high & D \\
x7 & weak & weak & medium & low & low & C \\
\hline \multicolumn{7}{c}{ Source: Our elaboration. }
\end{tabular}

With reference to the coding of attribute c5, linked to profitability level, the original quantitative values of c5 were classified as follows (Table 4 ):

Table 4. Classification of the level of profitability.

\begin{tabular}{cc}
\hline Original Values of $\mathbf{c 5}(\boldsymbol{\epsilon} / \mathbf{h a})$ & Coded Values of $\mathbf{c 5}$ \\
\hline $\mathrm{X}<€ 1.000$ & low \\
$1.000<X<€ 2.500$ & medium \\
$\mathrm{X}>2500$ & high \\
\hline \multicolumn{2}{c}{ Source: Our elaboration. }
\end{tabular}

The decision-making attribute " $\mathrm{d}$ " determines a set of alternatives according to the following ranking:

$\mathrm{A}=$ optimal culture for the maintenance of the ecosystem balance;

$\mathrm{B}$ = fully compatible culture;

$\mathrm{C}=$ crop with a low risk of disturbance of equilibrium; and

$\mathrm{D}=$ crop with a medium risk of disturbance of equilibrium.

The classifications originate from the set of information derived from previous studies and research carried out by naturalists and biologists on the influence of different crops that are present in the ecosystem in Etna Park.

Each type of farm is characterized by a different description in terms of the attributes considered: cl, c2, c3, c4, and c5. Therefore, based on the information derived from them, they were discernible. Formally, the relationship of indiscernibility based on all of the attributes considered was IQ $=\{(1,1)$, $(2,2),(3,3),(4,4),(5,5),(6,6),(7,7)\}$, so there were no two types of farm $x$ and $y$ so that $(x . y) \in I Q$.

From the evaluation of the information table, it can be observed that the farm types 1 and 2 are indiscernible in terms of the attributes of $\mathrm{P}=\{\mathrm{c} 2, \mathrm{c} 3, \mathrm{c} 4, \mathrm{c} 5\}$ because they have the same evaluation for each of them. Formally, we have Ip=\{(1,1), $(1,2),(2,1),(2,2),(3,3),(4,4),(5,5),(6,6),(7,7)\}$ for which $(1,2) \in \mathrm{Ip}$ (and obviously also $(2,1) \in \mathrm{Ip}$. Just as company types 3 and 4 are indiscernible in terms of attributes $\mathrm{P}^{\prime}=\{c 1, \mathrm{c} 2, \mathrm{c} 5\}$ and formally we have $\mathrm{Ip}^{\prime}=\{(1,1),(2,2),(3,3),(3,4),(4,3),(4,4),(5,5),(6,6),(7,7)\}$, so $(3,4)$ CE Ip' (and $(4,3)$ CE Ip'). In this way, it would be possible to proceed considering all possible sub-sets of Q.

Each subset $\mathrm{P}$ of the attributes of $\mathrm{Q}$ originates a partition of the universe $\mathrm{U}$ that classifies equivalent groups that have the same description in terms of the attributes of $\mathrm{P}$ as equivalent objects, for example, for $\mathrm{P}=\{\mathrm{cl}, \mathrm{c} 2\}$, we have $\mathrm{U} \mathrm{Ip}=\{\{1\},\{2\},\{3,4,5\},\{6,7\}\}$ and therefore $\{1\},\{2\},\{3,4,5\},\{6,7\}$ are the sets P-elementaries. 
Suppose that using the set of attributes $\mathrm{P}=\{\mathrm{cl}, \mathrm{c} 2, \mathrm{c} 3\}$ we intend to approximate the set of $\mathrm{X}$ farms types that involve a low risk of alteration to the ecosystem balance, $X=\{x 4, x 5, x 7\}$.

Then, $\mathrm{U} \mathrm{I} / \mathrm{Ip}=\{\{1\},\{2\},\{3\},\{4,5\},\{6\},\{7\}\}$, the approximations will be:

$$
\begin{aligned}
& \operatorname{PL}(X)=\{4,5,7\} \\
& \operatorname{PU}(X)=\{4,5,7\}
\end{aligned}
$$

The BNP frontier $(X)$ is empty, for which set $X$ can be described with the attributes of $P$ and is an ordinary set (i.e., it is precise), since the units contained certainly belong to the set of business types that determine a low risk of ecosystem alteration.

From the information table, let us consider the set of attributes $\mathrm{P}=\{\mathrm{cl}, \mathrm{c} 3, \mathrm{c} 4\}$, which determines the following indiscernibility relation $\mathrm{U} / \mathrm{Ip}=\{\{1\},\{2\},\{4,5,7\},\{3,6\}\}$. Now, let us consider the following subsets of $Q: P=\{c l, c 3, c 4\}, R=\{c l " c 3\}, S=\{c l, c 4\}, T=\{c 3, c 4\}$.

It is observed that IR = Ip, IS = Ip, while EN \# Ip.

What has been observed means that $\mathrm{R}$ and $\mathrm{S}$ are reduced of $\mathrm{P}$, while $\mathrm{T}$ is not, that is, $\mathrm{R}$ and $\mathrm{S}$ are sub-sets of $\mathrm{P}$ that have generated partitions of the universe $\mathrm{U}$ equal to those obtained by using all the attributes of $\mathrm{P}$. It is possible, therefore, to use any of the two determined reduced attributes without affecting the quality of information deduced with all the attributes present in $\mathrm{P}$.

If we had considered as a set of attributes $\mathrm{P}=\{\mathrm{cl}, \mathrm{c} 2, \mathrm{c} 3\}$, the relation of indiscernibility generated would have been $\mathrm{U} / \mathrm{Ip}=\{\{1\},\{2\},\{3\} ;\{4,5\},\{6,7\}$. By determining how subgroups of $\mathrm{P}, \mathrm{R}=\{\mathrm{c} 1, \mathrm{c} 2\}, \mathrm{S}=\{\mathrm{c} 2$, $\mathrm{c} 3\}, \mathrm{T}=\{\mathrm{c} 1, \mathrm{c} 3\}$, we would have identified only one reduced attribute, namely $\mathrm{T}$, as IT = IP, while IR \# IP and IS \# IP. It is therefore possible to identify the "superfluous" conditional attribute in P, which corresponds to $\mathrm{c} 2$.

From the intersection of the reduced $\mathrm{R}$ and $\mathrm{S}$, we can determine the core of $\mathrm{P}$ as core $\mathrm{P}=\mathrm{R} \cap \mathrm{T}$ or core $\mathrm{P}=\cap \mathrm{R}$ and $\mathrm{P}$, which in this case corresponds to the conditional attribute $\mathrm{c} 1$. Therefore, $\mathrm{c} 1$ is "the most important attribute" of the whole $\mathrm{P}$, and is the attribute without which the quality of the information would deteriorate, that is, it represents the most important attribute to describe the business types considered (we could define it as the essential one), while c3 and c4 can be exchanged without the quality of the information deteriorating.

The information table constructed containing conditional attributes $C=\{c 1, c 2, c 3, c 4, c 5\}$ and the decision attribute $\mathrm{D}=\{\mathrm{d}\}$ can be read as a decision table, through which it is possible to explain the evaluations of the decision attribute, by means of the values attributed to the conditional ones. It is possible, therefore, to interpret the information able as a table of decisions. Referring to the example given, the set of decision-making rules obtained from the illustrated decision table is as follows:

if $f(x, c 1)=$ high and $f(x, c 2)=$ strong and $f(x, c 3)=$ medium and $f(x, c 4)=$ low and $f\left(x^{\prime \prime} c 5\right)=$ low, then $f(x, d)=A$;

if $f(x, c 1)=$ average and $f(x, c 2)=$ strong and $f(x, c 3)=$ average and $f(x, c 4)=$ low and $f(x, c 5)=$ low, then $\mathrm{f}(\mathrm{x}, \mathrm{d})=\mathrm{B}$;

if $f(x, c 1)=$ : weak and $f(x, c 2)=$ medium and $f(x,(3)=$ high and $f(x, c 4)=$ medium and $f(x, c 5)=$ medium, then $\mathrm{f}(\mathrm{x}, \mathrm{d})=\mathrm{D}$;

if $f(x, c 1)=$ weak and $f(x, c 2)=$ medium and $f(x, c 3)=$ medium and $f(x, c 4)=$ low and $f(x, c 5)=$ medium, then $f(x, d)=C$;

if $f(x, c 1)=$ weak and $f(x, c 2)=$ medium and $f(x, c 3)=$ medium and $f(x, c 4)=$ low and $f(x, c 5)=$ low, then $f(x, d)=C$;

if $f(x, c 1)=$ weak and $f(x, c 2)=$ weak and $f(x, c 3)=$ high and $f(x,(4)=$ medium and $f(x, c 5)=$ high, then $f(x, d)=D$

if $f(x, c l)=$ weak and $f(x, c 2)=$ weak and $f(x, c 3)=$ medium and $f(x, c 4)=$ low and $f(x, c 5)=$ low, then $\mathrm{f}(\mathrm{x}, \mathrm{d})=\mathrm{C}$

The set of rules just described can be suitably reduced to a minimal set by using fewer attributes in each rule. Considering only attribute 1 , the following decision rules are reached: 
$\left.1^{\prime}\right)$ if $f(x, c 1)=$ strong, then $f(x, d)=A$;

$\left.2^{\prime}\right)$ if $f(x, c 1)=$ average, then $f(x, d)=B$;

$\left.3^{\prime}\right)$ if $f(x, c 1)=$ weak, then $f(x, d)=C$ or $D$;

and again, by adding attribute $c 4$, it is able to unambiguously explain both value $\mathrm{C}$ and the value $D$ of the decisional attribute. That is:

$\left.4^{\prime}\right)$ if $\mathrm{f}(\mathrm{x}, \mathrm{c} 1)=$ weak and $\mathrm{f}(\mathrm{x},(4)=$ low, then $\mathrm{f}(\mathrm{x}, \mathrm{d})=\mathrm{C}$;

$\left.5^{\prime}\right)$ if $f(x, c 1)=$ weak and $f(x, c 4)=$ average, then $f(x, d)=D$.

It is possible to observe that the rules $\left.\left.1^{\prime}\right), 2^{\prime}\right), 4^{\prime}$ ), and $5^{\prime}$ ) are certain and exact because they have a consequent univocal, instead rule $3^{\prime}$ ) is an approximate rule because it does not have a consequent univocal.

\section{Conclusions}

The multi-functionality in Etna Park highlights the related problems of the choice of location of the various activities in the area and the difficulties of managing such activities within an agricultural environment that assumes a fundamental role for both its traditional productive function and for the environmental and landscape services it provides. These functions are underlined and recognized in the latest community policy guidelines [22,44,46-48].

The multiplicity of activities in the Park also defines the presence of various operators who obviously tend to pursue diverse objectives that are often in conflict with each other [49]. Clashes of opinion are well known between naturalists, farmers, agro-industrial industry, tour operators, traders, urban planners, and so forth, relating to the allocation of human activity. The situation is even more complex when these problems surface within protected areas, where the protection of the natural environment and the use of resources must find a sustainable solution to coexist without stifling human activities that are subject to environmental restrictions [50]. Moreover, Etna Park is characterized by the presence of an active volcano, which frequently impacts the Park's management activities, agricultural production, and its collectivity.

The rough sets theory adopted in this research has allowed the treatment of data characterized by vagueness and imprecision, using qualitative and quantitative data, by adopting specific discretization to generate results based on the preferences expressed. These results are fundamental in the evaluation phase used by decision-makers. The resulting information table, even with inconsistent data, confirms that marginal data must not be eliminated as they highlight possible cause-effect relationships between the available data. These elements allow us to emphasize the importance and the strategic role of some attributes and the irrelevance of others (reduced and essential) [42].

The interventions on cultural heritage and, more generally, urban and territorial transformations determine direct effects in the physical-environmental system of a given area and indirect effects in the social and economic system [51-53]. Then, the construction of a model of preferences in terms of decision-making rules such as "if ... then" facilitates a model of understanding for decision-makers, which could become an operational tool to support land management policies. The final decision analysis allowed us to define aspects inherent to the decision (retrospective analysis) and to provide help to decision-makers on how to make decisions in the future (prospective analysis). The latter is based on decision-making rules obtained from the decision tables: "the explanation phase, therefore, prepares that of the prescription, giving useful information for the aid of decisions. In this respect, the rough sets approach is similar to an inductive process" [45].

The theory of rough sets in the case of Etna Park has made it possible to define more clearly that the crops present (apart from vegetables) are perfectly integrated with the landscape and have a limited impact on the ecosystem. Pistachios are the crops that integrate well and have no impact on the landscape, while other crops such as vines and olive trees, although showing a slight impact, represent crops of high landscape importance such as active components like woods and natural meadows.

In relation to the results obtained, using the rough sets approach to the analysis of decisions, agricultural cultivation appears to be perfectly sustainable as a whole. Recently, however, other 
applications of the original rough sets approach have been developed, based on approximations constructed using dominance relationships instead of indiscretions, which allows its application to all classes of multi-criteria problems.

The results of this study could be used by the local decision-makers and interested stakeholders to review and update management planning of the Etna Park, with the aim of being able to achieve what is indicated as recommendations by UNESCO (Decision: 37 COM 8B.15):

(a) Strengthen harmonization between the various management organizations and private sector partners inensuring that the outstanding geological features in the Park are not adversely impacted by increasing tourism pressures.

(b)Strengthen mechanisms to monitor visitor use so that the protection of natural heritage and enhanced visitor experience and safety are balanced.

(c) Encourage improved research and monitoring of the eco-balance by including technical staff (geologists, geomorphologists, and volcanologists) as an integral part of the site management team.

This study has shown that when the rough sets theory is applied to the conservation of resources in Etna Park, the model used provides an excellent eco-eco tool (ecological-economic) [48,54,55] for decision-makers where the application of multi-disciplinary contributions [56] is needed.

Author Contributions: Conceptualization, L.S., A.S. and B.M.; methodology, B.M.; software, S.A. and G.T. and B.M.; validation, L.S., A.S. and B.M.; investigation, L.S., A.S. and G.T.; data curation, A.S.; writing-original draft preparation, L.S. and A.S.; writing-review and editing, L.S., A.S.; funding acquisition, L.S. All authors have read and agreed to the published version of the manuscript.

Funding: This work was financed by the University of Catania within the project "Piano della Ricerca Dipartimentale 2016-2018 of the Department of Civil Engineering and Architecture".

Conflicts of Interest: The authors declare no conflicts of interest.

\section{References}

1. Bresso, M. Per un'Economia Ecologica; NIS: Roma, Italy, 1993.

2. Fusco Girard, L. I beni ambientali: Valutazioni e strategie di conservazione tra conflitto e cooperazione. Genio Rural. 1994, 5, 35-44.

3. Peano, A. Aree protette e governo del territorio. In Parchi D'Europa. Verso Una Politica Europea per Le Aree Protette; Gambino, R., Talamo, D., Thomasset, F., Eds.; Edizioni ETS: Pisa, Italy, 2008.

4. Shmelev, E.S.; Rodriguez-Labajos, B. Dynamic Multidimensional Assessment of Sustainability at the Macro Level: The Case of Austria. Ecol. Econ. 2009, 68, 2560-2573. [CrossRef]

5. Tempesta, T. La stima del valore ricreativo del territorio: Un'analisi comparata delle principali metodologie. Genio Rurale. 1995, 12, 15-34.

6. Salberini, G. L'evoluzione della legislazione italiana in materia di aree protette. In Codice Delle Aree Protette; Marchisio, S., Salberini, G., Della Fina, V., Ferrajolo, O., Eds.; Giuffrè Editore: Milan, Italy, 1999; pp. 177-194.

7. Pappalardo, G.; Pilato, M.; Bracco, S. To what extent are local communities involved in the governance of protected areas? Experiences from a case study in Sicily (Italy). Qual. Access Success 2015, 16, 102-109.

8. Scuderi, A.; Sturiale, L.; Bellia, C.; Foti, V.T.; Timpanaro, G. The redefinition of the role of agricultural areas in the city in relation to social, environmental, and alimentary functions: The case of Catania. Riv. Studi Sulla Sostenibilità 2017, 2, 1-10.

9. Gallenti, G.; Troiano, S.; Marangon, F.; Bogoni, P.; Campisi, B.; Cosmina, M. Environmentally sustainable versus aesthetic values motivating millennials' preferences for wine purchasing: Evidence from an experimental analysis in Italy. Agric. Food Econ. 2019, 7, 12. [CrossRef]

10. Munaretto, S.; Siciliano, G.; Turvani, M. Integrating adaptive governance and participatory multicriteria methods: A framework for climate adaptation governance. Ecol. Soc. 2014, 19, 74. [CrossRef]

11. Pearce, D.W.; Turner, R.K. Economia Delle Risorse Naturali e Dell'Ambiente; II Mulino: Bologna, Italy, 1991.

12. Romano, D.; Carbone, F. La valutazione economica dei benefici ambieniali: Un confronto fra approcci non di mercato. Riv. Econ. Agrar. 1993, 48, 19-62. 
13. Torrieri, F.; Concilio, G.; Nijkamp, P. Decision Support Tools for Urban Contingency Policy. A Scenario Approach to Risk Management of the Vesuvio Area in Naples-Italy. J. Contingencies Crisis Manag. 2002, 10, 95-112. [CrossRef]

14. Van Der Berghr, J.M.; Buttonn, K.J.; Nijkamp, P.; Pepping, G. Meta-Analysis in Environmental Economics; Kluwer Academic Publishers: Dordrecht, The Netherlands, 1997.

15. Tempesta, T.; Vecchiato, D. The value of a properly maintained hiking trail network and a traditional landscape for mountain recreation in the Dolomites. Resources 2018, 7, 86. [CrossRef]

16. Timpanaro, G.; Scuderi, A.; Foti, V.T.; Lo Giudice, V. The Social Relationships' effectiveness of "agrisocial" farms: A model of sustainable local development. Riv. Studi Sulla Sostenibilità 2015, 1, 99-116.

17. Soderberg, H.; Karman, E. MIKA: Methodologies for Integration of Knowledge Areas-The Case of Sustainable Urban Water Management; Dept. of Built Environment \& Sustainable Development, Chalmers Architecture, Chalmers University of Technology: Goeteborg, Sweden, 2003.

18. Sturiale, L. Possibili modelli di valutazione.della compatibilità tra sviluppo .e tutela con particolare attenzione alle aree protette. In Proceedings of the Atti del XXX Incontro di Studio CeSET. Gestione delle risorse naturali nei territori rurali e nelle aree protette: Aspetti economic, giuridici ed estimativi, Potenza, Italy, 5-6 October 2000; pp. 161-195.

19. Simoncini, A. Quadro giuridico e amministrativo. In The Ministry of the Environment and Ced-Ppn (European Centre for Documentation on the Planning of Natural Parks, Department of Territorial, of the Politecnic of Turin), Il Sistema Nazionale Delle Aree Protette Nel Quadro Europeo: Classificazione, Pianificazione E Gestione; Altra linea edizioni: Turin, Italy, 2002; pp. 22-34.

20. Vargas Isaza, O.L. La evaluación multicriterio social y suaporte a la conservación de los bosques social multicriteria. Rev. Fac. Nac. Agron. Medellín 2005, 58, 2665-2683.

21. WCED (World Commission on Environment and Development). Our Common Future; Oxford University Press: Oxford, UK, 1987.

22. Sturiale, L. Criteri di valutazione per la fruizionesostenibiledellerisorsenaturali in areeprotette. In Pagine Di Estimo, Allegato N. 19-20 Quaderni Del Dipartimento Patrimonio Architettonico Ed Urbanistico; Gangemi Editore: Roma, Italy, 2000; pp. 9-14.

23. Casini, L.; Bernetti, L.; Menghini, S. Teoria delle "libertà" e metodi multicriterio per l'analisi delle condizioni di sviluppo rurale. Riv. Econ. Agrar. 1997, 52, 29-59.

24. Maestrelli, S. I parchi: Una risorsa nazionale per lo sviluppo di qualità. Geogr. Ital. Rev. 2001, 2, $219-225$.

25. Scuderi, A.; Sturiale, L. Analysis of social network applications for organic agrifood products. Int. J. Agric. Resour. Gov. Ecol. 2014, 10, 176-189.

26. Carbone, F. La pianificazionemultifunzionaledelleforeste: l'applicazione di un modellointegrato MCDM-GIS. Aestimum 1999, 37, 67-101.

27. Nicoletti, A. Parchi e areeprotette. La Nuova Ecol. 1999, 2, 18.

28. Ferretti, V.; Bottero, M.; Mondini, G. A spatial decision support tool to study risk and opportunities of complex environmental systems. J. Environ. Account. Manag. 2015, 3, 197-212. [CrossRef]

29. MIPAAF. Le Aree Protette in Italia; MIPAAF: Roma, Italy, 2019.

30. Scuderi, A.; Sturiale, L.; Foti, V.T. The challenges and opportunity of protected natural areas in Italy: The case study of "Simeto oasis". Qual. Access Success 2017, 18, 401-408.

31. Foti, V.T.; Scuderi, A.; Stella, G.; Sturiale, L.; Timpanaro, G.; Trovato, M.R. The integration of agriculture in the politics of social regeneration of degraded urban areas. Green Energy Technol. 2018, 99-111.

32. Timpanaro, G.; Sturiale, L.; Foti, V.T. Sustainability as a business strategy in sicilian viniculture. Qual. Access Success 2016, 17, 186-191.

33. Scuderi, A.; Sturiale, L. Multi-criteria evaluation model to face phytosanitary emergencies: The case of citrus fruits farming in Italy. Agric. Econ. (Czech Republic) 2016, 62, 205-214. [CrossRef]

34. Papale, F.; Sturiale, C. Analisi del Settore Agro-Zootecnico; Parco dell'Etna: Nicolosi, Italy, 1994.

35. Calabrò, F.; Cassalia, G.; Tramontana, C. Evaluation Approach to the Integrated Valorization of Territorial Resources: The Case Study of the Tyrrhenian Area of the Metropolitan City of Reggio Calabria. In New Metropolitan Perspectives, ISHT 2018, Proceedings of the Smart Innovation, Systems and Technologies—SIST, Reggio Calabria, 22-25 May 2018; Calabrò, F., Della Spina, L., Bevilacqua, C., Eds.; Springer: Berlin/Heidelberg, Germany, 2019; Volume 101, pp. 3-12. 
36. Sturiale, C. L'esercizio dell'agricoltura nei Parchi e Riserve. In Proceedings of the Atti del Convegno Provincialesu"Parchi e Riserve: Quale modello per un corretto sviluppo del territorio locale", Castiglione di Sicilia, Italy, 18 October 1997; pp. 34-48.

37. Pawlak, Z. Rough set approach to knotoledge-based decision support. Eur. J. Oper. Res. 1997, 99, 48-57. [CrossRef]

38. Greco, S.; Lo Cascio, S.; Matarazzo, B.; Slowinski, R. A new rough set approach to evaluation of bankrupcty risk. In New Operational Tools in the Management of Financial Risks; Zopounidis, C., Ed.; Kluwer: Dordrecht, The Netherlands, 1997.

39. Greco, S.; Matarazzo, B.; Slowinski, R. Rough approximation of a preference relation by fuzzy dominance relations. In Proceedings of the 1st International Workshop on Preferences and decision, Trento, Italy, 5-7 June 1997; pp. 70-72.

40. Greco, S.; Matarazzo, B.; Slowinski, R. Rough approximation of a preference relation by dominance relations. In ICS Research Report; Warsaw University of Technology: Varsavia, Poland, 1996.

41. Greco, S.; Matarazzo, B.; Slowinski, R. Rough sets theory for multicriteria decision analysis. Eur. J. Oper. Res. 2001, 129, 1-47. [CrossRef]

42. Greco, S.; Matarazzo, B.; Slowinski, R. Dominance-based Rough Set Approach to decision under uncertainty and time preference. Ann. Oper. Res. 2010, 176, 41-75. [CrossRef]

43. Greco, S.; Matarazzo, B.; Slowinski, R. Rough sets methodology for sorting problems in presence of multiple attributes and criteria. Eur. J. Oper. Res. 2002, 138, 247-259. [CrossRef]

44. Matarazzo, B. L'approccio dei rough sets all'analisi delle decisioni. In Proceedings of the Atti del XXI Convegno Annuale, A.M.A.S.E.5., Roma, Italy, 10-13 September 1997.

45. Matarazzo, B.; Greco, S.; Slowinski, R. La teoria degli insiemi approssimati. In Strategie, Introduzione Alla Teorie Dei Giochi e Delle Decisioni; Stach, B.G., Ed.; Editore Giappichelli: Torino, Italy, 2019.

46. Chinnici, G.; Pecorino, B.; Scuderi, A. Environmental and economic performance of organic citrus growing. Qual. Access Success 2013, 14, 106-112.

47. Foti, V.T.; Scuderi, A.; Timpanaro, G. Organic Social agricoltur: A tool for rural development. Calit. Access Success 2013, 14, 266-271.

48. Sturiale, L.; Scuderi, A. The evaluation of green investments in urban areas: A proposal of an eco-social-green model of the city. Sustainability 2018, 10, 12. [CrossRef]

49. Fandel, G.; Gal, T. Multiple Criteria Decision Making Theory and Applications; Springer: Berlin/Heidelberg, Germany, 1980.

50. Borrini-Feyerabend, G.; Phillips, A. Politiche del paesaggio per la conservazione dellanatura. Diversità, equità e cambiamento. Urbanistica 2009, 139, 52.

51. Ferretti, V.; Bottero, M.; Mondini, G. Decision making and cultural heritage: An application of the Multi-Attribute Value Theory for the reuse of historical buildings. J. Cult. Herit. 2014, 15, 644-655. [CrossRef]

52. Sturiale, L.; Trovato, M.R. ICTs and smart territories. the knowledge and use of the UNESCO heritage by using the QR codes system. In Proceedings of the7th International Conference on Information and Communication Technologies in Agriculture, Food and Environment, HAICTA 2015, Kavala, Greece, 17-20 September 2015; Volume 1498, pp. 946-956.

53. Bresso, M. Riflessioni per la costruzione di un paradigma economico-ecologico. Oikos 1991, 43, $201-220$.

54. Sturiale, L.; Scuderi, A. The role of green infrastructures in urban planning for climate change adaptation. Climate 2019, 7, 119. [CrossRef]

55. Calabrò, F.; Mallamace, S.; Meduri, T.; Tramontana, C. Unused Real Estate and Enhancement of Historic Centers: Legislative Instruments and Procedural Ideas. In New Metropolitan Perspectives, ISHT 2018, Proceedings of the Smart Innovation, Systems and Technologies-SIST, Reggio Calabria, 22-25 May 2018; Calabrò, F., Della Spina, L., Bevilacqua, C., Eds.; Springer: Berlin/Heidelberg, Germany, 2019; Volume 101, pp. $464-474$.

56. Sturiale, L.; Scuderi, A.; Timpanaro, G.; Foti, V.T.; Stella, G. Social and inclusive "value" generation in metropolitan area with the "urban gardens" planning. Green Energy Technol. 2020, 285-302.

(C) 2020 by the authors. Licensee MDPI, Basel, Switzerland. This article is an open access article distributed under the terms and conditions of the Creative Commons Attribution (CC BY) license (http://creativecommons.org/licenses/by/4.0/). 


\title{
Comparative Analysis of Multi-Criteria Methods for the Enhancement of Historical Buildings
}

\author{
Antonio Nesticò * and Piera Somma \\ Department of Civil Engineering, University of Salerno, 84084 Fisciano (SA), Italy \\ * Correspondence: anestico@unisa.it; Tel.: +39-089-964318
}

Received: 26 July 2019; Accepted: 18 August 2019; Published: 21 August 2019

\begin{abstract}
The protection of cultural heritage is essential to preserve the memory of the territory and its communities, but its enhancement is also important. In this perspective, the theme of choosing the best use for historic buildings, which often make up a substantial and widespread part of real estate and which can become a driving force for the sustainable development of cities, is important. These decision-making processes find effective support tools in Multi-Criteria Decision Making (MCDM) methods, able to consider the multiple financial, social, cultural, and environmental effects that the enhancement project generates. In order to identify the most appropriate evaluation approach to select the best use of the building, this paper proposes a comparison between some of the best-known MCDM methods: Analitic Hierarchy Process (AHP), ELimination Et Choix Traduisant la REalité (ELECTRE), Tecnique for Order Preference by Similarity to Ideal Solution (TOPSIS), and the Compromise Ranking Method (VIKOR). The comparative analysis gives rise to the validity of the AHP, which is useful for reducing the problem into its essential components, so as to make a rational comparison among the design alternatives based on different criteria. The novelty of the research is the characterization of the hierarchical structure of the model, as well as the selection of criteria and indicators of economic evaluation. The application of the model to a real case of recovery and enhancement of a former convent in the province of Salerno (Italy) verifies the effectiveness of the tool and its adaptability to the specificities of the case study.
\end{abstract}

Keywords: economic evaluation of projects; Multi Criteria Decision Making (MCDM); historical building; economic enhancement

\section{Introduction}

In recent decades, growing interest in historic buildings has grown in the European context. In fact, these possess a cultural, social, and economic value capable of generating virtuous processes for the development of the surrounding territory [1,2]. In preserving this heritage, to interrupt the frequent degradation processes and promote recovery activities, it is necessary to give legal force to the actions necessary for its protection, as the historical environment is vulnerable [3].

However, it is important to highlight that just protection of the historic building alone does not guarantee the economic development of the surrounding communities, and does not always have a positive impact on their quality of life. Indeed, citizens often do not have the opportunity to access or use the historic building. It is therefore necessary to guarantee public space and accessibility, and more generally, the inclusion of architectural heritage in management and development programs aimed at meeting the needs of residents $[4,5]$.

The recovery and enhancement of the historic building can be important for the sustainable development of the city [6,7]. From this point of view, it is sufficient to think about the saving of soil that is generated where derelict buildings are recovered and reused [8]. The indication of the right function provides the decision-maker with indispensable data to evaluate the correctness of the intervention, the 
integration with the context, the respect of the building, and investment opportunities. In the process of recovery and enhancement of the real estate property, the identification of the best use maximizes the effects of the project, not only in purely financial terms, but also in social, cultural, and environmental terms [9-11].

On the one hand, the functional destination must satisfy the existing technical-urban planning constraints, which usually put significant limits on the possible alternatives; on the other hand, it must look at the financial sustainability of the investment project, and at maximizing the benefits for the community [12,13].

But what approaches are possible to establish the optimal destination with regard to the multiple needs of a community? The economic evaluation of the projects provides multi-criteria methodologies for analysis. A peculiarity of these methodologies is precisely the ability to consider the multi-dimensional character of the evaluation problem, allowing a comparative analysis of the alternatives according to multiple criteria, both quantitative and qualitative [14,15].

The recovery, protection, and valorization of historic buildings represent a demanding, yet unavoidable bet, where careful actions on the vast existing heritage can certainly contribute to the relaunch of the building sector and to the achievement of all the benefits of urban regeneration [16-18].

\section{Aim of the Paper}

The research mainly pursued three goals. Starting from a comparative evaluation of different methods of multicriterial analysis, the objective was to identify an effective approach to establish the best use of a historic building, despite the multiple technical-urbanistic and financial constraints that characterize any option of investment.

The second objective was to select multiple social, cultural, and financial criteria that would be useful for logically structuring the economic analysis model. This is fundamental to reconcile the multiple evaluative aspects involved and to rationally organize the available information.

The third objective was the characterization of a model based on the Analytic Hierarchy Process (AHP) algorithms able to compare functional alternatives based on different selected criteria, so as to choose the optimal use for the historic building.

The resolution of a real multicriterial evaluation problem concerning the recovery and enhancement of a former convent in the province of Salerno (Italy) allows for the verification of both the usefulness of the proposed model and its easy adaptability to the specificities of the case study.

\section{MCDM Methods Comparative Analysis}

With reference to urban and territorial planning interventions and the building heritage, the multi-criteria analysis methods allow for comparisons of different design solutions on the basis of multiple criteria (financial, social, cultural, and environmental), which can be expressed through quantitative or qualitative indicators [19]. Thus, unlike the cost-benefit analysis that expresses the judgment of economic convenience only on the monetary criterion, the multicriteria analysis rationalizes the selection process through the optimization of a multi-criteria vector, weighted according to the decision-maker's priorities. In this way, it is possible to include both monetizable and extra-economic criteria, measurable only in physical or qualitative terms, in a single evaluation process [20,21].

Through logical-mathematical algorithms structured on parameters specific to the technical, economic, managerial, social, environmental, and psychological disciplines, the methods of multicriterial analysis allow for the ordering of the possible solutions to the problem, even when there is no alternative that clearly prevails over the others-in other words, one that is capable of simultaneously maximizing all the evaluation criteria [22]. These methods make it possible to identify the optimal solution that can better combine the set of objectives [23].

The literature offers a wide range of techniques, such as: the Analytic Hierarchy Process (AHP), Analytic Network Process (ANP), ELimination Et Choix Traduisant la REalité (ELECTRE), Preference Ranking Organization METHods for Enrichment Evaluations (PROMETHEE), Tecnique for Order 
Preference by Similarità to Ideal Solution (TOPSIS), Compromise Rankimg Method (VIKOR), and EVAluation of MIXed criteria (EVAMIX). Use of the AHP, ELECTRE, TOPSIS, and VIKOR methods are certainly widespread, and these are the subject of this research [24-35].

In general, the choice of the most appropriate multi-criteria method to solve the evaluation problem depends on the characteristics of the case study, in terms of the objectives of the analysis, reference territory, and specificity of the intervention, nature, and quality of the information available to the analyst $[36,37]$.

The AHP method is more effective in the presence of the criteria and sub-criteria of evaluation, since through a hierarchical structure, at several levels, it allows the complex problem to be broken down into simpler sub-problems that can be analyzed in greater detail. The other three methods only consider criteria and do not include the presence of sub-criteria.

The hierarchical structure of the AHP has: the main goal of the decision-maker at the top; the sub-goals at the underlying level; and the criteria that lead to the achievement of sub-goals at an even lower level [30,33]. The criteria can be expressed by sub-criteria up to the most appropriate breakdown level for full understanding of the problem. The last level is that of alternatives $[29,32,33,38]$.

The hierarchical analysis requires the estimation of the weights $w$ to be associated with each criterion and the various alternatives. This estimate is carried out using evaluation matrices whose individual elements, $a_{i j}$, are obtained by comparing the criteria and the alternatives with reference to each criterion.

The $A$ matrices of pairwise comparisons are of the type:

$$
A=\left[\begin{array}{cccc}
a_{11} & a_{12} & \ldots & a_{1 m} \\
a_{21} & a_{22} & \ldots & a_{2 m} \\
\vdots & \vdots & \ddots & \vdots \\
a_{n 1} & a_{n 2} & \ldots & a_{n m}
\end{array}\right]
$$

These matrices are:

- Positive-that is, all the principal minors are positive, where by 'principal minor', we mean the determinant of the square sub-matrix formed by the first $n$ rows and $m$ columns (with $1 \leq m \leq n$ );

- $\quad$ Reciprocal, being $a_{i j}=1 / a_{j i}$ and therefore, the elements on the main diagonal are all unitary $\left(a_{i i}=1\right)$. This relationship of reciprocity arises from the need to guarantee the symmetry of judgments of importance;

- Constituted by finite elements, since for each criterion, $C$, considered, we have $a_{i j} \neq \infty$.

As suggested by Saaty, comparisons can be made according to the semantic scale with scores 1, 3 , 5,7 , and 9 [33].

Commonly, in real cases, the relationship $w_{i} / w_{j}$ is not known, so it is necessary to look for $a_{i j}$, such that $a_{i j} \approx w_{i} / w_{j}$. If $W$ is the matrix of the weights and $w$ the column vector of the sought variables, then:

$$
W \cdot w=n \cdot w
$$

In extended form:

$$
W \cdot w=\left[\begin{array}{cccc}
\frac{w_{1}}{w_{1}} & \frac{w_{1}}{w_{2}} & \cdots & \frac{w_{1}}{w_{n}} \\
\frac{w}{w_{1}} & \frac{w 2}{w_{2}} & \cdots & \frac{w 2}{w_{n}} \\
\vdots & \vdots & \ddots & \vdots \\
\frac{w_{n}}{w_{1}} & \frac{w w_{n}}{w_{2}} & \cdots & \frac{w_{n}}{w_{n}}
\end{array}\right] \cdot\left[\begin{array}{c}
w_{1} \\
w_{2} \\
\vdots \\
w_{n}
\end{array}\right]=\left[\begin{array}{c}
n w_{1} \\
n w_{2} \\
\vdots \\
n w_{n}
\end{array}\right]=n\left[\begin{array}{c}
w_{1} \\
w_{2} \\
\vdots \\
w_{n}
\end{array}\right]=n \cdot w
$$

Therefore, the matrix $W$ has, as its sole eigenvalue, its order $n$, and as a corresponding eigenvector, the vector of the sought variables. This means that it is possible to obtain the values of the variables $w_{1}$, $w_{2}, \ldots, w_{n}$, starting from the relationships between them, taken two at a time. This allows us to affirm 
that, if we do not have the exact value of the relations $w_{i} / w_{j}(i, j=1,2, \ldots, n)$, but their estimate, the method of the eigenvalue can still be usefully used for the approximate evaluation of the variables. The values thus obtained are closer to the exact ones if the estimates of the $w_{i} / w_{j}$ ratios are consistent between them [39]. In this case, all the $n$ eigenvalues are almost zero, and if the ratios are coherently estimated, the maximum eigenvalue $\lambda_{\max }$ is not far from the value $n$, which can therefore be assumed as an approximate estimate of the vector $w$ :

$$
W \cdot n=\lambda_{\max } \cdot w \quad \text { con } \quad \lambda_{\max } \geq n
$$

Alternatively, the vector $w$ can be determined by normalizing the matrix $W$ through the ratio of each of its elements to the sum of the elements placed in the same column. The arithmetic mean of each of its lines is then calculated [40].

The consistency measure of the values assigned to the $w_{i} / w_{j}$ ratios derives from the difference between $\lambda_{\max }$ and $n$. This difference is null for perfectly consistent estimates. The relationship consistency index $C I$ is defined as:

$$
C I=\frac{\lambda_{\max }-n}{n-1}
$$

The CI index must be compared to the RCI random consistency index, whose value is a function of the number $n$ of variables according to Table 1 .

Table 1. RCI values.

\begin{tabular}{cccccccccc}
\hline $\boldsymbol{n}$ & $\mathbf{1}$ & $\mathbf{2}$ & $\mathbf{3}$ & $\mathbf{4}$ & $\mathbf{5}$ & $\mathbf{6}$ & $\mathbf{7}$ & $\mathbf{8}$ & $\mathbf{9}$ \\
\hline$R C I$ & 0 & 0 & 0.58 & 0.90 & 1.12 & 1.24 & 1.32 & 1.41 & 1.45 \\
\hline
\end{tabular}

The relationship between $C I$ and $R C I$ is defined as the Consistency Ratio $(C R)$ :

$$
C R=\frac{C I}{R C I}
$$

Binary comparisons are sufficiently coherent with one another if:

$$
\begin{array}{ll}
- & C R<5 \% \text { for } n=3 ; \\
- & C R<9 \% \text { for } n=4 ; \\
- & C R<10 \% \text { for } n>4 .
\end{array}
$$

The final step is to calculate the overall weights (or priorities) of the actions. For this, it applies the principle of hierarchical composition, by virtue of which the local weights of each element are multiplied by those of the corresponding superordinate elements. Finally, the products obtained are added together [30].

Compared to AHP, the ELECTRE method is not preferred when there is a large number of alternatives and a large number of criteria. Depending on the number of alternatives, the values of the concordance threshold $\underline{c}$ and the discordance threshold $\underline{d}$ are calculated. On the basis of these values, we constructed the dominance matrix of concordance $F$, whose elements $f_{k p}$ are:

$$
f_{k p}=1 \text { if } c_{k p} \geq \underline{c} \quad f_{k p}=0 \text { if } c_{k p}<\underline{c}
$$

and the dominance matrix of discordance $G$, whose $g_{k p}$ elements are:

$$
g_{k p}=1 \text { if } d_{k p} \geq \underline{d} \quad g_{k p}=0 \text { if } d_{k p}<\underline{d}
$$

where $c_{k p}$ represents the set of all the criteria for which the alternative $A_{k}$ is preferable to the alternative $A_{p}$, and $d_{k p}$ is the complement of $c_{k p}$. The product of the homologous elements of the matrices $F$ and $G$ returns the aggregate dominance matrix, $E$ : 


$$
E=\left[\begin{array}{cccc}
e_{11} & e_{12} & \ldots & e_{1 p} \\
e_{21} & e_{22} & \ldots & e_{2 p} \\
\vdots & \vdots & \ddots & \vdots \\
e_{k 1} & e_{k 2} & \ldots & e_{k p}
\end{array}\right]
$$

which allows the construction of a partial ordering of the alternatives, since the columns in which the value 1 appears indicate the alternatives to be discarded because they are dominated by the others. Therefore, ELECTRE, unlike the other models in question, does not provide a final ranking of scores, but only an order of preference. Precisely, the peculiarities of the calculation algorithms can make ELECTRE easier to use if the number of evaluation criteria is particularly limited.

Despite being very versatile, as it can be applied even in the presence of a large number of criteria and alternatives, the TOPSIS method tends to reject those alternatives that have low values in most of the attributes. Specifically, for each criterion, TOPSIS searches for the ideal solution, $A^{*}$ and the ideal negative solution, $A^{-}$(respectively better and worse performance offered by the alternatives considered), with respect to which it calculates the distances $S_{i}{ }^{*}$ and $S_{i}{ }^{-}$. It is possible to determine the relative distance $C_{i}{ }^{*}$ of the generic alternative $A_{i}$, with respect to the ideal solution:

$$
C_{i}^{*}=\frac{S_{i}^{-}}{S_{i}^{-}+S_{i}^{*}}
$$

For the final classification, the best solution is the one that presents the minimum distance from $A^{*}$ and the maximum distance from $A^{-}$at the same time- - that is, the one with the highest value of $C_{i}^{*}$.

The VIKOR method substantially has the same characteristics of TOPSIS, but unlike the latter, does not provide for the presence of an ideal negative solution. In fact, VIKOR considers the optimal alternative to be the one closest to the ideal solution. The lowest value of the $Q_{i}$ scalar corresponds to this alternative:

$$
Q_{i}=v \frac{S_{i}-S^{*}}{S^{-}-S^{*}}+(1-v) \frac{R_{i}-R^{*}}{R^{-}-R^{*}}
$$

In (9), the variable $v$ is between 0 and 1 , and allows to give different weight to the single addends. Moreover:

$$
\begin{gathered}
S_{i}=\sum_{j=1}^{m} \frac{w_{j}\left(a_{j}^{*}-a_{i j}\right)}{a_{j}^{*}-a_{i j}} ; R_{i}=\max _{j}\left[\frac{w_{j}\left(a_{j}^{*}-a_{i j}\right)}{a_{j}^{*}-a_{i j}}\right] \\
S^{*}=\min _{i} S_{i} ; S^{-}=\max _{i} S_{i} ; R^{*}=\min _{i} R_{i} ; R^{-}=\max _{i} R_{i}
\end{gathered}
$$

According to VIKOR, the optimal solution must comply with two criteria of acceptability:

- Acceptable advantage, $Q\left(A^{\prime \prime}\right)-Q\left(A^{\prime}\right) \geq D Q$, with $D Q=1 /(n-1)$, where $n$ is the number of alternatives;

- Acceptable stability of the decision, that is, the alternative $A^{\prime}$ must also be the best, or in the ranking must present the minimum value in terms of $S_{i}$ and/or $R_{i}$.

If one of the two conditions is not verified, it is impossible to directly determine the best solution, which is obviously a limitation of the method. In this case, a set of compromised solutions is established in the set of alternatives under consideration. This set is given by:

- $\quad$ Alternatives $A^{\prime}$ and $A^{\prime \prime}$, if only condition 2 is not satisfied;

- $\quad$ Alternatives $A^{\prime}, A^{\prime \prime}, \ldots, A^{N}$, if only condition 1 is not met.

$A^{N}$ is the last solution, taken in the order obtained according to $Q_{i}$, for which the inequality is still valid:

$$
Q\left(A^{N}\right)-Q\left(A^{\prime}\right)<D Q
$$


The alternatives of the set thus determined are characterized by a sensitive reciprocal "closeness".

The essential examination of the four methods, AHP, ELECTRE, TOPSIS, and VIKOR aimed at a useful comparison to highlight the peculiarities of each of them, is summarized in Table 2.

Table 2. Comparison between multi-criteria methods.

\begin{tabular}{llllll}
\hline Method & $\begin{array}{l}\text { Number of } \\
\text { Criteria }\end{array}$ & $\begin{array}{l}\text { Number of } \\
\text { Alternatives }\end{array}$ & $\begin{array}{l}\text { Algorithm } \\
\text { Structure }\end{array}$ & Ranking & Solution \\
\hline AHP & Large & Limited & $\begin{array}{l}\text { Pairwise } \\
\text { comparison }\end{array}$ & $\begin{array}{l}\text { Score } \\
\text { ranking }\end{array}$ & $\begin{array}{l}\text { Best alternative of all } \\
\text { according to criteria and sub-criteria }\end{array}$ \\
\hline ELECTRE & Limited & Limited & $\begin{array}{l}\text { Preference } \\
\text { thresholds }\end{array}$ & $\begin{array}{l}\text { Preference } \\
\text { ranking }\end{array}$ & Not dominated alternative \\
\hline VIKOR & Large & Large & $\begin{array}{l}\text { Ideal solution and } \\
\text { negative ideal } \\
\text { solution }\end{array}$ & $\begin{array}{l}\text { Score } \\
\text { ranking }\end{array}$ & $\begin{array}{l}\text { Alternative closer to the ideal solution and } \\
\text { at the same time more distant from the } \\
\text { ideal negative solution }\end{array}$ \\
\hline
\end{tabular}

\section{Selection of the Best Use of a Historic Building. Characterization of a Hierarchical Analysis Model}

The Analytic Hierarchy Process is defined as "analytical" because it breaks down the complex decision problem into fundamental constituent elements [30,31,41,42]; "hierarchical" because the breakdown of the problem itself occurs at successive levels characterized by ever-increasing detail $[27,28,43,44]$. At the top of the hierarchy, there is the goal to be reached, which, in the case under consideration, is given by the best use for the historic building.

The choice of indicators is fundamental for the correct application of the model. Here, they have been selected from literature datasets [45-48].

At the first level, we consider the social (C1), cultural (C2), and financial (C3) criteria, which are described in turn in the sub-criteria. In particular, the social criterion includes the sub-criteria:

- $\quad$ Community involvement $\left(C_{1}\right)$, intended as the average number of daily users. It is measured as the average daily number of people who attend the structure;

- $\quad$ New workers $\left(C_{2}\right)$, measured as the number of new workers in the structure.

- The cultural criterion includes the sub-criteria:

- $\quad$ Cultural effects $\left(C_{3}\right)$, understood as the attraction of the structure with respect to cultural events;

- Compatibility of the function with the historical-architectural characteristics of the property $\left(C_{4}\right)$. This criterion depends on the three sub-criteria:

- Representativeness of the use function $\left(C_{41}\right)$, evaluated as the aptitude to express the cultural peculiarities of the reference territory, in respect of the material and spiritual reality of the architectural artefact;

- $\quad$ Residential impact $\left(C_{42}\right)$, understood as the average number of daily users, in this case with a negative meaning, in the sense that higher this number is, the lower the score attributed to the alternative is. In fact, with the aim of protecting the cultural asset, it is advisable to prefer functional activities with a moderate residential impact, in order to avoid excessive loads on the structure that may require interventions of static adaptation such as to compromise the authenticity of the historical matter;

- $\quad$ Respect of the criterion of minimum intervention $\left(C_{43}\right)$, that is, safeguarding the characteristics of the building. It is necessary to avoid intrusive interventions, such as new openings, tracks for installations, partitions, kitchens, and toilets, so as not to reduce the artistic-monumental quality of the property. This criterion represents one of the cardinal principles of architectural restoration and conservation of historic buildings. It is therefore a qualitative, rather than a quantitative criterion [49,50]. 
The financial criterion includes the sub-criterion:

- $\quad$ Return On Investment $\left(C_{5}\right)$. This is measured by ROI (Return On Investment), which expresses the rate of return on the total investments of a company for the assumed activity.

According to the existing technical-urban planning constraints and in relation to the socio-demographic and economic characteristics of the territory in which the recovery and enhancement project of the historic building is inserted, the analysts define the possible investment alternatives $A_{1}$, $A_{2}, \ldots, A_{n}$. Among these, it is necessary to make the choice that guarantees the optimal use, according to the multiple criteria $C 1, C 2, C 3$ and sub-criteria $C_{1}, C_{2}, C_{3}, C_{4}, C_{5}$, as well as $C_{41}, C_{42}$, and $C_{43}$ defined above. The logical-operative scheme outlined finds graphic representation in Figure 1.

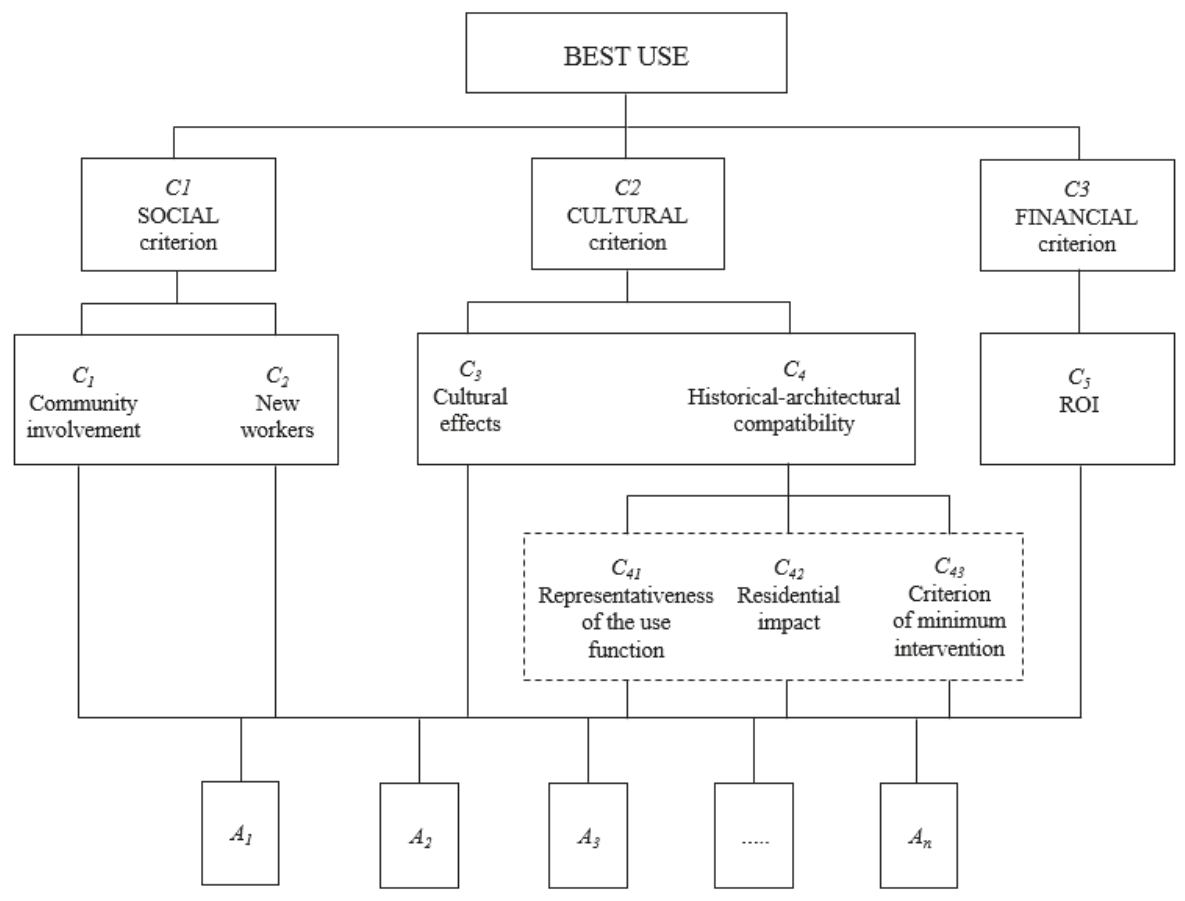

Figure 1. Hierarchical structure of the proposed model.

\section{Case Study and Results}

The aim of the paper was to define the best use for the recovery and enhancement of a former convent in the province of Salerno (Italy). For this purpose, in accordance with the provisions of paragraph 3, the Analogue Hierarchy Process was used. This is a multi-criteria approach able to take into account both the use value of cultural heritage as a source of economic growth in the short term, and its intrinsic value as an identity element of a community, to be preserved in the long term [51].

The monastic complex under study is of particular historical-architectural interest, but is currently in a very poor state of preservation. Thus, the public administration acquired the property with the aim of recovering it [52-55]. Figure 2 shows the plants, sections, and main elevations of the building in the actual state. 

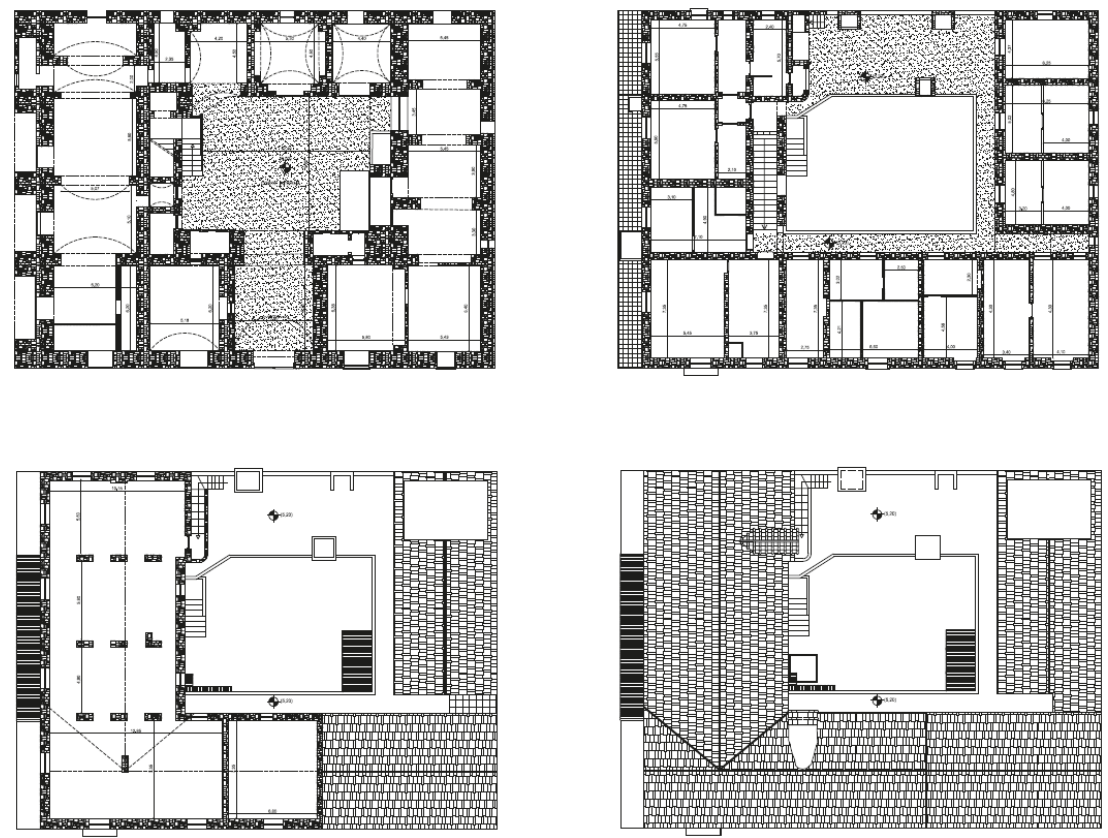

A.

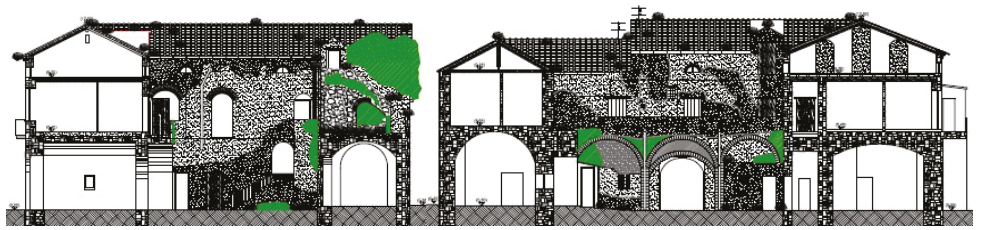

B.
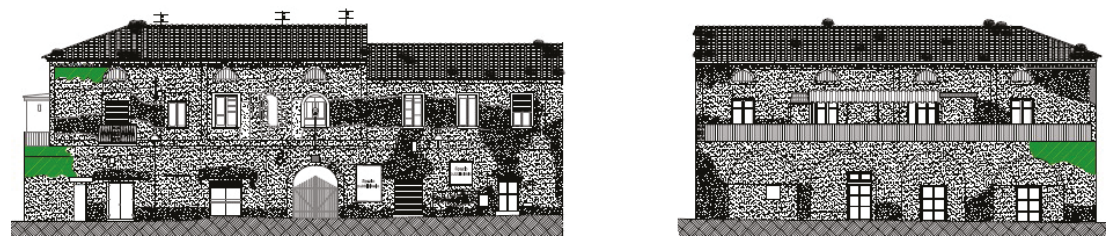

C.

Figure 2. Former convent in the province of Salerno (Italy): (A). plans; (B). sections; (C). elevations.

Depending on the technical-urban planning constraints and the economic characteristics of the area in which the historic building is located, possible uses are:

- $\quad$ Bed \& Breakfast $\left(A_{1}\right)$;

- $\quad$ Multi-purpose rooms $\left(A_{2}\right)$ for holding conferences, seminars, thematic meetings, exhibitions and multimedia workshops;

- $\quad$ Local cuisine restaurant $\left(A_{3}\right)$, which proposes traditional dishes, cooking workshops, as well as workshops for the valorization of the typical craftsmanship of the places, with an adjoining museum of rural civilization; 
- $\quad$ Public and private offices $\left(A_{4}\right)$.

The Figures A1-A12 in the Appendix A report the functional distribution of the internal spaces at the three levels of the building for each of the four solutions of use.

The AHP was developed according to the first-level evaluation criteria: social $\left(C_{1}\right)$, cultural $\left(C_{2}\right)$, and financial $\left(C_{3}\right)$. For the case in question, the sub-criteria are estimated as follows:

- Community involvement $\left(C_{1}\right)$. As there is no data on the average number of users, a filling index was envisaged as a rate of the maximum capacity of the structure;

- $\quad$ New workers $\left(C_{2}\right)$. This data was obtained through surveys carried out in similar structures in the territory;

- $\quad$ Cultural effects $\left(C_{3}\right)$. This is expressed as the ratio between the surface used for cultural activities $\left(S_{C}\right)$ and the total area of the building $\left(S_{T}\right)$;

- Compatibility of the function with the historical-architectural characteristics of the property $\left(C_{4}\right)$. This criterion depends on the three sub-criteria:

- $\quad$ Representativeness of the use function $\left(C_{41}\right)$, according to a judgment scale from 1-7;

- $\quad$ Residential impact $\left(C_{42}\right)$, assessed as for criterion $C_{1}$;

- Respect of the criterion of minimum intervention $\left(C_{43}\right)$. The judgment was assigned by the decision-maker according to a qualitative scale from 1-7, depending on the level of protection that is guaranteed to the original structure;

- $\quad$ Return On Investment, $R O I\left(C_{5}\right)$. For each of the four alternatives, the $R O I$ value was derived from information on the profitability of the reference economic sector in the area of investigation.

Once the alternatives, criteria, and sub-criteria for the evaluation was defined, the hierarchical decision model was outlined, as shown in Figure 3.

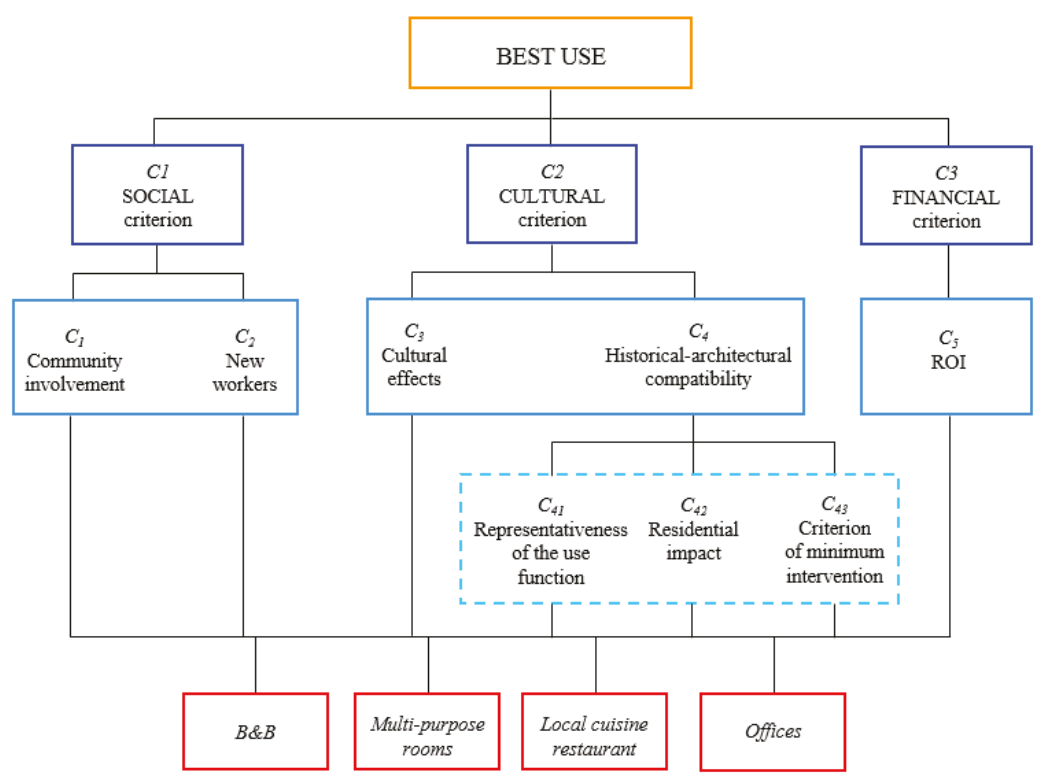

Figure 3. The hierarchical structure for the case study.

Table 3 shows the scalarized decision matrix, useful for the implementation of the AHP. In this matrix the generic element $a_{i j}$ (attribute) expresses the performance of the generic alternative $A i$ $(i=1,2, \ldots, n)$ with respect to the generic criterion $C_{j}(j=1,2, \ldots, m)[56,57]$. 
Table 3. Scalarized decision matrix.

\begin{tabular}{|c|c|c|c|c|c|c|c|}
\hline & $C_{1}$ & $C_{2}$ & $C_{3}$ & $C_{41}$ & $C_{42}$ & $C_{43}$ & $C_{5}$ \\
\hline & $\begin{array}{l}\text { Community } \\
\text { Involvement }\end{array}$ & $\begin{array}{c}\text { New } \\
\text { Workers }\end{array}$ & $\begin{array}{l}\text { Cultural } \\
\text { Effects }\end{array}$ & $\begin{array}{l}\text { Representativeness } \\
\text { of the Use Function }\end{array}$ & $\begin{array}{l}\text { Residential } \\
\text { Impact }\end{array}$ & $\begin{array}{l}\text { Criterion of } \\
\text { Minimum } \\
\text { Intervention }\end{array}$ & ROI \\
\hline & [n. users] & [n. workers] & {$\left[\mathrm{m}^{2} / \mathrm{m}^{2}\right]$} & & [n. users] & & {$[\mathbf{\epsilon} / \mathbf{\epsilon}]$} \\
\hline$A_{1}$ & 122 & 14 & 0.335 & 5 & 122 & 3 & 0.073 \\
\hline$A_{2}$ & 168 & 16 & 0.661 & 6 & 168 & 6 & 0.043 \\
\hline$A_{3}$ & 197 & 22 & 0.641 & 6 & 197 & 4 & 0.122 \\
\hline$A_{4}$ & 140 & 16 & 0.205 & 3 & 140 & 5 & 0.101 \\
\hline
\end{tabular}

The dominance coefficients were assigned according to Saaty's semantic scale in Table 4. They are representative of the relative importance between the compared elements [33].

Table 4. Saaty semantic scale.

\begin{tabular}{ccccccc}
\hline Intensity & $\mathbf{1}$ & $\mathbf{3}$ & $\mathbf{5}$ & $\mathbf{7}$ & $\mathbf{9}$ & $\mathbf{2 , 4 , 6 , 8}$ \\
\hline Linguistic & Equal & Moderate & Strong & Demonstrated & Extreme & Intermediate values \\
\hline
\end{tabular}

In paired comparisons of the alternatives with respect to the criteria, graduated scales were used, each divided into equal bands corresponding to the intensity of the Saaty scale, where the maximum value of the indicator in question corresponds to the upper end. On the scale thus defined, the numerical values of the indicators have been positioned for each alternative (Figure 4). If there is only one band between the indicators, then the strongest value was given coefficient 3 , and to the other, the reciprocal; if there are two bands, the strongest value was assigned coefficient 5 , and to the other, the reciprocal, and so on. In intermediate situations, there were intermediate intensities.
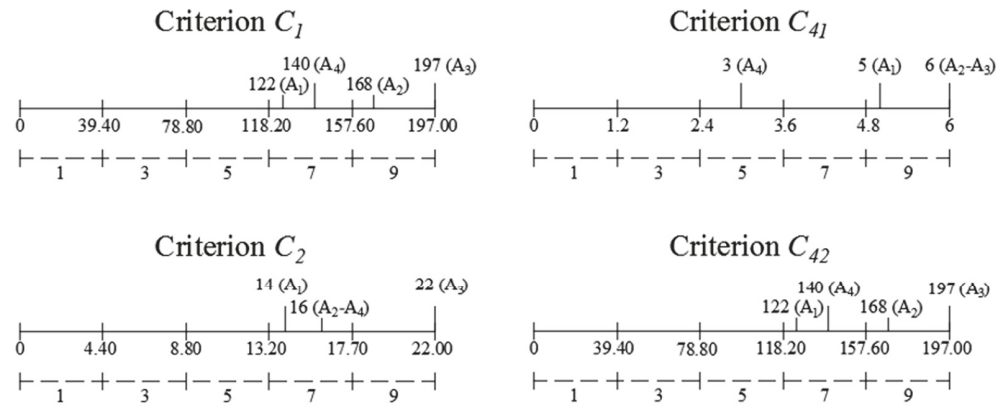

\section{Criterion $C_{3}$}
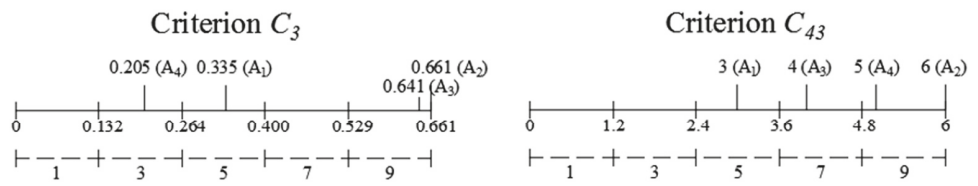

\section{Criterion $C_{5}$}

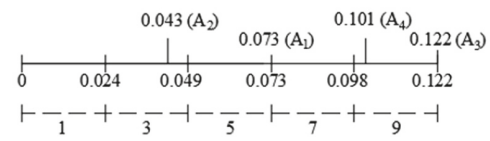

Figure 4. Scales for determining the dominance coefficients. 
Table 5 contains the matrices of pairwise comparisons and the matrices of normalized pair comparisons. From these matrices, processed by implementing Microsoft Excel software, it was possible to obtain the weights of each alternative with respect to each sub-criterion. In all cases, the matrices are consistent. In fact, being of rank 4, they have a value of consistency ratio lower than 9 .

Table 5. Comparison matrices in pairs between the alternatives with respect to each criterion.

\begin{tabular}{|c|c|c|c|c|c|c|c|c|c|c|}
\hline \multicolumn{5}{|c|}{$C_{1}$-Community Involvement } & \multicolumn{6}{|c|}{$C_{1}-$ Community Involvement } \\
\hline & $A_{1}$ & $A_{2}$ & $A_{3}$ & $A_{4}$ & & $A_{1}$ & $A_{2}$ & $A_{3}$ & $A_{4}$ & $w_{i}$ \\
\hline$A_{1}$ & 1.00 & 0.33 & 0.20 & 1.00 & $A_{1}$ & 0.100 & 0.071 & 0.112 & 0.111 & 0.099 \\
\hline$A_{2}$ & 3.00 & 1.00 & 0.33 & 3.00 & $A_{2}$ & 0.300 & 0.214 & 0.187 & 0.333 & 0.259 \\
\hline$A_{3}$ & 5.00 & 3.00 & 1.00 & 4.00 & $A_{3}$ & 0.500 & 0.643 & 0.561 & 0.444 & 0.537 \\
\hline$A_{4}$ & 1.00 & 0.33 & 0.25 & 1.00 & $A_{4}$ & 0.100 & 0.071 & 0.140 & 0.111 & 0.106 \\
\hline \multicolumn{5}{|c|}{$C R=3.80$} & \multicolumn{6}{|c|}{$C R=3.80$} \\
\hline \multicolumn{5}{|c|}{$C_{2}$-New workers } & \multicolumn{6}{|c|}{$C_{2}$-New workers } \\
\hline & $A_{1}$ & $A_{2}$ & $A_{3}$ & $A_{4}$ & & $A_{1}$ & $A_{2}$ & $A_{3}$ & $A_{4}$ & $w_{i}$ \\
\hline$A_{1}$ & 1.00 & 1.00 & 0.20 & 1.00 & $A_{1}$ & 0.125 & 0.143 & 0.118 & 0.143 & 0.132 \\
\hline$A_{2}$ & 1.00 & 1.00 & 0.25 & 1.00 & $A_{2}$ & 0.125 & 0.143 & 0.147 & 0.143 & 0.139 \\
\hline$A_{3}$ & 5.00 & 4.00 & 1.00 & 4.00 & $A_{3}$ & 0.625 & 0.571 & 0.588 & 0.571 & 0.589 \\
\hline$A_{4}$ & 1.00 & 1.00 & 0.25 & 1.00 & $A_{4}$ & 0.125 & 0.143 & 0.147 & 0.143 & 0.139 \\
\hline \multicolumn{5}{|c|}{$C R=0.38$} & \multicolumn{6}{|c|}{$C R=0.38$} \\
\hline \multicolumn{5}{|c|}{$C_{3}$-Cultural effects } & \multicolumn{6}{|c|}{$C_{3}-$ Cultural effects } \\
\hline & $A_{1}$ & $A_{2}$ & $A_{3}$ & $A_{4}$ & & $A_{1}$ & $A_{2}$ & $A_{3}$ & $A_{4}$ & $w_{i}$ \\
\hline$A_{1}$ & 1.00 & 0.17 & 0.20 & 3.00 & $A_{1}$ & 0.081 & 0.073 & 0.085 & 0.158 & 0.099 \\
\hline$A_{2}$ & 6.00 & 1.00 & 1.00 & 8.00 & $A_{2}$ & 0.486 & 0.436 & 0.427 & 0.421 & 0.443 \\
\hline$A_{3}$ & 5.00 & 1.00 & 1.00 & 7.00 & $A_{3}$ & 0.405 & 0.436 & 0.427 & 0.368 & 0.409 \\
\hline$A_{4}$ & 0.33 & 0.13 & 0.14 & 1.00 & $A_{4}$ & 0.027 & 0.055 & 0.061 & 0.053 & 0.049 \\
\hline \multicolumn{5}{|c|}{$C R=4.62$} & \multicolumn{6}{|c|}{$C R=4.62$} \\
\hline \multicolumn{5}{|c|}{$C_{41}-$ Representativeness of the use function } & \multicolumn{6}{|c|}{ 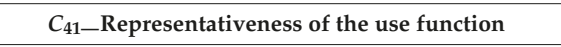 } \\
\hline & $A_{1}$ & $A_{2}$ & $A_{3}$ & $A_{4}$ & & $A_{1}$ & $A_{2}$ & $A_{3}$ & $A_{4}$ & $w_{i}$ \\
\hline$A_{1}$ & 1.00 & 0.33 & 0.33 & 5.00 & $A_{1}$ & 0.139 & 0.133 & 0.133 & 0.278 & 0.171 \\
\hline$A_{2}$ & 3.00 & 1.00 & 1.00 & 6.00 & $A_{2}$ & 0.417 & 0.400 & 0.400 & 0.333 & 0.388 \\
\hline$A_{3}$ & 3.00 & 1.00 & 1.00 & 6.00 & $A_{3}$ & 0.417 & 0.400 & 0.400 & 0.333 & 0.388 \\
\hline$A_{4}$ & 0.20 & 0.17 & 0.17 & 1.00 & $A_{4}$ & 0.028 & 0.067 & 0.067 & 0.056 & 0.054 \\
\hline \multicolumn{5}{|c|}{$C R=5.28$} & \multicolumn{6}{|c|}{$C R=5.28$} \\
\hline \multicolumn{5}{|c|}{$C_{42}$-Residential impact } & \multicolumn{6}{|c|}{$C_{42}$-Residential impact } \\
\hline & $A_{1}$ & $A_{2}$ & $A_{3}$ & $A_{4}$ & & $A_{1}$ & $A_{2}$ & $A_{3}$ & $A_{4}$ & $w_{i}$ \\
\hline$A_{1}$ & 1.00 & 3.00 & 5.00 & 1.00 & $A_{1}$ & 0.395 & 0.409 & 0.385 & 0.387 & 0.394 \\
\hline$A_{2}$ & 0.33 & 1.00 & 3.00 & 0.33 & $A_{2}$ & 0.132 & 0.136 & 0.231 & 0.129 & 0.157 \\
\hline$A_{3}$ & 0.20 & 0.33 & 1.00 & 0.25 & $A_{3}$ & 0.079 & 0.045 & 0.077 & 0.097 & 0.075 \\
\hline$A_{4}$ & 1.00 & 3.00 & 4.00 & 1.00 & $A_{4}$ & 0.395 & 0.409 & 0.308 & 0.387 & 0.375 \\
\hline \multicolumn{5}{|c|}{$C R=3.16$} & & & $C R$ & 3.16 & & \\
\hline$C_{43}$ & iterion & ninim & interv & ation & & 3-Crite & on of $m$ & mum i & rventat & \\
\hline & $A_{1}$ & $A_{2}$ & $A_{3}$ & $A_{4}$ & & $A_{1}$ & $A_{2}$ & $A_{3}$ & $A_{4}$ & $w_{i}$ \\
\hline$A_{1}$ & 1.00 & 0.17 & 0.33 & 0.20 & $A_{1}$ & 0.067 & 0.098 & 0.036 & 0.044 & 0.061 \\
\hline$A_{2}$ & 6.00 & 1.00 & 5.00 & 3.00 & $A_{2}$ & 0.400 & 0.588 & 0.536 & 0.662 & 0.546 \\
\hline$A_{3}$ & 3.00 & 0.20 & 1.00 & 0.33 & $A_{3}$ & 0.200 & 0.118 & 0.107 & 0.074 & 0.125 \\
\hline$A_{4}$ & 5.00 & 0.33 & 3.00 & 1.00 & $A_{4}$ & 0.333 & 0.196 & 0.321 & 0.221 & 0.268 \\
\hline & & $R=8.2$ & & & & & $C R$ & 8.26 & & \\
\hline & & $5-\mathrm{RC}$ & & & & & & OI & & \\
\hline & $A_{1}$ & $A_{2}$ & $A_{3}$ & $A_{4}$ & & $A_{1}$ & $A_{2}$ & $A_{3}$ & $A_{4}$ & $w_{i}$ \\
\hline$A_{1}$ & 1.00 & 3.00 & 0.20 & 0.33 & $A_{1}$ & 0.107 & 0.188 & 0.119 & 0.074 & 0.122 \\
\hline$A_{2}$ & 0.33 & 1.00 & 0.14 & 0.20 & $A_{2}$ & 0.036 & 0.063 & 0.085 & 0.044 & 0.057 \\
\hline$A_{3}$ & 5.00 & 7.00 & 1.00 & 3.00 & $A_{3}$ & 0.536 & 0.438 & 0.597 & 0.662 & 0.558 \\
\hline$A_{4}$ & 3.00 & 5.00 & 0.33 & 1.00 & $A_{4}$ & 0.321 & 0.313 & 0.199 & 0.221 & 0.263 \\
\hline & & $=6.5$ & & & & & $C R$ & 6.54 & & \\
\hline
\end{tabular}


In the preparation of the matrices of pairwise comparisons, both between criteria and between sub-criteria, we assigned equal weight to each criterion, and with reference to the single criterion, we assigned the same weight to each sub-criterion. All matrices were consistent.

The total priorities are shown in Table 6. The AHP therefore identifies the local cuisine restaurant as the best destination for the recovery and enhancement of the former convent.

Table 6. Priority matrix.

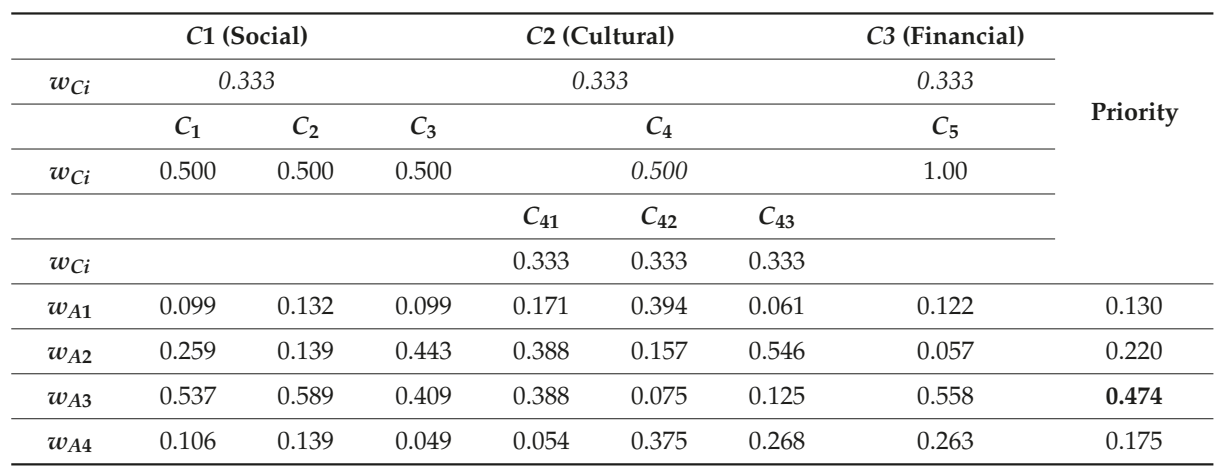

\section{Discussion}

The selection of possible alternatives of use for the historical building under study was derived from a careful analysis of the needs of citizenship, and from the economic and socio-demographic characteristics of the reference territory. In particular, the choice of the B\&B was born from the need to improve the reception services connected to the use of the existing historical, architectural, and archaeological heritage. The multi-purpose hall option was aimed at the cultural growth of the city, which was also poorly equipped with facilities for conferences, seminars, and professional training. The local cuisine restaurant destination with its museum of rural civilization intends to recover the building as the driving force of an economy capable of enhancing the typical local products, as in the past, the convent - thanks to the presence of Benedictine monks-was a point of reference for the agricultural development of a marginal area. The office function can allow the urban center to play a central economic role.

Table 5 of the priorities shows that, due to the multiple evaluation criteria considered, the AHP method considers $A_{3}$ as the best alternative. Thus, the optimal use is that of the local cuisine restaurant, with a score of 0.474 . In the order of the alternatives: $A_{2}$ (multi-purpose rooms) with a score of 0.220 , that is less than half of the valid score for the first solution in the ranking; $A_{4}$ (offices) with a score of 0.175 ; and $A_{1}$ (bed \& breakfast) with a score of 0.130 .

\section{Conclusions}

Historical environments are an exhaustible resource, so it requires great attention in terms of protection, but also of enhancement. This can happen through the functional recovery of the building with the identification of its best use, intended as a function able to simultaneously maximize the social, cultural, and financial effects that the intervention on the historic building generates on the reference territory. This approach respects the principles of sustainable urban development.

The aim of the research is to outline a multi-criteria evaluation model able to select the optimal function for a historic building in a state of neglect. From this point of view, a comparative analysis was carried out on some of the most well-known Multi-Criteria Decision Making methods: the Analitic Hierarchy Process (AHP), ELimination Et Choix Traduisant la REalité (ELECTRE), Tecnique for Order Preference by Similarity to Ideal Solution (TOPSIS), and the Compromise Ranking Method (VIKOR). The theoretical examination highlights that, due to its hierarchical structure, the AHP model allows the 
problem to be broken down to the level of detail necessary for the analysis, thus being more effective in solving the problem in question, where, very often, there are multiple criteria and sub-criteria of evaluation.

For the correct implementation of the hierarchical analysis algorithms, the rigorous selection of the evaluation criteria and sub-criteria, as well as the corresponding indicators, is fundamental. This selection represents an element of novelty of the research. The study led us to recognize: the social criteria, which can be expressed through the community involvement and the new workers; cultural criteria, translated in terms of cultural effects and compatibility of the function with the historical-architectural characteristics of the property; and financial criteria, to be expressed quantitatively by the Return On Investment. It should be noted that a careful study on the compatibility of the function with the historical-architectural characteristics of the property leads to a breaking down of this criterion into the three interventions.

The operational coherence of the evaluation model is verified through a case study, concerning the selection of the optimal destination for a former convent in the province of Salerno (Italy).

The multi-criteria analysis model proved to be an effective decision support tool, guaranteeing to both public administrations and private individuals an optimal allocation of available resources, with obvious and important implications of economic policy.

Author Contributions: Conceptualization, A.N.; Data curation, A.N and P.S.; Formal analysis, A.N. and P.S.; Methodology, A.N. and P.S.; Software, A.N. and P.S.; Supervision, A.N.; Validation, A.N.; Writing-original draft, A.N. and P.S.; Writing-review \& editing, A.N.

Funding: This research received no external funding.

Conflicts of Interest: The authors declare no conflicts of interest.

\section{Appendix A}

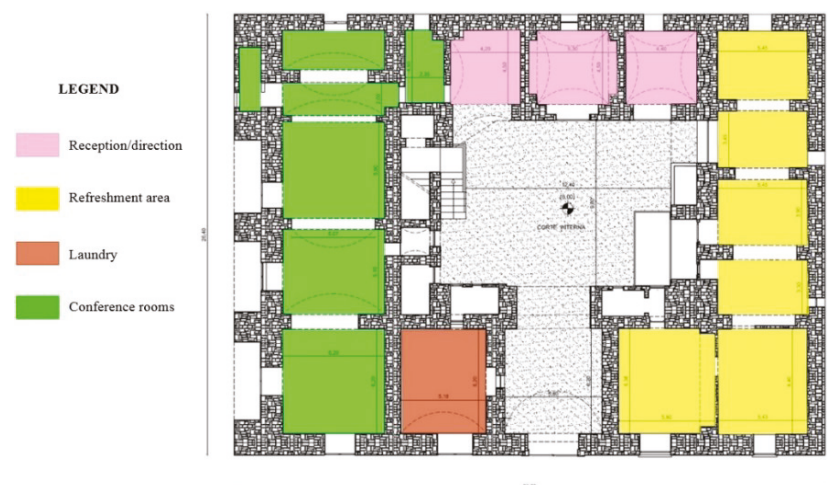

Figure A1. B\&B ground floor plan. 


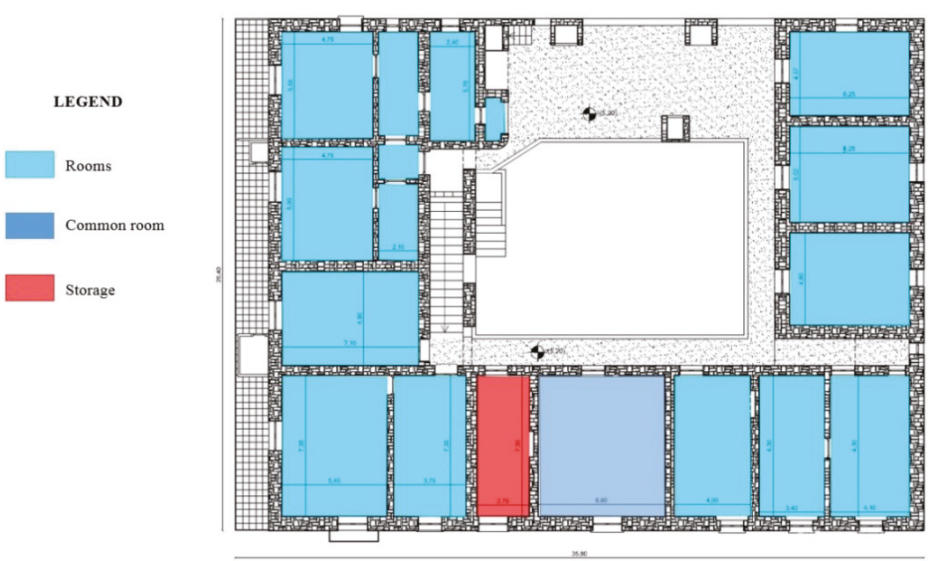

Figure A2. B\&B first floor plan.

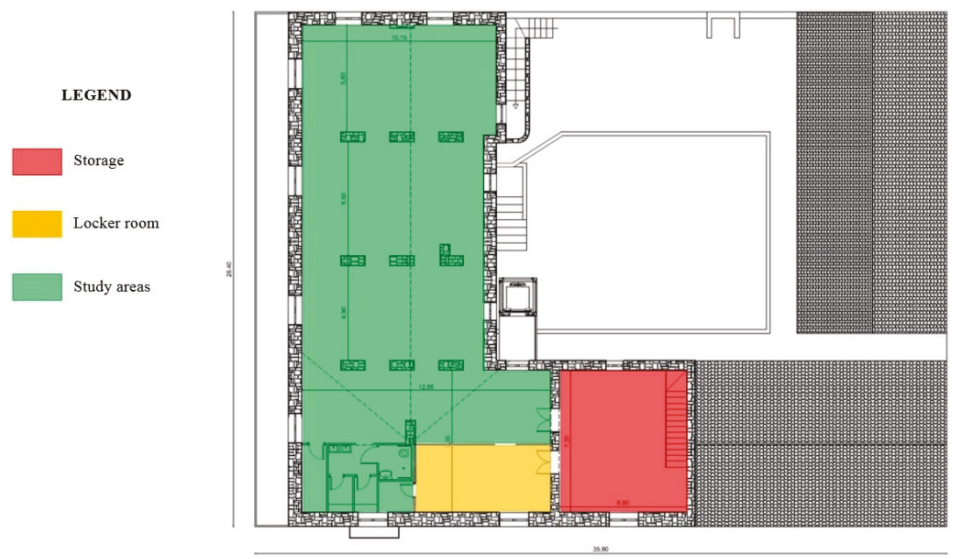

Figure A3. B\&B attic floor plan.

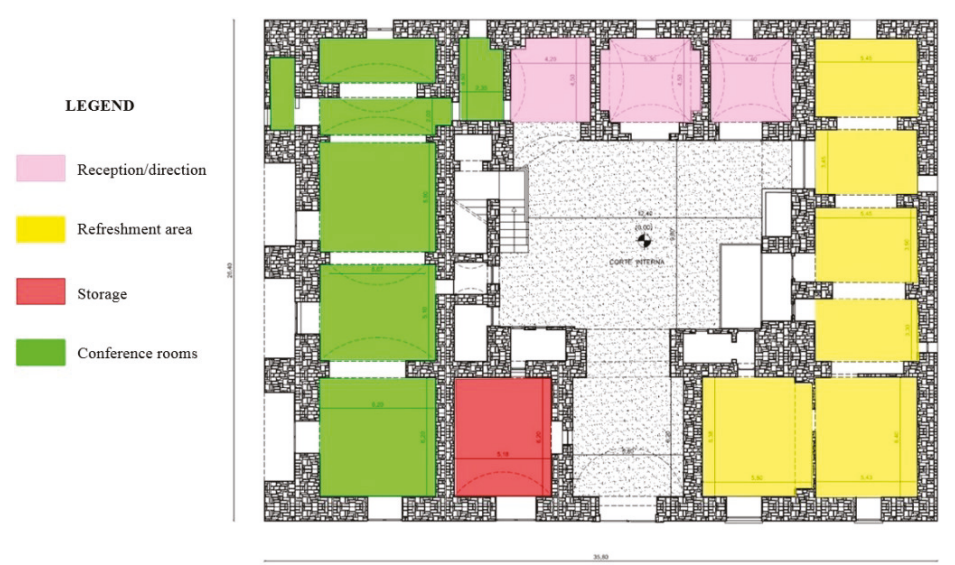

Figure A4. Multi-purpose rooms ground floor plan. 


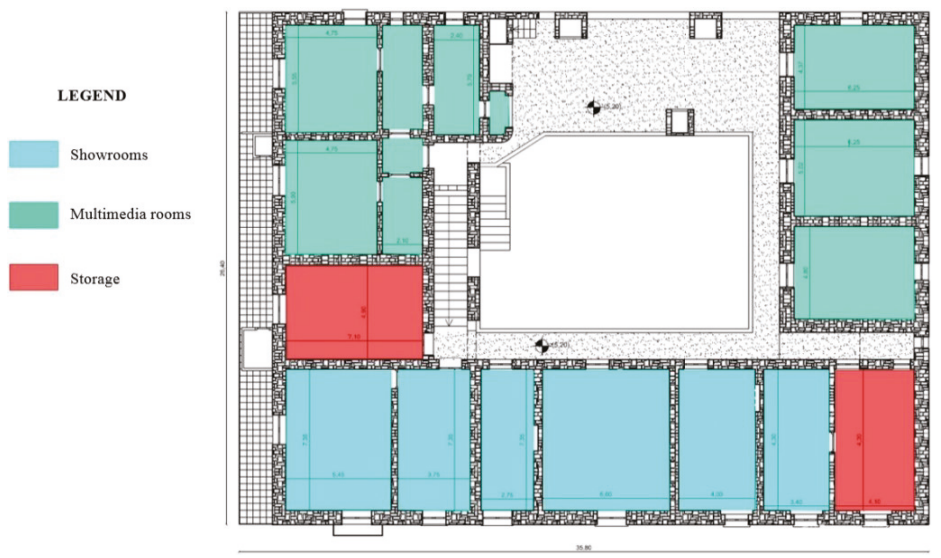

Figure A5. Multi-purpose rooms first floor plan.

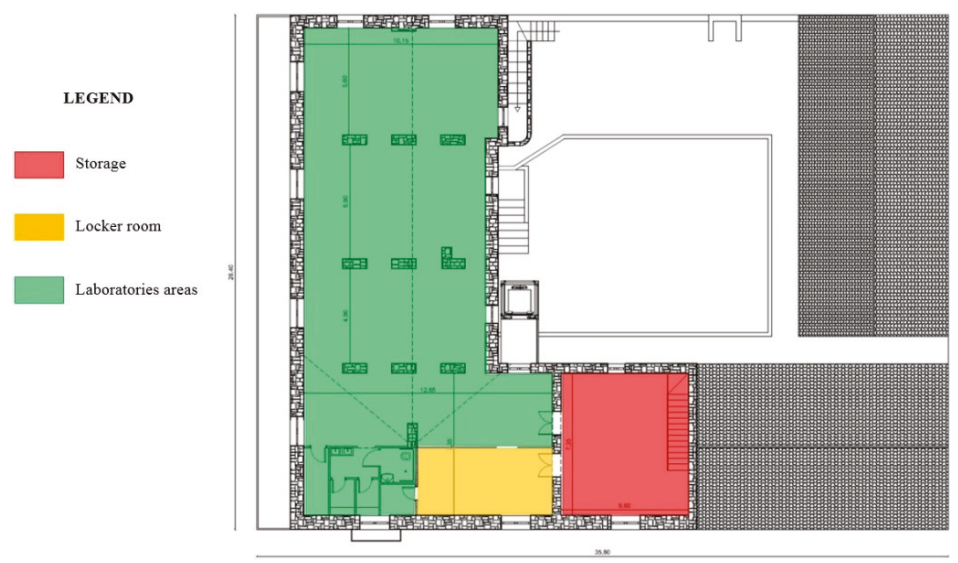

Figure A6. Multi-purpose rooms attic floor plan.

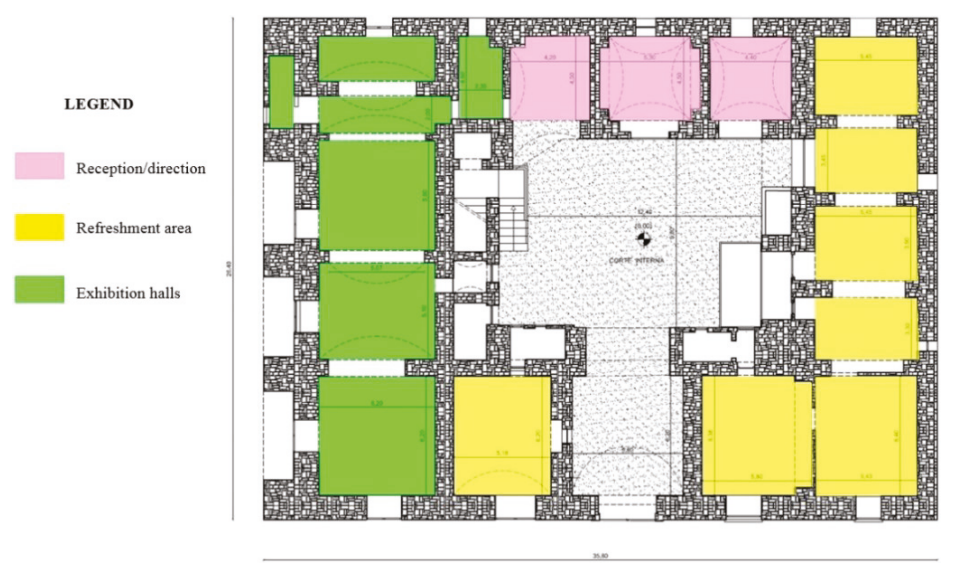

Figure A7. Local cuisine restaurant ground floor plan. 


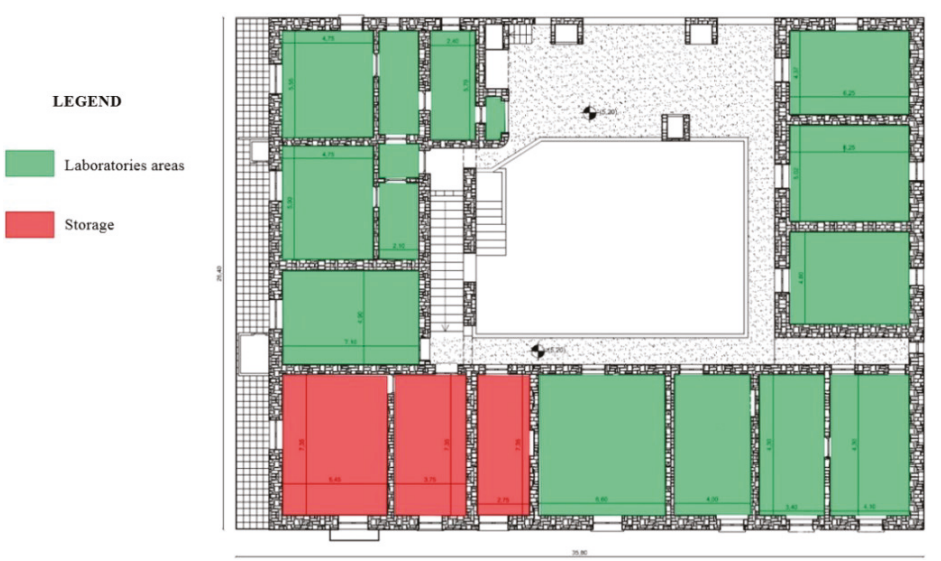

Figure A8. Local cuisine restaurant first floor plan.

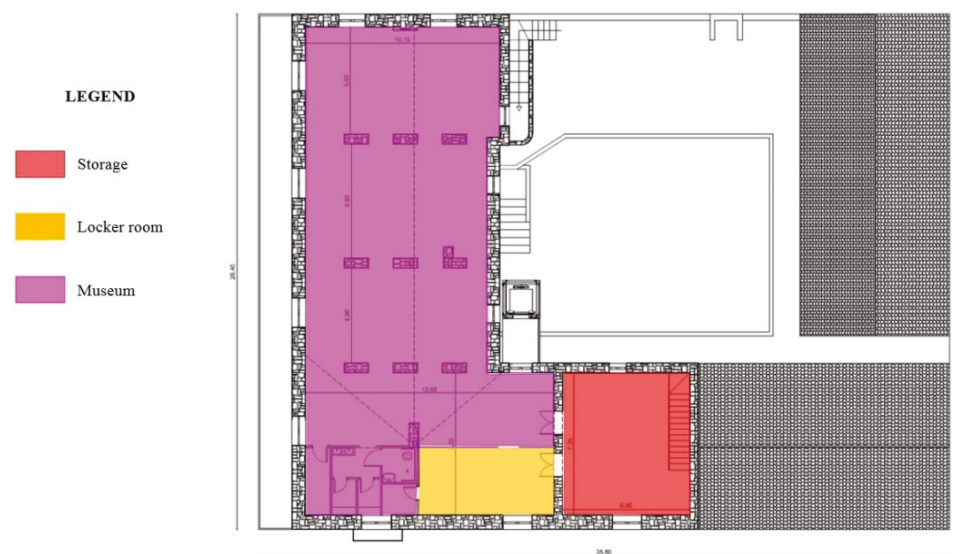

Figure A9. Local cuisine restaurant attic floor plan.

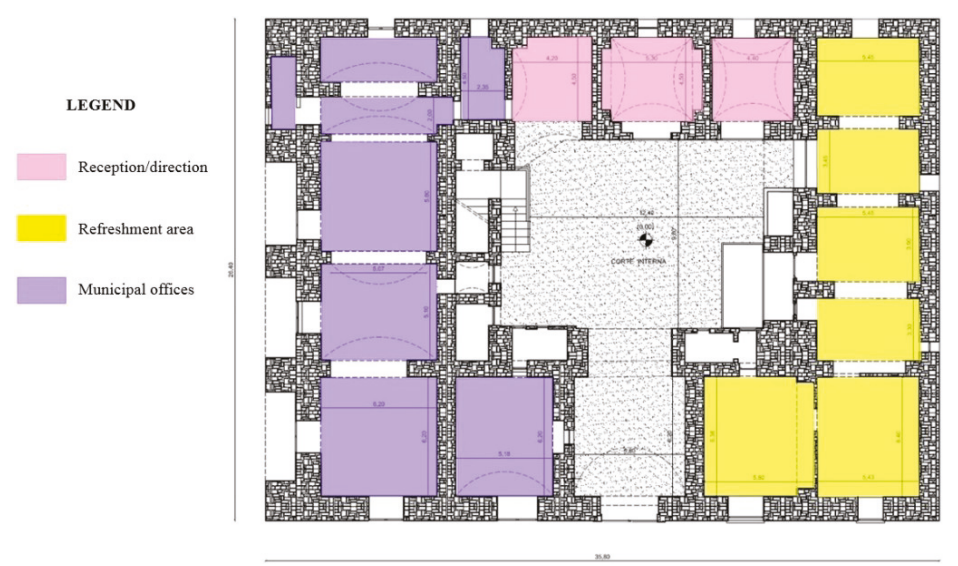

Figure A10. Offices' ground floor plan. 


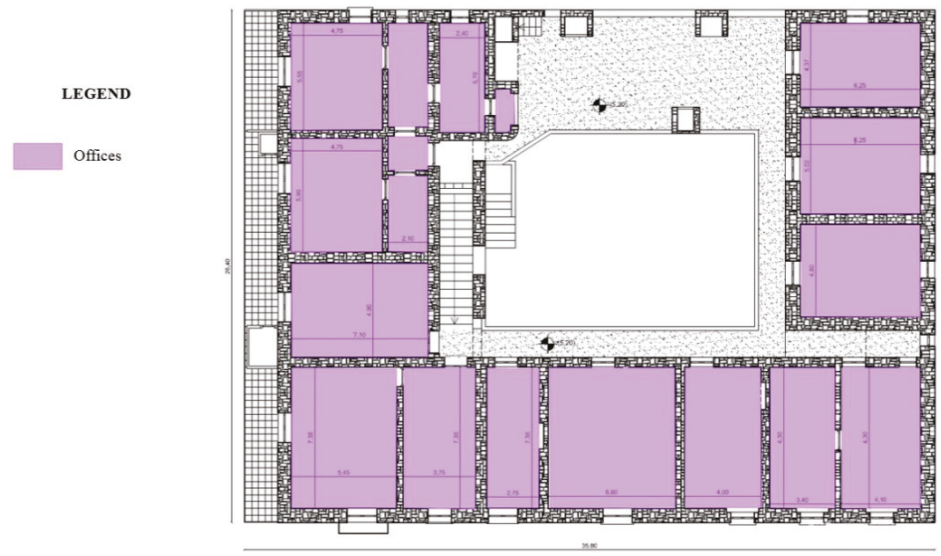

Figure A11. Offices' first floor plan.

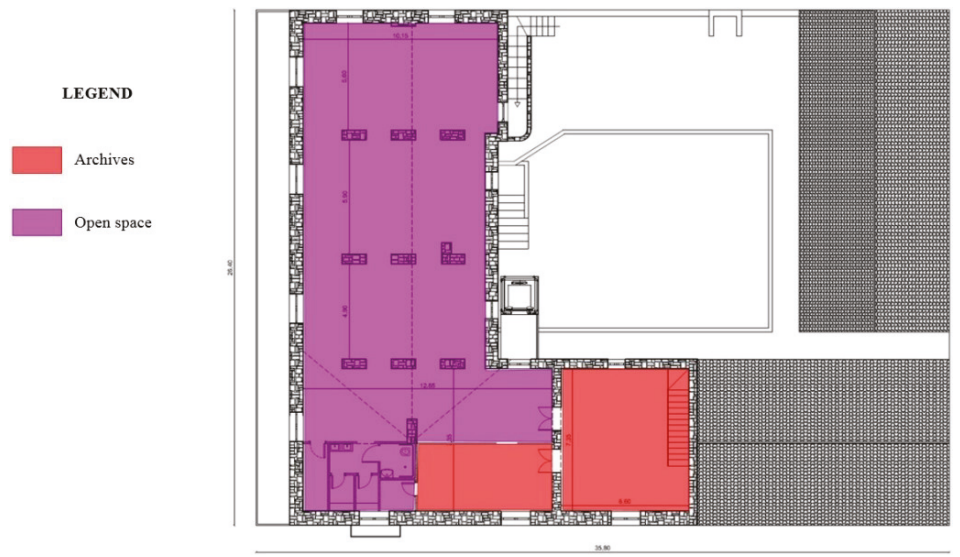

Figure A12. Offices' attic floor plan.

\section{References}

1. Nesticò, A.; Sica, F. The sustainability of urban renewal projects: A model for economic multi-criteria analysis. J. Prop. Invest. Financ. 2017, 35, 397-409. [CrossRef]

2. Di Ruocco, G.; Nesticò, A. Archaeological Site Conservation and Enhancement: An Economic Evaluation Model for the Selection of Investment Projects. Sustainability 2018, 10, 3907. [CrossRef]

3. Ministero dell'ambiente e della tutela del territorio e del mare. In Proceedings of the Le Infrastrutture verdi e i servizi Ecosistemici in Italia Come Strumento per le politiche Ambientali e la Green Economy: Potenzialità, Criticità e proposte, In Conference La Natura dell'Italia, Rome, Italy, 11-12 December 2013.

4. Maskey, V.; Brown, C.; Lin, G. Assessing factors associated with listing a historic resource in the national register of historic places. Econ. Dev. Q. 2009, 4, 342-350. [CrossRef]

5. Phillips, R.G.; Burdruk, M. Introduction to Quality of Life and Community Indicators for Parks, Recreation and Tourism Management; Springer: Dordrecht, The Netherlands, 2010.

6. Throsby, D. Heritage Economics: A conceptual framework. In The Economics of Uniqueness. Investing in Historic City Cores and Cultural Heritage Assets for Sustainable Development; Licciardi, G., Amirtahmasebi, R., Eds.; World Bank Group: Washington, DC, USA, 2012; pp. 45-74.

7. Nesticò, A.; Guarini, M.R.; Morano, P.; Sica, F. An Economic Analysis Algorithm for Urban Forestry Projects. Sustainability 2019, 11, 314. [CrossRef] 
8. Cascone, S.; Sciuto, G. Nuove identità per edifici dismessi in centro storico. Recupero Conserv. 2016, 133, 27.

9. Della Spina, L. Evaluation Decision Support Models: Highest and Best Use choice. Procedia Soc. Behav. Sci. 2016, 223, 936-943. [CrossRef]

10. Nesticò, A.; Macchiaroli, M.; Pipolo, O. Costs and Benefits in the Recovery of Historic Buildings: The Application of an Economic Model. Sustainability 2015, 7, 14661-14676. [CrossRef]

11. Ribera, F.; Nesticò, A.; Cucco, P.; Maselli, G. A multicriteria approach to identify the Highest and Best Use for historical buildings. J. Cult. Herit. 2019. [CrossRef]

12. Mosetto, G.; Vecco, M. Economia del Patrimonio Monumentale; FrancoAngeli: Milan, Italy, 2001.

13. De La Torre, M. Assessing the Values of the Cultural Heritage; Getty Conservation Institute: Los Angeles, CA, USA, 2002.

14. Fusco Girard, L.; Nijkamp, P. La valutazione per lo Sviluppo Sostenibile Della Città e del Territorio; FrancoAngeli: Milan, Italy, 1997.

15. Pompili, T.; Travisi, C.M.; Ruggeri, G. La valutazione di attività complementari alle attività agricole nelle aree rurali e periurbane della provincia di Milano: Un approccio multicriteri. In Proceedings of the XXV Conferenza Italiana di Scienze Regionali, Novara, Italy, 6-8 october 2004.

16. Nesticò, A. Risk-Analysis Techniques for the Economic Evaluation of Investment Projects. In Integrated Evaluation for the Management of Contemporary Cities. SIEV 2016. Green Energy and Technology; Mondini, G., Fattinnanzi, E., Oppio, A., Bottero, M., Stanghellini, S., Eds.; Springer: Cham, Switzerland, 2018; pp. 617-629. [CrossRef]

17. Nesticò, A.; Moffa, R. Economic analysis and Operational Research tools for estimating productivity levels in off-site construction [Analisi economiche e strumenti di Ricerca Operativa per la stima dei livelli di produttività nell'edilizia off-site]. Valori Valutazioni 2018, 20, 107-126.

18. Nesticò, A.; Morano, P.; Sica, F. A model to support the public administration decisions for the investments selection on historic buildings. J. Cult. Herit. 2018, 33, 201-207. [CrossRef]

19. Mavrotas, G. Effective implementation of the $\varepsilon$-constraint method in multi-objective mathematical programming problems. Appl. Math. Comput. 2009, 213, 455-465. [CrossRef]

20. Pompili, T. Metodologie di valutazione economica: Una rassegna sistematica. In Valutazione Economica e Strategica di Programmi e Progetti Territoriali; FrancoAngeli: Milan, Italy, 2006.

21. Nesticò, A.; He, S.; De Mare, G.; Benintendi, R.; Maselli, G. The ALARP Principle in the Cost-Benefit Analysis for the Acceptability of Investment Risk. Sustainability 2018, 10, 4668. [CrossRef]

22. Pons, O.; De la Fuente, A.; Aguado, A. The Use of MIVES as a Sustainability Assessment MCDM Method for Architecture and Civil Engineering Applications. Sustainability 2016, 8, 460. [CrossRef]

23. Figueira, J.; Greco, S.; Ehrgott, M. Multiple Criteria Decision Analysis: State of the Art Surveys; Springer Science \& Business Media: New York, NY, USA, 2005; Volume 78.

24. Figueira, J.; Greco, S.; Roy, B.; Stowinski, R. An overview of ELECTRE methods and their recent extensions. J. Multi Criteria Decis. Anal. 2013, 20, 61-85. [CrossRef]

25. Hwang, C.L.; Yoon, K. Multiple Attribute Decision Making, Lecture Notes in Economics and Mathematical System n. 186; Springer: Berlin, Germany, 1981.

26. Opricovic, S.; Tzeng, G.H. Extended VIKOR method in comparison with outranking methods. Eur. J. Oper. Res. 2007, 178, 514-529. [CrossRef]

27. Roy, B. How outranking relation helps multiple criteria decision making, Multiple Criteria Decision Making, Actes du Séminaire "Théorie de la Décision"; Beaulieu-Sainte-Assise: Francia, 1973.

28. Roy, B. Méthodologie Multicritére D'aide à la Decision; Economica: Paris, France, 1985.

29. Saaty, T.L. The Analytic Hierarchy Process; McGraw-Hill: New York, NY, USA, 1977.

30. Saaty, T.L. A Scaling Method for Priorities in Hierarchy Structures. J. Math. Psychol. 1980, 15, 234-281. [CrossRef]

31. Saaty, T.L. How to make a decision: The Analytic Hierarchy Process. Eur. J. Oper. Res. 1990, 48, 9-26. [CrossRef]

32. Saaty, T.L. Decision Making for Leaders: The Analytic Hierarchy Process for Decision in a Complex Word; RWS Publications: Pittsburg, CA, USA, 1999.

33. Tzeng, G.H.; Huang, J.J. Multiple Attribute Decision Making Methods and Applications; CRC Press, Taylor \& Francis Group: Boca Raton, FL, USA, 2011.

34. Velasquez, M.; Hester, P.T. An Analysis of Multi-Criteria Decision Making Methods. Int. J. Oper. Res. 2013, 10, 56-66.

35. Vincke, P. Multicriteria Decision-Aid; John Wiley \& Sons: Hoboken, NJ, USA, 1992. 
36. Zanakis, S.H.; Solomon, A.; Wishart, N.; Dublish, S. Multi-attribute decision making: A simulation comparison of select methods. Eur. J. Oper. Res. 1998, 107, 507-529. [CrossRef]

37. Mela, K.; Tiainen, T.; Heinisuo, M. Comparative study of multiple criteria decision making methods for building design. Adv. Eng. Inform. 2012, 26, 716-726. [CrossRef]

38. Forman, E.H. The Analytic Hierarchy Process: An Exposition, School of Business and Public Management; George Washington University: Washington, DC, USA, 2001.

39. Basak, I. Estimation of priority weights based on a resampling technique and a ranking method in analytic hierarchy process. J. Multi Criteria Decis. Anal. 2019. [CrossRef]

40. Fishburn, P.C. Additive Utilities with Incomplete Product Set: Applications to Priorities and Assignments; ORSA: Baltimora, MD, USA, 1967.

41. Saaty, T.L. Decision making with the analytic hierarchy process. Int. J. Serv. Sci. 2008, 1, 83-98. [CrossRef]

42. Calabrò, F.; Della Spina, L. Innovative Tools for the Effectiveness and Efficiency of Administrative Action of the Metropolitan Cities: The Strategic Operational Programme. In 1th international Symposium New Metropolitan Perspectives - The Integrated Approach of Urban Sustainable Development (ISTH2020), Advanced Engineering Forum; Trans Tech Publications: Switzerland, Zurich, 2014; Volume 11, pp. 3-10.

43. Yau, Y. Multi-criteria decision making for urban built heritage conservation: Application of the analytic hierarchy process. J. Build. Apprais. 2008, 4, 191-205. [CrossRef]

44. Nijkamp, P.; Rietveld, P.; Voogd, H. Multicriteria Evaluation in Physical Planning; North Holland Publications: Amsterdam, The Netherland, 1990.

45. UNESCO. Budapest Declaration on Word Heritage; UNESCO: Budapest, Hungary, 2002.

46. Chan, E.H.W.; Lee, G.K.L. Critical factors for improving social sustainability of urban renewal projects. Soc. Indic. Res. 2007, 85, 243-256. [CrossRef]

47. Zancheti, S.; Hidaka, L. Measuring urban heritage conservation: Indicator, weights and instruments (part. 2). J. Cult. Herit. Manag. Sustain. Dev. 2012, 2, 15-16. [CrossRef]

48. Phillips, R.G.; Stein, J.M. An indicator frameworkfor linking historic preservation and community economic development. Soc. Indic. Res. 2013, 113, 1-15. [CrossRef]

49. Petzet, M. Principles of Preservation: An Introduction to the International Charters for Conservation and Restoration 40 years after the Venice Charter. In International Charters for Conservation and Restoration Monuments \& Sites; ICOMOS: München, Germany, 2004.

50. Pellegri, P.C. Manuale del Riuso Architettonico: Sostenibilità e Compatibilità Ambientale Nella Scelta dei Materiali e Delle Tecnologie; Flaccovio Editore: Palermo, Italy, 2018.

51. Fusco Girard, L.; Nijkamp, P. Energia, Bellezza, Partecipazione: La sfida della Sostenibilità. Valutazioni Integrate tra Conservazione e Sviluppo; Angeli: Milano, Italy, 2004.

52. Fiore, P.; Nesticò, A.; Macchiaroli, M. The energy improvement of monumental buildings. An investigation protocol and case studies La riqualificazione energetica degli edifici monumentali. Un protocollo di intervento e caso studio. Valori Valutazioni 2016, 16, 45-55.

53. De Mare, G.; Granata, M.F.; Nesticò, A. Weak and Strong Compensation for the Prioritization of Public Investments: Multidimensional Analysis for Pools. Sustainability 2015, 7, 16022-16038. [CrossRef]

54. De Mare, G.; Manganelli, B.; Nesticò, A. Dynamic Analysis of the Property Market in the City of Avellino (Italy). In The Wheaton-Di Pasquale Model Applied to the Residential Segment; Murgante, B., Misra, S., Carlini, M., Torre, C., Nguyen, H.Q., Taniar, D., Apduhan, B.O., Gervasi, O., Eds.; Springer-Verlag: Berlin/Heidelberg, Germany, 2013; Volume 7973, pp. 509-523. [CrossRef]

55. Della Spina, L. Historical Cultural Heritage: Decision Making Process and Reuse Scenarios for the Enhancement of Historic Buildings. In New Metropolitan Perspectives. ISHT 2018. Smart Innovation, Systems and Technologies; Calabrò, F., Della Spina, L., Bevilacqua, C., Eds.; Springer: Cham, Switzerland, 2019; Volume 101. [CrossRef]

56. Triantaphyllou, E. Multi-Criteria Decision Making Methods: A Comparative Study, Applied Optimization 44; Kluwer Academic Publisher: Dordrecht, The Netherland, 2002.

57. Zimmermann, H.J. Fuzzy Set Theory and Its Applications; Third Revised Edition; Kluer Academy Publishers: Boston, MA, USA, 1996.

(C) 2019 by the authors. Licensee MDPI, Basel, Switzerland. This article is an open access article distributed under the terms and conditions of the Creative Commons Attribution (CC BY) license (http://creativecommons.org/licenses/by/4.0/). 

Article

\title{
An Operational Protocol for the Valorisation of Public Real Estate Assets in Italy
}

\author{
Sebastiano Carbonara * and Davide Stefano \\ Department of Architecture, G. d'Annunzio University, 65127 Pescara, Italy; davide.stefano@unich.it \\ * Correspondence: s.carbonara@unich.it; Tel.: +39-085-4537315
}

Received: 19 December 2019; Accepted: 15 January 2020; Published: 19 January 2020

\begin{abstract}
The Italian Treasury Department reports that a quota of the country's public real estate assets, with an estimated value of some 63 billion euros, consists of properties not directly utilised by the State Government and is therefore available for decommissioning alienation; in other words, for adaptive reuse. Numerous legislative initiatives dedicated to this issue over the past 30 years have produced very few comforting results. A plausible explanation for these shortcomings can be traced to the gap between established regulatory principles and the possibilities/capacities of local institutions to apply them. Put another way, legislation and indications, many of interest, have not been supported by adequate economic, structural, and organisational resources. The underlying question is, what is the structure of the decision-making process behind the sale or redevelopment of real estate assets? Beginning with these premises, this paper proposes an operational Business Process Modelling protocol that develops three different indexes—urban values index $\left(\mathrm{I}_{\mathrm{vu}}\right)$, use index $\left(\mathrm{I}_{\mathrm{ut}}\right)$, and technical-maintenance index $\left(\mathrm{I}_{\mathrm{tm}}\right)$-which may suggest three hypothetical scenarios of valorisation and three lines of action. A test of this model using a selection of public buildings owned by the City of Pescara showed it to be prognostic of some of the choices subsequently made by the municipal administration.
\end{abstract}

Keywords: public real estate property; operational protocol; model of choice; radar diagram

\section{Introduction}

The Italian Treasury Department published its Public Administration Assets report in 2018. This document examined the composition and value of public real estate assets more than 30 years after the first census made in Republican Italy by the Cassese Commission during the mid-1980s.

The information in the report, relative to publicly-owned real estate assets between 2011-2015, derives from the annual survey public administrations are obliged to produce since 2009 [1].

According to the report, the value of the Italian public real estate assets, for buildings alone, amounts to roughly 283 billion euros for a portfolio of 325 million square metres. Eighty percent of this area is used for strictly institutional purposes related to office functions. The remaining portion, with an estimated value of approximately 63 billion euros, is available and may be put to better use for social purposes or alienated.

In terms of estimated value this important figure makes the Italian State-at least potentially-the most important operator in the real estate market when compared to the assets held by the 450 real estate funds operating in the country, which total approximately 66 billion euros [2].

While this public real estate capital available for valorisation operations is very heterogeneous in its typologies, uses, and dimensions - not to mention highly fragmented and, often, in a significant state of disrepair-there is little doubt that it remains a resource with the possibility to generate additional revenue for public coffers. 


\section{The Real Estate Assets of Italy's Comuni}

In Italy, the reform of local government and the economic and financial crisis, together with the budgetary and spending constraints introduced by the European Fiscal Compact, have generated mounting financial pressure on the public administration and highlighted the role of public assets in debt management [3]. While these assets generate a negative balance because very often they are underutilised or not utilised at all, they represent a potential source of revenue in the event of sale [4] or improved use.

As described in the 2015 Ministry of Economy and Finance-Treasury Department report, the majority of these assets are owned by local administrations; the most relevant share belongs to the country's comuni (municipalities) $-67.42 \%$ by number and $59.51 \%$ by area. While seemingly large, in reality, these figures refer primarily to minor properties (apartments and related appurtenances, garages, commercial spaces, etc.). On the contrary, state, regional, and provincial governments and public healthcare authorities possess smaller though more valuable portfolios (historical buildings, structured offices, former hospitals, etc.).

Despite their small dimensions, in practical terms, the assets owned by Italy's municipalities can still make a significant financial and social contribution. In fact, it is not so much the asset itself that produces value, as much as the utility derived from it through proper valorisation— "the uses, public and private, economic and social, in any case multiple and diverse, emerge from the context in which the asset is situated" [5].

What follows is the need to develop design skills and visions that postulate possible new scenarios of use; new functions for spaces and sites within a more general notion of transformation [6].

This is particularly problematic for small municipalities. Unlike larger towns or metropolitan areas, they suffer from a lack of technical and financial resources and trained staff, which further complicates the implementation of any process of valorisation. It is clear that the complex factors linked to an adequate policy for the valorisation of public real estate assets cannot be a prerogative "solely of larger municipalities with financial and technical-operational capacities" [7]; hence there is a need to define an operational protocol that is particularly suited to small municipalities.

A further indicator of the need for tools in support of 'small municipalities' is offered by Law $n$. 158/2017. The principal interventions foreseen by this regulation include the establishment of a fund for the structural, economic, and social development of small municipalities. This fund was created to finance investments in the protection of the environment and cultural heritage, the preservation and urban redevelopment of historical centres, the promotion of economic and social development and the establishment of new productive activities. While this fund cannot count on large sums, nonetheless, the resources allocated may still aid small municipalities with the recovery and valorisation of their real estate assets.

\section{The Indispensable Role of Knowledge}

An adequate knowledge of real estate assets is the building block of a correct valorisation strategy. Two levels of in-depth analysis can be identified: the first is represented by the wealth of technical-financial information we can refer to as 'material knowledge'; the second, bound to the requirements, expectations and, needs of different communities—evidence of which should be present in political programmes-whose satisfaction can be traced, directly or indirectly, to available real estate assets, represents a level of knowledge that can only be referred to as 'immaterial'.

\section{1. "Material" Knowledge}

One of the principal factors hindering the success of valorisation projects is rooted in the Public Administration's scarce knowledge of its own assets [8,9].

Despite the issuance of several laws intent on rationalising this aspect, Italy continues to be plagued by a large number of public institutions with fragmented and often out of date information. 
Italy currently has no national information system [10] documenting the many aspects of its assets. Consequently, the country lacks a standardised procedure for their management. These are anything but secondary aspects: the size of a building and the layout of its interior spaces; the general state of maintenance and efficiency of plant systems; energy efficiency; current use and eventual revenues generated, etc. When such information is lacking, it is truly difficult to develop asset, property, and facility management activities [11], but also to adequately assess the option of selling off these assets.

\subsection{Immaterial Knowledge}

In the wake of the regulatory changes made during the 1990s and new forms of relationships between the Public Administration and citizens introduced by the principle of horizontal subsidiarity, the public sector has faced a growing demand for greater accountability which led, in turn, to the preparation of the Sustainability Report (or Social Report). This document permits stakeholders to be more informed, and consequently more involved, in the choices made by institutions. It represents a means for re-stitching the institutional 'rift' that developed over time between the public administration and citizens, especially after the "tangentopoli" (bribesville) scandal of the 1990s.

There has been a rapid shift from forms of "communication is participation" [12] involving citizens in public affairs, to even more direct forms of participatory budgeting. Inspired by the concept of participatory democracy, decisions are taken directly by citizens [13] and represent an approach to the development of budgets for local authorities through the direct participation of citizens in specific expenditures.

Furthermore, the declining demand for public assets to be alienated or valorised, the bureaucratic complexity linked to valorisation operations, and the complicated marketing of public properties that are both large and difficult to transform (such as former barracks, prisons or military hospitals) have directed public administrations "to test new ways of valorising assets, promoting the action of associations and self-organised groups interested in using buildings otherwise destined for abandonment" [14].

These new forms of bottom-up use (or better yet re-use), directly involve local communities through forms of civic engagement and civic crowdfunding.

While the former can be considered "active" for their civic engagement, the latter can be considered "passive" or "semi-passive" as they occur through fundraising to develop a specific project (or initiative). Without producing economic returns for investors, they are implemented solely due to the common belief that a project is worthy of being developed and implemented [15]. "Citizens will find bottom-up financing to be a tool for having a say in the management and valorisation of common goods, expressing their requirements and participating as active subjects in the decision-making processes of the public administration" [16].

Furthermore, the continuous development of new forms of activity, such as urban neo-manufacturing, digital craftsmanship, the creation of spaces for coworking and start-ups, and social services offered in refurbished spaces in former commercial or institutional buildings, paves the way for new policies of reuse in a form of so-called real estate upcycling.

Participatory budgets, civic engagement and civic crowdfunding undoubtedly represent direct forms of participation in decision-making processes; however, there are other forms that, while following indications provided by the community, are in fact implemented indirectly. Typical of all municipal administrations, they are represented by the implementation of an electoral programme.

This programme, comprised of the ideas underlying a political movement [17], contains the electoral promises made by a candidate during an election (to be respected in the case of victory). "Electoral promises should arise from stakeholder participation in the definition of priorities and the prior verification of reasonably available resources" [18].

Consequently, any process of valorisation or alienation involving public real estate assets must verify the satisfaction of the interests and expectations of the community in advance: these requests are (or should be) contained in an electoral programme. 
As a result, it is impossible to implement policies for the valorisation of public real estate when choices are not shared by the local community [19]; this prerogative appears to be essential to the success of any project fielded by the public administration [20]. In fact, many examples demonstrate that when projects are not shared by the community (for example the sale of public assets for immediate monetisation) they can meet with opposition from citizens. Demonstrations of dissent in the interest of protecting these assets, symbols of memory and tradition for an entire community, block (or considerably slow) procedures and compromise any initiative taken by public decision-makers.

The sum of these elements defines the concept of immaterial knowledge.

\subsection{Using the Third Sector to Favour the Recovery and Reuse of Public Buildings: A Bottom-Up Approach}

Since the 1990s, there have been numerous debates on public real estate and what contribution it could make to the consolidation of public finances. All of the procedures promoted by various legislators share one common element—seeking returns from the market in a relatively short time. Unfortunately, the complexity of regulations and the economic situation have impacted the ability to implement similar operations [21].

All the same, public buildings have always represented a resource for European cities: historically, they have permitted the production of public services and welfare in European cities, and represented one of the stabilising factors of their real estate markets [22]. Today, the "conditions of the European real estate market, the economic crisis and the widespread banking credit crunch oblige the identification of alternative solutions to the alienation of public assets" [23].

The models for valorising traditional public real estate assets have recently begun to make room for new approaches: "temporary uses and the revitalisation of small areas of public buildings are developing progressively, making the practices and management of these processes a possible new way to revitalise these assets" [24]. These bottom-up approaches arise from the requirements and needs of the local community and the development of forms of civic crowdfunding in which citizens, reunited primarily as non-profit associations, initiate real processes for the adaptive reuse of many abandoned public properties.

These forms of reuse are characterised for the most part by free concessions, for short periods of time, and a preference for small redevelopments over large investments. Nonetheless, these activities help foster a high social value by creating new forms of aggregation, such as urban laboratories for co-living, co-making, and co-working, as well as recovering, albeit in part, buildings otherwise destined for abandonment and deterioration.

Additionally, recent migratory movements have accelerated changes, already underway for several years, in the multi-ethnic city. This has generated "an entirely new demand for public and private assets compared to traditional needs" [25]. It is clear that the demand for new spaces in which to implement cultural integration projects can be satisfied through the use of abandoned public buildings by non-profit companies.

A greater impetus to the theme of re-use by non-profit associations came with the agreement signed in November 2017 between the Italian Ministry of Labour and Social Policies, the National Agency for the Administration and Allocation of Assets Seized from Organized Crime (ANBSC), the State Property Office, and the National Association of Italian Municipalities (ANCI). This agreement provides for the allocation of unused public real estate and fixed and mobile assets confiscated from organised crime to associations operating in the third sector to be used exclusively for activities of interest to the general public.

This opportunity sets a virtuous path in motion with a twofold objective: the valorisation of unused public buildings and the development of activities with an elevated social value [26]. Abandoned buildings are often in a poor state of repair. This degradation can expand to the entire neighbourhood in which they are located, generating a flywheel effect of a collective loss of value, [27] as demonstrated by the "Broken Windows Theory". 
While forms of valorisation of public real estate assets implemented through civic engagement procedures do not provide for the full recovery of an asset, nonetheless they ensure its use and self-maintenance, warding off (further) decay [28]; moreover, although they do not produce sizable financial resources for the property owner, they can instead heighten the attractiveness of the neighbourhood in which they are located by providing new services to the community that were not present before.

In this perspective, "the success of these bottom-up valorisation processes has highlighted the creation of a new economic and social value linked to redevelopment projects and new functions, restoring the attractiveness of assets" [29].

\section{An Operational Protocol}

A corporate vision and a managerial approach to the administration of public real estate assets requires the definition of a strategy capable of identifying the full range of activities necessary to reach a desired objective. With this in mind, the representation of the entire decision-making process, capable of guiding a public administration toward the definition of the most appropriate strategies for managing its real estate assets (valorisation, concession or sale), has been translated into a Business Process Modelling exercise (Figure 1). The modelling of this process makes it possible to define the necessary steps, responsibilities and methods for implementing the strategies to be adopted [30].

Starting from these premises, three hierarchical levels of action have been identified.

\subsection{The Technical Level}

The first phase, strictly the responsibility of public technical offices, focuses on identifying all of an administration's assets [31], followed by the drafting of a real estate due diligence for each asset.

The information to be provided for each property includes legal status (e.g., real or minor real rights held by a public body), eventual heritage listings, state of repair, building materials and construction methods, general maintenance of building plants, geolocation, and current occupancy (used by the public administration, leased, vacant).

This process must begin with the official lists already drawn up by public administrations (in compliance with laws on transparency and the drafting of the state budget). The next step is to verify the information collected against data present in revenue agency archives (land registry documents). This operation makes it possible to detect and, if necessary, correct inconsistencies in land registry documents, and to note the eventual presence of other property rights beyond those of full ownership_for example, co-ownership, usufruct, or surface rights.

In cases of co-ownership, for example, it is desirable to proceed with the immediate alienation of the relative percentage of ownership of assets with no specifically unique characteristics or when they are located in non-strategic positions. On the contrary, the public administration can proceed toward full ownership of the property and thus implement valorisation policies otherwise difficult to pursue.

When the public administration possesses only a surface right to a fixed asset, there is a need for policies designed to encourage property owners to purchase these rights in order to generate cash flows that can be allocated to budgetary needs [32] or to initiate valorisation operations involving other assets.

\subsection{The Political Level}

The second level involves planning and consultation activities. This level is used by political bodies to summarise the material knowledge of its assets and the wealth of immaterial knowledge represented by requests presented (directly and indirectly) by the community. This level involves the construction of two databases: one containing data relative to all buildings surveyed (material knowledge) and another containing the issues, suggestions and requests presented to the administration by associations, stakeholders and locally interested parties (immaterial knowledge). 
The elaboration of summary indexes (see Section 6) helps understand whether public real estate assets can satisfy the requirements and needs expressed by the community. For this reason, the first step involves the identification of properties that could be assigned to associations operating in the area, in order to guarantee the possibility to provide new services. For example, the analysis of requests received from stakeholders should be followed by the identification of properties whose dimensions, location or current state of maintenance are considered suitable. This activity may lead to the development of activities related to urban neo-manufacturing or digital craftsmanship, or propose the creation of spaces dedicated to coworking or the development of social housing, cohousing, etc.

This phase also includes the verification and reorganisation of spaces used by the public administration with the aim of eliminating inefficiencies; the new spaces that may be freed up by this activity can be valorised or sold.

A separate consideration must be made for buildings of notable cultural value [33]. Unfortunately, they are not always recognised as such and as a result not always listed as foreseen by Italian law: "the assets that represent the history and culture of the community do not always present nationally recognised values. In many cases, the identification and safeguarding of testimonials considered significant by local communities are subject to the sensitivity of decentralised local administrations" [34].

For assets of this type, the public administration is responsible for ascertaining the existence of these values beforehand and, if confirmed, initiating procedures for their valorisation. This operation allows for both the preservation of an asset's historical, artistic, or cultural interest and guarantees the possibility to introduce economic activities capable of generating new local economies and a new attractiveness for the territory [35].

In the event that an institution's assets are unable to respond to the requests advanced by stakeholders, it is possible to initiate a third phase, which envisages the use of a model of choice [36] to define the strategies to be adopted (valorisation, concession, or sale).

At this point, it is clear that any future choice made by the public administration should seek to avoid (at least in theory) any criticism and opposition from citizens, already amply involved during the second (political) level of the protocol and therefore in advance of any decision-making process.

\subsection{The Technical-Political Level}

The third and final level of the protocol, referred to as 'technical-political', is characterised by the implementation of the model of choice. This model is designed to provide a political body with indications regarding the most appropriate approach for properties lacking a strategy at the political level.

As the model provides general indications, it is clear that choices will have to be examined from both a technical and a political angle. We can consider, for example, the possibility of valorising a property for its future leasing or alienation: this requires both a technical evaluation of the operation (in financial terms), together with an evaluation of the opportunity to proceed with an activity that will require resources to achieve a particular objective (political decision). 


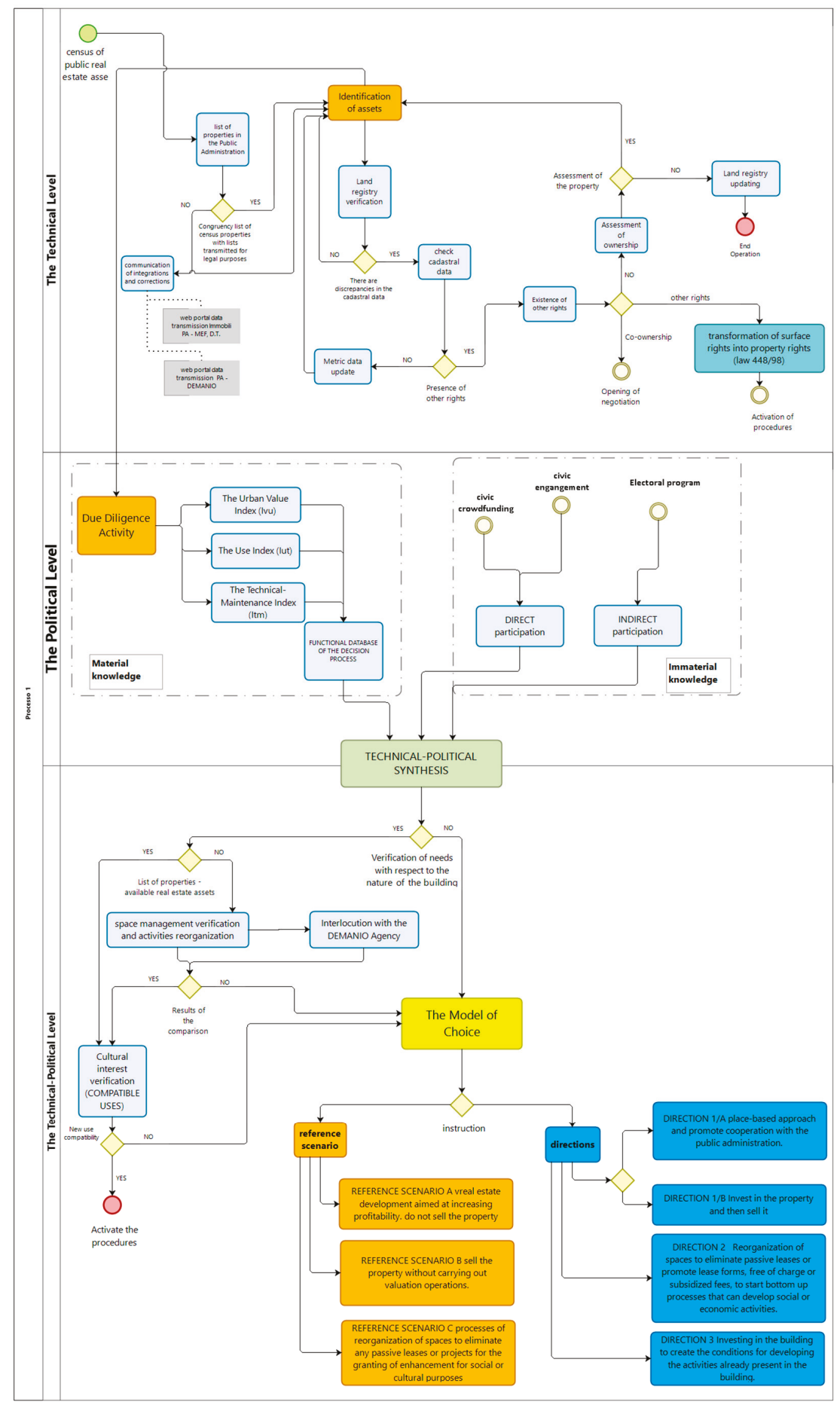

Figure 1. Business process modelling. 


\section{The Model of Choice}

While the Model tends to provide and/or consolidate a use for these assets, it does not develop a real estate rating capable of mathematically quantifying one specific use with respect to another. Based on objective criteria, this tool is easy to implement as the input data is readily obtainable by the public administration.

The process requires the identification of three thematic areas and three corresponding indexes: urban value $\left(\mathrm{I}_{\mathrm{vu}}\right)$, use $\left(\mathrm{I}_{\mathrm{ut}}\right)$, and technical maintenance $\left(\mathrm{I}_{\mathrm{tm}}\right)$ of an asset. Using a limited quantity of data, the intention is to maximise the desired objective, given the evident need to simplify the aspects characterising the three themes investigated.

\subsection{The Urban Value Index $\left(I_{v u}\right)$}

Urban quality and building quality are multidimensional concepts that can be interpreted from different points of view and perspectives [37]. One possible definition refers to the analysis of real estate values, to some degree a synthesis, effect and overall measure of these phenomena. The assumption is that the willingness to pay different prices suggests a greater or lesser appreciation of assets, not only in relation to their intrinsic characteristics, but also to the qualities expressed by the urban contexts in which they are located. This latter approach was used to construct the urban values index.

The starting point is defined by the OMI Zone (acronym for the Revenue Agency's Osservatorio del Mercato Immobiliare, Real Estate Market Observatory): a "continuous portion of the municipal territory that reflects a homogeneous sector of the local real estate market, with a uniform appreciation of economic and socio-environmental conditions". This uniformity is translated into a homogeneity of characteristics related to position, urban planning, historical-environmental and socio-economic qualities of settled areas, and the offering of urban services and infrastructures. The territorial delimitation of a homogenous market area thus passes through the analysis of the homogeneity of socio-environmental and economic conditions and location: first and foremost the requisite of centrality in terms of the presence of functions, accessibility to public and private facilities and services of varying degrees and levels, the level of urban and suburban transportation services, vehicular connections, the presence of schools, healthcare and sport facilities, shops, tertiary services, etc.

The index defines the distance (in terms of real estate values and therefore of market appreciation) between different urban contexts; it is calculated based on the maximum OMI market value on a municipal basis (for residential buildings in good condition) and the OMI market value where the property is located, according to the following formula:

$$
I_{v u}=\alpha V_{O M I}-q,
$$

- $\quad \alpha=1 /\left(V_{\text {OMI max }}-V_{\text {OMI min }}\right)$;

- $q=V_{\text {OMI min }} /\left(V_{\text {OMI max }}-V_{\text {OMI min }}\right)$;

- $V_{O M I} \max =$ maximum OMI quotation for the municipal territory;

- $V_{\text {OMI min }}=$ minimum OMI quotation for the municipal territory;

- $V_{O M I}=$ OMI listing where the property is located.

\subsection{The Use Index $\left(I_{u t}\right)$}

The use index represents the yield (or measure of its efficiency) tied to the use of an asset in relation to its occupancy. The index consists of two parameters describing both the actual degree of use in terms of surface area, and the ability of occupants to pay property management/utility costs (electricity, water, gas as well as services such as waste collection taxes, etc.).

The first parameter is linked to the management of spaces: parameter $\mathrm{P}_{1}$ expresses the relationship between the total building area and the portion occupied. This aspect indicates the degree of use of the 
property, or its underutilisation which permits the allocation of other activities (instrumental to the organisation of the Institution as well as social and/or cultural).

The second parameter, $\mathrm{P}_{2}$, expresses the capacity of the asset to achieve financial autonomy under current conditions of use. This parameter was envisaged as many public buildings are often granted (to non-profit associations) free of charge, meaning that users do not contribute to utility costs.

The index is expressed as follows:

$$
I_{u t}=\left\{\frac{P_{1}+P_{2}}{2}\right\}=\left\{\frac{\left(\frac{S_{o c c}}{S_{t o t}}\right)+\left(\frac{K_{o c c}}{K_{t o t}}\right)}{2}\right\},
$$

- $\quad S_{\text {occ }}=$ occupied area,

- $S_{\text {tot }}=$ total building area,

- $K_{o c c}=$ utility expenses incurred by users,

- $K_{\text {tot }}=$ total utility costs paid by the institution.

Structured in this way, the index will have a value of 1 when the building is completely occupied and when utility costs are fully paid by occupants. An index of 0.5 generally refers to intermediate situations, such as total occupancy but no payment of utility costs by occupants (buildings rented free of charge), or situations in which the building is only partially occupied (suggesting better strategies for organising spaces) and utility costs are not covered entirely by occupants.

\subsection{The Technical Maintenance Index $\left(I_{t m}\right)$}

The technical maintenance condition of an asset undoubtedly represents one of the fundamental elements for planning a correct valorisation strategy, even if not in absolute terms: elements such as the dimensions of the building itself (floor areas and the size of the lot), internal heights, and architectural constraints may take priority over technical maintenance requirements in relation to possible uses. In fact, for example, proceeding with an operation of valorisation through recovery and adaptive reuse to create social welfare or tourism/hospitality activities is highly improbable when building dimensions do not respect legally defined minimum standards. The same is true when future uses are incompatible with heritage listings and conservation projects.

In any case, these aspects must be carefully evaluated: prior to this process, through an initial screening that limits the sample of assets to be evaluated and identifies those best able to satisfy particular requirements; at the end of the process, they verify the congruity of proposed strategies.

Though the technical-maintenance index $\left(\mathrm{I}_{\mathrm{tm}}\right)$ expresses a plurality of parameters, an asset's current performance level can be analysed by subdividing the seven classes of needs suggested by Italy's UNI 8289:1981 standard. Furthermore, with a view toward simplification, a dichotomic variable can be proposed for each parameter related to the presence/absence of a specific requirement, evident even during a quick inspection. The following elements were analysed:

- Safety: a set of conditions relative to user safety, as well as protection and prevention against accidental damage to the operation of its technical systems. Specifically, this parameter looks exclusively at conditions of accessibility. The parameter will be set to 1 if the building is accessible and 0 otherwise.

- Well-being: a set of conditions relative to the asset's suitability to the life, health and activities of its users. Specifically, this parameter assesses the presence or absence of plant systems (mechanical, electrical and plumbing). The parameter will be set to 1 if the building is fully equipped with all systems, 0 when even only one system is absent.

- Usability: a set of conditions relative to the ability of the building system to adequately accommodate the activities of its users. Specifically, this parameter assesses aspects related to the flexibility of layouts offered by the structure; a building with a traditional frame structure 
(reinforced concrete or steel) imposes less constraints than buildings with a box-like structure (load-bearing masonry). The parameter will be set to 1 for framed structures and 0 otherwise.

- Appearance: a set of conditions relative to the perception of the asset by its users. Specifically, this parameter assesses aspects of heritage protection for listed buildings or assets declared of cultural interest. The parameter will be set to 1 if the building is of particular historical-artistic and/or architectural interest and 0 otherwise.

- Management: a set of conditions relative to the financial operation of the asset. Specifically, this parameter assesses aspects related to the presence of elements designed to limit energy consumption (PV systems, geothermal energy, insulation) resulting from recent refurbishments. The parameter will be set to 1 if the building has been refurbished and 0 otherwise.

- Integrability: a set of conditions relative to the ability to create functional connections between the units and elements of an asset. Specifically, this parameter expresses an overall assessment of the possibility to use the asset even when its individual parts may not fully respond to current regulations. The parameter will be set to 1 if the building is usable and 0 otherwise.

- Environmental Protection: a set of conditions relative to the maintenance and improvement of the state of the higher systems to which the asset belongs. Specifically, this parameter identifies eventual sources of pollution in the building (asbestos, waste abandonment, non-ionized radiation from electromagnetic fields, etc.). The parameter will be set to 1 when NO contaminants and/or waste are present in the area and 0 otherwise.

The index is expressed as follows:

$$
I_{s o}=\left\{\frac{\sum_{i=1}^{n} P_{i}}{n}\right\}
$$

- $\quad P_{i}=\mathrm{i}$-th parameter;

- $n=$ number of parameters analysed (7).

\section{Use and Interpretation of the Model}

The processing of the three indices is read simultaneously on a star (or radar) diagram that identifies three reference scenarios, three directions, and three uncertain situations for which the model is unable to provide an answer. Situations are defined as uncertain when the model is unable to provide exhaustive indications or when all three indicators share the same value (Figure 2).

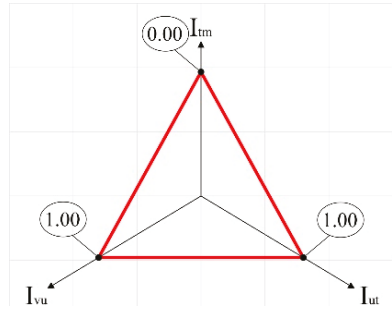

(a)

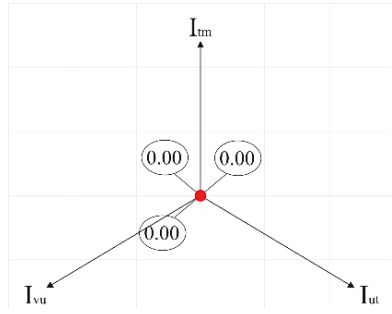

(b)

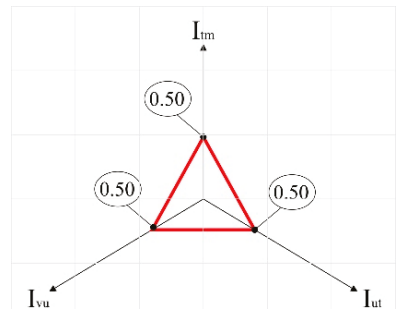

(c)

Figure 2. Situations are defined as uncertain when the model is unable to provide exhaustive indications because: (a) the three indices have a maximum value, (b) the three indices have a minimum value, and (c) the three indices have equal value. 


\subsection{Reference Scenarios}

Each of the areas defined by the reference axes identifies a strategic field in which to develop the process of valorisation (Figure 3). For greater clarity, limit situations are represented by hypothesising extreme values for the indices ( 1 or 0$)$. The following scenarios were identified:

- Reference Scenario A: An asset is located in a prestigious area, presents a poor technical maintenance level and, is occupied (occupants pay utility costs). The suggestion in this situation is to enhance the property to increase its profitability and maintain its ownership.

- Reference Scenario B: An asset is located in a prestigious area, presents a good technical maintenance level, and is unoccupied. The suggestion in this condition is to sell the asset at market value without proceeding with valorisation operations, as the property already presents good technical maintenance characteristics.

- $\quad$ Reference Scenario C: An asset is located in the area of no value, presents a good technical maintenance level, and is occupied (occupants pay utility costs). In this context, given the good condition of the asset, the suggestion is to reorganise its use to eliminate any passive leases or proceed with valorisation projects in concession for social or cultural purposes, given its location in an area that, despite a scarce offering of services, is capable of generating attractiveness and interests.

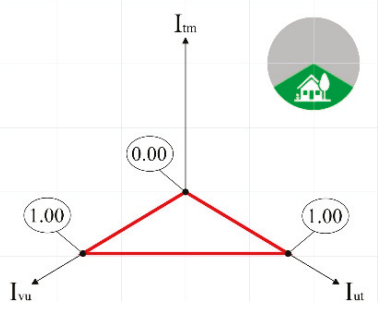

(a)

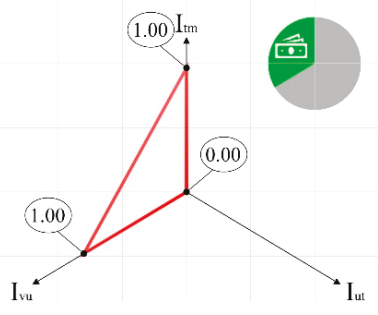

(b)

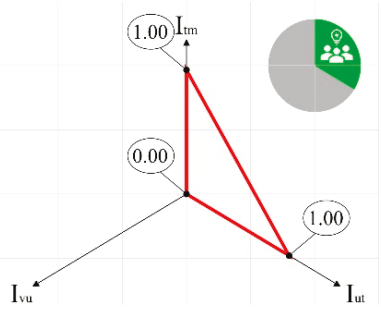

(c)

Figure 3. Representation of reference scenarios: (a) reference scenario A; (b) reference scenario B; (c) reference scenario $C$.

\subsection{Directions}

In addition to the scenarios presented above, it is possible to encounter limited conditions where only one of the three indicators prevails over the other two. In this situation, the public administration can proceed with activities to increase at least one of the other two indices to reflect one of the scenarios presented above (Figure 4). However, for a number of reasons, this is not always possible; while the indicators $\mathrm{I}_{\mathrm{tm}}$ (technical-maintenance) and $\mathrm{I}_{\mathrm{ut}}$ (use) can be modified by making small improvements to an asset or by offering incentives to rental through no-charge lease agreements, it is difficult to influence the $\mathrm{I}_{\mathrm{vu}}$ indicator (urban values) without making substantial changes to the urban setting (which require lengthy periods of time) sufficient to modify the structure and equilibriums of the real estate market. The directions identified are:

- Direction 1: An asset is located in a prestigious area, presents a poor technical-] maintenance level and is unoccupied. In this situation, it is possible to imagine Development Direction 1A: the public institution could adopt a place-based approach that involves local communities, using their knowledge, collaborating with all territorial stakeholders and promoting institutional cooperation. Another option is Development Direction 1B: the public institution may decide to invest in the redevelopment of the asset and then place it on the market (e.g., dilapidated buildings in prestigious urban contexts). 
- Single Direction 2: An asset is located in the area of no value but presents a good technical maintenance level and is unoccupied. In this context, the public institution could proceed with a reorganisation of the asset's spaces to eliminate any passive leases or decide to promote leases, free of charge or subsidised, to favour bottom-up processes that could develop into social or economic activities.

- Single Direction 3: An asset is located in the area of no value, presents a poor technical maintenance level, and is occupied (occupants pay utility costs). In this context, the public institution could invest in the asset to create the conditions required to further develop the activities already present in the building.

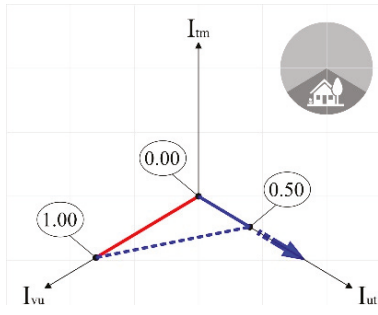

(a)

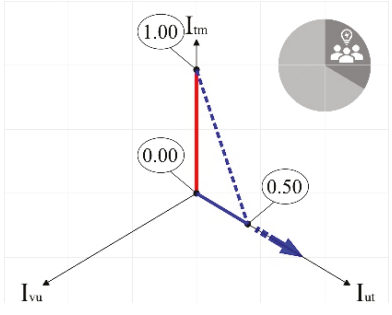

(c)

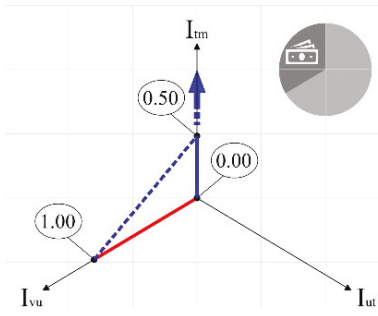

(b)

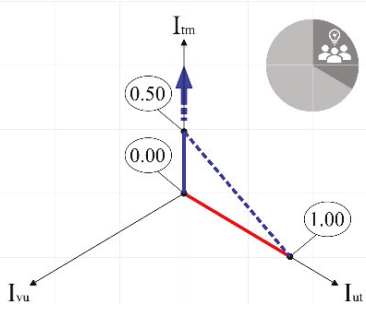

(d)

Figure 4. Representation of directions: (a) development 1A; (b) development 1B; (c) single 2; (d) single 3.

\section{Case Study}

The proposed model was applied to two public buildings in the City of Pescara (Italy): a former school building and a complex of office buildings. In one case the model proved predictive, defining the valorisation strategy eventually adopted by the municipal administration; the second, although not yet developed by the public decision-maker, has nevertheless earned the consent of the population residing in the district and could be incorporated by the public administration (Figure 5).

\subsection{Former School Building in Via Lago di Borgiano, Pescara}

This property, owned by the Municipality of Pescara, is located in Via Lago di Borgiano, near the former GTM garage. The site is situated in a public housing district between the Via Tiburtina Valeria and the so-called asse attrezzato urban highway. The building is currently home to various associations (some present under a regular free loan agreement, others illegally). One wing of the building is used as a kitchen serving the cafeterias of the neighbourhood's schools. Together, the associations and kitchen activity occupy roughly $85 \%$ of the available spaces. The costs of kitchen utilities are paid by the kitchen manager, while the utility costs of the associations are paid by the Municipality. The property is zoned D1 OMI. The indices are as follows: $\mathrm{I}_{\mathrm{VU}}=0.07 ; \mathrm{I}_{\mathrm{UT}}=0.40 ; \mathrm{I}_{\mathrm{TM}}=0.71$. 


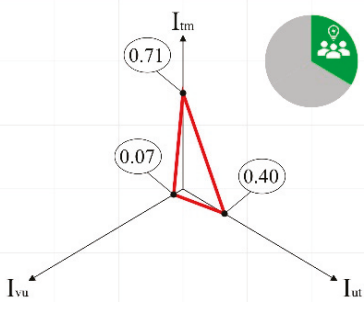

(a)

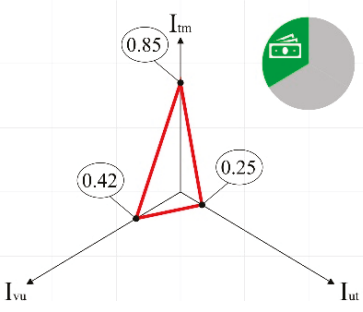

(b)

Figure 5. Reference scenarios: (a) former school in Via Lago di Borgiano; (b) offices in Via Tirino.

The model tends toward Scenario C-in this context, it is possible to hypothesize concession projects for the enhancement of social services or activities, given the location of the property in an area with a scarce offering of services, though capable of generating attractiveness and interests. The suitability of the proposal was confirmed by the neighbourhood's citizens. When interviewed about the possible solutions to be implemented, they confirmed the need for spaces and services for children, currently lacking in this densely populated area. In this scenario, a procedure could be activated to legalise occupancy, reorganise the areas assigned to the various associations, and promote, even though free lease agreements or reduced fees, the concession of spaces suitable for the construction of play areas or after-school services for children.

\subsection{Offices in Via Tirino, Pescara}

These properties are located on the ground floor of two adjacent blocks of flats in Via Tirino, near the G. d'Annunzio University campus. Constructed around the year 2000, they were sold to the Municipality of Pescara as part of an agreement with the builder. To date, they are partially unoccupied (the premises were once leased to the Pescara's ASL Health Authority) and partially occupied, under a regular contract, by the Misericordia di Pescara Srl company. The property is zoned C4 OMI. The indices are as follows: $\mathrm{I}_{\mathrm{VU}}=0.42 ; \mathrm{I}_{\mathrm{UT}}=0.25 ; \mathrm{I}_{\mathrm{TM}}=0.85$.

The model tends toward Scenario A: in this context, activities focused on selling the asset without proceeding with its valorisation are suggested, including a direct auction.

As confirmation of the hypothesis proposed by the model, it should be noted that the two buildings have already been included in Municipality of Pescara's Alienations and Valorisations Plan; the current idea is to sell the property to the university.

\section{Conclusions}

The Protocol and the model of choice represent a possible approach to the technical formalisation of the process, though without overriding the role of politics in decision making. Current literature offers no approaches of a global nature, but only experiments and case studies for individual properties or specific building types, such as barracks [38,39], buildings of historical and/or cultural value [40-43] or railway stations $[4,44]$.

The thick sediment of regulations accumulated over the past 30 years represents an obstacle to the analysis of the problem and the search for solutions. Instead of an organic corpus, it is more a of set of contingent approaches, proposed case-by-case by the legislator to confront urgent budgetary questions or remedy the failures of previous valorisation policies.

Given the conditions described above, only the most structured Institutions, with an adequate administrative apparatus, and to some degrees more farsighted and capable, can confront and govern the complex procedures underlying these processes. It is also true, however, that over the past 10 years, the technical equipment and human resources available to public administrations have proven insufficient: continuous spending cuts and turnover freezes have not allowed public bodies to 
modernise and hire new (and better trained) staff, or to acquire innovative tools capable of favouring better asset management (for example, property and facility management systems or more complex BIM systems).

This situation reaches its apex in the small municipalities that represent the vast majority in Italy: small towns, or those with a population of less than 5000 inhabitants, account for $69.83 \%$ of the total. The protocol elaborated and described here was imagined above all for these realties, precisely because, though they may suffer from an important or total lack of resources and means, they are not without legitimate needs and ambitions.

The purpose of the model of choice provided by the protocol is to guide political actors toward choices based on objective elements that, hopefully, should facilitate the identification of the most appropriate strategies. The validity of the model should be tested in a broad range of cases, beyond the comforting experiments carried out and proposed in this paper.

Author Contributions: Conceptualization, S.C. and D.S.; methodology, S.C. and D.S.; resources, S.C. and D.S.; data curation, S.C. and D.S.; writing-original draft preparation, S.C. and D.S.; writing-review and editing, S.C. and D.S. All authors have read and agreed to the published version of the manuscript.

Funding: This research received no external funding.

Conflicts of Interest: The authors declare no conflict of interest.

\section{References}

1. Art. 2, Comma 22, Law 191/2009 “Disposizioni Per La Formazione Del Bilancio Annuale e Pluriennale Dello Stato" (Legge Finanziaria 2010). Italy. 2009. Available online: http://www.edizionieuropee.it/LAW/HTML/9/ zn27_08_047.html\#_ART0002 (accessed on 20 October 2019).

2. Zirnstein, F.; Casadei, G.; Spano, F. I fondi immobiliari in Italia e all'estero - Rapporto 2019; Scenari Immobiliari: Milan, Italy, 2019.

3. Marona, B.; Van den Beemt-Tjeerdsma, A. Impact of Public Management Approaches on Municipal Real Estate Management in Poland and The Netherlands. Sustainability 2018, 10, 4291. [CrossRef]

4. Adisson, F. Clichy-Batignolles: L'autunno del regime pianificatore di Parigi. In Le Grandi Trasformazioni Urbane. Una Ricerca e un Dibattito per gli Scali Milanesi; Fondazione OAMi: Milan, Italy, 2018.

5. Mercati, L. Pubblico e Privato nella Valorizzazione del Patrimonio Immobiliare; Giappichelli: Doolin, Italy, 2009.

6. Camerin, F.; Gastaldi, F. Italian military real estate assets re-use issues and opportunities in three capital cities. Land Use Policy 2018, 78, 672-681. [CrossRef]

7. Vianello, D. Small steps to emerge from the tunnel. TECHNE J. Technol. Archit. Environ. 2012, 3, 45-54. [CrossRef]

8. Tronconi, O. La Valorizzazione del Patrimonio Immobiliare Pubblico; Franco Angeli: Milan, Italy, 2015.

9. Falanga, C.; Enzo, C.; Nasso, I. La dismissione del patrimonio immobiliare pubblico. Guida pratica per gli enti locali. La case history del Comune di Reggio Calabria; Maggioli Editore: Santarcangelo, Italy, 2013.

10. Rigamonti, B. Strategie e strumenti per la valorizzazione del patrimonio immobiliare pubblico; del Demanio, A., Ed.; Agenzia del Demanio: Roma, Italy, 2015.

11. Tronconi, O.; Baiardi, L. Valutazione, Valorizzazione e Sviluppo Immobiliare; Maggioli: Milan, Italy, 2010.

12. Alessandrini, A.; Alfonso, L.; Coruzzi, M.; De Fabritiis, F. La Nuova Comunicazione dell'Ente Locale. Organizzare l'Ascolto, l'Informazione e la Partecipazione degli Stakeholder; Franco Angeli: Milan, Italy, 2006.

13. Bruno, D.; Sarti, M. Rendicontazione e Partecipazione Sociale: Dal Bilancio Sociale al Bilancio Partecipativo. Cosa Sono E a Cosa Servono; Franco Angeli: Milan, Italy, 2009.

14. Mangialardo, A.; Micelli, E. The enhancement of public real-estate assets through participation and social innovation: Empirical data from Italy. Data Brief 2018, 21, 2379-2383. [CrossRef]

15. Piattelli, U. Il Crowdfunding in Italia. Una Regolamentazione All'avanguardia o Un'occasione Mancata? Giappichelli: Doolin, Italy, 2013.

16. Pais, I.; Peretti, P.; Chiara, S. Crowdfunding. La Via Collaborativa All'imprenditorialità; EGEA: Milano, Italy, 2018.

17. Meola, A. Il ruolo e le funzioni innovative degli amministratori pubblici locali nell'era dei social network. Sindaco, Giunta, Consiglio e Menagement: Le "locomotive". In Chiamati a Servire il Bene Comune. Vocazione, Cura e Impegno Civile; Di Bernardo, M., Ed.; Franco Angeli: Milan, Italy, 2012. 
18. Gagliardo, E.D. L Sistema Multidimensionale di Programmazione a Supporto della Governance Locale; Giuffrè: Milan, Italy, 2007.

19. Reviglio, E. La Dismissione del Patrimonio Immobiliare Pubblico: Una Grande Opportunità per il Paese; Istituto Bruno Leoni: Milan, Italy, 2008.

20. Bertolinelli, M.; Guzzoni, L.; Masseroni, S.; Pinti, L.; Utica, G. Innovative Participatory Evaluation Processes: The Case of the Ministry of Defense Real-Estate Assets in Italy. In Seminar of the Italian Society of Property Evaluation and Investment Decision; Springer: Cham, Switzerland, 2019; pp. 547-557. [CrossRef]

21. Boeri, T.; Pisauro, G. Patrimonio Pubblico: Basta con l'Ingegneria Finanziaria. 2011. Available online: https://www.lavoce.info/archives/27265/patrimonio-pubblico-basta-con-lingegneria-finanziaria/ (accessed on 20 October 2019).

22. Artioli, F. Le aree militari nelle città italiane: Patrimonio pubblico e rendita urbana ell'era dell'austerity e della crisi. La Rivista delle Politiche Sociali/Ital. J. Soc. Policy 2016, 1, 89-113.

23. Manganelli, B.; Tajani, F. Un modello di gestione per la valorizzazione di immobili pubblici extraordinari. Valori e Valutazioni. Teorie ed Esperienze 2014, 12, 139-153.

24. Bellè, M.B. Promesse e fallimenti nella valorizzazione degli immobili pubblici. Sci. Regional. Ital. J. Reg. Sci. 2017, 3, 463-472. [CrossRef]

25. Forte, F.; Torre, C.M. Città multietnica. Urbanistica e valutazione. Aestimum 2009, 32, 558-576. [CrossRef]

26. Bobba, L. A Enti Terzo Settore Beni Pubblici Inutilizzati o Confiscati a Mafie. 2017. Available online: https://www.adnkronos.com/lavoro/norme/2017/11/28/bobba-enti-terzo-settore-beni-pubbliciinutilizzati-confiscati-mafie_9JVUHXMdbb68RudALCAs0K.html (accessed on 29 March 2017).

27. Wilson, J.Q.; Kelling, G.L. Broken Windows: The Police and Neighborhood Safety. Atl. Mon. 1982, 249, 29-38.

28. Mollica, E.; Massimo, D.E. Valutazione degli strumenti del territorio e strumenti GIS. Aestimum 2002, 32, 710-757. [CrossRef]

29. Micelli, E.; Mangialardo, A. La partecipazione crea valore? Modelli di simulazione per la valorizzazione dal basso del patrimonio immobiliare pubblico. Valori e Valutazioni. Teorie ed Esperienze 2017, 19, 41-52.

30. Van der Aalst, W.M.P. Process Mining: Discovery, Conformance and Enhancement of Business Processes; Springer: Berlin/Heidelberg, Germany, 2011. [CrossRef]

31. Carbonara, S.; Stefano, D.; Di Ceglie, R.; Curcetti, S. Il censimento del patrimonio immobiliare pubblico della città di Pescara. In Versopescara2027. Vision e summer school; Spacone, E., Ed.; Gangemi: Roma, Italy, 2016.

32. Carbonara, S.; Stefano, D. Transforming surface rights into property rights: An analysis of current estimation procedures and a comparison with an alternative. Valori e Valutazioni. Teorie ed Esperienze 2020, 24. Soon to be published.

33. Della Spina, L.; Calabrò, F. Decision Support Model for Conservation, Reuse and Valorization of the Historic Cultural Heritage. In Proceedings of the Computational Science and Its Applications-ICCSA 2018. Lecture Notes in Computer Science, Melbourne, Australia, 2-5 July 2018; Volume 10962, pp. 3-17. [CrossRef]

34. De Medici, S. Nuovi Usi per la Tutela e la Valorizzazione del Patrimonio Costruito. La Privatizzazione dei Beni Immobili Pubblici; Franco Angeli: Milan, Italy, 2010.

35. Della Spina, L. Historical Cultural Heritage: Decision Making Process and Reuse Scenarios for the Enhancement of Historic Buildings. In Proceedings of the New Metropolitan Perspectives. ISHT 2018. Smart Innovation, Systems and Technologies, Reggio Calabria, Italy, 22-25 May 2018; Volume 101, pp. 442-453. [CrossRef]

36. Vandevyvere, H. From Scoring To Orienting: The Development OfA Compass For Evaluating Sustainable UrbanDevelopment At The Neighbourhood Scale. In Sustainable Development and Planning V; WIT Press: Southampton, UK, 2011; Volume 150, pp. 153-164. [CrossRef]

37. Carbonara, S. The Effect of Infrastructural Works on Urban Property Values: The asse attrezzato in Pescara, Italy. In Computational Science and Its Applications - ICCSA 2012; Murgante, B., Gervasi, O., Misra, S., Nedjah, N., Rocha, A.M.A.C., Taniar, D., Apduhan, B.O., Eds.; Springer: Berlin/Heidelberg, Germany, 2012. [CrossRef]

38. Gastaldi, F.; Camerin, F. Processi di dismissione degli immobili militari. Temi e problemi aperti per la rigenerazione urbana in Italia. Sci. Regional. 2017, 1, 103-120. [CrossRef] 
39. Carbonara, S.; Stefano, D.; Torre, C.M. The Economic Effect of Sale of Italian Public Property: A Relevant Question of Real Estate Appraisal. In Lecture Notes in Computer Science; Murgante, B., Misra, S., Rocha, A.M., Torre, C.M., Rocha, J.G., Falcão, M.I., Taniar, D., Apduhan, B.O., Gervasi, O., Eds.; Springer: Cham, Switzerland, 2014; pp. 459-470. [CrossRef]

40. Stanghellini, S. Valore dei beni e beni di valore:un contributo sulla valutazione dei beni immobili militari. Aestimum 2003, 43, 45-53. [CrossRef]

41. Leon, P. Valorizzazione del patrimonio storico-artistico. In Economia della Cultura, Rivista trimestrale dell'Associazione per l'Economia della Cultura; Il Mulino: Roma, Italiy, 2017; pp. 341-352. [CrossRef]

42. Della Torre, S. Una strategia di valorizzazione dei beni e della attività culturali. In Distretti Culturali: Dalla Teoria alla Pratica; Bologna, G.P., Barbetta, M.C., Della Torre, S., Eds.; Il Mulino: Abruzzo, Italy, 2013.

43. Giani, F. The ecclesiastical property assets: An analysis for its valorisation for social purposes. In XIV Congresso internazionale di riabilitazione del patrimonio. La conservazione del patrimonio artistico, architettonico, archeologico e paesaggistico; Porcari, V.D., Ed.; Luciano Editore: Napoli, Italy, 2018.

44. Oppido, S. La valorizzazione diffusa: il riuso del patrimonio ferroviario dismesso. BDC. Bollettino Del Centro Calza Bini 2014, 14, 221-235. [CrossRef]

(C) 2020 by the authors. Licensee MDPI, Basel, Switzerland. This article is an open access article distributed under the terms and conditions of the Creative Commons Attribution (CC BY) license (http://creativecommons.org/licenses/by/4.0/). 
Article

\title{
Strategic Planning and Management Model for the Regeneration of Historic Urban Landscapes: The Case of Historic Center of Novi Pazar in Serbia
}

\author{
Esad Muminović ${ }^{1}$, Uroš Radosavljević ${ }^{2, *}$ and Džemila Beganović ${ }^{1}$ \\ 1 Department of Technical Sciences, State University of Novi Pazar, Vuka Karadžića 9, 36300 Novi Pazar, \\ Serbia; arhesad@gmail.com (E.M.); dzbeganovic@np.ac.rs (D.B.) \\ 2 Department of Urbanism, Faculty of Architecture, University of Belgrade, Bulevar kralja Aleksandra 73/II, \\ 11000 Belgrade, Serbia \\ * Correspondence: yros@arh.bg.ac.rs
}

Received: 21 December 2019; Accepted: 7 February 2020; Published: 12 February 2020

\begin{abstract}
The purpose of this paper is to identify the role of strategic planning as a sustainable tool for regulating both the protection and development of historic urban landscapes, as well as developing an adequate and effective strategic model and management instruments for implementation. The role and importance of strategic planning are examined in the context of global transformative actions in the urban governance of community and private sector engagement and sustainable development on the local level. We argue that a specific-tailor-made—integrated strategic urban planning approach could be a useful model, both for development and urban regeneration and for the preservation of protected valuable historic urban landscapes, thus contributing to a sustainable urban revival of wider surrounding territories including cultural, social and economic development. We stand on the position that the sustainable approach to the protection and revitalization of the historic urban landscapes has to be in line with the acknowledgment of specific local community values, contemporary needs, their involvement, and, eventually, their satisfaction. The case study method was based on the example of a protected historic center of Novi Pazar in Serbia to test the possibilities of applying strategic planning model and management for the implementation tailored to the local context. Eventually, the scenario method was applied to test the possibilities of a simulation of the strategic planning model and management instruments for a protected historic center. We found that the appropriate combination and utilization of regulatory, economic and informational management instruments have to be in place in the specific context. We conclude and draw out theoretical and practical remarks from our research that integrated strategic urban planning model should consider the logic and the functioning of the competitive real estate markets, and the sustainable environmental, economic and social effects, potentials and benefits for the locality where they originate, in order to be utilized as the new generative value both for the protection and for the revival of historic city centers. The paper develops a conceptual strategic planning and management model for the regeneration of historic urban landscapes that capture the physical, environmental, economic, and social effects and indicators of a given space. Based on this input, an adequate initial stage of the conceptual strategy by the authors of the paper was developed for the regeneration of the historic urban city center.
\end{abstract}

Keywords: strategic planning; historic urban landscape; model; management instruments; Novi Pazar; Serbia

\section{Introduction and Research Methods}

The protection of architectural heritage within historic urban landscapes is a complex area that includes basic protection-research, valorization, legal protection—as well as protection through planning activities and documentation. 
The nature of the problem of treatment of protected historic urban landscapes is the fact that urban development and the preservation of architectural heritage in Serbian conditions are seen as the two opposing concepts, while in most Western European countries these two terms represent two sides of the same coin [1]. Part of the professional public often resents the traditional approach of protection and preservation of architectural heritage which hinders and slows down urban development, while, on the other hand, the pressure of development is often perceived as a threat to the survival of cultural heritage $[2,3]$. However, although there is an opinion that heritage preservation impedes the development of historic urban landscapes, a number of authors point out the role of architectural heritage as a driving force of economic and social development. Tweed and Sutherland [4] emphasize the contribution of cultural heritage in the development of local communities and in meeting human needs. Based on the examples of several cities which base their development strategy on cultural heritage values, Evans [5] points out the positive effects of regeneration and planning interventions in historic urban areas. Current research indicates that cities with cultural heritage have a soft advantage over the competition in the global market [4], which gives them a unique identity.

\subsection{Historic Urban Landscapes Interlinked with Strategic Spatial Planning}

The widespread understanding and appreciation of architectural heritage in recent decades, coupled with the increasingly complex ways of identifying, protecting, preserving and presenting it, has significantly increased the complexity of planning and managing historic urban landscapes. It is clear that a time of enormous uncertainty imposes the need to consider various possible planning patterns that could be continuously adapted to change. Many communities with cultural heritage face problems such as declining real estate values, economic downturns, and leaving the area. In order to preserve life in historic urban landscapes, we need to constantly change our approach and practice, because preserving historic urban landscapes today goes beyond the traditional sectors defined in economic, social and political systems. The consequences of mismanagement are often severe, and not only do they risk the destruction of a valuable architectural heritage, but they often threaten the socio-economic development of the local community.

Cities, including their historic urban landscapes, are at present the focus of economic, social and cultural activities, in which a diversity of interactions creates new initiatives and ideas. To preserve their vitality, historic urban landscapes need to be renewed through a planning approach based on modernization, adaptation and regeneration, while retaining entire complexes, or at least individual buildings, that give the city its character, identity and competitiveness. For these reasons, it is necessary to develop new strategies that can anticipate the complexity of change and create an adequate development and planning platform to act in the new, dynamic ambient [2].

In this sense, in recent decades, a new approach to heritage management has been developed worldwide that treats cultural and historic entities as urban landscapes and became more interlinked with spatial planning in general, and strategic planning in particular. This approach has been promoted by the United Nations Educational, Scientific and Cultural Organization (UNESCO) and International Council on Monuments and Sites (ICOMOS) regarding the integration of historic urban areas. Specific areas of integration and links between heritage management of urban landscapes and spatial planning have been strengthened in several characters and recommendations. As a result, the Nairobi Recommendations [6] brought several planning instruments for the regeneration of historic urban centers and a range of public, community organizations, experts and citizens as stakeholders, while the Washington Charter [7] introduced an integration of economic, cultural and social development policies and urban planning related to urban heritage conservation projects and urban regeneration. Eventually, historic urban landscape as a term was defined in the Vienna Memorandum [8], bearing in mind broader territorial context as the new method for heritage conservation and urban regeneration, and comprising assemblages of buildings, structures and open spaces, thus going beyond the traditional historic centers definition. 
Finally, Recommendations on the Historic Urban Landscape [9] envisioned that both urban heritage protection and its integration in local sustainable development using spatial planning could contribute to urban identity in the fundamentally changed globalization environment, traditional mass tourism, heritage exploitation, and climate change. The Recommendations recognize that the city is a dynamic organism influenced by economic, social, and cultural forces, and thus use the existing physical fabric, cultural diversity, and local community values integrally as assets in promoting sustainable goals of urban heritage conservation, social, environmental, and economic development. Therefore, this approach focuses on the specifics of local situations in the search for a balance between the protection of architectural heritage, and the economic development, functionality and vitality of the city. Furthermore, it responds to the present needs of residents while improving the city's natural and cultural resources for future generations, which is entirely in line with the notion of sustainability. Above all, it is assumed that the protection and presentation of heritage, economic, environmental and socio-cultural development goals are not conflicting but complementary, and that their long-term success depends on the ways that they are interconnected. An especially important aspect of the Recommendations on the Historic Urban Landscape for this particular research is the call for a range of traditional and innovative regulatory and legislative measures, as well as civic engagement and financial instruments which have to be locally adjusted.

On the urban level, the Recommendations on the Historic Urban Landscape [9] provide principles and guidance for implementing a comprehensive approach to local urban management. These recommendations indicate that the integration of heritage conservation and urban development is a major challenge at the urban level, as it is a very complex process that needs to be addressed at multiple levels [2].

The management of the historic urban landscapes is even more complex because, in addition to concepts related to intangible heritage, it has to include the management of complex social and economic processes [5,10]. This approach is holistic and aims to integrate architectural heritage management with a broader socio-economic development framework. This integration applies both to the vertical integration of heritage and non-heritage sectors in institutional terms, as well as to the horizontal integration of the different levels of related urban development and protection policies.

Integrating strategic urban planning with cultural planning is an approach that recognizes the unique value of the architectural heritage, but also the contemporary need for the development of communities related to historic landscapes. The architectural heritage should be seen as a vital ambient for both residents and visitors, especially in the light of the fast-growing cultural tourism. Regarding cultural tourism for increasing the attractiveness of tourist destinations, tourists are becoming more demanding in a globalized world with growing competition and, therefore, the management of any destination should strive to meet the multiple motivations and interests of tourists [11].

The preservation and enhancement of historic heritage are mostly dependent on a formulated local strategy for the protection and enhancement of historic urban landscapes. The strategy should clearly define the overall objectives, which should be based on the principles of sustainability, efficiency, fairness and transparency. The strategy needs to identify existing resources, both material and human resources. Following such a comprehensive analysis, concrete measures need to be developed in order to implement formulated objectives. What is specific to the historic urban landscapes, in relation to other cultural assets, is their dual character. First of all, this is the practical function of the buildings which represent part of the city, in most cases their most significant part, and the cultural functions that the objects themselves possess. Due to this specificity, in addition to the general strategy for the protection of cultural heritage at the national level, it is necessary to develop an integrated urban development strategy at the local level, whose essential aspect would be the preservation and enhancement of historic urban landscapes. 


\subsection{Strategic Plans for the Development of Historic Urban Landscapes}

Since the city is an arena where the fields of action of different sectorial strategies intertwine in the face of constant changes, an integrated strategic concept is proposed in this paper as an adequate planning model. Integrated strategic urban development planning in recent years, regardless of variations in economic structure and population, has been established as standard across Europe. It is based on a model of a spatially compact, mixed-use, socially and culturally integrated city in which the sustainable development conditions are met. This planning model was proposed at an informal meeting of European Ministers of Urban Development, held in the German city of Leipzig in 2007. The main objective of such planning is to pay equal attention to the economic, ambient, social and cultural dimensions through urban development. Planning should find a technical answer and a politically responsible balance between these areas of activity. Urban development planning should provide equal opportunity for various and different social and age groups through the participation of citizens and for the fulfillment of public interest.

The advantage of strategic versus traditional urban planning is reflected in several aspects. The choice of strategic objectives is more reliable due to the hierarchy of strategic planning, which allows the objectives to be defined at higher and implemented at lower levels. In the strategic planning process, the analysis of the situation and the creation of the framework are carried out within the framework of collaborative decision making, and therefore the relationship between local government officials and the public is far more transparent. Strategies should link the different sectors that affect each other and which, with the specific knowledge of experts and stakeholders, should agree on common goals. Of course, there are difficulties in reaching consensus when sectors are representing different interests [12].

A spatial dimension would be a key component for integrated urban development strategies for historic urban landscapes. For each part of the city, the specific integration of sectoral policies that are often interest-oriented would be specifically ensured. In an integrated approach, different thematic areas, levels of government, resources and other actors should be linked. In order to develop this strategy, coordination between different levels of government is needed, facilitated by communication with citizens, the participation of the local economic players, representatives of public institutions and other relevant actors in the planning and implementation of projects and activities. This strategy is expected to improve the process of urban planning and development by linking various institutions and the resources that can be provided. It should be based on the demographic and social parameters of development to ensure the preservation and improvement of quality of life, as well as ensuring social stability [13].

\subsection{Materials and Methods}

We used theoretical research and a literature review from the disciplines of cultural heritage, urban management, and planning for the heritage protection strategies, and experiences in managing protected areas in order to interlink those fields in the introductory part of the paper.

In the second chapter of the paper, we presented a conceptual strategic planning and management model for the regeneration and protection of the historic city centers.

In the third chapter of the paper, we presented the urban planning and governance context in relation to the preservation of protected heritage in Serbia. We have analyzed various types of documentation—reports, plans, documents, and personal experience of dealing with protected heritage and strategic planning.

In the fourth chapter, the strategic planning and management model for the regeneration of historic urban landscapes was tested by a case study method using the example of a protected historic center-Novi Pazar in Serbia. Yin [14] states that the case study method can be useful for more in-depth and comprehensive research for the analysis of a unique situation, like the one in this paper. Justification of a case study method is also underlined by Flyvbjergs [15] since it comes closer to real-life situations. Besides various local government reports, plans, and documents, we used interviews 
conducted with citizens, shop owners, and employees at the Institute for Urbanism and Planning of the City of Novi Pazar and Regional Institutes for the Protection of Monuments to gain their judgment on existing urban heritage values. Their value judgment was used as a starting point for an adequate initial stage of the conceptual strategy, which was done by the authors of the paper for the regeneration of historic city center in order to test the possibilities of applying strategic planning model and policy instruments for implementation tailored to the local context.

In the fifth chapter, the initial stage of the conceptual strategy and management model for the regeneration and protection of the historic center of Novi Pazar was prepared by the authors of the paper as a simulation for the future strategy. The process of the involvement, relation, and organization of different actors is involved in the model as an integral part of goals' and objectives' formulation.

In the concluding chapter of the paper, the sustainability indicators of regeneration strategies for historic city centers are provided in the Conclusion section of the paper. We found that the appropriate combination and utilization of regulatory, economic and informational management instruments have to be in place in the specific context. Additionally, the final section of the article builds on the preceding arguments to consider more directly the implications of the results for the theory and practice of strategic planning and the protection of historic urban landscapes.

The purpose of this paper is to identify the role of strategic planning as a sustainable tool for regulating both the protection and development of historic urban landscapes, as well as for developing an adequate and effective strategic model and management instruments for implementation. The goal is to capture the physical, economic, social and environmental indicators and effects of a given space.

\section{Conceptual Strategic Planning and Management Model for the Regeneration and Protection of the Historic City Centers}

With the purpose of developing the conceptual strategic planning and management model for the regeneration and protection of the historic city centers in this paper, we will use and adapt the theoretical model of urban management in the implementation of strategic projects in Serbia-developed and tested by Radosavljević [16], as shown in Figure 1. The model was tested on several case studies of urban regeneration through large-scale strategic projects and transformative actions in urban management, planning, and governance. Thus, urban management of sustainable urban projects in the model [16] is formulated in a threefold way:

1. As a specific combination of urban governance policy goals and instruments as different strategic options for the regeneration;

2. As a process of involvement, relations, and organization of different actors with often-conflicting interests, values and goals;

3. The range of indicators and effects that an actor's decisions and actions have on sustainability in terms of space/environment, society, and economy.

The purpose of adapting the model of urban management in the implementation of strategic projects [16] to heritage aspects is to make it relevant for the regeneration and protection of the historic city centers. The conceptual strategic planning and management model for the regeneration and protection of the historic city centers proposed in this paper (shown in Figure 2) consists of structural elements based on the aforementioned concepts of urban governance, strategic planning and participatory planning, and the protection and development of cultural heritage and historical urban landscapes.

The structural elements of the conceptual strategic planning and management model for the regeneration and protection of the historic city centers proposed in this paper (shown in the Figure 2) are as follows:

1. Spatial, Social, and Economic Context and the Level of Obsolescence and Dynamics of Historic City Centers;

2. Process of Involvement, Relations, and Organization of Different Actors; 
3. Formulating Strategic Options for the Regeneration of the Historic City Centers;

4. The Sustainability Indicators of Regeneration Strategies for Historic City Centers.

We argue that a specific — tailor-made-integrated strategic urban planning approach could be a useful model for the development and urban regeneration and the preservation of the protected valuable historic urban landscapes. Consequently, creating synergies between development and protection could contribute to the sustainable urban revival of wider surrounding territories, including the cultural, social and economic development of both the historic city center and the city as a whole. In the remainder of this chapter, we will present the structural elements of the conceptual strategic planning and management model for the regeneration and protection of historic city centers.

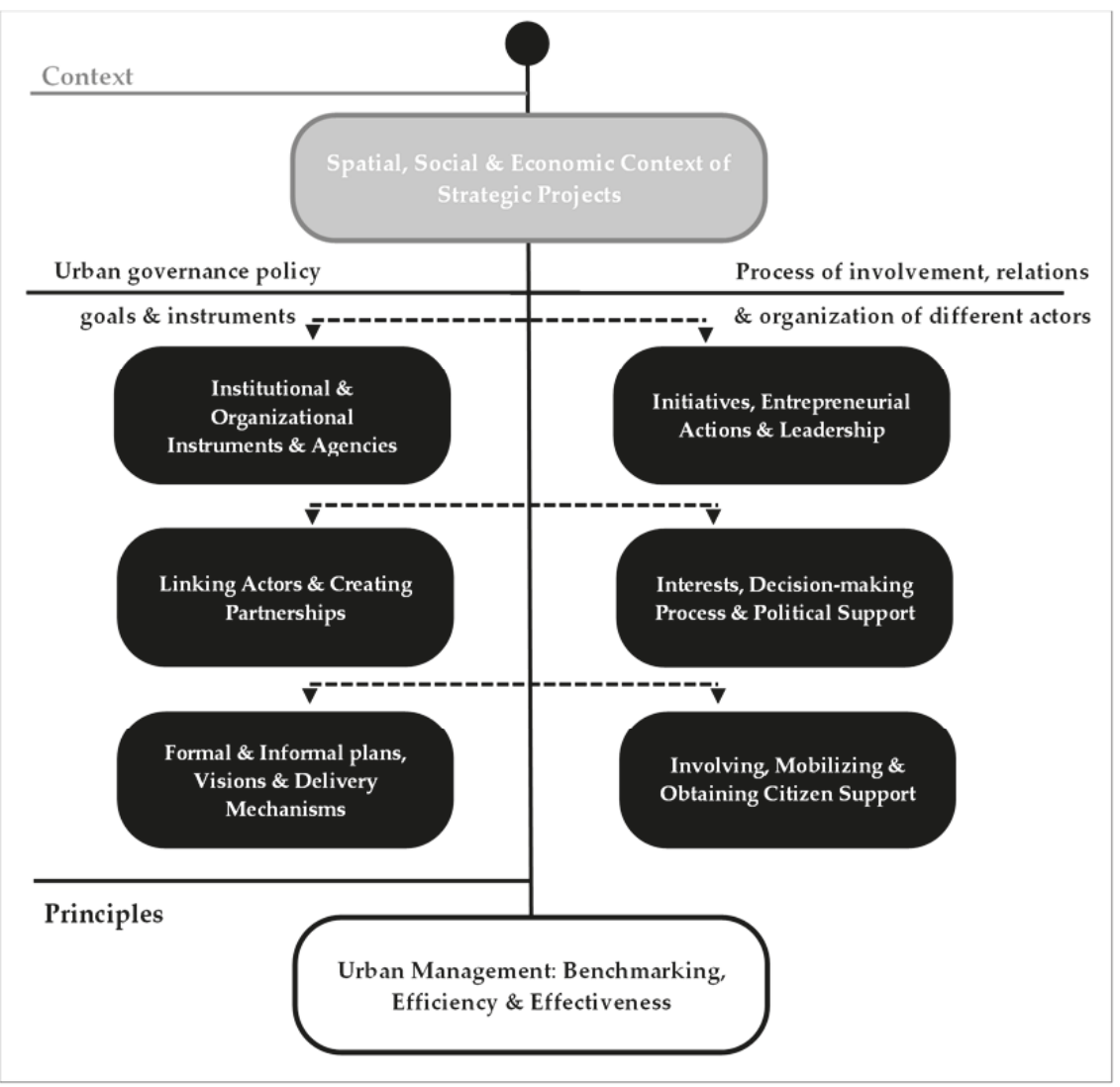

Figure 1. The Model of Urban Management in the Implementation of Strategic Projects in Serbia [16].

\subsection{Spatial, Social, and Economic Context and the Level of Obsolescence and Dynamics of Historic City Centers}

Fundamental changes in society in general, such as globalization and a transformation towards an information and network society and economy [17], as well as economic changes in western countries with the decline of traditional manufacturing industries, caused cities to shift their role from centers of production to centers of consumption [18]. Under those circumstances, historic city centers, regarding their spatial, functional, and physical structure, could not respond anymore to their new, contemporary needs and development dynamics.

Thus, regarding the protection and the development of cultural heritage and historic urban landscapes, Doratli [19] highlights that the proper diagnosis of two contextual characteristics during 
analyses plays a crucial role, even before deciding on the most appropriate strategic approach for the revitalization of historic urban quarters:

1. The level of obsolescence of physical, functional and locational characteristics;

2. The dynamics of the place.

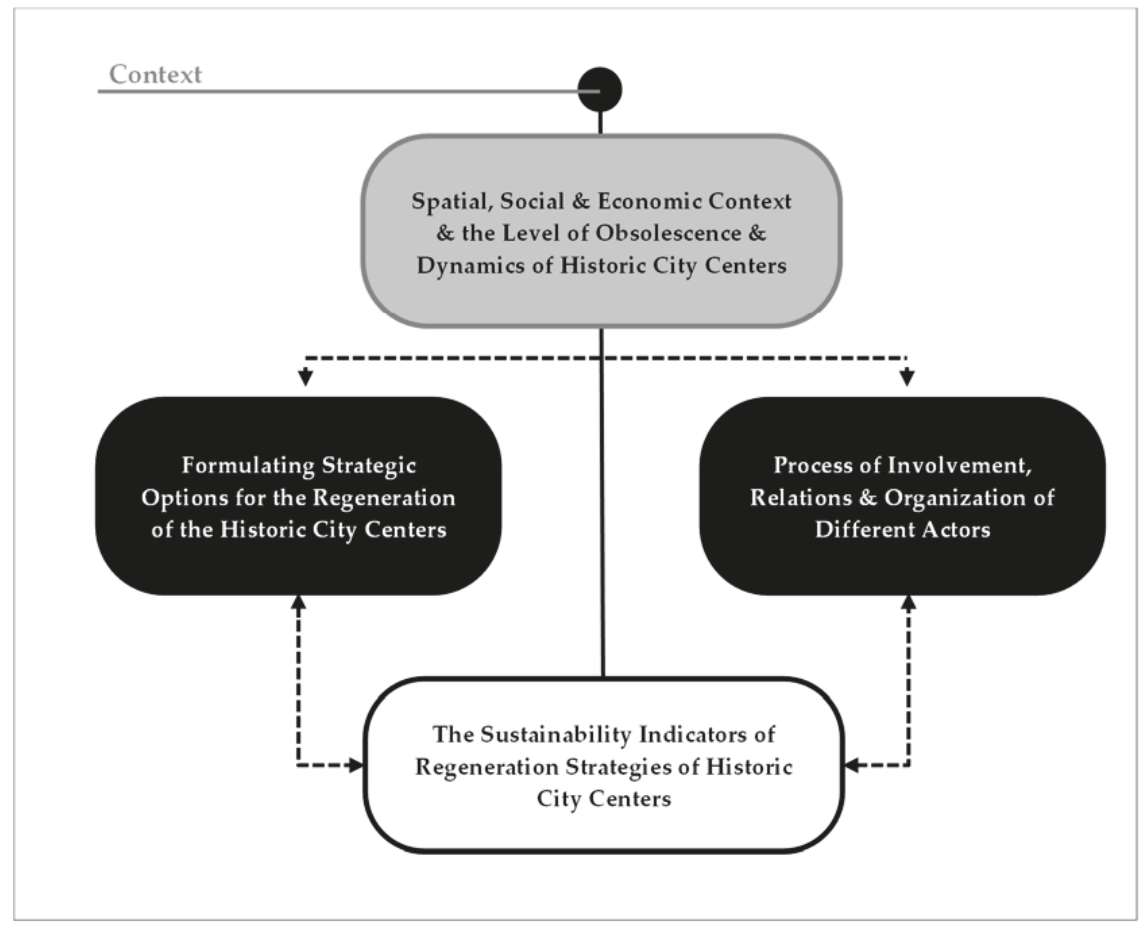

Figure 2. Conceptual Strategic Planning and Management Model for the Regeneration and Protection of Historic City Centers.

The level of obsolescence of historic city centers is directly related to the deterioration of the whole quarter, which usually has narrow streets and buildings used for less suitable purposes, in relation to the contemporary needs of standardization, traffic and energy efficiency and socio-economic, cultural, and political conditions. Thus, an investigation should be made to enlist the buildings in poor overall and sanitary conditions; scarce parking spaces; land uses in general, incompatible land uses, and which of the uses have left for more favorable city districts; changes in the ownership patterns; and surveys on tenure, property values and rent [19].

More specifically, according to Tiesdell, Oc, and Heath [18], the different aspects of obsolescence that have to be considered are:

- Physical and structural obsolescence of the building or an area, which happens with the impact of time, weather, traffic, or through reduced maintenance. Hence, the building needs refurbishment of the physical condition in order to be occupied and functional;

- Functional obsolescence, which might happen when the building is not suitable anymore for its planned function or current utilization by contemporary standards. The same goes for an entire historic area if it cannot comply with parking requirements on the site or in the surrounding streets, or if accessibility problems exist due to narrow streets and street network according to contemporary mobility and accessibility requirements; 
- Image obsolescence, which contributes to the perception and the level of unattractiveness of an area, regarding the uncomfortable traffic flow, noise, smell, and vibration in historic quarters. Such areas become unfashionable due to the lack of contemporary standards and fashionable image. Nevertheless, values change over time and older buildings might be more desirable due to the values of permanence and traditional expectations making deliberate image reconstruction an important element of the revitalization strategy;

- Legal obsolescence, which happens when a local jurisdiction determines new health, safety, fire or building control standards that make the building obsolete, or because the zoning ordinance for the area permits a larger building than the actual one;

- Locational obsolescence, which rests on the functional activities within the area regarding the original central city position of a historic area, where its location was determined in terms of accessibility to other land uses, markets, and transport infrastructure. In present times, due to contemporary polycentric development dynamics and the migration of the central business district, the area becomes unfavorable and obsolete for those activities due to the position of a particular location and shifting of accessibility patterns;

- Financial obsolescence, in which a good or service is no longer desired, especially when a new, better good or service becomes available. Specifically, regarding historic areas, this means that real estate does not have economic value and cannot be used for taxation-it makes the asset disposable. Hence, an issue for regeneration is to make real estate a renewable capital asset instead of a wasted one;

- Relative or economic obsolescence of the historic areas, which is a concept in relation to other buildings and areas, meaning that if the investments in the historic city center are higher than alternative investments in more attractive areas elsewhere, the area is economically obsolete.

The level of obsolescence of historic city centers can be in a close relationship with the economic development dynamics of the area itself and external dynamics of a whole city. These development dynamics, as argued by Doratli [19], can be in a:

- High state of development dynamics, where, although facing physical or functional obsolescence, the area is attractive for investment, with a range of existing employment opportunities and potentially high land values due to property market development pressures to demolish old buildings;

- Static state of development dynamics whilst facing physical or functional obsolescence, since there is neither economic pressure for the development nor for the demolition of historic buildings;

- Without development dynamics, meaning the area is declining in physical, social and economic terms, usually being a slum, or former industrial buildings and warehouses from 19th century urban industrial quarters used for manufacturing, production, and transportation.

\subsection{Process of Involvement, Relations, and Organization of Different Actors}

The public authorities around the world have led, in most cases, the revitalization projects of historic urban quarters in the last two decades of the 20th century-especially the physical component of regeneration-through which the quality of the built environment has been upgraded [19]. However, the preservation and conservation of historic city centers could not occur merely at the public expense [18], thus opening opportunities for the involvement and participation of the private and civil sector. Furthermore, the participation of new social and economic actors is demanded in the governance and urban management of historic quarters, which requires the coordination of multiple actors guided primarily by public institutions and the private sector [20].

The issue in urban regeneration became how urban governments actually stimulate the private sector to invest in public policies and programs [21], beyond the traditional role of the public sector through regulations and the provision of infrastructure. Local governments have, in general, two possible options, according to Vedung [22]: 
- Financial incentives to improve the conditions of actors or reduce the expenditures and risk actions;

- Financial disincentives to depress the condition of actors or to increase the costs and risks of actions.

There are several viable options for urban governments to provide economic incentives for private sector participation, such as different property tax exemptions; or the transfer of development rights from protected areas to new developments, which represent the transfer of floor space indexes that promote the efficient reuse of historic buildings; or as in-kind subsidies of the public sector as a reduction in building land development fees for land uses enabling tourism, cultural tourism, or adaptive reuse of protected buildings [23].

Another possible option in urban regeneration programs for historic areas is creating public-private partnerships. Public-private partnerships are a contractual agreement between a public agency and a private sector, in which the skills and assets, as well as risks and rewards, of each sector are shared in delivering a service or facility with the future benefit of public use [24]. In most cases of heritage protected areas projects, the private sector is not interested in participating in the beginning since there is a gap between the costs of renovation and the value of the property itself. Thus, public sector incentives should be attractive in order to attract private capital and to improve the overall investment environment that will reduce the risk and cost of investing and result in an increase of revenues. According to Rypkema [24], the physical improvement of the surrounding areas and the protected area itself, and the level of public services, can be a critical activity for private sector involvement in the regeneration projects. Public sector commitments to renew infrastructure, streets, and parking systems are often not sufficient to attract the private sector, although they might be a public partner's obligation in joint agreements. In order to increase the probability of the success of such projects, local authorities often adapt the local regulation of land use, zoning, and parking requirements according to the requirements of private partners.

Regarding citizen participation, they are increasingly involved in public life, policymaking, and project implementation at the local level. According to Radosavljević [16], participation can ensure the legitimacy of policies and projects, secure the public interest and introduce new creative ideas, while providing acceptance of and support for decisions made. The degree of involvement in and instruments to mobilize citizens and civic associations varies depending on the specificity of the cultural and social context, political will, and the importance and size of the projects and policies. In this regard, the various degrees of citizen participation refer to variations between:

- Information and communication through the media and other sources of information;

- Consultation, where citizens give their opinions on policies and projects;

- Participation in planning, where citizens participate in projects;

- Participation in decision-making, where citizens have a direct influence on decisions.

Therefore, the whole process of creating strategic urban projects should be transparent and communicate with the public, in order to obtain broad social support from citizens. Broader social support is possible through forums and public debates, live project presentations and the web, etc. [25].

Nevertheless, citizen participation can be controversial, particularly when the cultural heritage protection agencies inadequately perform their functions, usually due to private sector economic pressure, which generally opposes the sustainability and conservation criteria. Azpeitia Santander, Azkarate Garai-Olaun and De la Fuente Arana [20] criticize the involvement of social participation processes in the field of cultural heritage, and thus underline two different types that can be observed:

1. Developing artificial participation reduced to including actors without decision-making powers, only providing symbolic levels of participation in public hearings;

2. Participatory processes with adequate capacity to influence the outcomes of the process that require an in-depth prior debate. 
However, in the case of the citizen participation processes, we have to distinguish that, in the field of cultural heritage, differentiation has to be made concerning any other type of participation in planning and urban design. For instance, in the mid-ring of the city, in which new business or housing schemes make planning efforts easy for city governments and few developers, without strict conservation policies [18]. The same goes for peripheral city locations in which the revitalization and upgrading of depressed areas and public spaces pose elementary and less complicated tasks for city governments. The participation in central historic areas is influenced by contexts with various historic layers and often conflicting interests of different, mainly real-estate property market actors, local business and residents. Thus, participation has limited effectiveness regarding cultural assets in the case of the complex needs of the protection heritage values [20].

\subsection{Formulating Strategic Options for the Regeneration of the Historic City Centers}

Contemporary urban governance and planning considers the visions of development and strategies formulated with the involvement of main stakeholders in the context of understanding market operations, beside classical formal urban plans, land use and regulatory instruments.

In cases of historic urban quarters, Tiesdell, Oc, and Heath [18] argue that the economic problems of historic urban quarters are related to the topics of attracting private investment and encouraging dynamic growth. This is exactly why revitalizing historic urban quarters comprises strategies for the renewal of the physical fabric and the active economic utilization of buildings in historic city centres.

Thus, different aspects of revitalization can be recognized concerning the aspects of sustainability, which, according to Tiesdell, Oc, and Heath can be defined as [18]:

- $\quad$ Physical revitalization of the fabric, which is the adaptation of the physical fabric to contemporary standards using different policy options and instruments of renewal;

- Economic revitalization activities within the fabric of historic city centres, since the private realm and economic activities within the buildings compensate in the long-term for the maintenance of the public realm, including open spaces, thus providing the area with a competitive advantage over other city locations;

- $\quad$ Social public realm of the historic quarter, which must be revitalized and vibrant, which means that the rehabilitated physical attribute of the place and buildings form the frame for an authentic social public realm, which is also a social construct.

After thorough analyses of the level of obsolescence of buildings and areas of historic city centres, and according to the needs of contemporary needs, standards and trends, the improvement and the quality of the physical fabric may be realized through the following modes of renewal [18]:

- Refurbishment, as a form of conservation and consolidation in physical sense which affects the obsolescence of a building or an area to enable its future use;

- Conversion, as a form of adaptive re-use, which Latham [26] defines as a process of modifying a place to suit the existing or new use without diminishing its value;

- Demolition and redevelopment, which, according to Doratli [19], might be an unwanted method for historic centers.

Furthermore, for various levels of different obsolescence, distinctive types of strategic interventions of economic activity can be recognized to ensure revitalization in the long term $[18,19,27]$ :

- Functional restructuring, meaning that new land uses substitute existing uses;

- Functional diversification in terms of introducing new land uses and activities along with the existing uses;

- $\quad$ Functional regeneration as a method of running existing uses more efficiently or profitably.

Based on the identification of the contextual characteristics of the historic city centers and the thorough analyses regarding the levels of different types of obsolescence described previously, 
the relevant different strategic options for the revitalization of historic centers can be recognized. According to Doratli [19], we can distinguish three different strategic options regarding the level of obsolescence and the development dynamics:

1. The first option is for if the area has physical and functional obsolescence, meaning that most of the buildings are old and in poor structural condition, and the fabric of the buildings does not meet contemporary standards, especially sanitary conditions and size, despite monumental and architectural values, but nevertheless, development dynamics are high within the area and attractive for existing functions or other future land uses;

- Governance and policy response of the public authorities aims to create favorable conditions regarding the provision of infrastructure and public spaces, while the restoration and refurbishment of the old buildings should be encouraged in terms of physical revitalization, coupled with economic revitalization if the area in question is still attractive;

2. The second option is if the area is with physical and functional obsolescence and the development dynamics are without redevelopment pressures and static. This further stresses the decay and deterioration of the area, while property values begin to decline, and residents and businesses start to move out of the area, thus influencing changes in the socio-economic situation of the historic city center;

- Governance and policy response of the public authorities means that, besides the physical revitalization, existing land uses should be combined with new ones, used for rebranding and increasing the attractiveness of the area. Functional diversification of land uses and activities should be the right strategic alternative regarding economic revitalization;

3. The third option is characterized by multi-dimensional obsolescence with the declining area, inadequate buildings, incompatible uses that diminish the quality of the environment and decreasing owner-occupied buildings. Development dynamics are in decline and, with the high vacancy rate, low rents and property values, new investment is not present and bypasses the area. These trends contribute to image obsolescence, leaving a burden on the area showing that it is outmoded according to contemporary standards;

- Governance and policy response of the public authorities is to initiate a suitable long-term economic functional restructuring strategy, coupled with capital investments for physical revitalization related to infrastructure and open spaces in order to improve the image and public realm and build confidence in the historic area.

Finally, based on the identification of the contextual characteristics regarding the level of different types of obsolescence of historic city centers, we have to underline that economic, social and physical revitalization have to be considered as complementary in order to achieve a successful and sustainable revitalization of historic urban quarters irrespective of the different strategic options chosen.

\subsection{The Sustainability Indicators of Regeneration Strategies for Historic City Centers}

The evaluation and measurement of the quality and success of the implemented strategic urban regeneration projects according to the urban management model [16] depend on the angle of view, i.e., from public, private, or civil sector positions and, accordingly, the system of values and goals, which each actor defines individually or jointly in the partnership. This also raises questions of efficiency and effectiveness.

Effectiveness refers to issues related to the quality of implemented strategic urban regeneration projects. Issues regarding the effectiveness of strategic urban regeneration projects are related to the values that exist in a particular society and whether solely political representatives define the goals or 
whether goals are defined in interaction with other actors in society in collaborative ways. Thus, the model [16] distinguishes two essential elements concerning efficiency, such as:

1. Realization of goals, indicators and desired outcomes that are formulated in advance and which are directly related to strategic urban regeneration projects;

2. Effects that projects have in the socio-economic terms on the environment and context.

Efficiency refers to the process of implementing strategic urban regeneration projects. Thus, three essential elements concerning efficiency can be distinguished, such as:

1. Issues of direct costs of project realization, and also indirect costs for the residents of the wider territory;

2. The nature and length of the process of realization of certain phases of the project;

3. The proper choice of implementation process concerning the opportunities found in the local context.

Regarding the strategic urban regeneration projects in the urban management model [16], their successful implementation requires a system of developing new urban management and policy instruments and a continuous system of monitoring and evaluating the effects that projects have on urban development. It is necessary to distinguish between the interests and goals that actors have, both individually and jointly in partnerships, so that outcomes are also measured against individual outcomes, and to avoid an overflow of responsibility. Additionally, monitoring the effects of the implementation of strategic urban regeneration projects is important because the actions and projects that do not meet the formulated goals, objectives and performance can be evaluated, changed and adjusted, or even abandoned.

Regarding indicators, there are no standardized sustainability indicators for historic cities and heritage sites, since they have to be adapted to particular needs and specific contexts. Sustainability indicators constantly have to be modified and must be flexible and revisable to allow monitoring, control, and prediction [20].

However, in the quest for an all-embracing typology of sustainable indicators in relation to the revitalization of historic urban quarters in a changing context, we argue that, for the formulation and conception of the regeneration and protection strategy for the historic city centers, as well as for its implementation:

1. The indicators depend on the values and goals conceptualized and agreed through a process involving key actors from the public, private and civil sector in the local context;

2. The outputs and effects of physical, economic and social revitalization have public benefits and added value for all users [16].

Furthermore, the site-specific indicator selection process offers a methodology for combining goals' and objectives' formulation with community participation for the relevant, sustainable indicators of historic cities and heritage areas [27] as:

1. Identification of the community goals with community participation and consultation;

2. Identification of the specific objectives;

3. Determining the causing factors of a specific objective depending on the characteristics of an area;

4. Construction of indicators through enquiring about the causing factors;

5. Creating the initial indicators list;

6. Evaluation of indicators by testing their relevance for a specific case study area;

7. Deciding the final indicators list;

8. The meaning and objectives of the selected indicators;

9. Selection of a method for measuring the indicators; 
10. Analyzing the physical, economic, and social case study environment using multi-dimensional methods (historic, locational; land-use; architectural; transport; social analyzes, etc.)

11. Measuring the selected indicators and defining the sustainability level of the selected case study area and, after conducting the sustainability level measurement of a historic urban area, relating measurements to the revitalization process.

\section{Urban Planning and Governance in Relation to the Preservation of Protected Heritage in Serbia}

The problems facing Serbia are numerous and are mostly conditioned by the changing social, economic, and political ambient. Since the breakup of former Yugoslavia, Serbia has gone through a period of devastating economic, social and cultural crises. Problems with the preservation of cultural heritage have culminated over the last three decades, especially since the beginning of the wars in the territory of former Yugoslavia. Their causes are manifold, ranging from the most dangerous, such as war devastation, through to traditional negligence, the absence of a national strategy in this area and, finally, because of outdated and inapplicable legal regulations [28]. The restitution of several cultural and historical monuments to private owners introduced new actors in the heritage management process. Their behavior, apart from restrictive measures, has not been accompanied by an integral policy or by incentive measures. Due to the slow response of public institutions and low awareness of the new owners, there was an inexperienced restoration or even demolition of monuments and cultural heritage in order to obtain valuable construction land. The insufficient motivation of the decision-makers and lack of public funds for projects restoring historical urban landscapes led to the destruction of many valuable cultural monuments and their surroundings.

An integrative territorial approach to the protection of cultural and natural heritage and landscapes was introduced in the Spatial Plan of the Republic of Serbia from 2010 [29], in accordance with European standards of protection. Nevertheless, although this approach contributes greatly to the recognition of cultural heritage as a resource for sustainable development, the problem remains regarding the legal and institutional framework of protection. That framework is still inconsistent with contemporary conservation approaches, which raises the question of the possibility of implementing planning recommendations. Worth mentioning in this respect is that the Law on Cultural Properties from 1994 is still in power, and it does not comply with international recommendations, nor does it recognize categories of cultural landscapes and historic urban landscapes. Additionally, UNESCO's Recommendations on the Historic Urban Landscape form 2011 [9] have not yet been ratified in Serbia.

Nevertheless, there has been progress in Serbia regarding strategic planning. Priority areas for urban intervention have been identified in the Sustainable Urban Development Strategy of the Republic of Serbia until 2030 [30], linking recognized development problems with specific locations, thus directing local integrated urban development strategies to priority projects. Spatial units with cultural and architectural heritage, important urban settlements as reference points of cultural and historical development, and a group of urban settlements were individually set aside, following the perceived need to extend the subject of protection and the notion of cultural heritage. The following interventions are proposed within the Sustainable Urban Development Strategy — the rehabilitation and revitalization of buildings and architectural heritage; an integrative approach to the protection of cultural property and the urban protection of buildings and entities which are not under institutional protection; the adaptation of architectural heritage buildings according to modern requirements, such as energy efficiency; etc.

Nonetheless, Serbia has to incorporate a number of contemporary United Nations resolutions in its pursuit for its own model of heritage protection. HABITAT III Global Human Settlements Conference 2016 [31] promotes a new, integrative approach to urban planning and decision-making processes for the future of development. Out of the seven important areas for ensuring the sustainable development of cities, the first is urban regulation, land, and management, and the second is planning and design. The third component necessary for lasting prosperity is a financial plan that provides adequate revenue to achieve strategic objectives. 
Advantages and Limitations of the Transition from Conventional to Strategic Planning in Serbia in Relation to Heritage Protection

Following significant social, political and economic changes in Serbia in the early 1990s, there were, among other things, changes in planning and management policies that addressed the issue of protected historic urban landscapes. Although the management and planning of development in protected urban landscapes in Serbia is governed by a set of development and planning documents, as well as by the appropriate institutional framework, the practice has shown that this planning system is not efficient enough. Furthermore, the market pressures for land lead to unavoidable devastation of valuable architectural and urban heritage, which often leads to changes in their functional and spatial characteristics, causing the creation of so-called quasi cultural heritage. The physical degradation of heritage leads to its economic decline, but also to a decrease in real estate value. This loss of economic value has negative effects on the social component of cities, which is reflected in the impoverishment, deterioration, and eventual migration of the local population from historic centers of Serbian cities.

As there is no single strategy for urban development, due to the diversity of local conditions, development planning should address the development of guidelines, models, and action plans and programs. Management plans should provide policy instruments for implementation, as opposed to current project-oriented planning, and strategic plans should provide a link between strategy and implementation. Urban development planning should coordinate between sector strategies and sector policies with spatial resources.

Nevertheless, the transition from conventional to strategic planning is neither simple nor easy. When looking at the international experiences of prosperous cities and the global challenges that historic cities are facing, it is clear that Serbia needs to define fundamental mechanisms as soon as possible. This means that Serbia has to move from an ad-hoc style of making decisions regarding urban development to a systematically organized and defined way of planning development using strategic plans. Vujošević [32] states that the failure of strategic planning in Serbia is the responsibility of an elite that does not have a clear answer to the challenges our country faces. The slow development of civil society institutions is contributing to these processes, as well as poor education, which reduces the value of human capital.

Since citizens in almost all the countries of former Yugoslavia have not yet developed an awareness of their impact on decision-making, in Serbian circumstances the Serbian Government could oblige all local governments to develop a spatial development strategy with the participation of all relevant stakeholders. Based on these assumptions, local governments would develop action plans that could define the exact time and framework for the implementation of agreed goals and objectives. By providing financial assistance for development projects within action plans at the local level, the state would limit local governments to arbitrarily managing the development, with the mandatory condition that funds from the local budget can be spent solely on the implementation of the tasks specified in the action plans, which are derived from development strategies. Controlling the public budget expenditures would make local decision-makers more serious in the process of creating an integrated spatial development strategy as an umbrella document. Indeed, citizens would also be interested in getting involved in the development of the strategy, as the strategy sets priorities and budgetary resources for the implementation of projects. In this way, citizens could formally influence decision-making and the implementation of these projects, because, to date, it has been the practice for politicians to implement projects at their discretion.

Based on the experience of cities in other post-socialist countries, local authorities in Serbia could involve the private sector in the decision-making process and integrate comprehensive urban and financial planning through strategic planning. Furthermore, participation and collaboration should be integral parts of the decision-making process, since strategic plans involve the active participation of stakeholders, and the definition of problems, visions, objectives, and means of implementation [33].

A significant problem in the implementation of strategic urban planning in Serbia is the requirement for institutions to introduce consulting practices through which the legitimacy and diversity of inputs are 
established [34]. It is necessary to establish clear demarcations of responsibilities between institutions to prevent conflicts of interest, especially between governmental and non-governmental organizations, which have a crucial impact on strategic planning. Western Europe has a suitable institutional setting for strategic planning because it has a long tradition of involving all three sectors and embracing public interventions for the common good. Western countries include a large number of partners in urban spatial strategies in decision-making, policy formulation and implementation.

Local governments in Serbia have adopted comprehensive strategic development plans from the year 2000, which are called sustainable development plans in most municipalities, as well as individual sector strategies. A particular problem is that the priorities highlighted in the strategies are not precisely the projects for which they are competing and receiving external funding to finance or co-finance in the implementation process. The existence of development plans does not mean that development is also managed systematically. The main problem is budget preparation and the lack of financial and informational instruments for implementation. Without any reliance on a planning document, local governments often do not follow the plans and deadlines for implementation in strategic documents, and priorities are changed by the will of individuals [35]. Regarding implementation, the success of such plans varies in practice. Strategies are very often informal documents adopted by local governments on their own initiative. The quality of strategies depends on already established procedures, policies, institutionalized practices and forms, value systems and ways of observing phenomena, etc.

\section{The Case Study: Historic City Center of Novi Pazar in Serbia}

Novi Pazar is a town in southwestern Serbia, and the regional, economic, cultural and educational center of Raška region, which still retains its historic name-Sandžak. According to the 2011 census [36], Novi Pazar has 100,431 ethnically heterogeneous inhabitants, comprising $80 \%$ Bosniaks, $15 \%$ of Serbs and a small percentage of other nationalities. The city today covers an area of $742 \mathrm{~km}^{2}$, with an average density of 135 inhabitants per hectare.

The founding of Novi Pazar as a town is linked to the establishment of the Ottoman rule in these areas. The name Novi Pazar is related to the first official document from 1461, when the Republic of Ragusa-today's city of Dubrovnik in Croatia-sent its judges to the town of Novi Pazar. The name was probably due to the existence of the Old Trgovište town with the vast market-place-today's Pazarište site-a medieval settlement from the period of the old Serbian state of Raška located just a few kilometers from the present-day town of Novi Pazar. Both town names literally mean the market place in the Serbian language and are believed to have existed in parallel for some 100 years, when the Old Trgovište town was economically stifled and abandoned [37].

The favorable position along the Raška River on the crossroads and trading routes between neighboring regions of Zeta and Bosnia in the west, and Kosovo and Metohija in the south enhanced the strategic importance of the town of Novi Pazar and its rich cultural heritage. Its historic center as a business and commercial part of the town, with the fortress of Novi Pazar in the vicinity, as part of the once more significant market place, is one of the best-preserved urban heritage sites of its kind in Serbia and was declared the spatial cultural-historical unit of great importance in the Republic of Serbia. The creation of the historic center is related to the construction of the first 56 shops in the newly formed settlement built by the military commander and contributor Isa-beg Isaković. It is believed that during this period, the first public buildings were built, such as the central mosque and the hammam—Turkish bath—-that has been preserved to the present day. The historic center was built first on the left and then on the right bank of the Raška river, and reached its peak in the second half of the 17th century, when it was a large and rich oriental historic center, with several small and bigger lodgings, market places, hammams and about 1200 shops. The fact that during the time of the travel writer Evlija Čelebija in the sixteenth century, Novi Pazar had the status of a town—besides Belgrade, Skopje, Sarajevo and only 10 other cities in the occupied Serbian part of the Ottoman Empire-speaks of the richness and development of the city. From the 19th century until liberation from the Turks in 
1912, Novi Pazar achieved a significant degree of economic development, and most of the historical buildings from that period still exist today [37].

The city of Novi Pazar underwent a drastic urban transformation after World War II due to changed socialist ideological conditions, like most of the cities in the former Yugoslavia. The first general urban plan of the city of Novi Pazar was adopted in 1956, and shortly after that a city center project was created in 1968 that would give Novi Pazar a whole new modern spatial and visual identity. With this design of the city center, dominated by high-rise buildings along the arched road forming the wall, the city was symbolically divided into the center and the outskirts [38]. The settlement on the left bank of the Raška River-the historic city center-underwent significant changes during the implementation of this plan. A significant number of buildings were demolished around the town square to form roads and a new Municipal and Cultural Center building.

A significant change occurred in 1977 when the Institute for the Protection of Monuments from the city of Kraljevo recorded monuments and started the research and valorization of cultural heritage in this area. Moreover, a large group of architects and architecture students did their primary research in the period from 1971 to 1973, which served as the basis for the adoption of the General Plan for the Valorization and Protection of the Historic Center of Novi Pazar in 1975. Furthermore, the area of the medieval fortress of Old Ras-one of the first capitals of the medieval Serbian state of Raška-was listed on the UNESCO World Heritage List in the late 1970s. Consequently, a committee for the protection and management of cultural heritage in the municipality of Novi Pazar was established during this period. An important activity of the committee was to implement an appropriate approach for the planning of heritage protection, including the historic city center in Novi Pazar.

Thus, in 1986 and 1987, additional research and documentation of heritage began, which resulted in the development of a detailed plan for the protection and revitalization of the historic city center in Novi Pazar in 1988, under the leadership of Jovan Nesković, professor of architecture and a leading expert in the field of heritage protection and revitalization in the country at that time. Most of the buildings in the historic center were valorized and received legal protection [39]. As a result, significant public buildings in this area were restored during the period of the welfare state in former Yugoslavia.

Another critical period for the historic part of the city was during the 1990s, when the economic and social crisis in Serbia caused many substantial visual and spatial changes to it. The most evident changes were related to the great number of illegal interventions on housing stock in the historic center, e.g., without the consent of the Institute for the Protection of Monuments. Most of the shops modernized their storefronts during that period, which, although appealing in appearance, were not entirely in line with the character of the historic city center. The attitude that it is possible to perform works without obtaining a building permit has been maintained up to the present day, as evidenced by the latest interventions on the Arab Mosque, shown in Figure 3.

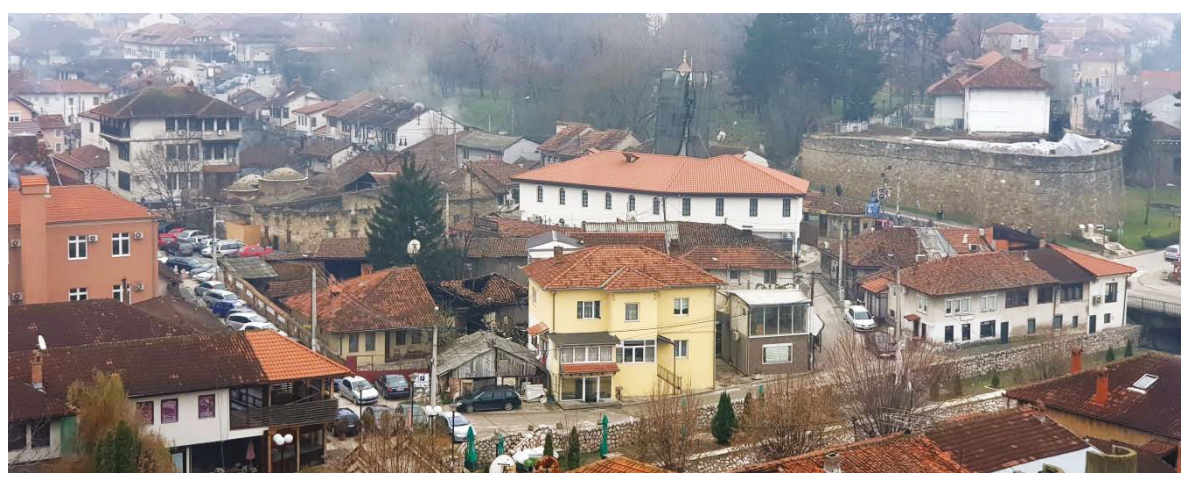

Figure 3. Panorama of the historic center in Novi Pazar with the Arab Mosque under reconstruction. 
From the 2000s, with the recovery of the state and with the market liberalization in Serbia, control over development processes began to be also established throughout the city of Novi Pazar, as well as in its historic part. Those circumstances have influenced a shift from physical to integral socio-economic territorial development, with the introduction of new planning concepts and instruments. Hence, new forms of planning in the form of participatory processes and strategic plans have emerged, including new models of cooperation between municipalities and educational institutions conducted as a simulation of a strategic planning exercise [40]. An essential event for the development of the historic center of Pazar is the adoption of the Plan of Detailed Regulation of the Historic Center with the park in 2017. Although this plan is primarily focused on the arrangement of the physical and spatial layout and land uses, it is an essential basis for the future development of the old historic city center of Novi Pazar.

\subsection{The Context and the Level of Obsolescence and Dynamics in the Historic Center of Novi Pazar: The} Physical, Social, Economic, Environmental and Governance Aspects

\subsubsection{The Spatial and Functional Aspect}

The historic center in Novi Pazar today is one of the rare and relatively well preserved urban heritage sites of its kind in Serbia. In the postwar period, the historic center underwent significant changes, such as its demolition and new construction. In the historic center and its surroundings, many objects of larger volume and inappropriate architecture were built, which did not comply with the standards and character of the historic center. The historic center has remained a vibrant and very active part of the city with a high level of development dynamics; however, many commercial and trade shops have replaced traditional, old wooden windows-for reasons of modernization, cost and energy efficiency-with new modern aluminum and plastic windows in a different color that is not suited to the character of the historic center. Furthermore, a large number of buildings on the 1st of May Street have problems with water supply, plumbing systems and sewage, while some stores do not have any sanitary facilities at all [39].

A significant problem is the fact that a large number of buildings are not in use, and houses in which the old crafts have been situated are being replaced with new uses that are often inconsistent with the historic character of the historic center. Most of the interventions have been done superficially, unprofessionally and without analysis and proper expert approach. The hammam building dating from the 15th century, being the oldest and most important building in the historic center, is not in proper function. The hammam building (Figure 4) was used for a long time as a warehouse, and one part of the facility was converted into a food preparation facility 15 years ago and used only during the summer months, since the roof was demolished. One of the most visually striking objects is the Han Smailbegovića building, popularly known as the Grenade. This facility has been out of service since the 1990s, further contributing to its decay and, eventually, this resulted in the facility burning completely in the 2013 fire.

The replacement of cobblestone asphalt on the 1st of May Street in 1965 and vibrant automobile traffic further shattered the ambiance and atmosphere of the historic center [41]. The condition of the infrastructure is reduced, as asbestos piping has been obsolete a long time ago, while numerous electrical power lines visually pollute this space.

During the 1990s, after the change in the social system and the weakening of the control over construction activities and inspection by the national and local authorities, the historic center has been left to the market forces, and many buildings were illegally constructed and reconstructed resulting in the devastation of the whole historic center. Another feature of modern interventions was the distinction of illuminated advertisements and billboards, which devastated the physical appearance and ambiance of the entire historic center (Figure 5).

Furthermore, due to the massive influx in population from the surrounding areas, the city of Novi Pazar has been exposed to an intense urbanization process in recent years. A large number of the old 
structures located in the contact zone of the historic center have been demolished to provide valuable building land for the construction of multistory residential and commercial buildings (Figure 6).

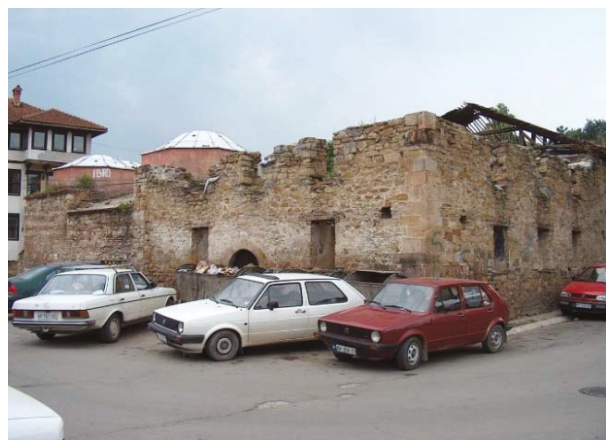

(a)

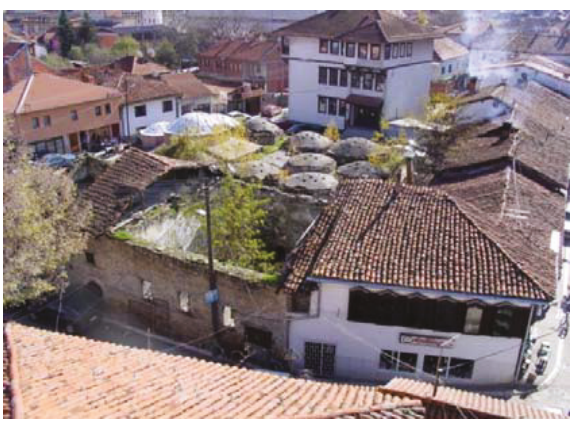

(b)

Figure 4. Hamam of Isa-beg Isaković, 15th century, (a) street view; (b) birds eye view.

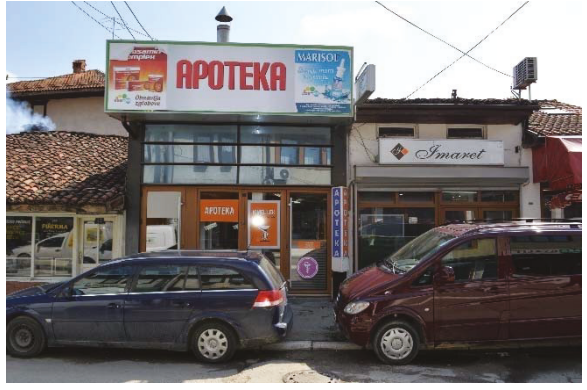

(a)

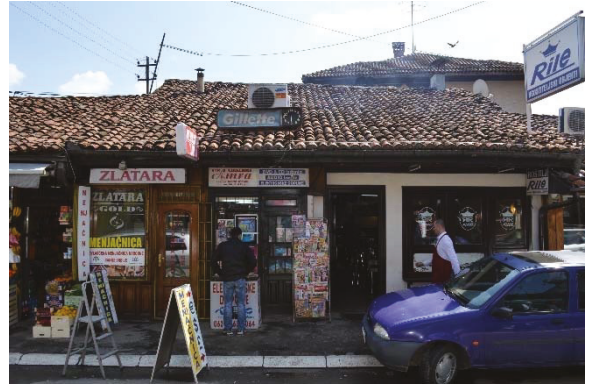

(b)

Figure 5. (a) Placing billboards in the historic center without any order; (b) new shop windows in a different color and material that are not suitable to the character of the historic center.

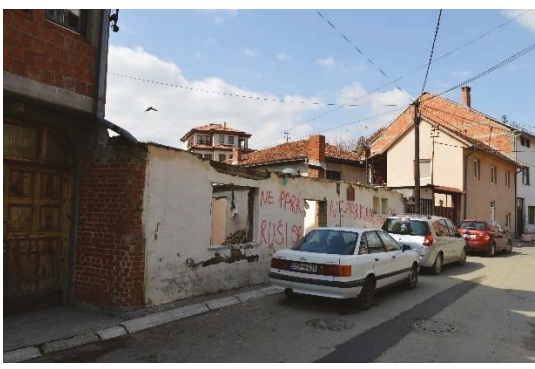

(a)

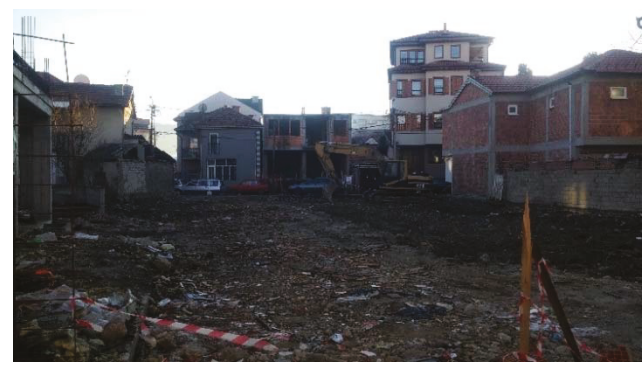

(b)

Figure 6. A cleared site for the construction of a multi-story building in the immediate vicinity of the historic center in the 7th of July Street (a) street view; (b) view of the lot.

These new buildings certainly do not fit the character of the historic center, regarding their height and size, and contribute to the image obsolescence. Another problem is the additional burden of the already existing infrastructure systems of the historic center. Parked vehicles in the narrow and 
winding streets inherited from the past create blockages for the smooth passage of pedestrians and utility vehicles, adding to the functional obsolescence.

\subsubsection{The Social Aspect}

Almost all of the buildings in the historic city center are privately owned. Currently, the biggest problem is the fact that a large number of objects have more than one owner, and a considerable number of them have more than three owners. A substantial number of buildings on the 1st of May Street are owned by the Islamic Community. Since the restitution process of returning confiscated property from the socialist period to its real owners has not yet been completed, court disputes prevent any activity on the objects. It is important to realize that a solution to property ownership issues is a fundamental prerequisite for beginning the process of regenerating the historic center at all.

Another key point regarding social issues is significant research about the inhabitant's and shop owner's satisfaction with the housing options and places of residence in the historic center done by Mulić [42]. The research reveals that the historic center is mainly populated by an older population which is over 50 years old, while a younger population of less than 40 years old lives in the new residential areas of the city [42]. This is explained by the fact that older families find it more challenging to move to new settlements, but there are also financial reasons behind this. Old-town residents are natives that have lived in these facilities for more than 40 years and they constitute 65 percent of the city center population. In terms of education, the older generations generally have completed secondary education, while the younger population living in new settlements have completed higher education. In a survey of 355 respondents, about satisfaction with living conditions and comfort, 15 percent of people answered that were satisfied, 20 percent were partially satisfied, while 65 percent of the surveyed responded that they were not satisfied with the levels of comfort of living in the old city center. Only one percent of residents would demolish their buildings to build new ones, 16 percent thought they could continue living in those buildings with minor renovations, while most, as many as of 64 percent, of residents replied that the facilities should be adaptively reused, while 16 percent of respondents would preserve objects for reasons of nostalgia and historic character and leave them without any use or purpose [42].

\subsubsection{The Economic Aspect}

The economic land uses—such as commercial and trade shops along the 1st of May Street, which is currently one of the busiest streets in the city-are extremely economically valuable as they carry out vibrant economic activity. The trend of opening new bars has recently become noticeable. The entire city, including this historic city center, has seen a rise in property values in recent years due to population growth, greater appreciation of the heritage values and the economic concentration of activities. This dynamic economic activity that puts particular pressure on property owners to modernize their stock. Nonetheless, important to this research is the fact that there is a conflict between the demands for modernization and the preservation of authenticity.

Regardless of this economic growth, the price of land is much lower in the historic center than in the areas directly adjacent to this part of the city. As an illustration of this trend-according to an interview conducted with a private investor who recently bought a building plot on the 1st of May Street-the price of the land in this part of the city ranges from 30 to 50 thousand euros per acre $\left(100 \mathrm{~m}^{2}\right)$. For comparison, the price of land in the new city center-which is about $100 \mathrm{~m}$ away from this part of the city-on the 8th of March Street is between 80 and 100 thousand euros per acre. The price of the land in the historic city center is lower precisely because of the imposed restrictions and the protection zoning regulations in terms of allowed urban coefficients-e.g., the lot coverage, height limitations and floor area ratio (FAR), as well as the poor condition of the existing infrastructure. 


\subsubsection{Environmental Aspect}

Traffic jams and lack of parking space are the biggest problems for the city of Novi Pazar in general and the historic center in particular, adding to the functional and locational obsolescence. This causes numerous environmental problems such as air pollution and damage to the facades of the old heritage buildings. A significant environmental problem is also the pollution of land and water from the sewage outflow from the old sewage system. Moreover, as municipal waste collection vehicles find it difficult to access certain parts of the historic center, the municipal waste containers are full and visually unsuitable for this ambiance. Since there are several butchers on the 1st of May Street, it is common to find trash of animal origin in municipal waste containers, and this situation is not sanctioned by the competent municipal services.

Although the Republic of Serbia has adopted the Ordinance on the Energy Performance of Buildings in 2011 [43], the City of Novi Pazar has only recently begun certifying objects, and therefore accurate data on the energy consumption of individual objects are still unknown. In the same way, while the city has started a few projects related to the reduction of public energy consumption in the form of introducing energy-efficient city lighting, the city's clear commitment to addressing these issues has not yet been seen. Likewise, the issue of increasing the energy performance of public buildings and open spaces in the city's historic core is also of paramount importance.

\subsubsection{Governance Aspect}

The local self-government is mainly committed to solving traffic problems, improving the quality of public spaces, creating preconditions for new investments and solving problems of coping with natural disasters, such as river flooding. Heritage preservation is not a priority issue to be addressed both at the national level and the local level of governance, as the state is confronted with the pressing issues of economic consolidation. Even though there is a department dealing with cultural heritage preservation within the Ministry of Culture of the Republic of Serbia, adequate models for the creation and implementation of a strategy for the preservation and promotion of architectural heritage have not been developed. Similarly, there is no strong political commitment to addressing issues related to heritage protection and revival at the local level. Although the city supports projects for the branding and promotion of heritage regarding tourism development, there are almost no funds from the local budget allocated to the research on and restoration of architectural heritage. This may be due to the complexity of historic center regeneration, since a large number of private actors need to be involved and significant financial resources invested. Another important aspect is that there is a low level of knowledge and skills among municipal experts in the field of heritage protection, management and regeneration related to the new trends and concepts of sustainable development. Additionally, another critical reason for the slow regeneration are the long-lasting and complicated procedures required to obtain building permits, since they have to be obtained at the national level. This is because the historic center is proclaimed a Spatial cultural-historical unit of great importance and falls under the jurisdiction of the republic level of governance.

According to a survey of a sample of 355 respondents [42], 62 percent of residents believe that the city government is responsible for the bad condition of the built heritage, and as many as 16 percent of the population consider that the state and owners themselves are responsible for this situation. Only five percent of the population was unsure who was responsible for this situation. This is important information for all NGOs dealing with cultural issues, to put more pressure on decision-makers to tackle heritage conservation issues in future.

\subsubsection{Problems Derived from the Physical, Social, Economic, Environmental and Governance Aspects}

The main problem was identified and elaborated using a problem tree method (Figure 7). It was done in a participatory manner with representatives of the Novi Pazar city authorities, the Institute 
for Urbanism and Planning of the City of Novi Pazar and Regional Institutes for the Protection of Monuments, and representatives of shop owners and citizens.

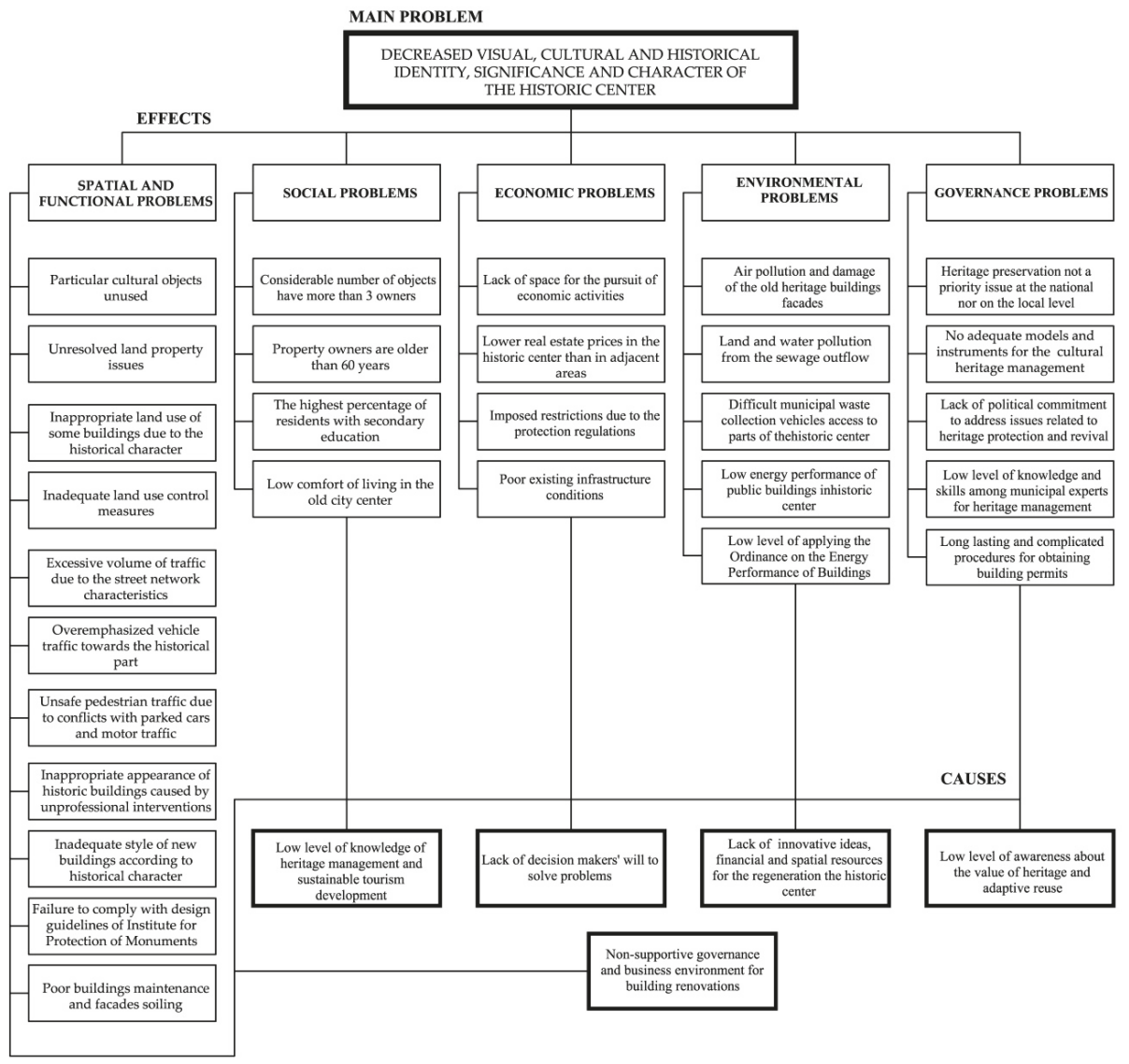

Figure 7. Problem tree.

The final product of this analysis is a graphical representation of problems, differentiated by causes and effects, which focuses on central or essential problems. This technique helps to understand the context and interdependence of problems, as well as the potential influences, when defining the goals of projects and programs. The problems described here were carefully recognized with the aim of identifying the essential main problems, and real causes and effects of dominant problems on different levels.

\section{Initial Stage of the Conceptual Strategy and Management Model for the Regeneration and Protection of the Historic Center of Novi Pazar}

The initial stage of the conceptual strategy and management model for the regeneration and protection of the historic center of Novi Pazar is envisioned as the combination of strategic options for regeneration. Thus, the public sector might create favorable conditions regarding the capital investments and the provision of infrastructure and public spaces for private sector involvement, along with the functional diversification and regeneration of economic activities. Additionally, the public sector can provide economic incentives to obtain private sector participation. Furthermore, goals, 
objectives and actions in this strategy are proposed with the time needed for the implementation. After the initial elaboration of goals and objectives, the strategy is presented in the form of a table with all the stakeholders that should be involved, as well as the particular stage of their involvement.

Renovating the historic city center in a multifunctional way and turning it into a place brand where everyone wants to live and which tourists want to visit by increasing its attractiveness is at the core of a future integrated urban development strategy. In order to achieve this, it is necessary to guide actions in two directions. The first one is an aesthetic, spatial and functional improvement, through the reconstruction of individual objects in order to restore historical character, while the second direction is to provide support and create a favorable environment that will motivate the owners and potential users to renovate their objects and transform them into comfortable objects.

The challenges in achieving these goals are numerous. The quality of the housing currently does not meet contemporary needs. Most of the buildings located in the historic center, are built of cob, which is traditionally referred to as an adobe-cerpič in the Serbian language—on a wooden skeletal structural system bondruk wall. This material is not weather-resistant enough, so frequent renovation of these walls is required. Additionally, a major problem is the lack of knowledge and skilled masons required for the reconstruction of these walls, since they have not been used for the construction of new buildings for many years. Furthermore, urban problems, reflected in the over-concentration of people and motor traffic on the 1st of May Street, places much pressure on the historic city center, as well as the construction of multi-story buildings near the historic center, which do not correspond to its cultural and historic identity, significance and character. Moreover, the urban quality of public spaces is deficient due to the enormous presence of parked cars and asphalt surface treatment, with the cobblestone underneath that was been used to pave streets in the past.

Although the city adopted a Plan of Detailed Regulation for the Historic Center, the plan envisions mainly the physical dimensions of heritage protection and regeneration, focusing only on regulatory instruments, while other aspects of the redevelopment are ignored, such as the economic and informational management instruments. Moreover, the city of Novi Pazar has not applied for heritage reconstruction projects in recent years on the open calls financed by the Ministry of Culture, since the city does not have an integral strategy in place to define the actions which ought to be implemented.

In essence, it is indisputable that the city should develop an integrated urban development strategy as soon as possible, in which the historic part of the city should be given a significant place. When designing the strategy, it is necessary to link all the goals of specific sectoral strategies that the city already has and propose a creative and contemporary way to solve the identified problems. Thereafter, it is necessary to adopt a local action plan for the implementation of the objectives defined in the strategy, using a participatory approach. All the future actions and small projects would involve citizens in some way in order to gain legitimacy and consensus for the implementation of those decisions.

In this respect, it is necessary to use numerous informational instruments in order to involve citizens in this project from the very beginning. Such participatory instruments would include door-to-door questionnaires with selected shop owners and households, online questionnaires, discussions, and meetings on workshop and roundtables with all relevant stakeholders, and through public consultations, in order to understand different users' various issues and their expectations of the particular place, value and identity of the historic center.

In a broader context, at the urban level of the city of Novi Pazar, it is necessary to update the current situation regarding the legal framework for protection, the issue of traffic and mobility in the city in general and in the historic city center, and the current conditions for the conservation of heritage buildings. Besides that, although it is still in the preparatory phase, it is necessary as soon as possible to enable the GIS system as a new digital platform in order to connect different data and information, which should include the current condition of the building conservation process within the historic city center. The use of different policy measures and instruments would prevent further deterioration of traditional buildings, while tighter control of land uses and activities would prevent the 
conflict of incompatible and unsuited uses in the historic city center. Poor quality in the design of new buildings needs to be improved and regulated using strict regulations for an adequate reinterpretation of traditional typologies, building construction and building materials. Additionally, an improved design of public spaces is expected to raise their quality and increase the attractiveness both of the historic city center and the wider territory, in order to reconstruct its image and create a recognizable place brand.

During the process of restoration of individual objects exposed to rapid decay, it is necessary to prevent further decay with various protection measures. Since the city does not have an accurate assessment of endangered vulnerable buildings, it is necessary to form an expert commission, which will enlist all the buildings in this zone and determine their condition. Based on these results, it is necessary to estimate the value and scope of work to be undertaken in order to protect these buildings from further deterioration. Furthermore, it is necessary to examine the energy performance and behavior of these buildings during seismic activity. A large number of buildings had improper interventions in the form of adding concrete elements; therefore, it is necessary to examine the impact of these reconstructions on the original structure. The city should, above all, using its public services and external expertise, examine the condition of local building materials and produce a brochure in the form of guidelines for the restoration of traditional buildings with an emphasis on environmental restoration and the increase of buildings' energy efficiency in this area.

The economic motivation for residents to renew their buildings is shallow, except on the 1st of May Street, in which lively trading activity leads to over-modernization. One way to increase the demand for historic buildings would be through increasing their energy performance. Another way would be to directly subsidize specific conservation works by the city. For example, the city of Novi Pazar-in cooperation with the Institute for the Protection of Monuments in Kraljevo, which has the jurisdiction for all the heritage buildings in this zone-could subsidize up to 70 percent of funds for the replacement of windows on the 1st of May Street. As an effect of this, the city could influence the design of new windows in order to restore the authentic appearance of the shops and increase the value of the entire protected area. Given that there is not a large amount of such buildings, it would not be a significant expenditure for local government in financial terms, while the benefits would be multiplied.

Regarding informational instruments, the need for expanding knowledge and awareness has to be considered crucial. The non-governmental sector, which is still in its development phase, should influence politicians to initiate a procedure for resolving the heritage problem. The strategy and the action plan should envision different activities and instruments, which should be targeted towards investors, architects, engineers, and technicians on the one hand, and towards all citizens on the other.

The city administration should facilitate and overcome numerous administrative procedures in order to reduce bureaucratic red tape and facilitate the issuance of building permits for construction activities. Since a large number of citizens and investors perceive the Institute for the Protection of Monuments in Kraljevo as an institution that slows down their intentions regarding building in the protected historic city center, the city of Novi Pazar should open a local office for the protection of monuments for the historic center. The task of the local office would be to organize meetings in order to increase co-operation between the institute for the protection, and urban planning departments, designers and other stakeholders. The mission of this office would be a significant contribution to overcoming problems and increasing understanding and trust between the institute and citizens. The work of this office could be aimed at increasing the availability of information to citizens and promoting the value of heritage. Furthermore, one of the resulting effects of this office would certainly be helping to coordinate activities when developing projects and seeking donor funding.

Formulating Goals, Objectives and Instruments for the Strategic Planning and Management Model the Regeneration and Protection of the Historic Center of Novi Pazar

The strategic planning and management model should be made for a period of 10 years, and it is desirable that it complies with the programming periods of the European Union (EU). Since this is a 
relatively long period, this plan should be used as a roadmap for achieving the goals defined in the strategy. Furthermore, it is of great importance that the local action plan remains open to revisions and improvements, in line with the challenges and perspectives of the present, thus addressing major urban, social, environmental, economic and governance challenges. Consequently, the main goals of the plan should be the following. One goal should be raising the awareness of municipal, private owners and citizens of the value of heritage, and the involvement of citizens in the decision-making process. Furthermore, the next goal should be the creation of a favorable environment that will stimulate the restoration and protection of traditional buildings for private owners in the historic center. Above all, improvement in the visual image, cultural and historical identity, significance, character and function of the entire historic center by renovating individual buildings, public spaces and inner courtyard private spaces should be the ultimate aim of the strategy.

When drafting the model, it is crucial to identify possible sources of funding. In addition to funds from the local and the national budget, it is important to initiate other direct financial mechanisms or in-kind instruments and credit financing mechanisms. An important source of funding could be European funds-for which Serbia has access to, as a country in the process of accession to the European Community - as well as various other dedicated funds, such as the Turkish Cooperation and Coordination Agency (TIKA) and others that support related activities. TIKA could be extremely interested since the urban matrix and individual cultural buildings in the historic center represent the valuable heritage of the Ottoman rule in these areas. Another financial source could be private donors, since many former residents of the Novi Pazar, Sandžak and the Raška region became wealthy citizens of the EU or Turkey and are willing to invest in and donate to the regeneration of the historic center.

Strategic Goal 1-Improvement of the visual image, cultural and historical identity, significance, character and function of the entire historic center by renovating individual buildings, public spaces and inner courtyard private spaces (Table 1)

The goal of improving the visual image could be achieved by organizing architectural competitions and other studies aimed at improving the urban and architectural quality of the place. Certainly, an important partner in achieving this goal, besides the city, could be the Department of Technical Sciences at the State University of Novi Pazar with its Architecture program. It is also advisable to include professional associations such as the Serbian Chamber of Engineers, Serbian Society of Architects, and the Society of Town Planners of Serbia. An important stakeholder in these processes should be the Institute for the Protection of Monuments from Kraljevo, which eventually needs to accept the proposed solutions.

Important actions should be focused on finding a solution to remove the road traffic from the 1st of May Street and to calm down the traffic in the whole protection zone of the historic center in order to achieve this goal. This has to be done in cooperation with the Institute for Urban Planning and Development Agency of Novi Pazar. The achievement of this goal likely requires the development of a citywide traffic study. An important issue for the management of parking areas should be to find locations for the construction of underground and surface parking garages on the outskirts of the historic city center. Fulfilling this task would reduce traffic in the protected zone and increase the area intended for pedestrians and cyclists, while also solving the problem of parking for the whole city. Additionally, one of the objectives and actions for improving the image and the identity of the historical part of the city should be focused on the aesthetic harmonization of modern buildings constructed after World War II-which are not in line with the spirit of the historic center-with the typical and traditional buildings. Moreover, many new buildings do not have any thermal envelope. By launching energy efficiency projects, many buildings could obtain a new thermal facade or another environmentally friendly cover, such as green and composite eco-materials. This would significantly alter the appearance of historic buildings and address the environmental problem. 
Table 1. Strategic Goal 1: improvement of the visual image, cultural and historical identity, significance, character and function of the entire historic center by renovating individual buildings, public spaces and inner courtyard private spaces.

\begin{tabular}{|c|c|c|c|}
\hline Objectives/Activities and Instruments & Actors/Stakeholders & Timeframe & Priority \\
\hline \multicolumn{4}{|c|}{ Specific objective 1: improving the quality of public spaces } \\
\hline $\begin{array}{l}\text { Organizing urban and architectural competitions for improving } \\
\text { public spaces }\end{array}$ & $\begin{array}{l}\text { State University of Novi Pazar, the Serbian } \\
\text { Chamber of Engineers, Serbian Society of } \\
\text { Architects, and the Society of Town } \\
\text { Planners of Serbia }\end{array}$ & $\begin{array}{l}\text { Mid-range } \\
\text { (6 months) }\end{array}$ & High \\
\hline $\begin{array}{c}\text { Organizing training courses for specialists for the design of buildings in } \\
\text { the historic environment }\end{array}$ & $\begin{array}{l}\text { Institute for Urban Planning of Novi Pazar, } \\
\text { State University of Novi Pazar }\end{array}$ & $\begin{array}{l}\text { Mid-range } \\
(12 \text { months) with } \\
\text { subsequent periodic } \\
\text { repetition }\end{array}$ & Middle \\
\hline $\begin{array}{l}\text { Amendment of the Plan of Detailed Regulation for the historic center, } \\
\text { on the basis of competition for proposed solutions }\end{array}$ & $\begin{array}{l}\text { Institute for Urban Planning of Novi Pazar, } \\
\text { Scientific Research Institute of the State } \\
\text { University of Novi Pazar, private urban } \\
\text { planning offices }\end{array}$ & $\begin{array}{l}\text { Mid-range } \\
\text { (12 months) }\end{array}$ & High \\
\hline Proclamation of the historic center as a business improvement district & $\begin{array}{l}\text { The City of Novi Pazar, Institute for Urban } \\
\text { Planning of Novi Pazar }\end{array}$ & $\begin{array}{l}\text { Mid-range } \\
\text { (12 months) }\end{array}$ & High \\
\hline $\begin{array}{c}\text { Completion of the inner ring road around the historic center to divert } \\
\text { all vehicle traffic from the area }\end{array}$ & $\begin{array}{l}\text { The City of Novi Pazar, Institute for Urban } \\
\text { Planning of Novi Pazar, Development } \\
\text { Agency of Novi Pazar }\end{array}$ & $\begin{array}{l}\text { Mid-range } \\
\text { (12 months) }\end{array}$ & High \\
\hline $\begin{array}{l}\text { Selection of locations and construction of underground garages in the } \\
\text { vicinity of the historical center }\end{array}$ & $\begin{array}{l}\text { The City of Novi Pazar, Institute for Urban } \\
\text { Planning of Novi Pazar, Development } \\
\text { Agency of Novi Pazar }\end{array}$ & $\begin{array}{l}\text { Mid-range } \\
\text { (12 months) }\end{array}$ & High \\
\hline Introduce time-limited parking in accessible local streets & $\begin{array}{l}\text { The City of Novi Pazar, Institute for Urban } \\
\text { Planning of Novi Pazar, Parking Service }\end{array}$ & $\begin{array}{l}\text { Short } \\
(1 \text { month) }\end{array}$ & High \\
\hline $\begin{array}{l}\text { Limit the time of parking for users of commercial and catering facilities } \\
\text { in the historic center }\end{array}$ & $\begin{array}{l}\text { The City of Novi Pazar, Institute for Urban } \\
\text { Planning of Novi Pazar, Parking Service }\end{array}$ & $\begin{array}{c}\text { Short } \\
(1 \mathrm{month})\end{array}$ & Middle \\
\hline $\begin{array}{l}\text { Monitor the traffic situation on the ground and control its progress } \\
\text { more frequently }\end{array}$ & Police, Parking Service, Municipal Police & Permanent & Middle \\
\hline $\begin{array}{l}\text { Hiring experts for the adaptive reuse of buildings and facades to the } \\
\text { historical and cultural context of the city }\end{array}$ & $\begin{array}{c}\text { The City of Novi Pazar, State University of } \\
\text { Novi Pazar }\end{array}$ & $\begin{array}{l}\text { Mid-range } \\
(6 \text { months })\end{array}$ & Middle \\
\hline $\begin{array}{l}\text { Media and promotion of heritage using flyers, posters, forums for } \\
\text { citizens and shop owners about the importance of preserving the } \\
\text { heritage and cultural identity of Novi Pazar }\end{array}$ & $\begin{array}{l}\text { The City of Novi Pazar, Media, NGOs, State } \\
\text { University of Novi Pazar, schools }\end{array}$ & $\begin{array}{l}\text { Mid-range } \\
(6 \text { months) with } \\
\text { subsequent } \\
\text { periodic repetition }\end{array}$ & High \\
\hline \multicolumn{4}{|c|}{ Specific objective 4: designating compatible and suitable land uses for the historic city center } \\
\hline $\begin{array}{l}\text { Stricter control of land uses, as defined in the Plan of Detailed } \\
\text { Regulation, in order to prevent conflict of uses }\end{array}$ & Building Inspection & Permanent & High \\
\hline $\begin{array}{l}\text { In-kind subsidies as a reduction in building land development fees for } \\
\text { land uses enabling cultural tourism }\end{array}$ & The City of Novi Pazar & Permanent & High \\
\hline $\begin{array}{l}\text { Land readjustment to allow for better-suited land uses to the } \\
\text { historic center }\end{array}$ & $\begin{array}{l}\text { The City of Novi Pazar and its planning } \\
\text { department, Institute for Urban Planning of } \\
\text { Novi Pazar }\end{array}$ & Long-range & High \\
\hline A land swap between municipal and privately owned land & The City of Novi Pazar, private owners & Long-range & High \\
\hline $\begin{array}{l}\text { Financial disincentives for land uses incompatible with and unsuited to } \\
\text { the historic center }\end{array}$ & $\begin{array}{c}\text { The City of Novi Pazar and its planning } \\
\text { department, Institute for Urban Planning of } \\
\text { Novi Pazar }\end{array}$ & Long-range & High \\
\hline
\end{tabular}

Furthermore, designating land uses compatible with and suitable for the historic center is an important task to improve the overall image of the historic urban landscape. It is certainly necessary to develop a study in collaboration with all relevant stakeholders in order to revise the existing legal framework of the Plan of Detailed Regulation, which regulates this area in order to preserve the multifunctional character and mixed uses of the historic center. Although the functional diversification of new land uses and activities along with existing uses might be a viable option, an important issue 
should be to control activities that are not prohibited but are not socially acceptable, compatible with, or suitable for the historic center. In recent years, numerous bookmakers and casinos obsessing over the most valuable sites close to the historical monuments are not exempted from this. Additional instruments in the form of regulatory and fiscal disincentives by the local government are needed, that should ban casinos in the cultural and historical part of the city because it sends a bad impression of the value of the heritage. The plan should provide subsidies to traditional crafts workers and their shops, which once inhabited this part of the city, as they are compatible land uses. Currently, these traditional crafts workers are almost completely gone as there is no economic interest for them anymore and they cannot withstand the high price of rent for their shops. Functional regeneration as a method of running existing uses more efficiently and profitably might be the best option.

Strategic Goal 2-Creation of favorable conditions that will stimulate the restoration and protection of traditional buildings for private owners in the historic center (Table 2)

Table 2. Strategic Goal 2: creation of a favorable environment that will stimulate the restoration and protection of traditional buildings for private owners in the historic center.

\begin{tabular}{|c|c|c|c|}
\hline Objectives/Activities and Instruments & Actors/Stakeholders & Timeframe & Priority \\
\hline \multicolumn{4}{|c|}{ Specific objective 1: improving the energy performance of historic buildings } \\
\hline List objects to be upgraded and their condition & Private companies, Building inspections & $\begin{array}{l}\text { Mid-range } \\
\text { (6 months) }\end{array}$ & High \\
\hline $\begin{array}{l}\text { Creating a guide with guidelines for improving the } \\
\text { energy efficiency of traditional buildings }\end{array}$ & $\begin{array}{l}\text { Institute for Urban Planning of Novi Pazar, } \\
\text { State University of Novi Pazar }\end{array}$ & $\begin{array}{l}\text { Mid-range } \\
(12 \text { months })\end{array}$ & Middle \\
\hline $\begin{array}{l}\text { Giving subsidies to improve the energy performance of } \\
\text { traditional buildings and promoting the results of } \\
\text { actions in order to encourage future actions }\end{array}$ & The City of Novi Pazar, Media, schools & $\begin{array}{c}\text { Mid-range } \\
\text { (6 months) with subsequent } \\
\text { periodic repetition }\end{array}$ & Middle \\
\hline \multicolumn{4}{|c|}{ Specific objective 2: introducing subsidies for restoration work and } \\
\hline $\begin{array}{l}\text { Subsidies for citizens who actively adhere to and } \\
\text { promote the recommendations of the Institutes for the } \\
\text { Protection of Monuments and the City of Novi Pazar }\end{array}$ & The City of Novi Pazar & Permanent & High \\
\hline $\begin{array}{l}\text { Subsidizing, by } 70 \% \text { of the value, the replacement of } \\
\text { PVC and aluminum windows in 1st of May Street with } \\
\text { traditional windows }\end{array}$ & $\begin{array}{l}\text { the City of Novi Pazar, Institutes for the } \\
\text { Protection of Monuments }\end{array}$ & Permanent & High \\
\hline \multicolumn{4}{|c|}{ Specific objective 3: improve technical protection measures for cultural heritage and building restoration } \\
\hline $\begin{array}{l}\text { Professional and financial support for private owners in } \\
\text { the design of conservation and restoration projects }\end{array}$ & Local offices of the historic center & $\begin{array}{c}\text { Mid-range } \\
(12 \text { months })\end{array}$ & High \\
\hline $\begin{array}{l}\text { Carrying out a pilot project for the restoration of a } \\
\text { cultural and historical object in order to effectively } \\
\text { promote restoration }\end{array}$ & $\begin{array}{l}\text { the City of Novi Pazar, Institutes for the } \\
\text { Protection of Monuments }\end{array}$ & $\begin{array}{l}\text { Mid-range } \\
(12 \text { months })\end{array}$ & High \\
\hline $\begin{array}{l}\text { Holding training workshops for heritage conservation } \\
\text { professionals }\end{array}$ & $\begin{array}{l}\text { the City of Novi Pazar, Institutes for the } \\
\text { Protection of Monuments, State University } \\
\text { of Novi Pazar }\end{array}$ & $\begin{array}{l}\text { Short } \\
(1 \text { month) }\end{array}$ & Middle \\
\hline \multicolumn{4}{|c|}{ Specific objective 4: speeding up procedures for issuing building permits } \\
\hline Opening of a local office for the historic center & Ministry of Culture, the City of Novi Pazar & $\begin{array}{l}\text { Mid-range } \\
(12 \text { months })\end{array}$ & High \\
\hline $\begin{array}{l}\text { Facilitating procedures for the renovation of buildings } \\
\text { by creating catalogs for typical interventions }\end{array}$ & $\begin{array}{l}\text { the City of Novi Pazar, Institute for Urban } \\
\text { Planning of Novi Pazar }\end{array}$ & $\begin{array}{l}\text { Mid-range } \\
\text { ( } 3 \text { months) }\end{array}$ & High \\
\hline
\end{tabular}

Actions aimed at improving the energy performance of historic buildings would increase the economic value of the buildings themselves and, henceforth, the whole area. Since the number of these buildings is not very large, funds from donor programs for the implementation of this action could be found relatively easily. The renovation and adaptive reuse of historic buildings costs more, than improving the energy performance of contemporary buildings. This discrepancy in the cost of the works could be covered by donor funds or public budget cash flows, and represents a key motivation and financial instrument for private owners to initiate the process of refurbishment and adaptive reuse of their shops and their buildings. Above all, it is imperative to promote the refurbishment and adaptive reuse of traditional buildings that are not under a direct protection regime in this process but represent an essential element of the vernacular architecture and character of the protected cultural and historical landscape. However, there has been a process during recent years of the demolition of these traditional, yet important buildings and the construction of new ones, which all look the same, without any elements of the vernacular and contextual style and architecture. The view of many international 
organizations is that it is crucial to preserve those traditional buildings in their original form and style. This can be achieved only by developing a stimulating and favorable environment where it will be economically viable for the facility to be renovated before it is demolished and rebuilt.

Moreover, to achieve this goal it is very important to obtain the technical support of heritage regeneration specialists, which can be achieved by organizing thematic workshops and training centers. The creation of a digital platform with recommendations and examples of good practice can make a big difference in that respect. Creating promotional materials in digital and hard copy with technical, financial and administrative information is also very important, as well as producing guidelines for the restoration and adaptive reuse of traditional buildings. Indeed, the implementation of this project requires the commitment of the city government, which can facilitate and speed up procedures for obtaining building permits, in the process of the renovation of shops and buildings for citizens and investors.

Strategic Goal 3-raising awareness of municipal, private owners and citizens about the value of heritage, and the involvement of citizens in the decision-making process (Table 3)

Table 3. Strategic Goal 3: raising awareness of municipal, private owners and citizens about the value of heritage, and the involvement of citizens in the decision-making process.

\begin{tabular}{|c|c|c|c|}
\hline Objectives/Activities and Instruments & Actors/Stakeholders & Timeframe & Priority \\
\hline \multicolumn{4}{|c|}{ Specific objective 1: promoting the values of heritage } \\
\hline $\begin{array}{c}\text { Promotion of the cultural and historical heritage of Novi Pazar } \\
\text { through various discussions, forums, media or through } \\
\text { promotional material }\end{array}$ & $\begin{array}{l}\text { The City of Novi Pazar, Media, schools, } \\
\text { NGOs }\end{array}$ & Permanent & High \\
\hline $\begin{array}{c}\text { Organizing discussions and forums about the importance of } \\
\text { preserving cultural heritage, with the inclusion of a large } \\
\text { number of stakeholders }\end{array}$ & $\begin{array}{l}\text { The City of Novi Pazar, Media, schools, } \\
\text { NGOs, State University of Novi Pazar }\end{array}$ & Permanent & High \\
\hline $\begin{array}{l}\text { Introduction of sections in local schools that would study the } \\
\text { culture and cultural heritage of Novi Pazar and the } \\
\text { whole region }\end{array}$ & $\begin{array}{c}\text { The City of Novi Pazar, secondary and } \\
\text { primary schools, State University of } \\
\text { Novi Pazar }\end{array}$ & $\begin{array}{c}\text { Mid-range } \\
\text { (12-24 months) }\end{array}$ & Small \\
\hline \multicolumn{4}{|c|}{ Specific objective 2: involvement of citizens in the decision-making process } \\
\hline $\begin{array}{c}\text { Surveys and questionnaires for all residents and shop owners } \\
\text { in the historic center during all stages of the } \\
\text { strategy development }\end{array}$ & The City of Novi Pazar, media & Permanent & High \\
\hline $\begin{array}{c}\text { Organizing public forums, workshops, individual encounters, } \\
\text { and interviews before making decisions to balance interests } \\
\text { and problems }\end{array}$ & The City of Novi Pazar, media, NGO's & Permanent & High \\
\hline $\begin{array}{l}\text { Informing citizens about all activities related to the } \\
\text { preservation of the architectural heritage and } \\
\text { historic urban landscape }\end{array}$ & $\begin{array}{l}\text { The City of Novi Pazar, media, NGO's, } \\
\text { Local offices of the historic center }\end{array}$ & Permanent & High \\
\hline \multicolumn{4}{|c|}{ Specific objective 3: Improve technical protection measures for cultural heritage and building restoration } \\
\hline $\begin{array}{l}\text { Professional and financial support of private owners in the } \\
\text { design of conservation and restoration projects }\end{array}$ & Local offices of the historic center & $\begin{array}{l}\text { Mid-range } \\
(12 \text { months) }\end{array}$ & High \\
\hline $\begin{array}{l}\text { Carrying out a pilot project for the restoration of a cultural } \\
\text { and historical object in order to effectively promote restoration }\end{array}$ & $\begin{array}{l}\text { the City of Novi Pazar, Institutes for the } \\
\text { Protection of Monuments }\end{array}$ & $\begin{array}{l}\text { Mid-range } \\
\text { (12 months) }\end{array}$ & High \\
\hline $\begin{array}{l}\text { Holding training workshops for heritage } \\
\text { conservation professionals }\end{array}$ & $\begin{array}{l}\text { the City of Novi Pazar, Institutes for the } \\
\text { Protection of Monuments, State University } \\
\text { of Novi Pazar }\end{array}$ & $\begin{array}{l}\text { Short } \\
(1 \text { month) }\end{array}$ & Middle \\
\hline \multicolumn{4}{|c|}{ Specific objective 4: Speeding up procedures for issuing building permits } \\
\hline Opening a local office for the historic center & Ministry of Culture & $\begin{array}{c}\text { Mid-range } \\
\text { (12 months) }\end{array}$ & High \\
\hline Opening a local office for the historic downtown & the City of Novi Pazar & $\begin{array}{l}\text { Mid-range } \\
\text { (3 months) }\end{array}$ & High \\
\hline $\begin{array}{l}\text { Facilitating procedures for the renovation of buildings by } \\
\text { creating catalogs for typical interventions }\end{array}$ & $\begin{array}{l}\text { the City of Novi Pazar, Institute for Urban } \\
\text { Planning of Novi Pazar }\end{array}$ & $\begin{array}{l}\text { Mid-range } \\
\text { (3 months) }\end{array}$ & High \\
\hline
\end{tabular}

Raising awareness about the value of cultural and architectural heritage should be directed to all citizens and all age groups. When implementing these programs, it is important to identify and involve all relevant actors, both when drawing up these plans and later in their implementation.

Furthermore, to achieve this goal it is of utmost importance to use a variety of participatory techniques, such as workshops, roundtables, and public consultations, that would all serve as a platform for establishing a dialogue among various stakeholders and their interests. If practiced in this particular way, the process can eventually gain legitimacy and, in the end, secure the commitment of various stakeholders. 


\section{Conclusions}

The existing model of planning and management of historic urban landscapes in Serbia does not contribute to the adequate preservation of the architectural heritage and does not correspond with the interests of local development. Numerous examples have shown that existing plans are inflexible, inefficient and unrealistic when concerning the requirements for the development of historic urban landscapes. Although this area is regulated by a whole set of development and planning decisions, the example presented in this paper, of the case of Novi Pazar, shows several functional, economic, social and ambient problems, and aspects of obsolescence, that these areas face. The physical changes that take place in these areas often do not match their historical character, thus the result is degradation, obsolescence, devastation or the creation of a so-called quasi-cultural heritage. Although some formal shifts have been made in the structure and content of various aspects of urban plans in recent years, there is still no positive step towards the protection and development of historic urban landscapes in Serbia.

All things considered, the research reveals that the most important goals of urban regeneration, protection and the development of historic urban landscapes are related to the potential for economic development, improved quality of life for residents and tourists, and the conservation of built and cultural heritage while promoting cultural and historical identity.

Given these points, it is important to underline that, although particular urban revitalization plans and projects have to be adapted to specific contexts and situations, in order to be successful and represent a benchmark for other places they should include the following objectives and instruments:

- Provide applicable methods, strategies, instruments, and procedures for the urban regeneration of historic city centers, including the protection and conservation of cultural heritage and the rehabilitation of protected buildings with historic value;

- Secure zoning and building regulations which are flexible to heritage issues, stimulate urban regeneration and development, and promote the refurbishment and adaptive reuse of built heritage, rather than demolition and redevelopment;

- Deliver economic and financial instruments for funding the reconstruction of public infrastructure, public spaces and the rehabilitation of private buildings;

- Provide economic and financial instruments for enabling the economic development of the heritage area in order to create a favorable environment for the involvement of the private sector through public-private partnerships;

- Obtain the participation of residents of historic city centers and other relevant civil sector stakeholders' in the regeneration process using a number of various participatory techniques, to gain legitimacy and secure their commitment, while taking their needs into account in relation to the potentials and limitations of the area in question in order to secure long-term public benefits for the society and the values of tangible heritage.

Monitoring and evaluation of the selected actions is necessary throughout the implementation phase in order to obtain an efficient and effective process. Therefore, it is necessary to form a criteria and indicators for the success of the realization in order to facilitate the monitoring and the accomplishment of tasks. Depending on the necessary time, resources and complexity of procedures, the ranking of the measures given could be easy, medium, and challenging. This process is essential to make recommendations for the design of future models in order to interlink strategic planning and development with historical urban landscapes.

In this respect, a useful innovative instrument for the governance of the urban regeneration projects-particularly the economic appraisal within projects that could be used in a public-private partnership—is the experimental model of the "economic feasibility project for the development of unused public buildings" called SOSTEC [44]. Besides the fact that the model provides an economic evaluation of the real estate adaptive re-use projects-including criteria to assess costs and revenues for better insight into economic sustainability-equally important is that the model allows for the involvement of local stakeholders' knowledge and points of view about the ongoing or implemented 
programs. These previously mentioned points of the model characteristics - in our view-provide possibilities for tailor-made solutions at the local level of governance. This model and approach could be a very useful instrument both during the conception of the strategic and action plan, as well as during the implementation phases for monitoring and reevaluation.

Besides the conceptual strategic planning and management model for the regeneration of historic urban landscapes, based on a methodology for the sustainable appraisal of the historic environment [45], measuring and evaluating culture's contribution to regeneration [5], the model for measuring the sustainability level of historic urban quarters [27] and our own research, the paper develops spatial, economic, and social goals, indicators and effects of regeneration strategies of historic city center, presented in Table 4.

Table 4. Spatial, economic, and social goals, sustainability indicators and effects of regeneration strategies of historic city centers.

\begin{tabular}{|c|c|c|}
\hline $\begin{array}{c}\text { Spatial and } \\
\text { Environmental } \\
\text { Regeneration }\end{array}$ & $\begin{array}{c}\text { Economic } \\
\text { Regeneration }\end{array}$ & $\begin{array}{c}\text { Social } \\
\text { Regeneration }\end{array}$ \\
\hline $\begin{array}{c}\text { Strategic goals } \\
\text { Sustainable development } \\
\text { Quality of life and } \\
\text { liveability } \\
\text { Mixed-use/ compact city (workplaces and residences) } \\
\text { Functional diversity } \\
\text { Heritage conservation } \\
\text { Spatial revitalization and adaptive reuse } \\
\text { Accessibility and mobility }\end{array}$ & $\begin{array}{c}\text { Strategic goals } \\
\text { Competitiveness and growth } \\
\text { Un/Employment, job quality } \\
\text { Inward investment } \\
\text { Wealth creation } \\
\text { SMEs/micro-enterprises } \\
\text { Innovation and knowledge } \\
\text { Clusters } \\
\text { Evening Economy }\end{array}$ & $\begin{array}{c}\text { Strategic goals } \\
\text { Social inclusion } \\
\text { Social cohesion } \\
\text { Neighborhood Renewal } \\
\text { Health and well-being } \\
\text { Cultural and historical identity } \\
\text { Social capital } \\
\text { Governance } \\
\text { Cultural diversity } \\
\text { Citizenship }\end{array}$ \\
\hline $\begin{array}{c}\text { Performance indicators (quantitative and/or } \\
\text { qualitative) } \\
\text { Quality of life indicators (economic environmental, } \\
\text { health, etc.) }{ }^{1} \\
\text { Reuse of derelict sites and developed property } \\
\text { concerning the historic character } \\
\text { Active use of individual buildings and open public } \\
\text { and inner courtyard spaces } \\
\text { Land/building occupation } \\
\text { Higher densities } \\
\text { Number of listed buildings } \\
\text { Use of traditional building materials } \\
\text { and techniques (new/adapted buildings) } \\
\text { Conservation areas } \\
\text { Vacancy rates } \\
\text { Accessibility level (block size, connecting streets, } \\
\text { sidewalks) } \\
\text { Reduced car-use and public transport/usage } \\
\text { Diversity of mobility modes (including } \\
\text { non-motorized transport) } \\
\text { Car parking spaces (restrictions on-street } \\
\text { parking/successful off-street and out of the historic } \\
\text { center solutions) } \\
\text { Pedestrian and bicycle-friendly streets }\end{array}$ & $\begin{array}{c}\text { Performance indicators (quantitative and/or } \\
\text { qualitative) } \\
\text { Level of income } \\
\text { Spending in an area } \\
\text { New and retained jobs } \\
\text { Employer (re)location } \\
\text { Public-private leverage } \\
\text { Cost-benefit analysis } \\
\text { Input-Output/Leakage } \\
\text { Willingness to pay for } \\
\text { cultural amenities/ } \\
\text { contingent valuation } \\
\text { Multipliers-jobs, spending } \\
\text { Employment diversity } \\
\text { (rate of privately owned business to } \\
\text { public business) } \\
\text { Local handcraft production rate } \\
\text { Land and property prices (minimum/maximum) } \\
\text { The ratio of tourism facilities in the area } \\
\text { Number and size of recreational, cultural and } \\
\text { spiritual sites } \\
\text { Development costs } \\
\text { Maintenance cost } \\
\text { Infrastructure improvements }\end{array}$ & $\begin{array}{c}\text { Performance indicators (quantitative } \\
\text { and/or qualitative) } \\
\text { Attendance/Participation in the } \\
\text { decision-making process } \\
\text { Civic Involvement } \\
\text { Cultural/Racial Diversity } \\
\text { Safety level, crime rates/fear of crime } \\
\text { Health, medical appointment } \\
\text { New community networks } \\
\text { Improved leisure options } \\
\text { Volunteering } \\
\text { Population increase } \\
\text { Income level } \\
\text { and leisure activities } \\
\text { The ratio of public open areas, recreational } \\
\text { Availability and accessibility of public } \\
\text { services } \\
\text { Existing laws and regulations on urban } \\
\text { revitalization } \\
\text { Mixed choice of housing } \\
\text { Housing price to income ratio } \\
\text { Housing tenure types } \\
\text { Housing affordability }\end{array}$ \\
\hline $\begin{array}{c}\text { Effects } \\
\text { Reuse of obsolete buildings and open spaces } \\
\text { Increased public use of space } \\
\text { Culture facilities, housing and } \\
\text { workspace in mixed-use } \\
\text { developments } \\
\text { High density (live/work)- } \\
\text { reduced environmental } \\
\text { impacts (traffic, pollution, health) } \\
\text { The attraction of old crafts, cultural and art uses } \\
\text { Design improvements } \\
\text { through public art and } \\
\text { architecture in public spaces } \\
\text { Accessibility, } \\
\text { public transport usage and } \\
\text { safety } \\
\text { Heritage identity, stewardship, } \\
\text { local distinctiveness } \\
\text { Environmental quality (water, air, noise, visual } \\
\text { pollution levels) } \\
\text { Energy consumption from transport and } \\
\text { cooling/heating of buildings }\end{array}$ & $\begin{array}{c}\text { Effects } \\
\text { Corporate involvement in the } \\
\text { local cultural sector } \\
\text { Retaining young graduates and attracting new } \\
\text { creative businesses/people } \\
\text { (including artists/old handcrafts) } \\
\text { Diversified land uses and workforce } \\
\text { (skills, social, gender and } \\
\text { ethnic profile) } \\
\text { Creative clusters and business improvement districts } \\
\text { Public-private-civil sector } \\
\text { partnerships } \\
\text { Investment and costs of adaptive reuse, public spaces } \\
\text { and infrastructure improvement (public and private) } \\
\text { Maintenance costs (public and private) } \\
\text { Increased land and property values/rents } \\
\text { (residential and business) } \\
\text { Residential and business income level } \\
\text { Higher cultural tourism } \\
\text { spending (residents and tourists) }\end{array}$ & $\begin{array}{c}\text { Effects } \\
\text { A feeling of safety, pride \& local identity } \\
\text { A positive change in residents' } \\
\text { perceptions of their area } \\
\text { Displacing crime and antisocial } \\
\text { behavior through } \\
\text { cultural activity } \\
\text { A clearer expression of } \\
\text { individual and shared ideas } \\
\text { and needs } \\
\text { Increase in volunteering and } \\
\text { organizational } \\
\text { capacity at a local level } \\
\text { A change in the image or } \\
\text { the reputation of a place or group } \\
\text { of people } \\
\text { Stronger public-private- } \\
\text { voluntary-sector partnerships } \\
\text { Increased appreciation of the } \\
\text { value and opportunities to } \\
\text { take part in arts projects } \\
\text { Greater individual confidence } \\
\text { and aspiration }\end{array}$ \\
\hline
\end{tabular}

${ }^{1} 8+1$ dimensions of quality of life [46]. 
Some lessons and recommendations could be drawn here based on the research presented in this paper, which could influence the implementation of an integrative approach to the protection and planning of cultural heritage in the frame of urban development and regeneration. However, an important prerequisite for such an integrative approach is the improvement of the institutional, legal and management framework and strategic planning methodology. Lessons and recommendations are as follows:

- Expanding the concept of cultural heritage to the urban heritage protection for objects and entities that are not declared as cultural heritage monuments in accordance with international recommendations and standards, especially with the Recommendations on the Historic Urban Landscape [9];

- Introducing a territorial approach: cultural heritage is an inseparable part of the living environment, both built and natural, and cannot be separated under the jurisdiction of sectoral institutions; it must be viewed in correlation with its immediate environment and satisfy contemporary functions and users' needs, including residents, investors and tourists;

- Improving the institutional and legal framework for the protection, planning and management of cultural heritage by expanding the subject of protection and strengthening cross-sectoral cooperation through the introduction of an integrative approach to the protection of the heritage;

- Improving the planning methodology through the introduction of an integrative strategic planning approach and concept of urban protection in formal statutory plans and the introduction of practical guides and methodologies for contextual analysis and valuation of the built cultural heritage;

- Introducing strategic planning models, management instruments and a financing system for the protection of heritage-both for individual buildings and historic urban landscapes_and for wider spatial, social, environmental and economic sustainable regeneration, with positive impacts and benefits for the localities from which they originate.

The strategic model for planning the protection and the development of historic urban landscapes is presented in this paper as an adequate planning model, which can represent an effective model for the implementation of a cultural heritage protection strategy in a sustainable way in the light of constant changes, crises, and uncertainties. The paper presents this planning model as a step forward from the conventional planning model, and in doing so, can respond to global and local, social, political and economic changes, aligning the needs and interests of a wide range of actors and stakeholders with realistic development opportunities.

It has been suggested [47] that heritage protection can learn from strategic planning by using various participatory instruments to include different actors' interests according to the contemporary community's needs, thus serving the public good. Their research also underlines the proper combination of various economic, social, and environmental instruments for achieving sustainable, comprehensive visions of development.

In order to secure long-lasting, sustainable and comprehensive visions of development, a condition is to have the significant relevant stakeholders engagement throughout the process, which "can bring new belief in the community that their specific places could be successfully branded and planned based on cohesive spatial visions" [40].

We conclude by giving theoretical and practical remarks from our research: integrated conceptual strategic urban planning model should consider the logic and the functioning of the competitive real estate markets on the one hand, and sustainable environmental, economic and social effects, as well as the potentials and benefits for the locality where they originate on the other hand, in order to be utilized as the new generative value both for the protection and for the revival of historic city centers.

Author Contributions: Conceptualization, E.M. and U.R.; Funding acquisition, E.M. and U.R.; Investigation, E.M., U.R. and D.B.; Methodology, E.M., U.R. and D.B.; Visualization, E.M. and U.R.; Writing-original draft, E.M. and U.R.; Writing-review and editing, E.M., U.R. and D.B. All authors have read and agreed to the published version of the manuscript. 
Funding: This research was funded by the Ministry of Education, Science and Technological Development of the Republic of Serbia, under the Grant number TR 36035 "Spatial, Environmental, Energy and Social Aspects of Development of Settlements and Climate Change".

Acknowledgments: This paper is partially the result of the ongoing doctoral research by Esad Muminović, and partially the result of the research within the TR 36035 "Spatial, Environmental, Energy and Social Aspects of Development of Settlements and Climate Change" by the Ministry of Education, Science and Technological Development of the Republic of Serbia conducted by Uroš Radosavljević.

Conflicts of Interest: The authors declare no conflict of interest.

\section{References}

1. Van Oers, R.; Pereira Roders, A. Historic cities as model of sustainability. J. Cult. Herit. Manag. Sustain. Dev. 2012, 2, 4-14. [CrossRef]

2. Bandarin, F.; Van Oers, R. The Historic Urban Landscape-Managing Heritage in an Urban Century; Wiley-Blackwell: Chichester, UK, 2012.

3. Getty Conservation Institute. Historic Urban Environment Conservation Challenges and Priorities for Action Experts Meeting; The Getty Conservation Institute: Los Angeles, CA, USA, 2010; Available online: http://www. getty.edu/conservation/publications_resources/pdf_publications/pdf/experts_mtg_mar09.pdf (accessed on 4 October 2019).

4. Tweed, C.; Sutherland, M. Built cultural heritage and sustainable urban development. Landsc. Urban Plan. 2007, 83, 62-69. [CrossRef]

5. Evans, G. Measure for Measure: Evaluating the Evidence of Culture's Contribution to Regeneration. Urban Stud. 2005, 42, 959-983. [CrossRef]

6. UNESCO. Recommendation Concerning the Safeguarding and Contemporary Role of Historic Areas; UNESCO: Warsaw, Poland; Nairobi, Kenya, 1976.

7. ICOMOS. Charter for the Conservation of Historic Towns and Urban Areas (Washington Charter); ICOMOS: Washington, DC, USA, 1987.

8. UNESCO. Vienna Memorandum on 'World Heritage and Contemporary Architecture-Managing the Historic Urban Landscape'; UNESCO World Heritage Centre: Paris, France, 2005.

9. UNESCO. Recommendation on the Historic Urban Landscape; UNESCO World Heritage Centre: Paris, France, 2011.

10. Jokilehto, J. International Charters on Urban Conservation: Some Thoughts on the Principles Expressed in Current International Doctrine. City Time 2007, 3, 23-42.

11. Huete-Alcocer, N.; López-Ruiz, V.R.; Grigorescu, A. Measurement of Satisfaction in Sustainable Tourism: A Cultural Heritage Site in Spain. Sustainability 2019, 11, 6774. [CrossRef]

12. Tsenkova, S. Urban Planning and informal cities in Southeast Europe. J. Archit. Plan. Res. 2012, 29, $292-305$.

13. German Association of Cities. Strategies and instruments for sustainable urban development. In Integrated Urban Development Planning and Urban Development Management; The German Association of Cities: Berlin, Germany, 2013.

14. Yin, R.K. Case Study Research: Design and Methods, 2nd ed.; Sage: Thousand Oaks, CA, USA, 2009.

15. Flyvbjerg, B. Five Misunderstandings About Case-Study Research. Qual. Inq. 2006, 12, 219-245. [CrossRef]

16. Radosavljević, U. Formation of the Urban Management Model in the Implementation of Strategic Projects. Ph.D. Thesis, University of Belgrade-Faculty of Architecture, Belgrade, Serbia, 2014.

17. Castells, M. The Rise of the Network Society, 2nd ed.; Blackwell Publishing: Oxford, UK, 2010; Original Work Published 1996; Volume I.

18. Tiesdell, S.; Oc, T.; Heath, T. Revitalizing Historic Urban Quarters; Architectural Press: New York, NY, USA, 1996.

19. Doratli, N. Revitalizing historic urban quarters: A model for determining the most relevant strategic approach. Eur. Plan. Stud. 2005, 13, 749-772. [CrossRef]

20. Azpeitia Santander, A.; Azkarate Garai-Olaun, A.; De la Fuente Arana, A. Historic Urban Landscapes: A Review on Trends and Methodologies in the Urban Context of the 21st Century. Sustainability 2018, 10, 2603. [CrossRef] 
21. Fainstein, S. The City Builders. Property Development in New York and London, 1980-2000, 2nd ed.; University Press of Kansas: Lawrence, KS, USA, 2001; Original Work Published 1994.

22. Vedung, E. Policy Instruments: Typologies and Theories. In Carrots, Sticks, and Sermons: Policy Instruments and Their Evaluation; Bemelmans-Videc, M.L., Rist, R.C., Vedung, E., Eds.; Transaction Publishers: Piscataway, NJ, USA, 1998; pp. 21-58.

23. Amirtahmasebi, R.; Orloff, M.; Wahba, S.; Altman, A. Regenerating Urban Land: A Practitioner's Guide to Leveraging Private Investment; Urban Development Series; World Bank: Washington, DC, USA, 2016.

24. Rypkema, D.D. Heritage and Development-The Role of Public-Private Partnerships. In Economics and Built Heritage-Towards New European Initiatives; Mälkki, M., Ed.; Helsinki University of Technology: Helsinki, Finland, 2008.

25. Radosavljević, U. Conditions Influencing Waterfront Development and Urban Actors Capacity as a Strategic Response. Spatium Int. Rev. 2008, 17-18, 78-83. [CrossRef]

26. Latham, D. Creative Re-Use: Working with the Building. J. Archit. Conserv. 1999, 5, 7-23. [CrossRef]

27. Oktay Vehbi, B.; Önal Hoşkara, Ş. A Model for Measuring the Sustainability Level of Historic Urban Quarters. Eur. Plan. Stud. 2009, 17,715-739. [CrossRef]

28. Despotović, J. Očuvanje kulturne baštine-U svetlu naših i evropskih propisa. Leskov. Zb. 2007, XLVII, 19-28.

29. The Government of the Republic of Serbia. The Spatial Plan of the Republic of Serbia (in Serbian); Official Gazette of the Republic of Serbia: Belgrade, Serbia, 2010; no. 88/10.

30. The Government of the Republic of Serbia. Sustainable Urban Development Strategy of the Republic of Serbia until 2030 (in Serbian); Official Gazette of the Republic of Serbia: Belgrade, Serbia, 2019; no. 47/19.

31. The United Nations Conference on Human Settlements. HABITAT III Global Human Settlements Conference. [Internet]. 2016. Available online: http://habitat3.org/the-conference/about-habitat-3/ (accessed on 17 October 2019).

32. Vujošević, M. Collapse of strategic thinking, research and governance in Serbia and possible role of the spatial plan of the Republic of Serbia (2010) in its renewal. Spatium Int. Rev. 2010, 23, 22-29. [CrossRef]

33. Stanilov, K. Urban Planning and the challenges of post-socialist transformation. In The Post-Socialist City-Urban Form and Space Transformations in Central and Eastern Europe after Socialism; Stanilov, K., Ed.; Springer: Dodrecht, The Netherlands, 2007; pp. 413-426.

34. Salet, W.; Faludi, A. The Revival of Strategic Spatial Planning; Royal Netherlands Academy of Arts and Sciences: Amsterdam, The Netherlands, 2000.

35. Križanić, T.P.; Šovljanski, R. Priručnik za Strateško Planiranje i Upravljanje Lokalnim Razvojem u Republici Srbiji; Friedrich Ebert Stiftung: Novi Sad, Serbia, 2010.

36. Statistical Office of the Republic of Serbia: Census 2011. Available online: https://www.stat.gov.rs/en-US/ oblasti/popis/popis-2011 (accessed on 15 November 2019).

37. Premović-Aleksić, D. Islamski Spomenici Novog Pazara; Muzej Ras Novi Pazar: Novi Pazar, Serbia, 2013.

38. Popadić, M. Architect Amir Ćorović and Project of City Center in Novi Pazar. Novopazar. Zb. 2009, 32, 209-225.

39. Nešković, J.; Folić, N.; Đorđević, S.; Radović, R. Stara Čaršija u Novom Pazaru; Zavod za Zaštitu Spomenika Kulture Kraljevo: Kraljevo, Serbia, 1988.

40. Radosavljević, U.; Đorđević, A.; Živković, J.; Lalović, K.; Đukanović, Z. Educational projects for linking place branding and urban planning in Serbia. Eur. Plan. Stud. 2019. [CrossRef]

41. Kadijević, A. O proučavanju i vrednovanju graditeljskog nasleđa novijeg doba u Novom Pazaru. Novopazar. Zb. 1995, 19, 193-203.

42. Mulić, I. Komponente Održivog Razvoja Tradicionalnog Neimarstva Sandžaka-Magistarska Teza; Evropski Centar za mir i Razvoj EPCD: Novi Pazar, Serbia, 2008.

43. The Government of the Republic of Serbia. Ordinance on the Energy Performance of Buildings [in Serbian]; Official Gazette of the Republic of Serbia: Belgrade, Serbia, 2011; No. 61/2011.

44. Calabrò, F.; Della Spina, L. The Public-Private Partnership for the Enhancement of Unused Public Buildings: An Experimental Model of Economic Feasibility Project. Sustainability 2019, 11, 5662. [CrossRef] 
45. Stubbs, M. Heritage-sustainability: Developing a methodology for the sustainable appraisal of the historic environment. Plan. Pract. Res. 2004, 19, 285-305. [CrossRef]

46. The European Commission: Quality of Life Indicators-Measuring Quality of Life. Available online: https://ec.europa.eu/eurostat/statistics-explained/index.php/Quality_of_life_indicators_-_measuring_ quality_of_life\#Framework_for_measuring_quality_of_life2019 (accessed on 12 September 2019).

47. Radosavljević, U.; Đorđević, A.; Lalović, K.; Živković, J.; Đukanović, Z. Nodes and Networks: The Generative Role of Cultural Heritage for Urban Revival in Kikinda. Sustainability 2019, 11, 2509. [CrossRef]

(C) 2020 by the authors. Licensee MDPI, Basel, Switzerland. This article is an open access article distributed under the terms and conditions of the Creative Commons Attribution (CC BY) license (http://creativecommons.org/licenses/by/4.0/). 



\title{
Paradoxes of the Italian Historic Centres between Underutilisation and Planning Policies for Sustainability
}

\author{
Paola Pellegrini ${ }^{1, *}$ and Ezio Micelli ${ }^{2}$ \\ 1 Department of Urban Planning and Design, Xi'an Jiaotong-Liverpool University, Suzhou 215123, China \\ 2 Department of Architecture and Arts, Università IUAV di Venezia, 30123 Venice, Italy; micelli@iuav.it \\ * Correspondence: paola.pellegrini@xjtlu.edu.cn; Tel.: +86-512-8188-4774
}

Received: 9 March 2019; Accepted: 16 April 2019; Published: 7 May 2019

\begin{abstract}
The paper presents the analysis of the statistical data on population and real estate in 20 small-to-medium-sized cities in Northern Italy and shows a high rate of vacancy of housing and significant shrinkage of businesses and institutions in the historic centres, where urban heritage is concentrated. Given these findings, the paper analyses the official city plans of the cities with the worst underutilisation conditions, to understand how the plans have reacted to the decline of the centre. The result shows the extensive planning and regulation activity has very limitedly registered the phenomenon and failed to propose the empty inner cores as resources to reduce land consumption and recycle valuable assets in a circular economic vision. Combining the statistical data and the findings from the city plans, the paper concludes that Italian historic centres are living paradoxes-a collection of beauty, icon of well-being, model of sustainability, but abandoned-and therefore, the dense regulatory mechanisms that were necessary to conserve urban heritage during the decades of economic and demographic growth must be reframed to implement a circular economy and adapt to new requirements for living conditions.
\end{abstract}

Keywords: urban heritage; historic centre; regulation; sustainability; city plan

\section{Introduction and Research Method}

Historic centres are the identity and heritage of Italian cities and have for decades been at the centre of cultural debate and administrative action, leading to extensive regulation activities and planning documents to conserve and restore them [1-9]. However, the interpretation of demographic movements and dynamics of uses over the past 30 years suggest that the effects of the planning policies and regulations on the settlement choices should be questioned because the internationally recognized heritage of the centres, one of the most valuable assets in urban Italy, is underutilized and therefore, devitalized and wasted. The issue of the underutilisation of the historic centres has not been studied in detail considering the long time span yet, even though the survey of the Associazione Nazionale Centri Storici Artistici has greatly contributed [10], its relation with plans and regulations have not been analysed; some literature deals with the problem of the regeneration of the historic centres [11-14], but none deals specifically with the reaction of planning instruments to underutilisation and little public discussion debates it. The research presented tries to fill this void and is the second phase of an on-going investigation.

The first phase of the research analyzed the Census data of 1991-2011 of demographic movements and property dynamics of 14 small-to-medium-sized cities in Northern Italy $[15,16]$. The findings show severe underutilisation in the city centres and urge to question the effectiveness of the planning approach and regulations. The research method of the second phase involved the following steps: 
- We increased the number of cities we investigated to 20 in order to cover the whole of Northern Italy; the same phenomena found in the first 14 cases were found also in the six new ones.

- Selection of the cities to further analyze according to the findings: The cities with the worst condition of vacancy were selected because the phenomenon in these cities is so evident that local planning activity should have tried to deal with the issue; the paper claims the city plans are not the main factor which caused the vacancy, but a mix of socio-economic causes. Therefore, the paper explores how planning activity reacts to the phenomenon where it is most observable.

- Collection of the city plans available in the official web page of the municipalities: The general planning documents and those specifically related to heritage conservation (if any) were collected.

- Analysis of the texts and maps of the plans, both in the descriptive parts (focusing on which phenomena are impacting the city according to the documents and if underutilisation is recorded among them) and in the design part (focusing on what development is proposed, what importance is given to sustainability, which actions are defined for the city centre and what role it is given in the future of the city). The selected cities are in different regions, and every region in Italy has a city planning law. Therefore, the planning processes are slightly different and the documents cannot be directly compared.

- Assessment of the conceptual and practical link between the phenomena of underutilisation and the choices of the plans for sustainability, that is to say if and how the "empty" built heritage in historic city centres is given a role to support the sustainable development of cities in the perspective of a circular economy and according to the International Charters and debate about urban development, which widely promote the connection of built urban heritage and sustainability [17-19].

- Conclusions, both expressed as paradoxes and challenges.

The structure of the paper is as follows: The second paragraph presents the results of the quantitative research about the underutilisation of the building stocks in the historic centres of small-to-medium-sized cities in Northern Italy between 1991 and 2011; the third paragraph presents the results of the qualitative research regarding the planning instruments, which define the future development of the city. The fourth paragraph introduces some paradoxes of the historic city centres, that is to say, the essential characters which should determine the value of the historic core, but fail to do so; these paradoxes should be the starting point for the re-framing of the cultural approach and the policies for the urban heritage, which is proposed in the last paragraph.

Cases of gradual decline over the last 20 years of the historic city centres in small-to-medium-sized cities can be found in the whole of Europe: Shrinkage in population, underutilized stock and diminished importance as places of exchange and congregation are widespread phenomena [20-24]. This paper wants to contribute to the academic and professional debate about this decline presenting the North Italian case, which is relevant because of the great value of its urban heritage, the extensive protection measures applied since the 1960s and it is a place where even centres enlisted in the United Nations Educational, Scientific and Cultural Organization (UNESCO) world heritage are underutilized.

\section{Evidence of Underutilisation of the Built Stock in Historic Centres of Small-to-Medium-Sized Cities in Northern Italy}

The research analyzed the Italian Census data from 1991 to 2011, the last comprehensive available one, of 20 small-to-medium-sized cities in six regions of Northern Italy: Friuli Venezia Giulia, Veneto, Trentino Alto Adige, Emilia Romagna, Lombardia, Piemonte [15,16]. There is no official classification in Italy of what a small-to-medium-sized city is; the research adopted the range between 30,000 and 200,000 inhabitants, which is pertinent to the dimension of the urban centres in Italy [25-27].

The analyzed cities are Udine, Pordenone, Treviso, Vicenza, Conegliano, Bassano del Grappa, Ravenna, Ferrara, Parma, Modena, Brescia, Mantua, Cremona, Bergamo, Pavia, Trento, Rovereto, Novara, Alba, Alessandria. All their city centres conserve a built heritage of exceptional value, some of them-Vicenza, Mantua, Ferrara-are listed as historic centres in the UNESCO World Heritage 
Sites, while some others-Modena, Pavia, Ravenna-have monuments included in the same list. The analyzed cities are a very relevant sample of the urban world of Northern Italy and host almost two million people of whom almost $10 \%$ lived in the historic centres in 2011, see Figure 1, Table 1).

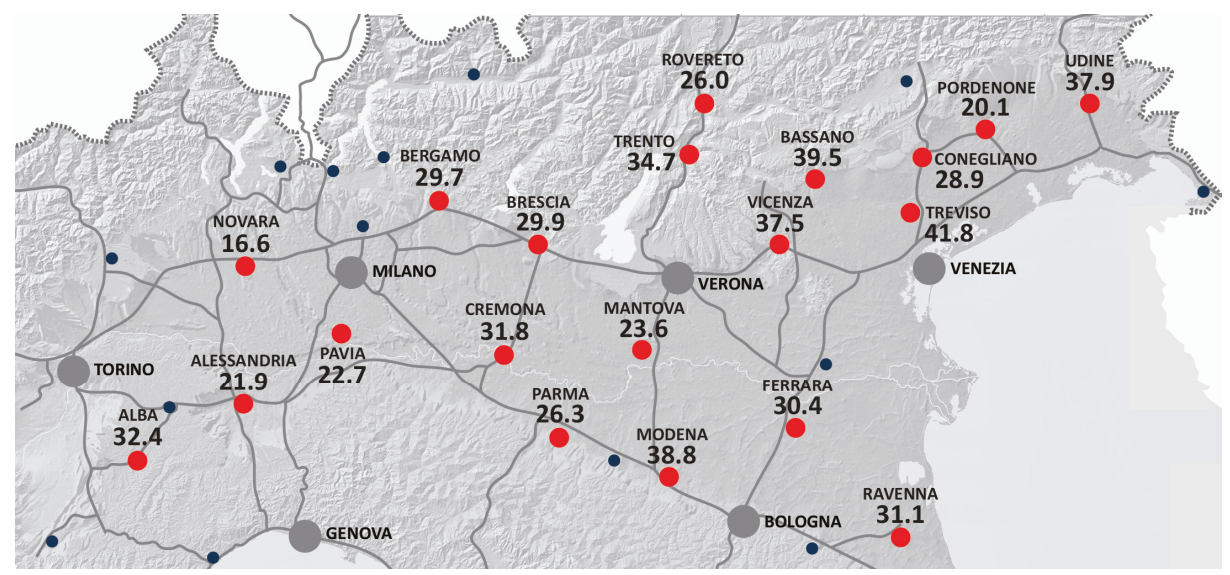

Figure 1. The 20 cities in Northern Italy; the numbers are the percentage of housing units not occupied in the ZTL.

The municipal territory of every city was divided into three areas to obtain specific results and compare dynamics: The inner part of the historic centre identified as the controlled traffic zone (ZTL), the rest of the historic centre defined by the last defensive walls and the rest of the municipality.

The analysis shows unoccupied housing (labelled "abitazioni vuote" by the Census) in the entire historic centre increased on average from $16 \%$ to $26 \%$ and systematically exceeded the other urban areas where the share of the unoccupied stock increased from $9 \%$ to $14.2 \%$. The situation is even more significant in the ZTL: In these areas, the percentage of unoccupied housing increased from $18.8 \%$ to $30 \%$.

The vacancy rate in ZTL increases significantly in some cities, such as Udine, Treviso and Modena when compared to the rate of the other historic centres; in Treviso, for example, from 1991 to 2011, the percentage of unoccupied housing reached $41.8 \%$, whereas it was $21.4 \%$ twenty years previously. The vacancy does not appear to be related to specific parts of the historic centre but appears to be a widespread process; in fact, in the majority of the centres it does not involve buildings in their entirety and this partial utilisation mitigates the perception of the phenomenon.

The vacancy rate, moreover, does not relate to the degradation of built stock nor is the decline of the population due to neglect: The large majority of buildings in northern Italian city centres, especially those whose heritage is particularly valuable, were largely restored and renovated in the 1980s and 1990s, as were the large majority of public open spaces; therefore, they are an available stock in acceptable conditions.

The value of these empty units has an absolute economic significance; in fact, the sum is 33,775 units out of a total of 130,084 in 2011, with an increase of 17,642 units since 1991. If this empty stock is assigned an under-estimated value of 1000 euros/sqm and an average surface of $100 \mathrm{~m} 2$-the average size resulting from the analysis the Census Data for the 20 city centres-it sums up to a value of 3.37 billion euros, $0.2 \%$ of the Italian GDP in 2011. 


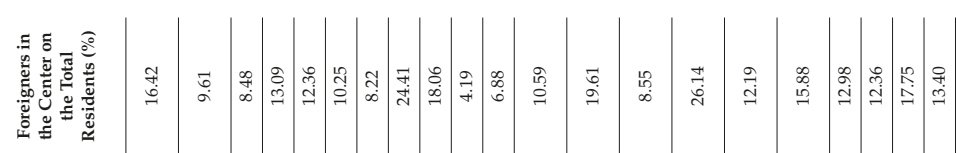

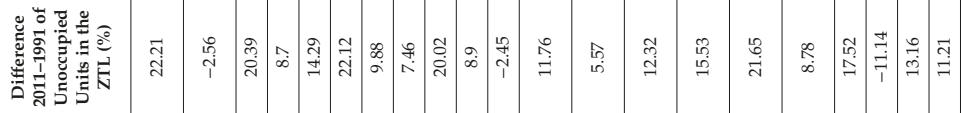

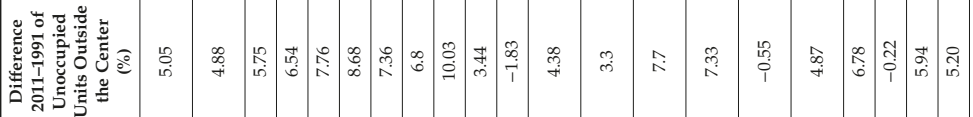

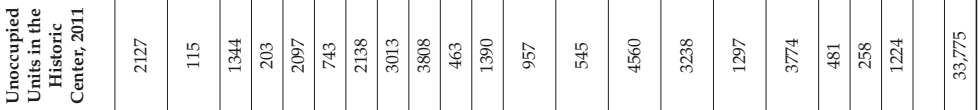

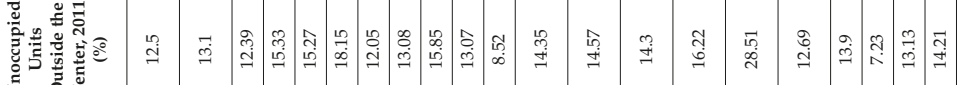

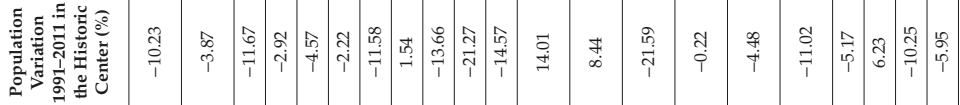

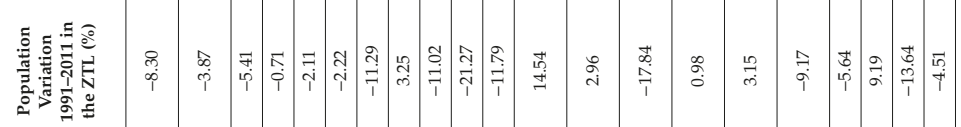

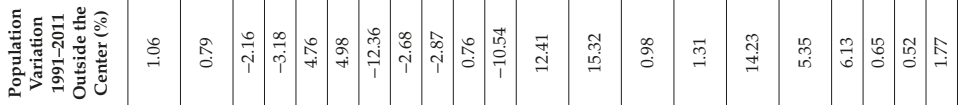

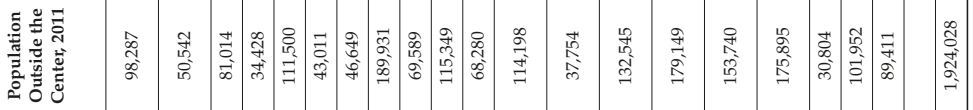

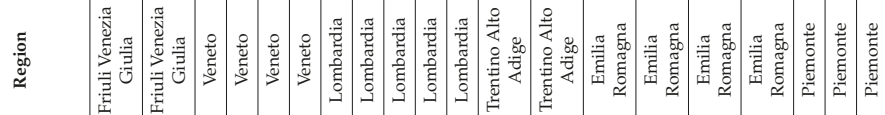

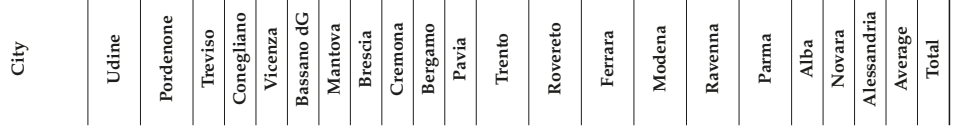


In the 20 centres that were analyzed, the population is decreasing: -20.118 units since 1991, on average $-10.35 \%$, but exceeds $-20 \%$ in Ferrara and Bergamo. This decrease is not directly proportional to the percentage of housing vacancy, and is clearly less relevant; this difference may be related to the deaths of single elderly people who often live in the historic centres and the arrival of foreigners. In fact, despite being almost irrelevant at the beginning of the 1990s, in twenty years, foreign residents have become a significant percentage of the inhabitants of the oldest parts of the city - in Brescia, foreign residents exceed a $24 \%$ share of the population, in Modena this share reaches $26 \%$-with an increase of twenty times compared to the figures of the early 1990 s.

The abandonment concerns not only residential units but also companies and institutions. The results regarding the companies operating in the historic centres are not homogeneous among the 20 cases and, very often, companies have not decreased in number, but have been re-organized with a significant reduction in employees, decreased by between one-fourth and one-fifth when compared to 1991 , with an average decrease of over twenty percentage points $(-20.1 \%)$.

The results regarding institutions in the historic centres are more clear-cut. The density of institutions in the historic centre-the units per hectare-continues to be higher than in the rest of the municipality, but has suffered a drastic shrinkage, decreasing between two-thirds and four-fifths compared to 1991 . The variation in institutions is homogeneous and indifferent to the regional location and the total local units declined by more than $70 \%(-73.9 \%)$ in the twenty years considered. Similarly, the institutions' employees decreased in all cases, with an average reduction of one-third. The average decrease in the rest of the municipal territories is also important, but minor: $-58.3 \%$ for the local units. This diminution is partly due to the rationalization of office premises following mergers and partly due to relocation in more accessible areas of the city.

The interpretation of demographic movements and property dynamics of the last thirty years in 20 small-to-medium-sized cities in the whole of Northern Italy reveals severe underutilization; original inhabitants have been abandoning the centres, partially substituted by immigrants, enterprises are significantly reduced, and many institutions have opted for new locations. The choice of new locations by households, public institutions and businesses is exceptional as well as paradoxical because the abandonment involves parts of the city that have most benefited from the community's resources for centuries, constantly improving infrastructures and public spaces both in the quality of service and beauty.

\section{The Missed Opportunity of Promoting the Re-cycling of the City Centres in the City Planning Instruments}

Has the city planning activity considered the underutilisation of the cores? Has it considered it as a problem? The theme is central for the development of Italian cities in this period of urban reuse, when a circular economy and sustainability concepts should be considered. In fact, if the cities' agenda takes on a more rational use of the land through the careful selection of projects which consume land, in particular, affecting the primary sector where there is a concern about food production, and if the regeneration of properties through physical as well as social redevelopment is considered a priority, then the historic centre of Italian cities becomes a fundamental test of adapting a stock of primary cultural, economic and social value to contemporary needs and wishes.

Even though precise numbers for a large sample of cities had not been produced until very recently $[10,15,16]$, the abandonment of the historic centre has been perceivable for several years in Italy. Therefore, it is plausible to hypothesize city planning documents have registered it and proposed to recycle the empty stock, adopting a circular economy approach, which integrates sustainable production as well as better use of resources. To understand the reaction to the phenomenon, the research explored the city planning documents of the cities with the worst condition of not-occupied units in the most valuable part of the city, the ZTLs. The paper claims the city plans are not the main factor which caused the underutilisation, but that it was a mixture of socio-economic causes, see paragraph 4.4 and Conclusions. Therefore, the paper explores how planning activity reacts to the phenomenon where it 
is most observable. The selected cases are Treviso, Modena, Udine, Vicenza where, in 2011, the empty units in ZTL reached $41.8 \%, 38.8 \%, 37.9 \%, 37.5 \%$ of the total units, respectively, see Table 1 .

The documents are available online in the official webpages of the municipalities. The research analyzed if and how the city planning documents react to the underutilisation phenomena, see Table 2:

- $\quad$ if the underutilization is reported;

- if sustainability is among the goals of the city;

- if the built heritage in the centre is considered an asset to exploit and reduce the consumption of land, establishing in this way a direct link between the phenomena and an efficient and sustainable use of resources;

- if innovative rules are established to both facilitate the regeneration process and conserve the heritage;

- if new developments and land consumption are planned.

Table 2. Table comparing the city plans.

\begin{tabular}{|c|c|c|c|c|}
\hline & Treviso & Udine & Modena & Vicenza \\
\hline $\begin{array}{l}\text { Region with a City } \\
\text { Planning Legislation }\end{array}$ & Veneto & $\begin{array}{l}\text { Friuli Venezia } \\
\text { Giulia }\end{array}$ & Emilia Romagna & Veneto \\
\hline Number of Inhabitants 2011 & 81,000 & 98,000 & 179,000 & 111,500 \\
\hline Empty Units in ZTL in 2011 (\% Total) & 41.8 & 38.8 & 37.9 & 37.5 \\
\hline Empty Units in the Historic Center & 2,127 & 1,344 & 3,238 & 2,097 \\
\hline Population Variation 1991-2001 in ZTL & -5.41 & -8.30 & 0.98 & -2.11 \\
\hline $\begin{array}{l}\text { Type of Plan Governing the } \\
\text { City Development }\end{array}$ & $\begin{array}{l}\text { Strategic plan PAT and } \\
\text { action plan PI }\end{array}$ & $\begin{array}{l}\text { Structure plan PS } \\
\text { and city plan PRGC }\end{array}$ & $\begin{array}{l}\text { Structure plan PCS and } \\
\text { action plan POC }\end{array}$ & $\begin{array}{l}\text { Strategic plan PAT, } \\
\text { action plan PI, } \\
\text { master plan of the } \\
\text { historic center PCS }\end{array}$ \\
\hline Date of Enforcement of the Plan & $\begin{array}{l}\text { PAT 2015, PI? (new adoption } \\
\text { 2018, not approved yet) }\end{array}$ & 2012 & $\begin{array}{c}\text { PCS 2013, POC 2014 } \\
\text { (new adoption 2018, not } \\
\text { approved yet) }\end{array}$ & $\begin{array}{l}\text { PAT 2010, PI 2013, } \\
\text { PCS } 1979\end{array}$ \\
\hline $\begin{array}{l}\text { The Plan Reports the Under-Utilization } \\
\text { of Units in the City Center }\end{array}$ & Only partially & NO & Yes, since 2006 & $\begin{array}{l}\text { the plan reports a } \\
\text { change in } \\
\text { population, not } \\
\text { underutilization }\end{array}$ \\
\hline $\begin{array}{l}\text { The Plan Defines } \\
\text { Restrictive/non-restrictive rules for the } \\
\text { Transformation of the Heritage }\end{array}$ & $\begin{array}{l}\text { Restrictive (categories of } \\
\text { action in compliance with } \\
\text { Law 456/78) }\end{array}$ & $\begin{array}{c}\text { Restrictive } \\
\text { (categories of } \\
\text { action in } \\
\text { compliance with } \\
\text { Law 456/78) }\end{array}$ & $\begin{array}{l}\text { Restrictive (categories of } \\
\text { action in compliance } \\
\text { with Law 456/78) }\end{array}$ & $\begin{array}{c}\text { Restrictive } \\
\text { (categories of } \\
\text { action in } \\
\text { compliance with } \\
\text { Law 456/78) }\end{array}$ \\
\hline $\begin{array}{l}\text { Main Goal for the Actions in the } \\
\text { Historic Center }\end{array}$ & Revitalization & $\begin{array}{l}\text { Natural } \\
\text { shopping mall }\end{array}$ & $\begin{array}{l}\text { Main reference for the } \\
\text { development of the } \\
\text { community }\end{array}$ & Open air museum \\
\hline $\begin{array}{l}\text { The Plan Introduces Some Innovation } \\
\text { in the Actions for Heritage } \\
\text { Conservation to Contrast the } \\
\text { Phenomenon of Under-utilization }\end{array}$ & $\begin{array}{l}\text { The } 2018 \text { update of the } \\
\text { action plan does. Some } \\
\text { actions are allowed in the } \\
\text { general restrictive approach, } \\
\text { such as flexible uses, } \\
\text { multistorey parking, tactical } \\
\text { transformations... }\end{array}$ & NO & $\begin{array}{l}\text { The } 2018 \text { update of the } \\
\text { action plan does. Some } \\
\text { actions are allowed in } \\
\text { the general restrictive } \\
\text { approach such as } \\
\text { improved accessibility, } \\
\text { touristic promotion... }\end{array}$ & NO \\
\hline $\begin{array}{l}\text { The Plan Considers the Historic Center } \\
\text { as a Relevant Resource to } \\
\text { Accommodate the Need of Housing }\end{array}$ & $\begin{array}{l}\text { It is declared, but the center } \\
\text { is mostly considered for } \\
\text { economic growth }\end{array}$ & NO & NO & Only partially \\
\hline $\begin{array}{l}\text { The Plan States the Need of Containing } \\
\text { Land Consumption for Sustainability }\end{array}$ & YES & $\begin{array}{l}\text { YES, zero growth is } \\
\text { the goal }\end{array}$ & YES & YES \\
\hline $\begin{array}{l}\text { New Developments Planned Enlarging } \\
\text { the Existing Urbanized Area }\end{array}$ & YES & YES & YES & YES \\
\hline $\begin{array}{l}\text { Forseen Increase in Population in the } \\
\text { Municipality Despite the Current } \\
\text { Population Decrease or Stability }\end{array}$ & $17 \%$ & $17 \%$ & $\begin{array}{l}\text { Information not } \\
\text { available in the } \\
\text { documents online }\end{array}$ & $13 \%$ \\
\hline
\end{tabular}

The reading focused on some key terms: Underutilization, heritage, centre, conservation, sustainability, sustainable development.

The research analyzed the spatial planning documents because they are the manifesto of the public and the official position assumed by the local governments in recent years: The research produced an 
exegesis of the texts and maps which compose the city plans as a critical assessment able to decipher urban planning stories and their coherence with the current reality.

The analysis shows the planning documents in force only recently started to acknowledge the problem and only a few policies were proposed to actively revitalize the centre. The research assumes it as evidence that the concept, tools and procedures of planning and regulatory practice-matured over the course of decades of economic and demographic growth—should be updated to be able to react to the structural changes in Italian society and economics and to the new-however, not really new-urban dynamics and mega-trends.

\subsection{City of Treviso, Acknowledgement of the Phenomenon in the Bigger Picture but Late and Limited Action}

Treviso, Veneto Region, has 81,000 inhabitants and 1600 inhabitants in the 24 ha of the ZTL in the the heart of the city centre [25]. Treviso has a strategic plan, approved in 2015 (Piano di Assetto del Territorio-PAT) and an action plan, adopted in 2018 (Piano degli Interventi-PI) [28,29].

PAT registers some cases of underutilized or unutilized building stock, but does not acknowledge the phenomena of abandonment of the historic centre, which was already evident when the drafting of the plan started. The document which defines how much the city can grow in relation to what already exists classifies only the un-let units as the unutilized stock, assessing it to be $8.5 \%$ of the total availability, and declares it is non-recoverable and, therefore, not relevant to answer the present and future demand [30,31].

Despite the general goals stated-intensified requalification of the existing stock and historic and cultural identities as strategic assets for economic growth - and despite the population decrease by $2.16 \%$ in the municipality and by $11 \%$ in the city centre between 1991 and 2011, PAT foresees an increase in population of 14,500 units, almost $17 \%$ of the existing population, and urbanizes new areas to accommodate this increase.

In the strategic actions for sustainable urban regeneration (ambiti dei programmi complessi) the historic centre is not included, even though its requalification would require minor urbanization investments compared to the targeted abandoned large areas, twentieth-century complexes in peripheral areas that no longer respond to any use; these are very evident to the inhabitants and represent a problem for the local governments, while the centre clearly does not.

More attention to the city centre is given in the action plan-PI adopted in 2018, see Figure 2. It claims the need for actions for containing land consumption and revitalizing the city centre, allowing more flexible uses, multistory parking in dismissed structures, public-private use of parking. PI almost halves the amount of new residential developments and pursues the return of inhabitants to within the perimeter of the historic centre, and in considering the centre as an asset, PI also mentions the tool for temporary reuse introduced by the regional legislation for the containment of land consumption: To activate a process of regeneration of the abandoned or unused building stock with tactical interventions, waiting for a more substantial and definitive restructuring operation [32,33]. PI is a step forward because it clearly identifies the problems associated with the city centre. However, this action is late and limited considering the significance of the issue.

\subsection{City of Udine, neither Acknowledgement nor Action}

Udine, Friuli Venezia Giulia Region, has 98,000 inhabitants and 1700 inhabitants in the 38 ha restricted traffic zone [25]. The city planning documents approved in 2012 (Piano Strutturale-PS and Piano Regolatore Generale Comunale-PRGC) do not acknowledge the abandonment of the city centre and, therefore, do not give it a role in the actions for future sustainable development; generally, the plans give a representation of a city without severe problems [34].

Considering the slow demographic trends, PRGC reasonably defines the population in 2025 as 101,666 inhabitants and defines the normal un-let units in the city at $8.8 \%$. Nevertheless, to answer the diverse housing needs, the plan adds 12,270 units to the existing ones, corresponding to a total 
population of 117,050 inhabitants $(+17 \%)$. To answer this future demand, the Piano Regolatore Generale Comunale does not specifically plan the requalification of the stock in the centre $[35,36]$.

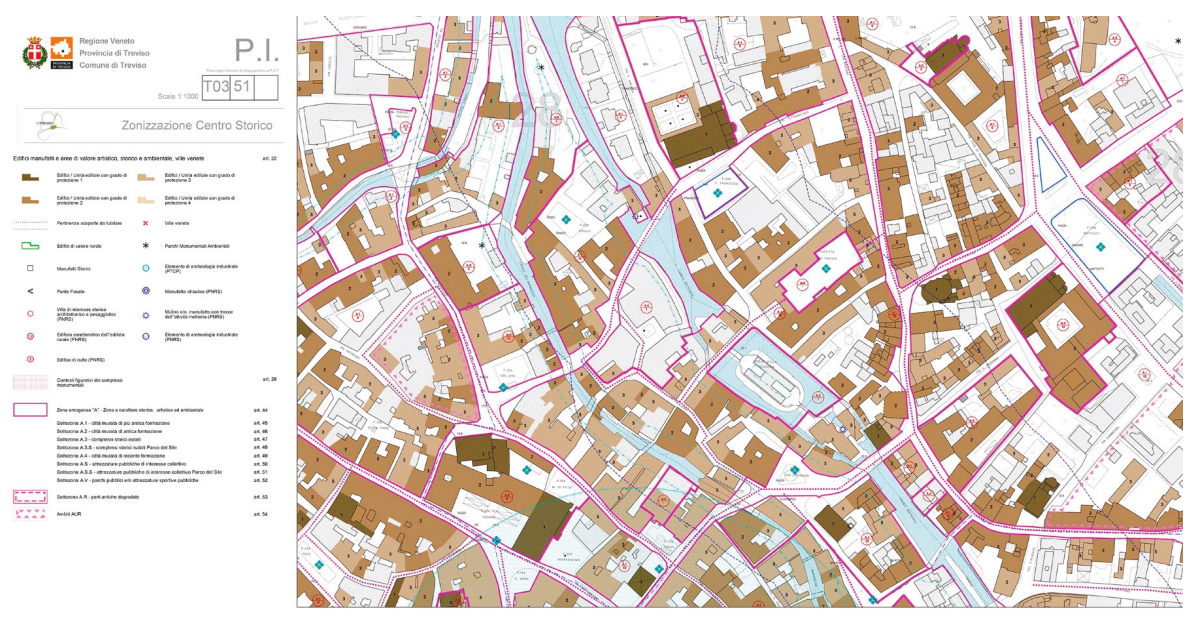

Figure 2. Excerpt from the Piano degli Interventi-PI of the city of Treviso, 2018; zoning and regulations of the historic centre.

PRGC mentions the goal of enhancing livability and re-qualifying the most valuable heritage to increase its attractiveness, but proposes some remarks that are unconnected to specific actions. The main idea, which shows some worries about the centre, is to consider the centre as a "natural shopping mall" in order to compete with the extremely successful shopping malls in the periphery, which have been massively draining customers and visitors since the 1990s. This idea proposes to reinforce the core without exploiting its identity, but adopting a model produced for different urban contexts. For this goal, PRGC proposes to enhance the public open spaces for pedestrians, mostly with projects of connections in the centre itself. This strategy seems to be out of focus, as the historic centre of Udine was designed and has always been a place for pedestrians and these connections are already there; in contradiction to this direction, the new local government in 2018 has approved to reintroduce car traffic in the ZTL.

The structural plan confirms the traditional interpretation of the territory around the Udine as a polycentric structure where the city is the most relevant core for its history and extraordinary urban quality; the plan also confirms Udine's historic centre as the centre of this territory, when in reality, it is in a crisis of representation and vitality with a rate of $39 \%$ of underutilized housing units in 2011, a $70 \%$ reduction in local units of public institutions, and an $8.30 \%$ decrease in inhabitants.

\subsection{City of Modena, Early Acknowledgement of the Phenomenon and Recent Attempt to Promote Actions}

Modena, Emilia Romagna Region, has 179,000 inhabitants and 8900 inhabitants in the 72 ha restricted traffic zone [25]. The structural plan, approved in 2013 (Piano Strutturale Comunale-PSC), reacts to the underutilisation phenomena impacting the historic centre, a problem studied since $2006[37,38]$.

PSC describes the constant transformation of the centre and focuses on two groups of actions, the first related to accessibility, rest areas, garages—declared as very relevant—and the second, to re-generation. For the first, the plan refers to further studies. For the second, it proposes to promote cultural and recreational activities, as well as commercial ones, and also refers to further studies for understanding the residents' needs. The plan connects the requalification of some areas with reduced land consumption, referring to further specific policies [39]. 
The action plan approved in 2014 (Piano Operativo Comunale-POC and the related Regolamento Urbanistico Edilizio-RUE) fails to go one step further, that is to say, it neither specifies actions nor adds studies but adopts the usual and well-established practice for conservation: Almost every building in the city centre is subject to restrictive regulations (i.e., the Italian law defining the types of intervention: law 456/1978, art. 31), see Figure 3. In addition to these usual restrictions of transformation, increasing the dimensions of the buildings is always prohibited, as well as the change in use from shops, bars and laboratories into garages [40].
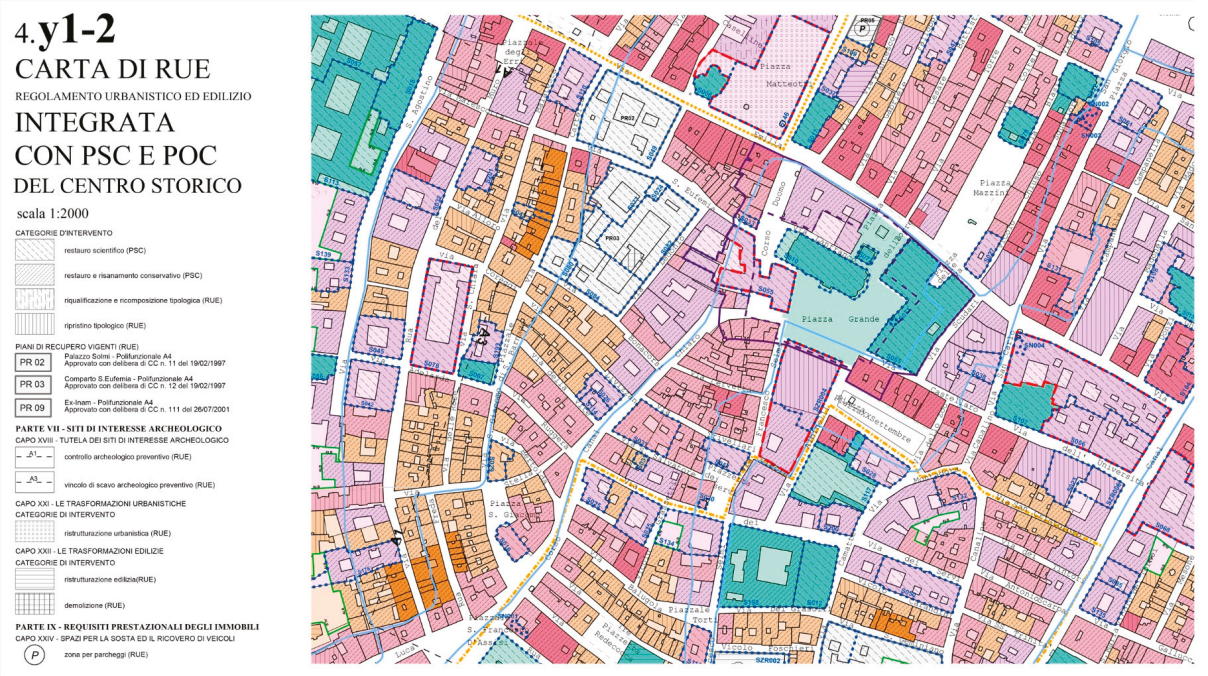

Figure 3. Excerpt from the Regolamento urbanistico edilizio-RUE of the city of Modena, 2013; zoning and regulations of the historic centre.

PSC gives a definition of the historic centre corresponding to the traditional idea of its values: The centre is a reference for the development of the local community, mostly for its public spaces and services which allow the inhabitants to be a community. According to this definition, the plan acts contradictorily: On one hand, it does not claim the recently diminished role, on the other, it includes the centre in the areas where public services are lacking, a new element to remark upon because the centre has traditionally been the place where services are concentrated.

Even if the plans in force do not propose an innovative approach to deal with the problems of the city centre, the revitalization of the historic centre with active policies has been discussed at a higher political level since the first months of 2018 and a new POC was adopted at the end of 2018: Facilities for the residents, improved accessibility and parking areas, economic support and touristic promotion are the solutions proposed.

\subsection{City of Vicenza, Some Acknowledgement and Some Action Limited in Purpose}

Vicenza, Veneto Region, has 111,500 inhabitants and 3019 inhabitants in the 50 ha restricted traffic zone [25]. The strategic city plan for Vicenza, approved in 2010 (Piano di Assetto del Territorio-PAT), declares a "crisis of identity" of the city centre for some of the reasons explained by the present research based on the interpretation of the Census Data: New, poor immigrants, weak public services, poor commercial supply and inadequate recreational areas [41,42]. To cope with this crisis, PAT suggests to improve cultural, recreational and shopping attractions and tourism and considers the city centre as an "open-air museum". The 'museumification' of the historic space is coherent with the age of the plan 
for the city centre in force (Piano particolareggiato per il Centro Storico-PCS), which dates back to 1979 (but was drafted in 1969, see Figure 4), even though it was partially updated in 1988 [43].

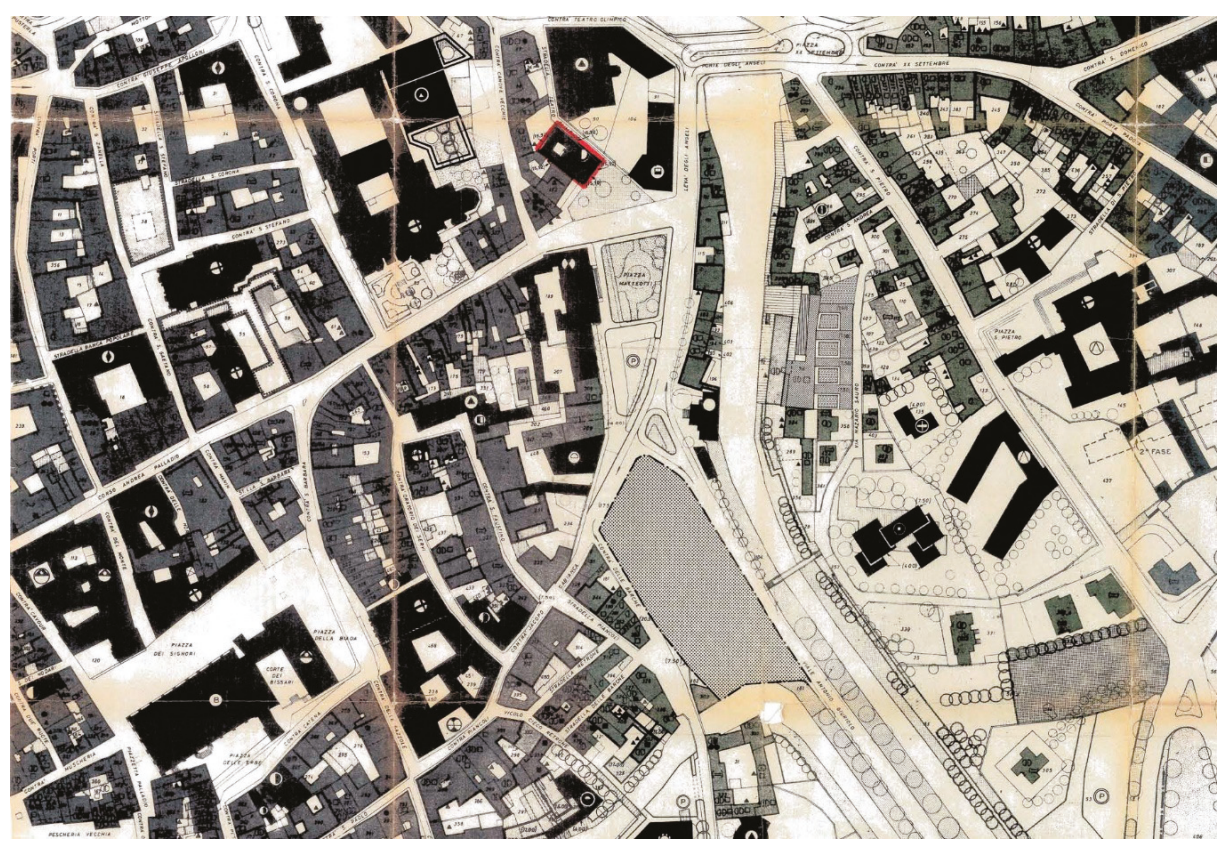

Figure 4. Excerpt from the Master plan of the city of Vicenza, 1969; zoning and regulations of the historic centre.

Similarly, PAT declares that the city centre must again become the place of high-quality residences, because the migrant and mobile population, that is increasingly occupying it, will cause its decay. PAT does not recognize in the new inhabitants a possible way to revitalize the centre, nor does it interpret the city centre as one of the "eco-neighbourhoods" which it defines their high-density and potential for regeneration: The main concern about the centre remains the conservation of its beauty and characteristics.

PAT promotes sustainability as a principle for a general urban re-structuring, and it stresses dwelling units must be obtained by re-using and re-qualifying the existing stock. However, the action plan approved in 2013 (Piano degli Interventi-PI) plans to accommodate only 600 of the future foreseen 14,269 new inhabitants in the city centre, while the present research found 2097 empty units potentially available [44].

Vicenza can be considered a paradigmatic example of the well-established approach to conservation in planning-resulting from an extensive technical, as well as conceptual theoretical reflection and definition of practices which goes back to the beginning of the XX century $[7,8,45,46]$ - consciously applied to deal with new conditions without updates or reframed regulations.

\subsection{Structural Difficulties and Conservational Approach}

Even though the numbers of vacancies are high and the value of the property not utilized seems to be of absolute economic significance, the city plans presented here fail to propose innovative policies to reuse the centre. A further step, i.e., to consider these actions as a way to reduce land consumption and recycle housing stocks, is only mentioned. 
The paper proposes that the examined plans express a structural difficulty in dealing with the historic centre within renewed cultural and economic coordinates. "Structural" means pertaining to the long-term cultural and organizational essence of the Italian regulation mechanism for protecting the heritage: In order to be in compliance with the law, regulations tend to deny transformation rather than to define an operational approach, allowing agreement and compensations.

Italian urbanism distinguishes between policies of regeneration based on cultural values-the building type, the permanence, the historical-architectural legacy-and actions based on scientific and technological values-sustainability, de-carbonisation, reduction of energy consumption, etc. This separation is highly inefficient, this is also because, in Italy, the conservational approach is often overwhelming due to the extension of the classification of heritage to essentially everything old $[47,48]$ and the idea that the historic centre-as a whole—is a work of art and, as such, it cannot be changed [49]. The risk of stagnation is increased by the lack of dialogue in Italy between the culture of design and the culture of conservation $[50,51]$.

\section{Four Paradoxes of the Italian Historic City Centres}

The results of the research show that some paradoxes exist in Italian historic centres. They are part of the most precious heritage of the country, locations of monuments and beauty, guarantee the cultural identity, icons of well-designed open spaces and well-being, and of a high-density and efficient use of land. Therefore, they should be successful but the interpretation of demographic movements and property dynamics over the past thirty years in small-to-medium-sized cities in Northern Italy reveal these positive and advantageous characteristics do not prevent the relocation of the population, businesses and institutions. The critical recognition of these paradoxes-presented in the following paragraphs-should be the starting point for the re-framing of the policies for urban heritage which do not seem able, as the previous paragraph shows, to give a dimension to the problems and react to transformation dynamics.

\subsection{High-density and Compactness}

The sustainability of high-density settlements has been a widely discussed topic together with the opportunity to drastically decrease land consumption for new settlements [52-55]. The historic centre has used land very efficiently because the settlement is compact and has a high-density: Generally, the centre is many times denser than the rest of the settlements in the municipality (in Udine, Brescia e Modena the inhabitants in the historic centre per square meter are roughly four times as many as in the rest of the city).

Compactness and high density produce also proximity to public services and can support public transportation, which is convenient and accessible because of the concentration of people and activities. It can be said the city centre has the required parameters to be sustainable because it is compact and of higher density, in a word, it has already the main characteristics of an "eco-neighborhood" [56]. But the issue is socio-cultural and related to changed desires for living condition: the heritage value and the human-scale value of the history centre are not enough to attract residents; failing to recognize it is misleading.

\subsection{Community Life and Local Identity}

The human-scale dimensions and the quality of the public spaces in the historic centre are generally praised and said to guarantee both individual well-being and community life in well-designed gathering places, as very influential scholars have been claiming for decades [57-59], reinforcing this myth in the Italian cities. These physical characters, where the symbols of the local community's identity are preserved, seem to reinforce the social bonds and cultural identity. The so called "New Urbanism" carefully considers these physical conditions and claims these should be re-proposed for contemporary settlements [60]. The image of the centre as a pleasant place is so anchored that even some shopping malls adopt the forms and dimensions of the traditional open-air historic centre. 


\subsection{Recurrent Re-generation and Accumulation of Resources or Embedded Energy}

The large majority of the built stock in the city centre was built to last, has resisted centuries and was recurrently transformed or adapted to new inhabitants, requests or functions. The high quality of the structures, as well as of some ancient urban tissue, has allowed regeneration and renovation; the Italian Aldo Rossi and Giancarlo De Carlo clearly highlighted these historical and architectural processes and opportunities, well before the idea of sustainable recycling [61-63].

In the old towns, the economy has been circular for the traditionally limited resources and for the great value of the buildings and their location, long-term resilient practices were constantly adopted over the centuries for preserving the assets. Open public spaces were constantly improved and maintained for the community gathering for market, justice, religious events.

Conditions are radically changed and nowadays, possibilities are not considered limited anymore, but the money and energy embedded in the historical structures must be recognized and valued, as well as the regeneration opportunity, to neglect this potentiality when the world is praising recycling and circularity would be a waste [64].

\subsection{Vanished Appeal}

If, over the centuries, the centre was the preferred and most prestigious location for inhabitants and institutions and the distinction between the centre and periphery was sharp, today the trend has changed. The paradoxes lay in the fact that universally recognized positive characteristics do not impact the choices of inhabitants and institutions today. Even though everyone recognizes the centre's great beauty, even if high-density settlements are praised as one of the best solutions for a sustainable urban environment, even though the surviving community spirit allowed by the centre's spatial peculiarities is considered an antidote against the metropolisation, the past appreciation has vanished and the historic centre is not appealing.

The paper suggests this lost social and economic attractiveness is due to the structural mutations in Italian society and economics, not only since the economic crisis in 2008 but progressively since the 1990s, expressed in urban terms by the phenomena of sprawl [65]. In times of climate change, of circular economy and search for a new ecological balance, where the urban world is a major actor, laws and regulations which govern the urban heritage should face the contradictions the paradoxes pose and be questioned. The research suggests it is urgent politicians and city officials give a different value to the historic city centre; this topic has long been neglected and it is high time the battle for preservation is followed by another battle for the recycling of a stock of primary cultural, economic and social value.

\section{Conclusion: A Renewed Cultural and Economic Approach to Recycle the Wasted Urban Heritage for a Sustainable Development}

The analyzed city plans clearly show that the challenge of connecting built urban heritage and sustainability is nowadays in Italy not to protect heritage as material facts-a goal already achieved thanks to a rich history of theories and actions-but to safeguard its socio-economic vitality. The failure is due not only to the mega-trends of globalization which re-structure the geography of urban hierarchy but also to the inadequacy of planning tools conceived for different socio-economic conditions.

The city plans show also that the regulation system finds it difficult to adapt to the unforeseeable and generally fast urban dynamics and to renewed cultural and economic conditions. The link between the built urban heritage and sustainability is often claimed in theory, but not taken to the operational level: The empty inner cores are not considered opportunities to reduce land consumption and recycle valuable assets.

In the Italian cultural and economic contemporary context -stagnation particularly affected the small-to-medium-sized cities since 2008- a new set of policies have to be framed together with new planning processes. A renewed cultural and economic approach must also be adopted. This task must 
be accomplished in the general frame defined by the guidelines of the international institutions for conservation and heritage but with operational details suitable for the developed Countries [66,67].

The new goals of the city plans should be to:

- $\quad$ survey in depth the phenomenon of abandonment and acknowledge its impact; consider the built stock in the historic centre as an essential asset to balance expansion and re-functionalize the built heritage resources;

- re-define what changes of the heritage can be allowed without diminishing its value; in doing so, the plan must consider if the dense regulatory mechanism, matured over the course of decades of economic and demographic growth, is an obstacle for innovative practices and what elements in the policies prevent revitalization;

- find a balance between "original" residents and the significant presence of foreign people;

- contrast mono-functionality connected to the development of specific services, tourism in particular, in order to rely not only on one source of revenues and maintain residents and vitality.

The future phase of the research will consider cases were the decline of the city centre has been successfully dealt with in Italy and in Europe; this research will not only consider spatial planning documents but how these are part of integrated policies: In fact, the city plan alone cannot solve socio-economic issues. For this goal, the financial mechanisms and fiscal incentives for promoting the conservation of the historic centres and, at the same time, maintaining the inhabitants and the activities in the historic centre will be investigated $[68,69]$.

Author Contributions: Conceptualization, P.P., E.M.; methodology, P.P., E.M.; resources, P.P.; data curation, P.P.; writing—original draft preparation, P.P.; writing—review and editing, P.P., E.M.

Funding: This research received no external funding.

Conflicts of Interest: The authors declare no conflict of interest.

\section{References}

1. ANCSA Associazione nazionale centri storici artistici. Salvaguardia e risanamento dei centri storico-artistici, Atti del convegno nazionale per la Salvaguardia e il Risanamento dei Centri Storici; ANCSA: Gubbio, Italy, 1960.

2. Tafuri, M. Il problema dei centri storici nella nuova dimensione urbana. In La città territorio, un esperimento didattico sul centro direzionale di Centocelle in Roma; AAVV; Leonardo da Vinci: Roma, Italy, 1964; pp. 38-40.

3. Carozzi, C.; Rozzi, R. Centri storici, questione aperta: il caso delle Marche; De Donato: Bari, Italy, 1971.

4. AAVV. L'intervento pubblico nei centri storici, problemi sociali, giuridici, economici, architettonici e tecnici; Edizioni di Edilizia Popolare: Roma, Italy, 1973.

5. Vassallo, E. Centri antichi 1861-1974: note sull'evoluzione del dibattito. Restauro 1975, 19, 3-96.

6. Ceccarelli, P.; Indovina, F. Risanamento e speculazione nei centri storici; Angeli: Milano, Italy, 1977.

7. Ciardini, F.; Falini, P. L'analisi dei centri storici, manuale per la formazione degli strumenti di intervento urbanistico; Officina: Roma, Italy, 1981.

8. Per una storia del restauro urbano: Piani, strumenti e progetti per i centri storici; Giambruno, M. (Ed.) Cittastudi: Torino, Italy, 2007.

9. La scoperta della città antica. Esperienza e conoscenza del centro storico nell'Europa del Novecento; Cutolo, D.; Pace, S. (Eds.) Quodlibet Studio: Macerata, Italy, 2016.

10. ANCSA Associazione nazionale centri storici artistici; Cresme. Centri storici e futuro del Paese. Indagine nazionale sulla situazione dei centri storici; Documenti ANCSA: Roma, Italy, 2017.

11. Balletti, F.; Ghersi, A. Abitare il centro storico: studi documenti e progetti per il cuore della città di Genova; Alinea: Firenze, Italy, 2014.

12. Marcoaldi, P. Per la città di Viterbo: Masterplan del centro storico; Quodlibet: Macerata, Italy, 2018.

13. Bellia, P.; Puma, P. Firenze, la trasformazione del centro antico; Edifir: Firenze, Italy, 2018.

14. Ferrigni, F.; Sorrentino, M.C. Il futuro dei territori antichi: problemi, prospettive e questioni di governance dei paesaggi culturali evolutivi viventi; Edipuglia: Bari, Italy, 2013. 
15. Micelli, E.; Pellegrini, P. Wasting heritage. The slow abandonment of the Italian Historic Centres. J. Cult. Herit. 2018, 31, 180-188. [CrossRef]

16. Micelli, E.; Pellegrini, P. Vuoto al centro. Impiego ed abbandono del patrimonio dei centri antichi italiani. Territorio 2017, 82, 157-170. [CrossRef]

17. Habitat-III. Third United Nations Conference on Housing and Sustainable Urban Development; New Urban Agenda, UN: Quito, Ecuador, 2016.

18. United Nations. Transforming our World: The 2030 Agenda for Sustainable Development; UN: New York, NY, USA, 2015.

19. Labadi, S.; Logan, W. Urban Heritage, Development and Sustainability; Routledge: Oxford, UK, 2016.

20. Klusáková, L. Small Towns in Europe in the 20th and 21st Centuries: Heritage and Development Strategies; Karolinum Press: Prague, Czech Republic, 2018.

21. Management of Historic Centres; (Conservation of the European Built Heritage Series Book 2); Pickard, R. (Ed.) Taylor \& Francis, e-book: Oxfordshire, UK, 2013.

22. Free Market, the Irish Pavilion at the 16th International Architecture Exhibition Freespace 2018. Available online: http://free-market.ie/ (accessed on 10 July 2018).

23. Ministère de la Cohésion des territoires et des Relations avec les collectivités territoriales. Action Coeur de Ville. Available online: https://www.cget.gouv.fr/sites/cget.gouv.fr/files/atoms/files/plaquette_actioncoeurdeville. pdf (accessed on 10 March 2019).

24. Nilsson, L. Can small towns survive in a global World? In Proceedings of the International Conference EAUH 2018 Urban Renewal and Resilience, Cities in Comparative Perspective, Rome, Italy, 28 August-1 September 2018.

25. Statistiche ISTAT. Censimento Popolazione Abitazioni. Available online: http://dati-censimentopopolazione. istat.it/Index.aspx (accessed on 10 May 2018).

26. Statistiche ISTAT. Censimento Industria e servizi. Available online: https://www.istat.it/it/censimentipermanenti/censimenti-precedenti/industria-e-servizi (accessed on 10 May 2018).

27. ISTAT-Istituto nazionale di statistica. Forme, Livelli e Dinamiche Dell'urbanizzazione In Italia; ISTAT: Roma, Italy, 2017.

28. Comune di Treviso. Piano di Assetto del Territorio Comunale (P.A.T.). Available online: http://www.comune. treviso.it/pat/ (accessed on 10 September 2018).

29. Comune di Treviso. Piano degli Interventi (P.I.). Available online: https://www.comune.treviso. it/rigenerazione-urbana-riqualificazione-quartieri-4/urbanistica/piano-degli-interventi-p-i/ (accessed on 10 September 2018).

30. Comune di Treviso. PAT, R03, Verifica del Dimensionamento; Comune di Treviso: Treviso, Italy, 2015.

31. Comune di Treviso. PAT, R01, Relazione tecnica; Comune di Treviso: Treviso, Italy, 2015.

32. Comune di Treviso. PI, R01, Relazione tecnica illustrative; Comune di Treviso: Treviso, Italy, 2018.

33. Comune di Treviso. PI, R02, Verifica del Dimensionamento; Comune di Treviso: Treviso, Italy, 2018.

34. Comune di Udine. Piano Regolatore Comunale Generale. Available online: http://www.comune. udine.gov.it/aree-tematiche/edilizia-territorio/urbanistica/piano-regolatore-generale-comunale (accessed on 15 September 2018).

35. Comune di Udine. PRGC, Relazione Generale; Comune di Udine: Udine, Italy, 2012

36. Comune di Udine. PRGC, Relazione sul dimensionamento; Comune di Udine: Udine, Italy, 2012.

37. Comune di Modena. PRG—Piano Regolatore Generale. Available online: http://urbanistica.comune.modena. it/ (accessed on 18 September 2018).

38. Comune di Modena. Rapporto sul centro storico. Available online: http://urbanistica.comune.modena.it/ index_Centro.html (accessed on 18 September 2018).

39. Comune di Modena. PRG 2003, Testo coordinato delle norme di PSC_POC-RUE; Comune di Modena: Modena, Italy, 2015.

40. Comune di Modena. PRG 2003, Carta di PSC del Centro Storico, 2.t1, Variante al POC-RUE approvata con delibera di C.C. $n^{\circ} 48$ del 07/05/2015; Comune di Modena: Modena, Italy, 2015.

41. Comune di Vicenza. Piano regolatore comunale (Pat-Pi) e piani urbanistici (Pua). Available online: https://www.comune.vicenza.it/servizi/prg.php (accessed on 20 September 2018).

42. Comune di Vicenza. PAT Relazione Tecnica; Comune di Vicenza: Vicenza, Italy, 2010. 
43. Comune di Vicenza. Relazione al piano particolareggiato di esecuzione del centro storico; Comune di Vicenza: Vicenza, Italy, 1969.

44. Comune di Vicenza. PI, Relazione Programmatica; Comune di Vicenza: Vicenza, Italy, 2013.

45. Pellegrini, P.; Micelli, E. Wasted heritage. Between policies for urban heritage and land consumption. In Proceedings of the 6th International Conference on Heritage and Sustainable Development HERITAGE 2018, Granada, Spain, 15 June 2018.

46. Cristinelli, G. Saverio Muratori e Egle Renata Trincanato. La nascita del restauro urbano in Italia; Ginevra Bentivoglio EditoriA: Roma, Italy, 2013.

47. Il progetto del passato; Pedretti, B. (Ed.) Bruno Mondadori: Milano, Italy, 1997.

48. Koolhaas, R. Preservation Is Overtaking Us; Carver, J., Ed.; Columbia Books on Architecture and the City, GSAPP Transcripts: New York, NY, USA, 2014.

49. Romano, M. La città come opera d'arte; Einaudi: Milano, Italy, 2008.

50. Ferlenga, A.; Vassallo, E. Antico e Nuovo. Architetture ed Architettura; Il Poligrafo: Venezia, Italy, 2008.

51. Cervellati, P. L'arte di curare la città; Il Mulino: Bologna, Italy, 2000.

52. Rogers, R. Cities for a Small Planet; Basic Books: London, UK, 1998.

53. Glaeser, E. Triumph of the City; Penguin Random House: New York, NY, USA, 2011.

54. Owen, D. Green Metropolis. Why Living Smaller, Living Closer, and Driving Less Are the Keys to Sustainability; Riverhead Books: New York, NY, USA, 2009.

55. Ng, E. Designing High-Density Cities: For Social and Environmental Sustainability; Earthscan Ltd.: London, UK, 2010.

56. Barattucci, C. La riqualificazione sostenibile dei centri storici come risposta italiana alla moltiplicazione degli ecoquartieri europei. Presented at the XXI Conferenza Nazionale Societa' Italiana degli Urbanisti, Firenze, Italy, 5 June 2018.

57. Sitte, C. The Art of Building Cities: City Building According to Its Artistic Fundamentals; Martino Fine Books: Eastford, CT, USA, 2013.

58. Whyte, W. The Social Life of Small Urban Spaces; Project for Public Spaces: New York, NY, USA, 1980.

59. Gehl, J. Life between Buildings: Using Public Space; Island Press: Washington, DC, USA, 1987.

60. Congress for the New Urbanism. Charter of the New Urbanism; McGraw-Hill Professional: San Francisco, CA, USA, 1999.

61. Rossi, A. L'architettura della città; Marsilio: Padova, Italy, 1966.

62. De Carlo, G. Urbino: la storia di una città e il piano della sua evoluzione urbanistica; Marsilio: Padova, Italy, 1966.

63. Re-cycle Italy. Available online: http://www.recycleitaly.net (accessed on 5 June 2018).

64. Ellen Macarthur Foundation. Available online: ellenmacarthurfoundation.org (accessed on 5 June 2018).

65. Indovina, F. La città diffusa. In La città diffusa; Indovina, F., Matassoni, F., Savino, M., Sernini, M., Torres, M., Vettoretto, L., Eds.; Daest-IUAV: Venezia, Italy, 1990; pp. 21-43.

66. Bandarin, F.; van Oers, R. Il paesaggio urbano storico: La gestione del patrimonio in un secolo urbano; CEDAM: Padova, Italy, 2014; pp. 32-33.

67. Operationalising the Historic Landscape, a Practitioner's View; Zhou, J.; van Oers, R. (Eds.) Tongji University Press: Shanghai, China, 2018.

68. Forte, F. Historic, Artistic and Cultural Patrimony for a "Habitable City": Incentives for Care. In Integrated Evaluation for the Management of Contemporary Cities, SIEV 2016; Mondini, G., Fattinnanzi, E., Oppio, A., Bottero, M., Stanghellini, S., Eds.; Springer: Cham, Switzerland, 2018; pp. 233-243. [CrossRef]

69. Pickerill, T.; Pickard, R. A Review of Fiscal Measures to Benefit Heritage Conservation; RICS Research Paper Series; Dublin Institute of Technology: Dublin, Ireland, 2007; Volume 7, p. 6. 



\title{
Valuation of the Vocationality of Cultural Heritage: The Vesuvian Villas
}

\author{
Fabiana Forte ${ }^{1}$, Vincenzo Del Giudice ${ }^{2}$, Pierfrancesco De Paola ${ }^{2, *}$ and Federica Troisi ${ }^{1}$ \\ 1 Department of Architecture and Industrial Design, University of Campania "Luigi Vanvitelli", \\ Via San Lorenzo ad Septimum, 81031 Aversa, Italy; fabiana.forte@unicampania.it (F.F.); \\ federicatroisi@outlook.com (F.T.) \\ 2 Department of Industrial Engineering, University of Naples "Federico II", Piazzale Vincenzo Tecchio 80, \\ 80125 Naples, Italy; vincenzo.delgiudice@unina.it \\ * Correspondence: pierfrancesco.depaola@unina.it; Tel.: +39-081-768-2933
}

Received: 20 December 2019; Accepted: 24 January 2020; Published: 28 January 2020

\begin{abstract}
The Vesuvian Villas are a system of architectural assets that, due to important artistic, historical and typological characteristics, have relevance that is not only local. However, due to ineffective management policies and insufficient financial resources, the system of the Vesuvian Villas is subject to abandonment or to invasive transformations for speculative purposes. The management policies for these real estate goods would require a profound theoretical and operational review that, together with the overcoming of the binding instrument as the only guarantee of protection, pursues conservation through synergies founded on appropriate uses of the Vesuvian Villas. This innovative path is difficult to implement due to the substantial rigidity of the architectural structures and the transformations aimed at renewing the forms of use, but mostly for the lack of available financial resources. Starting from the analysis of the relationships between conservation and transformation of the historical architectural asset, the paper proposes a multicriteria analysis model for the evaluation of the "vocational" nature of the "Villa Vesuviana" property, aimed to its conservative reuse. This suitability was assessed starting from a set of indicators explaining the actual state of the building (typological, morphological, structural and artistic characteristics) and its location. The indicators have been evaluated through qualitative judgments made using the hierarchical analysis technique. Particular attention was paid to the evaluation of synergies deriving from complementary uses.
\end{abstract}

Keywords: vocationality; cultural heritage; Analytic Hierarchy Process (AHP); Vesuvian Villas; valuation

\section{Introduction}

In accordance with the report "Cultural Heritage Counts for Europe", in the past few decades we have witnessed the main conceptual and policy developments that have recognized the multiple and valuable benefits that cultural heritage brings to society as a whole [1], making the conservation of cultural heritage a national priority for many countries. At a European Union level, starting from the Faro Convention (2005), there has been a growing awareness of the importance of cultural heritage, recognized as "a strategic resource for a sustainable Europe" (Council of the European Union, 2014 [2]) and "a shared resource and a common good", as in the document "Towards an integrated approach to cultural heritage for Europe" (European Commission, 2014 [3]). In this document it is also highlighted that the economic value of heritage has recently come into research focus, but only partial estimates of its importance are available.

It is clear that the issue of the evaluation of impacts connected with the valorization of Cultural Heritage has become relevant also in the policy agendas [4]. Most recently, during 2018, the EU has celebrated the importance of Cultural Heritage in Europe with a list of events hosted throughout Europe 
in order to remind its citizens of the linkage that binds each other and to enhance a common sense of belonging to the European Union (Heritage Hube, 2018). The Davos Declaration 2018, "Towards a high-quality Baukultur for Europe", recognized cultural heritage as "a crucial component of high-quality Baukultur. The way we use, maintain and protect our cultural heritage today will be crucial for the future development of a high-quality built environment."

With specific regard to Italy, the State, through the Ministry for Heritage, Cultural Activities and Tourism-MiBACT-is not only responsible for the strategic task involved in the protection of an extremely rich cultural heritage, but has direct responsibility for the management of a huge number of national heritage institutions. Protection and Management is regulated by the Italian Code of the Cultural and Landscape Heritage (Legislative Decree n. 42/2004). Public expenditures, allocated by several levels of government, have traditionally been the primary source in supporting Cultural Heritage; however, they have been progressively reduced, with Italy being, among the EU states, one of those who spends less on culture, despite its extraordinary heritage [5].

As in other European countries, the involvement of private subjects (both profit and non-profit) in the preservation of Cultural Heritage has become a relevant issue. As several studies and reports in the field of Cultural Economics have highlighted for a long time, the traditional approach that considered this heritage as "immutable" has been overcome by an approach that recognizes its vitality or productivity [6], as well as its preservation, and is understood as a "productive" activity carried out for "social use" [7]. It is in this sense that the notion of valorization should be interpreted.

In Italy, the theme of valorization has been particularly debated since there is a "culture of conservation" that prevails and it enforces a very ancient legislative tradition (it starts from the period that precedes the unification of Italy, passing from the 1939 legislation), which must be "proudly" defended, but there was no a "culture of valorization". In other words, the country has invested very little in its cultural heritage. As set out in the Italian Code of the Cultural and Landscape Heritage, valorization (or enhancement) consists of the exercise of the functions and regulation of the activities aimed at promoting knowledge of the cultural heritage as well as ensuring the best conditions for the use and public enjoyment of the heritage. A private subject may concur, co-operate or participate in such activities. Then, in a purely economic interpretation, it is possible to consider valorization as a way for the "entrepreneurial" management of the cultural heritage, capable of producing revenues for its preservation or care [8]. It is important to underline that this interpretation does not aim to increase the market value attributable to cultural asset, but rather to attract resources for financing its conservation and accessibility [9], providing it with the "social plus value" [10].

This obviously represents the economic gain and the social benefit yielded through the conservation, which - as it obtains a greater value - may be also understood as valorization. According to the Council of Europe, "private management is currently the most interesting area for involving the private sector in protection of the cultural heritage. It can take many forms and extend from management of State property to management of privately owned heritage" [11]. In this perspective, among the different forms of management of cultural property undertaken by private initiatives introduced by the Italian Code and aimed at the valorization, are Sponsorship and Additional Services; both have been analyzed, highlighting some critical aspects [12,13].

The most recent government reform on the overall system of cultural goods and activities, launched by the MiBACT with an "Art Bonus" Decree (Legislative Decreen. 83/2014 and successive modifications) encourages cash donations for the care of public cultural property, trying to align Italy to others European countries, but not to the USA where the fiscal incentives for the historic preservation are, traditionally, so much more consistent [14]. Together with this innovative form of incentives, the Legislative Decree n. 83/2014 introduces the Strategic Plan for "Major Cultural Patrimony Projects", with the aim of identifying goods or sites of exceptional cultural interest and of national relevance for which it is necessary and urgent to realize organic interventions of conservation, requalification, valorization and cultural promotion for the tourism sector, assigning several resources. Further, in 2012, 
the Italian Revenue Agency, with Invitalia and ANCI and other institutional subjects, the "Valore Paese-Dimore" program for the valorization of historical-artistic public patrimony was promoted.

The program aims to develop and put at work publicly owned properties for tourist accommodation that are of particular historic and artistic merit and located in sites of significance in terms of the environment and landscape. Among several properties that fall under the portfolio of immovable cultural properties of the "Valore Paese-Dimore" program, there is the bourbon Villa Favorita in Ercolano, in the metropolitan city of Naples and in the territorial development system "Miglio d'Oro-Area Torrese Stabiese", the object of analysis in the next paragraphs.

If all these recent institutional initiatives regard the cultural property owned by the Italian State, the question of financing the valorization of cultural property owned by private subjects is far more delicate and complex. Private cultural properties have a normative treatment that is different from the other private goods, since they are of public relevance. In fact, private subjects, owners of historic proprieties (historical villas, castles, palace, etc.) are obliged, by law (Legislative Decree n. 42/2004), to conserve and maintain their properties. The ministry may contribute to the expenses, both with capital grants and interest subsidies.

The owners who have received contributions are obliged to make the property accessible to the public, according to the modalities fixed, case by case, by special conventions. For each kind of intervention, there is a need for authorization from the competent authority and for the sale of the property the state has the power to purchase it by pre-emption. Against conservation obligations, for which the private subject must maintain the property at its own expense and under its responsibility (also penal), traditionally the state reserves to the private owner a less burdensome tax regime, a sort of "compensation". Nevertheless, in the last years, private cultural properties are facing a number of important challenges to survive.

Specifically, the property tax of listed buildings, which in the past was calculated on the basis of a symbolic value, the so called "figurative rent" from 2012 with the "Salva Italia" Decree, has been increased dramatically, making the taxable income the effective rent. This increase of the tax burden on the historical properties, conjointly with the forthcoming cadastral reform and the many bureaucratic bonds, are making the maintenance and valorization of the cultural property owned by private entities even more difficult. Thus, the ADSI ("Associazione Dimore Storiche Italiane" or Italian Association of Historical Houses) proposal presented to the government in 2015 is particularly efficacious.

It consists of the reduction by $30 \%$ of the property tax (the IUC- "Imposta Comunale Unica" or Municipal Single Tax-introduced in 2014 by Italian Stability Law) for owners of historic properties under the condition that they have to invest not only into the restoration but also in the valorization of properties, improving the services for their enjoyment, setting up a network with other realties in the territory and promotional activities.

This means that all "additional services" according to the analysis could generate a VAT revenue capable of compensating for the reduction of the property tax, but also, in the best case, further revenues for the state to re-invest in activities of valorization, communication and education for the enjoyment of the cultural heritage, in the form of multiplier effects [15].

However in Italy, where the private cultural properties are consistent (only the Italian Historic Houses, monitored by ADSI, are estimated to be around 45-50 thousand and diffused on all the national territory), the listed buildings are not all valuable historical mansions, localized in the central areas of the cities. Many listed buildings are located in secondary villages, in rural areas or in small municipalities in each part of the country. If they are of historic and cultural interest, they are degraded and of scarce economic and income value, as is the system of Vesuvian Villas, in the metropolitan area of Naples (Campania region). The opportunity to deepen some aspects regarding the care of private cultural property [15] derives from the participation in the Campus Project "Urban Ecotourism for the sustainable use of cultural heritage in Campania" (in implementation of the Operational Program ERDF Campania 2007/2013), managed by the Regional Center of Competence Benecon (Cultural Heritage, Ecology, Economics), of which one of the partners is the University of Campania "Luigi 
Vanvitelli. The project in fact involves the development system "Miglio d'Oro-Area Torrese Stabiese", with 12 municipalities that constitute a "landscape-cultural mosaic" of considerable interest, where there are concentrated not only goods of "outstanding universal value" (the UNESCO sites of the archeological areas of Pompei, Ercolano and Torre Annunziata), but also immovable goods of notable cultural interest, both public and private, such as the historic houses (villas and mansions) with gardens annexed, some designed and realized by famous architects (Vanvitelli, Sanfelice, Fuga, Vaccaro etc.) and an expression of one of the most florid Neapolitan periods (the Bourbon age).

Some of these historic houses are part of Authority for Vesuvian Villas (now a Foundation) instituted with state Law no. 578/1971 that provides to conserve, restore and valorize them. In 1976, with the Ministerial Decree of constrain, the work of the authority started for the preservation of the 122 monumental buildings in the territory of the municipalities of Naples, San Giorgio a Cremano, Portici, Ercolano and Torre del Greco. Only a restricted number of these historical buildings have been restored and valorized, or are under transformation, as Villa Favorita in Ercolano thanks to the "Valore Paese-Dimore" program. Many others are in a serious condition, with physical and functional obsolescence or have been abandoned.

The case showed in this article was developed by the authors on the base of the application made by D'Alpaos et al., concerning the possibility of the reconversion of the historic Venetian villas as museums [16].

\section{Multicriteria Decision Making}

The Analytic Hierarchy Process (AHP), developed by Saaty at the end of the 1970s, makes it possible to prioritize a series of alternative decisions or to relate criteria characterized by qualitative and quantitative evaluations (not directly comparable) combining multidimensional measure scales in a single scale of priorities [17].

The method is based on paired comparisons between the criteria, giving them a score of relative importance and ends with the assignment of a percentage weight.

The scores to be used for each comparison are arbitrary (random) and correspond to the number of quality levels to be considered during the pairwise comparisons.

The AHP uses an evaluation scale ranging from 1 to 9 , where each ordinal number corresponds to the following values:

- 1. Equal importance.

- 3. Moderate importance.

- 5. Strong importance.

- 7. Very strong importance.

- 9. Absolutely important.

- $2,4,6$ or 8 . Intermediate values.

The same is for the inverse comparisons.

The evaluation process is divided into phases:

- Structure the problem in the hierarchy model.

- Evaluation of comparative judgment and compilation of the analysis matrix; it consists of measuring the relative importance of the elements with respect to the overall goal.

- Construction of an evaluation matrix and a summary of the priorities.

The hierarchical organization of the AHP method allows a detailed decomposition of the problem in more levels.

The goal is set at the upper level of the structure; at the second level there are the objectives that specify the contents and meanings of the goal, factors, criteria or attributes that contribute to the result. Each of these can be subdivided into more specific elements. The actions to evaluate are located at the lowest level of the hierarchy. 
The subdivision of the problem in successive phases permits to define a series of sub-problems with reduced dimensions and complexity.

Each problem is explained by the compilation of a matrix of comparison in pairs that identify the level of convenience and importance of the same criteria.

The matrix is made up of as many rows as there are actions and columns corresponding to the significant attributes for the solution of the problem.

The comparisons are formulated by a verbal judgment, which is associated with a corresponding numerical value. From all these comparisons it is possible to obtain a result. The "best" is the one characterized by the highest level of convenience.

To detect the consistence of the matrix it is possible to calculate an "index of inconsistency" that shows the divergence of the judgments expressed with respect to an ideal situation where the consistence is perfect.

Saaty defines the "index of inconsistency" as

$$
\text { I.I. }=\frac{\lambda l-n}{n-l},
$$

in which the term $\lambda l$ is the maximum eigenvalue of the matrix $\mathrm{A}$ and represents the size of the same matrix. Saaty has shown that the pairwise comparison matrix is perfectly consistent when I. I. is equal to 0; the same matrix is perfectly inconsistent when I.I. is equal to 1. Generally, the acceptability threshold of the index of inconsistency is $10 \%$.

\section{Vesuvian Villas and Model Application}

The study aims to assess the vocationality of the Vesuvian Villas chosen for conservative and economic reuse, using a set of indicators explaining the state of fact of the buildings and their location.

The heterogeneous nature of the criteria considered (technical, economic or social) makes useful the application of an evaluation method of multi-criteria analysis that allows to summarize and analyze at best such complex information.

This method is applied to five villas not managed by the Fondazione Ente Ville Vesuviane, chosen because they have fewer owners.

The villas are all built along Resina Street, the main street of Ercolano that houses most of the eighteenth-century villas. Their distance from the highway exit of Ercolano ranges from $950 \mathrm{~m}$ from Villa Giulio de la Ville to $1150 \mathrm{~m}$ from Villa Lucia. The villas are far from the archaeological site of Herculaneum, $500 \mathrm{~m}$ minimum, $1100 \mathrm{~m}$ maximum.

The five villas studied are

- Villa Giulio de la Ville-38, A. Rossi Street;

- Villa Lucia-390, Resina Street;

- Villa Manes Rossi-321, Resina Street;

- Villa Arena-318, Resina Street;

- Villa Durante-308, Resina Street.

The number of owners of such dwellings varies from one to eleven.

\subsection{The Vesuvian Villas System}

The Vesuvian Villas system is a symbol of richness of the eighteenth century.

They are in the Ercolano municipality, known in the 18 th century as the "Golden Mile". It dates back to 1738 , when Charles Bourbon decided to move his residence from Naples to Portici (a municipality without interruption of its continuity with Ercolano). Antonio Canevari and Antonio Medrano were the architects who built the Royal Palace of Portici. During all of the eighteenth century the area knew the greatest splendor, with new luxurious buildings and farms. 
After the Second World War, the villas lost their main characteristics due to heavy urbanization. However, these buildings represent a unique concentrate, that can still be recognized today. The Vesuvian Villas are characterized by a central structure and a "double L" plan. Behind them, there are open spaces that define the atriums, the courtyards and the farms. The main façade can be oriented to the street (Golden Mile), to the sea, the Vesuvius or the countryside.

The villas have spectacular parks, with kiosks, fountains, seats and marble busts.

Inside the residences there are sculptures and frescoes with mythological and allegorical scenes.

Unfortunately, in the nineteenth century a series of events changed the configuration of the Vesuvian Villas System a lot.

In 1844 the railway was built; in 1904 and 1925 the Circumvesuviana railway and highway constructions broke down the gardens of the most recent villas.

Now the buildings do not have the function for which they were built; most of them are used as rustic houses, warehouses, restaurants or museums.

Some of these are now managed by the Fondazione Ente Ville Vesuviane, established on 29 July 1971 with Law n. 578/1971. This foundation was created to list, safeguard and enhance the conspicuous artistic and architectural heritage of the 122 Vesuvian Villas located between Naples and the province along the Golden Mile.

\subsection{Villa Giulio de la Ville (1795)}

The Villa was built in 1795 by an unknown architect, and it suffered important alterations over the years (see Figure 1).

The original planimetric configuration arranged the spaces around a court according to a symmetrical succession of buildings with respect to the axis passing through the entrance, the courtyard and the avenue of the original park. On the left side of the main building, there was a small chapel. Thereafter another two-story building was built against the chapel, while others overlooking the garden were demolished.

The condition of the villa can be considered very bad. In the central part of the facade, there are only a few elements of the original structure and the rest of the eighteenth-century decorations.

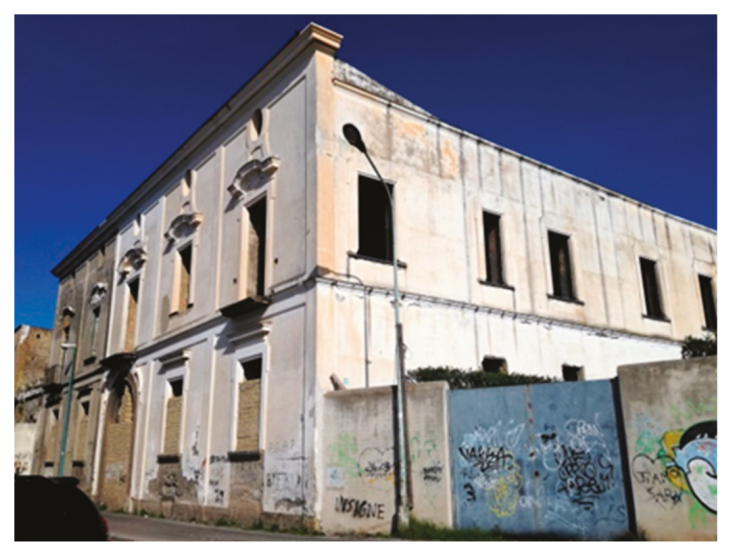

Figure 1. Villa Giulio de la Ville.

\subsection{Villa Lucia (1799)}

Built in 1799, Villa Lucia is in a very bad condition. The architect author of the building and the owners in the years are unknown (see Figure 2).

The " $\mathrm{C}$ " shaped building, on two levels, has a linear facade with openings surmounted by triangular tympanums. 
Inside the courtyard there is a narrow portal with doric pilasters and typical stucco decorations.

At the right edge of the inner facade stands an octagonal tower with a lowered sixth dome, and a pinnacle with volutes in Vesuvius black stone.

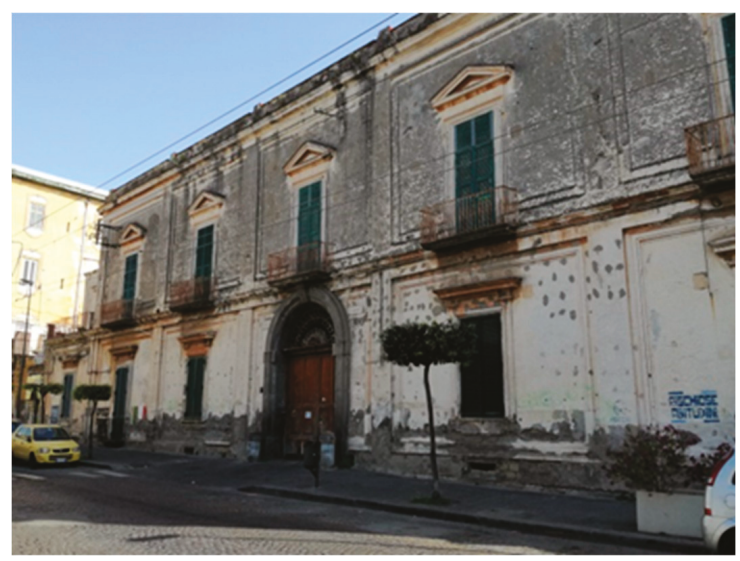

Figure 2. Villa Lucia.

\subsection{Villa Manes Rossi (1760)}

Villa Manes Rossi is a neoclassical building, built around 1760, and composed of a single level beyond the basement (see Figure 3).

The low facade on Corso Ercolano has a piperno base and decorations in the Pompeian style.

Beyond the entrance portal, framed by a pair of windows on each side, a long hallway leads to an open exedra on the well-preserved garden.

In the garden, after the construction of the villa, a two-story building was built.

The state of conservation of the villa can be considered discrete.

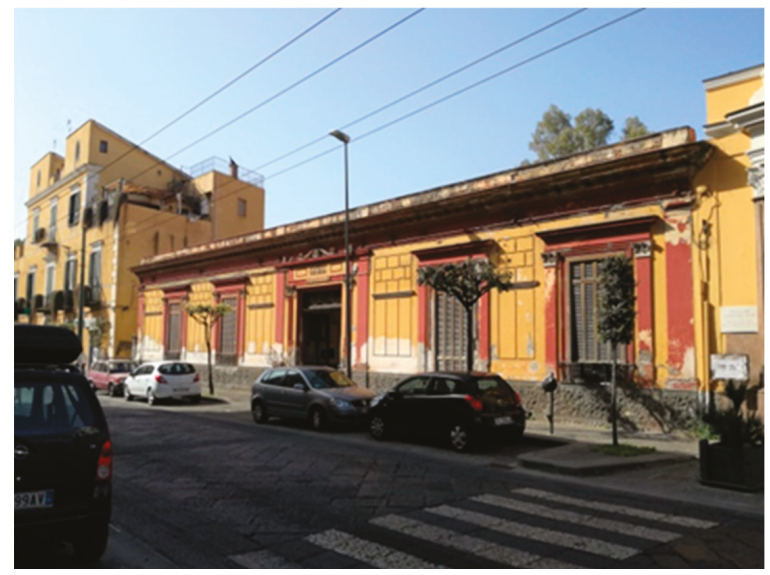

Figure 3. Villa Manes Rossi.

\subsection{Villa Arena (1740-1840)}

This building was built on an eighteenth-century layout of which nothing remains, except the staircase with shaped iron railings inside the courtyard (see Figure 4). 
Villa Arena is on three levels with a listed base.

The first one has balconies framed by triangular and curved tympanums, connected by a single balcony that runs along the entire front and extends towards the rear.

On the main front there are two long neoclassical railings.

Beyond the entrance gate with a piperno portal, there is a deep atrium covered by a coffered vault. Recently elevated structures have replaced the original roof.

The conservation state of the villa is discrete.

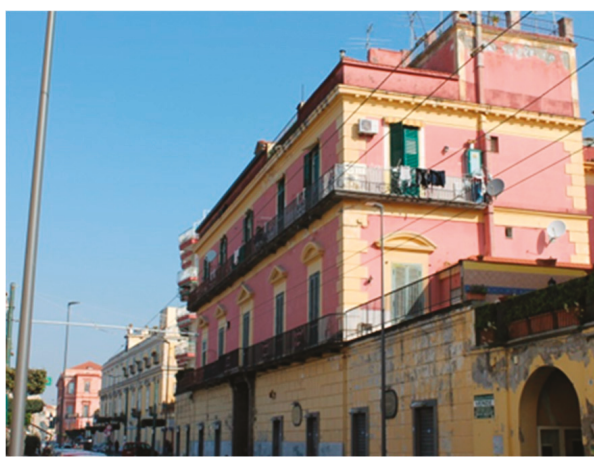

(a)

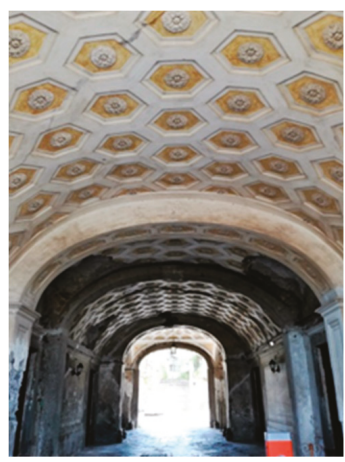

(b)

Figure 4. Villa Arena: main facade (a) and entrance hall (b).

\subsection{Villa Durante and Chapel (1700)}

The ancient structure was built for the Prince of Teora around 1700, and then became property of the Durante family in the 1800s (see Figure 5).

The villa, designed by the Neapolitan architect Ferdinando Sanfelice, is located in a large triangular area.

Its asymmetrical planimetric development is now unaltered, with the exception of the raised floor and the chapel, located in the courtyard.

The small sacred place, containing the statue of St. Gennaro, was also added by the Durante family. The family coat of arms is placed on the front of the building, and reports "Durante Deo domus est", with also a statue of St. Michael the Archangel.

Along the facade there is an alternation of triangular and semicircular tympanums, respectively, containing shells and laurel leaves.

The Villa has two portals with a smooth piperno frame that allow to access to two large atriums.

The garden is opposite, across the royal road. The entrance is characterized by a monumental portal, with a piperno structure and a curved architrave broken in key.

The conservation state of the villa is good. 


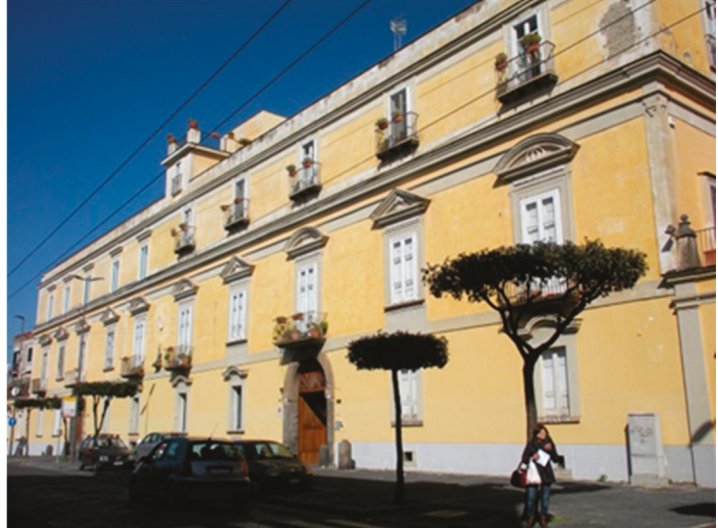

(a)

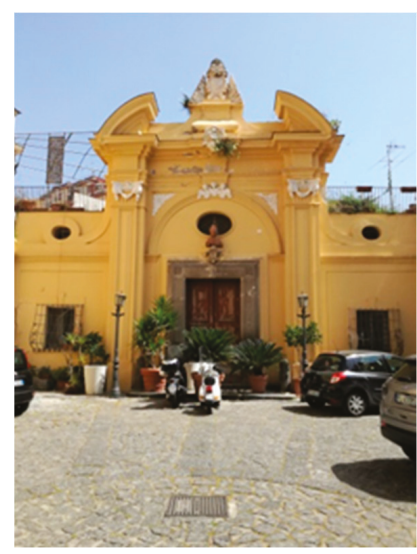

(b)

Figure 5. Villa Durante: main facade (a) and chapel entrance (b).

\subsection{The Evaluation Model (Voc)}

The global evaluation model (Voc) was created in reference to three Vesuvian Villas built in the eighteenth century, as a sub-unit of the larger universe of the Vesuvian Villas system in Ercolano.

These buildings are now managed by the Fondazione Ente Ville Vesuviane, namely, Villa Campolieto, Villa Ruggiero and Villa Favorita.

The model determines an index that represents the Villa's attitudes to a new economical destination. The result is a ranking of vocation of the elements that constitute the model.

The construction of the hierarchic model was done in the following stages:

- Creation of a database of villas with known economic destination (Villa Campolieto, Villa Ruggiero and Villa Favorita).

- Construction of a hierarchical model (Ahp1) for the evaluation of the success of compatible economic reuse and organization of villas according to this parameter $(\mathrm{u})$.

- Construction of hierarchical model (Ahp2) for the evaluation of vocationality (v) and determination of its ranking.

- Analysis of ranking (v) deviations from ranking $(\mathrm{u})$ and calibration of the vocational model.

- Application of the vocational model (Voc) to a real reference.

\subsection{The Assessment of Compatible Economic Use (Model Ahp1)}

The compatible economic use is the possible compromise between the profit of economic use and the conservation status of the historic building.

The villas considered in this part of the work are three villas managed by the Fondazione Ville Vesuviane (see above), because they are already planned for new use (two are museums, and one hosts the municipal library) and represent a significative subset of the Vesuvian Villas system.

The compatible economic use is valued from a group of parameters useful for the analysis of the current uses of buildings, their economic aspect and their general contribution to the conservation of the structure.

All these actions are necessary for the calibration of the reference model, to which will be made the calculations based on the villas chosen as case study.

The parameters used are (see Figure 6)

- Economic activity (ECON);

- $\quad$ Percentage of use of the building (GRU); 
- $\quad$ Building use function (MDU);

- Conservation status (SC).

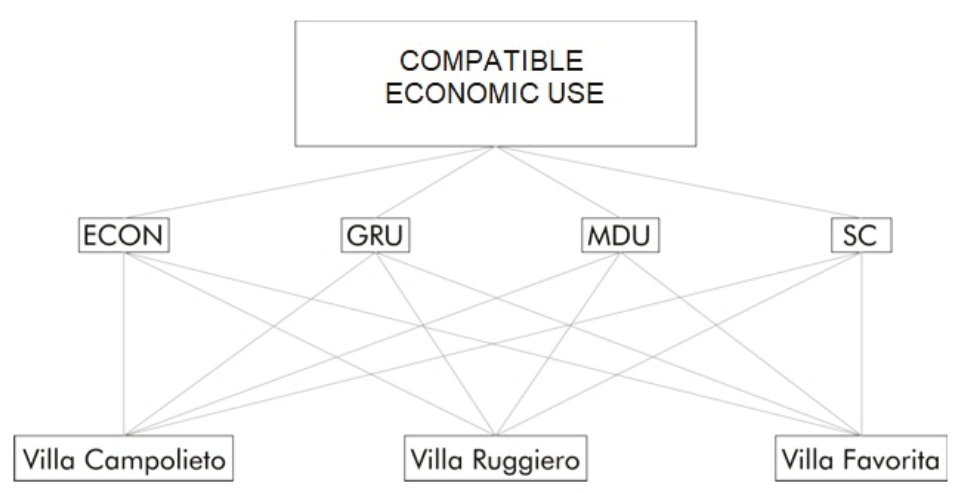

Figure 6. Model of the assessment of compatible economic use (Ahp1).

In particular:

- Villa Campolieto-ECON: museum; GRU: 80\%; MDU: museum, tourist purpose; SC: perfect.

- Villa Ruggiero-ECON: municipal library "G. Buonaiuto"; GRU: 80\%; MDU: public utility; SC: perfect.

- Villa Favorita-ECON: museum partially open to public (called "Casina dei Mosaici"); GRU: 25\%; MDU: tourist purpose; SC: mediocre.

\subsection{The Assessment of Vocationality (Model Ahp2)}

The assessment of vocationality of the Vesuvian Villas to an economic reuse is based on the analysis of a set of indicators in the creation of a potential offer for the building use.

The group of factors examined are the (see Figures 7 and 8 )

1. Characteristics about the Villa's localization (CLO): Useful for identifying the existence of potential demand for the different economic building uses. These factors are also influenced by the proximity of touristic sites (in this study the archaeological site of Herculaneum is considered). There are

- Importance of the touristic site, measured in yearly number of tourists (PPA);

- Touristic vocation of the site (TPA), indicated with values 1 (touristic site) or 0 (not touristic);

- Distance between the villa and the archaeological site of Herculaneum, measured in meters (DPA);

- Distance between the villa and the nearest highway exit measured in meters (DSA).

2. The intrinsic characteristics of the villa (CIN) is a group of indicators divided into two sub-families.

a. Typological characteristics (CTI): Parameters useful to define the current uses and the possible future ones with particular regard to the spatial composition of the building. There are

- $\quad$ Private garden (PG);

- $\quad$ Nearby parking (PPK); 
- $\quad$ Facade type (TF).

b. Historical and architectural characteristics of the villas (CSA): Parameters useful for an initial list of possible project choices when are planned functional and technological transformations:

- Detection of the architect author of the building (AC);

- Detection of the principal artists of inner decorations (AD);

- Presence of interior frescoes (PAFF);

- Presence of interior decorative stuccoes (PST);

- $\quad$ Presence of internal stone decoration (PDL);

- $\quad$ Presence of precious floors (PPP).

3. Characteristics about the ownership and legal structure (CLE): These factors take information about the ownership structure of the villas and the possible legal limitations. There are

- Type of property (ecclesiastic, private, public, etc.) (TPR);

- Presence of a safeguard limitation derived from National Law (VIN).

In particular, the parameters for the villas managed by the Fondazione Ente Ville Vesuviane, used as reference, are

$\underline{\text { CLO }}$

- Importance of touristic site: 534,328 tourists for the year 2018;

- Economic vocation of the site: Villa Campolieto: touristic; Villa Ruggiero: not touristic; Villa Favorita: touristic.

- Distance between the Villa and the archaeological site of Herculaneum: Villa Campolieto: 695 m; Villa Ruggiero: 752 m; Villa Favorita: 872 m.

- Distance between the Villa and the nearest highway exit: Villa Campolieto: $1115 \mathrm{~m}$; Villa Ruggiero: 975 m; Villa Favorita: 1117 m.

$\underline{\mathrm{CIN}}$

- Villa Campolieto-PG: yes; PPK: free parking 98 m away; TF: The villa has five levels; the main facade has a piperno plinth and the windows at the first floor have triangular tympanums; the back facade, on the sea, presents an elliptical large portico with round arches, connected to the building by two lowered arches with Tuscan capitals; AC: M. Giuffredo and L. Vanvitelli; AD: J. Cestaro and F. Fischetti; PAFF: yes; PDL: no; PPP: no; PST: yes;

- Villa Ruggiero-PG: yes; PPK: free parking 140 m away; TF: The building has three levels; the facade has stucco ornaments and tympanums around the windows on the first two levels; the portal is made with piperno and white marble, with a round arch; the back facade is typically Rococo; the balconies are framed by volutes in stucco and on the central one there is the niche with the bust of St. Gennaro, symbolically oriented to Vesuvius; AC: unknown; AD: unknown; PAFF: yes; PDL: no; PPP: no; PST: yes;

- Villa Favorita-PG: yes; PPK: free parking $84 \mathrm{~m}$ away; TF: The building, on two levels, has a main facade marked by pairs of pilasters and interrupted by two side portals in piperno and two central windows; on the back facade the protagonist is the large semicircular stone staircase, which the terrace with the park behind is connected; AC: F. Fuga; AD: P. Hackert; PAFF: yes; PPP: no; PDL: yes; PST: yes. 
- Type of property: All the buildings are managed by Fondazione Ente Ville Vesuviane.

- Presence of a safeguard limitation derived from National Law: all the buildings are limited by the Decree of the Ministry of Cultural and Environmental Heritage of 19.10.1976.

Then, following the previous indicators follow the same parameters for the five villas of the case study:

\section{$\underline{\text { CLO }}$}

- Importance of touristic site: 534,328 tourists for the year 2018;

- Economic vocation of the site: touristic;

- Distance between the Villa and the archaeological site of Herculaneum: Villa Giulio de la Ville: 725 m; Villa Lucia: 1254 m; Villa Manes Rossi: 1160 m; Villa Arena: 598 m; Villa Durante: $480 \mathrm{~m}$.

- Distance between the Villa and the nearest highway exit: Villa Giulio de la Ville: $950 \mathrm{~m}$; Villa Lucia: 1150 m; Villa Manes Rossi: 1.150 m; Villa Arena: 1060 m; Villa Durante: 1062 m.

- Villa Giulio de la Ville-PG: yes; PPK: free parking $150 \mathrm{~m}$ away; TF: The villa is on two levels; the main facade retains few elements of the original structure; on the entrance portal and on the windows are visible rests of the eighteenth-century decorations; AC, AD, PAFF, PDL, PPP: unknown; PST: yes;

- Villa Lucia-PG: yes; PPK: free parking 490 m away; TF: The building has two levels; the facade is linear and the windows have triangular tympanums; beyond the portal dominates an exedra courtyard; AC, AD, PAFF, PDL, PPP: unknown; PST yes;

- Villa Manes Rossi-PG: yes; PPK: free parking $450 \mathrm{~m}$ away; TF: The building is of clear neoclassical taste composed of unique floor; the facade has a piperno plinth and is decorated in Pompeian way; AC, AD, PAFF, PPP: unknown; PDL, PST: yes;

- Villa Arena-PG: yes; PPK: free parking $113 \mathrm{~m}$ away; TF: The facade is simple and with a piperno portal; at the first floor is decorated, the second one has a smooth surface with triangular and curved architraves; AC, AD, PPP: unknown; PAFF, PST, PDL: yes;

- Villa Durante-PG: yes; PPK: free parking 240 m away; TF: The continuous symmetrical curtain has an alternation of triangular and semicircular tympanums, respectively with shells and laurel leaves; in the center there is the Durante's family crest and a statue of St. Michael the Archangel; the villa has two portals with a smooth piperno frame that allow to access to two large atriums; AC: F. Sanfelice; AD, PAFF, PPP: unknown; PDL, PST: yes.

- Type of property: All the buildings are private.

- Presence of a safeguard limitation derived from National Law: All the buildings are limited by the Decree of the Ministry of Cultural and Environmental Heritage of 19.10.1976. 


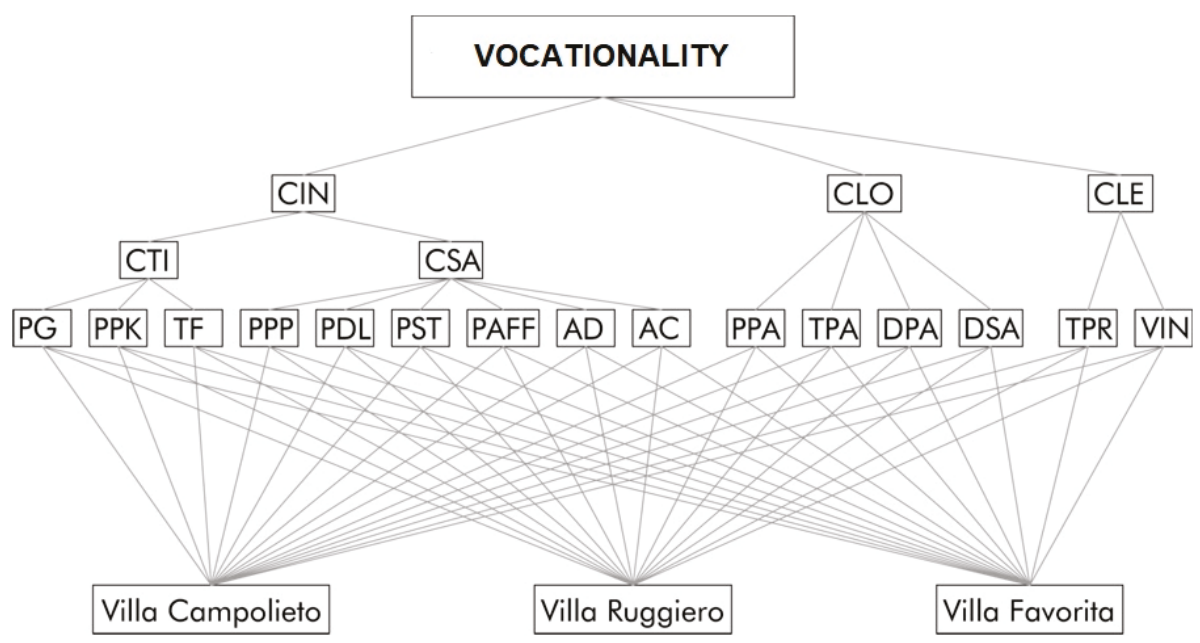

Figure 7. Model of assessment of the vocationality for the three villas managed by the Fondazione Ville Vesuviane, as a significant subset of the Vesuvian Villas system, and needed for the calibration of Model Ahp2.

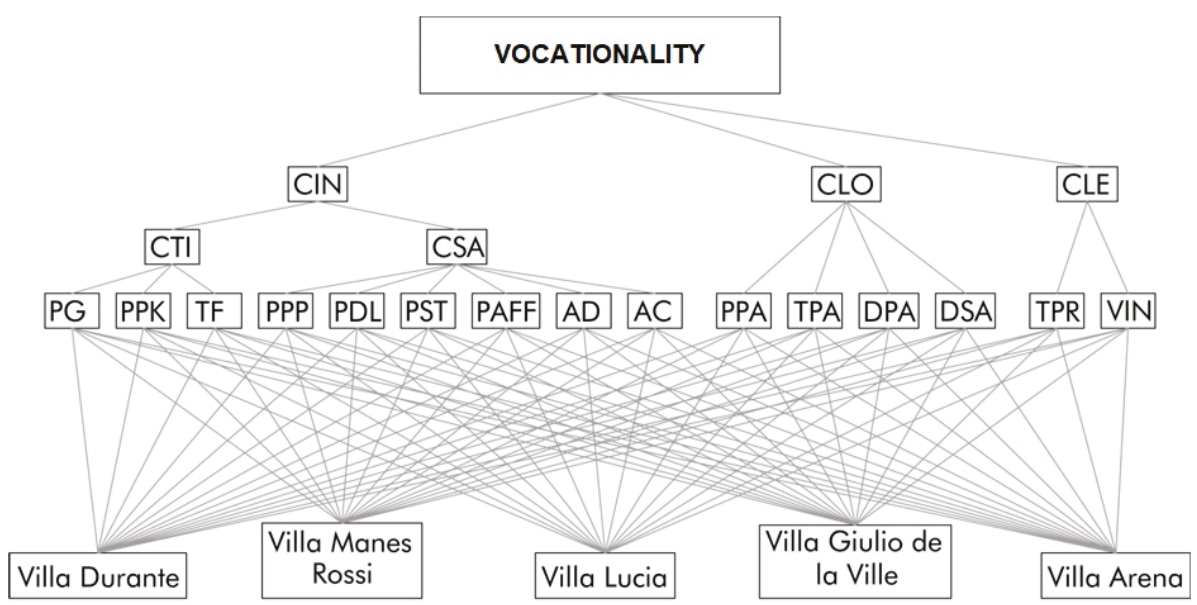

Figure 8. Model of assessment of the vocationality for the five villas of the case study (Ahp2).

\section{Model Calibration and Results}

The calibration of the model Ahp2 was carried out verifying the congruence of the ranking of the villas with known economic use (three villas managed by the Fondazione Ville Vesuviane: Villa Campolieto, Villa Ruggiero and Villa Favorita); more specifically, comparing the rankings obtained by models Ahp1 and Ahp2, or also comparing vector $u$ with vector $v$.

The results show that all the villas have a good level of economic vocation. The economic vocation model so validated is thus applied to the five villas of the case study. The results are in Figures 9 and 10. 


\begin{tabular}{|c|c|c|c|c|c|}
\hline New synthesis fo & isions Main V & TroisiAHP & $1 \ldots$ & $\square$ & $x$ \\
\hline $\begin{array}{l}\text { Here are the } \\
\text { alternatives. } \\
\text { Decisions } M\end{array}$ & $\begin{array}{l}\text { synthesiz } \\
\text { nthesized } \\
\text { low: Troi }\end{array}$ & $\begin{array}{l}\text { riorities } \\
\text { the ne } \\
\text { P1-Fon }\end{array}$ & $\begin{array}{l}\text { for the } \\
\text { etwork S } \\
\text { dazione }\end{array}$ & $\begin{array}{l}\text { Super } \\
\text { sdmod }\end{array}$ & \\
\hline Name & Graphic & Ideals & Normals & Raw & \\
\hline VILLA CAMPOLIETO & & 1.000000 & 0.653529 & 0.326765 & \\
\hline VILLA FAVORITA & & 0.081752 & 0.053428 & 0.026714 & \\
\hline VILLA RUGGIERO & & 0.448402 & 0.293043 & 0.146522 & \\
\hline $\begin{array}{ll}\text { Okay Copy Values }\end{array}$ & & & & & $v$ \\
\hline
\end{tabular}

Figure 9. Calibration phase: Overall synthesized priorities for the alternatives (Model Ahp1).

\begin{tabular}{|c|c|c|c|c|c|c|c|}
\hline 31 & lew synthesis fo & sions Main W & TroisiAHP2 & $2-F_{\ldots}$. & $\square$ & x & \\
\hline $\begin{array}{l}\text { Her } \\
\text { You } \\
\text { Win }\end{array}$ & $\begin{array}{l}\text { are the } \\
\text { synthesiz } \\
\text { dow: Trois }\end{array}$ & $\begin{array}{l}\text { inthesize } \\
\text { the netwo } \\
\text { ondazior }\end{array}$ & $\begin{array}{l}\text { urities fo } \\
\text { mod: fo }\end{array}$ & $\begin{array}{l}\text { for the al } \\
\text { ecisions } \\
\text { ormulaic }\end{array}$ & $\begin{array}{l}\text { Iternati } \\
\text { Main }\end{array}$ & & ^ \\
\hline & Name & Graphic & Ideals & Normals & Raw & & \\
\hline VILLA & CAMPOLIETO & & 1.000000 & 0.442216 & 0.944008 & & \\
\hline VILL & A FAVORITA & & 0.569822 & 0.251985 & 0.537917 & & \\
\hline VILL & A RUGGIERO & & 0.691516 & 0.305800 & 0.652797 & & \\
\hline Okay & Copy Values & & & & & & $v$ \\
\hline
\end{tabular}

Figure 10. Calibration phase: Overall synthesized priorities for the alternatives (Model Ahp2).

It is clear that the best potentials are those of Villa Durante with the value 0.866 (see Figure 11), and then Villa Giulio de la Ville (0.779), Villa Arena (0.721), Villa Lucia (0.660) and Villa Manes Rossi (0.650).

However, when trying to evaluate individual clusters of indicators, the final situation is different.

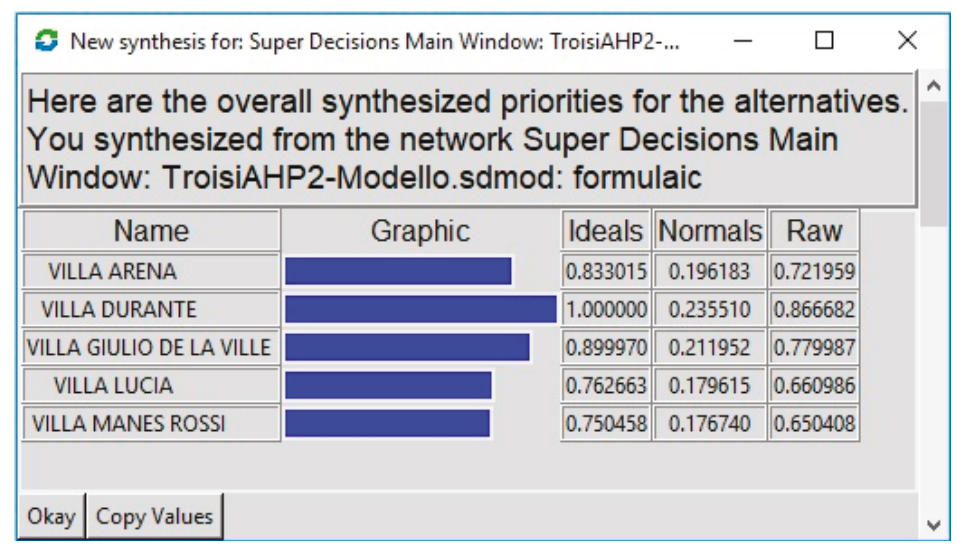

Figure 11. Results: Overall synthesized priorities for the alternatives (Model Ahp2).

Analyzing only the characteristics about the villa's localization (CLO) the results are shown in Figure 12. 
The highest value is still that of Villa Durante, but the other values are changed, and also the order of the villas.

\begin{tabular}{|c|c|c|c|c|c|c|}
\hline$\approx N$ & ew synthesis for: Su & der CLO & & - & $\square$ & $x$ \\
\hline & $\begin{array}{l}\text { Here are the } \\
\text { alternatives. } \\
\text { Subnet unde }\end{array}$ & $\begin{array}{l}\text { all synth } \\
\text { synthes }\end{array}$ & $\begin{array}{l}\text { priorit } \\
\text { om the }\end{array}$ & $\begin{array}{l}\text { ties for th } \\
\text { network }\end{array}$ & & \\
\hline & Name & Graphic & Ideals & Normals & Raw & \\
\hline VILI & A ARENA & & 0.915838 & 0.275860 & 0.137930 & \\
\hline VILL & A DURANTE & & 1.000000 & 0.301210 & 0.150605 & \\
\hline VILLA & GIULIO DE LA VILLE & & 0.828756 & 0.249630 & 0.124815 & \\
\hline & LA LUCIA & & 0.278505 & 0.083889 & 0.041944 & \\
\hline VILLA & MANES ROSSI & & 0.296841 & 0.089412 & 0.044706 & \\
\hline Okay & Copy Values & & & & & v \\
\hline
\end{tabular}

Figure 12. Overall synthesized priorities for the alternatives from the network characteristics about the villa's localization (CLO) (Model Ahp2).

We continue the verification with the other two categories of indicators, CLE (characteristics about the ownership and legal structure, see Figure 13) and CIN (intrinsic characteristics of the villa, see Figure 14), and the results change each time.

\begin{tabular}{|c|c|c|c|c|c|c|}
\hline \multicolumn{4}{|c|}{ New synthesis for: Subnet under CLE } & - & $\square$ & $x$ \\
\hline \multicolumn{6}{|c|}{$\begin{array}{l}\text { Here are the overall synthesized priorities for the } \\
\text { alternatives. You synthesized from the network } \\
\text { Subnet under CLE }\end{array}$} & ^ \\
\hline & Name & Graphic & Ideals & Normals & Raw & \\
\hline \multicolumn{2}{|c|}{ VILLA ARENA } & & 0.790717 & \begin{tabular}{|l||}
0.181441 \\
\end{tabular} & 0.090720 & \\
\hline \multicolumn{2}{|c|}{ VILLA DURANTE } & & 0.775421 & 0.177931 & 0.088965 & \\
\hline \multicolumn{2}{|c|}{ VILLA GIULIO DE LA VILLE } & & 1.000000 & \begin{tabular}{|l||}
0.229464 \\
\end{tabular} & 0.114732 & \\
\hline \multicolumn{2}{|c|}{ VILLA LUCIA } & & 0.895926 & 0.205582 & 0.102791 & \\
\hline \multicolumn{2}{|c|}{ VILLA MANES ROSSI } & & 0.895926 & \begin{tabular}{|l||}
0.205582 \\
\end{tabular} & 0.102791 & \\
\hline Okay & Copy Values & & & & & $v$ \\
\hline
\end{tabular}

Figure 13. Overall synthesized priorities for the alternatives from the network CLE (Model Ahp2).

The valuations highlight the more relevant indicators for the definition of the vocationality of the properties object to study: The aesthetic value, the presence of external areas and the more or less fractioned ownership regime undoubtedly have a very important weight. 


\begin{tabular}{|c|c|c|c|c|c|c|}
\hline \multicolumn{4}{|c|}{ New synthesis for: Subnet under CIN } & - & $\square$ & $<$ \\
\hline \multicolumn{6}{|c|}{$\begin{array}{l}\text { Here are the overall synthesized priorities for the } \\
\text { alternatives. You synthesized from the network } \\
\text { Subnet under CIN }\end{array}$} & ^ \\
\hline & Name & \multirow[t]{2}{*}{ Graphic } & Ideals & Normals & Raw & \\
\hline \multicolumn{2}{|c|}{ VILLA ARENA } & & 0.436105 & 0.190227 & \multicolumn{2}{|l|}{0.095113} \\
\hline \multicolumn{2}{|c|}{ VILLA DURANTE } & & 1.000000 & 0.436194 & \multicolumn{2}{|l|}{0.218097} \\
\hline \multicolumn{2}{|c|}{ VILLA GIULIO DE LA VILLE } & & 0.225393 & 0.098315 & \multicolumn{2}{|l|}{0.049158} \\
\hline \multicolumn{2}{|c|}{ VILLA LUCIA } & & 0.342519 & 0.149405 & \multicolumn{2}{|l|}{0.074702} \\
\hline \multicolumn{2}{|c|}{ VILLA MANES ROSSI } & & 0.288540 & 0.125859 & \multicolumn{2}{|l|}{0.062930} \\
\hline \multicolumn{6}{|c|}{ Okay Copy Values } & $\checkmark$ \\
\hline
\end{tabular}

Figure 14. Overall synthesized priorities for the alternatives from the network intrinsic characteristics of the villa (CIN) (Model Ahp2).

Finally, it is possible to evaluate how strong an alternative choice for the calculation of vocationality is through a sensitivity analysis (see Figures 15-17).

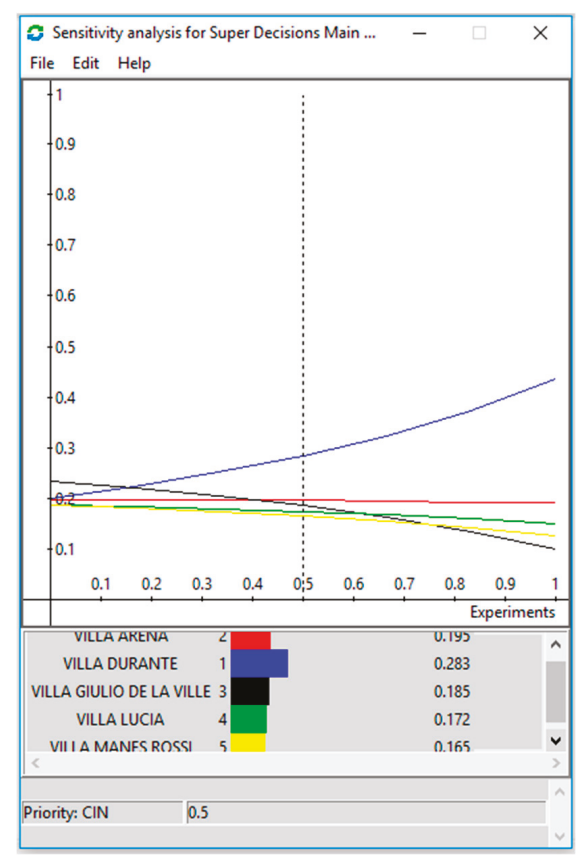

Figure 15. Sensitivity analysis based on the CIN criteria (intrinsic characteristics).

The diagram with colored bars in the table shows how the results of vocationality change when trying to modify the values of the CIN by $50 \%$ (see Figure 15). Following are similar examples but with variation in CLO (see Figure 16) and CLE criteria (see Figure 17). 


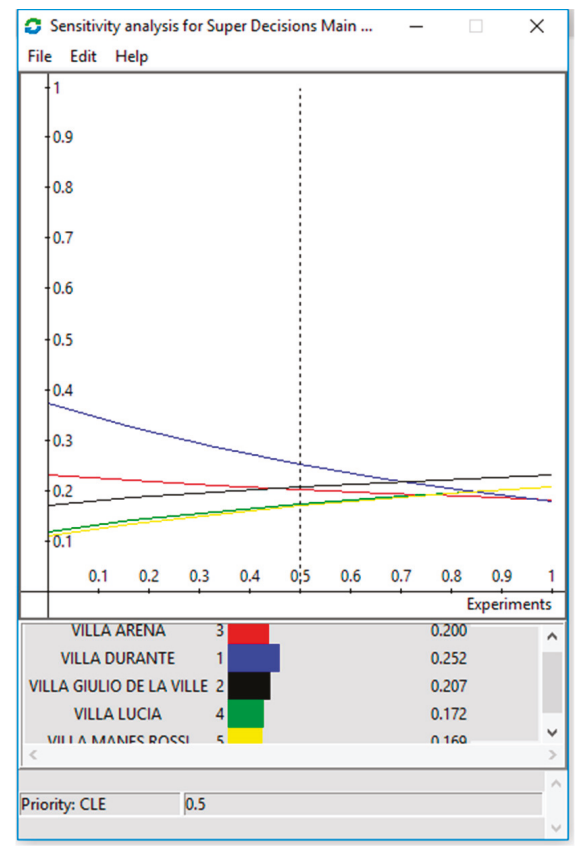

Figure 16. Sensitivity analysis based on the CLE criteria (characteristics about the ownership and legal structure).

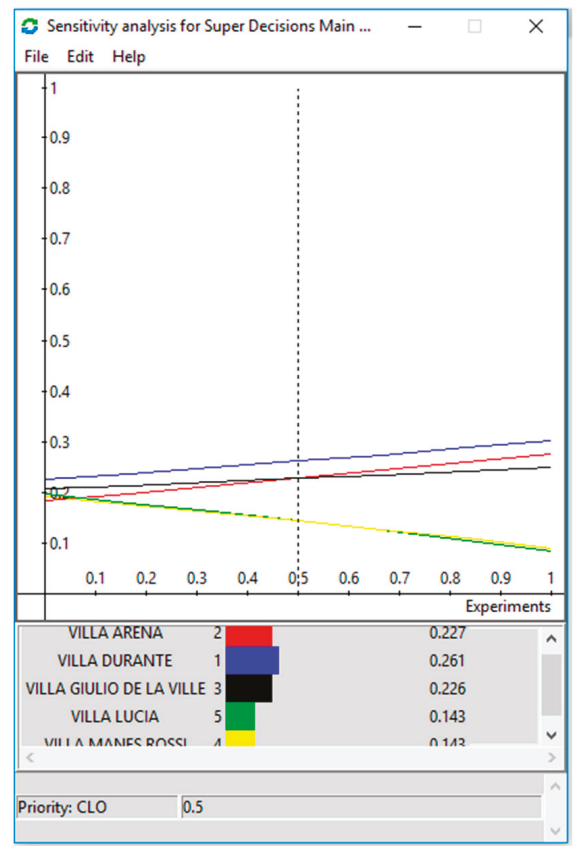

Figure 17. Sensitivity analysis based on the CLO criteria (characteristics of localization). 


\section{Conclusions}

The Vesuvian Villas, although a few are different from a functional and structural point of view, have unfortunately undergone several changes over time. Many of these villas are still used as homes today, but the original functional relationship between agricultural production and country residence has gone down (see Figure 18). This is due, in particular, to the poor profitability of the buildings and the division of properties, which, over time, have caused the alienation of soils and the worsening of environmental conditions. No villa performs the function for which it was built; most of them are used as a rustic house, warehouse, restaurant or, at best, in a converted house or museum.

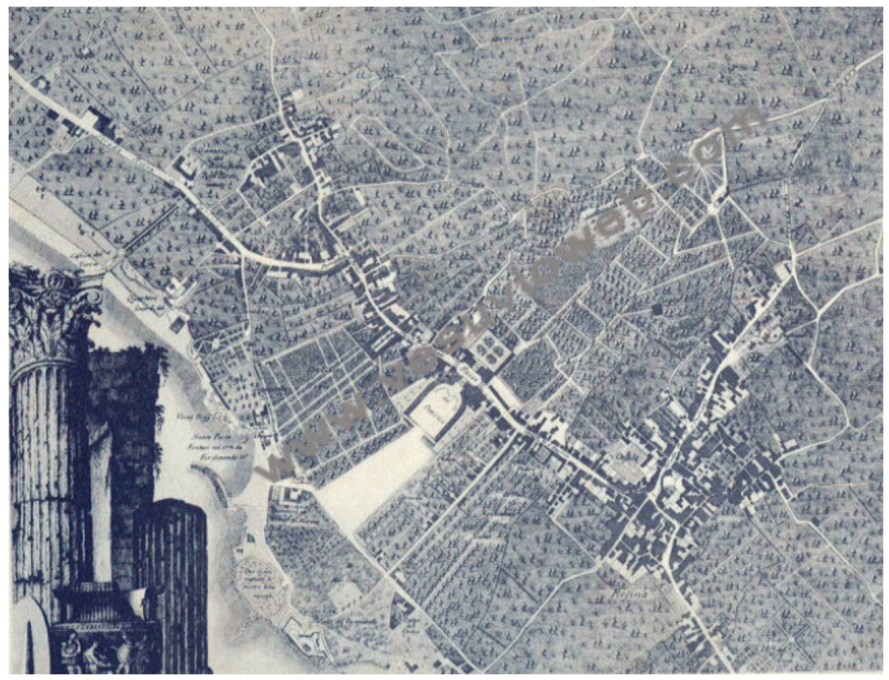

Figure 18. G. Carafa, Duke of Noja. Ancient topographic map of Naples and its hinterlands (1775), particularly of the "Strada Regia delle Calabrie".

In any case, they remain identity assets that connote geographic spaces and represent an inalienable patrimony of historical, environmental and artistic values to be protected and enhanced, since they nourish the identity of a community and guarantee a historical and cultural continuity.

The study proposes a model of analysis and evaluation of the vocationality of historical-architectural buildings in order to assess economic uses compatible with the needs of protection and conservation. Despite the evidence of these potentialities, the difficulties to convert qualitative variables into quantitative algorithms are a complex issue. These difficulties were particularly evident in the construction of the hierarchical model for the evaluation of vocationality. A further objective difficulty is the substantial absence of consolidated models of conservative economic re-use to be considered for the calibration of the potential vocationality. Not to underestimate, then, the usual and chronic lack of economic information: On the one hand, there are widely available information about historical and architectural aspects of the buildings, as well as their state of maintenance; on the other hand, there is total lack of information on the forms of buildings management and their consequent economic results [18-20]. This gap is only partially filled through the use of indirect indicators, such as revenue, grade and type of use. Only recently, due to high maintenance costs, the owners of the Vesuvian Villas have begun to consider economic use as a possible alternative to abandonment and, in this sense, the initiatives remain rather limited and still in the start-up phase. Therefore, the analysis carried out is a first attempt at reading a particularly changeable and unstable reality, where the use of the architectural artefact appears to be poised between distortive transformations (such as the intensive residential destination in peripheral urban areas) and recovery to original function as prestigious 
residence converted for public uses (libraries, corporate offices, museums, etc.). The applied model has demonstrated a good ability to identify the most favored realities to accommodate the forms of use compatible with conservation, also thanks to the possibility offered by the hierarchical analysis to build very articulated models, and being able to capture and incorporate into the evaluation more different aspects. Further model developments may be based on an improvement in the quality and quantity of economic data, in order to improve the fitting of the model. Evaluating the vocationality in relation to different forms of economic use can therefore provide useful indications to operate stratifications on the heritage of the Vesuvian Villas, in order to suggest private management strategies for individual assets; support the formation of adequate protection policies; and, last but not least, improve the efficiency of the few public funds available for the protection of a heritage unique for consistency and historical-architectural relevance.

Author Contributions: The paper is attributed in equal part to all authors. All authors have read and agreed to the published version of the manuscript.

Funding: This research received no external funding.

Conflicts of Interest: The authors declare no conflict of interest.

\section{References}

1. CHCfE, Cultural Heritage Counts for Europe, CHCfE Consortioum, 2015. Available online: https://www. ehhf.eu/ (accessed on 20 December 2019).

2. Council of the European Union. Conclusions on cultural heritage as a strategic resource for a sustainable Europe. In Proceedings of the Education, Youth, Culture and Sport Council Meeting, Brussels, Belgium, 20 May 2014.

3. European Commission. Towards an integrated approach to cultural heritage for Europe COM(2014) 477 final. In Proceedings of the Committee on Culture and Education, Brussels, Belgium, 22 July 2014.

4. Forte, F.; Listokin, D. Evaluating Impacts of Cultural Heritage Valorization - or Historic Preservation-: A Comparative Overview Italy - USA. In Proceedings of the XIV International Forum, Le vie dei Mercanti, La Scuola di Pitagora, Napoli, Italy, June 2016.

5. ISTAT, Rapporto BES 2018. Il benessere equo e sostenibile in Italia, Istat, Rome, 2018. Available online: https://www.istat.it/it/archivio/224669 (accessed on 22 January 2020).

6. Greffe, X. The economic Value of Heritage. Colloquio di Ahmedabad, National Trust of India. 1998. Available online: http://www.planningstudies.org/pdf/Raphael\%20Greffe-\%20E\%20(formatted).pdf (accessed on 22 January 2020).

7. Di Stefano, R. Il recupero dei valori; Edizioni Scientifiche Italiane: Napoli, Italy, 1979.

8. Forte, F. Historic, Artistic and Cultural Patrimony for a "Habitable City": Incentives for Care. In Green Energy and Technology; Springer: Heidelberg, Germany, 2018; pp. 233-243.

9. Papa, A. Valorizzazione e gestione del patrimonio culturale nel Codice dei beni culturali e del paesaggio. In BILANCIA, Paola Ed. La valorizzazione dei beni culturali. Modelli giuridici e di gestione integrata. Milano, Franco Angeli. 2006. Available online: https://www.francoangeli.it/Ricerca/scheda_libro.aspx?Id=14504 (accessed on 22 January 2020).

10. Forte, C. Valore di scambio e valore d'uso sociale dei beni culturali immobiliari; Editrice Arte Tipografica: Napoli, Italy, 1977.

11. Council of Europe. The private management of cultural property, Parliamentary Assembly Recommendation 1730 (2005). Available online: https://assembly.coe.int/nw/xml/XRef/Xref-XML2HTML-EN.asp?fileid= 17394\&lang=en (accessed on 22 January 2020).

12. Forte, F.; Formisano, R. Additional services in the management of Cultural Heritage: the paradox of archeological site of Pompei. In Proceedings of the XIII International Forum, Le vie dei Mercanti, La Scuola di Pitagora, Napoli, Italy, 11-13 June 2015.

13. Forte, F; Rupe, M. Sponsorship in the enhancement of Cultural Heritage and the role of Creative Industry: some evaluative aspects. In Proceedings of the XIII International Forum, Le vie dei Mercanti, La Scuola di Pitagora, Napoli, Italy, 11-13 June 2015. 
14. Listokin, D. Landmarks Preservation \& the Property Tax: Assessing Landmark Buildings for Real Taxation Purposes; Routledge: London, UK, 2012.

15. Monti, L. Il patrimonio storico e artistico private nel contesto dell'economia della cultura: una concreta opportunità di sviluppo. Intervention at the XXXVIII Members'Assembly of A.D.S.I. meeting Beni culturali: oneri o risorse? In Proceedings of the L'impatto economico del patrimonio storico-architettonico sull'economia del paese, Rome, Italy, 13 May 2015.

16. D'Alpaos, C.; Rosato, P.; Zanatta, V. Il riuso economico degli edifici storici: un modello di valutazione gerarchica. In Riconversione di Manufatti Storici in Musei, I musei di oggi negli edifici di ieri, Proceedings of the Atti delle giornate di studio, Torino, Genova, Italy, 7-8 May 2001; Ientile, R., Ed.; Name: Genova, Italy, 2002.

17. Saaty, T.L.; De Paola, P. Rethinking Design and Urban Planning for the Cities of the Future. Buildings 2017, 7, 76. [CrossRef]

18. Della Spina, L.; Calabrò, F. Decision Support Model for Conservation, Reuse and Valorization of the Historic Cultural Heritage. In Computational Science and Its Applications-ICCSA 2018, Proceedings of the ICCSA 2018. Lecture Notes in Computer Science, Melbourne, Australia, July 2018; Gervasi, O., Ed.; Springer: Cham, Switzerland, 2018; Volume 10962. [CrossRef]

19. Della Spina, L. Scenarios for a Sustainable Valorisation of Cultural Landscape as Driver of Local Development. In New Metropolitan Perspectives. ISHT 2018. Smart Innovation, Systems and Technologies; Calabrò, F., Della Spina, L., Bevilacqua, C., Eds.; Springer: Cham, Switzerland, 2018; Volume 100. [CrossRef]

20. Della Spina, L. Historical Cultural Heritage: Decision Making Process and Reuse Scenarios for the Enhancement of Historic Buildings. In New Metropolitan Perspectives. ISHT 2018. Smart Innovation, Systems and Technologies; Calabrò, F., Della Spina, L., Bevilacqua, C., Eds.; Springer: Cham, Switzerland, 2018; Volume 101. [CrossRef]

(C) 2020 by the authors. Licensee MDPI, Basel, Switzerland. This article is an open access article distributed under the terms and conditions of the Creative Commons Attribution (CC BY) license (http://creativecommons.org/licenses/by/4.0/). 
Article

\title{
Historical School Buildings. A Multi-Criteria Approach for Urban Sustainable Projects
}

\author{
Maria Rosaria Guarini ${ }^{1, *}$, Pierluigi Morano ${ }^{2}$ and Francesco Sica ${ }^{3}$ \\ 1 Department of Architecture and Design, "Sapienza" University of Rome, 00196 Rome, Italy \\ 2 Department of Science of Civil Engineering and Architecture, Polytechnic University of Bari, 70125 Bari, \\ Italy; pierluigi.morano@poliba.it \\ 3 Doctoral School of Architecture and Construction, Department of Architecture and Design, "Sapienza" \\ University of Rome, 00196 Rome, Italy; francesco.sica@uniroma1.it \\ * Correspondence: mariarosaria.guarini@uniroma1.it; Tel.: +39-347-3394586
}

Received: 20 December 2019; Accepted: 29 January 2020; Published: 3 February 2020

\begin{abstract}
It is recognized, in Europe and elsewhere, that there is a need to implement sustainable urban intervention policies based also on the recovery of existing public real estate assets. In Italy, the schools are a significant part of public property. At this time (2019), many buildings destined for teaching need to be redeveloped, both from a structural and plant engineering point of view, and with regard to the management of the spaces available for teaching and social activities. Although, there have been many attempts by the legislator to regulate the modus operandi in the school construction field, it is clear that there is a lack of a unique regulatory system in which the technical and functional-managerial aspects relating to the same school are considered together. On this basis, with this study a multi-criteria evaluation protocol to support intervention planning for the redevelopment of existing school buildings is proposed. The study defines an evaluation framework with which we can establish the design priorities to be carried out in accordance with the building features and community needs. The evaluation framework is tested on a renewal project regarding a school building located in the historic center of Rome (Italy).
\end{abstract}

Keywords: urban sustainable development; historical school buildings; multi-criteria evaluation

\section{Introduction}

The urban policies of many countries, both European and not, are characterized by sustainable intervention practices [1,2]. Among these, some concern specifically the preservation of ecosystems, while others concern the conservation of territorial infrastructure and existing buildings [3-7]. Since 2007, with the Leipzig Charter [8], the European Union Member States promoted development policies based on integrated planning actions, mainly for the regeneration and upgrading of both buildings and urban areas $[9,10]$. There are many European strategies and plans in which the aspects linked to the physical regeneration of territory and those regarding its economic, social and environmental system are considered jointly [11,12].

In Italy, the Legislative Decree 102/2014 [13], which implements the European Directive 2012/27/EU, outlines a strategic reference framework specifically aimed at promoting urban renewal projects on public real estate assets in order to improve their energy efficiency [14-17]. This also with the use of renewable resources present in nature, building systems and technological solutions capable of producing a low environmental impact [18-22].

With regard to the Italian context, the set of public buildings consists of 1,056,404 units divided into 11 homogeneous clusters [23]. The clusters with more than 10,000 buildings include offices $(48,376$ units), homes $(594,337)$, sheds and warehouses $(66,657)$, garages and car-parks $(184,532)$, shops $(28,794)$, barracks $(13,931)$, schools $(46,429)$, hospitals $(11,731)$, and sports facilities $(15,725)$ [24]. 
Along with dwellings and car-parks clusters, school buildings are one of the largest clusters. According to data in Anagrafe dell'Edilizia Scolastica (AES), made available (26 September 2018) through the portal of the Education, University and Research Ministry [25], in Italy there is a school building stock of 40,151 buildings in use, and 22,000 of these were built before 1970. A little more than half of the stock buildings are equipped with measures to reduce energy consumption $(57.5 \%)$ and have a static test certificate (53.2\%). The $22.3 \%$ of buildings without this certificate were built before 1970 . A total of $59.5 \%$ do not have a fire prevention certificate, and $53.8 \%$ lack a habitability certificate. The situation regarding the emergency plan, present in $78.6 \%$ of schools, and architectural barriers, removed in $74.5 \%$ of buildings, is better. On the basis of these data, the Italian school heritage is mainly composed of buildings built before 1975, not only with technological criteria typical of their construction time, but also conforming to educational needs different from the current ones. Only those built after 1975 are characterized by the typological specialization enshrined in the currently legislation that takes into account the new international educational-didactic guidelines. With reference to international guidelines, the school is no longer conceived only as a space for students and school staff offering services closely related to education, but also as a place to carry out activities in order to make the urban context in which the school is located more livable [26,27]. By providing the community with structures, spaces, and services, the school can be an aggregating territorial polarity (civic center), and it can improve the quality of life in the community [28-30].

Over time, there have been multiple attempts to direct the modus operandi of intervention on Italian school buildings with specific measures. Among these, those issued from 1960 onwards, and some currently in force are:

- the Circular $n^{\circ} 425 / 1967$ of the Public Works (Studies and Planning Service) Ministry concerning building and urban planning standards, in particular the Appendix $C$ of the aforementioned Circular contains indications on influence radius to be assumed for the identification of the territorial area in which the school is;

- the Ministerial Decree (MD) (Ministry of Public Works in agreement with the Ministry of Public Education) of 18/12/1975 (Updated technical standards relating to school building) [31];

- the Law $n^{\circ} 23 / 1996$ (Regulations on school building) [32];

- the Legislative Decree $n^{\circ}$ 106/2009 (concerning the protection of health and safety in the workplace) [33];

- the Agreement of 18 November 2010 according to Article 9 of Legislative Decree of 27 August $1997, n^{\circ} 281$, between the Government, regions, autonomous provinces of Trento and Bolzano, provinces and municipalities (Guidelines for the prevention of indoor risk factors for allergies and asthma in schools) [34];

- $\quad$ the Ministerial Decree (Ministry of Infrastructure and Transport) of 17 January 2018 (Technical Regulations for Construction, in particular Chapter 8, which contains the anti-seismic safety parameters to be followed by existing structures) [35];

- the Law $n^{\circ} 107 / 2015$ (Reform of the national education and training system and delegation for the reorganization of current legislative provisions) [36];

- the Law n ${ }^{\circ} 81 / 2019$ (art. 4-bis: Adaptation of school buildings to fire regulations) [37] (for a more specific description see Section 2).

Significant among the guidelines that directly affect the construction and redevelopment of school buildings are those relating to the:

- internal architecture of schools, by the Education, University and Research Ministry after hearing the Unified Conference (MD 11 April 2013), bearing the technical standards-framework, containing the minimum and maximum indices of urban functionality, construction, also with reference to technologies in the field of efficiency and energy saving and production from renewable energy sources, and teaching, to ensure appropriate and homogeneous reference design guidelines on the national territory [38]; 
- $\quad$ recommendations for the energy upgrading of school buildings, produced by the Italian Ente per le Nuove tecnologie, l'Energia e l'Ambiente (ENEA) aimed to disseminate knowledge and operational tools at the basis of the energy upgrading of buildings for training, following an updated approach to the latest regulations and the current possibilities for economic incentives [39] for a healthy school environment in Europe.

- $\quad$ environmental quality in external and internal spaces of European schools, in 2015 the pilot project SINPHONIE (Indoor Pollution in Schools and Health-European Observatory) [40], funded by the European Parliament and supported by the European Commission, aimed at investigating the quality of air inside and outside school environments, and the effects that pollutants can have on the health of school users. Through the SINPHONIE pilot project, it was possible to establish methodologies and define standardized tools for characterizing school interiors and assessing health risks for students and staff, in order to maintain a healthy and livable school environment [41-43].

Each of the legislative acts take into account one of the many aspects to be considered with reference to the life cycle of the school building. This is in relation to mono-dimensional logics that consider in a disjointed manner the design and construction components of the transformation or redevelopment project preferring a solving approach to that of integrated problem based-solving type [44-46].

The many investment programs aimed at upgrading the existing school heritage and new schools, promoted at the national and/or regional level since the second half of the last century, have followed a solving approach logic. The allocations for interventions on the school building stock have been directed to finance interventions on the school buildings for their construction, renovation, safety, anti-seismic adaptation, energy efficiency and innovation [47]. However, from 2015, according to Art. 10 of Legislative Decree $n^{\circ}$ 104/2013 (now Law n 128/2013), the three-year national planning of school building interventions was introduced. The substantial funding allocated to school building in recent years has preferred a modus operandi to solve specific problems, not often taking into account the mutual relations that there may be between multiple technical and regulatory aspects of a transformation/redevelopment intervention.

On the basis of such a complex and articulated technical-regulatory framework, due to the multiple aspects and mutual relations that characterize the reference system to be taken into account in the field of school building, the execution of actions on the same school building in different times in a non-integrated way may involve intervening in the same building in a way not perfectly congruent functionally and aesthetically with the need to interrupt or move educational activities with a consequent increase in the costs [48,49].

So, in the present work a multi-criteria evaluation protocol for the definition of integrated action strategies regarding new school buildings or existing ones is proposed. With reference to the part of the school building already in use, the implementation of the proposed methodology is aimed at identifying, on the one hand, the need and degree of a functional and structural adaptation of the school, and on the other, the re-modelling of the internal spaces and external ones to be used by the community. This takes into account both the technical and structural features of the building, and the socio-economic characteristics of the urban context. Using the proposed evaluation methodology, the types of intervention to be adopted in order to ensure the building conforms to the regulations in force and to satisfy the people's needs are defined. The validity and flexibility of the proposed method are tested by implementing the evaluation approach in the case of a redevelopment project concerning the school building located in the historic centre of Rome (Italy).

In the following sections, Section 2 describes the type of technical-regulatory material for the execution of initiatives on school buildings with particular reference to Italian case, Section 3 defines the phases of the multi-criteria methodology, and Section 4 illustrates the case study. Finally, conclusions are reached, and the potential for application of the proposed instrument and future research prospects are defined. 


\section{Materials}

\subsection{Premise}

In order to highlight the multi-dimensional character of initiatives aimed at enhancing and/or modifying the existing schools in an integrated manner, it is necessary to take into account, in the planning and design phase, multiple aspects according to a multicriteria logic [50-54].

For the recovery and/or enhancement of existing school buildings, it is advisable to verify at the planning and design stage the correct sizing of the spaces for teaching in compliance with the endowment of minimum areas per capita, the compliance with the parameters of seismic safety and fire prevention, as well as the overcoming of any architectural barriers present, and the energy efficiency of the building and the healthiness of the indoor spaces. These checks allow one to define the objectives to be pursued, and the actions to be carried out for the requalification/enhancement of the building. In addition to technical and regulatory considerations, it is also important to highlight which are the primary needs of the urban context to be answered, both through the reorganization of the existing educational offer and by allocating some areas to the exercise of extra-curricular activities. In the following, the technical and regulatory aspects of the Italian context are taken into account.

Thus, preferring a logic of integration between the design aspects related to the same intervention, the proposed multi-criteria evaluation approach includes each of them in order to establish the priorities for action in compliance with the current regulatory system and technical-structural characteristics of the building, as well as the economic and social conditions of the urban context in which the school is located. A careful survey of the state of the building allows us to establish the degree of transformability from the interventions that need to be carried out for the adaptation of the building in compliance with the technical and legislative provisions in force and the community's needs. It should be noted that the main aspects to be considered in order to upgrade the existing building in an integrated manner are listed below. We will not go into the details of each one, especially for those of technical nature, because for each aspect a specific sector study by experienced professionals (such as the one on the energy performance of the building, and/or the rehabilitation of its structure) is required. The methodology proposed supports the programming of sustainable interventions in existing school buildings, and also accessing the funds for the redevelopment and renovation of the schools, taking into account multiple aspects in an integrated way.

\subsection{Regulatory Overview and In-Depth Analysis of the Main Italian Legislative Measures for School Construction}

As previously specified, in Italy, since the 1970s, there have been many legislative measures in the field of school building aimed at providing planning and/or design indications for the execution of interventions on existing schools, preferring a problem-solving-based approach, and not one of integration and compensation between multiple effects deriving from the same settlement transformation intervention. The main reference standard documents currently in force (2019) for school building are the:

- $\quad$ Ministerial Decree (MD) of 18 December 1975, which illustrates the Technical Standards referring to school buildings, including the educational, building and urban planning functionality indices, to be observed in the design and verification of interventions on existing schools. These indices vary according to the type of study courses and the morphological-urban characteristics of the urban context in which the school building is located. The same MD also distinguishes between indoor spaces (units for teaching and special activities, sanitary facilities, indoor gyms, administrative offices, classrooms for common events) and outdoor ones (outdoor sports fields and parking areas);

- $\quad$ Law n ${ }^{\circ}$ 23/1996 (School Building Regulations), aimed at the construction of a national information system on existing school buildings resulting from the collection of data on the state of maintenance, the safety level of existing school structures and the rate of usability of the same by the community in extra-educational time. With the above law, Anagrafe dell'Edilizia Scolastica (AES), with which 
the management of interventions on school building at national and regional level is carried out, is established;

- Legislative Decree $n^{\circ} 106 / 2009$, which contains additional and corrective provisions to Legislative Decree $\mathrm{n}^{\circ} 81 / 2008$, on the protection of health and safety in the workplace, especially with regard to the presence of materials containing asbestos;

- $\quad$ Agreement of 18 November 2010 pursuant to Article 9 of Legislative Decree $n^{\circ}$ 281/1997, for the implementation of project initiatives aimed at preventing indoor risk factors for allergies and asthma in schools;

- Ministerial Decree of 14 April 2013, containing the new guidelines of reference for the construction of new schools, also with regard to technologies in the field of energy efficiency and saving and production from renewable energy sources, and teaching. The provisions contained in this MD, of a performance type, deviate from the prescriptive style of the previous illustrated in the MD of 18 December, 1975;

- Law n 107/2015 ("Good School Law”), specifically aimed at describing the objectives to be pursued in the design and implementation of "innovative schools" from an architectural, plant engineering, technical-construction, energy, anti-seismic and fire safety point of view. This law identifies the types of extra-educational functions at the service of the community to which to allocate part of the school space;

- $\quad$ ENEA guide published on 19 April 2016, which also illustrates the types of intervention related to the building envelope, lighting and daylight, heating systems, renewable energy and water management to be implemented for the redevelopment of a school building;

- Ministerial Decree of 17 January 2018, containing provisions on anti-seismic safety to be taken into account when intervening in the construction, as in the case of existing schools;

- $\quad$ Law $n^{\circ} 81 / 2019$, which illustrates certain provisions for the adaptation of existing school buildings to the requirements of the fire regulations.

These standards can be used as a reference both for existing buildings and new constructions. From each one, the technical-regulatory objectives to be pursued can be defined, and the corresponding parameters (Evaluation Criteria) can be identified with which one it is possible to express the degree of achievement of each objective.

Table 1 shows some of the main regulatory references in the field of Italian school buildings. The basic purpose is described for each of them, and the corresponding evaluation criteria is specified with which one can express the level of conformity between the actual state of the school building and the legal requirements to be complied with. With respect to the list of normative references described above, in Table 1 the normative references containing provisions for Italian school field still in force are taken into consideration.

Table 1. Main regulatory references for Italian school buildings and corresponding evaluation criteria.

\begin{tabular}{|c|c|c|}
\hline $\begin{array}{l}\text { Main References for } \\
\text { School Buildings }\end{array}$ & Objective to Be Pursued & Evaluation Criteria \\
\hline $\begin{array}{l}\text { Ministerial Decree of } \\
18 \text { December } 1975\end{array}$ & Provide technical standards for school buildings & $\begin{array}{l}\text { Respect of the per capita surface area allocation for each } \\
\text { space }\end{array}$ \\
\hline Law n ${ }^{\circ} 23 / 1996$ & Collect information on the building to renewal & $\begin{array}{l}\text { Check and acquisition of the data availability relating to the } \\
\text { school }\end{array}$ \\
\hline $\begin{array}{l}\text { Legislative Decree } \\
n^{\circ} 106 / 2009\end{array}$ & $\begin{array}{l}\text { Proposals of interventions for the safety of school } \\
\text { spaces from pollutants and harmful factors }\end{array}$ & $\begin{array}{c}\text { Control of the implementation of interventions to remove } \\
\text { polluting materials }\end{array}$ \\
\hline $\begin{array}{l}\text { Agreement } \\
18 \text { November } 2010\end{array}$ & $\begin{array}{l}\text { Establish lines of action for the prevention of indoor } \\
\text { risk factors }\end{array}$ & $\begin{array}{l}\text { Check of the implementation of measures to prevent surface } \\
\text { condensation inside the spaces used for teaching }\end{array}$ \\
\hline $\begin{array}{l}\text { Ministerial Decree of } \\
17 \text { January } 2018\end{array}$ & Define security parameters for existing buildings & $\begin{array}{c}\text { Possible inactivity of internal/external spaces to school } \\
\text { building }\end{array}$ \\
\hline
\end{tabular}

Description of the Information Contained in the Data Sheet for School Buildings

Among the normative references illustrated above, the Law $n^{\circ} 23 / 1996$ (Norms on school building) underlines the necessity to carry out a planning phase of interventions aimed at the conservation of the 
existing building considering the survey about the characteristics of the school structures in use and of the urban context. Through this law, the Anagrafe Regionale dell'Edilizia Scolastica (ARES) is established with the aim of systematizing the information system on the regional school property assets.

Each region and autonomous province independently manages access to ARES, and the provinces and municipalities are responsible for compiling, updating and implementing the data collection forms for each individual school building. This was done through direct surveys and inspections conducted at the school of interest.

The module for collecting data on school buildings contained the elements needed to acquire information ascertained through the completion of two questionnaires:

(i) Questionario Edificio (QE), aimed at collecting elements to evaluate quantitatively and qualitatively the school in use;

(ii) Questionario istituzione scolastica, aimed at collecting information on individual school units, i.e., whether or not there are several school units in the same building, what type and how they are organised.

The data that can be deduced from (i) on the school building are summarized in the Appendix of Questionario Edificio in ARES by Law 23/1996. For a more detailed specification, please refer to the Instruction Manual of QE in ARES for the compilation of the school building stock survey sheets.

In order to have a clear view of the current state of the suitability of the school building for its functions when new work is to be carried out on the building, the relevant data which are particularly interesting include the most recent work on the structure (Point 9.0-Subsequent transformations). For each intervention, it is important to establish the intervention class and the execution year. The classes of intervention referred to in the survey, aimed at determining the state of maintenance, and consequently the level of adequacy of the building to the functions for which the building is intended, both from the technological point of view and in terms of amount of space, are:

- $\quad$ extension and/or super-elevation: a complex of works that have the effect of enlarging an existing building, creating additional spaces or volumes. The extension can be done by "horizontal addition" (in which case it involves an increase in coverage), or "vertical addition" (i.e., elevation), or finally with actions of both expansion and elevation;

- building renovation: interventions aimed at transforming building organizations through a systematic set of works that can lead to a building organization in whole or in different parts. These interventions include the restoration or replacement of certain elements of the building, or even the elimination, modification and insertion of new elements and systems;

- integral and conservative restoration: interventions aimed at preserving the building organism and ensuring its functionality through a systematic works set that-in compliance with the typological, formal and structural elements of the building organism-allow its compatible use with them. These interventions include: the consolidation, restoration and renewal of the building's constituent elements; the insertion of ancillary elements and systems required by the use needs; the elimination of extraneous elements to the building organism;

- $\quad$ extraordinary maintenance: works aimed at renovating and replacing parts, including structural ones, of buildings, as well as the construction and integration of sanitary and technological services, while respecting the volumes and surfaces of the individual building units.

The state of maintenance regarding the building works and systems (Point 13.0 — State of conservation) is evaluated qualitatively by attributing a score according to the following classification: 6 = does not require any intervention; $5=$ requires partial maintenance; $4=$ requires complete maintenance; 3 = requires replacement or partial refurbishment; 2 = requires replacement or complete refurbishment; 1 = requires ex-novo installation; $\mathrm{X}=$ system is not necessary;

The functional and dimensional characteristics of the rooms include the location, functional destination, size of the rooms on each floor of the building, shape (when the floor plan of a room is clearly different from a rectangular square, and it is difficult to carry out the educational activities), 
natural and artificial lighting, hygienic conditions (dependent not on poor cleaning but on a physical deficiency of the inner shell), and the acoustic conditions of each space.

Depending on the observations and specifications previously made, it is clear that in order to plan interventions on existing school buildings, it is necessary to comply with a number of regulatory requirements, and to obtain information on the building to be recovered. In general, the information concerning the school structure can be of various types. Each one regards a specific school aspect, both technical and organizational-functional, especially regarding the way in which the available space is used.

On the basis of the information system proposed by Law 23/1996, it can be observed, however, that the phase of reconnaissance of the state concerning the school building does not include the acquisition of data in the urban context.

With reference to the Constitutional Court (judgments 62/2013, 284/2016 and, lastly, 71/2018), within the discipline on school buildings "more subjects intersect, such as "territorial governance", "energy" and "civil protection". Thus, the proposed evaluation methodology, as specified in the following paragraph, aims to jointly consider regulatory aspects, school building features, and characteristics of the territory.

Some of the information illustrated in Table 2 is identified from the data list contained in Questionario Edificio. For each one, an alternative reference document to the QE is indicated, from which the data relating to the information to be quantified can be extrapolated. This is with a view to creating an information system useful for the planning and execution of projects that respect the characteristics of the building to be recovered, and that can also satisfy the citizens' needs in view of the distinctive characteristics of the local economy expressed in terms of existing services and those potentially settled in the area. In order to establish what further activities can be offered to the community by taking advantage of the internal spaces and connected externally to the school, it is necessary to identify the users' class to which the type of service provided is allocated (as specified in the following Section 3.1.2).

\section{Evaluation Framework}

The proposed evaluation method seeks to verify the technical, regulatory and management conditions of existing school buildings (especially with regard to the use of available space for educational activities) in order to plan transformation and/or conservation activities, not only observing the regulatory requirements in force and the physical characteristics of the building, but also the needs of the community.

The method consists of two steps: (a) knowledge phase, (b) evaluation phase. In the first phase, the reference legislation is analyzed, and technical-management information on the school is collected. In the second phase, the regulatory requirements are verified, and the main methods of intervention are defined. This is with regard to both the physical and management system of the building and the services that the school can offer to the community.

Figure 1 shows the outline of the proposed evaluation methodology and highlights the mutual relations between each of its sub-phases. The diagram in Figure 1 aims to graphically illustrate how the physical and functional characteristics of the school buildings are checked and evaluated in accordance with the reference regulations, as well as to program interventions compatible with existing community's needs. Compatibility and consistency assessments provide useful information on the types of interventions to be implemented for the renovation/recovery of the building.

Each step (knowledge phase and evaluation phase) is made of specific sub-phases analysed in the following. 


\subsection{Knowledge Phase}

3.1.1. Collection of Data to Describe the School of Interest from a Historical, Technical (Structural, Technological, Plant Engineering) and Architectural Point of View, According to its Own Training Plan and the Active Extra-Didactic Services Offered to the Community

As already highlighted in the previous Section 2.1, it is through the ARES, established by Law no. 23/1996, that the verification and collection of information about the consistency (surface and volume) and the management system of the school space takes place, also for the purpose of planning interventions compatible with the building and the training system in force. In many cases, this database is still incomplete, inaccurate and not always updated in the information. So, it is necessary to verify the data on the school building that is the subject of the evaluation problem of intervention contained in the database through inspections and field surveys to obtain more up-to-date and complete data to be used in the implementation of the proposed methodology.

Taking into account the data contained in the QE of ARES, the main information to be considered when implementing the procedure concerns the geographical location and morphological characteristics of the building (year of construction; architectural layout, overall dimensions of the building, etc.), the type of training offered and information on the total number of students enrolled both in the last year of activity and in previous years, the superficial consistencies of the spaces (internal and external) used for teaching and not, the safety conditions (earthquake-proof, fireproof, hygienic-sanitary and environmental) of the school, and the types of services offered to students and/or people not attending school during ordinary teaching activities in teaching hours and not.

Table 2 specifies for each type of information the reference source in order to find and quantify the data of interest, even in the event that the descriptive sheet of the school in question is not present in the ARES information system. For each type of data, the usefulness (expressed in terms of objectives to be pursued) is also illustrated.

Table 2. Types of information to be collected for describing the state of the school.

\begin{tabular}{ccc}
\hline Information Type & Reference Source & Usefulness Information \\
\hline $\begin{array}{c}\text { Geographical location and } \\
\text { morphology of the building }\end{array}$ & $\begin{array}{c}\text { Data from: ARES questionnaire; } \\
\text { Research at the State Historical } \\
\text { Archives and inspections carried } \\
\text { out at the school }\end{array}$ & $\begin{array}{c}\text { The reconstruction of the history } \\
\text { of the school provides information } \\
\text { on its possible maintenance, } \\
\text { adaptation and renovation }\end{array}$ \\
$\begin{array}{c}\text { Type of educational offer and total } \\
\text { number of students enrolled }\end{array}$ & Data from ARES questionnaire & $\begin{array}{c}\text { The characterization of the school } \\
\text { in terms of education and number } \\
\text { of students provides an indication } \\
\text { of the type of education offered }\end{array}$ \\
\hline $\begin{array}{c}\text { Aggregate measurement of the } \\
\text { consistencies of the spaces } \\
\text { arranged on each floor of which } \\
\text { the school is composed }\end{array}$ & $\begin{array}{c}\text { Survey campaign conducted in } \\
\text { each space inside and/or outside } \\
\text { the school building }\end{array}$ & $\begin{array}{c}\text { Knowledge of the surface area of } \\
\text { each environment inside and/or } \\
\text { outside the school is necessary } \\
\text { information to verify the } \\
\text { per-capita budget that must be } \\
\text { guaranteed to each student. }\end{array}$ \\
\hline $\begin{array}{c}\text { Types of services offered (didactic } \\
\text { and extra) }\end{array}$ & School Self-Assessment Report \\
(SAR) & $\begin{array}{c}\text { The specification of the services } \\
\text { offered by the school during } \\
\text { extra-curricular hours shows how } \\
\text { the school is used }\end{array}$ \\
\hline
\end{tabular}




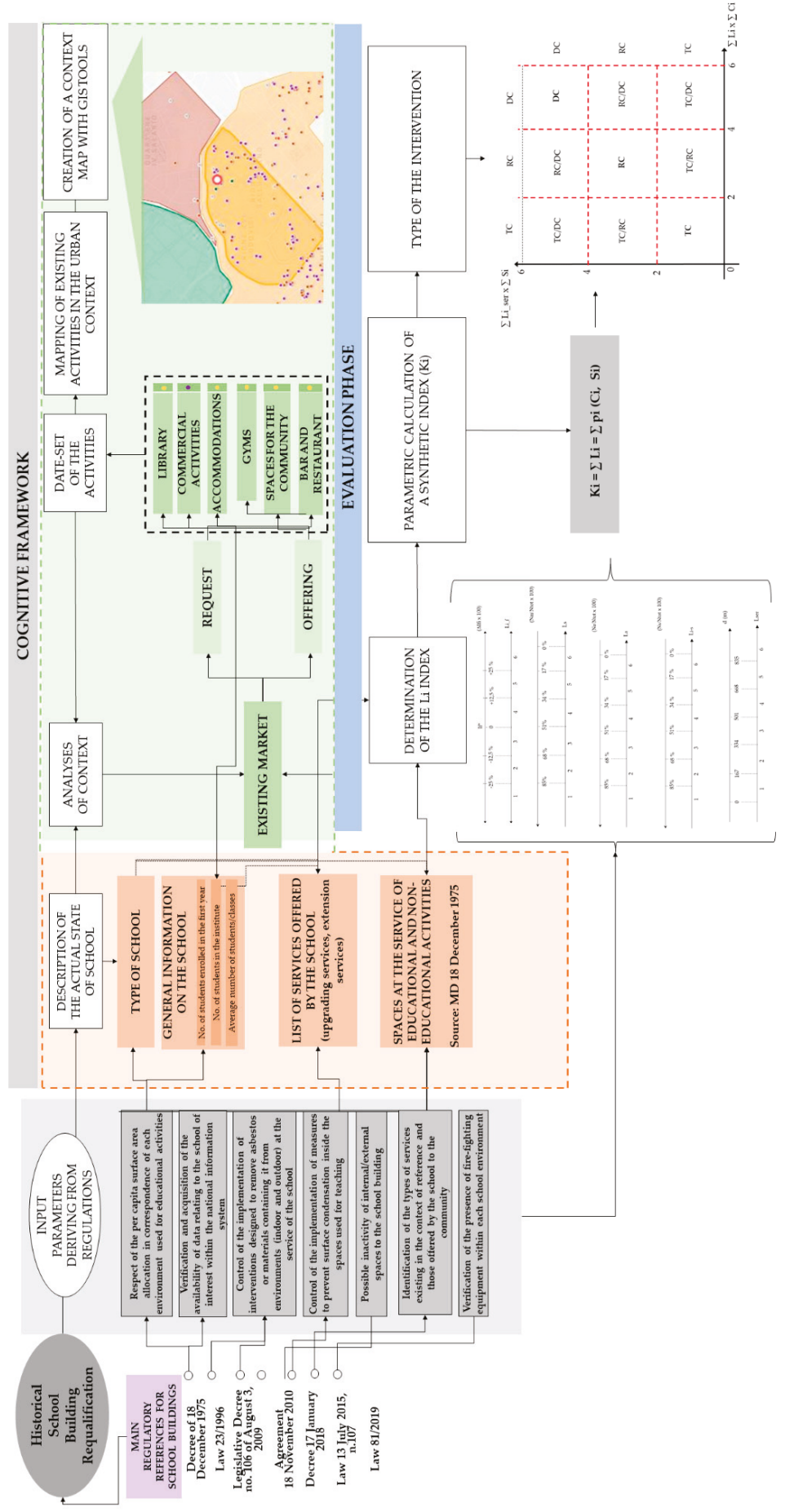

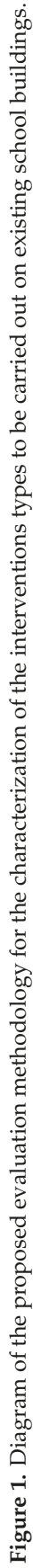




\subsubsection{Socio-Economic Analysis of the Urban Context}

With the aim of carrying out specific interventions that can also satisfy the needs of social aggregation of the community, it is appropriate to examine the urban context of reference in terms of services for the population. This is done by analyzing the market conditions in terms of demand and services supply that characterize the territory in which the school assumes a polarizing function.

To do this, it is necessary to demarcate the territorial area of interest in which it is necessary to detect and quantify the demand and services supply characterizing the market of the urban context of reference. The criteria for identifying the analysis area can specifically concern the composition and characteristics (economic, social, etc.) of the local population, the economic-productive system of the territory, the socio-cultural apparatus of the place, or even the morphology of the urban fabric of reference of which the school is a part. On the basis of morphological aspects, the field of investigation can coincide either with the perimeter of a single district, or of a part of it, in which the school can assume a catalytic and barycentric function with respect to the evolutionary dynamics of the surrounding urban fabric, or even with larger areas in the case of schools with locations located in the city and that are distant from each other. This, in part also depends on the demographic dimension and the territorial extension of the municipality and its parts in which the school structure is located. The definition of the gravitational field can therefore be carried out on the basis of data relating to: settlement type, socio-demographic information (age, sex, nationality, educational qualification/level, social class, employment, income, etc.), geographical information (region/province/common, urban/suburban/rural area, city size, population, climate, etc.), psychographic information (lifestyle, habits, etc.), and historical, cultural and productive information.

Operationally, the quantification of these indicators and, in particular, of the number and location of services present in the urban environment, can be carried out by identifying a cluster of circular analyses with a center at the point where the school of interest is located and a certain radius. In the Circular $N^{\circ} 425 / 1967$ in Appendix C, the measures of influence rays at each level of the school to be considered in order to identify the territorial area in which to include the catchment area referred to the school building considered are specified. Depending on the territorial scope of the survey thus identified and the type of supply/demand characterizing the cluster in which the school falls, the types of services offered by the school institution are compared with those present in the territory. Within the cluster analysis, the type of services considered are those illustrated in the Ministerial Decree of April 14, 2013 and include libraries, commercial activities, accommodations, gyms, bars and restaurants, and spaces for the community.

\subsection{Evaluation Phase}

3.2.1. Evaluations of Consistency According to the Technical and Regulatory Requirements to Be Complied with at the Design Stage and the Actual State of the School

From the identification and collection of data, both of the building to be redeveloped and of the urban context in which the school is located, it is necessary to proceed to the verification of congruence between the actual state of the school and the reference law provisions. Specifically, on the basis of the surface textures measured for each space intended for teaching (frontal and laboratory), the minimum surface area per student for each type of environment (classroom, laboratory, gym) is verified in correspondence with each floor of the building, in compliance with the minimum regulatory targets to be respected. The value of the surface area per capita to be considered during the verification phase varies with respect to the school training offer, as regulated in the Ministerial Decree of 18 December 1975; in compliance with the provisions of the law on school building, the level of compliance of the school's functional, seismic, fireproof and hygienic-sanitary system with the safety and use conditions to be guaranteed is assessed.

By means of an evaluation index $\left(\mathrm{L}_{\mathrm{i}}\right)$, the degree of adequacy of the spatial-functional $\left(\mathrm{L}_{\mathrm{f}}\right)$, structural $\left(\mathrm{L}_{\mathrm{s}}\right)$, fireproof $\left(\mathrm{L}_{\mathrm{a}}\right)$ and hygienic-sanitary $\left(\mathrm{L}_{\mathrm{i}-\mathrm{s}}\right)$ system of the school building to the requirements 
expressed in the i-th reference standard is qualitatively measured, as well as the level of correlation between the services currently present in the school and those found in the territorial area of investigation $\left(\mathrm{L}_{\mathrm{ser}}\right)$. For each aspect, the corresponding $\mathrm{L}_{\mathrm{i}}$ is measured qualitatively by assigning a score $\left(\mathrm{p}_{\mathrm{i}}\right)$ according to the scale of values from 1 to 6 used in the filling in of the questionnaire for ARES ( 6 = does not require any intervention; 5 = requires partial maintenance; $4=$ requires complete maintenance; 3 = requires partial replacement or renovation; 2 = requires replacement or complete renovation; 1 = requires ex-novo installation). On the basis of the scale of values used to fill in the ARES questionnaire, the attribution of the score to the $\mathrm{L}_{\mathrm{i}}$ parameter for each aspect is a function of the greater and/or lesser level of adaptation of the state (spatial, physical, functional, plant engineering, sanitation, environmental, structural) of the school to the reference regulatory requirements evaluated according to a suitable technical-regulatory criterion $\left(C_{i}\right)$ (see Table 2$)$, and the degree of correspondence between the types of services $\left(S_{i}\right)$ currently present in the school and those found in the urban context in which the building to be renovated is located.

Using an algebraic-linear formulation, the $\mathrm{L}_{\mathrm{i}}$ parameter can be expressed through the following mathematical function:

$$
\mathrm{L}_{\mathrm{i}}=\mathrm{p}_{\mathrm{i}}\left(\mathrm{C}_{\mathrm{i}}, \mathrm{S}_{\mathrm{i}}\right)
$$

In the following, for each $\mathrm{L}_{\mathrm{i}}$ concerning the structural, plant engineering, sanitary, environmental, and spatial-functional aspects of the building and the services currently present in the school, the corresponding scoring system is illustrated according to the scale of values from 1 to 6 . For each $\mathrm{L}_{\mathrm{i}}$, especially for those referring to the technical-regulatory aspects, the measurement parameters considered are such that it is possible to use a qualitative evaluation approach. For the evaluation of technical and plant engineering aspects of the sector, for which it will be necessary to take into account the act of implementing the planning of interventions to be carried out on the existing building, it is mandatory to take into account a judgment expressed through more detailed design drawings drawn up by technical professionals in the fields of seismic adjustment, energy, and plant engineering that give quantitative information. In the case of this work, aimed at providing an evaluation methodology to support the definition and planning of sustainable projects as compatible with the physical-spatial apparatus of the school building, also with a view to encouraging a more correct completion of the procedures for obtaining public funding, an evaluation methodology of a qualitative type is proposed. The use of a parameter L of reference allows us to express the level of correspondence between the state of affairs of the school building and the regulatory requirements to be complied with in the design phase. In particular, for each evaluation index:

a) the value of the parameter $\mathrm{L}_{\mathrm{f}}$ is assigned on the basis of the increase and decrease in the surface index deriving from the direct survey of school spaces (internal and external) (If) with respect to the parameter of law If*. Figure 2 below shows the extremes of the incremental and decremental intervals $(\Delta \mathrm{If})$, expressed in percentages, defined starting from the If* value and the average $\mathrm{L}_{\mathrm{i} \_f}$ score to be assigned to the $\mathrm{i}$-th space according to the corresponding $\Delta$ Ifi.

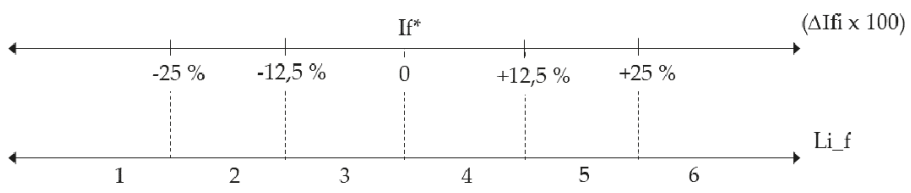

Figure 2. Scale of evaluation for the assignment of the score to the parameter $\mathrm{L}_{\mathrm{f}}$. If* is the value of law parameter of reference. It is the relative zero in the proposed scale of value.

b) With regard to the structural aspect, the corresponding evaluation index $\left(\mathrm{L}_{\mathrm{s}}\right)$ is measured according to the number of rooms, used for teaching and not, distributed on each floor of the school building, which at the time of the on-site inspection are unusable and risky for the safety of 
school users. Figure 3 below shows the interval extremes, expressed as a percentage, established on the basis of the number of currently unusable environments $\left(\mathrm{N}_{\mathrm{ai}}\right)$ defined with respect to the total number of spaces $\left(\mathrm{N}_{\mathrm{tot}}\right)$ present on each school level, and the average score to be assigned, according to the values scale [1-6], in descending order on the basis of the number of impassable spaces by the persons surveyed at the time of the inspection.

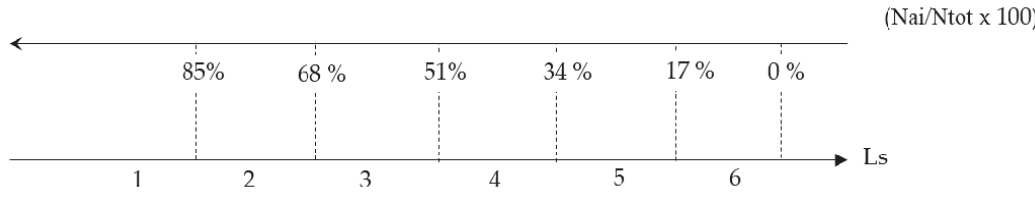

Figure 3. Evaluation scale for the assignment of the score to the parameter $\mathrm{L}_{\mathrm{s}}$.

c) As far as fire safety is concerned, the corresponding evaluation index $\left(\mathrm{L}_{\mathrm{a}}\right)$ is measured taking into account the obligation deriving from the law (Law 81/2019) to provide each environment with appropriate fire protection devices (e.g., fire extinguishers, which are not exhaustive) with particular performance characteristics. In particular, the attribution of a high, or low, numerical value to the $L_{a}$ index can be related to the frequency (in percentage terms) of spaces in correspondence with each floor of the school building, in which the presence, or absence, of fire extinguishers or other devices for fire risk prevention is found, compared to the total number of rooms on the same floor. Figure 4 below shows the reference diagram for the assignment of the score to the parameter $\left(\mathrm{L}_{\mathrm{a}}\right)$ as a function of the frequency of the rooms without fire-fighting devices $\left(\mathrm{N}_{\mathrm{e}}\right)$ compared to the total number $\left(\mathrm{N}_{\mathrm{tot}}\right)$ of spaces on the $\mathrm{i}$-th floor.

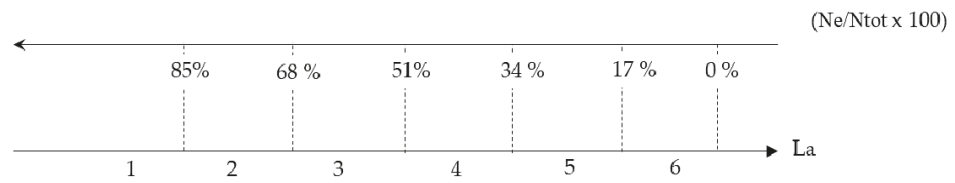

Figure 4. Scale of evaluation for the assignment of the score to the parameter La.

d) With reference, instead, to the hygienic-sanitary aspect, it is possible to refer to the possible presence of superficial condensation inside the spaces specifically destined to the carrying out of didactic activities. Similar to the previous evaluation indexes described above, the value assumed by $\mathrm{L}_{\mathrm{i}-\mathrm{s}}$ is related to the number of rooms on each floor within which there are forms of surface condensation $\left(\mathrm{N}_{\mathrm{s}}\right)$ compared to the total spaces $\left(\mathrm{N}_{\text {tot }}\right)$ on the i-th floor.

Figure 5 shows the scale of the scores (from 1 to 6 ) and the corresponding intervals of the measurement parameter considered $\left(\mathrm{N}_{\mathrm{s}} / \mathrm{N}_{\text {tot }}\right)$ expressed as a percentage.

(Ns/Ntot $\times 100)$

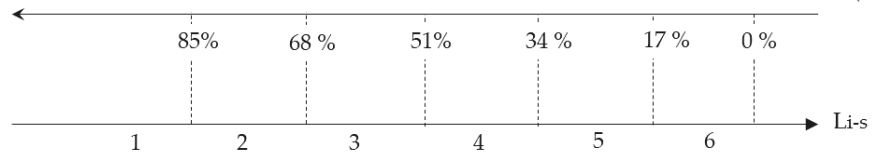

Figure 5. Evaluation scale for the assignment of the score to the parameter $\mathrm{L}_{\mathrm{i}-\mathrm{s}}$.

With reference, instead, to the evaluation of the congruence level between the services currently offered through the use of the internal environments of the building with those present in the analysis buffer (with a radius of $1000 \mathrm{~m}$ ), a qualitative score from 1 to 6 is attributed to the corresponding evaluation index $\left(\mathrm{L}_{\mathrm{ser}}\right)$ according to the number of similar services found in the urban area of interest 
and the relative distance (included in the analysis buffer of $1000 \mathrm{~m}$ ) measured with respect to the point where the building is located. Figure 6 shows the scale of values from 1 to 6 according to the distance of the i-th service from the school. The maximum distance is assumed to be $1000 \mathrm{~m}$, as indicated in Appendix C of Circular n 425/1967.

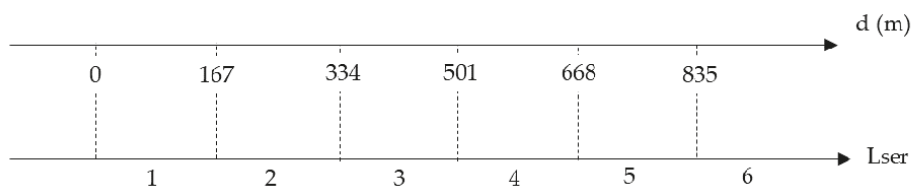

Figure 6. Scale of evaluation for the assignment of the score to the parameter Lser.

3.2.2. Identification of the Methods of Actions and Types of Interventions to Be Implemented in the Event of Non-Compliance with the Minimum Regulatory Requirements Regarding Security and Physical-Functional Management of the School Space

Following the $\mathrm{L}_{\mathrm{i}}$ measurement operation, the intensity of the intervention to be carried out for the overall requalification of the school is identified, also taking into account the characteristics (in terms of existing services) of the urban context in which the school is located. In order to encourage requalification practices according to an integrated logic, it is necessary to jointly consider both the actions of spatial reorganization of the didactic and laboratory environments (where the value of the minimum surface endowment per students for each environment is not satisfied), and the physical interventions aimed at adapting the school to the reference regulations, both of enhancement and/or integration of the services currently offered and/or potentially to be added to the existing ones.

From this perspective, three macro-types of intervention are outlined (Total Renovation, Regulatory Compliance, Distributional Challenges), which can be implemented in order to fully upgrade existing school buildings.

Total Renovation (TR) is carried out when the school needs substantial interventions in terms of both structure, plant engineering, health and hygiene, management and use of space for teaching, and integration of new services for the community. In the case of existing school buildings, when the date of construction is prior to the year in which the first anti-seismic regulations were issued, it goes without saying that it is mandatory to carry out preliminary consolidation and adaptation work on the structure in order to ensure the safety of direct and indirect users of the school.

Regulatory Compliance ( $\mathrm{RC}$ ) occurs when it is necessary to act on the school building in order to adapt it to some legal requirements regarding seismic risk, fire prevention, energy requalification, indoor quality improvement, together with a remodeling and re-functionalization of the internal and external spaces at the service of the school building and enhancement and/or integration of additional services identified according to the characteristics (supply/demand) of the analysis scope.

The Distributional Challenge (DC) is carried out in the event that it is necessary to carry out a complete and/or partial reorganization and redefinition of the intended use of the internal and/or external school spaces, and the correspondence between the structural-implant and hygienic-sanitary state of the school with the regulatory requirements that must be considered in the planning phase of the requalification interventions is verified.

It is possible to associate the identification of the intervention methods previously described (Total Renovation, Regulatory Compliance, Distributional Challenge) with a synthetic reference index $\left(\mathrm{K}_{\mathrm{i}}\right)$. This index allows us to express the level of intensity of the action type that needs to be implemented to upgrade the school, from the point of view of both functional and structural-implantistic-hygienic-sanitary $\left(\mathrm{K}_{\mathrm{t}}\right)$ factors, as well as in terms of services offered to the public $\left(\mathrm{K}_{\mathrm{ser}}\right)$. Both $\mathrm{K}_{\mathrm{t}}$ and $\mathrm{K}_{\text {ser }}$ are obtained by algebraically aggregating the corresponding $\mathrm{L}_{\mathrm{i}}$ values previously specified in Section 3.2.1. 
The $K_{i}$ parameter is obtained by means of a mathematical formulation such as:

$$
\mathrm{K}=\sum \mathrm{Li}=\sum \mathrm{p}_{\mathrm{i}}\left(\mathrm{C}_{\mathrm{i}}, \mathrm{S}_{\mathrm{i}}\right)
$$

The $\mathrm{K}_{\mathrm{i}}$ intervals for each intervention mode are shown in Table 3.

Table 3. Range of values of the $K_{i}$ parameter and corresponding switching modes.

\begin{tabular}{cc}
\hline Reference Range of Values of the Parameter $\mathbf{K}_{\mathbf{i}_{\_} \mathrm{t}, \mathrm{ser}}$ & Intervention Modalities \\
\hline$\sum_{i=0}^{n} 0\left(\mathrm{C}_{\mathrm{i}}, \mathrm{S}_{\mathrm{i}}\right) \leq \mathrm{K}_{\mathrm{i}_{\_} \mathrm{t}, \mathrm{ser}} \leq=\sum_{i=0}^{n} 2\left(\mathrm{C}_{\mathrm{i}}, \mathrm{S}_{\mathrm{i}}\right)$ & Total Renovation \\
$\sum_{\mathrm{i}=0}^{\mathrm{n}} 2\left(\mathrm{C}_{\mathrm{i}}, \mathrm{S}_{\mathrm{i}}\right) \leq \mathrm{K}_{\mathrm{i}_{-} \mathrm{t}, \mathrm{ser}} \leq \sum_{\mathrm{i}=0}^{\mathrm{n}} 4\left(\mathrm{C}_{\mathrm{i}}, \mathrm{S}_{\mathrm{i}}\right)$ & Regulatory Compilance \\
$\sum_{i=0}^{n} 4\left(\mathrm{C}_{\mathrm{i}}, \mathrm{S}_{\mathrm{i}}\right) \leq \mathrm{K}_{\mathrm{i}_{\mathrm{i}} \mathrm{t}, \mathrm{ser}} \leq \sum_{i=0}^{n} 6\left(\mathrm{C}_{\mathrm{i}}, \mathrm{S}_{\mathrm{i}}\right)$ & Distributional Challenge \\
\hline
\end{tabular}

Figure 7 below illustrates a double entry scheme according to which it is possible to identify the intervention mode (TC, RC, DC) referring to both the system of services and the technical-implantistic-hygienic-sanitary one on the basis of the corresponding score of $\mathrm{L}_{\mathrm{i}}$ parameter. The proposed diagram shows on the abscissa and ordinate axis the intervals of values, respectively referred to $\mathrm{K}_{\mathrm{t}}$ and $\mathrm{K}_{\mathrm{ser}}$, which identify the proposed macro-categories of intervention.

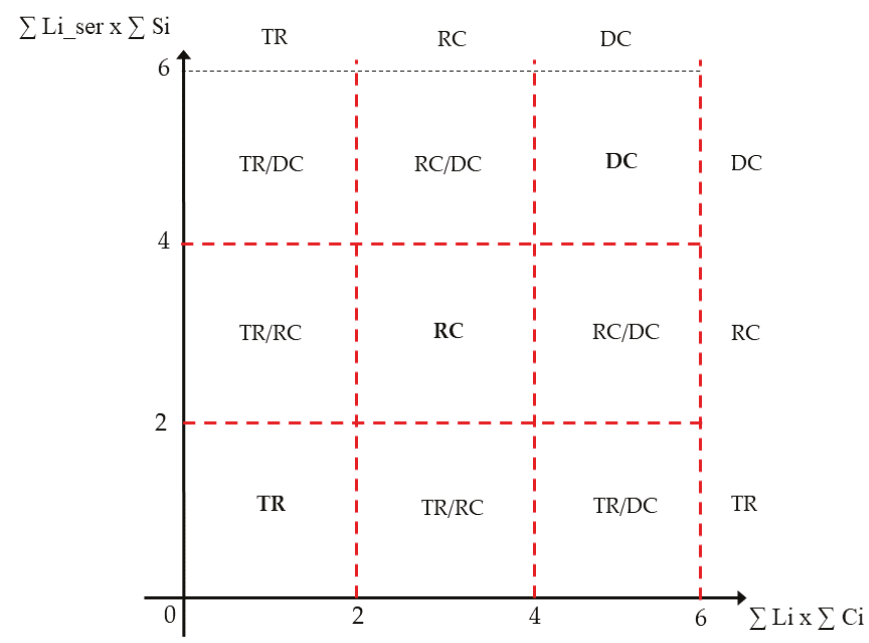

Figure 7. Double-entry diagram supporting the identification of the main intervention modalities for the integrated requalification of existing school buildings.

In Section 4, the proposed evaluation methodology is applied to the case study for the redevelopment of the Torquato Tasso classical high school located in the historic center of Rome (Italy).

\section{Case Study}

We intended to apply the proposed evaluation method considering the project of redevelopment, recovery and conservation of the school located in the historic center of Rome in Italy. The building is made of three institutes: Middle School, High School and Scientific High School. The methodology in Section 3 is tested on the part of the building relating to Torquato Tasso classical high school. Figure 8 shows (a) a historical picture of the building part where Torquato Tasso classical high school is, and (b) the façade on Sicilia Road where the principal entrance to the school is. 


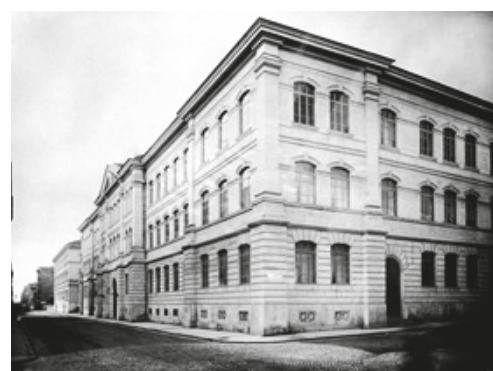

a. Historical picture of the school building Source: https://www.geoitaliani.it/2017/01/liceotasso.html (last accessed on 12 January 2020)

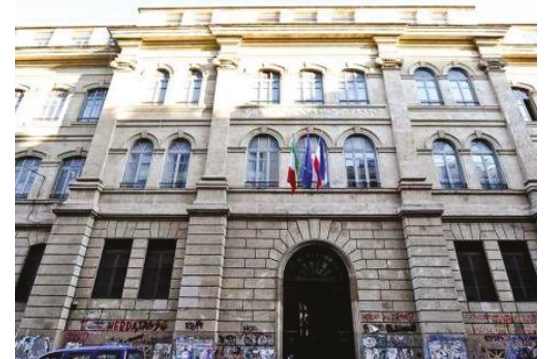

b. Main entrance to school by Sicilia Road

Source: http://www.romatoday.it/formazione/scuola/ migliori-licei-roma-2019.html (last accessed on 12 January 2020)

Figure 8. Pictures of Torquato Tasso classical high school.

It should be specified that the data on the student population in the last five years, the information on the educational offer of the institute in question, as well as the survey of the geometric dimensions of the spaces used for teaching were obtained by carrying out some investigation campaigns in the school.

\subsection{Knowledge Phase}

4.1.1. Description of the School from the Historical, Technical and Architectural Point of View, According to Its Own Training Plan and the Extra-Educational Services Offered to the Community

The school building is located inside the first Town Hall in the historic center of the city of Rome, specifically in the Ludovisi district. The building is strategically located with respect to both the main roads through which you can reach the city-center, and the train station of Roma Termini, as demonstrated by calculating the travel time between the school and the points of greatest infrastructural interest through Google Map.

With specific regard to the Torquato Tasso classical high school, the institute, founded in 1908, was proposed from the beginning as a school serving the Ludovisi district and neighboring ones. Over the years, a series of enlargements, transformations and building maintenance interventions were carried out, especially on the structure, such as the original internal and external conformation of the building is partially modified.

The school rooms are distributed over two floors as well as on the ground floor, where most of the administrative offices are located. As described in the Self-assessment Report (2019) of the institute, there are 44 classrooms for frontal teaching, three spaces for laboratories, a great hall, a Library and a Natural Sciences Museum, administrative offices and restrooms on each floor. Figure 9 shows a plan of a typical school floor plan from 1964. The plan in the figure was acquired from the school's administrative offices after an on-site inspection.

To date (2019), the school has 919 students enrolled in the first academic year (a.y.). Table 4 shows the number of students enrolled in the first year, the number of classes and sections from the academic year 2015-2016 to the academic year 2019-2020. 


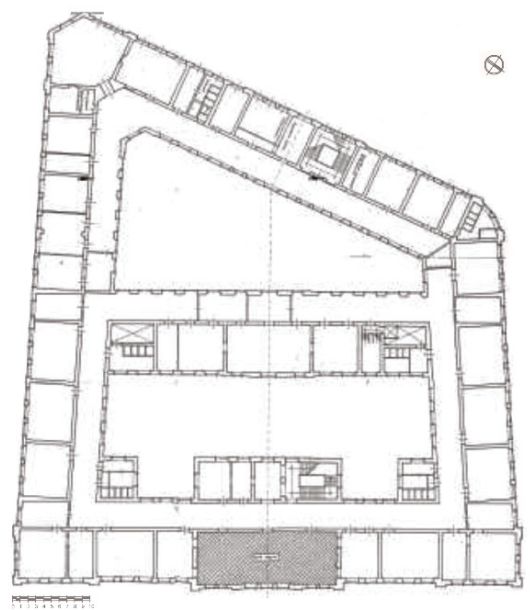

Figure 9. Typical floor plan of Torquato Tasso classical high school (1964).

Table 4. Time data on the students, number of classes and sections of the school.

\begin{tabular}{cccccc}
\hline Academic Year (a.y.) & 2015-2016 & 2016-2017 & 2017-2018 & 2018-2019 & 2019-2020 \\
\hline $\mathrm{N}^{\circ}$ of students enrolled in the first year & 795 & 800 & 820 & 942 & 319 \\
$\mathrm{~N}^{\circ}$ of classes & 35 & 35 & 35 & 35 \\
$\mathrm{~N}^{\circ}$ of sections & 7 & 7 & 7 & 11 & 7 \\
\hline
\end{tabular}

Source: Data obtained at the school administration offices and acquired during on-site inspections.

On the basis of the data in Table 4, a progressive increase in the student population is evident. In particular, the trend towards an increase in the number of students enrolled in the first year at each academic year has produced, over time, the gradual fragmentation of classrooms in environments characterized by different sizes. On-site inspections of three different types of large, middle and small size classrooms are revealed in Table 5, which shows the dimensions (both linear and superficial) of the three types of classroom together with those of the other rooms serving the school.

- Survey of the superficial consistencies of the internal and external spaces of the school

In order to verify the per capita surface endowment of each space inside and outside the school building, a preliminary phase of a consistency survey of the building was carried out. Table 5 shows the geometric measurements of the rooms located on a typical floor (the first and second floors on which teaching activities have the same planimetric and distributive-spatial composition). Table 6 , instead, shows the data of the surfaces of the internal and external spaces in an aggregate manner.

Table 5. Planimetric dimensions of rooms of a typical floor school.

\begin{tabular}{|c|c|c|c|c|c|c|}
\hline \multirow[b]{2}{*}{ Type of Spaces } & \multicolumn{5}{|c|}{ Geometric Dimensions } & \multirow{2}{*}{$\begin{array}{l}\mathrm{N}^{\circ} \text { of } \\
\text { Rooms }\end{array}$} \\
\hline & $\begin{array}{l}\text { Wide } \\
{[\mathrm{m}]}\end{array}$ & $\begin{array}{l}\text { Long. } \\
{[\mathrm{m}]}\end{array}$ & $\begin{array}{l}\text { Height } \\
{[\mathrm{m}]}\end{array}$ & $\begin{array}{l}\text { Sup. } \\
\text { [sqm] }\end{array}$ & $\begin{array}{l}\text { Vol. } \\
{[\mathrm{mc}]}\end{array}$ & \\
\hline SCHOOL CLASSES & & & & & & $\begin{array}{c}\mathrm{N}^{\circ} \\
\text { Class-Rooms }\end{array}$ \\
\hline Large & 10.50 & 7.54 & 4.70 & 79.17 & 372.10 & 1 \\
\hline Middle & 9.30 & 6.30 & 4.70 & 58.59 & 275.37 & 5 \\
\hline Small & 6.50 & 5.70 & 4.70 & 37.05 & 174.14 & 7 \\
\hline Tot. & & & & 631.47 & 821.607 & 13 \\
\hline
\end{tabular}


Table 5. Cont.

\begin{tabular}{|c|c|c|c|c|c|c|}
\hline \multirow{2}{*}{ Type of Spaces } & \multicolumn{5}{|c|}{ Geometric Dimensions } & \multirow{2}{*}{$\begin{array}{l}\mathbf{N}^{\circ} \text { of } \\
\text { Rooms }\end{array}$} \\
\hline & $\begin{array}{l}\text { Wide } \\
{[\mathrm{m}]}\end{array}$ & $\begin{array}{l}\text { Long. } \\
{[\mathrm{m}]}\end{array}$ & $\begin{array}{c}\text { Height } \\
{[\mathrm{m}]}\end{array}$ & $\begin{array}{c}\text { Sup. } \\
\text { [sqm] }\end{array}$ & $\begin{array}{l}\text { Vol. } \\
{[\mathrm{mc}]}\end{array}$ & \\
\hline \multicolumn{7}{|l|}{$\begin{array}{c}\text { SPACES FOR SPECIAL ACTIVITIES } \\
\text { (PHYSICS, CHEMISTRY, } \\
\text { NATURAL SCIENCES) }\end{array}$} \\
\hline Lab. ART & 4.60 & 8.00 & 4.70 & 36.80 & 172.96 & 1 \\
\hline Art Class-room & 6.41 & 5.27 & 4.70 & 33.78 & 33.78 & 1 \\
\hline Biology Classroom & 8.13 & 8.00 & 4.70 & 65.04 & 65.04 & 1 \\
\hline Tot. & & & & 135.62 & 271.78 & 3 \\
\hline BATHROOMS (B)-CONNECTIVE & & & & & & $\mathrm{N}^{\circ}$ restroom \\
\hline B 1 & 8 & 4.2 & 4.7 & 33.6 & 157.92 & 2 \\
\hline B 2 & 4.5 & 6.2 & 4.7 & 27.9 & 131.13 & 2 \\
\hline Connective & 4.2 & 223.24 & 4.7 & 937.61 & 4406.76 & \\
\hline TOT. & & & & 1060.61 & 4695.81 & 4 \\
\hline \multicolumn{7}{|l|}{ GYMS (G) } \\
\hline G 1 & - & - & - & - & - & \\
\hline Tot. & & & & - & - & \\
\hline \multicolumn{7}{|l|}{ SPACES FOR COLLECTIVE ACTIVITIES } \\
\hline Library & 13.71 & 12.91 & 4.70 & 177.00 & 831.88 & 1 \\
\hline Great Hall & 9.36 & 24.42 & 6 & 228.57 & 1371.43 & 1 \\
\hline Natural Science Museum & 8.00 & 23.22 & 4.7 & 185.76 & 873.07 & 1 \\
\hline Tot. & & & & 405.57 & 2203.31 & 3 \\
\hline \multicolumn{7}{|l|}{$\begin{array}{l}\text { SPACES FOR COMPLEMENTARY } \\
\text { ACTIVITIES }\end{array}$} \\
\hline Atrium & - & - & - & - & - & \\
\hline Administrative Offices & 8.51 & 57.61 & 4.70 & 490.26 & 2304.23 & 1 \\
\hline Tot. & & & & 490.26 & 2304.23 & 1 \\
\hline
\end{tabular}

Table 6. Surface consistencies of the school's internal and external aggregated spaces.

\begin{tabular}{ccc}
\hline Types of Spaces & Surfaces & No. of Rooms Per Floor \\
\hline 1. INDOOR SPACES & \\
\hline 1.1 School Classes & $1623.00 \mathrm{~m}^{2}$ & 13 \\
\hline 1.2 Spaces for special activities & $135.62 \mathrm{~m}^{2}$ & 3 \\
\hline 1.3 Bathrooms-Connectives & $2244.22 \mathrm{~m}^{2}$ & 4 \\
\hline 1.4 Internal gyms & $457.86 \mathrm{~m}^{2}$ & 0 \\
\hline 1.5 Spaces for collective activities & $716.85 \mathrm{~m}^{2}$ & 3 \\
\hline 1.6 Spaces for complementary activities & $490.26 \mathrm{~m}^{2}$ & 1 \\
\hline 2. EXTERNAL SPACES & & 1 \\
\hline 2.1 Surfaces used for outdoor sports activities & $311.25 \mathrm{~m}^{2}$ & 0 \\
\hline
\end{tabular}

4.1.2. Socio-Economic Analysis of the Urban Context in which the School Is Located

The Ludovisi district is characterized by the significant presence of elements with a strong historical-artistic and architectural value. Not far from the school, in fact, there are the Museum Boncompagni-Ludovisi, the National Gallery of Ancient Art of Barberini Building and the Borghese 
Gallery. The historical connotation of the territory contributes to define a context of strong cultural value through which the school encourage the education and training of students. The building's position in relation to some research institutes (for example, the National Research Centre) and university buildings (Sapienza University) has also made it possible to establish collaborative relationships by developing a number of joint educational initiatives in order to support the educational growth of students and also encourage the local development of the territory.

In order to analyze and characterize the territorial context of reference in which the school is placed also with regard to the types of existing services, the territorial area of analysis was included within a buffer with a radius of influence of $1000 \mathrm{~m}$ from the point where the school is located (Circular $\mathrm{N}^{\circ} 425 / 1967$ ). With the aim of identifying the prevailing services within the scope of the survey thus defined, a phase of georeferencing of the commercial, receptive, cultural and sports services was conducted.

After the georeferencing of the information, an information map (see Figure 10) was created using GIS instrumentation (Google Maps) to support the identification and analysis of services within the $1000 \mathrm{~m}$ buffer. Through the use of this cartography, it was possible to see that the territory is characterized by the high density of commercial activities and accommodation facilities. This is due to the presence of elements with strong tourist and infrastructural attractiveness (Roma Termini railway station) that influence the market dynamics. In the analysis buffer of 1000 meters, there are no services for the community (for example, non-exhaustive, neighborhood library, headquarters for cultural associations, spaces for social gathering).
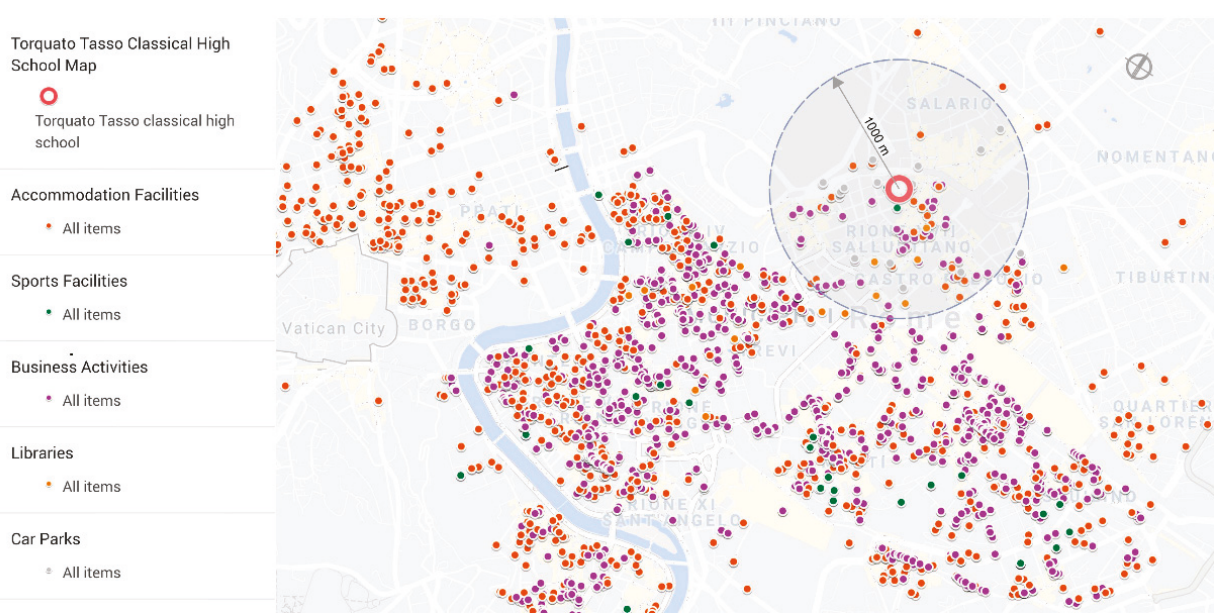

Figure 10. Map for market analysis of the study area made with GIS.

\subsection{Evaluation Phase}

4.2.1. Congruence Check between the Technical and Regulatory Requirements and the Actual State of the School

After the cognitive phase aimed at defining the state of the school, the congruence of the surface consistencies of the rooms surveyed (see Table 6) was verified, taking into account the regulatory requirements relating to their sizing in relation to the number of student that they host. This was done by comparing the value of the per capita equipment of each space (If), obtained by comparing the areas surveyed on site to the capacity of each space expressed in terms of number of students, with the corresponding regulatory standards (If*) contained in the Ministerial Decree of April 18 in 1975. For each type of space, the value of $\mathrm{L}_{\mathrm{i}_{-} \mathrm{fi}}$ was defined according to the corresponding percentage $\Delta \mathrm{Ifi}$. 
Table 7 shows the surface consistencies of each type of internal and external space used by the school, the theoretical surface indices, and those deriving from on-site measurements, the corresponding $\Delta$ Ifi and $\mathrm{L}_{\mathrm{i} \_\mathrm{f}}$. The score at $\mathrm{L}_{\mathrm{i}_{\mathrm{f}}}$ is assigned with the diagram in Figure 2. The final $\mathrm{L}_{\mathrm{f}}$ parameter to be considered was obtained from the arithmetic mean of the $\mathrm{L}_{\mathrm{i}_{\mathrm{f}}}$ of each type of space. In this case, the average value of $\mathrm{L}_{\mathrm{f}}$ was 2.3.

Table 7. Check of the surface textures of indoor/outdoor spaces of the school.

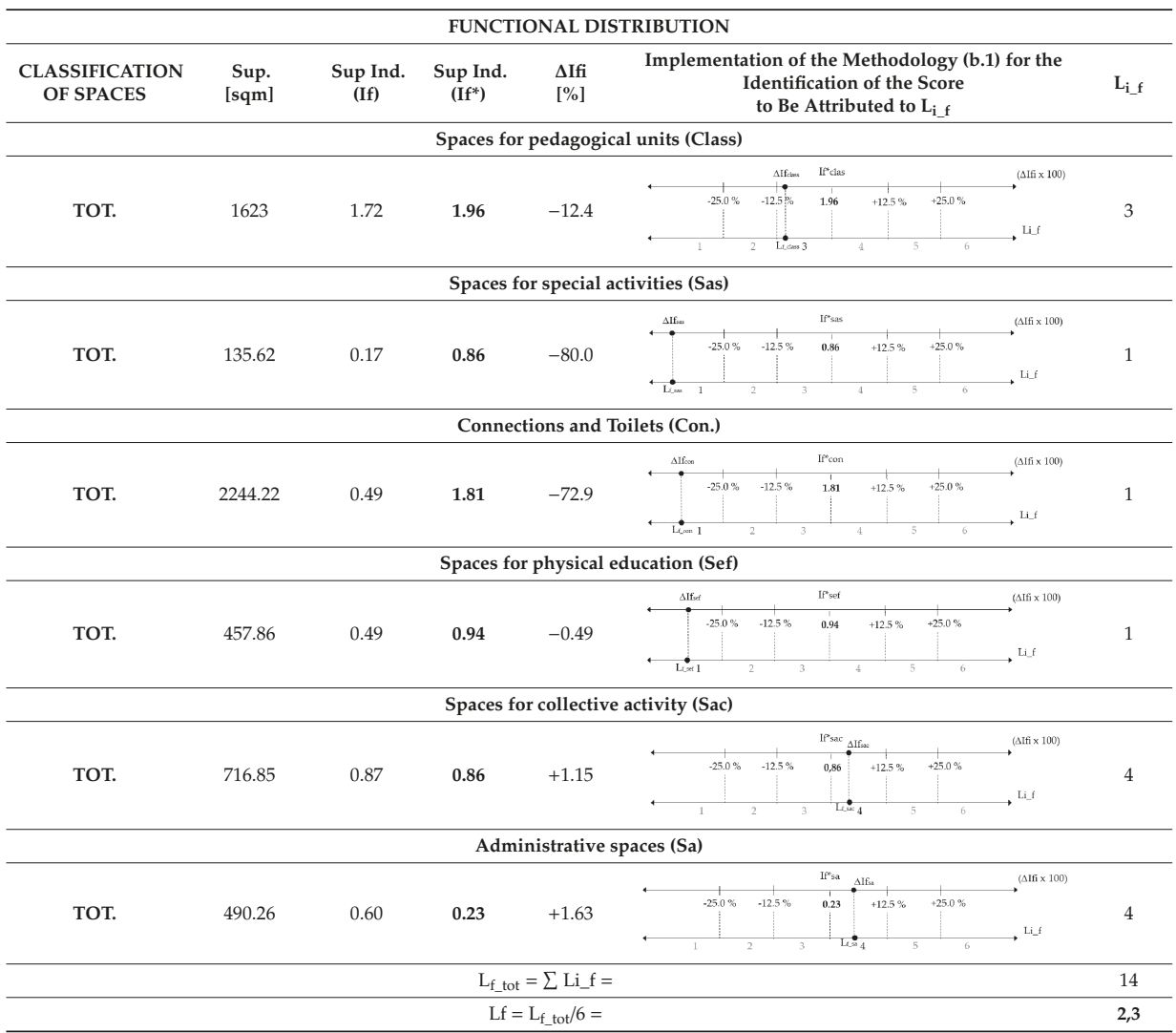

From a structural, plant engineering, environmental, health and hygiene points of view, on the other hand, a qualitative evaluation of the school's degree of conformity with the content expressed in the reference standards was carried out.

In order to estimate the level of compliance of the school with the laws on fire prevention, safety of the structure, indoor quality, the presence of fire-fighting devices in each room inside the school was verified, as well as the possible inability of some spaces at the time of on-site inspection, as well as the formation of condensation on the walls inside the building.

With regard to the type of actions implemented within the school in order to reduce the fire risk, at the date of the inspection in March 2019, the fire-fighting system serving the building had recently been brought into conformity with the technical regulations of reference. By carrying out a series of on-site inspections, it was possible to ascertain that each room was equipped with a special fire-fighting device.

With regard, however, to the safety of the school for the prevention of seismic risk, following a campaign of on-site inspections it was possible to find that some of its internal environments were not 
accessible (in particular two rooms on the first floor). The necessary interventions for their safety were being carried out in order to use them to carry out frontal and laboratory didactic activities.

Finally, again through visits to the school, it was possible to verify the presence of superficial condensation in the spaces of each floor. At the time of the inspection, some classrooms had spots of surface condensation on the internal perimeter walls (five on the first floor and two on the second).

On the basis of the ordinal scale of values used to assign a score to the parameter $\mathrm{L}_{\mathrm{i}}$ concerning the level of compliance with the content of the technical-normative system in force, the relative verification of compliance of the state with the fire regulations was measured by assigning a score of 5 . Instead, the level of congruence between the actual configuration of school building and the reference standard for the structural safety of the spaces was evaluated by assigning the corresponding parameter $\mathrm{L}_{\mathrm{S}}$ the score of 4 . From the sum of $\mathrm{L}_{\mathrm{a}}, \mathrm{L}_{\mathrm{s}}, \mathrm{L}_{\mathrm{i}-\mathrm{s}}$ and $\mathrm{L}_{\mathrm{f}}$ was obtained the $\mathrm{K}_{\mathrm{t}}$, which was equal to 15.3.

With reference, however, to the estimate of the level of correspondence between the services currently present in the school and those found in the context of reference, the $\mathrm{L}_{\mathrm{ser}}$ is defined for each type of service that you can see inside the building to be upgraded.

The services that are present in some rooms inside the school include a library, a space aggregative (aula magna), a museum of natural sciences, and an art laboratory. From the context analysis previously illustrated in Section 4.1.2, there are no similar services within the $1000 \mathrm{~m}$ analysis from the point where the school in question is located. Therefore, each $\mathrm{L}_{\mathrm{i} \text { sser }}$ is given a score of 6 . The corresponding $\mathrm{K}_{\text {ser, }}$, deriving from the aggregation of $\mathrm{L}_{\mathrm{i}_{-} \text {ser, }}$ is equal to 24 .

4.2.2. Identification of Types of Interventions to Be Implemented in Case of Non-Compliance with the Minimum Regulatory Requirements Regarding Safety and Use of School Space

After the phase of evaluation of the level of correspondence between the actual state of the school building and reference regulations, on the basis of the values obtained by $K_{\text {ser }}$ and $K_{i_{\_} t}$, the methods of intervention to be followed for the planning and/or design of interventions aimed at the functional and structural-plant requalification of the school were identified.

The phase of identification of the intervention modalities was carried out using the double entry diagram of Figure 7. Reporting on the $\mathrm{x}$-axis the value of $\mathrm{K}_{\mathrm{t}}$ and on $\mathrm{y}$-axis $\mathrm{K}_{\mathrm{ser}}$ is defined the combination of the types of action to be implemented for the integrated requalification of the school. In this case, four types of service were considered $\left(\mathrm{n}^{\circ} \mathrm{Si}=4\right)$ and four regulatory criteria were respected $\left(n^{\circ} \mathrm{Ci}=4\right)$. So, the extremes of the numerical intervals that identify the proposed types of intervention (TC, RC, DC) were multiplied respectively by the total number of services and technical-regulatory criteria considered.

Figure 11 shows the analysis scheme of Figure 7 for the part of the school building related to the Torquato Tasso classical high school.

In the light of the diagram in Figure 10, specifically concerning the school being studied, on the basis of the $K_{\text {ser }}$ and $K_{t}$ values obtained from the evaluation procedure described in Section 3.2.1, it is possible to observe that it would be appropriate to encourage interventions aimed, in particular, at making better use of the spaces dedicated to teaching and sports activities (Distributional Challenges), as well as actions for the safety of environments useful for carrying out training activities for students (Regulatory Compliance). From the analysis carried out on the types of service existing and falling within the territorial buffer of $1000 \mathrm{~m}$ (see Section 4.1.2), the need to enhance some environments inside and/or outside the institution to open up the cultural and social services to the community emerges. These include, for example, the opening to the public of the library inside the building, or even the use of some spaces (classrooms and/or corridors) for the exhibition of art and sculpture objects made by students in art education courses included in the educational training plan. 


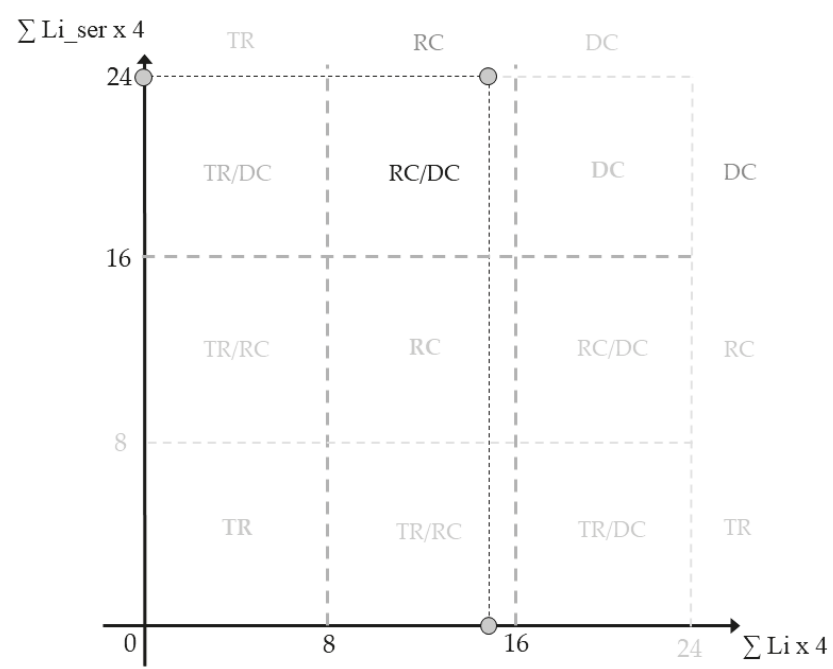

Figure 11. Double entry diagram for the definition of the intervention modalities to be implemented for the integrated requalification of Torquato Tasso classical high school.

\section{Conclusions}

In the context of the settlement transformation processes aimed at the redevelopment, recovery and enhancement of the existing building, the need to take into account, from the planning phase, multiple aspects of various kinds in an integrated manner considering the existing mutual relations among them is recognized. The complexity of jointly considering multiple characteristics of the same project, both technical and spatial-functional types, has encouraged the use of intervention practices often based on a problem-solving-based approaches and not on multidimensional ones.

This can be observed especially in the field of Italian school buildings (2019), where there is a lack of a single legislative framework in which to jointly include technical and functional evaluations with those that specifically concern the services that characterize the market of the urban context.

For supporting the planning, design and execution of interventions by preferring an integrated approach, especially with regard to the redevelopment of existing school buildings, the proposed methodology attempts to include within a single multi-criteria evaluation logic aspects of various kinds: structural, plant engineering, sanitation, spatial-functional, strengthening of existing services and integration of others on the basis of the market characterizing the city within the buffer of 1000 meters from the school is located. Appropriate criteria and qualitative measurement systems are used for each one.

The application of the evaluation methodology to the case of redevelopment of the school located in the historic center of the city of Rome (Italy) attests to the practicality of the proposed evaluation framework.

The in-depth analysis and study on a careful choice of the criterion with which to express the level of adaptation of the technical-structural features and the planimetric distribution of the schools places to the regulatory requirements and the possibility to express the use of spaces for additional services for the community outline future research prospects. Specifically, it would be interesting to implement participatory procedures for the identification of needs by the student community, for example through the administration of questionnaires, or even to return the proposed methodology in the form of mathematical expressions as the basis of the implementation of optimization linear systems of the operational research. 
Author Contributions: Conceptualization, M.R.G., P.M. and F.S.; Data curation, F.S.; Formal analysis, M.R.G., P.M. and F.S.; Investigation, M.R.G. and F.S.; Methodology, M.R.G., P.M. and F.S.; Resources, M.R.G. and F.S.; Supervision, M.R.G. and P.M.; Validation, M.R.G. and P.M.; Visualization, M.R.G., P.M. and F.S.; Writing-original draft, M.R.G., P.M. and F.S.; Writing—review \& editing, M.R.G., P.M. and F.S. All authors have read and agreed to the published version of the manuscript.

Funding: This research received no external funding.

Conflicts of Interest: The authors declare no conflict of interest.

\section{References}

1. Garett, S.; Portney, K.E. Sustainable Cities. Policies and Healthy Cities. In Integrating Human Health into Urban and Transport Planning; Nieuwenhuijsen, M., Khries, H., Eds.; Springer: Cham, Switzerland, 2019; pp. 31-49.

2. McCormick, K.; Anderberg, S.; Coenen, L.; Neij, L. Advancing sustainable urban transformation. J. Clean. Prod. 2013, 50, 1-11. [CrossRef]

3. Diaz-Sarachaga, J.M. Analysis of the Local Agenda 21 in Madrid Compared with Other Global Actions in Sustainable Development. Int. J. Env. Res. Pub Heal. 2019, 16, 3685. [CrossRef] [PubMed]

4. De Roo, G.; Donald, M. Compact Cities and Sustainable Urban Development: A Critical Assessment of Policies and Plans from an International Perspective; Routledge: New York, NY, USA, 2019.

5. Nesticò, A.; Sica, F. The sustainability of urban renewal projects: A model for economic multi-criteria analysis. J. Prop. Invest. Financ. 2017, 35, 397-409. [CrossRef]

6. Della Spina, L. Scenarios for a Sustainable Valorisation of Cultural Landscape as Driver of Local Development. In New Metropolitan Perspectives. ISHT 2018. Smart Innovation, Systems and Technologies; Calabrò, F., Della Spina, L., Eds.; Springer: Cham, Switzerland, 2018; Volume 100, pp. 113-122. [CrossRef]

7. Della Spina, L.; Calabrò, F. Decision Support Model for Conservation, Reuse and Valorization of the Historic Cultural Heritage. In Computational Science and Its Applications-ICCSA 2018. ICCSA 2018. Lecture Notes in Computer Science; Gervasi, O., Murgante, B., Eds.; Springer: Cham, Switzerland, 2018; Volume 10962, pp. 3-17.

8. European Commission. Leipzig Charter on Sustainable European Cities and Towns, CdR 163/2007 EN-COM/SAB/lc. 2007. Available online: https://ec.europa.eu/regional_policy/archive/themes/urban/ leipzig_charter.pdf (accessed on 20 January 2020).

9. European Commission. The State of European Cities: Cities Leading the Way to a Better Future; Publications Office of the European Union: Luxembourg, 2016. Available online: http://ec.europa.eu/regional_policy/ sources/policy/themes/citiesreport/state_eu_cities2016_en.pdf (accessed on 8 November 2019).

10. Della Spina, L.; Calabrò, F. Pianificazione strategica: Valutare per programmare e governare lo sviluppo. LaborEst 2015, 11, 3-4.

11. Directive 2012/27/EU of the European Parliament and of the Council of 25 October 2012 on energy efficiency, amending Directives 2009/125/EC and 2010/30/EU and repealing Directives 2004/8/EC and 2006/32/EC. Available online: https://eur-lex.europa.eu/legal-content/IT/TXT/PDF/?uri=CELEX:32012L0027\&from=EN (accessed on 8 November 2019).

12. Directive (EU) 2016/2284 of the European Parliament and of the Council of 14 December 2016 on the reduction of national emissions of certain atmospheric pollutants, amending Directive 2003/35/EC and repealing Directive 2001/81/EC. Available online: https://eur-lex.europa.eu/legal-content/IT/TXT/PDF/?uri=CELEX: 32016L2284\&from=EN (accessed on 8 November 2019).

13. Legislative Decree no. 102 of 4 July 2014 on Energy Efficiency (in the Official Journal 18 July 2014, $n^{\circ} 165$ ). Available online: https:/www.normattiva.it/uri-res/N2Ls?urn:nir:stato:decreto.legislativo:2014-07-04;102! vig= (accessed on 8 November 2019).

14. Balocco, C.; Colaianni, A. Assessment of Energy Sustainable Operations on a Historical Building. The Dante Alighieri High School in Florence. Sustainability 2018, 10, 2054. [CrossRef]

15. Corrado, V.; Ballarini, I.; Paduos, S.; Tulipano, L. A new procedure of energy audit and cost analysis for the transformation of a school into a nearly zero-energy building. Energy Procedia 2017, 140, 325-338. [CrossRef]

16. De Berardinis, P.; Rotilio, M.; Capannolo, L. Energy and Sustainable Strategies in the renovation of existing buildings: An Italian Case Study. Sustainability 2017, 9, 1472. [CrossRef]

17. Loreti, L.; Valdiserri, P.; Garai, M. Dynamic simulation on energy performance of a school. Energy Procedia 2016, 101, 1026-1033. [CrossRef] 
18. Guarini, M.R.; Morano, P.; Sica, F. Integrated Ecosystem Design: An Evaluation Model to Support the Choice of Eco-Compatible Technological Solutions for Residential Building. Energies 2019, 12, 2659. [CrossRef]

19. Asdrubali, F.; Calcagnini, L.; Evangelisti, L.; Guattari, C.; Marrone, P. Effectiveness of Materials, Technologies, and Renewable Energy in Educational Buildings Through Cluster Analysis of Energy Retrofitting. In Sustainable Building for a Cleaner Environment: Selected Papers from the World Renewable Energy Networks Med Green Forum 2017; Sayigh, A., Ed.; Springer: Cham, Switzerland, 2019; pp. 25-37. [CrossRef]

20. D'Agostino, D. Improving Energy Efficiency in Buildings: Challenges and Opportunities in the European Context. Sustainability through Energy-Efficient Buildings; CRC Press: New York, NY, USA, 2018; pp. 181-208.

21. Baggio, M.; Tinterri, C.; Dalla Mora, T.; Righi, A.; Peron, F.; Romagnoni, P. Sustainability of a historical building renovation design through the application of LeeDßrating system. Energy Procedia 2017, 113, 382-389. [CrossRef]

22. Della Spina, L. Historical Cultural Heritage: Decision Making Process and Reuse Scenarios for the Enhancement of Historic Buildings. In New Metropolitan Perspectives. ISHT 2018. Smart Innovation, Systems and Technologies; Calabrò, F., Della Spina, L., Bevilacqua, C., Eds.; Springer: Cham, Switzerland, 2019; Volume 101, pp. 442-453. [CrossRef]

23. Available online: http://www.dt.tesoro.it/export/sites/sitodt/modules/documenti_it/patrimonio_pubblico/ patrimonio_pa/RapportoImmobili_DatiAnno2016.pdf (accessed on 20 January 2020).

24. Ministry of Education, University of Research—Single School Data Portal. Available online: https://dati. istruzione.it/opendata/esploraidati/ (accessed on 19 December 2019).

25. Ministry of Education, University of Research—School building portal - registry office. Available online: https://www.istruzione.it/edilizia_scolastica/anagrafe.shtml (accessed on 19 December 2019).

26. Vitale, V.; Salerno, G. A numerical prediction of the passive cooling effects on thermal comfort for a historical building in Rome. Energy Build. 2017, 157, 1-10. [CrossRef]

27. Vogel, C. Recovery High Schools. Dist. Adm. 2009, 45, 37-40.

28. Patel, S.; Natalie, C. Student and staff social dynamics and transitions during school redesign. Improv. Sch. 2019, 22, 158-172. [CrossRef]

29. Green, T.L. School as community, community as school: Examining principal leadership for urban school reform and community development. Educ. Urban Soc. 2018, 50, 111-135. [CrossRef]

30. Salvado, F.; de Almeida, N.M.; Azevedo, Á.V.E. Historical analysis of the economic life-cycle performance of public school buildings. Build. Res. Inf. 2019, 47, 1-20. [CrossRef]

31. MD December 18, 1975 (in G.U. February 2, $1976 \mathrm{n}^{\circ}$ 29): Updated technical standards relating to school building. including building and town planning functionality indexes. to be observed when carrying out school building works. Available online: https://www.indicenormativa.it/norma/urn\%3Anir\%3Aministero. lavori.pubblici\%3Adecreto\%3A1975-12-18 (accessed on 20 January 2020).

32. Law $\mathrm{n}^{\circ} 23$ of January 11, 1996 (in G.U. no. 15-General Series—of January 19, 1996). Standards for school building. Available online: https://www.normattiva.it/uri-res/N2Ls?urn:nir:stato:legge:1996;23 (accessed on 20 January 2020).

33. Legislative Decree $n^{\circ} 106$ of August 3, 2009. Supplementary and corrective provisions of Legislative Decree $\mathrm{n}^{\circ} 81$ of April 9, 2008 on the protection of health and safety in the workplace (in G.U. $\mathrm{n}^{\circ} 180$ of August 5, 2009). Available online: http://www.bosettiegatti.eu/info/norme/statali/2009_0106.htm (accessed on 20 January 2020).

34. Agreement, pursuant to Article 9 of Legislative Decree n 281 of August 27, 1997, between the Government, regions, autonomous provinces of Trento and Bolzano, provinces, municipalities and mountain communities concerning "Guidelines for the prevention of indoor risk factors for allergies and asthma in schools" (in G.U. General Series no. 9 of 13-01-2011). Available online: https://www.normattiva.it/uri-res/N2Ls?urn:nir:stato: decreto.legislativo:1997-08-28;281 (accessed on 20 January 2020).

35. MD of January 17, 2018: Update of "Technical standards for construction" (in G.U. General Series n ${ }^{\circ} 42$ of 20-02-2018). Available online: https://www.gazzettaufficiale.it/eli/gu/2018/02/20/42/so/8/sg/pdf (accessed on 20 January 2020).

36. Law $\mathrm{n}^{\circ} 107$ of July 13, 2015 (1). Reform of the national education and training system and delegation for the reorganization of the current legislative provisions (in G.U. General Series n ${ }^{\circ} 162$ of 15-07-2015). Available online: https://www.gazzettaufficiale.it/eli/id/2015/07/15/15G00122/sg (accessed on 20 January 2020). 
37. Law $n^{\circ} 81$ of August 8, 2019 (art. 4-bis: Adaptation to fire regulations of school buildings in G.U. General Series $n^{\circ} 188$ of 12-08-2019). Available online: https://www.gazzettaufficiale.it/eli/id/2019/08/12/19G00090/sg (accessed on 20 January 2020).

38. MD April 11, 2013: Technical standards, containing the minimum and maximum indices of urban planning, building, also with reference to technologies for energy efficiency and energy saving and production from renewable energy sources, and teaching, to ensure adequate and homogeneous design guidelines on the national territory. Available online: https://www.edscuola.eu/wordpress/?p=20651 (accessed on 20 January 2020).

39. ENEA. Guide to energy efficiency in school buildings. Available online: http://www.efficienzaenergetica. enea.it/scuolesostenibili. (accessed on 20 January 2020.).

40. Csobod, E.; Annesi-Maesano, I.; Carrer, P.; Kephalopoulos, S.; Madureira, J.; Rudnai, P.; De Oliveira Fernandes, E.; Barrero, J.; Beregszászi, T.; Hyvärinen, A.; et al. SINPHONIE-Schools Indoor Pollution and Health Observatory Network in Europe-Final Report. EUR 26738; Publications Office of the European Union: Luxembourg, 2014; ISBN 978-92-79-39407-2. [CrossRef]

41. Lu, Y.; Zhang, X.P.; Huang, Z.; Lu, J.; Wang, D. Impact of introducing penalty-cost on optimal design of renewable energy systems for net zero energy buildings. Appl. Energy 2019, 235, 106-116. [CrossRef]

42. Pasimeni, M.R.; Valente, D.; Zurlini, G.; Petrosillo, I. The interplay between urban mitigation and adaptation strategies to face climate change in two European countries. Environ. Sci. Policy 2019, 95, 20-27. [CrossRef]

43. Iannone, F.; Carbonara, G.; Rinaldi, A.; D’Elia, M.; Dell'Osso, G.R. Building automation system to control natural ventilation in school buildings. The case study of "Michelangelo School" in Bari. Tema Technol. Eng. Mater. Archit. 2019, 5, 117-129.

44. Ozsezer, M.; Tufan, H.; Ozkul, A.E. Historical development of the school for deaf children which was opened in Cyprus under the British Administration. Qual. Quant. 2018, 52, 1421-1435. [CrossRef]

45. Campbell, C.; Sherington, G. The comprehensive Public High School: Historical Perspectives; Palgrave Macmillan: New York, NY, USA, 2013.

46. Cardinale, T.; Colapietro, D.; Cardinale, N.; Fatiguso, F. Evaluation of the efficacy of traditional recovery interventions in historical buildings. A new selection methodology. Energy Procedia 2013, 40, 515-524. [CrossRef]

47. National Institute for Documentation Innovation Educational Research, PON-GIES Project-Management of School Building Interventions. Available online: http://gies.indire.it (accessed on 19 December 2019).

48. Tarragüel, A.A.; Krol, B.; Van Westen, C. Analysing the possible impact of landslides and avalanches on cultural heritage in Upper Svaneti, Georgia. J. Cult. Herit. 2012, 13, 453-461. [CrossRef]

49. Huang, M.; Bo, W. Evaluating green performance of building products based on gray relational analysis and analytic hierarchy process. Environ. Prog. Sustain. Energy 2014, 33, 1389-1395. [CrossRef]

50. Pardo-Bosch, F.; Aguado, A.; Pino, M. Holistic model to analyze and prioritize urban sustainable buildings for public services. Sustain. Cities Soc. 2019, 44, 227-236. [CrossRef]

51. Nesticò, A.; Morano, P.; Sica, F. A model to support the public administration decisions for the investments selection on historic buildings. J. Cult. Herit. 2018, 33, 201-207. [CrossRef]

52. Macharis, C.; Turcksin, L.; Lebeau, K. Multi actor multi criteria analysis (MAMCA) as a tool to support sustainable decisions: State of use. Decis. Support Syst. 2012, 54, 610-620. [CrossRef]

53. Morano, P.; Locurcio, M.; Tajani, F.; Guarini, M. Fuzzy logic and coherence control in multi-criteria evaluation of urban redevelopment projects. In International Journal of Business Intelligence and Data Mining; Azevedo, A., Santos, M.F., Eds.; Business Science Reference: Hershey, PA, USA, 2015; pp. 73-93. [CrossRef]

54. Morano, P.; Tajani, F. Estimative analysis of a segment of the bare ownership market of residential property. In Computational Science and Its Applications, ICCSA 2013: 13th International Conference [...] Proceedings, Part IV. LECTURE NOTES IN COMPUTER SCIENCE; Murgante, B., Misra, S., Eds.; Springer: Berlin, Germany, 2013; Volume 7974, pp. 433-443. ISBN 978-3-642-39648-9. [CrossRef]

(C) 2020 by the authors. Licensee MDPI, Basel, Switzerland. This article is an open access article distributed under the terms and conditions of the Creative Commons Attribution (CC BY) license (http://creativecommons.org/licenses/by/4.0/). 


\title{
Citizen Action as a Driving Force of Change. The Meninas of Canido, Art in the Street as an Urban Dynamizer
}

\author{
María José Piñeira Mantiñán *, Francisco R. Durán Villa and Ramón López Rodríguez \\ Department of Geography, University of Santiago de Compostela, 15782 Santiago de Compostela, Galicia, Spain; \\ francisco.duran@usc.es (F.R.D.V.); ramonlopez.rodriguez@usc.es (R.L.R.) \\ * Correspondence: mariajose.pineira@usc.es; Tel.: +34-88181100 (ext. 12626)
}

Received: 20 December 2019; Accepted: 15 January 2020; Published: 20 January 2020

\begin{abstract}
The austerity policies imposed by the government in the wake of the 2007 crisis have deteriorated the welfare state and limited neighborhood recovery. Considering the inability and inefficiency on the part of administrations to carry out improvement actions in neighborhoods, it is the neighborhood action itself that has carried out a series of resilient social innovations to reverse the dynamics. In this article, we will analyze the Canido neighborhood in Ferrol, a city in north-western Spain. Canido is traditional neighborhood that was experiencing a high degree of physical and social deterioration, until a cultural initiative called "Meninas of Canido," promoted by one of its artist neighbors, recovered its identity and revitalized it from a physical, social, and economic point of view. Currently, the Meninas of Canido has become one of the most important urban art events in Spain and has receives international recognition. The aim of this article is to evaluate the impact that this action has had in the neighborhood. For this, we conducted a series of semi-structured interviews with the local administration, neighborhood association, the precursors of this idea, merchants, and some residents in general, in order to perceive the reception and evolution of this action.
\end{abstract}

Keywords: urban art; Neighborhood Regeneration; Social Empowerment; Right to the City; Ferrol (Spain)

\section{Introduction}

Over the last decade, citizen-administration relations in Spain have worsened. The austerity policies implemented in the wake of the 2007 financial crisis have resulted not only in political and institutional disaffection but also in a social and cultural crisis that has affected a high percentage of citizens. From the closing of companies and high unemployment rates (especially among young people) and eviction processes, the budget cutbacks (especially in personnel and basic services $[1,2]$ and the privatization or externalization by subcontracting of some of them) have led to a notable worsening in citizens' living conditions [3-5]. The result has been an increase in the dynamics of social exclusion that have deteriorated the social cohesion of cities, leaving families and individuals in precarious conditions and in situations of vulnerability. According to the Eighth Report on Poverty in Spain [6], $8 \%$ of the population suffer from cold at home (in 2009, it was 7.2\%), a quarter of those over 25 years old do not have their own income or earn less than 535 Euros a month, and 10\% of workers continue to lose $25 \%$ of their income each year due to precarious job conditions. Additionally, regarding the housing problem and according to judiciary statistics, evictions continue, with a total of 368,591 recorded since 2013 , of which $58.9 \%$ were for non-payment of rent and $41.1 \%$ for non-payment of mortgages [7].

In this context, it is not surprising that more and more cities and neighborhoods are immersed in a deep and long-lasting decline [8-12]. They are vulnerable neighborhoods where degradation 
and neglect have been noted in the urban frame [13]. Numerous researchers have echoed the situation, analyzing the impact of the processes of negotiation of disposition [14], how austerity policies affect citizens' rights [15,16], and how gentrification and tourism affect the aggravation of the population's vulnerability, limiting access to housing due to the increase in purchase and rental prices $[7,17,18]$. Likewise, the central government has tried to analyze the problem by creating a vulnerability observatory published by the Atlas of Urban Vulnerability and the Atlas of Vulnerable Neighborhoods. Thanks to them, an overview of the problem is available, but viable policies and initiatives that mitigate this vulnerability are still lacking.

Since 2011, civil society has organized itself in different movements, platforms, and associations to demand the stoppage of austerity policies and cuts and demand that access to basic social services be guaranteed. However, due the inability and inefficiency of administrations to carry out improvement actions, it is the neighborhood action itself that carries out a series of resilient social innovations to reverse neighborhood dynamics. To analyze them in this article we will focus on the Canido neighborhood in Ferrol, a city in the northwest of Spain. Canido is a traditional neighborhood affected by a high degree of physical and social deterioration, from which a cultural initiative has managed to reverse this deterioration, improving its physical aspect and energizing it from an economic, social, and cultural point of view. With its study, a double objective is pursued: first, to analyze to what extent the citizen movements of the neighborhood and their actions are the architects in the recovery of the neighborhoods and the reduction of their vulnerability, and also to study the degree of impact that these types of actions have in the neighborhood from a physical, economic, and social point of view and to what extent local administrations view them as a solution to the state of crisis in which they are still immersed.

To do this, we will first approach the urban policies that have been developed in Spain since the 1990s. We will check which urban sectors have been most interested in local administrations to make improvements and project an image of a concrete city. Later, we will study to what extent the social movements and their proposals for social innovation are helping to recover the quality of life in the neighborhoods. We will analyze some of them and consider if the administrations have supported them. Finally, we will analyze how the street art initiative Las Meninas de Canido has become the most attractive neighborhood in the city and has achieved international recognition.

\section{Objectives and Method}

To analyze the impact that the artistic experience of Las Meninas de Canido has had on the neighborhood, an eminently qualitative investigation that aims to accomplish the following objectives has been carried out:

- Analyze the extent to which cultural initiatives promoted by citizens are able to regenerate degraded spaces and increase the feeling of empowerment and identity of its inhabitants.

- Check to what extent these initiatives, which have arisen as popular resilience events, remain as such over the years, or if they have changed with respect to their beginnings.

- Know the perception that the inhabitants of the affected neighborhoods have towards such cultural initiatives and what their future prospects are.

To this end, an important review of the current literature on new models of urban governance, urban renewal and social innovation, social justice, neighborhood movements, and resilience and urban innovation initiatives (urban gardens, art in public space, etc.) was first carried out. The result was a theoretical approach to the issue.

Second, numerous websites and blogs have been consulted on Las Meninas de Canido, as well as the official websites of the municipality of Ferrol and neighborhood associations, to have some initial information on the projects that have been carried out in the neighborhood and thus be able to organize and design the questionnaire that laid the basis for the semi-structured interviews. 
The questions were organized into two large blocks (Table 1): one related to the Meninas event with questions that allowed the origin of the event to be known and to assess the degree of impact in the neighborhood, physically, socially, and economically; and another related to the neighborhood, to evaluate the sectors that were most degraded and see to what extent they were acting on them.

Table 1. Questions for interviews.

\begin{tabular}{cc}
\hline Las Meninas Block & Neighborhood Block \\
\hline $\begin{array}{c}\text { How did the idea come about? } \\
\text { Who participates? }\end{array}$ & $\begin{array}{c}\text { Has the identity of the neighborhood been reinforced } \\
\text { thanks to Las Meninas? }\end{array}$ \\
$\begin{array}{c}\text { In what areas are Las Meninas painted? How are the } \\
\text { areas to be painted organized (more degraded, } \\
\text { continuity with the previous ones)? }\end{array}$ & $\begin{array}{c}\text { Have Las Meninas been the true driving force of the } \\
\text { neighborhood? }\end{array}$ \\
$\begin{array}{c}\text { Is the popular character of the event maintained? } \\
\begin{array}{c}\text { To what extent have the Meninas de Canido promoted } \\
\text { the city of Ferrol and the neighborhood of Canido? } \\
\text { To what extent does the city council participate } \\
\text { (financial contribution, services, etc.)? }\end{array}\end{array} \quad \begin{array}{c}\text { Have you favored the opening of new businesses? } \\
\text { To what extent have they influenced urban } \\
\text { regeneration processes? }\end{array}$ \\
$\begin{array}{c}\text { What will happen when there are no walls left } \\
\text { to paint? }\end{array}$ & $\begin{array}{c}\text { Can gentrification processes associated with the } \\
\text { rehabilitation and sale of homes be observed? } \\
\text { Have prices increased? }\end{array}$ \\
How many artists and works are there? & What new projects are there for the neighborhood? \\
\hline
\end{tabular}

The people/institutions interviewed were Eduardo Hermida, the person who, in an act of protest against the state of ruin and degradation in which the Canido neighborhood had become, went out and painted the first Menina, thus becoming the promoter of the cultural event of Las Meninas de Canido. Jorge Suárez Fernández, Mayor of the city from 2015-2019 and the person who assessed the degree of involvement that the city council had with the event; the association of Neighbors of Canido, the cultural association O Muiño, hoteliers, and merchants. All of them were allowed to give their opinion on the event of Las Meninas in order to evaluate to what extent the neighborhood has been transformed for them in recent years, to know what aspects they value as positive and negative, in relation to both the event itself, and the situation in which the neighborhood is, and to know its future expectations.

Likewise, the field work carried out in May 2019 in the Canido neighborhood allowed us to check the state of deterioration, the humanization actions carried out through European funds through urban projects and to map the location of the existing Meninas paintings in the neighborhood.

\section{The Paradigm Shift in Urban Policies}

In each city, there is a trajectory that indicates the balances between the role of public authorities, the business world, social fabric, or citizens in the processes that have combined urban transformation, the construction of a city model, and the dynamics of urban governance [19]. If we review the period from the mid-1980s to the present, we can see that the 2007 crisis was a turning point, both in relation to the desired city model, and therefore, of urban policies and outbreaks of interest to be addressed, including the agents involved and the expected results. For two decades, local administrations opted to create competitive cities within the global and modern market, in which urban megaprojects—often linked to international events-were the protagonists and placed the city on the map [20]. Examples include the Olympic city of Barcelona, the equipment and facilities built in Seville and Zaragoza with the universal exhibitions, the Guggenheim in Bilbao, the city of arts and sciences of Valencia, or the city of culture in Santiago de Compostela. With the exception of Bilbao, the viability of these projects has been questioned since their inception. However, they have always defended themselves from the political spheres under the pretext of the economic profitability that they would bring to the city and the improvement that they would provide at an urban level. From the administrations, a neoliberal growth model was chosen, in which the market and the real estate developers marked the growth of the city, and there was a liberalization of the land and a total deregulation of urban growth. 
The creation of new infrastructure was considered to improve the positioning of cities in the urban hierarchy, and investment in housing was understood as a safe and highly profitable asset. The city looked like a business, and therefore, getting the maximum benefit was the key objective. Throughout this process, financial institutions played a key role, offering loans with generous conditions, both for real estate developers and for citizens, who were encouraged to apply for a mortgage to purchase their homes [21-23]. The result was unprecedented urban growth, in which the traditional city lost its meaning in the face of urban sprawl processes and in which the city was considered a consumer product, where all aspects that had to do with the social welfare and quality of life of citizens were relegated to the background. In this regard, it should be noted that while comprehensive development strategies have been implemented in Spanish cities since 1994, through initiatives funded with European funds. These include URBAN and URBAN II initiatives, Urban Innovative Actions (URBACT), and Integrated and Sustainable Urban Development Strategy (ISUDS). In relation to the former, its objective was to combat urban degradation and promote the regeneration of degraded neighborhoods. With them, it was possible to introduce in Spain a series of concepts that were not yet discussed. These include the concept of an integrated approach, the need to address the problems of cities based on collaboration between institutions, the need to involve the population and local actors through participatory processes and the introduction of a vision of urban regeneration based on the concept of sustainable urban development. In the period 1990-1994, there were 29 URBAN projects that involved an investment of $€ 16,748,739$; while with Urban II (2000-2007), only 10 were counted [24]. On the other hand, the URBACT initiatives (2014-2020) were aimed at promoting sustainable development in 28 Spanish cities, with an investment of $€ 351,820$ [25]; at the same time that for the Integrated and Sustainable Urban Development Strategy (ISUDS), more than one billion euros were allocated to improve the environment, promote social inclusion and the fight against poverty, improve access to ICTs and improve environmental quality [26]. Nevertheless, there are few studies on the impact that these have had when solving specific problems of a social, environmental, and economic nature in the sectors involved. In some cases, these funds were used to improve some sectors of downtown areas of cities, historic centers, or neighborhoods with degraded buildings, but they continued to be small urban acupuncture interventions [27]. The problem was that most of the municipal investments made were destined for actions that could attract a greater number of visitors or tourists; leaving the neighborhoods in practically the same state of degradation and vulnerability in which they started. In 2007, the urban development model changed completely, because:

- With the crises, the weaknesses of the neo-liberal growth model came to light, uncovering some cases of corruption linked to it [28], becoming, in 2015, the second problem that most worried Spanish citizens after unemployment, according to data from the Center for Sociological Research [29].

- Local administrations were aware of their degree of indebtedness derived from the construction of megaprojects and saw their budgets cut because debt forgiveness was a priority for the central government.

- Citizens demanded a new way of creating a city and recovering the right to the city and real democracy. According to Harvey [30], in Spain, the clamor for the right to the city meant insisting on the right to change and reinvent the city based on the wishes of the people who inhabit it. In this context, civil society took to the streets, following demonstrations and the emergence of citizen platforms from which the new leftist parties emerged that would arrive in the 2015 elections to the municipal governments of cities such as Madrid, Barcelona, Cádiz, A Coruña, Santiago de Compostela, and Ferrol. Some parties promised their citizens the defense of democratic rights, an end to corruption, a restoration of the well-being of citizens, a guarantee true democracy, and service to the vulnerable population [31-34].

From then on, talk about local government gave way to talk about urban governance, in which both the policies and the actors involved in them mattered. Among them were the citizens, who had 
become increasingly empowered. The interest of these policies focused on neighborhoods, especially those that were the most vulnerable and where there was greater unemployment and more evictions. It was based on the premise that the city should be a space to live and a place for citizens and that the administration had to be the one to guide the strategies that needed to be developed, with the help of citizens through real participation, in a way that decisions were not imposed but worked from the bottom up; transparency departments were created, and municipal budgets were redistributed by increasing the budget of the most disadvantaged neighborhoods to promote social rental housing, promote educational and cultural activities, and serve the most disadvantaged groups (youth, long-term unemployed, women, single parents) to improve their quality of life (Table 2).

Table 2. Keys of urban policies changes during the post-crisis period.

\begin{tabular}{|c|c|c|}
\hline Key Elements & 1990-2007 & 2008-2019 \\
\hline Model of urban governance & $\begin{array}{c}\text { Creating a modern and competitive city } \\
\text { image } \\
\text { Efficiency was established by private } \\
\text { sector measures } \\
\text { Contingent on the market }[22,23] \\
\text { Top-down control of power and } \\
\text { decision-making [19] }\end{array}$ & $\begin{array}{c}\text { City: a space to live and a place for } \\
\text { citizens [35] } \\
\text { Restoring the role of the state [27] } \\
\text { Multilevel governance that } \\
\text { incorporates social action [36] } \\
\text { Restoring democratic values } \\
\text { [37-39] } \\
\text { Need for a new economic order } \\
\text { Bottom-up decision-making and a } \\
\text { new institutional structure [40] }\end{array}$ \\
\hline Urban planning & $\begin{array}{l}\text { Subjecting planning to market interests } \\
\text { [41,42] } \\
\text { Builders define urban development [43] } \\
\text { Administration has a secondary role } \\
\text { Land liberalization and building at all } \\
\text { costs [23] } \\
\text { Deregulation of urban and spatial } \\
\text { planning [23] } \\
\text { Lack of coordination between urban } \\
\text { policies [19] }\end{array}$ & $\begin{array}{l}\text { Establishment of urban planning } \\
\text { criteria to halt deregulation [44] } \\
\text { Administration plays a leading } \\
\text { role in planning [23] } \\
\text { Encouraging participation in } \\
\text { neighborhoods Interaction in } \\
\text { actors' network [45] } \\
\text { Realistic budgets that conform to } \\
\text { actual needs } \\
\text { Focusing investments on } \\
\text { vulnerable neighborhoods }\end{array}$ \\
\hline Focus of interest & $\begin{array}{l}\text { Entrepreneurial city [46] } \\
\text { Construction of housing, installation } \\
\text { and infrastructure megaprojects [47] } \\
\text { Urban sprawl and metropolitan spaces }\end{array}$ & $\begin{array}{c}\text { Promotion of social and economic } \\
\text { innovations } \\
\text { Development of dynamic projects } \\
\text { Overcoming social, economic and } \\
\text { housing insecurity } \\
\text { Citizen mobility [48] } \\
\text { Cities being resilient and } \\
\text { alternative (ways of being resilient, } \\
\text { having alternative economies) } \\
\text { spaces [8] } \\
\text { Right to the city } \\
\text { Right to housing }\end{array}$ \\
\hline Key players & $\begin{array}{l}\text { Banks, financial institution and the } \\
\text { administration [23] }\end{array}$ & $\begin{array}{c}\text { The administration } \\
\text { Empowered citizens }[49,50]\end{array}$ \\
\hline Results & $\begin{array}{c}\text { Overextended growth of cities and } \\
\text { metropolitan areas } \\
\text { Loss of value in cities } \\
\text { City becoming a product } \\
\text { Dismantling of the welfare state } \\
{[9,51,52]}\end{array}$ & $\begin{array}{c}\text { Planning of model cities } \\
\text { Understanding the city as a whole } \\
\text { Value of neighborhoods } \\
\text { Priority for the well-being of } \\
\text { citizens } \\
\text { Greater quality of life }\end{array}$ \\
\hline
\end{tabular}

However, after four years since the arrival of these political parties, the interviews with policy makers in cities such as Madrid, Cádiz, Valencia, A Coruña, and Ferrol have revealed that the expected 
results have not been obtained. Among the causes is the budget deficit, the difficulty of changing work synergies established for decades in local administrations, excessive bureaucratization, and even reluctance on the part of the opposition parties when implementing some initiatives, such as those that favor the creation of public housing for social rent. Consequently, citizens continue to be the protagonist when it comes to implementing social innovation actions that mitigate the situation of degradation and vulnerability that still exists in neighborhoods.

\section{Citizen Movements and Social Innovation: Two Key Factors for the Recovery of Neighborhoods}

In Spain, neighborhood communities have become the mechanism to combat vulnerability and the lack of social cohesion. They are the nexus between the search for a democratic state guaranteeing basic rights on the one hand and the continuous reinvention of social life on the other [53]. They are the loci and drivers of social innovation aimed at improving the neighborhood environment and recovering the sense of a lost neighborhood, turning public spaces and abandoned buildings into self-managed meeting places. Consequently, a multiplicity of structures to govern/manage neighborhoods has emerged, in collaboration with the local administration, which has increasingly given greater importance to initiatives that encourage the revitalization of the urban environment arising from civil society $[40,53]$. Its success has been conditioned by the level of citizen mobilization, the degree of awareness and empowerment of neighbors, as well as their ability to learn. The result is that neighborhoods have become laboratories in which to test all kinds of innovative actions that result in dynamics of urban and social improvement.

But what does social innovation consist of? Depending on the discipline from which their interpretations are approached, they range from management, where social innovation is understood more as a product than a process [54] to which they have a more systematic approach, contemplating aspects such as the network of actors, the type of relationship and interdependence with public administrations, the overlapping of the territory and other contextual factors (social, cultural, political) as well as the objectives, the type of process and its social effects [55]. Another point of view is that which links social innovation to creative citizen practices that are carried out in neighborhoods and cities, conceived from social movements (community or civil platforms), considering that the welfare state has deteriorated and therefore there is an insufficient response capacity to meet the collective and individual needs of the community. Among them, we can highlight the following points:

- Innovative actions related to the different phases of the economic cycle (production, distribution, financing, or consumption). Among them, we can differentiate those of a formal and informal nature, from cooperatives and associations to organic produce consumer groups, where intermediaries are eliminated, or to projects such as social currency or barter markets. In this field, the population has mobilized looking for an alternative economy from which ethical banking or crowd funding are born. Financial alternatives to the existing ones, where the maximum benefit is not sought, are able to carry out projects thanks to the collaboration and cooperation of different agents. This group also includes actions such as time banks, in which participants exchange their skills for time instead of money, where hours are added when a service is provided to another participant and subtracted when you are the beneficiary of the service.

- Social innovation actions linked to participatory processes from which ideas are collected to carry out some urban interventions in the neighborhood. In this case, we want to highlight the Los Madriles project (Figure 1), started in 2015, which consists of an atlas of collectively generated neighborhood initiatives. Its objective is to generate a network between the different projects that are underway in Madrid and that share a social vision and community intervention. This initiative stands out for the diversity of promoters who managed to put the website into operation: the Federation of Neighborhood Associations of Madrid (FRAVM), Zuloark, the citizen initiatives incubator, all for praxis and Transversal Landscape collectives, social and neighborhood groups, 
and Intermediae, an experimental space devoted to socially engaged artistic practices approached from the angle of research and cultural innovation.

- Social innovation actions linked to the urban environment of the neighborhood, carrying out projects in the public space. In some cases, they are projects related to the environment-promoting more sustainable forms of access and use of resources - and the revitalization or recovery of the physical space, which will subsequently have a social impact. Self-management spaces that are developed in the public space and real estate. This group highlights the initiatives of urban gardens (Figure 2), small plots in which agricultural tasks are carried out with ecological farming methods, where the productive functions associated with family consumption are combined with social and environmental and which house a potential to develop activities of citizen participation. Its success has been so great that the number of urban gardens exceeds 15,000, in more than 300 municipalities. Among the initiatives aimed at recovering empty and degraded urban spaces, we can mention This is not a site in Zaragoza (Figure 2). It consists of the recovery of abandoned plots within the city, where a process of cleaning, rehabilitation, and maintenance makes them open spaces for neighbors. This results in the removal of abandoned spaces in urban and unused areas where rubbish usually accumulates. Empty plots are converted into public spaces, creating gardens, parks, or urban vegetable gardens among others.

- Social innovation action is linked to arts and creativity because culture can be the growth factor at the source of economic competitiveness and can also contribute to the social development and environmental sustainability of neighborhoods [56]. In this group, urban murals and paintings stand out. Artistic expressions are made in public space-buildings, facades, streets, and squares-with the aim of improving their image and recovering them not only from a physical point of view but also a social and economic one. In this sense, we must consider that some street art initiatives have resulted in urban regeneration, gentrification and tourism processes. However, while at first they can be valued positively since they revitalize the neighborhood, in the medium term, they can result in expulsion processes of the population due to the rise in housing and commercial real estate prices and the negative effects of mass tourism in specific urban sectors.

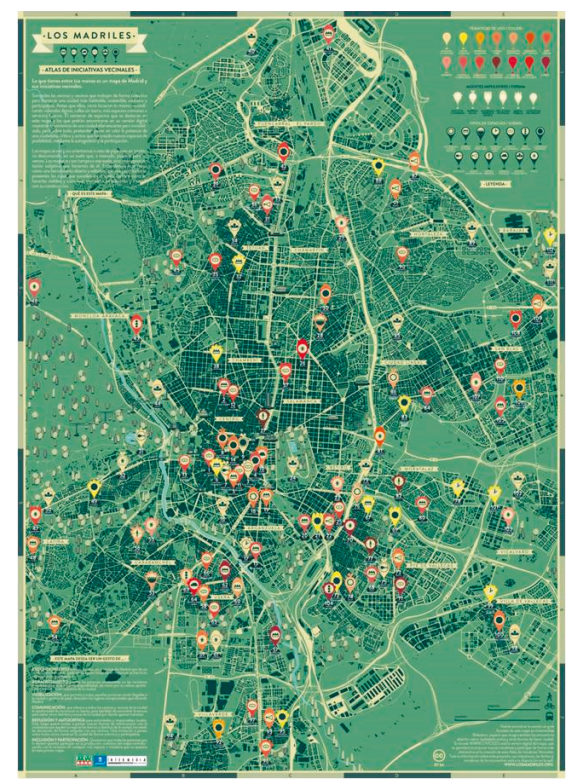

Figure 1. Los Madriles Map. 


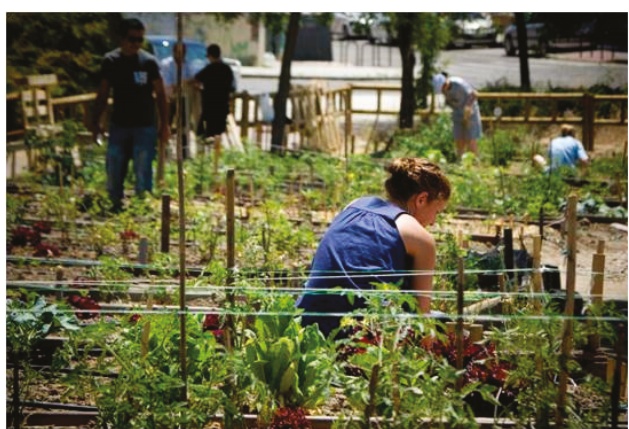

(a)

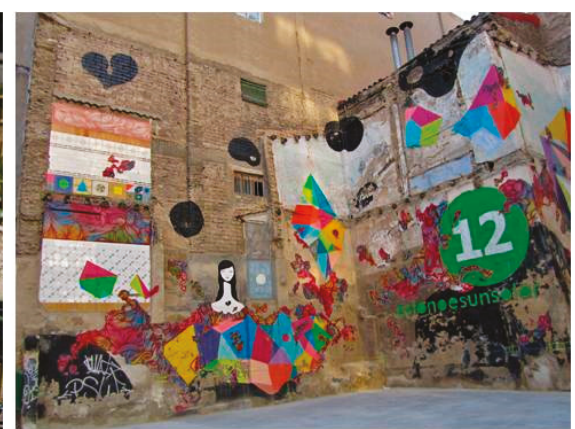

(b)

Figure 2. (a) Urban garden; (b) initiative in public space with the project This is not a site.

In the following sections, we will approach the regenerative effect that street art has had on urban spaces and later focus on the study of Las Meninas de Canido initiative and the impact it has had on the degraded Canido neighborhood.

\section{The Urban Recovery of Degraded Neighborhoods: The Effect of Street Art}

When talking about the impact of artistic performances in urban spaces, we must consider two key concepts: public art and urban art (also known as street art). Although both are developed in a common space, they differ in that public art is considered a legal artistic manifestation, insofar as it is part of the urbanization plans of the local administration and has a heritage character and is cared for; While urban art is performed by artists anonymously, it is considered illegal and has an ephemeral character [57]. Despite this, it is considered free and open access art [58].

However, some authors consider certain nuances between the concept of urban art and street art. They consider that the first is linked to the art of graffiti, as an artistic expression that generally contains a political or social criticism charge in its origins and associated with the imagery of vandalism, illegality, and protest, while street art, known as Postgrafiti, has a more artistic load, and its technique is more respectful than graffiti when choosing materials and support. Among its most used techniques are murals, stencils, stickers, and posters, some of which are commissioned $[59,60]$.

In any case, in this article, we do not intend to respond to the difference between these expressions or to debate whether graffiti is an artistic movement or not. What is intended is to highlight how an artistic activity such as murals or graffiti has become fashionable in recent years and is a common practice in those parts of the city most physically degraded. Thus, culture becomes a catalyst that triggers the reuse of abandoned spaces and the creation of new urban scenes; it can be a source of urban revitalization and social inclusion. Like a human body patterned with tattoos [61], degraded sectors are commonly covered with inscriptions that are already part of the urban imaginary [62]. Then, an aestheticization of everyday life is produced [63], in which images acquire a new and central role in the society of consumption. However, there is a debate about the contributions of the culture and public art industry for urban restructuring and regeneration [64]. There is a paradox that, on the one hand, urban art is considered a measure that contributes to the improvement of public space and, consequently, the quality of life of residents. In addition, it favors citizen empowerment, the strengthening of the neighboring identity — as it tries to adapt to the social and historical context of the neighborhood-and social commitment by questioning, discussing, or giving effective aesthetic responses to the needs of citizens [65]. Nevertheless, the fact that urban art moves between the allowed, the regulated, and the illegal cannot be ignored. Sometimes, it is accepted, even being conserved and promoted; in others, however, it is criminalized and prohibited. 
However, there is no doubt about the number of festivals, publications, and urban art galleries that have arisen in different cities, in which the participation of local administrations is increasing, reaching directly to encourage the realization of these practices in concrete spaces in the city, with a clear objective: to commercialize art as an advertisement for tourism. However, there are some practices in which these cultural productions revert to the benefit of the community, the neighborhood, or the city.

Cases like The Bushwick Collective in New York (Figure 3) are true outdoor art galleries, which have transformed a shady industrial neighborhood into a vibrant tourist destination. It has attracted numerous street, local, national, and international artists and thousands of street art fans since its first mural in 2011 [66].
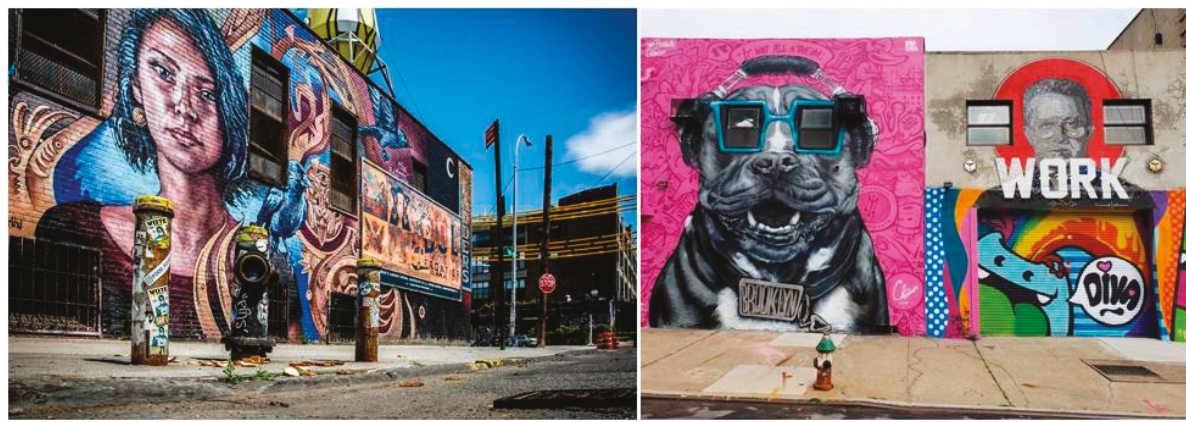

Figure 3. Murals of Bushwick Collective in New York.

Another similar initiative is Wynwood Walls, conceived in 2009 in Miami. The aim of the artist who had the idea was to look for something big to transform the Wynwood district, an area full of windowless warehouses, whose facades would soon become giant canvases in which to show the best street art. Since its inception, the Wynwood Walls program has had more than 50 artists representing 16 countries and has covered more than 80,000 square feet of walls. The New York Times, BBC News, Vanity Fair, and Forbes have included Wynwood in their list of the most modern neighborhoods in the United States [67].

\section{Street Art in the Canido Neighborhood: The Recovery of a Historically Stigmatized Neighborhood in the City of Ferrol}

The neighborhood of Canido, together with those of Ferrol Vello, La Magdalena, and the now-disappeared Esteiro, form the origins of an enlightened 18th century city, which emerged after the new naval policy of Felipe V came into force in 1726 in Spain (Figure 4). Its choice as the capital of one of the three maritime areas of Spain, the north, marked the beginning of the construction of an arsenal and shipyards, the orthogonal neighborhoods of Esteiro (known as the "frame " by its perimeter), the New Community of the Magdalena, and a whole series of defensive infrastructures materialized in the bastioned wall of the city and in the castles and batteries built on the margins of a natural port-the Ferrol estuary - an area of high geostrategic value until the first third of the 20th century [68].

The neighborhood is located on the top of a mountain to the north of the city with which it shares its name, between the cove of the Malata and the Magdalena neighborhood to the south, while in the west, it joins the early naval center of the old quarter. After successive proposals for fortification of the square, the one presented by the military engineer Pedro Torbé in 1768 definitively integrated this poorly populated rural space within the boundaries of the departmental city, being protected by one of the 12 bastions, known as Canido or Santiago. As well as its strategic nature, due to its altitude and exposure to the estuary, the peripheral and limit value in the administrative level can be added, 
a value that would play an important role in the process of expansion of the city from 1939 at the end of the civil war.

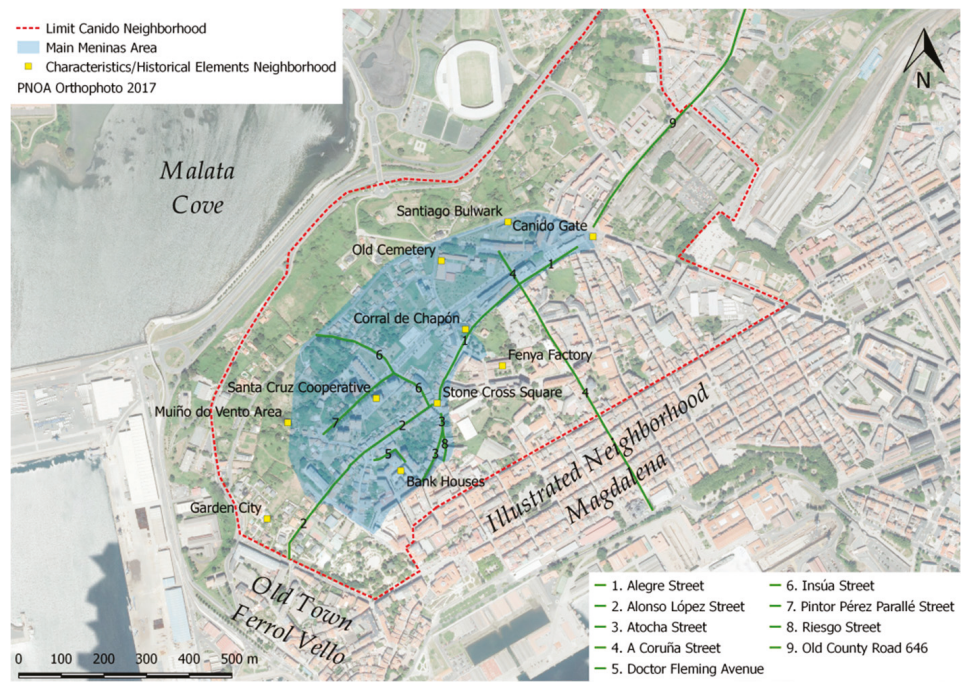

Figure 4. Canido neighborhood in the city of Ferrol.

Early reports refer to the place or village of Canido, which presupposes its earlier agricultural beginning, whose imprint is still noticeable in the traditional housing of the neighborhood. The rural exodus, attracted by the naval activity, would gradually turn this space into an alluvial neighborhood, capable of maintaining a part-time agricultural activity-unlike the one in Esteiro, erected from a new plant to house the shipyard workers and their families-supported mainly by women, who would have the opportunity to enter the labor market in one of the industrial initiatives (food, pencils, and textiles) from the 1940s. The male workforce was mainly concentrated in the shipyards of Bazán and Astano and participated in an increasingly diversified industrial sector, which was almost always related to the sea. Among others, the net factory (DEFER), PYSBE (Fisheries and Dryers of Cod of Spain), the Peninsular timber factory, the Hispania pencils factory, Soaps Pucho and properly in Canido the Fenya (Naval and Artillery Electrical Fabrications), the Piñon manufacturing company, and Industrial Pencils (ILASA). The settlement of workers in the neighborhood contributed to changing its physiognomy, causing profound internal social changes that were supported by the development of a militant commitment and solidarity strategies, which strengthened the affective and identity ties and generated a strong sense of belonging. While the bonding sentiment developed, initiatives at a parish level emerged to combat a daily life marked by the struggle against the difficulties of wages, hunger, poverty, inhuman housing, both parents working, and children in the street [69].

Its peripheral physical and structural origin, the peculiarities of its growth and development, as well as the recurring crises that plagued the city and its region were also accompanied by processes of social exclusion and segregation, materialized in perceptible forms of urban fragmentation and marginality, sometimes loaded with negative connotations and stereotypes, which were, without a doubt, defense strategies of the most favored groups to justify the existing social inequalities within the framework of the prevailing developmentalism. Stereotypes that can still be seen in the sociocultural imaginary and that condition the perception that some inhabitants of the city of Ferrol have of the neighborhood in general and in particular of its limits with the Magdalena neighborhood. The conformation as a structural periphery is related to the process of devaluation of the area that led to the opening of the municipal cemetery - the Canido cemetery-in 1804 after more than 25 years of heated controversies between the local authority and the neighborhood. On the other hand, 
the periphery, understood as a remote and disconnected space from the illustrated city, was favored with the opening of the street of San Eugenio in a north-south direction, which was immediately converted by citizens into the street of the dead and corresponds to the current street of La Coruña.

This fact, which responds to the implementation of sanitation budgets, was not isolated, as evidenced by the transfer or erection of new charitable welfare infrastructures in the open crossroads on the hillside, initiated with the opening of the new building of the charity hospital in March 1786, whose lands were acquired almost at the same time as those of the cemetery. Subsequently, this culminated with the nursery, the school for poor girls, the nursing home, and centers for social care and the company stores in the Dictatorship (1939-1975).

The presence of these institutions, together with the industrial enclaves, contained the prices of the abundant land and favored initiatives of private housing cooperatives-for example, the houses of the housing cooperative of Santa Cruz and, on a smaller scale, those of naval government officials, which were accompanied by the construction of the noncommissioned dwellings of the army at the door of Canido (currently Plaza de Canido, a newly created node) and by those built on the initiative of the Board of Trustees of Houses of the Navy for noncommissioned officers outside the entrance to Canido, extending the neighborhood to the east following the axis drawn by the old county road 646 and the newly built council housing. The emergence of these new promoters resulted in the emergence of new social upheavals and interior peripheries, degraded spaces located in the inner city, which in the past formed a periphery that was frozen in time [70] that materialized in an significant trade union and protest activity within the framework of specialized Catholic Action movements in the absence of democracy.

This construction fever began in the 1950s after the closure of the cemetery and fundamentally materialized in the decades of the 1960s and 1970s. This resulted in the expansion of the neighborhood to the east, and also to the west, coming into contact with the old quarter, where a small, upper class garden city arose, which was interwoven with the buildings that rise up the hillside as the northern extension of the Magdalena neighborhood, especially after the transfer from the hospital to the new dormitory suburb of Caranza and the opening of sections of the diagonal project dedicated to Doctor Fleming.

The increase in housing stock was accompanied by the improvement and opening of new urban roads and resulted in a significant increase in the population, whose immediate consequences were manifested in the erection of the new parish of the Holy Cross, which closed on 13 October 2019 after 55 years of operation, and the creation of public educational institutions for children and the current primary school—the Canido Infant and Primary Education Center (the former Reyes Catolicos school)-and the subsidiary of the women's institute on the grounds of the former graveyard, along with other private initiatives, all of which was accompanied by the proliferation of small commercial and artisanal activities to meet the demand of new residents.

To delimit the neighborhood is difficult. The Madoz dictionary [71] and the History of Ferrol de Montero Aróstegui [72] both break the space up as part of the Magdalena neighborhood or the historic center. At this time, the limits proposed by the city council coincided in the southern part with the layout of the grid of the enlightenment.

In line with its expansion process and its socio-economic peculiarities, urban deterioration processes do not affect all internal sectors equally. They are located mostly in the areas adjacent to the early roads that converge in the Stone cross square, the main node of the old enclave (Atocha-Riego, Alegre, Insúa, Alonso López), a crossing that has become an iconiclandmark of the neighborhoo, in the surroundings of the Plaza de la Tahona, Muiño do Vento, and in the corral of Chapón, which contrast more, if possible, with the recent estate built on the site of the old Fenya or on the new avenue of the painter Pérez Parrallé. It is worth noting that we are currently helping with the launch of major, new housing developments in the neighborhood [73], which support the recent prominence of this space in the framework of a city in perpetual crisis, due to both the decrease in the number of people living there and an aging population. It has been listed as the oldest city in Spain according to 2018 data from 
the Galician Institute of Statistics [74], since those over 64 represent $27.4 \%$ of the urban population, two points above the average of the region of Galicia (24.9\%).

This is also felt when you see that Canido currently has the lowest average age of the old walled enclosure- 45.4 years, only surpassed by two small districts located in the greater area outside the gates that develops on the axis traced along the road of Castile, the main (and until 1968 the only) communication route that links it to the state road network. The best neighborhood in Galicia [75], as was recently awarded by Galician Television, is the result and recognition of an important neighborhood action that has managed to combine the historical legacy, secular traditions such as the exaltation of nature in May - Los Maios -, the recovery of old icons that give it its own personality, such as the Chapon yard, the bulwark, or the public laundry, with the strength of identity and belonging ties, the result of a hard shared experience, to undertake a whole series of strategies that allowed the deterioration and abandonment initiated with the naval crisis of the late 1970s to be left behind. From an identity of resistance [76], collectively and jointly built during the dictatorship, the residents of Canido have managed to rebuild it through a project based on elements of their collective culture. Its objective is to implement a neighborhood project through different initiatives that combine the complaint and visibility of problems with actions aimed at the sustainable improvement of the living conditions of the inhabitants, the recovery of heritage and public spaces both from the environmental point of view and also for living side by side, and encouraging new buildings that attract and settle new residents who collaborate on the path of demographic recovery and in the process of economic revitalization.

There are many initiatives that have been carried out, among which we can highlight the second-hand market [77]; the flowery orchards of Canido [78] (in which the environmental component is combined with the principles of land Art, the Mercalegre art market [79]); and fundamentally the street art festival, Meninas de Canido, in which cultural activity has become an important local development strategy. With this, the neighborhood has been reborn as a neighborhood that tourists visit again and, above all, that neighbors can enjoy again; a neighborhood in which new small businesses are emerging, in which children go out to play in the street and in which adults meet again in the bars of the stone cross square to have a drink; and a neighborhood in which the neighbors no longer leave, but new ones arrive [80]. The increase of the neighborhood in a city that loses an average of 800 inhabitants in the last year (770 in 2018) supports this collective project, which we will analyze in depth in the following sections.

\subsection{Las Meninas de Canido: the Art of the Museum on the Street}

Las Meninas de Canido is an artistic and cultural festival where image and sound take to the streets as a vindication of a living neighborhood in permanent transformation. Its origin dates back to 2008, when it arose as a complaint mechanism to the situation of deterioration in the Canido neighborhood in Ferrol, a neighborhood in which the population did not want to reside and where there was no business or investment.

The artist Eduardo Hermida was its promoter. According to Hermida, it hurt to see how the neighborhood he had grown up in was degrading. That is why, remembering his childhood when he painted the wall of one of the houses in his neighborhood with chalk, he decided to begin to capture art in its streets. For this, he chose one of his favorite painters, Velázquez, and among his works, he chose Las Meninas, because it is perhaps his most recognizable work.

His goal was twofold: on the one hand, with the color of the works, he wanted to give light to the facades - victims of abandonment, deterioration, or vandalism; and on the other, he sought to take art from museums to the street in order to offer free culture to people who had no means to visit a museum or go to a concert. This is how, in 2008, the colorful performance of the Meninas de Canido began. In that year, Eduardo Hermida invited a group of artists from diverse, new, and renowned trajectories to participate in a pictorial marathon that would last 12 hours. The idea was to represent the Meninas painting on the walls of degraded homes, as if they were a canvas. It was an activity 
buoyed by music and one in which the local residents played an active role by offering supplies to the participants. The municipality collaborated by donating the material for the realization of the paintings, and thanks to the successful day, the initiative was repeated in later years.

Over the years, the Meninas de Canido has become more popular both nationally and internationally, and its organizational model has changed.

- The city council wanted to show its support for the initiative by providing funds for the purchase of material, although its contribution is not enough.

- The number of participants increased. While Eduardo Hermida initially called well-known artists to join him, there are currently many artists requesting to participate in the event.

- Artistic techniques and activities (short films, poetry, dance, games for children, etc.) have been expanded.

- The modality of large murals has been introduced to hide the ugliness of the large medians in the urban landscape of the neighborhood. Among the stand-out murals are the contemporary interpretations of Las Meninas by artist Shfir, with the unmasked Menina (Figure 5), or the work of Lily Brik with a Menina tribute to the Infanta Margarita (Figure 5).

- If at the beginning they were only painted on the walls of abandoned homes, now it is the owners of the houses and the catering businesses that request that the Meninas be painted on their walls or streets. Likewise, homeowners who have been affected by rehabilitation processes that have occasionally deteriorated or destroyed the paintings on them, are requesting that their Meninas be restored.

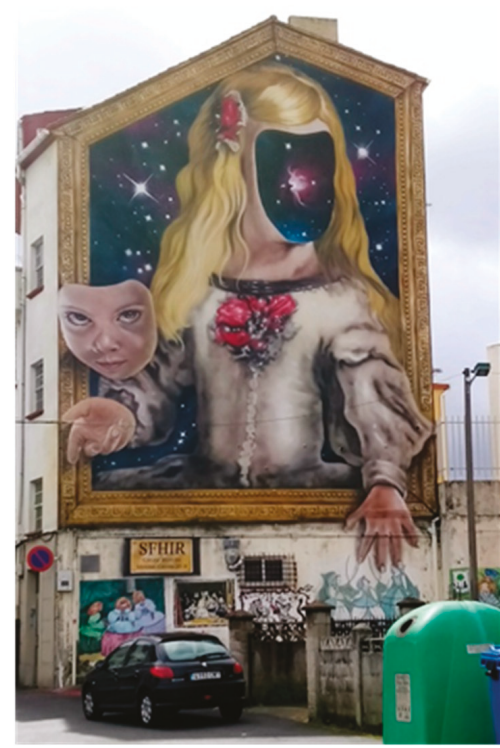

(a)

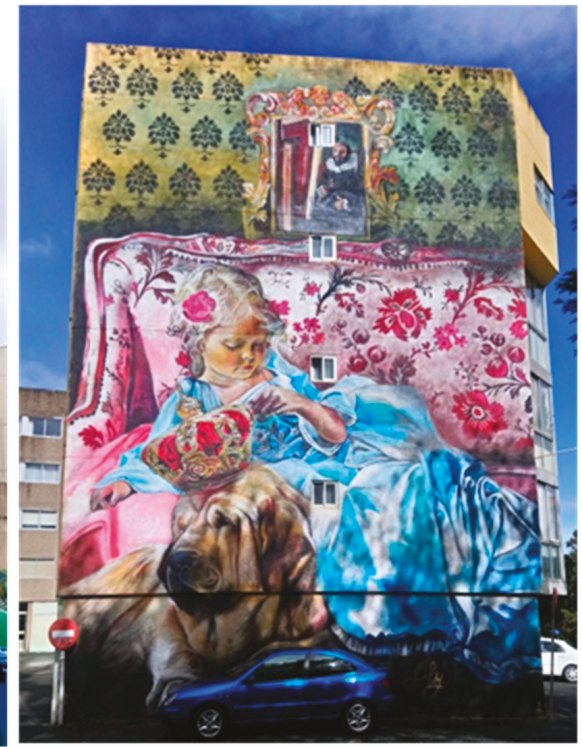

(b)

Figure 5. (a) Unmasked Menina Shfir; (b) Menina of Infanta Margarita Lily Brik.

The festival takes place in early September, and the 2018 edition welcomed more than 100,000 visitors. Since its inception, more than 1200 artists have participated in it, some from Syria, Slovenia, Poland, Brazil, or Taiwan, which gives the neighborhood a universality. Along its streets, there are more than 300 pictorial works that can be enjoyed (Figure 6). 

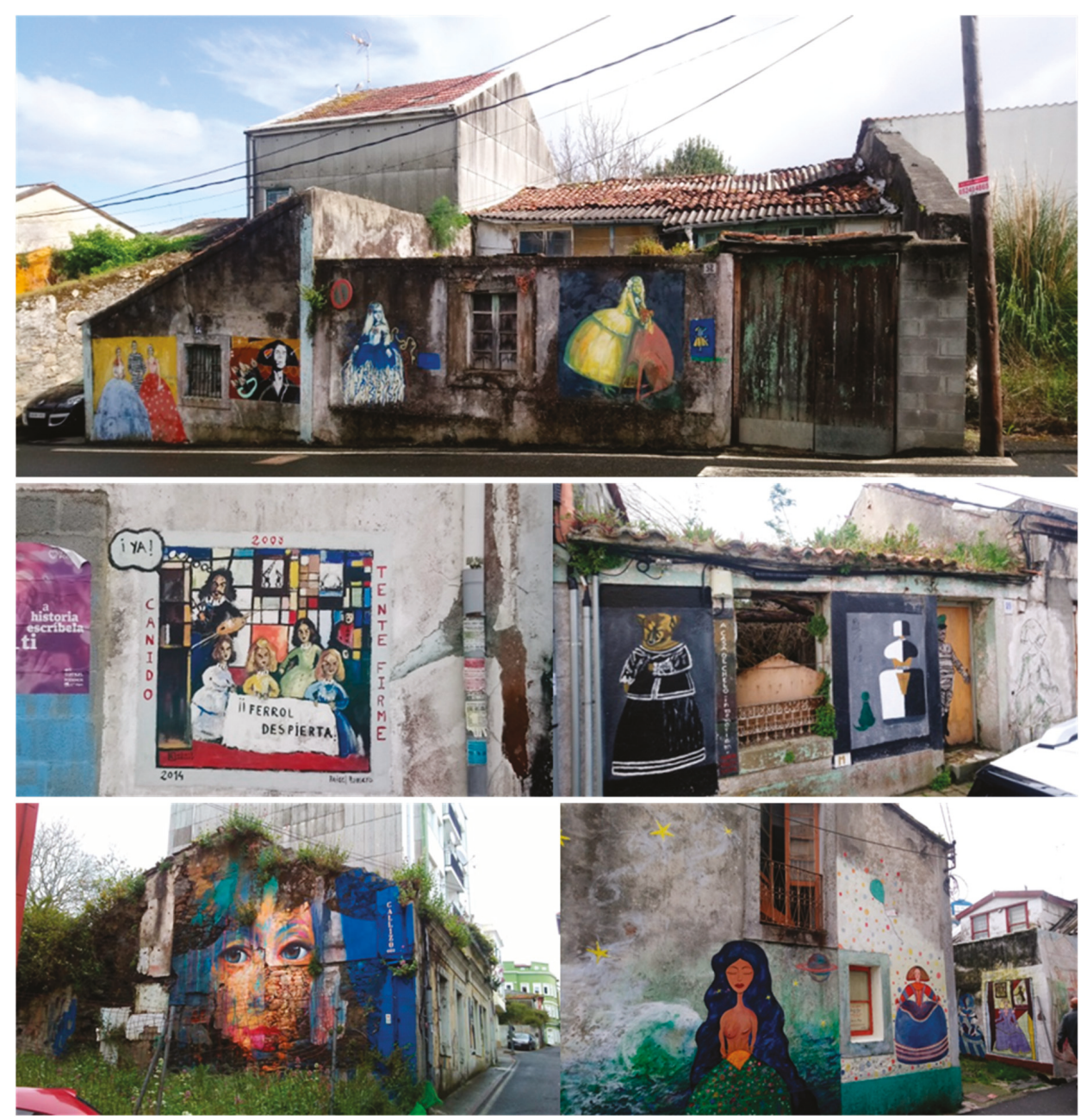

Figure 6. Meninas in the Canido neighborhood.

In addition, a mobile app known as VISUAR for IOS system and Visar Augmented Reality for Android mobiles have been created, through which information about the works can be obtained as well as some animations that can be seen by standing in front of them.

The festival has aroused interest in cities outside Spain, and Eduardo Hermida has given presentations in forums in Paris and Kiev. Other Spanish cities, such as Madrid, intend to develop a similar initiative in several districts, while other cities are also targeting chromatism. These include Cambre, with its Chromatic Mural Fest, or Carballo, with its initiative Breaking Down Walls with Paint (Figure 7). It has been shown that art has very positive effects, not only from the point of view of the physical improvement of neighborhoods but also of social valorization. 


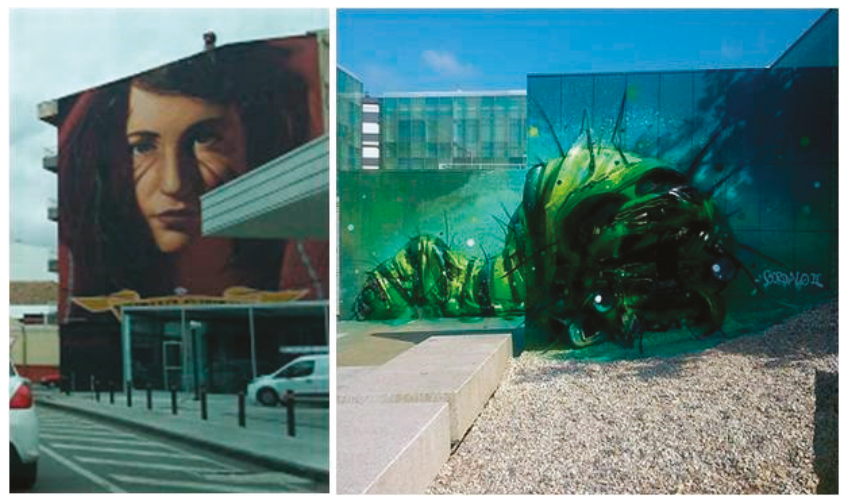

Figure 7. Murals in Carballo.

\subsection{The Impact of Meninas in the Neighborhood of Canido}

The interviews with the neighbors and entities of the neighborhood allow us to affirm that the cultural event of Las Meninas de Canido has managed to position the neighborhood on the map on an international scale. The neighborhood has regained its life and has become a fashionable space. As a consequence:

- The neighbors of Canido feel prouder than ever of their neighborhood and have reinforced a degree of identity with it. In the past, Canido was considered by the population of Ferrol as a degraded neighborhood, in which all the industries and amenities that "bothered people" (cemetery, factories, etc.) were historically located, where the working population was concentrated, and an whose residents were contrary to the regime of the dictator. No one wanted to live or say they lived in it.

- Canido has become a trendy neighborhood, which some people say resembles London's Soho. It is one of the most desired spaces in the city to live. It has become a different neighborhood: vital, enthusiastic, optimistic. It is a reconstructed neighborhood in which art itself is the identifying element and a tourist attraction. People want to live in the neighborhood, and this is how it has gone from having about 1300 inhabitants in 2008 to 7500 in 2018. The rise in land prices is another complement. While before you could buy a plot of land for less than $€ 20,000$, now, there is nothing below $€ 150,000$.

- It is currently an urban sector in which institutions are focusing their interest, understanding that the Meninas de Canido is an event that favors tourist activity, which was previously practically nonexistent in the city. Murals have become a focus of attraction for people who visit it (Figure 8). Everyday there is someone who walks the streets with a camera to take a picture of the murals. It is estimated that about 50,000 people visit the neighborhood every year in order to see Las Meninas.

- This momentum has also been supported by municipal urban renewal initiatives to provide it with more livable spaces: functional squares, benches, trees, or larger sidewalks are elements that improve livability. In some of them, Las Meninas are part of their urban furniture.

- Rehabilitation projects of some homes are being carried out in the key points of the neighborhood. Rehabilitations are mostly carried out by residents of the neighborhood with liberal professions and enough purchasing power to cover the cost of refurbishment and renovation. Without a doubt, it is the best way to keep residents and attract new ones.

- In the last five years, 27 businesses have opened. Hospitality and traditional commerce have noticed a revival in the neighborhood. The owners of traditional commerce affirm that this revitalization has allowed them to stay open and to have some stability. For its part, the hospitality sector acknowledges it has benefited and seen its bottom line improve, not only on the days when 
the Meninas are celebrated but also during the rest of the year, thanks to the activities being promoted since becoming a civic center of the neighborhood.

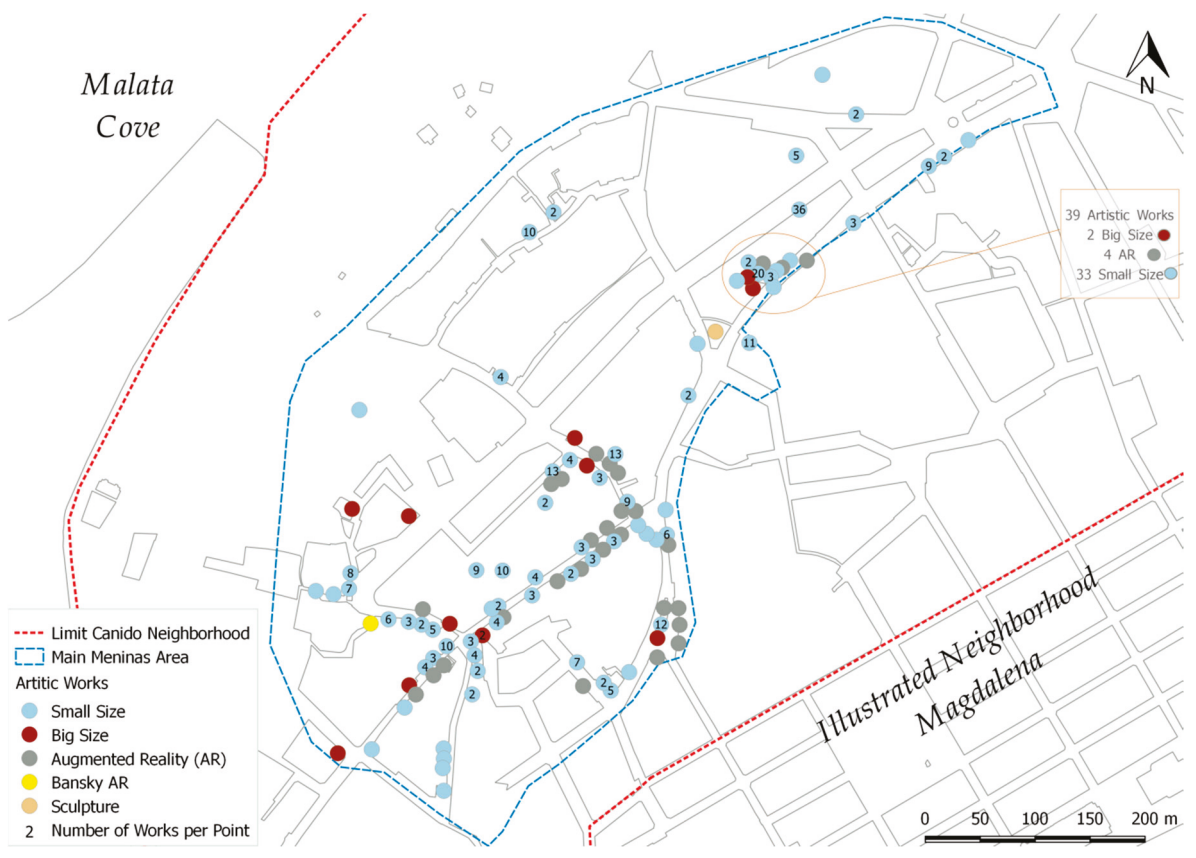

Figure 8. Distribution of the Meninas in Canido neighborhood.

Therefore, there is no doubt that Las Meninas have been a turning point whereby Canido is the fashionable neighborhood of Ferrol, and that both the city and the neighborhood have positioned themselves on the map at national and international levels. If Ferrol was the typical example of a shrinking city in permanent crisis as a result of the reconversion of the naval sector, the Meninas initiative has managed to transfer a new image of the city, more dynamic and with good practices in order to rehabilitate degraded spaces.

However, a part of the social and business fabric observes that the original idea has been progressively transformed, and that behind the Meninas event another reality is hidden that they want to show. They fear that the success of Las Meninas will be a screen that will leave other initiatives in the background that are not less important and launched by government institutions, neighborhood associations and the business community, as well as many other problems that remain unsolved.

First, they consider that, although Las Meninas began as a cultural initiative, a popular event, in which the neighbors collaborated with the artists and one in which the local hospitality industry played an active role organizing the meals, in recent years, it has become a more private event, with the emergence of a brand of beer as a sponsor, the commissioning of food services to companies outside the neighborhood and not to the bars in the area, and the hiring of personnel that do not belong to the neighborhood to attend said services. All this has meant a quantitative and qualitative change with respect to the original idea. Las Meninas have gone from being a neighborhood event, where the neighbors used to eat together in the street, with 400 people in the neighborhood sitting at a large table, to becoming an international event attended by more than 50,000 people in which the popular character has disappeared. Given this circumstance, and with the aim of recovering precisely that link with the neighborhood, the last edition decided to reduce the contribution of the sponsor and give more opportunities to the population and businesses of the neighborhood. 
On the other hand, if at the beginning the idea was for enthusiasts to paint Las Meninas on small walls or parts of houses, now, the tendency is for invited international artists to paint large murals on buildings. Obviously, these works are an element of attraction for visitors and tourists, who come to the neighborhood and have the opportunity to see real works of art in the street. However, the neighbors miss the freshness and initial spontaneity.

With regard to the neighborhood, it is true that Canido is now a pleasant and uncomplicated place to live. However, the Association of Neighbors state that this is not due to the Meninas movement so much but rather to an urban plan, of which little is said. It is a plan that modified the physiognomy of the neighborhood through the humanization of several streets (Alegre, A Rocha), the opening of new roads and the construction of a civic center. The interventions carried out in the Stone cross square turned this space (traditionally occupied by a small roundabout) into the epicenter of the neighborhood and a public space in which to socialize, taking away that functionality from Canido Square. In that same period, the plot occupied by the old Fenya factory was built upon. These homes were occupied by young families with medium-high purchasing power and with children, a circumstance that favored the socio-demographic rejuvenation of the neighborhood. On the other hand, there was an interest on the part of some neighbors to rehabilitate some homes in the neighborhood and stay there. In this sense, a study is being carried out on the existing properties in the neighborhood to analyze the possibility of rehabilitating them. More than 100 properties have been accounted for, all suffering from different levels of degradation. In any case, the recovery of some of them would be a significant improvement, and it would be a mechanism to improve the resident population in the neighborhood and to attract more residents with medium-high purchasing power. In this sense, it should be noted that gentrification processes have not yet been observed, as has happened in other Spanish or European cities, where cultural initiatives such as those of Las Meninas have been launched. At the moment, traditional rents remain stable, and there has been no replacement of the original population of the neighborhood.

There is a consensus on the part of retailers and hoteliers that the Meninas have managed to revitalize the neighborhood and generate a synergy of activism and participation by the business and associative fabric of the neighborhood, which was consolidated with the creation of Hoscompro, an association of merchants and hoteliers whose purpose was to energize life in the neighborhood. This association has always been collaborative with the association of neighbors of Canido, whose objective is to create a community project in the neighborhood, launching cultural activities, leisure and consolidating traditional festivals, which despite being less known than Las Meninas, provide equivalent income to the catering sector.

The neighborhood association seeks to generate and consolidate intra-neighborhood dynamics, collaborating with all the entities of the neighborhood (cultural, religious, sports) to seek a complicity that allows them to develop activities together and become a driver when requesting that institutions make greater investments in the neighborhood and improvement initiatives, both physically and socially. They insist that behind the festivity and the playful nature of Las Meninas lies a reality of sub-standard housing, streets affected by serious degradation, a high percentage of the population in a situation of endemic poverty, numerous elderly people living alone and without suitable services, while there is a deficit of spaces for young people who come to the neighborhood to play sports or games. Some successes have already been achieved, such as those derived from the "happy city" project, through which sports exhibitions, dance shows, musical performances, gastronomic routes, and even a curious slow bicycle race were held in different parts of the neighborhood.

What is definitively pursued by these processes of social construction of the urban space is to change the perception of the public about Canido. Thanks to Las Meninas, the urban imaginary of the neighborhood has been improved. The cultural action of the Girls has served as a loud protest against the situation of the crisis, as an element of tourist attraction that had not experienced before. However, we ask if the imaginary created on the barrio will not be surpassing the reality-hence, the insistence on the part of the association that we not see Canido as the new Soho, where everything is perfect and beautiful and all of the problems have been solved, but as a neighborhood where institutions need to 
continue investing to generate public spaces of coexistence, where people can play, dance, listen to music, or just chat with neighbors.

Eduardo Hermida, the promoter of Las Meninas, has the same opinion. He is aware that this event may continue for some time, but that it will have to come to an end. With the objective that the neighborhood continues to improve, from a physical, social, economic, and environmental point of view, several projects are already underway. Among them is the recovery of an agricultural green space, turning abandoned gardens into urban leisure spaces or environmental education spaces for schools in the area. For this they have already begun to plant fruit trees, turning this abandoned space into a meeting place for neighbors of all ages. Another collective artistic initiative consists of recovering the environment of the missing Hispania pencil factory by installing 40 wooden pencil-shaped posts, each with five faces and a height of $2.85 \mathrm{~m}$. This initiative aims to spread the experience of Las Meninas in the historic center of Ferrol, an area also affected by serious processes of urban deterioration; if successful, it could be used as a link between the two neighborhoods.

\section{Conclusions}

Street art can contribute to the physical sustainability of the degraded urban sector, one of the essential dimensions of the urban sustainability paradigm - together with economic, social, ecological, and political sustainability [81]. It has proven to be an effective alternative to recover urban spaces affected by abandonment, loss of population and identity, improving those spaces from a physical, social, and economic point of view and turning them into places of opportunity. Local administrations have found in it a tool for urban regeneration, which does not involve large investments and which, in return, brings great benefits, since they are spaces that increasingly attract more tourists and position their cities internationally. However, by way of conclusion, we must reflect on the following issues:

- How to manage/control the processes of gentrification, elitization, and tourism that can be registered in neighborhoods where street art is successful in order to preserve the harmony of the neighborhood and avoid as far as possible the expulsion of its residents.

- What situations are conducive to the success of street art, and what is the degree of dependence on this activity. Could there be a reversal of the dynamic process that these neighborhoods undergo if these artistic competitions end? Will the neighborhood still have the same social and economic strength?

- If we start from the fact that street art contests arise in most cases at the initiative of some artists or groups of citizens, with the support of the administrations, the future of the neighborhood should be designed together between all parties, promoting complementary actions that strengthen the quality of life of residents in the neighborhood and consolidate their economic and social revitalization in the medium term.

In the case of Canido, the Las Meninas movement has managed to save the neighborhood from a slow death and put it in the spotlight of international artists and tourists from around the world who visit the festival. However, the limit of availability of spaces in which to make the paintings is being reached, and although other artistic initiatives that try to have the same success in other nearby urban sectors have already been launched, it may not be the same Success achieved with Las Meninas. We hope that all the agents involved—neighbors, associations, and local administration-are able to jointly design a program of integral intervention in the neighborhood that guarantees its dynamism, quality of life, and urban sustainability.

Author Contributions: F.R.D.V., M.J.P.M., and R.L.R. contributed to the conception and design, data analysis, and manuscript preparation. M.J.P.M. and R.L.R. contributed to the interviews. F.R.D.V. and M.J.P.M. contributed to proofreading and editing. R.L.R. contributed to mapping. F.R.D.V., M.J.P.M., and R.L.R. contributed to the interpretation of the results. All authors have read and agreed to the published version of the manuscript.

Funding: This research was funded by the Spanish Ministry of Economy and Competitiveness (MINECO) New Models for Governing Cities and Intervention in Urban Spaces in the Post-Crisis Period (CSO2016-75236-C2-1-R). 
Conflicts of Interest: The authors declare no conflict of interest.

\section{References}

1. Eizaguirre, S.; Pradel, M.; Terrones, A.; Martinez-Celorrio, X.; García, M. Multilevel governance and social cohesion: Bringing back conflict in citizenship practices. Urban Stud. 2012, 49, 1999-2016. [CrossRef]

2. García-Cabeza, M.S. State-led real estate boosterism and the breakdown of the Spanish urban model. In Debating the Neo-Liberal City, 1st ed.; Pinson, G., Morel-Journel, C., Eds.; Routledge: London, UK, 2017; pp. 174-187.

3. Del Romero-Renau, L.; Valera-Lozano, A. From NIMBYsm to the 15M: A decade of urban conflicts in Barcelona and Valencia. Territ. Politics Gov. 2016, 4, 375-395. [CrossRef]

4. Koutrolikou, P. Governmentalities of urban crises in inner-city Athens, Greece. Antipode 2016, 48, 172-192. [CrossRef]

5. Vale, M. Economic crisis and the Southern European regions: Towards alternative territorial development policies. In Identity and Territorial Character. Re-Interpreting Local-Spatial Development, 1st ed.; Salom, J., Farinós, J., Eds.; Universidad de Valencia: Valencia, Spain, 2014; pp. 37-48.

6. Fernández Maíllo, G. VIII Informe Sobre Exclusión y Desarrollo Social en España; Fundación Foessa: Madrid, Spain, 2019.

7. Mínguez, C.; Piñeira, M.J.; Fernández-Tabales, A. Social Vulnerability and Touristification of Historic Centers. Sustainability 2019, 11, 4478. [CrossRef]

8. Méndez, R.; Abad, L.D.; Echaves, C. Atlas de la Crisis. Impactos Socioeconómicos y Territorios Vulnerables en España; Tirant lo Blanch: Valencia, Spain, 2015.

9. Piñeira, M.J.; Trillo, J.M. The effect of the crisis on vulnerable neighbourhoods: An approximation of the Spanish context through a case study (A Coruna, Galicia). In Crisis, Globalization and Social and Regional Imbalances in Spain; Spanish Commitee IGU: Madrid, Spain, 2016; pp. 136-148.

10. Piñeira, M.J.; Durán, F.R.; Taboada, J. Urban Vulnerability in Spanish Medium-Sized Cities during the Post-Crisis Period (2009-2016). The Cases of a Coruña and Vigo (Spain). Urban Sci. 2018, 2, 37. [CrossRef]

11. Alguacil, J.; Camacho, J.; Hernández, A. La vulnerabilidad urbana en España. Identificación y evolución de los barrios vulnerables. Empiria. Rev. Metodol. Cienc. Soc. 2014, 27, 73-94. [CrossRef]

12. Nel.lo, O. Transform the City with Citizenship. Criteria and Reflections for the Barrio. Plan of Barcelona; Ayuntamiento de Barcelona: Barcelona, Spain, 2017.

13. Hernández, A.; Matesanz, A.; García, C.; Alguacil, J.; Camacho, J.; Fernández, C. Atlas de Barrios Vulnerables de España: 12 Ciudades 1991/2001/2006; Instituto Juan de Herrera: Madrid, Spain, 2015.

14. Wehrhahn, R. Contentious urban housing politics in European metropolises between financialisation, dispossession and re-possession: Jahrbuch StadtRegion. In Housing and Housing Politics in European Metropolises; Wehrhahn, R., Pohlan, J., Hannemann, C., Othengrafen, F., Schmidt-Lauber, B., Eds.; Springer: Wiesbaden, Germany, 2019; pp. 3-20.

15. Koutrolikou, P. Changing landscapes of urban citizenship: Southern Europe in times of crisis. Citizsh. Stud. 2017, 21, 379-392.

16. Heernan, E.; McHale, J.; Moore-Cherry, N. Debating Austerity in Ireland: Crisis, Experience and Recovery; Royal Irish Academy: Dublin, Ireland, 2017.

17. Janoschka, M. Who Loses and Who Wins in a Housing Crisis? Lessons from Spain and Greece for a Nuanced Understanding of Dispossession. Hous. Policy Debate 2017, 28, 117-134.

18. Blanco, A.; Blázquez, M.; Canoves, G. Barcelona, Housing Rent Bubble in a Tourist City. Social Responses and Local Policies. Sustainability 2018, 10, 2043. [CrossRef]

19. Blanco, A.; Subirats, J. Políticas urbanas en España: Dinámicas de transformación y retos ante la crisis. Geopolitica(s) 2012, 3, 15-33.

20. Pradel Miquel, M.; García Cabeza, M. El Momento de la Ciudadanía: Innovación Social y Gobernanza Urbana; Catarata: Barcelona, Spain, 2018.

21. Burriel de Orueta, E. La década prodigiosa del urbanismo español (1997-2006). Scr. Nova Rev. Electrón. Geogr. Cienc. Soc. 2008, 12, 64. 
22. Lois González, R.C.; Piñeira Mantiñán, M.J. Urban development processes in Spain-From consolidated cities to urban regions with an over densified housing market. In Contemporary Problems of Urban and Regional Development; Mierzejewsa, L., Wdowicka, M., Eds.; Bogucki: Poznan, Poland, 2011; pp. 61-71.

23. Rullan Salamanca, O. Urbanismo expansivo en el Estado Español: De la utopía a la realidad. In Geografía y Desafíos Territoriales en el Siglo XXI.; Gozálvez, V., Marco, J.A., Eds.; Asociación de Geógrafos Españoles \& Universidad de Alicante: Alicante, Spain, 2012; pp. 165-209.

24. De Gregorio Hurtado, S. El desarollo de las iniciativas comunitarias Urban y Urban II en las periferias degradadas de las ciudades españolas. Una contribución a la práctica de la regeneración urbana en España. Ciudades 2010, 13, 39-59.

25. Ministry of Development. URBACT Program. Available online: www.rediniciativasurbanas.es/convocatoriade-ayudas/urbact (accessed on 3 January 2020).

26. Ministry of Development. Integrated and Sustainable Urban Development Strategy. Available online: www.rediniciativasurbanas.es/convocatoria-de-ayudas/estrategias-dusi (accessed on 3 January 2020).

27. Casagrande, M. Urban Acupuncture. Available online: http://thirdgenerationcity.pbworks.com/f/urban \% 20acupuncture.pdf (accessed on 3 January 2020).

28. Alcalá Agulló, F.; Jménez Sánchez, F. Los Costes Económicos del Déficit de Calidad Institucional y la Corrupción en ESPAÑA; Fundación BBVA: Bilbao, Spain, 2018.

29. Center for Sociological Research. Three Main Problems That Currently Exist in Spain (Multi-Response\%) (2007-2019). Available online: www.cis.es/cis/export/sites/default/-Archivos/Indicadores/documentos_html/ TresProblemas.html (accessed on 3 January 2020).

30. Harvey, D. Ciudades Rebeldes. del Derecho de la Ciudad a la Revolución Urbana; Akal: Madrid, Spain, 2012. [CrossRef]

31. Ekers, M.; Hamel, P.; Keil, R. Governing suburbia: Modalities and mechanisms of suburban governance. Reg. Stud. 2012, 46, 405-422. [CrossRef]

32. McLeod, G. Urban politics reconsidered: Growth machine to post-democratic city. Urban Stud. 2011, 48, 2629-2660. [CrossRef]

33. Romero, J.; Farinós, J. Redescubriendo la gobernanza más allá del buen gobierno. Democracia como base, desarrollo territorial como resultado. Boletín Asoc. Geógrafos Españoles 2011, 56, 295-319.

34. Swyngedouw, E. Governance innovation and the citizen: The Janus face of governance-beyond-the-state. Urban Stud. 2005, 42, 1991-2006. [CrossRef]

35. Peck, J.; Theodore, N.; Brenner, N. Neoliberalism resurgent? Market rule after the great recession. South Atl. Q. 2012, 111, 265-288. [CrossRef]

36. Storper, M. Keys to the City. How Economics, Institutions, Social Interaction and Politics Shape Development; Princeton University Press: Oxford, UK, 2013.

37. Lois, R.; Macía, C.; Piñeira, M.J.; Calvo, A. Smart cities and regions in Europe. In Life in a Changing Urban Landscape; Kotze, N., Donaldson, R., Visser, G., Eds.; University of Johannesburg: Johannesburg, South Africa, 2014; pp. 27-36.

38. European Commission. Local and Regional Partners Contributing to Europe 2020: Multi-Level Governance in Support of Europe 2020; European Commission: Brussels, Belgium, 2015.

39. Hatfield, S.; Nelson, R.; Cook, D. Adaptive Governance: An Introduction and Implications for Public Policy; Australian Agricultural and Resource Economics Society: Queenstown, Ireland, 2007.

40. Howlett, M.; Ramesh, M. Three orders of governance failure: Policy capacity, problem context and design mismatches. In Proceedings of the 23rd World Congress (Challenges of Contemporary Governance), Montreal, QC, Canada, 20-25 July 2014; IPSA: Montreal, QC, Canada, 2014.

41. Steurer, R. Disentangling governance: A synoptic view of regulation by government, business and civil society. Policy Sci. 2013, 46, 387-410. [CrossRef]

42. Cabreirizo, C.; Klett, A.; García, P. De alianzas anómalas a nuevos paisajes políticos. Madrid, Lavapiés y otras geografías de lo común. Urbs. Rev. Estud. Urbanos Cienc. Soc. 2014, 5, 163-178.

43. Allmendiger, P.; Haughton, G. Spatial planning, devolution, and new planning spaces. Environ. Plan. Gov. Policy 2010, 28, 803-818. [CrossRef]

44. Méndez, R. Crisis económica, vulnerabilidad urbana y desempleo en España. Ciudad Territ. Estud. Territ. 2013, 178, 649-667. 
45. Stocker, G.; Wilson, D. British Local Government: An Assessment of Labour's Reform Programme; Palgrave Macmillan: Basingstoke, UK, 2004.

46. Harvey, D. De la gestión al empresarialismo: La transformación de la gobernanza urbana en el capitalismo tardío. In Espacios de Capital, Hacia una Geografía Crítica; Harvey, D., Piña, C., Eds.; Akal: Madrid, Spain, 2007.

47. Lois, R.; Piñeira, M.J.; Vives, S. The urban bubble process in Spain: An interpretation from the theory of circuits of capital. J. Urban Reg. Anal. 2016, 8, 5-20.

48. Lois, R.C.; Piñeira, M.J. The revival of urban social and neighbourhood movements in Spain: A geographical characterization. DIE ERDE 2015, 146, 127-138.

49. Delgado Jiménez, A. De la participación ciudadana a la gobernanza urbana: Transformaciones políticas y territoriales. Ciudad. Para Futuro Más Sosten. Boletín Cf+S 2009, 44, 67-78.

50. Newman, J. Remaking Governance: Peoples, Politics and the Public Sphere; Policy Press: Bristol, UK, 2005.

51. Durán Villa, F.R.; Piñeira Mantiñán, M.J. Evictions and the social crisis in Spanish cities. In Urban Challenges in a Complex World: Resilience, Governance and Changing Urban; Moore-Cherry, N., Ed.; University College Dublin \& IGU: Dublin, Ireland, 2015; pp. 80-85.

52. Valiño, V. Emergencia Habitacional en el Estado Español: La Crisis de las Ejecuciones Hipotecarias y Los Desalojos Desde una Perspectiva de Derechos Humanos; Observatorio OESC, PAH: Barcelona, Spain, 2013.

53. Moulaert, F.; Martinelli, F.; Swyngedouw, E.; Gonzalez, S. Towards alternative model(s) of local innovation. Urban Stud. 2005, 42, 1969-1990. [CrossRef]

54. Murray, R.; Mulgan, G.; Caulier, J. How to Innovate: The Tools for Social Innovation; The Young Foundation and Nesta: London, UK, 2011.

55. Blanco, I.; Cruz, H.; Martínez, R.; Parès, M. El papel de la innovación social frente a la crisis. Ciudad Territ. 2016, 48, 49-260.

56. Sacco, P.L.; Ferilli, G. Il Distretto Culturale Evoluto Nell'economia Post Industriale; DADI, Università IUAV: Venezia, Italy, 2006.

57. Arte Urbano. Available online: www.significados.com/arte-urbano/ (accessed on 3 December 2019).

58. Ballaz, X. El graffiti como herramienta social. Una mirada psicosocial a las potencialidades críticas del arte urbano. In Violencia y Salud Mental Salud Mental y Violencias Institucional, Estructural, Social y Colectiva; Markez Alonso, I., Fernández Liria, A., Perez-Sales, P., Eds.; A.E.N Estudios: Madrid, Spain, 2009; pp. 131-144.

59. Allende, S. Street Art. Catálogo de la Exposición Arte Urbano de la Calle al Museo; Consejería de Cultura y Portavocía; Comunidad Autónoma de la Región de Murcia: Murcia, Spain, 2017.

60. Visconti, L.M.; Sherry, J.F.; Borghini, S.; Anderson, L. Street art, sweet art? Reclaiming the "Public" in public place. J. Consum. Res. 2010, 37, 511-529. [CrossRef]

61. Loeffler, S. City as Skin: Urban Imaginaries of Flesh and Fantasy. Archit. Des. 2013, 83, 114-119. [CrossRef]

62. Molina, G.; Guinard, P. Arts in Cities-Cities in Arts. Artic. J. Urban Res. 2017, 15.

63. Baudrillard, J. Le Système des Objets; Éditions Gallimard: Paris, France, 1968.

64. Tornaghi, C. Questioning the Social Aims of Public Art in Urban Regeneration Initiatives. The Case of Newcastle upon Tyne and Gateshead (UK); University of Milano-Bicocca: Milan, Italy, 2008.

65. Gómez Aguilera, F. Arte, Ciudadanía y Espacio Público. W@Terfront 2004, 5, 36-51.

66. The Bushwick Collective. Available online: https://artsandculture.google.com/exhibit/gRPwr3J5 (accessed on 5 November 2019).

67. Wynwood Walls. Available online: www.thewynwoodwalls.com/overview (accessed on 5 November 2019).

68. Ramil, E.; Llorca, G.; Rodríguez, J.A. Historia de Ferrol; Via Lactea: La Coruña, Spain, 1998.

69. Pérez, S. Gabriel Vázquez Seijas. Memoria de Vida. Salamanca; Gráficas Lope: Salamanca, Spain, 2001; pp. 393-414.

70. Kapstein, P. Vulnerabilidad y Periferia Interior. Cuad. Investig. Urban. 2010, 71, 7-114.

71. Madoz, P. Galicia. Diccionario Madoz; Breogán: Santiago de Compostela, Spain, 1986; Volume 3, pp. 537-538.

72. Montero, J. Historia y Descripción de El Ferrol; Gersan: Puentedeume, Spain, 1986; pp. 203-204.

73. Abeconsa Prepares to Launch Its Great Promotion in Canido. Available online: www.lavozdegalicia.es/noticia/ ferrol/2019/09/14/abeconsa-prepara-lanzar-gran-promocion-canido/0003_201909F14C2992.htm (accessed on 5 November 2019).

74. Galician Institute of Statistics. Available online: www.ige.eu/estatico/estat.jsp?ruta=html/gl/ PanoramaConcellos/02_Poboacion.html (accessed on 5 November 2019). 
75. Canido Will Receive the Award on Wednesday for Being Considered the Best Neighborhood in Galicia. Available online: www.diariodeferrol.com/articulo/ferrol/canido-recibira-miercoles-galardonser-considerado-mejor-barrio-galicia/20190610000218258368.html (accessed on 5 November 2019).

76. Castells, M. Globalización e Identidad. Quad. Mediterr. 2010, 14, 254-262.

77. The Neighborhood of Canido Is Once Again Pulled with Its Market. Available online: www.lavozdegalicia. es/noticia/ferrol/ferrol/2019/09/10/barrio-canido-vuelve-tener-tiron-mercado/0003_201909F10C12991.htm (accessed on 5 November 2019).

78. A Market That Took the Urban Garden to Canido. Available online: www.lavozdegalicia.es/noticia/ferrol/ 2013/09/21/mercado-llevo-huerta-canido/00031379775449214304300.htm (accessed on 5 November 2019).

79. The Art of Mercalegre Was Completed in This Edition with Music and Theater. Available online: www.diariodeferrol.com/articulo/ferrol/arte-mercalegre-completo-edicion-musica-teatro/ 20180708224309230793.html (accessed on 5 November 2019).

80. The Route of the Canido Meninas in Ferrol (with Banksy?). Available online: https://saltaconmigo.com/blog/ 2017/09/ruta-de-las-meninas-canido-ferrol-banksy/ (accessed on 5 November 2019).

81. Forte, F.; De Paola, P. How Can Street Art Have Economic Value? Sustainability 2019, 11, 580. [CrossRef]

(C) 2020 by the authors. Licensee MDPI, Basel, Switzerland. This article is an open access article distributed under the terms and conditions of the Creative Commons Attribution (CC BY) license (http://creativecommons.org/licenses/by/4.0/). 


\title{
Protection of Cultural Heritage Buildings and Artistic Assets from Seismic Hazard: A Hierarchical Approach
}

\author{
Chiara D'Alpaos ${ }^{1, *}$ and Maria Rosa Valluzzi ${ }^{2}$ \\ 1 Department of Civil, Environmental and Architectural Engineering, University of Padova, \\ 35131 Padova PD, Italy \\ 2 Department of Cultural Heritage, University of Padova, 35139 Padova PD, Italy; mariarosa.valluzzi@unipd.it \\ * Correspondence: chiara.dalpaos@unipd.it; Tel.: +39-049-8276717
}

Received: 15 December 2019; Accepted: 12 February 2020; Published: 21 February 2020

\begin{abstract}
The occurrence of natural disasters such as earthquakes represent a worldwide challenge in the conservation of cultural heritage $(\mathrm{CH})$, which suffer from damage due to high vulnerability conditions. Therefore, the protection of $\mathrm{CH}$ from seismic hazard is of paramount importance. Damage and vulnerability assessment of $\mathrm{CH}$ and artistic assets play a key role in the identification of conservation strategies. Effective strategies require the stabilization of severely damaged buildings and the preventive improvement of constructions structural response to seismic actions. Although the operation of emergency inspections is meant to classify buildings on the basis of buildings residual seismic capacity, investment decisions in restoration and conservation strategies of such vulnerable structures must take into consideration tangible and intangible values of both building structures and artistic goods as well as must combine objectives of verifying structural safety standards and preserving cultural heritage significance. Damage and vulnerability assessment depend on different criteria, which, on the one hand, are related to buildings structural characteristics, materials, and geometrical properties. On the other hand, to the peculiarities and uniqueness of artworks and artistic goods present on structural elements. In this paper, an AHP (absolute) model is proposed to rank multi-criteria prioritization of protection and restoration interventions on a set of 15 churches, which were damaged by earthquakes, occurring in Italy in the last decades. In detail, in order to structure the decision problem, identify key factors, and define the hierarchy, we conducted an extensive literature review and interviewed a pool of experts. Focus groups were organized to develop the set of criteria and sub-criteria and validate the hierarchy by dynamic discussion.
\end{abstract}

Keywords: cultural heritage; cultural heritage; artistic assets; multicriteria decision aid; AHP; seismic hazard

\section{Introduction}

The occurrence of natural disasters such as earthquakes represent a worldwide challenge in the conservation of cultural heritage $(\mathrm{CH})$, which suffer from damage due to high vulnerability conditions. Historical city centers are threatened by events referring to natural and anthropic causes, the occurrence of which can lead to important losses in terms of cultural and artistic goods. In such a context, historical masonry buildings constitute the most extended stock of $\mathrm{CH}$, including both the architectural structures and their contained artworks. Masonry buildings are particularly vulnerable to earthquakes as they often present weaknesses due to construction aspects (low quality materials, irregular arrangements, inaccurate architectural details, etc.) and lack of conservation [1,2]. This context also highly jeopardizes the preservation of artistic assets, both movable and unmovable, whose risk is connected not only to intrinsic or typological issues, but also to the behavior of the structural system. The seismic behavior of historical masonry buildings is evaluated by the macro-element approach, i.e., the structural system is considered composed of independent portions whose limits are identified among either crack patterns 
or construction/vulnerability defects (e.g., large voids, lack in connections, etc.) [3,4]. Such an approach is particularly effective for churches as macro-elements can also coincide with the architectural portions of the building (apse, façade, dome, etc.), whose brittle behavior has been observed even under earthquakes of moderate magnitude [5,6].

Hence, to face the seismic post-emergency phase [7] a comprehensive methodology able to contribute in managing the protection of both artistic assets and structural issues is needed. Damage and vulnerability assessment of $\mathrm{CH}$ and artistic assets plays a key role in the identification of effective conservation strategies, which require the stabilization of severely damaged buildings and the preventive improvement of constructions structural response to seismic actions. Investment decisions in restoration and conservation strategies of such vulnerable structures must take into consideration the tangible and intangible values of both building structures and artistic goods. It must also combine objectives of verifying structural safety standards and preserving $\mathrm{CH}$ significance. Protection of $\mathrm{CH}$ involves high investment costs, which usually exceeds available financial resources due to stringent budget constraints. It is therefore necessary to prioritize interventions [8-11]. In this context, in which decision-making involves high stakes and stochastic future implications, multicriteria approaches provide formal decision-making techniques to assess a finite set of criteria, evaluate alternatives on the basis of each criterion, and aggregating these evaluations to rank alternatives with respect to a specific objective (e.g., priority of intervention). Among the variety of multicriteria methods provided in literature, the Analytic Hierarchy Process (AHP) proposed by Saaty in the Eighties [12], is one of the most widely used across multiple domain worldwide (e.g., business, social studies, environmental studies, R\&D, etc.) in taking complex decisions in real-world situations. Due to its ease of use and understanding, it facilitates structuring the complexity, measurement, and synthesis of rankings [13]. Nonetheless, there are few contributions in literature, to our knowledge, on the implementation of the AHP to the valuation of $\mathrm{CH}$ and historic buildings preservation [14-17].

In this paper, a novel application of the AHP in the domain of $\mathrm{CH}$ vulnerability assessment is proposed. In detail, we develop and implement an AHP (absolute) model to rank multi-criteria prioritization of protection and restoration interventions on $\mathrm{CH}$, i.e., churches, damaged by earthquakes. Based on literature review and experts' judgements, tangible valuation criteria, sub-criteria, and ratings were identified, and weights were determined by pairwise comparisons of elements with tangible properties to create a one-dimensional index for representing the overall assessment of alternatives, and rank them from most to least vulnerable. Then the model was validated on a set of 15 churches, which were damaged by earthquakes, occurring in Italy in the last decades. These buildings present damage and vulnerability aspects related to structural components and their supported artistic assets, whose data (surveyed on site visual inspections have been collected in a new web archive called DataBAES [18]. Such a tool provides two levels of inspections: the former is limited to damage survey of the artistic asset, such as structural element pairs detected in a building, whereas the latter includes also vulnerability issues of the pairs in questions. Level I and level II survey forms are provided (see supplementary materials in [18] to support the onsite inspections. Therefore, level I corresponds to emergency phases (i.e., when more expeditious survey is in need), whereas level II can be applied in any other phase to deeply clarify the relationships between present vulnerabilities and potential further damage, thus providing useful information for the possible prioritization and identification of interventions.

The remainder of the paper is organized as follows. Section 2 describes materials and method, Section 3 provides the model and presents its implementation on a real-world situation where 15 Italian churches which suffered from damage caused by recent earthquakes in Italy are ranked according to priority of intervention to mitigate seismic hazard, Section 4 discusses results, and finally, Section 5 concludes. 


\section{Materials and Method}

\subsection{Materials}

A set of 15 churches were selected among the case studies included in the DataBAES web archive $[18,19]$ to validate the proposed AHP model and consequently implement it in the wider context of preservation of historical structures and their integral unmovable artworks (e.g., frescoes and mural paintings, stuccoes, and mosaics). Most of Italian $\mathrm{CH}$ assets, specifically churches, were struck by earthquakes along their history and their main characteristics, and in terms of both construction details and possible occurred damage, are taken into consideration in the modeling. Vulnerability is also taken into account (e.g., irregular arrangements, large openings, too slender piers, lack of connections, thrusting arch/roof, etc.), as well as the presence of possible earthquake-proof devices (e.g., ties, confining rings at floor/roof level, etc.). Table 1 lists the buildings examined in the study.

Table 1. Churches under investigation in this study.

\begin{tabular}{llc}
\hline \multicolumn{1}{c}{ Church } & Localization (Province) & Reference Earthquake \\
\hline Chiesa di Sant'Antonio & San Polito Ultra (AV) & 1980 \\
Complesso di Santa Maria ai Monti & Tricarico (MT) & 1980 \\
Chiesa dell'Annunziata & Laurino (SA) & 1980 \\
Chiesa di Santa Maria delle Grazie & Cassano Irpino (AV) & 1980 \\
Complesso della Madonna del Carmine & Tricarico (MT) & 1980 \\
Chiesa di San Michele Arcangelo & Saviano (NA) & 1980 \\
Cappella degli Scrovegni & Padova & - \\
Chiesa di San Marco & L'Aquila & 2009 \\
Chiesa di San Silvestro & L'Aquila & 2009 \\
Chiesa del Santo Rosario & Finale Emilia (MO) & 2012 \\
Chiesa di S. Egidio & Cavezzo (MO) & 2012 \\
Chiesa dei Santi Senesio e Teopompo & Medolla (MO) & 2012 \\
Chiesa di San Luca Evangelista & Medolla (MO) & 2012 \\
Chiesa dell'Immacolata Concezione & Crevalcore (BO) & 2012 \\
\hline
\end{tabular}

According to the macro-element approach, the typical portions whose behavior can be considered homogeneous under seismic actions have been identified. The recognition phase relied on the onsite application of the Italian survey form for churches, which includes 28 possible mechanisms [20] related to either out-of-plane or in-plane damage patterns, as well as to specific issues involving the main architectural components of the building (façade, triumphal arch, dome, transepts and naves, vaults, etc.) (Figure 1).
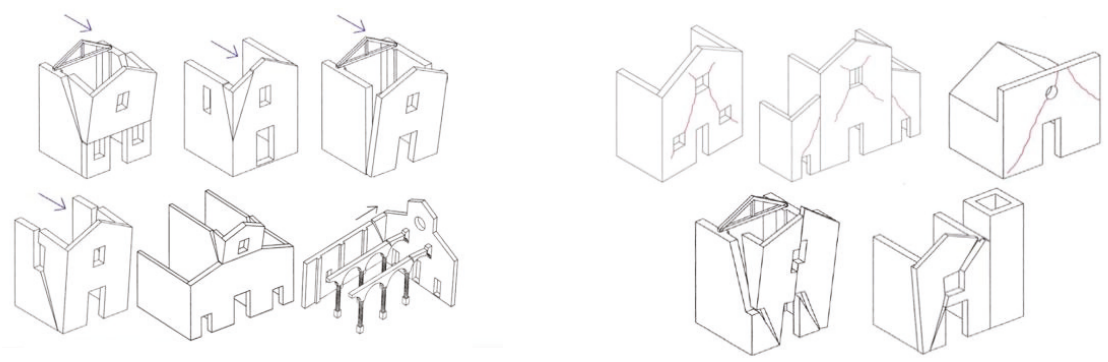

Figure 1. Example of out-of-plane (left) and in-plane (right) collapse mechanism of church façade [20].

Levels of damage are compared on a 1-to-5 scale, according to the European Macro Seismic Scale grading [21]. 
For the artistic assets, i.e., frescoes/mural paintings, stuccoes, and mosaics, the main aspects affecting damage were identified according to the Italian CNR-ICR recommendations [22]. As for vulnerability, no references are available as the study was carried out based on the comprehensive evaluation process provided by the reference survey forms of DataBAES [18]. They refer to both construction (composition, realization technique, etc.) and installation (application, position, intermediate support, etc.), as well as intrinsic variables (possible original defects).

\subsection{Method: The Analytic Hierarchy Process}

The Analytic Hierarchy Process (AHP), introduced by Saaty in the 1980s [12], is a general theory of measurement to obtain ratio scales from pairwise comparisons [23]. It is widely known in literature as a well-established multi-criteria approach and is applied by both academicians and practitioners in different context to systematize a wide range of decision problems. It proved to be useful when there is little quantitative information on the effects of the actions to be evaluated [24-28].

It grounds on the two basic principles that experience and people knowledge are as valuable as data in order to make a decision $[12,25,29]$ and that measurements can be taken from actual measurement or from a fundamental scale, which reflects the relative strengths/importance of preferences. In this respect, the AHP allows for the measurement of tangible and/or intangible criteria and factors and allows for evaluating quantitative and qualitative criteria and alternatives on the same preference scale.

The AHP is based on the assumption that the decision-maker is always able to express a preference and judge the relative importance of (or preference for) the evaluation parameters [30-32]. In other words, it orders a finite number of actions $\mathrm{Ai}[12,33,34]$, by evaluating them with respect to a finite number $\mathrm{k}$ of attributes aj $(\mathrm{j}=1, \ldots, \mathrm{k})$, each of which is assigned a judgment score qualifying its performance.

The individuals' ability to acquire and use information is used in the AHP process to determine relative magnitudes and importance through pairwise comparisons, which allows for constructing ratio scales on tangible and intangible factors and dimensions [35]. The AHP deconstructs the initial problem into several levels, constructing a hierarchy, which is an ordered set with unidirectional hierarchical relationships between different levels. The main goal of the decision problem represents the top of the hierarchy, whereas criteria and sub-criteria that contribute to the goal are positioned at lower levels. The bottom level is constituted by alternatives/actions to be evaluated. Then, a series of partial sub-decision problems are defined by structuring the problem through successive decomposition stages. These problems are smaller, less complex, and are easier to solve, since formulating a preference judgment is simpler when dealing with a limited number of decision criteria and expressing the individuals' opinions on two elements rather on all elements simultaneously. Consequently, relations within the hierarchical structure and elements relative importance are determined through pairwise comparisons. In detail, pairwise comparisons of the elements at each hierarchical level are conducted with respect to their relative importance towards their control criterion [35]. Expressed in semantic judgments are then converted into numerical values, according to Saaty's fundamental scale [12]. Saaty's scale is a scale of integers from 1 to 9 (Table 2), which has proven to be insensitive to small changes in numerical judgments [36,37].

Table 2. Saaty's fundamental scale (source Saaty, 1990).

\begin{tabular}{cc}
\hline Importance & Definition \\
\hline 1 & Equal importance \\
3 & Moderate dominance \\
5 & Strong dominance \\
7 & Demonstrated dominance \\
9 & Extreme dominance \\
$2,4,6,8$ & Intermediate values \\
\hline
\end{tabular}


Pairwise comparisons result in square matrices of preferences, where the dominance coefficient $\mathrm{a}_{\mathrm{ij}}$ represents the relative importance of the component on row i over the component on column $\mathrm{j}$. In detail, $\mathrm{a}_{\mathrm{ij}}$ represents the relative importance of a certain criterion, sub-criterion or action $\mathrm{A}_{\mathrm{i}}$ in comparison to another criterion, sub-criterion, or action $A_{j}$ (Figure 2): the score of 1 represents equal importance of the two components, and 9 represents extreme importance of component $i$ over component $j[12,25]$. Pairwise comparison matrices are square $n \times n$ positive reciprocal matrices of preferences, where the elements on the main diagonal are equal to 1 , since the binary preference relation is reflexive, and the elements in the lower triangular sub-matrix are the reciprocals of the elements in the upper triangular sub-matrix $\left(a_{j i}=1 /\right.$ aij $)$.

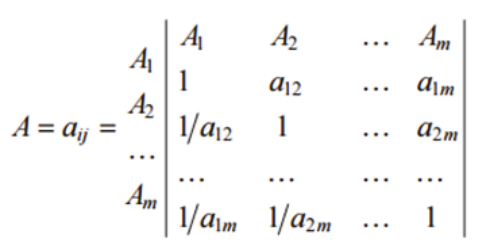

Figure 2. Comparison matrix-.

To solve each sub-decision problem, priorities (i.e., weights $\mathrm{w}_{1}, \mathrm{w}_{2}, \ldots, \mathrm{w}_{\mathrm{n}}$ ), which reflect recorded judgements in a pairwise comparison matrix, are determined for consistent or near consistent matrices, where the relations between weights $\left(\mathrm{w}_{\mathrm{i}}, \mathrm{w}_{\mathrm{j}}\right)$ and judgements $\left(\mathrm{a}_{\mathrm{ij}}\right)$ are given by:

$$
w_{i} / w_{j}=a_{i j}(\text { for } i, j=1,2, \ldots, n)
$$

If the matrix A would be perfectly consistent, then $\lambda_{\max }=\mathrm{n}$, where $\lambda_{\max }$ is the maximum eigenvalue à la Perron-Froebenius of the pairwise comparison matrix [12], which represents the priority vector of a consistent nxn matrix (A) and is computed as the unique solution of $\mathrm{Aw}=\lambda_{\max } \mathrm{w}$. According to Saaty [38], the priority vector of a near consistent matrix, derived by small and continuous perturbation of an underlying consistent matrix A, can be obtained as a perturbation of the corresponding principal eigenvector of A [38]. It is nonetheless necessary to test the consistency of comparison matrices by calculating the so-called consistency index $C I$ [39]. As small changes in $\mathrm{a}_{\mathrm{ij}}$ generates small changes in $\lambda_{\text {max }}$, the deviation of $\lambda_{\text {ma }}$ from $n$ (which coincides with the rank of matrix A) measures consistency. Therefore, the consistency index, which represents the "closeness to consistency" can be calculated as follows [12]:

$$
C I=\frac{\lambda_{\max }-\mathrm{n}}{n-1}
$$

In addition, Saaty recommends to determine the consistency ratio $C R$ :

$$
C R=\frac{C I}{R I}
$$

where $R I$ is a random consistency index which depends on the rank of matrix A $[12,40]$.

$C R$ is considered as an acceptable consistency ratio when it is less than $0.10[12,41]$, whereas whenever $C R>0.1$, a revision of pairwise comparison is recommended.

Absolute measurement is the comparison of some value on a scale with the unit value of the scale. Theories based on absolute require units of measurement to tradeoff weights for criteria or attributes. According to Saaty [35], measurements of phenomena on absolute scale serve as surrogates, indicators, or stimuli to the mind educated about the significance of magnitude of the number in terms of the goals and understanding of an individual. In a group, its members have to agree on how to interpret measurements to lend credence to objective acceptance. In the AHP absolute method an alternative is compared against an ideal property, i.e., a 'memory' of that specific property [42]. AHP absolute 
models are implemented to rank each independent alternative at a time in terms of rating intensities for each criterion/sub-criterion. In an absolute model, the hierarchy is structured as usual into criteria and sub-criteria, which are further decomposed to a final level hierarchical level, which accounts for intensities through ratings $[43,44]$. In other words, according to [45], rating categories were established for each criterion and categories were prioritized by pairwise comparisons in terms of their preference, and then alternatives were evaluated by identifying an appropriate rating on each criterion.

It is worth noting that the typology (e.g., qualitative vs quantitative) and number of ratings may vary according to different criteria/sub-criteria. Each criterion is evaluated by an "intensity". This intensity is identified by a numerical range of variation, which completes the bottom level or the hierarchy, and allows for numbering each alternative with respect to the criterion [46]. To define the relative weights for each criterion, according to the eigenvalue approach, the absolute measurement AHP requires a pairwise comparison procedure between indicator categories (i.e., high, low, etc.) and ideal preference synthesis: alternatives are compared to standard levels and are measured in an absolute scale, thus preserving rankings from rank-reversal issues and reducing the limitation on the number of alternatives to be compared [47-50].

\section{Model}

Firstly, to structure the decision problem, an extensive literature review was conducted. Secondly, to identify key issues and construct the hierarchy, a group of nine experts representing three main perspectives (knowledge, government, and business) were selected [51,52]. Group decision making benefits de facto from the plurality of its members [53] to capture as much diversity of thinking as possible and to reach consensus on the final decision in a systematic and credible way [54].

Focus groups were organized, and a Delphi survey-based process was implemented to create consensus on criteria and sub-criteria, obtain experts' judgments, and validate the final hierarchy through dynamic discussion $[25,31,51,54]$. In order to ensure the best representability, the group of nine experts consisted of six academicians and professionals with proven expertise in cultural heritage conservation and preservation, seismic risk assessment, structural dynamics, art history, history of architecture, a representative from construction companies specialized in restoration and retrofit of cultural heritage assets, a representative of Superintendence, and a representative from the Italian Civil Protection Department.

The panel of experts identified seven hierarchical levels from the goal at the top of the hierarchy (i.e., ranking alternatives according to priorities of intervention) to ratings at the bottom of the tree-like structure, and identified three main criteria which represent a first-level decomposition of the building structure into foundations, above-ground structure, and horizontal components. These criteria were then decomposed into nine sub-criteria and 26 sub-sub-criteria (Table 3). The graphical representation of the hierarchy is included in the Supplementary Materials of this paper.

In order to determine the weights of criteria, sub-criteria and sub-sub-criteria once the panel reached consensus on the hierarchy and validated it, the model was firstly implemented on the Super Decision Software, and each expert was asked to compile the entire set of pairwise-comparison matrices in a face-to-face interview. Secondly, the CI for each matrix was calculated and proved to be within the acceptability limit (i.e., $C I<0.1$ ). Subsequently, each expert's judgements were combined and weights were aggregated by calculating judgements geometrical mean (Table 4). According to group decision making theory, this procedure allows for making a synthesis of individual judgements expressed with respect a single pairwise comparison as the representative judgment for the entire group $[25,55,56]$. 
Table 3. Description of criteria, sub-criteria, and sub-sub-criteria.

\begin{tabular}{|c|c|}
\hline Criteria & Description \\
\hline D1-Foundation & Structure composing foundations \\
\hline D2-Above-ground structure & Structural elements composing the surface structure \\
\hline D3-Horizontal components & Floors and roof \\
\hline Sub-criterion D2 & Description \\
\hline D2.1-triumphal arch & Wall archway opposite to façade \\
\hline D2.2-apse & Semicircular or polygonal recess, arched or with a domed roof \\
\hline D2.3-nave & Main body of a church between façade and triumphal arch enclosed either between aisles or lateral walls \\
\hline D2.4-bell-tower & Tower with a belfry containing bells, included, adjacent or detached from the church \\
\hline D2.5-lateral chapel & Small room adjacent to the main walls of the church \\
\hline D2.6-façade & External main face of the church \\
\hline D2.7-transept & Transverse portion lying across the main body of the church \\
\hline Sub-criterion D2.1 & Description \\
\hline D2.1.1-mural painting & Decorative painting applied to immovable substrate \\
\hline D2.1.2-mosaic & Patterned surface composed of tesserae \\
\hline D2.1.3-none & Absence of decoration on substrate \\
\hline D2.1.4-stucco & Decorative plasterwork \\
\hline Sub-criterion D2.2 & Description \\
\hline D2.2.1-apse overturning & Out-of-plane rotation \\
\hline D2.2.2-shear mechanism & Shear deformation/cracking of masonry walls \\
\hline D2.2.3-presbytery/vaults of apse & Shear deformation/cracking of masonry vaults \\
\hline Sub-criterion D2.3 & Description \\
\hline D2.3.1-transverse response & Out-of-plane displacement of one or more lateral walls \\
\hline D2.3.2-shear mechanism & Shear deformation/cracking of masonry walls \\
\hline D2.3.3-response of colonnade & Shear deformation/cracking due to in-plane actions in colonnade \\
\hline D2.3.4-vaults of nave & Shear deformation/cracking of vaults of central nave \\
\hline D2.3.5-vaults of side aisle & Shear deformation/cracking of vaults of side aisles \\
\hline Sub-criterion D2.4 & Description \\
\hline D2.4.1-belfry & In-plane deformation of arches or pier ends \\
\hline D2.4.2-bell tower & Rotation of tower or in-plane deformation of walls \\
\hline D2.4.3-projections & Out-of-plane rotation or displacement of projections \\
\hline Sub-criterion D2.5 & Description \\
\hline D2.5.1-overturning & Out-of plane rotation of lateral chapels \\
\hline D2.5.2-shear mechanisms & In-plane shear deformation/cracking \\
\hline D2.5.3-vaults of chapels & Shear deformation/cracking on vaults of chapels \\
\hline D2.5.4-irregularities on plan and elevation & Deformations due to interaction with adjiacent structures \\
\hline Sub-criterion D2.6 & Description \\
\hline D2.6.1-overturning & Out-of-plane overturning of façade \\
\hline D2.6.2-in-plane mechanisms & In-plane shear deformation or tensile cracking of façade \\
\hline D2.6.3-mechanisms at top part & Out-of-plane flexural displacement at top \\
\hline D2.6.4-prothyrum or narthex & Out-of-plane flexural displacement of prothyrum or narthex \\
\hline Sub-criterion D2.7 & Description \\
\hline D2.7.1-overturning of end wall & Out-of-plane overturning of end walls of transept \\
\hline D2.7.2-shear mechanisms & Shear deformation/cracking \\
\hline D2.7.3-vaults of transept & Shear deformation/cracking of vaults \\
\hline $\begin{array}{l}\text { Sub-criterio D2.i.j } \\
\text { where } 2<i<7 \text { e } 1<j<5\end{array}$ & Description \\
\hline D2.i.j.1-mural painting & Decorative painting applied to immovable substrate \\
\hline D2.i.j.2-mosaic & Patterned surface composed of tesserae \\
\hline D2.i.j.3-none & Absence of decoration on substrate \\
\hline D2.i.j.4-stucco & Decorative plasterwork \\
\hline Sub-criterion D3 & Description \\
\hline D3.1-roofing & Roof structure of the church \\
\hline D3.2-matroneum (floors) & Balcony or porch for women; horizontal floor \\
\hline Sub-criterion D3.1 & Description \\
\hline D3.1.1-dome & Roofing cap covering squared, circular or poligonal rooms di vani a pianta quadrata, circolare o poligonale \\
\hline D2.1.2-roof of building & Main roof structure made of timber trusses covered by secondary wooden framework and roof tiles \\
\hline Sub-criterion D3.1.1 & Description \\
\hline D3.1.1.1-lantern & Shear deformation/cracking of cap or torsional rupture of base of pillars \\
\hline D3.1.1.2-lantern tower/drum & Shear deformation/cracking of dome with extension to drum \\
\hline $\begin{array}{l}\text { Sub-criterio D3.1.i.j } \\
\text { where } 1<i<2 \mathrm{e} 1<j<2\end{array}$ & Description \\
\hline D2.i.j.1-mural painting & Decorative painting applied to immovable substrate \\
\hline D2.i.j.2-mosaic & Patterned surface composed of tesserae \\
\hline D2.i.j.3-none & Absence of decoration on substrate \\
\hline D2.i.j.4-stucco & Decorative plasterwork \\
\hline
\end{tabular}


Table 4. Aggregation of experts' judgements on criteria, sub-criteria and sub-sub-criteria, and final priority vector.

\begin{tabular}{|c|c|}
\hline Goal & Priority Vector \\
\hline foundations & 0.3196 \\
\hline above-ground structure & 0.5584 \\
\hline horizontal components & 0.1220 \\
\hline $\mathrm{CI}$ & 0.01759 \\
\hline Criterion D2 & Priority vector \\
\hline triumphal arch & 0.1375 \\
\hline apse & 0.0609 \\
\hline nave & 0.2507 \\
\hline bell tower & 0.0222 \\
\hline lateral chapel & 0.0270 \\
\hline façade & 0.4170 \\
\hline transept & 0.0845 \\
\hline CI & 0.07515 \\
\hline Sub-criterion D2.1 & Priority vector \\
\hline mural painting & 0.4673 \\
\hline mosaic & 0.2772 \\
\hline none & 0.0954 \\
\hline stucco & 0.1601 \\
\hline $\mathrm{CI}$ & 0.01160 \\
\hline Sub-criterion D2.2 & Priority vector \\
\hline apse overturning & 0.6483 \\
\hline shear mechanisms & 0.2297 \\
\hline presbytery/vaults of apse & 0.1220 \\
\hline $\mathrm{CI}$ & 0.00355 \\
\hline Sub-criterion D2.3 & Priority vector \\
\hline transverse response & 0.5360 \\
\hline shear mechanisms & 0.0533 \\
\hline response of colonnade & 0.2246 \\
\hline vaults of nave & 0.1166 \\
\hline vaults of side aisle & 0.0696 \\
\hline $\mathrm{CI}$ & 0.05971 \\
\hline Sub-criterion D2.4 & Priority vector \\
\hline belfry & 0.2785 \\
\hline bell tower & 0.6630 \\
\hline projections & 0.0585 \\
\hline $\mathrm{CI}$ & 0.05156 \\
\hline Sub-criterion D2.5 & Priority vector \\
\hline overturning & 0.5781 \\
\hline shear mechanisms & 0.2282 \\
\hline vaults of chapels & 0.1336 \\
\hline irregularities on plan and elevation & 0.0601 \\
\hline $\mathrm{CI}$ & 0.02524 \\
\hline Sub-criterion D2.6 & Priority vector \\
\hline overturning of façade & 0.5610 \\
\hline in-plane mechanisms & 0.0963 \\
\hline mechanisms at top part & 0.2960 \\
\hline prothyrum or narthex & 0.0467 \\
\hline
\end{tabular}


Table 4. Cont.

\begin{tabular}{lc}
\hline Goal & Priority Vector \\
\hline CI & 0.07311 \\
\hline Sub-criterion D2.7 & Priority vector \\
\hline overturning of end wall & 0.6483 \\
Shear mechanisms & 0.2297 \\
vaults of transept & 0.1220 \\
\hline CI & 0.00355 \\
\hline Criterion D3 & Priority vector \\
\hline roofing & 0.8333 \\
matroneum (floors) & 0.1667 \\
\hline CI & 0.0001 \\
\hline Sub-criterion D3.1 & Priority vector \\
\hline dome & 0.750 \\
roof of building & 0.250 \\
\hline CI & 0.0002 \\
\hline Sub-sub-criterion D3.1.1 & Priority vector \\
\hline lantern & 0.125 \\
Lantern tower/drum & 0.875 \\
\hline CI & 0.0001 \\
\hline Sub-sub-criterion D2.i.j $(2<i<7$ and $1<j<5) ;$ & 0.09772 \\
Sub-sub-criterion D3.1.i.j $(1<\mathrm{i}<2$ and $1<j<2)$ & 0.01601 \\
\hline mural painting & \\
mosaic & \\
none & \\
stucco & \\
CI & 0.4673 \\
\hline & \\
\hline
\end{tabular}

Finally, the panel of experts subdivided the bottom level of the hierarchy into a level for intensities and listed ratings under each sub-criterion or sub-sub-criterion. Ratings were identified according to the Italian survey form for churches [20] and correspond to six damage levels: null, low, moderate, high, very high, and collapse. The experts subsequently pairwise compared the six levels of intensities (i.e., damage levels) above mentioned in terms of priority with respect to the parent node and set equal ratings for all of the sub-criteria (Table 5).

Table 5. Ratings priority.

\begin{tabular}{lc}
\hline & Priority Vector \\
\hline collapse & 0.4830 \\
very & 0.1921 \\
high & 0.1874 \\
high & 0.0646 \\
moderate & 0.0427 \\
low & 0.0302 \\
null & \\
\hline
\end{tabular}

\section{Results and Discussion}

The model was implemented to rank multi-criteria prioritization of protection and restoration interventions on a set of 15 Italian churches (i.e., alternatives), listed in the DataBAES and damaged by earthquakes, which occurred in Italy in the last decades. Prior to this, it was compiled in an evaluation 
matrix to identify for each alternative its characteristics and assess its damages to both structural elements and pieces of art. Subsequently, each alternative was rated by assigning it intensity ratings, which characterize the alternative with respect to criteria (sub-criteria, sub-sub-criteria).

Table 6 summarizes the global priority vector and the ranking of alternatives with respect to the goal.

Table 6. Global priority vector and ranking of alternatives.

\begin{tabular}{lcc}
\hline \multicolumn{1}{c}{ Church } & Priority (Normal Values) & Ranking \\
\hline Chiesa di Sant'Antonio & 0.233600 & 1 \\
Chiesa di San Marco & 0.130948 & 2 \\
Cappella degli Scrovegni & 0.121087 & 3 \\
Chiesa Madre & 0.100648 & 4 \\
Chiesa di San Silvestro & 0.073573 & 5 \\
Chiesa di Santa Maria delle Grazie & 0.072700 & 6 \\
Chiesa del Santo Rosario & 0.060274 & 7 \\
Chiesa dell'Annunziata & 0.037264 & 8 \\
Chiesa di S. Egidio & 0.034368 & 9 \\
Chiesa dei Santi Senesio e Teopompo & 0.034363 & 10 \\
Complesso di Santa Maria ai Monti & 0.019805 & 11 \\
Chiesa di San Luca Evangelista & 0.008256 & 12 \\
Complesso della Madonna del Carmine & 0.001544 & 13 \\
Chiesa di San Michele Arcangelo & 0.001054 & 14 \\
Chiesa dell'Immacolata Concezione & 0.000713 & 15 \\
\hline
\end{tabular}

According to our findings, the church which requires the most urgent intervention is "Chiesa di San Antonio", located in San Potito Ultra in Avellino (South of Italy), which was damaged by the earthquake that occurred in Irpinia in 1980. Its top position in the ranking is due to both the collapse of the dome, which in turn caused the loss of a fresco, and the activation of mechanisms involving the triumphal arches. This mechanism is probably related to the damage of the dome. However, although its priority is low, it must be taken into consideration to prevent from future worsening caused by a new seismic event. The "Chiesa di San Marco in l'Aquila" (central Italy), which was damaged by the earthquake that occurred in Abruzzo Region in 2009, is ranked as second. Several out-of-plane damage were identified, which involved stuccoes, although the limited extension did not affect the final evaluation for this church. The "Cappella degli Scrovegni" in Padova included 20 cases of damage to frescoes and is ranked as third, although at current no signs of mechanism activation can be observed. The priority, in this case, is due to the valuable cycle of Giotto's frescoes, although no significant risk for the structure is detected.

At the bottom of the hierarchy there are the "Chiesa di San Michele Arcangelo" in Saviano in Naples (South of Italy), and the "Chiesa dell'Immacolata Concezione" in Bologna (Central Italy) respectively. They both have limited damage for their stuccoes. The difference in ranking between the two churches is due to the damage mechanism, respectively referred to the more brittle mechanism of the vault of the transept than the in-plane behavior of the apse walls.

\section{Conclusions}

The occurrence of natural disasters such as earthquakes represent a worldwide challenge in the conservation of cultural heritage $(\mathrm{CH})$, which suffer from damage due to high vulnerability conditions. Therefore, the protection of $\mathrm{CH}$ from seismic hazard is of paramount importance. As the protection of $\mathrm{CH}$ involves high investment costs, which usually exceeds available financial resources, it is necessary to prioritize interventions and rank $\mathrm{CH}$ assets according to their vulnerability. In order to create a one-dimensional index for representing the overall assessment of alternatives, an AHP absolute model was developed. Based on literature review and expert judgements, six hierarchical levels (from goal to ratings), criteria, sub-criteria and ratings were identified, and local priorities and 
global priorities were determined according to the eigenvalue approach to pairwise comparisons. The model was then validated by ranking 15 Italian churches damaged by earthquakes occurred over the last decades. Our results mirror the results from DataBAES web archive, which correlates vulnerability and damage of artistic assets to damage and vulnerability of their related structural components. Once the model had been validated, it can be implemented to rank a wide set of $\mathrm{CH}$ churches, independently from the need to pairwise compare alternatives one another. The ranking de facto depends on data/information collected during on site visual inspection and can be extended to include new alternatives under investigation to the original set without requiring to re-set local and global priorities, thanks to absolute measurements here adopted. This model can provide a valid and robust decision support tool to Governments and Public Administrations in the design of effective conservation strategies, which require the stabilization of severely damaged buildings and the preventive improvement of constructions structural response to seismic actions and the management of the seismic post-emergency phase.

Supplementary Materials: The following are available online at http://www.mdpi.com/2071-1050/12/4/1608/s1.

Author Contributions: Conceptualization C.D. and M.R.V.; methodology C.D.; software C.D.; validation, C.D. and M.R.V.; formal analysis, C.D. and M.R.V.; investigation C.D. and M.R.V.; data curation, C.D. and M.R.V.; writing C.D. and M.R.V. All authors have read and agree to the published version of the manuscript.

Funding: This research received no external funding.

Acknowledgments: The authors are grateful to Anita Pezzolo for administering questionnaire to the panel of experts and data survey.

Conflicts of Interest: The authors declare no conflict of interest.

\section{References}

1. Valluzzi, M.R. On the vulnerability of historical masonry structures: Analysis and mitigation. Mater. Struct. 2007, 40, 723-743. [CrossRef]

2. Binda, L.; Cardani, G.; Saisi, A.; Valluzzi, M.R.; Munari, M.; Modena, C. Multilevel approach to the vulnerability analysis of historic buildings in seismic areas-Part 1: Detection of parameters for the vulnerability analysis through on site and laboratory investigations. Int. J. Restor. Build. Monum.IInternationale Zeitschrift für Bauinstandsetzen und Baudenkmalpflege 2007, 13, 413-426.

3. D'Ayala, D.; Speranza, E. Definition of collapse mechanisms and seismic vulnerability of historic masonry buildings. Earthq. Spectra 2003, 19, 479-509. [CrossRef]

4. Lagomarsino, S.; Podesta, S. Seismic Vulnerability of Ancient Churches: I. Damage Assessment and Emergency Planning. Earthq. Spectra 2004, 20, 377-394. [CrossRef]

5. Valluzzi, M.R.; Munari, M.; Modena, C.; Binda, L.; Cardani, G.; Saisi, A. Multilevel approach to the vulnerability analysis of historic buildings in seismic areas-Part 2: Analytical interpretation of mechanisms for the vulnerability analysis and the structural improvement. Int. J. Restor. Build. Monum.Internationale Zeitschrift für Bauinstandsetzen und Baudenkmalpflege 2007, 13, 427-441.

6. da Porto, F.; Silva, S.; Costa, C.; Modena, C. Macro-Scale Analysis of Damage to Churches after Earthquake in Abruzzo (Italy) on April 6, 2009. J. Earthq. Eng. 2012, 16, 739-758. [CrossRef]

7. Modena, C.; Valluzzi, M.R.; da Porto, F.; Casarin, F. Structural aspects of the conservation of historic stone masonry constructions in seismic areas. Int. J. Archit. Herit. 2011, 5, 539-558. [CrossRef]

8. Della Spina, L.; Calabrò, F. Decision Support Model for Conservation, Reuse and Valorization of the Historic Cultural Heritage. In Computational Science and Its Applications, Proceedings of the ICCSA 2018, Melbourne, Australia, 2-5 July 2018; Gervasi, O., Murgante, B., Misra, S., Stankova, E., Torre, C.M., Rocha, A.M., Taniar, D., Apduhan, B.O., Tarantino, E., Ryu, Y., Eds.; Springer: Cham, Switzerland, 2018; Volume 10962, pp. 3-17.

9. Bottero, M.; D'Alpaos, C.; Oppio, A. Ranking of adaptive reuse strategies for abandoned industrial heritage in vulnerable contexts: A multiple criteria decision aiding approach. Sustainability 2019, 11, 785. [CrossRef] 
10. Ribera, F.; Nesticò, A.; Cucco, P.; Maselli, G. A multicriteria approach to identify the Highest and Best Use for historical buildings. J. Cult. Herit. 2019, 41, 166-177. [CrossRef]

11. Bottero, M.; D'Alpaos, C.; Marello, A. An Application of the A'WOT Analysis for the Management of Cultural Heritage Assets: The Case of the Historical Farmhouses in the Aglié Castle (Turin). Sustainability 2020, 12, 1071. [CrossRef]

12. Saaty, T.L. The Analytic Hierarchy Process: Planning, Priority Setting, Resource Allocation; McGraw-Hill: New York, NY, USA, 1980.

13. Bhushan, N.; Rai, K. Strategic Decision Making Applying the Analytic Hierarchy Process; Springer: London, UK, 2004.

14. Yau, Y. Multi-criteria decision making for urban built heritage conservation: Application of the analytic hierarchy process. J. Build. Apprais. 2008, 4, 191-205. [CrossRef]

15. Kutut, V.; Zavadskas, E.K.; Lazauskas, M. Assessment of priority alternatives for preservation of historic buildings using model based on ARAS and AHP methods. Arch. Civ. Mech. Eng. 2014, 14, 287-294. [CrossRef]

16. Aliabadi, S.F.; Sarsangi, A.; Modiri, E. The social and physical vulnerability assessment of old texture against earthquake (case study: Fahadan district in Yazd City). Arab. J. Geosci. 2015, 8, 10775-10787. [CrossRef]

17. Vodopivec, B.; Žarnića, R.; Tamošaitienè, J.; Lazauskas, M.; Šelih, J. Renovation priority ranking by multi-criteria assessment of architectural heritage: The case of castles. Int. J. Strateg. Prop. Manag. 2014, 18, 88-100. [CrossRef]

18. Valluzzi, M.R.; Calò, S.; Giacometti, G. Correlation of vulnerability and damage between artistic assets and structural elements: The DataBAES archive for the conservation planning of $\mathrm{CH}$ masonry buildings in seismic areas. Sustainability 2020, 12, 653. [CrossRef]

19. Technologies for the Seismic Protection and Valorization of Cultural Heritage. Available online: https: //databaes.dicea.unipd.it/ (accessed on 18 November 2019).

20. D.P.C (Italian Department of Civil Protection); Work Group for Protection of Cultural Heritage against Natural Risks. Scheda per il Rilievo dei Beni Culturali-Danno alle Chiese-Modello A-DC (in Italian). 2001. Available online: http://www.beniculturali.it (accessed on 18 November 2019).

21. Grünthal, G. European Macroseismic Scale 1998. Cahiers du Centre Européen de Géodynamique et de Séismologie 1998, 15, 1-97.

22. CNR-ICR. Raccomandazioni NorMaL-1/88. Alterazioni Macroscopiche Dei Materiali Lapidei: Lessico. Roma (in Italian). 1990. Available online: https://www.unirc.it/documentazione/materiale_didattico/597_ 2011_287_13564.pdf (accessed on 10 January 2020).

23. Saaty, T.L. Decision making, new information, ranking and structure. Math. Model. 1987, 8, 125-132. [CrossRef]

24. Ferreira, F.A.; Santos, S.P.; Dias, V.M. An AHP-based approach to credit risk evaluation of mortgage loans. Int. J. Strateg. Prop. Manag. 2014, 18, 38-55. [CrossRef]

25. De Felice, F.; Petrillo, A. Absolute measurement with analytic hierarchy process: A case study for Italian racecourse. Int. J. Appl. Decis. Sci. 2013, 6, 209-227. [CrossRef]

26. Abastante, F.; Lami, I.M. An analytical model to evaluate a large scale urban design competition. Geoingegneria Ambientale e Mineraria 2013, 139, 27-36.

27. Nicu, I.C. Cultural heritage assessment and vulnerability using Analytic Hierarchy Process and Geographic Information Systems (Valea Oii catchment, North-eastern Romania). An approach to historical maps. Int. J. Disaster Risk Reduct. 2016, 20, 103-111. [CrossRef]

28. Ma, H.; Li, S.; Chan, C.-S. Analytic Hierarchy Process (AHP)-based assessment of the value of non-World Heritage Tulou: A case study of Pinghe County, Fujian Province. Tour. Manag. Perspect. 2018, 26, 67-77. [CrossRef]

29. Vargas, L.G. An overview of the analytic hierarchy process and its applications. Eur. J. Oper. Res. 1990, 48, 2-8. [CrossRef]

30. Banzato, D.; Canesi, R.; D'Alpaos, C. Biogas and biomethane technologies: An AHP model to support the policy maker in incentive design in Italy. In Smart and Sustainable Planning for Cities and Regions; SSPCR 2017. Green Energy and Technology; Bisello, A., Vettorato, D., Laconte, P., Costa, S., Eds.; Springer: Cham, Switzerland, 2018; pp. 223-231. [CrossRef] 
31. D'Alpaos, C.; Bragolusi, P. Multicriteria prioritization of policy instruments in buildings energy retrofit. Valori e Valutazioni 2018, 21, 15-25.

32. D'Alpaos, C.; Bragolusi, P. Prioritization of energy retrofit strategies in public housing: An AHP model. In New Metropolitan Perspectives; ISHT 2018. Smart Innovation, Systems and Technologies; Calabrò, F., Della Spina, L., Bevilacqua, C., Eds.; Springer: Cham, Switzerland, 2019; Volume 101, pp. 534-541. [CrossRef]

33. Roy, B. Métodologie Multicritère d'Aide à la Decision; Economica: Paris, France, 1985.

34. Vincke, P. Multicriteria Decision-Aid; John Wiley \& Sons: New York, NY, USA, 1992.

35. Saaty, T.L. Fundamentals of Decision Making and Priority Theory with the Analytic Hierarchy Process; RWS Publications: Pittsburgh, PA, USA, 2000.

36. Saaty, T.L. The Analytic Hierarchy Process in Conflict Management. Int. J. Confl. Manag. 1990, 1, 47-68. [CrossRef]

37. Ishizaka, A.; Labib, A. Review of the main developments in the analytic hierarchy process. Expert Syst. Appl. 2011, 38, 14336-14345. [CrossRef]

38. Saaty, T.L. Decision-making with the AHP: Why is the principal eigenvector necessary. Eur. J. Oper. Res. 2003, 145, 85-91. [CrossRef]

39. Saaty, T.L. A scaling method for priorities in hierarchical structures. J. Math. Psychol. 1977, 15, $234-281$. [CrossRef]

40. Alonso, A.J.; Lamata, M.T. Consistency in the Analytic Hierarchy Process: A New Approach. Int. J. Uncertain. Fuzziness Knowl. Based Syst. 2006, 14, 445-459. [CrossRef]

41. Perez-Gladish, B.; M'Zali, B. An AHP-based approach to mutual funds' social performance measurement. Int. J. Multicriteria Decis. Mak. 2010, 1, 103-127. [CrossRef]

42. Saaty, T.L.; Vargas, L.G.; Dellmann, K. The allocation of intangible resources: The analytic hierarchy process and linear programming. Socio-Econ. Plan. Sci. 2003, 37, 169-184. [CrossRef]

43. Saaty, T.L. Absolute and relative measurement with the AHP. The most livable cities in the United States. Socio-Econ. Plan. Sci. 1986, 20, 327-331. [CrossRef]

44. Saaty, T.L. Rank from comparisons and from ratings in the analytic hierarchy/network processes. Eur. J. Oper. Res. 2006, 168, 557-570. [CrossRef]

45. Saaty, T.L. Decision-making with the Analytic Hierarchy Process. Int. J. Serv. Sci. 2008, 1, 83-98. [CrossRef]

46. Rafikul, I.; Mohd Rasad, S. Employee performance evaluation by AHP: A case study. In Proceedings of the ISAHP 2005, Honolulu, HI, USA, 8-10 July 2005.

47. Saaty, T.L.; Odzemir, M.S. Why the magic number seven plus or minus two. Math. Comput. Model. 2003, 38, 233-244. [CrossRef]

48. Tramarico, C.L.; Marins, F.A.; Urbina, L.M.; Salomon, V.A.P. Benefit assessment of training on supply chain management. Int. J. Anal. Hierarchy Process. 2015, 7, 240-255. [CrossRef]

49. Saaty, T.L.; Wei, L. Should the UK have brexited the European Union? Int. J. Anal. Hierarchy Process 2016, 8, 206-223. [CrossRef]

50. Salomon, V.A.P. Absolute measurement and ideal synthesis on AHP. Int. J. Anal. Hierarchy Process. 2016, 8, 538-545. [CrossRef]

51. Saaty, T.L.; Peniwati, K. Group Decision Making: Drawing Out and Reconciling Differences; RWS Publications: Pittsburgh, PA, USA, 2012.

52. Dias, L.C.; Antunes, C.H.; Dantas, G.; de Castro, N.; Zamboni, L. A multi-criteria approach to sort and rank policies based on Delphi qualitative assessments and ELECTRE TRI: The case of smart grids in Brazil. Omega 2018, 76, 100-111. [CrossRef]

53. Kintarso, H.; Peniwati, K. Developing and selecting business strategy, and prioritizing strategic actions for a tool steel company with the analytic hierarchy process'. In Proceedings of the 7th Asia Pacific Management Conference, Kuala Lumpur, Malaysia, 27-31 January 2001.

54. Senge, P.M. The Fifth Discipline: The Art \& Practice of the Learning Organization; Currency Doubleday: New York, NY, USA, 2006. 
55. $\mathrm{Xu}, \mathrm{Z}$. On consistency of the weighted geometric mean complex judgement matrix in AHP. Eur. J. Oper. Res. 2000, 126, 683-687. [CrossRef]

56. Grošelj, P.; Zadnik Stirn, L. Acceptable consistency of aggregated comparison matrices in analytic hierarchy process. Eur. J. Oper. Res. 2012, 223, 417-4201. [CrossRef]

(c)

(C) 2020 by the authors. Licensee MDPI, Basel, Switzerland. This article is an open access article distributed under the terms and conditions of the Creative Commons Attribution (CC BY) license (http://creativecommons.org/licenses/by/4.0/). 


\title{
Cultural Heritage and Sustainable Development: Impact Assessment of Two Adaptive Reuse Projects in Siracusa, Sicily
}

\author{
Stefania De Medici ${ }^{1}$, Pasquale De Toro ${ }^{2}$ and Francesca Nocca ${ }^{2, *}$ \\ 1 Department of Civil Engineering and Architecture, University of Catania, Satellite Campus of Architecture, \\ Piazza Federico di Svevia, I-96100 Siracusa, Italy; sdemedi@unict.it \\ 2 Department of Architecture, University of Naples Federico II, via Toledo 402, I-80134 Naples, Italy; \\ pasquale.detoro@unina.it \\ * Correspondence: francesca.nocca@unina.it
}

Received: 30 November 2019; Accepted: 22 December 2019; Published: 31 December 2019

\begin{abstract}
In this period of increasing urbanization, cultural heritage can play a key role to achieve sustainable development, as widely recognized by international institutions (i.e., United Nations (UN), UN Educational, Scientific and Cultural Organization (UNESCO), International Council on Monuments and Sites (ICOMOS)). In this perspective, it is necessary to operationalize the principles stated at international level and thus new approaches and tools are required. The paper aims to understand the relationships between the implementation of adaptive reuse projects and their success (or not) in terms of impacts on the buildings themselves and on the urban context. The assessment framework for evaluating the impacts of heritage conservation and rehabilitation projects is described through the analysis and comparison of two Italian case studies: the Ancient Market and the Basilica of St. Peter the Apostle, in Siracusa (Italy). Although realized both in the same place (Ortigia, the historic centre of Siracusa), during the same period and by the same architect, these two interventions have produced different results in terms of urban development. A set of indicators, deduced from recent scientific studies, has been used to analyse the different impacts on physical, cultural, social, environmental and economic systems. To understand in depth the causes of these two different results, a survey has been carried out involving experts. The proposed indicators used for the ex-post evaluation can be also adopted in other contexts and for ex ante evaluation, in order to orient the strategic design choices in cultural heritage adaptive reuse projects.
\end{abstract}

Keywords: adaptive reuse; building rehabilitation; cultural heritage; impact assessment

\section{Introduction}

In this period, cities are facing many challenges related to different issues as climate change, demographic growth, social inequalities, economic crisis. The United Nations (UN) declared that $68 \%$ of the world population expected to live in urban areas by 2050 [1]. This means that the aforementioned challenges will be increasingly concentrated in urban areas. So, cities play a key role in the sustainability of our future. For this reason, international institutions (such as the $\mathrm{UN}$ ) are actively moving in promoting actions to achieve sustainable development.

In this paper the attention is focused, in particular, on the role that cultural heritage can play in sustainable development. Although the debate around this issue is very current, many discussions remain on a mostly theoretical level.

In the 2030 UN Agenda for Sustainable Development [2], cultural heritage is explicitly mentioned only once in goal 11 (one out of 17 goals) that referred to the cities, in particular to the need of making cities and human settlements "inclusive, safe, resilient and sustainable". It has a marginal role in 
the document, being mentioning in particular only in target 11.4 ("strengthen efforts to protect and safeguard the world's cultural and natural heritage"), one out of 169 targets. Furthermore, this specific target is referred only to the protection and safeguard of cultural heritage, and not to its valorization and regeneration [3].

The "translation" of the principles of the 2030 Agenda in the cities, that is their territorialisation, is represented by the New Urban Agenda [4]. Here cultural heritage takes on a more important role. In fact, there are many points (i.e., points 10, 26, 38, 45, 60) of the document highlighting the role of cultural heritage (both tangible and intangible) in urban sustainable development. For example, the roles that it can play in the humanization of cities (point 26) and in developing vibrant, sustainable and inclusive urban economies (point 45) are underlined.

Furthermore, there are some international organizations, such as the UN Educational, Scientific and Cultural Organization (UNESCO) and the International Council on Monuments and Sites (ICOMOS), that stress the importance of cultural heritage in the achievement of sustainable development [5-7]. A very significant contribution by UNESCO to the international debate about this issue are the Historic Urban Landscape (HUL) Recommendations [5]. These recommendations recognize the necessity of supporting the protection, safeguard, conservation, and valorization of the Historic Urban Landscape, that is the «historic layering of cultural and natural values and attributes» ([5], art. 8), incorporating the intangible dimension of heritage and the related economic processes. In this document, the attention shifts from the "monument" to the context, to the recognition of the importance of the social, cultural and economic processes in the conservation of urban areas ([5], art. 4).

In the same perspective, the European Commission (EC) has recognized cultural heritage as a common good representing a strategic resource for sustainable development. In Section 2 the EC identifies it as a key element in the global competition using, for the first time in an institutional context, the notion of "intrinsic and social value of heritage" [8].

Many issues at the heart of the contemporary world debate concerning our future intersect with the theme of building reuse, ranging from the research of sustainable development models to the protection of cultural roots. Reuse is consistent with a sustainable approach to urban and landscape management, according to a model of "circular economy" capable of turning "goods that are at the end of their service life into resources for others, closing loops in industrial ecosystems and minimizing waste» [9] (p. 435). Nowadays, in line with instances of climate justice and the living planet, «it is unthinkable for anything to have no function, to be useless, to make no contribution to the betterment of society. The contemporary mantra "Reduce, Reuse, Recycle" is testament to this essential prerequisite of contemporary life. Everything has to be useful, and existing building cannot escape from this agency of usefulness» [10] (p. xviii).

There is an urgent need to make this principal operational and thus "to move from principles to actions" (Agenda for Sustainable Development art. 39-46, New Urban Agenda points 126-160). To this end, new approaches and tools are required.

The research question aims to understand the relationships between the implementation of adaptive reuse projects and their success (or not) in terms of impacts on the buildings themselves and on the urban context. After a review of the relevant literature related to the adaptive reuse (Section 2), the assessment framework (including multidimensional indicators) for evaluating the impacts of heritage conservation and rehabilitation projects is described through the analysis and comparison of two Italian case studies by a survey (Sections 3 and 4). The choice of the case studies has been oriented to two similar adaptive reuse projects by the same architect and in the same place in order to highlight exclusively the relationships between the intended use and the impacts on the context. The results of the investigation are then examined and discussed to answer the research question (Sections 5 and 6).

\section{Review of Relevant Literature}

Adaptive reuse, defined by Douglas as «any building work and intervention aimed at changing its capacity, function or performance to adjust, reuse or upgrade a building to suit new conditions 
or requirements» [11], allows conserving the different values of cultural heritage, both the use value and the intrinsic one. It allows extending the life cycle of a building, in line with the circular economy principles [12] that aim to prolong the lifetime of resources as long as possible and avoid resources consumption.

Building reuse is considered a means for conveying the legacy of the past, which consists not only of heritage buildings as physical items, but mainly of the wealth of information provided by each item. The reuse experience dates back a long way, but today we have a deeper understanding of its strategic importance. Considering the built environment as a resource does not only mean acknowledging its value as a result of human work or as a useful object. The built heritage can also acquire value for its talent to tell stories, to transmit knowledge, to recall the past, to be a symbol of events, traditions, shared knowledge, religions; in other words, it can be a source of cultural value.

To redesign a heritage building for new uses, knowledge of its history and past uses is critically important. A careful identification of exterior and interior architectural elements is needed to define the building's identity and assess the impact of the changes that the new use requires.

Since the 19th century, scientific debate has focused on the significance of physical evidence from the past and on the conservation of architectural heritage with cultural value. At the end of the 18th century, the sale of ecclesiastical properties confiscated by the State during the French Revolution in many cases brought about a change in their use as a production or military site $[13,14]$, with transformations driven by functional and financial ends [15]. In the 19th century, «the needs linked to the new and even more complex functions, created as a result of the Industrial Revolution, as well as a new economic soils regime, whereby land value-rather than buildings-is the key to making a profit, encourage demolishing and rebuilding rather than adapting and adding» [16] (p. 6). As a reaction to these trends, the first theories on the relationship between conservation and transformation of the vestiges of past epochs have been developed; from these theories different positions have emerged.

To develop the idea of reusing the built heritage as a preservation strategy, the 20th century must be awaited. The Athens Charter, released by the International Museums Office in 1931, recommends «maintaining whenever it is possible, the occupation of monuments which can ensure the vital continuity, provided that the modern destination will be such as to respect the historic and artistic character» (art. 2). Nevertheless, only since the 1970s building reuse can be considered as an autonomous discipline. Indeed, just after the Venice Charter, which in 1964 affirmed the role of reuse as a means of conservation, the international scientific community focused its interest on this topic with conferences, articles and books. In 1972 a special issue of the journal Architectural Review entitled "New uses for old buildings", edited by Sherban Cantacuzino, was published. In 1977 two symposia entitled "Old into New" in Glasgow and "Old and New Architecture: Design Relationship" in Washington were held [17-20].

Research by Bie Plevoets and Koenraad Van Cleempoel $[14,20,21]$ has effectively described different theoretical approaches to the topic of adaptive reuse, coexisting throughout the scientific debate over the last 50 years: the typological approach, which examines compatible uses for specific building typologies [11,13,22]; the architectural approach, which analyses the morphological relationships between old and new and the different design strategies (for example, the addition of new volumes inside, above, around the existing building) [23-25]; the technical approach, which focuses on building adaptations required to meet the needs of safety, comfort and usability (fire resistance, thermal behaviour, acoustic performance, etc.) [26-29]; the programmatic approach, which starts from the choice of a specific function and compares it with buildings available for reuse, in order to select a building suitable to accommodate such function [15,30]; the approach of interior design, which focuses on the "soft" values of the building (immaterial aspects, atmosphere, narrative), with the focus on protecting the "meaning" of the building rather than preserving its physical integrity [31-33]. Each of these theoretical approaches can be related to many reuse projects, as a principle of inspiration or as a deduction from past experiences.

The work of Italian researchers, developed through studies dating back to the 1970s, 1980s and 1990s, is based on the performance-based building design approach as a key to forecasting the results 
of adaptive reuse at the project stage [34]. Such an approach aims at establishing a system of rules to evaluate the existing buildings, considered as «organisms able to evolve and live in symbiosis with the needs of the people» ([35] p. 2). The rehabilitation project should choose what to preserve and what to transform, through an iterative process of information/decision [36].

Since the 2000s, multi-criteria assessment methodologies have been applied in research aimed at choosing new uses for heritage buildings, to find a balance between conservation and adaptation needs, as well as to define the preferable new function in relation to the impacts on the urban context [37-41].

An important issue in adaptive reuse projects is related to the "admissible limits for changing", and thus related to the compatibility between intrinsic values (values linked to history and collective memory) and new use values (that is the problem related to the suitable "choices") [42]. The starting point for choosing the new functions (use value) in the adaptive reuse projects is thus represented by the intrinsic value. It can contribute to orient choices for the most appropriate use for cultural heritage regeneration, adapting its use to changes in the needs of society and, at the same time, conserving its intrinsic value, minimizing the negative impacts on the urban context [42].

The reuse project involves a preliminary evaluation of the building, to identify criteria for the preferable design choices. These derive from the system of values ascribed to the building and its potential use. A wide-ranging scientific literature on the subject of reuse highlights the potential benefits on an urban and territorial scale, such as the increase in the market value of soils and buildings, social promotion and economic development in the surroundings [26,43]. The adaptive reuse can contribute to sustainable development producing, at the same time, multidimensional benefits: «cultural benefits (conserving "alive" a symbol of community identity), economic benefits (in terms of increase of productivity), environmental benefits (i.e., reduction of resource consumption) and social benefits (i.e., employment)» [44].

New functions may encourage new public and private investments, promoting the development of related activities and the provision of support services in the area in which the reuse project is carried out [11,45].

\section{Case Studies: The Ancient Market and the Basilica of Saint Peter the Apostle in Ortigia}

Siracusa is a coastal town in the south-east of Sicily (about 121,000 inhabitants in 2019), founded by the Corinthians between 734 and 733 BC and called "Siracusa" by the ancient Siculan language Surraka (meaning abundance of water). Included in the UNESCO World Heritage List in 2005, the city preserves everywhere memories of its ancient history, classic and Baroque works in spectacular scenery (Figure 1). Thanks to an enviable geographic position, since the Greek period Siracusa has been the junction of commercial exchanges and melting pot of customs and traditions of the populations (and of the dominations), that have succeeded in the Mediterranean area: from the Byzantines to the Bourbons, from the Arabs to the Normans up to the Aragonese. During the Classical period it was one of the leading urban centres of the Mediterranean basin and, because of its geographical position, it played an outstanding role in trading and cultural exchanges until the Middle Ages. As a result of the heavy earthquake of 1693, the town was rebuilt in Baroque style. The homogeneity of the style and the widespread use of light-coloured limestone, as well as the particular location of the old town on Ortigia Island, strengthened the urban identity of Siracusa, coming from the fragile balance between tradition and innovation, technical knowledge and creativity, built environment and nature [46]. 

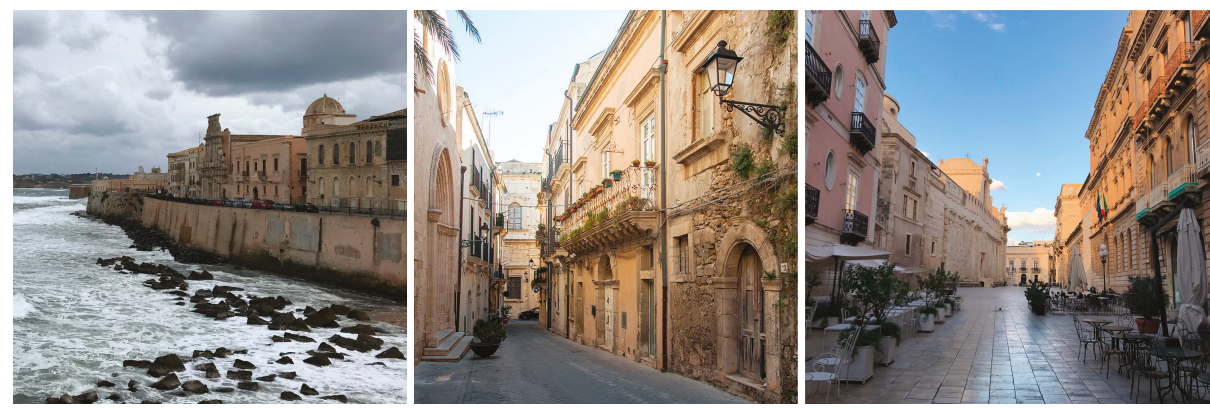

Figure 1. Three views of Ortigia: the eastern seafront, S. Martino and Minerva streets. Source: photos by the authors.

In the 1950s, the most important chemical and petrochemical industrial centre in Europe developed in the Siracusa area, where oil refining, derivative processing and energy production were carried out [47-49]. For decades this provided the main employment opportunity for citizens of Siracusa. Since the mid-eighties, industrial activities have been significantly reduced, leading to serious reconversion problems and the need for land decontamination. In more recent years the situation has changed. The decline of the chemical industry is driving investment in agriculture, food industry and tourism. In particular, this latter activity has developed as a result of the policies implemented by the local government over the last 30 years. Exceptional historical and artistic testimonies and the outstanding landscape value of the area, which combines cultural heritage harmoniously with natural resources, are driving an increasing number of people to visit the site and in 2005 this resulted in the inclusion of the town, along with the Rocky Necropolis of Pantalica, in the UNESCO World Heritage List.

Ortigia Island (Figure 2) is the main attraction for visitors, alongside the Neapolis area, which is almost completely devoid of tourist facilities. Ortigia is located at the eastern end of Siracusa in an area of 45 hectares. Two bridges across a narrow channel connect the island to mainland Sicily. Nowadays, the island is an extremely popular place for tourist accommodation, shopping, food and entertainment. This is the result of political choices and investments over the last fifty years.

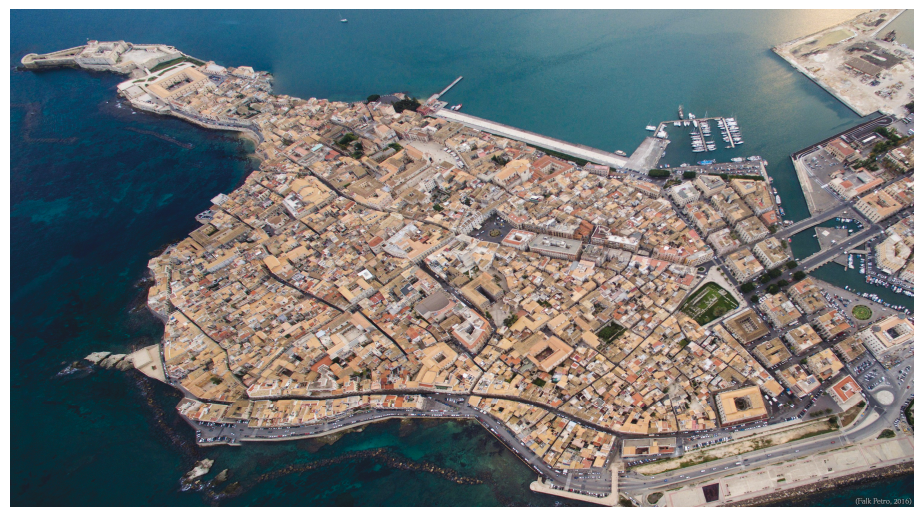

Figure 2. Ortigia Island. Source: www.flickr.com, author: Falk Petro, 2016, no changes were made [Attribution 2.0 Generic (CC BY 2.0)].

Since the beginning of the 20th century, Ortigia has been affected by a progressive decrease in the number of inhabitants. The depopulation process was particularly marked after the Second World War and overlapped with the expansion of the town on the mainland. The Ortigia inhabitants dramatically 
decreased between 1960 and 1970 from 23,000 to 12,000 units, up to 5994 in 1991 [50]. In recent years, data show that the number of residents has stabilised between 4450 and 4550 from 2003 to date [51].

The subsidies granted by the Regional Law for Ortigia (1971) and the special law no. 70 on "Protection of the historical centres and special regulations for the Ortigia district and for the historical centre of Agrigento" (1976) are the leading factor behind the depopulation of the town centre in the 1970s and 1980s [52]. Such laws had the purpose of implementing strategies of conservative restoration for heritage buildings and urban rehabilitation in the old town. In addition, the special law no. 70/1976 authorised the variant of the General Regulatory Plan of Siracusa through the Detailed Plan for Ortigia, drawn up by Giuseppe Pagnano and approved in 1990. The urban renewal policies arising from the Pagnano's plan were implemented in an area characterised by deep physical and socio-economic degradation. Most of Ortigia's population was destitute and its displacement to other neighbourhoods was considered a necessary condition to accelerate the process of urban rehabilitation. The strategy of the Detailed Plan is based on the reuse of the building heritage to combine housing, tourist and accommodation services, cultural activities, headquarters of local government, university and education. Minor physical transformations were planned for the old town centre, but significant changes were expected in the use of the buildings. The investments concerned not only the conservation of cultural heritage, but also the creation of culture, as "one of the most powerful engines for the functional and economic revival of the historic center [...] able to build significant processes of reuse in disused areas of the island" [53] (p. 167). The implementation of the plan mainly encouraged the development of tourist and cultural activities in the historical centre, and increased hotels, restaurants and commercial activities for guests.

The process of urban rehabilitation has progressively extended beyond the area of the main tourist attractions (Duomo, Fonte Aretusa, Castello Maniace, Museo Bellomo, etc.) towards other urban areas (such as the ancient Jewish district of Giudecca) and the waterfront. Nevertheless, Ortigia still retains degraded areas, borderline to the tourist circuits. In particular, marginal areas such as the Graziella district and the Market district retain a large number of abandoned buildings and are perceived by the inhabitants as unsafe areas. Even the reuse of heritage buildings for public use is not always as effective as it should have been in terms of increasing local vitality, attracting new users, and encouraging private businesses.

In order to verify the research hypotheses, the Ancient Market and the Basilica of St. Peter the Apostle have been analyzed as examples of reuse of public buildings in marginal areas of Ortigia. These reuse works, both designed by the architect Emanuele Fidone, professor at the Master's Degree Course in Architecture of the University of Catania, are good examples of a compatible adaptation. Although such cases have several common characteristics (location, designer, public use, public client, acknowledgements and awards for the quality of design), the impacts on the urban context seem different.

\subsection{The Ancient Market}

The Ancient Market of Siracusa is a building dating back to 1900, built on the remains of the Spanish city walls, next to the Temple of Apollo. Designed by Edoardo Troja, an engineer of the Municipal Technical Office, it follows the design of the Livorno Market, with large arches and windows with shutters. The internal rectangular courtyard has an ornamental fountain in the middle and a portico with 24 arches surrounded by shops. From the early 1950s to 1985 the building has been transformed in order to adapt the retail spaces. The building was used as a city market until the end of the 1980s, when the sale of food products and local handicrafts moved outdoors, in the nearby streets. The Ancient Market was restored in 2000 and reused for cultural and commercial events and trade fairs (e.g., farmers' market, display and sale of typical products, exhibitions, music and cultural events).

The reuse project included the repair of windows and walls, the restoration of stone surfaces by keeping the erosion of materials visible and replacing only unstable stones (Figures 3a and 4). The structural improvement works and the partial reconstruction of the roofs were carried out using 
traditional techniques. A large polyfunctional hall (Figure 3b) on the east side was created closing the portico with self-supporting panels, which frame the columns from the inside with high vertical cuts.

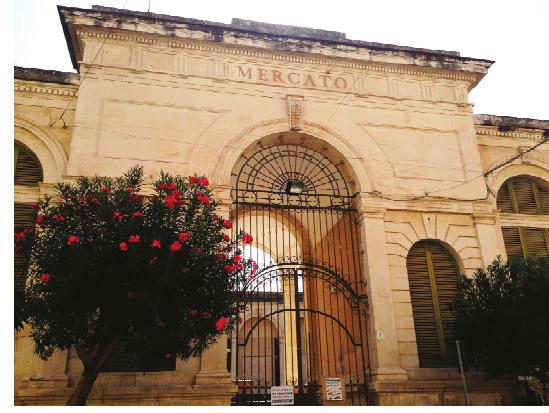

(a) Western façade

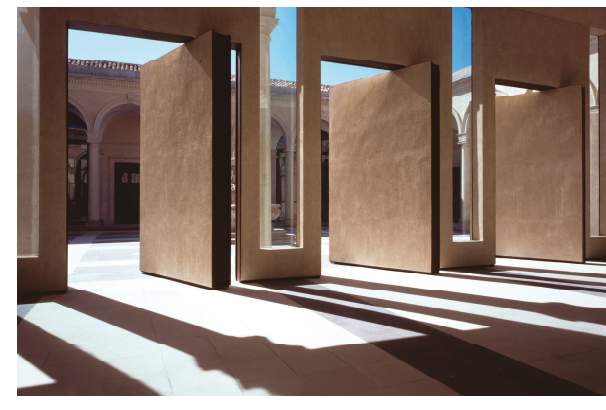

(b) The polyfunctional hall

Figure 3. The Ancient Market. Sources: Figure 3a, www.commons.wikimedia.org, author Davide Mauro, 2017, no changes were made [Creative Commons Attribution-Share Alike 4.0 International license]; Figure 3b, photo kindly provided by Professor Emanuele Fidone, the designer of the reuse projects of the two case studies.

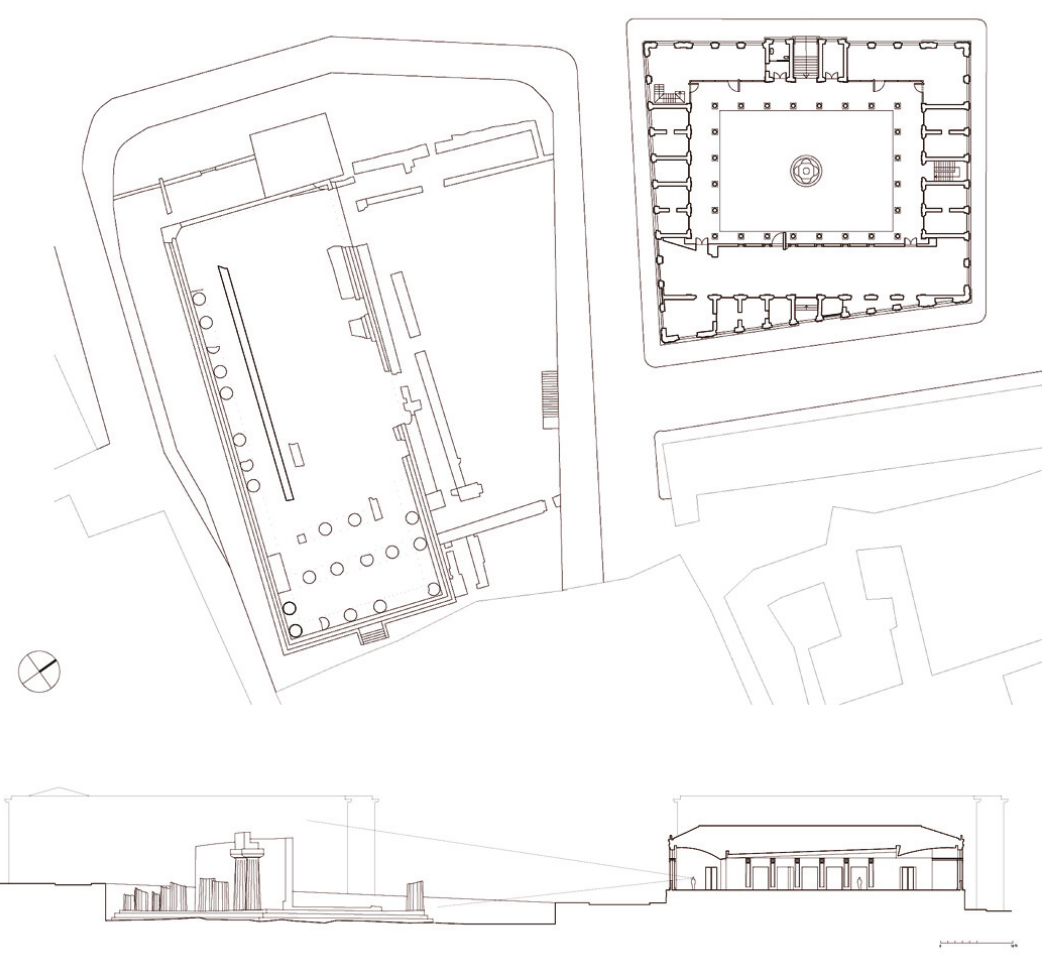

Figure 4. The Ancient Market, project drawings by Emanuele Fidone.

The roof of the new hall is divided into three sections, designed as fragments of large barrel vaults shaped like a telescope, and lead the eye towards the Temple of Apollo, which can be seen from the two large windows at the end of the hall. The western rooms have been redesigned with a structure in 
oxidized steel and glass which separates functions without altering the overall view. The building now houses a service centre for tourists.

\subsection{The Basilica of St. Peter the Apostle in Ortigia}

The early Christian Basilica of St. Peter the Apostle is one of the oldest churches in Ortigia, dating back to the end of the fourth century, and has been repeatedly transformed. The first adaptation, occurred in the seventh century, inverted the position of apse and facade, and added a tripartite transept. In the early 1400 s the Gothic portal was built, and the previous entries were closed. Many decorations were added in the Baroque period. The restoration of the 1950s has altered the interior spaces in an irreversible way, with the goal to restore the original appearance of the church.

The project of the architect Emanuele Fidone, awarded with the Italian Heritage Award in 2013, has reused the church as music hall (Figures 5 and 6). The project highlights the stratigraphy of the surfaces, showing traces of the Byzantine frescoes. The architect has built two new structures, the false ceiling and the large portal, clearly identifiable as a contemporary addition. The false ceiling restores the original dimension of the interior space: it is made of wooden lamellae and is at the height of the supports of the barrel vault of the early Christian age, apparently detached by the building's bearing structure. This lightweight wooden element filters the view of the roof trusses, modulates the daylight entering from the upper windows made in the 1950s and improves the acoustics of the church.

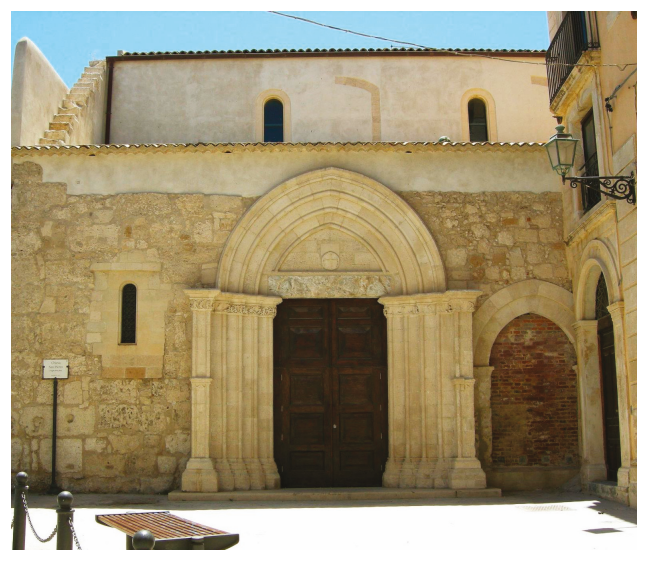

(a) Northern façade

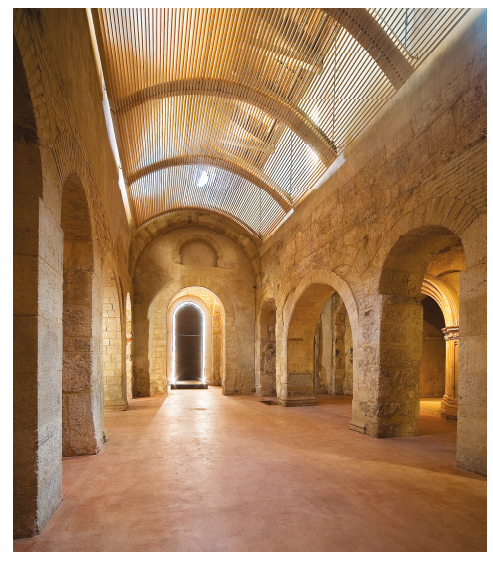

(b) The central nave

Figure 5. The Basilica of St. Peter the Apostle. Sources: Figure 5a, www.commons.wikimedia.org, author: Sailko, 2009, the picture has been cutted [GNU Free Documentation License, Version 1.2; Creative Commons Attribution-Share Alike 3.0 Unported license]; Figure 5b: photo by Emanuele Fidone.

The new portal consists of a thick corten steel panel, slightly spaced from the sides and the lower surface of the arch. The opening requires a frontal push of the lower part of the panel, which looks like a screen, a filter towards the internal space. The floor made in "cocciopesto", a flooring material made of a compound based on hydraulic lime and crushed bricks, with natural wax finishing, is a continuous surface, which highlights the preserved part of the Byzantine flooring.

In such building conservation goals are not restricted to safeguarding the building's exterior appearance, but are also taken on to spaces and interior elements. Moreover, we can clearly distinguish the new parts by the ancient ones. It demonstrates that good contemporary architecture is not incompatible with the needs of restoration and can improve building performance. Today the building can be visited daily, paying an entrance fee. 
Both of the case studies are in marginal areas as compared to the exceptional tourist development of Ortigia. Nevertheless, while the area of the Ancient Market seems to benefit from the users' flows attracted by the activities held in the building, the Basilica of St. Peter the Apostle is still visited only by a few people, moving away from the traditional tour of the island or involved in the seldom cultural events which take place in the building.

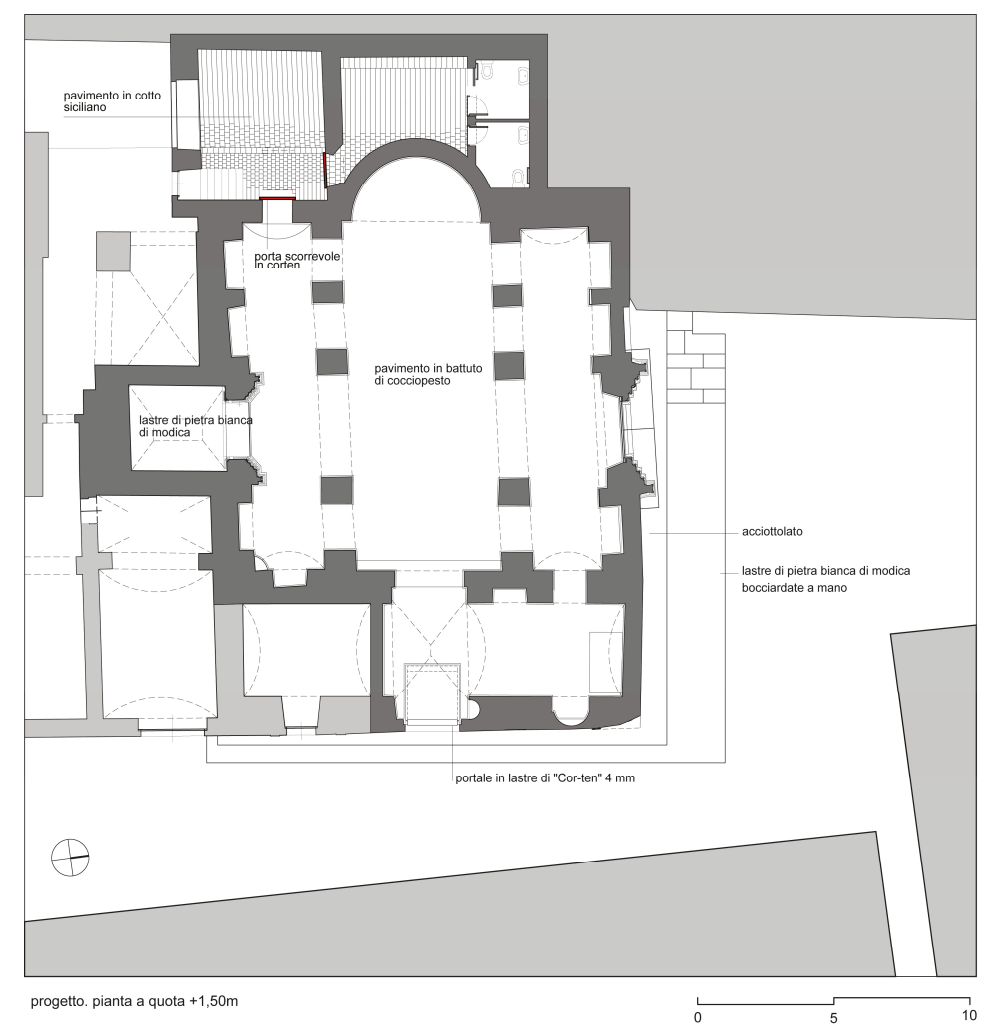

Figure 6. The Basilica of St. Peter the Apostle, project drawings by Emanuele Fidone.

\section{Materials and Methods}

There are many factors affecting the success (or failure) of an adaptive reuse project. The selected case studies, in fact, demonstrate as the same design approach in the same place can have different results on the socio-economic system.

The process for understanding and analysing the proposed case studies has been organized into three main steps (Figure 7):

- $\quad$ knowledge phase;

- critical analysis of indicators for assessing the impacts of adaptive reuse;

- participatory phase. 


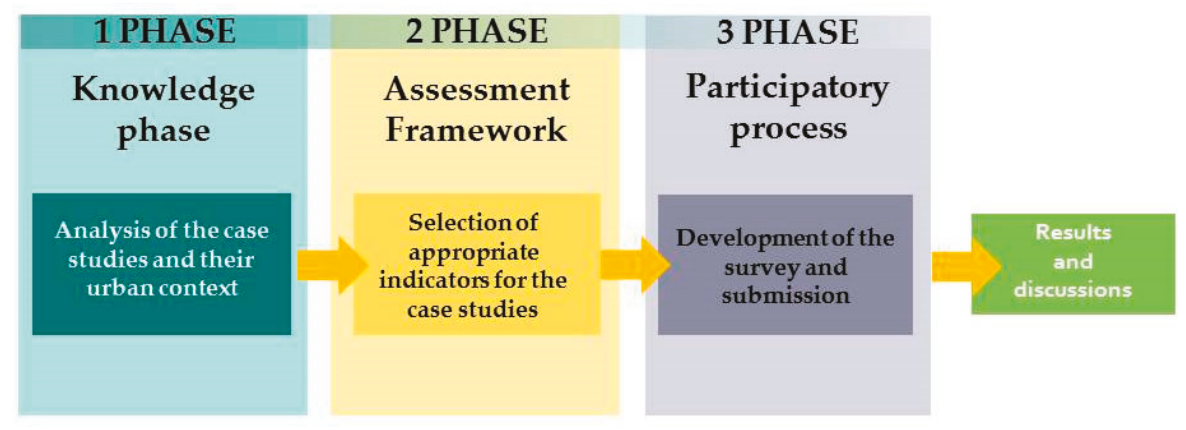

Figure 7. Methodology.

The first step was characterized by the analysis of the projects' area and thus data collection to understand in depth the two case studies and their contexts. During this phase, the context in which the Ancient Market and of St. Peter the Apostle are placed was analyzed, including the analysis of values, and existing regulatory, planning instruments.

The second step concerned the identification of indicators able to understand the reasons of the different performance of these two projects.

In particular, two studies and research works [54,55] about the multidimensional impacts that conservation and regeneration projects are able to produce were considered as the starting point. The first study [54] highlighted a set of multi-dimensional indicators for assessing the impacts of cultural heritage conservation and regeneration projects, both on cultural heritage and from cultural heritage on the entire city system (both in the short and medium and long term, whether direct, indirect or induced).

The indicators to assess these impacts were deduced from concrete experiences of conservation/regeneration projects from 40 case studies of cities (from all over the world) [54].

They were organized in the following 9 identified impact categories (with related sub-categories):

- tourism and recreation;

- creative, cultural and innovative activities;

- typical local productions;

- environment and natural capital;

- community and social cohesion;

- real estate;

- financial return;

- welfare/wellbeing;

- cultural value of properties/landscape.

In the present work, the indicators included in the aforementioned categories were integrated with other indicators identified by De Medici et al. [55] mainly focused on the protection of building's characteristics (and thus on impacts on cultural heritage). Such indicators are classified according to the following categories of characteristics they refer to: perceptual-cultural, morphological-dimensional and material-constructive characteristics.

The indicators selected from the sets of the two aforementioned research studies were deduced by expert knowledge on the basis of the context and of the expected goals of the two adaptive reuse projects. The survey (described below in the third phase) was based on the indicators that allow assessing and highlighting the difference among the effects produced by the two case studies.

- The selected indicators are the following [54,55]:

- No. of visitors per year or per day (attractiveness); 
- Percentage of crowding;

- Contribution of heritage tourism to the city;

- No. of visitors for cultural reason;

- No. of participants in cultural events;

- No. (or percentage) of projects of cultural programmes and events;

- No. of people involved in organising events;

- Percentage growth rate of cultural events and creative activities;

- No. of cultural (and creative) enterprises (attractiveness);

- No. of new start-ups (attractiveness);

- $\quad$ No. artists (attractiveness);

- Attraction of new investments in cultural heritage and cultural/creative events-activities;

- Economic impact generated by cultural events;

- No. (or percentage) of craft stores/new handcraft shops/small scale manufacture/local production activities;

- Percentage of crafts, small scale manufacture, production activities;

- No. of licenses granted in retail and services for artisan;

- New funds to support activities of non-profit organizations;

- No. of new businesses;

- Percentage of increase in employment in activities (or $n$. of new jobs) related to typical local production/distribution;

- $\quad$ Average price of properties;

- Average rent value for residential properties;

- Average rent value for commercial-use properties/offices;

- Average market value for residential properties;

- Average market value for commercial-use properties;

- Percentage of increase in private land/properties value;

- No. (or percentage) of commercial units;

- No. of use change of properties;

- No. of new constructions/rehabilitations;

- No. (or percentage) of well-preserved buildings;

- No. (or percentage) of buildings in poor condition;

- No. (or percentage) of historic building with minor problems;

- Percentage of used/partially used historic building;

- Percentage of vacant historic building;

- No. of restoration and adaptation works undertaken on historic buildings/sites;

- Percentage of re-functionalized historic buildings;

- Area of facades of historic buildings rehabilitated;

- Recognizability and acceptability of the transformations;

- Shape preservation of the building envelope;

- Conservation of the aesthetic relationship with the context;

- Conservation of the original dimensions of the building;

- Conservation of the geometric features;

- Conservation of the indoor spaces;

- Preservation of the finishes;

- Compatibility of the transformations;

- Percentage of citizens satisfied with historic buildings quality;

- Willingness to pay for a contribution to heritage restoration; 
- Percentage of citizens feeling safe in the city/perception of personal safety;

- Average number of crimes (murders, thefts in dwellings, pickpocketing, robberies);

- Percentage of citizens satisfied with cultural facilities supply.

These indicators are used in the following phase to assess both the impacts of the project on the buildings themselves and the urban context.

The third step, that is the participative phase, was based on the involvement of different stakeholders through interviews aiming at understanding the reasons why two projects by the same architect and in the same place have had different impacts and success. To this end, a questionnaire was developed and submitted to the different identified stakeholders.

The participative phase was, in turn, organized in different phases: identification of experts at local level; development of the questionnaire; submission of the survey to the identified experts; deduction and critical analysis of results.

The experts were representatives of institutions, technical-professional organizations and experts in the field of adaptive reuse.

The questionnaire was submitted through an on line survey tool by Google Form and 12 questionnaires were filled in over a time span of two weeks: a representative of the Professional List of Architects, Planners, Landscape Architects of Siracusa and its Province; a representative of the Chamber of Commerce of the Province of Siracusa; a representative of the General Confederation of Italian Industry (Confindustria) of Siracusa; a representative of the Municipal Administration (Councillor); an urban planning expert at the University of Catania, Siracusa headquarters; a building and urban rehabilitation expert at the University of Catania, Siracusa headquarters; an expert in adaptive reuse and valorization of the cultural heritage at the University of Catania, Siracusa headquarters; an expert in architectural design at the University of Catania, Siracusa headquarters; an architect, freelancer professional, expert in architectural design; an expert in history of architecture at the University of Catania, headquarters of Siracusa; an expert in architectural conservation at the University of Catania, Siracusa headquarters; and an expert in restoration and consolidation of cultural heritage at the University of Catania, Siracusa headquarters.

The survey was structured in 6 main sections:

- introduction about the object and aim of the questionnaire and respondent data;

- questions related to the urban quality perception;

- questions for understanding the interviewees' knowledge/opinion about the project area of the Ancient Market in Ortigia (knowledge and perceptive aspects of the project area);

- questions for understanding the interviewees' knowledge/opinion about the reuse project of the Ancient Market of Ortigia (knowledge and perceptive aspects of the project);

- questions for understanding the interviewees' knowledge/opinion about the project area of the Basilica of St. Peter the Apostle in Ortigia (knowledge and perceptive aspects of the project area);

- questions for understanding the interviewees' knowledge/opinion about the reuse project of the Basilica of St. Peter the Apostle in Ortigia (knowledge and perceptive aspects of the project).

The questionnaire aimed at assessing different aspects related to the analysed projects, with a particular focus on the multiple impacts that they have produced and are still producing. The perception of different experts and thus their different points of view were investigated.

The set of indicators for assessing the impacts of cultural heritage conservation and regeneration projects represented the starting point for structuring the questions of the survey, based on qualitative and perceptual aspects. In fact, based on these, we selected the most appropriate indicators for analysing and assessing the impacts of the two projects (Ancient Market and Basilica of St. Peter the Apostle in Ortigia) both on the buildings themselves and on the context in which they are placed.

The questionnaire addressed issues (in Sections 2 and 4) related to the project area and thus to the interviewees' perception about its state of conservation, accessibility level, attractiveness (both in 
terms of activities, initiatives and investments), crowding level, liveliness (or not), social and real estate values, capacity to provide public spaces and services. Moreover, the survey addressed issues (in Sections 3 and 5) closely related to the reuse projects. It particular, Sections 3 and 5 aimed to understand the interviewees' opinion about the suitability of the intended use both with the building and with satisfaction of local needs, their perception of the success (or not) of the projects, their opinion about the recognisability of the asset after the implementation of the adaptive reuse projects. Furthermore, through the questionnaire the willingness to pay for the conservation of the buildings was investigated.

At the end of these sections, there were some open questions. They aim to ask the interviewees some alternative uses (if any) that they considered more appropriate for the adaptive reuse of the Ancient Market and the Basilica of St. Peter the Apostle. Furthermore, they included questions to identify aspects of the projects that could be eventually improved to enhance the adaptive reuse of the two case studies (they will be shown in the results section).

\section{Results and Discussion}

The preliminary section of the survey concerned the introduction about the object and aim of the questionnaire and respondents' data. Then, some questions about the context of both projects were asked.

Most of the respondents perceived a positive improvement in the state of conservation of buildings and urban spaces in Ortigia in the last 20 years (58.3\% "good", 16.7\% excellent). Only 8.3\% of them considered it as "poor" (Figure 8). Furthermore, the results show a clear positive perception about the contribution of the two adaptive reuse interventions to the improvement of the area in the last 20 years (91.7\% of respondents) (Figure 8).

Among these interventions, the Ancient Market project was the one most frequently reported for the benefits produced ( $75 \%$ of respondents). Other projects mentioned were: Bellomo Museum and the rehabilitation of the nearby square, the Faculty of Architecture, recovery of "Porta Marina", Maniace Castle, Bottari Courtyard, rehabilitation of residential buildings, the convent of the "Ritiro", the reuse of some historic buildings as tourist accommodation, the paving of Piazza Duomo, Via Minerva, Piazza XXV Luglio and Corso Umberto. None of the interviewees recognized the reuse project of the Basilica of St. Peter the Apostle as an intervention that has significantly contributed to the improvement of the urban quality of Ortigia. This shows that there is a lack of perception of the positive impacts (if any) of the Basilica adaptive reuse project on the improvement of the area, in contrast to the Ancient Market project whose benefits were perceived by almost all the respondents.

If on the one hand the interviewees assessed on average positively both the selected reuse projects in relation to the investments made, on the other hand, there were significant differences in the assessment of the capacity to trigger new investments in building and urban rehabilitation. In fact, 50\% of interviewed experts declared as "good" and 8.3\% as "excellent" the capacity of the Ancient Market reuse project in contributing to generating new investments, while in the case of the Basilica of St. Peter the Apostle the negative percentage was higher (41.7\% declared it as "scarce") (Figure 9). The benefits of the project, as mentioned above, were not limited to the building itself, but also to the context: a reuse project can become an engine capable of triggering further investments that can contribute to the development and regeneration of the area. The new investments undertaken in relation to a reuse project are significant in terms of its attractiveness. 
At what level do you think that the state of conservation of buildings and urban spaces in Ortigia has improved over the last 20 years?

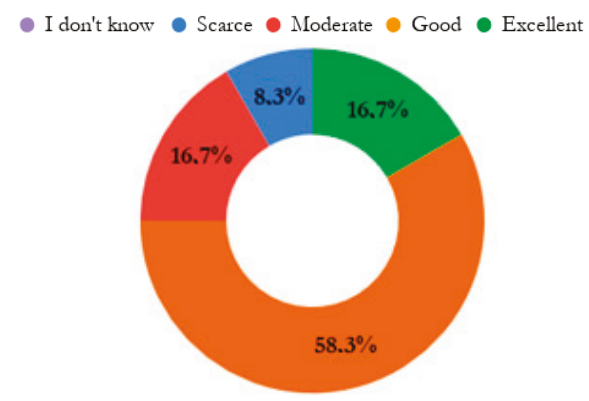

In your opinion, are there any interventions for the reuse of cultural heritage already carried out that have contributed to the improvement of the area in the last 20 years?

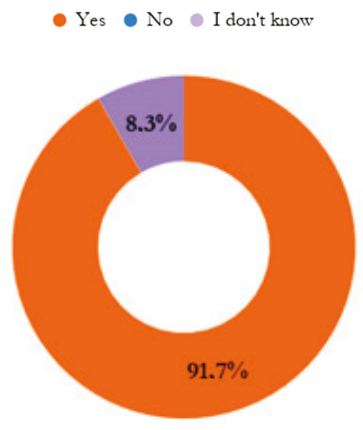

Figure 8. Results of the survey.

How do you consider the benefits produced by the implementation of the reuse project compared to the investment made?

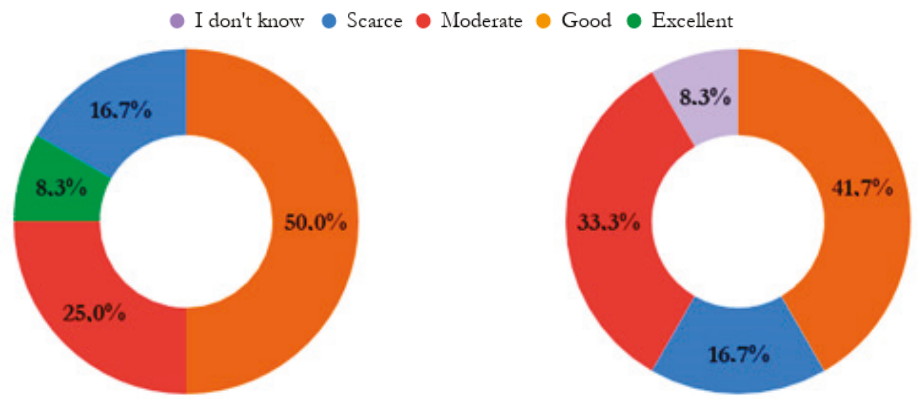

Figure 9. Cont. 

To what extent the implementation of the reuse project has triggered further investments (public and/or
private) in building or urban renewal projects in Ortigia?

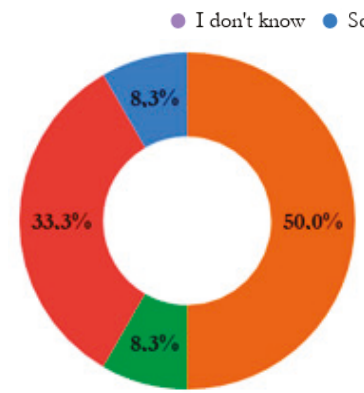

(a2) Ancient Market
Moderate Good Excellent

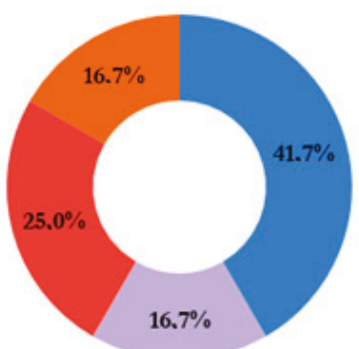

(b2) Basilica of St. Peter the Apostle

To what extent the implementation of the reuse project has contributed to reduce the crime rate in the area?

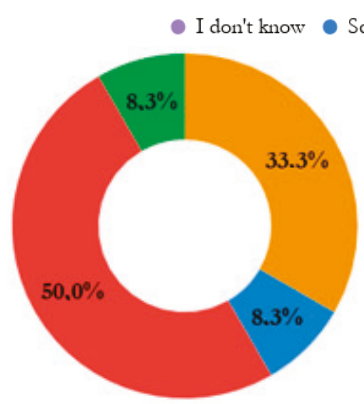

(a3) Ancient Market

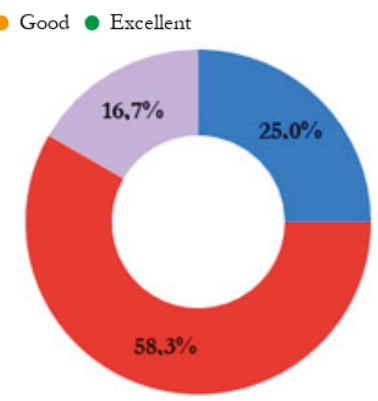

(b3) Basilica of St. Peter the Apostle

Figure 9. Results of the survey.

Moreover, the respondents expressed a different opinion about the two projects in terms of their effectiveness in reducing the crime rate in the area: $33.3 \%$ of the respondents considered the impact of the reuse of the Ancient Market to be "good" and 8.3\% to be "excellent" for this aspect, whereas $25 \%$ considered the impact of the reuse of the Basilica of St. Peter the Apostle to be "poor" (Figure 9). The safety perception can be affected by an adaptive reuse project and it is an important indicator of the wellbeing category [54] linking the project not only to the transformation of the space, but also to the human capital.

The sample of respondents deemed significant the change of use of the area as a consequence of the reuse of the Ancient Market (25\% "good", 25\% "excellent"), while in relation to the reuse of the Basilica of St. Peter the Apostle only a low percentage expressed a positive perception (8.3\% "excellent", 8.3\% "good") (Figure 10). The intended uses of both projects were considered compatible with the characteristics of the buildings. In fact, both the Ancient Market (41.7\% "excellent", 25\% "good") and the Basilica of St. Peter the Apostle (33.3\% "excellent", 50\% "good") reached a great consensus (Figure 10). So, there was a positive perception of the adaptive reuse projects with respect to the objectives of protecting the materic-constructive and morphological-dimensional characteristics (identified by De Medici as criteria for evaluating projects for reuse [55]). 
At what level do you consider that there has been a change in the vocation of use of the area after the reuse project?

I don't know - Scarce $\bullet$ Moderate $\bullet$ Good Excellent

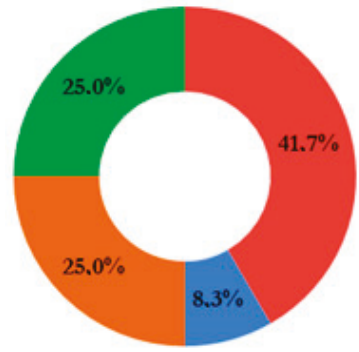

(a1) Ancient Market

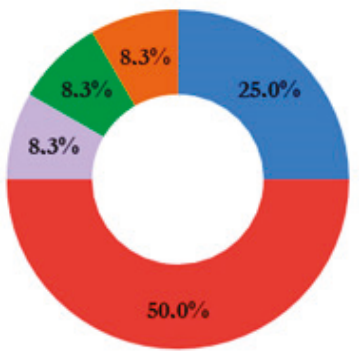

(b1) Basilica of St. Peter the Apostle

To what extent do you consider that the intended use is compatible with the building?

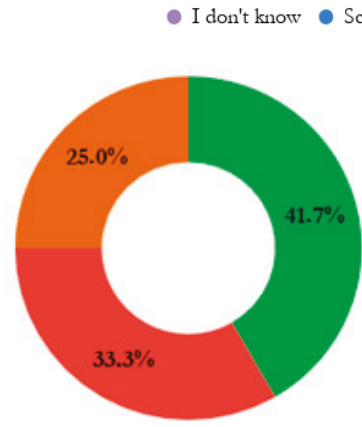

(a2) Ancient Market

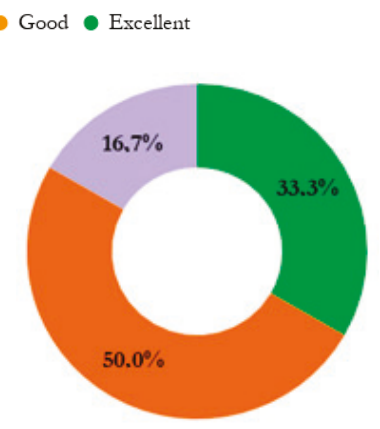

(b2) Basilica of St. Peter the Apostle

To what extent do you consider that the intended use chosen for the reuse project is adequate to meet needs of the area?

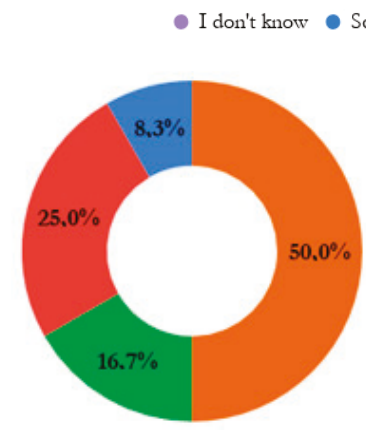

(a3) Ancient Market

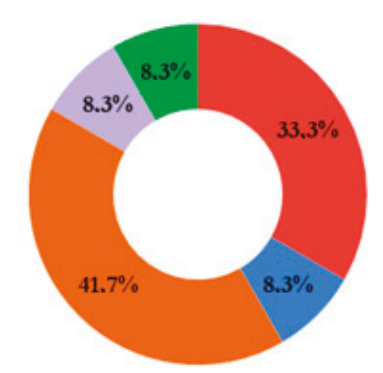

(b3) Basilica of St. Peter the Apostle

Figure 10. Results of the survey. 
The new functions play a key role in adaptive reuse projects as they should meet and satisfy the need of our ever-changing society and, at the same time, not to compromise the possibility of future generations to enjoy the resource. The new intended use of the Ancient Market was considered adequate for satisfying the local community needs (16.7\% "excellent", $50 \%$ "good"). Concerning the Basilica of St. Peter the Apostle, the number of positive answers was lower $(8.3 \%$ "excellent", $41.7 \%$ "good") (Figure 10). Indeed, 60\% and $40 \%$ of the respondents would have chosen the same new intended use respectively for the Ancient Market and the Basilica of St. Peter the Apostle. Other respondents proposed to enrich the Ancient Market with the retail of typical products or to consider a new function such as a municipal library. Concerning the Basilica of St. Peter the Apostle, it was proposed to integrate the chosen function with that of museum for the exhibition of religious collections, preserved in the Curia.

Overall, the reuse project of the Ancient Market was assessed to be almost as successful $(41.7 \%$ "good", 8.3\% "excellent") by the sample interviewed, while the project of the Basilica of St. Peter the Apostle was considered successful by only $30 \%$ (Figure 11). The answers that considered the success of such projects to be "scarce" were equal to $16.7 \%$ for the first and $25 \%$ for the second.

The panel of experts recognized in both projects a high capacity to conserve the cultural value of the building (Ancient Market: 50\% "excellent", 33.3\% "good"; Basilica of St. Peter the Apostle: 75\% "excellent", 16.7\% "good") (Figure 11). Furthermore, after the two interventions both the buildings remained almost recognizable, preserving their identity (Ancient Market: 75\% "excellent", 16.7\% "good"; Basilica of St. Peter the Apostle: 83.3\% "excellent", 8.3\% "good") (Figure 11). The questions of the survey concerning the perception of the recognizability of the buildings after the interventions and the ability to preserve the cultural value were related to the protection of the perceptual-cultural characteristics. Therefore, these results refer to the recognizability of the transformations (understood as the possibility of clearly distinguishing the new elements from pre-existence), acceptability of the transformations (understood as the ability to ensure that changes in the intended use and interventions upon the existing ones are admissible for the client and for the direct, indirect and potential users of the building) and respect for the collective memory (understood as the ability to ensure that the transformations do not alter the recognizability of the asset as an element representative of the identity of social groups, which attribute specific symbolic values to it) [55]. In fact, as mentioned in the previous paragraphs, the reuse project should, first of all, ensure the preservation of the intrinsic value of the building (representing the "limit in managing change" [5]), its identity and recognizability and ensure its enjoyment by future generations.

In both case studies analysed, as highlighted by the survey, some issues could be improved to enhance the success of the interventions. Among these, the need to increase the activities carried out in the buildings (in terms of type and duration) was a priority, guaranteeing continuity of use and balancing underutilisation characterising the two buildings today (in particular the Basilica of St. Peter the Apostle). Moreover, as underlined by the interviewees, the management of both buildings represented a weakness to be faced through maintenance and improvement of accessibility. The long-term success of a reuse project depends not only on the project itself, but also on a number of factors linked to its functioning and use. Therefore, it does not end with the project, but covers the entire life cycle of the "transformed" building, including aspects related, for example, to management and maintenance which, if not properly implemented, can produce negative impacts. 
At what level do you think that the reuse project was a success?

- I don't know $\bullet$ Scarce $\bullet$ Moderate $\bullet$ Good $\bullet$ Excellent

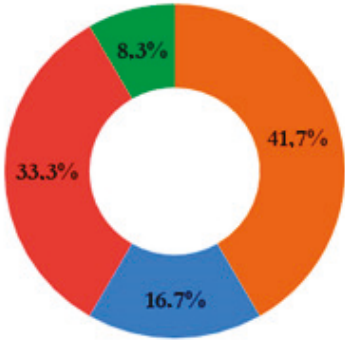

(a1) Ancient Market

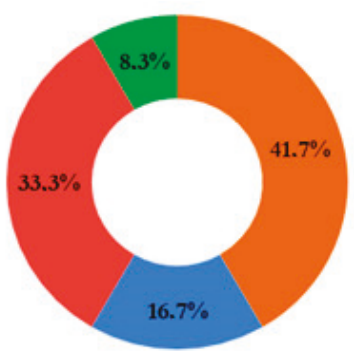

(b1) Basilica of St. Peter the Apostle

At what level do you think that the reuse project has contributed to preserving the cultural value of the building?

I don't know - Scarce $\bullet$ Moderate $\bullet$ Good Excellent

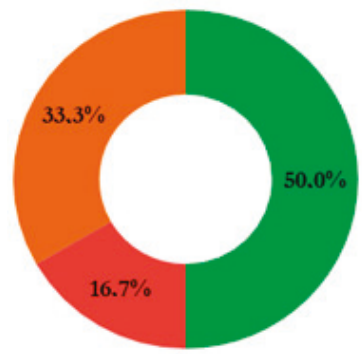

(a2) Ancient Market

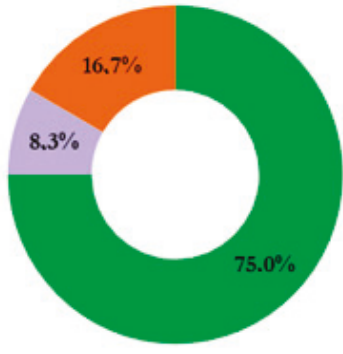

(b2) Basilica of St. Peter the Apostle

At what level do you consider that the project actions have been invasive for the building?

- I don't know - Scarce $\bullet$ Moderate Good Excellent

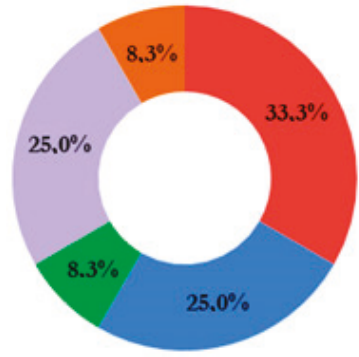

(a3) Ancient Market

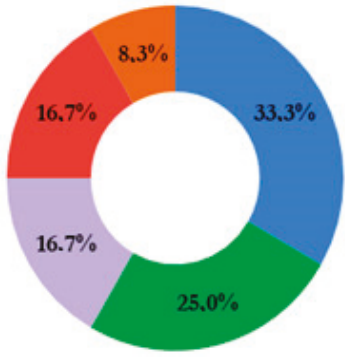

(b3) Basilica of St. Peter the Apostle

Figure 11. Cont. 
At what level do you think that the building is still recognisable in its original features after the reuse project implementation?

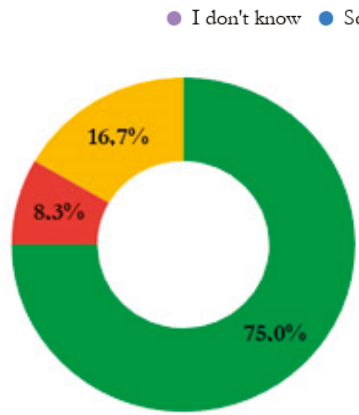

(a4) Ancient Market
- Moderate Good Excellent

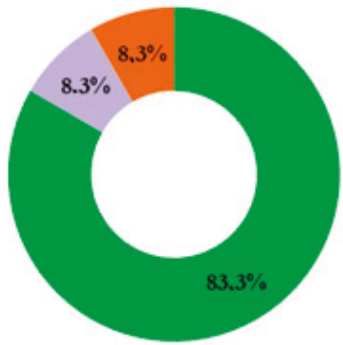

(b4) Basilica of St. Peter the Apostle

Would you have chosen a different use for the building?

- Yes No I don't know

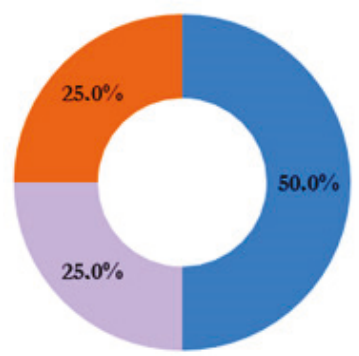

(a5) Ancient Market

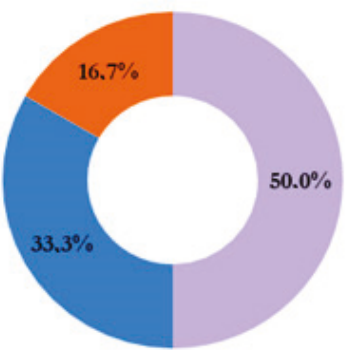

(b5) Basilica of St. Peter the Apostle

In relation to the transformation of the building, do you think that some aspects could be improved?

- Yes - No I don't know

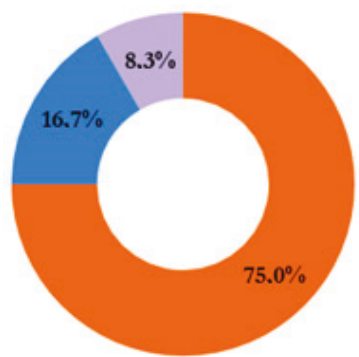

(a6) Ancient Market

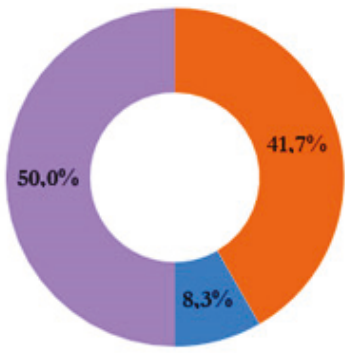

(b6) Basilica of St. Peter the Apostle

Figure 11. Results of the survey.

\section{Conclusions}

The global scientific community and the main international institutions recognise the role of cities and their cultural heritage for the sustainability of our future. Abandoned or under-used buildings are 
both a threat to urban quality [56] and a resource for triggering local development processes [54,57]. According to the circular economy model, adaptive reuse complies with sustainable management strategies for urban regeneration, as it helps to reduce the consumption of soil and resources that would be needed to construct new buildings.

The research discussed in the present paper investigates the impacts of adaptive reuse of heritage buildings, in terms of preserving the identity of cultural heritage, protecting its intrinsic values, and enhancing, at the same time, the development dynamics of the urban area in which the reuse project is implemented [58]. In particular, previous studies by the authors identify indicators able to describe the impacts of conservation, rehabilitation and adaptive reuse on heritage buildings and the urban context $[54,55]$. These research studies have represented the starting point to verify the following specific aspect of building reuse: how much a new function in an urban area can change its quality and trigger new value chains. The indicators selected allow verifying the compatibility of the project transformations in terms of safeguarding a building's identity, by preserving its shape, its construction systems, its materials, and above all the relationship between the building and the urban context. This investigation has been made possible by choosing two case studies which allow many of the variables involved in the adaptive reuse projects, such as the urban context, the design approach, the implementation date, the client, and the public/private use to be kept constant.

The results highlight that the choice of a new function for an abandoned building should be based on the attraction power of the building to its users, as well as on a prolonged and continuous attractiveness, both during the day and over the years. An unsuccessful reuse in terms of power of attraction condemns the building to a new abandonment.

As an additional validation of the outcome of the comparison between the two selected case studies, we can compare the impacts of the projects of the Ancient Market and the Basilica of St. Peter the Apostle with the effects of the settlement of the Faculty of Architecture on the island of Ortigia, in the same years. As shown by De Medici, Riganti and Viola [51], the Faculty of Architecture in Ortigia is an exceptional example because the university moved into old buildings without adapting them with a reuse project in the old town centre of Siracusa. Nevertheless, the establishment of academic research and teaching activities has significantly changed the dynamics of development of the area, triggering a virtuous process "based on knowledge sharing and resources' protection that promoted processes of social engagement and institutional empowerment for both new residents and entrepreneurs" [51] (p. 1).

Therefore, a reuse project does not necessarily have positive impacts on the abandoned building and its context. The quality of the reuse intervention depends both on the quality of the architectural project and on the adequacy of the new intended use both in relation to the building itself and to the urban context in which it is located. It has to be able, in a circular perspective, to satisfy the needs of the community, keep the building "alive" (use value) and, at the same time, preserve its intrinsic value.

Author Contributions: Conceptualization, S.D.M., P.D.T. and F.N.; Methodology, S.D.M., P.D.T. and F.N.; Formal Analysis, S.D.M. and F.N.; Investigation, S.D.M. and P.D.T.; Data Curation, S.D.M. and F.N.; Writing-Original Draft Preparation, S.D.M., P.D.T. and F.N.; Writing-Review and Editing, S.D.M., P.D.T. and F.N. All authors have read and agreed to the published version of the manuscript.

Funding: This research received no external funding.

Conflicts of Interest: The authors declare no conflict of interest.

\section{References}

1. United Nations. Available online: https://www.un.org/development/desa/en/news/population/2018-revisionof-world-urbanization-prospects.html (accessed on 15 October 2019).

2. United Nations. Transforming Our World: The 2030 Agenda for Sustainable Development; Unied Nations: New York, NY, USA, 2015.

3. Nocca, F. Hybrid Evaluation Tools for Operationalizing Unesco Historic Urban Landscape Approach; University of Naples Federico II: Naples, Italy, 2017. 
4. United Nations. Draft Outcome Document of the United Nations Conference on Housing and Sustainable Urban Development (Habitat III); United Nations: New York, NY, USA, 2016.

5. UNESCO. Recommendation on the Historic Urban Landscape; UNESCO World Heritage Centre: Paris, France, 2011.

6. Hosagrahar, J.; Soule, J.; Fusco Girard, L.; Potts, A. Cultural Heritage, the UN Sustainable Development Goals, and the New Urban Agenda. ICOMOS: Paris, France, 2016; Available online: http://www.usicomos. org/wp-content/uploads/2016/05/Final-Concept-Note.pdf (accessed on 15 October 2019).

7. Potts, A. The Position of Cultural Heritage in the New Urban Agenda A Preliminary Analysis Prepared for ICOMOS; ICOMOS: Charenton-le-Pont, France, 2016.

8. European Commission. Communication from the Commission to the European Parliament, the Council, the European Economic and Social Committee and the Committee of the Regions. Towards an integrated approach to cultural heritage for Europe. Brussels, 22.7.2014; 2014. Available online: https://ec.europa.eu/assets/eac/ culture/library/publications/2014-heritage-communication_en.pdf (accessed on 12 September 2019).

9. Stahel, W.R. The circular economy. Nature 2016, 531, 435-438. [CrossRef] [PubMed]

10. Plevoets, B.; Van Cleempoel, K. Adaptive Reuse of the Built Heritage: Concepts and Cases of an Emerging Discipline; Routledge: Abingdon, UK, 2019; pp. xvii-xix.

11. Douglas, J. Building Adaptation; Butterworth-Heinemann Publishing: London, UK, 2002.

12. European Commission. Communication from the commission to the European parliament, the council, the European economic and social committee and the committee of the regions. In Closing the Loop-An EU Action Plan for the Circular Economy; European Commission: Brussels, Belgium, 2015.

13. Cunnington, P. Change of Use: The Conversion of Old Buildings; Alpha Books: London, UK, 1988.

14. Plevoets, B.; Van Cleempoel, K. Adaptive reuse as a strategy towards conservation of cultural heritage: A survey of 19th and 20th Century theories. In Proceedings of the IE International Conference 2012 Reinventing Architecture and Interiors: The Past, the Present and the Future, London, UK, 29-30 March 2012.

15. Powell, K. Architecture Reborn: Converting Old Buildings for New Uses; Rizzoli International Publications: New York, NY, USA, 1999.

16. Pinto, M.R. Il Riuso Edilizio: Procedure, Metodi Ed Esperienze; UTET Libreria: Torino, Italy, 2004.

17. Cantacuzino, S. New uses for old buildings. Arch. Rev. 1972, CLI, 262-323.

18. Markus, T.A. Building Conversion and Rehabilitation: Designing for Change in Building Use; Butterworth: London, UK, 1979.

19. National Trust for Historic Preservation. Old \& New Architecture: Design Relationship; Society of American Historians Washington Metropolitan Chapter and American Institute of Architects, Ed.; The Preservation Press: Washington, DC, USA, 1980.

20. Plevoets, B.; Van Cleempoel, K. Adaptive Reuse of the Built Heritage: Concepts and Cases of an Emerging Discipline; Routledge: Abingdon, UK, 2019.

21. Plevoets, B.; Van Cleempoel, K. Adaptive reuse as a strategy towards conservation of cultural heritage: A literature review. In Structural Studies, Repairs and Maintenance of Heritage Architecture XII; Brebbia, C.A., Binda, L., Eds.; WIT Press: Southampton, UK, 2011; Volume 118, pp. 155-164.

22. Latham, D. Creative Re-Use of Buildings; Donhead: Shaftesbury, UK, 2000.

23. Robert, P. Adaptations, New Uses for Old Buildings; Princeton Architectural Press: New York, NY, USA, 1989.

24. Cramer, J.; Breitling, S. Architecture in Existing Fabric: Planning, Design, Building; Birkhäuser: Berlin, Germany, 2007.

25. Jäger, F.P. Old E New Design Manual for Revitalizing Existing Buildings; Birkhauser Verlag AG: Basel, Switzerland, 2010.

26. Highfield, D. The Rehabilitation and Re-Use of Old Buildings; Spon Press: London, UK; Taylor \& Francis: New York, NY, USA, 1987.

27. Gorse, C.; Highfield, D. Refurbishment and Upgrading of Buildings; Spon Press: London, UK; Taylor \& Francis: New York, NY, USA, 2009.

28. Rabun, J.; Kelso, R. Building Evaluation for Adaptive Reuse and Preservation; John Wiley \& Sons: Hoboken, NJ, USA, 2009.

29. Carroon, J. Sustainable Preservation: Greening Existing Buildings; John Wiley \& Sons: Hoboken, NJ, USA, 2010.

30. Fisher, A. New Life in Old Buildings; Verlag: Stuttgart, Germany; Zurich, Switzerland, 1992. 
31. Brooker, G.; Stone, S. Re-Readings Interior Architecture and the Design Principles of Remodelling Existing Buildings; RIBA Enterprises: London, UK, 2004.

32. Littlefield, D.; Lewis, S. Architectural Voices: Listening to Old Buildings; John Wiley \& Sons: Hoboken, NJ, USA, 2007.

33. Wong, L. Adaptive Reuse: Extending the Lives of Buildings; Birkhäuser: Basel, Switzerland, 2017.

34. Di Battista, V.; Fontana, C.; Pinto, M.R. Flessibilità E Riuso; Alinea: Firenze, Italy, 1995.

35. Galliani, G.V. Tecnologia Del Costruire; Alinea: Firenze, Italy, 2002.

36. Caterina, G. Tecnologia Del Recupero Edilizio; UTET: Torino, Italy, 1989.

37. Caterina, G.; Pinto, M.R.; Fabbricatti, K.; Oppido, S.; De Medici, S.; De Toro, P.; Bianchi, A. Reusing and Managing the "Real Albergo de' Poveri" of Naples: Evaluation and Re-Design for Improved Efficiency. In Proceedings of the CIB W70 International Symposium on the "Facilities, Management and Maintenance"; Shiem-Shin, D.T., Johnes, K., Hinks, J., Eds.; The Hong Kong Polytechnic University: Hong Kong, China, 2004; pp. 129-139.

38. Ferretti, V.; Bottero, M.; Mondini, G. Decision making and cultural heritage: An application of the Multi-Attribute Value Theory for the reuse of historical buildings. J. Cult. Herit. 2014, 15, 644-655. [CrossRef]

39. Pinto, M.R.; De Medici, S.; Senia, C.; Fabbricatti, K.; De Toro, P. Building reuse: Multi-criteria assessment for compatible design. Int. J. Des. Sci. Technol. 2017, 22, 165-193.

40. Ribera, F.; Nesticò, A.; Cucco, P.; Maselli, G. A multicriteria approach to identify the Highest and Best Use for historical buildings. J. Cult. Herit. 2019. [CrossRef]

41. Della Spina, L. Historical cultural heritage: Decision making process and reuse scenarios for the enhancement of historic buildings. In New Metropolitan Perspectives: ISHT 2018 Smart Innovation, Systems and Technologies; Calabrò, F., Della Spina, L., Bevilacqua, C., Eds.; Springer: Berlin/Heidelberg, Germany, 2019; Volume 100.

42. Fusco Girard, L.; Nocca, F.; Gravagnuolo, A. Matera: City of nature, city of culture, city of regeneration. Towards a landscape-based and culture-based urban circular economy. Aestimum 2019, 74, 5-42.

43. Simons, R.A.; Choi, E. Adaptive Reuse of Religious Buildings and Schools in the US: Determinants of Project Outcomes. Int. Real Estate Rev. 2010, 13, 79-108.

44. Fusco Girard, L.; Nocca, F. Moving Towards the Circular Economy/City Model: Which Tools for Operationalizing This Model? Sustainability 2019, 11, 6253. [CrossRef]

45. Amit-Cohen, I. Synergy between urban planning, conservation of the cultural built heritage and functional changes in the old urban center-The case of Tel Aviv. Land Use Policy 2005, 22, 291-300. [CrossRef]

46. Adorno, S. (a cura di) Syracuse 1880-2000: Città, Storia, Piani; Marsilio: Venezia, Italy, 2005.

47. Adorno, S. La Produzione di uno Spazio Urbano: Syracuse Tra Ottocento e Novecento; Marsilio: Venezia, Italy, 2005.

48. Cabianca, V. Vent'anni di Utopia Urbanistica a Syracuse; La Casa del Nespolo: Roma, Italy, 2013.

49. Urbact Syracuse Urbact-Driving Change for Better Cities. Available online: https://urbact.eu/syracuse (accessed on 21 October 2019).

50. Orlando, M. Le molteplici facce della periferia urbana: Il caso della città di Siracusa. In Proceedings of the Integrazione Delle Politiche: Territori E Città Del Mezzogiorno. Quante periferie, Convegno Nazionale Dell'inu Campania, Napoli, Italy, 23-24 March 2007; pp. 1-7. Available online: http://www.planum.net/ download/marilena_orlando-pdf (accessed on 20 October 2019).

51. De Medici, S.; Riganti, P.; Viola, S. Circular economy and the role of universities in urban regeneration: The case of Ortigia, Syracuse. Sustainability 2018, 10, 4305. [CrossRef]

52. Faraci, G. Recovery and enhancement of the historic centre of Syracuse: A balanced strategy of identity and development, with a positive impact on the local community. In Proceedings of the II International Conference on Best Practices in World Heritage: People and Communities, Menorca, Spain, 29 April-2 May 2015; Universidad Complutense de Madrid, Servicio de Publicaciones: Madrid, Spain, 2015; pp. 738-757.

53. Pagnano, G. Analisi e definizioni generali del piano particolareggiato di Ortigia. Recuperare 1989, 40, $164-173$.

54. Nocca, F. The role of cultural heritage in sustainable development: Multidimensional indicators as decision-making tool. Sustainability 2017, 9, 1882. [CrossRef]

55. De Medici, S.; Pinto, M.R.; Viola, S. Quality principles for heritage-led regeneration. The Ortigia case study. In Proceedings of the REHAB 2019-4th International Conference on Preservation, Maintenance and Rehabilitation of Historic Buildings and Structures, Guimarães, Portugal, 17-19 July 2019; pp. 83-92.

56. Greffe, X. Is heritage an asset or a liability? J. Cult. Herit. 2004, 5, 301-309. [CrossRef] 
57. De Medici, S.; Senia, C. Enhancement of Abandoned Buildings: Rudinì Winery in Pachino; FrancoAngeli: Milano, Italy, 2014.

58. Della Spina, L.; Calabrò, F. Decision Support Model for Conservation, Reuse and Valorization of the Historic Cultural Heritag. In Computational Science and Its Applications_ICCSA 2018. Lecture Notes in Computer Science; Gervasi, O., Murgante, B., Misra, S., Stankova, E., Torre, C.M., Rocha, A.M.A.C., Taniar, D., Apduhan, B.O., Tarantino, E., Ryu, Y., Eds.; Springer: Cham, Switzerland, 2018; pp. 3-17.

(C) 2019 by the authors. Licensee MDPI, Basel, Switzerland. This article is an open access article distributed under the terms and conditions of the Creative Commons Attribution (CC BY) license (http://creativecommons.org/licenses/by/4.0/). 



\title{
Time Overrun in Public Works-Evidence from North-East Italy
}

\author{
Giuliano Marella and Valentina Antoniucci * \\ ICEA, Department of Civil, Architectural and Environmental Engineering, University of Padova, via Venezia 1, \\ 35131 Padova, Italy; giuliano.marella@unipd.it \\ * Correspondence: valentina.antoniucci@unipd.it
}

Received: 15 October 2019; Accepted: 4 December 2019; Published: 10 December 2019

\begin{abstract}
Cost and time overruns in public mega-projects have been widely studied and considered as interdependent factors in the literature on project management and the public economy. On the other hand, small-scale projects for public works (costing under $€ 100$ million) are far more common and contribute to transforming cities and territories even more than mega-projects. Is the development of these kinds of projects affected in the same way by overrun issues? Do cost and time overruns always go hand in hand? The present contribution tries to answer these questions by means of an empirical study on a dataset of 4781 small public works planned and built in the Veneto Region (north-east Italy) from 1999 to 2018. Specifically, the analysis refers to the stage of development when the decision is made to outsource the work, that is, after the project's design and before its construction. Our sample of data is considered both as a whole and clustered by period, cost, contractor and category of work. The results of our analysis and statistical modeling are counterintuitive, suggesting that time overruns do not depend on the cost dimension, whereas norms and regulations play a crucial part in extending the duration of public works. The threshold by law of 1 million $€$ costs implies time-consuming procedures to verify abnormal offers in the bid, that double the average award time from 244 days to 479 days.
\end{abstract}

Keywords: risk assessment; public investment; economic feasibility; time overrun; public works

\section{Introduction}

A large body of literature is devoted to the adequacy of cost and time assessments for major public projects, focusing specifically on the cost overrun of such projects (see, among others, References [1-3]). Nevertheless, the performance of the project and design of public works does not seem to improve, regardless of location or typology of work. The present contribution is focused on the time needed for the procedures of the awards by public authorities, which is one of the less-studied phases of the development of public work. Specifically, the aim is to verify whether the law's requirements affect the time to fulfill the awards. In times of scarce economic resources and public funds, cost and time overruns are particularly damaging to communities. Worse still, "particular groups, who are often already disadvantaged, are sometimes forced to carry a disproportionate share of negative environmental and social impacts from projects that do not even deliver the promised benefits" [1]. The purchase of works and services by public authorities guarantees the homogeneous urban and regional development of cities and territories. Inefficiencies in public procurement consequently damage individuals and communities, while an efficient delivery of public works may "hamper the full satisfaction of public needs" ([4], p. 809). The cost and time overrun of projects is a relevant issue all over the world, affecting all categories of works, from civil engineering projects like highways, dams and railways to urban landmarks such as theaters, libraries and to urban brownfield restoration works.

Public works are not just a public service to the communities and they are also one of the most relevant issues for the sustainability of the National public economy. Public procurement represents in 
the OECD's Countries the $12 \%$ of Gross Domestic Product (GDP) (while in the EU public procurement is the $14 \%$ of GDP) and the $29 \%$ of government expenditure on average, which is a total amount of 6 trillion euros per year [5]. Looking at the values and goals of the public economy, "public procurement has been included in the Sustainable Development Goals, as part of Goal 12, Responsible consumption and production" ([5], p. 4).

Much attention has been paid to mega-projects [6], such as the Channel tunnel or Sydney Opera House, because of their vast impact on the public purse and their relevance to regional development. However, the idea that high cost overruns and long delays are only associated with major projects seems to be questionable. A few studies have already described different patterns of cost and time overrun depending on the size of the projects concerned [7] but the effectiveness of public procurement and development methods for small projects has been scarcely analyzed to date. It is well known, however, that most public works are relatively small in terms of cost, physical dimension and time of execution-especially in times of severe budget constraints-in Southern European countries, at least. Hence the relevance of assessing these kinds of project too, which mostly involve the ordinary maintenance of cities and territories. According to Bruzelius et al. [8], mega-projects can be defined as works with an expected lifetime of at least 50 years and implying investments of US $\$ 1$ billion and more. The bigger the project, the more difficult it is to forecast the uncertainty and volatility of features affecting the execution of the works. This means that any discussion of large-scale works often boils down to telling the unique story of a given construction and it is very hard to obtain a homogeneous historical track record of such works.

Small-scale projects should be more straightforward and more common than mega-projects, so it should be easier to forecast the cost and time of their completion. The aim of the present contribution is to see whether accountability for small projects is actually greater than for mega-projects by focusing on time overrun issues in public project management. In our scenario, we found that delays are at least partly due to Italian legislation on public works.

Unlike the case of other building works, a public works contract has to strictly follow the various phases imposed by legislation and the structure of public procurement. This aspect has rarely been considered in the existing literature, which focuses mostly on methods for predicting the uncertainty over time in a project's management. The procedures and timing required by public authorities have become more and more relevant in Italy, where the legislation governing public works has changed more than six times in the last 25 years.

To measure the influence of legislation on time overruns for public works in Italy, we collected and analyzed a large dataset concerning public works scheduled from 1999 to 2018 in the Veneto Region (north-east Italy). The maximum investment was always under $€ 100$ million and the works ranged from small urban maintenance works to highway projects.

The goals of this paper are-(1) to provide additional original data on the magnitude of time overruns in public works projects in northern Italy; (2) to highlight any differences between the time overruns affecting small-scale projects and the more often-studied major projects; and (3) to suggest an empirical framework to explain time overruns in Italy, focusing on the role of Italian legislation.

The remainder of the article is divided as follows-Section 2 provides an overview of the literature on the topic of time overruns in public works and a brief account of Italian legislation on public works; Section 3 illustrates our dataset and descriptive statistics; Section 4 discusses our model and the results of our statistical analyses; and the Conclusion provides some suggestions for further research on the topic.

\section{Schedule Delays-An Overview from Literature to Legislation}

\subsection{Time Overrun in the Scientific Literature}

Time overruns are generally represented as being a consequence of the more important project management issue, cost overrun, which has been amply investigated in the literature on public and private works. So, cost and time overruns are seen as related in most theoretical works (see, for 
instance, References [1,2,7]) and empirical research (see, among others, References [5,8-11]), though some studies, mostly developed in emerging countries, have addressed cost and time overruns as two separate issues [12-15].

Studies on underperformance in public works can be divided into three main groups. One comprises research conducted by modeling samples of project data to predict increases in the costs and time required to complete them [16-20]. Among others, the methods most often used in such studies are-fuzzy logic "to model the relationships between the characteristics of a project and the potential risk events that may occur" ([18], p. 503); econometric analyses, such as the multistep approach [21-23], the three stages square technique [24] or the stochastic Global Cost [25], which can be used to estimate the effects of factors associated with the contract bidding process, the type of project and the design; and artificial neural networks [26,27]. This kind of research is not extensively discussed here because it is beyond the scope of the present work but it is worth mentioning that the most common "root causes" of rising costs and delays are the size of the project and an underestimation, in the project design and development phase, of risks relating to conditions on-site [28,29]. The second group of studies examines the main causes of cost and time overruns mostly taking a qualitative approach, such as structured and semi-structured interviews with the actors involved in construction industries $[12,15,30]$. This type of analysis probably accounts for the bulk of the international literature and it mainly generates a taxonomy of causes, divided into categories ranging from project design to the structure of public contracts. The third group of studies includes statistical analyses and efforts to design a theoretical framework for cost and time overruns [31-33]. A general consensus identifies the underestimation of risks and uncertainties as the core reason for rising costs and delays, especially in major and mega-projects. Flyvbjerg defines public authorities' and developers' tendency to underestimate potential problems as an "optimism bias" [27] that prevails over any "strategic misinterpretation" [2] in explaining why the costs of a project are underestimated and its benefits are overestimated. Flyvbjerg's theoretical explanation is disputed by Love [34], who shifts the focus to distortions in competitive tendering to select project design firms and building contractors-the selection process tends to compress the fee excessively and to raise unrealistic expectations regarding design aspects and the paperwork involved. van Marrewijk et al. [33] also consider the restrictions imposed by project governance as interfering with the proper management of a project's development. This focus on the role of laws and norms in project management moves away from the majority of the literature on the topic of cost and time overruns, which tends to underscore the centrality of the design and planning phase of the process.

Far fewer research articles have concentrated on time overruns, in particular and on the role of procurement in causing them. After conducting a questionnaire-based survey, Larsen et al. [10] support the conviction that delays are largely due to poor project design and planning but they also highlight the different incidence of these factors on the time to completion, cost and quality of a project. Sepasgozar et al. [35] conduct a systematic bibliographic review on time delay in construction projects and highlight the potential of the new digital tools such as BIM to reduce the delays. Assaf and Al-Hejji [30] analyze major project delays in emerging economies. A survey conducted on owners, consultants and contractors identified several factors affecting time constraints-all three parties agreed that the most important causes of time overruns were changes in government regulations and laws, job site management issues and accidents during the construction phase. They also mentioned the relevance of workforce quality and productivity. These are issues more likely to affect building works in emerging economies than in Western countries (where the literature has focused on shortcomings in planning and an underestimation of the risks in the design phase). Nevertheless, studies conducted in the Far East have also pointed to the role of contractual commitments and the need to avoid litigations between owners and contractors as crucial factors influencing the expected time to complete a development [36].

As for the Italian context, Guccio et al. [37] identify the dimension of the public government body involved as a key factor in time overruns. They report empirical research leading to the conclusion 
that projects promoted by local authorities took longer to complete than works commissioned by the country's central government. Along the same lines, when Gori et al. [4] analyzed a dataset concerning public works in Tuscany (central Italy) for local authorities, they found that "insufficient procurement experience is associated with a higher probability of incurring delays and longer work durations" (p. 822). Public works require a level of specialization that not all local authorities possess [38].

The present empirical analysis contributes to the literature on these issues, focusing on small and medium-sized projects and highlighting the role of national regulations on public works as a potential cause of delays.

\subsection{Italian Legislation on Public Works}

The so-called Code of Public Works, Services and Supply Contracts (Legislative Decree n. 50/2016) is the latest in a number of revisions of the regulations covering the awarding of public works to have been implemented in Italy over the last 50 years. The revisions were needed partly to comply with EU laws aiming to improve the "design" of contract awarding procedures and to enforce "principles of publicity, transparency and equal treatment" ([32], p. 210). Since 1999, the first year covered by our analysis, the legislation on this matter has changed significantly at least three times.

The Code essentially considers three main aspects-the phase of the works to be awarded (i.e., design, construction, and/or works management); the procedure for selecting the contractor; and the selection criteria. The Italian regulations envisage two types of agreement-the procurement contract; and the concession contract. The former relates to the construction phase or, rarely, to the executive project design and the construction. The latter covers construction and works management. The competition procedures covered by the Code are the most important aspects of the process for our purposes here because they can play a remarkable part in explaining delays in the completion of the works. There are four types of tender:

1. open procedures;

2. restricted procedures;

3. restricted procedures with negotiation;

4. negotiation without a call for tenders.

The first, open procedure is one of the most often used. There is a call for tenders containing a detailed description of the object of the contract and the characteristics required of potential bidders. Fulfillment of all the requirements is only ascertained when the bids are examined. The restricted procedure is used for works costing less than $€ 1.5$ million. It involves an initial prequalification phase to ascertain what requisites a contractor will need and then identify at least five enterprises to invite on the basis of their expertise and technical endowment. Then only selected enterprises are invited to a second bidding phase. In the third type of procedure, invited enterprises make a bid on the grounds of which negotiation is conducted to improve the proposal both technically and economically.

As Decarolis and Giorgiantonio explained [39], in Italy there is not much difference between open and restricted procedures because all applicants fulfilling the requirements must be invited to make a bid when the value of the work is less than $€ 40$ million.

The last procedure applies to a very limited number of cases, mostly in emergencies or in cases where only one enterprise is capable of doing the work. The public administration asks the enterprise in question to make a bid for the work and then negotiates with them to improve on their proposal.

In the case of public-private partnerships (PPP), for project financing, for instance, the awarding of the contract is divided into two phases and the second phase involves a restricted procedure with negotiation.

There are basically two criteria for assigning the works - the "lowest price" and the "economically most advantageous offer." Clearly, the former only considers the lowest price of the works. The latter takes into account a set of other aspects of the bids as well as the price, such as the quality of the urban and project design, the duration of the building works, energy cost savings and lower maintenance 
costs. The selection is based on a scoring rule. In both cases, administrations must check for abnormal tenders or abnormally low offers. Checking for excessively low offers is a long and complicated process that varies depending on the type of bidding procedure used. The contracting authority has to set a threshold for the abnormal offer and ask the bidder for clarification. The bidder can dispute the authority's observations. Abnormal offers must be examined for works costing more than $€ 1$ million, while for works costing less abnormal offers may be excluded automatically. This screening of bids may give rise to delays that are impossible to account for in the design or construction phases but the building work may be postponed depending on the duration of the contractor selection process.

It is worth mentioning that, for PPPs, the documentation needed to present a bid includes a demonstration of the value for money (VfM) of the proposed works. We do not discuss the question of $\mathrm{VfM}$ here because it is beyond the scope of the present contribution.

\section{Public Works in Italy and the Veneto Region-Sample Data and Descriptive Statistics}

The present analysis considers the time elapsing between the conclusion of a project's design phase and the beginning of the works, that is, all the time it takes to complete the contract awarding procedure.

This enables us to isolate the phase in the timeline that is most affected by the norms and regulations governing the contract awarding process. Project design may be affected by technical and managerial issues, while the construction phase may come up against various problems, from design errors to a workforce's low productivity or lack of specialization.

We collected an original database from the Italian State's General Accounting Department covering 19,117 public works, planned and implemented between 1999 and 2018 in the Veneto Region in north-east Italy. As in similar Italian analyses [40], we chose to include unfinished works too. Otherwise, the sample could be biased by an over-representation of the more efficient (or smaller and/or more straightforward) projects. The database includes the following information:

- description of the work;

- location;

- $\quad$ category of works (e.g., infrastructure, soil protection, school and university buildings and so on);

- $\quad$ type of works (maintenance, restoration, renovation, new build and so on);

- $\quad$ state of progress (e.g., ongoing, completed);

- $\quad$ contractor (State, Region, municipality);

- $\quad$ estimated and actual dates of starting and completing each project design phase;

- $\quad$ estimated and actual dates of starting and completing construction;

- $\quad$ estimated and actual date of starting operation;

- $\quad$ estimated and actual costs.

The database is part of the Open Data on Italian public economy and finance collected by the State's General Accounting Department. Data on the single works are provided by the work's managers of each public contractor. So, often data are entered incomplete. We excluded projects for which we had incomplete data concerning the costs and each phase of the timeline, thus reducing the sample to 4781 public works. As shown in Figure 1 and Table 1, 55\% of the sample (i.e., 2625 projects) are extraordinary maintenance works, while new builds account for only $22 \%$ (1047 projects). Renovations of existing buildings involving a change of use amount to less than $9 \%$ and restoration work on historical buildings about $6.6 \%$. 
Table 1. Proportions of different types of project.

\begin{tabular}{ccc}
\hline Type of Project & Number of Projects & Relative Frequency (\%) \\
\hline Building extensions & 194 & 4.08 \\
Demolitions & 11 & 0.23 \\
Equipment and technology & 6 & 0.13 \\
improvement & 2.625 & 54.90 \\
Extraordinary maintenance & 1.047 & 21.90 \\
New builds & 155 & 3.24 \\
Other & 425 & 8.91 \\
Renovation with a change of use & 316 & 6.61 \\
Restoration & & \\
\hline
\end{tabular}

As shown in Table 2, the incomplete data we excluded from the sample has substantially the same proportion of the selected sample for the attribute "Type of project," with the clear predominance of extraordinary maintenance and new builds among other.

Table 2. Proportions of different types of projects of the incomplete and excluded data.

\begin{tabular}{ccc}
\hline Type of Project & Number of Projects & Relative Frequency (\%) \\
\hline Building extensions & 688 & 4.80 \\
Demolitions & 36 & 0.25 \\
Equipment and technology & 29 & 0.20 \\
improvement & 7.268 & 50.70 \\
Extraordinary maintenance & 3.441 & 24.00 \\
New builds & 738 & 5.15 \\
Other & 1.085 & 7.57 \\
Renovation with a change of use & 1.051 & 7.33 \\
Restoration &
\end{tabular}

We identified 33 categories of works (see Table 3 and Figure 2) but seven of them accounted for more than $70 \%$ of the sample. Road infrastructure is the largest category with 1365 works, followed by school buildings and buildings for social activities. Social infrastructure (urban and neighborhood theaters, libraries and cultural centers) make up $11.15 \%$ of the sample. These proportions are consistent with the trend for public works in northern Italy. Countrywide, the availability of public infrastructure varies enormously between north and south [41-43], so drawing comparisons on the whole of Italy would be misleading. 
Table 3. Proportions of categories of works.

\begin{tabular}{|c|c|c|}
\hline Category of Works & Number of Projects & Relative Frequency (\%) \\
\hline Agriculture and agro-industrial works, industrial plants & 4 & 0.10 \\
\hline Brownfield restoration & 14 & 0.30 \\
\hline Courts and penitentiaries & 11 & 0.20 \\
\hline Cultural heritage & 247 & 5.17 \\
\hline Defense department buildings & 11 & 0.20 \\
\hline Energy distribution & 66 & 1.38 \\
\hline Energy production & 38 & 0.80 \\
\hline Equipment and facilities for retail and service sectors & 12 & 0.30 \\
\hline Fishing facilities & 1 & 0.00 \\
\hline Public health buildings and facilities & 79 & 1.65 \\
\hline Housing & 298 & 6.23 \\
\hline ICT infrastructure & 8 & 0.20 \\
\hline Infrastructure-airport & 1 & 0.00 \\
\hline Infrastructure and facilities for industrial areas & 17 & 0.40 \\
\hline Multimodal transportation & 6 & 0.10 \\
\hline Offices and administrative buildings & 122 & 2.55 \\
\hline Protection, improvement and use of the environment & 120 & 2.51 \\
\hline Public security infrastructure & 13 & 0.30 \\
\hline R\&D works and infrastructure & 5 & 0.10 \\
\hline University R\&D projects & 1 & 0.00 \\
\hline Railways & 16 & 0.30 \\
\hline Religious sites & 20 & 0.40 \\
\hline Road infrastructures & 1365 & 28.55 \\
\hline Schools and buildings for social activities & 896 & 18.74 \\
\hline Social infrastructures & 533 & 11.15 \\
\hline Soil protection & 322 & 6.73 \\
\hline Sport, entertainment and free time activities & 339 & 7.09 \\
\hline Telecommunications infrastructures & 13 & 0.30 \\
\hline Tourist facilities & 2 & 0.00 \\
\hline Urban transportation & 10 & 0.20 \\
\hline Waste management & 47 & 0.10 \\
\hline Water resources and waste water & 111 & 2.32 \\
\hline
\end{tabular}

As for the previous feature, the category of works also has the same structure in the selected and the incomplete, excluded data (see Table 4). The latter also has the same seven prevalent categories, with mostly the same proportion of the selected data. 
Table 4. Proportions of categories of works of the incomplete and excluded data.

\begin{tabular}{|c|c|c|}
\hline Category of Works & Number of Projects & Relative Frequency (\%) \\
\hline Agriculture and agro-industrial works, industrial plants & 23 & 0.16 \\
\hline Brownfield restoration & 53 & 0.37 \\
\hline Courts and penitentiaries & 52 & 0.36 \\
\hline Cultural heritage & 615 & 4.29 \\
\hline Defense department buildings & 315 & 2.20 \\
\hline Energy distribution & 288 & 2.01 \\
\hline Energy production & 96 & 0.67 \\
\hline Equipment and facilities for retail and service sectors & 33 & 0.23 \\
\hline Fishing facilities & 7 & 0.05 \\
\hline Public health buildings and facilities & 315 & 2.20 \\
\hline Housing & 401 & 2.80 \\
\hline ICT infrastructure & 30 & 0.21 \\
\hline Infrastructure—airport & 10 & 0.07 \\
\hline Infrastructure and facilities for industrial areas & 112 & 0.78 \\
\hline Multimodal transportation & 17 & 0.12 \\
\hline Offices and administrative buildings & 648 & 4.52 \\
\hline Protection, improvement and use of the environment & 354 & 2.47 \\
\hline Public security infrastructure & 205 & 1.43 \\
\hline R\&D works and infrastructure & 42 & 0.29 \\
\hline University R\&D projects & 0 & 0.00 \\
\hline Railways & 146 & 1.00 \\
\hline Religious sites & 201 & 1.40 \\
\hline Road infrastructures & 3.676 & 25.64 \\
\hline Schools and buildings for social activities & 2.155 & 15.03 \\
\hline Social infrastructures & 1.359 & 9.48 \\
\hline Soil protection & 1.069 & 7.46 \\
\hline Sport, entertainment and free time activities & 1.005 & 7.01 \\
\hline Telecommunications infrastructures & 46 & 0.32 \\
\hline Tourist facilities & 20 & 0.14 \\
\hline Urban transportation & 50 & 0.35 \\
\hline Waste management & 353 & 2.46 \\
\hline Water resources and waste water & 529 & 3.69 \\
\hline
\end{tabular}

The contract awarding procedure takes 271 days on average. In other words, about nine months go by between the completion of the project design phase and the start of building work on site (Table 3). This time varies enormously, from just one day to about ten years. According to the Agency for Territorial Cohesion (Agenzia per la Coesione Territoriale) [40], the average time to assign works worth less than $€ 1$ million is about 5 months, so the Veneto Region seems to perform poorly compared with the national average, despite the public authority managing the procedure being reasonably well organized [44]. 
Sustainability 2019, 11, 7057

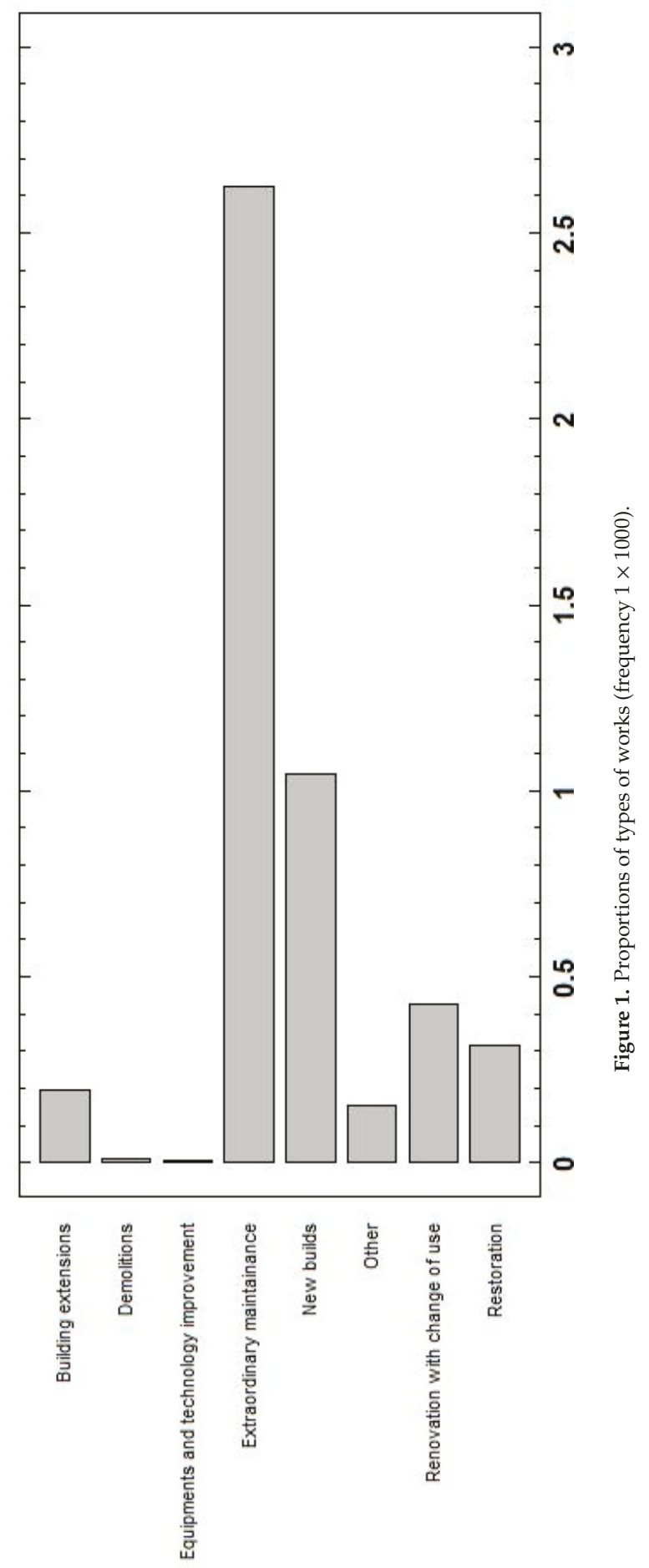



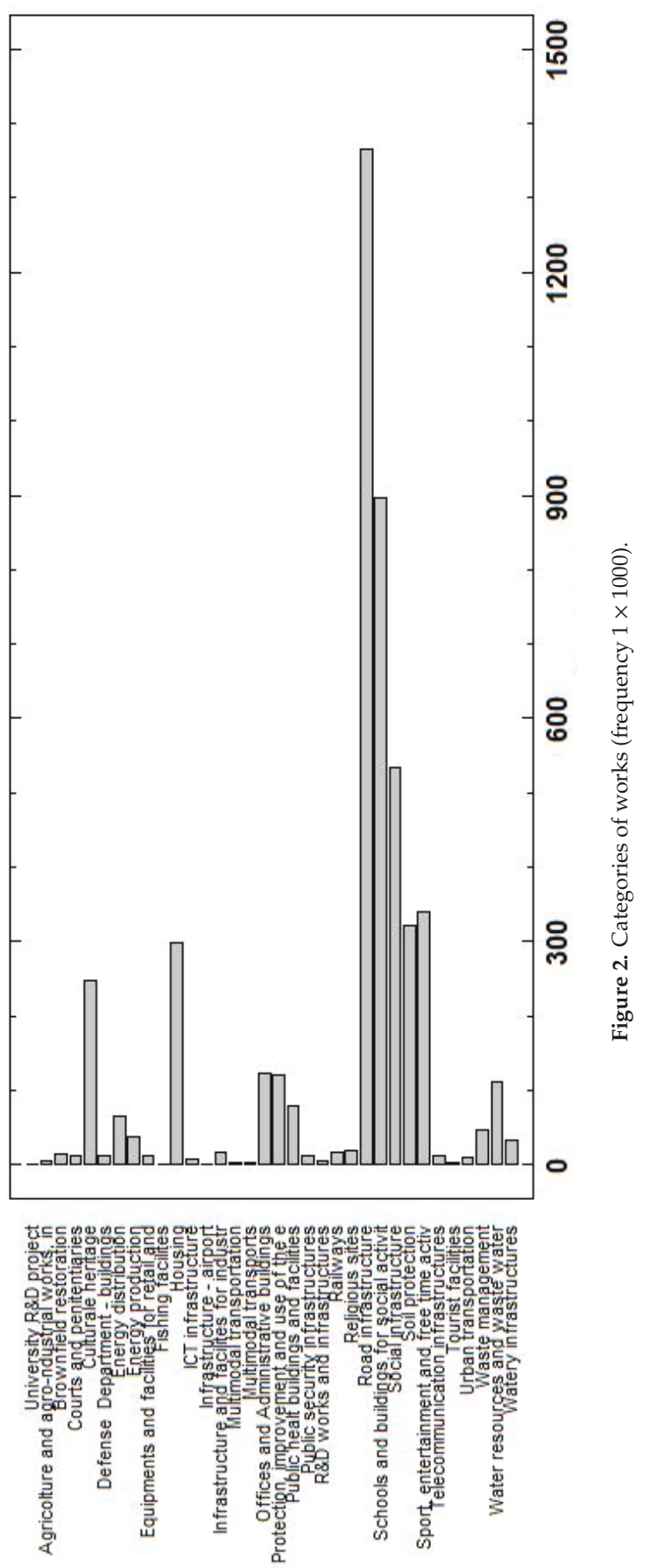
We used "population" as a proxy of the dimension of the public authority awarding the contract, assuming that it would be more structured and specialized, the larger the population it serves $[4,37]$. This may indicate the skills available for managing the contract awarding process and consequently of the time needed to complete the procedure. As shown in Table 5, the average population is 426,650, which is higher than that of any of the provincial capitals in the region. Although most of the contracts were awarded by local authorities ( $85.30 \%$, see Table 6 ), many of them (about 9\%) were promoted by territorial entities, such as Energy Service Companies (ESCOs) and utility consortia that cover larger territories than provinces. The Veneto Regional Authority and other regional bodies account for just $3 \%$ of the contracts awarded and national bodies (Ministries or the Italian Central Government) for just 9 contracts awarded in the Veneto Region during the period considered. There is marked variability in the 'local authorities' category, which reflects the different organizational capabilities of local government bodies.

Table 5. Descriptive statistics of cardinal variables.

\begin{tabular}{cccc}
\hline & $\begin{array}{c}\text { Time to Awarding } \\
\text { Contracts (Days) }\end{array}$ & Cost of Works $(\boldsymbol{\epsilon})$ & $\begin{array}{c}\text { Population Served by the Public } \\
\text { Authority (Number) }\end{array}$ \\
\hline No. & 4783 & 4783 & 4783 \\
Mean & 271 & 651,766 & 426,650 \\
Standard deviation & 306 & $2,821,999$ & $2,615,789$ \\
Coefficient of variation & $113.00 \%$ & $432.91 \%$ & $613.02 \%$ \\
Minimum & 1.0 & 104.72 & 201.0 \\
Maximum & 3673 & $83,820,524$ & $60,589,445$ \\
\hline
\end{tabular}

Table 6. Contracts awarded by public authorities in the sample data.

\begin{tabular}{ccc}
\hline Dimension of Public Authority & Number of Awards & Incidence (\%) \\
\hline Local authorities & 4080 & 85.30 \\
Provincial authorities & 116 & 2.45 \\
Territorial authorities & 430 & 9.01 \\
Regional authorities & 146 & 3.05 \\
National authorities & 9 & 0.19 \\
Total & 4781 & 100 \\
\hline
\end{tabular}

\section{The Threshold for Abnormal Offers-Clustering the Sample Data}

Given the variability of the sample and the different requirements for awarding contracts worth more or less than $€ 1$ million, we clustered the sample into two groups based on this $€ 1$ million cutoff.

The clusters confirm that small-scale works make up most of the sample, while the projects and works costing more than $€ 1$ million accounts for just $11 \%$ (see Table 7 ).

Table 7. Descriptive statistics for clusters of contracts worth more or less than $€ 1$ million.

\begin{tabular}{|c|c|c|c|c|c|c|}
\hline & \multicolumn{3}{|c|}{ Contract Worth Less Than $€ 1$ Million } & \multicolumn{3}{|c|}{ CONTRACT Worth More Than $€ 1$ Million } \\
\hline & $\begin{array}{c}\text { Time to } \\
\text { Award } \\
\text { Contract } \\
\text { (Days) }\end{array}$ & $\begin{array}{c}\text { Cost of } \\
\text { Works }(€)\end{array}$ & $\begin{array}{l}\text { Population } \\
\text { Served by } \\
\text { Public } \\
\text { Authority }\end{array}$ & $\begin{array}{l}\text { Time to } \\
\text { Award } \\
\text { Contract } \\
\text { (Days) }\end{array}$ & $\begin{array}{c}\text { Cost of } \\
\text { Works }(€)\end{array}$ & $\begin{array}{c}\text { Population } \\
\text { Served by } \\
\text { Public } \\
\text { Authority }\end{array}$ \\
\hline No. & 4244 & 4244 & 4244 & 537 & 537 & 537 \\
\hline Mean & 244.33 & 241,518 & 257,998 & 479.87 & $4,103,369$ & $1,755,459$ \\
\hline $\begin{array}{l}\text { Standard } \\
\text { deviation }\end{array}$ & 257 & 212,485 & 741,002 & 509 & $7,563,886$ & $7,394,556$ \\
\hline $\begin{array}{l}\text { Coefficient } \\
\text { of variation }\end{array}$ & $105.59 \%$ & $99.05 \%$ & $287.21 \%$ & $106.08 \%$ & $184.33 \%$ & $421.23 \%$ \\
\hline Minimum & 1.0 & 104.72 & 201.0 & 1.0 & $1,000,000$ & 755 \\
\hline Maximum & 2622 & 998,000 & $7,193,706$ & 3673 & $83,820,524$ & $60,589,445$ \\
\hline
\end{tabular}


Larger-scale works refer to a broader territory, although smaller works are also awarded on average by public bodies larger than the local authorities. The relevance of the $€ 1$ million contract value threshold emerges from the time taken to award the contracts, which is 244 days (about 8 months) for works worth less than $€ 1$ million but 480 days ( 16 months) for those worth more, that is, it takes twice as long to complete the contract awarding procedure for the latter.

Looking at the categories of works, nine categories account for more than $70 \%$ of the works in both contract value clusters but the incidence of the various categories differs significantly between the two. As shown in Table 8, "Road infrastructure" makes up 30\% of the works worth less than $€ 1$ million but only $16 \%$ of the costlier contracts. "Schools and buildings for social activities" and "social infrastructure" account for about $20 \%$ and $12 \%$ respectively of the cluster. For the works costing more than $€ 1$ million, the "Road infrastructure" and "Soil protection" are the largest categories (both making up more than $16 \%$ of the sample), followed by "Schools and buildings for social activities" and "Housing." This latter category accounts for less than $11 \%$ of the costlier projects and just under $6 \%$ of the works costing less than $€ 1$ million. The interpretation of the different incidence of each category in the two clusters is contentious. For instance, projects relating to soil protection and water resources tend to involve a broad territory and expensive activities but we might have expected an incidence of small-scale maintenance works in the "Housing" category higher than a mere 5.7\%. Projects relating to "Social infrastructure" and "Sports, entertainment and free time activities" are usually on a neighborhood scale, so they are unsurprisingly more relevant in the cluster of projects worth less than $€ 1$ million.

Table 8. Categories of works by cluster (works costing more vs less than $€ 1$ million).

\begin{tabular}{|c|c|c|}
\hline \multirow[t]{2}{*}{ Category of Works } & \multicolumn{2}{|c|}{ Relative Frequency (\%) } \\
\hline & $<€ 1$ Million & >€1 Million \\
\hline Cultural heritage & 4.85 & 7.64 \\
\hline Housing & 5.68 & 10.61 \\
\hline Protection, improvement and use of the environment & 2.43 & 3.17 \\
\hline Road infrastructure & 30.04 & 16.76 \\
\hline Schools and buildings for social activities & 19.56 & 11.92 \\
\hline Social infrastructure & 11.88 & 4.66 \\
\hline Soil protection & 5.54 & 16.20 \\
\hline Sport, entertainment and free time activities & 7.54 & 3.54 \\
\hline Water resources and waste water & 1.72 & 7.08 \\
\hline
\end{tabular}

\section{Discussion of the Statistical Analysis-The Relevance of Regulations and Norms}

We performed a simple regression to test whether the time taken to award a contract correlates with the cost of the project.

We tested this correlation on:

- $\quad$ the whole sample;

- the cluster of works costing less than $€ 1$ million;

- the cluster of works costing more than $€ 1$ million.

While the statistical significance of each variable is robust at $1 \%$ level, the adequacy of the model is unsatisfactory, with the $\mathrm{R}^{2}$ adj less than $10 \%$ (The $\mathrm{R}^{2}$ adj. is $1.93 \%$ for the whole sample, $4.04 \%$ for works costing less than $1 \mathrm{mil} €$ and $0.00 \%$ for works costing more than $1 \mathrm{mil} €$. Given the scarce magnitude of the $\mathrm{R}^{2}$ adj. we omitted the $p$-value for the independent variable.) This means that the variables do not contribute to explaining the phenomenon. In other words, in the sample considered here, there is not only no causal relationship but also no statistical correlation between time overruns and costs of the works. These results confirm the findings of other empirical research assessing cost and time overruns as separate issues [13-15]. We obtained the same results for the whole sample and when 
testing the model separately on the two clusters. Given the high variability of the data, we performed the same statistical analysis, clustering the sample by the contractor. We subdivided the sample into two groups-the first one contains works awarded by municipalities (n. 4080 works) and the second one, works awarded by the other public authorities (n. 701 works). Again, the results of regressions are unsatisfactory (The $\mathrm{R}^{2}$ adj. is $2.37 \%$ for the cluster with works awarded by municipalities and $1.01 \%$ for the works awarded by other public authorities. Given the scarce magnitude of the $\mathrm{R}^{2}$ adj. we omitted the $p$-value for the independent variables.).

As the dataset covers a lengthy period during which the legislation on the contract awarding procedure changed significantly three times, we divided the sample differently to check for bias relating to changes in the regulations. We considered three clusters:

- $\quad$ contracts awarded from 1999 to 2006;

- contracts awarded from 2006 to 2016;

- contracts awarded from 2016 to 2018.

The first cluster is the smallest, accounting for less than $1 \%$ of the sample. The second, covering the longest period makes up $76 \%$ of the whole sample and the third cluster includes $26 \%$ of the total dataset. The first cluster is too small to provide any robust statistical results while analyzing the other two clusters confirms the previous results. When we tried considering other independent variables-the cost of the works, the dimension of the public authority and the type of the works-the correlations are not strong enough to explain the time take to award the contracts The $\mathrm{R}^{2}$ adj. is $10.87 \%$ for the period 1999-2006, 3.02\% for the period 2006-2016 and 4.45\% for the period 2016-2018. Given the scarce magnitude of the $\mathrm{R}^{2}$ adj. we omitted the $p$-value for the independent variables. Given the substantial diversity of the works by type and category, we refined the sample again to test for the heterogeneity of the sample. Thus, we clustered the sample considering just the two most frequent categories of works, that are "Road infrastructures" (n. 1365 works) and "Schools and buildings for social activities" (n. 896 works) and we performed a multivariate regression for each group using cost of works and the dimension of public authority as independent variables. However, again, also in this case, the results are inadequate to demonstrate a statistical correlation between the award time and cost of works The $\mathrm{R}^{2}$ adj. is just $0.75 \%$ for the data of road infrastructures and is $5.04 \%$. Again, we omitted the $p$-value for the independent variables.

This result partly confirms the previously-mentioned literature [12-15,45] - the cost and the duration of public works, and-in the present sample - the time taken to award the contract is unrelated. The dimension of the public authority is also unrelated to the time taken to award the contract, even though the contractor's specialization is recognized as a crucial feature affecting the efficiency of the contract awarding procedure.

So what decides the amount of time it takes to award a contract? Clustering our sample of data by the abnormal offer cutoff shows that current Italian regulations on the matter, ceteris paribus, significantly influence this variable. This is also confirmed when we compared the first group of clusters (by costs) with the second ones (by time)—what does not change between the groups and represents the different regulations governing the contract awarding procedure, is the cutoff for abnormal offers (which is $€ 1$ million).

In short, the procedure needed to deal with the formalities associated with the examination of abnormal offers may double the time it takes to award a contract (244 days against 479 days for works over $€ 1$ million costs), in the sample considered here at least, as it is shown in the descriptive statistics. In fact, the procedure is time-consuming but also paves the way to litigations between bidders, that very often lead to the contract awarding procedure being suspended and/or the winner being changed by a decision of the courts. The Italian Government [40] also recognizes that the "passage time" takes up a mean $45.7 \%$ (range: $40.5 \%$ to $55.3 \%$ ) of the time needed to complete contract awarding procedures. The relevance of procedures required by law is a feature of time overrun scarcely studied. The results are underpinned by the several statistical tests performed-none of them have 
demonstrated a statistical correlation between award duration and cost, nor for the whole sample, nor for the diverse cluster tested. The findings confirm the part of the existing literature that considers time overrun unrelated with cost. This feature may not be valid for each phase of the duration of the works-during the construction phase, an increase of the costs is most of the time correlated with a time increase, due to the above-mentioned design errors and underestimation of risks, that is, in the construction site. Focusing on the procedure's duration, costs (increasing or not) may be irrelevant to the time increase, as the analysis here presented confirm. The compliance of estimated time depends on the requirements to perform the award procedures. These requirements do not change according to the kind of contractors, because they vary just for the ex-ante estimated work cost (above and below the 1 million euros threshold, as shown in Figure 3). Some public authorities, that is, small municipalities, do not have the technical expertise and the minimum staff too, to accomplish in time the procedural steps. Also, this kind of municipalities may be involved in the development of major works and/or infrastructures that they are not able to manage in time properly. Nevertheless, further and in-depth analysis is needed to extend the finding, here limited to a specific Italian Region. To generalize the result, the findings should be validated for Northern Italy at least.

\section{Conclusions}

Although there is plenty of literature on cost overruns in major and mega-projects, it does not seem to have improved the situation concerning the increase in costs and delays in completing public works. Public works are a relevant part of the National public economy and the efficiency of these investments contributes significantly to the sustainability of public accounts and finances. Moreover, the strategy adopted in public works may increase the spread of sustainable development and behavior at the local and territorial level. While there are several factors affecting the completion of public work-most of them in the design and construction phase, that is, at the beginning and end of the process—very little attention has been devoted to what happens in between. The present work considers the time taken to award contracts, which cannot be explained by considering the cost overrun (incurred largely during the construction phase, after the contract has been awarded). We provided empirical findings on the time taken to award contracts in a sample of 4781 public works projects planned and implemented from 1999 to 2018 in the Veneto Region, in north-east Italy, in an effort to find an explanation for time overruns in these procedures.

Our elaborations show that even small projects, defined here as public works costing less than $€ 100$ million, are liable to time overrun and this stems mostly from the administrative demands of laws and regulations on public works $[46,47]$. In particular, when we divided our sample into works using the $€ 1$ million cutoff for monitoring abnormal offers, the time taken to award public works below the cutoff is 244 days, while it almost doubles to 479 days for those above the cutoff (see Figure 3).

To check whether this difference in timeline is coincidental, we performed several statistical multivariate regressions where the time taken to award contracts is the dependent variable and the independent variables are other features conventionally assumed to affect time overruns, that is, the cost of the works, the dimension of the public authority and the category of the works. 


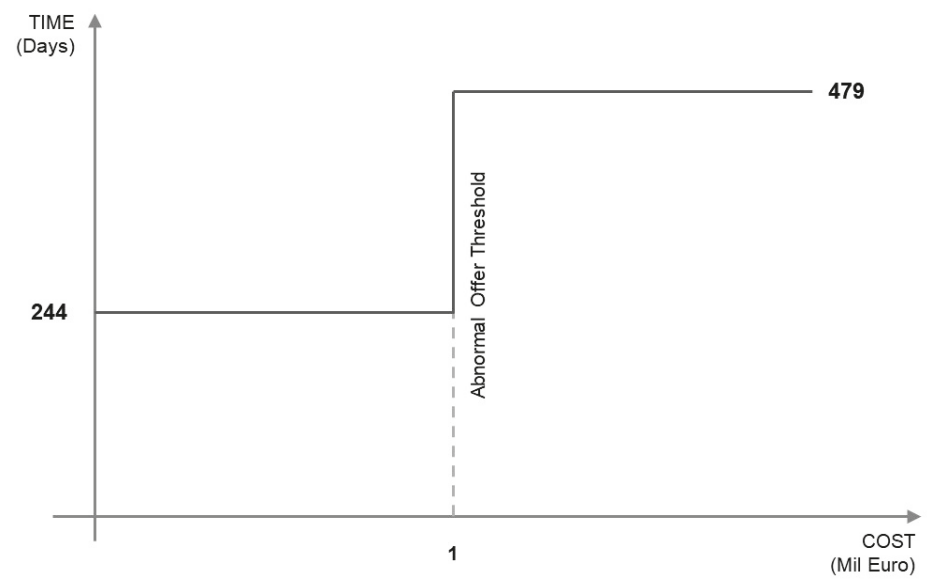

Figure 3. The effect of the abnormal offer threshold on time taken to award contracts.

We tested this model with five different specifications-in the whole sample; in two clusters by contract value (below vs. above $€ 1$ million); in two clusters by period (2006-2016 vs. 2016-2018); in two clusters by contractor (municipalities and other public authorities) and lastly testing just the two major category of works in the sample, which are "Road infrastructure" and "Schools and buildings for social activities."

All our statistical analyses demonstrate that the longer time taken to award contracts is unrelated to the cost of the works or the dimensions of the public authorities involved (as a proxy for their contract awarding expertise). This means that the extension of the time taken to award contracts can be attributed entirely to the related procedures required by law and especially to the checks on abnormal offers.

These findings point to the need for further research. To be generalizable, our findings would need to be tested on a larger sample of data and in different regions. The same set of data could also be useful for examining other phases of the public works process, such as design and construction. A further breakdown of the dataset, such as for works on historical buildings [47,48] and on improvement and use of the environment [49] could help to clarify the timeline of the public works process. Lastly, the features affecting the mismatch between the expected and actual costs and times to the completion of public works are worth analyzing, also by comparison with existing literature on the topic.

Author Contributions: Conceptualization, G.M.; methodology, G.M. and V.A.; formal analysis, G.M. and V.A.; data curation, V.A.; writing-original draft preparation, V.A.; writing-review and editing, G.M. and V.A.; visualization, G. M. and V.A.; supervision, G.M.

Funding: This research was funded by the Department of Civil, Architectural and Environmental Engineering of the University of Padova, research grant number 68/2018, prot. n. 560, date 29/03/2018.

Conflicts of Interest: The authors have no potential conflict of interest to disclose concerning the research, authorship, and/or publication of this article.

\section{References}

1. Flyvbjerg, B. Over Budget, over Time, over and over again. In The Oxford Handbook of Project Management; Morris, P.W.G., Pinto, J.K., Soderlund, J., Eds.; Oxford University Press: Oxford, UK, 2011; pp. 321-344. Available online: http://ssrn.com/abstract=2278226 (accessed on 6 September 2019).

2. Flyvbjerg, B.; Holm, M.S.; Buhl, S. Underestimating costs in public works projects: Error or lie? J. Am. Plan. Assoc. 2002, 68, 279-295. [CrossRef] 
3. Flyvbjerg, B.; Ansar, A.; Budzier, A.; Buhl, S.; Cantarelli, C.; Garbuio, M.; Glenting, C.; Holm, M.S.; Lovallo, D.; Lunn, D.; et al. Five things you should know about cost overrun. Transp. Res. A Policy Pract. 2018, 118, 174-190. [CrossRef]

4. Gori, G.F.; Lattarulo, P.; Mariani, M. Understanding the procurement performance of local governments: A duration analysis of public works. Environ. Plan. C Politics Space 2017, 35, 809-827. [CrossRef]

5. OECD. Reforming Public Procurement: Progress in Implementation 2015 OECD Recommendation. 2019. Available online: https://www.oecd.org/gov/public-procurement/public-procurement-progress-reporthighlights.pdf (accessed on 23 September 2019).

6. Priemus, H.; van Wee, B. Decision-Making on Mega-Projects. In International Handbook on Mega-Projects; Edward Elgar: Cheltenham, UK; Northampton, MA, USA, 2014; pp. 9-10. [CrossRef]

7. Shrestha, P.P.; Burns, L.A.; Shields, D.R. Magnitude of Construction Cost and Schedule Overruns in Public Work Projects. J. Constr. Eng. 2013, 2013, 935978. [CrossRef]

8. Bruzelius, N.; Flyvbjerg, B.; Rothengatter, W. Big decisions, big risks. Improving accountability in mega projects. Transp. Policy 2002, 9, 143-154. [CrossRef]

9. Ullah, K.; Abdullah, A.H.; Nagapan, S.; Suhoo, S.; Khan, M.S. Theoretical framework of the causes of construction time and cost overruns. IOP Conf. Ser. Mater. Sci. Eng. 2017, 271, 012032. [CrossRef]

10. Larsen, J.K.; Shen, G.Q.; Lindhard, S.M.; Brunoe, T.D. Factors Affecting Schedule Delay, Cost Overrun, and Quality Level in Public Construction Projects. J. Manag. Eng. 2015, 32, 04015032. [CrossRef]

11. Olawale, Y.A.; Sun, M. Cost and time control of construction projects: Inhibiting factors and mitigating measures in practice. Constr. Manag. Econ. 2010, 28, 509-526. [CrossRef]

12. Kazaz, A.; Ulubeyli, S.; Tuncbilekli, N.A. Causes of Delays in Construction Projects in Turkey. J. Civ. Eng. Manag. 2012, 18, 426-435. [CrossRef]

13. Aibinu, A.A.; Odeyinka, H.A. Construction Delays and Their Causative Factors in Nigeria. J. Constr. Eng. Manag. 2006, 132, 667-677. [CrossRef]

14. Al-Kharashi, A.; Skitmore, M. Causes of delays in Saudi Arabian public sector construction projects. Constr. Manag. Econ. 2009, 27, 3-23. [CrossRef]

15. Kaming, P.F.; Olomolaiye, P.O.; Holt, G.D.; Harris, F.C. Factors influencing construction time and cost overruns on high-rise projects in Indonesia. Constr. Manag. Econ. 1997, 15, 83-94. [CrossRef]

16. Love, P.E.D.; Wang, X.; Sing, C.; Tiong, R.L.K. Determining the Probability of Project Cost Overruns. J. Constr. Eng. Manag. 2012, 139, 321-330. [CrossRef]

17. Del Giudice, V.; de Paola, P.; Francesca, T.; Nijkamp, P.J.; Shapira, A. Real Estate Investment Choices and Decision Support Systems. Sustainability 2019, 11, 3110. [CrossRef]

18. Acampa, G.; Forte, F.; De Paola, P.B.I.M. Models and Evaluations; Springer: Cham, Switzerland, 2020; pp. 351-363. [CrossRef]

19. Nesticò, A.; He, S.; de Mare, G.; Benintendi, R.; Maselli, G. The ALARP Principle in the Cost-Benefit Analysis for the Acceptability of Investment Risk. Sustainability 2018, 10, 4668. [CrossRef]

20. Knight, K.; Fayek, A.R. Use of Fuzzy Logic for Predicting Design Cost Overruns on Building Projects. J. Constr. Eng. Manag. 2002, 128, 503-512. [CrossRef]

21. Gkritza, K.; Labi, S. Estimating Cost Discrepancies in Highway Contracts: Multistep Econometric Approach. J. Constr. Eng. Manag. 2008, 134, 953-962. [CrossRef]

22. Bisello, A.; Grilli, G.; Balest, J.; Stellin, G.; Ciolli, M. Co-Benefits of Smart and Sustainable Energy District Projects: An Overview of Economic Assessment Methodologies; Springer: Cham, Switzerland, 2017; pp. 127-164. [CrossRef]

23. Antoniucci, V.; Marella, G. Immigrants and the city: The relevance of immigration on housing price gradient. Buildings 2017, 7, 91. [CrossRef]

24. Bhargava, A.; Anastasopoulos, P.C.; Labi, S.; Sinha, K.C.; Mannering, F.L. Three-Stage Least-Squares Analysis of Time and Cost Overruns in Construction Contracts. J. Constr. Eng. Manag. 2010, 136, 1207-1218. [CrossRef]

25. Fregonara, E.; Pattono, S. A sustainability indicator for building projects in presence of risk/uncertainty over time: A research experience. Aestimum 2018, 173-205. [CrossRef]

26. Boussabaine, A.H. The use of artificial neural networks in construction management: A review. Constr. Manag. Econ. 1996, 14, 427-436. [CrossRef]

27. Attalla, M.; Hegazy, T. Predicting Cost Deviation in Reconstruction Projects: Artificial Neural Networks versus Regression. J. Constr. Eng. Manag. 2003, 129, 405-411. [CrossRef] 
28. Mangialardo, A.; Micelli, E.; Saccani, F. Does sustainability affect real estate market values? Empirical evidence from the office buildings market in Milan (Italy). Sustainability 2018, 11, 12. [CrossRef]

29. Torrieri, F.; Oppio, A. The Ex-Ante Evaluation of Flood Damages for a Sustainable Risk Management; Springer: Cham, Switzerland, 2019; pp. 542-550. [CrossRef]

30. Assaf, S.A.; Al-Hejji, S. Causes of delay in large construction projects. Int. J. Proj. Manag. 2006, 24, 349-357. [CrossRef]

31. Cantarelli, C.C.; Flybjerg, B.; Molin, E.J.E.; van Wee, B. Cost overruns in Large-Scale Transportation Infrastructure Projects: Explanations and Their Theoretical Embeddedness. arXiv 2013, arXiv:1307.2176.

32. Flyvbjerg, B.; Holm, M.K.S.; Buhl, S.L. Inaccuracy in Traffic Forecasts. Transp. Rev. 2006, 26, 1-24. [CrossRef]

33. Van Marrewijk, A.; Clegg, S.R.; Pitsis, T.S.; Veenswijk, M. Managing public-private megaprojects: Paradoxes, complexity, and project design. Int. J. Proj. Manag. 2008, 26, 591-600. [CrossRef]

34. Love, P.E.D.; Edwards, D.J.; Irani, Z. Moving Beyond Optimism Bias and Strategic Misrepresentation: An Explanation for Social Infrastructure Project Cost Overruns. IEEE Trans. Eng. Manag. 2012, 59, 560-571. [CrossRef]

35. Sepasgozar, S.M.E.; Karimi, R.; Shirowzhan, S.; Mojtahedi, M.; Ebrahimzadeh, S.; McCarthy, D. Delay Causes and Emerging Digital Tools: A Novel Model of Delay Analysis, Including Integrated Project Delivery and PMBOK. Buildings 2019, 9, 191. [CrossRef]

36. Lau, C.H.; Mesthrige, J.W.; Lam, P.T.I.; Javed, A.A. The challenges of adopting new engineering contract: A Hong Kong study. Eng. Constr. Archit. Manag. 2019. [CrossRef]

37. Guccio, C.; Pignataro, G.; Rizzo, I. Do local governments do it better? Analysis of time performance in the execution of public works. Eur. J. Political Econ. 2014, 34, 237-252. [CrossRef]

38. Bencardino, M.; Nesticò, A. Spatial Correlation Analysis among Land Values, Income Levels and Population Density; Springer: Cham, Switzerland, 2019; pp. 572-581. [CrossRef]

39. Decarolis, F.; Giorgiantonio, C. Local public procurement regulations: The case of Italy. Int. Rev. Law Econ. 2015, 43, 209-226. [CrossRef]

40. Agenzia per la Coesione Territoriale, I Tempi di Attuazione delle Opere Pubbliche. 2018. Available online: http://www.agenziacoesione.gov.it/dossier_tematici/i-tempi-delle-opere-pubbliche/ (accessed on 8 November 2019).

41. Deloitte, Gli Investimenti in Infrastrutture. Relazione con la Distribuzione del Reddito e Attese di Remunerazione Finanziaria Degli Investitori. 2018. Available online: https://economiaefinanza.luiss. it/sites/economiaefinanza.luiss.it/files/180116_Report-infrastrutture_lq.pdf (accessed on 12 September 2019).

42. Forte, F.; Antoniucci, V.; de Paola, P. Immigration and the housing market: The case of Castel Volturno, in Campania Region, Italy. Sustainability 2018, 10, 343. [CrossRef]

43. Antoniucci, V.; Marella, G. Is social polarization related to urban density? Evidence from the Italian housing market. Landsc. Urban Plan. 2017, 177, 340-349. [CrossRef]

44. Canesi, R.; Marella, G. Residential construction costs: An Italian case study. Int. J. Appl. Eng. Res. 2017, 12, 2623-2634.

45. Antoniucci, V.; Marella, G. The influence of building typology on the economic feasibility of urban developments. Int. J. Appl. Eng. Res. 2017, 12, 4946-4954.

46. Prizzon, F.; Rebaudengo, M. [Unfinished Public Works: A National Heritage to Develop?] Le Opere Pubbliche Incompiute: Un Patrimonio da Valorizzare? LaborEst 2015, 11, 55-59. [CrossRef]

47. Della Spina, L.; Calabrò, F. Decision Support Model for Conservation, Reuse and Valorization of the Historic Cultural Heritage; Springer: Cham, Switzerland, 2018; pp. 3-17. [CrossRef]

48. Della Spina, L. Scenarios for a Sustainable Valorisation of Cultural Landscape as Driver of Local Development; Springer: Cham, Switzerland, 2019; pp. 113-122. [CrossRef]

49. Sturiale, L.; Scuderi, A. The Evaluation of Green Investments in Urban Areas: A Proposal of an eco-social-green Model of the City. Sustainability 2018, 10, 4541. [CrossRef]

(C) 2019 by the authors. Licensee MDPI, Basel, Switzerland. This article is an open access article distributed under the terms and conditions of the Creative Commons Attribution (CC BY) license (http://creativecommons.org/licenses/by/4.0/). 

Article

\title{
An Integrated Decision Support System for the Sustainable Reuse of the Former Monastery of "Ritiro del Carmine" in Campania Region
}

\author{
Francesca Torrieri ${ }^{1, *}$, Marina Fumo ${ }^{2}$, Michele Sarnataro ${ }^{2}$ and Gigliola Ausiello ${ }^{2}$ \\ 1 Department of Industrial Engineering, University of Naples Federico II, Piazzale Tecchio 80, \\ 80125 Naples, Italy \\ 2 Department of Civil, Architectural and Environmental Engeneering, Piazzale Tecchio 80, 80125 Naples, Italy; \\ marina.fumo@unina.it (M.F.); michele.sarnataro@gmail.com (M.S.); ausiello@unina.it (G.A.) \\ * Correspondence: frtorrie@unina.it
}

Received: 31 July 2019; Accepted: 18 September 2019; Published: 25 September 2019

\begin{abstract}
Nowadays, many public administrations have abandoned and underused heritage buildings due to a lack of public resources, although the effective contribution of cultural heritage as a driver and enabler of sustainable development is strongly recognized. Currently, investments in cultural heritage have multidimensional impacts (social, economic, historical, and cultural) and can contribute to increasing overall local productivity; improving the wellbeing of inhabitants; and attracting funding from the public, private, and private-social sectors. Lack of public resources has pushed local administrations to favor new forms of valorization of public property that can promote the "adaptive reuse" of historic buildings in order to preserve their social, historical, and cultural values. At the same time, administrations seek to stimulate the experimentation of new circular business, financing, and governance models in heritage conservation, creating synergies between multiple actors; reducing the use of resources; and regenerating values, knowledge, and capital. The objective of this paper is to propose an integrated evaluation model, based on multicriteria analysis, and a financial model to support the choice of an alternative reuse of an ancient monastery in the municipality of Mugnano in the Campania region in order to define a "shared strategy" based on a "bottom-up" approach. This starts from the needs of the local community but does not neglect the historical and cultural values of the heritage building, as well as the economic and financial feasibility. The positive results obtained show that the model proposed can be a useful decision support tool in environments characterized by high complexity such as cultural heritage sites, where the objective is to precisely highlight the elements that influence the dynamics of choice for building shared bottom-up development strategies.
\end{abstract}

Keywords: integrated evaluation; multicriteria analysis; cultural heritage and circular economy; financial sustainability; Ritiro del Carmine; built heritage sustainable reuse

\section{Introduction: The Sustainable Reuse of Cultural Heritage from a Circular Economic Perspective}

In the current European context, the conservation of cultural heritage presents a real challenge for professional and public institutions at national and local levels, even if it is strongly recognized that the heritage sector makes a significant economic contribution [1-3].

In Italy, many public institutions have an enormous asset that is composed of several unused buildings [4]. In recent years, difficulties in finding new uses for these properties has led to their abandonment and ruin.

Lately, Italian authorities have experimented with new approaches to valorize these massive estates, which are otherwise destined to remain unused [5]. The new policies look at redevelopment as 
a possibility to enhance public welfare through the creation of new social hubs [6] and a new sense of community within the population [7]. The promotion of these types of actions, strictly linked to citizens' social needs and social capital $[8,9]$, allows the development of more sustainable and successful strategies [10]. In fact, investments in cultural heritage have multidimensional impacts (social, economic, historical, and cultural) and can contribute to increasing overall local productivity; improving inhabitants' wellbeing; and attracting funds from the public, private, and private-social sectors [11,12]. As Throsby highlights, cultural heritage can be considered as the "glue" between the different dimensions of sustainable development [13].

The redevelopment of ancient and unused buildings represents an opportunity to pursue new and innovative solutions [14].

In this context, the concept of adaptive reuse plays a significant role. Adaptive reuse helps to protect and preserve historical buildings against obsolescence, considering environmental, social, and financial aspects of sustainability and promoting the valorization of the surrounding society [15-17].

Currently, the conservation of cultural heritage can promote social cohesion and integration through regeneration of neglected areas, creation of locally rooted jobs, and promotion of a shared understanding and sense of community [18]. In this sense, cultural heritage must gain an active role both in today's society and the urban reality, especially in many small town and historic centers, as the case study presented here well represents.

Moreover, from a circular economic perspective, the efficient reuse of cultural heritage and the choice of recycled and natural materials help to reduce negative externalities and produce positive environmental, social, and cultural impacts which benefit the whole society [19].

The choice of functions compatible with the building structure and historical values, expressing the needs of local communities, helps to integrate cultural assets within the city context and to attract financial capital to ensure its management over time. Moreover, the use of recycled and natural materials is the starting point of a circular path that follows the environmental, economic, and social sustainability perspective [20-22].

The choice of a new function requires a systematic framework to evaluate the different feasible alternatives and sufficient information to identify the best solution, or at least, the best compromise solution [23]. The evaluation process has to handle the problem holistically by considering different perspectives, objectives, stakeholders, and values in a comprehensive manner; such a process may increase the quality of public decisions [24].

In this context, the objective of the paper is to propose an integrated evaluation model, based on multicriteria analysis, and a financial model to support the choice of the alternative reuse function of an ancient monastery in the municipality of Mugnano in the Campania region in order to define a "shared strategy" based on a bottom-up approach. This starts from the needs of the local community but does not neglect the historical and cultural values of the heritage building, as well as the economic and financial feasibility.

The methodology follows the general approach to decision problems [25], adapted to the case study analyzed in order to support the public administration - the owner of the monastery-to choose the best alternative reuse functions from a sustainable perspective.

The paper is organized as follows. In Section 2, we illustrate the framework adopted. In Section 3, we report the results of the social and financial evaluations tested on the case study of the Ex Ritiro del Carmine. In Section 4, the conclusion and discussion of future research are presented.

\section{Material and Methods}

\subsection{The Integrated Decision Support System for the Choice of Alternative Functions}

The redevelopment of heritage buildings is a complex design problem, in which several points of view need to be managed in a holistic evaluation process. 
Following the classical approach proposed by Simon (1972) to the decision-making process, an integrated methodological framework was developed for this case study, as Figure 1 illustrates.

Simon defines three main stages of the decision-making process:

- Intelligence, which deals with the problem of identification of and data collection for the problem.

- Design, which deals with the generation of alternative solutions to the problem at hand.

- Choice, that is, selecting the "best" solution from among the alternative solutions using some criterion.

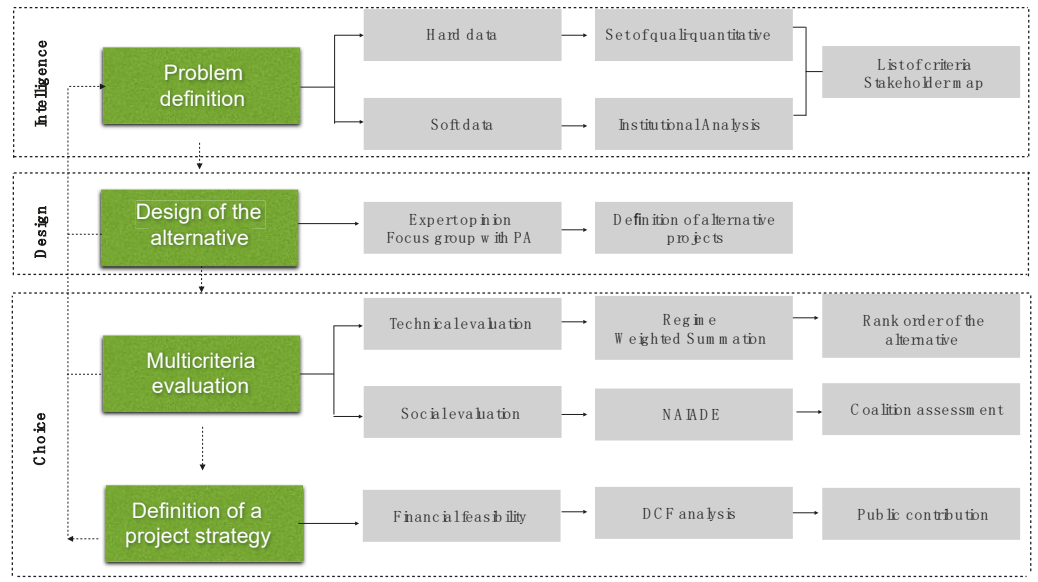

Figure 1. Methodological framework.

Starting from the main stages proposed by Simon, different evaluation methods have been proposed in order to support the definition of different reuse alternatives in redevelopment projects, including both technical problems related to the design projects [21-23,25], social issues, and financial feasibility. In the stated methodological framework, different multicriteria methods are combined with a financial model in the diverse stages of the decision-making process in order to develop a tool to enhance the quality and trustworthiness of the decision-making process itself.

The methodological framework is illustrated in Figure 1.

In the first stage of the decision-making process, the decision maker (DM) identifies problems, opportunities, and objectives regarding the reuse project of the heritage building. To accomplish this, a list of criteria was identified. The list of criteria was structured based on appropriate reference [26], which identified the main categories of analysis, but considering the peculiarity of the case study, this also emerged in a focus group with the public administration and the technicians.

Moreover, a map of relevant stakeholders was defined in accordance with the strategic objectives of the public administration oriented toward the valorization of the building as having symbolic value and being a meeting place for the local community.

Then, in the design phase, five alternatives were identified during a focus group organized by the municipality with the experts (architect, economist, and sociologist) and politicians.

The alternative solution was evaluated by means of a multicriteria method considering two types of analysis, namely a "technical evaluation" linked with the DM's preferences and a "social evaluation" linked with the stakeholders' points of view [27,28].

The technical evaluation was developed with the aim of defining a rank order of alternatives, considering the list of criteria previously defined with the help of two multicriteria methods, namely, regime analysis [29] and the weighted summation method (WSM) [30].

The regime method is used to start a dialogue between the DM and the experts, which has the advantage of simplifying the debate with the DM: at the beginning, he often does not have a clear idea 
of the problem and is not familiar with the decision-making tools. The regime method is a discrete multicriteria method with a partial compensatory structure based on pairwise operations. This method can handle mixed types of information, that is, both quantitative and qualitative. The fundamental input data are the impact matrix and the political weights, and these two elements are combined to calculate the probability that one alternative is preferred over another. The impact matrix includes the evaluation of each alternative with respect to each criterion. The set of weights is a qualitative assessment consisting of an ordinal evaluation of the criteria reflecting the DM's preferences.

Weighted summation is then used to check the previous results through the improvement of the set of weights: it regards a more complex process based on the pairwise comparison of the decision criteria. In this part, the main information is provided for better comprehension of the problem, the opportunities, and the potential of the alternative projects. Weighted summation is a compensatory approach based on a linear model. This method uses an indirect approach, in which the qualitative information is first transformed into cardinal information to be compared; this procedure is called "normalization" and it is necessary to handle different types of attributes. The input data are the impact matrix and the matrix of weights. The evaluations of each alternative with respect to each criterion are combined through the matrix of weights in one overall value, obtained by the addition of all the weighted scores together. The combination of the two previously described methodologies encourages a transparent dialogue between the public administration, which owns the building, and the architects in order to define a sustainable solution taking into account the different dimensions involved. Moreover, the methodologies proposed are included in the Definite software, which makes communication easier among different actors.

The Definite program was developed by Ron Janssen and Marine van Herwijnen in 1987 [31]. It is a multiobjective decision support system that supports the whole decision process from problem definition to report generation. The system performs the following functions: (1) structure the problem and generate alternatives; (2) compare alternatives; (3) rank and/or value alternatives; (4) support interpretation of the results; (5) present results. To perform these functions, the system contains five modules. Each module contains a variety of procedures (like the regime and weight summation methods) to perform these functions.

The social evaluation was developed with the aim of understanding the possible coalition generated by the choice of the best alternative [32,33]. This analysis was carried out with the help of the Novel Approach to Imprecise Assessment and Decision Environments (NAIADE) method [34]. The NAIADE method captures the preference of stakeholders and supplies indications of the distance of positions among the different interest groups. It evaluates the social compromise solution through the analysis of the possible coalitions. The NAIADE method is a discrete social multicriteria method, which includes mixed types of information and a conflict analysis in a fuzzy environment. Through pairwise linguistic evaluation, "based on semantic distance between linguistic qualities", two types of evaluation are provided. The first regards the assessment of the alternatives based on the social impact matrix, which contains a qualitative evaluation of each alternative with respect to a defined set of criteria, based on the stakeholder's preferences. The second analysis is performed by the completion of an equity matrix, where a similarity matrix is calculated. It sheds light upon the level of decision conflicts among the different interest groups and highlights the possible formation of coalitions (building a dendrogram of coalitions), showing the impact of each alternative as perceived by the social actors. In this way, NAIADE provides the following information: (a) distance indicators between the interests of the different social actor groups, as an indication of coalition formation possibility or interest convergence; (b) rankings of alternatives for every coalition, in accordance with the impacts on the social groups or the social compromise solution.

Then, the preferred alternative is developed, and the financial feasibility is evaluated. The method combines different assessment phases with sequential checks. This kind of structure allows the creation of an interactive framework able to incorporate the ideas of learning processes and the engagement of 
different stakeholders. These characteristics may provide better-informed decisions and a greater level of consent.

Multicriteria decision analysis (MCDA) can handle the complexity of the whole process. The MCDA methods provide tools for gauging stakeholders' preferences, comparing alternatives, and supplying useful indications to the DM [35]. From this perspective, MCDA has the principal aim to "create" instead of "find" solutions; therefore, it is a "constructive" approach [36].

Multicriteria analysis has the ability to compare the alternatives according to various conflicting stakeholder interests. The ability to involve several points of view in the early stage of the design problem through a participative process may help to avoid conflict and make more successful and transparent decisions [37,38].

Actually, the evaluation process is not a "one-shot activity"; instead, it is a constructive, dynamic process that advances in relation to continuous reinforcing gains along the various steps [39-43].

\subsection{Introduction to the Case Study}

The case study under analysis regards the reuse of the unused monastery Ex Ritiro del Carmine in the Campania region (Italy).

The ancient building is located in the old town of the municipality of Mugnano di Napoli, a town located in the northern area of the Metropolitan City of Naples. The city has approximately 35,000 inhabitants and is directly dependent on the City of Naples, where the main activities and public services are located. The municipality's future development strategy establishes the conservation and enhancement of the old town together with the creation of new residential areas and public services [44].

The monastery was built as an orphanage for girls in 1818 [45,46], far from the city center of that time. The growth of the congregation led to incremental construction: In 1860, the ground floor was completed, and the Church of Santa Maria del Carmine was built as an annex to it. In 1937, the original order left the building, which was acquired by a new congregation who enlarged the monastery building with two other floors and established a private elementary school. The order left the monastery in 2003 after many years of intense activity. The municipality acquired the monastery in 2010, even though it has been unused since 2003.

As mentioned above, the building is located in the old town, far from the main roads, in a quiet place alongside a by-road. The complex is composed of four main parts: the monastery, the church, the theater, and the garden. It is important to underline that the church is still open and is not part of the municipality project (Figure 2).
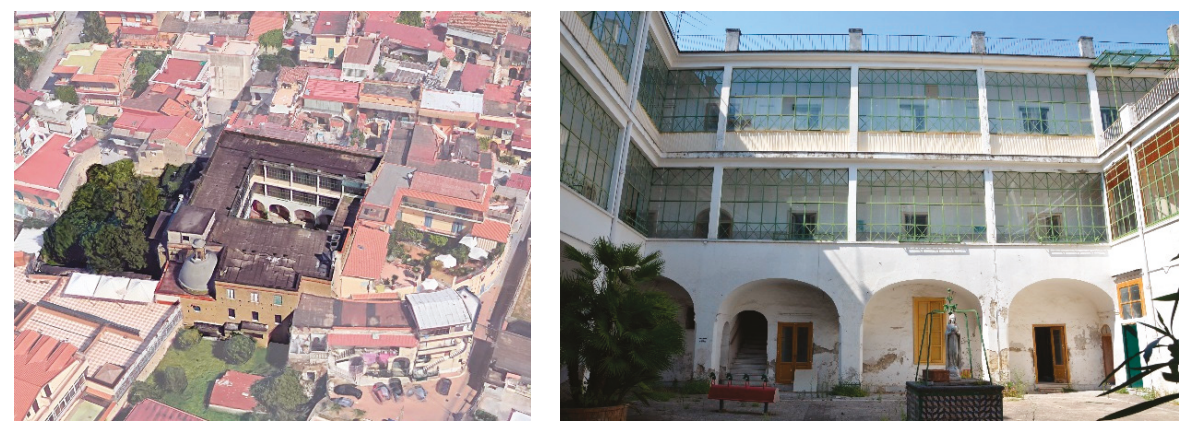

Figure 2. The monastery of Ex Ritiro del Carmine in the Campania region (Italy).

The monastery itself has three levels and a central courtyard, while the garden is on the western side together with the theater. On the ground floor (approx. GEA $1000 \mathrm{~m}^{2}$ ), there are the entrance hall, the reception, the porch, the kindergarten, the kitchen, and the canteen; on the first floor (approx. 
GEA $850 \mathrm{~m}^{2}$ ), there are the nuns' bedrooms; on the second floor (approx. GEA $800 \mathrm{~m}^{2}$ ), there are the classrooms and two other bedrooms.

\section{Results}

\subsection{Problem Definition}

The definition of the criteria is a process, which follows a hierarchical logic. Starting from the dialogue with the DM, the list of subcriteria are formulated by the technicians and reflect the DM's objectives and needs. For each fixed criterion, more specific elements are defined: the subcriteria are measurable and index-linked with the specific dimensions of the projects.

In our case study, the DM showed the intention of pursuing the social-sustainability-based criteria mentioned by the most appropriate references $[13,47,48]$. This vision is elaborated in a holistic view and includes the enhancement of social objectives, economic objectives, and urban development. At this high-level stage of project development, no specific environmental issues are considered. Environmental implications should be addressed at the more refined second stage of analysis.

The hierarchical structure of the criteria was formulated by a focus group, in which the different points of view arose and became clearer. The board session allowed the technicians to summarize the information in a limited number of clearly defined criteria. For the analyzed case study, the formulated criteria are represented in a tree chart, as shown in Figure 3.

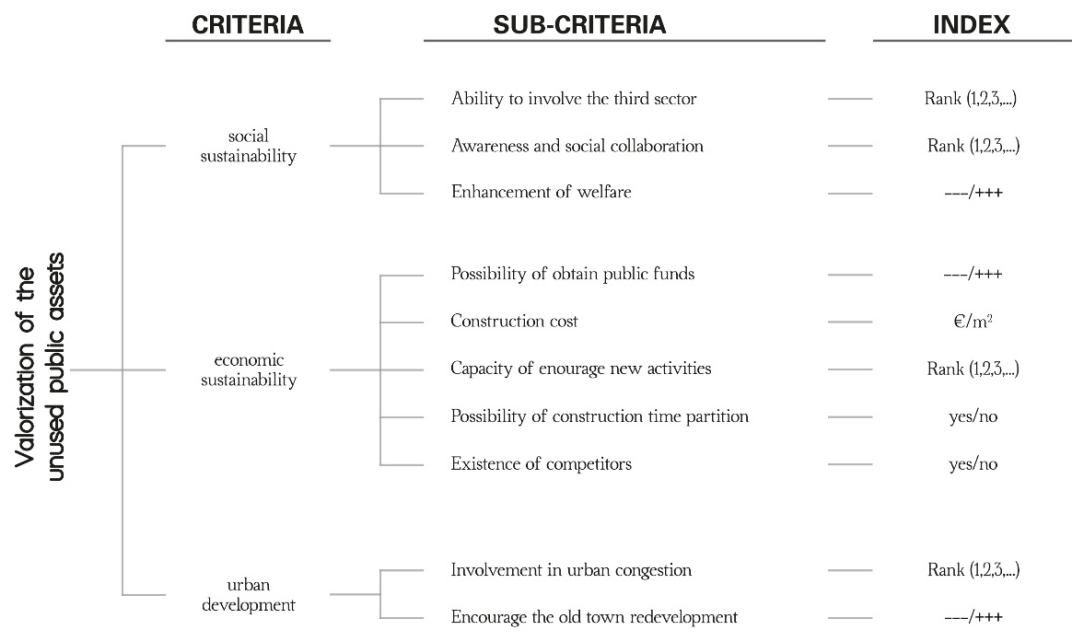

Figure 3. Tree chart of criteria.

As Figure 3 illustrates, the criteria identified are more oriented toward social issues and the integration of the building in the urban context. Currently, the main strategic objective of the public administration is to create a new catalyst for the entire urban development process.

The evaluation subcriteria are described below:

- $\quad$ Ability to involve the third sector: assesses the availability of nonprofit organizations to take part in such activities;

- Awareness and social collaboration: linked with the capability to involve citizens in social activities and attract interest in social issues;

- $\quad$ Enhancement of welfare: assesses the ability to answer social needs;

- Possibility of obtaining public funds: linked to the availability of public grants; 
- Construction cost: a parametric appraisal of the possible square-meter cost in relation to the specific characteristics as defined in the regulation;

- Capacity to encourage new activities: linked with private investment in new activities not necessarily directly linked with the project;

- Possibility of construction time partition: assesses the possibility of splitting the construction into several periods;

- $\quad$ Existence of competitors: assesses the existence of other similar activities in the municipality;

- Involvement in urban congestion: linked with the growth of traffic overcrowding;

- $\quad$ Encourage old town redevelopment: linked with the possibility of attracting new public and private investment for the redevelopment of the old town.

In a public project, key stakeholders have to be involved in order to help the DM in making better decisions and improving organization performance. The redevelopment of the monastery aims to enhance the welfare of the whole population of the municipality. For this reason, the population is divided into several groups in order to involve every social party.

As defined in [49], stakeholders are any group of people, organized or not, who share common interests, values, and behaviors and who can affect or be affected by the outcomes of the project. The representative groups are selected by the technicians to address all the social components involved in the project [50].

Here, the selected groups were

- public administration;

- political opposition;

- $\quad$ entrepreneurs;

- freelance professionals;

- business owners;

- $\quad$ social and cultural associations;

- students;

- the employed;

- the unemployed;

- the retired.

These groups were involved in the process to evaluate the developed alternative in order to understand the level of consensus among them on the rank order evaluation.

\subsection{Design of the Alternative}

In the early stage of the evaluation process, with respect to the objectives of the public administration, the designers formulated three alternatives. These are briefly described below.

\section{Alternative A: Antiviolence Center for Women}

This alternative is in response to the increasing phenomenon of violence against women observed by the municipal "antiviolence desk" and by the Italian National Institute of Statistics (ISTAT) [51]. The project would provide hospitality for both women and their underage children who were victims of or were exposed to violence. The center would grant several services, including expert consultation, legal advice, and psychological assistance and support groups. It would organize information meetings and public events to spread knowledge and tackle the spread of the problem.

\section{Alternative B: Refugee Center}

This alternative is in response to the "European migrant crisis" characterized by the increase in migrants arriving in the European Union. The project aims to provide a place in which the refugees may be hosted, and it would grant hospitality, social services, and legal support. The center may 
organize events and public meetings to promote the idea of a new multicultural society based on equality between people.

\section{Alternative C: Cultural Center and Library}

This alternative is in response to the lack of public social and cultural spaces, especially a public library in the municipality. The project aims to create a center of excellence to support citizens' education that would gather public services, study rooms, laboratories for shared workshops, and a conference room.

The redevelopment of the theater and the garden would provide two new recreational areas for entertainment and outdoor activities.

The above-presented alternatives derive from a static vision in which a single function absorbs the whole complex. These three alternatives were presented in a focus group to the public administration and the new idea emerged in a brainstorming section considering the characteristic of the building and the objectives of the public administration.

Actually, during the deliberation process, the possibility of taking advantage of the characteristics of the complex and a multifunctional building was suggested: the integration of different functions and resources in a hybrid building can enhance the social services provided and reduce public expenditure. In particular, the Ex Ritiro del Carmine has different characteristics, which support this idea:

- the partition in three levels allows for there to be three different activities with different modes and periods of operation. Each one would be separated from the others, but they would share the courtyard and the garden as places of social exchange;

- the courtyard represents a cornerstone which links all the activities and stakeholders together, creating a place of meetings and cultural exchanges;

- the shape of each story gives some suggestions about the use of the level: the ground floor could host shared functions with a food service; the first floor, which included the nuns' room, could host hospitality functions; the second floor, which has big free rooms, could host the library.

These features characterize the new formulated alternatives that present the integration of different activities, in particular, the organization rooms on the ground floor, the hospitality activities on the first floor, and the library on the second floor. The two other proposed alternatives differ in terms of the hospitality activities. They are presented below.

\section{Alternative D: Social Hub with Refugee Center}

This project aims to create a center in which to develop new procedures of cultural integration between refugees and citizens, which would help refugees and tackle the widespread forms of racism.

\section{Alternative E: Social Hub with Antiviolence Center for Women}

This project aims to create a multifunctional center in which the female guests would recover their independence through new forms of work integration. The center would also organize seminars to spread knowledge about the phenomenon of violence.

The five developed alternatives were then evaluated in a second stage of the decision process. They were evaluated from a technical point of view considering the list of criteria developed and with the help of regime analysis and weighted summation. Then, the social acceptability of the solution found was evaluated with the NAIADE method. The results obtained are reported in the next subsection.

\subsection{Multicriteria Analysis}

\subsubsection{Regime Method}

The regime method is based on two input data: the impact matrix and the weight vector to compare the alternative and to define a ranking of these. 
The impact matrix developed for the analyzed case study is illustrated in Table 1, starting from the list of criteria identified in Figure 1.

Table 1. Impact matrix.

\begin{tabular}{|c|c|c|c|c|c|c|c|c|c|}
\hline & & \multirow[b]{2}{*}{ Subcriteria } & \multirow[b]{2}{*}{ Index } & \multirow[b]{2}{*}{$\mathbf{c} / \mathbf{b}$} & \multicolumn{5}{|c|}{ Project Alternatives } \\
\hline & & & & & A & B & $\mathrm{C}$ & D & $\mathbf{E}$ \\
\hline \multirow{8}{*}{ CRITERIA } & \multirow{2}{*}{ 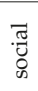 } & Ability to involve the third sector & rank & $\mathrm{b}$ & 2 & 5 & 3 & 4 & 1 \\
\hline & & Enhancement of welfare & $-/+++$ & $\mathrm{b}$ & ++ & + & ++ & ++ & +++ \\
\hline & \multirow{4}{*}{ 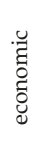 } & Possibility of obtaining public funds & $-/+++$ & $\mathrm{b}$ & ++ & 0 & 0 & + & ++ \\
\hline & & Construction cost & $€ / \mathrm{m}^{2}$ & $c$ & 793 & 793 & 975 & 904 & 904 \\
\hline & & Possibility of construction time partition & yes/no & $\mathrm{b}$ & no & no & yes & yes & yes \\
\hline & & Existence of competitors & yes/no & $c$ & no & yes & no & yes & no \\
\hline & \multirow{2}{*}{$\begin{array}{l}\Xi \\
\stackrel{\Xi}{\Xi} \\
\Xi\end{array}$} & Involvement in urban congestion & rank & $\mathrm{b}$ & 3 & 1 & 5 & 2 & 4 \\
\hline & & Encourage the old town redevelopment & $-/+++$ & $\mathrm{b}$ & - & - & ++ & + & ++ \\
\hline
\end{tabular}

As illustrated in Table 1, in order to evaluate the alternatives and to reflect the complexity of the design problem, each criterion uses a different scale of measurement, qualitative or quantitative, due to their nature and the available information. It is important to highlight that each criterion can be considered as a cost or a benefit, and for this reason, they have to be respectively minimized or maximized.

The main scales are nominal, ordinal, binary, and ratio. For simplicity, we refer to the first three as qualitative information and to the last one as a quantitative scale.

In the case of the Ex Ritiro del Carmine, the scale of measurement and the related unit of measurement used are

- $\quad$ rank: ordinal scale, ranks the alternatives with respect to the analyzed criterion; it goes from 1 , for the best alternative, to 5 , for the worst one;

- $\quad-/+++$ nominal scale, consists of a linguistic evaluation with respect to a seven-level qualitative scale; the levels are high $(+++/-)$, medium $(++/-)$, and low $(+/-)$, for both positive and negative values, and " 0 " for moderate;

- $\quad € / m 2$ : ratio scale, a statistical appraisal of the unitary cost of construction for each alternative;

- Yes/no: binary scale, it defines the existence of a certain condition for each evaluated alternative.

For each criterion, it is indicated whether the criterion represents a cost or a benefit $(\mathrm{c} / \mathrm{b})$.

The system of weights identifies the priority among the evaluation criteria, which is basically a political issue linked to the policy game and anyone involved in the decision-making process.

The weight vector consists of an ordinal assessment of the criteria. This type of evaluation allows for the simplification of the dialogue with the DMs, especially in the early stage.

During the decision-making process, the DM was asked to express his own preferences through the assessment of the set of weights associated with the evaluation criteria. The identified ordinal sets of weights are shown below in Figure 4.

The two sets highlight two different approaches: the first, which refers to the political class, attributes more importance to the social aspects, while the second, which refers to the technical experts, pays close attention to the economic feasibility.

The results obtained with the regime method are shown in Figure 5. 

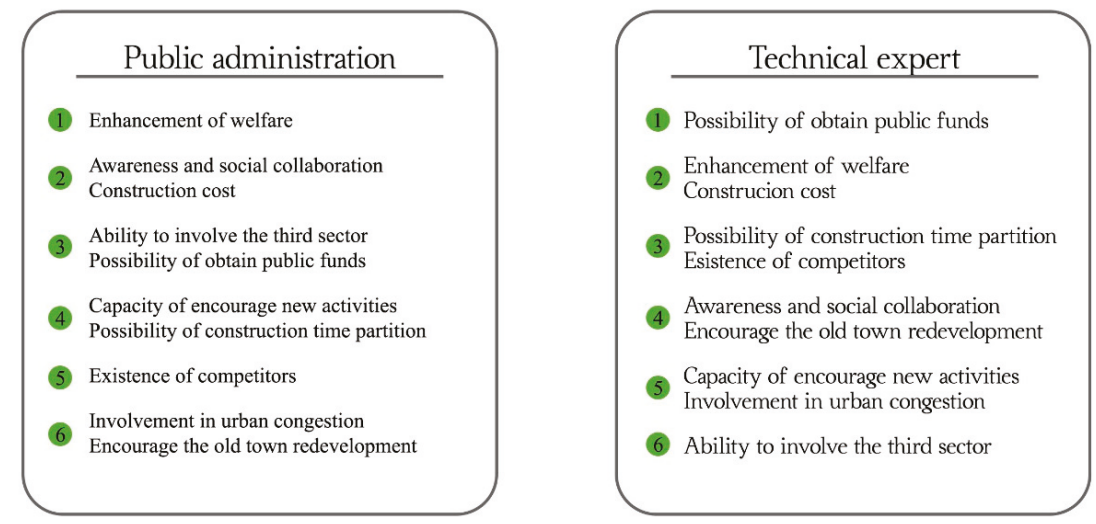

Figure 4. Rank order of criteria.
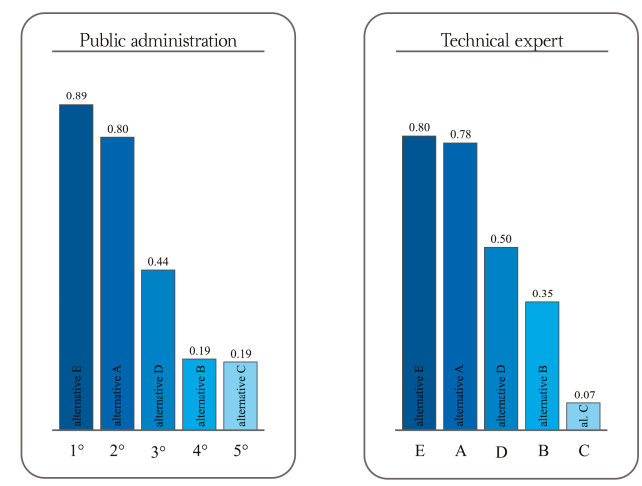

Figure 5. Results of the regime method: rank order of alternatives.

Both the rankings presented "Alternative E: Social hub with antiviolence center for women" as the best compromise solution. The other alternatives were in the same order, but they presented different scores.

\subsubsection{Weighted Summation Method}

The sensitivity of the rank order obtained with the regime analysis was then tested using the WSM, evaluating two new sets of weights through a pairwise process and using them to rank the alternatives.

The WSM is based on the impact matrix and a set of weights to compare the alternatives and to define a ranking of these. The set of weights consists of a matrix, in which the quantitative evaluation of the DM's preferences is reported; this type of evaluation allows the improvement of the design of the expressed preference through a quantitative pairwise evaluation process shown below (Table 2).

The application of weighted summation allows for verification of the robustness and stability of the results through a "sensitivity analysis". This process assesses the variation of the previous results through the variation of the weight matrix.

The weight matrix is assessed in a process of pairwise comparison among every possible couple of criteria. The DM is asked to express his preference through a numeric value that goes from 1 , when the considered criteria have the same importance, to 9, when a criterion is "extremely more important" than the other one. The matrix is not symmetrical, because if we compare $a$ with $b$, then the value of $b$ 
with respect to $a$ represents the reciprocal. For example, in our case, the criterion $a$ was more important than $b$, and the value was 1.2. So, the value of $b$ with respect to $a$ was the reciprocal $(1 / 1.2$, or 0.833$)$.

The identified weight matrixes are shown below in Table 2.

Table 2. Sets of weight matrix.

\begin{tabular}{|l|c|c|c|c|c|c|c|c|c|c|c|}
\hline \multicolumn{2}{|c|}{ Public administration } & $\mathrm{a}$ & $\mathrm{b}$ & $\mathrm{c}$ & $\mathrm{d}$ & $\mathrm{e}$ & $\mathrm{f}$ & $\mathrm{g}$ & $\mathrm{h}$ & $\mathrm{i}$ & $\mathrm{l}$ \\
\hline Ability to involve the third sector & $\mathrm{a}$ & & 1.200 & 0.588 & 1.000 & 0.714 & 1.200 & 1.000 & 1.500 & 1.500 & 1.700 \\
\hline Awareness and social collaboration & $\mathrm{b}$ & 0.833 & & 0.500 & 1.300 & 1.000 & 1.400 & 1.700 & 2.000 & 1.800 & 2.200 \\
\hline Enhancement of welfare & $\mathrm{c}$ & 1.700 & 2.000 & & 3.000 & 1.500 & 2.000 & 2.000 & 3.000 & 3.300 & 4.000 \\
\hline Possibility of obtain public funds & $\mathrm{d}$ & 1.000 & 0.769 & 0.333 & & 0.588 & 1.500 & 1.600 & 2.000 & 2.000 & 3.000 \\
\hline Construction cost & $\mathrm{e}$ & 1.400 & 1.000 & 0.667 & 1.700 & & 2.000 & 1.600 & 2.000 & 2.100 & 3.000 \\
\hline Capacty of encourge new activities & $\mathrm{f}$ & 0.833 & 0.714 & 0.500 & 0.670 & 0.500 & & 1.000 & 1.500 & 1.600 & 1.800 \\
\hline $\begin{array}{l}\text { Possibility of construction time } \\
\text { partition }\end{array}$ & $\mathrm{g}$ & 1.000 & 0.588 & 0.500 & 0.625 & 0.625 & 1.000 & & 1.400 & 1.800 & 2.100 \\
\hline Existence of competitors & $\mathrm{h}$ & 0.667 & 0.500 & 0.333 & 0.500 & 0.500 & 0.667 & 0.714 & & 1.500 & 1.300 \\
\hline Involvement in urban congestion & $\mathrm{i}$ & 0.667 & 0.556 & 0.303 & 0.500 & 0.476 & 0.625 & 0.556 & 0.667 & & 1.500 \\
\hline Encourage the old town redevelopment & $\mathrm{l}$ & 0.588 & 0.455 & 0.250 & 0.333 & 0.333 & 0.556 & 0.476 & 0.769 & 0.667 & \\
\hline
\end{tabular}

\begin{tabular}{|l|c|c|c|c|c|c|c|c|c|c|c|}
\hline \multicolumn{2}{|c|}{ Technical expert } & $\mathrm{a}$ & $\mathrm{b}$ & $\mathrm{c}$ & $\mathrm{d}$ & $\mathrm{e}$ & $\mathrm{f}$ & $\mathrm{g}$ & $\mathrm{h}$ & $\mathrm{i}$ & $\mathrm{l}$ \\
\hline Ability to involve the third sector & $\mathrm{a}$ & & 1.000 & 3.500 & 4.000 & 0.333 & 1.500 & 0.500 & 2.000 & 1.500 & 1.800 \\
\hline Awareness and social collaboration & $\mathrm{b}$ & 1.000 & & 0.500 & 2.500 & 0.667 & 1.500 & 0.714 & 0.714 & 2.000 & 1.000 \\
\hline Enhancement of welfare & $\mathrm{c}$ & 0.286 & 2.000 & & 1.000 & 1.000 & 2.000 & 1.700 & 1.500 & 3.000 & 1.700 \\
\hline Possibility of obtain public funds & $\mathrm{d}$ & 0.250 & 0.400 & 1.000 & & 1.500 & 3.000 & 2.000 & 2.000 & 3.500 & 3.500 \\
\hline Construction cost & $\mathrm{e}$ & 3.000 & 1.500 & 1.000 & 0.667 & & 2.000 & 1.500 & 1.700 & 3.000 & 2.100 \\
\hline Capacty of encourge new activities & $\mathrm{f}$ & 0.667 & 0.667 & 0.500 & 0.333 & 0.500 & & 0.667 & 0.500 & 1.000 & 0.769 \\
\hline $\begin{array}{l}\text { Possibility of construction time } \\
\text { partition }\end{array}$ & $\mathrm{g}$ & 2.000 & 1.400 & 0.588 & 0.500 & 0.667 & 1.500 & & 1.000 & 1.700 & 1.500 \\
\hline Existence of competitors & $\mathrm{h}$ & 0.500 & 1.400 & 0.667 & 0.500 & 0.588 & 2.000 & 1.000 & & 2.000 & 1.500 \\
\hline Involvement in urban congestion & $\mathrm{i}$ & 0.667 & 0.500 & 0.333 & 0.286 & 0.333 & 1.000 & 0.588 & 0.500 & & 0.714 \\
\hline Encourage the old town redevelopment & $\mathrm{l}$ & 0.556 & 1.000 & 0.588 & 0.286 & 0.476 & 1.300 & 0.667 & 0.667 & 1.400 & \\
\hline
\end{tabular}

The results of the calculation for the above sets of weights are shown in Figure 6. Both the evaluation rankings presented "Alternative E: Social hub with antiviolence center for women" as the best compromise solution, while the other positions were different in each ranking. These two rankings were different from the previous results due to an improved assessment of the sets of weights.
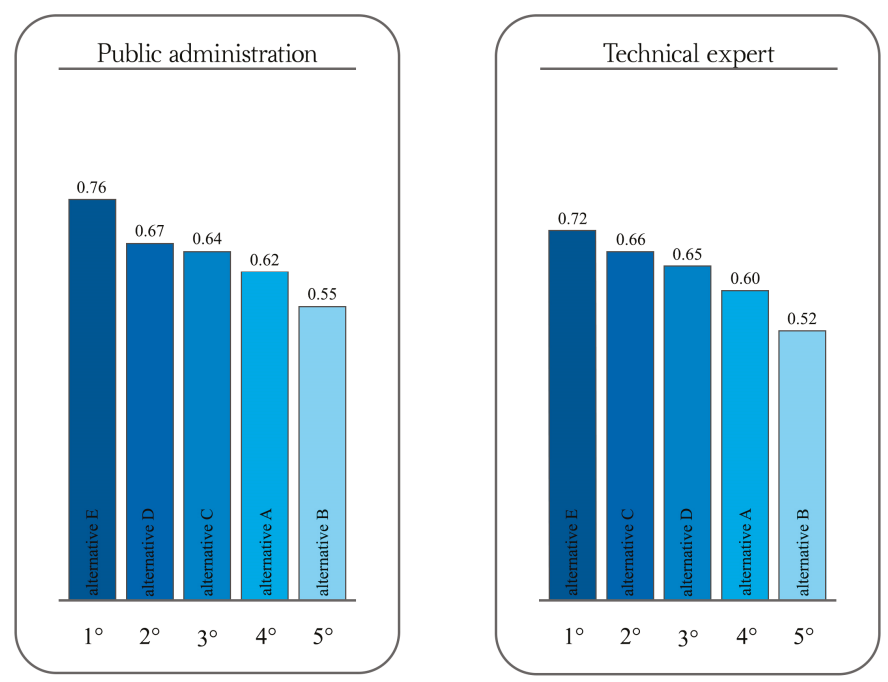

Figure 6. Results of the weighted summation method (WSM): rank order of the alternatives. 
The results obtained confirmed that the rank order of the alternative is not sensible to the weight vector assessed.

\subsubsection{Social Evaluation and Coalition Assessment}

Starting from the definition of the main social actors (see Section 3.1), the first step was the identification of their points of view in relation to the project alternatives. The information from each group, identified in the first stage of the process, was collected through an online survey supplied through Google Forms. The questionnaire was structured with close-ended questions; they had a limited number of answers, among which the respondent must choose the one which best matches his/her opinion. The form presented an initial introductory part with the aim of obtaining general information about the respondent; the second part described the case study and presented the project alternatives in simple and familiar language; in the third section, the respondent was asked to evaluate the presented alternatives through a linguistic evaluation. The linguistic assessment was expressed on a nine-level qualitative scale from "perfect" to "extremely bad".

Examples of the questions posed in each part of the questionnaire are here reported.

1) Are you a citizen of Mugnano di Napoli?
a) Yes, I am
b) I usually frequent the city
c) No, I am not

2) Do you know the ex-monastery of the Ritiro del Carmine
a) Yes, I do
b) I have heard of it
c) No, I don't

3) How do you evaluate the scenario "C: Cultural center and library" from 1 (perfect) to 9 (extremely bad)?

The collected evaluations were gathered and are displayed in the equity matrix shown in Table 3.

Table 3. Equity matrix.

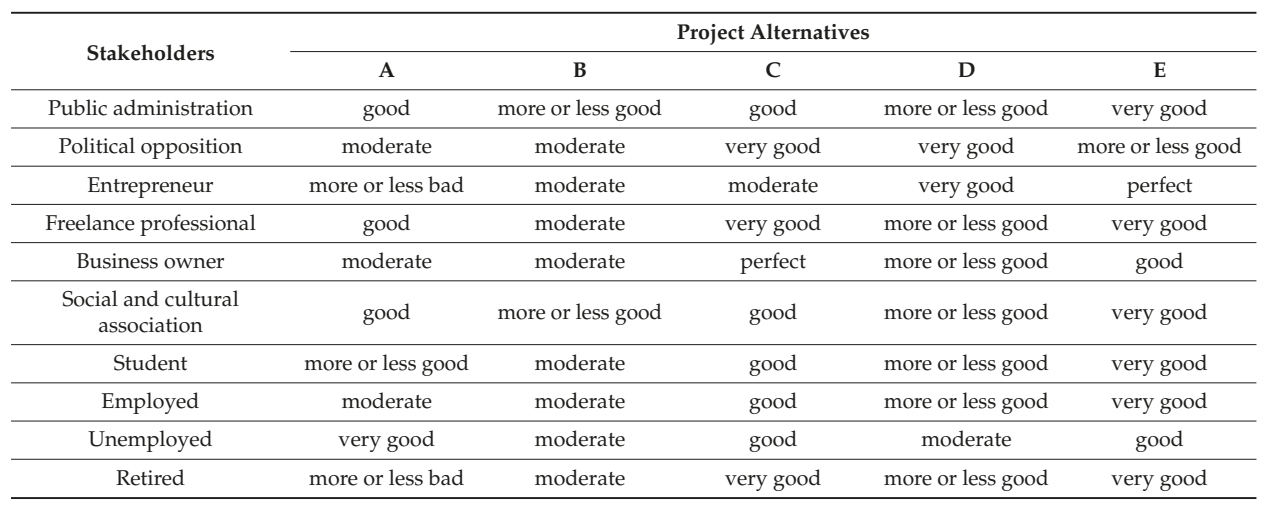

The equity matrix provided the linguist indication of the interest group judgments for each alternative. Semantic distance was also used in this case to calculate the similarity indexes among interest groups. A similarity matrix was then computed starting from the equity matrix. The similarity matrix provided an index, for each pair of interest groups $i, j$, of the similarity of judgement over the 
proposed alternatives. This index sij was calculated as sij $=1 /(1+\mathrm{dij})$, where dij is the Minkovsky distance between groups $i$ and $j$, which was calculated as follows:

$$
d i j=\sqrt[P]{\sum_{K=1}^{N}\left(S_{k}(i, j)\right)^{P}}
$$

where $S(i, j)$ is the semantic distance between groups $\mathrm{i}$ and $\mathrm{j}$ in the judgement of alternative $k, N$ is the number of alternatives, and $p>0$ is the parameter of the Minkovsky distance. Lastly, through a sequence of mathematical reductions, the dendrogram of coalition formation was built. It shows possible coalition formation for decreasing values of the similarity index and the degree of conflict among interest groups.

For the case study, we used the NAIADE software for the calculations. This software allowed for comparison of the preferences expressed and analysis of the similarity between the interests of each group. Furthermore, it supplied a graphic representation of the potential coalitions between the parties in the form of a dendrogram. Each meeting point has an associated numeric value, named "similarity index", which shows the similarity of the coalition, with values between 0 and 1 .

In the case of the Ex Ritiro del Carmine, the dendrogram (Figure 7) highlights good results in terms of agreement among the stakeholders.

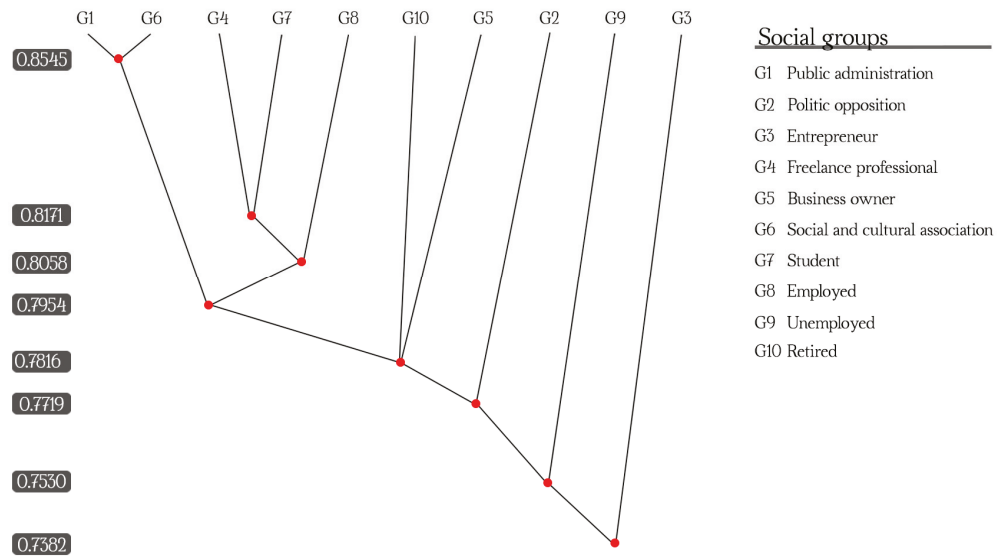

Figure 7. Dendrogram of the coalitions.

The first coalition, between G1 and G6 (public administration and social and cultural association), had a similarity index value of 0.8545 . The second coalition, between G4 and G7 (freelance professionals and students), had a the similarity index value of 0.8171 . The third coalition, between G8 (the employed) and the previous coalition (G4-G7), had a value of 0.8058 . The coalition between the two abovementioned groups (G1-G6 and G4-G7-G8) had a value of 0.7954 .

In Table 4, the possible coalitions referring to different rankings of the alternatives are shown. It is possible to note that for the ranking $(\mathrm{E}, \mathrm{C}, \mathrm{D}, \mathrm{A}, \mathrm{B})$, all the groups were in agreement, having a similarity index of 0.7382 , which is really high considering that the maximum value of similarity (total agreement) is 1 . 
Table 4. Possible coalitions on the ranking of the alternatives.

\begin{tabular}{ccccccccc}
\hline & & $\mathbf{0 . 8 5 4 5}$ & $\mathbf{0 . 8 1 7 1}$ & $\mathbf{0 . 8 0 5 8}$ & $\mathbf{0 . 7 8 1 6}$ & $\mathbf{0 . 7 7 1 6}$ & $\mathbf{0 . 7 5 3 0}$ & $\mathbf{0 . 7 3 8 2}$ \\
\hline $\boldsymbol{Z}$ & 1 & $\mathrm{E}$ & $\mathrm{E}$ & $\mathrm{E}$ & $\mathrm{E}$ & $\mathrm{E}$ & $\mathrm{E}$ & $\mathrm{E}$ \\
$\mathbf{Z}$ & 2 & $\mathrm{C}$ & $\mathrm{C}$ & $\mathrm{C}$ & $\mathrm{C}$ & $\mathrm{C}$ & $\mathrm{C}$ & $\mathrm{C}$ \\
$\mathbf{C}$ & 3 & $\mathrm{~A}$ & $\mathrm{~A}$ & $\mathrm{D}$ & $\mathrm{D}$ & $\mathrm{D}$ & $\mathrm{D}$ & $\mathrm{D}$ \\
$\mathrm{Z}$ & 4 & $\mathrm{D}$ & $\mathrm{D}$ & $\mathrm{A}$ & $\mathrm{A}$ & $\mathrm{A}$ & $\mathrm{A}$ & $\mathrm{A}$ \\
$\mathrm{A}$ & 5 & $\mathrm{~B}$ & $\mathrm{~B}$ & $\mathrm{~B}$ & $\mathrm{~B}$ & $\mathrm{~B}$ & $\mathrm{~B}$ & $\mathrm{~B}$ \\
\hline
\end{tabular}

So, the results of the calculation underline that the favorite solution is the "Alternative E: Social hub with antiviolence center for women".

\subsubsection{Results Comparisons}

In the previous sections, we established the rankings for each phase of the proposed framework with different methods. The obtained results supplied the same preference in every stage of the process (Table 5), so that the best compromise solution may be "Alternative E: Social Hub with antiviolence center for women".

Table 5. Comparison between different rankings.

\begin{tabular}{cccccc}
\hline \multirow{2}{*}{ Ranking } & \multicolumn{2}{c}{ Regime Method } & \multicolumn{2}{c}{ Weighted Summation } & \multirow{2}{*}{ NAIADE } \\
\cline { 2 - 5 } & P.A. & T.E. & P.A. & T.E. & \\
\hline 1 & E & E & E & E & E \\
\hline 2 & A & A & D & C & C \\
\hline 3 & D & D & C & D & D \\
\hline 4 & B & B & A & A & A \\
\hline 5 & C & C & B & B & B
\end{tabular}

P.A.-public administration; T.E.-technical experts; NAIADE-Novel Approach to Imprecise Assessment and Decision Environments.

In the next subsection, we evaluate the financial sustainability of the alternative chosen from the stakeholders involved in the decision-making process.

\subsection{Definition of a Project Strategy}

Nowadays, the lack of public resources complicates the financing of the redevelopment process. Therefore, public administrations have to assess the public expenditure in order to guarantee economic sustainability throughout the life of a project. Financial analysis allows the evaluation of both the construction cost and the cash flow and calculates the necessary public economic resources to effectively run a reused building [52,53].

Once the new function is defined, it is possible to evaluate the construction cost of the project. This is composed of several rates [50], in particular, technical construction cost (TCC), taxes (corresponding to $21 \%$ of TCC), professional costs, preliminary studies and surveys, tender notice, and accidents (corresponding to $5 \%$ of TCC).

The values of technical costs used here referred to the official "Listino Tipologico" published by DEI, (Tipografia del Genio Civile) in 2017.

For the Ex Ritiro del Carmine, each rate is shown in Table 6 
Table 6. Construction cost.

\begin{tabular}{lc}
\hline \multicolumn{1}{c}{ Construction Cost } & $\boldsymbol{\epsilon}$ \\
\hline Technical construction cost & $1,445,996.83$ \\
Taxes & $303,659.33$ \\
Professional costs & $113,727.65$ \\
Preliminary studies and surveys & $40,000.00$ \\
Tender notice & 5000.00 \\
Accidents $\quad$ Total & $72,299.84$ \\
& $1,980,683.66$ \\
\hline
\end{tabular}

While the cost of construction concerns the early years of the process, the management of the building spans a greater amount of time. Management costs and incomes are evaluated during a time period, which depends on the type and size of the project. For public projects, this is often a period of 20 years [54].

This evaluation requires the formulation of a mode of operation: for each new function, a management hypothesis is made between the "direct management", by the municipality, and the "indirect management", through private rent. Indirect management consists of the renting of the spaces to private companies for each specific activity.

For the monastery, the above hypotheses are reported for each function in Table 7.

Table 7. Management model.

\begin{tabular}{lc}
\hline \multicolumn{1}{c}{ Activity } & Management Model \\
\hline Ground floor & \\
Pooled space & Direct \\
Toy room & Direct \\
Association room & Indirect \\
Sacristy & Indirect \\
Restaurant & Indirect \\
Theater & Direct \\
First floor & \\
Antiviolence center & Direct \\
Second floor & \\
Cultural center & Direct \\
Conferences room & Direct \\
\hline
\end{tabular}

The costs and the incomes for each function were evaluated through a market analysis and referring to official list prices. The evaluation used both the comparison between historical data, when available, and an indirect approach, when there were no historical data [50].

The evaluated costs were the following:

- $\quad$ Ordinary management $(\mathrm{O})$ : linked to necessary things to run the activity;

- $\quad$ Staff management (SM): linked to the costs of the employees;

- Ordinary maintenance (OM): linked to the periodic activities to allow the normal use of the building;

- $\quad$ Extraordinary maintenance (EM): linked to long period for repairs and/or prolonged use of the building;

- $\quad$ Insurance and taxes (IT).

The evaluated incomes were the following:

- $\quad$ Rate income (RI): linked with the tickets sold for public events;

- $\quad$ Nonrate income (NRI): linked with the rent received for the activity management; 
The comparison between the costs and the incomes (Table 8) showed that the costs are greater than the incomes. Therefore, it is necessary to find new funds from public capital. These, which are reported in Table 8, derive from participation in public invitations to tender for single activities.

Table 8. Costs, incomes, and funds.

\begin{tabular}{|c|c|c|c|c|c|c|c|c|}
\hline & \multicolumn{5}{|c|}{ Costs } & \multicolumn{2}{|c|}{ Incomes } & \multirow{2}{*}{$\begin{array}{c}\text { Funds } \\
\mathrm{F}\end{array}$} \\
\hline & $\mathrm{O}$ & SM & OM & EM & IT & RI & NRI & \\
\hline & $€ /$ Year & k€/Year & $€ /$ Year & $€ / 10$ Year & $€ /$ Year & $€ /$ Year & $€ /$ Year & $€ /$ Year \\
\hline \multicolumn{9}{|l|}{ Ground floor } \\
\hline Pooled spaces & 2400 & 51,662 & 800 & 12,000 & & & & \\
\hline Toy room & 3.6 & 11,310 & 400 & 8000 & & 2400 & & \\
\hline Association room & & & & 5000 & 1155 & & 3000 & \\
\hline Sacristy & & & & 600 & 1680 & & 5400 & \\
\hline Restaurant & & & & 18,000 & 6006 & & 25,200 & \\
\hline Theater & 14,000 & 10,000 & 600 & 16,000 & & 39,230 & 12,500 & 10,000 \\
\hline \multicolumn{9}{|l|}{ First floor } \\
\hline Antiviolence center & 17,850 & 53,192 & 2000 & 50,000 & & & & 91,042 \\
\hline \multicolumn{9}{|l|}{ Second floor } \\
\hline Cultural center & 23,500 & 43,668 & 2500 & 55,000 & & & & 4500 \\
\hline Conferences room & 7000 & & 400 & 8000 & & & 12,000 & \\
\hline Total & 68,350 & 169,832 & 6700 & 178,000 & 8841 & 41,630 & 58,100 & 105,542 \\
\hline
\end{tabular}

$\mathrm{O}$-ordinary management; SM-staff management; OM-ordinary maintenance; EM-extraordinary maintenance;

IT-insurance and taxes; RI-rate income; NRI-nonrate income.

The cash flow is represented on a time line, which relates costs, incomes, and funds to each period of time (Table 9).

The comparison between the costs, incomes, and funds highlights the lack of resources for the management of the Ex Ritiro del Carmine. While the cost of construction is provided by public funds, the incomes do not entirely cover the costs. Therefore, the municipality has to cover the gap with its own resources.

The public financial statement is divided into "missions". The redevelopment of the monastery embraces three different missions, for which $€ 3.3$ million are allocated. Therefore, the Ex Ritiro requires at least $1.5 \%$ of the stated budget available. 


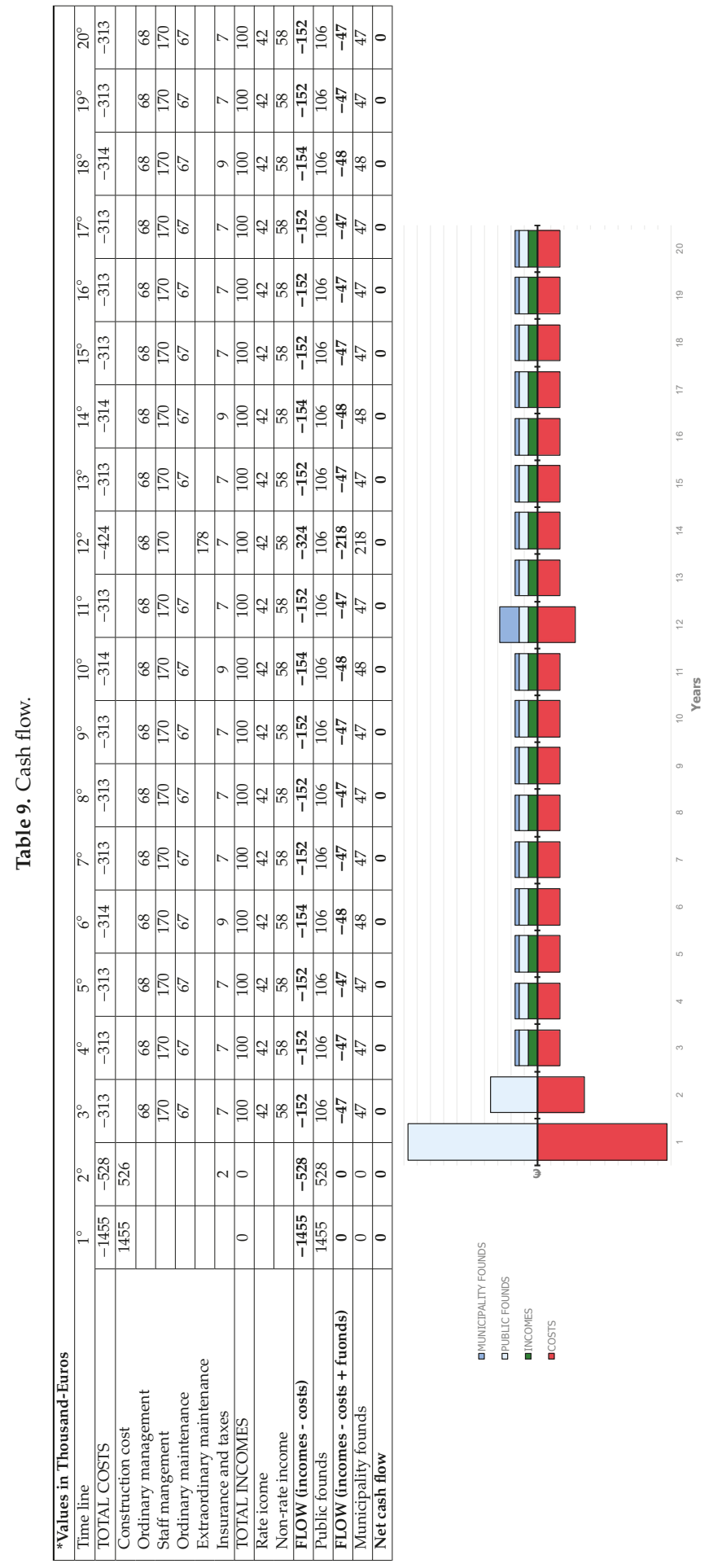




\section{Discussion and Future Research}

This paper presents an integrated methodological approach for the choice of sustainable alternative functions for the adaptive reuse of the former monastery of Ritiro del Carmine in the Campania region from a circular economic perspective.

The proposed methodology integrates different evaluation methods to support the whole decision-making process, from the phase of problem definition to the study of the financial feasibility of the chosen project function, in order to encourage the participation of all stakeholders and to define a "shared solution" that can meet the needs of the local community, as well as be sustainable in the long term from an economic point of view.

The presented case study allowed the exploration of the framework in order to support a flexible and adaptive decision-making process that can consider the complex value of cultural heritage and the multiplicity of actors involved in the decision-making process.

The obtained results showed that "Alternative E: Social hub with antiviolence center for women" was the preferred choice considering the defined evaluation criteria. Moreover, this alternative reached the highest level of consensus among the stakeholders involved in the decision-making process. Nevertheless, the solution found is not sustainable from a financial point of view considering the high investment costs; so, the financial analysis showed the need for access to public funding to cover investment costs. The public investment represents an opportunity to recover a building that is a symbol of the community in order to provide a space for growth and cultural exchange, creating the first cultural center of the city and providing a concrete response to the demand for protection of vulnerable women.

The abovementioned experimentation has shown the usefulness of the integration of different evaluation methods to support the decision-making process in the case of renovation of a heritage building characterized by several elements of complexity. In this sense, the proposed approach seemed appropriate to the case study.

Today, the new challenge for local authorities is to regenerate abandoned heritage buildings while involving different stakeholders and the local community in order to create new models of governance which are able to guarantee economic sustainability and the conservation of historical and cultural values, as in the case of the former monastery of Ritiro del Carmine, which has always been a symbolic building for the community.

From this perspective, future research in this field can be oriented toward the search for new circular financing models, particularly in the field of impact financing, as well as new circular governance models, based on the notion of "heritage as commons", starting from the Italian experimentations of municipal civic agreements for commons management.

Author Contributions: Conceptualization: F.T., M.F., and G.A.; Data curation: F.T., M.S. M.F.; Formal analysis: F.T., M.S.; Methodology: F.T.; Validation: F.T., M.F. and G.A.; Writing—original draft: F.T., M.F. G.A. and M.S. All authors read and approved the final manuscript.

Funding: This research received no external funding.

Conflicts of Interest: The authors declare no conflict of interest.

\section{References}

1. UN (United Nations). Transforming Our World: The 2030 Agenda for Sustainable Development; General Assembly; UN (United Nations): New York, NY, USA, 2015.

2. ICOMOS. ICOMOS Action Plan: Cultural Heritage and Localizing the UN Sustainable Development Goals (SDGs); ICOMOS: Paris, France, 2017.

3. European Commission. Towards an Integrated Approach to Cultural Heritage for Europe; European Commission: Brussels, Belgium, 2014.

4. Gastaldi, F.; Camerin, F. Immobili pubblici e militari dismessi: un'opportunità per la rigenerazione urbana. Eyesreg G. Sci. Reg. 2016, 6, 11-14. 
5. Ladu, M. Strategie e strumenti per rigenerare il patrimonio immobiliare pubblico nell'era della Smart City. In Proceedings of the 22nd Conferenza Nazionale ASITA, Bolzano, Italy, 27-29 November 2018; pp. 609-616.

6. Mingione, E.; Vicari, S. Politiche urbane e innovazione sociale. In Città tra Sviluppo e Declino: Un'agenda Urbana Per l'Italia; Calafati, A.G., Ed.; Donzelli Editore: Roman, Italy, 2015; pp. 97-108.

7. Mangialardo, A.; Micelli, E. From sources of financial value to commons: Emerging policies for enhancing public real-estate assets in Italy. Pap. Reg. Sci. 2018, 97, 1397-1408. [CrossRef]

8. Ausiello, G. Innovation in the field of Cultural Heritage. Towards a conservative approach more and more sustainable. In Sustainable Technologies for the Enhancement of the Natural Landscape and of the Built Environment; De Joanna, P., Passaro, A., Eds.; Luciano Editore: Napoli, Italy, 2019.

9. Yung, E.H.; Chan, E.H. Evaluation of the social values and willingness to pay for conserving built heritage in Hong Kong. Facilities 2015, 33, 76-98. [CrossRef]

10. Calvaresi, C.; Pederiva, I. Community hub: Rigenerazione urbana e innovazione sociale. In Agenda Milano Ric e Prat Per una Città Inclusiva; Fond Feltrinelli: Milano, Italy, 2016.

11. Valdes-Vasquez, R.; Klotz, L.E. Social sustainability considerations during planning and design: Framework of processes for construction projects. J. Constr. Eng. Manag. 2012, 139, 80-89. [CrossRef]

12. Dempsey, N.; Bramley, G.; Power, S.; Brown, C. The social dimension of sustainable development: Defining urban social sustainability. Sustain. Dev. 2011, 19, 289-300. [CrossRef]

13. Throsby, D. Investment in urban heritage conservation in developing countries: Concepts, methods and data. City Cult. Soc. 2016, 7, 81-86. [CrossRef]

14. Fusco Girard, L.; Gravagnuolo, A. Circular economy and cultural heritage/landscape regeneration. Circular business, financing and governance models for a competitive Europe. Bdc Boll. Cent. Calza Bini 2017, 17, $35-52$.

15. Latham, D. Creative Re-Use of Buildings; Donhead Publishing Ltd.: Shaftesbury, UK, 2000.

16. Plevoets, B. Retail-Reuse: An Interior View on Adaptive Reuse of Buildings. Ph.D. Thesis, Hasselt University, Hasselt, Belgium, 2015.

17. Bullen, P.; Love, P. Adaptive reuse of heritage buildings. Struct. Surv. 2011, 29, 411-421. [CrossRef]

18. Forte, F. Historic, Artistic and Cultural Patrimony for a "Habitable City": Incentives for Care. In Green Energy and Technology, Integrated Evaluation for the Management of Contemporary Cities, Results of SIEV 2016; Springer: Cham, Switzerland, 2018.

19. Fusco Girard, L. Creativity and the Human Sustainable City: Principles and Approaches for Nurturing City Resilience. In Sustainable City and Creativity. Promoting Creative Urban Initiatives; Girard, L.F., Baycan, T., Nijkamp, P., Eds.; ASHGATE: Farnham, UK, 2011; pp. 55-95.

20. Lazrak, F.; Nijkamp, P.; Rietveld, P.; Rouwendal, J. Cultural heritage and creative cities: an economic evaluation perspective. In Sustain City Creat Promot Creat Urban Initiat; Girard, P., Baycan, L.F., Nijkamp, T., Eds.; Ashgate Publishing Editor: Farnham, UK, 2012; pp. 225-243.

21. Geraedts, R.P.; van der Voordt, T.; Remøy, H. Conversion Potential Assessment Tools. In Building Resilience in Urban Settlements through Sustainable Change of Use; Remøy, H., Wilkinson, S.J., Eds.; Wiley-Blackwell: Hoboken, NJ, USA, 2018; pp. 121-151. ISBN 978-1-119-23142-4.

22. Celadyn, M. Interior Architectural Design for Adaptive Reuse in Application of Environmental Sustainability Principles. Sustainability 2019, 11, 3820. [CrossRef]

23. Pinto, M.R.; De Medici, S.; Senia, C.; Fabbricatti, K.; De Toro, P. Building reuse: multi-criteria assessment for compatible design. International Journal of Design Sciences and Technology. IJDST 2017, 22, 165-193.

24. Bottero, M.; D'Alpaos, C.; Oppio, A. Ranking of Adaptive Reuse Strategies for Abandoned Industrial Heritage in Vulnerable Contexts: A Multiple Criteria Decision Aiding Approach. Sustainability 2019, 11, 785. [CrossRef]

25. Simon, H. Theories of Bounded Rationality. In Decision and Organization; McGuire, C.B., Radner, R., Eds.; North-Holland: Amsterdam, The Netherlands, 1972; pp. 161-176.

26. Dyson, K.; Matthews, J.; Love, P.E. Critical Success Factors of Adapting Heritage Buildings: An Exploratory Study. Built Environ. Proj. Asset Manag. 2015, 6, 44-57. [CrossRef]

27. Munda, G. Social multi-criteria evaluation: Methodological foundations and operational consequences. Eur. J. Oper. Res. 2004, 158, 662-677. [CrossRef] 
28. De Marchi, B.; Funtowicz, S.O.; Cascio SLo Munda, G. Combining participative and institutional approaches with multicriteria evaluation. An empirical study for water issues in Troina, Sicily. Ecol. Econ. 2000, 34, 267-282. [CrossRef]

29. Nijkamp, P.; Rietveld, P.; Voogd, H. Multicriteria Evaluation in Physical Planning; Elsevier Science: Amsterdam, The Netherland, 1990.

30. Hinloopen, E.; Nijkamp, P.; Rietveld, P. Qualitative discrete multiple criteria choice models in regional planning. Reg. Sci. Urban Econ. 1983, 13, 77-102. [CrossRef]

31. Janssen, R.; van Herwinjen, M.; Beinat, E. DEFINITE for Windows. A System to Support Decisions on a Finite Set of Alternatives (Software Package and User Manual); Institute for Environmental Studies (IVM), Vrije Universiteit: Amsterdam, The Netherland, 2001.

32. Munda, G. Social multi-criteria evaluation for urban sustainability policies. Land Use Policy 2006, 23, 86-94. [CrossRef]

33. Munda, G.; Nijkamp, P.; Rietveld, P. Qualitative multicriteria methods for fuzzy evaluation problems: An illustration of economic-ecological evaluation. Eur. J. Oper. Res. 1995, 82, 79-97. [CrossRef]

34. Joint Research Centre of the European Commission. NAIADE. Manual and Tutorial. Joint Research Centre, EC, ISPRA Website, Version 1.0. ENG. Institute for Systems, Informatics and Safety: Italy, 1996. Available online: https://www.weadapt.org/sites/weadapt.org/files/naiade.pdf (accessed on 22 September 2019).

35. Ferretti, V.; Pluchinotta, I.; Tsoukiàs, A. Studying the generation of alternatives in public policy making processes. Eur. J. Oper. Res. 2019, 273, 353-363. [CrossRef]

36. Roy, B. Decision-aid and decision-making. Eur. J. Oper. Res. 1990, 45, 324-331. [CrossRef]

37. Tsoukiàs, A. From decision theory to decision aiding methodology. Eur. J. Oper. Res. 2008, 187, $138-161$. [CrossRef]

38. Del Giudice, V.; De Paola, P.; Torrieri, F. An integrated choice model for the evaluation of urban sustainable renewal scenarios. Adv. Mater. Res. 2014, 1030, 2399-2406. [CrossRef]

39. Del Giudice, V.; De Paola, P.; Torrieri, F.; Nijkamp, P.; Shapira, A. Real Estate Investment Choices and Decision Support Systems. Sustainability 2019, 11, 3110. [CrossRef]

40. Torrieri, F.; Batà, A. Spatial Multi-criteria Decision Support System and Strategic Impact Assessment: a case study. Buildings 2017, 7, 96. [CrossRef]

41. Rydin, Y.; Pennington, M. Public participation and local environmental planning: the collective action problem and the potential of social capital. Local Environ. 2000, 5, 153-169. [CrossRef]

42. Coenen, F.H.J.M. Public Participation and Better Environmental Decisions. In The Promise and Limits of Participatory Processes for the Quality of Environmentally Related Decision-Making; Springer: Berlin/Heidelberg, Germany, 2008.

43. Mathur, V.N.; Price, A.D.F.; Austin, S. Conceptualizing stakeholder engagement in the context of sustainability and its assessment. Constr. Manag. Econ. 2008, 26, 601-609. [CrossRef]

44. Buanne, M.; Squillacciotti, M.; Scognamiglio, I.; Fumo, M. Idee progettuali per rigenerare il patrimonio edilizio scolastico: due complessi architettonici storici nel cuore antico di Napoli. In Colloqui. AT. e 2018. Edilizia Circolare; Cuboni, F., Desogus, G., Quaquero, E., Eds.; Edicom Edizioni: Cagliari, Italy, 2018; pp. 219-228.

45. De Gargiulo, F. Mugnano di Napoli: Fra Storia e Tradizioni; Ferraro: Naples, Italy, 1982.

46. Marina, F.; Gigliola, A.; Violano, A.; Mariangela, B.; Veronica, V. Dall'anagrafica dell'edilizia scolastica alla valutazione degli interventi di riqualificazione: alcuni casi di studio nella città metropolitana di Napoli. In Proceedings of the ReUso-VI Convegno Internazionale Sulla Documentazione, Conservazione e Recupero del Patrimonio Architettonico e Tutela Paesaggistica, Messina, Cyprus, 29 October-3 November 2018; Gangemi Editore: Roman, Italy, 2018.

47. Trowers \& Hamlins. Highly Valued, Hard to Value: Towards an Integrated Measurement of Real Estate Development. 2016. Available online: https://www.trowers.com/insights/2016/march/Highlyvalued-hard-to-value---towards-an-integrated-measurement-of-real-estate-development (accessed on 22 September 2019).

48. Rojas, E. Urban Heritage for Sustainable Development. In Culture: Urban Future; Global Report on Culture for Sustainable Urban Development. UNESCO, 2016. Available online: https://unesdoc.unesco.org/ark: /48223/pf0000245999 (accessed on 22 September 2019).

49. Bryson, J.M. What to do when stakeholders matter: Stakeholder identification and analysis techniques. Public Manag. Rev. 2004, 6, 21-53. [CrossRef] 
50. Bovaird, T.; Löffler, E. The changing context of public policy. In Public Management and Governance, 2nd ed.; Bovaird, T., Löffler, E., Eds.; Routledge: Abington, UK, 2009; pp. 39-50.

51. Istituto Nazionale di Statistica (Istat) La Violenza Sulle Donne. Available online: https:/www.istat.it/it/ violenza-sulle-donne. (accessed on 22 September 2019).

52. Valenza, A.; Vignetti, S. Analisi finanziaria dei progetti di intervento pubblico. In Interventi Pubblici e Processi Decisionali; Parmentola, N., Rotondo, S., Eds.; Formez: Naples, Italy, 2004; pp. 85-103.

53. Del Giudice, V. Estimo e Valutazione Economica dei progetti; Loffredo Editore University: Naples, Italy, 2011.

54. Sartori, D.; Catalano, G.; Genco, M.; Pancotti, C.; Sirtori, E.; Vignetti, S.; Bo, C. Guide to Cost-Benefit Analysis of Investment Projects. Economic appraisal tool for Cohesion Policy 2014-2020; European Commission: Brussels, Belgium, 2014.

(C) 2019 by the authors. Licensee MDPI, Basel, Switzerland. This article is an open access article distributed under the terms and conditions of the Creative Commons Attribution (CC BY) license (http://creativecommons.org/licenses/by/4.0/). 

Article

\title{
Solutions for the Sustainable Management of a Cultural Landscape in Danger: Mar Menor, Spain
}

\author{
María Griñán Montealegre \\ Department of History of Art, University of Murcia, 30001 Murcia, Spain; mariagri@um.es; Tel.: +34-868-88-7770 \\ Received: 26 October 2019; Accepted: 24 December 2019; Published: 31 December 2019
}

\begin{abstract}
The focus of this paper is a case study on the municipality of San Javier in Spain. The aim of the analysis was to provide a working model for the integration of the assessment and conservation of built heritage into broader projects devoted to the sustainable restoration of natural spaces undergoing evident declines in habitability. With a population of 31,905, San Javier is located on the edge of the Mar Menor, which is one of the largest permanent salt water lagoons in the Mediterranean. It forms part of the coastal region of the Autonomous Community of the Region of Murcia in the southeast of the Iberian Peninsula. San Javier is one of four municipalities that administer this unique ecosystem. However, despite being designated as a protected natural site since its 14 beaches are one of the most important national and international tourist destinations in this Autonomous Community, since 2016, it has suffered one of the worst environmental crises in its history. One of the outcomes of this situation is that the government bodies involved have begun to seek new models for the area's complete regeneration that would enable sustainable growth and also include the social and economic sectors that have, to date, played a secondary role in managing the area. In this regard, cultural heritage should play a key role. The aim of this study was to demonstrate that the region's cultural heritage, despite the complex issues involved in its management, especially for the local administrative bodies, can contribute to the creation of new models for regeneration. Besides the added value of cultural prestige provided by this area's unique cultural landscape, which is a further legacy of the region's history and artistic development, engaging with cultural heritage facilitates the revival of traditional systems that contribute to environmental improvement. Finally, this paper provides tools that enable local groups, and, above all, the residents themselves, to identify with the values of their cultural heritage.
\end{abstract}

Keywords: cultural landscape; local governance; sustainability; immovable properties; bathing houses; Mar Menor; San Javier

\section{Introduction}

The municipality of San Javier is located on the edge of the Mar Menor, which is located in Spain's Murcia region, and is one of the largest permanent salt water lagoons in Europe. It is a medium-sized town, which, according to the 2018 census [1], had a population of 31,905, although the trend toward seasonal fluctuation in its population during the summer months has become pronounced. Mar Menor forms part of a unique ecosystem, and it is listed in the Ramsar Convention for wetlands of international importance [2], in addition to being designated a protected natural space and area of special interest. The lagoon forms part of three other municipalities: San Pedro del Pinatar, Los Alcázares, and Cartagena.

The region's unique natural features are combined with other types of cultural heritage that are in diverse states of conservation across the region, and together they form a cultural landscape that is unique in Spain. However, the Mar Menor and its surrounding landscape have undergone one of the worst environmental crises in its history, which was mainly due to the agricultural overexploitation 
of the coastal region; the municipality of San Javier alone generates around 21,500 jobs in the fishing and agricultural sector. The rapid development of tourism since 1950 has alarmingly transformed the traditional urban landscape. This has been due, firstly, to the high demand for second homes [3] intended primarily for seasonal use (the municipality of San Javier generates 10,145 jobs in the service sector and almost 700 in the building sector), and secondly, the lack of planning with regard to the public and private infrastructures that drain into the lagoon.

All this occurred with the emergence of a cultural dimension of tourism in Spain from the 1980s onwards - the consolidation of democracy and the social and cultural change that led to legislative developments and the creation of a complex administration that gave local governance an unprecedented role.

Subsequently, the 2007 financial crisis launched a new economic cycle that had major repercussions for the commercial and social fabric in the region. The drastic collapse of sectors, such as the construction sector, and the reactivation of agriculture in the Mar Menor region prompted the local administration to seek new forms of tourist attraction, including cultural heritage, which, after a gradual start, has succeeded. However, the natural environment, and with it the natural and human landscape, has been the location of natural disasters clearly linked to the widely predicted impact of local malpractice and the broader effects of climate change. Since 2016, various episodes of flooding have been caused by run-off water from the region's watercourses, and these have introduced noxious substances and waste into the Mar Menor, which has had a serious effect. In parallel, the land has been overly exploited, and especially the lagoon. Unorganized urban development, excessively intensive agriculture, the construction of artificial beaches, navigable canals, heavy metal runoff, construction of marinas, and the anchoring of hundreds of ships have been slowly destroying the delicate marine meadows of the Mar Menor state since the 1980s. Since 2017, this situation has been the main cause of the progressive decrease in traditional sun and beach tourism. The greatest impact cannot yet be quantified due to the disaster caused by the recent rains in the area that triggered the low-price sales of these second homes and the loss of attraction as a tourist destination.

As a result, the different levels of administration (general, regional, and local) have begun to examine new models of integral regeneration to ensure there is sustainable growth in the region [4]. As a starting point, the premise was taken that both the importance of cultural heritage and its conservation must play an active role in the creation of new social, economic, and urban models for the Mar Menor region (see Figure 1). The main objective of this study was to demonstrate that the area's heritage and especially the real estate should be considered in the creation of these new models, which will also provide a means to revive traditional systems capable of contributing to environmental improvements. In addition, through education programs, recovering and promoting traditional trades (such as the restoration and conservation of Latin sailboats, companies specializing in sustainable maritime construction, etc.), and increased awareness, members of society could play an active role in these new social models.

When the cultural heritage of the San Javier region is addressed, its protected cultural heritage can be identified and documented and, thereby highlighting its role in the region. This study demonstrates that the repercussions of the various regional regulations on cultural heritage have not been effective, and that, in some cases, the regulations have led to the destruction of built heritage. 


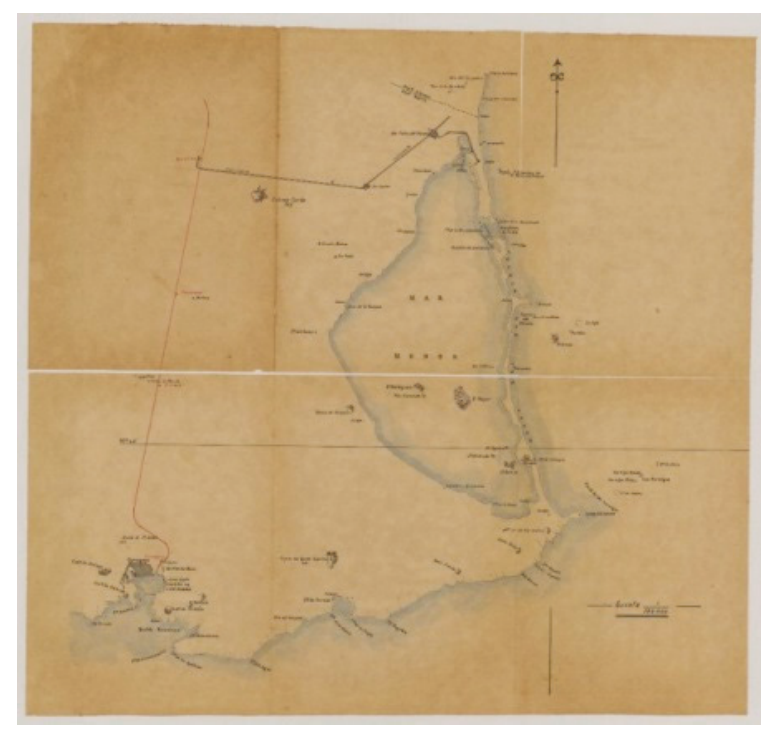

Figure 1. Mapa de la Comarca del Mar Menor by Gustavo Gilman. 1905 [5]. For more information, please entry the website at https://archivoweb.carm.es/archivoGeneral/arg.mostrar_visor3?idses=0\& $\mathrm{id}=4001373$.

\section{Materials and Methods}

The basis of this article was the findings of the research project called Analysis of the Impact of Regeneration Strategies on the Conservation of the Cultural Heritage of Historic Industrial Areas (HAR2014-58151-R), which was funded by the Spanish government, through the state $\mathrm{R}+\mathrm{D}+\mathrm{I}$ program Addressing the Challenges Faced by Society 2014 undertaken between 2015 and 2019. A major outcome of this project and its study of a select group of municipalities was the observation that a major blind spot exists in Spanish local government policies with regard to historical artistic and cultural heritage sites. Therefore, the analysis was extended to coastal spaces, such as that studied here: a medium-sized municipality whose growth was linked to the historic evolution of a maritime space, and, despite the environmental issues that have arisen, a municipality whose historic and cultural legacy could constitute an element of cohesion for its broader region. However, to achieve this, good local government practices are required, and a further issue to be addressed is the complex bureaucratic network that affects such practices. The bureaucracy has been proven to be inefficient in the conservation and promotion of San Javier's cultural legacy, due to the heritage regulations being affected by urban planning legislation.

In various municipalities, for the preservation of cultural heritage, knowledge of the relevant cultural assets in each region must be accompanied by the understanding of their use and impact in both their specific environment and in society as a whole. Above all, the potential and advantage offered by the assets for generating individual and collective values and experiences must be considered, as well their contribution to urban sustainability. The forms of governance must be analyzed to provide information, knowledge, and tools to those responsible for both decision-making and the managers to enable improvements in the design, implementation, and evaluation of strategies and actions, in which cultural heritage could be included as a core factor. Thereby, social and cultural values and the collective dynamics of identification need to undergo a series of social changes, especially with regards to the provision of increased knowledge concerning the evolution of cultural identities and common values amidst processes of change. Finally, I aimed to demonstrate that cultural heritage 
is a key element in the construction of citizenship values, the learning of one's own history, and the identification of solutions for the challenges faced by society [6].

For this purpose, I analyzed the complex administrative network that influences wealth management and how it is based on a wide range of regulations of different legal natures. In some cases, such as the municipality of San Javier, these regulations have been the reason for the loss of some of the most significant and representative examples of cultural heritage. The balnearios [7] merit special attention as they are specific local examples of the region's architecture. Despite their importance in the image of the cultural landscape of the Mar Menor, their disappearance throughout the last decade of the 20th century was the result of a conflict between original private property owners and the application of a new Coastal Law that transformed them into public spaces (Bienes Demaniales). The general administration was not interested in these elements that were historically constructed by individual owners who not only acquired property on land but also on the coast. Finally, the local community is concerned about the area becoming a tourist destination, but are not worried about its impact on the environment and future sustainable growth.

In this study, examples of immovable properties that have been granted various categories of heritage and/or urban protection were identified and studied, and the impact of this protection with regard to the region was analyzed, which until just a few decades ago was administratively referred to as the Comarca del Mar Menor.

\section{Results}

Since 1990, many authors have assessed cultural heritage as a fundamental piece in strategic local development operations [8-11]. The association between tourism and management of cultural heritage [12-14] has been studied as an alternative to local difficulties $[15,16]$.

In Spain, cultural tourism has been extended since 2000 as an alternative to the marked dependence on sun and beach tourism, which is strongly seasonal [8,17], improving the local economy by reducing seasonality and unemployment, and providing business opportunities to improve the standard of living of the population and attract European local development programs [18]. As a form of economic independence of heritage assets, cultural tourism guarantees their conservation [19], connecting heritage areas with international models that highlight the importance of cultural tourism [20-26].

Although the conceptualization and methods applied seem clear, the objectives of the International Charter on Cultural Tourism [27] indicate that its local application is basic and not yet fully effective, since the value and quality of historical and cultural assets are still measured only in terms of their ability to attract tourism [18]. Thus, except for sites with significant heritage values, museums and exhibitions have been the main devices used to attract cultural tourists. Starting a program to modernize the supply and management of museums [28-35] is a strategy that currently exists in Spain in almost all of the local heritage policies under the guidelines initiated by the Council of Europe and with the support from programs and structural funds of the European Union in its attempt to overcome regional imbalances [36,37].

In this work, the starting premise is that to preserve cultural heritage, it must be transformed into a product not only of tourist consumption, but also of cultural and identity. The disciplines of art history [38-40] and visual culture [41] are required to assist with this transition. According to Mirzoeff, the ways of seeing involve the practices of looking, with the senses of what we call the viewer, who is the one who looks or sees. The object or thing that is looked at may be an object of art or a series of things experienced by people in the present or in the past [42].

Historical, artistic, or cultural heritage [43,44] can be immovable, movable, or intangible [45-47], and is understood as a changing social creation and therefore has a relative attributed degree of value. As UNESCO indicated in its 1992 revision, during the commemoration of the 1972 Convention on the Protection of the World Cultural and Natural Heritage, the representation of European and North American cultural goods, strong predominance of religious buildings (mostly Christianity), and preponderance of historic cities and civilizations had disappeared, to the detriment of living cultures. 
In reality, the list only included a few countries, not all of humanity $[45,48]$. Also, the complexity of the local management of Spanish cultural heritage that is mainly due to an interdependence of diverse public and private sectors, which, in some cases, has led to the loss of a significant part of its cultural goods [49-52]. Both tangible and intangible cultural heritage aspects need to be used as an element of identity cohesion of the community, so that cultural heritage is valued as an effective tool that contributes to the sustainable development of the territory [53,54]. Through cultural goods, the different ways of life, customs, and beliefs are demonstrated, and therefore must be known and valued by the society that produced them. In this dialogue, society recognizes and protects not only its past but also its future.

In terms of space, certain studies Mar Menor have laid the foundation for the knowledge of its history and culture [55-59]. Conversely, research on the physical environment, its geological conformation, and its evolution and water characteristics have not recently been the object of study [60], with studies from 1978 [61-63], when natural changes and anthropogenic changes were occurring [61-68].

\section{Discussion}

A fundamental factor of the Mar Menor's historical evolution, which is crucial for understanding its current landscape, occurred over the course of the twentieth century. This was the entwined consolidation of the tourism boom and the modernization of the municipalities along the Mar Menor. The use of the Mar Menor as a place for leisure and for the therapeutic benefits of its waters by the privileged families of the kingdom of Murcia, dates back to the 17th century. The earliest extant records of the construction of leisure residences dates to this period, which were used take advantage of the water, although they were built some way from the sea due to the risk of piracy [69].

On this basis, in 1898, the village of Santiago de la Ribera was founded by the Barnuevo-Sandoval family, who owned the Torre Mínguez estate in San Javier. They transformed the ancient agrarian property into a new zone of holiday residences, equipped with the necessary maritime constructions including bathing houses (balnearios). Shortly after, something similar occurred in the other nearby villages, and especially in Los Alcázares, which, until 1990, had fallen under the jurisdiction of the municipality of San Javier. In Los Alcázares at the end of the 19th century, the Balneario de la Encarnación was built for seasonal summer use. However, in this case, the bathing house was intended for the public in order to accommodate the growing number of tourists who came from increasingly distant places, as a wide range of people enjoyed the benefits of the sea [70].

From 1960 onwards, the rate of development increased rapidly until it reached a peak in the first decade of the 21st century, not in terms of population, but in infrastructure and uncontrolled urban expansion, which considerably impacted the image of the region [71]. Until a few years ago, the popularity of Mar Menor as a unique natural space made it an increasingly important tourist destination, linked to the sea and sun, which only offered new cultural attractions in some cases, including summer events such as the Theater, Music, and Dance Festival in August and the Jazz Festival in July.

However, the elements that constituted the heritage image of the territory had been forgotten, such as the first modernist houses, navigation, and bathing houses, both by the local administration and by the community that had used them since their origin, which led to their abandonment and the loss of heritage value for neighbors. The same has not occurred with popular festivals and vernacular folklore, which have been maintained by the community $[9,46]$. This is the case for Romería de San Blas, which one of the religious traditions linked to the origins of the population in the 17th century, or Carnival. Both are celebrated in the winter months, at times of lower tourist influxes. This phenomenon indicates how the perception of heritage and its dynamic process of construction by local communities, which have already been analyzed in other parts of South America [72] or Europe [15], can guarantee the preservation of cultural heritage regardless of the support of the administration, and even beyond 
the intervention of external experts who can provide a scientific discourse on historical, artistic, and cultural content.

In this sense, this same community, in the face of conflict that involves the loss of environmental and cultural values that are fundamental to their way of life, can become an active defense agent. What role would cultural heritage have in trying to improve this situation? According to previous studies [8-10,14], the first function of cultural heritage is to legitimize a discourse based on scientific knowledge to publicize traditional uses that served to maintain the balance between humans and the environment. Simultaneously, these elements can be part of a responsible and sustainable tourism offering. The incorporation of heritage into tourism requires collaboration and coordination between the local community and tourism and cultural strategies, which should be planned according to the forms of governance [73].

In the regional regulations, the preamble of Law 4/2007 of the Cultural Heritage of the Region of Murcia defines Cultural Heritage as:

One of the main signs of identity and a testimony of the contribution made to the universal culture. The assets form an invaluable heritage and their conservation and enrichment corresponds to all the people of Murcia, and especially to the public authorities that represent them [74].

Therefore, cultural heritage is not something hieratic or ancient, but must be understood as a dynamic element, one that is part of our lives, and its value contributes to society, being incorporated into our daily lives. [75].

The history of the term cultural heritage highlights heritage as a discovery [76] in which the meaning of objects, beyond their timelessness due to their nature, is transformed according to their spectators. They can be understood by a social group as what their identity and history represents [77]. So, in our case, we propose a review through the appropriation of the community that not only recovers heritage through its rediscovery, but also feels the need to maintain it. Then, the value of equity and the contribution to the recovery of sustainable practices can be realized through external elements, especially tourism, and new technologies [72].

The core texts of international heritage doctrine underscore the concept that emerged from the UNESCO World Conference on Cultural Policies, which was held in Mexico in 1982. Despite being relatively old, this concept has not lost its contemporary relevance for defining how cultural heritage represents what we have the right to inherit from our predecessors and implies the obligation that we conserve for future generations [78]. With regard to San Javier, further insight is provided by considering the relationship between the natural environment and the landscape as a defining element of history and culture. The ICOMOS (International Council on Monuments and Sites) Burra Charter, revised and published in 1992, defends the overall conservation of cultural heritage in the places for and in which they were created, and that have been conserved in such a way that "they enrich people's lives, often providing a deep and inspirational sense of connection to the community and landscape, to the past and to lived experiences. Places of cultural significance reflect the diversity of our communities, telling us about who we are and the past that has formed us ... " [79]. In 2005, ICOMOS revised its concept of cultural sites as heritage areas on different scales, including: isolated buildings and spaces; historic cities; urban, rural, or maritime landscapes; cultural routes; and archaeological sites that gain their meaning and distinctive character from the perception of social, spiritual, historic, artistic, aesthetic, natural, and scientific values, or other factors of a cultural nature. Likewise, meaningful relationships are established between cultural heritage and its physical, visual, and spiritual surroundings. These relationships can be the result of a creative, conscious, and planned acts, spiritual belief, historical events, usage, or an organic cumulative process arising from cultural traditions over the course of time [80].

For the case study under discussion, in the Region of Murcia, concern has been growing regarding natural heritage, largely due to the impact of tourism on the local environment and the active role 
of environmental associations throughout the region. However, weaknesses continue to be detected regarding public awareness of the importance of cultural heritage and its role in local communities. Despite the publication of Law 12/2014 of 16 December 2014 on Transparency and Citizen Participation of the Region of Murcia, which provided a framework for the various forms of social participation, the Mar Menor currently lacks any non-governmental group, agency, or association whose objectives cover the partial or total protection of the cultural heritage of its territory.

Cultural goods that are protected by the local government are collected through its Urban Plan San Javier 2014 PGMO, which are the heritage assets that the local administration consider to be relevant and key elements of the municipality's identity according to the urban regulations, San Javier currently has 52 protected examples of cultural heritage (Table 1). Only 13 out of the 52 have received the maximum protection category of Cultural Interest Asset (abbreviated as BIC in Spanish) [81]. Most of these cultural assets are ethnographic in nature, especially windmills, although other examples of civil architecture are included such as the Villa Barnuevo and numerous residential summer buildings that originally included bathing houses within the Mar Menor. In Santiago de la Ribera where there is an entire catalogued urban area, the military city of Colonia Ruiz de Alda demonstrated the effects that legislation and protection have had on the heritage immovable cultural center of San Javier. Examples of religious cultural heritage are also present. These heritage sites are the most visited sites due to their artistic value and because they are where several popular shows and rites occur in the community [82]. Finally, the archaeological, terrestrial, and underwater heritage sites, located in La Manga and the Mar Menor Islands, today provide cultural value regardless of the lack of tourism prospects.

For this reason, and after analyzing the administration's proposals [83], two elements of the protected heritage were highlighted since, in a small way, they are still present in the daily lives of the local community. T first is the bathing houses because, although many have disappeared from the beaches, the regeneration expected to be conducted by the regional government includes the construction of new spas to minimize the amount of artificial beaches that have been built since 2000 to reduce negative impacts on the local environment. The second is the Vela Latina, which as a form of navigation can improve the local economy through development for tourism. 
Table 1. Cultural heritage sites protected by the San Javier PGMU (Plan General Ordenación Urbana) [84].

\begin{tabular}{lcl}
\hline BIENES & GRADO & POBLACIÓN \\
\hline ARQUITECTURA CIVIL & & \\
\hline Casa del Conde Campillo & 1 & Stgo. de La Ribera \\
Chalet Barnuevo & 1 & Stgo. de La Ribera \\
Hacienda de Roda & BIC & Roda \\
Conjunto de Edificaciones de la Isla del Barón & Incoado BIC & La Manga (San Javier) \\
Villa San Francisco Javier & 1 & Stgo. de La Ribera \\
Torre Javiera & 2 & Stgo. de La Ribera \\
Villa la Pinada & 2 & Stgo. de La Ribera \\
Casa Benimar & 2 & Stgo. de La Ribera \\
Torre García & 2 & Stgo. de La Ribera \\
Villa "El Retiro" & 2 & San Javier \\
Torre Saavedra & 3 & San Javier \\
Colonia Ruiz de Alda & 2 & Stgo. de La Ribera \\
Academia General del Aire & 3 & Stgo. de La Ribera \\
Torre Mínguez & 3 & Stgo. de La Ribera \\
Club Náutico & 1 & San Javier \\
Cuartel de la Guardia Civil & 2 & Stgo. de La Ribera \\
Casa en Paseo Colon, $n^{\circ} 48$ & 3 & San Javier \\
Grupo Escolar San Javier & 3 & San Javier \\
Torre Octavio & 3 & La Manga \\
Casa en Veneziola. & 3 & La Manga \\
Casa de la Encañizada & 1 & San Javier \\
Casa Los Urreas & 3 & Stgo. de La Ribera \\
Casa en Paseo Colon, $\mathrm{n}^{\circ}$ 46 & 3 & Stgo. de La Ribera \\
Casa en Paseo Colon, $\mathrm{n}^{\circ} 47$ & 3 & \\
\hline & & \\
\hline
\end{tabular}


Table 1. Cont.

\begin{tabular}{|c|c|c|}
\hline BIENES & GRADO & POBLACIÓN \\
\hline ARQUITECTURA POPULAR & & Stgo. de La Ribera \\
\hline Noria de sangre de la Torre García & 3 & La Manga \\
\hline Aljibe de cúpula de la Isla Grossa & 1 & \\
\hline MOLINOS & BIC & \\
\hline Molino de Sal & & La Manga \\
\hline Molino de Sal & & La Manga \\
\hline Molino de trasegar Agua & & La Manga \\
\hline Molino Finca la Máquina & & San Javier \\
\hline Molino de Agua & & San Javier \\
\hline Molino de Harina & & San Javier \\
\hline Molino de Agua & & La Grajuela \\
\hline Molino de Agua & & La Grajuela \\
\hline Molino de Agua & & La Grajuela \\
\hline \multicolumn{3}{|l|}{ ARQUITECTURA RELIGIOSA } \\
\hline Iglesia Parroquial de San Francisco Javier & 1 & San Javier \\
\hline Iglesia Nuestra Señora de EL Rosario & 2 & El Mirador \\
\hline Ermita de San José & 3 & Pozo Aledo \\
\hline Iglesia Parroquial de Santiago Apóstol & & Stgo. de La Ribera \\
\hline \multicolumn{3}{|l|}{ BALNEARIOS } \\
\hline Balneario. Concesión 522 & Incoado BIC & Stgo. de La Ribera \\
\hline Balneario. Concesión 474 & Incoado BIC & Stgo. de La Ribera \\
\hline Balneario. Concesión 397 & BIC & Stgo. de La Ribera \\
\hline Balneario. Concesión 445 & BIC & Stgo. de La Ribera \\
\hline \multicolumn{3}{|l|}{ ÁREAS DE INTERÉS ARQUEOLÓGICO } \\
\hline Isla Perdiguera I - Exp.208/90 45 & $\mathrm{C}$ & La Manga \\
\hline Isla Perdiguera II - Exp.830/98 46 & $\mathrm{C}$ & La Manga \\
\hline La Grajuela - Exp.877/90 47 & $\mathrm{~B}, \mathrm{C}$ & La Grajuela \\
\hline La Esparteña I - Exp.828/98 48 & $\mathrm{C}$ & Isla La Perdiguera \\
\hline La Esparteña II - Exp.829/98 & $\mathrm{C}$ & Isla La Perdiguera \\
\hline
\end{tabular}

\subsection{Balnearios}

To interpret the heritage landscape as it is seen today, transformation in this region has almost always coexisted with the sea, ranging from the maritime exploitation of the lagoon to the traditional medicinal or therapeutic uses of the inhabited regions of the Mar Menor. This is demonstrated by the remains of Roman baths, as well as the construction of the Hotel de la Encarnación, which occurred centuries before that of Los Alcázares [85]. On some of the Mar Menor islands, the remains of sites intended for relaxation and pleasure can still be found, such as the residential complex of the Isla Mayor, also known as Isla del Barón, which owes its nickname to the property and buildings constructed by Julio Falcó d'Adda, Baron of Benifayó, the descendent of a Savoy family. The island served as one of the family's summer residences in the region during the 19th and 20th centuries [86].

The development of maritime leisure activities from the end of the 19th century until 1960 produced the old agricultural villages in vacation locations, including Lo Pagán, Santiago de la Ribera, Los Nietos, and Los Alcázares, which were inhabited by a population that moved to the coast for several weeks in the summer months to take baths. During that time, companies and services were installed that were permanently established near the mid-20th century.

At the same time that the leisure houses were built, in front of them, the balnearios were also built. These were wooden platforms that entered the sea so that bathers could comfortably enter the water. Each family built a corresponding spa in front of their villa. This created spatial importance between the house and the sea. Thus, balnearios served as an extension of the privacy of the residence. This 
even extended to formal details, and especially in Los Alcázares and Los Nietos, until the end of the 20th century, where some family residences exhibited decorative features, colors, and portico designs, which were the for the house and the spa and, in some cases, details were included that identified the family.

As Gutiérrez-Cortines [87] has stated, these bathing platforms played a role from the point of view of land use, since they served to alleviate overcrowding on the beaches and provided a solution to the rocks and mud on the shore. As of 1965, high apartment buildings were constructed, and once again, spas were built in front of these buildings that were 500 to $1000 \mathrm{~m}^{2}$, and fulfilled the same social function as social interaction spaces, also serving as moorings for pleasure boats and jetties for water sports. However, with the construction of artificial beaches and the increased demand for space for bathrooms, the balnearios were considered to be obsolete pieces in the maritime landscape as of 1990.

These balnearios were not only intended for bathing or mooring boats, as is the case today, but some, practically from the outset, were also used for social gatherings in the form of literary or intellectual gatherings, dancing, and other leisurely pursuits. The construction of the yacht club in la Ribera in 1918, which was known as the Casino Marítimo de la Ribera, is an example of this trend. In 1971, this casino was transformed into the current building, the Real Club de Regattas de Santiago of la Ribera.

From the 1960s onward, the combination of the mass tourism phenomenon, new building techniques, the exhaustion of the traditional tourism model, and the development of extensive legislation under Spain's new democratic government meant that a series of legal and economic circumstances in the 1980s led to the disappearance of the majority of the bathing houses. Mar Menor underwent a form of intervention that transformed the region to fit the mold of modernity being sought at that time. The Project for the Rehabilitation of the Beaches was launched by the Coastal Commission, which depended on the Ministry for Public Works and Urban Planning [88]. Its aim was to create artificial beaches that would facilitate bathing and the enjoyment of the seaside, and this drastically affected the traditional image of the coast and led to the elimination of the majority of the maritime buildings.

In 1987, the Committee for Culture of the Region of Murcia began the process of designating the complex of the Mar Menor balnearios as a BIC to protect and conserve these fragile maritime architectural structures. However, this measure was never implemented and only a limited form of the initial project led to the conservation of some bathing houses, either due to their singular value, or their good state of conservation, or because their social or economic role permitted it. However, in the majority of the cases, this measure only managed to delay an inevitable decline, as was the case with the Lonja, the historic municipal fishmongers, and the restaurant the Pescadería de Mariche. In the latter case, despite its kitchens having conserved long-established seafood traditions of sampling the caldero de mújol (a stewed mullet dish) by the edge of the Mar Menor, the restaurant could not stave off demise following the Coastal Commission's cessation of the temporary concession of 50 to 100 years that it had been granted when it opened.

This is the aspect that has begun to work with the community of neighbors. The awareness of the planned construction being a solution for the regeneration and use of beaches through the construction of new maritime platforms is based on the tradition of the oldest construction in the area. Therefore, awareness campaigns are being conducted at the administration level through advice and meetings with experts. Conferences are being promoted within the communities with publication of informative brochures about the history and traditional uses of these popular locations [88].

What objectives have been set for these actions? At the level of local governance, the strategy of maintaining the affected and protected structures has been reported (Table 1), which would have been maintained according to traditional construction systems. On an economic level, this would entail a lowering of costs and their maintenance would require traditional crafts such as additional works of wood, network systems, and esparto. The strategy would help improve an innovative, unique offer for bathing in the sea and attract new tourism. The local community would benefit because it would lead to tourists continuing to be attracted to the area. 
The Hotel Los Arcos in La Ribera, opened in August 1960 by Adrián Viudes Guirao with its beach resort, is an example that could be recovered as an example of the contemporary policy of attracting quality national and international tourism. In 1981, after being closed for several years, Hotel Los Arcos was transformed into a hospital. In early 2011, the hospital was transferred to Pozo Aledo and the empty building, currently and provisionally, is a training center for the San Javier regattas, but is waiting for a project that to confirm its position as a historic element in Santiago de la Ribera (Figure 2).
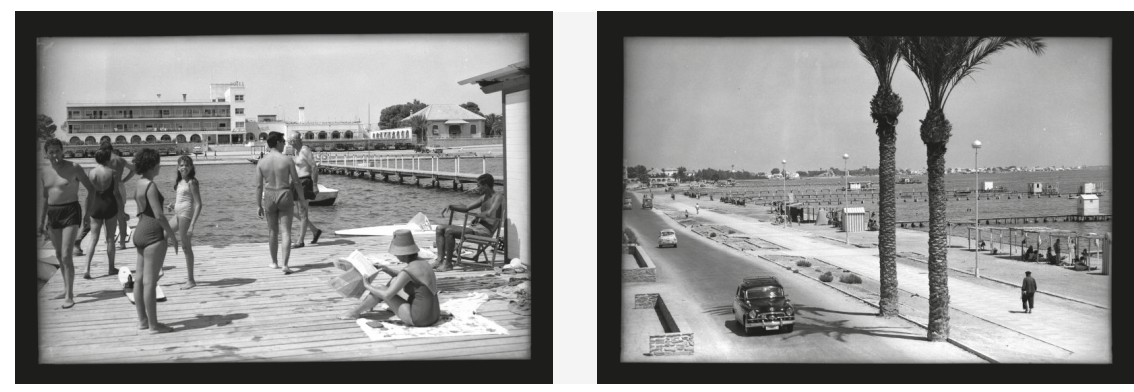

Figure 2. Bathing houses at Hotel Los Arcos and Santiago de la Ribera, part of the municipality of San Javier, 1965, Murcia [89].

\subsection{La Vela Latina}

Maritime, sports, and leisure activities are the best known aspects of identity in the region. The Latin Sail (Vela Latina) is a vessel with rigging and sails used for both professional fishing and marine sports without motor. They symbolize the knowledge and trades related to this practice. In February 2018, the Autonomous Community of the Region of Murcia (CARM) protected the Latin Candle as a BIC [90,91].

According to the extensive report provided by the Higher Technical School of Naval and Oceanic Engineering of the Polytechnic University of Cartagena, "Vela Latina sailing is the result of the uses and customs that involve a technical knowledge of undoubted value in the historical knowledge of navigation". The rich cultural heritage that results from this relationship between man and the environment deserves to be taken care of and conserved, especially when many of these practices are an example of sustainability, essential even for the conservation of biological diversity [92].

Villa Barnuevo, which was the first home built in the new city of Santiago de la Ribera, is an example of modernist architecture [93], which has become a center of restoration and entertainment of this sport. Although the Villa Barnuevo was declared BIC, in recent decades, its conservation status has decreased. Work is underway to complete a transformation into a study center of this art of navigation, including programming guided tours through which visitors can be informed about the history of this navigation system. Sailing with a Latin sail was inseparable from the primitive traditional and original fishing systems of the Mar Menor: the fishing weirs. This system is also practiced in the other Spanish salty lagoons, the Albudera of Valencia [94]. Therefore, the Valencian initiatives were taken as a reference, since this navigation system has also been protected since 2016. Its existing promotional initiatives, through increasing visibility of its sports activities and competitions, provide a reference for San Javier.

\section{Conclusions}

The administrative structure in which Spanish cultural heritage is framed is structured by a network of overlapping entities that affect different territorial sectors and management levels. Thus, the state coordinates the interventions in the assets that are under its jurisdiction, which include cultural 
heritage sites located on the peninsular coast. In this case study, the state has particular influence on the public domain, both on land and at sea, as stipulated by the powers assigned to the Secretary of State for the Environment, which cover the coasts, the sea, and the continental waters. The regulations covering Spanish coastal heritage as public domain assets and their application with respect to San Javier have resulted in the loss of most of their bathing houses between 1990 and 2015. Similarly, according to the Commission created by the Ministry for Ecological Transition (MPTE), despite Mar Menor being environmentally protected as a wetland inscribed on the Ramsar list, included in the Natura 2000 Network, categorized as a Community of Importance (LIC) and a Special Protection Area for Birds (ZEPA), and a Specially Protected Area of Interest for the Mediterranean (ZEPIM), these efforts have been insufficient to preserve the affected area.

In conclusion, the effectiveness of preserving and managing their cultural heritage does not depend on the degree of protection granted, since, in most cases, the effectiveness is not supported by the actual results. However, this inefficiency has proven extensible to the natural environment and its heritage values. Therefore, a series of measures have been initiated to involve not only the city council but also the local community in the recovery processes [9,11,15].

The concept of citizen participation has become a basis for protection proposals and this phenomenon could affect the case study region [95]. To achieve this, we must overcome the problem of a large percentage of the population only identifying in a limited way with cultural heritage. Above all, their perception of the past could become an element of cohesion and provide a means to distinguish San Javier from other nearby regions $[8,10]$.

In the case of San Javier, if this method has been effective for another the municipalities, it can be applied to other cities related to Mar Menor. The transformative process will affect a wider community, as the patrimonialization of their territories and common traditions, in addition to favoring the external visibility of the territory and, therefore, tourism [14,16,17], can become a fundamental basis for promoting sustainable growth based on the recovery of traditional forms of production in the lagoon.

In conclusion, the Mar Menor has a cultural heritage whose assets are unique. In accordance with the five objectives established by the UNESCO Convention of Budapest 2002 [95] for World Heritage, the community involved in this case, including the residents and the seasonal population that owns second residences in the area, must have an essential role in the discovery, valuation, and new uses of cultural heritage so that these buildings can become models of sustainable use of tourism since, through their cultural and identity potential, they provide motivation for a change in mentality about the use of the weakened natural environment. The enjoyment values of the lagoon can be increased through the use of responsible activities such as sailing sports, which eventually replace the tourism offer of the sun and the beach, and bathing houses, which contribute to lessening the impact of artificial beaches. For this, the population must be engaged in campaigns of active participation in defense of heritage, promoting associations with non-governmental organizations (NGOs) whose basic objective is heritage protection [96]. This is the aim of UNESCO through actions such as the World Heritage Volunteers Campaign 2020, and is one of the basic objectives, in accordance with the 2030 agenda, of sustainable development established by United Nations Program for Development (UNDP) since 2015, also known as the global objectives [97].

Now that we are aware of the dimension of the problem and the real situation of the natural and cultural values of the region have been analyzed, the cooperation of all the social agents involved is needed. With the basic objective of immediate action in addressing the situation and knowing that, according to UNESCO, this has led to many successful restorations [75], the support of the International Community should be requested to start the process for the inclusion of the Mar Menor as a global project for its declaration as a World Heritage Site.

Author Contributions: For research articles with several authors, a short paragraph specifying their individual contributions must be provided. The following statements should be used "conceptualization, M.G.M.; methodology, M.G.M.; formal analysis, M.G.M.; investigation, M.G.M.; resources, M.G.M.; data curation, 2019 M.G.M.; writing-M.G.M.; visualization M.G.M. All authors have read and agreed to the published version of the manuscript. 
Funding: This article presents findings of the research project Análisis del impacto de estrategias de regeneración urbana sobre la conservación del patrimonio cultural de zonas industriales históricas (Analysis of the impact of regeneration strategies on the conservation of the cultural heritage of historic industrial areas) (HAR2014-58151-R), which was funded by the Spanish government, through the State R+D+I programme 'Orientada a los Retos de la Sociedad 2014 (Addressing the Challenges Faced by Society 2014)' and undertaken between 2015 and 2019.

Conflicts of Interest: The author declares no conflict of interest.

\section{References and Notes}

1. Instituto Nacional de Estadística España. 30035 San Javier. Available online: http://www.ine.es/jaxiT3/Datos. htm?t=28831 (accessed on 12 August 2019).

2. Ramsar Convention Secretariat. An Introduction to the Ramsar Convention on Wetlands, 5th ed.; Ramsar Convention Secretariat: Gland, Switzerland, 2016; Available online: https:/www.ramsar.org/sites/default/ files/documents/library/handbook1_5ed_introductiontoconvention_e.pdf (accessed on 8 June 2019).

3. García Sánchez, A.; Artal Tur, A.; Ramos Parreño, J.M. El turismo del Mar Menor: Predominio de la segunda residencia. Cuad. Tur. 2002, 9, 33-44. Available online: https://revistas.um.es/turismo/article/view/21981 (accessed on 8 June 2019).

4. Barragán, J.M.; García Sanabria, J. Estrategia de Gestión Integrada de Zonas Costeras del Sistema Socio-Ecológico del Mar Menor y su Entorno; Consejería de Presidencia y Fomento CARM: Murcia, Spain, 2018.

5. Gustavo Gillman, B. Mapa de la Comarca del Mar Menor by Gustavo Gilman; Archivo General de la Región de Murcia (AGRM): Murcia, Spain, 1905; Gustavo Gillman Bovet, ingeniero y fotógrafo. 248.6.

6. The Manifiesto de Boadilla (Boadilla Manifiesto), February 2019, was launched by the civic groups and associations that met at the 16th Jornadas en Defensa del Patrimonio Cultural de España (Conference for the Defence of Spanish Cultural Heritage), where they declared their intention to create the Federación Nacional de Asociaciones (National Federation of Associations) which would act as an intermediary with the state administrative entities. They also advocated the urgent need to undertake conservation, dissemination, promotion and educational activities with regard to cultural heritage across all tiers of society. Manifiesto de Boadilla. Asociaciones en Defensa del Patrimonio Cultural, 2019; p. 2. Available online: https://madridciudadaniaypatrimonio.org/sites/default/files/pdf-embed-blog/manifiesto_boadilla_ asociaciones_en_defensa_del_patrimonio (accessed on 2 September 2019).

7. author's note: The translation of this term as bathing house does not do full justice to the architectural construction referred to by this Spanish term and described in this article, hence the Spanish term is used.

8. Ashworth, G.; Voodg, H. Marketing of tourism places: What are we doing? J. Int. Consum. Mark. 1994, 6, 5-19. [CrossRef]

9. Antón, S. Turismo y Gestión Municipal del Patrimonio Cultural; AECIT: Gijón, Spain, 1996.

10. Harvey, D.C. Heritage Pasts and Heritage Presents: Temporality, meaning and the scope of heritage studies. Int. J. Herit. Stud. 2001, 7, 319-338. [CrossRef]

11. Zamora Acosta, E. Sobre patrimonio y desarrollo. Aproximación al concepto de patrimonio cultural y su utilización en procesos de desarrollo territorial. Pasos 2011, 9, 101-113. [CrossRef]

12. Timothy, D.; Boyd, S. Heritage Tourism; Peqrson Education Lm.: Essex, UK, 2003.

13. McKercher, B.; Du Cros, H. Cultural Tourism: The Partnership between Tourism and Cultural Heritage Management; Rodledge-Haworth Press: New York, NY, USA, 2002.

14. Landorf, C. Managing for sustainable tourism: A review of six cultural World Heritage Sites. J. Sustain. Tour. 2009, 17, 53-70. [CrossRef]

15. Du Cros, H. A new model to assist in planning for sustainable cultural heritage tourism. Int. J. Tour. Res. 2001, 3, 165-170. [CrossRef]

16. Mydland, L.; Grahn, W. Identifying heritage values in local communities. Int. J. Herit. Stud. 2012, 18, 564-587. [CrossRef]

17. Smith, V.L. Anfitriones e Invitados. Antropología del Turismo; Endymion: Madrid, Spain, 1992.

18. Camarero, C.; Garrido, M.J. Marketing del Patrimonio Cultural; Prámide: Madrid, Spain, 2004.

19. Hernández, J.B.; Tresserras, J.J. Gestión del Patrimonio Cultural; Ariel: Barcelona, Spain, 2001.

20. Barre, J. Vendre le Tourisme Culturel. Guide Méthodologique; Institut d' Éstudes Superieurs des Arts: París, France, 1995. 
21. Carter, B.; Grimwade, G. Balancing use and preservation in cultural heritage management. Int. J. Herit. Stud. 1997, 3, 45-53. [CrossRef]

22. Kirshenblatt-Gimblett, B. Destination Culture: Tourism, Museums, and Heritage; University of California Press: Berkeley, CA, USA, 1998.

23. Bendix, R. Cultural tourism in Europe. Ann. Tour. Res. 1999, 26, 1031-1037.

24. Barre, H. Cultural Tourism And Sustainable Development. Mus. Int. 2002, 54, 126-133. [CrossRef]

25. Michael Hall, C. Geography, Marketing and the Selling of Places. J. Travel Tour. Mark. 1997, 6, 61-84. [CrossRef]

26. Dimanche, F.; Havitz, M.E. Consumer Behavior and Tourism: Review and Extension of Four Study Areas. J. Travel Tour. Mark. 1995, 3, 37-57. [CrossRef]

27. International Council On Monuments and Sites. Carta Internacional sobre Turismo Cultural La Gestión del Turismo en los sitios con Patrimonio Significativo. In Proceedings of the 12th Asamblea General ICOMOS en México, México, 17-23 October 1999.

28. Herreman, Y. Museum and Tourism: Culture and Consumption. Mus. Int. 1998, 50, 4-12. [CrossRef]

29. Lord, B.; Lord, G.D. Manual de Gestión de Museos; Ariel: Barcelona, Spain, 1998.

30. Goulding, C. The Museum Environment and the Visitor Experience. Eur. J. Mark. 2000, 34, 261-278. [CrossRef]

31. Gilmore, A.; Rentschles, R. Changes in Museum Mnagement. A Custodial or Marketing Emphasis? J. Manag. Dev. 2002, 21, 745-760. [CrossRef]

32. Chaumier, S. Des Musées en Quête d'Identité (Nouvelles Études Anthropologiques); L'Harmattan: Paris, France, 2003.

33. Silberberg, T. Cultural Tourism and Business Opportunities. For Museum Heritage Sites. Available online: https://www.lord.ca/Media/Artcl_Ted_CultTourismBusOpps.pdf (accessed on 12 December 2019).

34. Asenjo, E. (Ed.) Lazos de Luz Azul: Museos y Tecnologías 1, 2 y 3.0; UOC (Universitata Operta de Cataluya): Barcelona, Spain, 2011.

35. Moreré Molinero, N.; Perelló Oliver, S. Turismo Cultural. Patrimonio, Museos y Empleabilidad; FundaciónEOI: Madrid, Spain, 2013; Available online: https://www.eoi.es/es/savia/publicaciones/20726/turismo-culturalpatrimonio-museos-y-empleabilidad (accessed on 9 September 2019).

36. Marchena, M.J. Turismo Urbano y Patrimonio Cultural. Una Perspectiva Europea; Junta de Andalucía: Sevilla, Spain, 1998.

37. Verdugo Santos, J. El patrimonio histórico como factor de desarrollo sostenible. Una reflexión sobre las políticas culturales de la Unión y su aplicación en Andalucía. Cuad. Econ. Cult. 2003, 1, 55-90.

38. Morales, A.J. Patrimonio Histórico-Artistico; Historia 16: Madrid, Spain, 1996.

39. Hernandez Martínez, A. ¿Qué hace una chica como tú en un lugar como este?(Algunas reflexiones acerca de la relación entre Historia del Arte y Patrimonio Cultral). Artigrama 2000, 15, 543-564.

40. Cuetos, P. El Patrimonio Cultural. Conceptos Básicos; Prensas Universitarias de Zaragoza: Zaragoza, Spain, 2011.

41. Dikovitskaya, M. Visual Culture: The Study of the Visual after the Cultural Turn; The MIT Press: Cambridge, MA, USA, 2005.

42. Mirzoeff, N. Una Introducción a la Cultura Visual; Paidos: Madrid, Spain, 2003.

43. Debray, D. Vida y Muerte de la Imagen. Historia de la Mirada en Occidente; Paidós: Barcelona, Spain, 1994.

44. Brugère, F. Le musée entre la culture populaire et le divertissement. En quelle culture défendre? Sprit 2002, $283,46-58$.

45. Martí, H.I.; Manuel, G.; Santamarina Campos, B.; Moncusí Ferré, A.; Rodrigo, M.A. La Memoria Construida. Patrimonio Cultural y Modernidad; Tirant lo Blanch: Valencia, Spain, 2005.

46. Hobsbawm, E.; Ranger, T. (Eds.) La Invención de la Tradición; Crítica: Barcelona, Spain, 2002.

47. Fernández de Paz, E. De tesoro ilustrado a recurso turístico: El cambiante significado del. PASOS Rev. Tur. Patrim. Cult. 2006, 4, 1-12.

48. Matías, A.R. Historia del Arte y Patrimonio Cultural de España; Sintesis: Madrid, Spain, 1997.

49. Blanc Altemir, A. El Patrimonio Común de la Humanidad. Hacia un Régimen Jurídico Internacional de su Gestión; Bosch: Barcelona, Spain, 1992.

50. Cebrián Abellán, A. Turismo Cultural y Desarrollo Sostenible. Análisis de Áreas Patrimoniales; EDITUM: Murcia, Spain, 2002.

51. Abad Liceras, J.M. Administraciones Locales y Patrimonio Histórico; Montecorvo: Madrid, Spain, 2003.

52. Santana, A. Turismo cultural, culturas turísticas. Horiz. Antropol. 2003, 20, 31-57. [CrossRef] 
53. Prats, L. La mercantilización del patrimonio: Entre la economía turística y las representaciones identitarias. PH Bol. Inst. Andal. Patrim. Hist. 2006, 58, 72-80. [CrossRef]

54. Crespi Vallbona, M.; Planells Costa, M. Patrimonio Cultural; Síntesis: Madrid, Spain, 2010.

55. Amador de los Ríos, R. España, sus Monumentos y Artes. Murcia y Albacete; Tip. Daniel Cortezo: Barcelona, Spain, 1889.

56. Jiménez de Gregorio, F. El Municipio de San Javier en la Historia del Mar Menor; Academia alfonso X el Sabio: Murcia, Spain, 1984.

57. Torres-Fontes, J. Repartimiento y Repoblación en el Siglo XIII; Academia Alfonso X el Sabio: Murcia, Spain, 1990.

58. Cutillas Victoria, B. Proteger y defender la Manga del Mar Menor: Estudio histórico-arqueológico de la Torre de San Miguel del Estacio y la Torre de la Encañizada. Defensive Archit. Mediterr: XV XVIII centuries. 2015, 1, 95-102. [CrossRef]

59. Victoria Moreno, D. Los Alcázares y el Mar Menor: El complejo tránsito a la Modernidad. En AAVV, Historia de los Alcázares; CARM: Murcia, Spain, 2008; Volume II, pp. 1-34.

60. Buitigieg, J. La despoblación del Mar Menor y sus causas. Bol. Pescas Dir. Gral. Pesca Minist. Mar. Inst. Esp. Oceanogr. 1927, 133, 251-286.

61. Lillo Carpio, M. Geomorfologia litoral del Mar Menor. Pap. Dep. Geogr. 1978, 8, 9-46.

62. López-Bermúdez, F.; Ramírez, L.; Martín de Agar, P. Análisis integral del medio natural en la planificación territorial: El ejemplo del Mar Menor. Murcia (VII) 1981, 18, 11-20.

63. Pérez-Ruzafa, I.M. Fitobentos de una Laguna Costera. El Mar Menor. Ph.D. Thesis, University of Murcia, Murcia, Spain, 1989.

64. Arévalo, L. El Mar Menor como sistema forzado por el Mediterráneo. Control hidráulico y agentes fuerza. Bol. Inst. Esp. Oceanogr. 1988, 5, 63-95.

65. Mas Hernández, J. El Mar Menor.: Relaciones, Diferencias y Afinidades Entre las Lagunas Costeras yel Mar Mediterráneo Adyacente. Ph.D. Thesis, Universidad Autónoma de Madrid, Madrid, Spain, 1994.

66. Romero Díaz, A. Belmonte SerratoF. Los paisajes geomorfológicos de la Región de Murcia como recurso turístico. Cuad. Tur. 2002, 9, 103-122. Available online: https://revistas.um.es/turismo/article/view/21931/ 21221 (accessed on 24 June 2019).

67. Cabezas, F. Balance Hídrico del Mar Menor. El Mar Menor. Estado Actual del Conocimiento Científico; Fundación Instituto Euromediterráneo del Agua: Murcia, Spain, 2009.

68. Baudron, P. Anthropisation d'un Système Aquifère Multicouche Méditerranéen (Campo de Cartagena, SE Espagne). Approches Hydrodynamique, Géochimique et Isotopique. Ph.D. Thesis, Université de Montpellier 2, Montpellier, France, 2014.

69. Thompson, I.A. Guerra y Decadencia. Gobierno y Administracción en la España de los Austrias. 1560-1620; Crítica: Barcelona, Spain, 1981.

70. Larrinaga, C. Patrimonio del sector turístico: Los balnearios. El caso guipuzcoano. Áreas Rev. Int. Cienc. Soc. 2010, 29, 91-100.

71. Troitiño Vinuesa, M.Á.; Troitiño Torralba, L. Patrimonio y turismo. Scr. Nova Rev. Electrón. Geogr. Cienc. Soc. 2016, 20, 527-551.

72. Dormaels, M. Identidad, comunidades y patrimonio local: Una nueva legitimidad social. Alteridades 2012, 22, 9-19.

73. Griñán Montealgre, M.; Trigueros Molina, J.C. Patrimonio y Paisaje Cultural del agua en el Valle de Ricote. E-rph 2018, 22, 5-36.

74. Ley 4/2007 de Patrimonio Cultural de la Región de Murcia. Available online: http://noticias.juridicas.com/ base_datos/CCAA/mu-14-2007.html (accessed on 6 May 2019).

75. UNESCO. "Culture: Urban Future”. The Role of Cultural Heritage for Sustainable Local Development. 2017. Available online: https://whc.unesco.org/en/events/1395 (accessed on 12 February 2019).

76. Davallon, J. Comment se fabrique le patrimoine? Sci. Hum. Hors Sér. 2002, 36, 74-77.

77. Dormaels, M. The concept behind the word. In Understanding Heritage: Perspectives in Heritage Studies; Marie-Theres, A., Roland, B., Britta, R., Eds.; Walter de Gruyter: Berlin, Germany, 2013; pp. 107-115.

78. Informe Final de la Conferencia Mundial Sobre Politicas Culturales; UNESCO: Paris, France, 1982; p. 7. Available online: https://unesdoc.unesco.org/ark:/48223/pf0000052505_spa (accessed on 8 February 2019).

79. Carta de Burra para Sitios de significación Cultural. ICOMOS Australia. 1999. Preámbulo. Available online: https://www.icomos.org/charters/burra1999_spa.pdf (accessed on 8 February 2019). 
80. Declaración Sobre la Conservación del Entorno de las Estructuras, Sitios y Áreas Patrimoniales -Xi'an (China), ICOMOS. 22 October 2005. Available online: https://www.icomos.org/images/DOCUMENTS/Charters/xiandeclaration-sp.pdf (accessed on 8 February 2019).

81. 25 Ley 16/1985, de 25 de Junio, de Patrimonio Histórico Español. Available online: https://www.boe.es/ buscar/act.php?id=BOE-A-1985-12534 (accessed on 2 May 2019).

82. Ministerio de Cultura y Deporte. Gobierno de España, Regímenes Especiales de Protección del Patrimonio Histórico, Madrid, Spain. Available online: http://www.mecd.gob.es/cultura-mecd/areas-cultura/patrimonio/ bienes-culturales-protegidos/niveles-de-proteccion/regimenes-especiales.html. (accessed on 2 May 2019).

83. Comunidad Autónoma de la Región de Murcia. Estrategia de Gestión Integrada de Zonas Costeras del Sistema Socio-Ecológico del Mar Menor y su Entorno. Available online: https://www.carm.es/web/pagina? IDCONTENIDO=106035\&IDTIPO=10\&RASTRO=c $\$ \mathrm{~m} 122,70$ (accessed on 3 October 2019).

84. Ayuntameniento de San Javier. Bienes Inmuebles Protegidos Desde el Plan General Municipal de Ordenación de San Javier. In Registro General de Bienes Culturales dependiente de la Consejería de Educación y Cultura de la Región de Murcia; Ayuntameniento de San Javier: San Javier, España; Available online: http://www.pgmo. sanjavier.es/textos/02-3-1\%20Catalogo\%20de\%20Bienes\%20Protegidos-PGMO-AP\%202014.pdf (accessed on 15 May 2019).

85. VVAA. El Papel de Nuestra Historia. Los Alcázares, San Pedro del Pinatar, San Javier. Murcia; Tres Fronteras: Murcia, Spain, 2009; pp. 17-23.

86. Cegarra Beltrí, G. Arquitectura Modernista en la Región de Murcia; Mablaz: Cartagena, Colombia, 2013; p. 215.

87. Gutiérrez-Cortines Corral, C. La Arquitectura del Agua: Los Balneario del Mar Menor. Imafronte 1990, 19, 79-92.

88. irección General de Patrimonio Histórico. (Consejería de Cultura y Educación de la CARM). The Proyecto de Rehabilitación de las Playas (Project for the Rehabilitation of the Beaches) was launched by the Junta de Costas (Coastal Commission), which depended upon the Ministerio de Obras Públicas y Urbanismo (Ministry for Public Works and Urban Planning). Murcia, Spain. Its aim was to create artificial beaches that would facilitate bathing and the enjoyment of the seaside, and this drastically affected the traditional image of the coast and led to the elimination of the majority of the maritime buildings.

89. AGRM. ES.30030.AGRM/71. 71.12./Postales y Documentos de San Javier y La Ribera (1822-1965). Serie Costa de la Luz. Available online: https://archivoweb.carm.es/archivoGeneral/arg.muestra_detalle?idses=0\& pref_id $=4343958$ (accessed on 15 October 2019).

90. Decreto, n. 7/2018, de 31 de enero, del Consejo de Gobierno de la Comunidad Autónoma de la Región de Murcia, por el que se declara Bien de Interés Cultural inmaterial(BIC) la Vela Latina y los oficios y saberes relacionados con su práctica. Boletín Oficial Región de Murcia, 8 febrero. 2018, pp. 2898-2906. Available online: https:/www.borm.es/services/anuncio/ano/2017/numero/1619/txt?id=755120 (accessed on 1 October 2019).

91. Ley 10/2015 para la Salvaguardia del Patrimonio Cultural Inmaterial. (BOE 126, de 27 de mayo de 2015, art.1 45289). Available online: https://www.boe.es/buscar/doc.php?id=BOE-A-2015-5794 (accessed on 2 May 2019).

92. Available online: https://www.laopiniondemurcia.es/consejo-gobierno/2018/01/31/ (accessed on 22 September 2019).

93. La Spina, V.; Serrano Hidalgo, M.C. El Modernismo en la Región de Murcia; Universidad Politécnica de Cartagena, CRAI Biblioteca: Murcia, Spain, 2017.

94. El Levante (Valencia). Available online: https://www.levante-emv.com/valencia/2018/12/02/vela-latinareivindica-bic-segundo/1803368.htm (accessed on 9 May 2019).

95. UNESCO. Textos básicos de la Convención del Patrimonio Mundial de 1972. Available online: http: //www.icomos.es/wp-content/uploads/2017/05/DIRECTRICES.pdf (accessed on 22 October 2019).

96. UNESCO. Join the World Heritage Volunteers Campaign 2020. Available online: https://whc.unesco.org/en/ news/2061 (accessed on 12 October 2019).

97. UNDP. 2030 Agenda for Sustainable Development. Available online: https://www.undp.org/content/undp/ en/home/2030-agenda-for-sustainable-development.html (accessed on 12 October 2019).

(C) 2019 by the author. Licensee MDPI, Basel, Switzerland. This article is an open access article distributed under the terms and conditions of the Creative Commons Attribution (CC BY) license (http://creativecommons.org/licenses/by/4.0/). 
Article

\title{
An Application of the A'WOT Analysis for the Management of Cultural Heritage Assets: The Case of the Historical Farmhouses in the Aglié Castle (Turin)
}

\author{
Marta Bottero ${ }^{1}$, Chiara D'Alpaos ${ }^{2, *}$ and Alessia Marello ${ }^{1}$ \\ 1 Department of Regional and Urban Studies and Planning, Politecnico di Torino, 10129 Torino, Italy; \\ marta.bottero@polito.it (M.B.); alessia.marello@hotmail.it (A.M.) \\ 2 Department of Civil, Environmental and Architectural Engineering, University of Padova, \\ 35122 Padova, Italy \\ * Correspondence: chiara.dalpaos@unipd.it
}

Received: 20 December 2019; Accepted: 24 January 2020; Published: 3 February 2020

\begin{abstract}
In recent years, governments, public institutions, and local communities have devoted growing attention to the identification of promising strategies for the preservation and valorization of cultural heritage assets. Decisions on the management of cultural heritage assets based on multiple, often conflicting, criteria and on the stakes of various, and potentially non-consensual actors and stakeholders. In this context, in which the trade-offs between the preservation of assets historical symbolic values and the adaptation to alternative and economically profitable uses play a key role in investment decisions, multi-criteria analyses provide robust theoretical and methodological frameworks to support decision-makers in the design and implementation of adaptive reuse strategies for cultural heritage and public real estate assets. In this paper, we provide a multi-criteria decision aiding approach for ranking valorization strategies of cultural heritage assets aimed at promoting their restoration and conservation, as well as at creating cultural and economic benefits. In detail, we present a novel application of the A'WOT analysis to support the design and implementation of alternative management strategies of abandoned cultural heritage assets. The paper focuses on the potential reuse and management of four historical farmhouses (Cascina Mandria, Cascina Lavanderia, Cascina Gozzani, and Cascina Ortovalle) located in the Agliè Castle estate, one of the Residences of the Royal House of Savoy, currently listed in the UNESCO World Heritage Sites.
\end{abstract}

Keywords: cultural heritage; A'WOT analysis; multicriteria decision aid; valorization; management strategy

\section{Introduction}

The problems in the context of cultural heritage conservation and valorization refer to multi-faceted issues described by several dimensions, such as historic and artistic values, economic constraints, technical aspects, and a plurality of actors and stakeholders who play important roles in the decisional arena. Such as public authorities, practitioners, tourists, inhabitants, and so on. Of particular importance in this domain is the concept of adaptive reuse, which involves a change of use of existing buildings with the aim to achieve improvement in environmental, economic and social dimensions of sustainability, including resource efficiency, costs reduction and intrinsic values retention [1-6]. In the light of this complexity, decision-making processes in the domain of cultural heritage operations need to be addressed by specific evaluation tools, able to consider both the use-values related to the tangible benefits provided by Cultural Heritage to people and non-use values, accruing to current potential and future users, under the perspective of the Social Complex Value [7,8].

In particular, the evaluation of cultural heritage focused so far on two main directions: 
- non-market valuation by means of Stated/Revealed Preferences methods,

- Multicriteria evaluation methods and decision-making approaches.

As far as the first group of methods are concerned, they have been primarily introduced by economists to value public goods, which are non-excludable and non-rivaled in consumption [9]. Cultural heritage typically possesses the characteristics of public goods and thus can be valued by implementing non-market valuation methods. Broadly speaking, there are two categories of non-market valuation methods: Stated Preferences methods, such as Contingent Valuation Method or Choice Experiments, which estimate the value individuals place on public goods by means of direct elicitation queries in terms of Willingness To Pay [10], and Revealed Preferences methods, such as Travel Cost Method or Hedonic Models, which infer values from individual choices within real markets [11]. With reference to the context under investigation, it has to be noticed that heritage economics established as an area of analysis for the valuation of benefits provided by cultural heritage projects and different applications exist with reference both to Stated Preference and Revealed Preferences Methods [12-15].

Regarding multicriteria evaluation, these methods are used to make a comparative assessment of alternative projects or heterogeneous measures and they allow several criteria to be taken into account simultaneously, considering the opinions of the different stakeholders involved in the decision-making process [16]. In the context of cultural heritage, multi-criteria approaches proved to be able to provide a systematic vision of project effects, taking into account both the qualitative and the quantitative aspects of the decision problem [17-20].

The objective of the paper is to explore the use of a novel hybrid multi-criteria method named $\mathrm{A}^{\prime}$ WOT for supporting a real-world problem in the context of cultural heritage. In particular, the method combines two common approaches used in decision analysis, namely the Analytic Hierarchy Process (AHP) [21], and Strengths, Weaknesses, Opportunities and Threats (SWOT) Analysis. SWOT analysis bases on internal and external valuation criteria to identify strengths and opportunities, which can positively encourage the achieving of objectives, as well as weaknesses and threats, which can adversely affect achieving the objectives. Its contribution to the development of sustainable valorization strategies addressing abandoned cultural heritage is to maintain the strengths, find solutions for weaknesses, capitalize on opportunities and prevent threats. As it might become onerous to come to concordant decisions by solely implementing a SWOT analysis, due to the qualitative nature of its value judgments, the AHP is used to derive priorities and relative importance of criteria, sub-criteria and alternatives in a formal, analytical way, based on experts' judgment.

In the present paper, an A'WOT model has been developed with the purpose of addressing the design and management of the historic farmhouses in the Aglié Castle in Northern Italy. In our framework, SWOT analysis captures potential drivers (i.e., opportunities and strengths) and barriers (i.e., threats and weaknesses) to promote an in-depth understanding of the framework conditions of the ducal farmhouses system, whereas AHP is used to define the priorities of the elements under examination. Thanks to this understanding, we drew new sustainable strategies for the valorization of these assets and we prioritized ducal farmhouses according to their potential in successful strategy implementation.

The rest of the paper is organized as follows. Section 2 presents the methodological background and focuses on the A'WOT method. Section 3 presents the A'WOT model provided to identify top-priority targets among the farmhouses of the Aglié Castle. It describes the structuring of the decision problem thought the SWOT matrix and the evaluation procedure implemented to identify priorities of SWOT categories and factors and the final ranking of alternatives. Section 4 illustrates and discusses the results. Finally, Section 5 synthetizes conclusions and provides guidelines for the design of interventions and the identification of successful management strategies.

\section{Methodological Framework}

Strategic management of cultural heritage assets and their valorization involve the analysis of both interactions between these assets and the environment that surrounds them and internal and 
external factors, which affect the successful implementation of sustainable interventions to restore and enhance them.

In this respect, the Strengths, Weaknesses, Opportunities, and Threats (SWOT) analysis is a systematic approach to assess internal and external factors [22,23] as well as potentials and pitfalls, which guarantees a fact-based, data-driven insight on planning decisions and course of actions, thus providing a good basis for strategy definition $[24,25]$. The SWOT analysis was firstly developed for business and market analysis and it is currently extensively implemented in planning and management and adopted in many fields for research assistance in order to evaluate potential bottlenecks or opportunities to prioritize development and/or management strategies [26,27]. Strengths may enhance overall performances and provide an advantage, whereas Weaknesses may affect efficiency, profitability and competitive advantage. Opportunities are possibilities, which may contribute to exploit advantage or add additional advantage, whereas threats may generate problems and retrieve successful development and implementation of any strategy.

Nonetheless, SWOT analysis can mainly support in the accurate identification of key factors in the analysis, but it does not provide any analytical tool or algorithm to determine the relative importance of these factors in the decision-making process, as results are provided in terms of qualitative assessment $[25,28,29]$. To address this issue and to improve the quantitative information basis of strategic planning processes, Reference [28,29] proposed the A'WOT method, a hybrid tool, which combines the SWOT analysis and the Analytic Hierarchy Process (AHP), proposed by Saaty in the Eighties [21]. The AHP is a well-established technique to address complex decisions and obtain a priority ranking of alternatives. Criteria, sub-criteria, and alternatives relative importance are determined through pairwise comparisons expressed in semantic judgments converted into numerical values according to Saaty's fundamental scale [21].

In the A'WOT model, the AHP is therefore used to evaluate the SWOT factors, as well as the four SWOT categories (i.e., Strengths, Weaknesses, Opportunities, and Threats) and make them commensurable with respect to their priority intensities.

The integration of AHP with SWOT analysis provides analytically determined priorities, according to the eigenvalue approach to pairwise comparisons, and allows comparing alternatives with respect to each SWOT factor and ranking them from best to worse with respect to the goal of the decision-making problem [30]. The A'WOT decomposes a complex decision problem into a simple hierarchy. According to the AHP, at the top of the hierarchy, there is the goal, whereas SWOT categories (i.e., criteria) and SWOT factors (i.e., sub-criteria), which contribute to the goal are at lower levels and alternatives under investigation are at the bottom of the hierarchy.

According to [28], the A'WOT method is structured into the following subsequent phases:

1. SWOT analysis (identification of internal and external factors) and decomposition into hierarchical levels,

2. Pairwise comparisons of factors and SWOT categories,

3. Final ranking.

Firstly, the SWOT analysis is carried out, and internal and external factors are identified. Secondly, pairwise comparisons of factors (with respect to each SWOT category) and of SWOT categories (with respect to the goal) are performed and priorities (i.e., preference intensities) are calculated. At this stage, weights (i.e., priorities) are determined according to the eigenvalue approach to pairwise comparisons and pairwise comparisons of elements at each level are conducted with respect to their relative importance towards their parent node [31]. The consistency of pairwise comparison matrices is then verified by determining the inconsistency index IC, which is usually considered as acceptable whenever IC $<0.10$ [21]. Finally, we obtained the priority ranking of alternatives via a weighted sum aggregation procedure, from the bottom to the top of hierarchical levels.

Starting from the seminal works by [28,29], a significant strand of literature developed and the number of its applications to real-world decision problems increased significantly [32-34]. 
The applications of the A'WOT method are varied and cover as major fields natural resource management [32,35-37], sustainable tourism and tourism management [25,34,38,39], strategic assessment and group decision making (see among others, [40-43]). Although in the field of cultural heritage assessment, there are a flourishing set of recent contributions in literature on the sole application of AHP [44-51] and SWOT analysis [52-56], respectively. Nonetheless, to our knowledge, there are no contributions on the application of $\mathrm{A}^{\prime}$ WOT to strategic decisions on cultural heritage. This contribution complements the existing literature on $\mathrm{A}^{\prime}$ WOT methods with a novel application in cultural heritage and provides a new investigation of strategic choice on the valorization of abandoned and severely damaged assets.

\section{Case Study}

\subsection{Description of the Case Study: The Ducal Farmhouses in Aglié Estate}

The township of Agliè is located in the Canavese area, about $40 \mathrm{~km}$ from Turin. It has a varied territory and it is located partly in the hilly area of the Ivrea morainic amphitheater and partly in the plain to the south. The agricultural area features arable land, meadows and poplar groves on the plain and vineyards, as well as wooded areas, on the hills. The ducal farmhouses, established to serve Agliè Castle, together with a system of reserves, farms, and estates, represent the assets in possession of the Savoy family [57]. Their main function was agricultural management.

In 1764 Benedetto Maria Maurizio, Duke of Chiablese, bought the estate of Agliè Castle and its park and other possessions, including the "Cascina del Parco" (Lavanderia), owned by the Marquis San Germano, Cascina Valle owned by the Count of Agliè and Cascina Gozzani, belonging to poet Guido Gozzano's family (Figures 1 and 2).

The farmhouses, joined in one property, were called "Cascine Ducali" [58]. In 1769, Count Ignazio Birago di Borgaro planned new expansions of the existing buildings as well as new settlements, including Cascina Allea.

Cascina Allea (called Mandria) was designed between 1772 and 1773, thanks to the drawings and instructions of Birago di Borgaro, at the service of the Duke of Chiablese [59]. This building is interesting mainly because of its location within the context of the Castle Park and the architecture of its façade. The complex is formed by a closed court organized around a quadrangular space. A U-shaped volume forms the structure and it has an axis of symmetry passing through the large entrance arch. The structure is composed of three main volumes. The structure does not appear to be in an advanced state of degradation but it is damaged by a lack of maintenance and by exposure to the action of atmospheric agents.

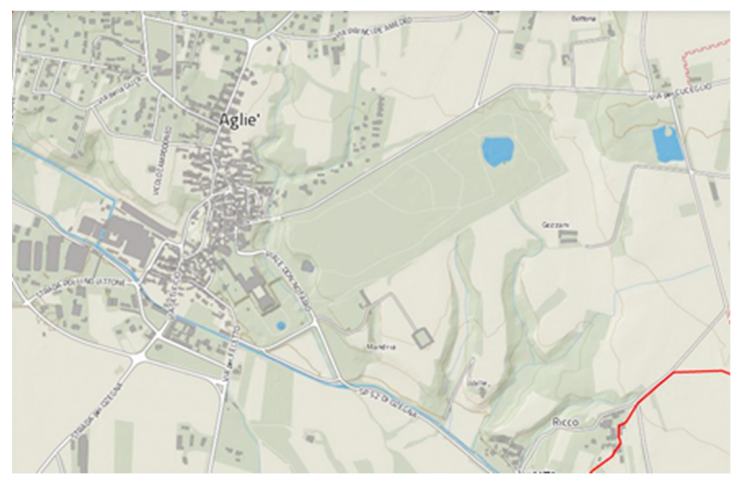

Figure 1. Cartography extrapolated from the geoportal representing the system of the Castle and ducal farmhouses, Municipality of Agliè, http://www.geoportale.piemonte.it/geocatalogorp. 


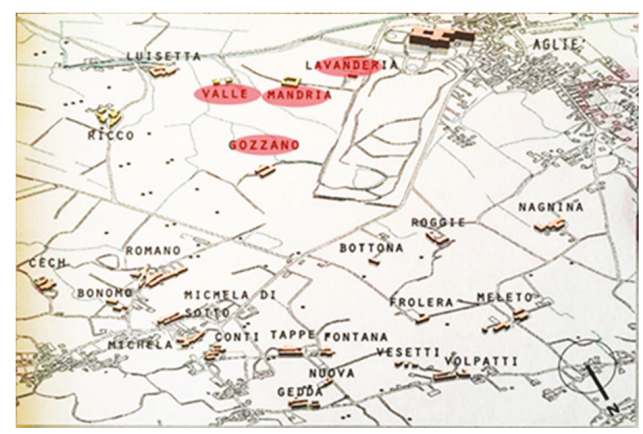

Figure 2. Cartography extrapolated from the Ancient Archive of Agliè Municipality ("Comune di Agliè").

Cascina Ortovalle has been represented on historical maps since 1745. It is a single building with simple wings and was mainly used for cultivations of fruit and vegetables. The structure is located on a hill and can be accessed by a poorly maintained dirt road. The building is in good condition since it is inhabited. It has a large terrace looking into the countryside of Agliè and a wall surrounds it. The context in which Cascina Ortovalle is located is characterized by large cultivated fields and the structure is well connected to the city through the main road. Presently the building can be used on all stories, it is not at risk of collapse and it does not present a compromised crack pattern. The architectural structure has a large barn with a trussed roof, which was restored a few months ago by the current tenants of the building.

Cascina Gozzani is composed of two buildings and is located south-east of Agliè, not far from the park. The historical cadastral maps attribute ownership of Cascina to Guido Gozzano's family. The complex is characterized by a planimetric layout composed of an internal courtyard with a double opposing row that is spread throughout the Canavese countryside. The two buildings face each other at close range and they are connected to each other through a surrounding wall. The position in which it is located is rather isolated because it is more to the north and far from the city center and the castle. The functional division between the two buildings reflects the theme in which they're inserted, on the northern side, there are the residential and service functions, including the rooms of the Lord, an area which stands out for its height and turns towards the east. The general situation of the complex is considerably compromised. A recent inspection revealed the definitive collapse of part of the roof, added to degradation phenomena of extended areas from the upper floors through the ground floor. The main reason related to this phenomenon is the total absence of maintenance by the State, which owns the property and has not tried to limit damages to both buildings. The main causes of deterioration of the structures concern substantial infiltrations of water through cracks and collapses of the roofs, which have caused considerable damage to the underlying areas.

Among the farmhouses, Cascina Lavanderia is the closest to the Park and, due to its location, on historical maps, it is referred to as "Cascina del Parco" [60]. Its role was of service to the Castle and it was destined for the cultivation of vegetables and food preservation. The farmhouse is situated in a strategic position with respect to the Park, in an area next to the south-west border and the main access road and numerous paths directly connect it to the Park. From an architectural point of view, the structure is a simple two-story wing, with a stairwell in its central part. The state of conservation of this building is almost totally compromised. The structure is secured thanks to a system of beams to help in stabilizing it and reducing the risk of collapse. The roof and ceiling are almost completely collapsed, thus damaging the horizontal surfaces of the lower floors, and some of these rooms host dense vegetation (Figure 3 ). 

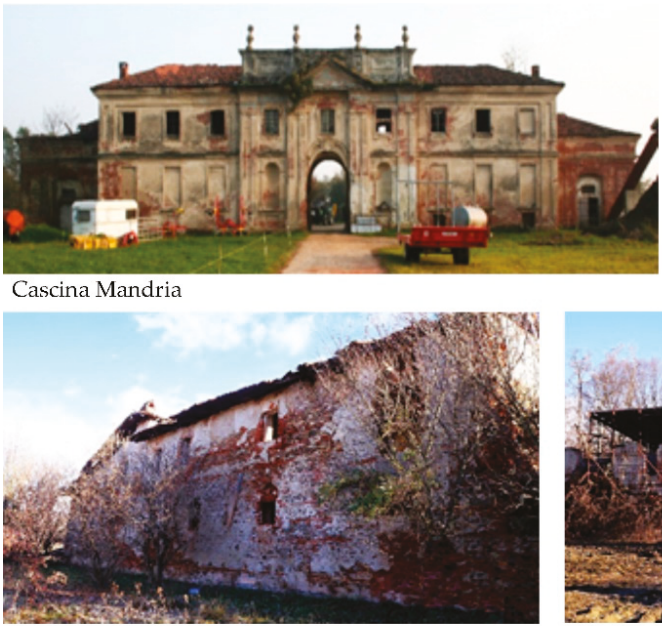

Cascina Gozzano

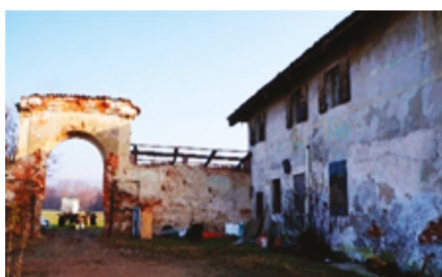

Cascina Ortovalle

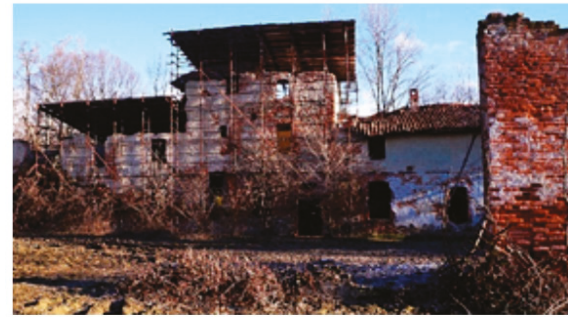

Cascina Lavanderia

Figure 3. The farmhouses of the Aglié Castel (photos by the authors).

\subsection{The $A^{\prime}$ WOT Model}

Starting from the analysis of the historic farmhouses of the Aglié Castle, in this paper we provide new management strategies of these assets aiming at their preservation and valorization. To this purpose, we developed an $A^{\prime}$ WOT model with the objective of ranking the four farmhouses according to their potential for successful valorization strategy implementation. They are ranked from best to worst in terms of priorities of potential successful intervention.

As described in Section 2, we structured the modeling into three main subsequent stages. The first step of the modeling consists of the implementation of the SWOT analysis and construction of the SWOT matrix in order to structure the decision problem. In particular, the SWOT categories (namely Strengths, Weaknesses, Opportunities and Threats) were further broken down into specific sub-criteria, which represent the key aspects in the evaluation of cultural heritage assets. To identify the above-mentioned SWOT factors, we conducted an extensive literature review on cultural heritage valorization and interviewed a panel of experts composed by an officer of the Superintendence of Turin and by three experts in the field of history of architecture, restoration, and structural analysis, and economic valuation, respectively.

The SWOT factors identified, grouped into to Strengths S, Weaknesses W, Opportunities O and Threats $\mathrm{T}$, can be described as follows.

- S1 accessibility: it considers the mobility network for reaching the farmhouses,

- S2 state of conservation: it is related $t$ to the current level of maintenance of the buildings,

- $\quad \mathrm{S} 3$ historical/cultural value: it considers the value of the building as resulting from specific documents and sources,

- W1 current use: it takes into account the current function of the asset or the abandoned condition,

- W2 ownership: it refers to the present ownership of the properties, whether of the Superintendence or of specific private entities,

- W3 operation and maintenance (O\&M) costs: they are related to building structure and materials, recreation, consumption and production activities host in the farmhouse,

- O1 flexibility: it takes into account the potential of transforming and restoring the building, whereas maintaining the readability of the original structures and functions,

- $\mathrm{O} 2$ quality of the context: it refers to the quality of the surrounding natural environment, 
- O3 network infrastructures: it takes into account whether the farmhouse is connected to public network infrastructures (e.g., utilities),

- T1 relationship with the context: it is related to the typology and state of maintenance of built environment surrounding the asset,

- T2 regulatory risks: it refers to changes in regulation which can affect the business or property use),

- T3 budget constraints: it refers to a credit crunch, lack of financial resources and potential investors.

According to the aforementioned structure, we then compiled four SWOT matrixes, one for each farmhouse under investigation. As an example, Appendix A reports the SWOT matrix that has been constructed for Cascina Lavanderia.

In the second phase, according to the AHP [21], the SWOT factors have been organized into a hierarchical structure (Figure 4), in which at the top of the hierarchy there is the goal (i.e., ranking of ducal farmhouse with respect to their potential in the implementation of successful, sustainable valorization strategies), at lower levels, there are criteria (i.e., SWOT categories) and sub-criteria (i.e., SWOT factors), respectively. At the bottom of the tree-like structure, there are the alternatives under investigation (i.e., the ducal farmhouses in the Aglié estate), to be compared in order to determine their relative importance and the final ranking.

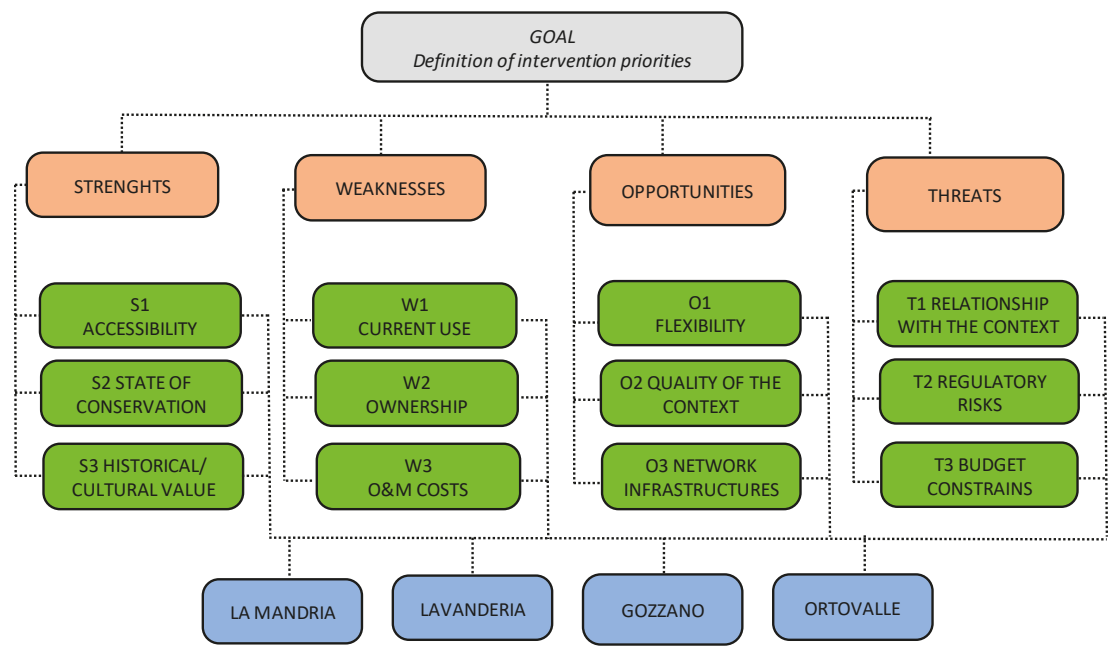

Figure 4. Structuring of the decision problem through the A'WOT (a hybrid tool, which combines the Strengths, Weaknesses, Opportunities, and Threats (SWOT) analysis and the Analytic Hierarchy Process (AHP) method.

As required by the AHP method, firstly it was necessary to evaluate the criteria level, i.e., the SWOT categories, and the sub-criteria level, i.e., the SWOT factors. To this purpose, being more strategic and general levels of the problem, we asked the officer of the Superintendence, involved in the panel of experts, to fill in the pairwise comparison questions related to the relative importance of SWOT categories and SWOT factors. Figures 5 and 6 report an example of questions for the evaluation of the SWOT categories and factors, respectively.

Once all the pairwise comparison questions were filled in, we calculated the priorities of SWOT categories and factors as resulting from the Superintendence officer's interview (see Section 4). 


With reference to the definition of the potential in asset valorization of the historical farmhouses in Aglie
Caste, which of the two criteria do you think is more important? And to what extent?
Strengths \begin{tabular}{|l|l|l|l|l|l|l|l|l|l|l|l|l|l|l|l|l|}
9 & 8 & 7 & 6 & 5 & 4 & 3 & 2 & 1 & 2 & 3 & 4 & 5 & 6 & 7 & 8 & 9 \\
Threats
\end{tabular}

Figure 5. Example of pairwise comparison questions for the evaluation of the SWOT categories.

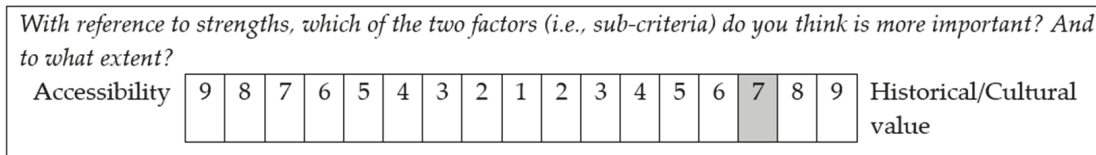

Figure 6. Example of pairwise comparison questions for the evaluation of the SWOT factors.

Subsequently, it was necessary to fill in the pairwise comparison questions for alternatives evaluation. In this case, being a specific technical issue, we submitted the questionnaire to the above-mentioned three experts in the field of history of architecture, restoration, and structural analysis, and economic valuation. They evaluated the farmhouses with respect to each selected factor (sub-criterion) and evaluated each factor with respect to SWOT categories (criteria). It worth noting that as far as Strengths and Opportunities are considered, the preference for an alternative means that the alternative is able to maximize the S/O elements, while as far as the Weakness and Threats elements are considered, the preference for an alternative indicates the alternative is able to minimize the W/T elements. As an example, Figures 7 and 8 report two of the questions administered to the panel of experts.

With reference to maximization of accessibility potential, which of the two farmhouses do you think is
preferable? And to what extent?
Mandria \begin{tabular}{|l|l|l|l|l|l|l|l|l|l|l|l|l|l|l|l|l|}
\hline 9 & 8 & 7 & 6 & 5 & 4 & 3 & 2 & 1 & 2 & 3 & 4 & 5 & 6 & 7 & 8 & 9 \\
Lavanderia
\end{tabular}

Figure 7. Example of pairwise comparison questions for the evaluation of the alternatives with respect to sub-criteria "accessibility".

With reference to the minimization of the threats related to the relationship with the context, which of the
two farmhouses do you think is preferable? And to what extent?
\begin{tabular}{|l|l|l|l|l|l|l|l|l|l|l|l|l|l|l|l|l|l|} 
Lavanderia & 9 & 8 & 7 & 6 & 5 & 4 & 3 & 2 & 1 & 2 & 3 & 4 & 5 & 6 & 7 & 8 & 9 \\
Gozzani \\
\hline
\end{tabular}

Figure 8. Example of pairwise comparison questions for the evaluation of the alternatives with respect to sub-criteria "relationship with the context".

Finally, in the third phase, once all the pairwise comparison matrices were replied, we calculated the priorities of the alternatives and multiplied the local weights of each farmhouse by the weights of the SWOT categories and factors previously defined, and obtained the final ranking (see Section 4).

\section{Results and discussion}

As aforementioned, we organized a focus group to validate the hierarchy and the weights by dynamic discussion [61-63]. During the brainstorming, experts processed data and contributed to discussion according to their expertise, and a moderator helped discussion among experts and favored the process of reaching consensus on the final set of weights. We obtained the final set of weights by calculating the geometrical mean of experts' judgments [64,65]. In detail, local priorities of objects are 
derived according to the eigenvalue approach to pairwise comparisons and then aggregated within the hierarchy in order to derive global priorities. We implemented the weighted geometric mean aggregation method in the computation of the global priorities as it reflects the preference information contained in local pairwise comparison matrices of alternatives properly [66]. In order to obtain the priority vectors and the final ranking, we implemented the A'WOT model in the Super Decision software. The overall set of weights of criteria and sub-criteria are displayed in Table 1.

Table 1. Criteria and sub-criteria priority vector.

\begin{tabular}{cccc}
\hline Criteria & Sub-Criteria & Priority of Criteria & Priority of Sub-Criteria \\
\hline \multirow{3}{*}{ Strengths } & S1-Accessibility & & 0.083 \\
& S2-State of conservation & 0.386 & 0.121 \\
& S3-Historical/cultural value & & 0.796 \\
\hline \multirow{3}{*}{ Weaknesses } & W1-Ownership & & 0.088 \\
& W2-O\&M costs & 0.034 & 0.243 \\
& W3-Current use & & 0.669 \\
Opportunities & O1-Flexibility & 0.479 & 0.149 \\
& O2-Quality of the context & & 0.065 \\
\hline \multirow{3}{*}{ Threats } & O3-Network infrastructures & & 0.279 \\
& T1-Relationship with the context & 0.100 & 0.072 \\
& T2-Regulatory risks & & 0.649 \\
\hline
\end{tabular}

According to criteria priorities displayed in the third column of Table 1, Opportunities play a major role (i.e., 0.479 ) in the successful implementation of valorization strategies, whereas Weaknesses are ranked as fourth (i.e., 0.034). This result is rather intuitive with respect to asset valorization processes, in which the asset potential resides mostly in Opportunities, which counterbalance the negative impact of asset's Weaknesses on strategy implementation. As far as sub-criteria priorities are concerned (fourth column of Table 1), historical/cultural value has the highest priority with respect to strengths (i.e., 0.785), followed by state of conservation (i.e., 0.121) and by accessibility (i.e., 0.083), whose impact is negligible with respect to other factors. As far as Weaknesses are concerned current use has the highest priority (i.e., 0.669), whereas the quality of the context is the most important opportunity factor (i.e., 0.785) and the connection to network infrastructures is the least important (0.066). As expected, experts considered budget constraints as the most crucial Threats (i.e., 0.649) and, although it might appear counterintuitive, regulatory risks are ranked as third (i.e., 0.072). It is worth noting that the above priorities represent the relative importance of SWOT factors with respect to the parent node SWOT category. Therefore, although regulatory risks are ranked as last, this does not imply that they are not worth considering in absolute terms, being identified as a Threat in the SWOT analysis.

Finally, Table 2 illustrates alternatives' priority vectors with respect to each sub-criterion as well as ducal farmhouses' final ranking.

According to results, Cascina Lavanderia has the highest potential in the successful implementation of asset valorization strategies. This is mainly because the asset is not currently in use, thus easing any potential intervention, and to the quality of the surrounding context. In fact, it is very close to the park and its favorable location is of paramount importance in the successful design and development of a touristic circuit connecting the four ducal farmhouses in a unitary management perspective. The remaining farmhouses reveal the same potential, with a small preference for Cascina Lamandria, which is ranked as second, due to its good state of conservation. By contrast, Cascina Gozzano is ranked as fourth due to both its state of abandonment and distance from the Castle.

The Lavanderia farmhouse has a great potential in creating a synergy with the park system due to its favorable location and its original reason for construction, as it was built to be of service to Aglié Castle. Although it fell into neglect, its relatively small size (approximately 1400 square meters) 
increases the potential for timely maintenance and refurbishment of the structures, as well as the renovation of the roads connecting it to the park. The classification of the asset state of conservation and the identification of pathologies and degradation represent a crucial phase in the knowledge process and they are preliminary operations to establish the urgency of restoration works, defining the works to be undertaken in a specific site and their progressive order of intervention (Figure 9).

Table 2. Alternatives priority vector and final ranking.

\begin{tabular}{|c|c|c|c|c|}
\hline \multirow{2}{*}{ Sub-Criteria } & \multicolumn{4}{|c|}{ Alternatives Priority Vector } \\
\hline & Lavanderia & Lamandria & Gozzano & Ortovalle \\
\hline Accessibility & 0.369 & 0.434 & 0.062 & 0.135 \\
\hline State of conservation & 0.066 & 0.571 & 0.060 & 0.303 \\
\hline Historical/cultural value & 0.250 & 0.250 & 0.250 & 0.250 \\
\hline Ownership & 0.406 & 0.068 & 0.476 & 0.050 \\
\hline O\&M costs & 0.051 & 0.104 & 0.422 & 0.422 \\
\hline Current use & 0.446 & 0.065 & 0.446 & 0.043 \\
\hline Flexibility & 0.410 & 0.072 & 0.446 & 0.071 \\
\hline Quality of the context & 0.483 & 0.288 & 0.076 & 0.153 \\
\hline Network infrastructures & 0.522 & 0.182 & 0.137 & 0.159 \\
\hline Relationship with the context & 0.198 & 0.069 & 0.507 & 0.225 \\
\hline Regulatory risks & 0.250 & 0.250 & 0.250 & 0.250 \\
\hline Budget constraints & 0.152 & 0.068 & 0.390 & 0.390 \\
\hline Overall Normal Priority & 0.348 & 0.248 & 0.203 & 0.201 \\
\hline Overall Ideal Priority & 1.000 & 0.710 & 0.583 & 0.578 \\
\hline
\end{tabular}

The main objective is to recover the interconnections of this system to the reality of the Castle and the Park and to establish a unique well-coordinated management strategy.

From this preliminary analysis, it emerged the scenario hypothesis a, in which territorial, social and cultural peculiarities are the most suitable to the characteristics of the asset under investigation, to the detriment of the scenario hypothesis whose characteristics do not fully respond to its valorization needs.

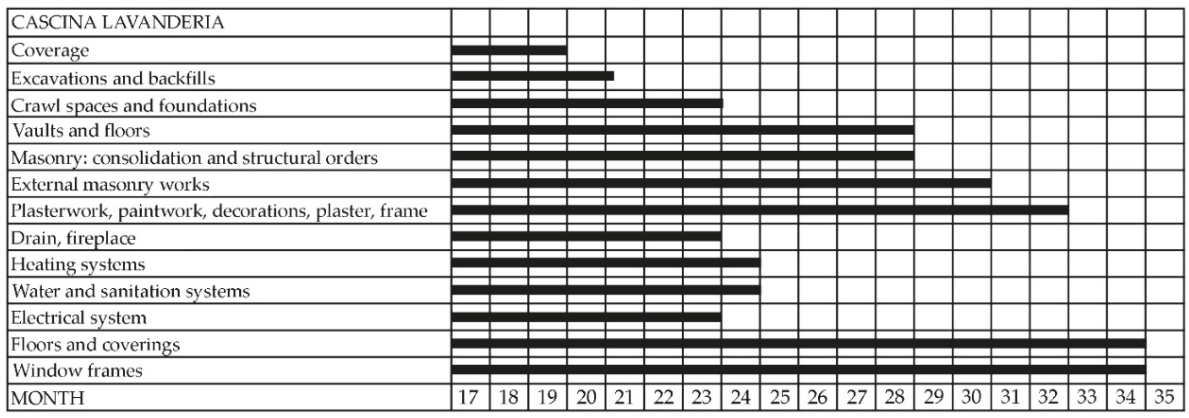

Figure 9. Gantt chart for the intervention works of the Lavanderia farmhouse.

\section{Conclusions}

The design of efficient strategies of cultural heritage assets valorization is a complex process, which requires a profound knowledge of its peculiarities and its identity through history and involves multiple decision variables, actors and stakeholders whose objectives are often conflicting and competing $[67,68]$. Enhancement of cultural heritage consists of giving forms and norms to those activities, which aim at both promoting its knowledge, use and public enjoyment and fostering conservation and restoration works. There is often a dichotomy between the economic and socio-cultural perspectives in defending human values and historical testimonies, which contribute to creating a cultural identity 
through centuries. Due to a lack of financial resources, it is fundamental to improve capacities for the sustainable use of cultural heritage by conserving, protecting, promoting and developing both natural and cultural heritage assets. Indeed, it is generally agreed in literature that sustainable development is a multi-dimensional concept and its evaluation is an interdisciplinary process, which involves cultural, social, environmental and economic disciplines, to show the impact of investment and allocation decisions on common resources. Integrated frameworks are thus necessary to cope with these issues and to support the decision-making processes [20,69]. In this respect, we provided a multi-criteria decision aiding approach for ranking valorization strategies of cultural heritage assets aimed at promoting their restoration and conservation, as well as at creating cultural and economic benefits by focusing on the ducal farmhouses of Aglié Castle estate in the Piedmont region, listed in the UNESCO World Heritage Sites in 1997. We ranked the assets according to their potential in implementing successful valorization strategies. In order to be effective, these strategies should combine, on the one hand, efficient re-use of the above-mentioned buildings (currently in state of abandonment and partially or totally collapsed) and restoration of the original functions they were designed for, with the enhancement of existing synergies between them and the creation of new opportunities for their interconnections with cultural tourism circuits, on the other.

In order to provide guidelines to support the policymaker, it is of paramount importance to structure the analysis into sequential phases. In this respect, we firstly analyzed data relating to the real estate assets under investigation, with the aim of segmenting them for types of feasible interventions. Secondly, we developed the masterplan of the operation of valorization. We then implemented feasibility studies of individual assets in collaboration with the panel of experts to support the policymaker in the implementation phase of valorization actions.

The meta-design phase is de facto a fundamental preliminary step in the design of a future final project meant to the valorization of the farmhouses complex: it consists in constructing a structured mental scheme to clarify the needs that emerged in the preliminary study phase and provide guidelines for valorization strategies implementation. Contribution by different disciplines and technical competency are necessary in order to define the best project proposals to be developed within the preliminary planning stage and designing the final project. Many aspects that interact need to be carefully evaluated in order to obtain a sustainable proposal for compatible re-functionalization and re-use of this set of farmhouses.

According to our analysis, the main aspects to investigate are:

- $\quad$ architectural features and priority ranking of action proposals,

- environmental and landscape issues or road and infrastructure characteristics,

- management and economic/financial outcomes,

- $\quad$ social concerns.

The main objective in the conceptualization of the interventions designed to the restoration and valorization of the Agliè ducal farmhouses is to re-set in place synergies and interconnections between the farmhouses, the city center, and the Castle. In this respect, the determinant of the valorization process identified through our analysis is the inclusion in the Agliè estate of cultural activities, accommodation facilities and the establishment of biodynamic agriculture and organic farming experimental centers.

Cultural activities are key drivers in the valorization process as they add value to memory and testimony of cultural and historical traditions and enhance the knowledge of the historical phases, which have characterized the estate from construction. The development of accommodation facilities is necessary in order to attract and welcome to the farms' tourists (e.g., cultural tourists) from the town and the Castle to the farm: tourists coming to the Castle are currently at 20,000 per year, with a trend destined to grow. Our findings reveal that agriculture and hospitality could be the keystones of the restoration, conservation and financial recovery process involving these assets. 
Based on our results, activities and functions with the highest potential in the implementation of successful management strategies relative to each farmhouse are listed below.

- Cascina Lavanderia: construction of hiking trails, accommodation, and reception facilities, aimed at acting as a filter between activities in the castle -park system and the other farmhouses. This is mainly due to its location directly connected to the park system,

- Cascina Mandria: construction of a center for experimentation in agriculture and horse-riding facilities,

- Cascina Ortovalle: construction of, an experimental/educational center for the growing of vegetables and recovery of its original vocation,

- Cascina Gozzani: construction of an agricultural production center and a cultural center to develop knowledge of Guido Gozzano's life and poetry.

Author Contributions: Conceptualization, M.B., C.D. and A.M.; methodology, M.B., C.D. and A.M.; formal analysis, M.B., C.D. and A.M.; investigation, M.B., C.D. and A.M.; data curation, M.B., C.D. and A.M.; writing M.B., C.D. and A.M. All authors have read and agreed to the published version of the manuscript.

Acknowledgments: Part of the research presented in this paper was carried out within the specialization thesis "Il sistema delle cascine ducali ad Agliè. Dallo studio di fattibilità strategica ad una nuova proposta di valorizzazione" by Alessia Marello, Postgraduate School of Beni Architettonici e del Paesaggio of Politecnico di Torino. Authors are grateful to Professor Monica Naretto for her contribution to the historical archive search and the analysis of the historical issues this study bases on.

Conflicts of Interest: The authors declare no conflict of interest. 


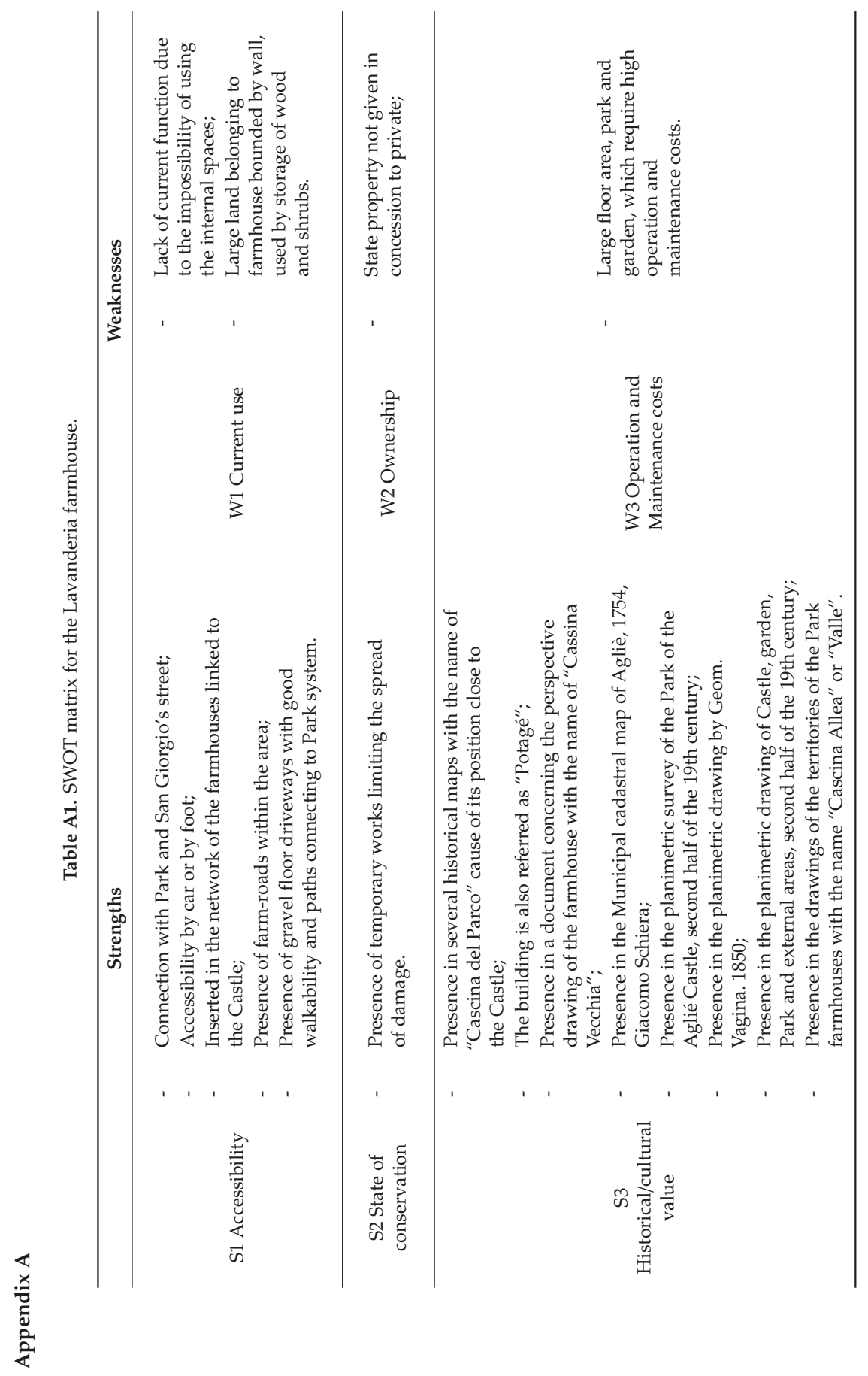




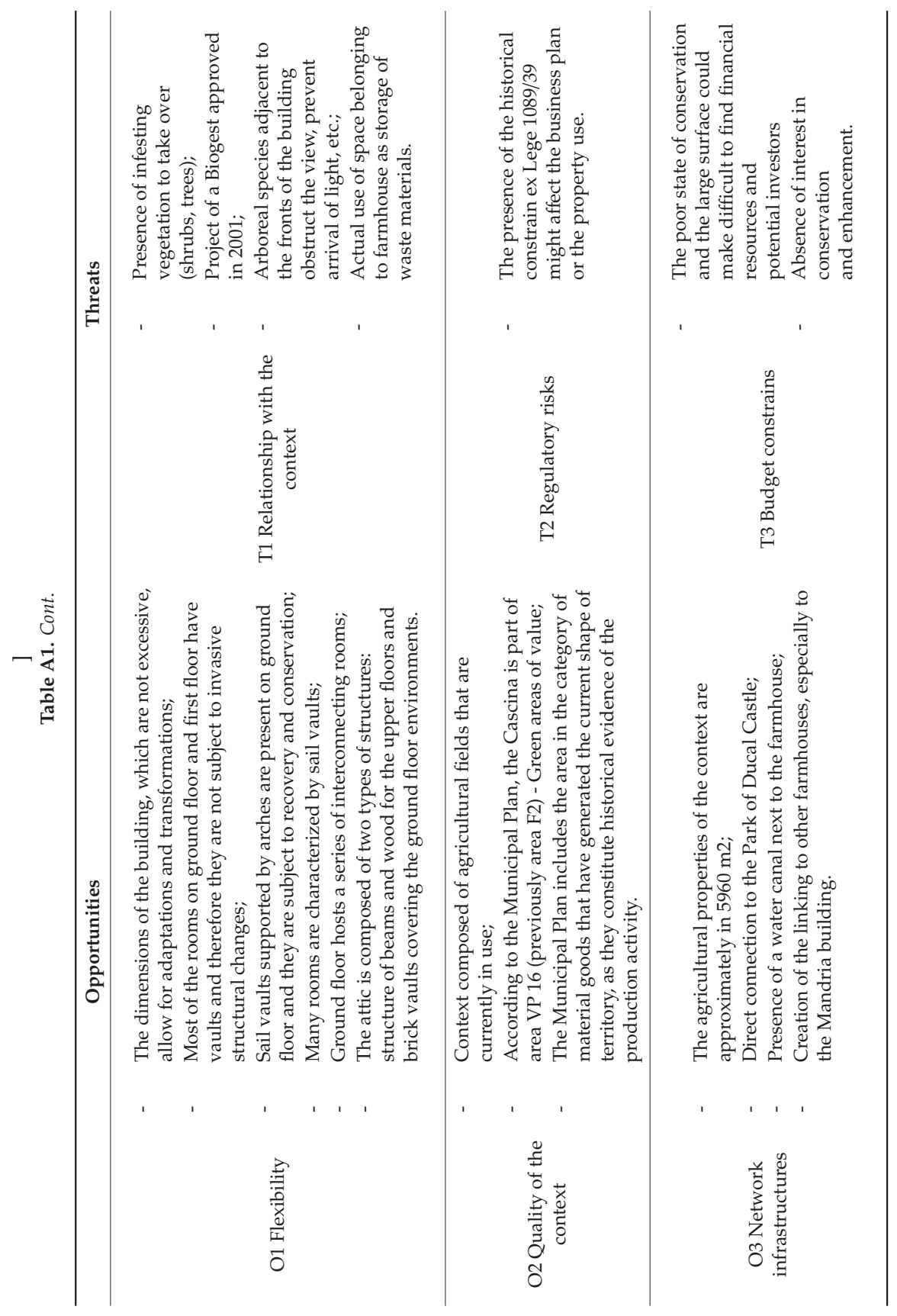




\section{References and Note}

1. Ball, R. Developers, regeneration and sustainability issues in the reuse of vacant industrial buildings. Build. Res. Inf. 1999, 27, 140-148. [CrossRef]

2. Langston, C.; Wong, F.K.; Hui, E.C.; Shen, L.-Y. Strategic assessment of building adaptive reuse opportunities in Hong Kong. Build. Environ. 2008, 43, 1709-1718. [CrossRef]

3. Bullen, P.A.; Love, P.E. Adaptive reuse of heritage buildings. Struct. Surv. 2011, 29, 411-421. [CrossRef]

4. Plevoets, B.; Van Cleempoel, K. Adaptive reuse as a strategy towards conservation of cul-tural heritage: A literature review. WIT Trans. Built Environ. 2011, 118, 155-164.

5. Mısırlısoy, D.; Günçe, K. Adaptive reuse strategies for heritage buildings: A holistic approach. Sustain. Cities Soc. 2016, 26, 91-98. [CrossRef]

6. Dolores, L.; Macchiaroli, M.; De Mare, G. Sponsorship for the Sustainability of Historical-Architectural Heritage: Application of a Model's Original Test Finalized to Maximize the Profitability of Private Investors. Sustainability 2017, 9, 1750. [CrossRef]

7. Forte, C.; De Rossi, B. Principi di Economia ed Estimo; Etas: Milano, Italy, 1974.

8. Mazzanti, M. Cultural heritage as multi-dimensional, multi-value and multi-attribute economic good: Toward a new framework for economic analysis and valuation. J. Socio-Economic. 2002, 31, 529-558. [CrossRef]

9. Pearce, D.W.; Turner, R.K. Economics of Natural Resources and the Environment; Johns Hopkins University Press: Baltimore, MD, USA, 1990.

10. Louviere, J.J.; Hensher, D.A.; Swaitt, J.D. Stated Choice Methods. Analysis and Application; Cambridge University Press: Cambridge, UK, 2000.

11. Voke, M.; Fairley, I.; Willis, M.; Masters, I. Economic evaluation of the recreational value of the coastal environment in a marine renewables deployment area. Ocean Coast. Manag. 2013, 78, 77-87. [CrossRef]

12. Bedate, A.; Herrero, L.C.; Sanz, J. Ángel Economic valuation of the cultural heritage: Application to four case studies in Spain. J. Cult. Heritage 2004, 5, 101-111. [CrossRef]

13. Báez, A.; Herrero, L.C.; Prieto, L.C.H. Using contingent valuation and cost-benefit analysis to design a policy for restoring cultural heritage. J. Cult. Heritage 2012, 13, 235-245. [CrossRef]

14. Wright, W.C.; Eppink, F.V. Drivers of heritage value: A meta-analysis of monetary valuation studies of cultural heritage. Ecol. Econ. 2016, 130, 277-284. [CrossRef]

15. Kee, T. Sustainable adaptive reuse - economic impact of cultural heritage. J. Cult. Heritage Manag. Sustain. Dev. 2019, 9, 165-183. [CrossRef]

16. Roy, B.; Bouyssou, D. Aide Multicritere a la Decision: Methodes et Cas; Economica: Paris, France, 1993.

17. Ferretti, V.; Bottero, M.; Mondini, G. Decision making and cultural heritage: An application of the Multi-Attribute Value Theory for the reuse of historical buildings. J. Cult. Heritage 2014, 15, 644-655. [CrossRef]

18. Nesticò, A.; Morano, P.; Sica, F. A model to support the public administration decisions for the investments selection on historic buildings. J. Cult. Heritage 2018, 33, 201-207. [CrossRef]

19. Bottero, M.; D'Alpaos, C.; Oppio, A. Multicriteria Evaluation of Urban Regeneration Processes: An Application of PROMETHEE Method in Northern Italy. Adv. Oper. Res. 2018, 2018, 1-12. [CrossRef]

20. Bottero, M.; D'Alpaos, C.; Oppio, A. Ranking of Adaptive Reuse Strategies for Abandoned Industrial Heritage in Vulnerable Contexts: A Multiple Criteria Decision Aiding Approach. Sustainability 2019, 11, 785. [CrossRef]

21. Saaty, T.L. The Analytic Hierarchy Process: Planning, Priority Setting, Resource Allocation; McGraw-Hill: New York, NY, USA, 1980.

22. Wheelen, T.L.; Hunger, J.D. Strategic Management and Business Policy, 5th ed.; Addison Wesley: Reading, MA, USA, 1995.

23. Hill, T.; Westbrook, R. SWOT analysis: It's Time for a Product Recall. Long Range Plan. 1997, 30, 46-52. [CrossRef]

24. Kajanus, M. A model for creating innovative strategies for an enterprise and its application to a rural enterprise. Manag. Decis. 2000, 38, 711-722. [CrossRef]

25. Kajanus, M.; Kangas, J.; Kurttila, M. The use of value focused thinking and the A'WOT hybrid method in tourism management. Tour. Manag. 2004, 25, 499-506. [CrossRef]

26. Falcone, P.M.; Tani, A.; Tartiu, V.E.; Imbriani, C. Towards a sustainable forest-based bioeconomy in Italy: Findings from a SWOT analysis. For. Policy Econ. 2020, 110, 101910. [CrossRef] 
27. Kamran, M.; Fazal, M.R.; Mudassar, M. Towards empowerment of the renewable energy sector in Pakistan for sustainable energy evolution: SWOT analysis. Renew. Energy 2020, 146, 543-558. [CrossRef]

28. Kurttila, M.; Pesonen, M.; Kangas, J.; Kajanus, M. Utilizing the analytic hierarchy process (AHP) in SWOT analysis-a hybrid method and its application to a forest-certification case. For. Policy Econ. 2000, 1, 41-52. [CrossRef]

29. Pesonen, M.; Kurttila, M.; Kangas, J.; Kajanus, M.; Heinonen, P. Assessing the priorities using A'WOT among resource management strategies at the Finnish Forest and Park Service. For. Sci. 2001, 47, 534-541.

30. Kangas, J.; Kangas, A.; Leskinen, P.; Pykäläinen, J. MCDM methods in strategic planning of forestry on state-owned lands in Finland: Applications and experiences. J. Multi-Criteria Decis. Anal. 2001, 10, 257-271. [CrossRef]

31. Saaty, T.L. Fundamentals of Decision Making and Priority Theory with the Analytic Hierarchy Process, 1st ed.; RWS Publications: Pittsburgh, PA, USA, 2000.

32. Kajanus, M.; Leskinen, P.; Kurttila, M.; Kangas, J. Making use of MCDS methods in SWOT analysis—Lessons learnt in strategic natural resources management. For. Policy Econ. 2012, 20, 1-9. [CrossRef]

33. Vacik, H.; Kurttila, M.; Hujala, T.; Khadka, C.; Haara, A.; Pykäläinen, J.; Honkakoski, P.; Wolfslehner, B.; Tikkanen, J. Evaluating collaborative planning methods supporting programme-based planning in natural resource management. J. Environ. Manag. 2014, 144, 304-315. [CrossRef]

34. Moharramnejad, N.; Rahnamai, M.-T.; Dorbeiki, M. Application of A'WOT Method in Strategic Management of Sustainable Tourism in a National Park. Environ. Eng. Manag. J. 2017, 16, 471-480.

35. Gallego-Ayala, J.; Juizo, D. Performance evaluation of River Basin Organizations to implement integrated water resources management using composite indexes. Phys. Chem. Earth Parts A/B/C 2012, 50, 205-216. [CrossRef]

36. Podimata, M.V.; Yannopoulos, P.C. Evaluating challenges and priorities of a trans-regional river basin in Greece by using a hybrid SWOT scheme and a stakeholders' competency overview. Int. J. River Basin Manag. 2013, 11, 93-110. [CrossRef]

37. Gallego-Ayala, J.; Juizo, D. Integrating Stakeholders' Preferences into Water Resources Management Planning in the Incomati River Basin. Water Resour. Manag. 2014, 28, 527-540. [CrossRef]

38. Chitsaz, N.; Azarnivand, A. Water Scarcity Management in Arid Regions Based on an Extended Multiple Criteria Technique. Water Resour. Res. 2017, 31, 233-250. [CrossRef]

39. Kişi, N. A Strategic Approach to Sustainable Tourism Development Using the A'WOT Hybrid Method: A Case Study of Zonguldak, Turkey. Sustainability 2019, 11, 964. [CrossRef]

40. Canto-Perello, J.; Curiel-Esparza, J.; Calvo, V. Strategic decision support system for utility tunnel's planning applying A'WOT method. Tunn. Undergr. Space Technol. 2016, 55, 146-152. [CrossRef]

41. Pellegrino, D.; Schirpke, U.; Marino, D. How to support the effective management of Natura 2000 sites? J. Environ. Plann. Man. 2017, 60, 383-398. [CrossRef]

42. Kittiyankajon, M.; Chetchotsak, D.; Ruangchoengchum, P. Group decision technique for multiple criteria evaluation problems: The preferential difference and rank approach through data envelopment analysis. IJBIR 2019, 18, 410-427. [CrossRef]

43. Bottero, M.; Comino, E.; Dell'Anna, F.; Dominici, L.; Rosso, M. Strategic Assessment and Economic Evaluation: The Case Study of Yanzhou Island (China). Sustainability 2019, 11, 1076. [CrossRef]

44. Roberti, F.; Oberegger, U.F.; Lucchi, E.; Gasparella, A. Energy retrofit and conservation of built heritage using multi-objective optimization: Demonstration on a medieval building. Build. Simul. 2015, 2, 189-197.

45. Roberti, F.; Oberegger, U.F.; Lucchi, E.; Troi, A. Energy retrofit and conservation of a historic building using multi-objective optimization and an analytic hierarchy process. Energy Build. 2017, 138, 1-10. [CrossRef]

46. Turskis, Z.; Morkunaite, Z.; Kutut, V. A hybrid multiple criteria evaluation method of ranking of cultural heritage structures for renovation projects. Int. J. Strateg. Prop. Manag. 2017, 21, 318-329. [CrossRef]

47. Bozic, S.; Vujicic, M.; Kennell, J.; Besermenji, S.; Solarevic, M. Sun, sea and shrines: Application of analytic hierarchy process (AHP) to assess the attractiveness of six cultural heritage sites in Phuket: Thailand. Geogr. Pannonica 2018, 22, 121-138. [CrossRef]

48. Keyvanfar, A.; Shafaghat, A.; Mohamad, S.; Abdullahi, M.; Ahmad, H.; Derus, N.M.; Khorami, M. A Sustainable Historic Waterfront Revitalization Decision Support Tool for Attracting Tourists. Sustainability 2018, 10, 215. [CrossRef] 
49. Liu, F.; Zhao, Q.; Yang, Y. An approach to assess the value of industrial heritage based on Dempster-Shafer theory. J. Cult. Heritage 2018, 32, 210-220. [CrossRef]

50. Ma, H.; Li, S.; Chan, C.-S. Analytic Hierarchy Process (AHP)-based assessment of the value of non-World Heritage Tulou: A case study of Pinghe County, Fujian Province. Tour. Manag. Perspect. 2018, 26, 67-77. [CrossRef]

51. Ribera, F.; Nesticò, A.; Cucco, P.; Maselli, G. A multicriteria approach to identify the Highest and Best Use for historical buildings. J. Cult. Heritage 2019, in press. [CrossRef]

52. Iliopoulou-Georgudaki, J.; Theodoropoulos, C.; Konstantinopoulos, P.; Georgoudaki, E. Sustainable tourism development including the enhancement of cultural heritage in the city of Nafpaktos-Western Greece. Int. J. Sustain. Dev. World 2017, 24, 224-235. [CrossRef]

53. Guzman, P.; Roders, A.P.; Colenbrander, B. Impacts of Common Urban Development Factors on Cultural Conservation in World Heritage Cities: An Indicators-Based Analysis. Sustainability 2018, 10, 853. [CrossRef]

54. Dastgerdi, A.S.; De Luca, G. The Riddles of Historic Urban Quarters Inscription on the Unesco World Heritage List. Archnet-IJAR: Int. J. Arch. Res. 2018, 12, 152-163. [CrossRef]

55. Ali, A.G.M.; Elsheikha, A.A.A.; Elbanna, E.M.; Peinado, F.J.M. An approach to conservation and management of Farasan Islands' Heritage sites, Saudi Arabia. Int. J. Conserv. Sci. 2018, 9, 245-256.

56. Lak, A.; Gheitasi, M.; Timothy, D.J. Urban regeneration through heritage tourism: Cultural policies and strategic management. J. Tour. Cult. Chang. 2019, in press. [CrossRef]

57. Vinardi, M.G. Scavalcò lo steccato e vide che la natura era tutto un giardino. In Il risveglio del giardino: Dall'hortus al paesaggio, studi, esperienze, confronti; Cornaglia, P., Giusti, M.A., Eds.; Pacini Fazzi: Lucca, Italy, 2015; p. 83.

58. Antoniono, R. Il castello d'Agliè: Una residenza aulica e un territorio. Master's Thesis, Politecnico di Torino, Turin, Italy, 1996; p. 149.

59. Comoli, V.; Vanelli, A. Le residenze sabaude: Dizionario dei personaggi; Roggero, C., Poletto, S., Eds.; Regione Piemonte: Accolade, Torino, 2008; p. 44.

60. Colla, G.B. Tipo regolare del Parco, Castello, Giardini e Valle e limiti in coerenza di codesto Castello spettante a S.A.R. il Sig. Duca del Chiablese, Agliè, lì 25 febbraio 1975, Paper drawing, Archivio Antico, Agliè.

61. Banzato, D.; Canesi, R.; D'Alpaos, C. Biogas and biomethane technologies: An AHP model to support the policy maker in incentive design in Italy. In Smart and Sustainable Planning for Cities and Regions. SSPCR 2017; Green Energy and Technology; Bisello, A., Vettorato, D., Laconte, P., Costa, S., Eds.; Springer: Cham, Switzerland, 2018; pp. 319-331. [CrossRef]

62. D'Alpaos, C.; Bragolusi, P. Multicriteria prioritization of policy instruments in buildings energy retrofit. Valori e Valutazioni 2018, 21, 15-25.

63. D'Alpaos, C.; Bragolusi, P. Prioritization of Energy Retrofit Strategies in Public Housing: An AHP Model. In New Metropolitan Perspectives. ISHT 2018; Smart Innovation, Systems and Technologies, vol 101; Calabrò, F., Della Spina, L., Bevilacqua, C., Eds.; Springer: Cham, Switzerland, 2019; pp. 534-541. [CrossRef]

64. Grošelj, P.; Stirn, L.Z. Acceptable consistency of aggregated comparison matrices in analytic hierarchy process. Eur. J. Oper. Res. 2012, 223, 417-420. [CrossRef]

65. Dong, Q.; Saaty, T.L. An analytic hierarchy process model of group consensus. J. Syst. Sci. Syst. Eng. 2014, 23, 362-374. [CrossRef]

66. Krejčí, J.; Stoklasa, J. Aggregation in the analytic hierarchy process: Why weighted geometric mean should be used instead of weighted arithmetic mean. Expert Syst. Appl. 2018, 114, 97-106. [CrossRef]

67. Della Spina, L. Scenarios for a Sustainable Valorisation of Cultural Landscape as Driver of Local Development In New Metropolitan Perspectives. ISHT 2018; Smart Innovation, Systems and Technologies, vol. 101; Calabrò, F., Della Spina, L., Bevilacqua, C., Eds.; Springer: Cham, Switzerland, 2019; pp. 113-122. [CrossRef]

68. Della Spina, L. Historical Cultural Heritage: Decision Making Process and Reuse Scenarios for the Enhancement of Historic Buildings. Development. In New Metropolitan Perspectives. ISHT 2018; Smart Innovation, Systems and Technologies, vol. 101; Calabrò, F., Della Spina, L., Bevilacqua, C., Eds.; Springer: Cham, Switzerland, 2019; pp. 442-453. [CrossRef]

69. Bottero, M.; Caprioli, C.; Cotella, G.; Santangelo, M. Sustainable Cities: A Reflection on Potentialities and Limits based on Existing Eco-Districts in Europe. Sustainability 2019, 11, 5794. [CrossRef]

(C) 2020 by the authors. Licensee MDPI, Basel, Switzerland. This article is an open access article distributed under the terms and conditions of the Creative Commons Attribution (CC BY) license (http://creativecommons.org/licenses/by/4.0/). 



\title{
Article \\ Tourist Flow Management: Social Impact Evaluation through Social Network Analysis
}

\author{
Giovanna Acampa *, Mariolina Grasso, Giorgia Marino and Claudia Mariaserena Parisi \\ Faculty of Engineering and Architecture, University "Kore" of Enna, 94100 Enna, Italy; \\ mariolina.grasso@unikore.it (M.G.); giorgia.marino@unikorestudent.it (G.M.); claudia.parisi@unikore.it (C.M.P.) \\ * Correspondence: giovanna.acampa@unikore.it; Tel.: +39-335-749-5736
}

Received: 20 December 2019; Accepted: 9 January 2020; Published: 19 January 2020

\begin{abstract}
The present paper was prompted by the activity carried out within the scope of an EU-funded project (WARMEST). It calls to analyse the reasons for the degradation of the Patio de Los Leones, which attracts over 2 million tourists per year to Granada in Spain. We review here the most advanced studies and regulations on the assessment of the social impact of mass tourism and present a novel methodology to analyse its effects. We dug into the material available on social networks-especially feedback to posts published on major relevant sites—and got a comprehensive picture of the thoughts that were expressed there and a comprehensive assessment of the citizens' opinion on the social impact of tourism in Granada. Thus, we obtained a new indicator called "C.1.2 index modified", which measures the level of dissatisfaction of citizens with the tourists' pressure; we propose to replace the existing ETIS index with C.1.2, which is mainly based on direct surveys that are often carried out with very limited resources. At the end of the research, we could point out topics that are especially important to the citizens, thus allowing us to define a strategic action plan with a bottom-up approach.
\end{abstract}

Keywords: tourist flow management; ETIS; carrying capacity; social impact; social network analysis

\section{Introduction}

The paper aims to define a new methodology to measure the attitude of the population living in major destinations of cultural tourism vis-à-vis the increasing number of visitors. This growing flow is strongly affecting residents' everyday lives as well as their attitude towards their cities, especially their city centres. While is it easier to establish the cities' capacity to properly host visitors by means of quantitative data that are often available, it is far more complex to follow the residents' attitude. To date, the main tools required, also at a European level, were direct interviews and questionnaires, which entail considerable time and effort to convey significant information. Our approach is to use social networks instead, which can be analysed-as we did-through proper tools. Such tools, more specifically the one that we selected, can deliver a larger and more representative set of data according to a well-specified procedure that can be repeated, thus enabling us to compare results over time and define a trend. The paper was prompted by the activity carried out within the scope of an EU-funded project (WARMEST) [1]. Its goal is to analyse monuments' degradation due to factors such as climate change and the growing number of tourists, create a model to describe and anticipate the phenomenon, and put in place a user-friendly decision support system that will help in setting up preventive maintenance plans. As part of the project, we had the chance to study in depth the degradation at the Patio de Los Leones, the core of the world-famous site of the Alhambra and Generalife, which attracts over 2 million tourists per year to Granada (whose population is around 232.000 individuals) in Spain, where they are located. 
The United Nations Environment Program (UNEP) and the World Tourism Organization (WTO) defined sustainable tourism as "tourism that takes full account of its current and future economic, social and environmental impacts, addressing the needs of visitors, the industry, the environment and host communities" [2].

The definition of sustainability can be applied to tourism by referring to the following three aspects: environmental, economic, and socio-cultural. The aim is to find a balance between the requirements thus defined, i.e., to make responsible use of environmental resources, respect the socio-cultural traditions of the hosting communities, and ensure long-term economic activities for all stakeholders, including employment opportunities and social services [3].

The World Tourism Organization (UNWTO), in its 2018 annual report, showed that tourists worldwide increased from 25 million in 1950 to 1.3 billion in 2017. This growth trend has advantages and disadvantages. On the one hand, the larger number of tourists contributes significantly to the socio-economic well-being and cultural development of cities. On the other hand, the negative attitude of the local population towards visitors increases due to perceived overcrowding, noise, and other resulting nuisances [4].

In Spain, this issue affects several cities. The citizens there tend to mobilise and publicly express their dissatisfaction; movements based on anti-tourism and tourist-phobia are growing $[5,6]$. Spanish mass media has been putting the topic at the forefront of public debate.

In this context, the present paper reviews the state of the art on the assessment of the social impact of mass tourism and analyses its effects through a novel methodology based on digging into the material published on social networks. The methodology is then applied to a case study, the city of Granada, where, in recent years, the threat posed by mass tourism to the preservation of its values is increasingly perceived.

\section{Literature Review}

Several studies were published regarding the social impact of an uncontrolled flow of tourists. Some of them refer to the definition given by UNWTO to the Tourism Carrying Capacity (TCC), i.e., "the maximum number of people that may visit a tourist destination at the same time without causing destruction of the physical, economic, or socio-cultural environment and an unacceptable decrease in tourist satisfaction" [7]. Various attempts were made to quantitatively assess the TCC, setting a maximum number of tourists, beyond which the destination begins to decay [8]. Yet, as Saveriades (2000) pointed out, there is no universally accepted definition of TCC [9] and no unique model is applicable to asses it for each tourist destination. The methodologies are serving many different purposes and are getting uneven results [10]. The reason for the lack of a unique method is that the approach is strongly influenced by the intrinsic characteristics of the venue taken into consideration.

The UNWTO identifies five types of TCC [11]:

- Ecological capacity: the ability of certain species to withstand disturbance thanks to biological and physical elements.

- Socio-cultural capacity: measured by the intolerable impacts on the local community or limitations due to the availability of human resources.

- Psychological capacity: given by the amount of crowding that tourists can endure without affecting the quality of their experience. This changes according to the types of tourist and the types of activity and their use.

- Infrastructural capacity: it refers to factors such as the number of bedrooms, the capacity of transport systems, or water supply. It can easily vary in the medium to long term.

- Management capacity: it is the number of tourists that can be handled in a city without creating economic and administrative issues.

Regarding socio-cultural capacity, UNWTO refers to the tourist's perception and the quality of experience without mentioning the social impact of tourism on resident citizens. 
Several authors evaluate the experience of tourists and the social impact that the flow of tourists generates on residents by means of survey-based questionnaires [12-14], the processing, administration, and analysis of which is very long.

Additionally, in recent years, the European Commission has been working to achieve the objectives of sustainable tourism development set out in Agenda 2030. In 2013, it introduced the European Tourism Indicators Systems (ETIS) [15]. It is a management, information, and monitoring tool specifically intended for tourism destinations, which supports the activity of collecting and analysing data with the overall goal of assessing the impact of tourism on a destination. The system aims to identify the key problems and determine the best intervention strategies for the management of the tourist flow.

ETIS lists 43 main indicators and other optional ones, dividing them in four categories:

- Destination management

- Economic value

- Social and cultural impact

- Environmental impact

Each of these categories is then divided into criteria and sub criteria. Table 1 is an extract of the ETIS indicators relating to the criteria required to assess the social and cultural impact of tourism.

Table 1. European Tourism Indicators Systems (ETIS) core indicators [15].

\begin{tabular}{ccc}
\hline & \multicolumn{2}{c}{ Social and Cultural Impact } \\
\hline \multirow{2}{*}{ Criteria } & $\begin{array}{c}\text { Indicator } \\
\text { Reference }\end{array}$ & ETIS Core Indicators \\
\cline { 2 - 3 } C.1 Community/social & C.1.1 & Number of tourists/visitors per 100 residents \\
\cline { 2 - 3 } impact & C.1.2 & $\begin{array}{c}\text { Percentage of residents who are satisfied with } \\
\text { tourism in the destination (per month/season) }\end{array}$ \\
\cline { 2 - 3 } & C.1.3 & $\begin{array}{c}\text { Number of beds available in commercial } \\
\text { accommodation establishments per 100 residents }\end{array}$ \\
\cline { 2 - 3 } & C.1.4 & Number of second homes per 100 homes \\
\hline
\end{tabular}

The first, third and fourth indexes are easy to compute because they require easily available data (the number of tourists, the number of beds in accommodations, and the number of second homes). The second index is more complex because the percentage of residents who are satisfied with tourism in the destination is not an easily measurable value.

For each indicator, the European Commission defines: the reason for measurement, the data requirements, the method of calculation, the frequency of data collection, the reporting format, and the suggestion of actions (regarding indicator C.1.2, see Table 2). When the data to quantitatively measure ETIS indicators is missing, the European Commission suggests using questionnaires as helping tools. The European Commission has also prepared a predefined questionnaire for interviewing a group of people (on average 15-20 individuals) living in the tourist destination and working in the tourism sector. 
Table 2. Data sheets for core indicators [16].

\begin{tabular}{cl}
\hline Indicator: C.1.2 & $\begin{array}{l}\text { Percentage of Residents Who are Satisfied with Tourism in } \\
\text { the Destination (Per Month/Season) }\end{array}$ \\
\hline Reason for Measuring & $\begin{array}{l}\text { Checking on resident satisfaction on a regular basis is an } \\
\text { important gauge for visitor experience. Being alert to changes in } \\
\text { the levels of resident satisfaction and taking action where } \\
\text { necessary is fundamental to sustainable tourism. }\end{array}$ \\
\hline Data Requirements & Resident surveys and tourism studies during peak season \\
\hline Units of Measurement & $\%$ \\
\hline Terms in Glossary & Resident satisfaction \\
\hline Data Collection Instructions & Resident Survey \\
\hline Method of Calculation & $\begin{array}{l}\text { Number of residents who responded "satisfied" or better } \div \text { total } \\
\text { number of residents who responded } \times 100=\% \text { of residents } \\
\text { satisfied with tourism } \\
\text { Repeat for each month/season/year }\end{array}$ \\
\hline Frequency of Data Collection & $\begin{array}{l}\text { Monthly } \\
\text { Seasonally } \\
\text { Annually }\end{array}$ \\
\hline Reporting Format & Bar graph \\
\hline Suggested Actions & N/A \\
\hline & Destination Managers \\
\hline Shational Benchmarks & $\begin{array}{l}\text { Should the results be less than an agreed percentage, destination } \\
\text { to determine how to better plan tourism development and } \\
\text { activities. }\end{array}$ \\
\hline &
\end{tabular}

Some researchers also relied on questionnaires as a survey method to assess the evaluation of the social and cultural impact of tourism. Tudorache et al. (2017) [17] and Modica et al. (2018) [18] used ETIS indicators to assess social impact in different environments and both concluded that their new indicators should be added to ETIS in order to analyse aspects that are difficult to compute.

Despite the widespread use of questionnaires as a method of data collection, many authors define it as an obsolete method $[19,20]$ and other ones defines it as a "static method" [21] that does not take into account the speed in which opinions and preferences change. Moreover, high-quality data requires high costs and a lot of time investment due to the needed number and time of the following groups: researchers, who design the surveys; interviewers, who collect the data; and respondents, who voluntarily provide answers. Moreover, the people interviewed often give superficial answers due to their lack of interest.

Our research proposes an alternative method to collect data and evaluate the C.1.2 indicator, which defines "the percentage of residents who are satisfied with tourism in the destination (per month/season)". We apply a method of social network analysis to assess the citizens' opinion about the social impact of tourist flows in Granada using feedbacks to posts.

\section{Materials and Methods}

\subsection{Social Network Analysis}

The analysis of the information provided by social networks can be useful to understand public opinion regarding the effects of mass tourism. It can also help to track changes in mass tourism continuously and in real time, while questionnaires can be used to deliver random surveys.

The advantages are:

- People's comments and reactions highlight issues of social relevance; 
- Free access to social network archives allows people to explore all published posts;

- The level of interest in a topic can be assessed by counting the number of people sharing, appreciating, or commenting on the posts related to that topic;

- The discussion's content can be analysed by classifying it as a positive or negative a message published on the web. This process is called sentiment analysis.

\subsection{Methodology}

Recently, the use of social networks (Facebook, Instagram, Twitter) has become obvious, allowing us to share thoughts or multimedia content and to create a wide interaction among users.

In this paper we propose a new approach to assess the local populations' degree of dissatisfaction with tourism flow through the analysis of the social network content.

The proposed procedure is divided into 8 steps:

1. Definition of the topic: the thorough and precise definition of the subject under investigation is key to clearly set the goal of the analysis.

2. Selection of the sources: once the investigation subject and the goal are defined, the available media (online newspaper, journals, blogs, etc.) must be listed, searched, and analysed. The result is the identification of the groups and associations discussing the relevant issues related to the city of the selected media. Neighbourhood associations, created to defend citizens, are especially relevant to our research topic are. These associations usually communicate and share news through social network pages, and on these pages, citizens share new ideas and deal with emerging problems.

3. Analysis of the social network pages: the analysis of the groups or associations on social pages allows us not only to evaluate the citizens' opinion about certain events but also to collect useful data for the investigation.

4. Definition of the keywords for post selection: key word selection is pivotal to pick up the most significant information related to the investigated topic. Within Facebook, it is possible to extract all posts containing a given keyword.

5. Selection of the social network analytical tool: social networks allow us to have an overview of the page's performance, but these data are confidential and can only be extracted by page administrators. Therefore, it is necessary to find other tools useful for defining the key metrics and to extract data from social networks. Several are available online.

6. Analysis of the key metrics: social network activity is measured through KPIs (Key Performance Indicators) that provide accurate information on the page performance of interest. Some helpful KPIs are:

- Activity metrics: KPIs measuring the page activity level (such as the number of posts, temporal frequency, and the recency of posting activity)

- Interaction metrics: KPIs measuring the level of interaction between posts and users (the number of comments, reactions, and shares).

7. Collection of reactions to the selected posts: the analysis of the feelings and thoughts of the citizens involved in an event is important to understand its effects in terms of social impact.

8. Dissatisfaction index calculation: in the last phase, the reactions collected over the selected period of time are quantified and a dissatisfaction index in relation to mass tourism is computed. The procedure required to achieve this, is divided into the following steps:

a. Within the selected social networks, the "angry" reactions related to tourist protests (A) should be picked and separated from the rest (the total number of posts with "angry" reactions) (B);

b. Calculate the functions $A=f(x)$ and $B=z(x)$ of reactions $\mathrm{A}$ and B over time (days/months) for each year $(n=a, a+1, \ldots, N)$; 
c. Calculate the integrals $T A_{a} e T A_{b}$ of the functions $f(x)$ and $z(x)$ for each $N$ year;

$$
\begin{aligned}
& \mathrm{TA} A_{a}=\int_{a}^{a+1} f(x) d x \\
& T A_{b}=\int_{a}^{a+1} z(x) d x
\end{aligned}
$$

d. Analyse the performance of $T A_{a}$ and $T A_{b}$ over time;

e. $\quad$ Replace the ETIS index C.1.2. (Percentage of residents who are satisfied with tourism in the destination (per month/season)) with the "C.1.2 index modified", which is the ratio between the "angry" reactions related to tourism flow and the total "angry" reactions in the period of interest (per month/season).

$$
I_{\text {ins }}=\frac{T A_{a}}{T A_{a R}}
$$

Thus, we can compute the dissatisfaction "C.1.2 index modified" without using surveys; we use the followers' reaction to obtain a quantitative value of the level of disaffection of local citizens vis-à-vis mass tourism in their cities. This value reflects the citizens' real opinions and can be analysed and compared over time.

\section{Results}

\subsection{Case Study-Granada}

The case study on which we have focused, as part of the research effort carried out within the European financed project H2020/MSCA/RISE2017/Warmest, is the city of Granada in Spain, which is among the most visited Spanish cities due to the Alhambra [22]. This monumental complex is located on a rocky hill on the banks of the River Darro. In the XIII century, the Nasrid kingdom was established and the first palace was built by the founder King Mohammed ibn Yusuf ben Nasr. In the XIII, XIV, and $\mathrm{XV}$ centuries, the fortress consisted of two main areas: the military area (Alcazaba) and the medina where there are the famous Nasrid Palaces and the remains of the houses of noblemen and plebeians. In the 1492, Catholic kings took the Alhambra, and later on the emperor Carlos V built a palace there. The complex also consists of the Generalife, a palace surrounded by orchards and gardens [23].

The monumental complex of the Alhambra and the Generalife has been a UNESCO World Heritage Site since 1984 and is Granada's main tourist destination, although not the only one. As history shows, the Alhambra has always been the core of the city of Granada. It was first a military and commercial resource, and today it is an economic resource for the large number of tourists it attracts. Figure 1 shows the annual trend of tourists visiting the Alhambra and Generalife. From 1991 to 2018 their number increased to almost 3 million.

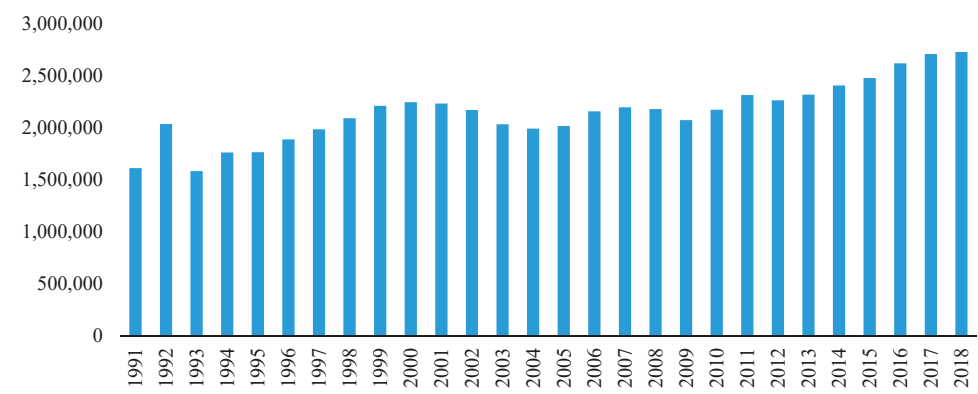

Figure 1. Annual tourists at the Alhambra and Generalife [1]. 
On the other hand, Figure 2 shows the average monthly distribution of visitors from 1991 to 2018. In terms of tourist flow, years may be divided into three different seasons: a low, a medium, and a high one. The low season includes January, February, November, and December (22\% of the annual flow). The medium season includes March, June, and July (27\% of the annual flow). The high season includes April, May, August, September, and October (51\% of the annual flow).

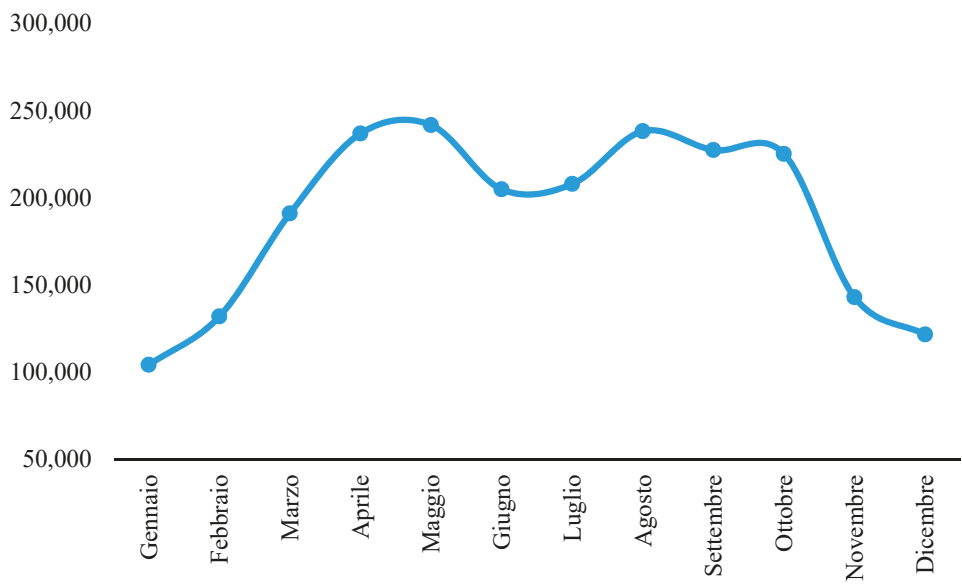

Figure 2. Average monthly trend of tourists (2000 to 2018) [24].

According to official data, the resident population in Granada is 232,000 inhabitants [25] and tourists are more than 4.5 million [22]. The latest annual Exceltur report (Alliance for Excellency in Tourism) shows how tourist pressure in Granada is the highest of the 15 most visited destinations in Spain [26]. The report states that "the main factor determining the increase in tourist pressure in Spanish cities is the transformation of housing into tourist accommodation. In fact, in cities where there has been a greater social response to tourism are those with a significant percentage of tourist accommodation".

In Granada, 55\% of tourists choose to stay in apartments found through peer-to-peer hosting, such as Airbnb. The remaining 45\% still choose "traditional" accommodation in hotels and hostels.

Homesharing platforms have become very powerful tools for the development, increase in incomes, and the promotion of tourist destinations; at the same time, they have brought significant disadvantages when used in an unregulated manner. As in many cities in Europe, in Granada citizens move from the old town to the so-called new towns [27], pushed by the increase in nightlife, restaurants, souvenir shops, and city traffic. In addition, there is a growing trend in flat owners to invest in their apartments and transform them into tourist accommodation to receive a profit from them. The result of the rise in the number of apartments for homesharing is an increase in the price of rents, which tends to keep the local population even further away.

One of the most famous neighbourhoods of Granada is the Albayzin, located on a hill opposite the Alhambra and separated from it by the Darro River. It is characterized by narrow winding streets built during its Medieval Moorish period dating back to the Nasrid Kingdom of Granada. It was declared a World Heritage Site in 1984, along with the Alhambra. The socio-economic activities of the Albayzin district consist of typical North African shops with restaurants and small shops selling Moroccan goods (carpets, fabrics, jewellery, and coloured lamps). In recent years, most of the apartments in the neighbourhood have been converted into tourist apartments, losing their original social characteristics.

Nevertheless, Granada should not be considered as a case by itself but rather a good, though not extreme, case study of a tourist pressed city. In Italy, this phenomenon is even more serious in various venues. In Florence and Venice, the ratio among tourists and residents is even higher, at 26 and 45 , respectively, which means that for each resident there are 26 tourists in Florence and 45 tourists in 
Venice [28-30]. Historical cities live a seemingly unstoppable process of "Disneyfication" [31]. The New York Times also defined Venice as "Disney on the sea" [5] because it is invaded by tourists from all over the world attracted by a venue that today is crushed by noise and landscape pollution.

Generally speaking, historic centres are undergoing a transformation process and are increasingly adapted to the needs of tourists and decreasingly to the citizens' ones. Therefore, the risks are the loss of identity and the progressive abandonment of the historical centre [32].

\subsection{The Proposed Methodology Applied to the Case of Granada}

The methodology described above was applied, point by point, on the case study of Granada.

1. Definition of the topic: the subject selected for the research was the attitude of the residents towards the growing tourist flow in Granada, especially in the light of the outbursts of tourism-phobia that affected the city in recent times. To do this, we analysed the negative or positive impacts of tourists on Granada as perceived by the citizens.

2. Selection of the sources: local newspapers frequently report news on citizens' complaints about mass tourism. Among these citizens, the majority live in the Albayzin, a historical district of Granada. The most striking protest event reported by local newspapers took place in May 2019. The walls of the main Albayzin streets were covered by graffiti "@tourist: go away" or "@tourist: flamenco is not a show" [24]. A few months later (August 2019) a group of citizens complained about the tourists' lack of respect for the neighbourhood (or district) and its inhabitants. [25]. Several news reports also concern the inconvenience and damage caused by tourist buses that run along the typical streets of the neighbourhood [26].

We carried out a web investigation on social media that collects the citizens' opinion on this matter. We identified two associations spreading information on the topic through their blogs and social platform (Facebook e Twitter). The first one, "Bajo Albayzin", is a neighbourhood association founded in January 2000. It aims to improve the cultural, historical, social, urban, and environmental values of the Albayzin district. The second association, "SOS Alhambra", was founded in July 2015 following a citizen's protest against an architectural design involving a major transformation of the "atrium" area of the Alhambra, in front of the Carlos V palace. The aim of the organization is to warn about the possible environmental damage that the project could cause to the site, which is one of the UNESCO World Heritage Sites and it also generally provides information on the main problems affecting the monumental complex.

3. Analysis of the social network pages: To date, among the "Bajo Albayzin" communication platforms (blogs, Facebook, and Twitter), the Facebook page (2000 followers) is the most active in terms of published posts and reactions/comments to them. Although the Twitter page collects a thousand more followers than Facebook, it has not been active since August 2019. As far as the blog is concerned, it turned out to only be a data archive. There is no interaction (comments or shares) to the published posts there. The Facebook page of "SOS Alhambra" (1700 followers) is the most active platform compared to the blog and the Twitter page that counts only 450 followers. Thus, we chose to focus our attention on analysing data extracted from these Facebook pages.

4. Definition of key words for post selection: the data investigation was carried out through the following selected keywords: "protestas", "turistas", "turismo", "turismo masivo", "Alhambra" (Figure 3). Since 2016 to date, the followers of the "Bajo Albayzin" and "Sos Alhambra" pages published 370 posts with negative tourism reactions to the tourist flow. 


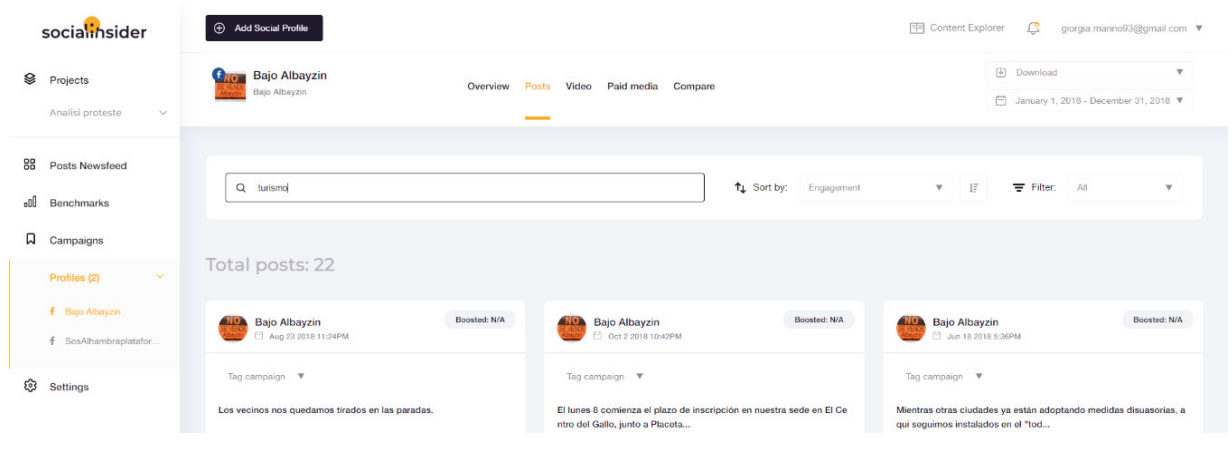

Figure 3. Search keywords—screen application from Social Insider.

We decided to focus on "Bajo Albayzin" because it hosts more articles regarding the citizens' protests than "Sos Alhambra", which appears to prefer political and administrative topics. The "Bajo Albayzin" association is in a direct relationship with the residents of the district and their problems, being themselves active in the neighbourhood. Moreover, Figure 4 shows that the engagement trend in "Bajo Albayzin" is higher than in "Sos Alhambra" in terms of shares, comments, publications, and reactions.

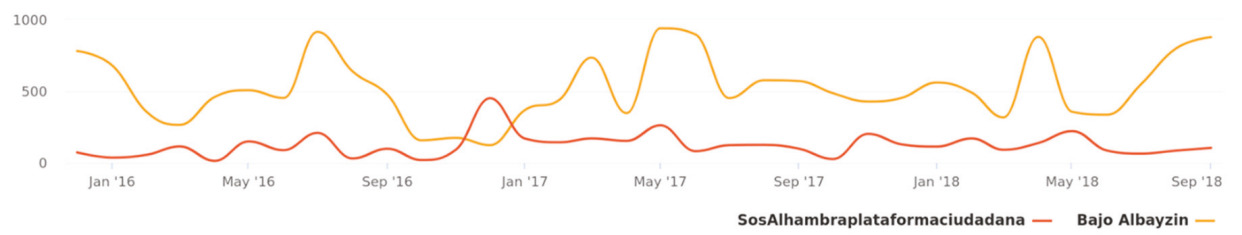

Figure 4. See the distribution of total engagement for each page-report Social Insider.

5. Selection of the social network analytical tools: Data collection was carried out through the platform called Social Insider. The co-operation between Kore University of Enna and the company allowed us to use freely the Pro version (generally it is charged). This tool is used to provide information for marketing companies in order to create more competitive benchmarks. We used it to analyse the two Facebook pages: "Bajo Albayzin" and "SoS Alhambra". Social Insider offers advanced analysis functions for social networks, including:

- $\quad$ saving data for unlimited time;

- identification of the most performing posts;

- multi-page comparisons;

- analysis of own posts and those of competitors;

- extraction of reports in CSV, PDF, PPT format.

The tool can create charts automatically, providing information about the posts and the distribution of shares and reactions to posts over time.

6. Analysis of the key metrics: we analysed the key metrics of posts, fans, fan growth, and engagement from 2015 to date; as we can see (Figure 5) the fan growth remained unchanged, while the page remains active for posts publication. 


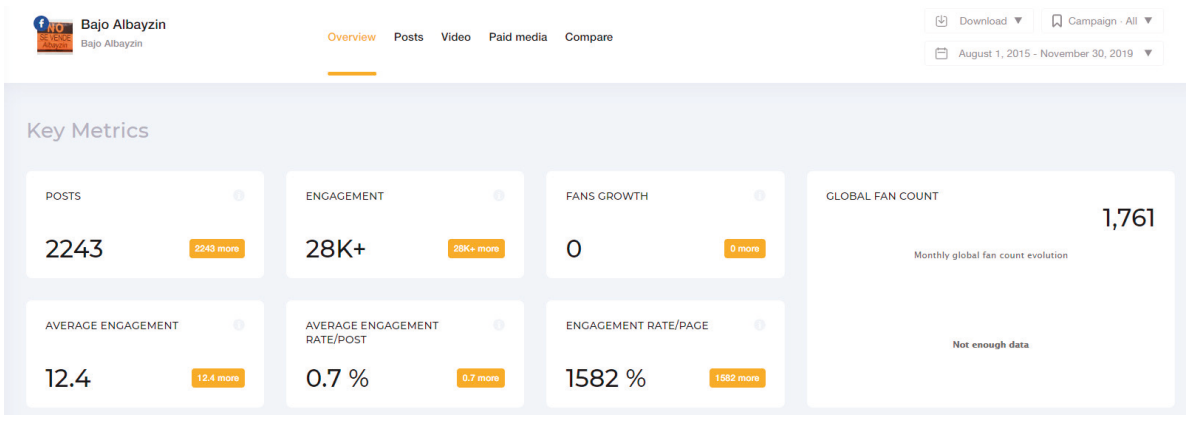

Figure 5. Key Metrics "Bajo Albayzin"—report from Social Insider.

In particular, owing to the machine learning tool of the Social Insider, we were able to trace the distribution of the various posts-links, photos, videos, events, albums-in the time frame of interest.

The orange histogram bars, compared to the much shorter azure and blue, suggest which type of post is preferred: links are published significantly more often than other multimedia content (Figure 6).

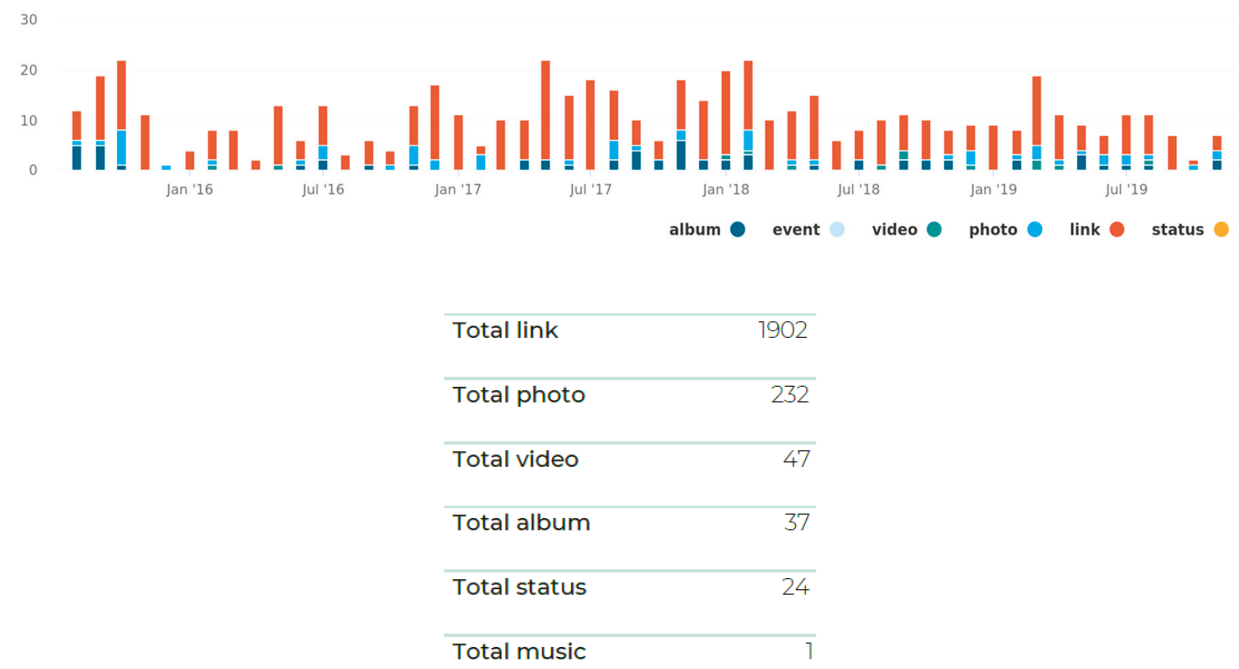

Figure 6. Total post by type "Bajo Albayzin"—-report from Social Insider.

The association, in fact, uses Facebook as a vehicle to spread information. Any new content, already made known by blogs or newspaper articles, is re-shared on the page to increase its circulation rate.

7. Collection of reactions to selected posts. The analysis of reactions was carried out through Facebook Reactions (Figure 7).

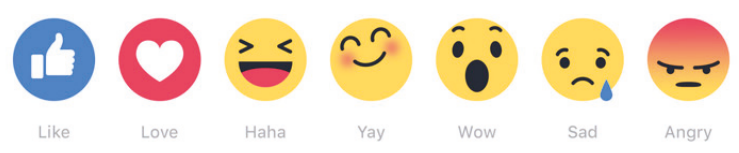

Figure 7. Reactions from Facebook. 
Social Insider automatically generates a graph that expresses the reactions trend excluding like reactions. Figure 8 shows that the peaks of the function "angry" (in blue) increase over the years. Instead, the others (ahah, sad, love, wow) remain stable over time. The "angry" reaction curve refers to several topics and not only to those related to tourism protests.

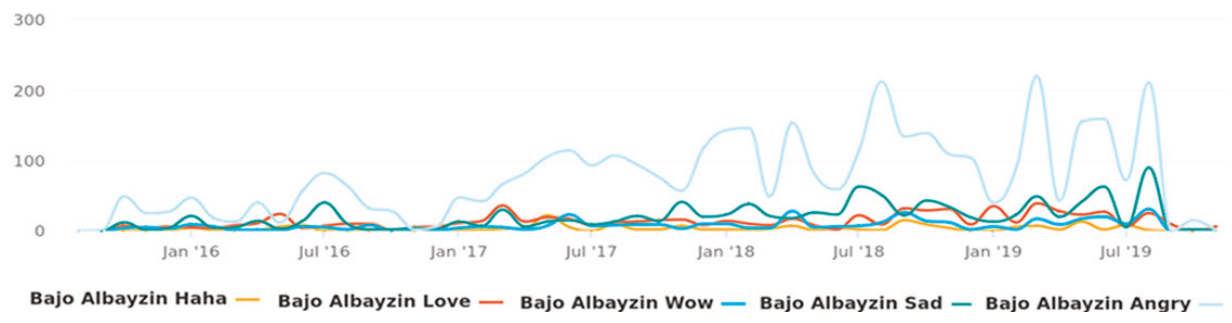

Figure 8. "Angry" reaction curve trend on "Bajo Albayzin" page.

8. Dissatisfaction Index Calculation:

a. For each month of each year taken into account (2016, 2017, 2018, 2019), from the total number of posts with "angry" reactions (B), we selected the "angry" reaction number referring only to posts related to tourism (A). In the four years analysed, the number of (A) is 1449 and the number of (B) is 4008 .

b. The distribution of "angry" reactions relating to tourism over time defines the function $A=$ $f(x)$, while the distribution of total "angry" reactions over time defines the function $B=z(x)$. The latter can also be obtained from Social Insider (Figure 8). Figure 9 (a-b-c-d) shows the trend of both functions over time.
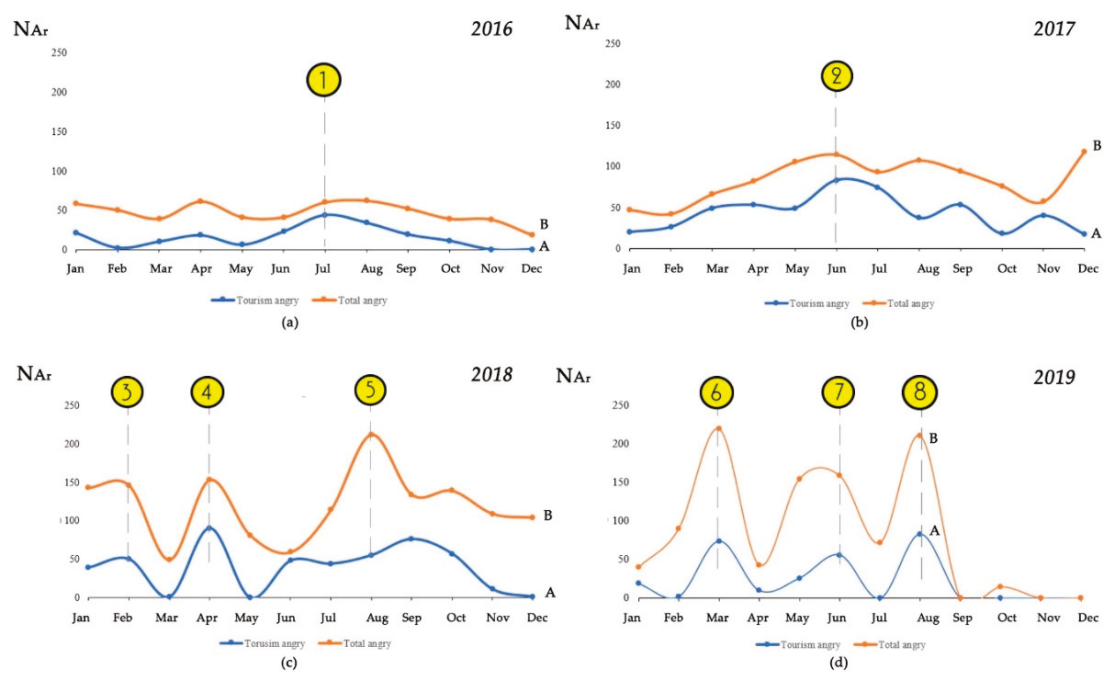

Figure 9. Comparison between $A=f(x)$ and $B=z(x)$.

c. In order to compute the dissatisfaction index, we calculated the integrals of both functions $A=f(x)$ and $B=z(x)$. For example, the integral referred to in 2016 is:

$$
T A_{a}=\int_{2016}^{2017} A d x
$$




$$
T A_{b}=\int_{2016}^{2017} B d x
$$

d. Figure 10 shows the areas under the A curve for each year. The "angry" reactions have a peak in conjunction with the triggering events. As we can see, the highest peak is in 2017. Instead, in 2019 there is a lower value probably due to the citizens' acquiescence to the flow of tourists in Granada.

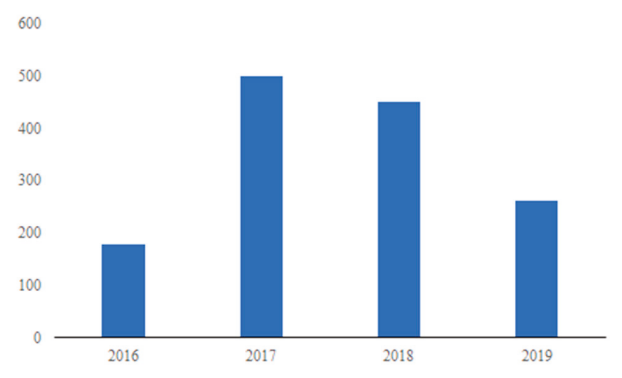

Figure 10. "Angry" reactions trend over time.

e. The "C.1.2 Index modified", for example, for the year 2017, is as follows:

$$
I_{\text {diss }}=\frac{T A_{a}}{T A_{a R}}=\frac{\int_{2016}^{2017} A d x}{\int_{2016}^{2017} B d x}
$$

The ratio $I_{\text {diss }}$ defines the incidence of citizens' dissatisfaction with the flow of tourism in relation to the totality of the topics on the page. The higher percentage is in 2016 (Table 3).

Table 3. Comparison among dissatisfaction indexes over four years.

\begin{tabular}{ccccc}
\hline Year & $\mathbf{2 0 1 6}$ & $\mathbf{2 0 1 7}$ & $\mathbf{2 0 1 8}$ & $\mathbf{2 0 1 9}$ \\
\hline $\boldsymbol{I}_{\text {diss }}$ & $34 \%$ & $54 \%$ & $34 \%$ & $26 \%$ \\
\hline
\end{tabular}

We analysed the posts related to mass tourism for each peak of curve (A) and found that they are related to the following main events:

1. A high risk of damage to historical heritage. The huge flow of tourists is considered to be a cause of damage to the historical heritage [33].

2. Little trains in a historic city. Residents are against little trains because they are held to be not appropriate for the Granada's territorial morphology [34-36].

3. The illegal occupation of parking. The violations of traffic road regulations are frequent. There many vehicles that park on sidewalks or in reserved parking lots. "Bajo Albaycin" said "Unos llegan en furgonetas, otros en caravanas, todos invadiendo y saturando el espacio público con sus vehículos para vivir con vistas a la Alhambra" ("Many tourists come by camper and roulette invading the public roads with their vehicles in order to visit the Alhambra") [37,38].

4. The invasion of public spaces. Souvenir shops and street vendors occupy main streets and walls of buildings from Elvira Street and Grifos de San José to Plaza Nueva [39].

5. The demolition of a kiosk in "Plaza Nueva". The Authorities ordered the demolition of a kiosk where there was a bakery. The residents claimed that this decision was affecting small local businesses [40]. 
6. Lack of apartments for residents. Granada's historic centre is increasingly left by local citizen. Residents move from the old city to suburbs and rent their apartment to tourists. The Realejo and Albaycín neighbourhoods are becoming large "low-cost hotels". The rise in the number of apartments meant that homesharing has increased, which in turn has increased the price of the rents that boosted this trend in the first place $[2,41]$.

7. The demolition of a building in "Plaza de Santa Ana". The building was located in the A2 area, where buildings with significant architectural, historical, and cultural characteristics are located. The demolition rose the debate on heritage conservation. The residents wanted to "preserve its [Granada's] architectural elements and qualities" [42,43].

8. The residents' rebellion against tourists. Graffiti against tourists was written on street walls in Albayzin. They were signed by "@tourist". They called visitors to leave the city, accusing them of destroying it. The association "Bajo Albayzin" agreed with the graffiti's content but did not agree with the manner in which the citizens protested [44,45].

9. Undisciplined tourists. The increase in nightlife, music, and parties are among the major causes of citizen dissatisfaction. Residents understand that tourism is an economic resource for Granada but would like tourists to be more sympathetic and respectful [46].

\section{Conclusions and Strategic Indications}

The continuous growth of the tourists' pressure on historical city centres causes an increase in dissatisfaction among local residents. We applied a method based on the analysis of the reactions posted on the social networks by cities' citizens to assess their opinion on the social impact of mass tourism on Granada. Thus, we replace the ETIS index C.1.2 with a new indicator called "C.1.2 index modified" to define the level of dissatisfaction among citizens experiencing the tourist pressure.

From this analysis, we can also outline a strategic action plan aimed at countering the negative outcomes of mass tourism through a bottom-up approach.

We suggest that the Granada authorities makes use of the following six intervention strategies (Figure 11):

- Cultural heritage management

- Sustainable mobility

- Urban planning

- The promotion of local businesses

- The promotion of hotels rather than the rent of private flats

- Taking care of public orders and standards of behaviour

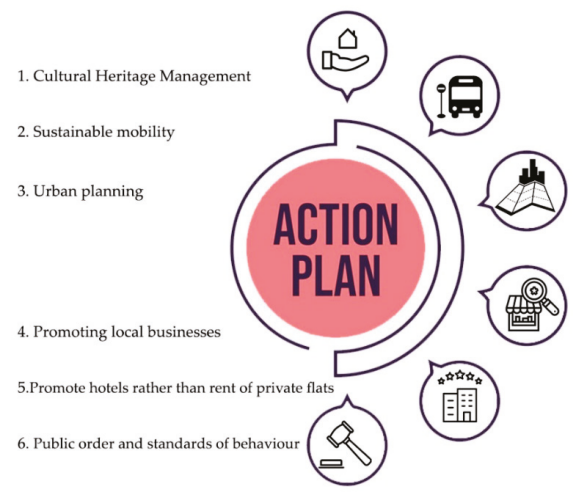

Figure 11. Proposal of Action Plan. 
As next step, a possible development of the present research could be to create a hierarchy among the proposals arising from the citizens' reactions.

According the Granada Tourist Plan 2018-2022 (Plan Turístico de Grandes Ciudades de Granada) [47] there are six objectives to achieve through strategic guidelines:

- Enhancing tourism resources from a cultural, social, environmental, and economic point of view;

- Adapting the urban environment to tourism by promoting universal accessibility;

- Increasing the quality of the city's tourist services;

- Improving the existing tourism products and creating new products based on the innovative exploitation of resources;

- Raising awareness and involving the population and local agents in a culture of quality tourism;

- Strengthening the competitiveness of the local tourism sector.

When comparing the action plan adopted by the Municipality of Granada with what we propose in this paper, it becomes clear that there are some common points regarding urban planning and the promotion of local business.

Author Contributions: Conceptualization, G.A.; methodology, G.A.; investigation, M.G., G.M. and C.M.P.; data analysis, M.G., G.M. and C.M.P.; writing-original draft preparation M.G., G.M. and C.M.P.; writing-review and editing, G.A.; supervision, G.A. All authors have read and agreed to the published version of the manuscript.

Funding: This research was funded by H2020 Marie Skłodowska-Curie Actions, grant number 777981 and The APC was funded by Kore University of Enna.

Conflicts of Interest: The authors declare no conflict of interest

\section{References}

1. WARMEST Project. WARMEST EU PROJECT_Low Altitude Remote Sensing for the Monitoring of the State of Cultural Heritage Sites: Building an Integrated Model for Maintenance, 2017. Available online: http://warmestproject.eu/ (accessed on 5 December 2019).

2. UNWTO. Sustainable development | UNWTO. Available online: https://www.unwto.org/sustainabledevelopment (accessed on 10 December 2019).

3. Why Tourism? | World Tourism Organization UNWTO. Available online: http://www2.unwto.org/content/ why-tourism (accessed on 22 November 2019).

4. World Tourism Organization. 'Overtourism'?-Understanding and Managing Urban Tourism Growth beyond Perceptions, Executive Summary; WTO, Centre of Expertise Leisure, Tourism \& Hospitality, NHTV Breda University of Applied Science, NHL Stenden University of Applied Sciences, Eds.; World Tourism Organization (UNWTO): Madrid, Spain, 2018.

5. Coldwell, W. First Venice and Barcelona: Now Anti-Tourism Marches Spread across Europe, 2017. Available online: https:/static1.squarespace.com/static/53109b11e4b05040160f0a8f/t/59bfcc8b90badeccf7cc1564/ 1505741995422/First+Venice+and+Barcelona_+now+anti-tourism+marches+spread+across+Europe+_+ Travel+_+The+Guardian.pdf (accessed on 10 January 2020).

6. Milano, C.; Novelli, M.; Cheer, J.M. Overtourism and Tourismphobia: A Journey Through Four Decades of Tourism Development, Planning and Local Concerns. Tour. Plan. Dev. 2019, 16, 353-357.

7. Lück, M. Sustainable Development of Tourism: A Compilation of Good Practices: Una Compilación de Buenas Prácticas: Une Compilation de Bonnes Pratiques = Desarrollo Sostenible del Turismo = Développement Durable du Tourisme; World Tourism Organization: Madrid, Spain, 2000.

8. Brown, K.; Turner, R.K.; Hameed, H.; Bateman, I. Environmental carrying capacity and tourism development in the Maldives and Nepal. Environ. Conserv. 1997, 24, 316-325. [CrossRef]

9. Saveriades, A. Establishing the social tourism carrying capacity for the tourist resorts of the east coast of the Republic of Cyprus. Tour. Manag. 2000, 21, 147-156. [CrossRef]

10. Cimnaghi, E.; Mondini, G.; Valle, M. La Capacità di Carico Turistica. Uno Strumento per la Gestione del Patrimonio Culturale; Ministero dei Beni e delle Attività Culturali e del Turismo: Rome, Italy, 2017.

11. WTO; UNEP. Making Tourism more Sustainable: A Guide for Policy Makers; United Nations Environment Programme: Nairobi, Kenya, 2005. 
12. Gonzalez, V.; Coromina, L.; Galí, N. Overtourism: Residents' perceptions of tourism impact as an indicator of resident social carrying capacity-Case study of a Spanish heritage town. Tour. Rev. 2018, 73, 277-296. [CrossRef]

13. Gonson, C.; Pelletier, D.; Alban, F. Social carrying capacity assessment from questionnaire and counts survey: Insights for recreational settings management in coastal areas. Mar. Policy 2018, 98, 146-157. [CrossRef]

14. Joshi, S.; Dahal, R. Relationship between Social Carrying Capacity and Tourism Carrying Capacity: A Case of Annapurna Conservation Area, Nepal. J. Tour. Hosp. Educ. 2019, 9, 9-29. [CrossRef]

15. European Commission. European Tourism Indicators System for Sustainable Destination Management | Internal Market, Industry, Entrepreneurship and SMEs, 2016. Available online: https://ec.europa.eu/growth/ sectors/tourism/offer/sustainable/indicators_en (accessed on 2 December 2019).

16. European Commission. DocsRoom-European Commission, 2016. Available online: https://ec.europa.eu/ docsroom/documents/15849 (accessed on 14 December 2019).

17. Tudorache, D.; Simon, T.; Frenț, C.; Musteaţă-Pavel, M. Difficulties and Challenges in Applying the European Tourism Indicators System (ETIS) for Sustainable Tourist Destinations: The Case of Braşov County in the Romanian Carpathians. Sustainability 2017, 9, 1879. [CrossRef]

18. Modica, P.; Capocchi, A.; Foroni, I.; Zenga, M. An Assessment of the Implementation of the European Tourism Indicator System for Sustainable Destinations in Italy. Sustainability 2018, 10, 3160. [CrossRef]

19. Savage, M.; Burrows, R. The Coming Crisis of Empirical Sociology. Sociology 2007, 41, 885-899. [CrossRef]

20. Mayer-Schönberger, V.; Cukier, K. Big Data: A Revolution That Will Transform. How We Live, Work, and Think; Houghton Mifflin Harcourt: New York, NY, USA, 2013.

21. Fedrigo, F. Le Potenzialità Dell'analisi Dell'utilizzo dei Social Network a Fini di Marketing Caso Studio Sulle Sigarette Elettroniche; Tesi di Laurea Magistrale, Università Ca'Foscari Venezia: Venezia, Italy, 2013.

22. Junta de Andalucía. Instituto de Estadística y Cartografía de Andalucía, 2019. Available online: https: //www.juntadeandalucia.es/institutodeestadisticaycartografia (accessed on 11 December 2019).

23. Alhambra.org. La Alhambra de Granada—alhambra.org. Available online: https://www.alhambra.org/ alhambra/alhambra-diaria.html (accessed on 22 November 2019).

24. Junta de Andalucía. Junta de Andalucía—Estadística de la Red de Espacios Culturales de Andalucía; Consejeria de Cultura u Patrimonio Historico: Seville, Spain, 2019; Available online: https://www.juntadeandalucia.es/organismos/ culturaypatrimoniohistorico/servicios/estadistica-cartografia/actividad/detalle/175086/175547.html (accessed on 22 November 2019).

25. Eurostat. Statistics Illustrated-Eurostat. 2018. Available online: https://ec.europa.eu/eurostat/en/web/ population-demography-migration-projections/statistics-illustrated (accessed on 11 December 2019).

26. Exceltur $\mid$ Alliance for Excellency in Tourism. Perspectivas Turisticas $n^{\circ} 67$. Valoracion Turistica Empresarial de 2018 y Perspectivas para 2019. Available online: https://www.exceltur.org/wp-content/uploads/2019/ 01/Informe-Perspectivas-N67-Balance-del-a\%C3\%B1o-2018-y-previsiones-para-2019.pdf (accessed on 10 January 2020).

27. Montanari, T. Le Pietre e il Popolo: Restituire ai Cittadini L'arte e la Storia delle Città Italiane; I Edizione; Minimum Fax: Roma, Italy, 2013.

28. Becheri, E.; Micera, R.; Morvillo, A. Rapporto sul Turismo Italiano; XXII Edizione 2017/2018; Rogiosi: Napoli, Italy, 2019.

29. Romano, I.M. Pressione Turistica sul Centro Storico di Firenze-sito UNESCO: Un Modello per la Valutazione Dell'impatto Percettivo; Firenze University Press: Florence, Italy, 2018.

30. Zannini, A. Il turismo a Venezia dal secondo dopoguerra ad oggi. Lab. Ital. Polit. Société 2014, 15, 191-199. [CrossRef]

31. Pollice, F. Valorizzazione dei Centri Storici e Turismo Sostenibile nel Bacino del Mediterraneo. Boll. Della Soc. Geogr. Ital. 2018, 41-56.

32. Acampa, G.; Mattia, S. Marginal Opportunities: The Old Town Center in Palermo. In Integrated Evaluation for the Management of Contemporary Cities; Mondini, G., Fattinnanzi, E., Oppio, A., Bottero, M., Eds.; Springer International Publishing: Cham, Switzerland, 2018; pp. 441-451.

33. Lucas, M.A.O. El nuevo director de la Alhambra: El turismo tiene también un límite que, si se rebasa, empieza a ser depredador. eldiario.es. 3 October 2015. Available online: https://www.eldiario.es/andalucia/ nuevo-director-Alhambra-gestion-hacer_0_435707492.html (accessed on 19 December 2019). 
34. El Turismo Colapsa los Autobuses del Barrio, que se han Visto Reducidos en Favor de la Línea Turística, y Desespera a los Vecinos, Bajo Albayzín. Available online: http://albayzin.info/2018/08/el-turismo-colapsalos-autobuses-del-barrio-que-se-han-visto-reducidos-en-favor-de-la-linea-turistica-y-desespera-a-losvecinos/ (accessed on 11 December 2019).

35. Bajo Albayzín. Atasco en San Isabel la Real a causa de un autobús turístico cuyas dimensiones le impiden seguir a San Miguel Bajo. Bajo Albayzín. 21 April 2018. Available online: http://albayzin.info/2018/04/atasco-en-san-isabella-real-a-causa-de-un-autobus-turistico-cuyas-dimensiones-le-impiden-seguir-a-san-miguel-bajo/ (accessed on 19 December 2019).

36. Bajo Albayzín. Peligroso e ilegal aparcamiento de autobuses en la Cuesta del Chapiz. Bajo Albayzín. 2 July 2017. Available online: http://albayzin.info/2017/07/peligroso-e-ilegal-aparcamiento-de-autobuses-en-lacuesta-del-chapiz/ (accessed on 19 December 2019).

37. Bajo Albayzín. PRENSA: Los otros 'okupas' del Albaicín. Bajo Albayzín. 16 May 2017. Available online: http://albayzin.info/2017/05/prensa-los-otros-okupas-del-albaicin/ (accessed on 19 December 2019).

38. Bajo Albayzín. PRENSA: Los Vecinos Claman Contra las Acampadas Ilegales en el Albaicín. Bajo Albayzín. 1 July 2017. Available online: http://albayzin.info/2017/07/prensa-los-vecinos-claman-contra-las-acampadasilegales-en-el-albaicin/ (accessed on 19 December 2019).

39. Bajo Albayzìn. Invasión de la vía pública por los comercios. Bajo Albayzín. 11 Feburary 2017. Available online: http://albayzin.info/2017/02/invasion-de-la-via-publica-por-los-comercios/ (accessed on 19 December 2019).

40. Bajo Albayzín. Kiosco del pan de Plaza Nueva, SI. Terrazas Abusivas y tantos Chirimbolos, NO. Bajo Albayzín. 27 April 2018. Available online: http://albayzin.info/2018/04/kiosco-del-pan-de-plaza-nueva-si-terrazasabusivas-y-tantos-chirimbolos-no/ (accessed on 19 December 2019).

41. Moreno, P.S. El problema del Turismo en Granada, igual que en Barcelona, Bajo Albayzín. Available online: http://albayzin.info/2014/09/el-problema-del-turismo-en-granada-igual-que-en-barcelona/ (accessed on 10 January 2020).

42. Granada Hoy. El derribo de Santa Ana levanta el debate sobre la conservación del patrimonio. Granada Hoy. 7 March 2019. Available online: https://www.granadahoy.com/granada/derribo-plaza-Santa-Anaconservacion-patrimonio_0_1333967227.html (accessed on 19 December 2019).

43. Battisti, F.; Campo, O. A methodology for determining the profitability index of real estate initiatives involving public-private partnerships. A case study: The integrated intervention programs in rome. Sustainability 2019, 11, 1371. [CrossRef]

44. Bajo Albayzín. PRENSA: Las Calles del Albaicín se Llenan de Pintadas Contra el Turismo. Bajo Albayzín. 29 May 2019. Available online: http://albayzin.info/2019/05/prensa-las-calles-del-albaicin-se-llenan-depintadas-contra-el-turismo/ (accessed on 19 December 2019).

45. Bajo Albayzín, PRENSA: Las Pintadas Contra los Turistas del Albaicín Llegan Hasta la Prensa Inglesa. Bajo Albayzín. 1 June 2019. Available online: http://albayzin.info/2019/06/prensa-las-pintadas-contra-los-turistasdel-albaicin-llegan-hasta-la-prensa-inglesa/ (accessed on 19 December 2019).

46. Opdenhoff, J.-H. PRENSA: «No Tienen Ningún Respeto por el Barrio»: Los Vecinos del Albaicín Cargan Contra Turistas Incívicos. Bajo Albayzín. Available online: http://albayzin.info/2019/08/prensa-no-tienenningun-respeto-por-el-barrio-los-vecinos-del-albaicin-cargan-contra-turistas-incivicos/ (accessed on 19 December 2019).

47. LabIN Granada. Plan Turístico de Granada 2018-2022, LabIN Granada. Available online: https://labingranada. org/foroalbaicinsacromonte-foro2/ (accessed on 3 January 2020).

(C) 2020 by the authors. Licensee MDPI, Basel, Switzerland. This article is an open access article distributed under the terms and conditions of the Creative Commons Attribution (CC BY) license (http://creativecommons.org/licenses/by/4.0/). 


\title{
Correlation of Vulnerability and Damage between Artistic Assets and Structural Elements: The DataBAES Archive for the Conservation Planning of $\mathrm{CH}$ Masonry Buildings in Seismic Areas
}

\author{
Maria Rosa Valluzzi ${ }^{1, *}$, Sergio Calò ${ }^{2}$ and Gianluca Giacometti ${ }^{3}$ \\ 1 Department of Cultural Heritage, University of Padova, 35139 Padova, Italy \\ 2 Venetian Cluster, 30124 Venice, Italy; sergio.calo@venetiancluster.eu \\ 3 Department of Civil, Environmental and Architectural Engineering, University of Padova, 35131 Padova, \\ Italy; gianluca.giacometti@unipd.it \\ * Correspondence: mariarosa.valluzzi@unipd.it
}

Received: 20 December 2019; Accepted: 13 January 2020; Published: 16 January 2020

\begin{abstract}
Historical buildings in seismic hazard-prone regions need specific measures in safety protection, largely due to the presence of artistic assets and/or decorations, both movable (e.g., statues, pinnacles, etc.) and unmovable (e.g., frescoes, valuable plasters or wall paintings, mosaics, and stuccoes). A correlation of damage between structural systems and artworks is fundamental for defining limit states, which can identify the proper conditions for interventions. Nevertheless, several vulnerability aspects can be identified before a seismic event occurs, the study of which can provide the basic dataset for setting up preventive measures in conservation programs. In this paper, the vulnerability and damage conditions related to structural elements (SE) and unmovable artistic assets (AA) belonging to historical masonry buildings are analysed. Optimized survey forms for the onsite detection of either intrinsic (e.g., compositional) defects or deterioration phenomena for both materials and structure are proposed, and results are provided in a web data system (called DataBAES). This enables us to compare the current levels of vulnerability and damage of AA and SE on a scale of five increasing grades. This procedure has been validated on a series of buildings struck by earthquakes in Italy and can be used for correlations of the seismic behaviour of SE and AA in predictive analyses.
\end{abstract}

Keywords: artworks; macro-elements; vulnerability; seismic damage; deterioration; web archive

\section{Introduction}

Masonry buildings in historic city centres are recognised as being structures that are prone to damage from earthquakes due to vulnerabilities associated with their architectural type (e.g., church, palace, tower) as well as specific constructional aspects (e.g., the quality of materials, construction details, connections) and current state conditions (e.g., decay, previous interventions). The concurrence of these factors can cause severe damage scenarios and losses of cultural heritage $(\mathrm{CH})$ assets even under medium-intensity earthquakes, as demonstrated by several episodes of seismic activity in Italy in recent decades (e.g., 1997 Umbria-Marche, 2009 L'Aquila, 2016 Central Italy earthquakes) [1,2].

A post-earthquake emergency in historical city centres often requires the implementation of safety measures for unmovable artistic assets, i.e., those which are integral to damaged structural components. This is the case with the frescoes, decorated plasters, mural paintings, mosaics and stuccoes, which are supported by vaults, columns, ceilings, or walls of a historical building [3,4]. This phase involves both structural engineers and experts on the preservation of $\mathrm{CH}$ assets, who usually can make decisions based on the compliance with safety conditions according to their specific knowledge (i.e., on the 
artistic asset or the structure) and suggest appropriate actions if necessary. Nevertheless, due to the close interconnection between the artwork and its structural support, the evaluation of the current conditions of the former heavily depends on the latter, whose worsened conditions can jeopardise the safety of the whole building.

As an example, a typical severe and, unfortunately, common condition is that of a fresco borne by a wall that has no constraints (e.g., ties, effective connections with adjacent walls and the floor/roof) to counteract overturning under seismic loads, or has low masonry quality (e.g., multi-leaf rubble section). The fresco is in the highest risk condition, because the structural element (the wall to which the fresco is connected) behaves according to the weakest and most brittle mechanism (i.e., out-of-plane collapse) (Figure 1a). On the contrary, in a building where the activation of overall behaviour is provided by more earthquake-resistant conditions, limited structural damage is expected (e.g., in-plane shear cracking in a wall), although it can provide severe patterns, which could affect the fresco integrity (Figure 1b).

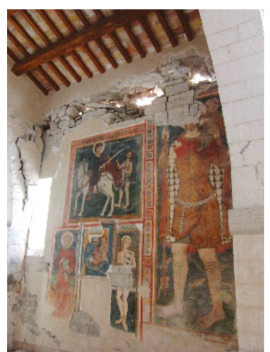

(a)

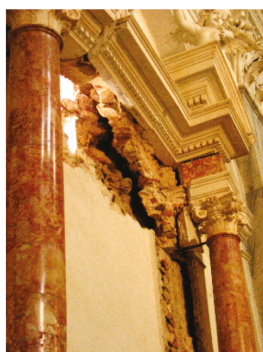

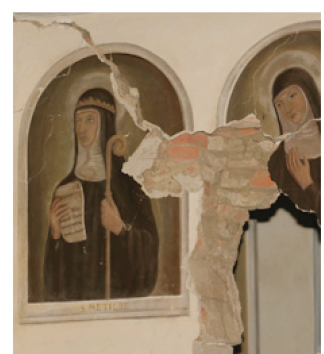

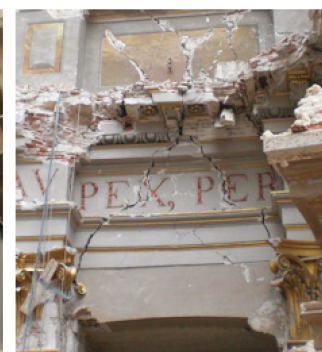

(b)

Figure 1. Examples of damage of artworks due to out-of-plane (a) and in-plane (b) mechanisms for frescoes and stuccoes.

Studies on the correlation of damage between artistic assets (AA) and structural elements (SE) are very limited in literature, especially if related to historic/traditional materials (e.g., plasters). Calderini et al. [5] examined the mechanical interaction between stone masonry panels and covering plasters simulating frescoes by either diagonal compression or shear tests, integrated by non-destructive techniques (NDT, e.g., infrared thermography and ground-penetrating radar). The study highlighted the damage propagation from the wall to the plaster and contributed to the definition of the limit states of plaster regarding the condition of the SE. Valluzzi et al. [6] measured the overall interaction between a fresco and its supporting wall under laboratory compression tests. Various plasters (in terms of composition and finishing quality) applied to the surfaces of a rubble stone masonry panel were taken into consideration, and laser scanning and infrared thermography were also applied to monitor the damage progression during loading. The test caused surface detachments and spalling, which was limited in the plaster that complied with better application criteria. Both studies highlighted the high fragility of plasters in terms of either in-plane or out-of-plane behaviour. More contributions in literature concern the application of NDT for the evaluation of the conservation conditions of CH assets [7-10], or are mainly focused on modern render products, both in terms of the theoretic approach of elasticity applied to multi-layer systems [11] and of the effect of weathering on durability [12,13]. A significant contribution aimed at supporting the design of strengthening interventions for the preservation of architectural buildings comes from comprehensive EU research, e.g., PERPETUATE [14,15] and NIKER [16] projects. The former included the study of buildings with the contained artworks, and the latter provided guidelines for strengthening actions in the form of a structured web catalogue [17]. Recent developments in web-based tools [18] also provide decision-making processes in assessing failure modes through the observation of post-earthquake scenarios. 
Nevertheless, at present, while the knowledge of the mechanical behaviour of structural components has been clarified by several studies and has been supported by reliable evaluation procedures (for both damage and vulnerability aspects), there are not yet any prediction tools encompassing the vulnerability of artworks. In particular, the parameters affecting the combined behaviour between AA and SE need to be identified, and their mutual influence must be properly quantified to optimise integrated and all-encompassing solutions for historic buildings in seismic zones.

Therefore, there is a need for a comprehensive procedure, to use contextually among different areas of expertise, which can encompass both specific aspects related to single issues of both AA and $\mathrm{SE}$ and to combine their evaluations. With this aim in mind, an extensive interdisciplinary research was carried out between the University of Padova and the Venetian Cluster. The research focused on the proposal of an integrated approach that was able to take into consideration architectural (for structural elements) and compositional (for artistic assets) details, as well as the critical aspects related to execution or deterioration/damage conditions. This information was structured in a web database (called DataBAES) so that both aspects of vulnerability and damage in a building may be correlated between artworks and their specific supporting structural components.

The procedure provides a multilevel approach through two survey forms, which are to be used for basic (level I form) or deeper (level II one) onsite visual inspections. The level I form provides the correlation of damage between AA and SE, whereas at level II, both the correlation of damage and vulnerability are proposed. Level II corresponds to an in-depth autopsic analysis to understand the state of conservation of the artworks and the bearing structure, as well as to the possibility of carrying out further studies (e.g., in terms of the quality and quantity of present salts, biological attacks, as well as the presence of 'bottaccioli' or 'calcinaroli'). Of course, particularly at level II, ND procedures (e.g., the above-mentioned georadar, infrared thermography, and laser scanning) can be used to help in the identification of the aspects and parameters (in relation to both materials and structure) needed in the following phases of investigation, according to a more detailed knowledge plan.

The applicability of levels I or II might correspond to different conditions, respectively, e.g., (i) the surveying of emergency conditions for preliminary and quick evaluations just after the earthquake occurrence, or (ii) as a predictive tool, focused on collecting data before an event on each possible mechanism of the various SE and related AA. The results are correlated in terms of five degrees of vulnerability/damage (increasing from the lowest to the highest) to provide prioritisation in the possible interventions for the building. Furthermore, according to information collected on various buildings in a certain area through DataBAES, prioritisation evaluations can be extended on a wider scale to integrate possible conservation plans.

The basic reference for the new procedure consists of the survey forms currently applied in Italy, specifically to churches (form A-DC) [19], palaces (form B-DP) [20], and artistic assets (form C-BM) [21] in the emergency post-earthquake phases, as well as the recommendation/classification charts available for evaluating surface decay [22,23] and structural damage (EMS-98, European Macro Seismic Scale) [24].

The procedure was applied to case studies in various conditions for its validation. At present, the archive includes about 100 case studies (i.e., evaluated AA) belonging to about 30 historic masonry buildings. Results are provided in either quick or more extended charts/summaries, including main pictures and descriptions, which can be easily exported in common formats (e.g., pdf documents) for further elaborations.

\section{The DataBAES Archive}

'DataBAES' is a web archive created within the framework of the Italian project ProVaCi ('Technologies for the Seismic Protection and Valorisation of Cultural Heritage', 2011-2015 [25]) aimed at promoting multi-disciplinary actions for the seismic protection and valorisation of cultural heritage, this term meaning an integrated and broad extent of assets. The tool correlates the vulnerability and damage aspects between artistic assets and their supporting architectural components for a given 
historical building that is either exposed to seismic risk or has already been damaged by an earthquake. The acronym BAES stands for the Italian words 'Beni Artistici' (BA) and 'Elementi Strutturali' (ES), i.e., 'artistic assets' and 'structural elements', respectively.

DataBAES was developed in PHP and JavaScript with a MySQL DBMS backend. Its content is available at https://databaes.dicea.unipd.it/ [26] to registered users. Registration is free of charge and is open to students, scientists, and researchers who deal with historical and cultural heritage preservation.

The archive structure is based on survey forms representing two levels of investigation for historical and cultural heritage assets, i.e., the "Evaluation and correlation of damage of unmovable artistic assets and structural elements" and "Evaluation and correlation of damage and vulnerability of unmovable artistic assets and structural elements", respectively. These new forms are included in the Supplementary Materials of this paper. Each form refers to a specific building. Each building contains one or more case studies, each of which are defined by the following four components: the artistic asset, its supporting structural component, a possible failure mechanism for the structural element, and its localisation within the building. Artistic assets are defined by a sequence of capital letters (i.e., A ... Z , . ) , whereas structural elements are defined by integers $(1 \ldots \mathrm{N})$, so that each case study is identified by a letter-number label (i.e., A1, B2, C3, ... ). In case of two artworks borne by the same macro-element (e.g., a fresco in a stucco frame against a wall), the two AA will be connected to the same SE by using, as an example, A1 and B1 (for the fresco and the stucco, respectively).

The forms are composed of the following four sections: (1) general information on the building and identification of the significant case studies (AA related to SE); (2) a survey of the damage and vulnerability of the architectural components; (3) a survey of the damage and vulnerability of the artworks; (4) a schematic of the concise judgment of the damage and vulnerability for the list of case studies in the building in question. Sections 1 and 4 , being the introductory and the conclusive parts, respectively, have a grey background. This distinguishes them from the two central sections, which can be replicated for the number of case studies identified in the same building.

The options for items that relate to damage and vulnerability aspects are square or round check boxes, which correspond to multiple or single choice, respectively. Detailed degrees of vulnerability and damage are inserted by users, while the average levels are automatically calculated by the system according to given algorithms and/or evaluation scales.

As a result, a table with an overview of the correlation of vulnerability and damage between AA and $\mathrm{SE}$ is generated for each building; a scale of colours of five degrees, from green to red, indicates the increasing condition (of damage and vulnerability), so that the most critical artistic assets can be identified and prioritisation measures may be applied if necessary. Levels equal to or greater than three indicate that an emergency threshold has been passed for specific actions to apply to AA and/or SE. Figure 2 shows the process flow diagram of the archive. 


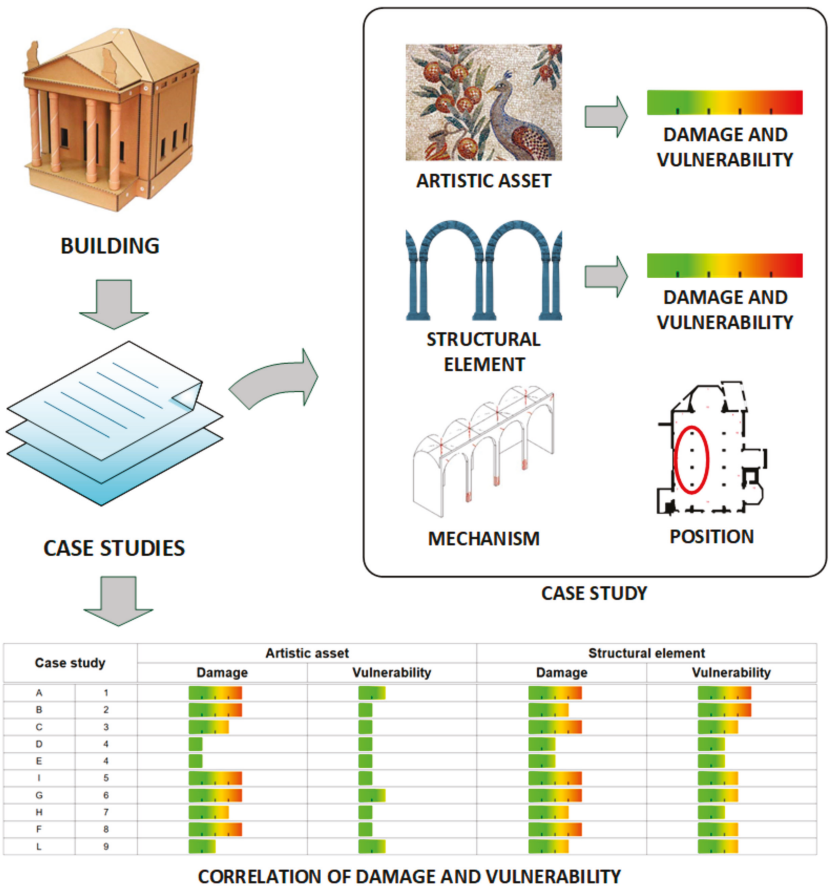

Figure 2. Overview of DataBAES' functioning.

\subsection{Structural Element Data}

The evaluation of the seismic behaviour of structural components in masonry buildings is based on the macro-element approach, which means on the identification of architectural portions of the building 'characterised by a substantial autonomous structural response' [27] compared to the whole building. Macro-elements are defined on the basis of archive documentation and constructive investigations, and are often recognised as typical architectural elements (e.g., for a church: façade, apse, bell-tower, triumphal arch, etc.). In fact, in the absence of good connections (both wall-to-wall and wall-to-floor/roof), sufficient stiffness of the horizontal components, and regularity (both at geometric and behavioural level), the masonry building cannot activate the ideal 'box-like' behaviour, and single brittle rigid body mechanisms can occur, mainly out-of-plane (the so called 'mode 1'). These mechanisms can provoke severe losses (also related to supported artworks) and collapses, before any in-plane ('mode 2') behaviour can be activated [28,29]. Nevertheless, a primary fundamental condition to be checked is the presence of possible indicators of masonry quality [30], as brittle masonry disaggregation (the so called 'mode 0 ', [31]) can occur before any other mechanisms. Evidently, a low masonry quality also severely affects the safety and integrity of the artworks that are integral to the architectural element, even with a very low seismic hazard.

With regards to the SE, the new survey forms collect values as optimized parameters derived from the church and palace damage forms $[19,20]$. Levels I and II differ for the type and extension of information, so that they can support quick or more extensive onsite evaluations, respectively. The case study, as named in the forms, is the AA, to which the various conditions of the connected SE are considered. Both damage and vulnerability indicators are included, as follows.

At first, the building in question is identified, and the possible availability of previously filled forms (the aforementioned forms for churches and palaces) regarding a specific earthquake can be mentioned. 
At level I, the AA and its related SE are identified only with their localisation in the building. Then, each SE (e.g., wall, column, vault, or other) is described in terms of masonry type (material and dimensions, the latter could be measured or estimated) and with the identification of the main damage mechanisms, the origin (i.e., due to seismic action, pre-existing, or simply because of deterioration) and importance (i.e., either primary or secondary) of which should be specified. The possible mechanisms are listed as selected items from the form available for churches (28 mechanisms) and for palaces (22 mechanisms), and the damage level is identified on a scale of 1 to 5 , according to the EMS-98. Figures 3 and 4 show the damage mechanisms included in the DataBAES forms, and the five damage levels as provided by EMS-98, respectively.

For the primary mechanisms, additional data can be provided about in-plane or out-of-plane damage details for walls (e.g., overturning activation, spalling, crushing, or collapse), as well as about the extension of potential cracking for vaults and columns. Details on cracking are also included, since these can affect the integrity of the AA in terms of extension and type (crazing, passing through, rough estimation of crack opening, etc.). At level I, damage evaluation is predominant, hence the presence of vulnerability indicators and of earthquake-proof devices is simply declared and kept as an overall recognition of potential additional issues for the building.

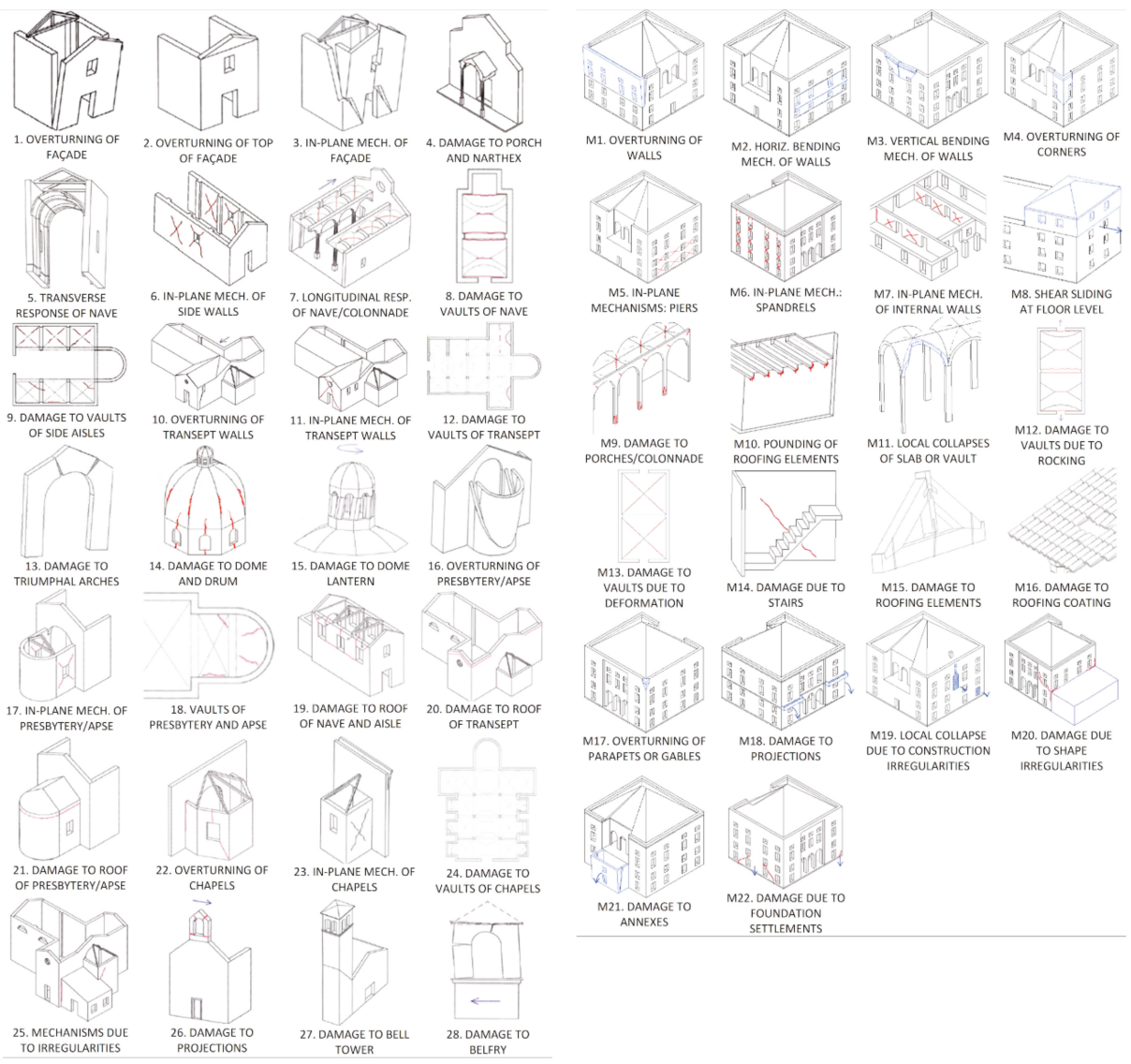

Figure 3. Example of mechanisms for masonry churches and buildings adopted in DataBAES forms, as shown in church (A-DC, 28 mechanisms) [19] and palace (B-DP, 22 mechanisms) [20] survey forms. 


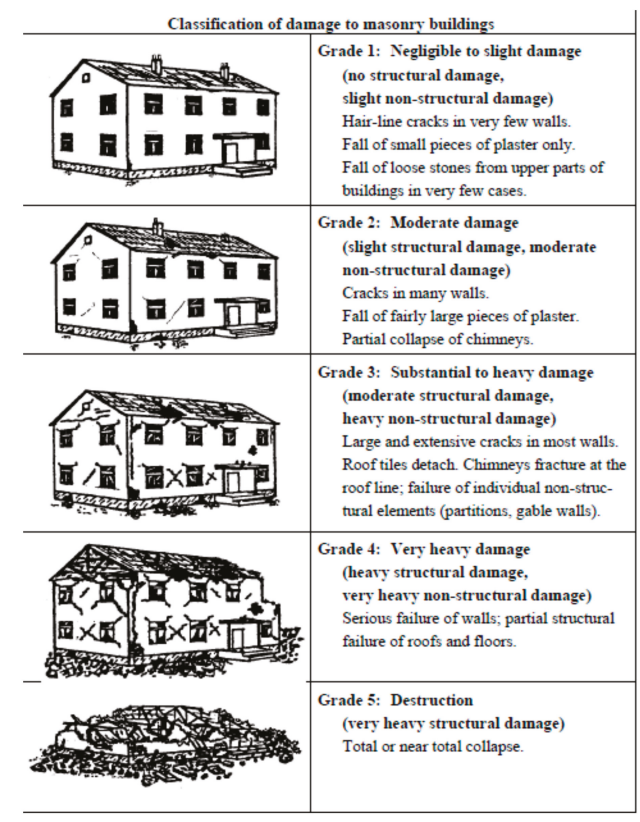

Figure 4. Damage degrees for masonry structures according to EMS-98 (reproduced with permission from G. Grünthal [24]).

At level II, the form requires the identification of the AA and its related SE with the primary or secondary mechanism having already been identified (Figure 5). In fact, at this level, each of the 28 mechanisms for churches or the 22 mechanisms for the other types of buildings are analysed in detail, both in terms of damage and the presence of specific vulnerability/devices. In the case of non-structural elements supporting artworks (e.g., camorcanna vaults), the mechanism should be identified as that of the macro-element, which could affect the one of the non-structural elements in question (e.g., the possible overturning of walls supporting the camorcanna vault). For the increasing damage evaluation, a grade from 1 to 5 is required, whereas for the vulnerability assessment the increasing overall scale of 1 to 5 is derived from the detailed evaluation of each item based on a three-grade scale, according to the effectiveness of potentially good construction details and/or earthquake-proof systems (e.g., ties, buttresses, connections and anchors, light bracing, and lintels). This scale corresponds to 'ineffective' (any check box marked), 'moderate' (grade 1), 'good' (grade 2), and 'completely effective' (grade 3). Vulnerability concerns the presence of thrusting elements (roof struts, arches, and vaults), slender piers, large openings close to corners, stiff ridge beams, heavy floors/roof, high-span thin vaults, etc., and is expressed on the scale of 1 to 5 corresponding to the grades 'very low'-'low'-'average'-'high'-'very high'.

Space for notes and schematics is available in the form where needed. The specific items required for SE are reported in the level I and level II forms included in the Supplementary Material of this paper. 


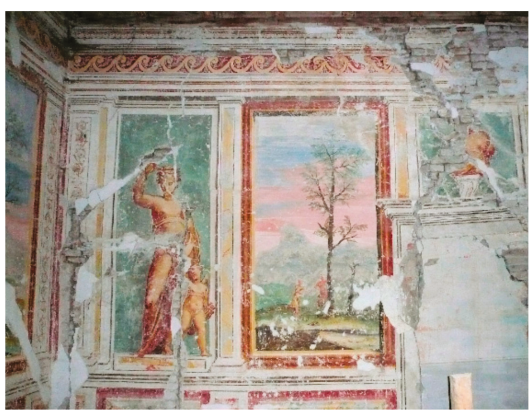

(a)

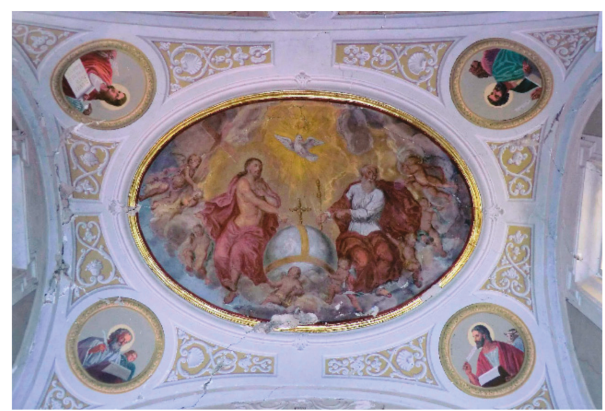

(b)

Figure 5. Example of primary and secondary mechanisms associated with a damaged fresco located in apse of a church: (a) apse is subjected to 'overturning', but the main mechanism affecting fresco is 'shear'; therefore, 'in-plane mechanism of apse' and 'overturning of apse' are, respectively, primary and secondary mechanisms; (b) damage to the vault of the central nave affects the painting as a primary mechanism.

\subsection{Artistic Asset Data}

Artworks in historical buildings are exposed to deterioration and damage according to their specific features and conservation conditions. Calò et al. [32] defined a methodological approach for the restoration of artistic assets. In particular, the initial phases of anamnesis and analysis of the application techniques were clarified; nevertheless, the study does not include any evaluation of intrinsic risk parameters. Application techniques, such as the half-dry mural technique, have always been considered an initial factor in degradation $[33,34]$. However, also in this case, the comparative analysis of risk is missing.

In this paper, the risk of damage has been parametrized on the basis of the following three factors: (i) the construction technique and any application (i.e., intrinsic) faults; (ii) the position where the artworks are located (e.g., wall support, canopy, vertical and horizontal position); (iii) the state of conservation (the passage of artefacts over time). This approach allows predictions to be made and therefore enable us to identify where the greatest damage to artworks could occur. As a result, information for the planning of maintenance and interventions is provided. Moreover, in the DataBAES archive, for a more comprehensive risk analysis, the specific issues of artworks are also combined with the possible effect of earthquakes on the supporting structural element according to two levels of investigation.

At level I, wall paintings/frescoes, stuccoes, and mosaics are recognised as immovable artworks integral to the building structure. The possibility of identifying an artistic cycle is also taken into consideration. At first, the overall maintenance state should be defined, then 12 items derived from $[22,23]$ are selected as a representative list for damage evaluation (Figure 6 shows some examples). The surveyor should also in this case check whether the detected damage has been caused by seismic activity or is a consequence of pre-existing damage or an effect of direct deterioration. A specific judgment on a scale of 1 to 5 is required for each item on the list, followed by an overall judgment related to all the 12 items. At such a level, as for the structural evaluation, the vulnerability does not require any quantification; nevertheless, the main aspects to inspect are defined so that they can be recognised for further analysis provided by level II. These aspects are specified for the three types of AA (i.e., wall paintings/frescoes, stuccoes, and mosaics) and are related to application technique, i.e., compositional and finishing issues, and vulnerability, by taking into consideration the placement position and the interaction with possible additional interlay elements (e.g., camorcanna, reed wattle, etc.). 


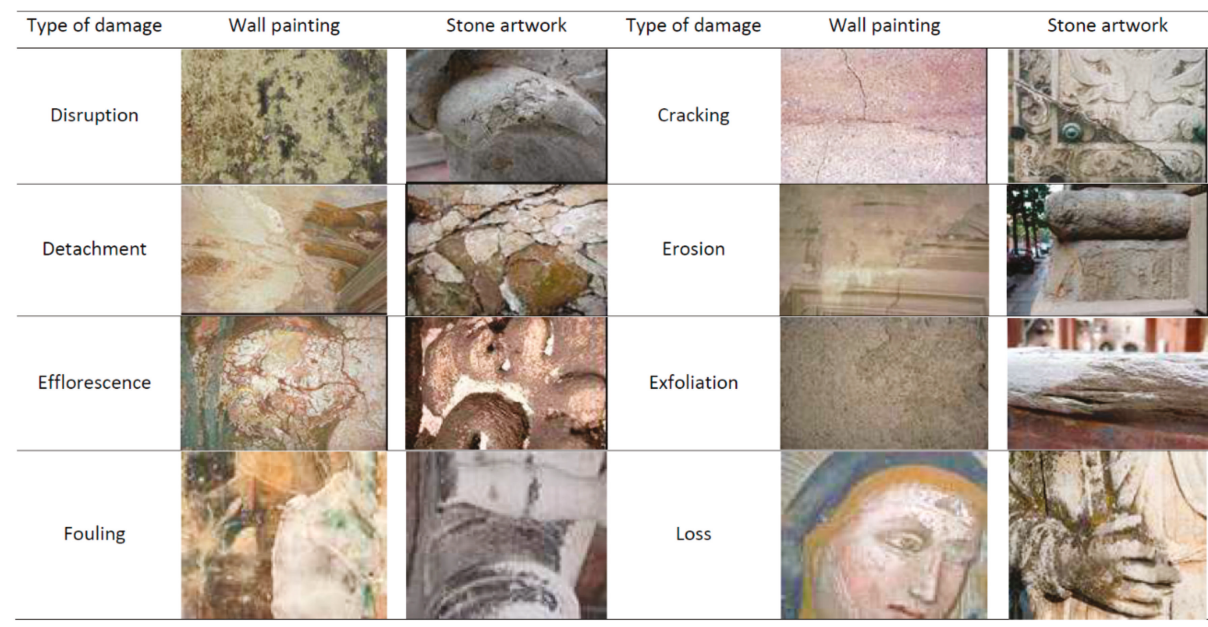

Figure 6. Examples of the deterioration of wall paintings and stone artworks according to current charts for classification of surface decay $[22,23]$.

At level II, the description and the evaluation of damage is similar to that of level I, but the list of possible types of damage is increased up to 25 items. Vulnerability is distinguished by those derived by the application technique and other inherent variables. In both cases, specific parameters are taken into consideration, each of them quantified by a coefficient. For each type of AA (i.e., wall painting/fresco, stucco, and mosaic), in addition to general information, such as finishing or placement technique, the thickness, inert/binder ratio, and grain size are specified, which represent potential critical aspects. The other inherent variables are related to the presence of sulphation, solubilisation, organic material, crazing, wood or metal inclusions, and of products due to the incorrect mixing of basic components. These products can be: 'bottaccioli' (i.e., inert agglomerate not mixed with the binder inside the mortar causing the punctual fall of original material due to the loss of adhesion); 'calcinaroli' (i.e., binder agglomerate without bonding that, with the absorption of humidity, causes binder swelling and original material to fall); 'gessaroli' (i.e., plaster agglomerates in powder dispersed in the mixture of chalk and water caused by bad mixing; the absorption of chalky material provokes swelling and the fall of original material in humid conditions). The presence of these variables higher than $30 \%$ must be accompanied by the parameter to which it refers. Table 1 shows the quantification of parameters for vulnerabilities related to wall paintings. It is important to underline how the judgment on the overall damage must be expressed based on the extent or quantity of the artefact that has been damaged, regardless of its artistic relevance.

A proportional calculation that balances the risks with respect to extrinsic and intrinsic factors (executive techniques, the state of conservation, and the effects of the earthquake) based on a scale of 1 to 5 was proposed. This approach results in a weighted average, which is useful for defining intervention priorities and monitoring for the best preservation of the cultural asset. Lastly, the presence of intrinsic variables caused by degradation, which may have influenced the progression of the lesions and contributed to the detachment of portions, is checked. Any further information can be inserted into the 'notes'. The item's 'thickness' for the stuccoes and the 'finishing technique' for stuccoes and frescoes can be compiled with more than one value, since these characteristics can be present to an equal extent.

At the end of the II level form, a list of damage and vulnerability levels evaluated for each AA and its related SE is provided, so that a comparison of those levels can be made for all case studies (i.e., AA) identified in the building. This evaluation is processed automatically in the DataBAES archive once the forms have been filled out on the web platform, so that a colour scale may appear immediately 
for quick correlations. The same procedure also applies to the level I form, although it is limited to a damage comparison.

The specific items required for AA are reported in the level I and level II forms included in the Supplementary Materials of this paper.

Table 1. Example of quantification of vulnerability of wall paintings/frescoes/plasters (all compositional stratigraphy) with respect to the application technique and inherent variables.

\begin{tabular}{|c|c|c|}
\hline Vulnerability for Application Technique & Item & Parameter \\
\hline \multirow{3}{*}{ Finishing technique } & Fresco & 0.00 \\
\hline & Half fresh & 0.25 \\
\hline & Dry & 0.50 \\
\hline \multirow{4}{*}{ Thicknesses } & $<10 \mathrm{~mm}$ & 0.00 \\
\hline & $>11 \mathrm{~mm}<30 \mathrm{~mm}$ & 0.75 \\
\hline & $>31 \mathrm{~mm}<50 \mathrm{~mm}$ & 2.50 \\
\hline & $>51 \mathrm{~mm}$ & 4.50 \\
\hline \multirow{5}{*}{ Aggregate-binder ratio } & $3-1$ & 0.00 \\
\hline & $2-1$ & 1.75 \\
\hline & $1-1$ & 3.00 \\
\hline & $4-1$ & 3.50 \\
\hline & $5-1$ & 4.00 \\
\hline \multirow{4}{*}{ Granulometry (average value) } & $<3 \mathrm{~mm}$ & 0.80 \\
\hline & $>4 \mathrm{~mm}$ a $<8 \mathrm{~mm}$ & 0.00 \\
\hline & $>8 \mathrm{~mm}$ a $<10 \mathrm{~mm}$ & 1.30 \\
\hline & $>10 \mathrm{~mm}$ & 3.50 \\
\hline \multirow{4}{*}{ Interlay element with support } & Seamless in historical overlaps & 3.00 \\
\hline & Reed wattle & 4.00 \\
\hline & Camorcanna & 4.50 \\
\hline & Seamless in working days & 0.80 \\
\hline \multirow{3}{*}{ Placement } & Vertical & 0.75 \\
\hline & Horizontal & 3.00 \\
\hline & Vault & 2.00 \\
\hline \multirow{7}{*}{ Vulnerability for Inherent Variables } & 'Bottaccioli' & 0.75 \\
\hline & 'Calcinaroli' & 0.75 \\
\hline & Crazing & 2.00 \\
\hline & Sulfation of plaster & 1.50 \\
\hline & Solubilisation & 1.50 \\
\hline & Organic material & 2.00 \\
\hline & If greater than $30 \%$ & $3.00 *$ \\
\hline
\end{tabular}

* applicable to all inherent variables.

\section{Application and Validation}

The procedure set on DataBAES has been applied to about 30 buildings for a total of about 100 case studies, i.e., AA examined in terms of vulnerability and damage (level II) in relation to their bearing SE. Figure 7 shows how the analysed buildings appear on the main page of the web archive. Visualisation of the quick results of the present damage and vulnerability conditions for both AA and SE is possible (Figure 8), thus providing a comparison at a glance among case studies and the possibility of ranking them in order to manage an emergency or in order to identify a priority in intervention actions. 


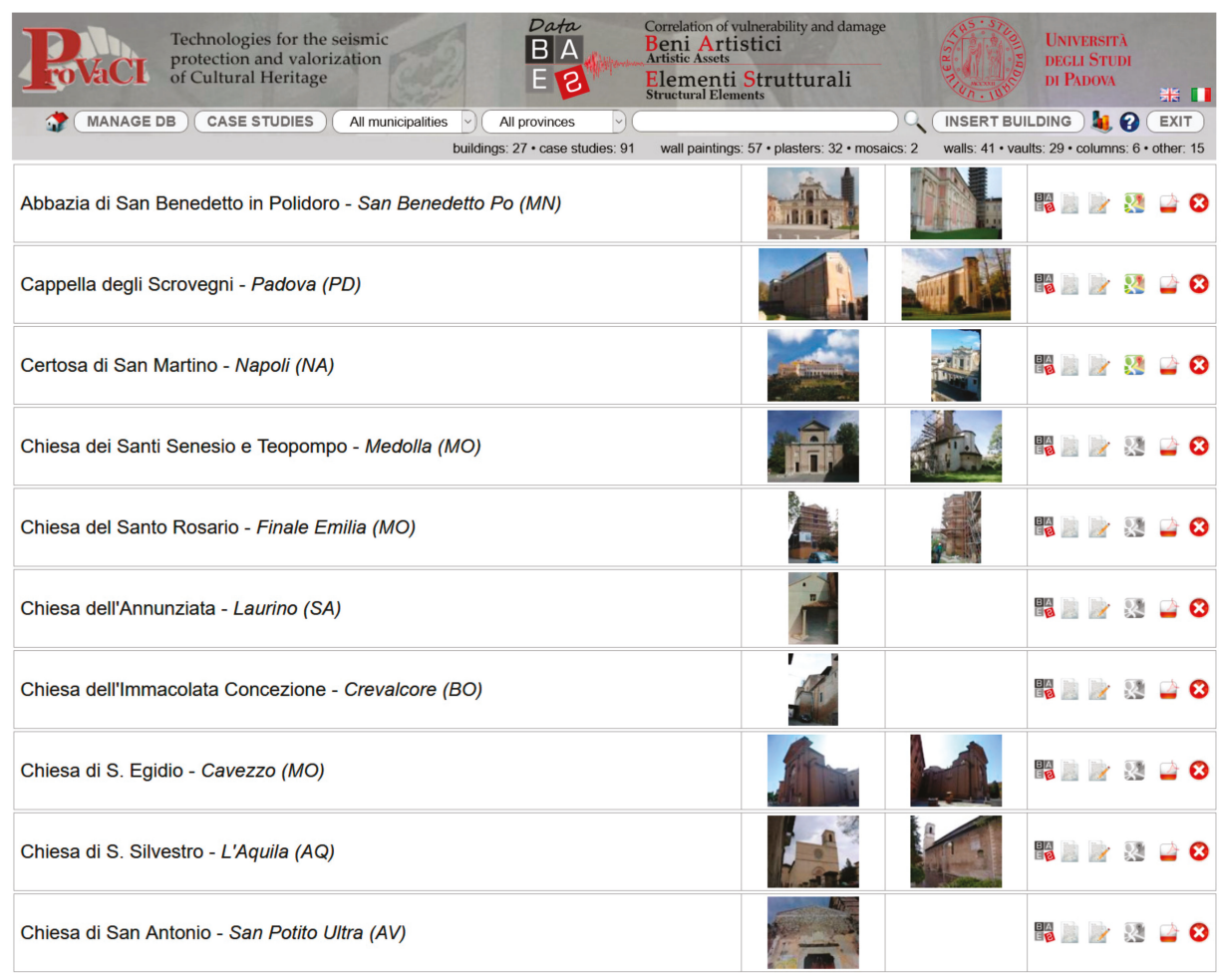

Figure 7. Screenshot from DataBAES archive listing buildings analysed in terms of both artistic assets (AA) and structural elements (SE). 


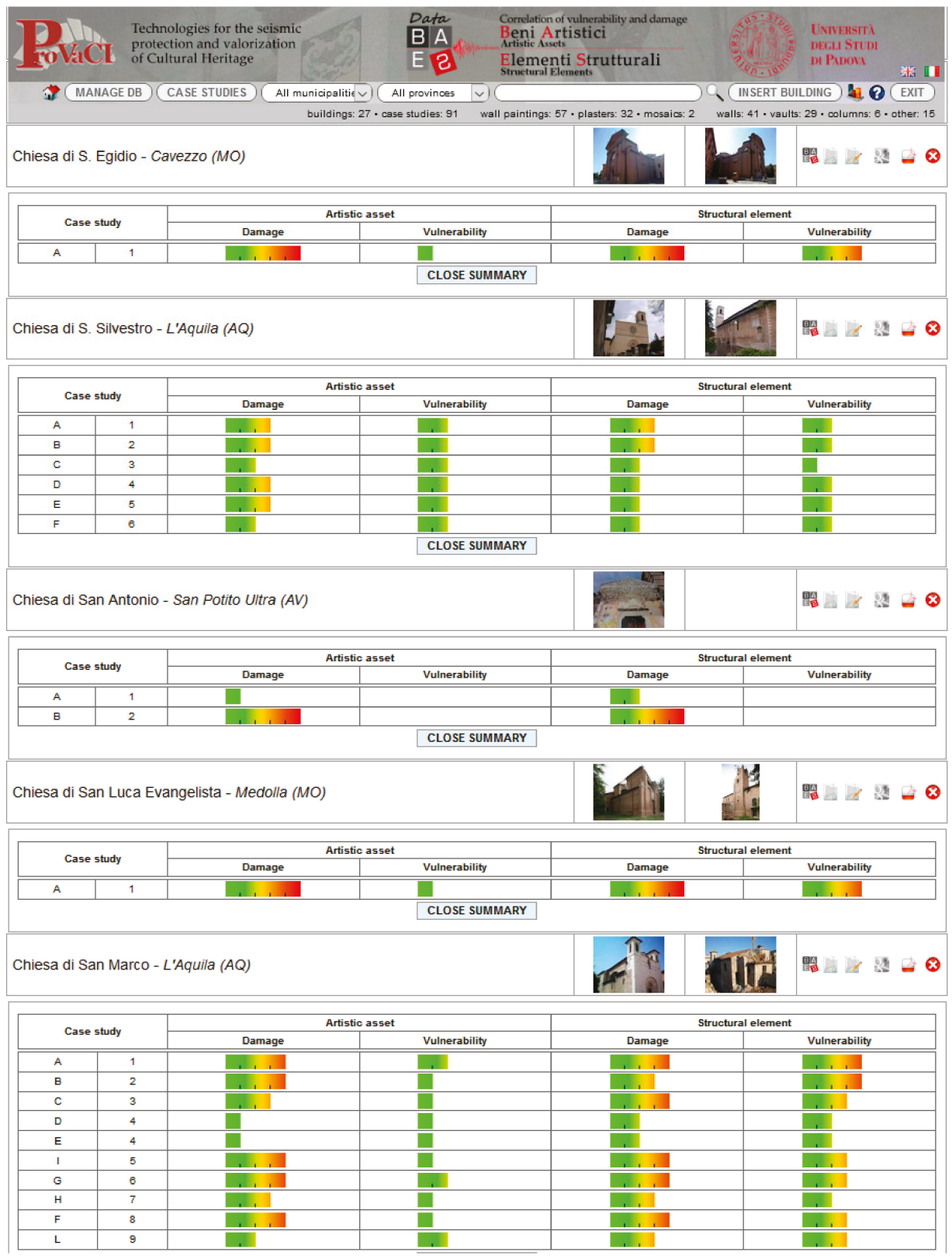

Figure 8. View of the summary of results of case studies for analysed buildings.

Furthermore, for each building, additional data for the evaluation of damage and vulnerability conditions for each of the identified case studies is summarised and easy to consult (see example in Figures 9 and 10). 


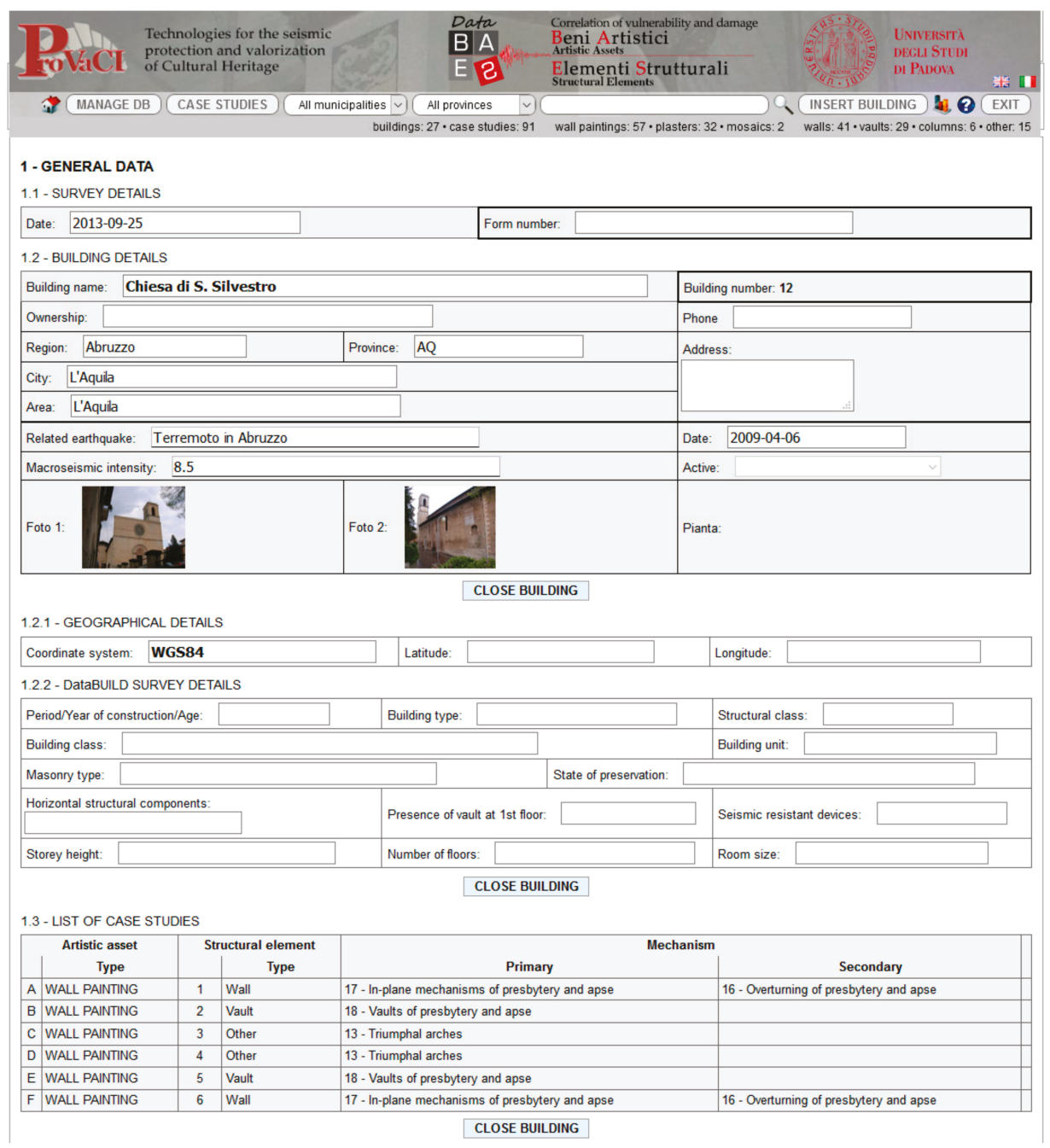

Figure 9. Church of S. Silvestro (L'Aquila, Italy): general data and identification of the case studies of the building. 


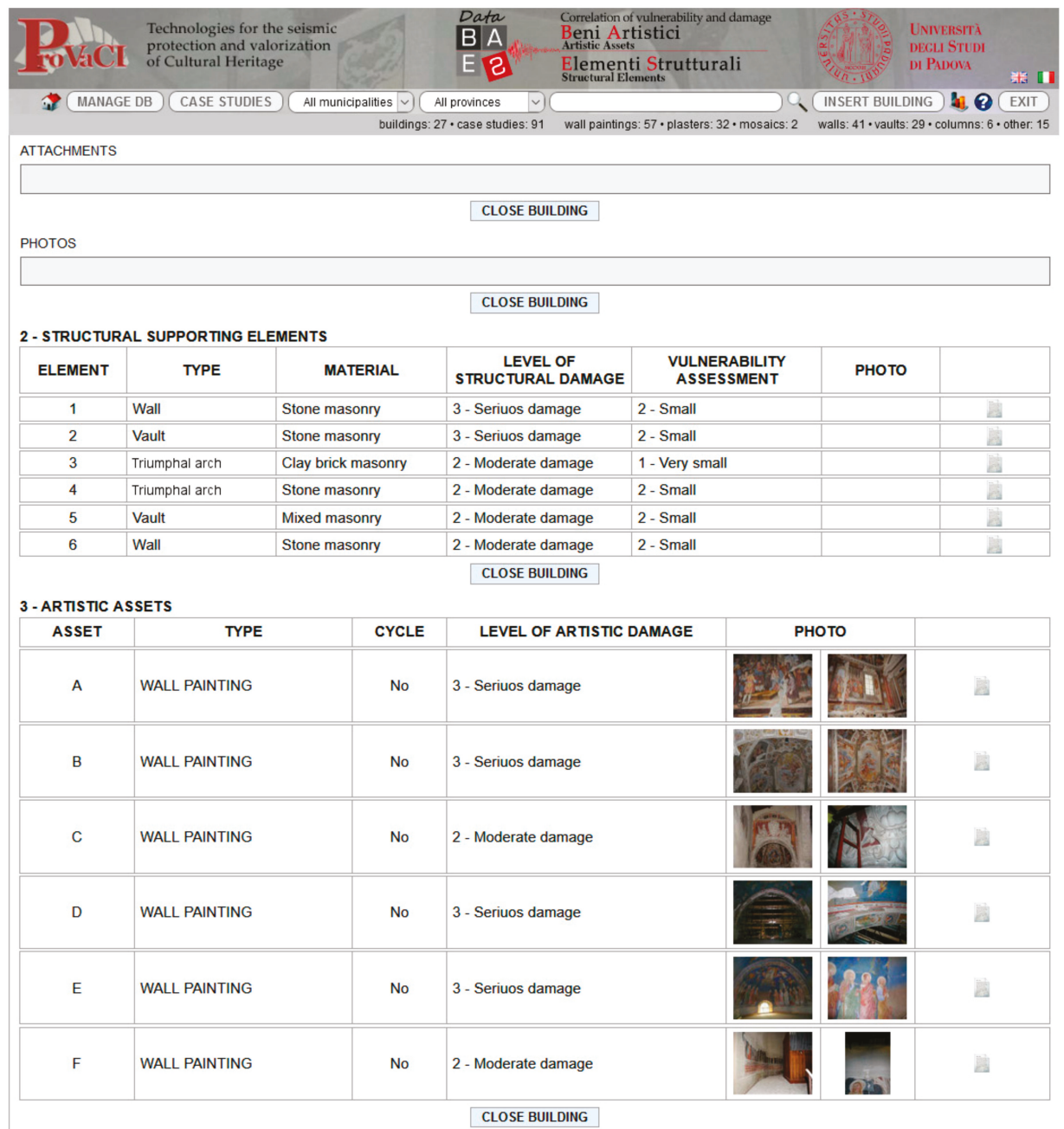

4 - VULNERABILITY AND DAMAGE CORRELATION FOR ARISTIC ASSETS AND STRUCTURAL ELEMENTS

4.1 - DAMAGE LEVELS AND VULNERABILITY ASSESSMENTS

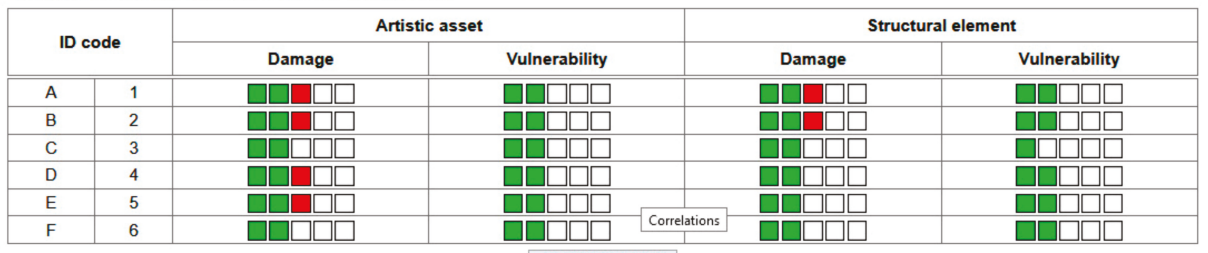

4.2 - NOTES

Figure 10. Church of S. Silvestro (L'Aquila, Italy): damage and vulnerability data for each AA and SE pairs. 
This systematic approach provided a general view of combined information between AA and SE (the detailed data of which are included in the level II survey form) for rapid comparative evaluations, but also allowed for two developments in the research, as specified in the following. The data collected from selected case studies have been used to structure a hierarchical approach and identify key factors to predict the prioritization of interventions (this analysis is described in [35]). Furthermore, the collected data have been analysed in terms of the frequency of parameters, which occurred in similar contexts (e.g., the type of building and the main structural bearing material), in order to find possible trends and correlation curves. This analysis is described in the following.

\section{Damage Correlation between Artworks and Structural Macro-Elements}

The study focused on eight Italian masonry buildings struck by earthquakes, which occurred in 2009 in the Abruzzo region (with a Richter scale magnitude of $M=5.8$ ) and in 2012 in Emilia-Romagna and Lombardia $(M=5.9)$. The basic structural material of those buildings is typical of buildings in mountainous (Abruzzo) and level areas (Emilia-Romagna and Lombardia) where there is prevalence of stone or clay brick masonries, respectively. A group of multidisciplinary experts surveyed both AA and the connected SE, so that a list of case studies (as defined by the DataBAES archive) was identified. Mural paintings (including frescoes) and stuccoes were found as AA in the buildings; therefore, the following analysis refers to this type of artwork. Artistic assets were inspected to detect a series of typical surface alterations (i.e., lack, lacunae, detachment, and cracking) that can be influenced by structural damage. For structural macro-elements, the out-of-plane and in-plane mechanisms of the walls, as well as the damage to the columns and vaults, were taken into consideration. Table 2 lists the mechanisms detected for the selected buildings according to the cataloguing of the II level survey form (see also Figure 3).

Table 2. Identification of macro-element mechanisms involving artworks in analysed buildings.

\begin{tabular}{|c|c|c|c|c|c|c|c|c|c|}
\hline \multirow{2}{*}{ Building } & \multirow{2}{*}{ Place and Region } & \multicolumn{4}{|c|}{ Mural Paintings } & \multicolumn{4}{|c|}{ Stuccoes } \\
\hline & & $\begin{array}{c}\text { Wall } \\
\text { (Out-of-Plane) }\end{array}$ & $\begin{array}{c}\text { Wall } \\
\text { (In-Plane) }\end{array}$ & Vault & Column & $\begin{array}{c}\text { Wall } \\
\text { (Out-of-Plane) }\end{array}$ & $\begin{array}{c}\text { Wall } \\
\text { (In-Plane) }\end{array}$ & Vault & Column \\
\hline $\begin{array}{c}\text { Theatre of } \\
\text { S. Filippo Neri }\end{array}$ & $\begin{array}{c}\text { L'Aquila } \\
\text { (Abruzzo) }\end{array}$ & 2,10 & 23 & 24 & 5 & 2,10 & 17,23 & 24 & 5 \\
\hline $\begin{array}{l}\text { Church of } \\
\text { S. Marco }\end{array}$ & $\begin{array}{l}\text { L'Aquila } \\
\text { (Abruzzo) }\end{array}$ & & 6 & 24 & & 3,22 & 6,17 & 14 & 5 \\
\hline $\begin{array}{l}\text { Church of } \\
\text { S. Silvestro }\end{array}$ & $\begin{array}{l}\text { L'Aquila } \\
\text { (Abruzzo) }\end{array}$ & & 17 & 18 & & & & & \\
\hline $\begin{array}{l}\text { Convent of } \\
\text { S. Angelo }\end{array}$ & $\begin{array}{c}\text { Ocre } \\
\text { (Abruzzo) }\end{array}$ & & 6 & 8 & & 1 & & & 8 \\
\hline $\begin{array}{l}\text { Church of } \\
\text { S. Rosario }\end{array}$ & $\begin{array}{c}\text { Finale Emilia } \\
\text { (Emilia-Romagna) }\end{array}$ & 5 & & & & 16 & 3 & & \\
\hline $\begin{array}{l}\text { Church of } \\
\text { Immacolata } \\
\text { Concezione }\end{array}$ & $\begin{array}{c}\text { Crevalcore } \\
\text { (Emilia-Romagna) }\end{array}$ & & & & & & 17 & & \\
\hline $\begin{array}{l}\text { Abbey of } \\
\text { S. Benedetto } \\
\text { in Polirone }\end{array}$ & $\begin{array}{l}\text { S. Benedetto Po } \\
\text { (Lombardia) }\end{array}$ & & & 8 & & & & & \\
\hline Villa Galvagnina & $\begin{array}{c}\text { Moglia } \\
\text { (Lombardia) }\end{array}$ & M01, M10 & M07 & & & & & & \\
\hline
\end{tabular}

The combination of the case studies representing mural paintings (30 case studies) and stuccoes (20 case studies) in the buildings allowed preliminary elaborations of data regarding the frequency of the occurrence of the deterioration of AA in relation to the mechanisms of their supporting SE. The majority of case studies detected in the buildings referred to the in-plane damage of the walls, for either mural paintings or stuccoes, followed by the damage of the vaults and the out-of-plane mechanisms of walls, respectively, for mural paintings and stuccoes. Figure 11 shows the results obtained for the artworks detected in the buildings. Most deterioration for AA refers to cracking and detachment, either for the cases of mural paintings or stuccoes. Especially in mural paintings, the lacunae are not connected to out-of-plane mechanisms, as they do not involve substrate layers; 
on the contrary, as expected, lacunae are more frequent in the case of in-plane mechanisms. Lack and detachment particularly affect the stuccoes, due to loss of the material of their typical slender protruding portions.

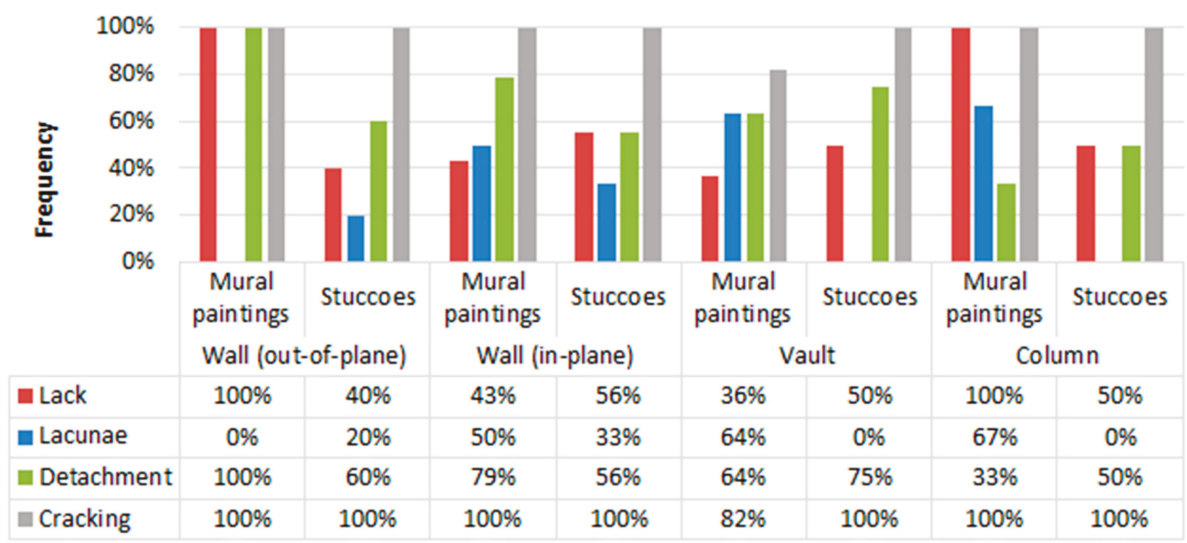

Figure 11. Frequency of the occurrence of the deterioration types of AA combined with mechanisms of SE detected for mural paintings and stuccoes.

Figure 12 shows the evaluation of average damage according to the indications of the II level survey form, performed for both mural paintings and stuccoes, as well as an overall judgment encompassing the condition of the structural macro-element (i.e., wall, vault, or column). The highest values of average damage (higher than 2) mainly refer to the out-of-plane mechanisms of walls for stuccoes, and the damage of the vaults, followed by the in-plane damage of the walls, for mural paintings.

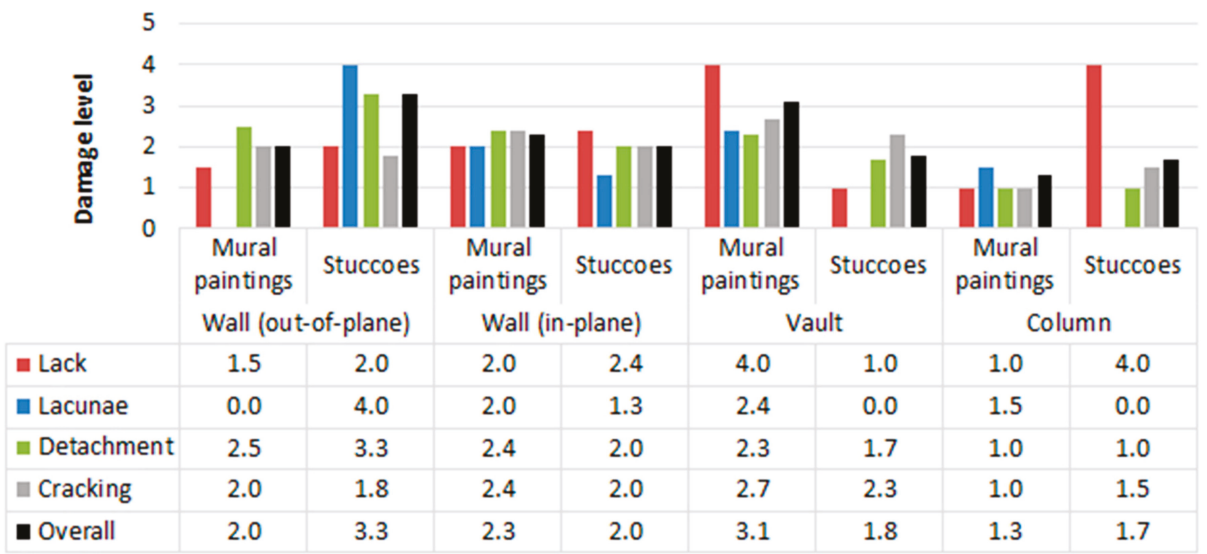

Figure 12. Average damage levels of AA combined with mechanisms of SE identified for mural paintings and stuccoes.

The analysis of the levels of overall damage associated with the macro-element supporting each artistic asset provided the distributions given in Figure 13. The results refer to the overall damage of both mural paintings and stuccoes and are based on the assumption that the damage of the AA (d1, $\mathrm{d} 2, \ldots, \mathrm{d} 5)$ is induced by that of the SE (D1, D2, .., D5) [24]. In general, the increase of damage in AA corresponds to an increasing damage in the supporting SE, with significant distribution at D2-D3 levels, i.e., from moderate (D2) to substantial to heavy damage (D3). 


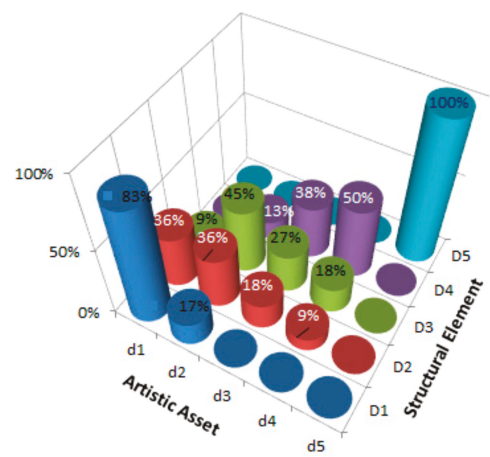

Figure 13. Distribution of the damage levels of AA versus each degree of SE damage.

The same trend is expressed by the curves in Figure 14, which better clarify the strong correlation between the damage of AA and SE.

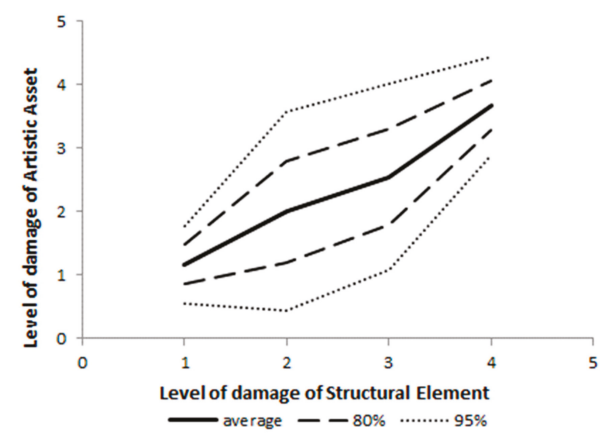

Figure 14. Joint distribution of damage levels between AA and SE for both mural paintings and stuccoes.

\section{Conclusions}

Artistic assets are commonly an important component in the evaluation of masonry buildings belonging to historic city centres for defining safety measures after the occurrence of an earthquake. However, these safety actions are often evaluated with the risk connected to the bearing components not being taken into account. In reality, the preservation of artistic heritage strongly depends on the mechanical behaviour of the building structure, the knowledge of which, together with collectable data on the joined assets, can provide useful information for possible conservation plans. A new web archive, called DataBAES, which aims to collect damage and vulnerability data of unmovable artworks (frescoes, stuccoes, mosaics) for priority ranking evaluations has been proposed. It is based on survey forms focused on two increasing levels of direct visual inspections applicable to AA that are integral to their bearing SE.

The I level form, called "Evaluation and correlation of damage of unmovable artistic assets and structural elements", allows for an expeditious survey of the damaged condition of artworks and related architectural elements after an earthquake occurs. At level I, the objectives of the visual inspection are as follows: helping the data collection in the post-emergency phases, qualifying the current damage level of artistic assets taking into consideration the behaviour of the supporting structural macro-elements, and allowing for potential safety measures. At such a level, possible significant vulnerability aspects (for both AA and SE) are simply identified, and do not contribute to the final judgment. A deeper evaluation of both damage and vulnerability aspects is allowed at level II, by means of the form "Evaluation and correlation of damage and vulnerability of unmovable artistic 
assets and structural elements". It completes the investigation by detailing their mutual description, with reference to 50 possible mechanisms of macro-elements representing SE, as well as 25 potential deterioration indicators for AA. For both levels, the proposed approach provides a judgment based on a 1 to 5 scale, which shows the severity of increasing damage/vulnerability.

The procedure has been implemented on the web archive platform DataBAES, which provides a comprehensive view of both single and multiple case studies detected in different buildings. Hence, this approach can be applied for ranking artistic assets in a building, as well as whole buildings belonging to a historic city centre, according to the results obtained for their related assets, in order to plan preventive measures and the management of $\mathrm{CH}$ to a greater extent. Further developments in DataBAES could focus on the evaluation of structural interventions applied to buildings after previous earthquakes in order to highlight possible negative effects on the mechanical behaviour and, consequently, on the integrity of their joined artworks.

Supplementary Materials: The following are available online at http://www.mdpi.com/2071-1050/12/2/653/s1, DataBAES I level survey form: "Evaluation and correlation of damage of unmovable artistic assets and structural elements", DataBAES II level survey form: "Evaluation and correlation of damage and vulnerability of unmovable artistic assets and structural elements".

Author Contributions: Conceptualization, M.R.V.; Data curation, M.R.V., S.C. and G.G.; Funding acquisition, M.R.V.; Methodology, M.R.V. and S.C.; Software, G.G.; Supervision, M.R.V.; Validation, M.R.V.; Writing-original draft, M.R.V.; Writing-review \& editing, M.R.V., S.C. and G.G. All authors have read and agreed to the published version of the manuscript.

Funding: The research was funded by ProVaCi (Technologies for the Seismic Protection and Valorization of Cultural Heritage, Italy, 2011-2015).

Acknowledgments: The authors wish to acknowledge F. Vanin, A. Rinaldin, M. Munari, for their collaboration in collecting and processing data, and S. Ulizio for her contribution to the technical evaluation of the risk parameters. $\mathrm{M}$. Giaretton and H. Branch are also acknowledged for their help with the translation phase of the survey forms and website.

Conflicts of Interest: The authors declare no conflict of interest.

\section{References}

1. Lagomarsino, S. Damage assessment of churches after L'Aquila earthquake (2009). Bull. Earthq. Eng. 2012, 10, 73-92. [CrossRef]

2. Fiorentino, G.; Forte, A.; Pagano, E.; Sabetta, F.; Baggio, C.; Lavorato, D.; Nuti, C.; Santini, S. Damage patterns in the town of Amatrice after August 24th 2016 Central Italy earthquakes. Bull. Earthq. Eng. 2018, 16, 1399-1423. [CrossRef]

3. Parisi, F.; Augenti, N. Earthquake damages to cultural heritage constructions and simplified assessment of artworks. Eng. Fail. Anal. 2013, 34, 735-760. [CrossRef]

4. Penna, A.; Calderini, C.; Sorrentino, L.; Carocci, C.F.; Cescatti, E.; Sisti, R.; Borri, A.; Modena, C.; Prota, A. Damage to churches in the 2016 central Italy earthquakes. Bull. Earthq. Eng. 2019, 17, 5763-5790. [CrossRef]

5. Calderini, C.; Degli Abbati, S.; Cotic, P.; Kržan, M.; Bosiljkov, V. In-plane shear tests on masonry panels with plaster: Correlation of structural damage and damage on artistic assets. Bull. Earthq. Eng. 2014, 13, 237-256. [CrossRef]

6. Valluzzi, M.R.; Salemi, G.; Deiana, R.; Faresin, E.; Giacomello, G.; Giaretton, M.; Panizza, M.; Pasetto, M.; Calò, S.; Battistella, M.; et al. Integrated ND methodologies for the evaluation of the adhesion of frescoes on stone masonry walls. In Emerging Technologies in Non-Destructive Testing VI, Proceedings of the ETNDT6-Emerging Technologies in NonDestructive Testing 6, Brussels, Belgium, 27-29 May 2015; Aggelis, D.G., van Hemelrijck, D., Vanlanduit, S., Anastasopoulos, A., Philippidis, T.P., Eds.; Taylor \& Francis Group: London, UK, 2016; pp. 505-510.

7. Theodorakeas, P.; Ibarra-Castanedo, C.; Sfarra, S.; Avdelidis, N.P.; Koui, M.; Maldague, X.; Paoletti, D.; Ambrosini, D. NDT inspection of plastered mosaics by means of transient thermography and holographic interferometry. NDT E Int. 2012, 47, 150-156. [CrossRef] 
8. Cotic, P.; Jaglicic, Z.; Bosiljkov, V. Validation of non-destructive characterization of the structure and seismic damage propagation of plaster and texture in multi-leaf stone masonry walls of cultural-artistic value. J. Cult. Herit. 2013, 15, 490-498. [CrossRef]

9. Moropoulou, A.; Labropoulos, K.C.; Delegou, E.T.; Karoglou, M.; Bakolas, A. Nondestructive techniques as a tool for the protection of built cultural heritage. Constr. Build. Mater. 2013, 48, 1222-1239. [CrossRef]

10. De Freitas, S.S.; de Freitas, V.P.; Barreira, E. Detection of façade plaster detachments using infrared thermography-A nondestructive technique. Constr. Build. Mater. 2014, 70, 80-87. [CrossRef]

11. Kovler, K.; Frostig, Y. On the problem of cracking in plaster layers. Constr. Build. Mater. 1998, 12, $251-258$. [CrossRef]

12. Galvão, J.; Flores-Colen, I.; de Brito, J.; Veiga, M.R. Variability of in-situ testing on rendered walls in natural ageing conditions-Rebound hammer and ultrasound techniques. Constr. Build. Mater. 2018, 170, 167-181. [CrossRef]

13. Bochen, J.; Nowoświat, A. Service life assessment of renders on the basis of changes of physical and mechanical properties during simulated weathering. Constr. Build. Mater. 2019, 229, 117003. [CrossRef]

14. Lagomarsino, S.; Modaressi, H.; Pitilakis, K.; Bosjlikov, V.; Calderini, C.; D’Ayala, D.; Benouar, D.; Cattari, S. PERPETUATE project: The proposal of a performance-based approach to earthquake protection of cultural heritage. Adv. Mater. Res. 2010, 133, 1119-1124. [CrossRef]

15. D'Ayala, D.; Lagomarsino, S. Performance-based assessment of cultural heritage assets: Outcomes of the European FP7 PERPETUATE project. Bull. Earthq. Eng. 2015, 13, 5-12. [CrossRef]

16. Modena, C.; da Porto, F.; Valluzzi, M.R.; Munari, M. Criteria and technologies for the structural repair and strengthening of architectural heritage. Int. J. 3R's-Trans. Repair Restor. Renew. Built Environ. 2013, 4, 606-621.

17. Valluzzi, M.R.; da Porto, F.; Giacometti, G.; Lorenzoni, F.; Modena, C. Knowledge-based data warehouse of interventions for the protection of masonry historical heritage. In Brick and Block Masonry —Trends, Innovations and Challenges, Proceedings of the 16th IB2MaC-International Brick and Block Masonry Conference, Padova, Italy, 26-30 June 2016; Modena, C., da Porto, F., Valluzzi, M.R., Eds.; CRC Press: Boca Raton, FL, USA, 2016; pp. 787-796. [CrossRef]

18. Novelli, V.I.; D'Ayala, D. Use of the knowledge-based system LOG-IDEAH to assess failure modes of masonry buildings, damaged by L'Aquila earthquake in 2009. Front. Built Environ. 2019, 5, 95. [CrossRef]

19. D.P.C (Italian Department of Civil Protection); Work Group for Protection of Cultural Heritage against Natural Risks. Scheda per il Rilievo dei Beni Culturali-Danno alle Chiese-Modello A-DC (in Italian). 2001. Available online: http://www.beniculturali.it (accessed on 18 November 2019).

20. D.P.C (Italian Department of Civil Protection); Work Group for Protection of Cultural Heritage against Natural Risks. Scheda per il Rilievo del Danno ai Beni Culturali-Palazzi, Modello B-DP (in Italian); Ministero per i Beni e le Attivita Culturali e per il Turismo: Rome, Italy, 2006. Available online: http://www.beniculturali.it (accessed on 18 November 2019).

21. M.I.B.A.C.T (Italian Ministry for Cultural Heritage). Direttiva del 23 aprile 2015Procedure per la Gestione delle Attività di Messa in Sicurezza e Salvaguardia del Patrimonio Culturale in caso di Emergenze Derivanti da Calamità Naturali (in Italian). Available online: http://www.beniculturali.it (accessed on 18 November 2019).

22. CNR-ICR. Raccomandazioni NorMaL-1/88. Alterazioni macroscopiche dei materiali lapidei: Lessico. Roma (In Italian). 1990. Available online: https://www.unirc.it/documentazione/materiale_didattico/597_ 2011_287_13564.pdf (accessed on 10 January 2020).

23. UNI 11182. Beni culturali. Materiali lapidei naturali ed artificiali. Descrizione della forma di alterazione. Termini e definizioni (in Italian). 2006. Available online: http://www00.unibg.it/dati/corsi/60057/70709UNI11182.pdf (accessed on 10 January 2020).

24. Grünthal, G.; Musson, R.M.W.; Schwarz, J.; Stucchi, M. European Macroseismic Scale 1998 (EMS-98). Cahiers du Centre Européen de Géodynamique et de Séismologie; Centre Europèen de Géodynamique et de Séismologie: Luxembourg, 1998; Volume 15, p. 99. [CrossRef]

25. Tecnologie per la PROtezione sismica e la VAlorizzazione di Complessi di Interesse culturale. Available online: http://www.stress-scarl.com/en/innovation/projects/provaci.html (accessed on 18 November 2019).

26. Technologies for the Seismic Protection and Valorization of Cultural Heritage. Available online: https: //databaes.dicea.unipd.it/ (accessed on 18 November 2019). 
27. M.I.B.A.C.T. (Italian Ministry for Cultural Heritage). Guidelines for Evaluation and Mitigation of Seismic Risk to Cultural Heritage with Reference to Technical Construction Regulation; Gangemi, A., Ed.; Italian Ministry for Cultural Heritage: Rome, Italy, 2011.

28. Giuffrè, A. Safety and Conservation of Historical Centers: The Ortigia Case; Laterza Press: Rome, Italy, 1993.

29. Doglioni, F.; Moretti, A.; Petrini, V. Churches and Earthquakes; Lint: Trieste, Italy, 1994.

30. Borri, A.; Corradi, M.; Castori, G.; De Maria, A. A method for the analysis and classification of historic masonry. Bull. Earthq. Eng. 2015, 13, 2647-2665. [CrossRef]

31. Valluzzi, M.R.; Sbrogiò, L. Vulnerability of architectural heritage in seismic areas: Constructive aspects and effect of interventions. In Cultural Landscape in Practice. Conservation Vs. Emergencies; Amoruso, G., Salerno, R., Eds.; Lecture Notes in Civil Engineering; Springer: Cham, Switzerland, 2019; Volume 26, pp. 203-218. [CrossRef]

32. Calò, S.; Куковальська, Н.M.; Баратін, Л; Тедескі, К.; Скляр, С.; Володимирівна, Т.; Ольга, М.; Марченко, Г; Анатоліївна, Р.; Ольга, О. Methodological Approach for the Conservative Restoration of Wall Paintings, Frescoes and Mosaics; Серджіо Кало: Kiev, Ukraine, 2016.

33. EwaGlos. European Illustrated Glossary of Conservation Terms for Wall Paintings and Architectural Surfaces Documentation; M. Imhof Verlag: Petersberg, Germany, 2015. Available online: http://openarchive.icomos. org/id/eprint/1706 (accessed on 10 January 2020).

34. ICOMOS. Tangible Risks, Intangible Opportunities: Long-Term Risk Preparedness and Responses for Threats to Cultural Heritage 2013 Theme: Reducing risks to Cultural Heritage from Uncontrolled Development in a Globalized World. ICOMOS International. 2015. Available online: http://openarchive.icomos.org/id/eprint/ 1533 (accessed on 10 January 2020).

35. D'Alpaos, C.; Valluzzi, M.R. Protection of cultural heritage buildings and artistic assets from seismic hazard: A hierarchical approach. Sustainability. under review.

(C) 2020 by the authors. Licensee MDPI, Basel, Switzerland. This article is an open access article distributed under the terms and conditions of the Creative Commons Attribution (CC BY) license (http://creativecommons.org/licenses/by/4.0/). 
Article

\title{
Analysing the Mediating Effect of Heritage Between Locals and Visitors: An Exploratory Study Using Mission Patrimoine as a Case Study
}

\author{
Luc Beal ${ }^{1}$, Hugues Séraphin ${ }^{2}$, Giuseppe Modica ${ }^{3, *}$ and Marco Platania ${ }^{4}$ \\ 1 Excelia Group, La Rochelle Cedex 17024, France; beall@esc-larochelle.fr \\ 2 Department of Marketing, events management, and project management, University of Winchester, \\ Winchester, SO22 5HT, UK; hugues.seraphin@winchester.ac.uk (H.S.); \\ manuela.pilato@winchester.ac.uk (M.P.) \\ 3 Dipartimento di Agraria, Università degli Studi Mediterranea di Reggio Calabria, Località Feo di Vito, \\ I-89122 Reggio Calabria, Italy \\ 4 Department of Educational Sciences, University of Catania, Catania 95100, Italy; marco.platania@unict.it \\ * Correspondence: giuseppe.modica@unirc.it
}

Received: 29 April 2019; Accepted: 23 May 2019; Published: 28 May 2019

\begin{abstract}
The connection among firms and tourists within cultural tourism clusters (CTC) is particularly strong in historical and World Heritage Cities destinations due to the ability of these destinations to contribute to the development of social capital (SC). This ability is explained from the fact there is a strong connection between cultural heritage, identity and sense of belonging. In recent years the meaning of heritage has shifted from national to local importance, based on cultural value rather than on architectural or historical value. Therefore, the participation of local communities is essential in the heritage of sustainable tourism. This allows them not only to express their opinions, but also to actually take part in the processes of planning and management of heritage conservation. Local communities are those that are closely linked to cultural heritage. On the one hand, by applying an ambidextrous management approach to Mission Patrimoine (French lottery launched in 2018 aiming at generating revenue to restore build heritage) the French government has the opportunity to initiate a social capital (SC) initiative associating local stakeholders, namely the local government and the local population, and on the other hand, visitors or tourists. In this paper, a community-based heritage conceptual model is suggested to strengthen the identity sense and to combat the negative effects of tourism. Organisational ambidexterity has been identified as the most suitable approach, due to its ability to contribute to the development of a dialogical spaces. The findings of this research are going beyond the topic of heritage. They are relevant to any research related to sustainability.
\end{abstract}

Keywords: ambidextrous management; creative tourism; tourismphobia and anti-tourism movements; social capital; heritage

\section{Introduction}

Half of the French population is gambling or playing the lottery at least once a year. As a result, gambling and lottery generate 40 billion euro per year, and 100,000 jobs are created [1]. Despite the income generated by the sector and the job creations, Costes et al. [1] are arguing that the money spent by players can not be fully considered as rational, because it does not benefit the wellbeing of the player or the society/community. In this study, the opposite is argued. More specifically, this study is arguing that the lottery, Mission Patrimoine, launched in 2018 by the French government, could play the role of a mediator between the government, the locals and visitors. This is all the more important in the current international context of anti-tourism movements and tourismphobia [2-4]. Additionally, 
Gombault [5], is arguing that there is a gap in the application and research on the topic of creative tourism applied to France.

Additionally, this paper is arguing that Mission Patrimoine could play a role in the creative dimension of tourism in France. Creative tourism is defined as 'travel directed toward an engaged and authentic experience, with participative learning in the arts, heritage, or special character of a place, and it provides a connection with those who reside in this place and create this living culture' ([5], p. 2). Part of the objectives of this research is to establish a link between Mission Patrimoine and creative tourism. Equally important, for the success of Mission Patrimoine, a balance needs to be found between all parties involved in order to get some benefits out of it. When an activity is not beneficial to all parties involved it can lead to conflicts and social issues [6,7].

At this stage, it is important to mention the fact that in English, the word patrimoine means 'heritage'. This paper is therefore establishing a connection between heritage and lottery, what will be more obvious in the following section. France is the most visited destination in the world, as such, the tourism industry is very important for the local economy [8]. Heritage plays a significant role in the competitive advantages of the destination. For instance, "The French gastronomy and 'art de vivre' are among the segment of heritage that contributes to the positive image of the destination abroad [9]. To maintain in good condition its (built) heritage (castles etc.), the French government launched the project Mission Patrimoine. Thus, using Mission Patrimoine as a case study, this paper has two objectives:

- Discuss the limitations of Mission Patrimoine

- Discuss the potential benefits of adopting an ambidextrous management approach for Mission Patrimoine

From a methodological point of view, this paper is based on a qualitative approach. Indeed, this method focuses 'on words rather than numbers in the collection of data' ([10], p. 286). Additionally, in this paper, an inductive approach has been adopted as the study is 'exploring, explaining uncovering phenomena and for generating new theoretical insights' ([11], p. 107). It is also worth adding that on top of being inductive, qualitative research can also be constructivist and interpretivist ([10], p. 286). Because of the topic of this research study (impact of the management of lottery on the interaction among different groups) and based on the fact that the subject considered for this study was recently created (as highlighted before, Mission Patrimoine was created in 2018), this research is already calling for further research, and more specifically for longitudinal studies. As stated by Silver et al. ([12], p. 75) 'The primary objective of longitudinal research is to monitor behaviour over time and thereby identify behavioural or attitudinal changes'. This view is further supported by Costes et al. [1], who carried out some research on lottery (and gambling) in France.

\section{Contextual Framework}

Société Française des Jeux (FDJ, The French Games) is a semi-public company, $72 \%$ owned by the French government. Heir to the French National Lottery created in 1933, the FDJ was founded in 1976, the year when the National Lottery was created. It holds, under the control of the Minister in charge of the budget, the monopoly of lottery and sports of betting games on the French territory, including the overseas territories (Martinique, Guadeloupe, etc.) and Monaco. However, since 2010 online betting activities have been open to competition. In 2018, the French State announced a plan to sell part of the FDJ's capital, to keep only 25 to $30 \%$. In 2015, betting activities made 15 billion euros ( $5 \%$ yearly growth) for a customer base of 26 million and 31,000 points of sale. The company, which employs about 16,000 people, generated gross gaming revenue of 5 billion euros for a net profit of 180 million in 2017, and a contribution to public finances of 3.4 billion euros, or 1\% of the French government's budget.

The first draw of the Heritage Lotto took place on 15 and 16 September 2018 during the European Heritage Days, which granted free access to 12 million visitors to sites such as the Presidential Palace, the Senate, etc. Therefore, the issue of cultural heritage is now a popular cause due to the fact it was 
for long neglected. The idea of a heritage lotto was initiated in 2001 by the Foundation du Patrimoine (heritage foundation), in response to the recurrent observation that it is difficult for the French Ministry of Culture and local governments to maintain a budget sufficient for them to fulfil their heritage conservation mission [13]. The project is inspired by the British Heritage Lottery Fund, which has raised nearly $£ 8$ billion since its inception in 1994, used namely for the restoration of nearly 20,000 historic buildings and monuments.

The FDJ was identified to create and distribute bespoke lottery games aimed at new audience groups, in order to generate new revenues to be earmarked for the restoration of historic and cultural monuments [14]. The Heritage Lotto is designed to raise funds for the Heritage Foundation to ensure the maintenance of monuments. The criteria for allocating the collected funds are the result of a consultation between the Ministry of Culture and the Heritage Foundation: 270 monuments have been designated as beneficiaries, including 18 sites considered emblematic ( 1 site for each of the 18 regions and metropolitan and overseas France territories) appearing on the lotto tickets sold [15]. The choice of beneficiary sites aims at highlighting local heritage in each territory, the vernacular heritage, the threatened heritage (e.g., house of the writer Aimé Césaire on the island of Martinique, in the French Caribbean).

The matter is of the utmost importance in terms of the attractiveness of French destinations, and in general terms of the overall performance of France, as a destination for tourists. Specifically, the French government's objective of welcoming 100 million foreign tourists in 2020 is in fact confronted with the rising constraints induced by a massive number of visitors to certain monuments, natural sites, etc. The General Manager of Atout France, the government agency in charge of promoting France overseas, points out that $80 \%$ of tourists visit Parisian cultural sites along the Seine, now close to saturation for an increasing number of dates during the year. The question also concerns Versailles or Mont-Saint-Michel.

\section{Conceptual Framework}

\subsection{Organisational Ambidexterity and Tourism Management}

Regardless the industry or sector, organisational ambidexterity (OA) is about combining exploratory and exploitative innovation [16]. On one hand, exploratory innovation requires a departure from existing knowledge and implies experimentation [17]; on the other hand, exploitative innovations are incremental and represent refinements of existing knowledge [18]. The topic of OA is closely related to the topics of innovation; performance and sustainability [16]. Indeed, 'Innovation can help firms to improve (1) how they serve an existing consumer base and (2) further exploit their current competencies, in order to gain prolonged benefits ( ... ) from the potential of organisational ambidexterity, as a key driver of sustained performance in the tourism industry' ([16], p. 1-2). Because of the benefits of this management tool, many sectors and/or industries have opted for this mentioned tool. Among these are: higher education; media studies; information technology; human resources management; professional services; leadership; management control system; entrepreneurship; customer service; finance; agriculture; pharmacy; etc. [19]. That said, in tourism and hospitality, the use of OA is extremely limited. In Events and leisure, OA is not used at all. Thorough a systematic literature review of the 52 journals in tourism, hospitality, leisure and events listed in the Association of Business School (ABS) list (now 'Academic Journal Guide', since 2018), reveals that 12 articles have been published between 2014 and 2018 with the words: 'Ambidexterity' or 'Ambidextrous' either in their title and/or keywords. A summary of existing research on $\mathrm{OA}$ in the area of hospitality and tourism is summarised in the table below (Table 1). There is a dearth of research in this area which this work is going to contribute to address. The same could be said about cultural tourism and innovation [20]. 
Table 1. Systematic literature review on organisational ambidexterity (OA) in hospitality and tourism.

\begin{tabular}{cccc}
\hline Area & Author & Year & Journal \\
\hline \multirow{3}{*}{ Destination Management } & Mihalache \& & 2016 & Annals of Tourism Research \\
& Mihalache & 2016 & Int. Journal of Cont. Hosp. Mgt \\
& Martinez-Perez et al. & $2018 \mathrm{a}$ & Journal of Dest. Marketing \& Mgt. \\
& Seraphin et al. & $2018 \mathrm{~b}$ & Journal of Dest. Marketing \& Mgt. \\
\hline Seraphin et al. & 2014 & Inter. Journal of Hosp. Mgt. \\
& Tang & 2015 & Current Issues In Tourism \\
Tsai & 2016 & Cornell Hospitality Quarterly \\
Ubeda-Garcia et al. & 2016 & Inter. Journal of Hosp. Mgt. \\
Cheng et al. & 2017 & Int. Journal of Cont. Hosp. Mgt. \\
& Bouzari \& Karatepe & 2018 & Int. Journal of Cont. Hosp. Mgt. \\
& Wang et al. & 2018 & Int. Journal of Cont. Hosp. Mgt. \\
& Ubeda-Garcia et al. & Inter. Journal of Hosp. Mgt. \\
\hline
\end{tabular}

\subsection{Heritage/Cultural Tourism Management}

Seraphin et al. ([21]: 92) explain that "heritage" "covers a wide range of areas (including culture; history; politics; religions and communications) that can be classified into three main groups: built heritage (forts, relics, etc.); scientific heritage (plants, birds, natural habitats, etc.); and cultural heritage (fine art, customs, languages, etc.)." They also added that: "heritage tourism, which typically falls under the purview of cultural tourism (and vice versa), is one of the most notable and widespread types of tourism and is among the oldest forms of travel." As highlighted by Di Fazio and Modica [22], what ethnologists, anthropologists, geographers, and other researchers in Europe had started documenting, collecting and valorising from the end of the 19th century, today can be seen as a common heritage of largely recognised and consolidated value. Today, heritage should be considered as an economic resource to be used in view of long-term sustainability [22]. Additionally, as a field of research, heritage and tourism is well developed and play a major role in the marketing strategies of many destinations, as central in the attractiveness of destination and satisfaction level of visitors [21]. The topics associated with "heritage" are as follows: sustainability; stakeholders; identity; culture; ecology; sustainability; corporate social responsibility; food; authenticity; identity; politics; tourist consumption; world heritage; local community; environment; and interpretation; lifestyle; attraction; experience; tourist motivation; nostalgia; interpretation; and thanatourism or dark tourism [23]. That said, research associating heritage with marketing is rather limited [2]. The same could be noted about research associating heritage/cultural tourism and OA. Martinez-Perez, Garcia-Villaverde and Elche [20], provided the only paper in the area.

\subsection{Cultural Tourism Clusters}

Cultural tourism clusters (CTCs) "entail engaging networks of collaborative and competitive relationships with other local companies, such as, for example, travel agencies, tour operators and institutions" (Sainaghi \& Baggio, 2014 cited in [20], p. 1485). Moreover, Martinez-Perez, Garcia-Villaverde and Elche [20], explain that the connection among firms and tourists within CTC is particularly strong in historical and World Heritage Cities destinations due to the ability of these destinations to contribute to the development of social capital (SC). This ability is explained from the fact there is a strong connection between cultural heritage, identity and sense of belonging [20]. Putman (2000, cited in [20]) argues that SC puts together two opposites, namely bonding SC (Reciprocity and solidarity between individuals sharing similar characteristics) and bridging SC (connection from networks of different individuals) (Figure 1). As for Martinez-Perez, Garcia-Villaverde and Elche [20], they argue that bonding and bridging SC are complementary (not opposite) and source of innovation. Based on the preceded information, СTC could be related to OA as "CTC are able to drive the 
combination of bonding and bridging capital to develop a strategy from exploration and exploitation of knowledge simultaneously, this would achieve better results in innovation" ([20]: 1491).
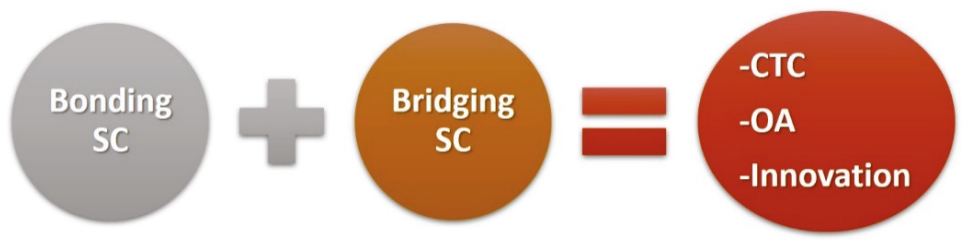

Figure 1. Cultural Tourism Cluster and Organisational Ambidexterity.

\subsection{Hypothesis}

Based on preceded literature, this research paper is going a step further by inferring that within, a bridging SC strategy, can emerge groups that are often presented as heterogeneous. But when combined, the result is an Ambidextrous Recovery Strategy (ARS), which is a source of greater experience and benefits for all stakeholders involved within the cluster. More specifically, this research paper is arguing that by developing the lottery Mission Patrimoine, the French government has the opportunity to initiate a bridging SC strategy. On the one hand, associating local stakeholders, namely the local government and the local population; and on the other hand, visitors or tourists. This segmentation could also be depicted as providers of an experience (local populations and government) and recipients of a service (visitors or tourists). Or even as individuals (locals and tourists) vs government body (FdJ). The trinity locals, FdJ and visitors is made of partners belonging to different spheres, and therefore perfectly fits the characteristics of bridging SC defined by Putnam (2000 cited in [20]). In a nutshell, this study moves from the hypothesis that the different spheres generated within the bridging SC strategy of Mission Patrimoine are complementary rather than opposed dimensions. From this complementarity can emerge the topic of Ambidextrous Recovery Strategy (ARS). Figure 2 summaries the hypothesis and the related process.

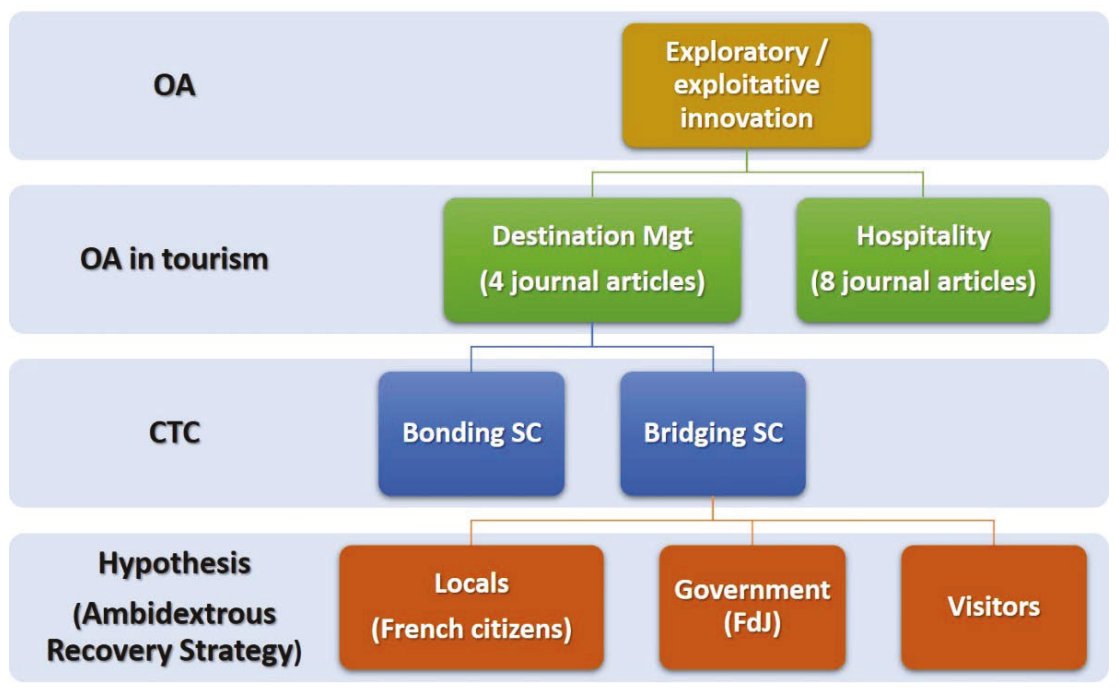

Figure 2. Ambidextrous Recovery Strategy (ARS). 


\section{Methodology}

In this paper, the French Lottery Mission Patrimoine is used as an exploratory case study. A single case exploratory study "focus on one individual, business or organisation to develop research questions and objectives" ([24], p. 43). The case study which "has become increasingly associated with an in-depth exploration of a particular context ( . . ) in order to explain how and why about a phenomenon, (... ) it is literally an example of something, a unit of analysis" ([11], p. 16-18), and has subsequently been used to test the hypothesis indicated in Section 3.4. Indeed, 'there is no reason why case studies, in particular multiple cases, should not be used to test a hypothesis' ([11]: 18). Thus, to address the research question regarding the benefits and limitations of Mission Patrimoine, key stakeholders were interviewed (semi-directive interviews). To some extent, this approach could be assimilated to the Delphi method, in other words, it was implemented on "the construction of a panel of expert and collecting and interpreting experts responses" ([11], p. 43). The interviewees were told that: the results of the study would be used for research purpose; that their answers would be kept anonymous; and finally that they could withdraw from the study at any time. The following paragraph provides an overview of the panel interviewed.

1. A regional director for the Fondation du Patrimoine. This non-profit organization is eligible to receive donations suitable for a tax deduction. This regional director leads an important local network of donors and companies involved in skills-based sponsorship. He has also played an important role in identifying the monuments eligible for the heritage lottery.

2. An academic, specialist of cultural tourism in France, who also happens to be knowledgeable about monuments restoration initiatives in France, as well as the challenges of cultural tourism. This academic is also familiar with the various financing mechanisms existing in France for heritage conservation.

3. The co-founder of a non-profit association, whose purpose is to restore endangered castles and create sustainable communities around them. He also created a crowdfunding platform dedicated to raising funds for buildings that need to be restored. This platform is used by the NGO, as well as by other restoration project leaders. The founder is an expert in strategies for creating and animating communities around heritage sites (digital strategy, communities around restoration sites).

4. The owner of a heritage site. In order to secure funding for the conservation of his domain, he became at creating events aimed at attracting national and foreign visitors, all over the year.

5. The founder of a destination management company (DMC). Specialist of a tourist destination including 2 castles of major touristic and heritage importance, she also leads a local NGO bringing together donors and sponsors in skills.

\section{Results}

\subsection{The Heritage Lottery as a Tool to Raise Funds for the Restoration of Pre-Identified Sites}

They were collected 15 million euros for the benefit of the Mission Patrimoine. To quote the president of FDJ, the "French are [with the Loto du "Patrimoine] twice winners, since they can win as players but also because they will financially support something useful". The amounts collected, supplemented by donations made directly to the Fondation du Patrimoine for the rehabilitation of identified sites, will make it possible to reach a budget of around $€ 35$ million [25]. This amount represents $10 \%$ of the annual budget allocated by the Ministry of Culture to historic monuments. On 15 January 2019, the Mission Patrimoine disclosed the exact amount of funding allocated to each of the 269 earmarked sites: while some project leaders were disappointed by the amounts they would finally receive, others were fully satisfied.

The other sources of funding for the restoration of the sites are both public (city, administrative district, the Ministry of Culture via its regional delegation) and private, namely by way of tax deductible 
sponsorship. The Mission Patrimoine proves to be a powerful vehicle to facilitate the involvement of private stakeholders: financial donations earmarked to the Foundation are automatically recognized as tax-deductible, and donations in kind are also facilitated (for example, a catering service offered for an event aimed at promoting the endangered site, then invoiced to the Mission Patrimoine, is tax exemption eligible in the same way as a financial donation). Despite the absence of a tax rebate, the expectation of a gain with the heritage lottery was a strong enough incentive to secure a significant budget. The sums collected might have been higher if a communication campaign had succeeded in giving greater prominence to the sites, particularly via mass media, of which Stéphane Bern is a specialist.

\subsection{On the Relevance of the List of Sites to Receive the Proceeds from the Heritage Lottery}

Established by the Ministry of Culture and the Heritage Foundation, the list contains sites of significant historical importance, as well as other sites of more questionable historical importance. Greater transparency in the selection process, and perhaps also the involvement of other stakeholders-particularly within local communities-in the selection of sites, could have a significant impact on the amounts collected in future occurrences of the lottery.

\subsection{On its Ability to Nurture the Public's Interest in National Heritage}

The public, whether local residents or not, show little awareness of the beneficiary sites. This first occurrence of the lottery seems to have an effect which limited fundraising for monument restoration, with little effect, on communities, potentially interested in the site. Besides offering tickets in the effigy of the 18 emblematic sites, the lottery could have been more effective in developing the general public's awareness and interest for the 269 restauration projects, for example using TV documentaries. As such, the decision to purchase a lottery ticket might be merely motivated by the hope for winning than the keen intention to sponsor heritage. Because of the large number of tickets sold and the strong notoriety of the project leader, the lottery is nonetheless a potentially powerful means for structuring communities around the designated sites. If "sponsorship in skill" (mécènat de compétence) were to be made a mandatory component of the financing plan, it would result in enhancing the local community's involved and trust in heritage management [26,27], whilst stimulating the local economy and contributing to the preservation of craftsman skills.

\subsection{On the Heritage Lotto's Ability to Contribute to National Heritage Besides the Restoration and Conservation Missions}

If we define heritage as the "features belonging to the culture of a particular society, such as traditions, languages, or buildings, that were created in the past and still have historical importance" (https://dictionary.cambridge.org, accessed on 26 April 2019), such communities are liable to play a role beyond restoration building in order to preserve or even resuscitate events and traditions directly associated with the monument. It is considered regrettable that this first occurrence of the lottery did not play a more important mediating role with the general public. Instead of leading the latter to express a more lasting interest than the ephemeral act of buying a lottery ticket bearing the image of one of the 18 chosen sites. This occurrence of the heritage lottery fails to contribute in building a real community of individuals, close or remote, willing to visit the site, to get involved in a prolonged and tangible way.

Based on the findings of this research, the following section is going to provide some recommendations. Indeed, Section 6.1 is going to provide recommendations and further discussed Sections 5.1 and 5.2. Section 6.2 will address Sections 5.3 and 5.4 . 


\section{Recommendations and Discussions}

\subsection{Building a Community Around the Heritage Site}

A community, geographically defined, which is involved in preserving the heritage associated with a monument, is characterized by the diversity of its stakeholders, as well as the nature and impact of their contributions (Figure 3).

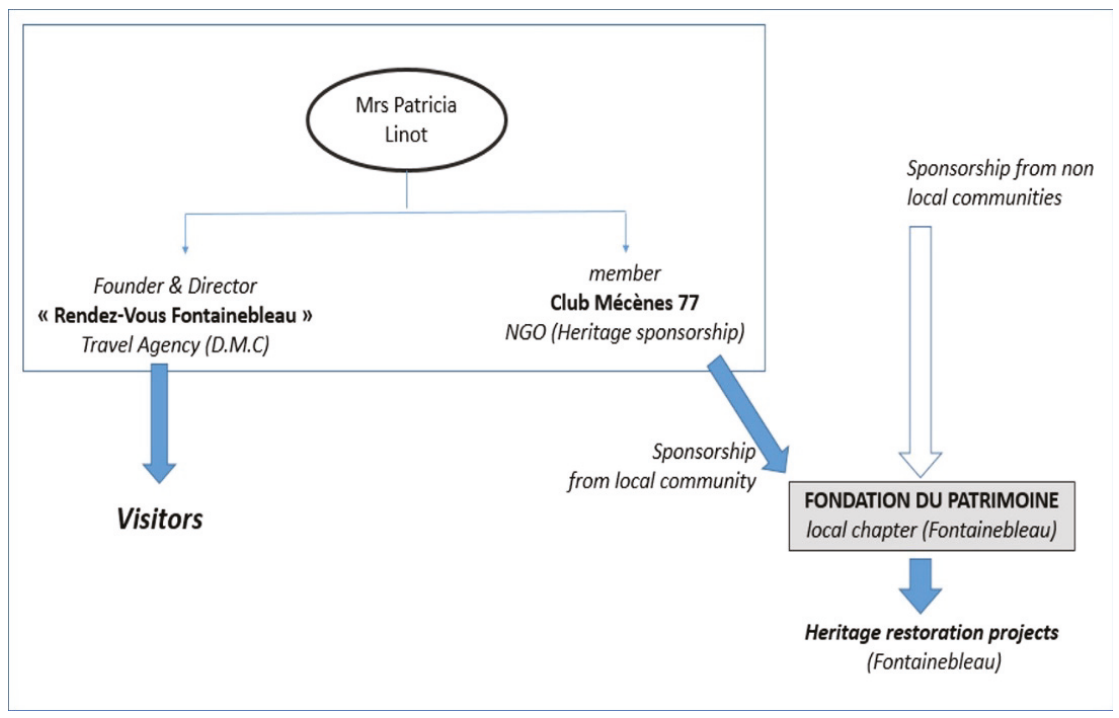

Figure 3. Stakeholders from the tourism industry. A key actor in a local heritage community. Example of the founder of a Destination Management Company (DMC)-Fontainebleau, France.

The first occurrence of the heritage lottery brought members (general public) into a loosely defined community, with a common interest: getting a chance to win than contributing to the restoration. An interesting illustration of successfully engineering community is the one of Adopte un Château (Adopt a Castle). Established in 2015, this French association aiming at restoring castles has laid-out a very specific strategy for collecting funds, intensifying donors' involvement into the project, and, last but not least, attracting stakeholders into the site, or during the restoration phase, or after its completion. With its crowdfunding platform named Dartagnans, the NGO offers to the general public in France and overseas the opportunity to sponsor the restoration of monument from a list of selected sites. This initiative is remarkable in many aspects.

First, the overall communication strategy relies on rich contents (video footages filmed from a drone, etc.) made widely available via video-sharing platforms and abundantly commented by national media. More importantly perhaps, the promise made to the donor is very different from the usual tax-deductible donation scheme: Adopte un Château offers the opportunity to become the owner, amongst many others, of the castle to be restored. Each individual donor is therefore symbolically more profoundly engaged into the restoration project:

"I did not want to let 'my' castle down, the castle from my childhood." (A local resident, during a visit organized by Adopte un Château. Château de l'Ebaupinay: http://bit.ly/2RtI0wV, accessed on 26 April 2019).

By turning donors into committed owners, Adopte un Château carves around each project a community: participation to the restoration, taking part into events aimed at attracting visitors: 
"We will do our best to restore [the castle], and organize events, like in the Middle Age (A local resident, future owner, during a visit organized by Adopte un Château." Château de l'Ebaupinay: http://bit.ly/2RtI0wV accessed on 26 April 2019).

The community is expected to play a large and long lasting role: finance and volunteer restoration work, activities and events to attract visitors. For such communities, bringing back a castle to its original function and attractiveness is as important as restoring it. The relationship between heritage and tourism is well established [28] and it is generally assumed that culture and tourism are interdependent.

The growing interest in cultural resources is likely to benefit culturally rich destinations, and simultaneously to provide the tourism industry with challenges of managing heritage facilities and attractions. Tourism is also an opportunity to generate income for the local community while simultaneously supporting the preservation of its heritage [29].

Stakeholders from the tourism industry are therefore meant to be an important part of such local communities, contributing to the preservation and protection of heritage, whilst incorporating the latter into tourism products and attracting visitors, local or foreign. With local knowledge, expertise and resources, they work in the design and implementation of events, activities, tours, transportation and program logistics.

Together with local chapters of Fondation du Patrimoine (offering the advantage of guaranteeing tax rebates to donor), stakeholders of the tourism industry have a vested interest in the preservation of local cultural heritage, as illustrated in the exhibit above. Another category of stakeholder of the tourism industry are companies managing heritage sites: with the responsibility of bearing the cost of preserving the site, they lay out business models with tourism as the main source of revenue. As an illustration, the company managing the Vaux le Vicomte castle offers a very wide array of events and attractions throughout the year, as well as unique venues for the organization of private events.

The relationship between heritage and tourism is frequently characterized by contradictions and conflicts whereby conservationists perceive heritage tourism as compromising conservation goals for profit [28]. However, the importance of preserving cultural heritage through tourism is receiving increasing attention and has been discussed in relation to sustainable tourism (ibid.). Fostering heritage tourism throughout an extended territory (nationwide) is also likely to efficiently address the rising issue of overtourism and related perverse impacts (tourismphobia and anti-tourism movements) in major cities.

\subsection{Community Based Heritage as a Tool to Reduce Tourismphobia, Anti-Tourism Movements}

The relationship between heritage and the identity of a community makes it possible to address some issues of modern tourism. Here, we talk about the problems related to tourist pressure and its effects.

Public participation in the management of cultural heritage can solve some social conflicts [30] and it also helps to define the meaning of heritage in which the visions of experts and communities are often different [31,32]. As stated by Yung \& Chan [31], in recent years the meaning of heritage has shifted from national to local importance, based on cultural value rather than on architectural or historical value.

It must also be said that the management of cultural heritage has evolved [33] by making public participation an essential element in the decision-making process [34]. Active participation at local level and social relevance are, therefore, dominant characteristics of cultural heritage practice of the recent years [35]. The participation of local communities in heritage management and in planning should extend the involved scientific expertise by adding local experiences that allows to recognize the importance of social judgement [36]. Therefore, the participation of local communities is essential for their involvement in the decision-making process. This allows them not only to express their opinions, but also to actually take part in the processes of planning and managing heritage conservation. Local communities are those that are closely linked to cultural heritage. Heritage conservation 
should be for local communities and they should have the right to decide what and how to maintain and manage.

The presence of a community and its relation with the heritage could develop also a wellbeing condition. Power \& Smyth [37] argue that it is important understanding the space that is created by community-based heritage groups and whether it is likely to promote social welfare. Gleeson [38] state that in the era of globalization and ecological risk it is certainly understandable that there is a new desire for social values based on community, belonging and place. That is what Gleeson call the new social yearning: the desire for a safe place in a social networks based on reciprocity, trust and mutual respect [37].

All those elements represent the formal condition to a social and economic development under sustainable condition. In this sense the tourism could be managed in a sustainable form. The importance of the relationship between community and sustainable tourism has been clearly recognized in the last two decades through studies on community-based tourism (CBT). These communities aim to create a more sustainable tourism industry by focusing on hospitality in terms of planning and maintaining tourism development. This idea came to the fore in the 1990s, with Pearce [39] suggesting that CBT represents a way to provide a fair flow of benefits to all those interested in tourism through consensus-based decision-making and local control of development. The CBT, therefore, proposes a symbolic or reciprocal relationship in which the tourist is not given central priority but becomes an equal part of the system [40]. According to Salazar [41] the most promising niche to develop CBT programs is cultural tourism, one of the major growth markets in global tourism.

Communities can also be based on heritage. Power \& Smyth [37] state that the preservation of community-based cultural heritage refers to the increasingly popular activity of uniting with community members to research local historical "assets". It involves the development of a stronger relationship with your local area and is potentially open to everyone, regardless of location.

Unfortunately, participatory protection and enhancement are often linked only to high-value cultural assets. In relation to the low value attributed to local and less important sites and buildings, there is a tendency to separate communities from their local history. Furthermore, oral traditions and local knowledge which in the past supported a sense of place and it was often associated with specific sites and monuments, it has been eroded by the socio-economic and demographic discontinuities that have occurred in both rural and urban communities over the past years.

In many countries, participatory approaches to heritage conservation and management have had success and are more culturally and socially sustainable than using top-down approaches. Experiences of community involvement are related to the integration among urban planning, conservation and public involvement in the field of heritage, also with a growing role in the decision-making process of the voluntary and community sector [31]. These communities are able to support the tourist pressure and the negative impacts of the overtourism. Actually, the concept of overtourism is not easy to define. Recently, the European Parliament's Committee on Transport and Tourism (TRAN) [42] defined overtourism as "the situation in which the impact of tourism, at certain times and in certain locations, exceeds physical, ecological, social, economic, psychological, and/or political capacity thresholds".

As highlighted by Seraphin et al. [43], local communities are being aware of negative effects caused by overtourism. Therefore, they are increasingly interested in their quality of life rather than simply in the additional income generated by the tourism industry [43]. The consequences of overtourism are witnessed in various forms, namely pollution; littering; destruction of amenity of a place; degradation of landscapes; congestion; vandalism; disorders and anxiety among the local population [44]. Overtourism also causes risks to the UNESCO (United Nations Educational, Scientific and Cultural Organization) World Heritage status of some destinations; negative impacts on the quality of life, loss of identity, etc. [2]. Finally, it is important to remember the crowding out effects between residents' and tourism needs related to high pressure on touristic destinations. That said, Moscardo et al. [45], argued that conflicts between residents and visitors can contribute to the development of 
social capital among local residents who gather within organizations or associations (anti-tourism movements) to defend their shared values and their sense of community and identity.

By involving local communities in heritage management, the benefits can be generated not only for the communities themselves, but also for society as a whole. At social level, community life can be improved. Community participation helps communities to build a sense of identity offering common identity, history and heritage.

Furthermore, social inclusion, cohesion and understanding can be strengthened by promoting a sense of shared responsibility towards the places where people live. Social capital and trust between people and government can be increased [46]. Furthermore, the relationships between not only within the communities but also between generations can be improved. And, above all, it helps the heritage to continue and maintain the value of the heritage of the past in the current life of local communities [47]. In addition to social impacts, through the involvement of local communities, it is possible to create more job opportunities for them. The economic profits of historical tourism can also go directly to the communities. Finally, the fact of being involved in heritage is good for health issues, which is a significant component.

Overall, we can therefore state that a community based heritage is characterized by the fact that it is the same community that has control over the management of tourism and receives a significant part of the benefits generated by this activity [48]. Therefore, this type of tourism has emerged as a possible solution to the negative effects of mass tourism and overtourism, allowing it to simultaneously become a strategy for social organization for the local community.

\section{Conclusions}

\subsection{Key Findings}

Despite the good intention behind Mission Patrimoine, the success could be considered to be mitigated by the fact that apart form the financial aspect, the project has failed to foster a sense of community, hence, the importance of the recommendation formulated in this research. The sense of community developed through the development and fostering of social capital is very important. The key findings of this research are directly related to the topic of sustainability.

\subsection{Theoretical Implications}

Carlisle et al. [49] are arguing that when multi-stakeholders are collaborating on a project, it often leads to innovation and success, which means that they are putting together resources and more importantly a shared vision. They took the example of academic institutions working with private and public sector organisations to support their view. Indeed, they argued that this collaboration led to knowledge creation; innovation; and growth in knowledge-based firms. In particular, they used the Triple Helix model [49] to theorise their findings. 'At the heart of this triangulation is the transfer of knowledge and knowledge management to promote the diffusion of ideas from universities adding value to the private sector via innovation' ([49], p. 62). Furthermore, 'the triple helix model is being widely used as a source of inspiration for policies and programmes aimed at fostering innovation. This is evolving across the range of policymaking at geographical scales, as well as independently of the geographies of context that determine different framework conditions for promoting innovation' ([50], p. 1675). The Helix model has also proven to be a suitable leverage tool particularly in less developed countries [49-51]. The case of Mission Patrimoine, support the fact that the collaboration between the government, locals and tourism (triple helix), can be beneficial for each stakeholders and contribute to the creation of a theoretical and practical dialogical space. In other words, a space (Figure 4) that would create positive relationships between the three groups, what might prevent issues like tourismphobia and anti-tourism movements [4]. 


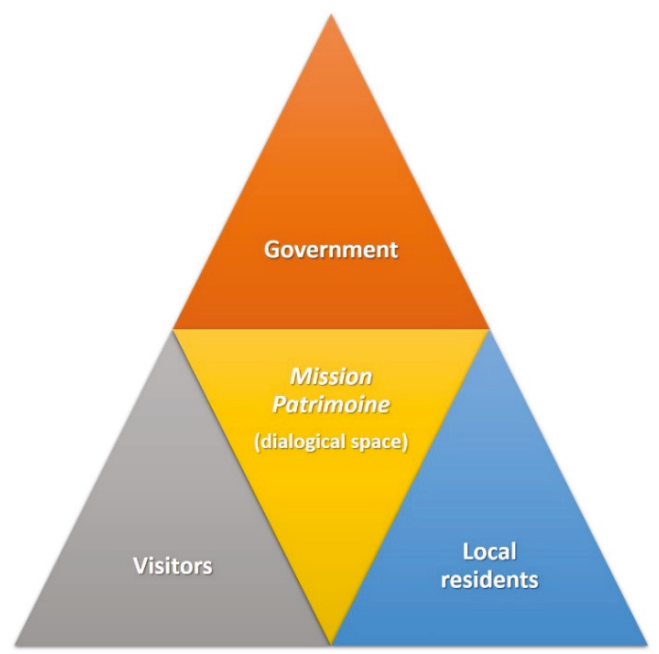

Figure 4. Mission Patrimoine as a dialogical space.

\subsection{Practical Implications}

An ambidextrous management approach applied to the lottery could improve its outcomes and impacts. Among these are:

- Involvement of all stakeholders. Experts disagree on how monuments are selected in order to be restored. They are arguing the lack of transparency in the process. Additionally, not all stakeholders were involved in the selection process. Among these, there are expert in local heritage; or local residents living close to the selected monuments for restoration. Section 7.1 and Figure 4 are clearly highlighting the importance of having all stakeholders working hand in hand. An ambidextrous management approach could help with the development of a dialogical space. Local community involvement offers stronger ties with a common identity, history and heritage. Furthermore, social inclusion, cohesion and understanding can be strengthened by promoting a sense of shared responsibility towards the places where people live.

- Sense of belonging. From the interviews that we carried out, it appears that locals are mainly interested with the financial aspect of the project (winning the lottery), more than the social and cultural aspect. Indeed, in Section 3.3. we highlighted the importance of having a sense of belonging and, as highlighted in Section 7.2, community participation helps communities to build a sense of identity. A communication strategy (as part of an overall ambidextrous management approach) would have helped. Indeed, the organisation Adopte un Château in charge of Vaux Le Vicomte castle is a good example of organisation that has managed to gather locals around a project. Indeed, every member of a local community has the opportunity to invest in a local heritage site and subsequently to be an owner of this attraction. This strategy has contributed to develop a strong connection between the heritage site and the locals. Moreover, Vaux Le Vicomte castle, supports the models developed in Figures 1, 2 and 4.

Author Contributions: Conceptualization, H.S. and L.B.; Formal analysis, H.S., G.M., M.P. (Marco Platania) and M.P. (Manuela Pilato); Methodology, L.B. and H.S.; Supervision, H.S., L.B. and M.P. (Marco Platania); Writing—original draft, H.S., L.B. M.P. (Marco Platania) and G.M.; Writing—review \& editing, H.S., G.M. and M.P. (Marco Platania).

Funding: This research received no external funding. 
Acknowledgments: The authors are gratefully to all stakeholders who kindly participated in the survey. The Authors would like to express their great appreciation to the three anonymous reviewers for their very helpful comments provided during the revision of the paper.

Conflicts of Interest: The authors declare no conflicts of interest.

\section{References}

1. Costes, J.-M.; Massin, S.; Etiemble, J. Première Évaluation de l'Impact Socio-Économique des Jeux d'Argent et de Hasard en France; Observatoire des jeux, Ministère de l'économie et des Finances: Paris, France, 2014.

2. Seraphin, H.; Sheeran, P.; Pilato, M. Over-tourism and the fall of Venice as a destination. J. Destin. Mark. Manag. 2018, 9, 374-376. [CrossRef]

3. Seraphin, H.; Gowreesunkar, V.; Zaman, M.; Bourliataux-Lajoinie, S. Community based festivals as a tool to tackle tourismphobia and antitourism movements. J. Hosp. Tour. Manag. 2018. [CrossRef]

4. Séraphin, H.; Zaman, M.; Olver, S.; Bourliataux-Lajoinie, S.; Dosquet, F. Destination branding and overtourism. J. Hosp. Tour. Manag. 2019, 38, 1-4. [CrossRef]

5. Gombault, A. Tourisme et création: Les hypermodernes. Mondes Tour. 2011, 4, 18-35. [CrossRef]

6. Haifeng, Y.; Jing, L.; Mu, Z. Rural community participation in scenic spot. A case study of Danxia Mountain of Guangdong, China. J. Hosp. Tour. 2012, 10, 76-112.

7. Järvinen-Tassopoulos, J. Les jeux d'argent: Un nouvel enjeu social? Pensée Plurielle 2010. [CrossRef]

8. World Tourism Organization. UNWTO Annual Report 2017; World Tourism Organization (UNWTO): Madrid, Spain, 2018.

9. Seraphin, H. Terrorism and tourism in France: The limitations of dark tourism. Worldw. Hosp. Tour. Themes 2017, 9, 187-195. [CrossRef]

10. Quinlan, C.; Babin, B.J.; Carr, J.; Griffin, M.; Zikmund, W.G. Business Research Methods, 1st ed.; Cengage Learning EMEA: Hampshire, UK, 2015.

11. Hammond, M.; Wellington, J.J. Research Methods: The Key Concepts; Routledge Key Guides; Routledge: London, UK; New York, NY, USA, 2013.

12. Silver, L.; Stevens, R.; Wrenn, B.; Loudon, D. The Essentials of Marketing Research, 3rd ed.; Routledge: Abingdon/Oxford, UK, 2012.

13. Ministère de la Culture. Création d'un Loto du Patrimoine. Available online: http: //www.culture.gouv.fr/Presse/Archives-Presse/Archives-Communiques-de-presse-2012-2018/Annee2017/Creation-d-un-loto-du-patrimoine (accessed on 23 April 2019).

14. Française des Jeux Mission Patrimoine. Available online: https://www.groupefdj.com/actualite/une-offre-dejeux-pour-sauver-le-patrimoine-francais.html (accessed on 26 April 2019).

15. Ministère de la Culture. List of Sites and Number of Visitors. Available online: http://www.culture. gouv.fr/content/download/170070/1890806/version/2/file/20170917_MC_CP-bilan-JEP-2017.pdf (accessed on 23 April 2019).

16. Mihalache, M.; Mihalache, O.R. Organizational ambidexterity and sustained performance in the tourism industry. Ann. Tour. Res. 2016, 56, 142-144. [CrossRef]

17. Levinthal, D.A.; March, J.G. The myopia of learning. Strateg. Manag. J. 1993, 14, 95-112. [CrossRef]

18. Jansen, J.J.P.; Van Den Bosch, F.A.J.; Volberda, H.W. Exploratory Innovation, Exploitative Innovation, and Performance: Effects of Organizational Antecedents and Environmental Moderators. Manag. Sci. 2006, 52, 1661-1674. [CrossRef]

19. Séraphin, H.; Butcher, J. Tourism Management in the Caribbean. Caribb. Q. 2018, 64, 254-283. [CrossRef]

20. Martínez-Pérez, Á.; García-Villaverde, P.M.; Elche, D. The mediating effect of ambidextrous knowledge strategy between social capital and innovation of cultural tourism clusters firms. Int. J. Contemp. Hosp. Manag. 2016, 28, 1484-1507. [CrossRef]

21. Seraphin, H.; Yallop, A.C.; Capatîna, A.; Gowreesunkar, V.G. Heritage in tourism organisations' branding strategy: The case of a post-colonial, post-conflict and post-disaster destination. Int. J. Cult. Tour. Hosp. Res. 2018, 12, 89-105. [CrossRef]

22. Di Fazio, S.; Modica, G. Historic Rural Landscapes: Sustainable Planning Strategies and Action Criteria. The Italian Experience in the Global and European Context. Sustainability 2018, 10, 3834. [CrossRef] 
23. Séraphin, H.; Smith, S.M.; Scott, P.; Stokes, P. Destination management through organisational ambidexterity: Conceptualising Haitian enclaves. J. Destin. Mark. Manag. 2018, 9, 389-392. [CrossRef]

24. Brunt, P.; Horner, S.; Semley, N. Research Methods in Tourism, Hospitality and Events Management, 1st ed.; SAGE Publication: Thousand Oaks, CA, USA, 2017.

25. La Croix Succès pour le Tirage du Loto du Patrimoine. Available online: http://bit.ly/LaCrxLotoPatrimoine (accessed on 23 April 2019).

26. World Heritage and Sustainable Development. New Directions in World Heritage Management; Larsen, P.B., Logan, W., Eds.; Routledge: London, UK, 2018.

27. Li, Y.; Hunter, C. Community involvement for sustainable heritage tourism: A conceptual model. J. Cult. Herit. Manag. Sustain. Dev. 2015, 5, 248-262. [CrossRef]

28. Alazaizeh, M.M.; Hallo, J.C.; Backman, S.J.; Norman, W.C.; Vogel, M.A. Value orientations and heritage tourism management at Petra Archaeological Park, Jordan. Tour. Manag. 2016, 57, 149-158. [CrossRef]

29. Aas, C.; Ladkin, A.; Fletcher, J. Stakeholder collaboration and heritage management. Ann. Tour. Res. 2005, 32, 28-48. [CrossRef]

30. Sirisrisak, T. Conservation of Bangkok old town. Habitat Int. 2009, 33, 405-411. [CrossRef]

31. Yung, E.H.K.; Chan, E.H.W. Problem issues of public participation in built-heritage conservation: Two controversial cases in Hong Kong. Habitat Int. 2011, 35, 457-466. [CrossRef]

32. Pignataro, G.; Rizzo, I. The Political Economy of Rehabilitation: The case of the Benedettini Monastery. In Economic Perspectives on Cultural Heritage; Palgrave Macmillan UK: London, UK, 1997; pp. 91-106.

33. Hobson, E. Conservation and Planning; Routledge: London, UK, 2003.

34. Clarck, K. From Regulation to Participation: Cultural heritage, sustainable development and citizenship. In Forward Planning: The Function of Cultural Heritage in a Changing Europe; Council of Europe: Strasbourg, France, 2000; pp. 103-110.

35. Heritage, Conservation and Communities; Chitty, G. (Ed.) Routledge: Abingdon/Oxford, UK, 2016.

36. Modica, G.; Zoccali, P.; Di Fazio, S. The e-Participation in Tranquillity Areas Identification as a Key Factor for Sustainable Landscape Planning. In Computational Science and Its Applications-ICCSA 2013; Murgante, B., Misra, S., Carlini, M., Torre, C.M., Nguyen, H.-Q., Taniar, D., Apduhan, B.O., Gervasi, O., Eds.; Lecture Notes in Computer Science; Springer: Berlin/Heidelberg, Germany, 2013; Volume 7973, pp. 550-565.

37. Power, A.; Smyth, K. Heritage, health and place: The legacies of local community-based heritage conservation on social wellbeing. Health Place 2016, 39, 160-167. [CrossRef]

38. Gleeson, B. Deprogramming planning: Collaboration and inclusion in new urban development. Urban Policy Res. 2004, 22, 315-322. [CrossRef]

39. Pearce, D.G. Alternative tourism: Concepts, classifications, and questions. In Tourism Alternatives: Potentials and Problems in the Development of Tourism; Eadington, W.R., Smith, V.L., Eds.; University of Pennsylvania Press: Philadelphia, PA, USA, 1992; pp. 15-30.

40. Wearing, S.; McDonald, M. The Development of Community-based Tourism: Re-thinking the Relationship Between Tour Operators and Development Agents as Intermediaries in Rural and Isolated Area Communities. J. Sustain. Tour. 2002, 10, 191-206. [CrossRef]

41. Salazar, N.B. Community-based cultural tourism: Issues, threats and opportunities. J. Sustain. Tour. 2012, 20, 9-22. [CrossRef]

42. Peeters, P.; Gössling, S.; Klijs, J.; Milano, C.; Novelli, M.; Dijkmans, C.; Eijgelaar, E.; Hartman, S.; Heslinga, J.; Isaac, R.; et al. Research for TRAN Committee-Overtourism: Impact and Possible Policy Responses; European Parliament, Policy Department for Structural and Cohesion Policies: Brussels, Belgium, 2018.

43. Séraphin, H.; Platania, M.; Spencer, P.; Modica, G. Events and Tourism Development within a Local Community: The Case of Winchester (UK). Sustainability 2018, 10, 3728. [CrossRef]

44. Singh, T.V. Is over-tourism the downside of mass tourism? Tour. Recreat. Res. 2018, 43, 415-416.

45. Moscardo, G.; Konovalov, E.; Murphy, L.; McGehee, N.G.; Schurmann, A. Linking tourism to social capital in destination communities. J. Destin. Mark. Manag. 2017, 6, 286-295. [CrossRef]

46. Labadi, S. Impacts of culture and heritage-led development programmes. In Urban Heritage, Development and Sustainability. International Frameworks, National and Local Governance; Labadi, S., Logan, W., Eds.; Routledge: London, UK, 2015; pp. 137-150. 
47. Huong, P.T.T. Living heritage, community participation and sustainability. Redefining development strategies in the Hoi An Ancient Town World Heritage property, Vietnam. In Urban Heritage, Development and Sustainability. International Frameworks, National and Local Governance; Labadi, S., Logan, W., Eds.; Routledge: London, UK, 2015; pp. 274-290.

48. Trejos, B.; Chiang, L.-H.N. Local economic linkages to community-based tourism in rural Costa Rica. Singap. J. Trop. Geogr. 2009, 30, 373-387. [CrossRef]

49. Carlisle, S.; Kunc, M.; Jones, E.; Tiffin, S. Supporting innovation for tourism development through multi-stakeholder approaches: Experiences from Africa. Tour. Manag. 2013, 35, 59-69. [CrossRef]

50. Rodrigues, C.; Melo, A.I. The Triple Helix Model as Inspiration for Local Development Policies: An Experience-Based Perspective. Int. J. Urban Reg. Res. 2013, 37, 1675-1687. [CrossRef]

51. Saad, M.; Zawdie, G. Triple helix in developing countries-Issues and challenges. Technol. Anal. Strateg. Manag. 2008, 20, 649-652. [CrossRef]

(C) 2019 by the authors. Licensee MDPI, Basel, Switzerland. This article is an open access article distributed under the terms and conditions of the Creative Commons Attribution (CC BY) license (http://creativecommons.org/licenses/by/4.0/). 



\title{
How to Assess Urban Regeneration Proposals by Considering Conflicting Values
}

\author{
Stefano Capolongo, Leopoldo Sdino, Marta Dell'Ovo *, Rossella Moioli and Stefano Della Torre \\ Department of Architecture, Built environment and Construction Engineering (A.B.C.), Politecnico di Milano, \\ 20133 Milan, Italy; stefano.capolongo@polimi.it (S.C.); leopoldo.sdino@polimi.it (L.S.); \\ rossella.moioli@polimi.it (R.M.); stefano.dellatorre@polimi.it (S.D.T.) \\ * Correspondence: marta.dellovo@polimi.it; Tel.: +39-02-2399-5140
}

Received: 11 June 2019; Accepted: 4 July 2019; Published: 17 July 2019

\begin{abstract}
Urban regeneration has to be based on rigorous methodological frameworks able to find a balance among preservation instances, economic development, urban quality and the well-being of the population. Considering these premises, this research is focused on the definition of the decision-aiding process for the reuse of an abandoned health care facility with several historic buildings. Both public and private interests have been taken into consideration, since they play an important role for the urban regeneration project and for the definition of urban regeneration policies. Given the complexity of this issue, the evaluation process has been structured by combining different methodologies to support the policy cycle: Stakeholder Analysis, to identify the actors engaged (Social sustainability); Nara Grid for the values elicitation of the Built Cultural Heritage (Cultural and environmental sustainability); and the subsequent definition of different sustainable scenarios evaluated by the Discounted Cash Flow Analysis (Economic sustainability). Four alternatives have been assessed with the support of a Multi-Criteria Analysis (MCA) aimed at defining the most balanced one considering heritage significance retention and urban regeneration. This work contributes to the literature on soft OR by exploring interactions among different stakeholders and addresses policy instances by providing a transparent methodology based on value elicitation.
\end{abstract}

Keywords: territorial health center; urban regeneration; Multi-Criteria Analysis; built cultural heritage; values

\section{Introduction}

The complexity present in decision problems is given by the coexistence of sometimes conflicting values elicited by stakeholders involved in the process [1]. To overcome and facilitate complex and uncertain situations, Problem Structuring Methods (PSMs) are widely used by soft OR scholars [2] in order to support the interaction among stakeholders [3] in managing ill-structured problems [4,5].

When the problem has to face an urban regeneration processes, the complexity is the result of the contribution of different dimensions and layers embedded, such as urban, cultural, social and economic [6]. Moreover, these urban interventions not only have interdependent actors with individual and common goals, but they are also the result of public and private interest and internal political dynamics [7]. What deserves to be further analyzed in this context concerns the externalities generated by these processes from which the community can benefit and that can have positive effects by improving the general well-being of the area, also considering the economic side [6]. In fact, as has been suggested by [8], problems concerning architecture choices are characterized by direct consequences on both the territory and the society. In addition, when the urban regeneration process is placed in a context characterized by cultural heritage, the complexity is the result of tangible and intangible values that deserve to be preserved and maximized [9]. The role and power of cultural policy and cultural heritage in promoting and driving urban regeneration programs [10-13] by the recognition of 
strategies able to integrate both conservation and innovation has been widely discussed. According to [11], the key to a sustainable and successful process is given both from a balance between public and private parties and from the reconciliation between tradition and innovation. In detail, [13] recognized the presence of four different approaches aimed at enhancing cultural heritage that are able to support city regeneration:

1. policy to develop high-profile projects: aimed at attracting private investment and to redesign the image of the city;

2. policy to promote cultural policymaking: aimed at promoting bottom-up revitalization processes;

3. policy for cultural industries: aimed at exploiting arts and artists to design and promote cultural quarters with a high concentration of cultural and entertainment facilities;

4. policy for urban heritage: aimed at fostering the potentialities of historical resources by developing tourism activities and conserving the sense of place.

This complexity and the presence of conflicting preferences makes decision support methods structured to collect information by the stakeholders involved necessary, in order to better shape the problem [8]. The objective of the current contribution is to present the results of a case study based on the regeneration of a disused area in the Municipality of Vimercate (MB), Italy, where several dimensions have been taken into consideration. In detail, the paper aims at developing an evaluation process structured by combining different methodologies in order to support the Decision Maker (DM) in taking complex decisions when conflicting values are involved. Values engaged in the process consider both tangible (Functional and Economic Sustainability) and intangible aspects (Environmental and Socio-Cultural Sustainability). Another aspect considered in the research has been the investigation of the most suitable location for the new territorial health center within the areas of the "former hospital of Vimercate" to promote its urban development; indeed, the site hosts an abandoned health care facility and historic buildings. In fact, in this context, it is important to explore not only how the design of the project can achieve the objective of the work but also how different combinations of functions can better exploit the potentialities of the area. Complementarity and compatibility are two key concepts analyzed in order to develop a conscious mixitè by boosting both the attractiveness of investment, considering the economic dimension, and limiting the presence of incompatible uses to protect sensitive users, considering the social dimension. Here, private and public interests play a strategic role in the definition of its regeneration process and, as has already been stressed by [8], these kind of interventions are able to shape both the society and the territory, which implies that the decisions should be transparent and justified in order to be communicated to the citizens involved.

This paper is organized into five sections. The first part presents the theoretical background of the methodological framework proposed by explaining its main phases; the case study aims at illustrating the area where the project has to be developed; in the third section, the methods are explained and then, in the fourth section, the results are presented and discussed. In the last section, the policy implications and potentialities of the approach proposed are further explained and validated.

\section{Methodological Approach}

The evaluation framework proposed combines different methodologies in order to include several aspects in the analysis and with the aim to consider the multi-dimensional characteristics of the problem. In detail, as has been represented in Figure 1, the approach is structured according to three phases: i) intelligence, ii) design and iii) decision [14]. In the i) intelligence phase, the state of the art is analyzed by considering the actors involved, the current situation of the $\mathrm{BCH}$ and by investigating real case studies. In the ii) design phase, a set of alternatives is proposed as the result of the previous analysis, and to conclude, in the iii) decision phase, alternatives are evaluated.

In detail, considering the first phase, it is important to highlight how the Stakeholders Analysis allows understanding and prioritizing actors engaged in the process; in fact, by performing the power/interest matrix, it has been possible to clarify which actors deserve to be mostly satisfied since 
they have a direct relation with the project [15]. Once identified, the categories of actors with a key role in the decision problem, their needs and expectations have been elicited [16]; this phase is strategic in order to design alternatives in the second phase that are able to represent and meet their demands. This step has been combined with the Nara Grid, aimed at defining artistic, historic, social, scientific/cultural and economic values expressed by the built cultural heritage (BCH) [17], and able to identify most compatible uses for each building considering its typology. The last method of the intelligence phase implies a comparative analysis of case studies, where existing territorial health centers have been deeply investigated in order to fully comprehend their functional aspects, both intrinsic and extrinsic.

The output of the first phase, becomes the object of investigation of the second one. In fact, the three analyses previously described, combined together, resulted in the design of a set of alternatives aimed at regenerating the area under analysis by considering all the aspects elicited in the introduction. The alternatives considered have been obtained by the interaction with the actors involved, compliance with the regulations and the limits given by the conservation and use of historical buildings and their level of authenticity.

The last phase concerned the evaluation of alternatives generated. It has been carried on, first of all, under an economic point of view, performing a Discounted Cash Flow (DCF) analysis and afterwards under a multi-dimensional point of view, with the support of the Multi-Criteria Analysis (MCA). In detail, a value tree divided into criteria and sub-criteria has been developed in order to assess which one, among the set of alternatives, was the most satisfying by considering the following dimensions: 1. Functional Sustainability; 2. Socio-Cultural Sustainability; 3. Environmental Sustainability; 4. Economic Sustainability. Moreover, a Sensitivity Analysis has been performed in order to validate the results obtained. The methodological flowchart described is a first attempt to combine multi-disciplinary methodologies belonging to different fields of research with the aim to not disregard any important dimensions.

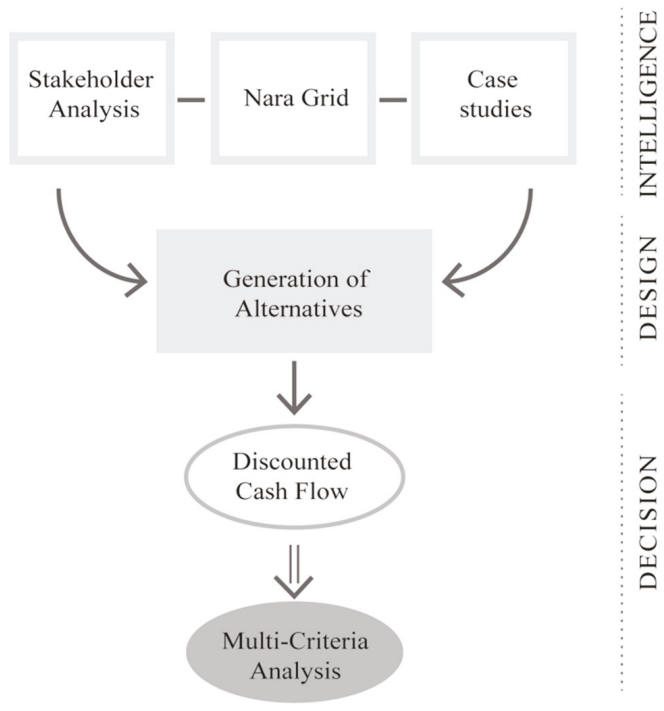

Figure 1. Methodological flowchart.

\section{Case Study}

Given the aim of the research to define the most suitable location, inside the site of the "former hospital of Vimercate", for a new territorial health center by considering the urban regeneration, the $\mathrm{BCH}$ and the economic attractiveness of the development, it is necessary to understand the context of 
where the area is located. In fact, the site analyzed in the context of this paper, and a priori identified by the Municipality to host this intervention, is located in the city of Vimercate (MB), in the Lombardy region, $25 \mathrm{~km}$ far from Milan, Italy (Figure 2).

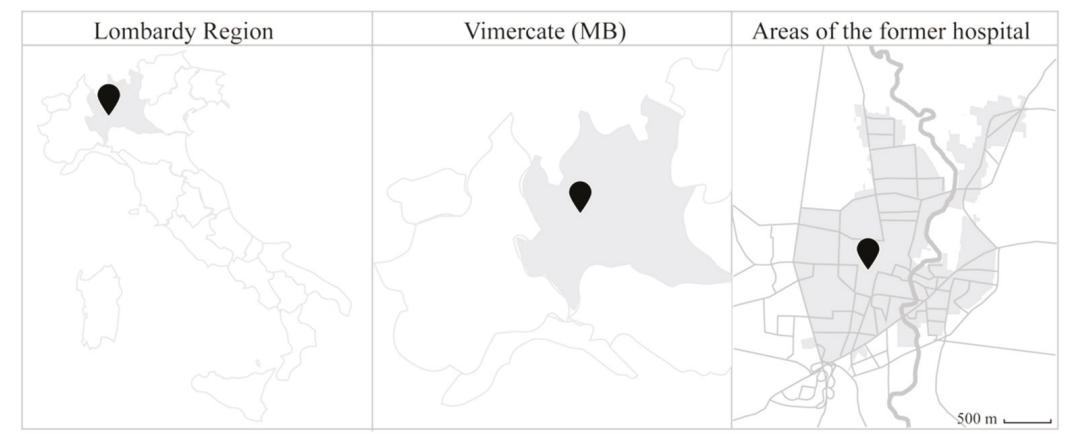

Figure 2. Location of the area.

The area of the "former hospital" consists of two parts: in the first, there is a large building called a mono block, some service buildings and three small villas; in the second, there are some historic buildings and a church. Currently it emerges that only two buildings are used, and they host a psycho-social center and the house of the chaplain.

The analysis detected its central position with respect to the municipal territory and its importance as part of the historical fabric of the city.

The analysis of the context allowed to focus in detail on:

- $\quad$ investigating the accessibility and identifying existing services;

- $\quad$ exploring the historical stratification of the area and of its buildings.

For what concerns the first focus, the area is accessible, by both private and public transport, and it is also well served by main services, e.g., schools, post offices, supermarkets, restaurants, while it is not very close to green areas and public offices.

The second focus underlined its high historical value since it is in proximity to the ancient core of the city; in fact, there are buildings characterizing the ancient nucleus of the town that are of medieval origin, which also determines the perception of continuity with the historic center.

\section{Multi-Dimensional Evaluation}

In this section, the methodological approach previously presented is applied to the case study. In detail, it is organized by following the structure proposed by [14] concerning the division of a decision problem in three main stages.

\subsection{Intelligence Phase}

This part considers a deep analysis of the state of the art, which means understanding the actors involved, the current state of the area and the existing case studies.

\subsubsection{Stakeholder Analysis}

It is important to underline that the area is part of a broader Urban Development Agreement (UDA) signed between the Lombardy Region, the Hospital of Vimercate- the current owner of the area-and the Municipality. Moreover, several developers can be identified as interested in investing in its regeneration and others can also be considered since they are affected by the impact of the project. It is possible to mention people living nearby the site, the entire population of the Municipality 
of Vimercate, local traders and the University Politecnico di Milano, the support of which has been requested by the Municipal Administration in order to conduct research about the proposal of an urban regeneration model. In Figure 3, the matrix of power/interest [15] is presented, considering different roles played by the stakeholders previously identified and their interest in this intervention.

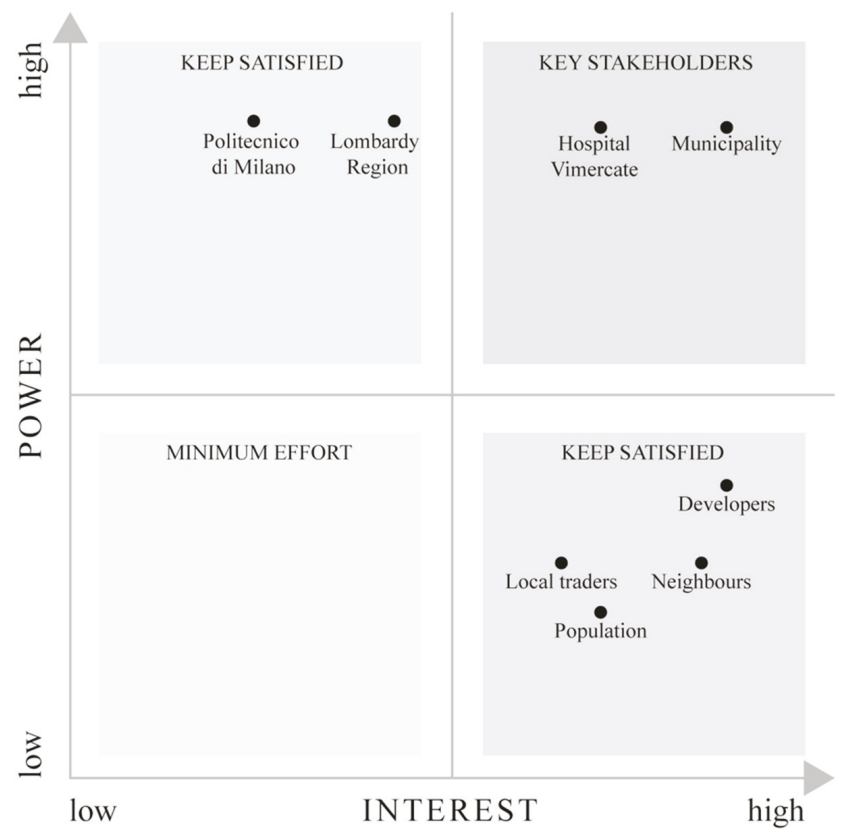

Figure 3. The matrix of power/interest developed.

The position of different stakeholders is in relation to the case study analyzed and depends on the different consultations organized. Since the Municipality is in charge of the final decision and the Hospital of Vimercate is the owner of the area, they have been identified as key stakeholders. Furthermore, since in Italy decisions about health management are taken by regions, the Lombardy Region holds much power, but its interest lays in its bureaucratic role. Politecnico di Milano has a relevant role since it is in charge of developing a feasibility study and evaluating different scenarios; its decision can strongly affect the key stakeholders, but it does not have a specific interest on this case study, while developers, local traders, neighbors and the population of the Municipality of Vimercate have a strong interest in this specific project since it is going to affect their daily life, but they have lower power in influencing the final decision. The position of developers is higher compared to the other "keep satisfied" actors, for what concerns the power since they manage economic resources [15].

\subsubsection{Nara Grid}

To define the threshold of transformability and compatibility with a range of uses and functions suitable for urban regeneration, an analytic approach has been implemented based on a broader understanding of the cultural values to be protected and the related strategies.

The Nara Grid has been developed based on the Nara Documents on Authenticity [18] to support in evaluating the multi-layered concept of authenticity $[19,20]$. This methodology has been implemented in order to identify the systemic relations of the area with the building fabric. In detail, for each historic building located inside the site under evaluation, a qualitative table has been developed aimed to define their performance against the artistic, historic, social, scientific/cultural and economic values. 
To fulfil the description, a detailed survey and investigation of the historic buildings was also required, leading to recognition of some of the buildings and the relics of the medieval hospital-that is a value definitely worth protecting and taking into account in the urban policies.

The analysis enabled the highlighting of the potential of the $\mathrm{BCH}$ in order to inspire functions, which could be more feasible in the regeneration process, because of the easy match of the functional requirements with the actual performances offered by historic buildings. Thus, among the selected scenarios, it has been possible to design alternatives oriented to consider profitable facilities with limited transformation required in order to minimize the cost, while enhancing the conservation of the authentic historical structures.

\subsubsection{Case Studies}

Ten territorial health centers in the national context and seven in the European context have been analyzed in order to underline best practices and to elicit intrinsic and extrinsic characteristics to consider [21]. The sample has been selected by considering the year of the projects-in fact the most recent ones have been chosen - and those placed in a territorial context comparable to the Municipality of Vimercate. In detail, the investigation allowed us to elicit important criteria able to support the design phase, both regarding the location and the outdoor spaces, e.g., accessibility, proximity to residential areas, proximity to green areas, flexibility, etc., and regarding the indoor spaces, e.g., harmonization with context, accesses, architectural barriers, flexibility of the structure, natural light, etc. This part allowed us to understand how territorial health centers should be designed and in which context they can be located. In fact, this analysis gave us the possibility to develop a comparative table where both intrinsic and extrinsic characteristics have been evaluated to assess trends and best practices and specifically pros and cons, with the aim to frame notions to guide the design and the location phase.

\subsection{Design Phase}

The previous phase supported the generation of different alternatives [22,23]. They have been agreed with the Municipality and with the parties involved in the enhancement of the area; in particular, they have been designed with the possibility of recovering and using the buildings that housed the former hospital or with the possibility of creating a new building to accommodate the new territorial health centers.

Alternatives have been generated by eliciting the needs and expectations of stakeholders engaged in the decision problem, trying to satisfy the main important ones identified through the matrix of power/interest, by the transformability threshold detected for each building according to the function to allocate and by the criteria to consider during the design phase, resulting from the investigation of existing case studies.

In detail, four alternatives, in addition to the business as usual scenario, have been generated:

- Scenario_0: Existing situation;

- Scenario_1: Masterplan proposed by the UDA, territorial health centers located on the ground and first floors of the former hospital building;

- Scenario_2: Territorial health centers located on the first and second floors of the former hospital building;

- Scenario_3: Territorial health centers located in a new building close to the villas in C. Battisti street;

- Scenario_4: Territorial health centers located in a new building close to the entrance of Ospedale street.

In Figure 4, it is moreover possible to understand other functions characterizing each scenario and, selected together with the Municipality, in order to regenerate the whole area. 

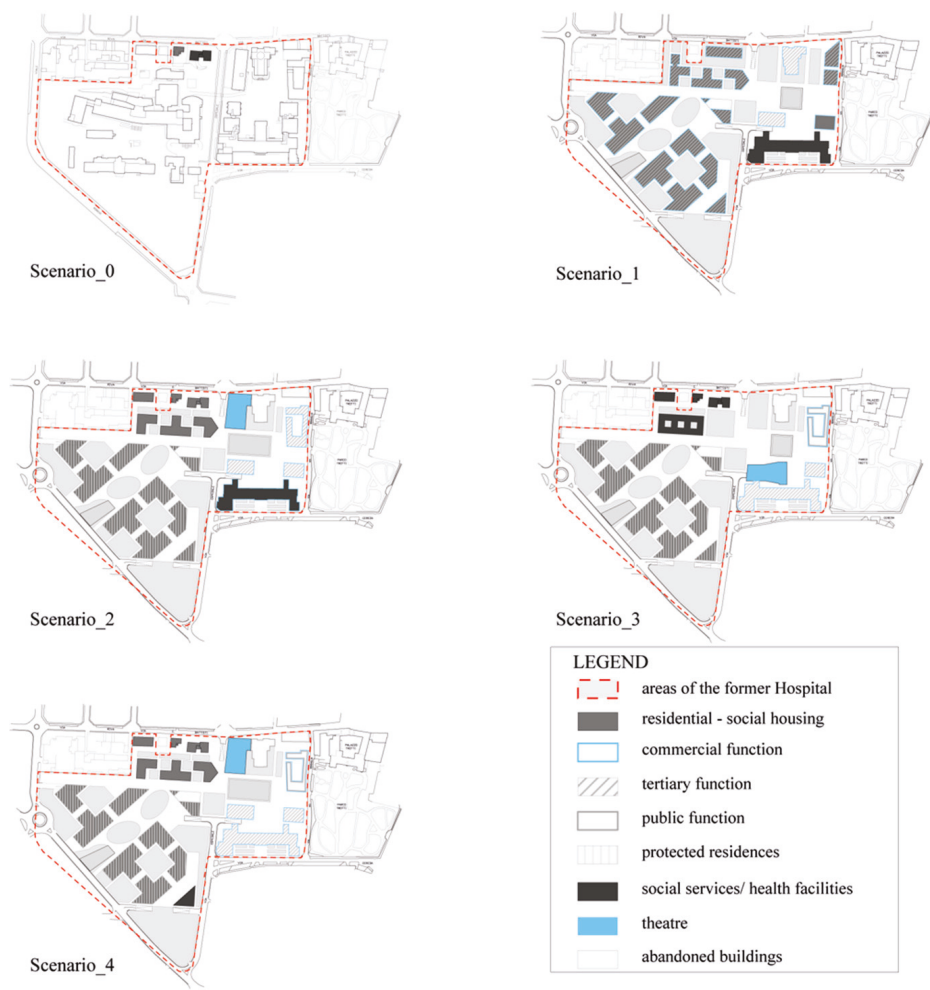

Figure 4. Masterplan of scenarios defined.

\subsection{Decision Phase}

The first phase of the evaluation of the alternatives developed considered the economic dimension, while the second one multi-dimensional aspects.

\subsubsection{Discounted Cash Flow Analysis (DCF)}

A DCF analysis has been performed by considering the value generated by each intervention. It has to be specified that the analysis has been carried out on a desktop in a parametric form and could be further explored by better defining the functions, also in relation to the indications of the Administrations involved. The results will be also presented in Table 2, but it is possible to highlight that Scenario 2 and 3 performed with a higher value given by their functional mix, followed by Scenario 1 and Scenario 0 . Since the area is actually abandoned, it does not generate value.

\subsubsection{Multi-Criteria Analysis}

Considering the coexistence of different objectives, which have been elicited by the needs and expectations of the actors involved, and by considering values expressed by the $\mathrm{BCH}$, it has been decided to perform an MCA in order to take into consideration this complexity [24]. The decision problem has been structured into four criteria, further divided into sub-criteria. The framework has been developed by both taking into account the final objective of selecting the most sustainable project and combining phases developed during the intelligence phase.

Table 1 presents the value tree defined and there is also information about the nature of the sub-criteria selected. 
Table 1. Value tree.

\begin{tabular}{lll}
\hline Criteria & Sub-criteria & Nature \\
\hline \multirow{3}{*}{ 1. Functional Sustainability } & 1.1 Flexibility & qualitative \\
\cline { 2 - 3 } & 1.2 Usability/Accessibility & qualitative \\
\cline { 2 - 3 } & 1.3 Buffer and common space & qualitative \\
\cline { 2 - 3 } 2. Socio-Cultural Sustainability & 2.4 Transformability index & qualitative \\
\cline { 2 - 3 } & 2.1 Functional mix & qualitative \\
\cline { 2 - 3 } 3. Environmental Sustainability & 2.3 Aggregation spaces & qualitative \\
\cline { 2 - 3 } & 3.1 Harmonization with the context & qualitative \\
\cline { 2 - 3 } & 3.2 Energetic quality & qualitative \\
\cline { 2 - 3 } 4. Economic Sustainability & 3.3 Consistency with constraints & qualitative \\
\hline & 4.1 Construction cost & quantitative \\
\cline { 2 - 3 } & 4.2 Maintenance cost & qualitative \\
\cline { 2 - 3 } & 4.3 Profitability of the intervention & quantitative \\
\hline
\end{tabular}

Each criterion has been defined regarding the purpose to achieve in order to be satisfied, while for each sub-criterion, a three-part evaluation sheet has been prepared and divided into:

- objective: to describe what has to be pursued;

- $\quad$ score: to explain how the sub-criterion is measured;

- evaluation of alternatives: a score is assigned to each scenario by considering the description previously provided.

In detail, 1. Functional Sustainability specifically takes into account the characteristics of the structure that will host the territorial health center; in fact, 1.1 Flexibility analyses the ability of the structure to modify its configuration over time according to the needs, therefore the availability of outdoor spaces for future expansions; 1.2 Usability/Accessibility evaluates the easy access to the building by all users, with particular reference to people with disabilities; 1.3 Buffer and common spaces considers the presence of an area in front of the building that allows to facilitate the passage between its interior and exterior area; 1.4 Transformability index is the ability of the structure to modify its internal configuration over time as needed. Then, 2. Socio-cultural Sustainability investigates the functional program of different scenarios; 2.1 Functional mix promotes the coexistence of several functions; 2.2 Social attractiveness is aimed at involving all age groups of the population through the creation of specific functions; 2.3 Aggregation spaces focuses on the creation of open spaces designed to facilitate interaction and living in the open air. Then, 3. Environmental Sustainability considers the external and internal spaces of the territorial health center, with a focus on the design solutions envisaged; 3.1 Harmonization with the context aims at minimizing the interference of the new project with the context; 3.2 Energetic quality evaluates the orientation of the building, the predisposition to accommodate photovoltaic panels and the ratio between the surface and the volume; 3.3 Consistency with constraints takes into consideration the regulations in place in the area. The last criterion, 4 . Economic Sustainability, measures the feasibility of the intervention; in fact, 4.1 Construction cost assesses the ex-novo works, the recovery works and the services that will be set up there; 4.2 Maintenance cost considers in a qualitative way the costs for the maintenance of the intervention; 4.3 Profitability of the intervention evaluates the market value generated by different projects. 
Given this framework, the alternatives previously generated have been measured and their performances are presented in Table 2 where it is possible to visualize, moreover, the U.M. selected and if the performance has to be maximized (benefit) or minimized (cost).

Table 2. Performance matrix.

\begin{tabular}{|c|c|c|c|c|c|c|c|c|}
\hline Criteria & Sub-criteria & U.M. & $\begin{array}{c}\text { Cost/ } \\
\text { Benefit }\end{array}$ & Scen_0 & Scen_1 & Scen_1 & Scen_3 & Scen $\_4$ \\
\hline \multirow{4}{*}{$\begin{array}{l}\text { 1. Functional } \\
\text { Sustainability }\end{array}$} & 1.1 Flexibility & $+/-$ & B & - & 0 & 0 & + & 0 \\
\hline & 1.2 Usability/Accessibility & $+/-$ & B & - & 0 & - & + & - \\
\hline & $\begin{array}{l}1.3 \text { Buffer and } \\
\text { common space }\end{array}$ & $+/-$ & B & - & 0 & 0 & + & 0 \\
\hline & 1.4 Transformability index & $+1-$ & B & - & 0 & 0 & + & + \\
\hline \multirow{3}{*}{$\begin{array}{l}\text { 2. Socio-cultural } \\
\text { Sustainability }\end{array}$} & 2.1 Functional mix & $+/-$ & B & - & 0 & + & + & + \\
\hline & 2.2 Social Attractiveness & $+/-$ & B & - & 0 & + & + & + \\
\hline & 2.3 Aggregation spaces & $+1-$ & B & - & + & + & + & + \\
\hline \multirow{3}{*}{$\begin{array}{l}\text { 3. Environmental } \\
\text { Sustainability }\end{array}$} & $\begin{array}{c}\text { 3.1 Harmonization with the } \\
\text { context }\end{array}$ & $+/-$ & B & 0 & - & + & + & + \\
\hline & 3.2 Energetic quality & $+/-$ & B & - & 0 & 0 & 0 & 0 \\
\hline & $\begin{array}{l}\text { 3.3 Consistency with } \\
\text { constraints }\end{array}$ & $+/-$ & B & + & - & 0 & 0 & 0 \\
\hline \multirow{3}{*}{$\begin{array}{l}\text { 4. Economic } \\
\text { Sustainability }\end{array}$} & 4.1 Construction cost & $€ /$ sqm & $\mathrm{C}$ & 0.00 & $1,905.00$ & $1,965.00$ & $2,011.00$ & $2,011.00$ \\
\hline & 4.2 Maintenance cost & $+/-$ & B & - & - & - & 0 & 0 \\
\hline & $\begin{array}{l}\text { 4.3 Profitability of the } \\
\text { intervention }\end{array}$ & $€$ & B & 0.00 & $4,200,000$ & $8,741,156$ & $10,200,000$ & $10,200,000$ \\
\hline
\end{tabular}

Notes: $+=$ high; $0=$ medium; $-=$ low.

Given the qualitative and quantitative nature of the different sub-criteria and, consequently, the lack of homogeneity of the units of measurement and of the scoring scales, the performances have been standardized with the aim of using the same a-dimensional scale between 0 and 1 for all the values. Consequently, each sub-criterion has been evaluated by means of a specific performance scale appropriate to the object of the evaluation and then standardized in a range from 0 (the worst performance) to 1 (the best performance) in order to be compared synergistically in the final evaluation of the alternatives. The value functions have been discussed with experts with specific knowledge about the procedure to develop during a focus group and it has been decided to use the maximum standardization that means:

$$
\text { stadardized score }=\frac{\text { score }}{\text { highest score }}
$$

if the value has to be maximized (benefit), while:

$$
\text { stadardized score }=-\frac{\text { score }}{\text { highest score }}+1
$$

if the value has to be minimized (cost) [25].

The Multi-Criteria Analysis has been carried out with the support of the DEFINITE software (decisions on a finite set of alternatives) [26]. Once the problem has been structured, alternatives measured according to the value tree defined and performances standardized, the next phase, involved in the procedure selected concerns criteria weights elicitation. In order to assign weights to the defined criteria and sub-criteria, one round of questionnaires has been administered to a selected group of experts. In detail, for the criteria, a group of eight experts have been selected, who answered individually to the questionnaire, while for the sub-criteria, only one expert answered for each macro area. The choice of experts has been based on their previous experiences on the proposed topic. The method applied for the weights elicitation has been the point allocation [27]; in fact, the experts have been asked to allocate 100 points among the criteria or sub-criteria proposed, assigning a higher number of points to criteria or sub-criteria with a higher importance. The results of the interaction have been then aggregated in order to obtain a unique weighing (Table 3). 
Table 3. Weights assigned by experts.

\begin{tabular}{|c|c|c|c|}
\hline Criteria & Weights_1 & Sub-criteria & Weights_2 \\
\hline \multirow{4}{*}{ 1. Functional Sustainability } & \multirow{4}{*}{$32 \%$} & 1.1 Flexibility & $18 \%$ \\
\hline & & 1.2 Usability/ Accessibility & $5 \%$ \\
\hline & & 1.3 Buffer and common space & $56 \%$ \\
\hline & & 1.4 Transformability index & $21 \%$ \\
\hline \multirow{3}{*}{ 2. Socio-cultural Sustainability } & \multirow{3}{*}{$15 \%$} & 2.1 Functional mix & $32 \%$ \\
\hline & & 2.2 Social Attractiveness & $46 \%$ \\
\hline & & 2.3 Aggregation spaces & $22 \%$ \\
\hline \multirow{3}{*}{ 3. Environmental Sustainability } & \multirow{3}{*}{$14 \%$} & 3.1 Harmonization with the context & $20 \%$ \\
\hline & & 3.2 Energetic quality & $60 \%$ \\
\hline & & 3.3 Consistency with constraints & $20 \%$ \\
\hline \multirow{3}{*}{ 4. Economic Sustainability } & \multirow{3}{*}{$39 \%$} & 4.1 Construction cost & $10 \%$ \\
\hline & & 4.2 Maintenance cost & $26 \%$ \\
\hline & & 4.3 Profitability of the intervention & $64 \%$ \\
\hline
\end{tabular}

In order to solve the problem, the Multi Attribute Value Theory (MAVT) [28] has been applied. The method allows to handle both qualitative and quantitative data by analyzing a finite set of alternatives [29]. Since there were no specific thresholds to respect, it has been chosen to aggregate standardized scores and weights by the use of an additive method as the MAVT_-this means a bad performance is compensated by a good one.

\section{Results}

Figure 5 shows both the partial results obtained for each criterion and the overall results. The ranking is the result of the weighted sum of the scores of each alternative multiplied by the influence assigned by the experts to criteria and sub-criteria. According to the defined decision framework, the most suitable alternative is Scenario_3, followed by Scenario_4, Scenario_2, Scenario_1 and Scenario_0.

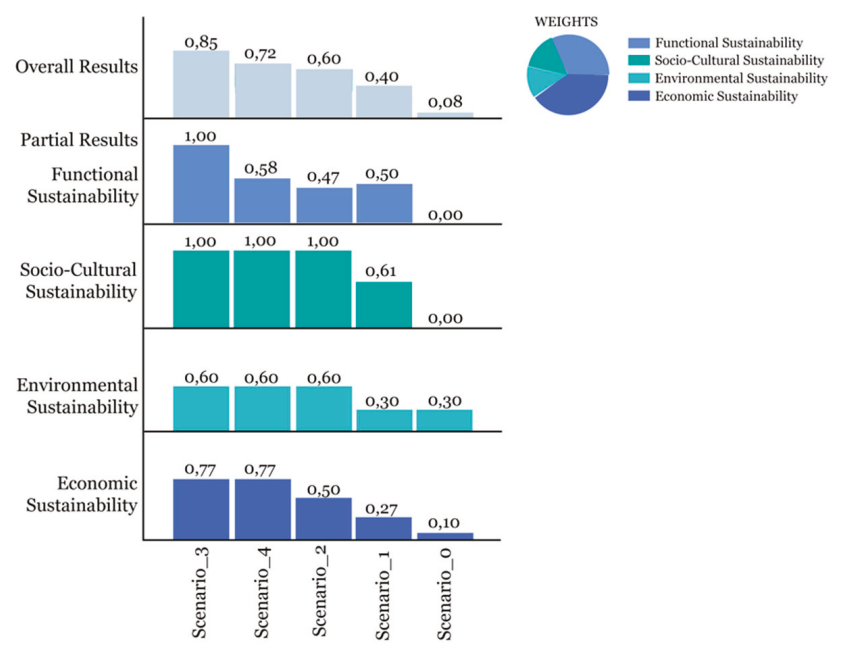

Figure 5. Partial and Overall results. 
By reading the results, it is possible to highlight some considerations:

- Scenario_3 had an overall score of $85 \%$. The criteria Functional Sustainability and Socio-Cultural Sustainability obtained the maximum score, $100 \%$. Environmental Sustainability obtained a score of $60 \%$, as with Scenario_4 and Scenario_2. Economic Sustainability maintained a priority position with respect to the other scenarios $(77 \%)$, together with Scenario_4. From this picture, a propensity to select this alternative emerges as the most suitable for the urban regeneration of the area and for the location of the territorial health center; in particular, this tendency has been underlined by the partial scores and in particular under the Functional Sustainability point of view.

- Scenario_4 obtained an overall score of $72 \%$, while Functional Sustainability obtained a score of $58 \% ; 100 \%$ for Socio-Cultural Sustainability; $60 \%$ for Environmental Sustainability and $77 \%$ for Economic Sustainability. From this picture, a propensity of this hypothesis to a greater Socio-Cultural Sustainability emerges, placing criticalities on Functional Sustainability.

- Scenario_2 obtained an overall score of $60 \%$ for Socio-Cultural Sustainability and Environmental Sustainability obtained a good evaluation, as with Scenario_3 and Scenario_4, whereas Functional Sustainability obtained a lower score $(47 \%)$. From this picture, functional criticality for what concerns its suitability in locating the territorial health center at the first and second floors of the former hospital building emerges.

- Scenario_1 obtained an overall score of $40 \%$, while Functional Sustainability obtained a score of $50 \% ; 61 \%$ for Socio-Cultural Sustainability; $30 \%$ for Environmental Sustainability and $27 \%$ for Economic Sustainability. From this picture, criticalities for all the criteria involved in the analysis emerges.

- Scenario_0 obtained the lowest score in all the criteria involved and can be disregarded from the evaluation.

This result is also confirmed by performing a sensitivity analysis. In fact, Figure 6 shows the results obtained by changing the weights assigned to the four criteria, and four different perspectives are illustrated by the "What if" Scenario.

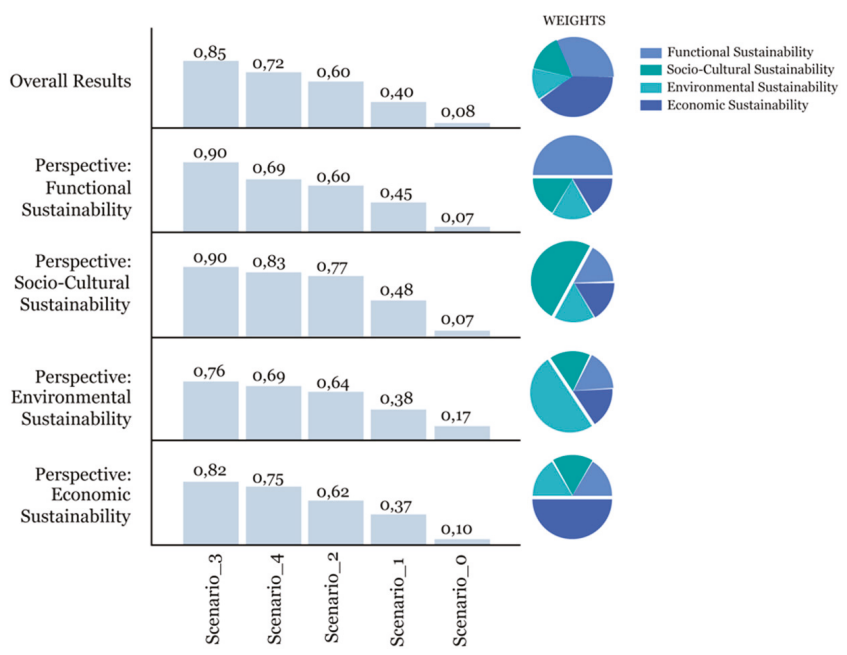

Figure 6. "What if" Scenario

Despite changes in the influence assigned, the rank obtained is stable and robust; in fact, the alternatives maintain the same position. Given the complexity and multi-dimensionality of the decision-making framework, it is important to verify the sensitivity of the result to possible changes. 
The sensitivity analysis is a fundamental step in the MCA that is able to improve the quality of the decision and it is a powerful tool when embedded in all the phases of a decision-making process, assigning a higher accuracy to the evaluation [30,31]. In fact, rankings are often conditional, given the uncertainty of data, criteria and also weights, since DMs are not always aware about their preferences. In particular, when multiple stakeholders are involved, it is difficult to select the best procedure to aggregate their weights and to elicit them $[32,33]$. The role of the sensitivity analysis is strategic in this context for the validation of the output and to reduce the uncertainty.

According to the results obtained, further confirmed by the sensitivity analysis, and trying to provide an answer to the research question stated in the introduction, it is possible to underline the following remarks:

- $\quad$ it is suggested the location of the health functions in new buildings given by the flexibility of its internal spaces and the possibility to provide and design external areas both to be accessed by users and in case of future expansions;

- the location of health functions in a protected area promotes both the privacy of the patients and the compatibility of different functions. In fact, given the high mixitè provided by different alternatives, it is fundamental to understand how they can be combined to preserve the well-being of people and the livability of the neighborhood;

- the complementarity of functions identified and the analysis of their compatibility in the Scenario_3, increase the attractiveness of the area and, moreover, its value.

\section{Conclusions}

The methodology proposed aimed at supporting the DM in the selection of the most suitable scenario for an urban regeneration that involves the location of a territorial health center and the enhancement of the $\mathrm{BCH}$. One of the main focuses of the contribution has been to present a multi-methodological approach and to explain each phase by illustrating how it has been developed. In fact, when multiple stakeholders are involved, multiple and sometimes conflicting values and expectations are at stake and it is fundamental to understand which are the most urgent to satisfy. At the same time, when the process of regeneration embeds tangible cultural values and intangible values, the support of a robust method able to elicit them and to recognize which have to be maximized or implemented and which are the most critical becomes strategic. In fact, both the approaches stated in the intelligence phase, together with the analysis of existing case studies, are functional to the generation of alternatives. These are the results of a strict cooperation with the actors involved and of a strict comprehension of values carried on by the BCH. Moreover, the combination of the DCF Analysis with the MCA for the evaluation of alternatives, given their transparency and robust methods, supports the DM in understanding the feasibility of the projects with their strengths and weaknesses. The final decision is, moreover, enhanced by the sensitivity analysis able to validate the results obtained.

The methodology and the approach proposed, if applied appropriately, could moreover facilitate the interaction and the satisfaction of both public and private parties and improve the policy-making process. In fact, since it is supported by evidence, it could lead to the concept of Evidence-Based Policy Making, where the consensus about policies is obtained through evidences [34]. Following this idea and by the active participation of many different actors, the DM is even more justified in taking a final decision. The policy implication becomes fundamental considering the context of application described in this paper since the location of a territorial health center is also evaluated. This public service is aimed at serving the population and, in the current scenario of increasing urbanization, health facilities play an important role as urban elements that can trigger and stimulate benefits throughout the territory [35]. Their location can also strongly affect the success of the whole project and may have negative impacts in several respects, such as patient well-being and the service's efficiency [36].

The methodology is able to consider all the aspects recognized as urgent to investigate and could facilitate the whole decision process, contributing to the literature on soft OR and covering all the four different approaches aimed at enhancing the cultural heritage and able to support city regeneration 
described in the introduction. Moreover, this first attempt could be investigated and different MCA approaches can be explored in order to better understand which is the most suitable one in this context. For example, if the DM has a deeper knowledge of the project and owns more information, it could be possible to test the aggregation by defining thresholds of acceptability (partially compensatory or non-compensatory methods) or by applying other additive models. In addition, the topic regarding the possible uses of historical buildings could be facilitated by exploring the methodology proposed by [37].

Given these conclusions, it is possible to perceive the flexibility of the methodology proposed and the iterative nature of the process. In fact, according to the case study, to the context of application and to the stakeholders involved and their values, a different path can be processed considering the general framework previously shaped.

Author Contributions: Conceptualization, S.C.; Data curation, R.M.; Formal analysis, L.S.; Methodology, M.D.; Project administration, R.M.; Supervision, S.C. and S.D.T.; Validation, S.D.T.; Visualization, M.D.; Writing-original draft, M.D.; Writing—review and editing, S.C., L.S., R.M. and S.D.T.

Funding: This research received no external funding.

Acknowledgments: This study was partially supported by the Municipality of Vimercate (MB), Italy, for which the authors are most grateful.

Conflicts of Interest: The authors declare no conflict of interest in the results.

\section{References}

1. Franco, L.A. Problem structuring methods as intervention tools: Reflections from their use with multi-organisational teams. Omega 2009, 37, 193-203.

2. Lami, I.M.; Tavella, E. On the usefulness of soft OR models in decision making: A comparison of Problem Structuring Methods supported and self-organized workshops. Eur. J. Oper. Res. 2019, 275, 1020-1036. [CrossRef]

3. Franco, L.A.; Montibeller, G. Facilitated modelling in operational research. Eur. J. Oper. Res. 2010, 205, 489-500. [CrossRef]

4. Smith, C.M.; Shaw, D. The characteristics of problem structuring methods: A literature review. Eur. J. Oper. Res. 2019, 274, 403-416. [CrossRef]

5. Rosenhead, J.; Mingers, J. (Eds.) Rational Analysis for A Problematic World Revisited; Wiley: Chichester, UK, 2001.

6. Cervelló-Royo, R.; Garrido-Yserte, R.; Segura-García del Río, B. An urban regeneration model in heritage areas in search of sustainable urban development and internal cohesion. J. Cult. Herit. Manag. Sustain. Dev. 2012, 2, 44-61. [CrossRef]

7. Baarveld, M.; Smit, M.; Dewulf, G. Negotiation processes in urban redevelopment projects: Dealing with conflicts by balancing integrative and distributive approaches. Plan. Theory Pract. 2015, 16, 363-384. [CrossRef]

8. Abastante, F.; Corrente, S.; Greco, S.; Ishizaka, A.; Lami, I.M. Choice architecture for architecture choices: Evaluating social housing initiatives putting together a parsimonious AHP methodology and the Choquet integral. Land Use Policy 2018, 78, 748-762. [CrossRef]

9. Iavarone, R.; Alberico, I.; Gravagnuolo, A.; De Vita, G.E. The Role of Cultural Heritage in Urban Resilience Enhancement. In Proceedings of the International Symposium on New Metropolitan Perspectives, Reggio Calabria, Italy, 22-25 May 2018; Springer: Cham, Switzerland; pp. 369-377.

10. Boeri, A.; Bortoli, G.; Longo, D. Cultural heritage as a driver for urban regeneration: Comparing two processes. WIT Trans. Ecol. Environ. 2018, 217, 587-598.

11. Della Lucia, M.; Trunfio, M.; Go, F.M. Heritage and urban regeneration: Towards creative tourism. In Tourism in the City; Springer: Cham, Switzerland, 2017; pp. 179-191.

12. Della Lucia, M.; Franch, M. Culture-led urban regeneration and place brand building in Alpine Italian cities. In Harnessing Place Branding through Cultural Entrepreneurship; Go, F.M., Lemmetyinen, A., Hakala, U., Eds.; Palgrave Macmillan: New York, NY, USA, 2014; pp. 122-140. 
13. Lee, C.B. Cultural policy as regeneration approach in western cities: A case study of Liverpool's RopeWalks. Geogr. Compass 2009, 3, 495-517. [CrossRef]

14. Sharifi, M.A.; Rodriguez, E. Design and development of a planning support system for policy formulation in water resources rehabilitation: The case of Alcazar De San Juan District in Aquifer 23, La Mancha, Spain. J. Hydroinform. 2002, 4, 157-175. [CrossRef]

15. Dente, B. Who decides? Actors and their resources. In Understanding Policy Decisions; Springer: Cham, Switzerland, 2014.

16. Gamboa, G.; Munda, G. The problem of windfarm location: A social multi-criteria evaluation framework. Energy Policy 2007, 35, 1564-1583. [CrossRef]

17. Della Torre, S.; Moioli, R.; Cantini, L. The Historic Centre of Vimercate: Investigation, Education, Community Involvement. In Proceedings of the International Conference on Transdisciplinary Multispectral Modeling and Cooperation for the Preservation of Cultural Heritage; Springer: Cham, Switzerland, 2018; pp. 319-328.

18. ICOMOS. Nara Document on Authenticity 1994. Available online: http://icomos.org/index.php/en/chartersand-texts (accessed on 15 May 2019).

19. Van Balen, K. The Nara grid: An evaluation scheme based on the Nara document on authenticity. Assoc. Preserv. Technol. Int. (APT) 2008, 39, 39-46.

20. Eshrati, P.; Bahramjerdi, S.F.N.; Mahabadi, S.E.; Azad, M. Evaluation of Authenticity on the Basis of the Nara Grid in Adaptive Reuse of Manochehri Historical House Kashan, Iran. ArchNet-IJAR Int. J. Archit. Res. 2017, 11, 214-231. [CrossRef]

21. Capolongo, S.; Mauri, M.; Peretti, G.; Pollo, R.; Tognolo, C. Facilities for territorial medicine: The experiences of Piedmont and Lombardy Regions. TECHNE J. Technol. Archit. Environ. 2015, 9, 230-236.

22. Colorni, A.; Tsoukiàs, A. What is a decision problem? designing alternatives. In Preference Disaggregation in Multiple Criteria Decision Analysis; Springer: Cham, Switzerland, 2018; pp. 1-15.

23. Colorni, A.; Ferretti, V.; Luè, A.; Oppio, A.; Paruscio, V.; Tomasini, L. Rethinking feasibility analysis for urban development: A multidimensional decision support tool. In International Conference on Computational Science and Its Applications; Springer: Cham, Switzerland, 2017; pp. 624-638.

24. Sdino, L.; Rosasco, P.; Novi, F.; Porcile, G. The evaluation of actions aimed at enhancing the cultural heritage: The case study of the Colosseum roofing. Valori E Valutazioni 2018, 20, 1-13.

25. Figueira, J.R.; Greco, S.; Ehrgott, M. (Eds.) Multiple Criteria Decision Analysis: State of the Art Surveys; Springer Science \& Business Media: Berlin/Heidelberg, Germany, 2005; Volume 78.

26. Janssen, R.; Van Herwijnen, M.; Beinat, E. DEFINITE for Windows. A System to Support Decisions on A Finite Set of Alternatives (Software and Package and User Manual); Kluwer Academic: Aarhus, Denmark, 1994.

27. Bottomley, P.A.; Doyle, J.R.; Green, R.H. Testing the reliability of weight elicitation methods: Direct rating versus point allocation. J. Mark. Res. 2000, 37, 508-513. [CrossRef]

28. Keeney, R.L.; Raiffa, H. Decisions with Multiple Objectives: Preferences and Value Trade-Offs; Wiley: New York, NY, USA, 1976.

29. Oppio, A.; Bottero, M.; Arcidiacono, A. Assessing urban quality: A proposal for a MCDA evaluation framework. Ann. Oper. Res. 2018, 1-18. [CrossRef]

30. Samson, D. Managerial Decision Analysis; CRC Press: Boca Raton, FL, USA, 1992.

31. Triantaphyllou, E.; Sánchez, A. A sensitivity analysis approach for some deterministic multi-criteria decision-making methods. Decis. Sci. 1997, 28, 151-194. [CrossRef]

32. Chen, Y.; Yu, J.; Khan, S. Spatial sensitivity analysis of multi-criteria weights in GIS-based land suitability evaluation. Environ. Model. Softw. 2010, 25, 1582-1591. [CrossRef]

33. Dell'Ovo, M.; Frej, E.A.; Oppio, A.; Capolongo, S.; Morais, D.C.; de Almeida, A.T. FITradeoff Method for the Location of Healthcare Facilities Based on Multiple Stakeholders' Preferences. In Group Decision and Negotiation in an Uncertain World. GDN 2018. Lecture Notes in Business Information Processing; Chen, Y., Kersten, G., Vetschera, R., Xu, H., Eds.; Springer: Cham, Switzerland, 2018; Volume 315, pp. 97-112.

34. Blair, T. Labour Party Manifesto. 1997. Available online: http://www.labour-party.org.uk/manifestos/1997/ 1997-labour-manifesto.shtml (accessed on 22 May 2019).

35. Dell'Ovo, M.; Capolongo, S. Architectures for health: Between historical contexts and suburban areas. Tool to support location strategies. TECHNE J. Technol. Archit. Environ. 2016, 12, 269-276. 
36. Sdino, L.; Rosasco, P.; Magoni, S. True, Fair and Beautiful: Evaluative Paradigms Between the Encyclical Letter Laudato Sì and Keynes. In Green Energy and Technology; Springer: Cham, Switzerland, 2018; pp. 87-98. [CrossRef]

37. Bottero, M.; D'Alpaos, C.; Oppio, A. Ranking of Adaptive Reuse Strategies for Abandoned Industrial Heritage in Vulnerable Contexts: A Multiple Criteria Decision Aiding Approach. Sustainability 2019, 11, 785. [CrossRef]

(C) 2019 by the authors. Licensee MDPI, Basel, Switzerland. This article is an open access article distributed under the terms and conditions of the Creative Commons Attribution (CC BY) license (http://creativecommons.org/licenses/by/4.0/). 



\title{
Cultural Heritage and Sustainable Development Targets: A Possible Harmonisation? Insights from the European Perspective
}

\author{
Luigi Petti ${ }^{1}$, Claudia Trillo ${ }^{2}$ and Busisiwe Ncube Makore ${ }^{2, *}$ \\ 1 Department of Civil Engineering, University of Salerno, 84084 Fisciano, Italy; luigi.petti@unisa.it \\ 2 SEE: Science, Engineering and Environment, University of Salford, Salford M5 4WT, UK; \\ c.trillo2@salford.ac.uk \\ * Correspondence: B.C.Makore@salford.ac.uk
}

Received: 2 November 2019; Accepted: 23 January 2020; Published: 27 January 2020

\begin{abstract}
The Agenda 2030 includes a set of targets that need to be achieved by 2030. Although none of the 17 Sustainable Development Goals (SDGs) focuses exclusively on cultural heritage, the resulting Agenda includes explicit reference to heritage in SDG 11.4 and indirect reference to other Goals. Achievement of international targets shall happen at local and national level, and therefore, it is crucial to understand how interventions on local heritage are monitored nationally, therefore feeding into the sustainable development framework. This paper is focused on gauging the implementation of the Sustainable Development Goals with reference to cultural heritage, by interrogating the current way of classifying it (and consequently monitoring). In fact, there is no common dataset associated with monitoring SDGs, and the field of heritage is extremely complex and diversified. The purpose for the paper is to understand if the taxonomy used by different national databases allows consistency in the classification and valuing of the different assets categories. The European case study has been chosen as field of investigation, in order to pilot a methodology that can be expanded in further research. A cross-comparison of a selected sample of publicly accessible national cultural heritage databases has been conducted. As a result, this study confirms the existence of general harmonisation of data towards the achievement of the SDGs with a broad agreement of the conceptualisation of cultural heritage with international frameworks, thus confirming that consistency exists in the classification and valuing of the different assets categories. However, diverse challenges of achieving a consistent and coherent approach to integrating culture in sustainability remains problematic. The findings allow concluding that it could be possible to mainstream across different databases those indicators, which could lead to depicting the overall level of attainment of the Agenda 2030 targets on heritage. However, more research is needed in developing a robust correlation between national datasets and international targets.
\end{abstract}

Keywords: cultural heritage; Sustainable Development Goals; SDG 11; heritage database; heritage value; heritage classification

\section{Introduction}

All United Nations (UN) member states unanimously adopted the Sustainable Development Goals (SDGs) in 2015. This resulted in a comprehensive set of 17 goals and 169 targets aimed at reducing poverty and advancing health and wellbeing for all by 2030, Agenda 2030 [1]. The compelling need for action to create inclusive cities has been recognised in commitments and recommendations set out in the Sustainable Development Goals, World Humanitarian Summit and the New Urban Agenda from Habitat III (2016). The Millennium Development Goals (MDG) era came to an end in December 2015, and the global community decided to look back at the value of a unifying agenda underpinned by goals 
and targets and use the lessons learnt to effectively implement the Sustainable Development Goals (SDGs) from 2016 to 2030. The SDGs, otherwise known as the Global Goals, build on the Millennium Development Goals (MDGs), eight antipoverty targets that the world committed to achieving by 2015. The lack of focus on urban areas and disaggregation is addressed at an international level through the introduction of the SDGs [2]. Successful global campaigning by a network of civil society, cities and the United Nations was a campaign that recently culminated in a New Urban Agenda [1] and a specifically Urban Sustainable Development Goal (USDG) as part of the United Nations 2030 Agenda for Sustainable Development. The SDGs were intended to redress many of the shortfalls of the MDGs. While the MDGs committed governments and international agencies to reducing the number of people living in poverty or lacking access to essential services and infrastructure, the SDGs commit these actors to poverty eradication and universal access to these services and infrastructure. The SDGs are a United Nations-sponsored effort to create a common set of development goals for all communities in every country, with a deadline for attainment of 2030. The idea is to get governments, aid organisations, foundations and NGOs on the same page about what global problems most urgently need to be solved and how to measure progress and solutions.

While these efforts symbolise an important start, similar to the UN system, the platform lacks any data on indicators related to cultural heritage. Consequently, reflecting the challenges in attaining adequate data and developing systematic methodologies on cultural heritage is needed to realise the SDGs [3]. In this paper, we argue that the availability and harmonisation of data from member states is central to localising SDG 11.4.

The purpose of this study is to better understand if the taxonomy used by different databases allows consistency in the classification and valorisation of the different assets categories. In this context, the potential for a common approach to the protection and safeguarding of European heritage [4] is explored with the aim to feed into a methodological framework for the calculation of the 11.4 target. In order to do this, national heritage databases and inventories are identified for a cross-comparison review of what these states recognise as heritage and how they assign value to it. There are an increasing number of studies investigating the development of harmonised data in order to successfully achieve the SDGs [5-8]. Similarly, there is an established discourse on the documentation of cultural heritage and the development of heritage databases in implementing sustainable development for urban and rural areas [9-13]. However, there is a paucity of studies that have explored the development of a heritage database in Europe with the aim of harmonising data for the achievement of the SDGs. This paper argues that the way in which cultural heritage is perceived and conceptualised by national and local government and heritage stakeholders has a direct effect on the way it is managed, interpreted and understood. Consequently, this impacts how local communities associate themselves with heritage and value it. Acknowledging the crucial role of enforcing and monitoring the implementation of the legal heritage framework, it is also important to understand how common frameworks designed to protect and safeguard cultural heritage have translated into the local management practice of heritage assets and databases. It is anticipated that this review has two possible applications: (1) supporting national authorities in finding a suitable conceptual framework and methodology for the development of SDG culture indicators and (2) supporting the UN in understanding inconsistencies that may arise from different system of calculations of heritage-related targets according to different databases in Europe.

This paper is structured into six broad sections. Section 1 introduces the background of the paper, while Section 2 discusses the overall methodology used for developing this paper. Section 3 explores the classification of cultural heritage, culture in the Sustainable Development Goals, current heritage indicators and efforts to move beyond it through the development of heritage indicators. This is followed by the results in Section 4, which is divided into four thematic areas: (1) cultural heritage's multidimensional impact; (2) safeguarding built cultural heritage; (3) safeguarding intangible heritage; and (4) culture and environmental sustainability. Section 5 discusses these areas, and Section 6 concludes the paper. 


\section{Methodology}

Based on this paper's research aim to develop an understanding on the role of culture in sustainable development its implementation of the Sustainable Development Goals with reference to cultural heritage, the methodology of this paper can be understood in three parts: (1) scientific discourse analysis; (2) database cross-comparison analysis; and (3) a heritage expert informal online survey.

\subsection{Discourse Analysis}

In order to facilitate the exploration of the conceptualisation and the role of culture in sustainable development with a focus on countries in the European region, an academic and policy discourse analysis was conducted. A document analysis was conducted of documents linked to the databases found on the national authoritative agency website. The main aim of this analysis was to provide a broad picture of cultural sustainability in the context of Europe and the challenges of integrating culture in sustainable development. Targeted internet searches were conducted for documents through snowballing, identifying publications in reference lists and through expert recommendations. NVivo 10, a well-known qualitative data analysis software tool, was used to help the systematic storing, retrieval, evaluation and interpretation of the texts.

\subsection{Database Cross-Comparison Analysis}

As a first step for this analysis, known websites of national agencies responsible for the management of cultural heritage in Europe were explored and searched to determine the existence of heritage databases. Other websites were also used for this initial search, such as UNESCO, COE and ICOMOS. Any noteworthy details related to the conceptualisation of heritage were tracked and archived. As a second step to support add accuracy, the HEREIN database was used to identify and verify sources for heritage databases, information and management. HEREIN is a European Cultural Heritage Information Network developed in 2014 within the Council of Europe, which brings together European public administrations in charge of national cultural heritage policies and strategies to form a unique co-operation network in the domain of Cultural Heritage [14]. A comprehensive search for the details of the authoritative database was conducted of the heritage national report submitted from European members. The national reports on cultural heritage are based on information collected by HEREIN Coordinators among resource persons in all ministries and cultural heritage entities as well as additional experts in the field. Each national heritage report contains information about the inventories and databases developed by the nation state, including details about the content and level of detail. The search was supported by information from country profiles from the monitoring system, Compendium of Cultural Policies and Trends [15]. Each national report (29) and country profile (44) was reviewed for details about the national database and the classification and value system used. Searches were carried out in the language of the report aided by translation. The key search words included "inventory", "database", "Classification", "Values". "Repository", "Data", and "Geoportal". Resulting from this search, 20 countries were identified with national inventories and databases that were publicly accessible. Following this, we accessed each database and navigated with more detail through the site and the documents found on the site. We began this review by specifying important attributes to ensure comparability. Four attributes for the review of heritage databases were prioritised that would be essential for this methodology as highlighted below:

1. Publicly accessible online, freely available and having the ease of retrieval of information in English;

2. Searchable by keywords and user friendly;

3. Regularly updated;

4. Having a broad scope (including intangible heritage where possible)

According to the above criteria, we eliminated results that were ineligible for comparability, such as those that (1) were inaccessible due to permissions or site issues or other reasons and (2) lacked 
comprehensive information to support the purpose of the paper. After this review, 16 national heritage databases were found to be meeting the criteria as represented in Figure 1. These 16 online databases have been chosen to contain information to different extents of the conceptualisation of cultural heritage. As part of the analysis, a search for the classification and conceptualisation used in the database was conducted by analysing the list of heritage and categorisation used. Details concerning tangible and intangible heritage were identified. The values, significance and interest used for the listed cultural heritage in the database was explored in parallel to the step detailed above. Additionally, the inclusion of the public in determining the database was noted where mentioned. Other details include the access to a geoportal and spatial data. The databases were assessed against the conceptualisation of cultural heritage used by the UIS UNESCO Framework for Cultural Statistics for the SDG 11.4 target: "artefacts, monuments, and groups of buildings and sites that have a diversity of values including symbolic, historic, artistic, aesthetic, ethnological or anthropological, scientific and social significance."

\begin{tabular}{|c|c|c|c|c|c|c|c|c|}
\hline No & 1 & 2 & 3 & 4 & 5 & 6 & 7 & 8 \\
\hline Country & England & Scotland & Ireland & France & Italy & Sweden & Netherlands & Denmark \\
\hline $\begin{array}{l}\text { Name of } \\
\text { Database }\end{array}$ & $\begin{array}{l}\text { The National } \\
\text { Heritage List for } \\
\text { England } \\
\text { (NHLE) }\end{array}$ & $\begin{array}{l}\text { Heritage } \\
\text { register } \\
\text { (combined) }\end{array}$ & $\begin{array}{c}\text { National } \\
\text { Inventory of } \\
\text { Architectural } \\
\text { Heritage \& } \\
\text { Architectural } \\
\text { Heritage Garden } \\
\text { Survey }\end{array}$ & $\begin{array}{l}\text { Base Mérimée: } \\
\text { Immeubles } \\
\text { protégés au titre } \\
\text { des Monuments } \\
\text { Historiques }\end{array}$ & $\begin{array}{c}\text { Catalogo } \\
\text { Generale dei } \\
\text { Beni Culturali }\end{array}$ & $\begin{array}{c}\text { Building } \\
\text { Registry - BeBR }\end{array}$ & $\begin{array}{l}\text { National } \\
\text { Monument } \\
\text { Register }\end{array}$ & $\begin{array}{l}\text { Buildings } \\
\text { register }\end{array}$ \\
\hline No & 9 & 10 & 11 & 12 & 13 & 14 & 15 & 16 \\
\hline Country & Estonia & Finland & Portugal & Czech Republic & Poland & Slovenia & Ukraine & Lithuania \\
\hline $\begin{array}{l}\text { Name of } \\
\text { Database }\end{array}$ & $\begin{array}{l}\text { National } \\
\text { Register of } \\
\text { Cultural } \\
\text { Monuments }\end{array}$ & $\begin{array}{c}\text { Nationally } \\
\text { Constructed } \\
\text { Cultural } \\
\text { Environments } \\
\text { (RKY) } \\
\text { inventories }\end{array}$ & $\begin{array}{l}\text { Lista de } \\
\text { património } \\
\text { edificado em } \\
\text { Portugal }\end{array}$ & $\begin{array}{l}\text { National } \\
\text { Heritage } \\
\text { Institute }\end{array}$ & $\begin{array}{l}\text { Heritage board } \\
\text { of Poland }\end{array}$ & $\begin{array}{c}\text { Register of } \\
\text { Slovene cultural } \\
\text { heritage }\end{array}$ & $\begin{array}{l}\text { State Register of } \\
\text { Real Estate }\end{array}$ & $\begin{array}{c}\text { The Ministry } \\
\text { of Culture of } \\
\text { The Republic } \\
\text { of Lithuania } \\
\text { Register }\end{array}$ \\
\hline
\end{tabular}

Figure 1. Heritage databases.

\subsection{Heritage Expert Informal Online Survey}

Additionally, an informal online survey was sent to representatives of institutions working in the field of culture and heritage, academics and organisations' employees. They were encouraged to fill in the online questionnaire. A total of 10 experts in the field of heritage conservation in local governments for 10 European member states responded to the survey. The online survey was based on 8 open questions, asking for opinions on the conceptualisation of cultural heritage, value and impact of heritage, classification of cultural heritage in Europe and the recommendations to integrate culture in sustainable development.

\section{Positioning Culture within Sustainable Development}

\subsection{Classifying Cultural Heritage}

International standards of classification and heritage documentation are created by international organisations such as UNESCO and the Council of Europe. International heritage charters, conventions and recommendations have encouraged the development of inventories and current databases of heritage. This includes the Athens Charter, the UNESCO World Heritage Convention, the UNESCO recommendation concerning the protection, at national level, of the cultural and natural heritage, the ICOMOS (1996) principles for the recording of monuments, groups of buildings and sites, the UNESCO (2001) convention on the protection of underwater cultural heritage, the UNESCO (2003) convention for the safeguarding of the intangible cultural heritage, the ICOMOS $(2008,2011)$ charter on cultural routes and the Valletta principles for the safeguarding and management of historic cities, towns and urban areas. The identification of cultural heritage to be protected and inventoried is further recognised in European regional heritage norms, such as the Council of Europe's $(1985,1992)$ Convention for the Protection of the Architectural Heritage of Europe, known as the Granada Convention, and the European convention on the protection of the archaeological heritage. Aligned with the international 
policy landscape is the current social, economic and political crises, particularly prevalent in the Arab region. The prominent attacks on cultural heritage have stirred the urgent need to study and explore imminent risks to cultural heritage. A comparison of the international and European ratification status of different EU countries is illustrated in Figure 2. This context emphasises the need for the development of accurate databases in protecting heritage from threats to the urban fabric and spaces of heritage value and interest through the documentation of the state of conservation and the condition of the urban fabric. As a result, UNESCO has continued to place increasing scrutiny on the immaterial and material representations of heritage. This eventually led to a formal acknowledgment of the deeply rooted interdependence between the intangible and the tangible heritage through the 2003 Convention for the Safeguarding of the Intangible Cultural Heritage [16]. The Convention emphasises the crucial role of intangible cultural heritage as a mainspring of cultural diversity and a guarantee of sustainable development. Traditional knowledge and social practices and processes contribute to addressing fundamental needs and social issues leading to the achievement of inclusive social development $[17,18]$. In particular, among other measures of safeguarding in the Convention, it is recommended that the role of state parties is to identify and define the various elements of the intangible and draw up, in a manner geared to its own situation, one or more inventories of the intangible cultural heritage present in its territory (articles 11 and 12) [16].

\begin{tabular}{|c|c|c|c|c|c|c|c|c|}
\hline No & 1 & 2 & 3 & 4 & 5 & 6 & 7 & 8 \\
\hline Country & England & Scotland & Ireland & France & Italy & Sweden & Netherlands & Denmark \\
\hline \multicolumn{9}{|l|}{ UNESCO } \\
\hline World Heritage Convention of 1972 & $29 / 05 / 1984$ & 29/05/1984 & $16 / 09 / 1991$ & $27 / 06 / 1975$ & $23 / 06 / 1978$ & $22 / 01 / 1985$ & $26 / 08 / 1992$ & $25 / 07 / 1979$ \\
\hline $\begin{array}{l}\text { Convention for the Safeguarding of the } \\
\text { Intangible Heritage of } 2003\end{array}$ & & & $22 / 12 / 2015$ & $11 / 07 / 2006$ & $30 / 10 / 2007$ & $26 / 01 / 2011$ & $15 / 05 / 2012$ & $30 / 10 / 2009$ \\
\hline \multicolumn{9}{|l|}{ Council of Europe } \\
\hline $\begin{array}{l}\text { Granada Convention for the Protection } \\
\text { of the Architectural Heritage of } 1985\end{array}$ & $13 / 11 / 1987$ & $13 / 11 / 1987$ & $10 / 01 / 1997$ & $17 / 03 / 1987$ & $05 / 04 / 2006$ & 05/10/1990 & $15 / 02 / 1994$ & $23 / 07 / 1987$ \\
\hline $\begin{array}{l}\text { Valletta Convention for the Protection } \\
\text { of the Archaeological Heritage (revised) } \\
\text { of } 1992\end{array}$ & $19 / 09 / 2000$ & $19 / 09 / 2000$ & $18 / 03 / 1997$ & $10 / 07 / 1995$ & $30 / 06 / 2015$ & $11 / 10 / 1995$ & $11 / 06 / 2007$ & $16 / 11 / 2005$ \\
\hline $\begin{array}{l}\text { Florence European Landscape } \\
\text { Convention of } 2000\end{array}$ & $21 / 11 / 2006$ & $21 / 11 / 2006$ & $22 / 03 / 2002$ & $17 / 03 / 2006$ & $04 / 05 / 2006$ & $05 / 01 / 2011$ & $27 / 07 / 2005$ & $20 / 03 / 2003$ \\
\hline $\begin{array}{l}\text { Faro Convention on the Value of } \\
\text { Cultural Heritage for Society of } 2011\end{array}$ & & & & & $\begin{array}{r}27 / 02 / 2013 \\
\text { (signature) }\end{array}$ & & & \\
\hline No & 9 & 10 & 11 & 12 & 13 & 14 & 15 & 16 \\
\hline Country & Estonia & Finland & Portugal & $\begin{array}{l}\text { Czech } \\
\text { Republic }\end{array}$ & Poland & Slovenia & Ukraine & Lithuania \\
\hline \multicolumn{9}{|l|}{ UNESCO } \\
\hline World Heritage Convention of 1972 & $27 / 10 / 1995$ & 04/03/1987 & $30 / 09 / 1980$ & $26 / 03 / 1993$ & $29 / 06 / 1976$ & 05/11/1992 & $12 / 10 / 1988$ & $31 / 03 / 1992$ \\
\hline $\begin{array}{l}\text { Convention for the Safeguarding of the } \\
\text { Intangible Heritage of } 2003\end{array}$ & $27 / 01 / 2006$ & $21 / 02 / 2013$ & $21 / 05 / 2008$ & $18 / 02 / 2009$ & $16 / 05 / 2011$ & $18 / 09 / 2008$ & $27 / 05 / 2008$ & $21 / 01 / 2005$ \\
\hline \multicolumn{9}{|l|}{ Council of Europe } \\
\hline $\begin{array}{l}\text { Granada Convention for the Protection } \\
\text { of the Architectural Heritage of } 1985\end{array}$ & $15 / 11 / 1996$ & $17 / 10 / 1991$ & 27/03/1991 & $06 / 04 / 2000$ & $22 / 11 / 2011$ & $02 / 07 / 1992$ & $21 / 12 / 2006$ & $07 / 12 / 1999$ \\
\hline $\begin{array}{l}\text { Valletta Convention for the Protection } \\
\text { of the Archaeological Heritage (revised) } \\
\text { of } 1992\end{array}$ & $15 / 11 / 1996$ & $15 / 09 / 1994$ & 05/08/1998 & $22 / 03 / 2000$ & $30 / 01 / 1996$ & 07/05/1999 & $26 / 02 / 2004$ & $07 / 12 / 1999$ \\
\hline $\begin{array}{l}\text { Florence European Landscape } \\
\text { Convention of } 2000\end{array}$ & $08 / 02 / 2018$ & $16 / 12 / 2005$ & $29 / 03 / 2005$ & $03 / 06 / 2004$ & $27 / 09 / 2004$ & $25 / 09 / 2003$ & $10 / 03 / 2006$ & $13 / 11 / 2002$ \\
\hline $\begin{array}{l}\text { Faro Convention on the Value of } \\
\text { Cultural Heritage for Society of } 2011\end{array}$ & & $31 / 05 / 2018$ & $28 / 08 / 2009$ & & & $17 / 09 / 2008$ & $09 / 01 / 2014$ & \\
\hline
\end{tabular}

Figure 2. Comparison of international and European ratification status.

The 1954 Hague Convention for the Protection of Cultural Property in the Event of Armed Conflict classifies cultural property according to the identification of three typological categories: (1) movable or immovable property of great importance to the cultural heritage of every people; (2) buildings that contain cultural objects, such as museums, libraries and archives; and (3) historical centres containing monuments [19]. UNESCO broadens this classification of cultural heritage through the World Heritage Convention. The World Convention reconciles previous definitions of cultural heritage and presents immoveable cultural heritage within three categories: (1) monuments, (2) groups of buildings and (3) sites as illustrated in Figure 3 below. Intangible heritage is conceptualised into five categories 
focused on traditional social practices and processes, craftsmanship, rituals and arts. The protection of moveable cultural heritage is foregrounded through key legislation such as the 1970 UNESCO Convention on the means of prohibiting and preventing the illicit import, export and transfer of ownership of cultural property and at a later point, the 1995 UNIDROIT Convention on stolen or illegally exported cultural objects.

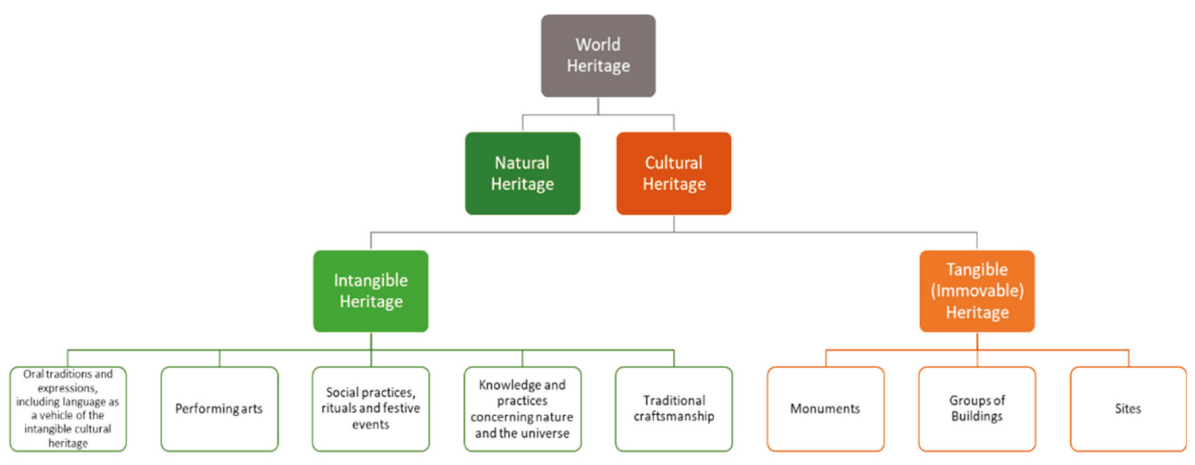

Figure 3. UNESCO cultural heritage classification.

3.2. Towards achieving the SDG 11.4: Strengthening efforts to protect and safeguard the world's cultural and natural heritage

Of the 17 finalised SDGs, one of those, Goal 11, centres on a pledge to "make cities and human settlement inclusive, safe, resilient and sustainable" and includes a series of 11 targets, each with politically negotiated indicators [2]. That goal is backed by specific targets and indicators (currently under negotiation), such as eliminating slum-like conditions, reducing urban sprawl and ensuring universal access to safe and sustainable urban transit. Goal 11 marks the United Nations' strongest expression ever of the critical role that cities will play in the world's future. Although none of the 17 SDGs focuses exclusively on culture, the resulting Agenda includes several explicit references to cultural aspects (Figure 4). Under goal 11 is an important indicator for cultural heritage: target 11.4 "Strengthen efforts to protect and safeguard the world cultural and natural heritage" [20]. Other references include target 4.7 , which focuses on promoting knowledge and skills and the appreciation of cultural diversity; targets 8.9 and $12 . \mathrm{b}$, which promote sustainable tourism and local culture aligned with target 14.7, promoting the sustainable use of aquaculture and tourism; targets 16.3, 16.8 and 16B promote the enforcement of the rule of law and strengthening global governance [20]. All targets have specific implications in the field of culture. These targets give light to the role that local heritage (that determines the cultural heritage) can play in this sustainable development framework. Indeed, SDGs are supported by international instruments and actions such as the establishment and mandate for a new special procedure entitled "independent expert in the field of cultural rights" through the Human Rights Council, resolution 10/23 [21]. In this context, cultural heritage is centralised in the enjoyment of human rights and in combating fundamentalism and extremism. While national governments of UN member states have set the USDG, the goal and its targets will need to be realised at the local scale. The USDG thus raises the question of the relationship and coordination between cities and other subnational as well as national governments in relation to the implementation of the goals and monitoring. What is uncertain is how another set of goals and targets will enable the appropriation of urban heritage at a local level. 


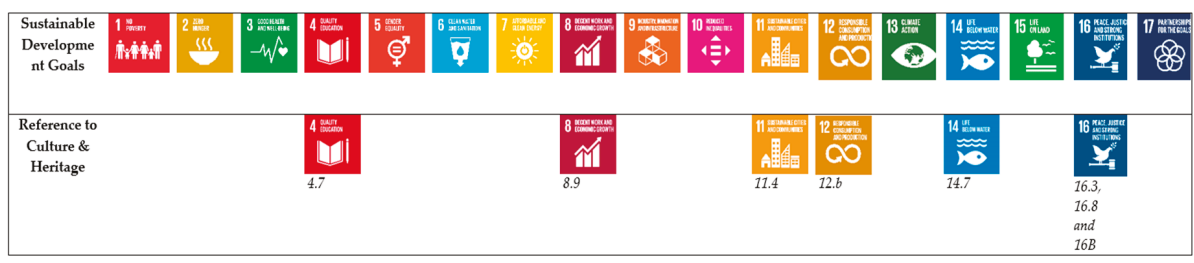

Figure 4. Culture in the Sustainable Development Goals.

The SDGs focus explicitly on heritage in one specific goal and indicator: Goal 11, target number 11.4. As illustrated in Figure 5, the indicator is concerned with developing a global picture of financial actions to safeguard cultural and natural heritage made by organisations in the private sector and public authorities at local and national levels. By identifying the spending of public authorities and private expenditure related to heritage, comparisons between countries can be made at a global scale to provide a complementary measure of the level of development and capacity of a nation [20]. Disaggregation for the indicator includes the type of heritage, World Heritage designation; level of government and type of private funding. Therefore, several sources of information are required for the assessment of this indicator. No internationally established methodology or standards are yet available for the indicator, but methodology and standards are being developed; therefore, this indicator is classed as Tier 3 [22]. According to the agency in charge, the UNESCO Institute for Statistics (UIS), work for the development of this indicator began in 2016 [23]. The first step toward the development of a global data collection instrument was to collect information about the availability of data through a completed survey by each country in 2017. The survey response rate varied greatly between global regions [22]. The majority of the results came from Europe and North America (59\%) followed by Northern and Western Africa (38\%). The results revealed a limitation in the provision of private expenditure and great variations in the level of detail for public expenditure. Initial results show that $71 \%$ of responding countries had at least one source of public heritage expenditure data, and $29 \%$ of countries had a least one source of private heritage expenditure data [23]. In addition, the UIS SDG 11.4.1 Heritage Statistics Pilot Survey was launched in October 2017 and sent to 14 National Statistical Offices around the world to test and assess the proposed data collection instrument, as well as the data collection process and response burden on countries. Based on the results of the UIS SDG 11.4.1 Metadata and Pilot Surveys undertook in 2017, UIS is designing a detailed global data collection tool that matches the needs of the indicator. Thus, the conceptual accounting treatment of heritage assets faces many challenges [22].

\section{SDGs Indicator 11.4.1}

Goal 11: Make cities and human settlements inclusive, safe, resilient and sustainable

Target 11.4: Strengthen efforts to protect and safeguard the world cultural and natural heritage.

Total expenditure (public and private) per capita spent on the preservation, protection and conservation of all cultural and natural heritage

\begin{tabular}{|c|c|c|c|}
\hline $\begin{array}{c}\text { Type of heritage (cultural, natural, } \\
\text { mixed, World Heritage Centre } \\
\text { designation) }\end{array}$ & $\begin{array}{c}\text { Level of government (national, } \\
\text { regional, and local/municipal) }\end{array}$ & $\begin{array}{c}\text { Expenditure (operating } \\
\text { expenditure/investment) }\end{array}$ & $\begin{array}{c}\text { Private funding (donations in kind, } \\
\text { private non-profit sector, } \\
\text { sponsorship) }\end{array}$ \\
\hline
\end{tabular}

Figure 5. Decomposition of SDG 11.4.

Indeed, there are some existing definitional challenges with the indicator and the development of a methodology. The UIS uses the UNESCO 2000 Framework for Cultural Statistics cultural definitions which define Cultural Heritage as including "artefacts, monuments, and groups of buildings and sites that have a diversity of values including symbolic, historic, artistic, aesthetic, ethnological 
or anthropological, scientific and social significance" [23]. This definition is fundamental to the identification and calculation of heritage expenditure for achieving the SDG 11.4. There is a need, therefore, to identify if classifications of cultural heritage in different nations align with this definition and according to what values. This determines what type of cultural heritage public authorities and private organisations will dedicate to protect and conserve and also why this is done. The UNESCO Framework for Cultural Statistics used for the methodology of SDG 11.4 defines Cultural Heritage as having a diversity of values, including "symbolic, historic, artistic, aesthetic, ethnological or anthropological, scientific and social significance." The articulation of heritage values allows for the consideration of the decision to give a "heritage status and significance", and therefore, the assessment of these values attributed to heritage is a very important activity for the achievement of the SDGs. The Australia ICOMOS (1979) Burra Charter introduced the concept of cultural value-based approaches by defining cultural significance as aesthetic, historic, scientific, social or spiritual value for past, present or future generations [24].

\subsection{European Heritage Indicators}

At a European level, Eurostat collects statistical data for culture from member states. It compiles databases with information on the main developments for culture-related education, cultural employment, cultural enterprises, international trade in cultural goods, cultural participation, the use of information and communication technology (ICT) for cultural purposes, as well as household and government expenditure on culture for all European Union member states. There are challenges related to the compilation of data on culture from all member states. This includes challenges related to the conceptualisation and definition of culture and cultural heritage as well as methodological and infrastructural challenges [25]. Furthermore, the exact culture indicators used for each country are difficult to obtain [26]. Recent culture statistics 2019 include statistics on the economic dimensions of culture (employment, enterprises and international trade) and cultural participation (from the perspective of individuals) [25]. Although the data give a more detailed picture of the impact of culture in member states, they remain purely quantitative and inconsistent in many cases. This focus on the economic dimension positions culture as having a significant economic impact on society, thereby justifying public expenditure for culture on the grounds of the advantages it can bring to a nation [26]. This can be in the form of socioeconomic factors related to urban regeneration, wealth and job creation and even an increase in cultural participation that can lead to social cohesion and community development. Figure 6 illustrates the percentage total share of expenditure that was devoted to cultural services for the selected countries (Depending on data availability for each country. Source: Eurostat online) in 2017 (Figure 1). The highest percentages are in countries such as Lithuania $(2 \%)$ and Poland $(1.7 \%)$, and the lowest countries below the European country average include Portugal $(0.5 \%)$, Italy $(0.6 \%)$ and the United Kingdom $(0.6 \%)$. Public sector expenditure demonstrates the public investment and priorities made. With the growing constraints of the public sector, achieving SDGs requires public-private alliances, and therefore, data should be made available that reflect these joint strategies of action.

Increased employment possibilities, job creation in other sectors and even social belonging and cohesion can all be an indirect consequence of the historic environment [27]. In 2018, there were 8.7 million (3.8\%) people across the 28 countries of the European Union working in a cultural activity or a cultural occupation [25]. Figure 7 shows the total number of people working in a cultural activity or a cultural occupation in 2018 in selected countries. Several countries are above average in cultural service employment, including Estonia (5.6\%), Slovenia (4.7\%) and all of the Nordic countries. The challenge in assessing the scale of employment requires various means of data collection and their categorisation in different countries. Therefore, these numbers should be treated only as indicative. 


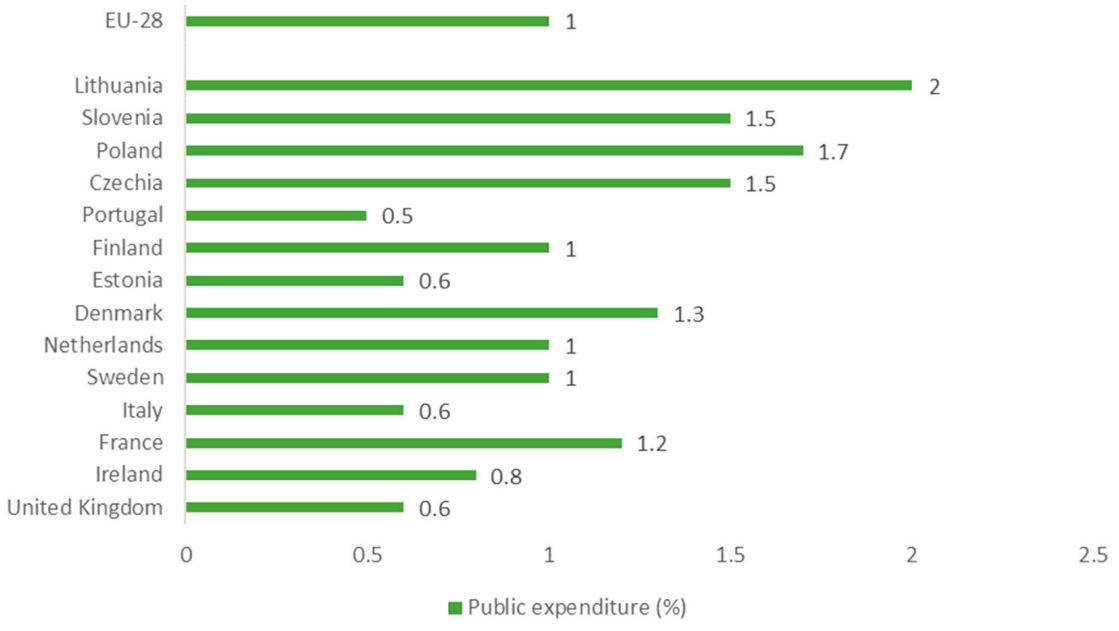

Figure 6. Percentage share of public expenditure (2017).

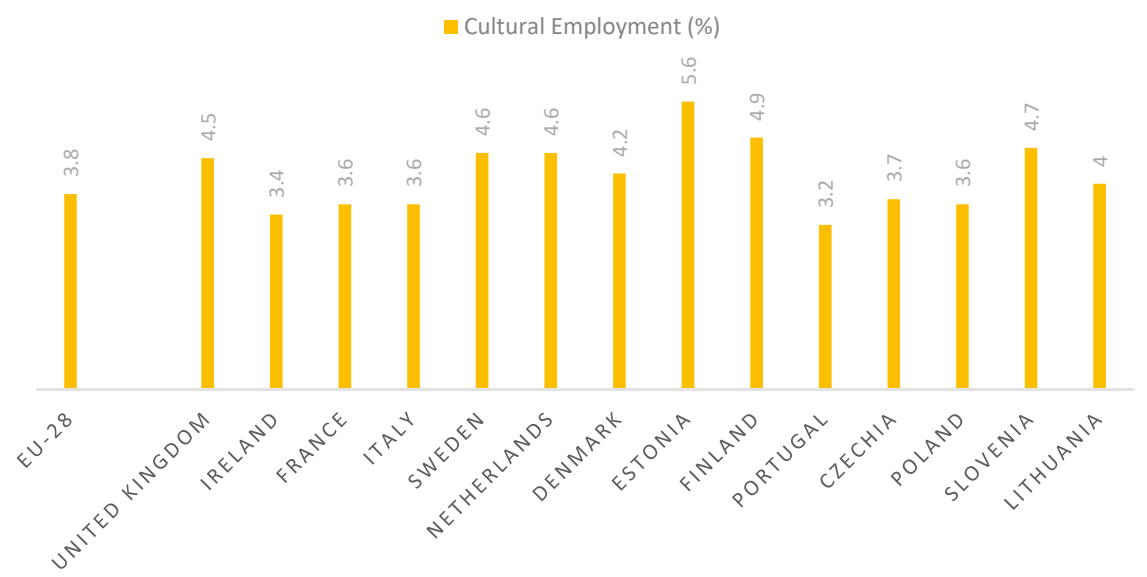

Figure 7. Percentage share of cultural employment (2018).

Figure 8 below illustrates the frequency of participation in cultural activities (cinema, live performances or cultural sites) for over 16-year-olds based on the results of a 2015 survey on social and cultural participation that formed part of EU statistics on income and living conditions (EU-SILC) [25]. The results show that more than half of the adult populations in the selected countries, except, surprisingly, for Italy, participated in cultural activities. Cultural participation can translate to an increase in civic awareness, the knowledge of tradition and history, the awareness of identity and local belonging, as well as influencing the development of other tourism-related activities, such as restaurants and hotel businesses $[27,28]$. 


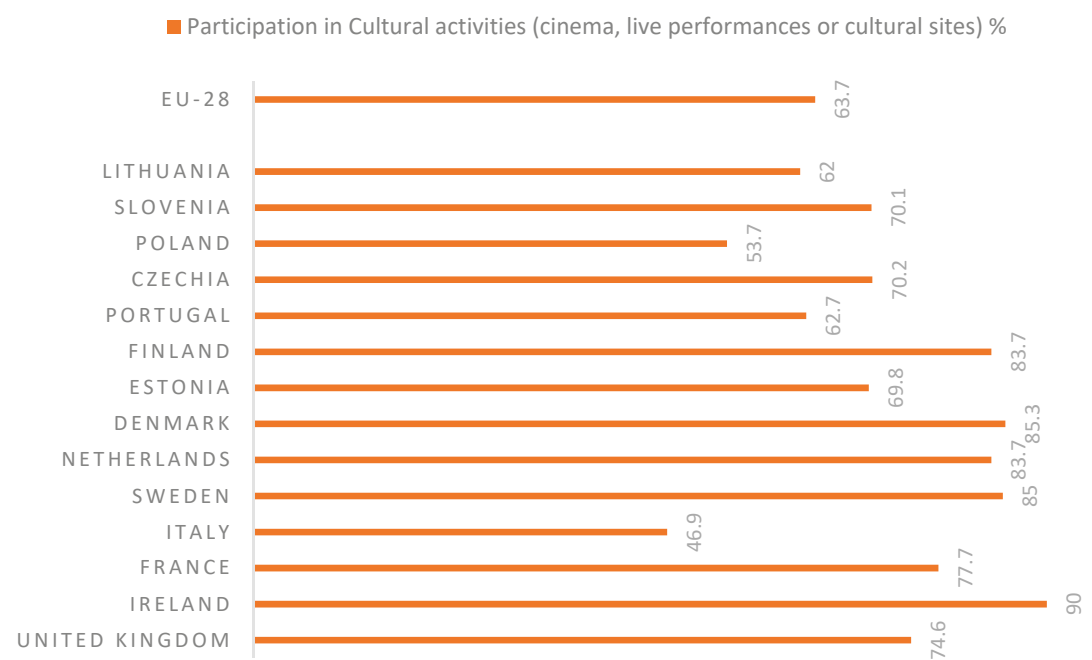

Figure 8. Percentage share of participation in cultural activities (cinema, live performances or cultural sites) (2015).

A frequently used indicator is the number of visitors to cultural sites, often referring to tourism demand and supply [29]. An example of this is the European Group on Museum Statistics (EGMUS), which has maintained a database that contains statistics on museums in Europe. Figure 9 below presents data on the total number of admissions from the five most visited museums in 2018. The highest numbers are found in the United Kingdom, France, Italy and Poland, indicating high levels of tourism interest. Nordic countries such as Denmark and Finland have lower numbers, although the statistics do not give further detail about visits from local and international visitors. Within this context is an acknowledgment of the central role that cultural heritage plays in heritage tourism and sustainable development [17,30-32]. The promotion of tourism results in economic impacts as well as contributing to the wellbeing of local communities [26]. However, the limited mention of the relationship between tourism, heritage and climate change in academic discourse suggests an absence of exploration of the full impacts of cultural tourism. Nocca [29] suggests that there is a double relationship between the tourism sector and climate change where, firstly, climate change represents a threat to cultural heritage, and consequently for cultural tourism (e.g., reducing attractiveness of places) and secondly, the increase of global $\mathrm{CO} 2$ emissions and global warming is a result of tourism (e.g., flights to visit cultural sites), therefore arguing for further development of cultural indicators related to tourism and subsequently accurate data that can support this. 


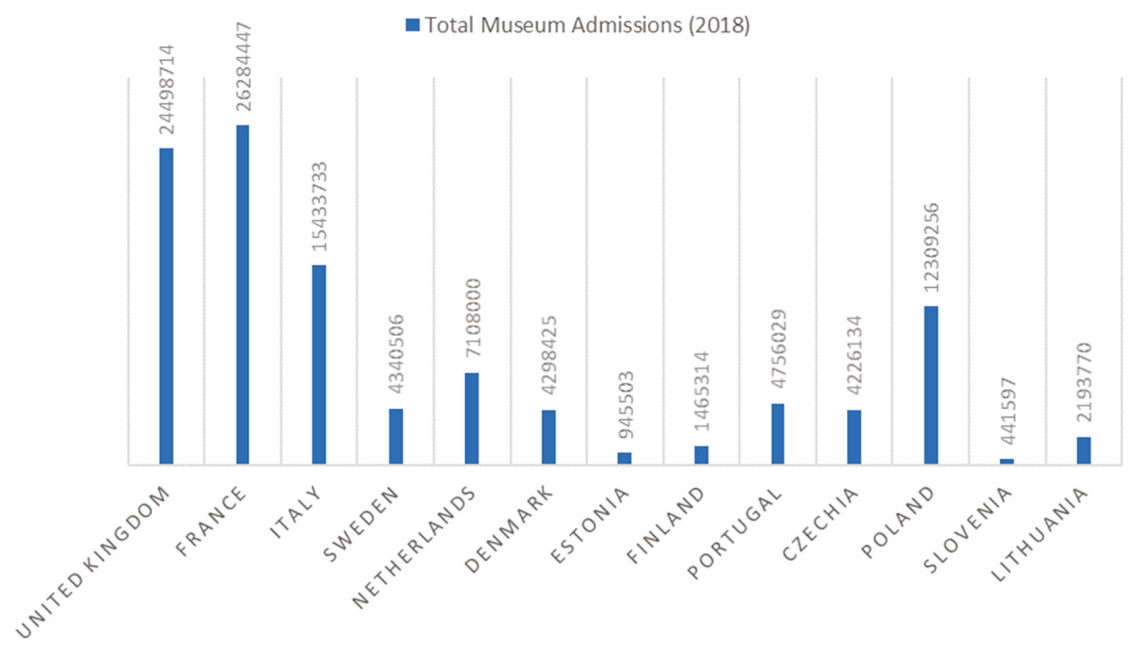

Figure 9. Total number of admissions from the five most visited museums.

\subsection{Beyond SDG 11.4: Developing Harmonised Heritage Data in Europe}

There is a growing discourse on contextualising the SDGs and ensuring that an international agenda can be translated at a local, community level $[3,29,33]$. For attempts to answer these questions and achieve Agenda 2030, local governments, heritage stakeholders and institutions must have sufficient data concerning their tangible and intangible heritage assets and the ability to infrastructure to monitor. In an effort to capture the progress of member states toward achieving the SDGs, the UN developed a global SDG Indicators Database. This database is regularly updated and is only limited to the country/area level and not the local level. Furthermore, the number of indicators represented in the database is limited. Notably, all the indicators explicitly mentioning culture as illustrated in Figure 5 lack any data. Another monitoring system is the World Bank Development Indicators platform, which allows users to explore the data for the targets and selected indicators.

Therefore, several initiatives and projects have been introduced to build evidence-based indicators that will help to build a multidimensional, coherent and strong narrative on culture and development. One of these projects is the UNESCO Culture 2030 Agenda for Sustainable Development Indicators initiative. This project deals with the integration of culture in the implementation of the 2030 Agenda [7]. Its aims to establish a methodology and conceptual framework for countries and cities to assess the contribution of culture to the SDGs as part of the existing implementation mechanisms of the 2030 Agenda at the national or local level. Responses from UNESCO member states in the European region challenge the achievability of these indicators, as illustrated from the member state survey which was launched in May 2019 to develop the framework of thematic indicators. There is unified agreement on the importance of measuring culture and the development of indicators to support and enhance the role of culture in their countries' efforts to implement the 2030 Agenda. However, responses emphasised that the "role and influence of culture is quite difficult to measure" (Estonia) with undeveloped understanding of the "interlinks between culture and human development and their possible undisclosed potential" (Portugal). Furthermore, harmonisation of data is perceived as logistically problematic, requiring "a consensus on a consistent approach so that the different agencies and institutions speak essentially the same language ... it is a compromise result of long-term negotiations" (Czechia). A response from the Netherlands describes the harmonisation and integration of data on culture as "time (and money) consuming ... it needs a central player with a clear mandate to make progress" (Netherlands). Member states such as Latvia and Portugal emphasised the need for qualitative data to give adequate insight on culture's impact on sustainability. 


\section{Results}

This section discusses the results from the analyses in four interdependent thematic areas: (1) cultural heritage's multidimensional impact; (2) safeguarding built cultural heritage; (3) safeguarding intangible heritage; and (4) culture and environmental sustainability. The discourse and the analysed databases demonstrate that culture plays a crucial and diverse role in the sustainable development of urban and rural environments. There is general harmonisation of the conceptualisation and classification of cultural heritage with international frameworks. These results are supported by Figure 10; Figure 11, which illustrate the classification and values of heritage. The responses from the expert informal survey support this finding, with $90 \%$ of respondents agreeing that assets, values and instruments in their country reflect the international and European framework of cultural heritage. The safeguarding of built cultural heritage is a central focus in the databases, although the association with intangible heritage is not yet established. Databases for intangible heritage have been developed according to international standards and created from collaboration with local citizens. Evidence of spatial mapping of cultural heritage emerged during the analysis with GIS-based geoportals and the accessibility of spatial data for the public. This last thematic area also explores the relationship between environmental and cultural processes and practices within the area of sustainability.

\subsection{Cultural Heritage's Mulitidimensional Impact}

The analysis revealed a strong focus on both tangible and intangible cultural heritage as independent and interdependent concepts. Articles included in the analysis presented cultural heritage as evolving, complex and multifaceted. What cultural heritage means and how it has been presented, represented, developed and protected, set against a backdrop of demands and motivations, is depicted as multidimensional [26,29]. Similarly, all the databases used a broad and diverse categorisation of heritage as illustrated in Figures 10 and 11. Built heritage (historical buildings, monuments, etc.) dominates the databases, whereas the academic discourse covers a broader representation of cultural heritage often associated with social aspects of sustainability. The significance of a place embraces the diverse cultural and natural heritage values that people associate with it, or which prompt them to respond to it [34]. The emphasis of layered values is further emphasised in the definition of urban heritage by UNESCO, "urban heritage is for humanity a social, cultural and economic asset, defined by an historic layering of values that have been produced by successive and existing cultures and an accumulation of traditions and experiences, recognized as such in their diversity" [35]. Valuing heritage has become a popular method for the conservation of tangible and intangible heritage in recent years. An alternative definition given by Gravari-Barbas, Bourdeau and Robinson [36] is that value relates to the material being of a site with emphasis upon issues such as integrity and authenticity. In order to make decisions on the preservation of cultural heritage, often undertaken by governments and public administrations, an evaluation of the value of the type of heritage is required [37]. Value-based approaches have been adopted in European countries for an increasing range of categories of cultural heritage, including archaeological and historic objects and sites [24,38,39], historic buildings [40] and urban and rural landscapes [34,41,42]. Often, predominance is given to what Michael Petzet [43] refers to as classical values. This emphasis is illustrated in value typologies used for the databases such as: "architectonic and urbanistic" (Czech Republic); "archaeological" (Poland, Scotland, Ireland, Italy, Estonia, Portugal); "historical" (England, Scotland, Ireland, France, Italy, Estonia, Portugal, Czech Republic); and "cultural-historical" (Belgium, Sweden, Ireland, Denmark, Czech Republic). The academic discourse rather demonstrates a move beyond the material bias of 19 th and 20th century practitioners to a more holistic view of interpreting cultural heritage [31]. Values of cultural heritage are considered plural [44] due to the fact that heritage is considered significant for multiple reasons. This is reflected in other value types included in the design for the databases: "environmental" (Denmark); "artistic" (Scotland, Poland, Ireland, Italy, Portugal); "Evidential" and "communal" (England); "social" (Scotland, Ireland, Portugal); "traditional" (Scotland); "technical" (Ireland, Portugal); Demo-ethno-anthropological (Italy); "Scientific and Technological" 
(Italy), "civilizational" (Slovenia) and "creative" (Ukraine). This representation aligns with the cultural heritage storyline described in the scientific analysis work of Soini and Birkeland [45]. It demonstrates heritage as temporally and spatially embedded, emphasising the importance for the future. Supporting this perspective is the work of Nocca [29], who demonstrates the multidimensional role that cultural heritage plays, including contributing to community wellbeing and social cohesion. Although stating that this impact is poorly considered, the author identifies an indicator from analysis which includes the engagement and participation in cultural activities. Participating in creative and cultural activities may have a considerable impact on strengthening mutual understanding, an individual's quality of life, contributing towards overall wellbeing and enhancing the sense of belonging within society [25].

In introducing the notion of interpretation [32] in this view of culture, the concept of heritage can be broadened into notions of local identity, ethnicity, nationalism, liveability of urban areas and social cohesion. Such an example is given in the context of the sustainability of Lithuanian towns and cities [46] and the sustainable development of Scottish towns [47]. Considering urban planning and development policies, the literature confirms that the holistic integration of cultural heritage is still relatively immature in many European countries. For example, Axelsson et al. [6] showed that more work needs to be done in Sweden to identify cultural indicators and target levels to support the inclusion of cultural values in a planning context. Some of the challenges of this integration from a case study of Slovenia [48] are described as inadequate public participation that relies on conventional strategies instead of bottom-up, direct involvement of users in the spatial planning process. The value of cultural heritage is often constructed through processes of selection criteria appropriated internationally or nationally and [29] then objectified to become worthy of political, economic and tourist attention and conservation. There is therefore a need to safeguard and respect the inherited values and significance of cultural heritage in cities. Analysis of cultural practice in the context of sustainable tourism shed some empirical light on its relevance and utility. The examination of the integration of cultural sustainability in Denmark [49], particular in areas of material and nonmaterial wellbeing, equitable relationships between host communities and tourists and quality of life concludes that cultural practice is not only a useful mechanism for successful competence development but a legitimate and integral part of sustainable tourism.

\subsection{Safeguarding Built Cultural Heritage}

Preserving and protecting built heritage is revealed as a central focus in the analysis. In particular, all the databases showed a significant effort in developing and maintaining monuments and protected historic buildings of national importance and significance. This is often supervised and updated by national Government ministries or Government-funded agencies. Countries such as Italy, France and England lead in the availability of data pertaining to cultural heritage. This is largely because these countries own a noteworthy number of world cultural heritage sites listed by UNESCO under the Convention concerning the protection of the world cultural and natural heritage as ratified in 1972 (Figure 2) [50]. In fact, Italy has a comprehensive database catalogue called SIGECweb which contains over 2,700,000 records of archaeological, architectural, historical heritage and intangible assets. In England, The National Heritage List for England (NHLE) is the only official, up to date, spatial database of all nationally protected historic buildings and sites in England led by Historic England, the UK Government-funded agency for heritage in England. The statutory list is a public platform that is continuously updated and holds over 400,000 entries which allows for both text and map-based searching. The lists were proposed to support the enactment of the Ancient Monuments Protection Acts of 1882 and 1900 by providing definitive lists of buildings and monuments that could be used to identify those most worthy of protection [10]. Scotland has a similar categorisation of heritage within their monument register, created and maintained by Historic Environment Scotland. The classification of heritage resembles England's with the exception of historic marine protection areas and conservation areas. Historic Environment Scotland maintains a schedule (a list) of monuments of national importance. The classification of cultural heritage in France is quite different to that of other 
European countries. The databases are managed by the Department of studies, and documentation and inventory and assisted by the General Inventory of Cultural Heritage, Historical Monuments and the Media Library of Architecture and Heritage in maintaining them. Firstly, the database categorises "Architecture" (Mérimée), which lists buildings in which movable heritage such as Furniture (Palissy) can also be found. Further categories include "Images" (Memory), which contains still images, and "Bibliography" (Archidoc), which contains bibliographic records which can also be related to the records of Mérimée and Palissy.

Building surveys and registers are used in some countries to act as a publicly accessible national inventory of buildings worth national importance and protection. This is the case in Denmark, Sweden and the Netherlands. In Sweden, the National Heritage Board is the authority that is responsible for issues concerning cultural heritage and cultural environments. The Buildings Register (BeBR) contains information on the built cultural heritage. The information comes from regional museums, the Swedish Church, county administrative boards, municipalities, universities and colleges in collaboration with the National Heritage Board. The information in the Settlement Register is updated continuously. The building typologies used are wide-ranging and include industrial sites, sites of agriculture and sites related to folk movement. In the Netherlands, The National Service for Cultural Heritage manages the national monument register. This website contains data on all real estate in the Netherlands that are a national monument, because they are of national significance. These categories range from administrative buildings, farms and mills to animal enclosures and mooring provisions. In Denmark, the FBB is a building register which contains information for approximately 9000 protected buildings and approximately 355,000 buildings whose conservation value has been assessed. In some cases, basic information is provided for buildings which have historical value and are not nationally protected. For example, the Denmark building and housing register has listings of more than 4 million buildings.

Other countries such as Portugal, the Netherlands, Ukraine, Lithuania and Poland have developed databases using the UNESCO framework as a significant catalyst. The cultural heritage database in Portugal is managed by the DGPC (Direcao-Geral do Patrimonio Cultural/Directorate-General for Cultural Heritage) in Portugal. The DCPC is the heritage authority that ensures the management, safeguarding, enhancement, conservation and restoration of assets. Similarly, the database in Poland uses the same language found within the UNESCO Heritage definition. According to the Monument Protection Act of 2003, three types of register of monuments: A (architecture and construction), B (movable monuments) and C (archaeological monuments) are used in the geoportal. Subcategories of immovable monuments include: cultural landscape, small architectural forms, spatial layout, sacred, defensive, industrial, park and garden, residential, public, utility, site of remembrance, cemetery and other. Although, in this classification, there is a certain freedom and for example, immovable archaeological monuments included modern earth bastion fortifications, and in the group of movable monuments, there were some roadside shrines or monuments. The Republic of Lithuania distinguishes between immovable and movable cultural heritage and is inscribed in the Register of Cultural Property, which is a state database.

The analysis showed that built heritage databases are populated from multiple sources and require cooperation from different departments. Estonia's national register of cultural monuments is linked to several other databases and registers, including the population and land registers, the database of the rural architectural heritage and the register of shipwrecks and external registers, such as the burial site register and war graves register. The Finnish Heritage Agency developed the built cultural environment database through cooperation and consultation with municipalities, regional environment centres, regional museums and the Regional Councils. The database is included among the national land-use objective inventories defined in the Land-Use and Building Act, forming the foundation for the land-use planning. The online database provides a free download of spatial data and research reports on the sites. 


\subsection{Safeguarding Intangible Heritage}

The interdependent relationship between tangible and intangible cultural heritage and its crucial position in sustainability is established in the results from the discourse analysis. Indeed, intangible heritage is often represented as being materialised and constructed by tangible heritage as well as playing a vital role in creating tangible heritage itself $[17,18,51-53]$. When considering the databases, the inclusion of intangible heritage assets is inconsistent in the approaches. UNESCO's 2003 Convention for the Safeguarding of the Intangible Cultural Heritage gives recommendations for signatories to develop a database of national intangible cultural heritage (Figure 2). The classification used the databases reflects the UNESCO classification of intangible cultural heritage (Figures 10 and 11) and includes diverse categories such as: oral tradition and folk literature, performances, customs and habits, knowledge of nature and the environment, economic knowledge and skills, traditional physical practices and games. In some cases, intangible heritage is classified and included in the main heritage database to be viewed and understood as a holistic representation of cultural heritage. This is the case for the PACI Integrated Project for Intangible Cultural Heritage and Cultural Diversity in Italy, which maintains over 300 intangible heritage entities integrated in the General Information System of the SIGECweb Catalog. This database has developed into a collaborative platform for the cataloguing of archaeological, architectural and landscape, demo-ethno-anthropological, photographic, musical, naturalistic, numismatic, scientific and technological, historical and artistic assets. In France, the Directorate General of Heritage maintains the online intangible cultural heritage databases made available on the website of the Ministry of Culture linked to the main built heritage database. In most other cases, intangible heritage assets are represented as seemingly separate from Government departments and national databases. This can be seen as a means to allow the public to engage and contribute to the development of the database. For example, Scotland has a separate database for its intangible cultural heritage, populated by contributions made by anyone and assessed by the Museums Galleries Scotland. Collaboration and participation in the development of the site is promoted through social networks and platforms. Another database is through the Netherlands Intangible Heritage Network and Register of Inspiring Examples of Heritage. Communities and individuals can register intangible heritage with the Intangible Heritage Network, showing the variety of cultural expressions through which heritage is recognised locally. The Register of Inspiring Examples of Heritage illustrates examples developed by heritage communities, groups or individuals. Similar local participation approaches can be found in Slovenia, Sweden, Denmark, Poland, Finland and Estonia. There is an absence of intangible heritage data included in any publicly accessible database for countries such as Ukraine, Ireland and England.

\subsection{Culture and Environmental Sustainability}

Findings from the discourse analysis suggest that the cultural aspects (social and spatial) are increasingly being considered in achieving environmental sustainability. Discourse suggests that the concept of landscapes has an identity that has historical, geomorphological, cultural and other aspects that are complementary to ecological aspects [54]. Sustainability is therefore achieved through intentional interaction with cultural aspects such as in the case study of sustainable rural development of a high mountain national park in France. Thompson [55] describes the careful balance needed to take actions to conserve the built environment and advance public awareness of cultural heritage and achieving ecological sustainability in a national park of high tourist interest. Intangible living practices and traditional knowledge are increasingly valued in the sustainable development of forest and woodland practices. However, this integration and the systems that can support it are being threatened. This is the case in Ukraine's mountain villages which are experiencing socioeconomic and technological changes in agriculture, industrial forestry and natural resource management [56]. In a place transitioning from socialism to market economy, the traditional village system based on sociocultural values is at risk. The authors argue for further evaluation of local and regional concepts that satisfy economic, ecological and cultural dimensions of sustainable landscapes. However, there 
is little mention of the impact of climate change and its relationship with culture within the context of sustainability. The impact of climate change on heritage has wide consequences ranging from structural damage, atmospheric moisture and temperature changes, new interactions between natural and anthropogenic factors to more socioeconomic factors, such as tourism demand and supply, as mentioned earlier in this paper.

Many of the databases analysed developed or are in the process of developing geoportals that use geographic information systems (GIS). The National Heritage Institute (NHI), as part of the Ministry of Culture of the Czech Republic, organises and manages cultural heritage in the Czech Republic. In 2015, a new geoportal was developed to ensure the availability of map services and geodata of the NHI from one single platform. The geoportal combines several map applications, including the historical catalogue for the Czech Republic and interests of monument care in terms of the current legal status of protection, and particularly relevant for risk management is the information for monuments threatened by natural and anthropogenic influences. Similar GIS-based heritage maps include databases from England, Scotland, Sweden and the Netherland register, which can be searched online and downloaded as GIS software packages free of charge for the public. Portugal's ATLAS database integrates the country's immovable, movable and intangible cultural heritage and constantly updates the ATLAS of classified heritage. The ATLAS can perform georeferencing searches of immoveable heritage with spatial information about the exact location of the monuments and sites as well as general searches. The information in the geodatabases is structured based on the INSPIRE (Infrastructure for Spatial Information in Europe) Directive 2007, which produced further guidelines for heritage assets in 2017, "INSPIRE data specification on Protected Sites-Technical Guidelines". In accordance with the Inspire Directive, the Member States of the European Union should provide spatial geographic data according to consistent standardised format. INSPIRE aims to develop "interoperability" across the European Community, which is the possibility to combine spatial data and services from different European countries in a singular way. However, much of the focus on research and development from INSPIRE is upon the management of natural environmental and security/asset information. Although there is a clear mandate for increased information to be published under INSPIRE, the relevance of information about built heritage is still largely ambiguous [57].

Systematic geographical positioning of cultural heritage can assist in effectively manage risk and contributing to the sustainability of urban and rural landscapes. The application of GIS allows for the integration of different data from multiple sources at an early point in planning processes that can allow for allowing for increased understanding and participation from stakeholders through planning and development conflicts. Furthermore, data on climate change can be incorporated with information about the historic environment. The overlay of spatial maps of vulnerable heritage and risk factors can communicate in a simple and effective manner, the overall scale of the problems presented by climate change. To successfully utilise the potential of GIS in achieving sustainability, there must be a reliable knowledge of European cultural heritage stock [58], and this includes, for example, a technical description of the materials and structures applied, or any information about its current state, all of which is decisive for its vulnerability to adverse natural actions. Furthermore, challenges are present-a lack of availability, quality, organisation, accessibility and sharing of spatial information is common to a large number of policies and activities and experienced across the various levels of public authority in Europe. 


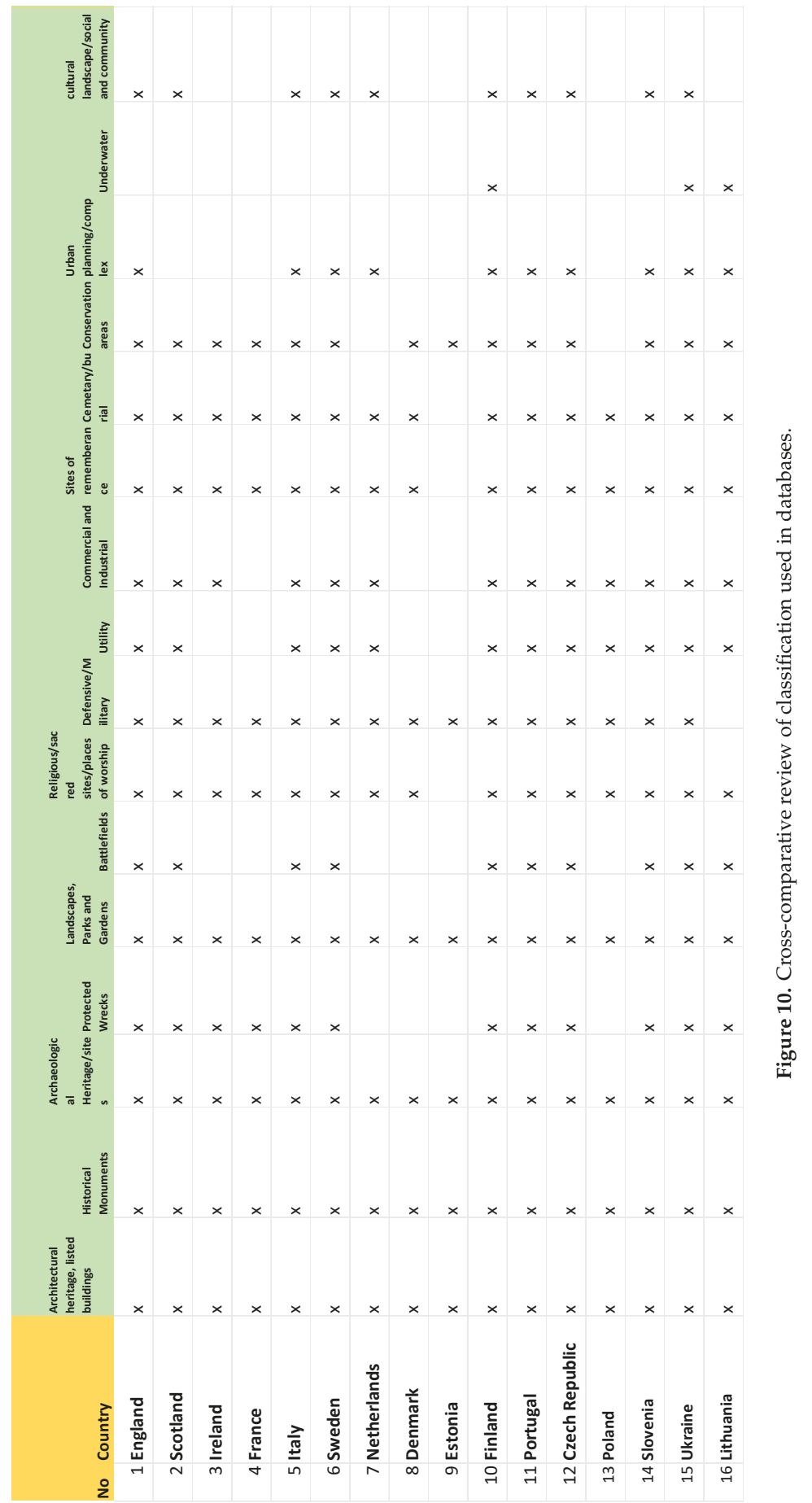




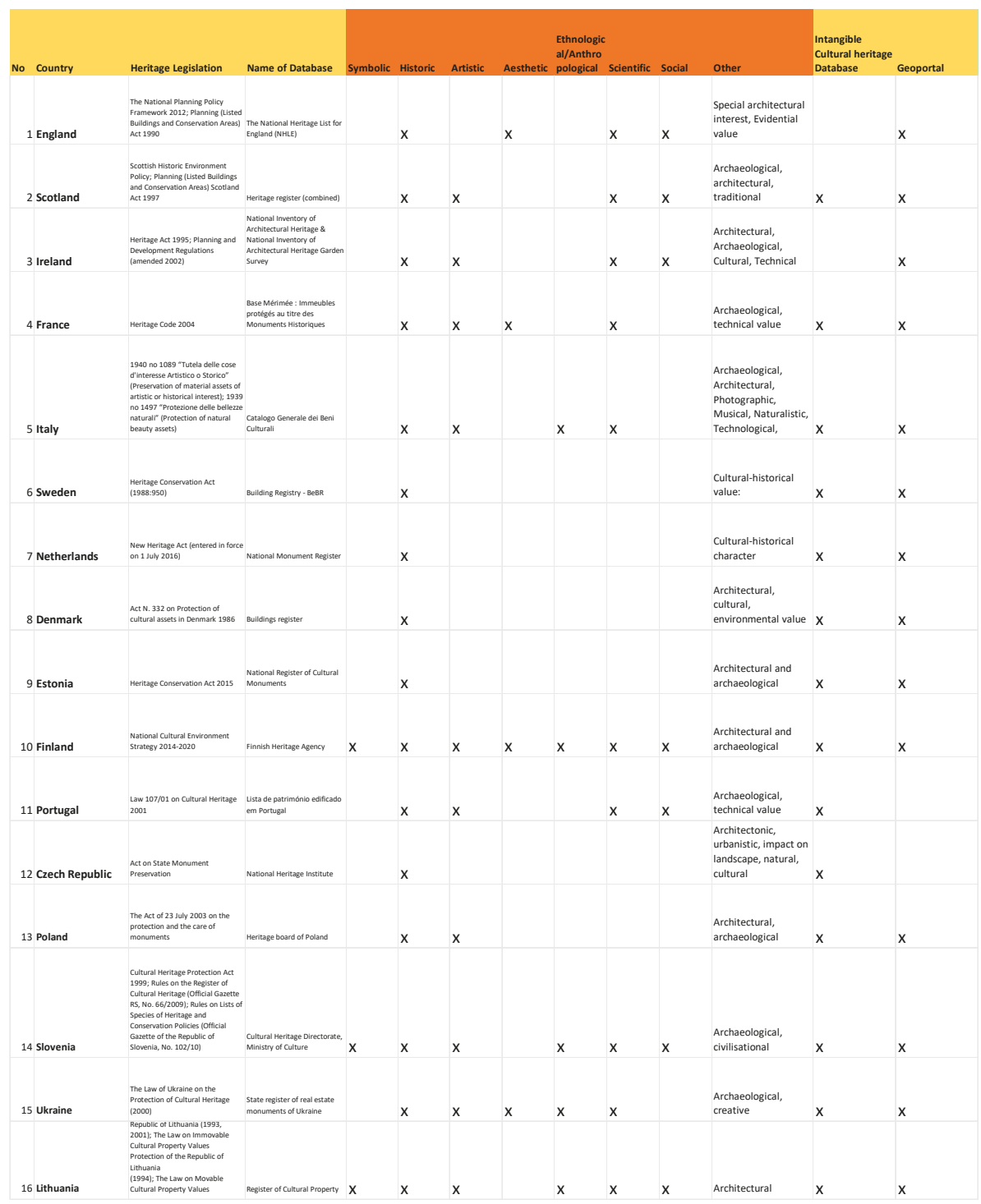

Figure 11. Cross-comparative review of value typologies used in databases.

\section{Discussion}

There is consensus in the culture-focused discourse of the transformative role culture plays in the economic, social and environmental dimensions of development. However, this contribution is yet to be truly visible and tangible in broader sustainability discourses. Cultural sustainability as a concept is still hidden within the agenda of social sustainability and often viewed in tandem. Part of this invisibility is due to the paucity and fragmentation of cultural data and evidence-based research $[7,26]$ that can be used for advocacy of culture in sustainability as well as for integration into development plans and policies at the national and urban levels. 
This cross-comparison study of national heritage databases has highlighted that heritage conceptualisations in their broad context are generally aligned with the international framework built by organisations such as UNESCO, ICOMOS and the Council of Europe. However, some differences in approach are reflected in how significance and value is assigned to cultural heritage and what classification is used. The results suggest that certain heritage assets that have significant attached values have been left out of government efforts to raise awareness and promote heritage. There is a larger focus on tangible heritage with historic value and architectural and/or artistic values. This is generally a traditional focus based on the articulation by experts' analysis of heritage. The cross-comparison demonstrates how states are embracing other sociocultural, less visible factors such as the ethnological, anthropological and community values. None of the databases analysed for this paper included the "symbolic" value in their approach as mentioned in the FCS definition, although "cultural" significance is integrated into most of the approaches. The scope of values illustrates the diversity of values used in the heritage conservation management and planning processes, thereby encouraging the widening of the circle of stakeholders involved in value assessment for heritage projects. Consequently, this recognition of multidimensional and interdependent values improves both the process and the outcome. Therefore, based on the results, this paper proposes the integration of a typology of values embracing this diversity in values as a means to facilitate the assessment and integration of different heritage values for the planning and management of tangible and intangible heritage. Multilayered and interdependent values may not be appropriate for all heritage sites and situations. However, it is an attempt to facilitate discussions and understanding of the different valuing processes at play in heritage conservation toward the development of methodological approaches for the SDG indicators, thus bringing new considerations to the discussions about what to conserve, how to conserve it, where to set priorities and how to handle conflicting interests. The working assumption is that these value types encompass most of the heritage values that shape decision making and must be considered within the context of tangible and intangible heritage. The values overlap and are interconnected and therefore should be viewed as different because they correspond to different ways of conceptualising the value of the heritage to different stakeholder groups [29,41].

Indeed, the benefits as discussed earlier from cultural heritage conservation are wide-ranging [29]. The issue of the protection of cultural heritage when considering the contribution to sustainable development is due to the fragmentation and inconsistency of existing national databases, which do not contain some data that are essential. Moreover, they are not standardised, harmonised or coordinated for effective exploitation [59].

Cultural heritage is making a growing contribution to urban economies globally, although a significant limitation is the availability and accessibility of data which varies from country to country. A comprehensive, publicly accessible database on heritage assets for each member state would provide an essential resource to support the SDG monitoring and achievement of the goals. These databases allow for the compilation of heritage in one place, but more importantly, they reflect the classification and valorisation used by the member state. Therefore, the details provided can be used as a comparative tool and data source for analysis. The findings demonstrate broad classification of tangible heritage within the reviewed national databases. These databases are the underpinning for establishing mechanisms for protection. In this regard, databases of local tangible and intangible cultural heritage are critical tools for the management of these resources. They are a key component of cultural management plans and critical in order to know, protect and preserve what is found in a specific area. Documentation and analysis of local knowledge systems, sociocultural practices and values must be documented and analysed as a means to achieve a comprehensive understanding of urban realities.

In the last decade, numerous organisations, meetings and research projects have turned their attention on various aspects related to protecting cultural heritage in Europe and the improvement of methods. Organisations such as ICOMOS (International Council on Monuments and Sites) and ICCROM (International Centre for the Study of Preservation and Restoration of Cultural Property) recommend strengthening the enabling framework for heritage protection through numerous measures, 
such as the improvement of databases at local, regional, provincial, national and international levels. From as early as the 1980s, the European Commission and the Council of Europe has been supporting documentation on architectural heritage, with the Direction du Patrimoine (France), the Nantes Colloquy on Inventory and documentation methods. Its purpose was to determine practical forms of co-operation between heritage documentation centres throughout Europe and to prepare a definition of common standards on the basis of comparing the inventory methods used in different countries [60]. As part of a funded project by the Getty Conservation Institute, Myers [12] described six characteristics of effective heritage database and management systems. The first characteristic is accuracy; records should have accurate information (such as location and significance/designation status) in order to make decisions and manage risk affecting heritage sites. The second is comprehensiveness; to aim to safeguard all heritage at risk within a particular area, there should be wide-ranging information about the geographic area, as gaps in coverage could significantly increase the risk on heritage. Thirdly, databases need to have up-to-date and current information. The fourth characteristic is authoritativeness; databases and inventory systems should be a definitive system of record for that context. Controlled accessibility is the fifth characteristic, which refers to information from that database that needs to be accessible, such as including data export functionality and expanded search tools. Security of information from corruption or intentional damage is the sixth characteristic. However, spatial content in databases is largely restricted to generalised locational data rather than representing the spatial extents of records.

The sustainability of cultural heritage and its management is strongly dependent on the national, regional and local government and the participation and support from local communities. Involving local communities includes reconciling international and local values of heritage which can sometimes be contested. The involvement of the public in developing the intangible inventories as described in many of the cases reflects the increasing focus on identifying, recognising and valuing the local community as a key actor in the process of sustainable heritage management. Local authorities play a crucial role in enabling this dialogue. Indeed, the local government in many cases, such as in England, acts as a landowner of historic buildings, a facilitator for growth and development and an advocate for heritage. Often set within the context of constrained public finances, the local government must ensure that heritage assets are managed in a sustainable manner in order that the benefits the can be provided be realised.

\section{Conclusions}

The findings allow concluding that it could be possible to mainstream those indicators across different databases, which could lead to depicting the overall level of attainment of the Agenda 2030 targets on heritage. However, more research is needed to develop a robust correlation between national datasets and international targets. This study confirms the existence of harmonisation of data toward the achievement of the SDGs. The cross-comparison review of the databases identified a broad agreement of the conceptualisation of cultural heritage with international frameworks. The value approaches and classification confirm that states are diversifying in their recognition and documentation of cultural heritage, thereby recognising cultural heritage as an important resource for sustainable urban development, although some inconsistencies still exist when considering the harmonisation of heritage data, such as the use of sociocultural values in assessing heritage and the classification used in identifying tangible heritage. Based on the results from this cross-comparison, the statistical definition of heritage from the UIS UNESCO Framework for Cultural Statistics for the SDG 11.4 target is limiting. It does not fully reflect the conceptualisation that is used across different countries. The current SDG 11.4 indicator is inadequate in representing the challenges and opportunities of cultural heritage within the context of sustainable development. To enhance the comparability of heritage data across cities and countries, there is a crucial requirement for standardised methods for perceiving, valuing, measuring and monitoring heritage. Therefore, national and local capacity development is needed to ensure the sustainability of national and local processes. The harmonisation of these processes using similar 
standards and conceptualisation can allow for the comparison of data among countries toward the achievement of the SDGs.

A limitation of the methods used in this paper is that this can only be considered as a "snapshot", as the databases are constantly updated and changed. Therefore, not all information will be available publicly, and this is not a complete reflection of the processes and information that are available. The authors selected a small sample of countries in the European region intended to illustrate the level of harmonisation. It is important to highlight the contextualisation of these areas and the differences that exist between the countries in this region. This could not be covered sufficiently in this paper. Furthermore, this paper did not use national statistics data for culture in its entirety due to the lack of comparable data, particularly qualitative data, for multiple European countries. However, this cross-comparison acts as an indication of the gaps and possible harmonisation that exists within European cultural heritage practices and processes.

Future research is recommended into the pluralistic values and impact of cultural heritage in achieving sustainable development. There are many emerging studies and projects that present various aspects of the vital role of heritage and the development of heritage indicators. However, many of these are concerned with only selected aspects of the potential impact of cultural heritage and tend to lack evidence. Future research development can therefore include the development of evidence-based indicators for European countries with emphasis on highlighting the multifaceted role of cultural heritage in sustainable development. This paper also acknowledges that future findings from heritage-related research projects such as those funded through the current Horizon 2020 research programme [60] will fill gaps of knowledge. The continuous development of cultural data and the international efforts towards data interoperability open up possibilities for new research and unique comparisons of the impact of culture between European countries. In conclusion, the evaluation of cultural heritage at multi-levels to the achievement of sustainable development as emerging from the analysis requires the recognition that the benefits from cultural heritage conservation are multivalent, pluralistic and layered from state to state, thus calling for national, regional and local government and heritage stakeholders to carefully integrate of these aspects into the development of multidimensional SDG indicators.

Author Contributions: Conceptualisation, L.P., C.T. and B.N.M.; Methodology, L.P., C.T. and B.N.M.; Supervision, L.P.; Writing—original draft, B.N.M.; Writing—review and editing, C.T. and B.N.M. All authors have read and agreed to the published version of the manuscript.

Funding: This research is financed by the PRIN program-Research Projects of National Interest, Italian Ministry for University and Research, n. 2015EAM9S5, Project name "Protecting the Cultural Heritage from water-soil interaction related threats".

Conflicts of Interest: The authors declare no conflict of interest. The funders had no role in the design of the study; in the collection, analyses, or interpretation of data; in the writing of the manuscript, or in the decision to publish the results.

\section{References}

1. United Nations. The New Urban Agenda; United Nations: San Francisco, CA, USA, 2017.

2. United Nations. Sustainable Development Goals. 2016. Available online: http://www.un.org/ sustainabledevelopment/sustainable-development-goals/ (accessed on 17 January 2020).

3. Guzmán, P.; Roders, A.P.; Colenbrander, B. Measuring links between cultural heritage management and sustainable urban development: An overview of global monitoring tools. Cities 2017, 60, 192-201. [CrossRef]

4. Petti, L.; Trillo, C.; Makore, B.C.N. Towards a Shared Understanding of the Concept of Heritage in the European Context. Heritage 2019, 2, 2531-2544. [CrossRef]

5. Chiabrando, F.; Colucci, E.; Lingua, A.; Matrone, F.; Noardo, F.; Spanò, A. A European Interoperable Database (Eid) to Increase Resilience of Cultural Heritage. ISPRS-Int. Arch. Photogramm. Remote. Sens. Spat. Inf. Sci. 2018, 151-158. [CrossRef] 
6. Axelsson, R.; Angelstam, P.; Degerman, E.; Teitelbaum, S.; Andersson, K.; Elbakidze, M.; Drotz, M.K. Social and cultural sustainability: criteria, indicators, verifier variables for measurement and maps for visualization to support planning. Ambio 2013, 42, 215-228. [CrossRef] [PubMed]

7. Hosagrahar, J. UNESCO Thematic Indicators for Culture in the 2030 Agenda for Sustainable Development, in Analytical Report of the Consultation with the Member States; UNESCO World Heritage Centre: Paris, France, 2019.

8. Koch, F.; Krellenberg, K. How to Contextualize SDG 11? Looking at Indicators for Sustainable Urban Development in Germany. ISPRS Int. J. Geo-Inf. 2018, 7, 464. [CrossRef]

9. Bala, S. Digital inventories on Cultural Memories and Intangible Cultural Heritage: Case study of Yadav community of Haryana, India. Incl. Bibl. 2012, 1, 11-19.

10. Carlisle, P.; Lee, E. Recording the past: heritage inventories in England. J. Cult. Herit. Manag. Sustain. Dev. 2016, 6, 128-137. [CrossRef]

11. Daele, K.V.; Meganck, L.; Mortier, S. On data-driven systems and system-driven data: Twenty years of the Flanders heritage inventory. J. Cult. Herit. Manag. Sustain. Dev. 2016, 6, 153-165. [CrossRef]

12. Myers, D. Heritage inventories: promoting effectiveness as a vital tool for sustainable heritage management. J. Cult. Herit. Manag. Sustain. Dev. 2016, 6, 102-112. [CrossRef]

13. Shah, K. Creation of cultural heritage inventories: case of the historic city of Ahmadabad. J. Cult. Herit. Manag. Sustain. Dev. 2016, 6, 166-194. [CrossRef]

14. Council of Europe. European Heritage Network (HEREIN). 2019. Available online: https://www.coe.int/en/ web/herein-system/home (accessed on 17 January 2020).

15. Compendium. Compendium of Cultural Policies and Trends in Europe. 2019. Available online: https: //www.culturalpolicies.net/web/index.php (accessed on 17 January 2020).

16. UNESCO. Convention For The Safeguarding Of The Intangible Cultural Heritage; UNESCO: Paris, France, 2003.

17. Jigyasu, R. The Intangible Dimension of Urban Heritage, in Reconnecting the City: The Historic Urban Landscape Approach and the Future of Urban Heritage; Bandarin, F., Oers, R.v., Eds.; Wiley-Blackwell: Hoboken, NJ, USA, 2014.

18. Lipp, T. Materializing the Immaterial. On the Paradox of Medializing Intangible Cultural Heritage. In Understanding Heritage: Perspectives in Heritage Studies; Albert, M.-T., Bernecker, R., Rudolf, B., Eds.; UNESCO Digital Library: Paris, France, 2013.

19. UNESCO. Convention for the Protection of Cultural Property in the Event of Armed Conflict with Regulations for the Execution of the Convention 1954; United Nations Educational Scientific and Cultural Organisation: Paris, France, 1954

20. UN-Habitat. Metadata on SDGs Indicator 11.4.1 Indicator category: Tier III; United nations human settlements program (UN-Habitat): Nairobi, Kenya, 2018.

21. The Office of the High Commissioner for Human Rights. Special Rapporteur in the field of cultural rights. 2019. Available online: https://www.ohchr.org/en/issues/culturalrights/pages/srculturalrightsindex.aspx (accessed on 17 January 2020).

22. United Nations. Goal 11; United Nations: San Francisco, CA, USA, 2018.

23. UNESCO Institute for Statistics. UNESCO Framework for Cultural Statistics; UNESCO Institute for Statistics: Montreal, QC, Canada, 2009.

24. Burra Charter. The Burra Charter: The Australia ICOMOS Charter for Places of Cultural Significance. Available online: http://openarchive.icomos.org/2145/ (accessed on 17 January 2020).

25. Eurostat. Culture Statistics 2019 Edition; European Union: Luxembourg, Publications Office of the European Union; Available online: https://ec.europa.eu/eurostat/documents/3217494/10177894/KS-01-19-712-EN-N. pdf/915f828b-daae-1cca-ba54-a87e90d6b68b (accessed on 17 January 2020).

26. Giraud-Labalte, C. Cultural Heritage Counts for Europe Report; CHCfE Consortium: Krakow, Poland, 2015; Available online: https://www.academia.edu/29155756/Cultural_Heritage_counts_for_Europe._Executive_ Summary (accessed on 17 January 2020).

27. Pendlebury, J. Conservation values, the authorised heritage discourse and the conservation-planning assemblage. Int. J. Herit. Stud. 2013, 19, 709-727. [CrossRef]

28. Pendlebury, J.; Townshend, T.; Gilroy, R. The Conservation of English Cultural Built Heritage: A Force for Social Inclusion? Int. J. Herit. Stud. 2004, 10, 11-31. [CrossRef] 
29. Nocca, F. The Role of Cultural Heritage in Sustainable Development: Multidimensional Indicators as Decision-Making Tool. Sustainability 2017, 9, 1882. [CrossRef]

30. Girad, L. Toward a Smart Sustainable Development of Port Cities/Areas: The Role of the "Historic Urban Landscape" Approach. Sustainability 2013, 5, 4329-4348. [CrossRef]

31. Bandarin, F.; Oers, R. The Historic Urban Landscape: Managing Heritage in an Urban Century; Wiley-Blackwell: Hoboken, NJ, USA, 2012.

32. Hitchcock, M.; King, V.; Parnwell, M. Heritage Tourism in Southeast Asia; NIAS Press: Copenhagen, Denmark, 2010.

33. Girard, L.F.; Nocca, F. Integrating cultural heritage in urban territorial sustainable development. In ICOMOS 19th General Assembly and Scientific Symposium "Heritage and Democracy"; ICOMOS: New Delhi, India, 2018.

34. English Heritage. Conservation Principles, Policies and Guidance: For the sustainable management of the historic environment. Available online: https://historicengland.org.uk/images-books/publications/ conservation-principles-sustainable-management-historic-environment/ (accessed on 17 January 2020).

35. UNESCO. Global Report on Culture for Sustainable Urban Development; United Nations Educational, Scientific and Cultural Organization (UNESCO): Paris, France, 2016.

36. Gravari-Barbas, M.; Bourdeau, L.; Robinson, M. World Heritage and Tourism: From Opposition to Co-production, in World Heritage, Tourism and Identity: Inscription and Co-Production; Bourdeau, L., Ed.; Routledge: London, UK, 2015; pp. 1-24.

37. Frey, B. The Evaluation of Cultural Heritage: Some Critical Issues. In Economic Perspectives on Cultural Heritage; Rizzo, M.H.A.I., Ed.; Palgrave MacMillan: Basingstoke, UK, 1997; pp. 31-49.

38. Reigl, A. The Modern Cult of Monuments: Its Character and Its Origin. Available online: https://marywoodthesisresearch.files.wordpress.com/2014/03/riegl_the-modern-cult-of-monuments_ sm.pdf (accessed on 17 January 2020).

39. Darvill, T. Value systems and the archaeological resource. Int. J. Herit. Stud. 1994, 1, 52-64. [CrossRef]

40. Robles, L.G. A Methodological Approach Towards Conservation. Conserv. Manag. Archaeol. Sites 2010, 12, 146-169. [CrossRef]

41. Mason, R. Assessing Values in Conservation Planning: Methodological Issues and Choices. In Assessing the Values of Cultural Heritage; Torre, M.d.l., Ed.; Getty Conservation Institute: Los Angeles, CA, USA, 2002.

42. Stephenson, J. The Cultural Values Model: An integrated approach to values in landscapes. Landsc. Urban Plan. 2008, 84, 127-139. [CrossRef]

43. Petzet, M. Introduction. In The World Heritage List: What is OUV? Defining the Outstanding Universal Value of Cultural World Heritage Properties; Jokilehto, J., Ed.; Hendrik Bäßler Verlag: Berlin, Gemany, 2008; pp. 7-10.

44. Fredheim, L.H.; Khalaf, M. The significance of values: heritage value typologies re-examined. Int. J. Herit. Stud. 2016, 22, 1-17. [CrossRef]

45. Soini, K.; Birkeland, I. Exploring the scientific discourse on cultural sustainability. Geoforum 2014, 51, $213-223$. [CrossRef]

46. Karvelyte-Balbieriene, V.; Grazuleviciute-Vileniske, I. Sustainability of Lithuanian villages with churches and landscape. J. Cult. Herit. Manag. Sustain. Dev. 2014, 4, 57-79. [CrossRef]

47. MacLeod, D. Scottish theme towns: have new identities enhanced development? J. Tour. Cult. Chang. 2009, 7, 133-145. [CrossRef]

48. Uršič, M. The Diminished Importance of Cultural Sustainability in Spatial Planning: The Case of Slovenia. Cult. Local Gov. 2011, 3, 101-116. [CrossRef]

49. Liburd, J.J. Sustainable Tourism, Cultural Practice and Competence Development for Hotels and Inns in Denmark. Tour. Recreat. Res. 2007, 32, 41-48. [CrossRef]

50. UNESCO. Convention Concerning the Protection of the World Cultural and Natural Heritage; United Nations Educational Scientific and Cultural Organization (UNESCO): Paris, France, 1972.

51. McCleery, A. Scoping and Mapping Intangible Cultural Heritage in Scotland Final Report 2008. Available online: https://www.semanticscholar.org/paper/Scoping-and-mapping-intangible-cultural-heritage-inMccleery-McCleery/7aea5211e840d08c51d27ae43c8ab66701901f55 (accessed on 17 January 2020).

52. Vecco, M. A definition of cultural heritage: From the tangible to the intangible. J. Cult. Herit. 2010, 11, 321-324. [CrossRef]

53. Yahaya, A. The Scope and Definitions of Heritage: From Tangible to Intangible. Int. J. Herit. Stud. 2006, 12, 292-300. 
54. Vos, W.; Meekes, H. Trends in European cultural landscape development: perspectives for a sustainable future. Landsc. Urban Plan. 1999, 46, 3-14. [CrossRef]

55. Thompson, I.B. Sustainable Rural Development in The Context Of A High Mountain National Park: The Parc National De La Vanoise, France. Scott. Geogr. J. 2008, 115, 297-318. [CrossRef]

56. Elbakidze, M.; Angelstam, P. Implementing sustainable forest management in Ukraine's Carpathian Mountains: The role of traditional village systems. For. Ecol. Manag. 2007, 249, 28-38. [CrossRef]

57. McKeague, P.; Scotland, H.E.; Corns, A.; Posluschny, A. Why the Historic Environment needs a Spatial Data Infrastructure. Internet Archaeol. 2017, 43, 7. [CrossRef]

58. Bumbaru, D. Initiatives of ICOMOS to Improve the Protection and Conservation of Heritage Sites Facing Natural Disasters and Climate Change. In Cultural Heritage and Natural Disasters Risk Preparedness and the Limits of Prevention; Meier, H.-R., Petzet, M., Will, T., Eds.; ICOMOS-International Council on Monuments and Sites: Paris, France, 2007; pp. 203-213.

59. Drdácký, M. Protecting the Cultural Heritage from Natural Disaster. Available online: http://www. europarl.europa.eu/thinktank/en/document.html?reference=IPOL-CULT_ET(2007)369029 (accessed on 17 January 2020).

60. European Commission. Communication from the Commission to the European Parliament, the Council, the European Economic and Social Committee and the Committee of the regions. Towards an integrated approach to Cultural Heritage for Europe. 2014. Available online: https://eur-lex.europa.eu/legal-content/en/ TXT/?uri=CELEX:52014DC0477 (accessed on 17 January 2020).

(C) 2020 by the authors. Licensee MDPI, Basel, Switzerland. This article is an open access article distributed under the terms and conditions of the Creative Commons Attribution (CC BY) license (http://creativecommons.org/licenses/by/4.0/). 
Article

\title{
New Public Institutional Forms and Social Innovation in Urban Governance: Insights from the "Mayor's Office of New Urban Mechanics" (MONUM) in Boston
}

\author{
Carmelina Bevilacqua *, Yapeng Ou, Pasquale Pizzimenti and Guglielmo Minervino \\ CludsLab PAU Department, Mediterranea University of Reggio Calabria, 89124 Reggio Calabria, Italy; \\ yapeng.ou@unirc.it (Y.O.); pasquale.pizzimenti@unirc.it (P.P.); guglielmo.minervino@unirc.it (G.M.) \\ * Correspondence: cbevilac@unirc.it
}

Received: 30 September 2019; Accepted: 12 December 2019; Published: 18 December 2019

\begin{abstract}
This paper investigates how public sector institutions change their form and approach to achieve a socially innovative urban governance. The "Mayor's Office of New Urban Mechanics" (MONUM) in Boston, Massachusetts (USA) proves a representative case of innovation in the public sector. As a new type of government agency, it is essentially an open innovation lab dedicated to innovative evidence-based policymaking. Following a new dynamic organizational pattern in urban governance, MONUM is conducive to project-oriented social innovation practices and horizontal multi-sectoral collaboration among the three societal sectors: public, private, and civil. Its results suggest that first, the peculiarity of MONUM lies in its hybrid and boundary-blurring nature. Second, new institutional forms that experiment with urban governance can rely on multi-sectoral collaboration. Third, MONUM has experimented with a systemic approach to social innovation following the "design thinking theory." The MONUM case can contribute to the current debate in Europe on the need to harmonize EU policies for an effective social inclusion by promoting the application of the place-sensitive approach.
\end{abstract}

Keywords: urban governance; new public institutional forms; multi-sectoral collaboration; social innovation; MONUM

\section{Introduction}

The innovation gap in the private sector is a subject of study that has raised much interest over the last 15 years [1-9]. Meanwhile, at a local scale, the public sector starts showing a growing interest in addressing the issue of urban governance. Public institutions are increasingly considered as important for driving systemic innovation and local development [10,11], which, as facilitators of social cooperation, can concretize social aspirations into realities and promote civil and political participation. Indeed, recent research has demonstrated that weak institutions, in general, and poor-quality government, in particular, constitute a crucial obstacle to development [12]. This has opened a broad research path concerning the relationship between public institutions and social innovation [13-17]. Social innovation can be spurred by new institutional forms, including experimental activities, largely relying on greater involvement of all stakeholders [18-20].

Since the 1990s, socio-economic changes affected social policies in "re-drawing the boundaries of social citizenships" and "giving a more prominent role to cities" [21] (p. 302). Murray and others [22] clarified that social innovation manifests in boundary-blurring activities developed through the interaction of the three conventional spheres of society: the public, the private, and civil society. The linkage among these sectors has created space for the formation of new dynamic organizational patterns of urban governance underpinned by people-centered "design thinking" [23] and participatory 
methods enhanced by Information and Communication Technologies (ICTs) [24-26]. Such a process is driven by a horizontal multi-sectoral collaboration among actors (Figure 1a) that responds to specific socio-economic issues with socially innovative practices [19]. Lemke [27] and Swyngedouw [28] consider multi-sectoral collaboration as a new form of governmentality. Actors from the three societal sectors have to interact (Figure 1b) within a "fuzzy area," namely, an "institutional void" of unclear rules and norms, and incomplete information and ambiguous knowledge [29,30]. Such practices, often characterized by blurred boundaries between public and private initiatives, result in flexible governmental forms [31] wherein actors can occasionally assume different roles. This flexibility is critical to provide positive contributions to innovation [32-34].
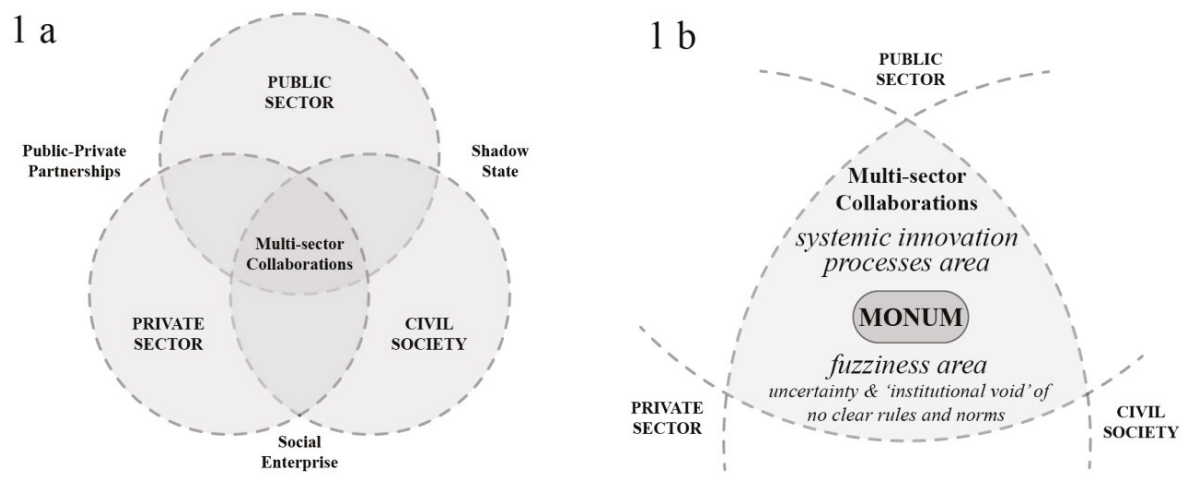

Figure 1. (a) New dynamic organizational pattern in urban governance; (b) Fuzziness emerges from multi-sectoral collaboration patterns. MONUM: "Mayor's Office of New Urban Mechanics." (Source: Drawing by the authors after Nicholls and Murdock [19]).

In line with the objectives of the Cohesion Policy, social innovation is widely promoted by the European Union [35]. According to the EU, social innovation is a main driver of socio-economic development, in that it is "highly focused on meeting social needs by enhancing social interactions and integrating ideas, knowledge and vision of civil society with urban development" [36] (p. 110). It is acknowledged that cities have a key role to play in driving social innovation at the local level. This is because that they are drivers of technological development, economic growth, and citizen empowerment to collaboratively mobilize locally embedded tangible and intangible assets [36]. Since 2011, the EU has stressed the importance for cities to adopt a different organizational paradigm based on seven principles: (1) holistic approach; (2) long-term strategic planning; (3) foresight and vision-building; (4) community involvement; (5) collective mobilization; (6) long-term objectives; and (7) inter-city partnerships and co-operation [36]. To drive a more social innovation-oriented development, cities need to (1) mobilize tangible and intangible resources; (2) adopt new organizational forms and urban governance models; and (3) promote multi-sectoral interactions and multi-stakeholder partnerships.

In this regard, this paper is aimed to explore how innovative public organizational paradigms can lead to social innovation at the local level. Specifically, it provides an analytical outline of the innovative project-oriented public organizational paradigm designed to implement a more effective approach to delivering tailored public services based on citizens' actual needs through social innovation initiatives. It proposes the Boston-based (Massachusetts, USA) "Mayor's Office of New Urban Mechanics" (MONUM) as a representative explanatory case of innovation in the public sector, following a new dynamic organizational pattern in urban governance. Such a pattern is conducive to project-oriented social innovation practices and horizontal collaboration among the three societal sectors (Figure 1b). Similar initiatives have been observed in other cities in the USA and Europe, which have established relationships and exchange with MONUM. 
The paper first conducts a critical review of the literature on social innovation from the public administration perspective, considering that cities need a more effective urban governance to address complex socio-economic and environmental challenges. Then, the MONUM case is investigated with a qualitative approach according to the eight characteristics of innovation in public government elaborated by Borins $[7,8]$. It is analyzed as a single exploratory case study using primary and secondary data to identify its main operational elements. The discussion section carries out a preliminary comparative analysis between the findings of MONUM and two cases selected by the EU as best practices of social innovation in 2015 [35]. Synthetically, this analysis reveals their main similarities and differences with the aim to gain a broad understanding of the urban governance approaches to social innovation practices between the USA and EU. Finally, the paper suggests new research trajectories to better address this topic, especially concerning the post-2020 European Union's Cohesion Policy. The topic explored may be relevant for the current European debate on leveraging cities' potential of place-based innovation by promoting inclusive multi-sectoral interactions, multi-stakeholder governance and strategic investment [36]. Lessons learnt from the MONUM case can provide useful references to the current need to harmonize EU policies to effectively reduce socio-economic inequalities by shifting towards a place-sensitive approach [12].

The study, by presenting the analysis of the MONUM case together with the preliminary comparison with the two EU best practices, offers a possible framework to structure the change factors that public organizations address concerning social innovation rationale. This framework intends to guide further analysis of the soundness of this change in terms of social benefits for local communities. The output of the latter activity may result in the definition of likely measurable indicators of social innovation-oriented policies (in terms of effects/impacts) to test and evaluate the transferability of the lessons learnt to European cities.

\section{Social Innovation in Public Administration}

Neoliberalism [37], in antithesis to the antecedent Fordist-Keynesian consensus [38], shifted economic power away from manufacturing to financial institutions [39]. This reduced the state to a mere organizer and protector of an institutional framework characterized by strong private property rights, free markets and free trade [40]. Truly, the changing territorial dimension at which social policies are designed and implemented, together with the horizontal involvement of increased and variegated actors, has led to a vertical decentralization of regulatory powers [21]. In this globalized context, two parallel phenomena occurred in cities. First, the role of cities and their governance morphed into a market-enterprise model [41,42]. The subsequent need of achieving competitive advantages led to the adoption of business management principles, concepts and tools $[43,44]$. Second, since the beginning of the 21st century, an increasing number of people have committed to active innovation processes of development and social well-being through shared networks and agendas [20,45]. Originally, these are grassroots initiatives aimed to produce solutions through atypical combinations of people, ideas and tools by mobilizing resources and knowledge around local issues [46-48]. Among others, Fressoli [49] argues that the encounter of innovative grassroots organizations with public and private institutions can lead to the development of new models of inclusion and knowledge production.

According to Brandsen and others [50], it is better to conceptualize rather than define social innovation as a complex societal process. Social innovation is therefore referred to as all activities, created mainly by networks and joint action in social realms beyond business and government routines, at any given moment, to raise the hope and expectations of progress towards something "better" (a more socially sustainable/democratic/effective society). Social innovation, as a participatory process and related outcome, is believed to be able to support progress and lead to the improvement of the urban system [51,52]. Common improvements may include products, services, procedures, policies, design and models that simultaneously meet social needs and create new collaborative relationships [22,53-56]. The horizontal relationship-based approach to problem-solving is prone to generate effective, efficient, 
and sustainable solutions [25]. However, the link between social innovation and organizational forms should be an empirical question, not a presupposition [50].

Innovation in the public sector aims at creating shared value [17,57], thereby achieving not only a more effective economic yield [6], but more democratic and just results, such as responsiveness, legitimacy and legality [58]. A fundamental aspect of innovation within the public sector refers to the "logic of appropriateness," meaning the care for local specificities [59]. Community responsiveness to this kind of innovation largely depends on if: (1) innovation is legitimate and politically sustainable [57]; (2) it refers to democratic, shared, and relevant values such as accessibility, participation, empowerment, transparency, accountability, and equality [17]; and (3) its results address citizens' needs [60].

Social innovation requires a systemic approach. This is because that socio-economic issues as it addresses cannot be perceived as isolated; rather, they must be considered within a "system" showing the inter-dependence between their own subsystems and the broader external ecosystem [61]. This explains why social model-based programs tend to fail to remedy complex socio-economic problems [62]. Indeed, without an overall strategy, they have isolated the areas under intervention from their adjacent economy and social context. A systemic approach is critical to create a good social innovation environment, as it can create, by promoting "systems thinking" and interactions among societal sectors, opportunities for co-creation of shared social values [63]. Besides, such an approach is conducive to multi-sectoral collaboration, which is most likely to trigger large-scale social change rather than isolated intervention by individual organizations [64]. In this sense, coupling public, private and civil institutions and local communities is critical to drive social innovation [65].

The City of Boston is leading and fostering a social innovation ecosystem $[66,67]$ while embracing an experimental challenge. Its new urban governance mechanism led by the public administration addresses cross-cutting socio-economic issues through a horizontal collaborative approach involving all the three societal sectors. Such a mechanism enables the public administration to actively engage the civil society and meanwhile, effectively play its intermediation role in the direction of the private sector. MONUM operates exactly as an interface between the public and private sectors and civil society where the opportunity for experimenting and capturing social innovation is higher $[19,20,22]$.

\section{Research Method}

The paper is aimed to explore how innovative public organizational paradigms can lead to social innovation at the local level. Specifically, it provides an analytical outline of the MONUM innovative project-oriented public organizational paradigm. In pursuing this objective, the research (Figure 2) follows a qualitative approach. Primary data were collected by four in-person interviews and by attending MONUM public presentations. Secondary data were collected by examining official public documents, the MONUM website, and online videos about MONUM and its project.

The literature review sets the ground for framing the case study analysis, highlighting the relationship between social innovation and public institutions. The desk analysis of the MONUM website, published projects [68] and video documents [69-72] facilitated an initial description of the case. It also provided a basis for preparing semi-structured interviews focused on MONUM's role within Boston's public administration, institutional structure, and methodology to operate. Two semi-structured interviews were conducted with key Office members, plus one with another member retrieved from an online article [73], as well as an additional interview with an external MONUM collaborator. The latter allowed for the comparison with the information obtained from internal sources. Lastly, a public consultation meeting was also attended to gain first-hand information on the implementation of MONUM projects. 


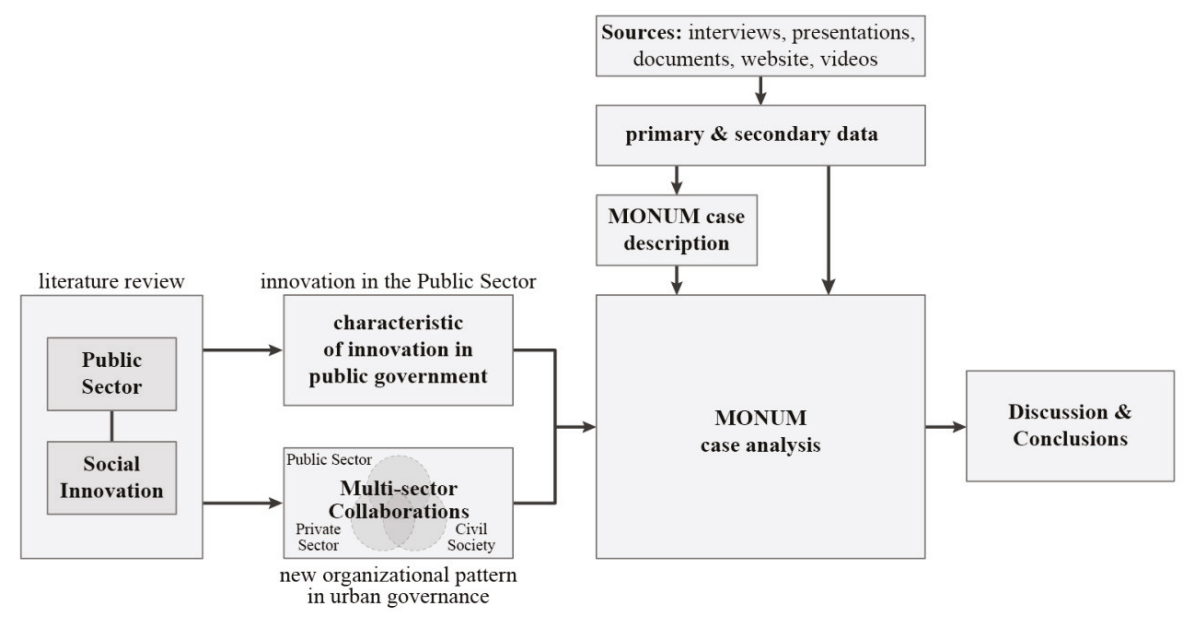

Figure 2. Map of the Methodology (Source: Drawing by the authors).

The explanatory case study analysis is based on primary and secondary data. The main sources are: semi-structured interviews, official documents, and online data on the MONUM initiatives and projects. The case study analysis is aimed to, rather than outlining a model to be transferred and replicated in EU context, point out the key aspects related to governance that can serve as useful references for triggering social innovation in EU lagging cities/regions. The investigation sheds light on the relevance of social innovation mechanisms led by the public administration for addressing local socio-economic problems. This is done mainly by conceptualizing and operationalizing an inclusive and integrated form of urban governance that promotes the collaboration among actors from public and private sectors and civil society. The MONUM case is argued as an improved urban governance pattern led by public institutions that respond better to the emerging socio-economic challenges by spurring social innovation with the horizontal collaboration amongst the three societal sectors.

The MONUM case is analyzed according to the eight characteristics of innovation in public governments elaborated by Borins $[7,8]$, namely organizational change in the public sector, process improvement, collaboration within the government, external collaboration, citizen empowerment, information technology, use of market incentives, and use of volunteers. The following is a brief description of each characteristic retrieved from Borins' works [7,8,24,74] (Table 1). His work analyzed the changes in the innovation in the American public sector since the early 1990s and found three significant points: (1) a major increase in inter-organizational collaboration; (2) shifts in the innovation agenda regarding its content; and (3) an increased societal awareness of public sector innovation that has become more transparent, with increased media attention, more external evaluation, and more transfer of innovative ideas and practices [7] (p. 8). The strength of Borins' framework lies in that the eight characteristics of innovation in public governments were derived from continuous comprehensive questionnaire surveys of local governments that allow facilitating research on current innovation and longitudinal comparison $[7,8]$. These characteristics emerged from a systematic analysis of real cases that provide a reliable reference for practical research on public sector innovation $[7,8]$. This is consistent with MONUM's innovative nature in the public sector. 
Table 1. Characteristics of innovation in public government according to Borins.

\begin{tabular}{|c|c|}
\hline Characteristics & Description \\
\hline Organizational change in the public sector & $\begin{array}{l}\text { Internal organizational improvement, often by importing } \\
\text { private sector practices (e.g., customer service enhancement, } \\
\text { total quality management, etc.). }\end{array}$ \\
\hline Process improvement & $\begin{array}{l}\text { When a process was made faster, more accessible, and friendlier, } \\
\text { or procedures for dealing with problems were simplified. }\end{array}$ \\
\hline Collaboration within the government & $\begin{array}{l}\text { Collaboration within one government and/or across levels of } \\
\text { government. }\end{array}$ \\
\hline External collaboration & Collaboration with the private and/or the nonprofit sector \\
\hline Citizen empowerment & $\begin{array}{l}\text { programs enhance the ability of individuals or groups to } \\
\text { overcome problems through their own initiative. }\end{array}$ \\
\hline Information technology & $\begin{array}{l}\text { ICT-supported institutional innovation by improving } \\
\text { transparency and citizens' access to and interactions with the } \\
\text { government. }\end{array}$ \\
\hline Use of market incentives & $\begin{array}{l}\text { Along with, or as substitutes for, regulation to bring about } \\
\text { socially desired behavior. }\end{array}$ \\
\hline Use of volunteers & To gain program objectives. \\
\hline
\end{tabular}

MONUM has planned and implemented a number of projects in five categories: streets, housing, engagement, education, and civic research. Table 2 describes each category according to MONUM's specific mission and objective.

Table 2. Category, Mission and Objective of the projects.

\begin{tabular}{cll}
\hline Category & \multicolumn{1}{c}{ Mission } & \multicolumn{1}{c}{ Objective } \\
\hline Street & $\begin{array}{l}\text { Making Boston's streets safer, more efficient, } \\
\text { and more delightful for all. }\end{array}$ & $\begin{array}{l}\text { To improve the flow of people } \\
\text { throughout the city. }\end{array}$ \\
\hline Housing & $\begin{array}{l}\text { Pioneer innovative housing models and } \\
\text { systems, as well as accelerating the pace of } \\
\text { innovation in the housing sector. }\end{array}$ & To increase housing affordability. \\
\hline Engagement & $\begin{array}{l}\text { Government must create an open culture in } \\
\text { finding ways to make civic engagement } \\
\text { more meaningful for more people through } \\
\text { new technologies. }\end{array}$ & $\begin{array}{l}\text { To find new ways to create } \\
\text { democratic action in the 21st } \\
\text { century. }\end{array}$ \\
\hline Education & $\begin{array}{l}\text { Test ideas to improve education for people } \\
\text { in the city, including students, parents, } \\
\text { teachers, and other community members. }\end{array}$ & $\begin{array}{l}\text { To improve the systems that } \\
\text { support learning experiences in } \\
\text { Boston. }\end{array}$ \\
\hline \multirow{2}{*}{ Civic Research } & $\begin{array}{l}\text { Quality and quantity of data and focus on } \\
\text { short-term and long-term results. }\end{array}$ & $\begin{array}{l}\text { To understand and explain civic } \\
\text { behaviors and needs for city } \\
\text { departments. }\end{array}$ \\
\hline
\end{tabular}

In order to investigate the agency's systemic approach to developing new urban policies, all projects have been re-categorized into eight common areas of intervention and analyzed through a correlation matrix (Table A1a-e, see the Appendix A). Intervention areas include ICTs, social relationships, built environment, mobility, health and safety, business, urban visioning and planning, and procedures and policies. The Table Ala-e in the Appendix A show the correlation matrix for each of the five categories listed in Table 2. 


\section{The Mayor's Office of New Urban Mechanics}

To understand the organizational and institutional peculiarities of the MONUM case, the first step was to examine its activities according to the eight innovation characteristics in public government $[7,8]$.

The characteristics of collaboration within the government and external collaboration were merged to better correspond to the systemic logic through which MONUM operates. Data derived from both primary and secondary sources demonstrated a correspondence between data sources and characteristics of innovation in the government, as is shown in Table 3.

Table 3. Correspondence between data sources and characteristics of innovation in the government.

\begin{tabular}{cc}
\hline Data Sources & Innovation Characteristics \\
\hline Interviews & Organizational change in the public sector \\
Web site, interviews, documents & Process improvement \\
Web site, interviews, public presentations & Collaboration within the government \\
Web site, interviews, public presentations & External collaboration \\
Web site, interviews, public presentations & Citizen empowerment \\
Web site & Information technology \\
Interviews, videos & Use of market incentives \\
\hline
\end{tabular}

In 2010, Thomas M. Menino, then Mayor of Boston, decided to establish the "Mayor's Office of New Urban Mechanics" (MONUM) to improve citizens' quality of life. Located in Boston's City Hall, the agency is part of the Mayor's Office. At that time, the Menino administration partially worked on innovation though never with a specific focus. Therefore, a work team devoted to innovation was established to better approach the future of Boston. The concept of "urban mechanics" came into being and was meant to overcome the averseness of risk and innovation often present in public administrations. The new team needed to explore the ground on which the government and citizens could work together to plan their city and respond to their needs.

By the beginning of Walsh's mayoral term in 2014, the size of MONUM's team and its scope had changed dramatically. The agency was not only kept but provided with more authority and support. The team received wide-ranging autonomy to experiment in new areas. This changed the MONUM's approach and organization, even though its main goal remained responding to people's needs with innovative solutions.

MONUM, as a public agency, takes risks that traditional city departments tend to be reluctant to do $[75,76]$. Pilot projects always follow a problem-solving rationale. When an experimentation succeeds, MONUM partners with one or more city departments to transform it into a permanent service. In case of failure, the team documents the lessons learned and shares them with partners and other cities around the world. In addition to pilot projects, MONUM acts as a "front-door" for start-ups, universities, and residents willing to experiment social innovation activities with the city. A mentoring activity is also carried out for other cities on how to establish innovation offices in their local governments.

Potential negative effects due to MONUM's high autonomy to experiment social innovation with risk-taking activities, such as confusion, chaos and poor quality of outcomes, are mitigated with highly organized and coordinated interventions from the public administration. Such interventions include systematic monitoring and assessment of the pilot projects to provide references to evidence-based public policy making.

\subsection{Organizational Change in the Public Sector}

Organizational change is the first characteristic of innovation in government that distinguishes MONUM from other types of public sector innovation. Public organizational change generally refers to internal improvement of the organization, which often occurs by introducing private sector 
practices, such as customer service improvement and service quality management [7]. The City of Boston had already made considerable progress in that direction when it introduced a collaborative problem-solving approach.

MONUM shows a reformed organizational structure (Figure 3). On the one hand, it has a traditional hierarchical structure: the agency is directly led by the Mayor and the team reports to two co-chairs who are in direct contact with the Mayor's staff and meet the Mayor weekly (Figure 3, right). This simplified vertical organization has enhanced the efficiency of the office in activating initiatives and projects, by allowing independence from other administrative departments and a direct connection to the political power. On the other hand, MONUM enjoys a considerable degree of autonomy. The work attitude and duties within MONUM are flexible. Rather than giving strong directives, MONUM encourages team members to pursue what they consider as relevant in producing evidence for public policies, being creative, and networking. However, they must meet the Mayor's priorities. One major difference from a traditional agency that usually operates on safe ground is that MONUM allows "failing": team members are encouraged to take risks, and failure is considered as important for the learning and knowledge-building process. This reformed organizational structure has created a "fuzzy area" of creativity and supported a horizontal collaborative approach to social innovation. This has ensured MONUM's agency to interact with all other public, private and civil entities in the city.

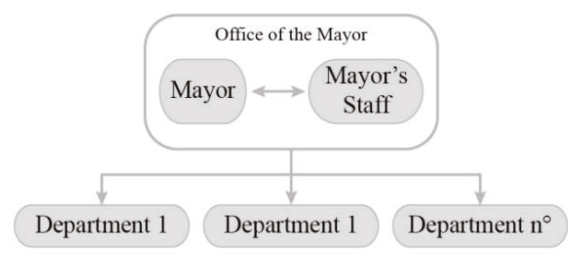

Administrative organizational structure before MONUM establishment

(a)

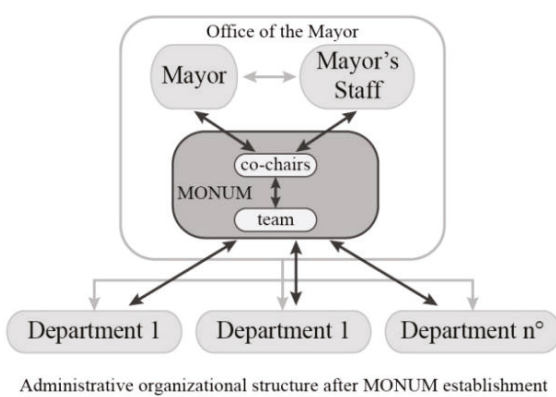

(b)

Figure 3. Administrative and organizational structure before (a) and after (b) MONUM's creation. (Source: Illustration by the authors).

\subsection{Improvement Process}

Improving an existing process means making it faster, more accessible, and friendlier [24] for citizens and employees. It also indicates simplification via ICTs and a better use of human resources for improved procedures.

MONUM is committed to its problem-solving civic research agenda [76]. It addresses questions raised during daily work, problems challenging the team, and required partnerships for problem solving. This work attitude moves within a paradigm that widely embraces an ecological perspective and systems theory [77-79]. Here, solutions to problems are achieved holistically. Such solutions treat the society as a whole, as opposed to the specialized, sectoral approach of the mechanized worldview [79]. MONUM's agenda democratizes the research process by being open to feedback and interaction. Unanswered questions and initial assumptions from the community's different perspectives are always welcome in urban policy [80,81]. For MONUM's team, civic research works as a conversation where observations and experiences count as much as hard numbers. Under the ecological paradigm, MONUM shifts from a hierarchy to a network [82]. Therefore, the public sector is seen as part of the community system where all elements produce and shape knowledge through a participatory process.

MONUM works on three levels: (1) project development; (2) technological transfer; and (3) knowledge transfer. Those levels come together under the broader philosophical umbrella of a new, 
innovative way of addressing public issues through multi-sectoral collaboration and social relationships among partners. The improvements achieved by MONUM benefit from methodologies such as "design thinking" and ICT-supported participatory processes.

A new project starts by raising questions and continues by listening to partners and potential stakeholders in order to reach an initial step of understanding. Eventually, local experts on the project's relevant matters are identified. Next, more questions are asked and data gathered. At this point, the established relationships allow for ideas and solutions to emerge. These are usually further analyzed by academics with the goal of developing an experimental prototype. Whether or not an experimental project is successful, the resulting knowledge is shared and made available for future use. If a project produces good results, it goes to the next stage, becoming permanent and eventually improved.

Table A1a-e in the Appendix A demonstrate that MONUM adopts "systems thinking" for project planning and implementation. Indeed, 46 projects implemented have addressed social, environmental, and economic issues in a systematic way, as all except for three projects had more than one intervention area that showed the interconnectedness between the three spheres. Intervention areas included social relationship (26 projects out of 46), ICTs (21/46), built environment (20/46), procedures and policies (18/46), urban visioning and planning (13/46), mobility (11/46), health and safety (8/46), and business (2/46) (Figure 4a). Twenty out of 46 projects cover three different intervention areas, 18 two, 5 four, and 3 projects only one (Figure 4 b) (to be discussed in the following section).
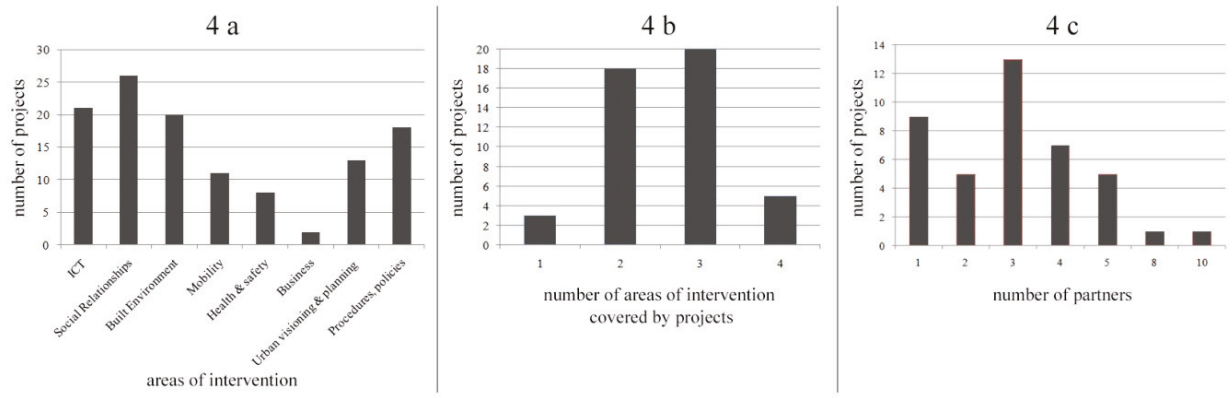

Figure 4. Evidence of the systemic approach adopted by MONUM: (a) number of projects per area of intervention; (b) number of projects per number of covered areas of intervention; (c) number of projects per number of partners that collaborated on a project. (Source: Image by the authors).

Another evidence of MONUM's "systems thinking" comes from the diversity of partnerships established in various projects. Its "systems thinking" approach benefits from cooperation among the actors from the civil society, which, having put them together into experimental projects of common interest, produced positive evidence (number and quality of the outcomes) regarding the adoption of a new dynamic organizational pattern in urban governance.

\subsection{Internal and External Collaboration}

Collaboration may occur within the government and/or across different governmental levels "internal" and/or with the private sector and/or nonprofit sector, which are considered "external" [7]. MONUM has established valuable partnerships and a know-how exchange mechanism, both at home and abroad. As component of the Mayor's Office, MONUM has been able to connect with various departments and people working on the city's problems. MONUM engages people with multidisciplinary expertise and shared values and vision. This broadens its projects' scope, areas of intervention, and partnerships. Although MONUM does not operate as a consultant beyond the scope of its own projects, it often helps the departments that fall into a gray area when it comes to responsibility. Consequently, it also works as a facilitator between public offices, researchers, start-ups, and local communities. 
Internal and external collaboration has been promoted by establishing partnerships. In fact, all of the 46 projects developed by MONUM, except for one, were implemented with partnership(s) (Figure 4c). A total of 119 partnerships were established, most of which were public-private. Yet, thirteen projects saw a partnership solely between public entities. In six cases, MONUM only partnered with private entities.

Apart from the internal and external collaboration at the local level, MONUM also established partnerships with other similar public agencies around the world. The most significant international partnerships were with public administrations of Copenhagen (Denmark) and Cluj-Napoca (Romania). Both cities established a municipal office focused on civil innovation [82,83], like MONUM does. To expand its international connections, MONUM has disseminated its work at various occasions in the USA and Europe. According to the interviewees, those international relationships are as valuable to MONUM as to their international partners. Such external collaboration has played a key role in promoting valuable knowledge exchange about similar models operating in different geopolitical contexts.

\subsection{Citizen Empowerment and Information and Communication Technologies}

Programs that help individuals or groups of people address problems by relying on their own initiative and resources tend to lead to citizen empowerment [24]. The experimental and participatory methods applied by MONUM require engaging a variety of actors from different societal sectors and making strong connections. The planning of new MONUM projects usually involves on-site investigations and direct communication with residents. The information helps conceptualize and implement strategies of empowerment. An example of citizen empowerment is the Community Plan IT project based on a game to find solutions to real-life problems by engaging more people in community issues and improving the feedback mechanism for urban planning.

Similarly, the 10-year master plan for the Chinatown area involved residents through the exploration of their living area by a character-driven virtual world. These kind of participatory method, using online interaction and in-person meetings, helped change the way in which people formulate and negotiate civic affairs with beneficial effects on both the community and municipal institutions. With regard to ICTs, the experimental nature of these projects facilitates learning based on citizens' use and modifies the technological design during construction. Peer-to-peer systems were also tested, for example, in the BOS:311 App project (later called Citizens Connect). This application nourishes residents' care for their communities by allowing them to report non-emergency issues and receive related solutions via smartphones.

These MONUM projects highlight how citizen empowerment activities became distinctive features of the Boston innovative urban governance model. Particularly, a sense of responsibility is fostered among citizens through certain programs which, by using ICTs, raise awareness of current issues and encourage people to proactively seek solutions. The use of ICTs can facilitate innovation in the public sector [8] by improving citizens' access to and interactions with various levels of government. For example, supporting public feedback to proposed regulations, facilitating public participation in the planning process, and contributing to guarantee public sector transparency.

In this perspective, the municipalities of San Francisco and Philadelphia established an innovation office [84] for a proactive utilization of ICTs. The rationale is to harness available open data and technological solutions to stimulate citizen participation in generating new ideas from those open sources [84]. The MONUM case is different in that ICTs have a complementary role in the innovation process. They are widely used, even though through a high infusion of research, relationship building, and a permanent learning process based on well-defined projects in which innovative solutions are generated. In the MONUM case, people are the main medium while ICTs and technological devices have a more passive role. They are adopted to produce data and information through their interaction with human beings, such as using applications and virtual reality. 
The initiative "Boston's Safest Driver Competition" was developed as a smartphone application in partnership with three public departments of the City of Boston and a private company. In order to obtain useful data for improving street security threatened by bad drivers, MONUM decided to leverage a competition among people in order to provide citizens with driving feedback. The idea included a driving competition motivating citizens to download and use the data-gathering app. A private insurance company provided monetary prizes as an additional incentive. Citizens competed with their family and friends while covering three million miles in three months. The experiment showed a reduction of risky behaviors, such as a $47 \%$ decrease in phone use and $35 \%$ speed reduction for the top 25 users. It also generated an amount of data that would be used to further develop street security projects and policies. Likewise, the Citizens Connect app facilitated the process of residents' empowerment in caring for their communities. Several apps developed within the MONUM projects were code-free, allowing other cities that use them to avoid the initial effort and development costs.

These examples show that MONUM distances itself from the narrow view of technology as a primary and undisputed role in producing innovation. Rather, it focuses on an ecologic worldview $[77,79]$ in which ICTs complement the experimentation process with new projects exploiting innovation-based solutions. This frees team members from focusing too much on technological solutions, allowing them to be more open-minded and creative in taking advantage of any available resources. This vision puts Boston's case among the public innovation programs that indicate the systemic approach as one of their main characteristics of innovation $[24,75]$.

\subsection{Market Incentives and Volunteers}

Borins [24] recognized the use of market incentives as substitutes for regulation to bring about socially desired behavior. Market incentive strategies are sometimes applied by MONUM to projects when the participation of the private sector plays a relevant role. This was the case for the Density Bonus Pilot project. Launched in January 2017, this program incentivized developers in exchange for more affordable housing units. The strategy allowed developers into the program in order to increase the height or floor area of their units in exchange for an income restriction on a percentage of their residential units. For that purpose, the City created new density bonus zoning for two Strategic Planning Areas. The reasons for that program is that the City has been increasingly losing funding for affordable housing every year. Therefore, it is always looking for new ways to create more affordable housing units. These kinds of policies can create lots of new affordable housing without using public funds as incentives and will push developers to join the pilot program. Market incentives were implemented by MONUM in projects that needed leverage to gain the attention and involve necessary partners to make them successful, especially when the private sector constitutes the main partner.

The use of a volunteer workforce to gain program objectives is detected by Borins' study [7] with a frequency of $12 \%$ among the characteristics of innovation in the government. MONUM's public agency has no volunteers working within the office but, periodically, there are fellowships offered for collaborations open to individuals from different backgrounds and with a strong interest in innovation. Such individuals show creativity, curiosity, and entrepreneurial spirit. Further, a summer program offers the possibility of being involved in MONUM activities for a defined time. Overall, there is collaboration with people on a voluntary basis for specific projects due to on-site work with individuals and organizations from civil society.

We can conclude that although the Office does not envisage volunteers, the collaborative spirit of a common agenda that characterizes volunteering is present and strong under more defined forms. This can be found in several projects developed by MONUM.

\section{Discussions}

This research argues that MONUM can be seen as an evolution of public institutions that acknowledge a new governmentality aimed at tackling local socio-economic issues through innovative 
approaches. MONUM is essentially an open innovation lab dedicated to innovative evidence-based policy making.

Public sector organizations, being fuzziness-averse, are used to working with limited uncertainty where everything was framed by codes and clear agreements and no space is left for "institutional voids" or unclear rules and norms. MONUM is unconventional in that it supports a new urban governance pattern conducive to social innovation by taking advantage of the fuzziness [32,33] that comes from the systemic nature of the innovation processes [31] and the dynamic organizational pattern (Figure 1b). At the organizational level, the fuzziness stems from a horizontal multi-sectoral collaboration mechanism (Figure 1a) made up of various entities from all societal sectors. The fuzziness on the condition of a multi-sectoral collaboration mechanism allows for a process of "open learning" and knowledge sharing among actors from all societal sectors. This complies with the modern innovation theory that emphasizes the open character of the learning process [85-89]. Through such a learning process, the production of innovation requires the cooperation among different entities that share ideas, knowledge and experience, and exchange staff and resources beyond traditional organizational borders [90,91]. This process plays a crucial role in strengthening its capability of addressing nested socio-economic issues through socially innovative practices [19]. In this respect, MONUM demonstrates the possibility of an effective urban governance through a new dynamic organizational pattern based on a horizontal multi-sectoral collaboration (Table 4). The new public agency's set-up, represented by MONUM, allowed for the pragmatic management of fuzziness in governance mechanisms by working with experimental projects and clear partnerships.

Table 4. Elements of the new dynamic organizational pattern in urban governance that emerged from the case study.

\begin{tabular}{cl}
\hline Characteristics & \multicolumn{1}{c}{ MONUM Characteristics } \\
\hline Change & $\begin{array}{l}\text { MONUM's organizational structure represents evidence of the need for an } \\
\text { institutional form of the public sector that operates in a new form of dynamic } \\
\text { organizational pattern in urban governance based on multi-sectoral societal } \\
\text { collaboration. }\end{array}$ \\
\hline Thprovement & $\begin{array}{l}\text { The process improvement derived from adopting a systems thinking approach } \\
\text { produced positive evidence (number and quality of the outcomes) about the } \\
\text { adoption of a new dynamic organizational pattern in urban governance based on a } \\
\text { horizontal multi-sectoral collaboration and socially innovative practices. }\end{array}$ \\
\hline Collaboration & $\begin{array}{l}\text { Collaboration activity produced valuable results for MONUM as it has led to } \\
\text { establishing new partnerships and exchange know-how with similar agencies } \\
\text { worldwide. }\end{array}$ \\
\hline Empowerment & $\begin{array}{l}\text { Citizen empowerment at MONUM manifests through programs that foster } \\
\text { responsibility in citizens, raising their awareness about issues and then pushing } \\
\text { them to seek solutions. }\end{array}$ \\
\hline Innovation & $\begin{array}{l}\text { In the Boston MONUM case, ICT has a complementary role of data gathering and } \\
\text { support of project developments for local engagement. }\end{array}$ \\
\hline Leverage & $\begin{array}{l}\text { MONUM uses market incentives as leverage to engage necessary partners for } \\
\text { starting and developing projects. This is more frequent in private sector } \\
\text { collaborations. }\end{array}$ \\
\hline \multirow{2}{*}{ Civic engagement } & $\begin{array}{l}\text { The use of volunteers within the Office is not envisaged, but the spirit of } \\
\text { collaboration characterizing volunteering is reflected in the action and behavior of } \\
\text { individuals and civil society groups engaged with MONUM on specific projects. }\end{array}$ \\
\hline &
\end{tabular}

The table above (Table 4) shows that the MONUM case complies with the eight characteristics of innovation in public government, revealing a new dynamic organizational pattern in urban governance in addressing societal problems. These findings are in line with the EU's objectives of promoting social innovation to enhance social cohesion. European cities face a common challenge, that is, how to collaborate with citizens and harness the potential benefits of social innovation. It is therefore 
commendable to conduct a preliminary comparison between the MONUM findings and the key characteristics of two European best practices of social innovation, i.e. Amersfoort (Netherlands) and Gdańsk (Poland) highlighted in the URBACT report "Social Innovation in Cities" [36]. This paper chose Amersfoort and Gdańsk as two contrasting cases: while the former is an advanced one which is experimenting with leading-edge innovations, the latter is a developing one which is starting engagement with social innovation and citizens.

The preliminary comparison presented in Table 5 is meant to reveal the similarities and differences of urban governance approaches to social innovation between the USA and the EU. It also allows outlining the organizational response of the public sector to citizens' needs that may differ with respect to the different social, economic, political and, above all, administrative contexts. Other studies would be needed to assess the impacts of these approaches in order to set out useful indicators for testing and evaluating the transferability of the lessons learnt to European cities.

Table 5. Similarities and differences from the governance perspective between MONUM case key characteristics and EU best practices [36].

\begin{tabular}{|c|c|c|}
\hline $\begin{array}{c}\text { MONUM } \\
\text { Characteristics }\end{array}$ & Amersfoort & Gdańsk \\
\hline Change & $\begin{array}{l}\text { Changing towards a more collaborative urban } \\
\text { governance between the city administration and } \\
\text { citizens. }\end{array}$ & $\begin{array}{l}\text { Reforming the traditional hierarchical } \\
\text { city administration system. }\end{array}$ \\
\hline Improvement & $\begin{array}{l}\text { Public administration is shifting from a power role' } \\
\text { to one of a 'learning administration'; fostering } \\
\text { multi-disciplinarity and collaboration between the } \\
\text { different departments; promoting transparency in } \\
\text { public action; being less expert and more able to } \\
\text { connect; making interdependent and integrated } \\
\text { policies; fostering responsibility beyond silos; and } \\
\text { learning how to learn from failures. }\end{array}$ & $\begin{array}{l}\text { City administration is promoting } \\
\text { horizontal multi-sectoral collaboration } \\
\text { in order to avoid administrative silos. }\end{array}$ \\
\hline Collaboration & $\begin{array}{l}\text { New forms of collaboration between citizens and the } \\
\text { city administration, such as the New Collaboration } \\
\text { conference, the G1000, Project Start-up. }\end{array}$ & $\begin{array}{l}\text { The municipality has extended its } \\
\text { cooperation and partnership with } \\
\text { NGOs for which a specific unit was set } \\
\text { up within the City administration. }\end{array}$ \\
\hline Empowerment & $\begin{array}{l}\text { Empowerment was achieved through the } \\
\text { collaborative design and implementation of } \\
\text { citizen-driven projects (e.g., the Elisabeth project, the } \\
\text { Sustainable Food process). }\end{array}$ & $\begin{array}{l}\text { Citizens were involved in (1) } \\
\text { consultation processes and enabled to } \\
\text { choose which city projects should take } \\
\text { priority for funding; and (2) co-creation } \\
\text { process. }\end{array}$ \\
\hline Innovation & $\begin{array}{l}\text { The Municipality deployed ICTs to support citizen } \\
\text { reorganization. }\end{array}$ & $\begin{array}{l}\text { The municipality, together with NGOs, } \\
\text { has supported innovation by creating } \\
\text { online platforms for co-creation. }\end{array}$ \\
\hline Leverage & No market incentives were reported. & No market incentives were reported. \\
\hline Civic engagement & $\begin{array}{l}\text { Citizens are not only users of social innovation } \\
\text { results, but co-designers and co-creators of solutions. }\end{array}$ & $\begin{array}{l}\text { Citizens are not only users of social } \\
\text { innovation results, but co-designers } \\
\text { and co-creators of solutions. }\end{array}$ \\
\hline
\end{tabular}

The two best practices selected by URBACT show some similarities with and differences from the MONUM case. This allows for a preliminary comparative analysis of key characteristics of social innovation under different geopolitical contexts. The first similarity lies in the common need for "change" coming from citizens and local communities. Both in the USA and the EU, cities' traditional operational system of public administration seems inadequate to address the current socio-economic needs. This is especially true in Europe where the public administration regulates all the aspects of citizens' lives. There is an evident mismatch between the multi-level socio-economic needs of citizens and public administrations' limited ability to respond. The current response by public administrations both in the USA and the EU is characterized by multi-sectoral and horizontal collaborations among departments to prevent silos effect (especially in the EU), citizen involvement and empowerment through a proactive participation in leading social innovation projects, and multi-stakeholder collaboration among all the 
three societal sectors. Another similarity is that ICTs have been deployed in all cases as an important tool to empower and engage citizens in social innovation initiatives.

One noticeable difference is the leverage factor of social innovation. Market incentives in the MONUM case have played a stronger role than in the European cases. In the MONUM case, market incentives were provided to make social innovation projects attractive to the private sector, and therefore build up public-private partnerships to promote the social innovation process. In the European cases, the public sector was the key funder with a minor role played by the private sector. Additionally, the social innovation process has been leveraged mainly through operating initiatives that stimulate citizen engagement and participation [36].

The emerging new role of the public sector, as MONUM represents in facilitating social innovation in urban governance through public institutional reforms, poses two main questions: (1) how much innovation will be generated, inclusively used, and fairly distributed by initiatives such as MONUM?; and (2) how far the new approach will take root? The first question is about change in usual practice and equity of social innovation, whereas the second is about how to innovate within practice and the public administration in a continuous process. The answer to these two questions demands a critical lens of MONUM's operation, which, despite all its organizational innovation and cross-cutting social innovation practices, is not free of potential limitations and risks. First of all, power asymmetries due to the predominant market-oriented context where such social innovation initiatives take place could undermine the effectiveness of citizen participation and multi-stakeholder partnerships. Indeed, participatory circumstances are decidedly non-ideal in many cases, marked by power asymmetries that often undermine the negotiation power of underprivileged social groups regarding issues of their interests. Consequently, the uneven interaction amongst stakeholders in terms of know-how and influence tends to pave the way for manipulative orchestrations in networking governance platforms [28]. Second, it is not unrealistic to question the sustainability of the participatory and multi-stakeholder partnership approach to lead social innovation initiatives, considering the inevitable presence of competing perspectives in the highly variegated civil society like Boston. All in all, how to prevent social innovation initiatives facilitated by public institutional reform from blurring competing perspectives, creating policy closures, and absorbing dissident societal voices, proves a highly relevant issue to investigate $[92,93]$.

\section{Conclusions}

MONUM represents a new urban governance model in the form of a public agency operating in a market-oriented society and conducive to processes of social innovation. On the public sector side, the MONUM case positively indicates a way to approach the need for changes in contemporary urban governance. This approach requires the evolution of existing institutional forms from a static, hierarchical organization towards an organizational structure based on evidence-based and socially innovative practices and horizontal collaboration across the three societal sectors (Figures $1 \mathrm{~b}$ and 5 ). One of the most significant innovative practices of MONUM is its evidence-based (project-based) and multi-stakeholder partnership approach to problem-solving that constitutes a significant change in usual practice within the public sector. This working model operates in a non-linear way, incorporating any elements of the urban governance system that can potentially influence positively the final result. Therefore, the dynamic of the new organizational paradigm of MONUM requires new operating methods that embrace creativity in problem solving and the acceptance of risk-taking in a climate of uncertainty. The case study analyses suggest that, first, the peculiarity of MONUM lies in its hybrid and boundary-blurring nature. Such a nature manifests itself as public responsiveness towards civil society, while operating through methods and techniques common to the private sector. Second, new institutional forms that experiment with urban governance can rely on a multi-sectoral collaboration. Through flexible alignment of actors from public, private and civil entities with coherent political will and budget support, MONUM has activated a series of community-based projects to address cross-cutting socio-economic issues. These aspects demonstrate reorganization, interdisciplinarity 
and interrelationship as three fundamental characteristics of innovation [54]. Besides, multi-sectoral collaboration seems to help improve the quality of project outputs. According to the opinion of the interviewees, the best projects are those with a collaboration between various societal entities. Third, MONUM has experimented with a systemic approach to social innovation in accordance with the "design thinking theory." Murray and others [22] expounded on this theory with six key stages in the nonlinear process that social innovation usually goes through: prompts, proposals, prototyping, sustaining, scaling and diffusion, as well as systemic change [94,95]. MONUM's methodology is a systemic one as it aims at developing evidence-based policies by following all such stages (Figure 5), thereby creating a system of "feedback loop." This assures a permanent learning process producing outputs with systemic impact and sensitive to local context and community needs, as is shown from the analysis of MONUM projects (Figure $4 \mathrm{a}-\mathrm{c}$ ). Its systemic approach is also evidenced by the fact that its projects were all, firstly, based on a multi-sectoral collaboration among all the three societal sectors; and secondly, with cross-cutting intervention areas that cover all social, economic and environmental spheres.

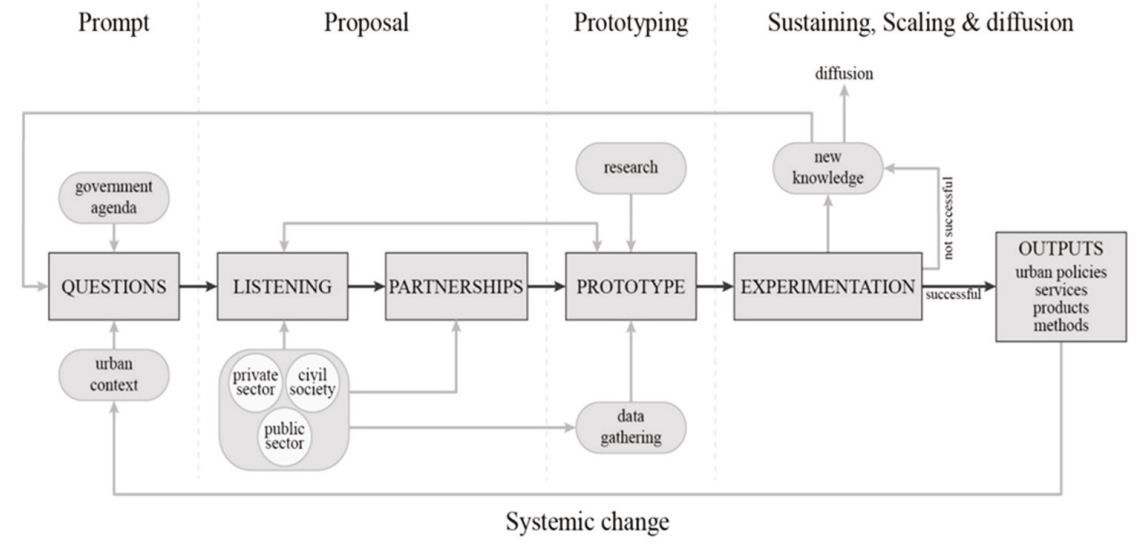

Figure 5. MONUM's operative process (Source: Image by the authors).

The implications of the MONUM case appear twofold. From the public sector's perspective, they can inspire public officers seeking to improve the efficiency and transparency of public administration, and address local socio-economic issues with a systemic approach. From the community's perspective, they can offer more empowerment and engagement opportunities for citizens, allowing for the dynamic interaction with a responsive public sector. Policy makers from the public sector and researchers who explore the future of cities and urban governance with a close attention to innovation in all fields may benefit from this research. The boundary-blurring MONUM case makes it ideal for further research from a civil society and private sector perspective, which is unchartered here. However, the analysis of the MONUM case indicates market incentives as a leveraging element in the social innovation projects. The leveraging mechanism consisted in a bonus formula for private developers involved in the projects committed to realizing affordable housing units. This process helped pool private financial resources for the social innovation initiatives promoted by the MONUM office.

The MONUM case can contribute to the current debate in Europe on the need to harmonize EU policies for an effective social inclusion by promoting the application of place-sensitive approach [12]. To meet such a need, promoting institutional improvement, rather than merely enhancing education and training, is crucial to build up the innovation capacities and stimulate entrepreneurship at the local level [12]. If the place-sensitive approach is characterized by the need of improvements in government, for instance through the promotion of e-government to improve transparency and effectively engage the 
civil society in any development intervention [12], then the MONUM case provides useful references for EU lagging regions and cities. For example, the application of ICTs in urban governance could support knowledge generation and sharing in those areas where agglomeration and density are lacking. Mindful of the socio-economic and political differences between the USA and the EU, this research is not meant to replicate the MONUM model in European cities. Rather, it is aimed to identify contributing factors to social innovation initiatives from the urban governance perspective. These factors can be useful references for designing social innovation initiatives and being adaptable to different institutional and administrative systems. Moreover, experimenting the model as MONUM represents under non-market-oriented contexts could inspire and help European cities develop more effective social innovation policies and projects (e.g., integrating market incentives).

Despite that the present work marks the initial step of a research pathway, the MONUM case has provided helpful evidence for identifying key elements of a new public organizational form supportive of social innovation practices. This, combined with the preliminary analytical comparison with EU best practices, represents the first step for further researches offering a possible framework to structure the change factors that public organizations need to address to spur social innovation. Such a framework is critical to guide further analyses on how public organizational change can favor social innovation to generate socio-economic benefits for local communities. Starting from this initial research work, upcoming research activities may, on the one hand, further the analysis of MONUM's socio-economic impacts on local communities. On the other hand, it is desirable to carry out a more critical and systematic comparative analysis of MONUM and EU cases. These two fields of work will help identify and define indicators for measuring social innovation-oriented policies (in terms of their impacts), and test and evaluate the transferability of the lessons learnt to European cities.

In this direction, further research work may, first, integrate quantitative data on socio-economic, financial and physical contexts into the analytical discussions to understand how the implementation of these ICT-based solutions in urban governance affect local communities and how such initiatives are funded. A preliminary analysis of the City of Boston Fiscal Year 2020 revealed how the Operating Budget for the New Urban Mechanics Initiatives increased constantly since its operation in 2014 (over $\$ 400,000.00)$. Second, deepened comparative analyses of social innovation initiatives supported by public institutional reforms from the USA and the EU seems a promising research branch. Thereby, it is expected to develop and improve existing social innovation models applicable to EU lagging cities and regions. In view of the reform agenda of the post-2020 programming of the EU Cohesion Policy, this is critical to help narrow the gap between urban and inner areas, and between core and lagging cities and regions.

Author Contributions: C.B. conceived and designed the case study analysis, methodology, supervision; Y.O. performed the literature review, writing-review \& editing; P.P. performed the analysis and data curation and G.M.: writing - original draft preparation, conceptualization and performed the analysis. All authors have read and agreed to the published version of the manuscript.

Funding: This research is part of the TREnD project (Transition with Resilience for Evolutionary Development), which has received funding from the European Union's Horizon 2020 research and innovation program under the Marie Skłodowska-Curie grant agreement No. 823952.

Acknowledgments: The authors extend their appreciation to the Mayor's Office at New Urban Mechanics in Boston, Massachusetts and the School of Public Policy and Urban Affairs at Northeastern University, also of Boston. Everyone provided assistance during this research.

Conflicts of Interest: The authors declare no conflict of interest. 


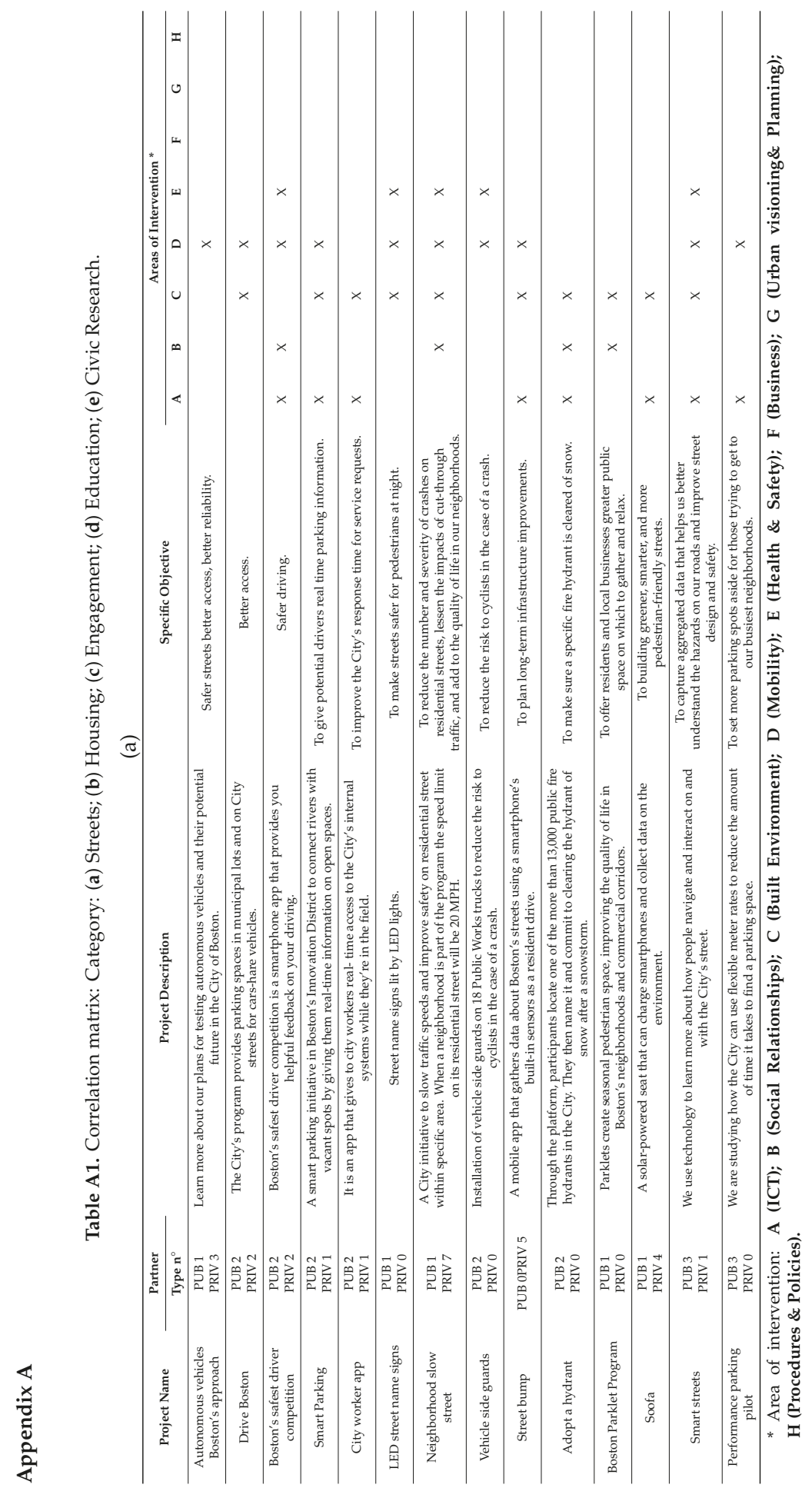


Sustainability 2020, 12, 23

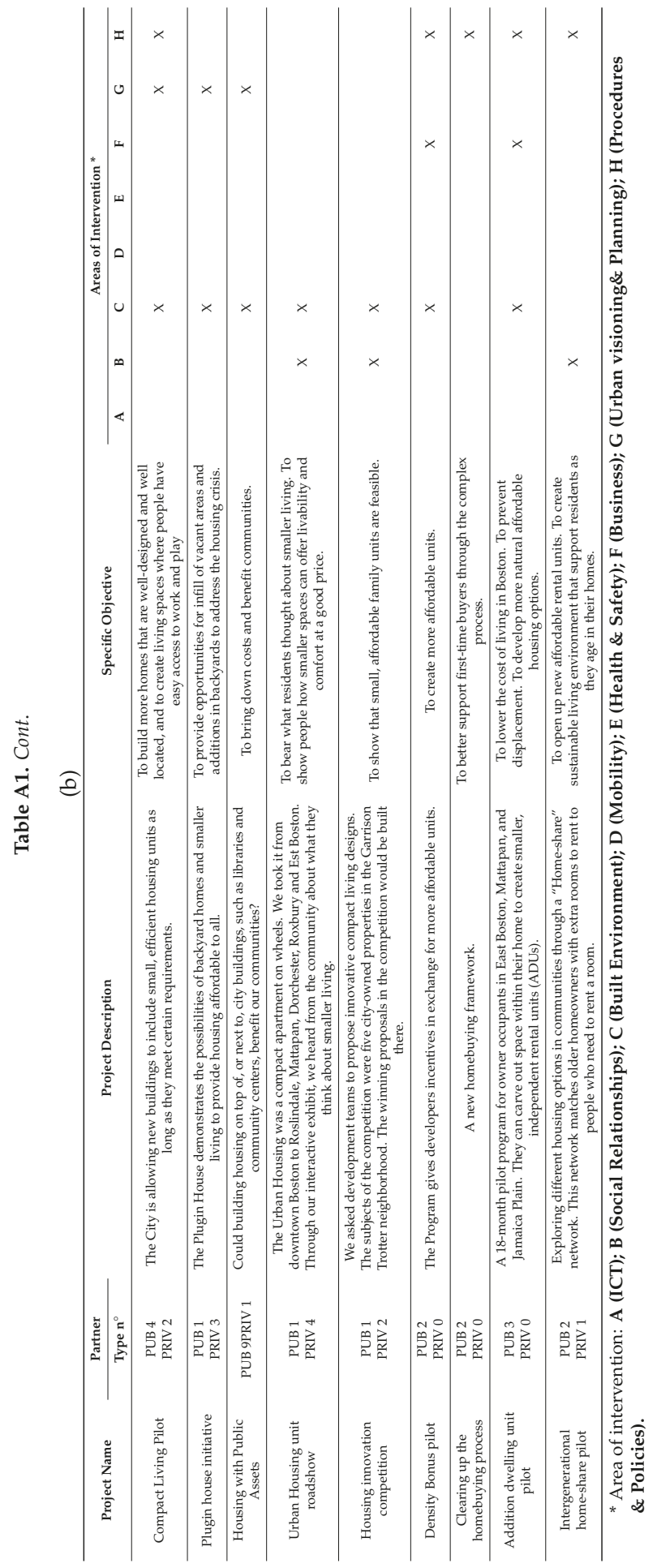


Sustainability 2020, 12, 23

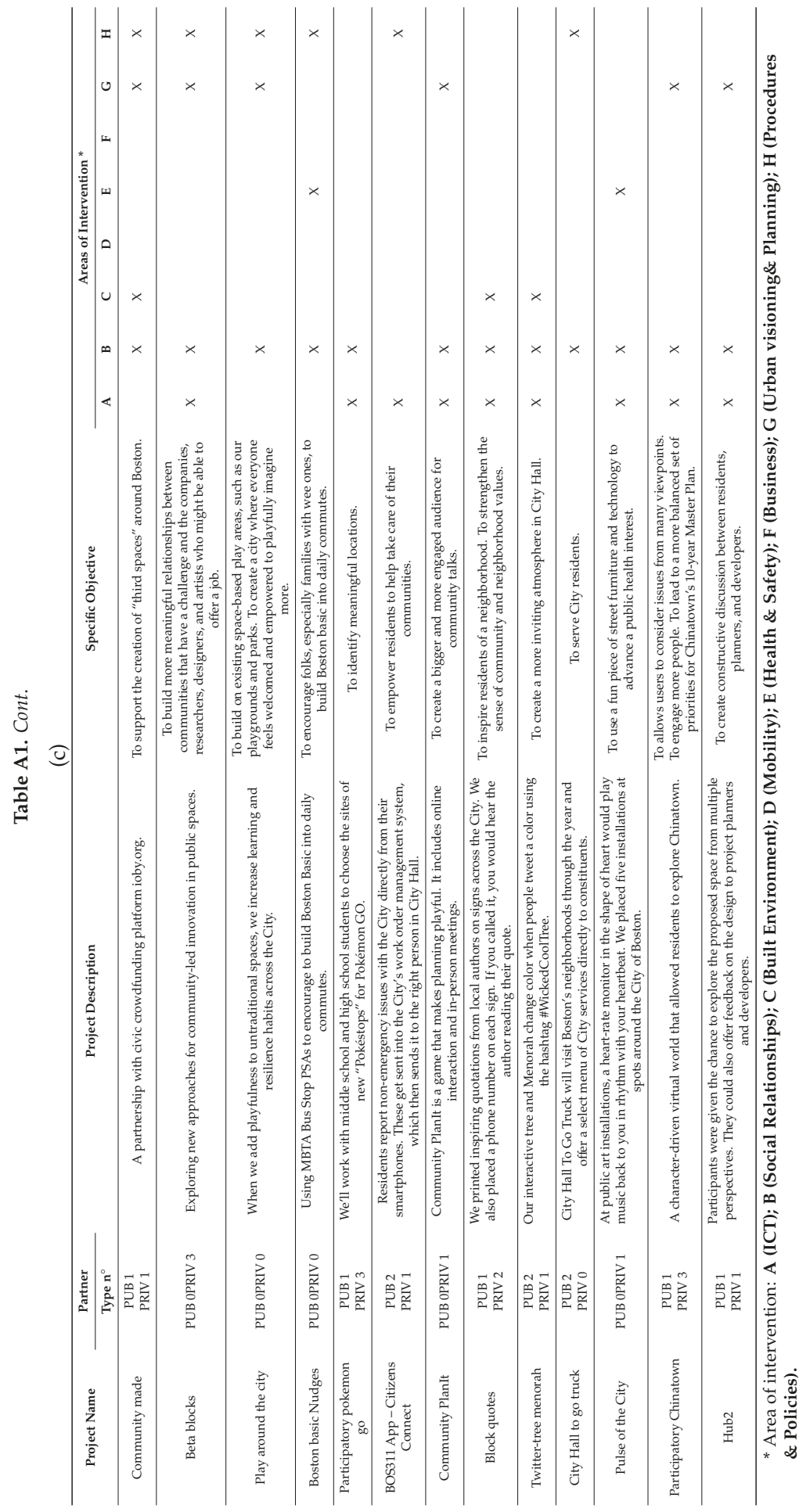


Sustainability 2020, 12, 23

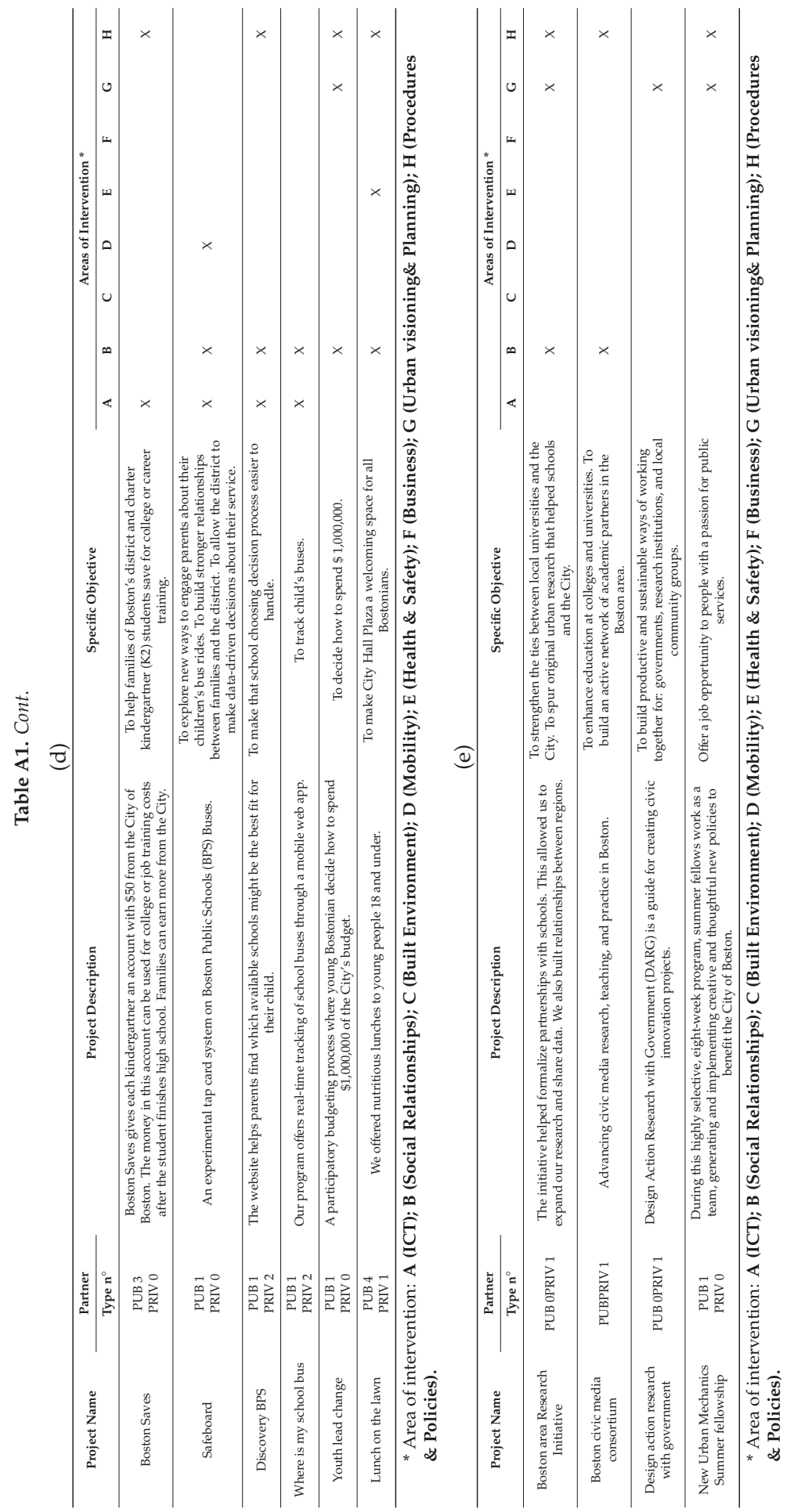




\section{References}

1. Hartley, J. Innovation in governance and public services: Past and present. Public Money Manag. 2005, 25, 27-34.

2. National Health Service Audit Commission. Seeing the Light: Innovation in Local Public Services; Audit Commission for Local Authorities, the National Health Service: London, UK, 2007.

3. Verhoest, K.; Verschuere, B.; Bouckaert, G. Pressure, legitimacy, and innovative behavior by public organizations. Governance 2007, 20, 469-497. [CrossRef]

4. Brown, K.; Osborne, S.P. Managing Change and Innovation in Public Service Organizations; Routledge: London, UK, 2012.

5. Osborne, S.P.; Brown, L. Introduction: Innovation in public services. In Handbook of Innovation in Public Services; Stephen, P.O., Brown, L., Eds.; Edward Elgar: Cheltenham, UK, 2013; pp. 1-11.

6. Sahni, N.R.; Wessel, M.; Christensen, C.M. Unleashing breakthrough innovation in government. Stanf. Soc. Innov. Rev. 2013, 11, 27-31.

7. Borins, S. The Persistence of Innovation in Government. A Guide for Innovative Public Servants; IBM Center for the Business of Government: Washington, DC, USA, 2014; Available online: http://www.businessofgovernment. org/sites/default/files/The\%20Persistence\%20of\%20Innovation\%20in\%20Government.pdf (accessed on 6 November 2018).

8. Borins, S. The Persistence of Innovation in Government; Brookings Institution Press with Ash Center for Democratic Governance and Innovation: Washington, DC, USA, 2014.

9. Bugge, M.M.; Bloch, C.W. Between bricolage and breakthroughs-Framing the many faces of public sector innovation. Public Money Manag. 2016, 36, 281-288. [CrossRef]

10. Raco, M. Competition, Collaboration and the New Industrial Districts: Examining the Institutional Turn in Local Economic Development. Urban Stud. 1999, 36, 951-968. [CrossRef]

11. Widestrom, A. Displacing Democracy: Economic Segregation in America; University of Pennsylvania Press: Philadelphia, PA, USA, 2015.

12. Iammarino, S.; Rodriguez-Pose, A.; Storper, M. Regional Inequality in Europe: Evidence, Theory and Policy Implications [PDF File]; Urban and Regional Research Center Utrecht University: Utrecht, The Netherlands, 2018; Available online: http://econ.geog.uu.nl/peeg/peeg.html (accessed on 10 July 2019).

13. Verschuere, B.; Brandsen, T.; Pestoff, V. Co-production: The State of the Art in Research and the Future Agenda. Volunt. Int. J. Volunt. Nonprofit Organ. 2012, 23, 1083-1101. [CrossRef]

14. Voorberg, W.H.; Bekkers, V.J.J.M.; Tummers, L.G. A Systematic Review of Co-Creation and Co-Production: Embarking on the social innovation journey. Public Manag. Rev. 2014, 17, 1333-1357. [CrossRef]

15. Arundel, A.; Casali, L.; Hollanders, H. How European public sector agencies innovate: The use of bottom-up, policy-dependent and knowledge-scanning innovation methods. Res. Policy 2015, 44, 1271-1282. [CrossRef]

16. De Vries, H.; Bekkers, V.; Tummers, L. Innovation in the public sector: A systematic review and future research agenda. Public Adm. 2016, 94, 146-166. [CrossRef]

17. Bason, C. Leading Public Sector Innovation: Co-Creating for a Better Society; Policy Press: Bristol, UK, 2017.

18. Moulaert, F.; Martinelli, F.; Swyngedouw, E.; Gonzalez, S. Towards Alternative Model(s) of Local Innovation. Urban Stud. 2005, 42, 1969-1990. [CrossRef]

19. Nicholls, A.; Murdock, A. Social Innovation: Blurring Boundaries to Reconfigure Markets; Palgrave Macmillan: Basingstoke, UK, 2012.

20. Nicholls, A.; Simon, J.; Gabriel, M. New Frontiers in Social Innovation Research; Palgrave Macmillan: London, UK, 2015.

21. Kazepov, Y. The territorial dimension of social policies and the new role of cities. In Handbook of European Social Policy; Kennett, P., Lendvai-Bainton, N., Eds.; Edward Elgar Publishing: Chelthenam, UK; Northampton, MA, USA, 2017.

22. Murray, R.; Calulier-Grice, J.; Mulgan, G. The Open Book of Social Innovation; National Endowment for Science, Technology and the Art: London, UK, 2010.

23. Brown, T. Design Thinking. Harv. Bus. Rev. 2008, 86, 84. Available online: https://readings.design/PDF/ Tim\%20Brown,\%20Design\%20Thinking.pdf (accessed on 29 September 2019). [PubMed]

24. Borins, S. Innovating with Integrity: How Local Heroes Are Transforming American Government; Georgetown University Press: Washington, DC, USA, 1998. 
25. Phills, A.; Deiglmeier, K.; Miller, D. Rediscovering Social Innovation. Stanf. Soc. Innov. Rev. 2008, 6, $34-43$.

26. Bazurli, R.; Cucciniello, M.; Mele, V.; Nasi, G.; Valotti, G. Determinants and Barriers of Adoption, Diffusion and Upscaling of ICT-Driven Social Innovation in the Public Sector [PDF File]. In Proceedings of the EGPA Conference, Speyer, Germany, 10-12 September 2014; Available online: http://www.lipse.org/upload/ publications/Determinants\%20and\%20Barriers\%20of\%20Adoption\%20Diffusion\%20and.pdf (accessed on 8 October 2018).

27. Lemke, T. Foucault, governmentality, and critique. Rethink. Marx. 2002, 14, 49-64. [CrossRef]

28. Swyngedouw, E. Governance Innovation and the Citizen: The Janus Face of Governance-beyond-the-State. Urban Stud. 2005, 42, 1991-2006. [CrossRef]

29. Hajer, M. Policy without polity? Policy analysis and the institutional void. Policy Sci. 2003, 36, 175-195. [CrossRef]

30. Hajer, M.A.; Wagenaar, H. Deliberative Policy Analysis: Understanding Governance in the Network Society; Cambridge University Press: Cambridge, MA, USA, 2003.

31. Jalonen, H. Clarifying 'fuzziness' in systemic welfare service innovation. In Proceedings of the 5th International Conference on Entrepreneurship, Innovation and Regional Development (ICEIRD), Sofia, Bulgaria, 1-2 June 2012; pp. 370-382.

32. Nonaka, I. A dynamic theory of organizational knowledge creation. Organ. Sci. 1994, 5, 14-37. [CrossRef]

33. Rogers, E.M. Diffusion of Innovations, 5th ed.; Free Press: New York, NY, USA, 2003.

34. Bevilacqua, C.; Pizzimenti, P. Living Lab and Cities as Smart Specialisation Strategies Engine. Procedia-Soc. Behav. Sci. 2016, 223, 915-922. [CrossRef]

35. Saad-Filho, A.; Johnston, D. Neoliberalism: A Critical Reader; Pluto Press: London, UK, 2005.

36. Jégou, F; Bonneau, M. Social Innovation in Cities. URBACT II Capitalisation; URBACT Secretariat 5, rue Pleyel 93283 Saint Denis Cedex: Paris, France, 2015.

37. Vandecasteele, I.; Baranzelli, C.; Siragusa, A.; Aurambout, J.P.; Alberti, V.; Alonso Raposo, M.; Attardo, C.; Auteri, D.; Barranco, R.; Batista e Silva, F.; et al. The Future of Cities-Opportunities, Challenges and the Way Forward; EUR 29752 EN; Publications Office: Luxembourg, 2019; ISBN 978-92-76-03847-4. JRC116711. [CrossRef]

38. Heilbroner, R.; Milberg, W. The Crisis of Vision in Modern Economic Thought; Cambridge University Press: New York, NY, USA, 1995.

39. Chomsky, N. Understanding the Crisis. Markets, the State and Hypocrisy; Foreign Policy in Focus: Washington, DC, USA, 2009; Available online: https://chomsky.info/20090210/ (accessed on 1 November 2018).

40. Harvey, D. A Brief History of Neoliberalism; Oxford University Press: New York, NY, USA, 2005.

41. Harvey, D. From Managerialism to Entrepreneurialism: The Transformation in Urban Governance in Late Capitalism. Geogr. Ann. Ser. B Hum. Geogr. 1989, 71, 3-17. [CrossRef]

42. Parkinson, M. The rise of the entrepreneurial European city: Strategic responses to economic changes in the 1980s. Ekistics 1991, 58, 299-307.

43. Jensen-Butler, C.N.; Shachar, A.; Van Weesep, J. European Cities in Competition; Avebury: Aldershot, UK, 1996.

44. Saez Vegas, L.; Periáñez Cañadillas, I. Market Orientation in Local Government through the Analysis of Municipal Website Content: A Framework for its Measurement. Glob. J. Bus. Res. 2013, 7, 47-58.

45. Organization for Economic Co-operation and Development. Fostering Innovation to Address Social Challenges; Workshop proceedings [PDF File]; OECD: Paris, France, 2011; Available online: https://www.oecd.org/sti/ inno/47861327.pdf (accessed on 6 September 2018).

46. Gupta, A.K.; Sinha, R.; Koradia, D.; Patel, R.; Parmar, M.; Rohit, P.; Patel, H.; Patel, K.; Chand, V.S.; James, T.J.; et al. Mobilizing grassroots' technological innovations and traditional knowledge, values and institutions: Articulating social and ethical capital. Futures 2003, 35, 975-987. [CrossRef]

47. Seyfang, G.; Smith, A. Grassroots innovations for sustainable development: Towards a new research and policy agenda. Environ. Polit. 2007, 16, 584-603. [CrossRef]

48. Smith, A.; Fressoli, M.; Abrol, D.; Around, E.; Ely, A. Grassroots Innovation Movements; Routledge: New York, NY, USA, 2017.

49. Fressoli, M.; Arond, E.; Abrol, D.; Smith, A.; Ely, A.; Dias, R. When grassroots innovation movements encounter mainstream institutions: Implications for models of inclusive innovation. Innov. Dev. 2014, 4, 277-292. [CrossRef] 
50. Brandsen, T.; Cattacin, S.; Evers, A.; Zimmer, A. Social Innovations in the Urban Context; Springer: Cham, Switzerland, 2016.

51. MacCallum, D.; Moulaert, F.; Hillier, J.; Haddock, S.V. Social Innovation and Territorial Development; Ashgate: Aldershot, UK, 2009.

52. Moulaert, F. The International Handbook on Social Innovation: Collective Action, Social Learning and Transdisciplinary Research, Paperback, Reprinted eds.; Edward Elgar: Cheltenham, UK, 2015.

53. Westley, F.; Zimmerman, B.; Quinn Patton, M. Getting to Maybe: How the World Is Changed; Random House: Toronto, CA, USA, 2006.

54. Mulgan, G.; Tucker, S.; Ali, R.; Sanders, B. Social Innovation: What It Is, Why It Matters and How It can Be Accelerated; Skoll Centre for Social Entrepreneurship: Oxford, UK, 2007.

55. European Commission. This Is European Social Innovation. Retrieved September 2017. 2010. Available online: http://ec.europa.eu/DocsRoom/documents/19042 (accessed on 22 November 2018).

56. Terstriep, J.; Totterdill, P. Economic Foundation of Social Innovation-New Ways of Policy Production. In Proceedings of the RIP 2014 9th Regional Innovation Policy Conference, Stavanger, Norway, 16-17 October 2014; University of Stavanger: Stavanger, Norway, 2014.

57. Moore, M. Creating Public Value. Strategic Management in Government; Harvard University Press: Cambridge MA, USA, 1995.

58. Bekkers, V.; Tummers, L.G.; Stuijfzand, B.G.; Voorberg, W. Social Innovation in the Public Sector: An Integrative Framework; LIPSE Working papers (no. 1); Erasmus University Rotterdam: Rotterdam, The Netherlands, 2013.

59. March, J.G.; Olsen, J.P. Rediscovering Institutions: The Organizational Basis of Politics; Fee Press: New York, NY, USA, 1989.

60. Vigoda-Gadot, E.; Shoham, A.; Schwabsky, N.; Ruvio, A. Public sector innovation for Europe: A multinational eight-country exploration of citizens' perspectives. Public Adm. 2008, 86, 307-329. [CrossRef]

61. Nobbs, C.L. Economics, Sustainability, and Democracy: Economics in the Era of Climate Change; Routledge: Abingdon, Oxon; New York, NY, USA, 2013.

62. Porter, M.E. The Competitive Advantage of the Inner City. Harv. Bus. Rev. 1995, 73, 55-71.

63. Baltazar Herrera, M.E. Creating Competitive Advantage by Institutionalizing Corporate Social Innovation. J. Bus. Res. 2015, 68, 1468-1474. [CrossRef]

64. Kania, J.; Kramer, M. Collective Impact. In Stanford Social Innovation Review; Winter: Berlin/Heidelberg, Germany, 2011; pp. 36-41.

65. Bevilacqua, C.; Ou, Y. Place, Relationships, and Community-Controlled Capital: On Ecosystem-Based Innovation towards an Equitable Competitive Advantages Distribution, the Boston Ujima Project Case. Int. J. Sustain. Dev. Plan. 2018, 13, 1072-1089. [CrossRef]

66. Bloomberg. Here Are the Most Innovative States in America. 2016. Available online: https://www.bloomberg. com/news/articles/2016-01-07/here-are-the-most-innovative-states-in-america (accessed on 16 October 2018).

67. Harrison, J.D.; Gorman, S. Innovation that matters [PDF File]; US Chamber Foundation: Washington, DC, USA, 2017; Available online: https://www.uschamberfoundation.org/sites/default/files/media-uploads/ITM\% 202017.pdf (accessed on 20 October 2018).

68. City of Boston. New Urban Mechanics Department. 2017. Available online: www.boston.gov/departments/ new-urban-mechanics (accessed on 17 October 2018).

69. The URBAN Vision [Video File]; Boston's Mayor's Office of New Urban Mechanics: Boston, MA, USA, 2013; Available online: https://www.youtube.com/watch?v=-LyaivtnCmg (accessed on 17 October 2018).

70. City of Boston. New Urban Mechanics Summer Fellows [Video File]. 19 December 2014. Available online: https://www.youtube.com/watch?v=rNL03-CJv7o (accessed on 20 October 2018).

71. TM Forum. Nigel Jacob, Co-Chair, Mayor's Office of New Urban Mechanics, City of Boston [Video File]. 7-9 September 2016. Available online: https://vimeo.com/182986435 (accessed on 26 October 2018).

72. School of Public Policy and Urban Affairs-Northeastern University. Innovative Uses of Data for Managing Boston Today, and in 2030-Myra Kraft Open Classroom [Video File]. 29 November 2017. Available online: https://www.youtube.com/watch?v=oNrLvP2zWR4\&t=1493s (accessed on 28 October 2018).

73. Jacob, N. NIGEL JACOB: From the Smart City to the Learning City (Unknown, Interviewer). 15 September 2013. Available online: http://lombardia.forumpa.it/en/news/nigel-jacob-smart-city-learning-city (accessed on 8 September 2018). 
74. Borins, S. The Challenge of Innovating in Government [PDF File]; IBM Center for The Business of Government: Washington, DC, USA, 2016; Available online: http://www.businessofgovernment.org/sites/default/files/ BorinsInnovatingInGov.pdf (accessed on 12 November 2018).

75. MONUM-Mayor's Office of New Urban Mechanics. Civic Research Agenda [PDF File]. 2018. Available online: https://www.boston.gov/departments/new-urban-mechanics/civic-research-agenda (accessed on 20 November 2018).

76. MONUM-Mayor's Office of New Urban Mechanics. New Urban Mechanics Year in Review [PDF File]. 2018. Available online: https:/www.boston.gov/sites/default/files/document-file-02-2018/monum_yearinreview_ 2017_finalv2.pdf (accessed on 22 November 2018).

77. Bertalanffy, L. General System Theory: Foundations, Development, Applications, 18th ed.; Braziller: New York, NY, USA, 2015.

78. Capra, F.; Luisi, P.L. The Systems View of Life: A Unifying Vision, 6th ed.; Cambridge University Press: Cambridge, UK, 2016.

79. Capra, F. The Web of Life; Flamingo: London, UK, 1996.

80. Cole, R.L. Citizen Participation and the Urban Policy Process; Lexington Books: Lexington, KY, USA, 1974.

81. Raco, M.; Imrie, R. Governmentality and Rights and Responsibilities in Urban Policy. Environ. Plan. Econ. Space 2000, 32, 2187-2220. [CrossRef]

82. City of Copenhagen. Available online: https://innovationshuset.kk.dk (accessed on 10 January 2019).

83. City of Cluj-Napoca, Centrul Cultural Clujean. Available online: http://www.cccluj.ro (accessed on 6 December 2018).

84. Badger, E. The Dawn of the Municipal Chief Innovation Officer. 19 May 2012. Available online: https://www. citylab.com/solutions/2012/03/dawn-municipal-chief-innovation-officer/1516/ (accessed on 18 October 2018).

85. Chesbrough, H. The era of open innovation. MIT Sloan Manag. Rev. 2003, 44, 35-41.

86. Chesbrough, H. Open Innovation. A New Paradigm for Understanding Industrial Innovation. In Open Innovation. Researching a New Paradigm; Chesbrough, H., Vanhaverbeke, W., West, J., Eds.; Oxford University Press: Oxford, UK, 2006.

87. Von Hippel, E. The dominant role of users in the scientific instrument innovation process. Res. Policy 1976, 5, 212-239. [CrossRef]

88. Von Hippel, E. Democratizing Innovation; MIT Press: Cambridge, MA, USA, 2005.

89. Von Hippel, E. Horizontal innovation networks-By and for users. Ind. Corp. Chang. 2007, 16, $293-315$. [CrossRef]

90. Bekkers, V.; Edelenbos, J.; Steijn, A.J. Linking innovation to the public sector: Contexts, concepts and challenges. In Innovation in the Public Sector: Linking Capacity and Leadership; Bekkers, V., Edelenbos, J., Steijn, A.J., Eds.; Palgrave Macmillan: Basingstoke, UK, 2014; pp. 3-34.

91. Bekkers, V.; Fenger, M.; Scholten, P. Public Policy in Action: Perspectives on the Policy Process; Edward Elgar Publishing: Cheltenham, UK, 2017.

92. Mayer, M. Manuel Castells' the city and the grassroots. Int. J. Urban Reg. Res. 2006, 30, 202-206. [CrossRef]

93. Chorianopoulos, I.; Tselepi, N. Austerity governance and bifurcated civil society: The changing matrices of urban politics in Athens. J. Urban Aff. 2019. [CrossRef]

94. Stickdorn, M.; Schneider, J. This Is Service Design Thinking: Basics, Tools, Cases, 6th ed.; BIS: Amsterdam, The Netherlands, 2016.

95. Leifer, L.; Meinel, C.; Plattner, H. Design Thinking Research: Making Design Thinking Foundational; Springer International Publishing: Cham, Switzerland, 2016. 


\title{
Cultural Heritage and Tourism Basis for Regional Development: Mapping of Scientific Coverage
}

\author{
José Álvarez-García ${ }^{1}$, Claudia Patricia Maldonado-Erazo ${ }^{2}$, María de la Cruz Del Río-Rama ${ }^{3, *}$ \\ and Francisco Javier Castellano-Álvarez ${ }^{4}$ \\ 1 Financial Economy and Accounting Department, Faculty of Business, Finance and Tourism, University of \\ Extremadura, 10071 Cáceres, Spain; pepealvarez@unex.es \\ 2 Facultad de Recursos Naturales, Escuela de Ecoturismo de la Superior Politécnica de \\ Chimborazo_ESPOCH, Riobamba 060155, Ecuador; claudia.maldonado@espoch.edu.ec \\ 3 Department of Business Organization and Marketing, Faculty of Business Sciences and Tourism, University \\ of Vigo, 32004 Ourense, Spain \\ 4 Economy Department, Faculty of Business, Finance and Tourism, University of Extremadura, 10071 Cáceres, \\ Spain; fcocastellano@unex.es \\ * Correspondence: delrio@uvigo.es; Tel.: +34-988-368-727
}

Received: 23 September 2019; Accepted: 25 October 2019; Published: 30 October 2019

\begin{abstract}
The aim of this research is to carry out a bibliometric and bibliographic study of the scientific production indexed in the international databases Scopus and Web of Science (WoS) on the use of cultural heritage by tourism as an alternative for regional development. This research allows us to observe the current situation of this area of study and to develop a research roadmap on this subject. The methodology used focuses on applying productivity, dispersion, collaboration, and citation indicators to a set of 103 articles identified through an advanced search of terms, in addition to applying an iterative analysis for the bibliographic study. The main findings of this study show that the documents are mostly analytical, mainly signed by a single author, and the productivity rate per author is 1.04. The co-author index in the subject is 2.34 , and the subject is in an exponential growth phase that began in 2004, with a ratio of 6.53 articles/year, with the majority of the production being by a single author per article. The country with the highest production is China, with 28 articles, 26 authors, 28 authorships, and 15 centers, followed by the Russian Federation, with 21 articles. Universiti Sains Malaysia (Malaysia) is the most productive institution, with 15 authorships, and there is a group of aspiring authors (between 2 and 4 articles) whose geographical affiliation is Malaysia, a group that represents $3 \%$ of the total of authors and concentrates 17 articles.
\end{abstract}

Keywords: cultural heritage; cultural tourism; regional development; rural areas; bibliometric analysis; bibliographic analysis

\section{Introduction}

The concept of cultural heritage is subjective and evolves over the years. It is subjective in the sense that cultural heritage is made up of those goods that the values that prevail in each society at a given time establish must be susceptible of being protected and preserved as a legacy of the past for future generations. This approach is reflected in the definition of cultural heritage proposed in The Charter of Krakow [1] (p. 5): “heritage is that complex of man's works in which a community recognizes its particular and specific values and with which it identifies. Identification and specification of heritage is therefore a process related to the choice of values". Smith [2] defines the concept of cultural heritage as the footprint of memory and oblivion, as a cultural practice involved in the construction and regulation of a spectrum of values and ways of understanding. This footprint is composed of the set of products of 
human activity, and its remains, intentional and unintentional, tangible intangibles, represent historical and social processes.

Therefore, in the first place it is important to establish the concept of "cultural heritage" and the elements that make it up. First, it is important to refer to the work done by the United Nations Educational, Scientific and Cultural Organization-UNESCO. UNESCO aims to encourage "the identification, protection, conservation, presentation and transmission to future generations of the cultural and natural heritage" throughout the world that is considered of great value to humanity [3]. The work was shown in the international treaty adopted in 1972, the "Convention Concerning the Protection of the World Cultural and Natural Heritage" [3], which lays the foundation for the first definition of the term "cultural heritage".

In this sense, one of the first definitions is the one proposed by UNESCO in the Declaration of Mexico on Cultural Policies [4] (p. 3): "The cultural heritage of a people includes the works of its artists, architects, musicians, writers and scientists and also the work of anonymous artists, expressions of the people's spirituality, and the body of values which give meaning to life. It includes both tangible and intangible works through which the creativity of that people finds expression: languages, rites, beliefs, historic places and monuments, literature, works of art, archives and libraries".

Subsequently, UNESCO approved The Convention for the Safeguarding of the Intangible Cultural Heritage [5] (p. 4) in 2003, which specifically defines what is meant by intangible cultural heritage: "the 'intangible cultural heritage' means the practices, representations, expressions, knowledge, skills-as well as the instruments, objects, artefacts and cultural spaces associated therewith-that communities, groups and, in some cases, individuals recognize as part of their cultural heritage. This intangible cultural heritage, transmitted from generation to generation, is constantly recreated by communities and groups in response to their environment, their interaction with nature and their history, and provides them with a sense of identity and continuity, thus promoting respect for cultural diversity and human creativity ... ". According to Timothy and Boyd [6] (p. 94), the conservation of cultural heritage can be carried out in different ways: by the preservation, restoration, renovation of goods, and urban regeneration.

According to UNESCO, cultural heritage is subdivided into the tangible (movable and immovable property), which can be preserved and restored by some kind of intervention [4], and intangible, which "is manifested inter alia in the following domains: (a) oral traditions and expressions, including language as a vehicle of the intangible cultural heritage; (b) performing arts; (c) social practices, rituals, and festive events; (d) knowledge and practices concerning nature and the universe; (e) traditional craftsmanship" [5] (p. 4).

Continuing with the contextualization of this research work, the next step is to relate cultural heritage and tourism. Thus, cultural heritage, composed of a wide variety of tangible and intangible assets, can be enhanced in order to articulate new development alternatives by the tourism activity. Tourism management of cultural heritage enables it to be transformed into tourist resources.

Thus arises the concept of heritage tourism and cultural tourism. Timothy [7] (pp. 4-5) points out that both terms are often used in the literature as distinct but related concepts. For Timothy and Boyd [6] (p. 14), the terms cultural and heritage tourism overlap and, in their opinion, are interchangeable (see the overlapping of the concepts cultural and heritage tourism in Timothy and Boyd [6] (p. 9)). Along the same lines, Timothy [7] suggests that heritage tourism is based on "old things" and usually occurs in rural areas and in more specific places, while cultural tourism predominates in urban areas and less determined places (see the exhaustive classification of the different attractions offered by heritage tourism in Timothy [7] (p. 49)). For Timothy [7], all definitions of heritage tourism include elements of the human past as a resource, and all collect a variety of reasons why tourists move (tourists move for reasons that may stem from the desire to improve their own personal culture, learn something new, spend time with friends or family, satisfy a curiosity, or simply spend the free time available).

In this sense, there are several studies that show that knowledge of the culture of other spaces is one of the main motivations that drive tourists [8-11]. Therefore, exploiting cultural heritage, being 
either tangible or intangible, through tourism activity becomes a fundamental means throughout the world for the development of spaces where this heritage is located. According to the World Tourism Organization (UNWTO), it was identified that within the practice of tourism activities related to culture, those related to intangible heritage are the most important, closely followed by tangible ones [12]. In short, tourism becomes an attractive option to take advantage of the local resources available, including heritage [13].

Focusing on cultural wealth as attractions of tourist interest allows for the creation of several hubs through which the cultural heritage and tourism relationship is developed, including: (1) the recovery or conservation of heritage, (2) economic growth focused on the commercialization of goods, (3) the increase of tourist facilities from internal or external investment ad an improved image for the community, and (4) social benefit for communities in order to improve living conditions [14-17]. In this way, cultural heritage becomes an element capable of generating benefits for the development of societies, the relationship with tourism being the most often used to achieve this goal. Thus, cultural tourism becomes an essential strategic feasibility for tourism development. This type of tourism may reduce the seasonality of the sector and favor the opening to new markets.

Developing tourism around cultural heritage is considered an action that is not focused on mass culture that homogenizes, imposing one culture over another, or that mythologizes or fragments the value of heritage. In this sense, it is proposed by the popular culture in which its vital particularities are created and preserved, and it is not based on selling but on participating with the purpose that this is produced and consumed by the human group itself. This approach allows for an increase in the quality of life, which, at the same time, enables the strengthening of the transmission and revitalization networks of elements contained in the collective memory of groups [14,15]. With this, it is argued that each community has something unique to offer that manages to generate considerable tourist flows based on the interests that they arouse for the existence of a vibrant culture.

The participation of the community is essential, and it should not be left out; the empowerment of processes leads to a more efficient development of heritage [18]. This must be the pillar in the process of tourism development and heritage management [19], always supported by local identity, that is, heritage [20,21].

In this context, this research aims to analyze and examine the scientific production developed in relation to exploiting cultural heritage by tourism activities for regional development. To achieve the objective, a bibliometric analysis is first performed, which is complemented by a bibliographic analysis, allowing for a systematic and structured analysis of production. The novelty of the study is based on the fact that there is no research of similar characteristics that provides a complete view of the research carried out in this field of study. The findings will be very useful for researchers as they provide a better understanding of current research and serve as a guide for future research.

This article is structured into five sections. The topic is contextualized, the literature is reviewed, and the objective is presented in the introduction. In the second section, the methodology is introduced. The results are shown in the third section and in the fourth section, the bibliographic analysis. Finally, to conclude, the conclusions and limitations of the investigation are discussed.

\section{Methodology}

The methodology used in this research is an exploratory quantitative bibliometric and bibliographic analysis, focused on identifying and analyzing scientific production on cultural heritage and its use by the tourism activity for regional development. This methodology is the most adequate to organize scientific information; on the one hand, it enables the identification and analysis of bibliographical sources of interest, while on the other hand, it provides an evaluation structure for this scientific production [22].

In the bibliometric analysis, indicators are applied [23] that arise from different mathematical models that are based on the relationship of two or more variables [24], which enables the measurement of the bibliographic material in quantitative terms. The indicators that will be used in this research 
are classified according to two criteria: activity indicators that provide information on quantity, productivity, dispersion, and collaboration, among others, and impact indicators, which provide information on the citation level of the documents through the impact factor or immediacy index, the $\mathrm{H}$ index, among others [25].

\subsection{Databases}

As in most research, the chances of covering a complete universe of study are small (the number of databases is very large). Therefore, three conditions should be complied with to choose the database. These are set out by Rueda et al. [26]: availability, relevance, and reliability of information.

Thus, the selection of databases was based on three criteria: (a) application of rigorous quality standards through Relative Quality Indexes, SJR (Scimago Journal Rank) for Scopus and JCR (InCites Journal Citation Report) for WoS (Web of Science); (b) extensive coverage over time compared to other databases; and (c) they allow for simultaneous downloading of a considerable number of references stored in detail as metadata. According to Harzing and Alakangas [27], the presence of these characteristics validates the information indexed in the database and are sufficient to justify its use.

\subsection{Tracking Methodology}

To construct the data matrix with which to work, it is necessary to establish the search criteria of the bibliographic production. Firstly, the coverage time was determined; in this study, this was set as equal to or less than the year 2018, with the purpose of considering the information published in full years. Secondly, the scientific article published in journals was determined as the documentary unit of analysis. This choice was based on the consideration of six criteria: (1) speed within publication processes, (2) rigorous arbitration process, (3) visibility and impact at different levels (local, national, and international), (4) specific outline for presenting the information, (5) acceleration in the positioning of the authors within the scientific community, and (6) increase of the feedback [28,29]. The articles from conferences, books and chapters, editorials, notes, letters or errata contained in WoS or Scopus were excluded from the search. Finally, the keywords and search equation were determined for the advanced search (Table 1).

Table 1. Search strategy.

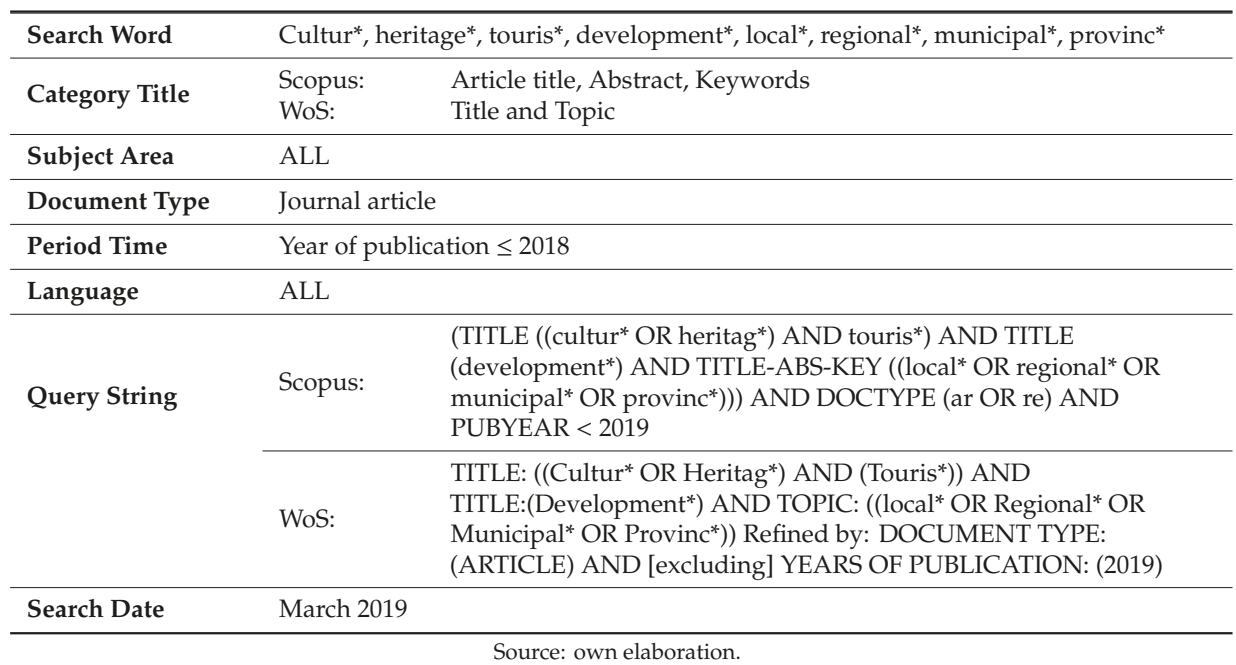




\subsection{Methodology of Calculations}

When working with two databases (WoS and Scopus), it is essential to determine the overlap level between both databases in relation to the identified articles and their degree of coverage. For this purpose, Meyer's index, traditional overlapping (TO), and relative overlapping (RO), which are the appropriate tools to identify the overlap level of indexed articles [30,31], were applied (Table 2). Although the overlap phenomenon has been known for more than 50 years, it is only in the last decade that there has been a greater interest in its study and application [30].

Table 2. Formulas to determine the level of overlap and degree of coverage between databases.

\begin{tabular}{|c|c|c|}
\hline Index & Calculation Formula & Objective and Observations \\
\hline Meyer index & $\begin{array}{l}\text { Meyer's index }(\mathrm{MI})= \\
\frac{\sum \text { sources*weight }}{\text { Total sources }} \\
\text { (A weight must be assigned to } \\
\text { each documentary unit based on } \\
\text { the number of repetitions that are } \\
\text { recorded between the databases. } \\
\text { Weight = 1; single character units } \\
\text { within each base. } \\
\text { As repetitions increase depending } \\
\text { on the number of databases used } \\
\text { for comparison, the weight will } \\
\text { gradually be reduced; duplicates } \\
\text { (weight =0.5), triplicates (weight } \\
=0.3 \text { ), and so on.) }\end{array}$ & $\begin{array}{l}\text { - To determine the degree of coverage that each } \\
\text { database has on the scientific production of a } \\
\text { specific subject or specialty [31,32]. } \\
\text { - It is established based on the number of } \\
\text { repetitions that are recorded for each document } \\
\text { among the chosen databases [33,34]. } \\
\text { - The result of Meyer's index is interpreted as } \\
\text { the singularity level for each base, with the } \\
\text { understanding that the higher the value, the } \\
\text { greater the originality of the database [34]. }\end{array}$ \\
\hline Traditional overlapping (TO) & $\% \mathrm{TO}=100 *\left(\left|\frac{A \cap B}{A \cup B}\right|\right)$ & $\begin{array}{l}\text { - To determine the percentage of overlap or the } \\
\text { degree of similarity between two databases (the } \\
\text { similarity that database A has within database } \\
\text { B is established). } \\
\text { - The higher the resulting percentage, the } \\
\text { greater the similarity [35]. }\end{array}$ \\
\hline Relative overlapping (RO) & $\% \mathrm{RO}$ in $\mathrm{A}=100 *\left(\frac{|A \cap B|}{|A|}\right)$ & $\begin{array}{l}\text { - Weight of repeated documents between the } \\
\text { bases compared with those that are single [26]. } \\
\text { - The result is the percentage of overlap of } \\
\text { database A with database B [36]. }\end{array}$ \\
\hline
\end{tabular}

Note: calculations complementary to Meyer's index. The creation algorithms used allow us to consider all the possible existing unions between the databases selected for the analysis [35], covering with them all the necessary edges to determine the overlapping of current information. Source: own elaboration.

\subsection{Systematization of Information}

To finish the construction of the study matrix (final ad hoc database) through the review of the identified bibliographic sources, the data collected was refined and normalized. This process led to the elimination of duplicate documents or those lacking any relationship with the subject of study, the latter situation generated by an inappropriate use of keywords by the authors during their indexation. As a result, the joint study matrix built in Microsoft Office Excel software was made up of 103 articles, and for the individual analysis of each of the databases, the original matrices constituted by 84 articles published in Scopus and 51 in WoS were maintained. The bibliographic references were managed with EndNote.

The methodological process of the study is shown in Figure 1. 


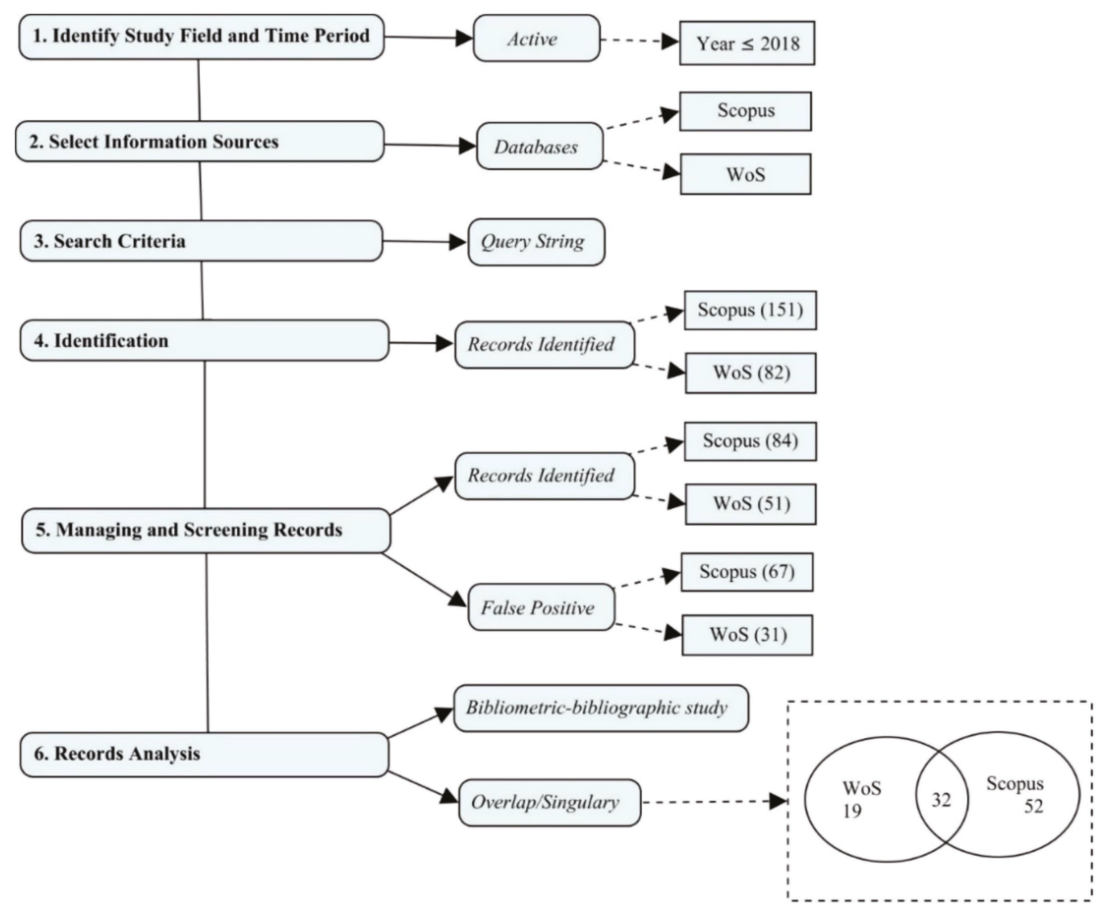

Figure 1. Bibliometric methodological procedure. Source: own elaboration.

\section{Results}

\subsection{Overlap of Databases}

The linear correlation coefficient is 0.73 , which indicates a strong and direct correlation between Scopus and WoS. Of the 135 articles (84 Scopus and 51 WoS), it was observed that 32 articles were indexed in both databases, which represents 38\% of the articles from Scopus and 63\% of those from WoS. Consequently, the remaining 52 articles from Scopus and 19 from WoS were classified as single documents as they are present in only one of the two bases. Table 3 shows the results related to the singularity of the databases, measured through the Meyer index (MI), with Scopus being the database with the highest singularity index with a $\mathrm{MI}=0.81$, while in WoS it reaches $\mathrm{MI}=0.69$.

Table 3. Singularity of the databases.

\begin{tabular}{ccccc}
\hline & \multicolumn{2}{c}{$\%$ Single Documents } & \multicolumn{2}{c}{ Meyer Index } \\
\hline Databases & Articles & Journals & Articles & Journals \\
\hline Scopus & $61.90 \%$ & $56.25 \%$ & 0.81 & 0.78 \\
WoS & $37.25 \%$ & $34.88 \%$ & 0.69 & 0.67 \\
\hline \multicolumn{3}{c}{ Source: own elaboration. }
\end{tabular}

The traditional overlapping (TO) percentage between Scopus and WoS determined a similarity of $31.07 \%$ between the databases, which is also understood as the existence of a $68.93 \%$ disparity between them. Likewise, in addition to the previous calculations, it is necessary to determine the percentage of coverage that Scopus shows in relation to WoS and vice versa [35], for which relative overlapping (RO) was applied. The percentages obtained show that $38.10 \%$ of Scopus was covered by WoS, while the RO 
of WoS shows that $62.75 \%$ of this database was covered by Scopus. As a result, Scopus has a lower overlap than WoS.

\subsection{Productivity Per Years}

The joint database consists of 103 articles (32 duplicated articles were eliminated). Figure 2 shows that scientific production covers 25 years (1994-2018), the first indexed study being Market-Based Product Development in Heritage Tourism, by Light and Prentice [37]. During this period, 2017 is consolidated as the year of greatest productivity, with 21 published articles.

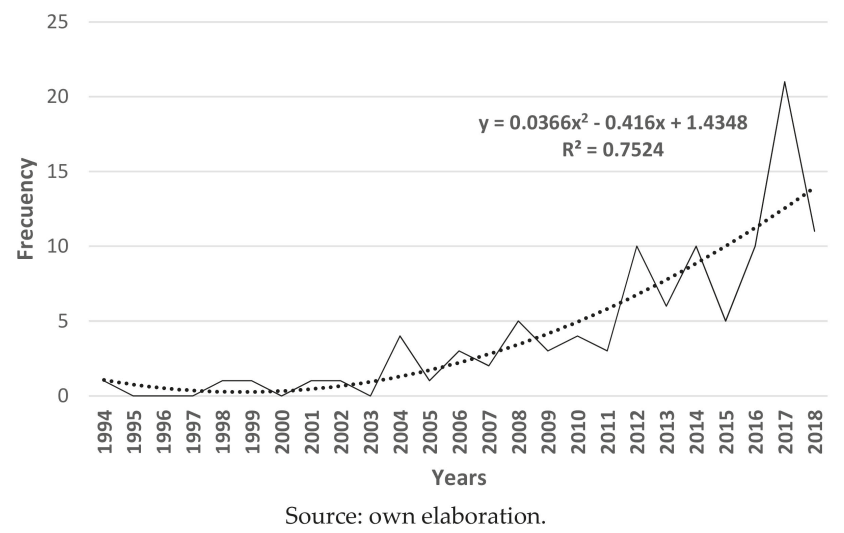

Figure 2. Trend of publications Scopus $\cup$ WoS.

Considering the four stages of Price [38] in the evolution of scientific production, precursors, exponential growth, linear growth, and collapse of the scientific field, the presence of two of them in the growth process are observed here. The first part or stage of precursors lasts from 1994 to 2003 and corresponds to 0.50 articles/year (60\% with a single signature), with slow growth being evident. The second stage goes from 2004 to 2018, with a ratio of 6.53 articles/year, and most of the production involving a single author per article. It is observed that the Price law is fulfilled: between 10 and 15 years after the first publication, the information developed on the subject at a global level is duplicated [38].

Finally, Figure 3 shows an average correlation between Scopus and WoS regarding articles that have been indexed, with $\mathrm{R}^{2}=0.5364$.

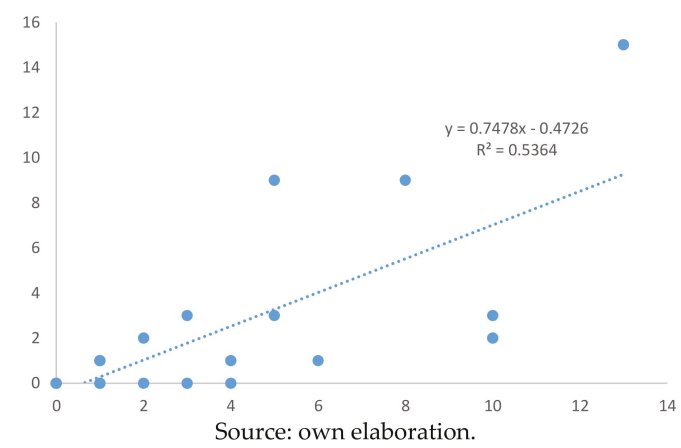

Figure 3. Correlation between the numbers of articles published in Scopus and WoS. 


\subsection{Citations}

During the 25 years of study, a cumulative total of 1135 citations (13.51 citations/article) are identified regarding the 84 Scopus articles indexed in Scopus. In WoS, 687 citations (51 articles), 13.47 citations/article, are recorded. Scopus has an $\mathrm{h}=14$, which means that at least 14 of the total articles identified have obtained 14 citations or more, and WoS an $\mathrm{h}=8$. The year that reaches the highest number of citations in both databases is 1998, with 378 citations in Scopus and 317 in WoS, concentrating $33 \%$ and $47 \%$ of the total citations, respectively.

Of the Scopus and WoS articles, $65 \%$ and 51\%, respectively, obtained between 24 and 1 citations, while $27 \%$ and $43 \%$ of the articles, respectively, do not have any citations recorded during the analysis period. On the other hand, more than 100 citations were recorded in 3 articles. It was observed that documents published in recent years have not received a significant number of citations; surely because these studies did not achieve the necessary dissemination to be consolidated as referents of the subject, a fact that limits the amount of citations they can receive [39].

The most-cited articles on the subject were Local Development and Heritage: Traditional Food and Cuisine as Tourist Attractions in Rural Areas, by Bessiere [20], with 378 citations in Scopus (18.0 citations per year) and 314 in WoS (15.1 citations per year), Tourism Development of World Heritage Sites in China: A Geographic Perspective, by Li et al. [40] with 156 citations (14.2) in Scopus and 138 in WoS (12.5), and The "Vicious Circle" of Tourism Development in Heritage Cities, by Russo [41], with 130 in Scopus (7.6) and 106 in WoS (6.2). In addition, three articles that have received a considerable number of citations were identified, but these are only indexed within Scopus: Industrial Heritage: A Nexus for Sustainable Tourism Development, by Jonsen-Verbeke [42], Resident Attitudes towards Heritage Tourism Development, by Chen and Chen [43], and "Heritagisation", a Challenge for Tourism Promotion and Regional Development: An example of Food Heritage, by Bessiere [44].

\subsection{Authors}

A total of 231 authors were identified in the scientific productions analyzed in the joint analysis matrix (103 articles), which corresponds to a productivity index per author of 1.04 articles. The most productive authors in the subject were Rasoolimanesh and Jaafar, with four articles belonging to the Universiti Sains Malaysia (Malaysia). Both authors have an average citations/article of 15.33 in Scopus and 8.25 in WoS. The second most productive author was Bessiere, of the University of Toulouse II (France), with 2 articles; however, he has a better citation average of 202.5 in Scopus and 317 in WoS. Authors such as Huibin, Marzuki, Razak, Min, and Sun are also in this second position, with 2 articles, but they are indexed only in the Scopus database and the number of citations is very small.

Total author productivity can be analyzed by means of different types of processes, which enables them to be classified according to the contribution that each author provides within the subject of study. The classification of Crane [45] is used to fulfill this purpose in this study, in which the production by authors can be explained by four groups of authors: (1) large producers-those who have a production greater than 10 articles, (2) moderate producers-authors who have produced between 5 and 9 documents, (3) aspiring authors-authors who have between 2 and 4 studies, and (4) transition authors-authors who have only produced one work. There are no large or moderate producers in this area; 223 are transition and 8 are aspiring.

The total transience index $(\mathrm{TI}=[\mathrm{PI}($ productivity index $)=0])$ of the total set of documents under study is $96.5 \%$; that is, this index is the same or represents the total of occasional authors who only arise once within the subject and who do not continue making contributions throughout the rest of the line of evolution, a figure which is based on the classification of Crane [45].

Another important element of analysis is the collaboration trend in scientific production, which makes it possible to analyze current relationships. In this sense, Berelson [46] determines that the more varied and the greater the collaboration shown within the development of documents, the greater the maturity of the subject of study [47]. This study revealed that $35 \%$ (36) of the articles are of a single authorship, and the remaining $65 \%$ (67) are the product of collaboration. In the case of collaboration, 
$29 \%$ (30) are signed by two authors, $17 \%$ (18) of papers are signed by three authors, $12 \%$ (12) by four authors, and $7 \%$ (7) are signed by five or more authors. With these data, the co-authorship index is 2.34 authors/article.

\subsection{Productivity by Type of Institution and Country}

Establishing productivity based on the affiliation registered by the authors allows the evaluation and understanding of the information nodes that are being developed based on the geographical and institutional affiliations registered during the study period. In this sense, geographical affiliation is established by country, with China being the country with the highest productivity, with 28 articles, 26 authors, 28 authorships, and 15 centers. The second most productive country is the Russian Federation, with 21 articles, 21 authors, 21 authorships, and 9 centers. In relation to the number of citations, France is the country that concentrates the largest number of citations, with 410 in Scopus and 317 in WoS, with 9 and 2 articles identified in each database, respectively (Table 4).

Table 4. Number of centers, authors, and authorships by their country of affiliation.

\begin{tabular}{|c|c|c|c|c|c|c|c|c|c|c|c|c|c|}
\hline \multirow{2}{*}{$\mathbf{R}$} & \multirow{2}{*}{ Country } & \multicolumn{4}{|c|}{ Scopus $\cup$ WoS } & \multicolumn{4}{|c|}{ Cites Scopus } & \multicolumn{4}{|c|}{ Cites WoS } \\
\hline & & $\mathrm{f}$ & $\mathrm{C}$ & A & As & $\mathrm{f}$ & hi $\%$ & TC & h-Index & $\mathrm{f}$ & hi $\%$ & TC & h-Index \\
\hline 1 & China & 28 & 15 & 26 & 28 & 28 & 13.8 & 206 & 4 & 13 & 10.7 & 151 & 2 \\
\hline 3 & United States & 19 & 11 & 19 & 19 & 15 & 7.4 & 350 & 5 & 9 & 7.4 & 291 & 3 \\
\hline 4 & Malaysia & 15 & 1 & 8 & 15 & 15 & 7.4 & 157 & 6 & 11 & 9.1 & 100 & 5 \\
\hline 6 & Spain & 13 & 8 & 13 & 13 & 8 & 3.9 & 51 & 4 & 10 & 8.3 & 13 & 1 \\
\hline 7 & France & 9 & 7 & 8 & 9 & 9 & 4.4 & 410 & 2 & 2 & 1.7 & 317 & 1 \\
\hline 8 & Italy & 9 & 8 & 9 & 9 & 9 & 4.4 & 38 & 4 & 2 & 1.7 & 6 & 1 \\
\hline 9 & United Kingdom & 8 & 7 & 8 & 8 & 8 & 3.9 & 86 & 6 & 4 & 3.3 & 31 & 3 \\
\hline 13 & Poland & 6 & 3 & 6 & 6 & 3 & 1.5 & 6 & 2 & 5 & 4.1 & 0 & 0 \\
\hline 14 & Ghana & 5 & 3 & 5 & 5 & 5 & 2.5 & 40 & 2 & - & 0.0 & - & - \\
\hline 15 & Kazakhstan & 5 & 1 & 5 & 5 & 5 & 2.5 & 0 & 0 & - & 0.0 & - & - \\
\hline 16 & Thailand & 5 & 2 & 5 & 5 & 5 & 2.5 & 2 & 1 & - & 0.0 & - & - \\
\hline 17 & Argentina & 4 & 1 & 4 & 4 & 4 & 2.0 & 12 & 3 & 4 & 3.3 & 12 & 3 \\
\hline 18 & Croatia & 4 & 3 & 4 & 4 & 4 & 2.0 & 0 & 0 & - & 0.0 & - & - \\
\hline 19 & Greece & 4 & 2 & 4 & 4 & 4 & 2.0 & 8 & 2 & 4 & 3.3 & 8 & 2 \\
\hline 20 & Hungary & 4 & 4 & 4 & 4 & 1 & 0.5 & 2 & 1 & 4 & 3.3 & 3 & 1 \\
\hline 21 & Norway & 4 & 3 & 4 & 4 & 4 & 2.0 & 8 & 2 & 4 & 3.3 & 4 & 1 \\
\hline 27 & Cuba & 2 & 2 & 2 & 2 & 2 & 1.0 & 2 & 1 & 2 & 1.7 & 2 & 1 \\
\hline 28 & Finland & 2 & 1 & 2 & 2 & 2 & 1.0 & 12 & 2 & 2 & 1.7 & 6 & 1 \\
\hline 29 & Germany & 2 & 2 & 2 & 2 & 2 & 1.0 & 8 & 2 & 1 & 0.8 & 3 & 1 \\
\hline 30 & South Africa & 2 & 2 & 2 & 2 & 2 & 1.0 & 16 & 2 & 2 & 1.7 & 10 & 1 \\
\hline 31 & South Korea & 2 & 1 & 2 & 2 & 2 & 1.0 & 0 & 0 & - & 0.0 & - & - \\
\hline 32 & Taiwan & 2 & 1 & 2 & 2 & 2 & 1.0 & 76 & 1 & - & 0.0 & - & - \\
\hline 33 & Belgium & 1 & 1 & 1 & 1 & 1 & 0.5 & 45 & 1 & 0 & 0.0 & 0 & 0 \\
\hline 34 & Brazil & 1 & 1 & 1 & 1 & - & 0.0 & - & 0 & 1 & 0.8 & 0 & 0 \\
\hline 35 & Canada & 1 & 1 & 1 & 1 & 1 & 0.5 & 5 & 1 & - & 0.0 & - & - \\
\hline
\end{tabular}


Table 4. Cont.

\begin{tabular}{|c|c|c|c|c|c|c|c|c|c|c|c|c|c|}
\hline \multirow{2}{*}{$\mathbf{R}$} & \multirow{2}{*}{ Country } & \multicolumn{4}{|c|}{ Scopus $\cup$ WoS } & \multicolumn{4}{|c|}{ Cites Scopus } & \multicolumn{4}{|c|}{ Cites WoS } \\
\hline & & $\mathrm{f}$ & $\mathrm{C}$ & A & As & $\mathrm{f}$ & hi $\%$ & $\mathrm{TC}$ & h-Index & $\mathrm{f}$ & hi $\%$ & TC & h-Index \\
\hline 36 & Ecuador & 1 & 1 & 1 & 1 & - & 0.0 & - & 0 & 1 & 0.8 & 0 & 0 \\
\hline 38 & Indonesia & 1 & 1 & 1 & 1 & 1 & 0.5 & 0 & 0 & - & 0.0 & - & - \\
\hline 39 & Mexico & 1 & 1 & 1 & 1 & 1 & 0.5 & 0 & 0 & - & 0.0 & - & - \\
\hline 41 & Netherlands & 1 & 1 & 1 & 1 & 1 & 0.5 & 130 & 1 & 1 & 0.8 & 106 & 1 \\
\hline 42 & Nigeria & 1 & 1 & 1 & 1 & 1 & 0.5 & 0 & 0 & - & 0.0 & - & - \\
\hline 43 & Senegal & 1 & 1 & 1 & 1 & - & 0.0 & - & 0 & 1 & 0.8 & 1 & 1 \\
\hline 44 & Slovenia & 1 & 1 & 1 & 1 & 1 & 0.5 & 5 & 1 & - & 0.0 & - & - \\
\hline
\end{tabular}

With regard to productivity by institution, the presence of 47 different types of affiliation centers was registered (universities, institutes, private companies, public institutions, international organizations, etc.). However, universities are the ones that concentrate the highest number of affiliations, with $70.2 \%$ (33).

Table 5 shows the ranking of the most productive institutions, considering the number of authors. Universiti Sains Malaysia (Malaysia) occupies the first position followed by the University of Novi Sad (Serbia) and the Institute Of Geographic Sciences and Natural Resources Research (China), with 8 authors, respectively.

Table 5. Most productive institutions measured by authors and authorships.

\begin{tabular}{|c|c|c|c|c|c|c|c|c|}
\hline \multirow{2}{*}{$\mathbf{R}$} & \multirow{2}{*}{ Institution } & \multirow{2}{*}{ Country } & \multicolumn{2}{|c|}{ Scopus $\cup$ WoS } & \multicolumn{2}{|c|}{ Scopus } & \multicolumn{2}{|c|}{ WoS } \\
\hline & & & A & As & A & As & $\mathbf{A}$ & As \\
\hline 1 & Universiti Sains Malaysia & Malaysia & 8 & 15 & 8 & 15 & 5 & 11 \\
\hline 2 & University of Novi Sad & Serbia & 8 & 8 & 8 & 8 & - & - \\
\hline 3 & $\begin{array}{l}\text { Inst. of Geographic Sciences and Natural } \\
\text { Resources Research }\end{array}$ & China & 6 & 8 & 6 & 8 & 2 & 2 \\
\hline 4 & Anhui Normal University & China & 6 & 6 & 6 & 6 & 6 & 6 \\
\hline 5 & $\begin{array}{l}\text { L.N. Gumilyev Eurasian National } \\
\text { University }\end{array}$ & Kazakhstan & 5 & 5 & 5 & 5 & - & - \\
\hline 6 & Russian State Social University & Russia & 5 & 5 & 5 & 5 & - & - \\
\hline 7 & University of Texas at San Antonio & United States & 5 & 5 & 5 & 5 & - & - \\
\hline 8 & $\begin{array}{l}\text { Scientific Research Commission of the } \\
\text { Prov. of Buenos Aires }\end{array}$ & Argentina & 4 & 4 & 4 & 4 & 4 & 4 \\
\hline 9 & Xiangnan University & China & 4 & 4 & 4 & 4 & - & - \\
\hline 10 & West Virginia University & United States & 4 & 4 & - & - & 4 & 4 \\
\hline
\end{tabular}

${ }^{*} \mathrm{R}=$ ranking; $\mathrm{A}=$ authors; $\mathrm{As}=$ authorships. Source: own elaboration

In relation to collaboration networks, collaboration at the institutional and geographical level is considered in the analysis. In 79\% (53) of the 67 articles signed by several authors, the authors are affiliated to the same country, and the remaining $21 \%$ (14) are articles written by authors from different countries. In the first case, affiliated to the same country, $64 \%$ (34) of the articles are signed by authors from the same center and $36 \%$ (19) by authors from different centers. 


\subsection{Journals}

The total set of articles (103) was published in 80 journals, 64 of which only published one article and the remaining 16 journals publishing two or more. Therefore, the index of dispersion is 1.29 articles/journal. The most productive journal is the Journal of Heritage Tourism, with five published articles (Table 6). However, in relation to the total number of citations received, Sociologia Ruralis leads with 378 citations accumulated within the only published study. Regarding the geographical origin of the journals, it can be seen that these are published mainly in the United Kingdom, with $39.1 \%$ (25) of Scopus journals and $32.6 \%$ (14) of WoS, followed by the United States with $9.4 \%$ of Scopus and $16.3 \%$ of WoS articles.

Table 6. Ranking of the most productive journals.

\begin{tabular}{|c|c|c|c|c|c|c|c|c|c|c|c|c|}
\hline \multirow{2}{*}{$\mathbf{R}$} & \multirow{2}{*}{ Title } & \multirow{2}{*}{ Country } & \multirow{2}{*}{$\mathbf{f}$} & \multirow{2}{*}{ hi $\%$} & \multicolumn{4}{|c|}{ Scopus (SJR) } & \multicolumn{4}{|c|}{ WoS (JCR) } \\
\hline & & & & & $f$ & TC & h-Index & $Q$ & $\mathrm{f}$ & TC & h-Index & $\mathrm{Q}$ \\
\hline 1 & Journal of Heritage Tourism & $\begin{array}{l}\text { United } \\
\text { Kingdom }\end{array}$ & 5 & 4.85 & 5 & 44 & 19 & 1 & 2 & 2 & 7 & 0 \\
\hline 2 & Tourism Management & $\begin{array}{c}\text { United } \\
\text { Kingdom }\end{array}$ & 3 & 2.91 & 3 & 194 & 143 & 1 & 3 & 164 & 157 & 1 \\
\hline 3 & Tourism Geographies & $\begin{array}{c}\text { United } \\
\text { Kingdom }\end{array}$ & 3 & 2.91 & 3 & 90 & 45 & 1 & 1 & 5 & 36 & 2 \\
\hline 4 & $\begin{array}{c}\text { WIT Transactions on Ecology and } \\
\text { the Environment }\end{array}$ & $\begin{array}{c}\text { United } \\
\text { Kingdom }\end{array}$ & 3 & 2.91 & 3 & 6 & 17 & - & & & & \\
\hline 5 & Anuario Turismo y Sociedad & Colombia & 3 & 2.91 & - & - & - & - & 3 & 0 & 2 & 0 \\
\hline 6 & Current Issues in Tourism & $\begin{array}{l}\text { United } \\
\text { Kingdom }\end{array}$ & 2 & 1.94 & 2 & 34 & 50 & 1 & - & - & - & - \\
\hline 7 & $\begin{array}{l}\text { Asia Pacific Journal of Tourism } \\
\text { Research }\end{array}$ & $\begin{array}{c}\text { United } \\
\text { Kingdom }\end{array}$ & 2 & 1.94 & 2 & 28 & 24 & 1 & 1 & 6 & 22 & 3 \\
\hline 8 & Life Science Journal & China & 2 & 1.94 & 2 & 19 & 19 & 4 & 1 & 0 & 15 & 4 \\
\hline 9 & Tourism & Croatia & 2 & 1.94 & 2 & 14 & 16 & 4 & 1 & 5 & 2 & 0 \\
\hline 10 & Estudios Geográficos & Spain & 2 & 1.94 & 1 & 10 & 7 & 3 & 1 & 0 & 3 & 0 \\
\hline 11 & Journal of Sustainable Tourism & $\begin{array}{c}\text { United } \\
\text { Kingdom }\end{array}$ & 2 & 1.94 & 2 & 8 & 76 & 1 & 2 & 3 & 60 & 1 \\
\hline 12 & Sustainability & Switzerland & 2 & 1.94 & 2 & 6 & 42 & 2 & 2 & 1 & 42 & 2 \\
\hline 13 & $\begin{array}{c}\text { Theoretical and Empirical } \\
\text { Researches in Urban Management }\end{array}$ & Romania & 2 & 1.94 & 2 & 5 & 9 & 2 & - & - & - & - \\
\hline 14 & Geographica Pannonica & Serbia & 2 & 1.94 & 2 & 4 & 6 & 3 & - & - & - & - \\
\hline 15 & $\begin{array}{c}\text { International Journal of } \\
\text { Heritage Studies }\end{array}$ & $\begin{array}{c}\text { United } \\
\text { Kingdom }\end{array}$ & 2 & 1.94 & 2 & 3 & 33 & 1 & 2 & 3 & 25 & 2 \\
\hline 16 & Chinese Geographical Science & China & 2 & 1.94 & 2 & 1 & 23 & 2 & 1 & 0 & 30 & 4 \\
\hline
\end{tabular}

To conclude with the analysis of productivity by type of institution and country, the concentration cores generated in relation to the scientific production of this subject can be identified, for which the law of Bradford [49] is applied, making it possible to identify a high percentage of studies concentrated in a small number of journals when analyzing the scientific production of a specific subject. First, the minimum Bradford zone (MBZ), which takes the value of 32, is calculated. The ranking of journals is arranged in descending order according to their productivity. Thus, the Bradford core corresponds to the group of journals whose summed productivity is equal to 32. In this area, the Bradford core consists of 41 journals (Figure 4 ). 


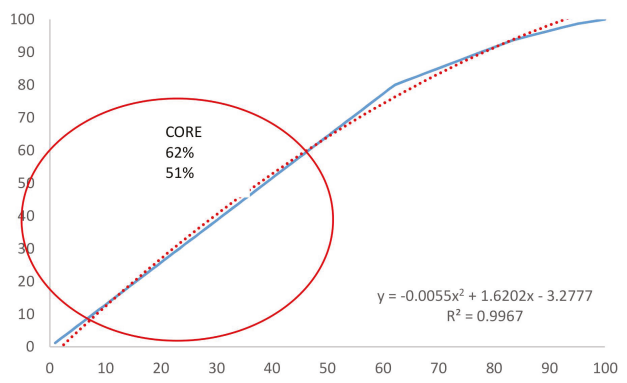

Figure 4. Lorenz curve-Bradford core of the most productive journals. Source: own elaboration.

\subsection{Thematic Areas}

Next, the thematic areas by which the resources within Scopus and WoS are classified are analyzed. In relation to the area of knowledge, the social sciences is the area that predominates, with 39 articles $(46 \%)$ and a total of 932 citations accumulated in Scopus; in WoS it accounts for 19 (37\%) articles and 327 citations (Table 7).

Table 7. Classification of articles by subject area.

\begin{tabular}{|c|c|c|c|c|c|c|c|c|c|}
\hline \multicolumn{5}{|c|}{ Scopus } & \multicolumn{5}{|c|}{ WoS } \\
\hline Area & $\mathbf{J}$ & $\mathrm{f}$ & TC & $\mathrm{C} / \mathrm{f}$ & Area & $\mathbf{J}$ & f & TC & $\mathrm{C} / \mathrm{f}$ \\
\hline Social Sciences & 30 & 39 & 932 & 23.9 & Social Sciences & 14 & 19 & 327 & 17.2 \\
\hline $\begin{array}{c}\text { Earth and Planetary } \\
\text { Sciences }\end{array}$ & 10 & 12 & 27 & 2.3 & $\begin{array}{c}\text { Environmental } \\
\text { Sciences \& Ecology }\end{array}$ & 6 & 6 & 8 & 1.3 \\
\hline Arts and Humanities & 6 & 11 & 56 & 5.0 & Arts \& Humanities & 5 & 6 & 4 & 0.7 \\
\hline $\begin{array}{c}\text { Business, } \\
\text { Management, and } \\
\text { Accounting }\end{array}$ & 6 & 7 & 27 & 3.9 & Geography & 5 & 5 & 321 & 64.2 \\
\hline Environmental Science & 6 & 8 & 32 & 4.0 & Area Studies & 2 & 2 & 0 & 0.0 \\
\hline $\begin{array}{l}\text { Biochemistry, Genetics, } \\
\text { and Molecular Biology }\end{array}$ & 2 & 3 & 39 & 13.0 & $\begin{array}{l}\text { Business \& } \\
\text { Economics }\end{array}$ & 2 & 2 & 5 & 2.5 \\
\hline $\begin{array}{c}\text { Economics, } \\
\text { Econometrics, and } \\
\text { Finance }\end{array}$ & 2 & 2 & 9 & 4.5 & $\begin{array}{l}\text { Science \& } \\
\text { Technology }\end{array}$ & 2 & 4 & 4 & 1.0 \\
\hline $\begin{array}{l}\text { Agricultural and } \\
\text { Biological Sciences }\end{array}$ & 1 & 1 & 9 & 9.0 & Agriculture & 1 & 1 & 1 & 1.0 \\
\hline Computer Science & 1 & 1 & 4 & 4.0 & Archaeology & 1 & 1 & 7 & 7.0 \\
\hline
\end{tabular}

${ }^{*} \mathrm{R}=$ ranking; $\mathrm{J}=$ journals; $\mathrm{f}$ = frequency (number of articles published); $\mathrm{TC}=$ total number of citations received for published articles; $\mathrm{C} / \mathrm{f}=$ average of citations received for published articles. Source: own elaboration.

\subsection{Keywords}

In recent years, keywords have been the most used mechanism for the identification of documents by the scientific community. Despite their relevance, today it is possible to observe articles that lack keywords, on the one hand, because the dissemination structure does not include this criterion, which makes it difficult to place them within the metadata of the different bases. In this study, 45 Scopus and 14 WoS documents do not have metadata in relation to the authors' keywords. The rest of the documents show that the term "tourism development" is the central descriptor, with a frequency of 31 in Scopus and 19 in WoS (Table 8). 
Table 8. Classification of articles by keywords.

\begin{tabular}{cccccc}
\hline \multicolumn{1}{c}{ Scopus } & \multicolumn{4}{c}{ WoS } \\
\hline $\mathbf{R}$ & Keywords & $\mathbf{f}$ & $\mathbf{R}$ & Keywords & $\mathbf{f}$ \\
\hline 1 & Tourism Development & 31 & 1 & Tourism Development & 19 \\
\hline 2 & Heritage Tourism & 22 & 2 & Cultural Heritage & 10 \\
\hline 3 & Sustainable Development & 15 & 3 & Sustainable Development & 10 \\
\hline 4 & Cultural Heritage & 13 & 4 & Heritage Tourism & 9 \\
\hline 5 & World Heritage Site & 12 & 5 & Perception & 8 \\
\hline 6 & Ecotourism & 10 & 6 & World Heritage Site & 8 \\
\hline
\end{tabular}

\section{Bibliographic Analysis}

The bibliographic analysis is based on the methodology of iterative analysis by Madden and Shipley [13], which proposes a documentary organization based on categories and subcategories, which are then reflected in an easy-to-understand relational concept map. The categories of analysis are established in: (a) intention, type of process applied; (b) study area, geographical perspective of study; and (c) analysis element, central theme of study. Within these three categories, a series of subcategories are generated that allow them to deepen their analysis. In relation to the intention, they are determined as subcategories: analytical, theoretical, and application; the study area is divided into urban, rural, or both (urban-rural); and the analysis element is classified into heritage, tourism, society, and economic development.

\subsection{Intention}

The distribution in this category was 64 analytical works, 26 case studies, and 13 theoretical studies. The analytical works show a greater concentration in rural areas, with 30 documents [50-52]. This data is of interest because these areas tend to be of less interest for the development of research due to the absence of regulation or planning. On the other hand, 13 documents refer to urban areas, highlighting the works of Kodir [53], which show an analysis of economic development from tourism experienced by the city of Batu, Indonesia; as well as the work of Kranjčević et al. [54], whose aim is to determine the potential for the development of cultural heritage, and in particular, the urban and architectural heritage of Lički (Croatia). Finally, 21 documents with considerably large areas of study are identified, covering both urban and rural areas. This is the case of the work by Ledo et al. [55], whose aim is to contribute to territorial development from both rural (the path) and urban development (the city of Santiago de Compostela, Spain) of the Camino de Santiago. In addition, the work by Lemmi and Tangheroni [56] is included in this category. These authors propose to take advantage of cultural heritage in general, and religion in particular, in the different areas that make up the region of Tuscany in order to start new tourism market segments.

The case studies (26) are very diverse, and most models or applications were developed for the different study areas they address. This is the case of Arthur and Mensah [57], who formulate and implement the plan called "Estrategia Elimina 2015" in Ghana. On the other hand, Armaitiene et al. [58] develop a discriminant analysis model on the conditions of erosion, entry, and accumulation of sediments in order to propose a model of tourism development for the transboundary Curonian Spit World Heritage site, which is based on the results.

Finally, the 13 theoretical studies address the conceptualization of culture and heritage within tourism development [20,59], relationships with society and key actors [60], as well as the conceptualization of the relationships between rural development, cultural heritage, and tourism [61]. 
Due to the geographical scope that can be covered, 5 works can be seen in urban-rural contexts, 6 in rural contexts, and only 1 in an urban context.

\subsection{Element of Analysis}

This category includes 43 documents on tourism, 27 on cultural heritage, 20 on society, and 13 on economic development. Tourism includes works whose general objective is to address tourism as a tool for local development [62-65], the development of products or tourist uses of heritage [66-70], and the formulation of strategic planning or development plans [71-73], in addition to sustainable strategies for the enhancement of heritage within tourism [74,75].

Within the cultural heritage category, there are studies that propose strengthening the identity of the areas through the use and enhancement of material or intangible heritage for tourism activities $[76,77]$. Other studies analyze the impact of heritage on local development [78-80] or the conservation and recognition of the heritage value [81-84]. Finally, studies on creating policies or cultural heritage planning are also collected $[85,86]$.

In the society category, we find works that address the perception of the resident population in relation to the use of heritage [87-91], the relationships or levels of community participation in exploitation processes [92-94], and the benefits perceived by society from the use that heritage gets $[95,96]$.

Economic development is the last subcategory. Included here are studies that highlight the economic and territorial achievements reached [97-100], as well as studies that propose or analyze economic development plans based on heritage [101,102]. Studies that are related to business networks based on heritage that generates economic development are also collected [103].

\subsection{Study Area}

This category includes 51 papers that address rural areas for the application of research [104-109]. A total of 23 studies consider heritage located in urban centers [110-114]. Finally, 29 documents cover entire countries, as well as national and international regions composed of several countries [115-119] (Figure 5).

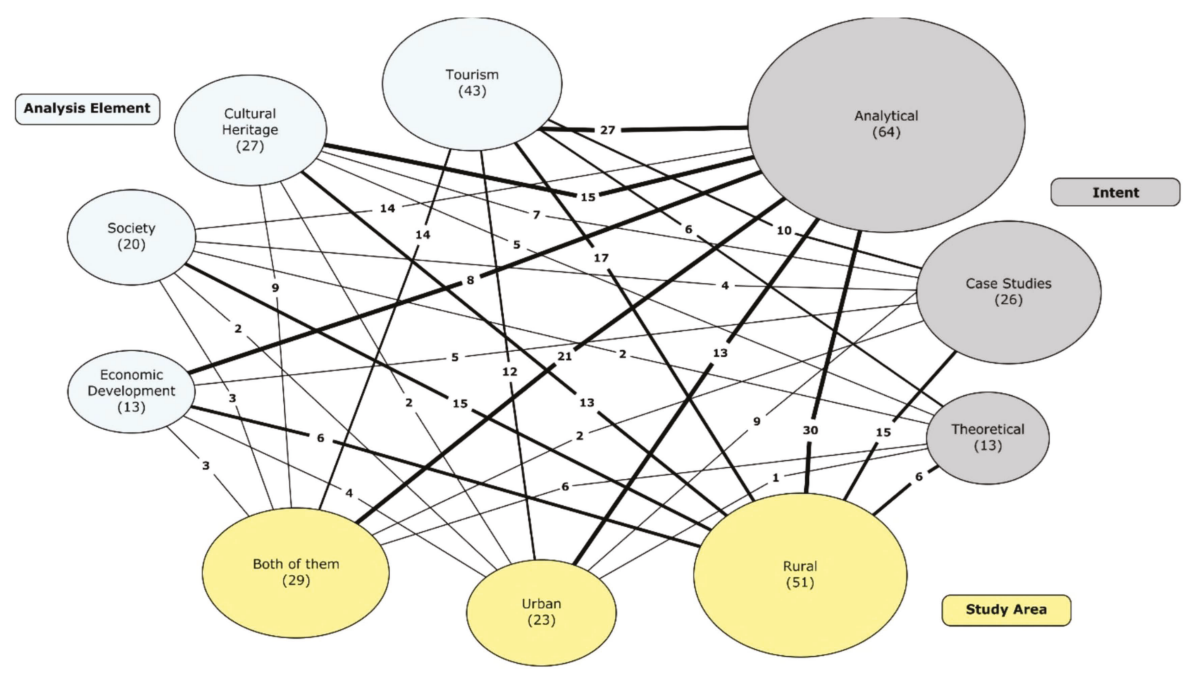

Figure 5. Concept map of themes. Source: own elaboration. * The circles represent the subcategories of analysis. The size corresponds to the proportion of items they contain. The lines detail the quantity relationships that connect each subcategory. 


\section{Conclusions}

These types of studies have, in recent years, constituted a highly valuable consultation tool for new researchers, as this analysis shows a detailed overview of the evolution and current state of the subject under study. In this way, there is information available on the following: the most productive authors based on citations and documents produced, the main journals whose interest is in the publication of topics related to the subject, and countries and institutions focused on the development of related research, among others.

The bibliometric analysis shows that the study of cultural heritage and regional development in tourism began in 1994. Currently, 25 years of scientific literature are consolidated in 103 articles, of which 50 in Scopus and 19 in WoS are unique to each database, recording an overlap of 32 articles between the databases.

The highest production year is 2017, during which no collaboration networks were developed due to the fact that of the 21 published documents, 6 of them were developed individually; while the year with the highest number of citations is 1998, with 378 citations. The line of evolution of the subject determines that it is in an exponential growth stage, and due to its distribution, it is far from reaching a linear adjustment growth, which shows that the subject is still very new, mainly due to the presence of a greater number of qualitative exploratory studies.

In relation to the production by authors, there is a predominance of transient researchers, and an absence of large producers is clear, which confirms that the subject is new. On the other hand, depending on the growth presented by the subject, the emergence of a small group of aspiring authors is shown, each presenting between 2 and 4 studies, of which $63 \%$ have Malaysia as their geographical affiliation. This group represents $3 \%$ of the total of authors and is responsible for $17 \%$ of the articles. The co-authorship index in this subject is 2.34 authors/article.

As for affiliations, a wide variety of latitudes can be seen regarding both geographical and institutional affiliation. In the latter case, universities are the type of center with the highest number of researchers (70.2\% of the total), with the Universiti Sains Malaysia (Malaysia) leading the ranking. In relation to geographical affiliation, China is the leading country, comprising 26 authors, 28 authorships, and 15 centers; its collaboration networks are nationwide.

The Bradford core is 64 and is made up of $62 \%$ of the articles in $51 \%$ of the journals, which determines the absence of a concentration core. The journal with the highest concentration of articles is the Journal of Heritage Tourism, with $4.85 \%$ of articles. The predominant classification area in the journals identified is social science, in both databases. It was also observed that $31 \%$ of the total of Scopus resources are indexed in the third quartile of their respective areas, while $53 \%$ of WoS resources lack the quartile calculation due to having recently been re-entered or removed from their indexation.

On the other hand, the correct use of keywords in studies can become a complex task to achieve, and in many cases is poorly recognized. It can be seen that the authors tend to use simple terms that allow them to achieve the greatest coincidence of their studies in the general searches that are developed in the scientific community. Unfortunately, this does not ensure that the terms fully express the objective achieved by the document, which is why should be highlighted that in order to obtain quality results in advanced searches, it is necessary to develop a greater effort so that these terms become correct descriptors of the investigation.

Regarding the limitations presented by the research, several points can be mentioned: (1) other databases are not taken into account, although their inclusion presents challenges to overcome, these could offer a more complete view of the subject (EBSCOhost, REBID, GoogleSchoolar, ProQuest, etc.); (2) the problem of homogenizing the names of the authors (different authors with the same name); (3) the bias of using a certain search equation. Finally, this study has contributed to describing the current situation of this subject in a descriptive and quantitative way, ruling out the intention of assessing the quality of the articles. 
Author Contributions: All authors contributed equally to this work. All authors wrote, reviewed, and commented on the manuscript. All authors have read and approved the final manuscript.

Funding: The dissemination of this work was possible thanks to the funding granted by the European Regional Development Fund (ERDF) and by the Junta de Extremadura to the DESOSTE research group through the aid with reference GR18052.

Conflicts of Interest: The authors declare no conflict of interest.

\section{References}

1. The Charter of Krakow. Principles for Conservation and Restoration of Built Heritage. 2000. Available online: http://smartheritage.com/wp-content/uploads/2015/03/KRAKOV-CHARTER-2000.pdf (accessed on 9 September 2019).

2. Smith, L. Uses of Heritage; Routledge: Abingdon, UK, 2006.

3. UNESCO. Convention Concerning the Protection of the World Cultural and Natural Heritage. Adopted by the General Conference at its Seventeenth Session Paris, 16 November 1972. Available online: http: //whc.unesco.org/archive/convention-en.pdf (accessed on 9 September 2019).

4. UNESCO. Mexico City Declaration on Cultural Policies World Conference on Cultural Policies Mexico City, 26 July-6 August 1982. Available online: https://culturalrights.net/descargas/drets_culturals401.pdf (accessed on 9 September 2019).

5. UNESCO. The Convention for the Safeguarding of the Intangible Cultural Heritage. 2003. Available online: https://ich.unesco.org/doc/src/01852-EN.pdf (accessed on 9 September 2019).

6. Timothy, D.J.; Boyd, S.W. Heritage Tourism; Prentice Hall: Harlow, UK, 2003.

7. Timothy, D.F. Cultural Heritage and Tourism: An Introduction; Chanel View Publications: Bristol, UK, 2011.

8. Chhabra, D.; Healy, R.; Sills, E. Staged authenticity and heritage tourism. Ann. Tour. Res. 2003, 30, 702-719. [CrossRef]

9. Pearce, P.L.; Lee, U.I. Developing the travel career approach to tourist motivation. J. Travel Res. 2005, 43, 226-237. [CrossRef]

10. Tan, S.K.; Luh, D.B.; Kung, S.F. A taxonomy of creative tourists in creative tourism. Tour. Manag. 2014, 42, 248-259. [CrossRef]

11. Pereira, G.A.; de Sevilha Gosling, M. Los viajeros y sus motivaciones. Un estudio exploratorio sobre quienes aman viajar. Estudios y Perspectivas en Turismo 2017, 26, 62-85.

12. World Tourism Organization. Tourism and Culture Synergies; World Tourism Organization (UNWTO): Madrid, Spain, 2018.

13. Madden, M.; Shipley, R. An analysis of the literature at the nexus of heritage, tourism, and local economic development. J. Herit. Tour. 2012, 7, 103-112. [CrossRef]

14. Clements, C.J.; Schultz, J.H.; Lime, D.W. Recreation, tourism, and the local residents: Partnership or co-existence? J. Park Recreat. Adm. 1993, 11, 78-91.

15. Weikert, B.; Kertstetter, D. Resident's Attitudes toward Tourism: An Applied Study in a Historic Community. In Proceedings of the 1995 Northeastern Recreation Research Symposium, Radnor, PA, USA, 9-11 April 1995; USFS: Washington, DC, USA, 1996; pp. 218-227.

16. Tuan, T.H.; Navrud, S. Capturing the benefits of preserving cultural heritage. J. Cult. Herit. 2008, 9, 326-337. [CrossRef]

17. Cano, M.; Garzón, E.; Sánchez-Soto, P.J. Preservation and conservation of rural buildings as a subject of cultural tourism: A review concerning the application of new technologies and methodologies. J. Tour. Hosp. 2013, 2, 1-23.

18. Elkington, J. Cannibals with Forks: Triple Bottom Line of 21st Century Business; New Society Publishers: Gabriola Island, BC, Canada, 1998.

19. Choi, H.S.C.; Sirakaya, E. Measuring Residents' Attitude toward Sustainable Tourism: Development of Sustainable Tourism Attitude Scale. J. Travel Res. 2005, 43, 380-394. [CrossRef]

20. Bessiere, J. Local development and heritage: Traditional food and cuisine as tourist attractions in rural areas. Sociol. Rural. 1998, 38, 21-34. [CrossRef]

21. Wachter, S. Etat, Décentralisation et Territoires; L'Harmattan: Paris, France, 1987. 
22. Andrés, A. Measuring Academic Research: How to Undertake a Bibliometric Study; Elsevier: Amsterdam, The Netherlands, 2009.

23. Spinak, E. Diccionario Enciclopédico de Bibliometría, Cienciometría e Informática; Unesco: Paris, France, 1996.

24. Hubert, J.J. General Bibliometric Models. Libr. Trends 1981, 30, 65-81.

25. Escorcia-Otálora, T.A.; Poutou-Piñales, R.A. Análisis bibliométrico de los artículos originales publicados en la revista Universitas Scientiarum (1987-2007). Univ. Sci. 2008, 13, 236-244.

26. Rueda, G.; Gerdsri, P.; Kocaoglu, D. Bibliometrics and Social Network Analysis of the Nanotechnology Field. Paper Presented at the Portland International Conference on Management of Engineering \& Technology (PICMET), Portland, OR, USA, 6-9 August 2007.

27. Harzing, A.W.; Alakangas, S. Google Scholar, Scopus and the Web of Science: A longitudinal and cross-disciplinary comparison. Scientometrics 2016, 106, 787-804. [CrossRef]

28. Rojas-Sola, J.I.; Navarrete-Cortes, J.; Fernandez-Lopez, J.A.; Chaichio-Moreno, J.A. Scientific production in the graphic expression in engineering area at the Spanish universities: An approach to ISI database. Revista Española de Documentación Científica 2008, 31, 190-204. [CrossRef]

29. Goldschmidt, P.G. Information synthesis: A practical guide. Health Serv. Res. 1986, 21, 215.

30. Gavel, Y.; Iselid, L. Web of Science and Scopus: A journal title overlap study. Online Inf. Rev. 2008, 32, 8-21. [CrossRef]

31. Pulgarín, A.; Escalona, M. Medidas del solapamiento en tres bases de datos con información sobre ingeniería. Anales de Documentación 2008, 10, 335-344.

32. Costas, R.; Moreno, L.; Bordons, M. Solapamiento y singularidad de MEDLINE, WoS e IME para el análisis de la actividad científica de una región en Ciencias de la Salud. Revista Española de Documentación Científica 2008, 31, 327-343.

33. Cañedo, R. Estudios de solapamiento en la selección de las publicaciones seriadas y las bases de datos. ACIMED 1999, 7, 164-170.

34. Meyer, D.E.; Mehlman, D.W.; Reeves, E.S.; Origoni, R.B.; Evans, D.; Sellers, D.W. Comparison study of overlap among 21 scientific databases in searching pesticide information. Online Rev. 1983, 7, 33-43. [CrossRef]

35. Gluck, M. A review of journal coverage overlap with an extension to the definition of overlap. J. Am. Soc. Inf. Sci. 1990, 41, 43-60. [CrossRef]

36. Bearman, T.; Kunberger, W. A Study of Coverage Overlap among Fourteen Major Science and Technology Abstracting and Indexing Services; National Federation of Abstracting and Indexing Services: Philadelphia, PA, USA, 1977.

37. Light, D.; Prentice, R. Market-based product development in heritage tourism. Tour. Manag. 1994, 15, 27-36. [CrossRef]

38. Price, D.J. The exponential curve of science. Discovery 1956, 17, 240-243.

39. Merigó, J.M.; Mas-Tur, A.; Roig-Tierno, N.; Ribeiro-Soriano, D. A bibliometric overview of the Journal of Business Research between 1973 and 2014. J. Bus. Res. 2015, 68, 2645-2653. [CrossRef]

40. Li, M.; Wu, B.; Cai, L. Tourism development of World Heritage Sites in China: A geographic perspective. Tour. Manag. 2008, 29, 308-319. [CrossRef]

41. Russo, A.P. The "vicious circle" of tourism development in heritage cities. Ann. Tour. Res. 2002, 29, 165-182. [CrossRef]

42. Jonsen-Verbeke, M. Industrial heritage: A nexus for sustainable tourism development. Tour. Geogr. 1999, 1, 70-85. [CrossRef]

43. Chen, C.F.; Chen, P.C. Resident attitudes toward heritage tourism development. Tour. Geogr. 2010, 12, 525-545. [CrossRef]

44. Bessiere, J. 'Heritagisation', a challenge for tourism promotion and regional development: An example of food heritage. J. Herit. Tour. 2013, 8, 275-291. [CrossRef]

45. Crane, D. Social Structure in a Group of Scientists: A Test of the "Invisible College" Hypothesis. American Sociological Rev. 1969, 34, 335-352. [CrossRef]

46. Berelson, B. Content Analysis in Communication Research; Free Press: New York, NY, USA, 1952.

47. López López, P. Introducción a la Bibliometría; Promolibro: Valencia, Spain, 1996.

48. Hirsch, J.E. An index to quantify an individual's scientific research output. Proc. Natl. Acad. Sci. USA 2005, 102, 16569-16572. [CrossRef] [PubMed]

49. Bradford, S.C. Sources of information on specific subjects. Engineering 1934, 137, 85-86. 
50. Zúñiga, B. The territorial changes of Totonacapan Veracruzano, Mexico and their heritage cultural based on the touristic activity as a regional development strategy. Cuadernos de Turismo 2014, 34, 351-442.

51. Ćopić, S.; Đorđević, J.; Lukić, T.; Stojanović, V.; Đukičin, S.; Besermenji, S.; Tumarić, A. Transformation of industrial heritage: An example of tourism industry development in the Ruhr area (Germany). Geogr. Pannonica 2014, 18, 43-50. [CrossRef]

52. Kisiel, R.; Zielińska-Szczepkowska, J.; Tradejna, D. Natural and Cultural Resources of Green Kurpie as Drivers of Tourism Development. Ekonomia i Środowisko 2018, 2, 231-245.

53. Kodir, A. Tourism and development: Land acquisition, achievement of investment and cultural change (case study tourism industry development in Batu City, Indonesia). Geoj. Tour. Geosites 2018, 21, $253-265$. [CrossRef]

54. Kranjčević, J.; Marković, I.; Božić, N. Lički Osik-Urban and Architectural Heritage as Tourism Development Potential. Sociologija i Prostor Časopis za Istraživanje Prostornoga i Sociokulturnog Razvoja 2016, 54, 103-126. [CrossRef]

55. Ledo, A.P.; Bonín, A.R.; Iglesias, A.M. The cultural tourism as strategic factor of development: The Santiago route. Estudios Geográficos 2007, 68, 205-234.

56. Lemmi, E.; Tangheroni, M.S. Cultural and religious heritage and place names in Tuscan areas crossed by Via Francigena for a sustainable tourist development. Riv. Geogr. Ital. 2013, 120, 155-169.

57. Arthur, S.N.; Mensah, J.V. Urban management and heritage tourism for sustainable development: The case of Elmina cultural heritage and management programme in Ghana. Manag. Environ. Qual. Int. J. 2006, 17, 299-312. [CrossRef]

58. Armaitiene, A.; Boldyrev, V.L.; Povilanskas, R.; Taminskas, J. Integrated shoreline management and tourism development on the cross-border World Heritage Site: A case study from the Curonian spit (Lithuania/Russia). J. Coast. Conserv. 2007, 11, 13-22. [CrossRef]

59. Alinejad, M.E.; Razaghi, Z. Culture and its role in tourism development. Life Sci. J. 2012, 9, 1593-1597.

60. Rasoolimanesh, S.M.; Jaafar, M. Community Participation toward Tourism Development and Conservation Program in Rural World Heritage Sites. In Tourism-From Empirical Research Towards Practical Application; IntechOpen: London, UK, 2016.

61. Condesso, F. Rural development, cultural heritage and tourism. Cuadernos de Desarrollo Rural 2011, 8, 197-222.

62. Chakravarty, S.; Irazábal, C. Golden geese or white elephants? The paradoxes of world heritage sites and community-based tourism development in Agra, India. Community Dev. 2011, 42, 359-376. [CrossRef]

63. Damir, D. The importance of the Danube strategy for tourism and culture development of the Croatian Danube region. Geogr. Pannonica 2012, 16, 112-125.

64. Fonseca, F.P.; Ramos, R.A.R. Heritage Tourism in Peripheral Areas: Development Strategies and Constraints. Tour. Geogr. 2012, 14, 467-493. [CrossRef]

65. Fournier, L.S. Local fêtes as cultural heritage in provence: New means for local development policies and tourism. Nottm. Fr. Stud. 2011, 50, 31-43. [CrossRef]

66. Gabrielli, C. "Fundacao Casa Grande" and the touristic development of Nova Olinda/CE: New chances for the dialogue between the local cultura and tourism. Turismo Estudos e Praticas 2015, 4, 74-95.

67. Londono, M.L.; Medina, F.X. Effects of Cultural and Tourism Policies on Local Development: The Case of Food Trails in Medellin, Colombia. Almatour. J. Tour. Cult. Territ. Dev. 2017, 8, 89-106. [CrossRef]

68. Nzeda Tagowa, W. Rural tourism as a factor of sustainable development: A case study of Sukur World Heritage Site in Adamawa State, Northeastern Nigeria. WIT Trans. Ecol. Environ. 2010, 142, 675-688. [CrossRef]

69. Saiken, A.; Zhaoping, Y.; Mazbayev, O.; Duissembayev, A.; Izenbaev, B.; Nassanbekova, S. Ethnic cultural tourism resources evaluation and development: Kazakh cultural tourism resources analysis. J. Environ. Manag. Tour. 2017, 8, 467-475. [CrossRef]

70. Vistad, O.I.; Wold, L.C.; Daugstad, K.; Haukeland, J.V. Mimisbrunnr Climate Park-A network for heritage learning, tourism development, and climate consciousness. J. Herit. Tour. 2016, 11, 43-57. [CrossRef]

71. Beloborodova, D.G.; Unagaeva, N.A.; Kukina, I.V. Spatial and Architectural Heritage of Yeniseysk Town Fringe Belts in the context of the development of cultural tourism. Vestn. Tomsk State Univ. J. Cult. Stud. Art Hist. 2017, 27, 220-228. [CrossRef] 
72. Iliopoulou-Georgudaki, J.; Theodoropoulos, C.; Konstantinopoulos, P.; Georgoudaki, E. Sustainable tourism development including the enhancement of cultural heritage in the city of Nafpaktos-Western Greece. Int. J. Sustain. Dev. World Ecol. 2017, 24, 224-235. [CrossRef]

73. Sun, Y.H.; Min, Q.W.; Zhong, L.S.; Cheng, S.K.; Zhang, D.; Long, D.Y. Agricultural heritage tourism development in minority areas: Taking congiian county in guizhou province as a case. China Popul. Resour. Environ. 2009, 19, 120-124.

74. Huibin, X.; Marzuki, A.; Razak, A.A. Conceptualizing a sustainable development model for cultural heritage tourism in Asia. Theor. Empir. Res. Urban. Manag. 2013, 8, 51-66.

75. Morales-Yago, F.J. Landscape and Heritage: Key Elements for Tourism Development in an Interior Space: The Case of Yecla (Murcia). Revista de Estudios Andaluces 2017, 34, 399-428. [CrossRef]

76. Kravanja, B. Selling and sharing culture: On relations between cultural heritage, nature conservation and tourism development institutions in the Upper Soča Valley, Slovenia. Narodna Umjetnost 2014, 51, 89-112. [CrossRef]

77. Valdez, L.M.C.; Fontecha, J.F. Gastronomy: A source for the developmente of tourism and the strengthening of the cultural identity in Santander. Anuario Turismo y Sociedad 2018, 22, 167-193. [CrossRef]

78. Duval, M.; Smith, B.W. UNESCO world Heritage list inscription and tourist development: The Ukhahlamba-Drakensberg Park world Heritage site (South Africa). Ann. De Geogr. 2014, 697, 912-934. [CrossRef]

79. Nieves, A.E.; Vargas, M.B.; Quesada, E.B. The potential of cultural assets associated to tourism activity as a local factor development at the Getsemani neighborhood, Cartagena de Indias. Anuario Turismo y Sociedad 2017, 21, 107-143. [CrossRef]

80. Rogerson, C.M.; van der Merwe, C.D. Heritage tourism in the global South: Development impacts of the Cradle of Humankind World Heritage Site, South Africa. Local Econ. 2016, 31, 234-248. [CrossRef]

81. Awuah-Nyamekye, S.; Sarfo-Mensah, P.; Amisah, S.; Owusu-Bi, A. Environmental conservation and preservation of cultural heritage: Assets for tourism development in the Akyem Abuakwa traditional area of Ghana. Worldviews Environ. Cult. Relig. 2014, 18, 30-53. [CrossRef]

82. Franch, M.; Irimias, A.; Buffa, F. Place identity and war heritage: Managerial challenges in tourism development in Trentino and Alto Adige/Südtirol. Place Branding Public Dipl. 2017, 13, 119-135. [CrossRef]

83. Herrera, O.J.M. Cultural heritage and tourism, a development alternative: Case Viota, Cundinamarca. Anuario Turismo y Sociedad 2016, 18, 99-116. [CrossRef]

84. Łach, J. Using the geographical space of the Little Beskid Mts. for the development of cultural tourism with the aim of protection of the landscape heritage. Annales Universitatis Mariae Curie-Sklodowska 2017, 72 , 103-119.

85. Fredholm, S. Assets in the age of tourism: The development of heritage planning in Ghanaian policy. J. Contemp. Afr. Stud. 2016, 34, 498-518. [CrossRef]

86. Popa, D.; Popa, A. Development of the cultural heritage tourism by rehabilitating the Sancraia Castle, Alba County. J. Environ. Prot. Ecol. 2016, 17, 1443-1451.

87. Deng, J.Y.; McGill, D.; Arbogast, D.; Maumbe, K. Stakeholders' perceptions of tourism development in Apalachian Forest Heritage Area. Tour. Rev. Int. 2016, 20, 235-253. [CrossRef]

88. Di Lernia, S. Incoming tourism, outgoing culture: Tourism, development and cultural heritage in the Libyan Sahara. J. North. Afr. Stud. 2005, 10,441-457. [CrossRef]

89. Gunjić, L. Local perceptions of cultural heritage and tourism development-Case study Bač, Serbia. Ge-Conserv. 2017, 1, 57-62.

90. Kulcsar, L.; Bodrogai, L.A.; Vizi, I.G. Tourism development and cultural heritage: The stakeholders' opinion on the rol of the restored Esterhazy Palace in western Hugary. Econ. Thought Pract. 2017, 26, 813-827.

91. Quyen, L.; Khanjanusthiti, P. The cultural impact of tourism development in a dong hoa hiep local community, Cai Be District, Vietnam. Asian Soc. Sci. 2015, 11, 203-214. [CrossRef]

92. Draper, J.; Oh, C.O.; Harrill, R. Preferences for heritage tourism development using a choice modeling approach. Tour. Anal. 2012, 17,747-759. [CrossRef]

93. Francis-Lindsay, J. From fashion to 'tangible-intangible' action: Local communities 'culturizing' new tourism development. WIT Trans. Ecol. Environ. 2010, 139, 489-500. [CrossRef]

94. Frolova, E.V.; Rogach, O.V.; Medvedeva, N.V.; Kabanova, E.E.; Ryabova, T.M. Volunteer activity as a factor in the development of cultural tourism in the Russian Federation. Acad. Strateg. Manag. J. 2017, 16, 1-12. 
95. Chand, M. Residents' perceived benefits of heritage and support for tourism development in Pragpur, India. Tourism 2013, 61, 379-394.

96. Su, M.; Sun, Y.; Min, Q.; Jiao, W. A Community livelihood approach to agricultural heritage system conservation and tourism development: Xuanhua Grape Garden Urban Agricultural Heritage Site, Hebei Province of China. Sustainability 2018, 10,361. [CrossRef]

97. Biville, Q. Enhancing the heritage value of small cities in the western region of sichuan province: The dynamics of development in dujiangyan and qingchengshan. Espace Geogr. 2017, 46, 364-379. [CrossRef]

98. Boujrouf, S. Heritage resources and the development of tourist areas in the High Atlas and southern regions of Morocco. Revue de Geographie Alpine 2014, 2, 102-112. [CrossRef]

99. Camelia, T.; Laurenţiu Ştefan, S. The ethno-creativity in the pilot centers in Romania and their role in the development of cultural tourism and the educational process. Forum Geogr. 2017, 16, 88-97. [CrossRef]

100. Lloyd, K.; Morgan, C. Murky Waters: Tourism, Heritage and the Development of the Ecomuseum in Ha Long Bay, Vietnam. J. Herit. Tour. 2008, 3, 1-17. [CrossRef]

101. De Montis, A.; De Montis, V. Planners in the face of mining cultural heritage: Tourism development at L'Argentiera, Italy. Int. J. Serv. Technol. Manag. 2008, 10, 128-146. [CrossRef]

102. Delaplace, M.; Gatelier, E. Individual and collective heritage strategies and development of the wine tourism in Burgundy. Territoire en Mouvement 2014, 21, 40-53. [CrossRef]

103. Ferguene, A.; Idir, S. Heritage, tourism and sustainable territorial development in the Algerian Sahara: The case of Tassili N'Ajjer. Geo Regards 2012, 5, 95-109.

104. Kocaman, M.; Kocaman, E.M. The importance of cultural and gastronomic tourism in local economic development: Zile sample. Int. J. Econ. Financ. Issues 2014, 4, 735-744.

105. Lenao, M.; Saarinen, J. Integrated rural tourism as a tool for community tourism development: Exploring culture and heritage projects in the North-East District of Botswana. South. Afr. Geogr. J. 2015, 97, 203-216. [CrossRef]

106. Morosi, J.; Amarilla, B.; Conti, A.; Contin, M. Estancias of buenos aires province, Argentina: Rural heritage, sustainable development and tourism. Int. J. Herit. Stud. 2008, 14, 589-594. [CrossRef]

107. Olya, H.G.; Alipour, H.; Gavilyan, Y. Different voices from community groups to support sustainable tourism development at Iranian World Heritage Sites: Evidence from Bisotun. J. Sustain. Tour. 2018, 26, 1728-1748. [CrossRef]

108. Salinas Chavez, E.; Delgado Mesa, F.A.; Henthorne, T.L.; Miller, M.M. The Hershey sugar mill in Cuba: From global industrial heritage to local sustainable tourism development. J. Herit. Tour. 2018, 13, 426-439. [CrossRef]

109. Xiao, H.; Li, L. Villagers' Perceptions of Traditions: Some Observations on the Development of Rural Cultural Tourism in China. Tour. Recreat. Res. 2004, 29, 69-80. [CrossRef]

110. Brebbia, C.A.; Doganer, S.; Dupont, W.; Doganer, S.; Dupont, W. Accelerating cultural heritage tourism in San Antonio: A community-based tourism development proposal for the missions historic district. Int. J. Sustain. Dev. Plan. 2015, 10,1-19. [CrossRef]

111. Rivera, M.; Hernández, R. MSMEs craft, tourism and local development strategies: Challenges and opportunities in a historical-heritage city (Cordoba, Spain). Estudios Geográficos 2018, 79, 529-553. [CrossRef]

112. Tur, J.N.; Martínez, A.F.; Spiegelhalder, M.R. From complement to motor: The changing role of leisure and tourism in local development strategies. The case of the recovery and valorisation of cultural heritage. Arbor 2012, 188, 379-393. [CrossRef]

113. Wang, Y.P. A study on Kinmen resident's perception of tourism development and culture heritage impact. Eurasia J. Math. Sci. Technol. Educ. 2016, 12, 2909-2920. [CrossRef]

114. Xu, H. Managing side effects of cultural tourism development-The case of Zhouzhuang. Syst. Anal. Model. Simul. 2003, 43, 175-188. [CrossRef]

115. Alonso, A.D.; O’Neill, M.A. Muscadine Grapes, Food Heritage and Consumer Images: Implications for the Development of a Tourism Product in Southern USA. Tour. Plan. Dev. 2012, 9, 213-229. [CrossRef]

116. Huibin, X.; Marzuki, A.; Razak, A.A. Protective development of cultural heritage tourism: The case of Lijiang, China. Theor. Empir. Res. Urban. Manag. 2012, 7, 39-54.

117. Montanari, A. Geography of taste and local development in abruzzo (Italy): Project to establish a training and research centre for the promotion of enogastronomic culture and tourism. J. Herit. Tour. 2009, 4, 91-103. [CrossRef] 
118. Seidl, A. Cultural ecosystem services and economic development: World Heritage and early efforts at tourism in Albania. Ecosyst. Serv. 2014, 10, 164-171. [CrossRef]

119. Zaiane, S. Heritage tourism in Tunisia: Development one-way choice. Tour. Rev. 2006, 61, 26-31. [CrossRef]

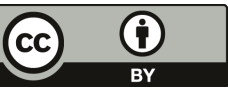

(C) 2019 by the authors. Licensee MDPI, Basel, Switzerland. This article is an open access article distributed under the terms and conditions of the Creative Commons Attribution (CC BY) license (http://creativecommons.org/licenses/by/4.0/). 



\title{
Promoting Research and Landscape Experience in the Management of the Archaeological Networks. A Project-Valuation Experiment in Italy
}

\author{
Salvatore Giuffrida ${ }^{1, *}$, Filippo Gagliano ${ }^{2}$, Enrico Giannitrapani ${ }^{3}$, Carmelo Marisca ${ }^{4}$, \\ Grazia Napoli ${ }^{5}$ and Maria Rosa Trovato ${ }^{1}$ \\ 1 Department of Civil Engineering and Architecture, University of Catania, 95125 Catania, Italy; \\ mrtrovato@dica.unict.it \\ 2 ITS G. Quarenghi, 24125 Bergamo, Italy; fmgagliano@gmail.com \\ 3 Arkeos-Integrated Services for Cultural Heritage, 94100 Enna, Italy; e_giannitrapani@alice.it \\ 4 Department of Economics, University of Messina, 98122 Messina, Italy; carmelo.marisca@unime.it \\ 5 Department of Architecture, University of Palermo, 90128 Palermo, Italy; grazia.napoli@unipa.it \\ * Correspondence: salvatore.giuffrida@unict.it; Tel.: +39-335-799-5263
}

Received: 15 April 2020; Accepted: 10 May 2020; Published: 14 May 2020

\begin{abstract}
Archaeological sites are part of the history and identity of a community playing a strategic role on the different scales of the cultural and economic common life. Whereas on the one end the most famous archaeological sites attract huge flows of tourists and investment, on the other hand, many minor archaeological sites remain almost ignored and neglected. This study proposes a project-evaluation approach devoted to the "minor" archaeological site development, outlining a territorial, socio-economic, and landscape communication pattern aimed at creating an archaeological network integrating other cultural and natural resources. As such, these networks get able to match the demand of customers who shy away from iper-consumerist tourism and want to deepen their knowledge of a place. The proposed approach integrates knowledge, evaluation, and design in a multiscale pattern whose scope is to foster and extend the archaeological research program, involving public and private stake/stockholders to widen the cultural-contemplative experience and promote further educational events concerning the themes of the local identity. With reference to the archaeological basin of Tornambè, Italy, a Web-GIS knowledge system has been drawn to provide the territorial information requested by the economic-evaluation multiscale pattern implemented to verify the cost-effectiveness of the project. The expected negative results of the economic valuation supported the allocation pattern of the considerable investment costs, as well as the hypothetic scenarios about the evolution of the cultural-contemplative experience due to the extension of the archaeological estate. Some disciplinary remarks propose a heterodox approach for a further interpretation of the economic results and financial indexes, by introducing the monetary dimension of such a social capital asset.
\end{abstract}

Keywords: archaeological basins; Web-GIS and Geodatabases; territorial marketing; cultural economics; land economy; tourism experience management; cultural estate; landscape heritage

\section{Introduction}

The management of the cultural real estate heritage is de facto a central issue of territorial policies as this heritage plays an important role in creating the community identity, supporting local economic development [1], driving sustainable innovation of local businesses, and redefining the very concept of social well-being. The preservation and enhancement of cultural heritage, as a 'common good passed from previous generations as a legacy for those to come' [2,3], is promoted on global, European, 
and national levels through specific programs, laws, and actions. The European Framework for Action on Cultural Heritage promotes an integrated and participatory approach to cultural heritage and-supported by the European Parliament—contributes to the mainstreaming of cultural heritage across European Union policies [4]. In Italy, where a great cultural heritage and an extraordinary archaeological heritage is concentrated, the high number of exceptional sites has contributed to spreading a general interest for art and culture, creating the basis for the concept of cultural basins [5] in which minor archaeological sites, that otherwise would remain marginal, form networks together with other resources.

The tourist sites of global or national importance attract huge financial resources and millions of tourists from all over the world every year, but they often generate territorial polarization that accentuates an unfair wealth distribution and affects peripheral areas. These sites face the impacts of overtourism [6-9], such as pollution and congestion, so much some limits on tourists access and stay need to be imposed for the preservation of the site itself, as in the case of the archaeological site of Machu Picchu in Peru, or in high naturalistic interest areas such as the Galapagos Islands in Ecuador. Conversely, the minor archaeological sites risk being abandoned due to the small tourist flows and the consequent public fund reduction.

The stratification and combination of multiple values and functions [10-12] in a territorial network connecting archaeological as well as ethno-anthropological and architectural features is coherent with the World Tourism Organization suggestions [13] on an alternative to predatory mass tourism, responding to the demand for slow tourism valuing the profound knowledge of a place, such as local traditions and products. Accordingly, alternative touristic itineraries should be identified as a success factor for reducing the effects of competition form the art cities and the most important tourist sites.

Among alternative models of tourism, such as Sustainable tourism - that can assure long term benefits to the local community and environment, as well as the tourists and tourist operators-, or Ecotourism - focused on travelling to wild and fragile areas and has the ethic goal of educating the traveller to the respect toward different cultures-, Slow tourism allows the traveller to get possession of time again, to be in tune with whatever surrounds him, interact to with local people, history and tradition $[14,15]$. Adopting a model of slow tourism allows putting into network different type of resources connected to a common theme and/or to a same territorial system in order to promote local resources and products which, otherwise, would not be economically feasible, as well as minor touristic sites which would not be able to attract flows of tourists. The implementation of this model requires appropriate marketing strategies, to build the supply and put it in the national and international tourism market in order to meet the demand. The marketing strategy should apply a systematic analysis of the slow tourist profile's and the most favourite activities, and verify strengths and weaknesses of the proposed territorial network in order to select the best actions to improve the supply. An application of this approach was proposed for the valorization of slow tourist itineraries between Italy and Slovenia in a project financed by the European Union and Slovenia within a cross-border cooperation program [16]. Moreover, marketing strategy could include the building of a brand to allow immediate identification of the 'territorial product', as proposed for the archaeological sites of the Pompeian pole $[17,18]$.

So that demand for territorial networks evaluation and management tools $[19,20]$ from the public institutions has grown, as well as, in response, the commitment from the science of appraisal and valuation in supporting political-administrative decision-making processes on the planning, programming and designing scale [21-28].

The strong connection of knowledge, valuation and project allows planners and decision-makers to generate multiple strategies aimed at promoting public consensus thus stimulating the participation of local communities [29], and at the same time to verify the consistency between outputs and outcomes, in terms of economic results [30].

The administration of the archaeological territory—concerning research (surface research, findings analysis and excavation campaigns programming) administration (the archaeological constraint subjection of the areas to be investigated) technical management (asset worksite and remains restoration) 
protection/promotion (on/off-site musealization of the remains)—requires specific professional and managerial know-how, and in Italy it is the exclusive responsibility of Superintendence of Cultural Heritage, applying generalized top-down decision-making processes. Some studies, however, promote the participation of tourists supporting a demand-oriented heritage management process complementary to the traditional supply-oriented one [31-33]. Such an approach focuses on the heterogeneous tourists' expectations outlining customer-oriented ways of fruition aimed at enhancing the customer service supply [34,35]. Other researches remark the need to include in management process other stakeholder groups and especially local communities [36] mostly concerned about the integration of the cultural heritage issue in the local socio-economic development $[37,38]$. Such an extension of the consensus deals with the natural conflict of values, expectations and scopes supported by different types of stakeholders (internal and external, private and public, economic and cultural, professional and common, etc.) to be involved [39] in the social communication process (public hearings, focus groups, citizen review panels, surveys, etc.) for new proposals ideas and perspectives [40,41].

In general, the economic profile of the archaeological assets is characterized by high investment costs and low and deferred streams of benefits [42] increasing over time. With the exception of some important archaeological parks, the negative results of their economic management is covered by public expenditure, whose amount can be considered the monetary measurement of the value a local community attributes [43-45] to the memory of its "ancient greatness", as well as a cultural-historic identity indicator.

The archaeological assets are quite different from primary goods, as so far as:

- $\quad$ stable assets, that are capable of providing cultural services in the long term,

- $\quad$ unique goods, that are fragile, not substitutable and non-reproducible, given the irreversibility of natural processes, or transformative or invasive reuse actions,

- $\quad$ sources of intangible services, thus mainly information-based assets as they can be considered the main and original contents of the longest-run intergenerational communication.

In the ground of the cultural heritage economics and in the prospect of a "circular economy" aimed at the progressive dematerialization of production and consume and mainly intended to create a culture of sustainability, valuation is committed to verifying how, in which extent, by and for whom economic development of land cultural assets characterized by mainly unspoiled potential and contextual territorial value, can be feasible.

In such prospect, this study proposes an integrated approach to knowledge, evaluation and design on two scales: on the territorial scale, a Web-GIS [46-49] Geodatabase-based knowledge approach allowed us to outline the landscape profile of the sites over the Province of Enna. On the local scale, it provided the spatial data describing the main "Value bearers" [50], involved in the project of research extension powering the cultural-contemplative experience supporting the promotion of the local economic activities and players.

Accordingly, the economic-financial analysis [51-54] aimed at outlining the efficiency profile of the development project proposed, although performed from the private player's perspective, tries to stress the favourable conditions of a coordinated land-economy-oriented policy integrating the visions and the missions of multiple, mostly public, players providing the socio-economic and political-decision context favourable to develop the archaeological basins culture and economy.

An archaeological basin is a territorial-landscape unit having a specific identity whose anthropology (including the economic framework) can be still related to the fundamental historic permanency as integrated into the multiple landscape dimensions [55]. So that the different basins constituting the archaeological asset of territory, should be defined as externally (from the landscape point of view) and internally (from an economic point of view) coherent territorial entities.

In such a prospect - that we assume as the political, institutional and economic condition for the sustainable development of low-value density wide territorial areas-the entities responsible for the protection and valorisation of the cultural asset, also according to the Italian Code of Cultural 
Heritage and Landscape currently in charge, demand behavioural patterns integrating multiple scales, subjects, scopes, and skills toward the common overall end of identifying in the permanence of the main outcrops of the ancient local civilization, the programmatic references of the socio-economic development of the settled communities.

Accordingly, the proposed approach outlines a project for the enhancement and musealization of a wide Sicilian inland archaeological basin (whose original core is the identified in the site of Tornambé in the Province of Enna, Italy). The general objective is the definition of the different dimensions of the project, involving the wide, intermediate and detail scales, public and private corporate players, and different kinds of actions, targeted to archaeological research development, to the opportunities of cultural and contemplative experiences, and to the promotion of entrepreneurial initiatives aimed at better finalizing the aforesaid activities. The specific object is the critical application of the cost-effectiveness analysis tools taking into account multiple scenarios validated by the prospect of connecting the multiple purposes of the subject involved, starting from the accounting of the investment and operating costs [56], revenues, and proposing the comparison of two different and somehow complementary interpretations of the specific findings. In fact, the application of the Discount Cash Flow Analysis confirmed that, despite the synergistic participation of public and private capitals and of various stakeholders, the economic feasibility remains a critical issue and the contribution of public expenditure is necessary. Rather, the socio-territorial and landscape context, powered by the public $[57,58]$ intentionality and illuminated by the prevailing of the collective intelligence characterizing the local community identity, are assumed as the decisional long-run prospect supporting a more challenging entrepreneurial action in spite of the modest economic short-run results.

This share of public expenditure, which is a non-productive investment according to the market law [59], can be defined as a sort of equalization monetary transfer from major archaeological sites, that have active balance sheets, to minor archaeological heritage, and would implicitly constitute the minimum social value [60] given to historical and identity heritage of local communities.

The paper presents, in Section 2, the basic descriptive aspects of the basin of Tornambè. In Section 3, the methodological approach, articulated according to three dimensions of communication-territorial, socio-economic and landscape-and focusing on the Discounted Cash Flow Analysis (DCFA) carried out according to the arrangement of public/private commitments. In Section 4, the description of the results in the above-mentioned triple communicative pattern. In Section 5, a critical and proactive discussion of the results of the valuation output based on the comparison between two different (orthodox and heterodox) approaches. In Section 6, the conclusions that frame the different points of view and highlight in the valuation the prospects outlined based on the related results, concerning the commitment of public-private players in the creation of the identity of this landscape unit.

\section{Materials. The Archaeological Basin of Tornambé}

The archaeological basin of Tornambé (municipality of Pietraperzia, Italy) (Figures 1 and 2) was chosen as an example of the archaeological heritage that is not part of the main tourist itineraries. In Italy, parks or archaeological sites of great importance are visited every year by several million tourists, e.g., the "Colosseum and Roman Forum" archaeological park in Rome had about 7.6 and Pompeii 3.6 million visitors in 2018, with revenues of 53.8 and 39.6 million euros respectively [61]. In Sicily, which is the Italian region where Tornambé is located, the numbers of visitors to the archaeological sites are lower. In fact, in 2018 the visitors were 928,952 and 354,941, with revenues of 6.6 and 2.6 million euros respectively in the "Valle dei Templi" archaeological park in Agrigento and in the "Villa romana del Casale" archaeological area in Piazza Armerina [62].

The Tornambé site is located in central Sicily on the top of a rocky ridge in a system of the hills Monte Grande, Tornambè, Rocche di Tornambè, Monte Cane, Cozzo Cialandria e Parcazzo that delimit the valley of the Imera river. The area is of great naturalistic value, in fact, the ridge above the Imera river has been recognized as a Site of Community Importance (according to the Ministry of the Environment Decree 3 April 2000) in order to preserve biological diversity, as it constitutes 
an emblematic example of the Mediterranean bio-geographic region characteristics. In addition, the area is part of the "Monte Capodarso and Imera valley" nature reserve.

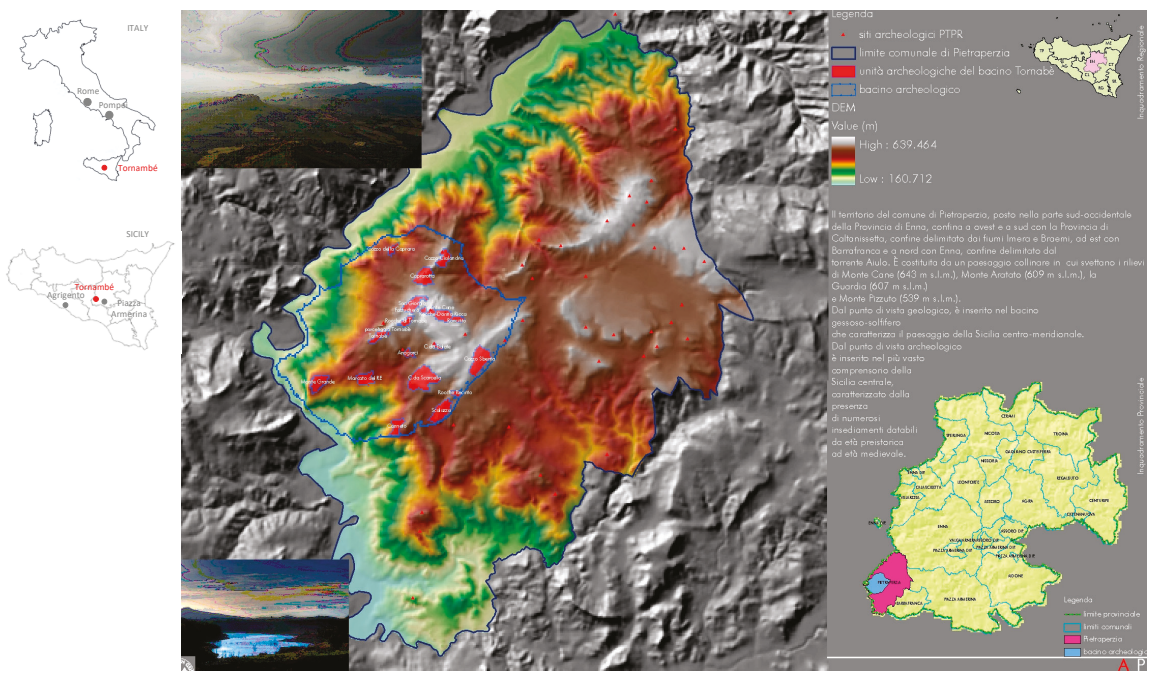

Figure 1. Geographical location of the archaeological basin of Tornambé.

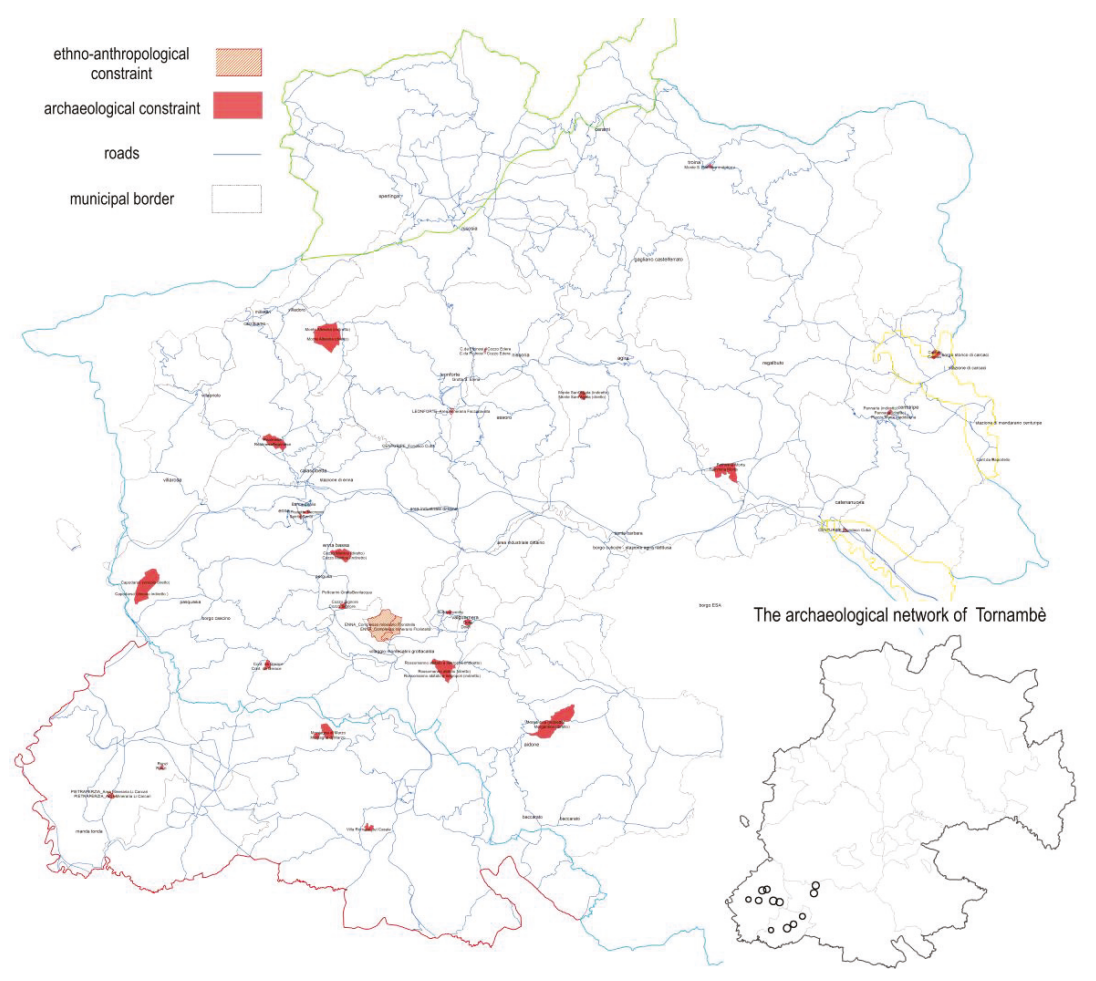

Figure 2. Ethno-anthropological and archaeological constraints in the Province of Enna, and the archaeological basin of Tornambé. 
This area is of archaeological interest as it has been inhabited for millenniums, since the prehistoric age, given their strategic location of controlling the Imera valley [63]. In fact, several excavation campaigns, from 2002 to 2012, found evidence of settlements dating back to the third millennium BC until the Greek age in the VII-VI century BC [64]. The surface reconnaissance activities and excavation campaigns from 2003 to 2007 (financed by Progetti Integrati Territoriali-PIT 11.496 of Programma Operativo Regionale-POR Sicilia 2000-2006) and another two excavation campaigns in 2008-2009, that were carried out thanks to a cooperation between the Municipality of Pietraperzia, the Sopritendenza BB.CC.AA. of Enna and a local Centro studi archeologici, allowed to expose the remains of a large necropolis and a village from the Copper Age (2700-2300 BC), the latter is formed of numerous huts having circular structures [65-67]. In this area, many pottery sherds, as glass or pot fragments, are present. Petrographic and mineralogical analyses carried out on ceramic samples dated them from the Copper Age to the Early and Middle Bronze and evidenced a certain continuity regarding the supply of raw material for a very long period, independently of the different cultural phases [68]. Moreover, in the site, there are numerous 'oven' tombs from the Bronze Age (2300-1600 BC), as well as some chambered tombs even monumental in size and other architectural elements dating back to the archaic Greek age (VII-V century BC). Furthermore remains of a phrourion, that was a military outpost consisting of rectangular rooms and cisterns dug into the rock, are located on the top of a hill to protect of the settlement [69]. In 2012 another excavation campaign started thanks to an agreement between local public authorities and a private local cultural association. Nevertheless the numerous archaeological finds, currently, accessibility to the site is difficult and there are no services for tourists, with the exception of some information panels and some paths inside the area, and only a few people sporadically visit the site on the initiative of local cultural associations.

The site of Tornambé is located in a wider territorial, environmental and landscape context which is the result of the essential integration between cultural and natural values, as it can be seen from the thematic maps, that have identified the territorial resources of the network within an area of approximately 2200 hectares, such as other minor archaeological sites, some places of naturalistic value, e.g., the San Giorgio or Monte Grande springs, or of testimonial interest such as the Monte Cane mine.

The Province of Enna government has mainly focused on the cultural policy of the archaeological heritage on the most important sites of the Province, Piazza Armerina (Villa del Casale) and Aidone (Morgantina). However, in recent years, the growing eco-environmental sensitivity and the general touristic demand diversification trend, integrating cultural and landscape heritage, as well as social events, local food and wine products, etc., have segmented users and induced the redistribution of a share of tourist demand towards inland areas of Sicily. In this context, the Tornambe site has the potential to capture part of the existing tourist flows to which to propose a composite offer, according to the concept of integrated conservation of the territory, thanks to numerous factors such as the proximity to the massive tourist flows of Piazza Armerina and Aidone and to a high-speed thoroughfare, the favourable altitude position with consequent landscape value. Furthermore, the site presents a remarkable complex of geomorphological, hydrogeological and vegetation values, integrated into the ethno-anthropological and cultural context of a still unspoiled territory, to which such an ancient and rare archaeological framework can give a primary and original significance.

This opportunity for enhancement generates, as a virtuous aspect, the involvement of local economic activities and a broader awareness of the value identity of places and also the strengthening of social cohesion. This prospect fits the progressive dematerialisation trend of an economy as for the contribution that the increase of the cultural-contemplative services demand can provide: 1 . to rebalance the town/countryside relationship as for the location demand of people and businesses, 2. to rebalance the relationship between mass and cultural-as well as between inland and coastal-tourism, in support of the preservation of the cultural estate territorial framework. 


\section{Methods. Territorial, Economic and Landscape Communication}

This study explores some aspects of multiscale integrated analysis, valuation and project approach to the protection and promotion of widespread archaeological heritage, in a territory, such as the Province of Enna, characterized by low-value-density cultural networks, and as a consequence by the emotional rarefaction of the "tactile experience".

In this context, the weak structure of the archaeological outcrops constitutes the main and original resource of cultural heritage which rediscovers its "original magnificence" in the shared awareness of the complex value expressed by the entirety of the landscape unit [70].

Accordingly, a general framework connecting territorial knowledge, economic valuations and cultural policies have been the methodological reference of this experience (Figure 3).

\begin{tabular}{|c|c|c|c|c|}
\hline $\begin{array}{l}\text { Territorial } \\
\text { communication } \\
\text { Regional Landscape } \\
\text { Territorial Plan }\end{array}$ & \multicolumn{2}{|c|}{$\begin{array}{l}\text { Geographic } \\
\text { Information } \\
\text { System } \\
\text { GeoDatabase }\end{array}$} & $\begin{array}{l}\text { Quantitative } \\
\text { features } \\
\text { Qualitative } \\
\text { features }\end{array}$ & $\begin{array}{l}\text { Areas } \\
\text { Infrastructures }\end{array}$ \\
\hline $\begin{array}{l}\text { Socio-economic } \\
\text { communication } \\
\text { Values/interests }\end{array}$ & $\begin{array}{l}\text { Project } \\
\text { Scale } \\
\text { Territory } \\
\text { Basin } \\
\text { Site }\end{array}$ & $\begin{array}{l}\text { Dothesis } \\
\text { Actions } \\
\text { Research } \\
\text { Experiencing } \\
\text { Promoting }\end{array}$ & $\begin{array}{l}\text { Appraisal } \\
\text { Assessment } \\
\text { Accounting }\end{array}$ & $\begin{array}{l}\text { Works and Real estate Estimates } \\
\text { Discounted Cash-Flow Analysis } \\
\text { Budget Analysis }\end{array}$ \\
\hline $\begin{array}{l}\text { Landscape } \\
\text { communication } \\
\text { Community identity }\end{array}$ & \multicolumn{2}{|c|}{$\begin{array}{l}\text { Stock-holders } \\
\text { Promoters }\end{array}$} & Scenario analysis & $\begin{array}{l}\text { Orthodox } \\
\text { Interpretation } \\
\text { Heterodox } \\
\text { Re-interpretations }\end{array}$ \\
\hline
\end{tabular}

Figure 3. The general valuation-project communicative framework.

This evaluation experience tries to connect aspects of the organization of knowledge, formalized with the use of GIS [71-77], with the economic analysis tools for efficient and effective management of the territorial cultural heritage, in a territorial context characterized by a low-value density.

The well-known economic unbalance and financial unfeasibility of the cultural enterprise typically aimed at providing streams of contemplative services, thus pursuing the growth of the immaterial component of the social territorial estate, is mostly due to the asymmetry between monetary, certain, initial, point and higher costs, on the one hand, non-monetary, uncertain, differed continuous and minimal, although widespread, benefits on the other hand.

Accordingly, the proposed analyses aim at defining the conditions of the cost-effectiveness of an archaeological-landscape network plan as conceptual and operative support to the cultural-asset-oriented territorial-landscape policies.

In such a complex decision-making prospect, one of the main theoretical and methodological criticalities of appraisal/valuation science arises, that is its relationship with project/planning activity [78]. Even today, valuation is considered to be a simple tool of design, whose output-at the end of a somehow self-referential design process-is verified by valuation, turning this design output into a social outcome. From such a perspective, a project is connected to representation and the decisions have a weak relation to the layer of shared values [79]. In this case, a top-down communication process is established, in which economic valuation plays the role of validating the choices coming from the top $[80,81]$.

The basic hypothesis of this perspective inversion is that this valuation/project relationship can be reversed so that the integration of economic valuation in the project process can integrate top-down and bottom-up decision-making processes, thus improving social-economic communication. 
Accordingly, this experiment covers three "communication areas":

- the "territorial communication" concerns the identification of the different archaeological networks of the Province of Enna and has been carried out in a previous study developed on a territorial scale. These networks consist of landscape contexts coherent with the underling archaeological sites and have been characterized on the basis of the geodatabases based on which the Guidelines of the Regional Territorial Landscape Plan have been created,

- the "social-economic communication" concerns the economic calculation aimed at connecting the cultural-contemplative instances and the social-territorial economic development opportunities. Once identified the archaeological network on which to test the economic potential of a "research, experiencing and promoting" program hypothesis-a DCFA was carried out aiming at estimating the number of costs and revenues, and therefore verifying the conditions of cost-effectiveness and financial sustainability $[82,83]$ of a typical enhancement and development hypothesis of an archaeological basin. The evidence of the economic-financial imbalance of the cash flow required the development of a subsequent in-depth phase,

- the "landscape communication" concerns the connection of the different players of the archaeological-oriented landscape redevelopment process. Two types of hypotheses were made: the first concern the allocation of the costs among the players identified as both stakeholders and especially stockholders. The second concerns the dynamics of the economic potential of the program, as a result of the development of the activities planned in the three action areas-research, experiencing and promoting.

\subsection{Territorial Communication. Representation as a Prospect for Identifying the Archaeological Basins}

The representation of territory through the prism of the landscape has been carried out in Sicily by means of the Guidelines of the Regional Landscape Territorial Plan [84] approved in 1999. Each of the nine Sicilian Provinces has or should have, draw its own Provincial Landscape Territorial Plan (PTLP) as the province of Enna did [85].

This document can be considered a territorial communication tool as for the standardized basic land information basing on which the activities of transformation and preservation of territorial and landscape units should be ruled.

Accordingly, the identification of the archaeological constraints of the Province of Enna [86] was carried out by first identifying the sites surveyed by the above-mentioned PTLP, from whose geodatabase the information and assessments characterizing their landscape relevance were extracted. Subsequently, the various sites were grouped into clusters-internally homogeneous and externally, that is compared to each other, heterogeneous—on the basis of similarity and complementarity relationships, whose parameters can be modified so as to allow the generation [87] of alternative configurations.

The relations of similarity and complementarity concern the original distinction of the basic three landscape components:

- abiotic (the biotopes), i.e., the geo-lithological and morphological components of the landscape,

- biotic (the biotypes), i.e., the vegetational, faunal and agricultural components of the landscape,

- anthropic, i.e., the cultural, built and infrastructural assets.

The 229 sites of the Province have been included in a database, of which they are the records and whose fields are the relevant characteristics extracted from the PTLP, the territorial profile of each of them has been defined in terms of compliance with a specific landscape matrix: 1. Agricultural, 2. Vegetational, 3. Geological, 4. Anthropic, 5. Cultural, 6 Infrastructural, 7. Architectural.

The landscape profile is represented by measurements that define value functions such as distances from infrastructures, presence of attractors or detractors, presence and consistency of valuable areas included within an established buffer. The themes are: historical, contemporary and railway roads; territorial and archaeological constraints; the presence of isolated goods; quarries and landfills; reserves, 
forests, Sites of Community Interest (SIC) and Special Protection Areas (SPAs); land uses; habitats; geological structure.

Furthermore, in order to define and identify the archaeological basins, the matrix of the mutual geometric distance of the sites was calculated to verify the spatial continuity of the basin with reference to the usability of the sites included in it.

The value functions of the individual thematic characteristics were subsequently defined for each of the seven landscape matrices, and for each of the latter, a specific system of weights has been established, that allows aggregating the characteristics in the seven scores for each site, with reference to each landscape matrix [88].

Among all the basins of the Province of Enna, the archaeological network of Tornambè (Figure 2) has been identified as an archaeological network currently characterized by a low-density archaeological value but having a relevant potential due to the multilayer connections between the landscape matrixes above described.

Once identified, the Tornambè basin was characterized through a detailed representation of its axiological and aesthetic layout, on the basis of which it was considered possible to prefigure a general scenario of development of the archaeological research, sustainable touristic experience and culture-oriented economic promotion.

\subsection{Socio-Economic Communication Project as a Valuation Tool in the Archaeological Asset Development}

The creation of a social-economic communication tool is the operational core of this experiment, aimed at measuring the economic profitability and the financial feasibility [89] of a standard archaeological basin development project by carrying out a Discounted Cash Flow Analysis [90]. The project hypothesis includes the actions (investments and management activities) requested to connect and develop the works aimed at creating the cultural assets and the related management operational unit involving a wide platform of private and public players.

In addition to the results of the territorial quantitative and qualitative analysis, the functions of the Geodatabase allowed us to extract and coordinate the spatial data necessary for the drafting of the preliminary project and the economic calculation of the development hypothesis.

Although the archaeological basins of the Province of Enna are quite different from each other, this hypothesis can be considered a sort of pilot project whose economic-monetary ratios provides standardized information about the interactions of the different players and the related instances and interests.

As well known, the DCFA is the comparison of revenues and costs of an investment within a given time span, the time horizon of the project [91]. The first step of this economic knowledge analysis has been the calculation of revenues and costs that have been carried out basing on their unit size.

The unit revenues were taken basing on specific market surveys of the goods and services that can be placed on the market. The total revenues were calculated according to a prudent scenario [92] concerning the users that could be attracted by implementing the activities included in the contemplative, recreational and educational program.

The unit costs have been taken both from specific market analyses and (especially for the building works) from the Bill of quantities for the Public Works of Sicilian Region 2018. The total costs have been calculated based on the dimensions of the involved geo-referenced areas and paths which have included in the geodatabase and whose development or renovation is supposed.

In order to make comparable the investment (point) costs $\left(C_{p}\right)$ to the annual operating (continue) costs and revenues, the former have been transformed in streams of constant annuities $\left(\bar{C}_{i}\right)$ by associating to each of them the economic lifetime $(n)$ of the corresponding asset and, according to the current financial market interest rate $\left(r_{d}\right)$ [93], the annuity was calculated by the following amortization formula:

$$
\bar{C}_{i}=C_{p} \frac{r_{d}\left(1+r_{d}\right)^{n}}{\left(1+r_{d}\right)^{n}-1}
$$


Furthermore, in order to quickly develop scenario analyses, the economic evaluation model is set up to enable or disable items, to attribute these items to different actors, to modify all the financial variables involved, such as the discount rate $[94,95]$, the interest rate on amortization and so on.

The cost-effectiveness for the private entrepreneurial player, in this case, a Special Purpose Vehicle (SPV), can be represented according to the following results and indices:

1. the Net Present Value (NPV) is the sum of the incoming and outgoing cash flows, that is revenues $(R)$ and costs $(C)$, over a defined time horizon $(T)$, discounted at the discount rate $r$. NPV is less, equal or more than the (net) Future Value (FV) if the discount rate $(r)$ is more, equal or less to 0 , $N P V$ is expected to be significantly positive in case of a private player:

$$
N P V=\sum_{i=0}^{T} \frac{R_{i}-C_{i}}{(1+r)^{i}} \geq 0
$$

2. the Total Rate of Return (TRR), that is the more significant index of profitability thus the ratio between NPV and the present cost, TRR should be greater than the opportunity cost of capital $c$.

$$
\operatorname{TRR}=\frac{\sum_{i=0}^{T} \frac{R_{i}-C_{i}}{(1+r)^{i}}}{\sum_{i=0}^{T} \frac{C_{i}}{(1+r)^{i}}} \geq c
$$

3. the Internal Rate of Return (IRR), that is the discount rate $r_{I R R}$ at which $N P V=0$, that is the maximum rate of return that can be extracted by an investment. It only depends on the distribution of the stream along the time horizon of the investment:

$$
\sum_{i=0}^{T} \frac{R_{i}-C_{i}}{\left(1+r_{I R R}\right)^{i}}=0
$$

4. the External Rate of Return (ERR)—also called Modified Internal Rate of Return (MIRR)—refers to both the cost of the investment and the interests on reinvested cash, and is calculated on the basis of an interest rate external to the investment, at which net (positive) cash flows generated by the investment over its time horizon can be invested or borrowed $r^{*}$. The External Rate of Return $r_{e}^{*}$ is the rate at which the investment costs discounted at the rate $r$ equals the future value at time $T$ of the positive Cash Flows $\left(C F_{i(>0)}\right)$ deferred at the rate $r^{*}$, given $C F_{i}=R_{i}-C_{i}$, in other words, ERR is the IRR of an ideal investment whose unique cost is the initial investment cost calculated as the NPV at the rate $r$ of the negative cash flows over the time horizon $T$, and whose unique revenue is the future value (at year T) of the positive cash flows at the rate $r^{*}$, this particular IRR is $r_{e}$.

$$
\sum_{i=0}^{T} \frac{C F_{i(<0)}}{\left(1+r_{e}\right)^{i}}=\sum_{i=0}^{T} C F_{T-1(>0)}\left(1+r^{*}\right)^{T-i}
$$

5. the Elasticity $\left(E_{r}\right)$, that is the marginal $N P V$ at the discount rate $r$ :

$$
E_{r}=\frac{\frac{\delta N P V_{r}}{N P V_{r}}}{\frac{\delta r}{r}}
$$

6. the Discounted Payback Period (DPP) is the number of years it takes to break even from undertaking the investment cost $\left(I_{0}\right)$ by discounting future cash flows and recognizing the time value of money $(r>0)$, the higher the discount rate, the longer the DPP, more simply, a Payback Period $(P P)$ can be calculated without taking into account the time preference rate $(r=0)$. 
In general, $P P$ is the ratio between the total investment cost and the annual constant or average cash flow. Often, the variability of the cash flow over the lifetime of the project reduces the reliability of the formulas usually implemented for $D P P$, so that a more general formula can be proposed considering $N P V(i)$, and then:

$$
D P P=i_{N P V(i)=0}
$$

7. the Average Period at the rate $r\left(A P_{r}\right)$ [48], that is a sort of time elasticity, that can be considered as the average period of deferral of the $i_{\text {th }}$ annual net discounted Cash Flows $\left(C F_{i}\right)$ given the discount factor,

$$
A P_{r}=\frac{\sum_{i=0}^{T} i \frac{C F_{i}}{(1+r)^{i}}}{\sum_{i=0}^{T} \frac{C F_{i}}{(1+r)^{i}}}
$$

The discount rate $r$ is an important indicator of the intertemporal social solidarity practised with the implementation of the project, and it enables two different and complementary prospects, the private one as means, the public one as scope.

Concerning the first one, the discount rate can be assumed as the well-known Weighted Average Cost of Capital (WACC), referred to the funds in terms of Debt $(D)$ and Equity $(E)$,

$$
W A C C=\frac{i_{d} D+i_{e} E}{D+E}
$$

where $i_{d}$ is the interest rate for debt and $i_{e}$ is the opportunity cost of equity, that can be respectively referred to the active and passive interest rates charged to households and consumers, according to the statistics of Bank Italia (2017), set at $4.66 \%$ (over a 5 years life of the loan) and $0.12 \%$, assuming leverage of $50 \%$, WACC is $2.39 \%$.

Concerning the second one, although the economic-financial valuation is carried out here form the private player perspective, it should be remarked that the whole project involves a wide range of public players providing the private one with the political-administrative support and the socio-cultural context allowing the business success.

As a consequence, as for the size as well as for the role it plays in the socio-economic intergenerational communication, the discount rate can be considered to be a sort of Social Discount Rate that the extensive literature on the subject [96] agrees should have a nearly zero value. The prospect of an economic communication able to involve as many players as possible encourages researchers to support a low-discount rate territorial-economic culture as one of the fundamental pillars of sustainability involving the relationship between the social system and the environment in the prospect of establishing of an actual and effective landscape communication.

\subsection{Landscape Communication. Valuation as a Programming Tool in the Archaeological Asset Protection}

The central role that landscape should play in the social and economic communication, in the specific field of the enhancement of the land cultural asset, demands the convergence of the social-economic players' perspectives (the viewpoints) towards sustainability (the vanishing point), once outlined the actions and the financial-economic profile of the archaeological basin development hypothesis.

The early comparison of revenues and costs provides the decision-makers with:

1. a significant measurement of the overall imbalance between revenues and total cost,

2. the degree of - and the conditions for-the cost-effectiveness and financial feasibility of the investment limited to the revenues of goods and services that can be placed on the market. 
Both the results encourage and guide the decision-makers (as part of the political-administrative social sub-system) the stockholders (as part of the economic-financial social sub-system) and the stakeholders (as parts of the cultural sub-system) to converge towards a shared arrangement of tasks and economic-financial commitments. This convergence is supported by the hoped shared awareness that the amount of costs that cannot be covered by the revenues represents the economic measurement of the minimum social value of this archaeological-landscape asset. Such awareness supports the improvement of the landscape communication if: 1 . this cost surplus is actually incurred by the political-administrative and cultural sub-systems, 2 . such political-cultural choice is shared by the public. Now, concerning the two complementary functions of the revenue-cost comparison:

1. as for the first one, starting from the evident revenue/costs imbalance that no further study could reduce, a cost allocation pattern between the players can be hypothesized in order to support and encourage the private player initiative. The costs have been allocated according to the specific functions and institutional scopes of the public players involved, as explained in Section 4.3,

2. the second one-however, concerning the cost-effectiveness analysis for the private player, that is the entrepreneurial layout of the archaeological basin management—can take advantage of some further studies concerning the percentage incidence of the different cost types, the scenario analysis and the sensitiveness analysis aimed at identifying the strategic economic and financial variables and their connection to the cultural, management features influencing the outputs of the DCFA.

The focus on the stake/stock-holders relationship is one of the several oppositions which the economic valuation usually deals with, each of these oppositions can often also result in a form of complementarity. The unavoidable overall economic unprofitability and financial infeasibility of the development of a landscape-archaeological unit intended to cultural, contemplative and recreational uses, is a measurement of the opposition, and somehow of the complementarity, between:

- the transformative actions, such as the development of the built environment, recreational functions, commercial activities and so on,

- the conservative ones, such as the expansion of the excavation areas for archaeological research, the widespread musealization in situ, the creation of panoramic equipped areas, the development of the cyclo-pedestrian road network and so on.

This conflict, in turn, highlights the intrinsic complementarities of different dimensions of the landscape that is an overarching entity including and displaying in a typically multifaceted shape its own complexities and contradictions.

Accordingly, the economic-financial variables have been assumed as project/program items and as such, they have been organized within a general conceptual and operative framework (Table 1) coordinating:

1. research, concerning the extension and dissemination of the archaeological knowledge,

2. experiencing, concerning the development and differentiation of the direct experience of tourism and education,

3. promoting, concerning the consideration of secondary and indirect economic activities that characterize all the sites as a unitary archaeological basin, also characterized in a socio-economic and political-communicative sense.

Each of the three above-mentioned general objectives has been represented outlining the related programming actions grouped according to the multiscale approach involving activities devoted to:

1. the territory, such as social communication and territorial marketing,

2. the basin, such as archaeological research and attraction of stakeholders and stockholders,

3. the site, such as protection, maintenance, development of the involved areas. 
Table 1. Framework of the project.

\begin{tabular}{|c|c|c|c|}
\hline & 1. Territory & 2. Basin & 3. Site \\
\hline 1. Research & $\begin{array}{l}\text { Geo-Database/Web-GIS platform } \\
\text { creation }\end{array}$ & $\begin{array}{c}\text { Field survey of } \\
\text { archaeological settlement }\end{array}$ & $\begin{array}{l}\text { Extension of the } \\
\text { archaeological } \\
\text { excavations }\end{array}$ \\
\hline 2. Experiencing & $\begin{array}{l}\text { Information customizable WebGIS } \\
\text { tool packages for different users } \\
\text { and institutions. } \\
\text { Coordination of these tools and } \\
\text { development of the Geo-Database } \\
\text { by including the pieces of } \\
\text { information provided by the users }\end{array}$ & $\begin{array}{l}\text { Maintenance of existing } \\
\text { roads and development } \\
\text { of cyclo-pedestrian paths. } \\
\text { Riding school } \\
\text { Management of } \\
\text { purchases of vehicles, } \\
\text { tools, commodities Wine } \\
\text { and food paths } \\
\text { arrangement. }\end{array}$ & $\begin{array}{l}\text { Expropriation of land } \\
\text { areas and renovation of } \\
\text { ancient country } \\
\text { buildings. } \\
\text { Theatrical and } \\
\text { recreational facilities. } \\
\text { Museum exhibitions and } \\
\text { theatrical performances } \\
\text { programming }\end{array}$ \\
\hline 3. Promoting & $\begin{array}{l}\text { Dissemination of information } \\
\text { through website, social media, } \\
\text { tour operators, etc. } \\
\text { Attraction of tourists. } \\
\text { Commercial agreements on prices } \\
\text { and tickets for standard and } \\
\text { customized packages of services. } \\
\text { Creation of the Archaeological } \\
\text { Basin Brand integrating } \\
\text { naturalistic trips, cultural } \\
\text { experiences, cultural } \\
\text { environmental education } \\
\text { meetings, and food-wine events. }\end{array}$ & $\begin{array}{l}\text { Archaeological, } \\
\text { ethno-anthropological, } \\
\text { botanical laboratories } \\
\text { Involvement of schools } \\
\text { and University for } \\
\text { training }\end{array}$ & $\begin{array}{l}\text { Exhibitions or theatrical } \\
\text { performances. } \\
\text { Musealization of the } \\
\text { archaeological sites and } \\
\text { finds. } \\
\text { Workshops. } \\
\text { Processing of } \\
\text { information for the } \\
\text { restoration of finds. } \\
\text { Restoration didactic } \\
\text { laboratories }\end{array}$ \\
\hline
\end{tabular}

The path along the main diagonal of the matrix reports the stages of actions ranging from the immaterial services to the material goods, and from the general context to the particular places, as described below.

1-1 Research/territory. The creation of a descriptive Geo-Database/Web-GIS of the basin is one of the basic tools of territorial marketing. It should be organized according to the description of the different levels included in the local landscape units with reference to abiotic, biotic and anthropic systems, and could be available for queries from scholars, entrepreneurs, designers and public administrations.

1-2 Research/basin. Surface reconnaissance activities are planned to detect the presence of not yet known archaeological sites.

1-3 Research/site. Stratigraphic research and excavation campaigns are planned to increase the cultural offer of each site with reference to the volume and archaeological value of the remains. These activities must be diversified between the sites in relation to their potential but also with respect to the organization within the basin which is aimed at differentiating potential itineraries.

2-1 Experiencing/Territory. A section of the Web-Gis, by means of specific toolbars, could allow the potential users to customize the itinerary according to specific interests, preferences and cultural themes. Specialistic information can be stored and made available for scholars and institutions after prior accreditation and certification.

2-2 Experiencing/Basin. Maintenance works are planned for the main connection routes to improve the coordinated fruition of the sites included in the itineraries of greatest overall cultural value. Actions are also planned to improve touristic accommodation and services (agritourism, catering, riding stables, bicycle shelter and rental), infrastructures (roads and car parks), entertainment (temporary and removable structures).

2-3 Experiencing/Site. The following actions are planned: the realization of lighting systems, panels and fences, the redevelopment of internal paths, etc. 
3-1 Promoting/Territory. The enhancement of the territory is obtained by creating connections with regional and provincial cultural events. The Web-GIS platform stores information on the most important cultural events and provides the potential itinerant archaeological-naturalistic events such as exhibitions, festivals and theatrical performances.

3-2 Promoting/Basin. Temporary and permanent laboratories will be set up in the archaeological, ethno-anthropological, botanical, geological, etc. fields, in collaboration with associations and universities.

3-3 Promoting/Site. Didactic and cultural events and performances are planned compatibly with the features of the different archaeological sites. In the most important sites, the remains can be musealized in new exhibition open spaces and new or reused ancient buildings. Other buildings are supposed to be intended to the restoration of the most important archaeological remains, also within workshops and open laboratories.

Accordingly, all the actions envisaged by the project hypothesis, have listed in the rows of a database divided into three sections each of which is sub-divided into three sub-sections. The columns, i.e., the fields, of the database report the characteristics arranged so as to perform scenario and sensitiveness analyses coherent with the above-mentioned three-parts subdivision, as below:

- $\quad$ extensions, such as size, number of items or people involved or interested in,

- design, such as the category of intervention (new building, renovation, maintenance, restoration),

- type of goods, such as ground, properties, buildings, furniture, instalments, tools, vehicles, materials,

- type of right supposed for the use of properties, such as expropriation, rent, purchase,

- type of task: advice, management, job,

- class of workers, such as director, officer, archaeologist, restorer, GIS professional, archaeological remain analyst,

- $\quad$ economic variables, such as unit revenues and costs, the latter distinguished by investment and operative costs, the lifetime of the works or goods,

- $\quad$ socio-political variables, concerning the player by which is supposed the action has to be promoted and the costs incurred, such as Special Purpose Vehicle, Sicilian Regional Authorities, Regional Province of Enna, Municipality of Pietraperzia, University, Archaeological Associations, Local Bank).

\section{Application and Results}

\subsection{Territorial Communication: The Geo-Database}

The Geo-database basing which the Guidelines of the RTLP and the PTPL refer to, allowed us to characterize the studied area, the archaeological basin of Tornambè that has been delimited as a landscape unit as follows.

As first the 229 archaeological sites of the Province of Enna have been individuated in the PTLP. Then, each of them has been characterized as for its landscape relevance and scored according to a metric representing the value of each category of the theme, which it belongs to. Finally, relations of similarity and complementarity have been established according to which the basins have been delimited and identified [97].

Once delimited the basin, its land area is divided into parcels that can be considered homogeneous with respect to each category of the related theme. Each parcel is scored according to a standard metric, set up in the PTLP, measuring the value of the parcel. By overlapping the different themes, the weighted average score of a generic item is calculated. For example, the relevance of a category of agricultural land use, such as almond grove, olive grove, pasture, etc., is expressed through the weighted average of the values of ecological, historical and agricultural values ranging from 1 to 5 . The watercourses were assessed on a scale of 1 to 3 depending on the flow rate. Other landscape elements have been classified solely on the basis of location and size. 
The geodatabase for the studied area reports the landscape themes from the PTLP, such as the geo-lithological and morphological ones, consisting of 14 categories, the vegetation and fauna themes consisting of 24 categories, the agricultural theme consisting of 21 categories and the cultural, real estate and infrastructure heritage themes consisting of 10 categories (Figure 4).

\begin{tabular}{|c|c|c|c|}
\hline \multirow[b]{2}{*}{ id } & \multicolumn{3}{|c|}{ Basins } \\
\hline & Basin name & Surface (ha) Per & Perimeter $(\mathrm{km})$ \\
\hline 1 & Tornambè & 21.95 & 20.27 \\
\hline \multicolumn{4}{|c|}{ Archaeological units } \\
\hline & Site name & Surface (mq) & $\begin{array}{c}\text { Perimeter } \\
(\mathrm{ml})\end{array}$ \\
\hline Ranc & & 42637 & $\begin{array}{ll}37 & 905\end{array}$ \\
\hline Mont & e Cane & 26945 & 728 \\
\hline Fastu & ichera & 26483 & 753 \\
\hline $\operatorname{San} C$ & Giorgio & 128837 & 1872 \\
\hline Cozz & o della Caprara & 41235 & 1060 \\
\hline Anag & & 37025 & 883 \\
\hline C.da & Balate & 21861 & 655 \\
\hline Cozz & o Cialandria & 104818 & 1482 \\
\hline Rocc & he di Tornabè & 53203 & 1140 \\
\hline Marc & ato del RE & 112353 & 1641 \\
\hline Mont & Grande & 140376 & 1557 \\
\hline Rocc & he-Donna Ricca & 120326 & 1350 \\
\hline C.da & Scarcella & 356335 & 2584 \\
\hline Cozz & o Sbenta & 258147 & 2184 \\
\hline Scala & & 190474 & 1933 \\
\hline Capre & arotta & 158964 & 1815 \\
\hline Cann & & 93025 & 1597 \\
\hline Rocc & he Recinto & 30756 & 717 \\
\hline Torna & ambè & 75639 & 1516 \\
\hline Torna & ambè site parking & 6215 & 320 \\
\hline
\end{tabular}

\begin{tabular}{|c|c|c|c|c|c|c|c|}
\hline \multirow[b]{2}{*}{ id } & \multicolumn{7}{|c|}{ Agricoltural areas } \\
\hline & Land Intended use & Area (sq.m) & $\begin{array}{l}\text { Perimet } \\
\text { er (ml) }\end{array}$ & $\begin{array}{l}\text { Ecologic } \\
\text { al value }\end{array}$ & $\begin{array}{c}\text { Historical } \\
\text { value }\end{array}$ & $\begin{array}{l}\text { Agricoltur } \\
\text { al value }\end{array}$ & $\begin{array}{l}\text { W.A. } \\
\text { score }\end{array}$ \\
\hline 22 & arable land & 39,562 & 825 & 2 & 4 & 2 & 2.9 \\
\hline 23 & arable land & 53,403 & 1607 & 2 & 4 & 2 & 2.9 \\
\hline 24 & urban area & 6576 & 363 & 0 & 0 & 0 & 0 \\
\hline 25 & pasture/uncultivated & 110,453 & 2987 & 4 & 4 & 1 & 3.7 \\
\hline 26 & pasture/uncultivated & 48,239 & 1439 & 4 & 4 & 1 & 3.7 \\
\hline 27 & arable land & 156,177 & 3355 & 2 & 4 & 2 & 2.9 \\
\hline 28 & arable land & 10,608 & 751 & 2 & 4 & 2 & 2.9 \\
\hline 29 & urban area & 1508 & 183 & 0 & 0 & 0 & 0 \\
\hline 30 & arable land with trees & 35,970 & 969 & 4 & 4 & 2 & 3.8 \\
\hline 31 & arable land & 50,056 & 1027 & 2 & 4 & 2 & 2.9 \\
\hline 32 & almond grove & 14,420 & 563 & 3 & 4 & 1 & 3.25 \\
\hline 33 & pasture/uncultivated & 3419 & 238 & 4 & 4 & 1 & 3.7 \\
\hline 34 & almond grove & 24,599 & 679 & 3 & 4 & 1 & 3.25 \\
\hline 35 & olive grove & 31 & 28 & 3 & 5 & 2 & 3.8 \\
\hline 36 & arable land & 190,784 & 3514 & 2 & 4 & 2 & 2.9 \\
\hline 37 & almond grove & 34,493 & 1065 & 3 & 4 & 1 & 3.25 \\
\hline 38 & arable land with trees & 73,211 & 1427 & 4 & 4 & 2 & 3.8 \\
\hline 39 & almond grove & 15,435 & 638 & 3 & 4 & 1 & 3.25 \\
\hline 40 & almond grove & 4312 & 262 & 3 & 4 & 1 & 3.25 \\
\hline 41 & arable land with trees & 56,745 & 1356 & 4 & 4 & 2 & 3.8 \\
\hline 42 & pasture/uncultivated & 5607 & 354 & 4 & 4 & 1 & 3.7 \\
\hline 43 & almond grove & 32,008 & 919 & 3 & 4 & 1 & 3.25 \\
\hline 44 & almond grove & 13,821 & 716 & 3 & 4 & 1 & 3.25 \\
\hline 45 & arable land with trees & 54,908 & 1556 & 4 & 4 & 2 & 3.8 \\
\hline 46 & pasture/uncultivated & 241,909 & 5501 & 4 & 4 & 1 & 3.7 \\
\hline 47 & arable land & 688 & 143 & 2 & 4 & 2 & 2.9 \\
\hline 48 & pasture/uncultivated & 11,306 & 584 & 4 & 4 & 1 & 3.7 \\
\hline 49 & arable land with trees & 123,053 & 1752 & 4 & 4 & 2 & 3.8 \\
\hline 50 & urban area & 11,913 & 542 & 0 & 0 & 0 & 0 \\
\hline
\end{tabular}

\begin{tabular}{|c|c|c|c|c|}
\hline \multicolumn{5}{|c|}{ Vegetation } \\
\hline id & Code & $\begin{array}{c}\text { Area } \\
\text { (ha) }\end{array}$ & $\begin{array}{c}\text { Per. } \\
\text { (km) }\end{array}$ & $\begin{array}{c}\text { Botanical } \\
\text { value }\end{array}$ \\
\hline 1 & 510 & 18.60 & 0.63 & 1 \\
\hline 2 & 400 & 4.54 & 0.29 & 1 \\
\hline 3 & 510 & 2.38 & 0.21 & 2 \\
\hline 4 & 510 & 51.64 & 1.55 & 1 \\
\hline 5 & 320 & 3.51 & 0.24 & 2 \\
\hline 6 & 312 & 43.50 & 1.27 & 2 \\
\hline 7 & 400 & 718.55 & 7.14 & 2 \\
\hline 8 & 600 & 2.19 & 0.19 & 1 \\
\hline 9 & 510 & 1.74 & 0.18 & 3 \\
\hline 10 & 312 & 25.54 & 0.92 & 3 \\
\hline 11 & 312 & 17.36 & 0.59 & 2 \\
\hline 12 & 400 & 244.69 & 2.34 & 3 \\
\hline 13 & 163 & 10.90 & 0.48 & 2 \\
\hline 14 & 400 & 2.32 & 0.19 & 4 \\
\hline 15 & 163 & 7.03 & 0.50 & 2 \\
\hline 16 & 312 & 49.29 & 1.45 & 3 \\
\hline 17 & 510 & 0.76 & 0.12 & 3 \\
\hline 18 & 320 & 10.19 & 1.14 & 2 \\
\hline 19 & 320 & 2.80 & 0.45 & 2 \\
\hline 20 & 310 & 10.26 & 0.41 & 2 \\
\hline 21 & 510 & 1.51 & 0.18 & 1 \\
\hline 22 & 510 & 0.91 & 0.12 & 1 \\
\hline 23 & 400 & 13.34 & 0.44 & 2 \\
\hline 24 & 400 & 23.72 & 0.79 & 2 \\
\hline 25 & 312 & 3.42 & 0.24 & 1 \\
\hline 26 & 312 & 318.48 & 4.19 & 1 \\
\hline 27 & 400 & 13.56 & 0.61 & 1 \\
\hline 28 & 400 & 108.25 & 2.67 & 2 \\
\hline 29 & 400 & 20.09 & 0.65 & 4 \\
\hline & & & & \\
\hline
\end{tabular}

Figure 4. Example excerpts of the Geo-database (geographical references omitted).

The thematic maps extracted from the Geo-database allowed us to outline a sort of "value-graphy" of the archaeological basin of Tornambè. Figures 5-10 display some samples of geo-graphy (as for geology, geomorphology and hydrology), historio-graphy (as for the historic land framework of the archaeological network), anthropo-graphy (as for land intended uses, urbanization and road network).

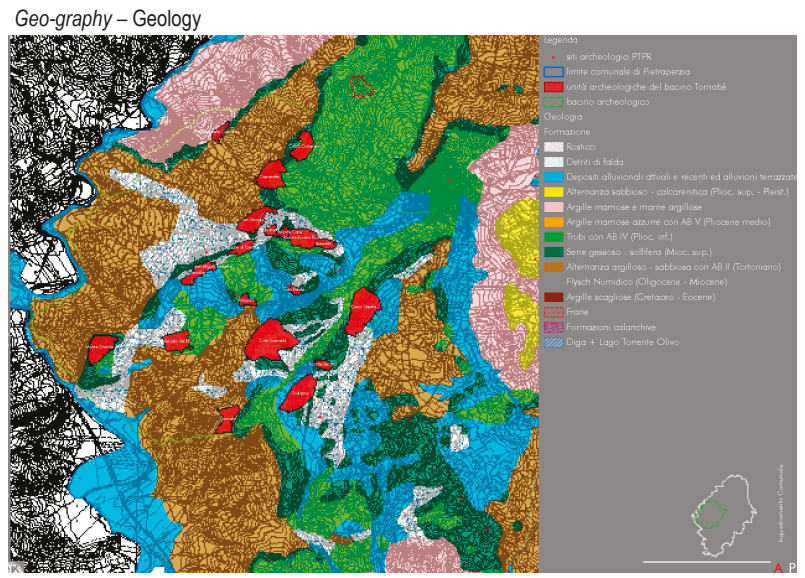

Figure 5. The Archaeological basin of Tornambè: Geology. 


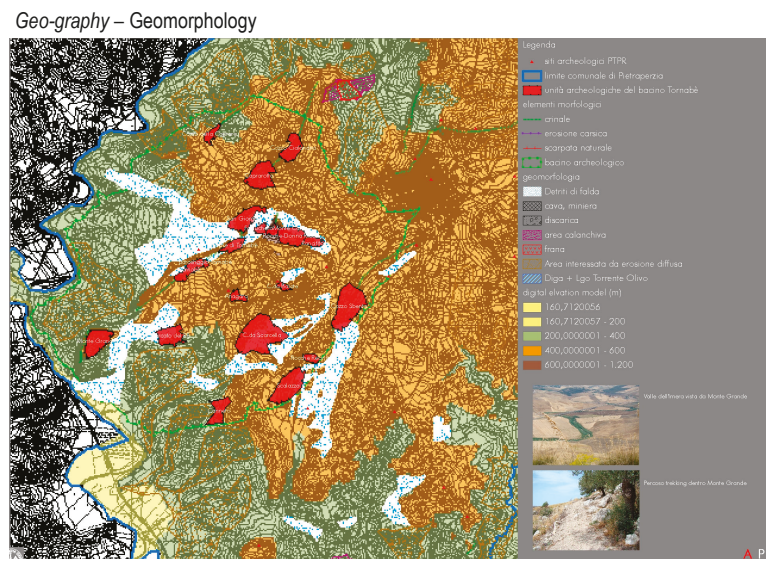

Figure 6. The Archaeological basin of Tornambè: Geomorphology.

Geo-graphy - Hydrology and lithological complexes

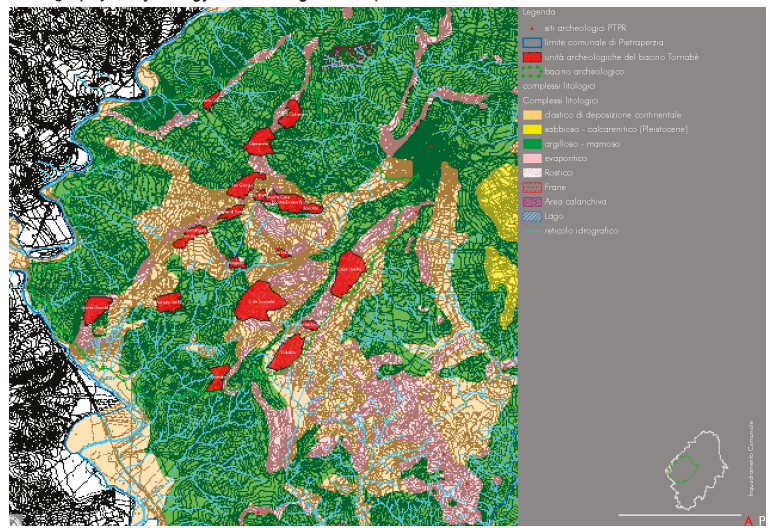

Figure 7. The Archaeological basin of Tornambè: Hydrology.

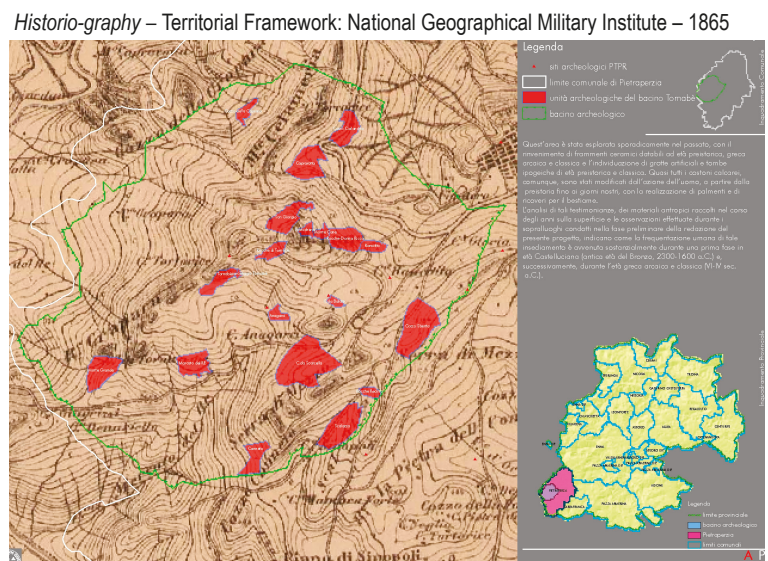

Figure 8. Historical territorial framework-IGM, 1865. 


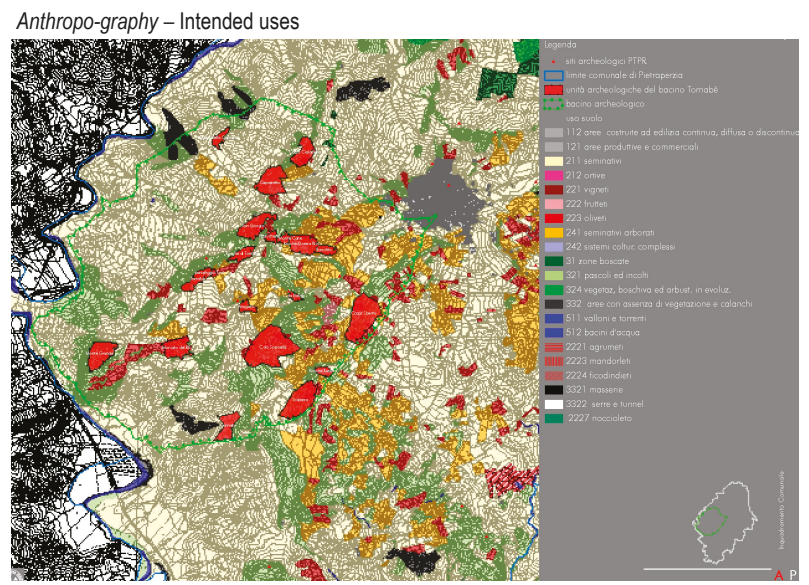

Figure 9. Intended uses.

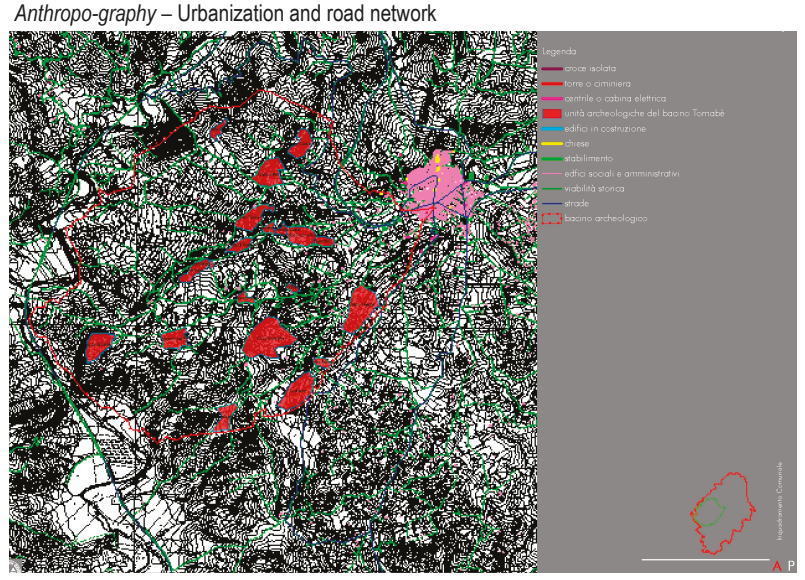

Figure 10. Urbanization and road network.

The landscape connection between these different value dimensions, or ways to be wort, concerns the direct and/or indirect experience of the territorial context, the former related to the accessibility, the latter to the intervisibility map of the different archaeological sites.

The accessibility can be represented through the matrix of the distances of the sites from each other, the intervisibility map is the land area that is visible from each site and from which each site is visible. Figures 11 and 12 sample the intervisibility maps of Montegrande and Fastuchera sites.

The key findings of this step are multiple interpretations of the territorial context given by the possibility to overlap the landscape (abiotic, biotic and anthropic) value maps and the intervisibility maps concerning the different archaeological sites included in the basin. 


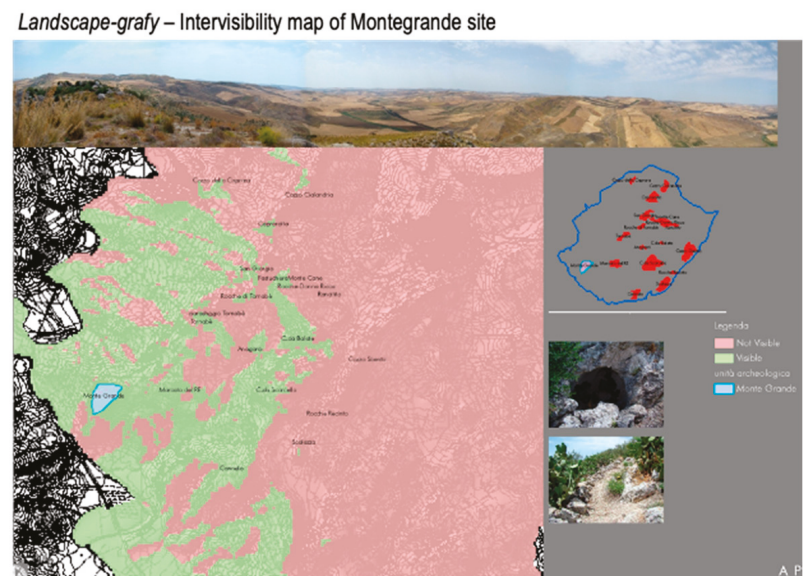

Figure 11. Intervisibility map of Montegrande archaeological site.

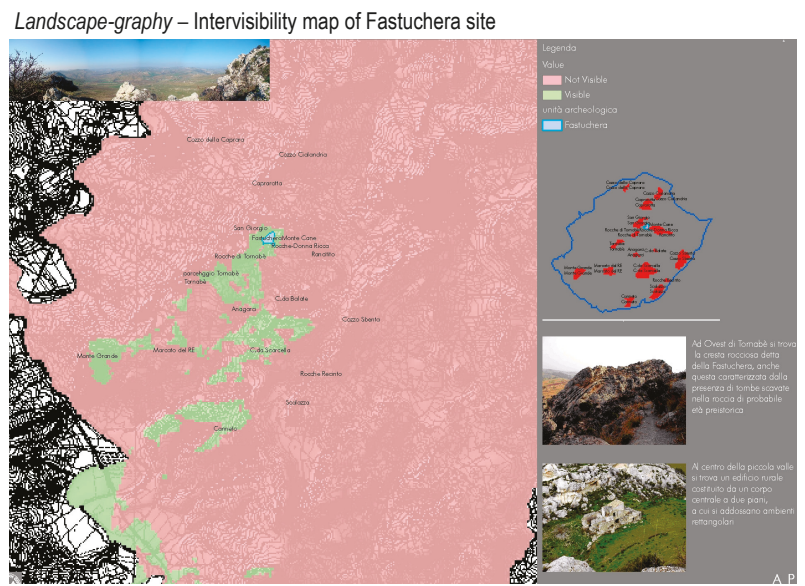

Figure 12. Intervisibility map of Fastuchera archaeological site.

\subsection{Socio-Economic Communication: Appraisal, Accounting, Assessment}

The development project was detailed grouping the actions to be carried out: (a) in the areas of research, use and enhancement, (b) on the scales of territory, basin, site, taking into account the characteristics of the archaeological settlements and the potential of the relative territorial and landscape contexts.

For each action, the investment and/or operating costs, and the annual revenues have been appraised, as summarized in Tables $2-4$.

Further synthesis of the above program allows us to distinguish and compare revenues and costs by policy [98] and scale (Figure 13). The amounts and the corresponding histograms display the expected clear unbalance between the scarce annual revenues (99,293 euro) and the huge annual costs $(855,932$ euro)that, more in general, characterizes the cultural-landscape asset economy, due to the emotional rarefaction of the contemplative experience in such landscape contexts.

As a consequence, the DCFA has been extended only to the economic goods and services that can be placed on the market, thus considering the economic-financial profile for a private player, a Special Purpose Vehicle (SPV), that could coordinate and manage all the actions needed for the 
development and protection of territory whose exceeding costs could be incurred by the other public players, as following explained.

Once selected the private components of the project (as proposed in the next sub-section), the cash flow has been drawn and the economic-financial results and indices have been calculated.

According to a basic hypothesis (H1), revenues have been considered constant over the entire 30-years lifetime of the project. This early simulation compares two possible distributions of the costs:

- the first one (SPV 30-y variable cash flow) considers the cost for durable goods and works to be incurred again at the end of their lifetime,

- the second one considering the costs to be constant each of them turned in a yearly depreciation charge.

Figure 14 displays both the not-discounted and discounted costs and revenues in order to show the effect of different costs and revenues, as well as the annual and cumulated cash flow.

Table 2. Summary of the actions, revenues and costs of the archaeological research program.

\begin{tabular}{|c|c|c|c|c|c|c|}
\hline Policy & Scale & Area & Action & Item & $\begin{array}{c}\text { Annual } \\
\text { Revenues }\end{array}$ & $\begin{array}{c}\text { Annual } \\
\text { Costs }\end{array}$ \\
\hline \multirow{25}{*}{ 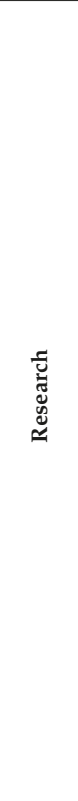 } & \multirow{2}{*}{ territory } & \multirow{2}{*}{$\begin{array}{c}\text { Enna } \\
\text { Province }\end{array}$} & \multirow{2}{*}{$\begin{array}{c}\text { webgis creation } \\
\text { website } \\
\text { maintenance }\end{array}$} & GIS professionals & & $€ 2321$ \\
\hline & & & & GIS professionals & & $€ 6000$ \\
\hline & \multirow{3}{*}{ basin } & \multirow{3}{*}{ whole basin } & \multirow{3}{*}{$\begin{array}{c}\text { surface } \\
\text { archaeological } \\
\text { research }\end{array}$} & director & & $€ 16,800$ \\
\hline & & & & archaeologists & & $€ 37,800$ \\
\hline & & & & GIS professionals & & $€ 1238$ \\
\hline & \multirow{20}{*}{ site } & \multirow{5}{*}{ Tornambè } & \multirow{5}{*}{$\begin{array}{l}\text { stratigraphy } \\
\text { research }\end{array}$} & director & & $€ 12,000$ \\
\hline & & & & archaeologists & & $€ 45,000$ \\
\hline & & & & restorers & & $€ 12,000$ \\
\hline & & & & GIS professionals & & $€ 6000$ \\
\hline & & & & archaeological remains analyst & & $€ 1500$ \\
\hline & & \multirow{5}{*}{ Montegrande } & \multirow{5}{*}{$\begin{array}{l}\text { stratigraphy } \\
\text { research }\end{array}$} & director & & $€ 12,000$ \\
\hline & & & & archaeologists & & $€ 45,000$ \\
\hline & & & & restorers & & $€ 12,000$ \\
\hline & & & & GIS professionals & & $€ 6000$ \\
\hline & & & & archaeological remains analyst & & $€ 1500$ \\
\hline & & \multirow{5}{*}{ Fastuchera } & \multirow{5}{*}{$\begin{array}{l}\text { stratigraphy } \\
\text { research }\end{array}$} & director & & $€ 12,000$ \\
\hline & & & & archaeologists & & $€ 36,000$ \\
\hline & & & & restorers & & $€ 12,000$ \\
\hline & & & & GIS professionals & & $€ 6000$ \\
\hline & & & & archaeological remains analyst & & $€ 1500$ \\
\hline & & \multirow{5}{*}{ Anagargi } & \multirow{5}{*}{$\begin{array}{l}\text { stratigraphy } \\
\text { research }\end{array}$} & director & & $€ 12,000$ \\
\hline & & & & archaeologists & & $€ 36,000$ \\
\hline & & & & restorers & & $€ 12,000$ \\
\hline & & & & GIS professionals & & $€ 6000$ \\
\hline & & & & archaeological remains analyst & & $€ 1500$ \\
\hline
\end{tabular}


Table 3. Summary of the actions, revenues and costs of the cultural-contemplative experience program.

\begin{tabular}{|c|c|c|c|c|c|c|}
\hline Policy & Scale & Area & Action & Item & $\begin{array}{l}\text { Annual } \\
\text { Revenues }\end{array}$ & $\begin{array}{l}\text { Annual } \\
\text { Costs }\end{array}$ \\
\hline \multirow{16}{*}{ 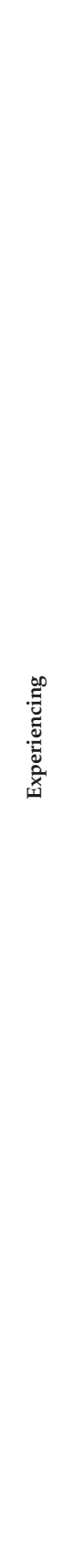 } & territory & $\begin{array}{c}\text { Enna } \\
\text { Province }\end{array}$ & $\begin{array}{l}\text { archaeological } \\
\text { laboratory }\end{array}$ & $\begin{array}{l}\text { facilities } \\
\text { personnel }\end{array}$ & & $\begin{array}{c}€ 7200 \\
€ 15,000 \\
€ 12,000\end{array}$ \\
\hline & & whole basin & accessibility & $\begin{array}{c}\text { roadways } \\
\text { pedestrian roads } \\
\text { cycle paths } \\
\text { horse paths }\end{array}$ & & $\begin{array}{l}€ 7669 \\
€ 8217 \\
€ 20541 \\
€ 5478 \\
€ 8217 \\
€ 2739 \\
€ 1369\end{array}$ \\
\hline & & $\begin{array}{l}\text { Montegrande } \\
\text { Montecane }\end{array}$ & parks & $\begin{array}{c}20 \text { cars park. area } \\
2 \text { buses } 30 \text { cars park. area }\end{array}$ & & $\begin{array}{l}€ 3492 \\
€ 5649\end{array}$ \\
\hline & basin & $\begin{array}{l}\text { Rocche di } \\
\text { Tornambè }\end{array}$ & $\begin{array}{l}\text { entertainment } \\
\text { equipment }\end{array}$ & 50 seats theatre & $\begin{array}{l}€ 8400 \\
€ 3360\end{array}$ & $\begin{array}{l}€ 1833 \\
€ 8000\end{array}$ \\
\hline & & $\begin{array}{l}\text { Masseria } \\
\text { D'arrigo }^{\prime}\end{array}$ & $\begin{array}{l}\text { recreational } \\
\text { equipment }\end{array}$ & $\begin{array}{c}\text { property expropriation } \\
\text { building renovation } \\
\text { external area enhancement } \\
\text { rent }\end{array}$ & $€ 30,600$ & $\begin{array}{c}€ 2,739 \\
€ 21,692 \\
€ 548\end{array}$ \\
\hline & & Montegrande & $\begin{array}{l}\text { riding } \\
\text { school, } \\
\text { bicycle } \\
\text { shelter, } \\
\text { catering }\end{array}$ & $\begin{array}{c}\text { country house renovation } \\
\text { depend. renov. (bike, stabl.) } \\
\text { riding school area renov. } \\
\text { property expropriation } \\
\text { rent }\end{array}$ & $€ 17,100$ & $\begin{array}{l}€ 7669 \\
€ 2629 \\
€ 2739 \\
€ 2739\end{array}$ \\
\hline & & $\begin{array}{l}\text { whole basin } \\
\text { management }\end{array}$ & $\begin{array}{l}\text { personnel } \\
\text { vehicles } \\
\text { materials }\end{array}$ & $\begin{array}{c}\text { personnel } \\
\text { all-terrain vehicles } \\
\text { supplies }\end{array}$ & & $\begin{array}{l}€ 108,000 \\
€ 6222 \\
€ 2000\end{array}$ \\
\hline & \multirow{9}{*}{ site } & $\begin{array}{l}\text { Donna Ricca } \\
\text { - Rancitito }\end{array}$ & $\begin{array}{l}\text { maintenance, } \\
\text { development, } \\
\text { valorization }\end{array}$ & $\begin{array}{l}\text { expropriation of land } \\
\text { necropolis cleaning } \\
\text { road panel installation } \\
\text { site panel installation }\end{array}$ & & $\begin{array}{l}€ 234 \\
€ 274 \\
€ 985 \\
€ 59\end{array}$ \\
\hline & & Monte Cane & $\begin{array}{l}\text { development, } \\
\text { valorization }\end{array}$ & $\begin{array}{l}\text { expropriation of land } \\
\text { panel installation }\end{array}$ & & $\begin{array}{l}€ 148 \\
€ 394\end{array}$ \\
\hline & & $\begin{array}{l}\text { Miniera di } \\
\text { Monte Cane }\end{array}$ & $\begin{array}{l}\text { maintenance, } \\
\text { development, } \\
\text { valorization }\end{array}$ & $\begin{array}{c}\text { expropriation of land } \\
\text { mine, scatt. houses recov. } \\
\text { site area pedestrian paths } \\
\text { panel installation }\end{array}$ & & $\begin{array}{c}€ 1,643 \\
€ 16,433 \\
€ 411 \\
€ 1182\end{array}$ \\
\hline & & Fastuchera & $\begin{array}{l}\text { development, } \\
\text { protection }\end{array}$ & $\begin{array}{l}\text { expropriations, land use } \\
\text { fencing }\end{array}$ & & $\begin{array}{l}€ 145 \\
€ 548\end{array}$ \\
\hline & & Rocche & $\begin{array}{l}\text { maintenance, } \\
\text { development, } \\
\text { valorization }\end{array}$ & $\begin{array}{l}\text { expropriation of land } \\
\text { roads maintenance } \\
\text { panel installation } \\
\text { necropolis cleaning }\end{array}$ & & $\begin{array}{l}€ 659 \\
€ 616 \\
€ 394 \\
€ 1096\end{array}$ \\
\hline & & Tornambè & $\begin{array}{l}\text { development, } \\
\text { valorization }\end{array}$ & $\begin{array}{c}\text { property expropriation } \\
\text { ruins recovery } \\
\text { photovoltaic panels }\end{array}$ & $€ 8333$ & $\begin{array}{l}€ 329 \\
€ 3,287 \\
€ 5641\end{array}$ \\
\hline & & Anagargi & $\begin{array}{l}\text { development, } \\
\text { protection }\end{array}$ & $\begin{array}{l}\text { expropriations, land use } \\
\text { fencing }\end{array}$ & & $\begin{array}{c}€ 2,028 \\
€ 548\end{array}$ \\
\hline & & $\begin{array}{l}\text { Sorgente } \\
\text { Giorgio }\end{array}$ & $\begin{array}{l}\text { development, } \\
\text { valorization }\end{array}$ & $\begin{array}{l}\text { expropriations, land use } \\
\text { panel installation }\end{array}$ & & $\begin{array}{l}€ 706 \\
€ 591\end{array}$ \\
\hline & & Montegrande & $\begin{array}{l}\text { maintenance, } \\
\text { development, } \\
\text { valorization }\end{array}$ & $\begin{array}{l}\text { expropriations, land use } \\
\text { panel installation } \\
\text { maintenance of the lane }\end{array}$ & & $\begin{array}{l}€ 769 \\
€ 1182 \\
€ 205\end{array}$ \\
\hline
\end{tabular}


Table 4. Summary of the actions, revenues and costs of the promoting program.

\begin{tabular}{|c|c|c|c|c|c|c|}
\hline Policy & Scale & Area & Action & Item & $\begin{array}{c}\text { Annual } \\
\text { Revenues }\end{array}$ & $\begin{array}{c}\text { Annual } \\
\text { Costs }\end{array}$ \\
\hline \multirow{27}{*}{ 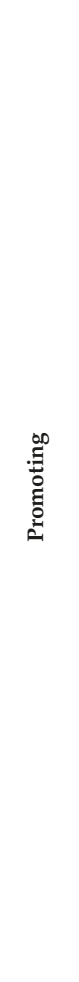 } & \multirow{8}{*}{ territory } & \multirow{8}{*}{$\begin{array}{c}\text { Enna } \\
\text { Province }\end{array}$} & \multirow{8}{*}{ territorial marketing } & tour operators & & $€ 4000$ \\
\hline & & & & Azienda Provinciale Turismo & & $€ 4000$ \\
\hline & & & & editing & & $€ 6000$ \\
\hline & & & & & & $€ 4,000$ \\
\hline & & & & tourist attraction & & $€ 6000$ \\
\hline & & & & event organization (exhibitions, & & $€ 6000$ \\
\hline & & & & book presentations, etc.) & & $€ 3000$ \\
\hline & & & & service packages organization & & $€ 2000$ \\
\hline & \multirow{8}{*}{ basin } & Tornambè & internet web page & GIS professionals & & $€ 2000$ \\
\hline & & \multirow{7}{*}{ whole basin } & \multirow{4}{*}{ botanical laborarory } & expropriation and use of land & & $€ 164$ \\
\hline & & & & shelter & & $€ 1972$ \\
\hline & & & & supplies & & $€ 2400$ \\
\hline & & & & teachers & & $€ 2000$ \\
\hline & & & ordinary tours & $\begin{array}{l}\text { full tickets } \\
\text { reduced tickets }\end{array}$ & & \\
\hline & & & guided tours & $\begin{array}{c}\text { tour guides trainer } \\
\text { tour guides refresher courses } \\
\text { visits }\end{array}$ & & $\begin{array}{l}€ 1575 \\
€ 300\end{array}$ \\
\hline & & & audio guides & gps audio guide & & $€ 657$ \\
\hline & \multirow{11}{*}{ site } & \multirow{11}{*}{ all sites } & \multirow{11}{*}{$\begin{array}{c}\text { archaeological } \\
\text { tourism, } \\
\text { archaeological } \\
\text { laboratories, school } \\
\text { camps }\end{array}$} & teachers & \multirow{11}{*}{$€ 31,500$} & $€ 50,400$ \\
\hline & & & & facilities & & $€ 3150$ \\
\hline & & & & users & & \\
\hline & & & & vehicles & & $€ 4200$ \\
\hline & & & & tools & & $€ 420$ \\
\hline & & & & shelter & & $€ 1200$ \\
\hline & & & & supplies & & $€ 1050$ \\
\hline & & & & $\begin{array}{c}\text { director } \\
\text { archaeologists }\end{array}$ & & $\begin{aligned} € 8400 \\
€ 25,200\end{aligned}$ \\
\hline & & & & restorers & & $€ 16,800$ \\
\hline & & & & $\begin{array}{l}\text { GIS professionals and } \\
\text { topographers }\end{array}$ & & $€ 16,800$ \\
\hline & & & & archaeological remains analyst & & $€ 25,200$ \\
\hline
\end{tabular}

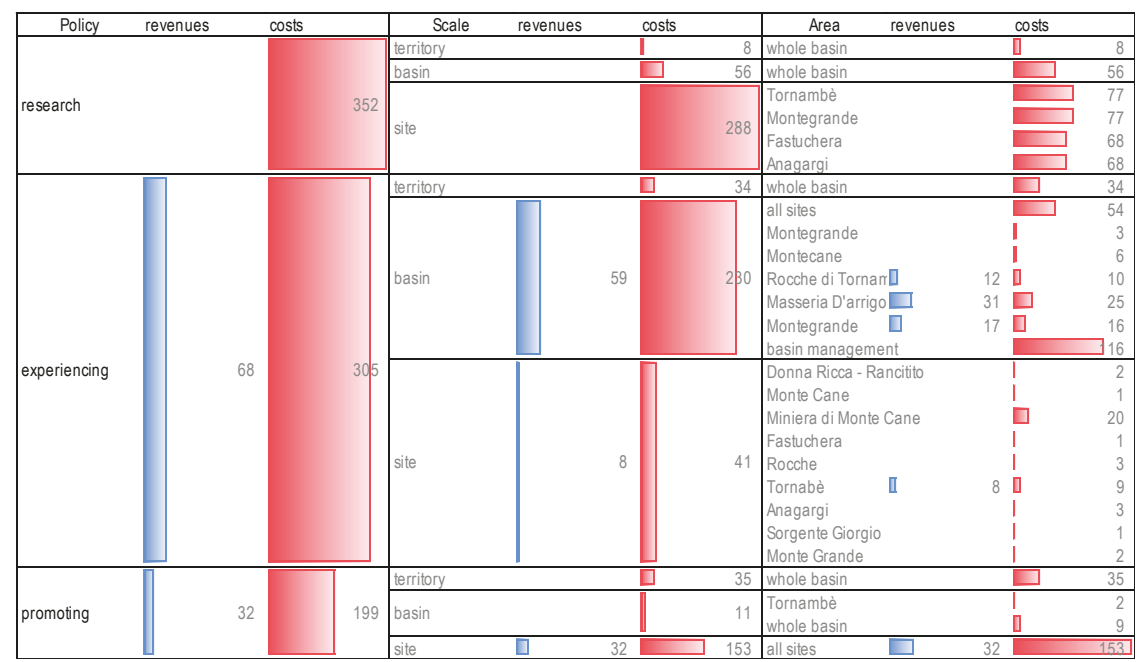

Figure 13. Summary and histogram of the main components of costs and revenues by policy and scale (EUR'000). 


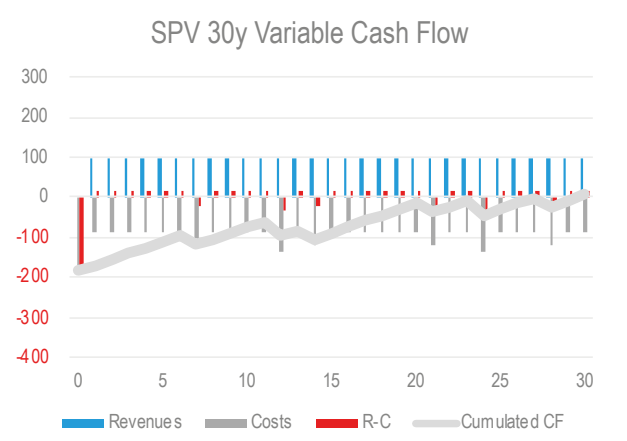

SPV 30y Discounted Variable Cash Flow

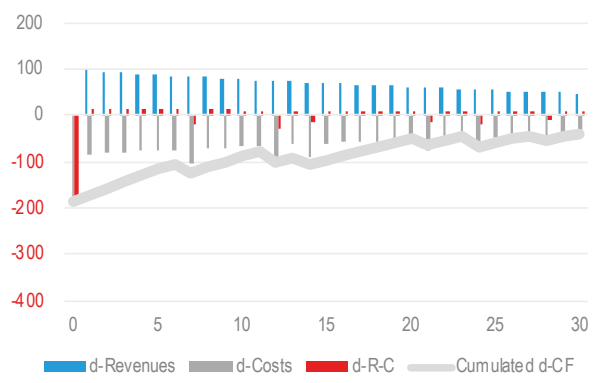

SPV 30y Constant Cash Flow

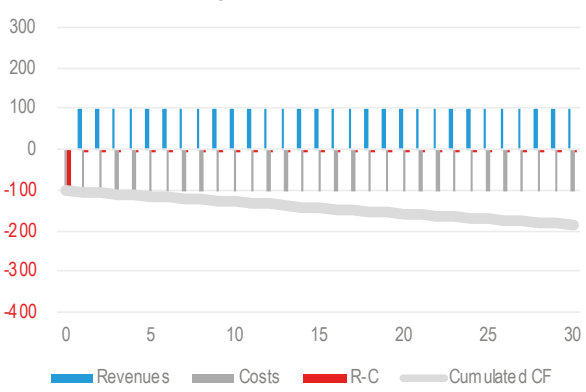

SPV 30y Discounted Constant Cash Flow

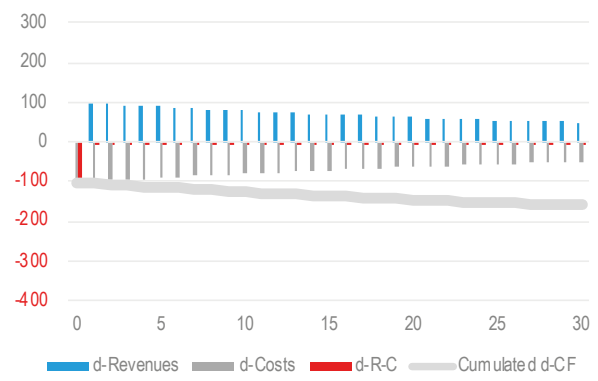

Figure 14. Variable and constant not discounted and discounted costs, revenues, annual and cumulated, cash flows (R-C) for the private player (SPV) over the 30y lifetime (abscissa: years; ordinate: EUR'000).

This early hypothesis has been evaluated according to the above-explained economic results (NPV and TRR) and financial indices (IRR, ERR, AP, DPP) calculated taking into account the four above mentioned arrangements of the cash flow (Table 5).

Table 5. Actions, revenues and costs of the promoting program.

\begin{tabular}{|c|c|c|c|c|c|c|c|}
\hline \multirow[b]{2}{*}{ Type of Cash Flow } & \multirow[b]{2}{*}{$\begin{array}{c}N P V \\
\text { (EUR'000) }\end{array}$} & \multicolumn{6}{|c|}{ Hypothesis 1} \\
\hline & & $T R R$ & $I R R$ & $E R R$ & $E_{r}$ & $\begin{array}{c}A P \\
\text { (years) }\end{array}$ & $\begin{array}{c}D P P \\
\text { (years) }\end{array}$ \\
\hline Variable cash flow & 6 & $0.2 \%$ & $0.3 \%$ & $1.89 \%$ & - & 389 & 29 \\
\hline Discounted Variable cash flow & -39 & $-1.8 \%$ & $-2.1 \%$ & $1.32 \%$ & 0.89 & n.s. & $>30$ \\
\hline Constant cash flow & -185 & $-5.8 \%$ & n.s. & n.s. & - & 7 & n.s. \\
\hline Discounted Constant cash flow & -161 & $-7.1 \%$ & n.s. & n.s. & 0.11 & 5 & n.s. \\
\hline
\end{tabular}

These early results and the comparison between variable and constant cash flow show the advantage of the absence of interests for amortizations charges in the former hypothesis, while the comparison between not discounted and discounted cash flow shows the advantage of a zero-discount approach in the field of "landscape impact investments" in which, i.e., the public interest is the primary and original scope and the cultural heritage is the original asset of the fixed overhead capital.

At this stage is clearly shown the non-cost-effectiveness of the hypothesis that revenues keep being constant, that is contrary to the main concern of a multiscale/multiplayer process supporting the development of research (through the extension of the excavation campaigns) as the original condition 
to trigger a self-powering information-based economic process [99] starting from the most structural of the cultural patterns, the archaeological one.

\subsection{Landscape Communication: Political, Cultural, Social and Entrepreneurial Accountability}

As above mentioned, such a complex project involves landscape as the general cognitive/project dimension of the common act. The landscape is the ontological reference basing on which the identity of the settled communities arises: the more featured the local identity, the stronger the global connection and the opening to different people and cultures: as such, landscape involves and coordinates the political, cultural, social and entrepreneurial accountability.

In the proposed project, the subjects sharing this responsibility are a private Special Purpose Vehicle (SPV), the Sicilian Regional Authorities, the Enna Regional Province, the Municipality of Pietraperzia, the regional Universities, the local banks.

The management of the actions foreseen by the project could be carried out by a project company with the following tasks:

- dialogue with local administrations to obtain public funding,

- $\quad$ negotiation of credit lines with local private banks,

- negotiation of concessions for the management of strategic areas of the basin,

- promotion of activities that provide financial income such as visits, shows, workshops, workshops, excursions, etc.,

- dialogue with cultural associations and public institutions (schools and universities) for the organization of cultural and educational activities,

- integration of the activities of the local food and wine sector and tourism services (catering, accommodation, tourist guides, etc.).

Given the central role played by the SPV, a risk profile can be outlined assuming that a private corporate takes:

- minor technical risks coming from the archaeological settlements, such as the enhancement of the road networks, the on-site cultural and educational activities, as for some logistic unexpected events due to possible meteorological instability and so on,

- relevant commercial risk, due to the uncertainty of the tourists'/users' attraction response, especially in the short run,

- relevant management risk, concerning the continuity of the cash flow, due to the multiplicity of the players directly and indirectly involved, and to the articulation of the commercial and company policy as for tickets, fees and prices, given the lack of a single demand function, and to the interdependence of the different ones,

- medium financial risk, given the complementarity of two opposite circumstances: the generally low-level of the interest rate on the one hand, and the unbalance between the little revenues and great costs on the other hand,

- relevant political risk, due to the long run of the time span typically involved by cultural asset heritage development programs, and the generally high level of social time preference rate in a fragile socio-economic territorial context.

In order to control the aforementioned risk factors, the SPV can implement a multiplayer strategy coordinating the action of the complementary subjects, basing on their areas of competence and responsibility:

- the SPV should include tour operators with territorial marketing skills, restaurateurs and hoteliers offering innovative tourist packages or products, owners of land and properties included in the project area, construction companies that carry out infrastructure and building works, 
- cultural or youth associations can participate in surveillance, visits, shows and special events, publishers can promote information products on the basin,

- $\quad$ the Sicilian Region would constitute the main source of public funding, which can also come from the European Structural Funds,

- $\quad$ the Enna Regional Province could promote and finance cultural and recreational activities such as workshops,

- $\quad$ the Municipality of Pietraperzia could start the expropriation process of land and buildings to be granted to the SPV,

- $\quad$ Sicilian Universities could guide archaeological research, edit publication of results and provide teachers for educational laboratories,

- $\quad$ local banks could open subsidized credit lines as a guarantee from the Municipality and sponsor theatrical events by supporting the costs of building temporary structures.

Accordingly, two project hypotheses have been proposed.

The first one concerns the cost allocation between the private and public players (Figure 15):

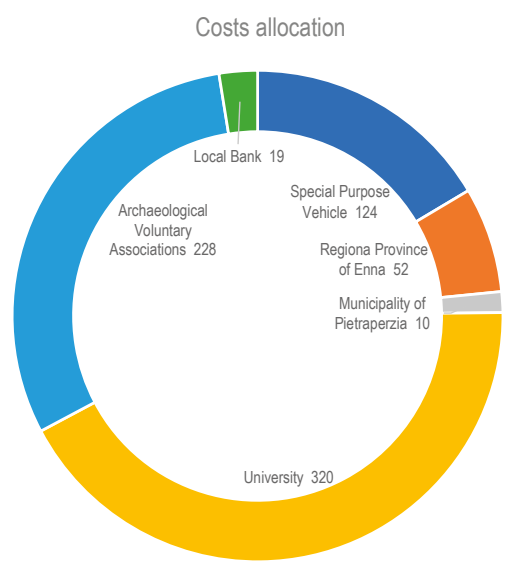

Figure 15. Cost allocation (EUR'000).

The second one concerns the revenues dynamics as a result of the progressive extension of the archaeological research and the excavation campaigns.

Four further scenarios have been outlined trying to take into account the advantages of a wider cultural/contemplative experience thus the greater attraction of the archaeological-landscape basin.

All of the scenarios assume revenue growing according to variable rates and more or less intensely over the project lifetime, in particular a multiplier of the basic revenue, assumes year by year values less, equal or greater than 1 , and constant or variable in different time spans of the 30-years project lifetime.

The five scenarios (including the basic one, whose multiplier is the constant value 1) are displayed in Figure 16, comparing the non-discounted layouts to the discounted ones.

For each of these layouts, the economic results and the financial indices have been calculated. The graphs of Figure 17 compare the scenarios 2-5 displaying the streams of revenues, costs, and annual and cumulated cash flows, only for the constant discounted cash flow, that is the less cost-effective. The tables underneath report the indices for each revenue layout (hypotheses 2-5) and for all the types of cash flow, as well as shown in Table 4. 

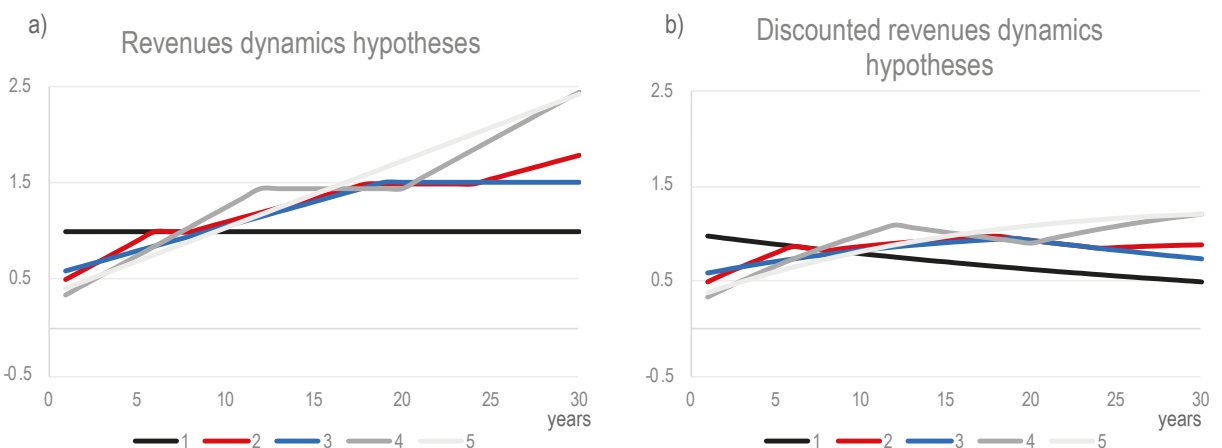

Figure 16. Revenue dynamics scenarios: (a) non-discounted layouts, (b) discounted layouts.
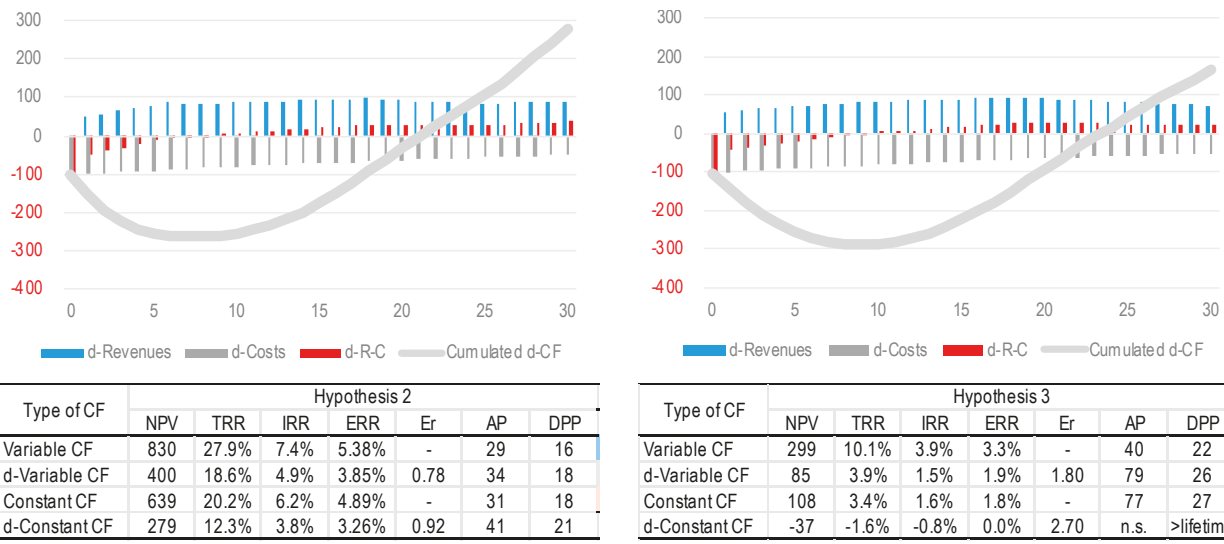

\begin{tabular}{|l|c|c|c|c|c|c|c|}
\hline \multirow{2}{*}{ Type of CF } & \multicolumn{7}{|c}{ Hypothesis 3 } \\
& NPV & TRR & IRR & ERR & Er & AP & DPP \\
\hline Variable CF & 299 & $10.1 \%$ & $3.9 \%$ & $3.3 \%$ & - & 40 & 22 \\
\hline d-Variable CF & 85 & $3.9 \%$ & $1.5 \%$ & $1.9 \%$ & 1.80 & 79 & 26 \\
\hline Constant CF & 108 & $3.4 \%$ & $1.6 \%$ & $1.8 \%$ & - & 77 & 27 \\
\hline d-Constant CF & -37 & $-1.6 \%$ & $-0.8 \%$ & $0.0 \%$ & 2.70 & n.s. & >lifetime \\
\hline
\end{tabular}
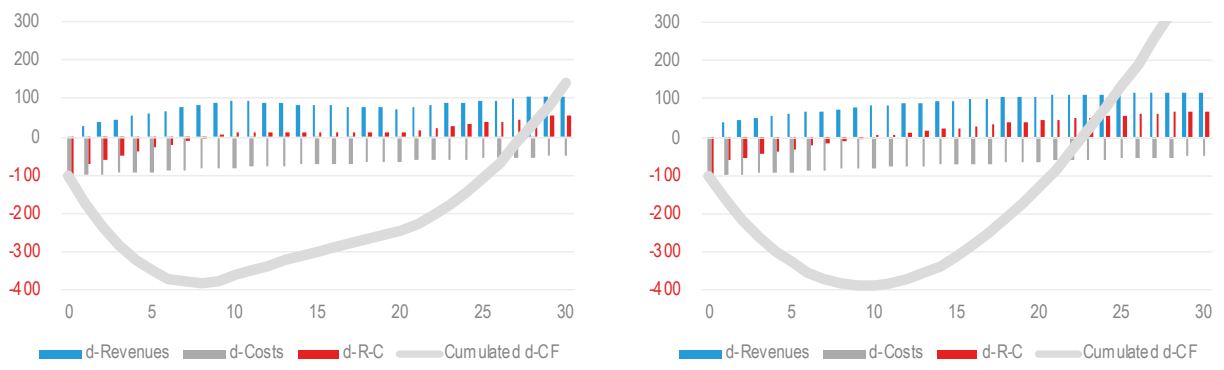

\begin{tabular}{|l|c|c|c|c|c|c|c|}
\hline \multirow{2}{*}{ Type of CF } & \multicolumn{7}{|c|}{ Hypothesis 4 } \\
& NPV & TRR & IRR & ERR & Er & AP & DPP \\
\hline Variable CF & 701 & $23.6 \%$ & $5.1 \%$ & $4.1 \%$ & - & 35 & 22 \\
\hline d-Variable CF & 261 & $12.2 \%$ & $2.6 \%$ & $2.5 \%$ & 1.18 & 52 & 25 \\
\hline Constant CF & 510 & $16.1 \%$ & $3.9 \%$ & $3.5 \%$ & - & 41 & 24 \\
\hline d-Constant CF & 140 & $6.2 \%$ & $1.5 \%$ & $1.8 \%$ & 1.83 & 81 & 27 \\
\hline
\end{tabular}

\begin{tabular}{|c|c|c|c|c|c|c|c|}
\hline \multirow{2}{*}{ Type of CF } & \multicolumn{7}{|c|}{ Hypothesis 5} \\
\hline & NPV & TRR & IRR & ERR & Er & AP & DPP \\
\hline Variable CF & 1242 & $41.8 \%$ & $7.2 \%$ & $5.5 \%$ & - & 30 & 18 \\
\hline d-Variable CF & 577 & $26.8 \%$ & $4.7 \%$ & $3.9 \%$ & 0.82 & 36 & 20 \\
\hline Constant CF & 1051 & $33.2 \%$ & $6.4 \%$ & $5.1 \%$ & - & 32 & 20 \\
\hline d-Constant CF & 456 & $20.1 \%$ & $3.9 \%$ & $3.4 \%$ & 0.92 & 41 & 22 \\
\hline
\end{tabular}

Figure 17. Economic-financial patterns of the discounted continuous cash flow of hypotheses 2-5. Tables: first column EUR'000; graphs: abscissa: years; ordinate: EUR'000.

Some comments on these results concerns:

- as first, the strategic role played by the stream of revenues and, indirectly, by the public recognition of the archaeological basin that should be created connecting the more strategic territorial values in an internally coherent landscape unit characterized by a strong identity, 
- as second, in order to better focus the following discussions, the difference between variable and constant cash flow, the former providing better results, thus the role played by the stockholders and, as a consequence, the impact of the interests of the amortizations,

- as third—starting from the results above displayed providing a wide range of information-the complementary relation between profitability and risk that assumes a specific connotation in the field of the social capital and typically in the case of the cultural asset, as more in-depth discussed below.

These final multifaceted results, corresponding to different hypotheses of revenue dynamics, discounting, and costs distribution (with and without amortization) are the basis for the following critical interpretation of the economic-financial indices listed and compared above, in the prospect of supporting a future-oriented entrepreneurial behavioural pattern.

\section{Discussions. The Economic-Financial Variables as Cultural and Social Communication Media}

As anticipated, a detailed discussion on the results of this experiment integrating territorial, socio-economic and landscape communication, can be carried out by considering the multiple interpretations of each scenario from the perspective of the different type of indices. Between the four types of cash flows, only the discounted cash flows have been considered in this comparison, in order to take into account the impact of the interests included in the amortisations.

The comparison of the four scenarios has been carried out by normalizing the results and indices-expressed in different measurement units (EUR, percentage, years) (Figure 18a)-according to a standard scale of dimensionless scores (Figure 18b). A sort of "average scenario" has been outlined by calculating the average values of all the indices (18a) and scores (18b) in order to provide a benchmark for each of the four scenarios.

Two further final indexes have been calculated:

- $\quad$ the "rate of deferral" $\left(R_{\text {Defer }}\right)$, calculated as the weighted average time of deferral of the revenues (second to the last column of the table in Figure 18a),

- $\quad$ the "overall revenue amount rate" $\left(R_{A m}\right)$, calculated as the average multiplier of the revenue considered equal to 1 in the basic scenario (last column of the table in Figure 18a).

The discussion of these results proposes a comparison between the orthodox economic-financial approach and a possible heterodox perspective, the latter coming from the Keynesian and post-Keynesian financial speculative approach. Particularly, especially in the field of public economy, speculation needs to be assumed in its original meaning (excluding any ethical bias) mostly aimed at the monetary dynamics of a capital asset—basing on its risk/return profile-as well as the financial situation of the of players involved, that in this case define the social, cultural and political-administrative reference territorial framework. 
a)

\begin{tabular}{|c|c|c|c|c|c|c|c|c|c|}
\hline & NPV & TRR & IRR & ERR & Er & AP & DPP & RDefer & $R A m$ \\
\hline Hypothesis 2 & 279 & $12.3 \%$ & $3.8 \%$ & $3.26 \%$ & 0.9 & 41 & 21 & 15.5 & 1.3 \\
\hline Hypothesis 3 & 165 & $7.3 \%$ & $2.4 \%$ & $2.39 \%$ & 1.3 & 55 & 23 & 17.7 & 1.2 \\
\hline Hypothesis 4 & 485 & $21.4 \%$ & $4.7 \%$ & $3.81 \%$ & 0.8 & 36 & 21 & 17.6 & 1.4 \\
\hline Hypothesis 5 & 456 & $20.1 \%$ & $3.9 \%$ & $3.42 \%$ & 0.9 & 41 & 22 & 18.7 & 1.4 \\
\hline Average & 346 & $15.2 \%$ & $3.7 \%$ & $3.22 \%$ & 1.0 & 43 & 22 & 17.4 & 1.3 \\
\hline
\end{tabular}

b)

\begin{tabular}{|l|c|c|c|c|c|c|c|}
\hline & NPV & TRR & IRR & ERR & Er & AP & DPP \\
\hline Hypothesis 2 & 1.36 & 1.36 & 1.59 & 1.61 & 1.76 & 1.77 & 2.00 \\
\hline Hypothesis 3 & 1.00 & 1.00 & 1.00 & 1.00 & 1.00 & 1.00 & 1.00 \\
\hline Hypothesis 4 & 2.00 & 2.00 & 2.00 & 2.00 & 2.00 & 2.00 & 2.00 \\
\hline Hypothesis 5 & 1.91 & 1.91 & 1.67 & 1.72 & 1.78 & 1.78 & 1.50 \\
\hline Average & 1.57 & 1.57 & 1.57 & 1.58 & 1.64 & 1.64 & 1.63
\end{tabular}

d)

\begin{tabular}{|l|c|c|c|c|c|c|c|}
\hline & NPV & TRR & IRR & ERR & Er & AP & DPP \\
\hline Hypothesis 2 & 1.36 & 1.36 & 1.59 & 1.61 & 1.24 & 1.23 & 1.00 \\
\hline Hypothesis 3 & 1.00 & 1.00 & 1.00 & 1.00 & 2.00 & 2.00 & 2.00 \\
\hline Hypothesis 4 & 2.00 & 2.00 & 2.00 & 2.00 & 1.00 & 1.00 & 1.00 \\
\hline Hypothesis 5 & 1.91 & 1.91 & 1.67 & 1.72 & 1.22 & 1.22 & 1.50 \\
\hline Average & 1.57 & 1.57 & 1.57 & 1.58 & 1.36 & 1.36 & 1.38 \\
\hline
\end{tabular}
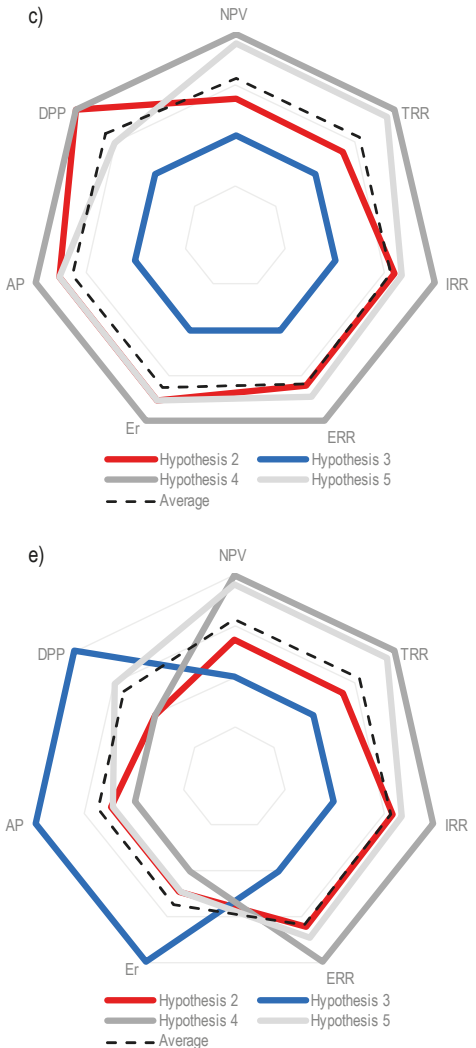

Figure 18. Orthodox and heterodox interpretations of the economic, financial and monetary performances of the four scenarios. (a) Economic financial results. (b) Orthodox interpretation: normalization of the economic and financial indices. (c) Graphic comparison of the four scenarios according to the orthodox interpretation. (d) Heterodox interpretation: normalization of the economic and financial indices. (e) Graphic comparison of the four scenarios according to the heterodox interpretation.

Furthermore, although this DCFA experiment focuses on the goods and services that can be placed on the market, the economic-financial-monetary representation tool needs to be placed within a process that is triggered and guided by the political-administrative system as a consequence of a preliminary agreement between all the players involved, converging toward the logic of the "Cultural Asset Impact Investments".

This approach is concretely applied here assuming the seven indices according to their specific economic, financial and monetary significance:

- $\quad$ NPV and TRR can be considered as "economic" choice criteria whose significance concerns the amount of revenue and costs, and whose reference is the "opportunity cost of capital",

- $\quad$ ERR and IRR can be considered "financial" criteria whose significance mostly concerns the time allocation of revenues and costs, and whose reference are respectively the Minimum Acceptable Rate of Return (MARR) and the discount rate,

- $\quad E_{r}, A P$ and $D P P$, mostly concerning the amount and at the same the deferral of the revenues (given the costs), although commonly considered as financial indices as well, have been here assumed as for their further "monetary" significance. This particular interpretation refers to the 
"propensity to expect" whose higher level typically characterizes low-risk or free-risk investments, such as the one developing in specific situations of complementarity and convergence of private and public interest, according to the concept of "social benefit".

Figure 18 displays the comparison of the orthodox and heterodox approaches. The first one, synthesized in the radar graph of Figure 18c, shows the almost concentric placement and size of the four scenarios hypotheses within the indices polygon area and with respect to the aforementioned "average scenario".

In such an interpretation, we notice that:

- $\quad$ hypothesis 4 results the most profitable and sustainable mostly due to the highest value of $R_{A m}$ (1.4), despite the significant deferral $\left(R_{\text {Defer }}=17.6\right)$. Symmetrically, hypothesis 3 results the less profitable and sustainable mostly due to the lowest value of $R_{A m}$ (1.2), and the significant deferral $\left(R_{\text {Defer }}=17.7\right)$. Both hypotheses and the average layout are perfectly concentric,

- $\quad$ the asymmetry of hypotheses 2 and 5 suggest a sort of complementarity of economic and monetary performances supporting some reflections about a possible heterodox approach.

Such an approach can be better discussed by assuming the deferral of the revenues as a positive value, once the ability of the public to guarantee the continuity of the project is recognized by the private player. As a consequence the monetary performances such as Elasticity $\left(E_{r}\right)$, Average Period $(A P)$ and Discounted Payback Period $(D P P)$ can be appreciated according to Figure $18 \mathrm{~d}$ so that the ranking of the hypotheses changes according to the radar graph of Figure 18e.

As a result, two new asymmetries are highlighted: on the one hand the minor between hypotheses 2 and 4 . On the other hand, the former poorest hypothesis 3 overcomes the average layout as well as hypothesis 2 , and a more evident asymmetry in respect of all the other layout is highlighted. At the same time hypothesis, 4 is slightly preferable to the others.

Some final remarks highlight the main limitation of this research. A first limitation concerns the uncertainty of the costs of the project implementation, that could vary based on the more detailed description of the intervention area. A second uncertainty factor concerns the impact of the information and communication system (WebGIS and territorial marketing) on the shared knowledge of the activities promoting the archaeological-cultural basin.

Finally, the dialectic between the orthodox and heterodox interpretations could be more widely founded by detecting the integration of the private and public commitments in similar investments over the regional territory. Such a further study would provide basic information about of the amount of the marketable goods and services, in reference to the composition and the agreements between the private and public players about the budgeting and the cost allocation.

\section{Conclusions}

The conceptual premises of this project-valuation experiment, focus on the potential virtuous circle connecting research, experiencing and promoting in the perspective of increasing the scientific knowledge and public awareness of the cultural-territorial value. This work takes up the challenge of integrating the contemplative and cultural experience in the promotion of activities involving a composite set of public and private players.

This prospect outlines the conditions for hypothesizing that, despite the evidence of an orthodox economic approach, typically inspired by a microeconomic vision, the context feeds-back on the perception of profitability by the entrepreneurs involved in a more general "landscape impact investment" experience. In fact, a traditional approach inspired by the logic of environmental responsible investments (according to the Social Responsible Investments—SRI—approach [100,101]) focuses on the compatibility between private revenues and social benefits. In this case, instead, we envisaged actions, players and scales able to trigger a positive territorial impact (the end) basing on the individual economic cost-effectiveness (the means). 
In fact, in such a project-decision context, mainly aimed at triggering local impact over a vast land area, this feed-back involves the decisions (concerning the expectations) as well as the underlying evidence (concerning the observations) that can be interpreted differently by the entrepreneurs according to the shared awareness to be part to an overarching socio-economic subject.

The convergence of natural evidence (the cultural landscape values) and cultural intentionality (the perspectives and prospects of players and users) encourage the individual entrepreneurial profiles to join giving rise to a territorial-economic unitary context where the sum of the individual choices creates a sort of collective intelligence typical of the social advanced communities.

In such a research area, a final remarkable issue concerning the achievements of evaluation science is the mutual causality - that is the expression of the two-way exchange of information -between valuation and project, surprisingly by means of economic-financial analysis when the project involves the cognitive/emotional individual/collective sphere of the land cultural asset.

In fact, whereas usually, the financial analysis holds creativity to the limitation of economic efficiency and financial feasibility, in this case, the collective project prospect, powered by the coordination of the landscape multiple dimensions, influence in turn the entrepreneurial creativity and the interpretation of the economic, financial and monetary indices.

According to the results of the economic analysis, further studies could be carried out in the field of operational research, thus transforming the aforementioned normalization of the indices in a Multi-Criteria Decision Making (MCDM) process involving the scores of the four scenario hypotheses, and the relative importance of each of them compared to the others, measured by a weight system. Furthermore, MCDM could be assumed as the communicative platform enveloping the whole cognitive, evaluative and creative process over which stake-holders and stock-holders meet combining and integrating cultural, professional and managerial resources: in this widened study context, the monetary abstraction would cover just a part of the whole decision-making path, actually involving the concrete values as the contents of specifically outlined value functions, and their relative importance according to an overarching weight system.

Typically, a weight system implements some theoretical assumptions coming from the constructivist philosophical approach, according to which the social reality-by definition, a linguistic elaboration-can be represented, and as a consequence constructed, as a result of an epistemic agreement between "standard observer" [102] (scientists). In an extended edition of this approach, influenced by pragmatism, the agreement involves social players converging toward shared objectives, thus prescinding from the truth. In fact, it should be noticed that the aforesaid agreement involves the programmes and the selective codes of conflicting and/or and cooperating social sub-systems. In general, such an extension values the predictive success, or at most, utility, that in the general context of land-urban policy, characterizes the programming approach based on standardized and shared communication patterns.

Accordingly, the landscape communication-here assumed as the final stage of the social abstraction of "things" in the "identity feeling" bringing together a settled community — can provide further social evidence for an economic-evaluation approach integrating the orthodox perspective (ordinary interpretation of the DCFA indices) and the heterodox prospect (valuing the extraordinary opportunity of a growing social agreement), thus highlighting the importance of the "monetary shape" [103] of the private-public investment. The monetary interpretation of the territorial development programmes takes into account and values the intertemporal communication [104], thus connecting further and further generations by means of the recognition of the settled communities' ancient greatness, which is the primary and original shape of the social capital asset.

Author Contributions: Conceptualization, S.G., E.G., F.G. and C.M.; methodology, S.G. and F.G.; software, S.G., F.G. and C.M.; validation, S.G., M.R.T., C.M.; formal analysis, S.G. and M.R.T; investigation, E.G. and F.G.; resources, E.G., C.M. and F.G.; data curation, S.G. F.G.; writing-original draft preparation, S.G. G.N.; writing-review and editing, S.G., G.N. and M.R.T.; visualization, S.G., F.G. and G.N.; supervision, S.G., G.N., M.R.T.; project administration, S.G., E.G., F.G., C.M., G.N. and M.R.T. All authors have read and agreed to the published version of the manuscript. 
Funding: This research received no external funding.

Conflicts of Interest: The authors declare no conflict of interest.

\section{References}

1. Orbaşli, A. Archaeological Site Management and Local Development. Conserv. Manag. Archaeol. Sites 2013, 15, 237-253. [CrossRef]

2. Ringbeck, B. The World Heritage Convention and Its Management Concept, Aspects of Management Planning for Cultural World Heritage Sites: Principles, Approaches and Practices; Springer International Publishing: Cham, Switzerland, 2017; pp. 15-24. [CrossRef]

3. Frey, B.S.; Steiner, L. World Heritage List. In Handbook on the Economics of Cultural Heritage; Edward Elgar Publishing Ltd.: Cheltenham, UK, 2013; pp. 171-186. [CrossRef]

4. European Commission. European Framework for Action on Cultural Heritage. 2018. Available online: https: //ec.europa.eu/culture/library/commission-swd-european-framework-action-cultural-heritage_en (accessed on 31 January 2020).

5. Rizzo, F. Nuova Economia; Aracne: Roma, Italy, 2013.

6. Koens, K.; Postma, A.; Papp, B. Is Overtourism Overused? Understanding the Impact of Tourism in a City Context. Sustainability 2018, 10, 4384. [CrossRef]

7. Thomas, B.; Langlitz, M. Archaeotourism, Archaeological Site Preservation, and Local Communities, Feasible Management of Archaeological Heritage Sites Open to Tourism; Springer International Publishing: Cham, Switzerland, 2018; pp. 69-78. [CrossRef]

8. Sodangi, M.; Khamdi, M.F.; Idrus, A.; Hammad, D.B.; AhmedUmar, A. Best Practice Criteria for Sustainable Maintenance Management of Heritage Buildings in Malaysia. Procedia Eng. 2014, 77, 11-19. [CrossRef]

9. Zhang, Y.; Li, X.R.; Su, Q. Does spatial layout matter to theme park tourism carrying capacity? Tour. Manag. 2017, 61, 82-95. [CrossRef]

10. Giuffrida, S.; Trovato, M.R.; Giannelli, A. Semiotic-Sociological Textures of Landscape Values. Assessments in Urban-Coastal Areas. In Information and Communication Technologies in Modern Agricultural Development, Communications in Computer and Information Science; Salampasis, M., Bournaris, T., Eds.; Springer: Cham, Switzerland, 2019; Volume 953, pp. 35-50. [CrossRef]

11. Giuffrida, S.; Trovato, M.R. A Semiotic Approach to the Landscape Accounting and Assessment. An Application to the Urban-Coastal Areas. In Proceedings of the 8th International Conference on Information and Communication Technologies in Agriculture, Food and Environment, HAICTA 2017, Chania, Crete Island, Greece, 21-24 September 2017; Volume 2030, pp. 696-708.

12. Giannelli, A.; Giuffrida, S.; Trovato, M.R. Madrid Rio Park. Symbolic Values and Contingent Valuation; Valori e Valutazioni 21/2018, E-Flow Dei Tipografia del Genio Civile; 2018; pp. 75-85. Available online: https://siev.org/numero-21-2018/ (accessed on 20 February 2019).

13. UNWTO. 'Overturism'? Understandind and Managing Urban Tourist Growth beyond Perception. Available online: https://www.e-unwto-org/doi/book/10.18111/9789284420070 (accessed on 31 January 2020).

14. Moira, P.; Mylonopoulos, D.; Kondoudaki, A. The Application of Slow Movement to the Tourism: Is Slow Tourism a New Paradigma? J. Tour. Leis. Stud. 2017, 2, 1-10. [CrossRef]

15. Dickinson, J.E.; Lumsdon, L.; Robbins, D. Slow travel: issues for tourism and climate change. J. Sustain. Tour. 2011, 19, 281-300. [CrossRef]

16. 2007-2013 Italia-Slovenia. Available online: http://2007-2013.ita-slo.eu/progetti/progetti_2007_2013/ 2010083015331456 (accessed on 4 May 2020).

17. Zamparelli, G.; Del Gaudio, G. 'Destination Branding' di un Sistema Archeologico: Proposte per il Polo Pompeiano. Rivista di Scienze del Turismo 2011, 3, 131-136.

18. Calderón, H.; Cervera-Taulet, A.; Molla, A. Brand assessment: A key element of marketing strategy. J. Prod. Brand Manag. 1997, 6, 293-304. [CrossRef]

19. Cameron, C.; Rössler, M. Introduction of Management Planning for Cultural World Heritage Sites, Aspects of Management Planning for Cultural World Heritage Sites: Principles, Approaches and Practices; Springer International Publishing: Cham, Switzerland, 2017; pp. 3-13. [CrossRef]

20. Blandford, C. Management Plans for UK World Heritage Sites: Evolution, lessons and good practice. Landsc. Res. 2006, 31, 355-362. [CrossRef] 
21. Holler, M.J.; Mazza, I. Cultural Heritage: Public Decision-Making and Implementation. In Handbook on the Economics of Cultural Heritage; Rizzo, I., Mignosa, A., Eds.; Edward Elgar Publishing Ltd.: Cheltenham, UK, 2013; pp. 17-36. [CrossRef]

22. Trovato, M.R.; Giuffrida, S. A DSS to Assess and Manage the Urban Performances in the Regeneration Plan: The Case Study of Pachino. In Computational Science and Its Application ICCSA 2014; LNCS 8581; Murgante, B., Ed.; Springer International Publishing: Cham, Switzerland, 2014; Part III; pp. 224-239. [CrossRef]

23. Trovato, M.R.; Giuffrida, S. The choice problem of the urban performances to support the Pachino's redevelopment plan. Int. J. Bus. Intell. Data Min. 2014, 9, 330. [CrossRef]

24. Guarini, M.R.; D’Addabbo, N.; Morano, P.; Tajani, F. Multi-criteria analysis in compound decision processes: The AHP and the architectural competition for the Chamber of Deputies in Rome (Italy). Buildings 2017, 7, 38. [CrossRef]

25. Snowball, J.D. The Economic, Social and Cultural Impact of Cultural Heritage: Methods and Examples. In Handbook on the Economics of Cultural Heritage; Rizzo, I., Mignosa, A., Eds.; Edward Elgar Publishing Ltd.: Cheltenham, UK, 2013; pp. 438-455. [CrossRef]

26. Della Spina, L.; Calabrò, F. Decision Support Model for Conservation, Reuse and Valorization of the Historic Cultural Heritage. In Computational Science and Its Applications-ICCSA 2018; Lecture Notes in Computer Science; Gervasi, O., Ed.; Springer: Cham, Switzerland, 2018; Volume 10962. [CrossRef]

27. Trovato, M.R. A multi-criteria approach to support the retraining plan of the Biancavilla's old town. In Smart Innovation, Systems and Technologies, 3rd International New Metropolitan Perspectives, 2018; Bevilacqua, C., Ed.; Springer: Cham, Switzerland; Reggio Calabria, Italy, 2019; Volume 101, pp. 434-441. [CrossRef]

28. Elsorady, D.A. Adaptive Reuse Decision Making of a Heritage Building Antoniadis Palace, Egypt. Int. J. Arch. Heritage 2018, 14, 658-677. [CrossRef]

29. Napoli, G.; Leone, M. The urban park as a "social island". The ANP in the participatory project of Parco Uditore in Palermo. In Values and Functions for Future Cities, Green Energy and Technology; Mondini, G., Stanghellini, S., Oppio, A., Bottero, M., Abastante, F., Eds.; Springer International Publishing: Cham, Switzerland, 2019; pp. 229-248. [CrossRef]

30. Napoli, G.; Giuffrida, S.; Trovato, M.R. Efficiency versus Fairness in the Management of Public Housing Assets in Palermo (Italy). Sustainability 2019, 11, 1199. [CrossRef]

31. Hang, P.L.K.; Kong, C. Heritage management and control. J. Qual. Assur. Hosp. Tour. 2001, 2, $105-117$. [CrossRef]

32. Balaawi, F.A. Evaluating visitor management at the archaeological site of Petra. Mediterr. Archaeol. Archaeom. 2013, 13, 77-87.

33. Young, C. Understanding management in a world heritage context: Key current issues in Europe. Hist. Environ. Policy Pr. 2016, 7, 1-13. [CrossRef]

34. Apostolakis, A.; Jaffry, S. A choice modeling application for Greek heritage attractions. J. Travel Res. 2005, 43, 309-318. [CrossRef]

35. Mazzanti, M. Cultural Heritage as Multi-Dimensional, Multi-Value and Multi-Attribute Economic Good: Towards a New Framework for Economic Analysis and Valuation. J. Socio-Economics 2002, 31, 529-558. [CrossRef]

36. Bruyere, B.L.; Beh, A.W.; Lelengula, G. Differences in perceptions of communication, tourism benefits, and management issues in a protected area of rural Kenya. Environ. Manag. 2008, 43, 49-59. [CrossRef]

37. Klamer, A. The values of cultural heritage. In Handbook on the Economics of Cultural Heritage; Rizzo, I., Mignosa, A., Eds.; Edward Elgar Publishing Ltd.: Cheltenham, UK, 2013; pp. 421-437. [CrossRef]

38. Della Spina, L. Scenarios for a Sustainable Valorisation of Cultural Landscape as Driver of Local Development. In New Metropolitan Perspectives; ISHT 2018, Smart Innovation, Systems and Technologies; Calabrò, F., Della Spina, L., Bevilacqua, C., Eds.; Springer: Cham, Switzerland, 2019; Volume 100. [CrossRef]

39. Seymour, E.; Curtis, A.; Pannell, D.; Allan, C.; Roberts, A. Understanding the role of assigned values in natural resource management. Australas. J. Environ. Manag. 2010, 17, 142-153. [CrossRef]

40. Byrd, E.T. Stakeholders in sustainable tourism development and their roles: applying stakeholder theory to sustainable tourism development. Tour. Rev. 2007, 62, 6-13. [CrossRef]

41. Alazaizeh, M.M.; Hallo, J.C.; Backman, S.J.; Norman, W.C.; Vogel, M.A. Value orientations and heritage tourism management at Petra Archaeological Park, Jordan. Tour. Manag. 2016, 57, 149-158. [CrossRef] 
42. Naselli, F.; Trovato, M.R.; Castello, G. An Evaluation Model for the Actions in Supporting of the Environmental and Landscaping Rehabilitation of the Pasquasia's Site Mining (EN); ICCSA 2014, LNCS 8581; Murgante, B., Ed.; Springer International Publishing: Cham, Switzerland, 2014; Part III; pp. 26-41. [CrossRef]

43. Vlami, V.; Kokkoris, I.P.; Zogaris, S.; Cartalis, C.; Kehayias, G.; Dimopoulos, P. Cultural landscapes and attributes of "culturalness" in protected areas: An exploratory assessment in Greece. Sci. Total. Environ. 2017, 595, 229-243. [CrossRef] [PubMed]

44. Giuffrida, S. City as Hope. Valuation Science and the Ethics of Capital. In Green Energy and Technology; Mondini, G., Fattinnanzi, E., Oppio, A., Bottero, M., Stanghellini, S., Eds.; Springer: Cham, Switzerland, 2018; pp. 411-424. [CrossRef]

45. Giuffrida, S. A Fair City. Value, Time and the Cap Rate. In Green Energy and Technology; Springer: Cham, Switzerland, 2018; pp. 425-439. [CrossRef]

46. Conolly, J.; Lake, M. Geographical Information Systems in Archaeology; Cambridge University Press: Cambridge, UK, 2006; pp. 1-338. [CrossRef]

47. Maatouk, M.M.H. Management of the preservation of heritage buildings using gis (the case of historic jeddah in Saudi Arabia). J. Eng. Appl. Sci. 2018, 65, 77-99.

48. Tantillo, M.D. GIS application in archaeological site of solunto, International Archives of the Photogrammetry, Remote Sensing and Spatial Information Sciences-ISPRS Archives, Volume 36 (5/C53), 2007. In Proceedings of the 21st International CIPA Symposium 2007, Athens, Greece, 1-6 October 2007.

49. Giuffrida, S.; Gagliano, F.; Napoli, G. Agriculture and Sustainability: A GIS Based Model to Appraise Incentive Policy. In Proceedings of the 7th International Conference on Information and Communication Technologies in Agriculture, Food and Environment (HAICTA 2015), Kavala, Greece, 17-20 September 2015; Volume 1498, pp. 912-921.

50. Giuffrida, S. The True Value. On Understanding Something. In Appraisal: From Theory to Practice; Mondini, G., Fattinnanzi, E., Oppio, A., Bottero, M., Stanghellini, S., Eds.; Springer: Cham, Switzerland, 2016; pp. 1-14. ISBN 978-3-319-49675-7. [CrossRef]

51. Eppich, R.; Grinda, J.L.G. Sustainable financial management of tangible cultural heritage sites. J. Cult. Herit. Manag. Sustain. Dev. 2019, 9, 282-299. [CrossRef]

52. Wright, W.C.C.; Eppink, F.V. Drivers of heritage value: A meta-analysis of monetary valuation studies of cultural heritage. Ecol. Econ. 2016, 130, 277-284. [CrossRef]

53. Giuffrida, S.; Trovato, M.R. From the Object to Land. Architectural Design and Economic Valuation in the Multiple Dimensions of the Industrial Estates; ICCSA 2017, LNCS 10406; Borruso, G., Cuzzocrea, A., Apduhan, B.O., Rocha, A.M.A.C., Taniar, D., Misra, S., Gervasi, O., Torre, C.M., Stankova, E., Murgante, B., Eds.; Springer: London, UK, 2017; Volume III, pp. 591-606. [CrossRef]

54. Giuffrida, S.; Trovato, M.R.; Falzone, M. The Information Value for Territorial and Economic Sustainability in the Enhancement of the Water Management Process; ICCSA 2017, LNCS 10406; Borruso, G., Cuzzocrea, A., Apduhan, B.O., Rocha, A.M.A.C., Taniar, D., Misra, S., Gervasi, O., Torre, C.M., Stankova, E., Murgante, B., Eds.; Springer: London, UK, 2017; Volume III, pp. 575-590. [CrossRef]

55. Napoli, G. The Value of the Useless in the Urban Landscape of Small Islands. In Proceedings of the 18th IPSAPA/ISPALEM International Scientific Conference: The Usefulness of the Useless in the Landscape Cultural Mosaic: Liveability, Typicality, Biodiversity, Catania, Italy, 3-4 July 2014; pp. 333-339.

56. Giuffrida, S.; Trovato, M.R.; Circo, C.; Ventura, V.; Giuffrè, M.; Macca, V. Seismic Vulnerability and Old Towns. A Cost-Based Programming Model. Geosciences 2019, 9, 427. [CrossRef]

57. Gabrielli, L.; Giuffrida, S.; Trovato, M.R. From Surface to Core: A Multi-Layer Approach for the Real Estate Market Analysis of a Central Area in Catania; ICCSA 2015, LCNS 9157; Gervasi, O., Murgante, B., Misra, S., Eds.; Springer: London, UK, 2015; Volume III, pp. 284-300. [CrossRef]

58. Gabrielli, L.; Giuffrida, S.; Trovato, M.R. Functions and Perspectives of Public Real Estate in the Urban Policies: The Sustainable Development Plan of Syracuse; ICCSA 2016, LNCS 9789; Gervasi, O., Ed.; Springer: London, UK, 2016; Volume IV, pp. 13-28. [CrossRef]

59. Gabrielli, L.; Giuffrida, S.; Trovato, M.R. Gaps and overlaps of urban housing sub market: a fuzzy clustering approach. In Appraisal from Theory to Practice, Green Energy and Technology; Stanghellini, S., Morano, P., Bottero, M., Oppio, A., Eds.; Springer: Cham, Switzerland, 2017; pp. 203-219. [CrossRef] 
60. Napoli, G. The complexity of value and the evaluation of complexity: social use value and multi-criteria analysis. In Integrated Evaluation for the Management of Contemporary Cities; Results of SIEV 2016, Serie: Green Energy and Technology; Mondini, G., Fattinnanzi, E., Oppio, A., Bottero, M., Stanghellini, S., Eds.; Springer International Publishing: Cham, Switzerland, 2018; pp. 187-198. [CrossRef]

61. MIBACT, Ministero dei Beni e delle Attività Culturali e del Turismo, Ufficio Statistica, 2017. Available online: http://www.statistica.beniculturali.it (accessed on 4 May 2020).

62. Regione Siciliana, Dipartimento dei Beni Culturali, 2018. Available online: http://www.regione.sicilia.it/ beniculturali/dirbenicult/musei/museifruizione.html (accessed on 4 May 2020).

63. Giannitrapani, E. Aspetti culturali e dinamiche del popolamento di età preistorica della provincia di Enna. Quaderni del Patrimonio Culturale Ennese 2012, 145-181.

64. Giannitrapani, E.; Iannì, F. Tornambé, Pietraperzia, prov. di Enna-Campagne di scavo 2012-2013. Notiziario di Preistoria e Protostoria 2014, 1, 104-105.

65. Giannitrapani, E.; Iannì, F. La tarda età del Rame nella Sicilia centrale. In Proceedings of the Atti della XLIII Riunione Scientifica dell'I.I.P.P., Bologna, Italy, 26-29 Novembre 2008; pp. 271-278.

66. Giannitrapani, E. Dalla capanna alla casa. L'architettura domestica nella preistoria nella Sicilia centrale. In Mito e Archeologia degli Erei. Museo Diffuso Ennese: Itinerary Archeologici; Bonanno, C., Valbruzzi, F., Eds.; 2012; pp. 69-75. Available online: https://www.academia.edu/7596010/Mito_e_Archeologia_degli_Erei. _Museo_Diffuso_Ennese_-_Itinerari_Archeologici_2012_(accessed on 12 March 2018).

67. Iannì, F. L'Eneolitico nella media valle del Salso. In Dai Ciclopi agli Ecisti: Società e Territorio Nella Sicilia Preistorica e Protostorica; Istituto Italiano di Preistoria e Protostoria: Firenze, Italy, 2012; pp. 1141-1143.

68. Fragnoli, P.; Manin, A.L.; Giannitrapani, E.; Iannì, F.; Levi, S.T. Indagine archeometrica sulla tecnologia produttiva e la composizione della ceramica preistorica e protostorica di Tornambè (EN). In Proceedings of the Atti del VII Congresso Nazionale di Archeometria, Modena, Italy, 22-24 Febbraio 2012; pp. 137-149.

69. Giannitrapani, E.; Iannì, F.; Pirrera, G. Indagini archeologiche e interventi di restauro del paesaggio antico nell'insediamento di età greca arcaica di Rocche a Pietraperzia (EN). Geologia dell'Ambiente 2015, 2, 64-77.

70. Napoli, G.; Giuffrida, S.; Trovato, M.R. A Paradigm Interpreting the City and the Analytic Network Process for the Management of Urban Transformations; ISTH 2018; Bevilacqua, C., Calabrò, F., Della Spina, L., Eds.; Springer Science and Business Media Deutschland GmbH: Cham, Switzerland, 2019; Volume 100, pp. 672-680. [CrossRef]

71. Evans, K.; Fielding, L. Giza (Egypt): The use of GIs in managing a World Heritage Site, Visitor Management; Taylor and Francis Inc.: Abingdon-on-Thames, UK, 2009; pp. 82-99.

72. Aronoff, S. Geographic Information Systems: A Management Perspective; WDL Publications: Ottawa, ON, Canada, 1989; pp. 1-29.

73. Van Orshoven, J.; Kint, V.; Wijffels, A.; Estrella, R.; Bencsik, G.; Vanegas, P.; Muys, B.; Cattrysse, D.; Dondeyne, S. Upgrading geographic information systems to spatial decision support systems. Math. Comput. For. Nat. Resour. Sci. 2011, 3, 36-41.

74. De Meyer, A.; Estrella, R.; Jacxsens, P.; Deckers, J.; Van Rompaey, A.; Van Orshoven, J. A conceptual framework and its software implementation to generate spatial decision support systems for land use planning. Land Use Policy 2013, 35, 271-282. [CrossRef]

75. Bica, I.; Tache, A.; Popescu, O.; Topoleanu, F.; Juganaru, G.; Tocanie, P.; Manole, S.D. Integrated Geospatial Type for Location and Protection of Archaeological Sites; Development Pilot Area: County Tulcea, Romania, 2008; pp. 361-366.

76. Brovelli, M.A.; Magni, D. An Archaeological Web Gis Application Based on Mapserver and Postgis; International Archives of the Photogrammetry, Remote Sensing and Spatial Information Sciences-ISPRS Archives; Fangi, F., Malinverni, E.S., Eds.; IPSR: Portonovo, Ancona, Italy, 2003; Volume 34, pp. 89-94.

77. Pareschi, M.T.; Stefani, G.; Varone, A.; Cavarra, L.; Giannini, F.; Meriggi, A. A geographical information system for the archaeological area of Pompeii. Geol. Soc. London Spéc. Publ. 2000, 171, 143-158. [CrossRef]

78. Makuvaza, S. Aspects of Management Planning for Cultural World Heritage Sites: Principles, Approaches and Practices; Springer International Publishing: Cham, Switzerland, 2017; pp. 1-261. [CrossRef]

79. Clark, K. Values in cultural resource management. In Heritage Values in Contemporary Society; Taylor and Francis: Abingdon-on-Thames, UK, 2017; pp. 89-99.

80. Kountouri, E.; Benissi, C.; Papageorgiou, J. Management plans: A tool for participative decision-making. Internet Archaeol. 2018. [CrossRef] 
81. Benkari, N. Archaeological site of Bat-Oman, management and public perception: Community involvement in archaeological heritage management and planning. J. Cult. Heritage Manag. Sustain. Dev. 2018, 8, $293-308$. [CrossRef]

82. Trovato, M.R.; Nocera, F.; Giuffrida, S. Life-Cycle Assessment and Monetary Measurements for the Carbon Footprint Reduction of Public Buildings. Sustainability 2020, 12, 3460. [CrossRef]

83. Giuffrida, S.; Ventura, V.; Nocera, F.; Trovato, M.R.; Gagliano, F. Technological, axiological and praxeological coordination in the energy-environmental equalization of the strategic old town renovation programs. In Values and functions for future cities. Green Energy and Technology; Mondini, G., Oppio, A., Stanghellini, S., Bottero, M., Abastante, F., Eds.; Springer: Cham, Switzerland, 2020; pp. 425-446. [CrossRef]

84. RTLP. Available online: http://www.regione.sicilia.it/beniculturali/dirbenicult/bca/ptpr/LineeGuida.pdf (accessed on 3 April 2005).

85. Provincial Landscape Territorial Plan (PTLP) as the Province of Enna Did. Available online: http://www.opendataterritorioenna.it/dati-scaricabili/ambiti-territoriali-del-p-t-p-r-piano-territorialepaesistico-regionale-della-provincia-di-enna/ (accessed on 15 February 2019).

86. Giuffrida, S.; Nocera, F.; Gagliano, F.; Trovato, M.R. Landscape Assessment and Economic Accounting in Wind Farm Programming: Two Cases in Sicily. Land 2018, 7, 120. [CrossRef]

87. Giuffrida, S. The grammar of the house and of the city. Theoretical approaches for generating project. Valori e Valutazioni 2019, 23, 65-75.

88. Giuffrida, S.; Gagliano, F.; Trovato, M.R. Identifying Archaeological Districts. A topology of the Landscape Values. Laborest 2019, 68-74.

89. Napoli, G. Financial Sustainability and Morphogenesis of Urban Transformation Project. In Computational Science and Its Applications_ICCSA 2015; Gervasi, O., Murgante, B., Misra, S., Gavrilova, M., Coutinho Rocha, A.M.A., Torre, C., Taniar, D., Apduhan, B., Eds.; Springer International Publishing: Cham, Switzerland, 2015; Volume 9157, Parte III; pp. 178-193. [CrossRef]

90. Nocera, F.; Giuffrida, S.; Trovato, M.R.; Gagliano, A. Energy and New Economic Approach for Nearly Zero Energy Hotels. Entropy 2019, 21, 639. [CrossRef]

91. Giuffrida, S.; Ferluga, G.; Valenti, A. Capitalisation rates and 'real estate semantic chains': An application of clustering analysis. Int. J. Bus. Intell. Data Min. 2015, 10, 174. [CrossRef]

92. Napoli, G.; Mamì, A.; Barbaro, S.; Lupo, S. Scenarios of climatic resilience, economic feasibility and environmental sustainability for the refurbishment of the early 20th century buildings. In Values and Functions for Future Cities, Green Energy and Technology; Mondini, G., Stanghellini, S., Oppio, A., Bottero, M., Abastante, F., Eds.; Springer International Publishing: Cham, Switzerland, 2019; pp. 89-115. [CrossRef]

93. Valenti, A.; Giuffrida, S.; Linguanti, F. Decision Trees Analysis in a Low Tension Real Estate Market: The Case of Troina (Italy); LNCS 2015, 9157; Gervasi, O., Rocha, A.M.A.C., Murgante, B., Taniar, D., Apduhan, B.O., Gavrilova, M.L., Misra, S., Torre, C., Eds.; Springer: London, UK, 2015; pp. 237-252. [CrossRef]

94. Napoli, G.; Giuffrida, S.; Trovato, M.R.; Valenti, A. Cap rate as the Interpretative Variable of the Urban Real Estate Capital Asset: A Comparison of Different Sub-Market Definitions in Palermo, Italy. Buildings 2017, 7, 80. [CrossRef]

95. Giuffrida, S.; Ventura, V.; Trovato, M.R.; Napoli, G. Axiology of the Historical City and the Cap Rate. The Case of the Old Town of Ragusa Superiore; Valori e Valutazioni 18/2017, E-Flow Dei Tipografia del Genio Civile; 2017; pp. 41-55. Available online: https://siev.org/numero-18-2017/ (accessed on 4 May 2020).

96. Trovato, M.R.; Giuffrida, S. The protection of territory in the perspective of the intergenerational equity. In Integrated evaluation for the management of contemporary cities. Green Energy and Technology; Mondini, G., Fattinnanzi, E., Oppio, A., Bottero, M., Stanghellini, S., Eds.; Springer: Cham, Switherland, 2018; pp. 469-485. [CrossRef]

97. Giuffrida, S.; Gagliano, F.; Trovato, M.R. An Unconceivable Abstraction. The Archaeological Landscape and the Landscape Archaeology of the Erei Mount Chain, Italy. In Proceedings of the 21th IPSAPA/ISPALEM International Scientific Conference: Paradise lost of the Landscape-cultural Mosaic: Attractiveness, Harmony, Atarassia, Venice, Italy, 6-7 July 2017.

98. Trovato, M.R.; Giuffrida, S. The Monetary Measurement of Flood Damage and the Valuation of the Proactive Policies in Sicily. Geosciences 2018, 8, 141. [CrossRef]

99. Giuffrida, S.; Casamassima, G.; Trovato, M.R. Le norme EMAS-ISO nella valutazione della qualità del servizio idrico integrato. Aestimum 2017, 70, 109-134. [CrossRef] 
100. Capelle-Blancard, G.; Monjon, S. Socially Responsible Investing: It Takes More than Words. Available online: https://www.researchgate.net/publication/46455477_Socially_Responsible_Investing_it_Takes_More_ than_Words (accessed on 5 March 2019).

101. Junkus, J.; Berry, T.D. Socially responsible investing: A review of the critical issues. Manag. Finance 2015, 41, 1176-1201. [CrossRef]

102. Maturana, H.; Varela, F. El árbol del Conocimiento; Rolf Behnchke Inscripción: Santiago, Chile, 1984.

103. Rizzo, F. Dalla Rivoluzione Keynesiana Alla Nuova Economia. Dis-Equilibrio, Tras-Informazione e Co-Efficiente di Capitalizzazione; FrancoAngeli: Milano, Italy, 2002.

104. Rizzo, F. Etica dei Valori Economici o Economia dei Valori Etici; FrancoAngeli: Milano, Italy, 2004.

(C) 2020 by the authors. Licensee MDPI, Basel, Switzerland. This article is an open access article distributed under the terms and conditions of the Creative Commons Attribution (CC BY) license (http://creativecommons.org/licenses/by/4.0/). 

Erratum

\title{
Erratum: Forte, F., et al. Valuation of the Vocationality of Cultural Heritage: The Vesuvian Villas. Sustainability 2020, 12, 943
}

\author{
Fabiana Forte ${ }^{1}$, Vincenzo Del Giudice ${ }^{2}$, Pierfrancesco De Paola ${ }^{2, *}$ and Federica Troisi ${ }^{1}$ \\ 1 Department of Architecture and Industrial Design, University of Campania "Luigi Vanvitelli", \\ Via San Lorenzo ad Septimum, 81031 Aversa, Italy; fabiana.forte@unicampania.it (F.F.); \\ federicatroisi@outlook.com (F.T.) \\ 2 Department of Industrial Engineering, University of Naples "Federico II", Piazzale Vincenzo Tecchio 80, \\ 80125 Naples, Italy; vincenzo.delgiudice@unina.it \\ * Correspondence: pierfrancesco.depaola@unina.it; Tel.: +39-081-768-2933
}

Received: 4 March 2020; Accepted: 5 March 2020; Published: 7 March 2020

The authors would like to make the following corrections to the published paper [1]. The changes are as follows:

Replacing the authors' name:

Forte Fabiana ${ }^{1}$, Del Giudice Vincenzo ${ }^{2}$, De Paola Pierfrancesco ${ }^{2, *}$ and Troisi Federica ${ }^{1}$

with

Fabiana Forte ${ }^{1}$, Vincenzo Del Giudice ${ }^{2}$, Pierfrancesco De Paola ${ }^{2, *}$ and Federica Troisi ${ }^{1}$

The authors and the Editorial Office would like to apologize for any inconvenience caused to the readers by these changes. The change does not affect the scientific results. The manuscript will be updated, and the original will remain online on the article webpage.

\section{Reference}

1. Forte, F.; Del Giudice, V.; De Paola, P.; Troisi, F. Valuation of the Vocationality of Cultural Heritage: The Vesuvian Villas. Sustainability 2020, 12, 943. [CrossRef]

(C) 2020 by the authors. Licensee MDPI, Basel, Switzerland. This article is an open access article distributed under the terms and conditions of the Creative Commons Attribution (CC BY) license (http://creativecommons.org/licenses/by/4.0/). 

MDPI

St. Alban-Anlage 66

4052 Basel

Switzerland

Tel. +41616837734

Fax +41 613028918

www.mdpi.com

Sustainability Editorial Office

E-mail: sustainability@mdpi.com

www.mdpi.com/journal/sustainability

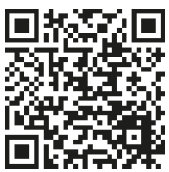



MDPI

St. Alban-Anlage 66

4052 Basel

Switzerland

Tel: +41 616837734

Fax: +41 613028918 